\title{
F-Area Hazardous Waste Management Facility Semiannual Correction Action Report, Vol. I and II
}

by

J. Chase

Westinghouse Savannah River Company

Savannah River Site

Aiken, South Carolina 29808

Exploration Resources

RECEIVED

NOV 291999

OS T I

DOE Contract No. DE-AC09-96SR18500

This paper was prepared in connection with work done under the above contract number with the U.S.

Department of Energy. By acceptance of this paper, the publisher and/or recipient acknowledges the U. S.

Government's right to retain a nonexclusive, royalty-free license in and to any copyright covering this paper, along with the right to reproduce and to authorize others to reproduce all or part of the copyrighted paper. 
WSRC-TR-99-60th

Undasidnta

\section{F-Area Hazardous Waste Management Facility \\ Semiannual Corrective Action Report (U)}

First and Second Quarter 1999

Volume I

October 1999

Westinghouse Savannah River Company, LLC Savannah River Site

Aiken, SC 29808

Prepared for the U.S. Department of Energy under

Contract No. DE-AC09-96SR18500 


\section{DISCLAMMER}

This report was prepared as an account of work sponsored by an agency of the United States Government. Neither the United States Government nor any agency thereof, nor any of their employees, makes any warranty, express or implied, or assumes any legal liability or responsibility for the accuracy, completeness, or usefulness of any information, apparatus, product, or process disclosed, or represents that its use would not infringe privately owned rights. Reference herein to any specific commercial product, process, or service by trade name, trademark, manufacturer, or otherwise does not necessarily constitute or imply its endorsement, recommendation, or favoring by the United States Government or any agency thereof. The views and opinions of authors expressed herein do not necessarily state or reflect those of the United States Government or any agency thereof.

This report has been reproduced directly from the best available copy.

Available to DOE and DOE contractors from the Office of Scientific and Technical Information, P.O. Box 62, Oak Ridge, TN 37831; prices available from (615) 576-8401.

Available to the public from the National Technical Information Service, U.S. Department of Commerce, 5285 Port Royal Road, Springfield, VA 22161.

\section{Printed in the United States of America}

Prepared for

U. S. Department of Energy

and

Westinghouse Savannah River Company, LLC 


\section{DISCLAIMER}

Portions of this document may be illegible in electronic image products. Images are produced from the best available original document. 


\section{EXECUTIVE SUMMARY}

The groundwater in the uppermost aquifer beneath the F-Area Hazardous Waste Management Facility (HWMF) at the Savannah River Site (SRS) is routinely monitored for selected hazardous and radioactive constituents as required by Module III, Section C, of the 1995 Resource Conservation and Recovery Act (RCRA) Renewal Permit (South Carolina Hazardous and Mixed Waste Permit SC1-890-008-989), effective October 5, 1995 (hereafter referred to as the RCRA permit). Currently, the F-Area HWMF monitoring network consists of 86 FSB wells, 9 FSL wells, and $1 \mathrm{HSB}$ well, each of which is screened in one of the three hydrostratigraphic units that make up the uppermost aquifer beneath the F-Area HWMF (Figures 2-5, Appendix B). This report presents the results of the required groundwater monitoring program as identified in provision IIIC.H.11.c of the RCRA permit and Section C of the Underground Injection Control permit application (hereafter referred to as the UIC application).

The following constituents exceeded the Groundwater Protection Standards (GWPS) during the first half of 1999 in samples from monitoring wells at the F-Area HWMF: cadmium, cobalt, gross alpha, lead, mercury, nickel, nitrate-nitrite as nitrogen, nonvolatile beta, sum of alpha, sum of beta, trichloroethylene, trichlorofluoromethane, tritium, and total radium (sum of radium-226 and radium-228). Although vanadium exceeded GWPS in one well, associated field data suggest that this result is not valid.

The following radionuclides exceeded the GWPS for either sum of alpha activity or sum of beta activity: americium-241, carbon-14, cesium-137, curium-243/244, iodine-129, strontium-90, technetium-99, uranium-233/234, uranium-235, and uranium-238. In addition, radium-226 and radium-228 each individually exceeded the standard for total radium.

At least one constituent exceeded GWPS in $73(76 \%)$ of the 96 F-Area wells. Tritium was the primary constituent detected above the GWPS; it exceeded the GWPS in $68(71 \%)$ of the wells. 
Cobalt, gross alpha, nitrate-nitrite as nitrogen, nonvolatile beta, strontium-90, and sum of betas each exceeded GWPS in 30 or more wells.

Constituents exceeding the GWPS were found primarily in Upper Aquifer Zone of the Upper Three Runs Aquifer (UAZ of the UTRA) and Lower Aquifer Zone of the Upper Three Runs Aquifer (LAZ of the UTRA). However, tritium exceeded the GWPS in 11 of the wells in the Gordon Aquifer (GA). Isoconcentration maps included in Volume II of this report indicate both the concentration or activity and the extent of the contamination during the first half of 1999 in each of the three hydrostratigraphic units.

Groundwater flow directions have remained relatively constant since the basins were closed in 1991. Groundwater flow in the UAZ and LAZ of the UTRA is generally south or southeast toward Fourmile Branch. Flow in the GA is generally northwest.

The estimated maximum groundwater flow rate in the UAZ of the UTRA during first quarter 1999 was approximately $274 \mathrm{ft} /$ year and during second quarter 1999 it was approximately 292 $\mathrm{ft} /$ year. The flow rate estimate in the LAZ of the UTRA ranged from approximately $23 \mathrm{ft} / \mathrm{year}$ during first quarter 1999 to approximately $37 \mathrm{ft}$ /year during second quarter 1999. The flow rate in the GA was estimated at $321 \mathrm{ft} /$ year during first quarter 1999 and $251 \mathrm{ft} /$ year during second quarter 1999.

The operating permit for the F-Area Wastewater Treatment Unit (WTU) was received on April 18, 1997, and shakedown activities began upon receipt of RCRA approval on April 22, 1997. The WTU operated in a shakedown mode through the second quarter of 1999. 


\section{CONTENTS}

VOLUME I

PAGE

EXECUTIVE SUMMARY iii

LIST OF FIGURES AND MAPS vii

LIST OF TABLES. ix

SEMIANNUAL CORRECTIVE ACTION REPORT …................................................... 1

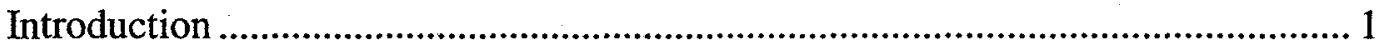

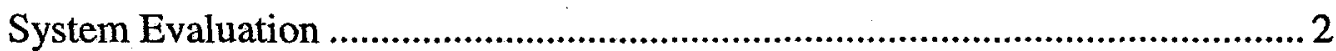

Water Quality and Elevation Data Tables (IIC.H.11.b.i)....................................... 2

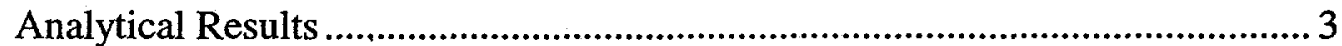

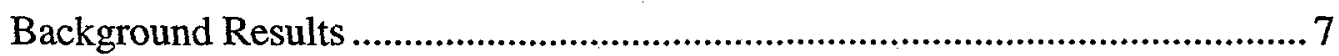

Appendix IX Analyses (Third Quarter 1998) …………................................... 7

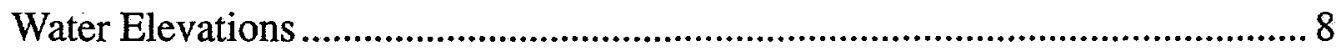

Hydrographs (IIIC.H.11.b.ii) ........................................................................... 8

Time vs. Concentration Plots (IIIC.H.11.b.iii) ........................................................... 8

Isoconcentration Maps (IIIC.H.11.b.iv)................................................................. 9

Potentiometric Maps (IIIC.H.11.b.v)................................................................ 10

Volume and Rates of Groundwater Pumped (IIIC.H.11.b.vi) ................................ 11

Volume and Rates of Groundwater Injected (IIIC.H.11.b.vii)................................ 12

Rainfall and Recharge (IIIC.H.11.b.viii) ............................................................ 13

Summary of System Downtime (IIIC.H.11.b.ix).................................................. 14

Minor Modifications to the System (IIIC.H.11.b.x) ……....................................... 16

Nature and Extent of Contamination.................................................................. 17

Upper Aquifer Zone of the Upper Three Runs Aquifer..................................... 17

Lower Aquifer Zone of the Upper Three Runs Aquifer .................................... 21

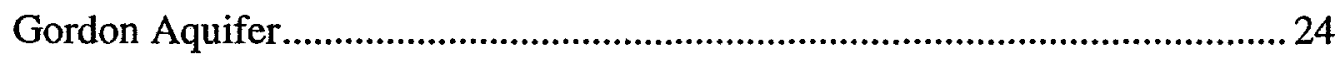

Underground Injection Control (UIC) Sampling Results........................................25

Tritium Activities at Selected Locations in Fourmile Branch ...............................26 
APPENDIX A-GROUNDWATER PROTECTION STANDARD A-1

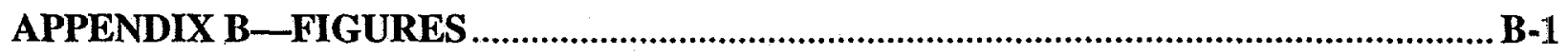

APPENDIX C_GROUNDWATER MONITORING RESULTS …......................................

Key to Reading the Tables ..........................................................................................

Data Qualification .................................................................................................. C-3

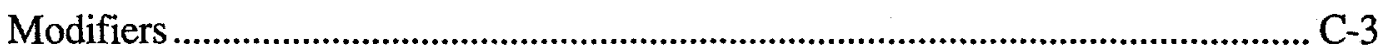

Results Below Detection...................................................................................... C-5

Data Filtering ........................................................................................................ C-5

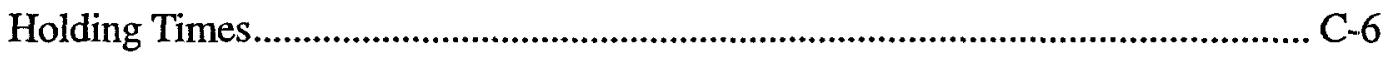

Sampling Dates ....................................................................................................... C-6

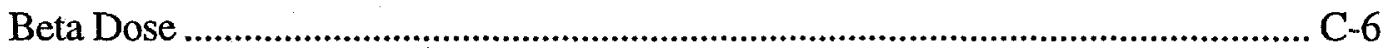

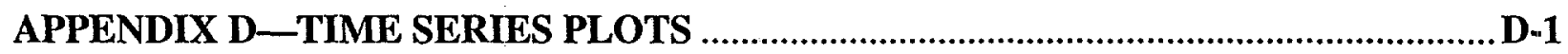

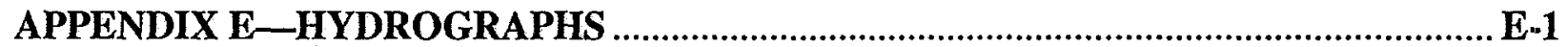

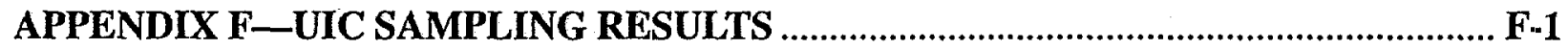

APPENDIX G-OPERATIONAL DOWNTIME .............................................................

VOLUME II

APPENDIX H-GENERAL SEPARATIONS AREA MAPS FOR THE F-

AND H-AREA HAZARDOUS WASTE MANAGEMENT FACILITIES

SEMIANNUAL CORRECTIVE ACTION REPORTS 


\section{LIST OF FIGURES AND MAPS}

\section{VOLUME I, APPENDIX B}

\section{FIGURES}

PAGE

1. Location of the F-Area HWMF at the Savannah River Site B-3

2. Location of the Groundwater Monitoring Wells at the F-Area HWMF B-5

3. Location of Monitoring Wells in the UAZ of the UTRA at the F-Area HWMF

4. Location of Monitoring Wells in the LAZ of the UTRA at the F-Area HWMF

5. Location of Monitoring Wells in the GA at the F-Area HWMF. B-11

6. Fourmile Branch Surface Water Sampling Locations B-13

7. Tritium Activity at Fourmile Branch B-15

\section{VOLUME II, APPENDIX H}

MAPS

PAGE

1. Cadmium Concentrations at the General Separations Area, Second Quarter 1999..............H-3

2. Gross Alpha Activities at the General Separations Area, Second Quarter 1999 ...................H-4

3. Iodine-129 Activities in at the General Separations Area, First Quarter 1999 ......................H-5

4. Lead Concentrations at the General Separations Area, Second Quarter 1999.......................H-6

5. Mercury Concentrations at the General Separations Area, Second Quarter 1999................H-7

6. Nitrate Concentrations at the General Separations Area, Second Quarter 1999 ...................H-8

7. Nonvolatile Beta Activities at the General Separations Area, Second Quarter 1999 ............H-9

8. Strontium-90 Activities at the General Separations Area, First Quarter 1999 $\mathrm{H}-10$ 
9. Technetium-99 Activities at the General Separations Area, First Quarter 1999 H-11

10. Tritium Activities at the General Separations Area, Second Quarter 1999 . $\mathrm{H}-12$

11. Uranium-233/234 Activities at the General Separations Area, First Quarter 1999 H-13

12. Uranium-238 Activities at the General Separations Area, First Quarter 1999 $\mathrm{H}-14$

13. Potentiometric Surface Map of UAZ of the UTRA at the General Separations Area, First Quarter 1999 $\mathrm{H}-15$

14. Potentiometric Surface Map of UAZ of the UTRA at the General Separations Area, Second Quarter 1999 H-16

15. Potentiometric Surface Map of LAZ of the UTRA at the General Separations Area, First Quarter 1999 $\mathrm{H}-17$

16. Potentiometric Surface Map of LAZ of the UTRA at the General Separations Area, Second Quarter 1999 H-18

17. Potentiometric Surface Map of GA at General Separations Area, First Quarter 1999...... H-19

18. Potentiometric Surface Map of GA at General Separations Area, Second Quarter 1999.. H-20 


\section{LIST OF TABLES}

$\underline{\text { IN-TEXT }}$

PAGE

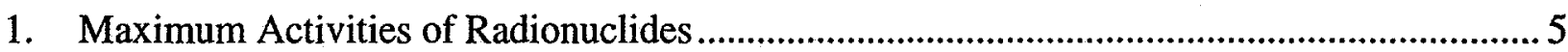

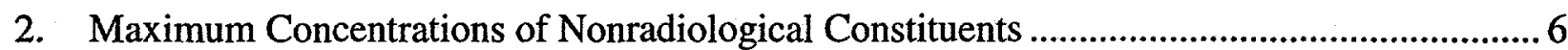

3. Horizontal Groundwater Velocities in the Hydrostratigraphic Units beneath the F-Area HWMF 11

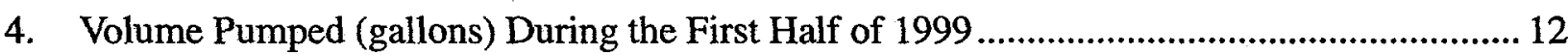

5. Volume Injected (gallons) During the First Half of 1999 ....................................................... 13

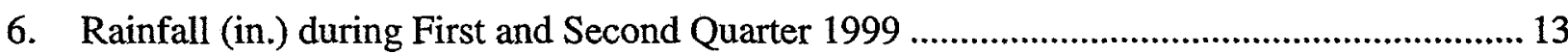

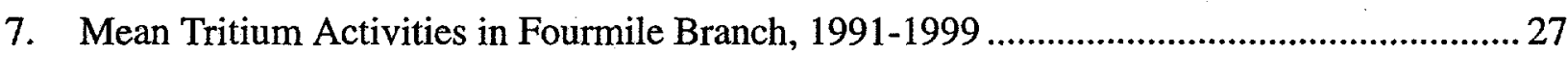

APPENDIX A

PAGE

A-1. Groundwater Protection Standard

A-1

APPENDIX C

PAGE

Activity to Dose Conversion Factors for F-Area HWMF Beta Emitters ..................................... C-7

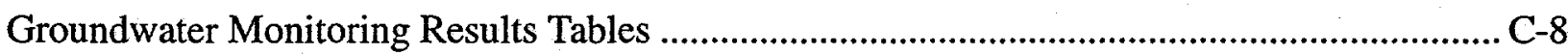

APPENDIX F

PAGE

F-Area WTU Sampling Results: January-June 1999 .............................................................F-2

F-Area WTU Sampling Results: January 1999 ........................................................................F-4

F-Area WTU Sampling Results: February 1999 _...................................................................5-5

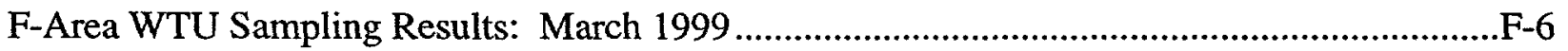




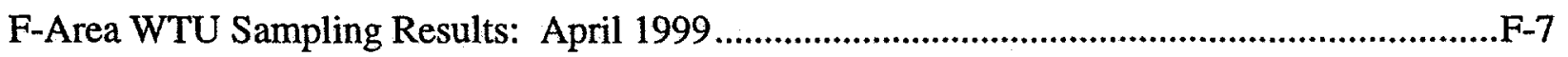

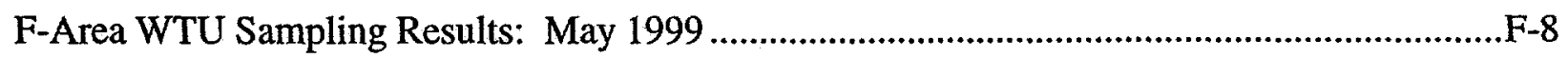

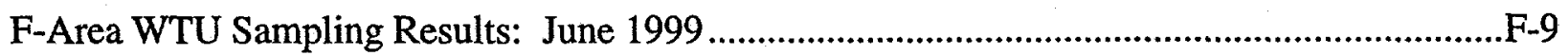

\section{APPENDIX G}

PAGE:

Operational Downtime $\mathrm{G}-1$ 


\section{SEMIANNUAL CORRECTIVE ACTION REPORT}

\section{Introduction}

This report addresses groundwater quality and monitoring data during First and Second Quarter 1999 for the F-Area Hazardous Waste Management Facility (HWMF). The report fulfills the semiannual reporting requirements of Module III, Section C, of the 1995 Resource Conservation and Recovery Act (RCRA) Renewal Permit (South Carolina Hazardous and Mixed Waste Permit SC1-890-008-989), effective October 5, 1995 (hereafter referred to as the RCRA permit), and Section $\mathrm{C}$ of the Underground Injection Control Permit Application (hereafter referred to as the UIC permit). The F-Area HWMF is described in the introduction to Module III, Section C, of the RCRA permit.

Savannah River Site (SRS) monitors groundwater quality quarterly at the F-Area HWMF and provides results of this monitoring to the South Carolina Department of Health and Environmental Control (SCDHEC) semiannually. Contaminant concentrations in the groundwater are compared to the GWPS list provided in the RCRA permit. SRS samples effluent from the Wastewater Treatment Unit (WTU) monthly in accordance with the UIC permit.

The F-Area HWMF well network monitors three distinct hydrostratigraphic units in the uppermost aquifer beneath the facility. The hydrostratigraphy at the F-Area HWMF is described in section IIC.H.2 of the RCRA permit. The groundwater monitoring system is described in section IIIC.H.4 and Appendix IIIC-B of the RCRA permit. A detailed description of the uppermost aquifer is included in the 1992 RCRA Part B Renewal Application for the F-Area HWMF. 


\section{System Evaluation}

The F-Area HWMF operated in a shakedown mode during the First and Second Quarter 1999. Approximately 12.7 million gallons were treated and injected. During the first half of 1999 , significant time was dedicated to modification of the Wastewater Treatment Unit (WTU).

In the first half of 1999, no changes in historical contaminant trends and/or levels were observed, which is expected because of the limited operation of the WTU. The most evident event in the hydrographs is the large recharge to the shallow aquifer systems that occurred during 1998 . The resulting water elevation increases and decreases are seen in almost every well, with deeper zones showing lesser responses as would be expected (e.g., well cluster FSB112). The water elevations began to decrease from these high levels in late 1998 and have continued to decrease in the first half of 1999.

The seepline in the area of the F-Area HWMF has experienced a significant reduction of water in the past six-months. This condition could be due to the current regional drought and/or the operation of nearby extraction wells of the remediation system. However, the specific cause has not yet been identified. The seepline will continue to be observed.

\section{Water Quality and Elevation Data Tables (IIIC.H.11.b.i)}

Water quality, elevation data, and field parameters for all F-Area monitoring wells during First and Second Quarter 1999 are presented in Appendix C. The data tables also include modifiers that explain laboratory accuracy and precision. Definitions of the abbreviations and the modifiers used in the data as well as descriptions of holding times, data rounding, and data qualification practices are provided in Appendix C. Analytical results are rounded to two significant digits. Constituents that exceeded either the GWPS or EPA-approved holding times are noted by symbols in columns headed ST and H, respectively. 
Variable-speed pumps have been installed in wells with a history of elevated analytical results for metals. Samples from wells with variable-speed pumps can be collected at a slower rate to minimize turbidity, which has been associated with elevated metals levels. The chart below identifies those wells that currently have variable-speed pumps as well as those with single-speed pumps.

\begin{tabular}{|l|l|}
\hline \multicolumn{1}{|c|}{ Variable-Speed Pumps } & \multicolumn{1}{|c|}{ Single-Speed Pumps } \\
\hline FSB 77, 78, 78C, 79, 79C, 88D, 89D, 90D, & All remaining FSB wells and well HSB 85A \\
91D, 92C, 92D, 93D, 94C, 97C, 97D, 98C, & have single-speed pumps \\
98D, 102C, 104D, 105C, 105DR, 106C, & \\
107D, and 110D & \\
FSL 1D, 2D, 3D, 4D, 5D, 6D, 7D, 8D, and & \\
9D & \\
\hline
\end{tabular}

Minor maintenance,problems at several wells, such as broken flowmeters, did not interfere with scheduled sampling during First and Second Quarter 1999. The well maintenance program was effective in that there were no instances of mechanical problems carrying over from one quarter to another. During First and Second Quarter 1999, 100\% of the F-Area monitoring wells were sampled as scheduled.

\section{Analytical Results}

All data received from the contracted laboratories are validated and verified according to EPA guidelines. Analytical results that exceeded the GWPS (Appendix A) during First and Second Quarter 1999 in the F-Area HWMF wells are indicated in Appendix C by a black square in the column headed ST. Due to analytical limitations, curium-244/246 activities are reported as an upper limit of curium-246 activities and uranium-233/234 activities are reported as an upper limit of uranium-234. Curium-246 and uranium-234 are the contaminants of concern, not curium-245 and uranium-233. 
Data received from the laboratories for the following radionuclide analyses were rejected during First Quarter due to analytical difficulties: americium-241 in two wells, carbon-14 in one well, cesium-137 in 11 wells, cobalt-60 in one well, curium-242 in one well, curium-243/244 in two wells, curium-245/246 in 23 wells, iodine-129 in one well, plutonium-238 in 15 wells, plutonium239/240 in two wells, technetium-99 in two wells, thorium-228 in 24 wells, thorium-230 in 18 wells, uranium-233/234 in four wells, and uranium-238 in four wells. Corrective measures have been implemented to reduce the number of rejected values due to analytical difficulties in the future.

SRS began implementing a data filtering process to better evaluate the data collected during third and fourth quarter 1998, after permission to implement the data filtering process was granted by SCDHEC (Taylor to Cook, April 21, 1998). The data tables in Appendix C include both a column for all data collected and a column for filtered data for comparison purposes. For the purposes of regulatory decision making, data that are qualified during validation by either the laboratory or WSRC contracted validators as rejected (R), not detected $(U)$, or estimated $(J)$ are filtered from the data set for purposes of discussion in this report. Appendix $\mathrm{C}$ includes additional information on data filtering.

The following constituents exceeded the GWPS during the first half of 1999 in monitoring wells at the F-Area HWMF: americium-241, carbon-14, cesium-137, curium-243/244, gross alpha, nonvolatile beta, total radium, iodine-129, radium- 226 , radium- 228 , strontium- 90 , sum of alpha, sum of beta, technetium-99, uranium-235, uranium-233/234, uranium-238, tritium, cadmium, cobalt, lead, mercury, nickel, nitrate-nitrite (as nitrogen), trichlorofluoromethane, and trichloroethylene.

The following radionuclides individually exceeded the standard for either sum of alpha or sum of beta: americium-241, curium-243/244, technetium-99, uranium-235, carbon-14, iodine-129, uranium-233/234, uranium-238, cesium-137, and strontium-90. Radium-226 and radium-228 
each individually exceeded the standard for total radium $(5 \mathrm{pCi} / \mathrm{L})$ in one or more wells. Although beta dose was exceeded in 44 wells, the sum of beta was only exceeded in 37 wells. Conversely, beta dose was not exceeded in two wells that did exceed the sum of beta standard.

Table 1 summarizes the maximum activities detected for indicator radionuclides in the F-Area groundwater during First and Second Quarter 1999, and Table 2 summarizes the maximum concentrations detected for other constituents that exceeded the GWPS during First and Second Quarter 1999.

Table 1. Maximum Activities of Radionuclides

\begin{tabular}{|l|c|c|c|c|c|}
\hline \multicolumn{1}{|c|}{ Radionuclide } & Maximum Activity & Quarter & Well & Aquifer Unit & GWPS \\
\hline Tritium & $14,000 \mathrm{pCi} / \mathrm{mL}$ & First & FSB 94C & LAZ of the UTRA & $20 \mathrm{pCi} / \mathrm{mL}$ \\
\hline Gross alpha & $1,400 \mathrm{pCi} / \mathrm{L}$ & First & FSB 119D & UAZ of the UTRA & $15 \mathrm{pCi} / \mathrm{L}$ \\
\hline Nonvolatile beta & $2,400 \mathrm{pCi} / \mathrm{L}$ & Second & FSB 94C & LAZ of the UTRA & $50 \mathrm{pCi} / \mathrm{L}$ \\
\hline Total radium & $140 \mathrm{pCi} / \mathrm{L}$ & First & FSB 95CR & LAZ of the UTRA & $5 \mathrm{pCi} / \mathrm{L}$ \\
\hline Sum of alpha & $1,200 \mathrm{pCi} / \mathrm{L}$ & First & FSB 119D & UAZ of the UTRA & $15 \mathrm{pCi} / \mathrm{L}$ \\
\hline Sum of beta & $1,400 \mathrm{pCi} / \mathrm{L}$ & First & FSB 78 & UAZ of the UTRA & $50 \mathrm{pCi} / \mathrm{L}$ \\
\hline
\end{tabular}


Table 2. Maximum Concentrations of Nonradiological Constituents

\begin{tabular}{|l|c|c|c|c|c|}
\hline \multicolumn{1}{|c|}{ Constituent } & $\begin{array}{c}\text { Maximum } \\
\text { Concentration }\end{array}$ & Quarter & Well & Aquifer Unit & GWPS \\
\hline Cadmium & $48 \mu \mathrm{g} / \mathrm{L}$ & First & FSB 112C & LAZ of the UTRA & $5 \mu \mathrm{g} / \mathrm{L}$ \\
\hline Cobalt & $630 \mu \mathrm{g} / \mathrm{L}$ & First & FSB 94C & LAZ of the UTRA & $3 \mu \mathrm{g} / \mathrm{L}$ \\
\hline Lead & $68 \mu \mathrm{g} / \mathrm{L}$ & Second & FSL 1D & UAZ of the UTRA & $15 \mu \mathrm{g} / \mathrm{L}$ \\
\hline Mercury & $17 \mu \mathrm{g} / \mathrm{L}$ & First & FSB 90D & UAZ of the UTRA & $2 \mu \mathrm{g} / \mathrm{L}$ \\
\hline Nickel & $170 \mu \mathrm{g} / \mathrm{L}$ & First & FSB 94C & LAZ of the UTRA & $100 \mu \mathrm{g} / \mathrm{L}$ \\
\hline $\begin{array}{l}\text { Nitrate/nitrite as } \\
\text { nitrogen }\end{array}$ & $320,000 \mu \mathrm{g} / \mathrm{L}$ & First and & FSB 94C & LAZ of the UTRA & $10,000 \mu \mathrm{g} / \mathrm{L}$ \\
\hline Trichloro- & Second & & & \\
fluoromethane & $7.2 \mu \mathrm{g} / \mathrm{L}$ & First & FSB 107C & LAZ of the UTRA & $5 \mu \mathrm{g} / \mathrm{L}$ \\
\hline Trichloroethylene & $76 \mu \mathrm{g} / \mathrm{L}$ & First & FSB 99C & LAZ of the UTRA & $5 \mu \mathrm{g} / \mathrm{L}$ \\
\hline
\end{tabular}

Tritium is the most widespread contaminant and exceeds the GWPS in all three aquifer zones (maps 28-30). The highest activities are downgradient of the seepage basins in UAZ and LAZ of the UTRA. Tritium is also present in the UAZ of the UTRA along the inactive process sewer line to the basins. Tritium exceeded GWPS in 7 GA wells during First Quarter 1999 (FSB-97A, 98AR, 99A, 100A, 112A, 113A, and 120A) and eight GA wells during Second Quarter 1999 (FSB- 96AR, 97A, 98AR, 99A, 100A, 112A, 113A, and 120A).

The extent of the nitrate plume, maps $16-18$, is very similar to the extent of the tritium plume in the UAZ and LAZ of the UTRA. The cadmium, gross alpha, and nonvolatile beta plumes (maps 1-F6 and 19-F21) in the UAZ and LAZ of the UTRA are also similar in shape but are less extensive than the tritium plume. 
Individual radionuclide plume maps for the UAZ and LAZ of the UTRA between the basins and Fourmile Branch are provided in maps 7-9, 22-24, and 31-36. These include technetium-99, strontium-90, uranium-234, and uranium-238.

Lead is elevated in several UAZ and LAZ wells (maps 10-12) near the basins. There is no clear pattern to the distribution of elevated lead.

\section{Background Results}

Wells FSB 76C and 108D and well HSB 85A are the background wells for the F-Area HWMF. These wells are screened in the LAZ of the UTRA, UAZ of the UTRA, and GA, respectively. The only analysis from the background wells to exceed GWPS during the first half of 1999 was nonvolatile beta in HSB 85A during Second Quarter. This result is not considered valid because it was extremely high compared to historical data from HSB 85A, and a second sample result on the same date was below detection.

\section{Appendix IX Analyses (Third Quarter 1998)}

Appendix IX analyses were performed during third quarter 1998, as required by the RCRA permit. Beryllium was detected in wells FSB 94C and FSB 95CR. Confirmation sampling was performed in January 1999, and the analytical results indicated that beryllium was present. Consequently, a modification to the 1992 RCRA Part B Renewal Application was submitted to SCDHEC on August 1, 1999, to add beryllium to the quarterly GWPS list with a standard of 4 $\mu \mathrm{g} / \mathrm{L}$. 
In addition, SCDHEC added aluminum to the quarterly groundwater monitoring list in the RCRA permit because aluminum is a suspected toxin in the tree kill area downgradient of the F-Area HWMF. However, aluminum is not on the GWPS list and there is no standard.

\section{Water Elevations}

Synchronous water-level elevations were measured on March 22 and 23 during First Quarter 1999 and on June 18 through 28 during Second Quarter 1999 in compliance with permit condition IIIC.H.7. Synchronous water levels could not be obtained from well FSB 90D during First Quarter and FSB 87D, FSB 90D, and FSB106D during Second Quarter because the wells were dry.

\section{Hydrographs (IIIC.H.11.b.ii)}

Hydrographs showing the water elevations for the F-Area HWMF wells are provided in Appendix E . Hydrograph data include both synchronous measurements and measurements taken during sampling. Clustered wells are shown on a single graph.

\section{Time vs. Concentration Plots (IIC.H.11.b.iii)}

Appendix D contains time series plots for cadmium, lead, nitrate (or nitrate/nitrite), gross alpha, nonvolatile beta, strontium-90, technetium-99, tritium, uranium-233/234, and uranium-238 for selected wells. These wells were selected to delineate the extent of the constituent distribution, to monitor the migration of the constituents, and to follow trends of constituents present at high levels. Rejected data and results below the detection limit do not appear on the plots. Beginning Third Quarter 1998, estimated (J-qualified) values do not appear on the plots. Only unfiltered, consecutive data points are connected. 
There is one minor revision to the Time vs. Concentration Plots in this report. The technetium-99 plots now begin in First Quarter 1994 to eliminate scaling problems from the anomalous 1993 data. There was also an analytical method change for technetium-99 in 1994, and the subsequent results are considered more reliable than the earlier results.

\section{Isoconcentration Maps (IIIC.H.11.b.iv)}

Isoconcentration maps for strontium-90, technetium-99, uranium-233/234, and uranium-238 in the three hydrostratigraphic units during First Quarter 1999 and for cadmium, lead, nitrate (or nitrate-nitrite), gross alpha, nonvolatile beta, and tritium during Second Quarter 1999 are presented in Appendix $\mathrm{H}$.

The contaminant plume maps have been revised from those provided in previous reports to reflect both physical/chemical changes in the aquifer and changes in interpretation. The most significant changes are due to interpretative methods. As discussed with SCDHEC hydrogeological staff during a previous technical meeting, a matrix has been developed to summarize the historical data through 1996. This baseline, along with current knowledge of contaminant levels and aquifer properties, allows for better interpretation of contaminant extent and migration pathways in two dimensions. A time trend analysis of tritium (a conservative tracer) and the spatial distribution of a representative suite of contaminants were used to interpret pathways of migration.

The contaminant concentrations/activities posted on the plume maps have undergone data filtering as described in Appendix C. The filtered maximum value for a given quarter is posted on the isoconcentration and isoactivity maps. However, SRS has made use of some "NDD" (not decision data) values on a case-by-case basis in accordance with SCDHEC guidance. NDD values that are consistent with surrounding data points have been considered in the positioning of the isoconcentration/isoactivity contours, but the NDD values are not posted on the maps because 
there is a lower level of certainty associated with the NDD points. All of the values (filtered and unfiltered) are provided in the Appendix $\mathrm{C}$ data tables.

\section{Potentiometric Maps (IIIC.H.11.b.v)}

Piezometric and potentiometric surface maps for the monitored water-bearing units during First and Second Quarter 1999 are located in Appendix H. Synchronous water elevations measured within a two-week time span were used to construct the piezometric and potentiometric maps. The maps illustrate groundwater flow patterns beneath the F-Area HWMF for First and Second Quarter 1999.

Groundwater velocity calculations provide a basis for estimates of the transport rate for constituents originating from the F-Area HWMF. Velocities in the LAZ of the UTRA are calculated along two flow paths (designated flow paths $\mathrm{A}$ and $\mathrm{B}$ ) to characterize the approximate maximum and minimum groundwater velocities within these units in areas downgradient from the basins. The maximum value has been reported. The velocity in the GA is calculated along a single flow path because the velocity within this unit is generally more uniform than in the overlying units.

Groundwater velocities are estimated using the following equation:

$$
\text { Velocity (ft/day) }=\frac{\text { Hydraulic Conductivity }(\mathrm{ft} / \text { day) }}{\text { Porosity (unitless) }} \times \frac{d h(\mathrm{ft})}{d l(\mathrm{ft})}
$$

The value $d h$ is the difference in head, and $d l$ is the length of the flow path. Flow path length is calculated to the nearest $50 \mathrm{ft}$. Velocity estimates vary depending on the vertical gradient between wells, the size of the area under consideration, the number of data points, and the length and location of the flow path. Because these calculations are based on inferred or estimated parameters, velocity estimates are only accurate to an order of magnitude. 
Horizontal flow rate estimates for the hydrostratigraphic units during First and Second Quarter are provided in Table 3.

Table 3. Horizontal Groundwater Velocities in the Hydrostratigraphic Units beneath the FArea HWMF

\begin{tabular}{|l|c|c|c|}
\hline & UAZ of the UTRA & LAZ of the UTRA & GA \\
\hline $\mathrm{K}_{\mathrm{h}}$ (ft/day) & 25 & 2 & 40 \\
\hline Effective porosity & 0.20 & 0.20 & 0.25 \\
\hline$d h / d l$ & Varies & Varies & Varies \\
\hline Groundwater velocity (ft/yr) (1099) & 274 & 23 & 321 \\
\hline Groundwater velocity (ft/yr) (2Q99) & 292 & 37 & 251 \\
\hline
\end{tabular}

Flow in the UAZ of the UTRA was south or southeast along both paths during First and Second Quarter 1999. Flow in the LAZ of the UTRA was southeast during both quarters. Flow in the GA was northwest.

\section{Volume and Rates of Groundwater Pumped (IIIC.H.11.b.vi)}

Table 4 summarizes the average pump rates, monthly volumes, and total 6-month volumes pumped from each extraction well associated with the F-Area WTU during First and Second Quarter 1999. 
Table 4. Volume Pumped (gallons) During the First Half of 1999

\begin{tabular}{|l|l|l|l|l|l|l|l|l|}
\hline Well & $\begin{array}{l}\text { Avg. Pump } \\
\text { Rate* (gpm) }\end{array}$ & $\begin{array}{l}\text { January } \\
1999\end{array}$ & $\begin{array}{l}\text { February } \\
1999\end{array}$ & $\begin{array}{l}\text { March } \\
1999\end{array}$ & $\begin{array}{l}\text { April } \\
1999\end{array}$ & May 1999 & $\begin{array}{l}\text { June } \\
1999\end{array}$ & $\begin{array}{l}\text { 6-Month } \\
\text { Total }\end{array}$ \\
\hline FEX 1 & 18.02 & 118,431 & 67,735 & 145,251 & 581,538 & 623,312 & 224,664 & $1,760,931$ \\
\hline FEX 2 & 18.57 & 138,816 & 90,985 & 143,190 & 584,193 & 615,001 & 126,664 & $1,698,849$ \\
\hline FEX 3 & 21.74 & 142,568 & 58,745 & 147,311 & 796,627 & 694,569 & 126,975 & $1,966,795$ \\
\hline FEX 5 & 5.91 & 122,308 & 79,825 & 133,912 & 791,316 & 811,692 & 179,896 & $2,118,949$ \\
\hline FEX 6 & 16.83 & 68,783 & 66,495 & 55,628 & 154,014 & 166,217 & 102,423 & 613,560 \\
\hline FEX 7 & 16.09 & 105,040 & 66,805 & 67,990 & 586,849 & 534,663 & 74,218 & $1,435,565$ \\
\hline FEX 8 & 7.41 & - & - & 37,085 & 196,702 & 229,163 & 77,757 & 540,707 \\
\hline FEX 9 & 10.77 & 85,791 & 68,956 & 42,236 & 355,826 & 362,906 & 35,284 & 950,999 \\
\hline FEX 10 & 0.26 & 3,876 & - & - & - & - & - & 3,876 \\
\hline FEX 11 & 0.38 & 5,627 & - & - & - & - & - & 5,627 \\
\hline Totals & & 842,443 & 573,016 & 912,739 & $4,620,570$ & $4,626,362$ & $1,106,080$ & $12,681,210$ \\
\hline
\end{tabular}

*Estimated due to instrument flow errors. Totals based on recorded well performance and extraction totals.

\section{Volume and Rates of Groundwater Injected (IIIC.H.11.b.vii)}

Table 5 summarizes the pump rates, monthly volumes, and total volumes pumped into each injection well associated with the F-Area WTU during First and Second Quarter 1999. The difference in total volume extracted (Table 4) and total volume injected (Table 5) is due to water retained in the WTU system and instrumentation accuracy of $+/-1 \%$ for all flow meters. 
Table 5. Volume Injected (gallons) During the First Half of 1999

\begin{tabular}{|l|l|l|l|l|l|l|l|l|}
\hline Well & $\begin{array}{l}\text { Avg. Pump } \\
\text { Rate* (gpm) }\end{array}$ & $\begin{array}{l}\text { January } \\
1999\end{array}$ & $\begin{array}{l}\text { February } \\
1999\end{array}$ & $\begin{array}{l}\text { March } \\
1999\end{array}$ & $\begin{array}{l}\text { April } \\
1999\end{array}$ & May 1999 & June 1999 & $\begin{array}{l}\text { 6-Month } \\
\text { Total }\end{array}$ \\
\hline FIN 1 & 24.07 & 103,921 & 77,151 & 139,520 & 864,688 & 807,235 & 224,664 & $2,217,179$ \\
\hline FIN 2 & 15.47 & 94,061 & 49,969 & 97,446 & 543,855 & 525,387 & 126,664 & $1,437,382$ \\
\hline FIN 3 & 15.38 & 95,488 & 47,773 & 95,228 & 516,657 & 547,278 & 126,975 & $1,429,399$ \\
\hline FIN 4 & 22.07 & 117,544 & 76,876 & 132,876 & 734,239 & 809,971 & 179,896 & $2,051,402$ \\
\hline FIN 5 & 14.19 & 98,991 & 55,049 & 94,158 & 511,217 & 456,977 & 102,423 & $1,318,815$ \\
\hline FIN 6 & 19.01 & 106,386 & 73,307 & 139,520 & 614,558 & 695,043 & 158,199 & $1,787,013$ \\
\hline FIN 7 & 11.36 & 83,293 & 64,796 & 90,799 & 359,801 & 383,095 & 74,218 & $1,056,002$ \\
\hline FIN 8 & 10.32 & 82,644 & 50,103 & 53,150 & 369,789 & 325,631 & 77,757 & 959,074 \\
\hline FIN 9 & 5.24 & 65,250 & 80,171 & 55,365 & 122,484 & 128,610 & 35,284 & 487,164 \\
\hline FIN 10 & 0.46 & 6,876 & - & - & - & - & - & 6,876 \\
\hline Totals & & 854,454 & 575,195 & 898,062 & $4,637,288$ & $4,679,227$ & $1,106,080$ & $12,750,306$ \\
\hline
\end{tabular}

*Estimated due to instrument flow errors. Totals based on recorded well performance and injection totals.

\section{Rainfall and Recharge (IIIC.H.11.b.viii)}

Table 6 summarizes the rainfall data for F-Area during First and Second Quarter 1999. Recharge to groundwater is estimated to be $30 \%$ of rainfall in the General Separations Area.

Table 6. Rainfall (in.) during First and Second Quarter 1999

\begin{tabular}{|c|l|l|l|l|l|c|}
\hline January & \multicolumn{1}{|c|}{ February } & March & April & May & June & Total \\
\hline 5.82 & 2.6 & 3.04 & 1.34 & 2.55 & 8.67 & 24.02 \\
\hline
\end{tabular}


Summary of System Downtime (IIIC.H.11.b.ix)

Appendix G summarizes the downtime for First and Second Quarter 1999.

The WTU went down on 1/9/99 due to high differential pressure across the roughing filters. In order to clean out the system and prepare for an extended run, exchange media was changed in the polishing system, sludge was removed from the system, and the flocculation tank and clarifier were cleaned of sludge and polymer. This shutdown extended until 1/18/99.

The WTU was down from $1 / 19 / 99$ to $1 / 28 / 99$ due to problems with the ferric chloride addition system, and problems obtaining a code certified rupture disk on the discharge line. This applied to both facilities.

On 1/29/99, several bolts failed at the sludge pump surge supressor mounting flange. The unit was repaired and returned to service on 2/2/99.

Sludge buildup, excessive sludge generation, and solids carryover to the polishing system continued to be a problem throughout the month of February. The unit was down intermittently for cleaning and/or sludge removal.

The unit was down from $2 / 22 / 99$ through $3 / 19 / 99$ for a scheduled outage. This included sludge pump modifications, grouting of the RO feed skid, air compressor relocation, installation of new chemical mixing tanks, installation of a flocculation tank platform, and lightening protection upgrades to the injection and extraction well systems. Ion exchange media was changed out prior to restart.

During the period from $3 / 20 / 99$ through $3 / 30 / 99$, the WTU was down intermittently in support of a special Process Test Plan conducted to evaluate WTU performance under conditions of reduced 
chemical feed rates. The objective of the test was to determine the contaminant removal effectiveness of the WTU under test conditions, with and without the use of a reroute for RO concentrate flow, while proving the system's capability in maintaining a continuous well system flow rate of 165 GPM.

From $3 / 31 / 99$ to $4 / 6 / 99$ the facility was down to conduct electrical inspections to detect and correct code violations resulting from WSRC subcontractors equipment installation.

From $4 / 7 / 99$ through $4 / 10 / 99$, the WTU was down intermittently due to problems with the sludge transfer pump, PolyBlend assembly, loss of the ferric chloride discharge rupture disk (due to corrosion), and removal of plated material from the process piping and tanks.

Other problems resulting in short term downtime included the following:

- Caustic buildup in the caustic addition feed lines.

- PolyBlend system cleaning and minor repairs.

- Fouling and corrosion in the ferric chloride feed lines.

- Piping lateral failure inside the roughing filters.

- Cleaning or replacement of the filter press screens.

- Poor flocculation quality with excessive solids carryover.

The WTU was down from 6/9/99 through 6/30/99 for a scheduled outage. Modifications performed during this outage will be reported in the next semi-annual report. 


\section{Minor Modifications to the System (IIIC.H.11.b.x)}

The sludge pump configuration for the flocculation tank sludge discharge was modified to reduce pressure drop, minimize bends and restrictions in the piping, improve system air supply, and improve the sludge pump and surge supressor mounting brackets.

The skid for the RO feed pumps was filled with grout to help reduce vibration harmonics in that area. In addition, flexible joints were added to the inlet, discharge and recirculation lines to reduce pump induced vibration in the piping. The pipe supports for the discharge piping were moved from the skid to the pad, and accumulators were added to the pump inlet piping.

The pulleys and sheaves on the RO feed pumps were changed to optimize flow rates for the system.

The two air compressors were relocated to a new pad north of the WTU unit. This was done to reduce noise at the WTU, and to provide room on the pad for other modifications (i.e., chemical mix tanks).

A new ferric chloride addition and mixing tank, and a new caustic addition and mixing tank, were added to the clarification system. These new tank systems included discharge pumps, agitators, and $\mathrm{pH}$ and level control instrumentation. The PLC software was revised to show these new tanks. The new tanks provided improved chemical mixing, greater residence time for chemical reactions, and improved $\mathrm{pH}$ control of the process. The polymer system feed piping was revised and static mixers added to improve injection mixing and chemical residence time.

A new access stairway and platform were fabricated and installed to provide improved and safer access to the top of the flocculation tank. 
A lightning protection upgrade was added to the F-Area instrumentation for the injection and extraction well systems, extraction and injection tanks, and the PLC controllers external to the WTU.

\section{Nature and Extent of Contaminantion}

\section{Contaminant Trends}

Time trends of key contaminants are provided in Appendix D and plume maps of key contaminants are provided in Appendix H. Each map illustrates the contaminant plumes in the UAZ and LAZ of the UTRA and posts concentration/activity values in the GA. Information from all three aquifer zones is combined on a single map for each contaminant to allow easy comparison of contaminant extent in all three aquifer zones. The contour intervals on each map include the PDWS for that contaminant and additional contours to adequately represent the contaminant distribution

\section{Upper Aquifer Zone of the Upper Three Runs Aquifer (UAZ of the UTRA)}

The tritium and nitrates plume maps represent the maximum extent of contamination in the aquifer zones below the HWMF. The primary contaminant migration pathways in the UAZ of the UTRA are clearly visible on the maps. These pathways likely represent areas of higher permeability in the aquifer zone. However, the three-dimensional extent and geological cause of the pathways has not been resolved.

Tritium and nitrate contamination is elevated along the southern portion of the F-Area Inactive Process Sewer Line (FIPSL) and beneath the upper basins and their eastern POC wells. The 
pathways diverge at the southern end of Basin F-3. The first pathway migrates directly to Fourmile Branch through wells FSB-110D, FSB-117D, and FSB-79. Maps of other GWPS contaminants show the highest levels of contamination migrating through this pathway. The second pathway is not as visible in the tritium data due to the natural decline in contaminant levels in this area of the aquifer zone. It shows up as an area of anomalous low activity in FSB$112 \mathrm{D}$ on the current tritium map. However, tritium activities in previous quarters have been elevated in FSB-95DR and FSB-97D, and southwest in FSB-112D, FSB-122D and FSB-104D. The 100 picocuries per liter $(\mathrm{pCi} / \mathrm{L}$ ) isoactivity contour on the Gross Alpha map also illustrates the two pathways.

The tritium and nitrates plumes are the most extensive contaminant plumes, extending from the seepage basins to Fourmile Branch in the UAZ and LAZ of the UTRA. The both plumes extend from the southern area of the FIPSL to the seepline of Fourmile Branch. The $1,000 \mathrm{pCi} / \mathrm{mL}$ tritium contour and $50 \mathrm{mg} / \mathrm{L}$ nitrates contour outline the eastern pathway. The highest tritium activities in First and Second Quarter 1999 were in the POC wells on the eastern and southern sides of Basins F-2 and F-3, and in wells FSB-119D and FSB-79. The highest nitrate concentrations in First and Second Quarter 1999 were in the POC wells on the eastern and southern sides of Basins F-2 and F-3, in wells on the southwestern side of Basin F-3, and in wells FSB-119D, FSB-92D, FSB-78, and FSB-79. Tritium activities and nitrate concentrations have both declined significantly in the UAZ of the UTRA since 1992.

The cadmium plume extends from the northern part of Basin F-2 beneath Basin F-3 and through FSB-119D, FSB-117D, and FSB-79 to the seepline of Fourmile Branch. The $5 \mu \mathrm{g} / \mathrm{L}$ contour outlines the eastern pathway. The highest cadmium concentrations in First and Second Quarter 1999 were in POC wells to the east and southeast of Basin F-3. Cadmium in the UAZ of the UTRA has not changed significantly since 1992, suggesting that cadmium is not highly mobile in the aquifer. 
Evaluation of historical data reveals that lead concentrations are variable across the monitoring well network and are highly dependent on field parameters indicating the presence of particulate contamination (e.g., turbidity and low purge volumes). Since these parameters vary between monitoring wells, reported values are not considered representative of the aquifer as a whole. Lead is best evaluated on a well-by-well basis. During the First Quarter of 1999, lead values in eight FSB (FSB-76, 97D, 98D, 106D, 111D, 115D, 116D, and 121DR) and two FSL (FSL-1D and 2D) wells exceeded the GWPS limit of $15 \mu \mathrm{g} / \mathrm{L}$ in the UAZ of the UTRA. Lead concentrations in the FSL wells and FSB-76 probably originated from the FIPSL. Lead in the remaining wells either originated from the seepage basins or is not representative of the aquifer.

FSB-76 has historically reported lead concentrations in the $30-50 \mu \mathrm{g} / \mathrm{L}$ range at low turbidity levels. FSL-1D has historically ranged from below detect to over $400 \mu \mathrm{g} / \mathrm{L}$, the higher values being associated with elevated dilution factors in the analysis and therefore not considered representative of the aquifer. FSL-4D has historically ranged from below detect to over 1,400 $\mu \mathrm{g} / \mathrm{L}$, the higher values being associated with elevated turbidity and therefore not considered representative of the aquifer.

Lead concentrations in FSB-97D have historically ranged from $18.8 \mu \mathrm{g} / \mathrm{L}$ to $130 \mu \mathrm{g} / \mathrm{L}$. FSB-98D has historically ranged from below detection to $125 \mu \mathrm{g} / \mathrm{L}$, with most results being less than 50 $\mu \mathrm{g} / \mathrm{L}$. Since field parameters for these wells were normal during $1 \mathrm{Q} 99$, the results probably reflect contamination from the facility.

FSB-111D has historically reported lead below detection in many events, the elevated results being associated with low purge volumes. FSB-106D has historically ranged between $9 \mu \mathrm{g} / \mathrm{L}$ and $222 \mu \mathrm{g} / \mathrm{L}$, with most events being associated with low purge volumes and moderately elevated turbidity. FSB-121DR has historically ranged from below detection to over $80 \mu \mathrm{g} / \mathrm{L}$. There is a clear trend of decreasing lead concentrations over the last three years. 
FSB-115D and FSB-116D are located across Fourmile Branch from the HWMF. They are shallow wells and have historically yielded elevated turbidity values in association with low purge volumes. Lead concentrations have varied significantly ranging from less than detect to over $80 \mu \mathrm{g} / \mathrm{L}$ in FSB-115D and from $13.5 \mu \mathrm{g} / \mathrm{L}$ to $94 \mu \mathrm{g} / \mathrm{L}$ in FSB-116D. Samples from these wells are not considered representative of the aquifer. Lead would have to be a more mobile contaminant than tritium to migrate from the HWMF into these wells since tritium activities in these wells are comparable to background tritium activities.

The gross alpha plume extends from the southern portion of the FIPSL to the seepline of Fourmile Branch, similar to tritium and nitrates. The $100 \mathrm{pCi} / \mathrm{L}$ contour outlines both pathways of migration, although the highest levels of gross alpha appear to be migrating through the eastern pathway. The highest gross alpha activities in First and Second Quarter 1999 were in FSB-78, FSB-95DR, FSB-92D and FSB-119D. Gross alpha in the UAZ of the UTRA has not decreased significantly since 1992 . The primary contributors to gross alpha activity in the groundwater are uranium-234 and uranium-238.

The uranium-234 and uranium-238 plumes are similar in configuration to the gross alpha plume, extending from well FSL-9D to the seepline of Fourmile Branch. The $200 \mathrm{pCi} / \mathrm{L}$ contour suggests that the eastern pathway carries most of the uranium-238 contamination. This is consistent with the gross alpha plume, and is also true of uranium-234. The highest uranium-238 activities in First Quarter 1999 are found in FSB-95DR and FSB-119D. The highest uranium-234 activities in First Quarter 1999 are found in FSB-77, FSB-95DR, and FSB-117D.

The nonvolatile beta plume is similar to the tritium and nitrates plumes, extending from FSL-9D to the seepline of Fourmile Branch. The $500 \mathrm{pCi} / \mathrm{L}$ contour outlines the eastern pathway. The highest nonvolatile beta activities in First and Second Quarter 1999 are found in FSB-78, FSB-90D, FSB-92D, FSB-93D, and FSB-119D. Nonvolatile beta in the UAZ of the UTRA continues to decrease at this facility. 
Strontium-90 is the primary contributor to nonvolatile beta activity levels in the groundwater. The strontium-90 plume is similar to the nonvolatile beta plume, and extending from FSL-9D to the seepline of Fourmile Branch. The $100 \mathrm{pCi} / \mathrm{L}$ contour outlines both migration pathways, and the $200 \mathrm{pCi} / \mathrm{L}$ contour shows that most of the strontium-90 contamination is migrating through the eastern pathway. The highest nonvolatile beta activities in First Quarter 1999 are found in FSL-7D, FSB-77, FSB-93D, and FSB-78. Strontium-90 in the UAZ of the UTRA has decreased since 1992.

Technetium-99 does not contribute to nonvolatile beta activity in the groundwater. However, evaluation of groundwater data indicate that technetium-99 is a significant contributor to gross beta activity. The technetium-99 plume is smaller than the nonvolatile beta plume, extending from FSB-90D to the seepline of Fourmile Branch. It is currently restricted to the eastern pathway. The highest technetium-99 activities in First Quarter 1999 are found in FSB-90D and FSB-110D. Historical technetium-99 data were severely distorted by analytical methods in use at that time. Method EPIA-005 has been in use since 1994, and has been producing more consistent results.

Lower Aquifer Zone of the Upper Three Runs Aquifer (LAZ of the UTRA)

The tritium and nitrates plume maps represent the maximum extent of contamination in the LAZ of the UTRA. The primary pathways of contaminant migration in the UAZ of the UTRA are also visible in the LAZ of the UTRA. This could imply either a duplication of relative aquifer permeability in the LAZ of the UTRA, or that the contaminant trends in the UAZ of the UTRA are superimposed on the LAZ of the UTRA via vertical migration through the Tan Clay Confining Zone. The latter explanation currently appears more likely. Historical aquifer testing has demonstrated the lower permeability and subsequent slower migration through the LAZ of the UTRA relative to the UAZ of the UTRA. Contaminant distributions in the LAZ of the UTRA support this conclusion in three ways: (1) the primary pathways of the UAZ appear "imprinted" on the LAZ over time, (2) greater "spread" between similar contour intervals in the LAZ 
suggesting a more significant component of diffusion in transport through the LAZ, and (3) the persistence of elevated levels of contamination in the LAZ.

Tritium and nitrates contamination are most elevated beneath Basin F-3, but extends from the northern end of the upper basins to Fourmile Branch. The concentrations/activities of mobile contaminants (e.g., tritium) are commonly elevated in the LAZ over those in UAZ. Tritium contamination is also more widespread in the LAZ relative to the UAZ based on a comparison of the $20 \mathrm{pCi} / \mathrm{mL}$ tritium activity contour in both aquifer zones.

The tritium plume extends from Basin F-1 to Fourmile Branch. The low tritium activities in FSB-115C and FSB-116C strongly suggest that Fourmile Branch is a hydrologic barrier to contaminant migration in the LAZ as well as in the UAZ. The $5,000 \mathrm{pCi} / \mathrm{mL}$ contour outlines the superimposed pathways from the UAZ. The highest tritium activities in First and Second Quarter 1999 are found in wells on the southern side of Basin F-3, in wells FSB-78, FSB-94C, and FSB-95CR. Tritium in the LAZ of the UTRA has declined significantly since 1992 in the wells north of Basin F-3. Tritium has only declined slightly in the wells south of Basin F-3. Tritium activities in FSB-78, FSB-94CR, and FSB-95CR have not changed significantly. The dramatic decrease in the UAZ in these well clusters demonstrates that continued high tritium activity levels in the LAZ are not based on ongoing vertical migration from the UAZ. The slow migration of tritium through the LAZ provides additional time for decay prior to discharging to Fourmile Branch.

The nitrates plume extends from the southern portion of the FIPSL to Fourmile Branch. The 100 $\mathrm{mg} / \mathrm{L}$ contour outlines the "imprinted" migration pathways of the UAZ. The highest nitrate concentrations in First and Second Quarter 1999 were in wells on the southern side of Basin F-3, in wells FSB-78C, FSB-94C, and FSB-95CR. Nitrates concentrations in the LAZ of the UTRA have not dropped significantly since 1992, and concentrations near Basin F-3 continue to exceed $200 \mathrm{mg} / \mathrm{L}$. 
The cadmium plume extends from the southern end of Basin F-2, beneath Basin F-3, and through the two UAZ migration pathways to Fourmile Branch. The highest cadmium concentrations in First and Second Quarter 1999 are found in wells to south of Basin F-3, FSB-94C, FSB-78C, and FSB-95CR, and in well FSB-112C. Cadmium in the LAZ of the UTRA has not changed significantly since 1992.

Lead concentrations in the LAZ of the UTRA only exceeded GWPS in one well during First and Second Quarter 1999, FSB-97C.

The gross alpha plume extends from the southern end of Basin F-2 to Fourmile Branch, similar to the tritium and nitrates plumes. The $100 \mathrm{pCi} / \mathrm{L}$ contour outlines both $\mathrm{UAZ}$ pathways of migration. The highest gross alpha activities in First and Second Quarter 1999 were in FSB-94C, FSB-95CR, FSB-78C, FSB-79, FSB-98C, and FSB-112C. Gross alpha in the LAZ of the UTRA has not decreased significantly since 1992 . The primary contributors to gross alpha activity levels in this aquifer zone are uranium-234 and uranium-238.

The uranium-234 and uranium-238 plumes are somewhat similar in configuration to the gross alpha plume, but extend from beneath Basin F-3 through the eastern UAZ pathway, through FSB-79C to Fourmile Branch. The $15 \mathrm{pCi} / \mathrm{L}$ contour outline suggests that uranium-238 contamination is restricted to the eastern UAZ pathway. This is consistent with uranium-234 contamination. The highest uranium-238 activities in First Quarter 1999 were in FSB-95CR and FSB-98C. The highest uranium-234 activities in First Quarter 1999 were in FSB-95CR. Uranium-234 and uranium-238 activities have decreased slightly since 1992.

The nonvolatile beta plume is similar to the tritium and nitrates plumes, extending from Basin F-2 to Fourmile Branch. The $1,000 \mathrm{pCi} / \mathrm{L}$ contour outlines the superimposed pathways from the UAZ. The highest nonvolatile beta activities in First and Second Quarter 1999 were in wells on the southern side of Basin F-3, in wells FSB-79, FSB-94C, FSB-95CR, and FSB-112D. 
Strontium-90 is the primary contributor to nonvolatile beta activity in the groundwater. The strontium-90 plume is similar to the nonvolatile beta plume, extending from Basin F-2 to Fourmile Branch. The $500 \mathrm{pCi} / \mathrm{L}$ contour outlines both $\mathrm{UAZ}$ migration pathways. The highest strontium-90 activities in First Quarter 1999 were in FSB-78C, FSB-94C, and FSB-112C. Strontium-90 in the LAZ of the UTRA has not declined significantly since 1992.

Technetium-99 is not a contributor to nonvolatile beta activity levels in the groundwater. However, evaluation of groundwater data indicate that technetium- 99 is a significant contributor to gross beta activity. The technetium-99 plume is similar to the nonvolatile beta plume, and extending from Basin F-3 to Fourmile Branch. The highest technetium-99 activities in 1Q99 are found in FSB-78C, FSB-94C, FSB-95CR, and FSB-112C. Historical (1992) technetium-99 data were severely distorted by analytical methods in use at that time. Method EPIA-005 has been in use since 1994, and has been producing more consistent results.

\section{$\underline{\text { Gordon Aquifer (GA) }}$}

Although contaminant values are posted, contaminant plumes are not illustrated in the GA. Evaluation of historical data indicates that the Green Clay is an effective confining zone relative to many contaminants. Although nitrates and strontium-90 have a couple scattered hits above GWPS, tritium is the only contaminant that is consistently elevated above the GWPS in the GA. Tritium exceeds the GWPS in ten wells, but the distribution is irregular and the source of tritium in this aquifer zone is not currently understood. Lead, gross alpha, and nonvolatile beta do not exceed GWPS in the GA. Cadmium, technetium-99, uranium-234, and uranium-238 are not detected in the GA. 
Underground Injection Control (UIC) Sampling Results

SRS remained in compliance with the F-Area UIC permit during First and Second Quarter 1999. Monthly compliance samples were collected from the F-Area WTU injection tank, and the results are provided in Appendix $\mathrm{F}$ along with other pertinent data.

All of the non-radionuclide constituents were below the UIC permit limits during First and Second Quarter 1999. The detection limit for thallium was slightly greater than the UIC permit limit during the first four months of 1999, but SRS has since implemented a new laboratory method for thallium to achieve an appropriate detection limit. The detection limit for vanadium was greater than the UIC permit limit during April because the laboratory used a dilution factor of 10x. Barium, cobalt, and copper were consistently detected during First and Second Quarter 1999, but at concentrations below the UIC permit limits. No organic constituents were detected in the UIC compliance samples during First and Second Quarter 1999.

Uranium isotopes were the most commonly detected alpha-emitting radionuclides in the F-Area UIC compliance samples during First and Second Quarter 1999. Curium, thorium, and plutonium isotopes were not detected in the compliance samples during First and Second Quarter 1999. Americium-241 was detected in April and radium-226 was detected in June, but both were below the UIC permit limits. Uranium-234 was detected in four samples, uranium-235 was detected in two samples, and uranium-238 was detected in five samples. However, all of the uranium detections were below the UIC permit limits. Uranium-234 and uranium-238 were both elevated over previous months during June, but still below the UIC permit limits.

Iodine-129 was the most commonly detected beta-emitting radionuclide in F-Area. Cesium-137, cobalt-60, and radium-228 were not detected during First or Second Quarter 1999. Carbon-14 was detected in April and June, but below the UIC permit limit. Iodine-129 was present every month, and exceeded the UIC permit limit in January. However, there was no confirmed 
exceedence since the February result was below the UIC permit standard. Strontium-90 was detected in four samples and technetium-99 was detected in three samples, but all were below the UIC permit limits.

\section{Tritium Activities at Selected Locations in Fourmile Branch}

Tritium activities in Fourmile Branch, a tributary of the Savannah River, are measured monthly at the Savannah River Site as part of routine monitoring activities conducted by the Environmental Monitoring Section of Westinghouse Savannah River Company. Four locations have been monitored by the SRS and the U.S. Geological Survey since 1991, and provide a long term record of tritium movement in Fourmile Branch. These include: (1) FM-2 at Road 4, (2) FM-2B above F-Area, (3) FM-3A below F-Area, and (4) FM-A7 at Road A7. These locations are sampled continuously with water samples being collected over a one week period for analysis of tritium activities. The objective of this analysis is to compare mean tritium activities measured in the First and Second Quarter 1999 with those recorded for years 1991 through 1998 in the SRS Environmental Monitoring Reports.

Mean activities and sample size at the four locations from 1991 to 1999 are given in Table 7. Mean tritium activities at FM-2 and Road 4 have declined each year since 1991 (Appendix B, Figure 7). Although the mean tritium activities for the first two quarters of 1999 have increased slightly at locations FM-2B and FM-A7, the general trend has been one of decreasing activities (Appendix B, Figure 7). The mean activity in 1999 also decreased at FM-3A (Appendix B, Figure 7). The general trend since 1991 at these four locations has been declining activity levels. However, it should be noted that the 1999 mean activity is based on a six month period whereas means prior to 1999 are based on a entire year of monitoring. Monitoring of the four locations will continue through 1999. Additionally, USGS and SRS will monitor additional locations on Fourmile Branch to evaluate tritium activities in surface water and its potential effect, if any, on human health and ecological resources. 
Table 7. Mean Tritium Activities (pCi/mL) in Fourmile Branch, 1991-1999.

\begin{tabular}{|c|c|c|c|c|c|c|c|c|}
\hline \multirow{2}{*}{ Year } & \multicolumn{3}{|c|}{ FM-2 at Rd 4 } & \multicolumn{2}{l|}{ FM-2B } & \multicolumn{2}{c|}{ FM-3A } & \multicolumn{2}{c|}{ FM-A7 } \\
\hline & Mean & n & Mean & n & Mean & n & Mean & N \\
\cline { 2 - 9 } & 14.7 & 13 & 60.8 & 13 & 1,189 & 13 & 349 & 13 \\
\hline $\mathbf{1 9 9 8}$ & 15.8 & 26 & 48.7 & 26 & 1,305 & 26 & 319 & 26 \\
\hline $\mathbf{1 9 9 7}$ & 18.8 & 27 & 68.4 & 25 & 1,167 & 26 & 354 & 26 \\
\hline $\mathbf{1 9 9 6}$ & 22.4 & 15 & 90.7 & 27 & 1,589 & 27 & 469 & 27 \\
\hline $\mathbf{1 9 9 5}$ & 22.6 & 25 & 88.4 & 26 & 1,856 & 26 & 452 & 26 \\
\hline $\mathbf{1 9 9 4}$ & 33.0 & 25 & 108.1 & 26 & 2,055 & 26 & 491 & 26 \\
\hline $\mathbf{1 9 9 3}$ & 43.3 & 26 & 127.4 & 26 & 2,052 & 26 & 519 & 26 \\
\hline $\mathbf{1 9 9 2}$ & 105 & 26 & 250.6 & 9 & 2,888 & 9 & 644 & 9 \\
\hline $\mathbf{1 9 9 1}$ & 117 & 27 & 249.9 & 26 & 2,567 & 26 & 652 & 26 \\
\hline
\end{tabular}


This Page was Intentionally Left Blank. 


\section{APPENDIX A: GROUNDWATER PROTECTION STANDARD}

\section{Table A-1. Groundwater Protection Standard}

Established for the F-Area Hazardous Waste Management Facility by the 1995 RCRA Renewal Permit and its revisions.

\begin{tabular}{|c|c|c|}
\hline Analyte & Concentration/Activity Limit & Unit \\
\hline \multicolumn{3}{|l|}{ Inorganic Constituents } \\
\hline Antimony & 6 & $\mu \mathrm{g} / \mathrm{L}$ \\
\hline Arsenic & 50 & $\mu \mathrm{g} / \mathrm{L}$ \\
\hline Barium & 2,000 & $\mu g / L$ \\
\hline Beryllium & 4 & $\mu \mathrm{g} / \mathrm{L}$ \\
\hline Cadmium & 5 & $\mu \mathrm{g} / \mathrm{L}$ \\
\hline Chromium & 100 & $\mu \mathrm{g} / \mathrm{L}$ \\
\hline Cobalt & $3^{\mathrm{a}}$ & $\mu \mathrm{g} / \mathrm{L}$ \\
\hline Copper & 1,300 & $\mu \mathrm{g} / \mathrm{L}$ \\
\hline Cyanide & $20^{\mathrm{b}}$ & $\mu \mathrm{g} / \mathrm{L}$ \\
\hline Lead & 15 & $\mu \mathrm{g} / \mathrm{L}$ \\
\hline Mercury & 2 & $\mu \mathrm{g} / \mathrm{L}$ \\
\hline Nickel & 100 & $\mu \mathrm{g} / \mathrm{L}$ \\
\hline Nitrate & 10,000 & $\mu g / L$ \\
\hline Selenium & 50 & $\mu g / L$ \\
\hline Silver & 50 & $\mu \mathrm{g} / \mathrm{L}$ \\
\hline Thallium & 2 & $\mu \mathrm{g} / \mathrm{L}$ \\
\hline Vanadium & $3.5^{\mathrm{a}}$ & $\mu g / L$ \\
\hline Zinc & 5,000 & $\mu \mathrm{g} / \mathrm{L}$ \\
\hline \multicolumn{3}{|l|}{ Organic Constituents } \\
\hline Benzene & 5 & $\mu g / L$ \\
\hline Bis(2-ethylhexyl) phthalate & $10^{c}$ & $\mu \mathrm{g} / \mathrm{L}$ \\
\hline $\begin{array}{l}\text { Dichloromethane (methylene } \\
\text { chloride) }\end{array}$ & 5 & $\mu g / L$ \\
\hline Phenols & $2^{\mathrm{d}}$ & $\mu g / L$ \\
\hline Tetrachloroethylene & 5 & $\mu g / L$ \\
\hline Trichloroethylene & 5 & $\mu \mathrm{g} / \mathrm{L}$ \\
\hline
\end{tabular}




\begin{tabular}{|c|c|c|}
\hline Analyte & Concentration/Activity Limit & Unit \\
\hline Trichlorofluoromethane & $5^{e}$ & $\mu g / L$ \\
\hline \multicolumn{3}{|l|}{ Radiological Constituents } \\
\hline Gross alpha & 15 & $\mathrm{pCi} / \mathrm{L}$ \\
\hline Nonvolatile beta & 50 & $\mathrm{pCi} / \mathrm{L}$ \\
\hline Tritium & 20,000 & $\mathrm{pCi} / \mathrm{L}$ \\
\hline Americium-241 & Sum of alphas $<15 \mathrm{pCi} / \mathrm{L}$ & $\mathrm{pCi} / \mathrm{L}$ \\
\hline Carbon-14 & Sum of beta dose $<4 \mathrm{mrem} / \mathrm{yr}$ and $<50 \mathrm{pCi} / \mathrm{L}$ & $\mathrm{pCi} / \mathrm{L}$ \\
\hline Cesium-137 & Sum of beta dose $<4 \mathrm{mrem} / \mathrm{yr}$ and $<50 \mathrm{pCi} / \mathrm{L}$ & $\mathrm{pCi} / \mathrm{L}$ \\
\hline Cobalt -60 & Sum of beta dose $<4 \mathrm{mrem} / \mathrm{yr}$ and $<50 \mathrm{pCi} / \mathrm{L}$ & $\mathrm{pCi} / \mathrm{L}$ \\
\hline Curium-242 & Sum of alphas $<15 \mathrm{pCi} / \mathrm{L}$ & $\mathrm{pCi} / \mathrm{L}$ \\
\hline Curium-243/244 & Sum of alphas $<15 \mathrm{pCi} / \mathrm{L}$ & $\mathrm{pCi} / \mathrm{L}$ \\
\hline Curium-245/246 & Sum of alphas $<15 \mathrm{pCi} / \mathrm{L}$ & $\mathrm{pCi} / \mathrm{L}$ \\
\hline Iodine-129 & Sum of beta dose $<4 \mathrm{mrem} / \mathrm{yr}$ and $<50 \mathrm{pCi} / \mathrm{L}$ & $\mathrm{pCi} / \mathrm{L}$ \\
\hline Plutonium-238 & Sum of alphas $<15 \mathrm{pCi} / \mathrm{L}$ & $\mathrm{pCi} / \mathrm{L}$ \\
\hline Plutonium-239/240 & Sum of alphas $<15 \mathrm{pCi} / \mathrm{L}$ & $\mathrm{pCi} / \mathrm{L}$ \\
\hline Radium-226 & Total radium $<5 \mathrm{pCi} / \mathrm{L}$ & $\mathrm{pCi} / \mathrm{L}$ \\
\hline Radium-228 & $\begin{array}{l}\text { Sum of beta dose }<4 \mathrm{mrem} / \mathrm{yr} \text { and }<50 \mathrm{pCi} / \mathrm{L} \text { and } \\
\text { total radium }<5 \mathrm{pCi} / \mathrm{L}\end{array}$ & $\mathrm{pCi} / \mathrm{L}$ \\
\hline Strontium-90 & $\begin{array}{l}\text { Sum of beta dose }<4 \mathrm{mrem} / \mathrm{yr} \text { and }<50 \mathrm{pCi} / \mathrm{L} \text { and } \\
\text { strontium- } 90<8 \mathrm{pCi} / \mathrm{L}\end{array}$ & $\mathrm{pCi} / \mathrm{L}$ \\
\hline Technetium-99 & Sum of beta dose $<4 \mathrm{mrem} / \mathrm{yr}$ and $<50 \mathrm{pCi} / \mathrm{L}$ & $\mathrm{pCi} / \mathrm{L}$ \\
\hline Thorium-228 & Sum of alphas $<15 \mathrm{pCi} / \mathrm{L}$ & $\mathrm{pCi} / \mathrm{L}$ \\
\hline Thorium-230 & Sum of alphas $<15 \mathrm{pCi} / \mathrm{L}$ & $\mathrm{pCi} / \mathrm{L}$ \\
\hline Thorium-232 & Sum of alphas $<15 \mathrm{pCi} / \mathrm{L}$ & $\mathrm{pCi} / \mathrm{L}$ \\
\hline Total radium & 5 (the sum of radium- 226 plus radium- 228 ) & $\mathrm{pCi} / \mathrm{L}$ \\
\hline Uranium-233/234 & Sum of alphas $<15 \mathrm{pCi} / \mathrm{L}$ & $\mathrm{pCi} / \mathrm{L}$ \\
\hline Uranium-234 & Sum of alphas $<15 \mathrm{pCi} / \mathrm{L}$ & $\mathrm{pCi} / \mathrm{L}$ \\
\hline Uranium-235 & Sum of alphas $<15 \mathrm{pCi} / \mathrm{L}$ & $\mathrm{pCi} / \mathrm{L}$ \\
\hline Uranium-238 & Sum of alphas $<15 \mathrm{pCi} / \mathrm{L}$ & $\mathrm{pCi} / \mathrm{L}$ \\
\hline
\end{tabular}

a Concentrations are observed background levels.

b Concentration is the practical quantitation limit (PQL) for EPA Method 335.2 (used by WA) and 335.3 (used by GE).

c Concentration is the Appendix IX PQL for EPA Method 8270.

d Concentration is the PQL for EPA Method 420.1 (used by GE) and 420.2 (used by WA).

c Concentration is the Appendix IX PQL for EPA Method 8240. 
APPENDIX B: FIGURES 
THIS PAGE WAS LEFT BLANK INTENTIONALLY. 


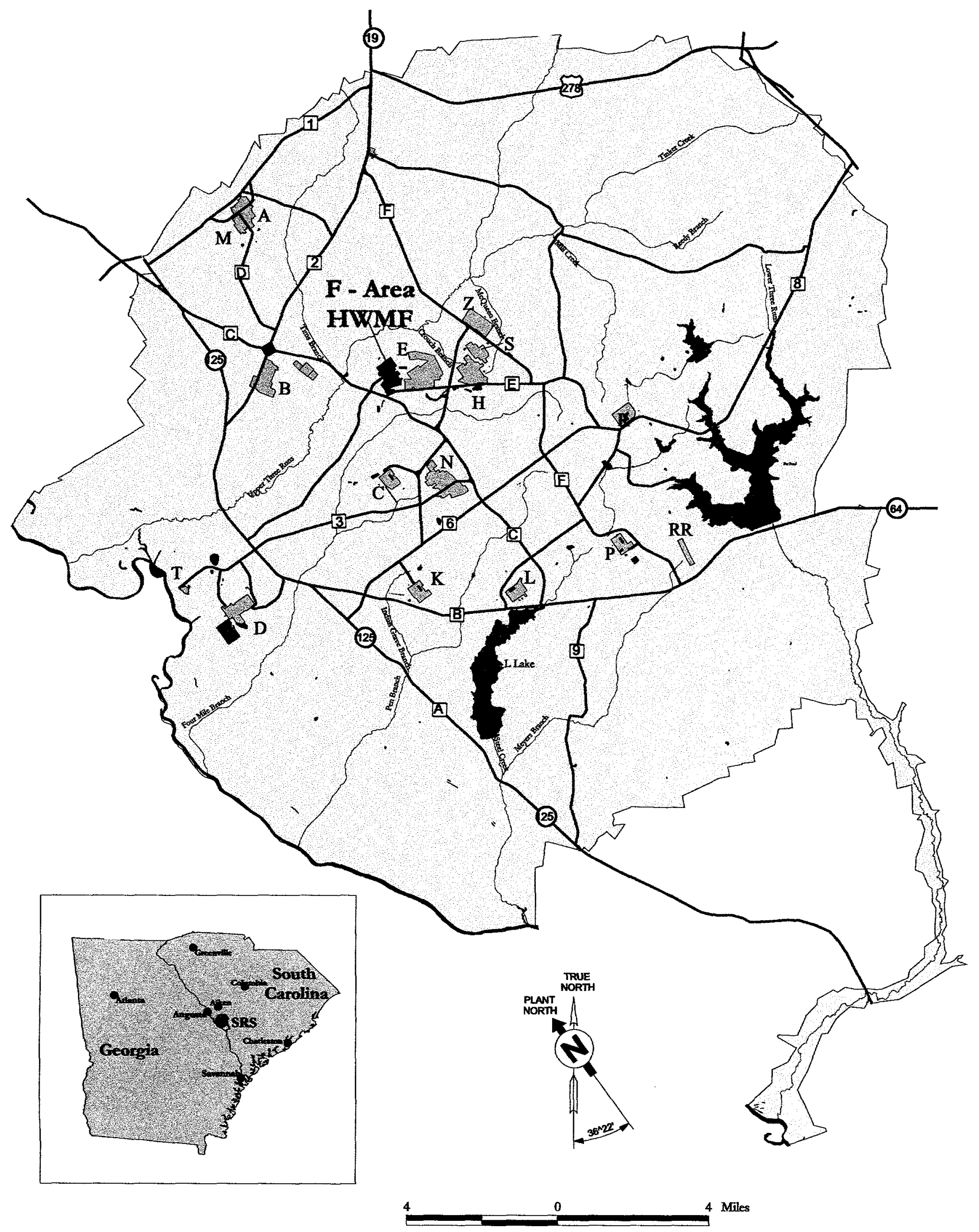

Figure 1. Location of the F-Area HWMF at the Savannah River Site 
THIS PAGE WAS LEFT BLANK INTENTIONALLY. 


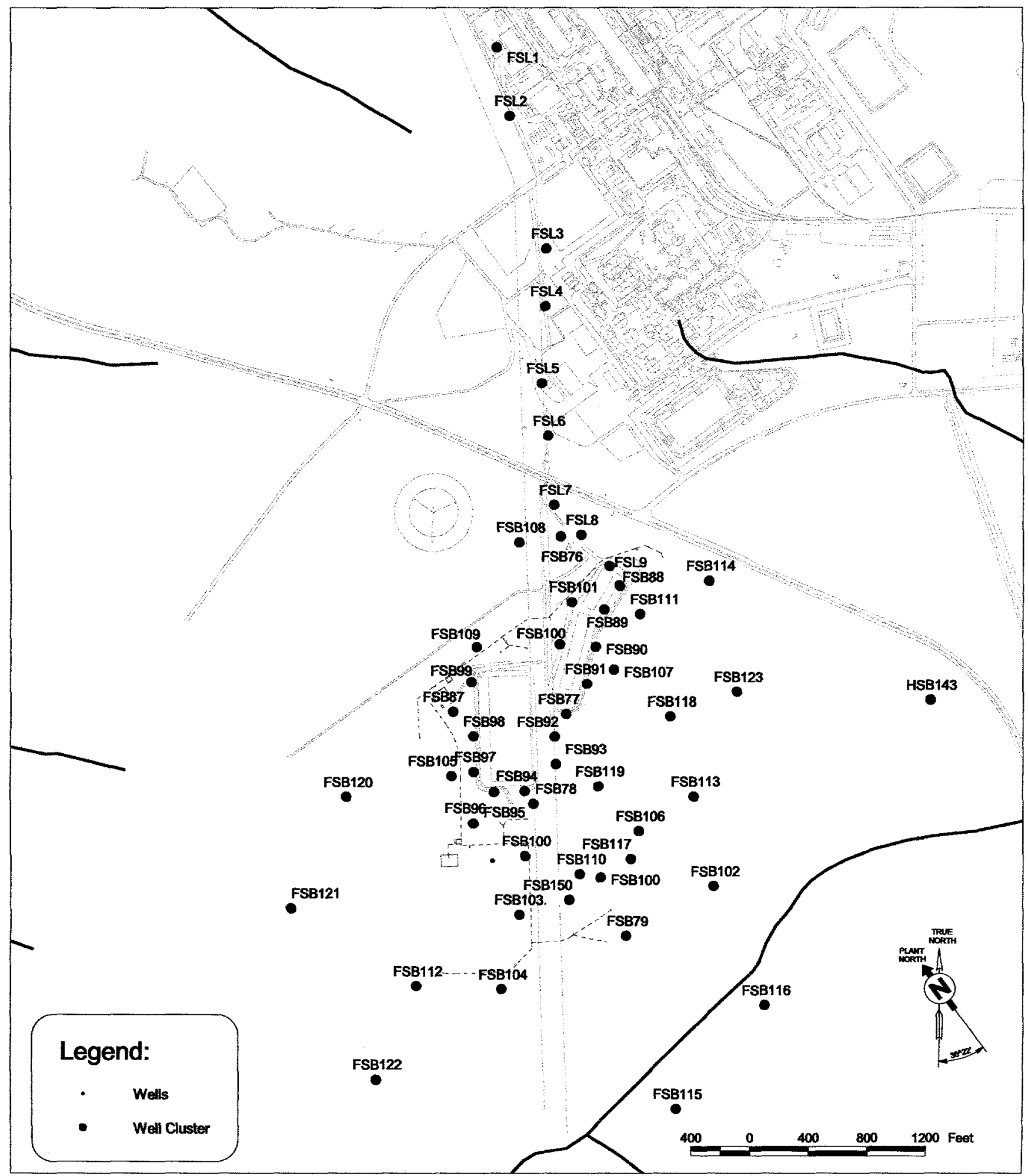

Figure 2. Location of Groundwater Monitoring Wells at the F-Area HWMF 
THIS PAGE WAS LEFT BLANK INTENTIONALLY. 


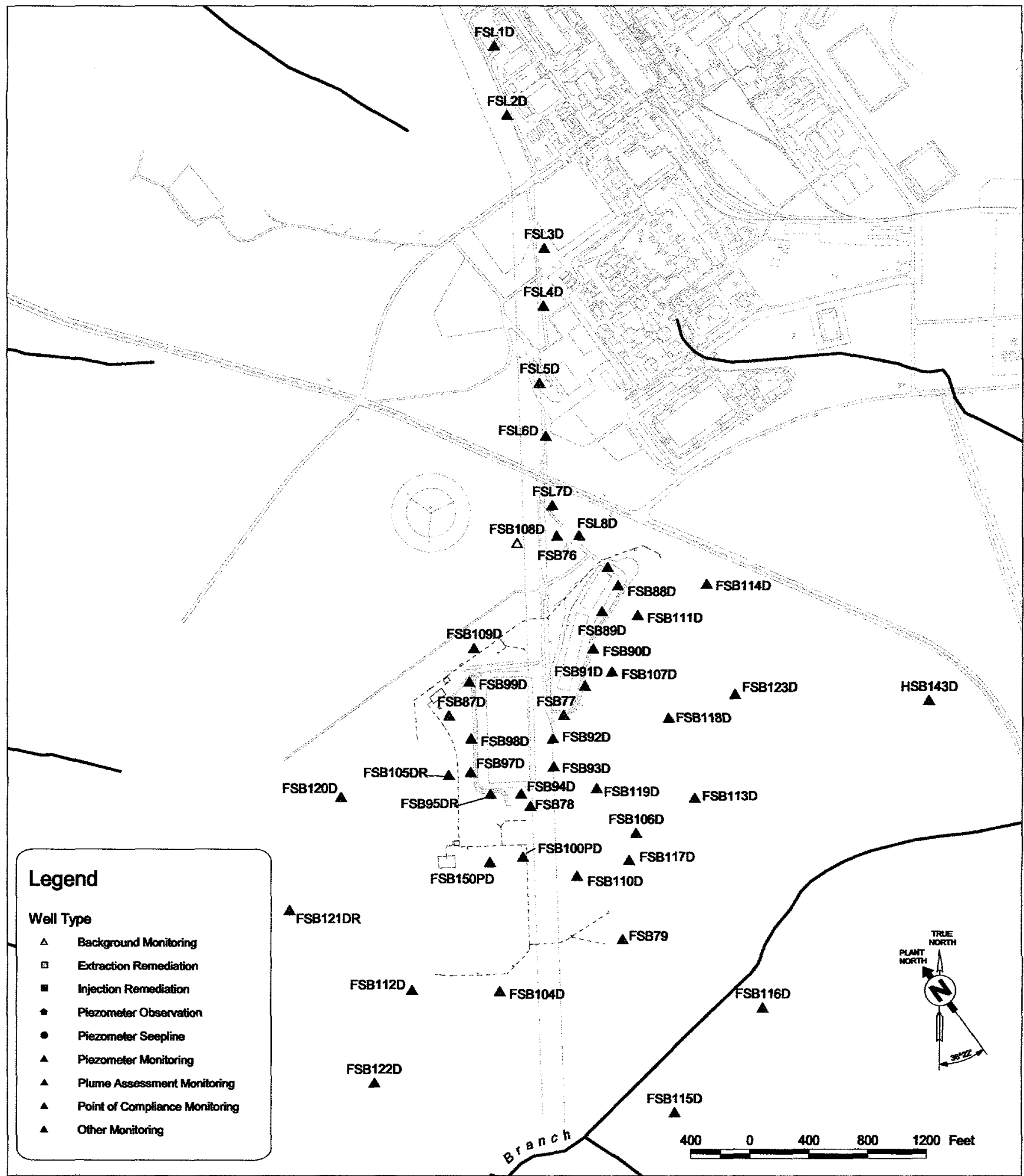

Figure 3. Location of Groundwater Monitoring Wells in the UAZ at the F-Area HWMF 
THIS PAGE WAS LEFT BLANK INTENTIONALLY. 


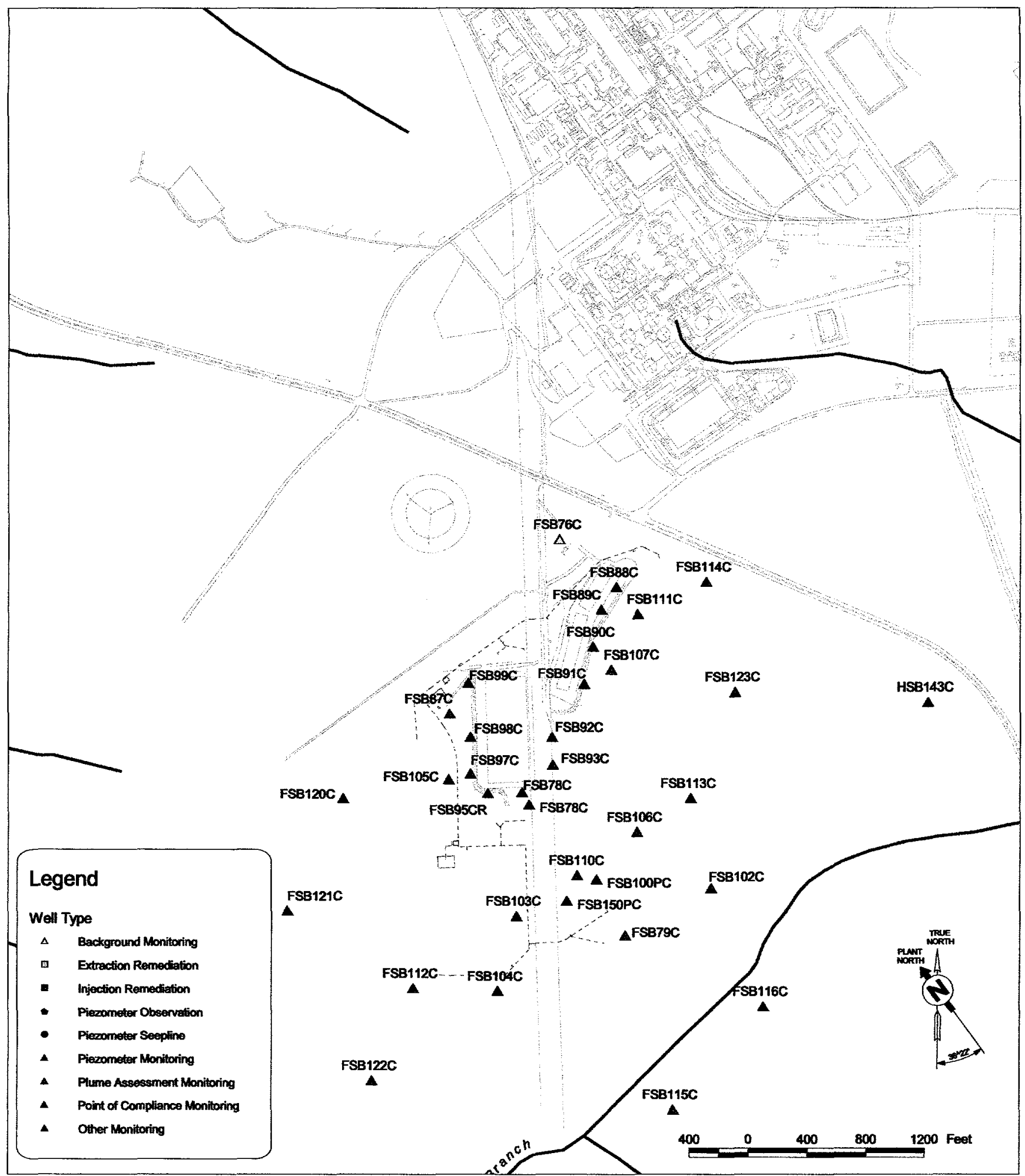

Figure 4. Location of Groundwater Monitoring Wells in the LAZ at the F-Area HWMF 
THIS PAGE WAS LEFT BLANK INTENTIONALLY. 


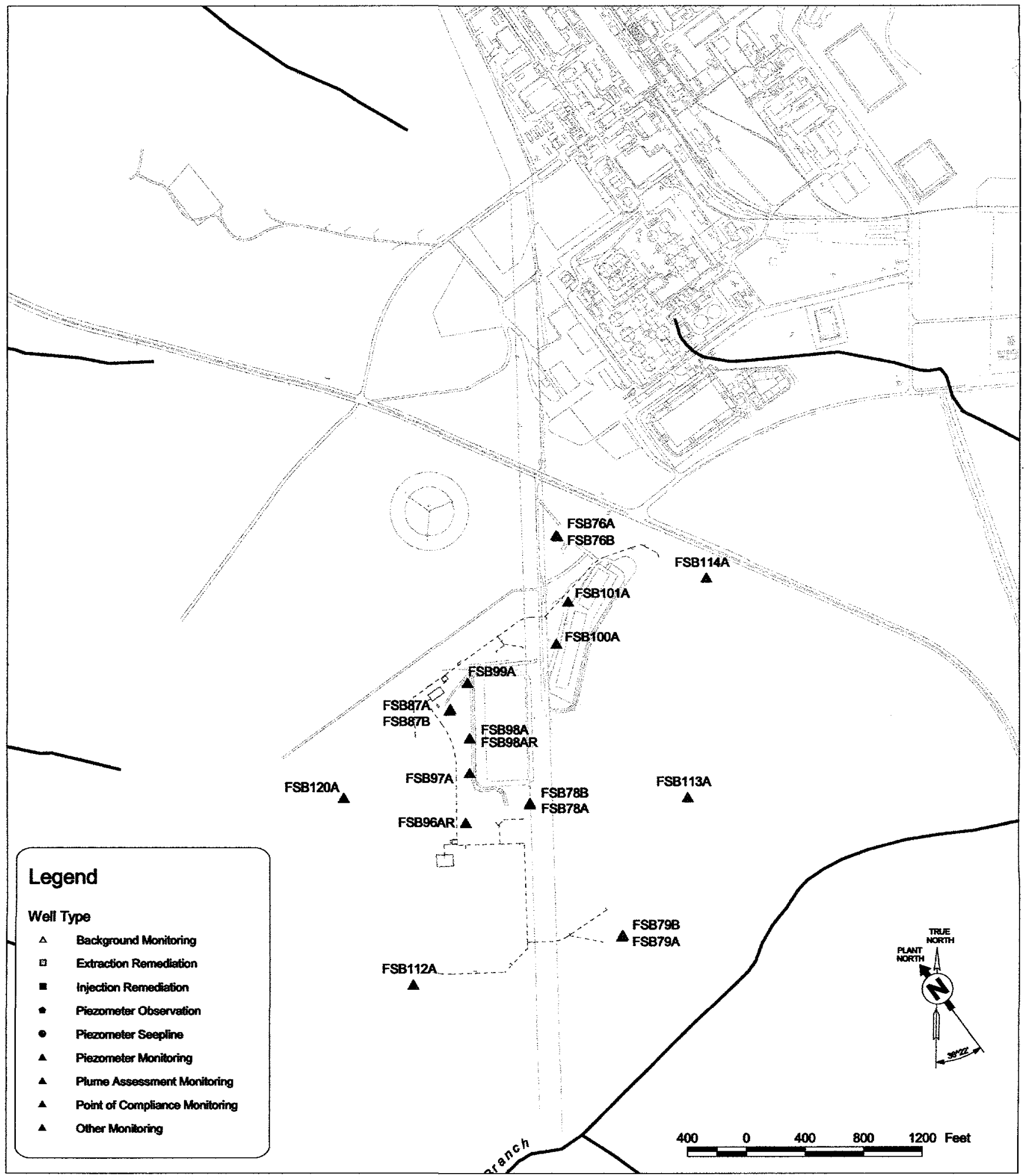

Figure 5. Location of Groundwater Monitoring Wells in the GA at the F-Area HWMF 
THIS PAGE WAS LEFT BLANK INTENTIONALLY. 


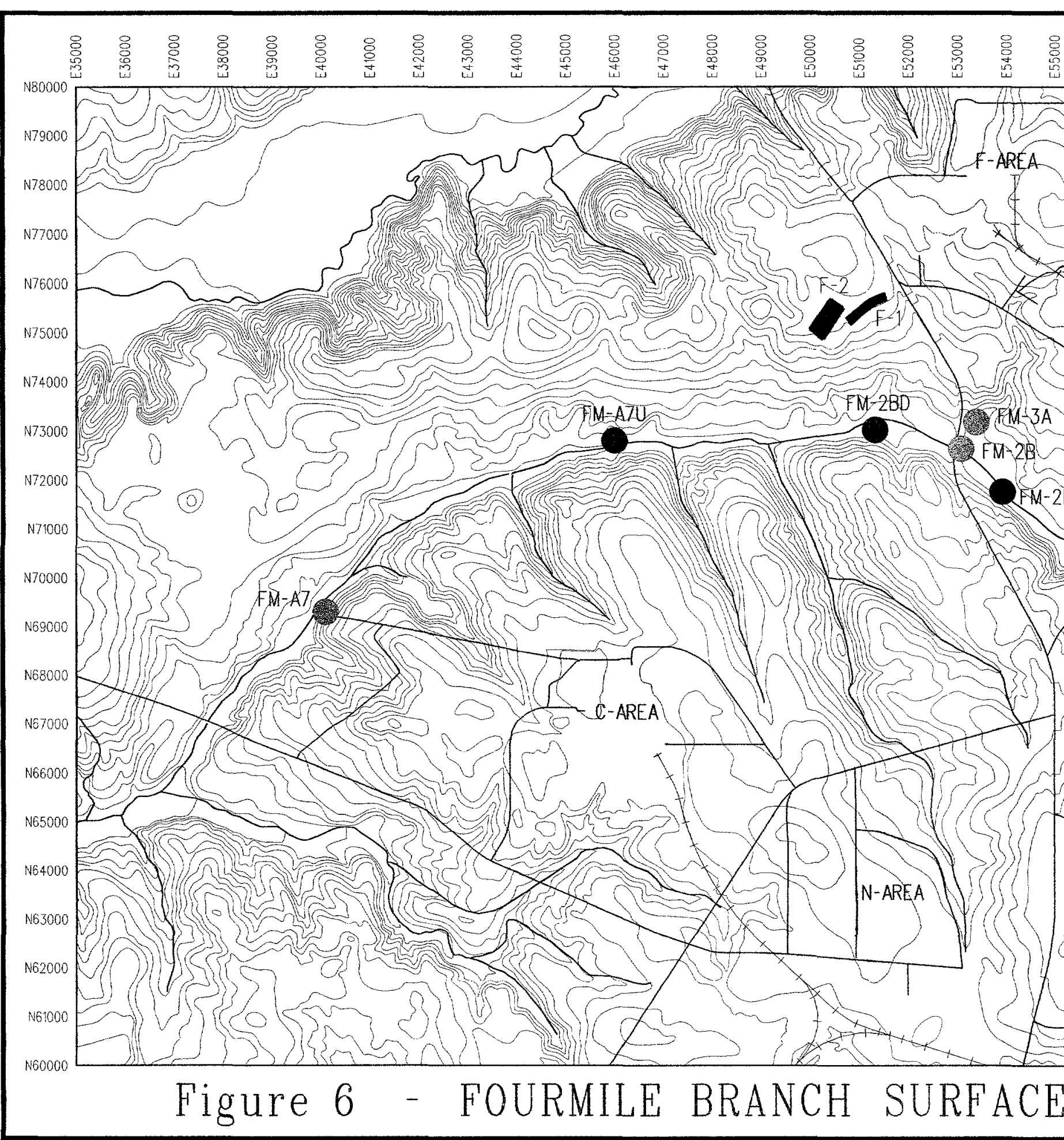




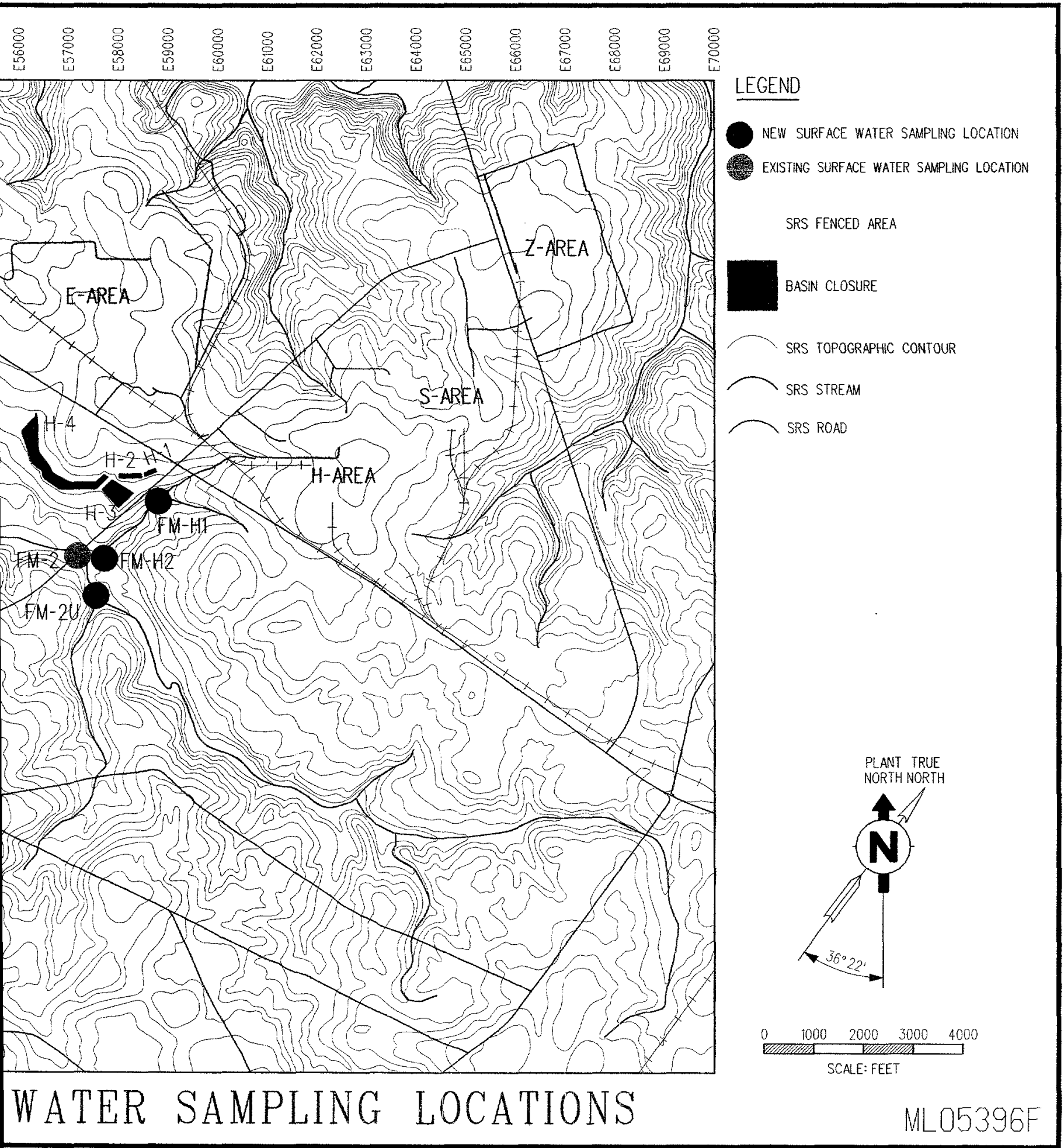




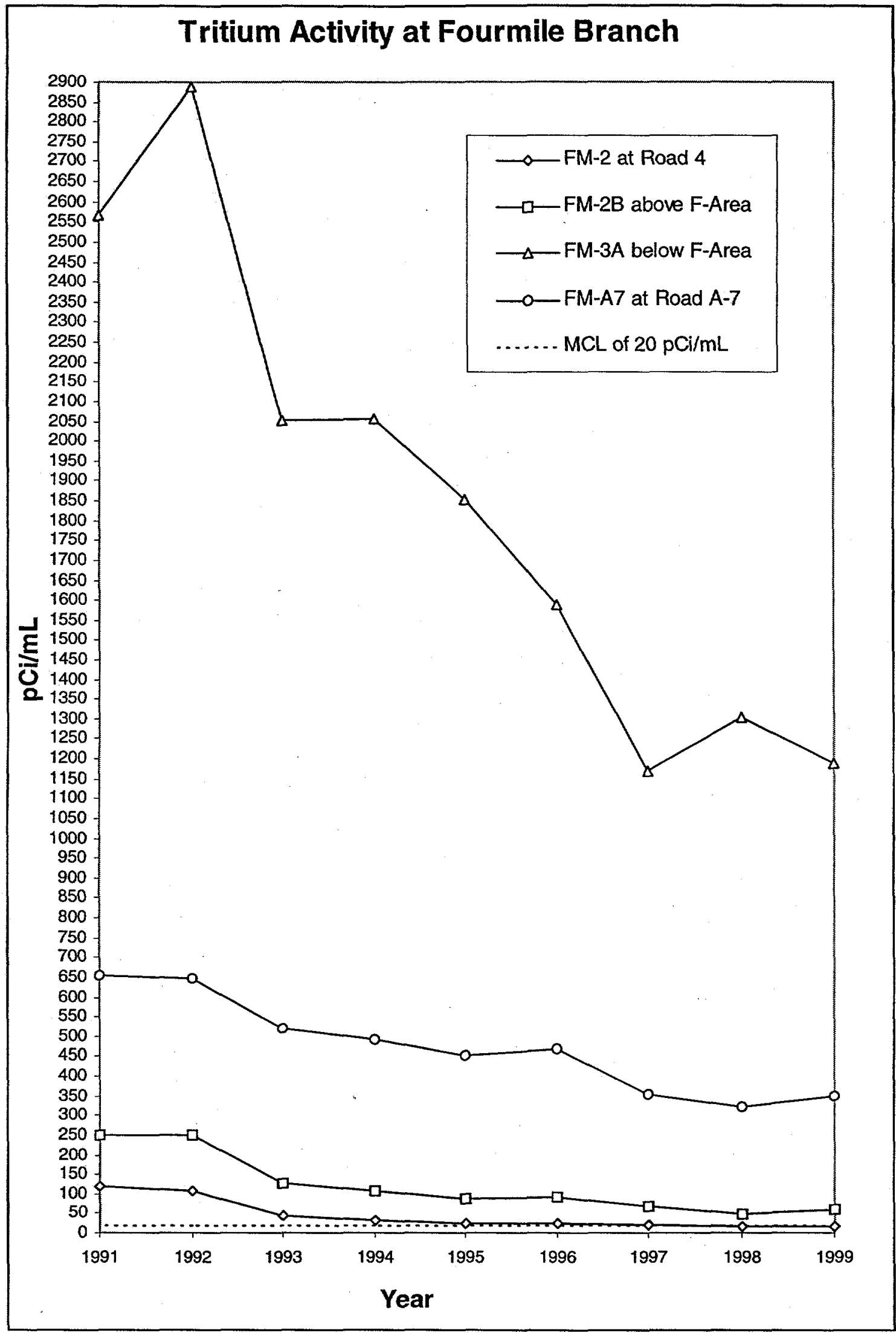

Figure 7. Mean Tritium Activities (pCi/mL) in Fourmile Branch, 1991-1999. 


\section{APPENDIX C: GROUNDWATER MONITORING RESULTS TABLES}

\section{Key to Reading the Tables}

The following abbreviations may appear in the data tables:

\section{Constituents}

Sp. conductance specific conductance

\section{Laboratories}

EM

EX

GE and GP

TM

WA

\section{Sampling Codes}

A

B

C

D

$\mathrm{E}$

I

L

N

$\mathrm{P}$

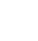

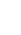

D

E

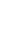

$\mathrm{L}$

N

$\mathrm{P}$

Environmental Protection Department/Environmental Monitoring Section (EPD/EMS) Laboratory

EMAX Laboratories, Inc.

General Engineering Laboratories, Inc.

Thermo NUtech

Recra LabNet Philadelphia (Roy F. Weston, Inc., until June 1997) 
$\mathrm{S}$

W

$\mathrm{X}$

\section{Sampling Methods}

B

$\mathrm{O}$

$\mathbf{P}$

S

V

\section{Units}

$\mathrm{mg} / \mathrm{L}$

msl

NTU

$\mathrm{pCi} / \mathrm{L}$

$\mathrm{pCi} / \mathrm{mL}$

$\mathrm{pH}$

$\mu \mathrm{g} / \mathrm{L}$

$\mu \mathrm{S} / \mathrm{cm}$ no water in standpipe; for water-level events only

unable to sample because of stabilization or sampling equipment failure; only water-level measurements were obtained well went dry during purging; samples were collected after well recovered

sample collected using an open-bucket bailer

sample collected by method other than bailer or pump

sample collected using a bladder pump

sample collected using a single-speed centrifugal downhole pump

sample collected using a variable-speed pump milligrams per liter

mean sea level

nephelometric turbidity unit

picocuries per liter

picocuries per milliliter

$\mathrm{pH}$ units

micrograms per liter

microsiemens per centimeter

\section{Data Table Column Headings}

DF dilution factor

$\mathrm{H}$

exceeded the holding time

Mod

modifier

ST

exceeded the GWPS 


\section{Other}

$\begin{array}{ll}\text { CS } & \text { carbon steel } \\ \text { E } & \text { exponential notation (e.g., } 1.1 \mathrm{E}-09=1.1 \times 10-9=0.0000000011) \\ \text { PVC } & \text { polyvinyl chloride } \\ \text { TOC } & \text { top of casing }\end{array}$

\section{Data Qualification}

The contract laboratories submit sample- or batch-specific quality assurance/quality control information either at the same time as analytical results or in a quarterly summary. Properly defined and used data modifiers (also referred to as qualifiers) can be a key component in assessing data usability. In the data tables, these modifiers appear under the column Mod. Three possible fields for modifiers for each quarter are separated by slashes. Functional Guideline qualifiers (FG) may appear before the first slash, STORET codes between the two slashes, and EMS codes after the second slash. For further information on modifiers and their definitions, contact EMS.

\section{Modifiers}

\section{Functional Guideline Codes (FG)}

(Blank) Data are not qualified. Numbers should be interpreted exactly as reported.

$J$ The analyte was positively identified; the associated numerical value is an estimated concentration of the analyte in the sample.

$\mathrm{R}$

The sample results are rejected due to serious deficiencies in the ability to analyze the sample and meet quality control criteria. The presence or absence of the analyte cannot be verified.

$\mathrm{U}$

Material was analyzed for, but not detected. The analyte concentration is less than the sample-specific estimated quantitation limit (ssEQL). 


\section{STORET Codes}

C

I

K

L

Q

V

Y

\section{EMS Codes}

A

C

D

H

I

K

$\mathrm{L}$

$\mathrm{O}$

$\mathbf{P}$

S

W
The result is calculated.

The result is less than the ssEQL but equal to or greater than the MDL.

The actual concentration is known to be less than the reported result.

The actual concentration is known to be greater than the reported result.

The sample was held beyond the normal holding time prior to analysis.

The analyte was detected in both the method blank and the sample.

The result is from an unpreserved or incorrectly preserved sample; the data may not be accurate.

Compound identification criteria were not met.

Laboratory control sample (LCS) or blank spike (BS) criteria were not met.

ICP serial dilution criteria were not met.

Internal standard (IS) criteria were not met when the IS was used for quantitation.

Matrix spike recovery was not within the control limits.

A tentatively identified compound is a suspected aldol-condensation product.

Initial or continuing calibration criteria were not met.

Surrogate or tracer spike recovery is out of specification.

Graphite furnace atomic absorption QC

a. Duplicate injection criteria were not met.

b. Post-digestion spike recovery was not within control limits and the sample absorbance is $>50 \%$ of the post-digestion spike absorbance.

The sample was analyzed by the method of standard additions.

Graphite furnace atomic absorption QC: the post-digestion spike 
recovery is not within control limits and the sample absorbance is $<50 \%$ of the post-digestion spike absorbance.

$\mathrm{X}$

4

5

6

8

9

The laboratory duplicate RPD or MS/MSD RPD was not within control limits.

Matrix interference is present.

Matrix spike concentration was $<0.25 \times$ the sample concentration, and the percent recovery cannot be determined.

The analyte was detected in both the sample and associated field blank.

The analyte was detected in both the sample and associated trip blank.

The field duplicate RPD was not within control limits.

\section{Results below Detection}

For radiological analyses, the analytical result field contains the result recorded on the analytical instrument and reported by the laboratory, even if it is negative. For nonradiological analyses, if the analyte is not detected, the ssEQL is entered into the result field and is reported with a less than $[<]$ sign. The ssEQL is defined as the lowest concentration that can be achieved reliably within specified limits of precision and accuracy during routine laboratory operating conditions. The ssEQL is modified for sample concentration or dilution or unusual aliquot size that affects analytical sensitivity.

\section{Data Filtering}

Data in the column headed Filt. have been filtered to clarify their usability. In this column, Rej. is used to indicate rejected data.

For nondetects, this column contains the less-than symbol and the sample-specific method detection limit or sample-specific minimum detectable activity. For chemical analyses, this is the same numeric value as appears in the unfiltered result column with a few exceptions, generally data that were qualified as nondetects during validation because of contamination in an associated blank. 
For all data qualified with the Functional Guideline code $J$, indicating an estimated quantity, the Filt. column contains the letters $N D D$, meaning that these data are considered not "decision" data.

None of the filtered data (rejected, undetected, or estimated) are considered in discussions of results above standards in this report.

\section{Holding Times}

Standard analytical methods include a limit, called holding time, on the maximum elapsed time between sample collection and extraction or analysis by the laboratory. In the data tables, a large bullet $(\bullet)$ in the $H$ (holding time) column indicates that holding time was exceeded. Analyses performed beyond holding times may not yield valid results.

The South Carolina Department of Health and Environmental Control (SCDHEC) allows only 15 minutes to elapse between sampling and analysis for $\mathrm{pH}$. Thus, only field $\mathrm{pH}$ measurements can meet the holding time criterion; laboratory $\mathrm{pH}$ analyses always will exceed it.

\section{Sampling Dates}

Samples for field data are collected once each quarter, but samples for analytical data may be collected more than once each quarter. Because the results tables present the highest analytical results for each quarter, the date of collection for reported analytical results may not coincide with the date of collection for field data.

\section{Beta Dose}

If any beta-emitting radionuclides on the GWPS exceeded their minimum detectable activity (MDA), beta dose was calculated and entered into the data tables in this appendix. If the data were filtered out because they were below MDA, rejected, or estimated for any reason, they were not included in the calculation of beta dose. 
A number greater than 1 in the beta dose column indicates that the $4 \mathrm{mrem} / \mathrm{yr}$ standard for beta dose has been exceeded. The values were obtained by dividing each detected, unfiltered betaemitting radionuclide's measured concentration by the EPA's conversion factor, shown in the table below.

\section{Activity to Dose Conversion Factors for F-Area HWMF Beta Emitters}

\begin{tabular}{|l|l|}
\hline Analyte & EPA 1976 dose (4mrem/yr) limit \\
\hline Carbon-14 & $2,000 \mathrm{pCi} / \mathrm{L}$ \\
\hline Cesium-137 & $200 \mathrm{pCi} / \mathrm{L}$ \\
\hline Cobalt-60 & $100 \mathrm{pCi} / \mathrm{L}$ \\
\hline Iodine-129 & $1 \mathrm{pCi} / \mathrm{L}$ \\
\hline Radium-228* & $5 \mathrm{pCi} / \mathrm{L}$ \\
\hline Strontium-90 & $8 \mathrm{pCi} / \mathrm{L}$ \\
\hline Technetium-99 & $900 \mathrm{pCi} / \mathrm{L}$ \\
\hline
\end{tabular}

*The EPA's 1976 guidance document gives no equivalent for radium-228, so the GWPS of $5 \mathrm{pCi} / \mathrm{L}$ for the sum of radium-226 and -228 is used for radium-228.

For each well, the unfiltered activities (in $\mathrm{pCi} / \mathrm{L}$ ) for each of the beta emitters on the GWPS is divided by its individual limit (also in $\mathrm{pCi} / \mathrm{L}$ ), and all of the resulting ratios (unitless) are added as shown below:

$\mathrm{A}_{\mathrm{C}-14} / 2,000+\mathrm{A}_{\mathrm{Cs}-137} / 200+\mathrm{A}_{\mathrm{Co}-60} / 100+\mathrm{A}_{\mathrm{I}-129} / 1+\mathrm{A}_{\mathrm{Ra}-228} / 5+\mathrm{A}_{\mathrm{Sr}-90} / 8+\mathrm{A}_{\mathrm{TC}-99} / 900=$ Beta dose factor

where $A$ is the measured activity for each radionuclide.

If the beta dose factor exceeds 1.0 , the beta dose for a theoretical person drinking $2 \mathrm{~L} /$ day of that well's water exceeds the EPA standard of $4 \mathrm{mrem} / \mathrm{yr}$. 


\section{WELL FSB 76}

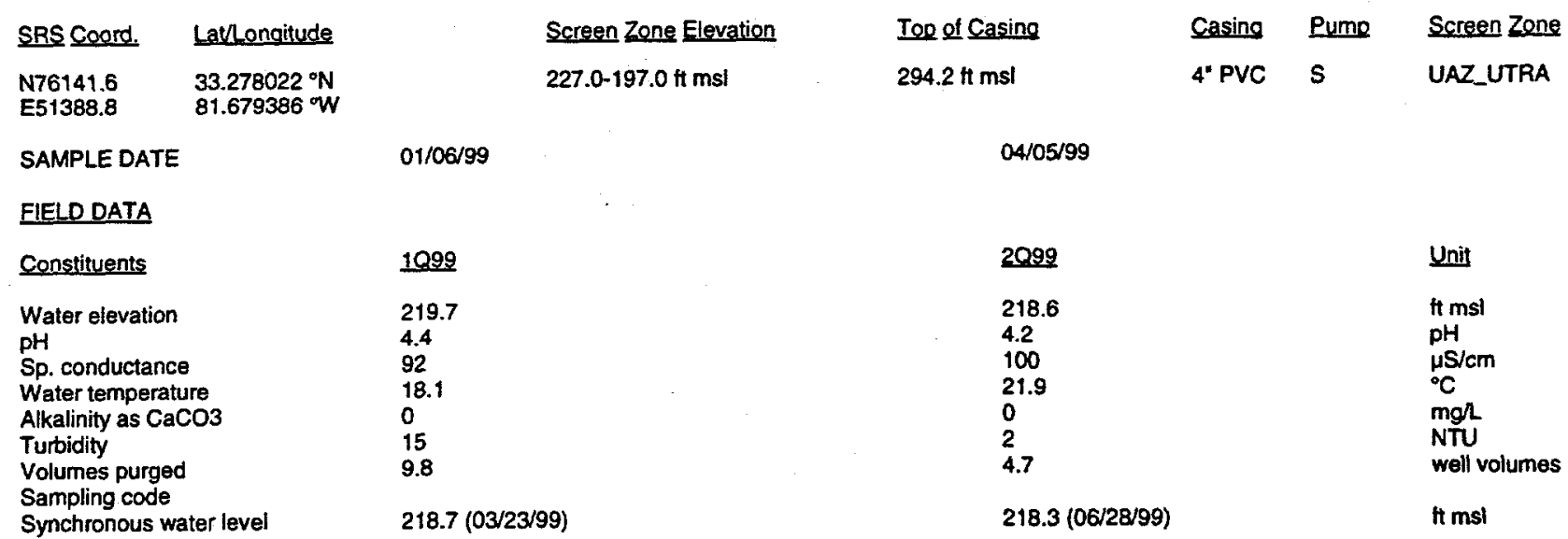

\section{ANALYTICAL DATA}

Inorganic Constituents

\begin{tabular}{|c|c|c|c|c|c|c|c|c|c|c|c|c|c|}
\hline Constituents & 1099 & Mod & Filt. & ST보 & DF & Lab & 2099 & Mod & Filt. & ST H & DF & Lab & Unis \\
\hline Aluminum, total recoverable & 410 & $/ I$ & & & 1 & GE & 280 & $\|$ & & & 1 & GE & pg/. \\
\hline Antimony, total recoverable & $<0.61$ & $\mathrm{JU} / / 4$ & $<0.89$ & & 1 & GE & & & & & & & sgh \\
\hline Arsenic, total recoverable & $<3.0$ & $\mathrm{U} / /$ & $<3.0$ & & 1 & GE & & & & & & & $\mu g h$ \\
\hline $\begin{array}{l}\text { Barium, total recoverable } \\
\text { Beryllium, total recoverable }\end{array}$ & 18 & II & & & 1 & GE & & & & & & & jgh \\
\hline Cadmium, total recoverable & 0.31 & $J y$ & NDD & & 1 & GE & $<1.0$ & $\mathbf{U} / /$ & $<1.0$ & & 1 & GE & \\
\hline Chromium, total recoverable & $<2.6$ & $U / / 6$ & $<3.0$ & & 1 & GE & & & & & & & \\
\hline Cobalt, total recoverabie & 0.76 & & & & 1 & GE & & & & & & & L \\
\hline Copper, total recoverable & 520 & & & & 1 & GE & & & & & & & L \\
\hline Cyanide & $<10$ & $U / \prime$ & $<10$ & & 1 & GE & & & & & & & \\
\hline Lead, total recoverable & 49 & 11 & & $\mathbf{z}$ & 1 & GE & 46 & $/ /$ & & $\mathbf{\square}$ & 1 & GE & \\
\hline Mercuny, total recoverable & $<0.20$ & U/I & $<0.20$ & & 1 & GE & & & & & & & \\
\hline $\begin{array}{l}\text { Nickel, total recoverable } \\
\text { Nitrate as nitrogen }\end{array}$ & 4.7 & $/ 1$ & & & 1 & GE & & & & & & & Hh \\
\hline Nitrate-nitrite as nitrogen & 10,000 & $\|$ & & $\mathbf{\square}$ & 10 & GE & 11,000 & $/ /$ & & a & 5 & GE & \\
\hline Selenium, total recoverable & $<5.0$ & $U / I$ & $<5.0$ & & 1 & GE & & & & & & & \\
\hline Silver, total recoverable & $<1.0$ & $\mathbf{u} / !$ & $<1.0$ & & 1 & GE & & & & & & & \\
\hline Thallium, total recoverable & $<2.5$ & $U / I$ & $<2.5$ & & 1 & GE & & & & & & & \\
\hline Vanadium, total recoverable & $<10$ & $v / /$ & $<10$ & & 1 & GE & & & & & & & $g / 2$ \\
\hline Zinc, total recoverable & 120 & $\|$ & & & 1 & GE & & & & & & & \\
\hline
\end{tabular}

Organic Constituents

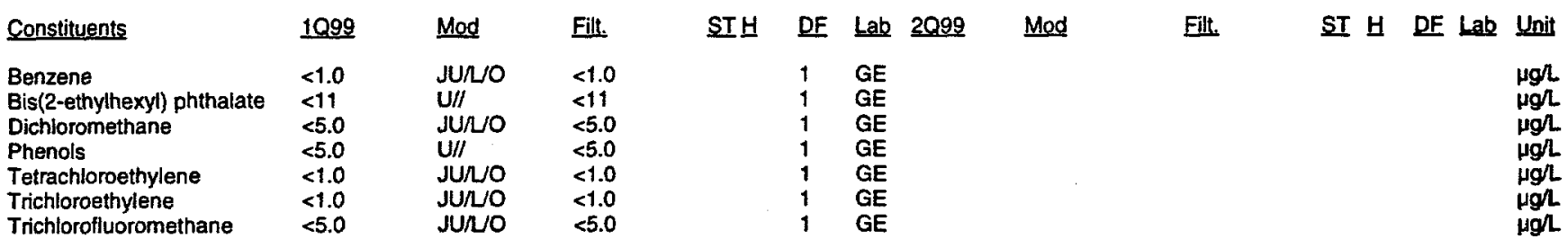

Notes:

- exceeded holding time

= exceeded groundwater protection or monitoring constituent standard (See Appendix A.)

NA - Not applicable. Applies to beta dose and sum of betas if there are no beta-emitting radionuclides above detection limits; to sum of alphas if there are no alphaemitting radionuclides above detection limits; and to total radium if neither radium-226 or radium-228 was above detection limit

UAZ UTRA - Upper Aquifer Zone of the Upper Three Runs Aquifer, LAZ_UTRA - Lower Aquifer Zone of the Upper Three Runs Aquifer, Gordon - Gordon Aquifer 


\section{WELL FSB 76 (cont.)}

Radioactive Constituents

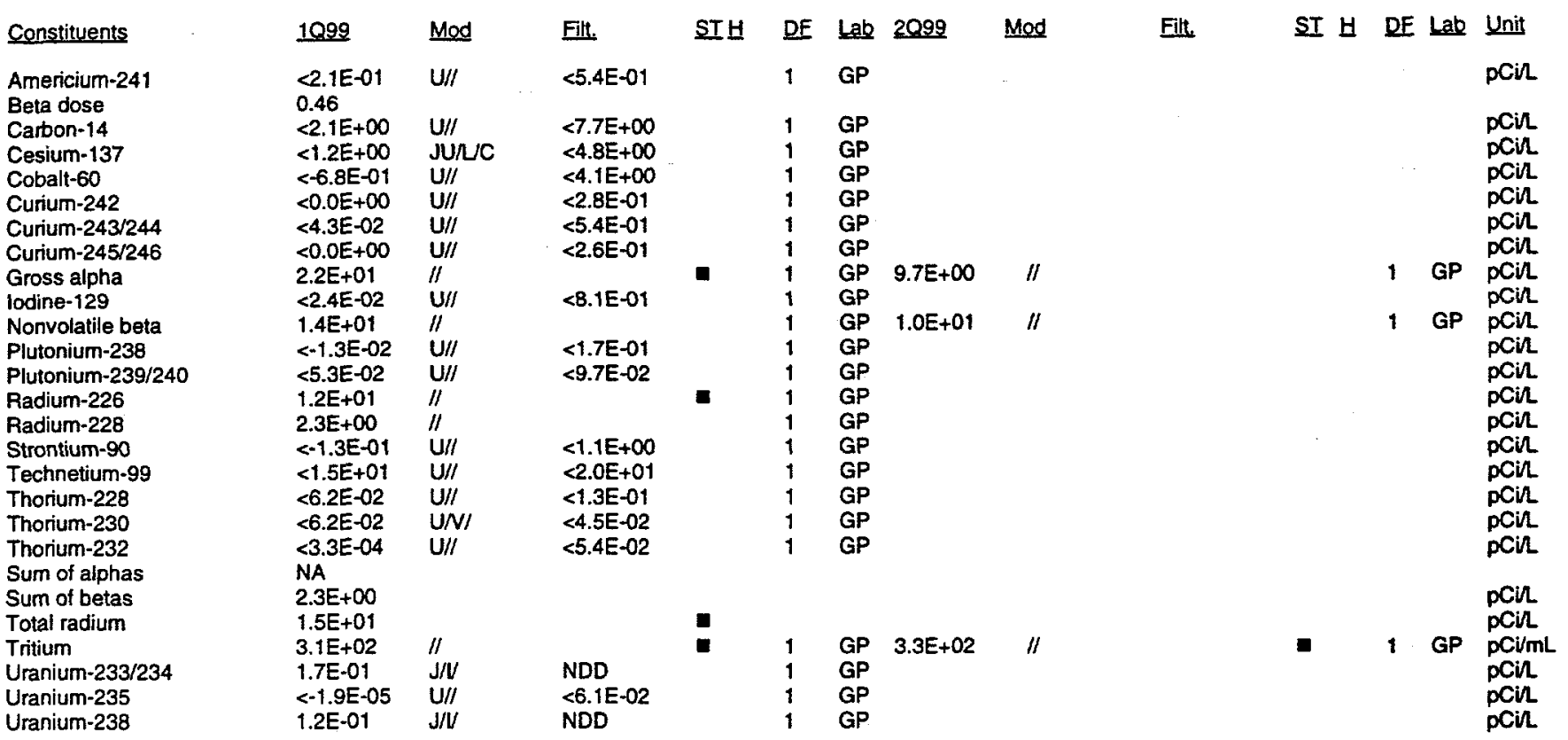

Notes:

- exceeded holding time

- exceeded groundwater protection or monitoring constituent standard (See Appendix A.)

NA - Not applicable. Applies to beta dose and sum of betas if there are no beta-emitting radionuclides above detection limits; to sum of alphas if there are no alphaemitting radionuclides above detection limits; and to total radium if neither radium-226 or radium-228 was above detection limit

UAZ UTRA - Upper Aquifer Zone of the Upper Three Runs Aquifer, LAZ_UTRA - Lower Aquifer Zone of the Upper Three Runs Aquifer, Gordon - Gordon Aquifer 


\section{WELL FSB 76A}

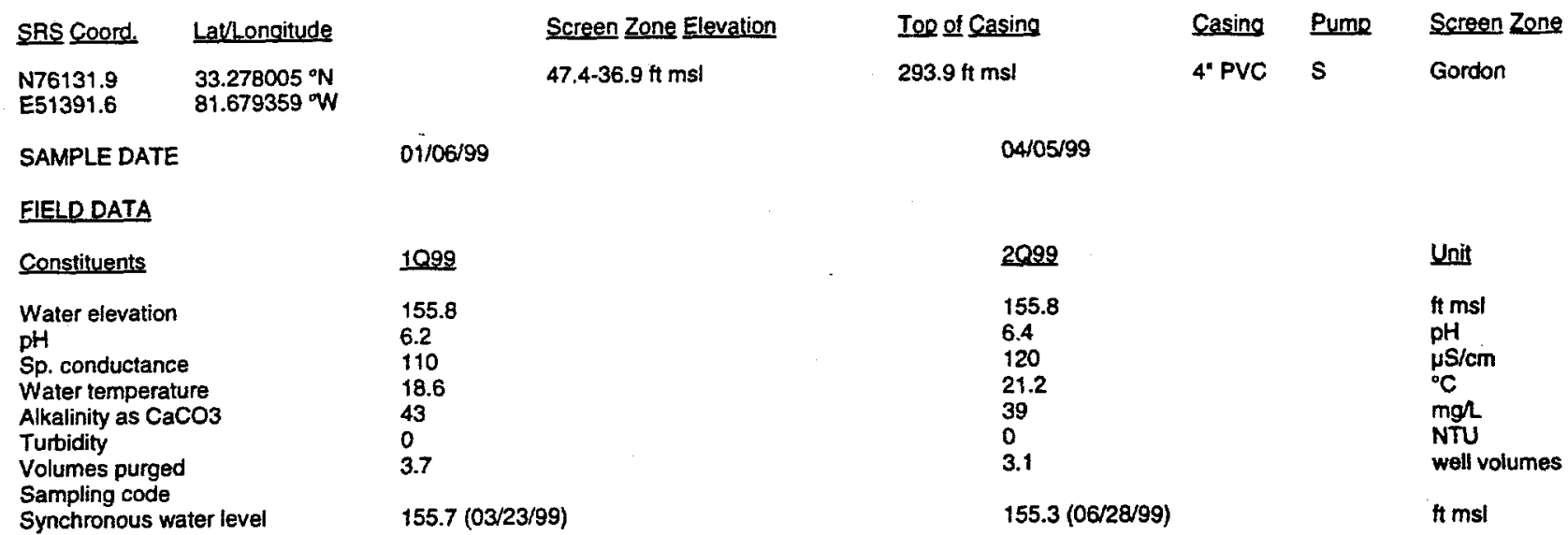

\section{ANALYTICAL DATA}

Inorganic Constituents

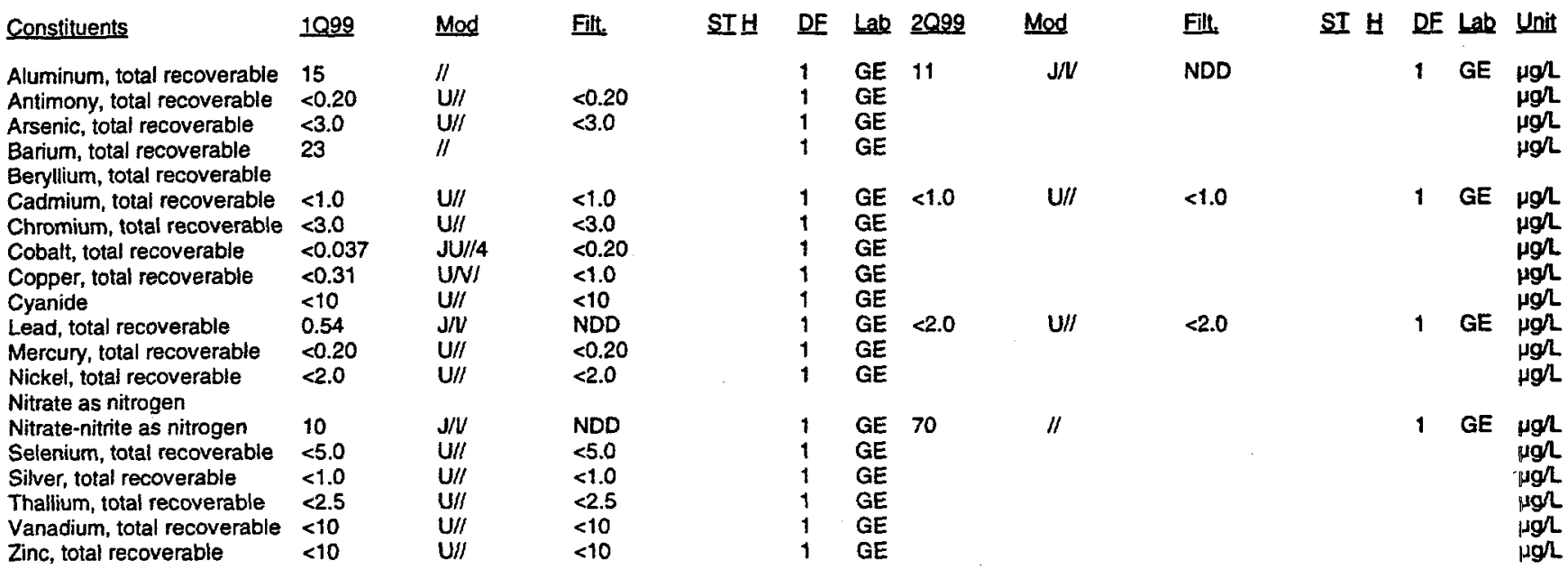

Organic Constituents

\begin{tabular}{|c|c|c|c|c|c|c|c|c|c|c|c|}
\hline Constituents & 1099 & Mod & Filt, & ST보 & DF & $\underline{\text { Lab }}$ & 2099 & Mod & Filt. & ST $\mathrm{H}$ DF Lab & Unit \\
\hline Benzene & $<1.0$ & JU/LO & $<1.0$ & & 1 & GE & & & & & $\mu g / L$ \\
\hline Bis(2-ethylhexyl) phthalate & $<10$ & $\mathrm{U} / /$ & $<10$ & & 1 & GE & & & & & $\mu g /$ \\
\hline Dichloromethane & $<5.0$ & JU/LO & $<5.0$ & & 1 & GE & & & & & $\mu g / L$ \\
\hline Phenols & $<5.0$ & $U / /$ & $<5.0$ & & 1 & GE & & & & & $\mu g h$ \\
\hline Tetrachloroethylene & $<1.0$ & JU/LO & $<1.0$ & & 1 & GE & & & & & $\mu g / L$ \\
\hline Trichloroethylene & $<1.0$ & JU/RO & $<1.0$ & & 1 & GE & & & & & $\mu g / l$ \\
\hline Trichlorofluoromethane & $<5.0$ & JUルO & $<5.0$ & & 1 & GE & & & & & \\
\hline
\end{tabular}

Notes:

- exceeded holding time

- exceeded groundwater protection or monitoring constituent standard (See Appendix A.)

NA - Not applicable. Applies to beta dose and sum of betas if there are no beta-emitting radionuclides above detection limits; to sum of alphas if there are no alphaemitting radionuclides above detection limits; and to total radium if neither radium-226 or radium-228 was above detection limit

UAZ UTRA - Upper Aquifer Zone of the Upper Three Runs Aquifer, LAZ_UTRA - Lower Aquifer Zone of the Upper Three Runs Aquifer, Gordon - Gordon Aquifer 


\section{WELL FSB 76A (cont.)}

Radioactive Constituents

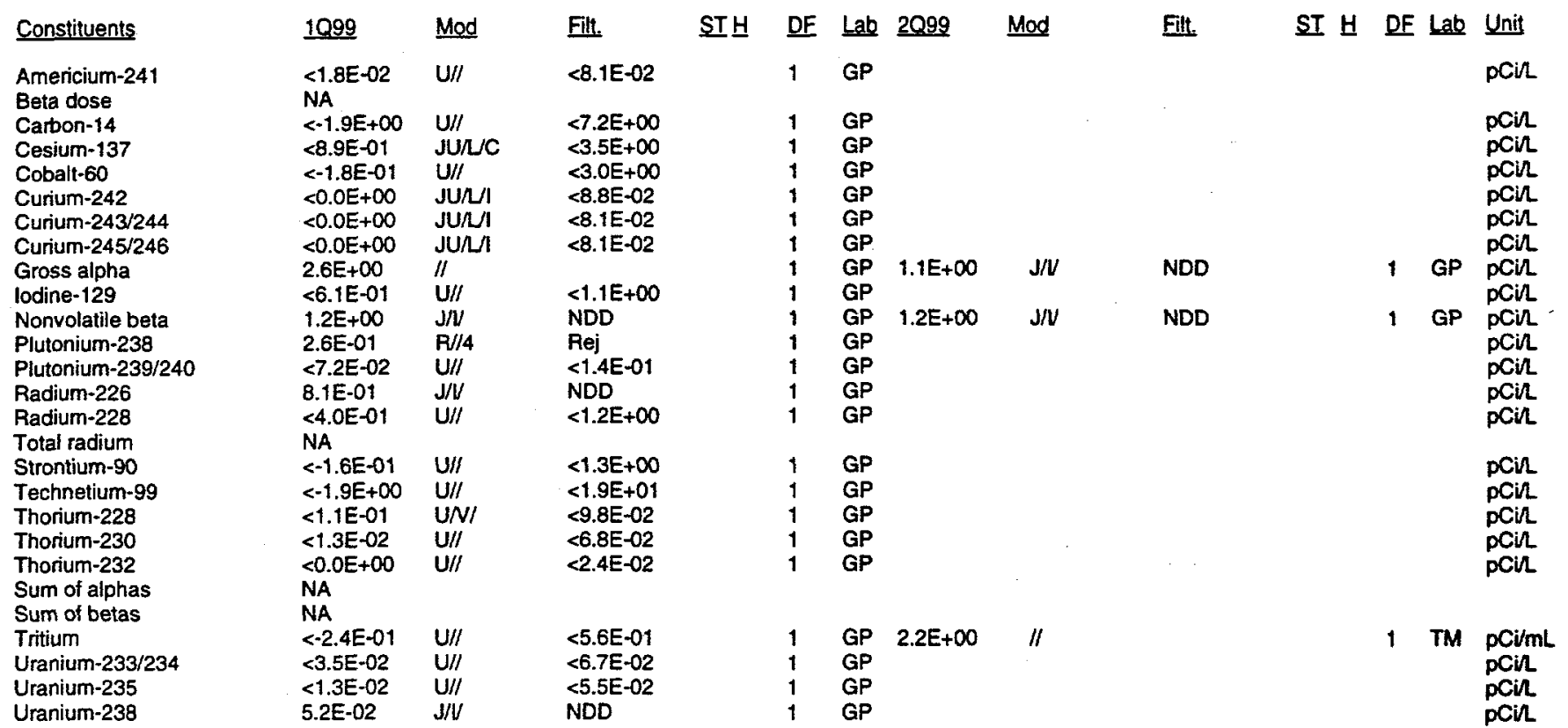

\section{Notes:}

- exceeded holding time

E = exceeded groundwater protection or monitoring constituent standard (See Appendix A.)

NA - Not applicable. Applies to beta dose and sum of betas if there are no beta-emitting radionuclides above detection limits; to sum of alphas if there are no alphaemitting radionuclides above detection limits; and to total radium if neither radium-226 or radium-228 was above detection limit

UAZ_UTRA - Upper Aquifer Zone of the Upper Three Runs Aquifer, LAZ UTRA - Lower Aquifer Zone of the Upper Three Runs Aquifer, Gordon - Gordon Aquifer 


\section{WELL FSB 76B}

\begin{tabular}{|c|c|}
\hline SRS Coord. & Lat'Longitude \\
\hline $\begin{array}{l}N 76122.4 \\
\text { E51394.0 }\end{array}$ & $81.679335 \mathrm{~W}$ \\
\hline
\end{tabular}

SAMPLE DATE

1099

152.0

6.6

130

19.1

44

1

4.9

$151.9(03 / 23 / 99)$

Volumes purged

Sampling code

Synchronous water level

\section{Screen Zone Elevation}

109.7-99.2 ft ms!

Top of Casing

$293.8 \mathrm{tt} \mathrm{msl}$

04/05/99

2099

152.0

120

21.6

3.2

$151.5(06 / 28 / 99)$
Casing Pumo

4" PVC 5
Screen Zone

Gordon

ANALYTICAL DATA

Inorganic Constituents

\begin{tabular}{|c|c|c|c|c|c|c|c|c|c|c|c|c|c|}
\hline Constituents & 1099 & Mod & Filt. & STH & DF & Lab & 2099 & Mad & Filt. & ST $\underline{H}$ & DF & Lab & Unit \\
\hline Aluminum, total recoverable & $<15$ & $\mathrm{U} / /$ & $<15$ & & 1 & GE & $<15$ & $u / /$ & $<15$ & & 1 & GE & $\mu g / L$ \\
\hline Antimony, total recoverable & $<0.17$ & $\mathrm{JU} / / 4$ & $<0.89$ & & $i$ & GE & & . & & & & & \\
\hline Arsenic, total recoverable & $<3.0$ & $\mathrm{U} / /$ & $<3.0$ & & 1 & GE & & & & & & & $\mu g /$ \\
\hline $\begin{array}{l}\text { Barium, total recoverable } \\
\text { Beryllium, total recoverable }\end{array}$ & 17 & $/ 1$ & & & 1 & GE & & & & & & & \\
\hline Cadmium, total recoverable & $<1.0$ & $\cup / l$ & $<1.0$ & & 1 & GE & $<1.0$ & $\mathrm{U} / !$ & $<1.0$ & & $\mathbf{1}$ & GE & \\
\hline Chromium, total recoverable & $<1.3$ & $U / / 6$ & $<3.0$ & & 1 & GE & & & & & & & \\
\hline $\begin{array}{l}\text { Cobalt, total recoverable } \\
\text { Copper, total recoverable }\end{array}$ & $\begin{array}{l}<0.035 \\
9.1\end{array}$ & $\mathrm{JU} / / 4$ & $<0.20$ & & $\begin{array}{l}1 \\
1\end{array}$ & $\begin{array}{l}\text { GE } \\
\text { GE }\end{array}$ & & & & & & & 1 \\
\hline Cyanide & $<10$ & $\mathrm{U} / /$ & $<10$ & & 1 & GE & & & & & & & \\
\hline Lead, total recoverable & $<2.0$ & $\mathrm{U} / I$ & $<2.0$ & & 1 & GE & $<2.0$ & $\mathrm{U} / /$ & $<2.0$ & & 1 & GE & \\
\hline Mercury, total recoverable & $<0.20$ & $\mathrm{U} / /$ & $<0.20$ & & 1 & $\overline{G E}$ & & & & & & & \\
\hline $\begin{array}{l}\text { Nickel, total recoverable } \\
\text { Nitrate as nitrogen }\end{array}$ & $<2.0$ & $U / !$ & $<2.0$ & & $i$ & GE & & & & & & & \\
\hline Nitrate-nitrite as nitrogen & 500 & $\|$ & & & 1 & GE & 540 & /I & & & 1 & GE & $\mu g$ \\
\hline Selenium, total recoverable & $<5.0$ & $U / /$ & $<5.0$ & & 1 & $\mathrm{GE}$ & & & & & & & \\
\hline Silver, total recoverable & $<1.0$ & $\mathrm{U} / /$ & $<1.0$ & & 1 & GE & & & & & & & \\
\hline Thallium, total recoverable & $<2.5$ & $\mathrm{U} / /$ & $<2.5$ & & 1 & GE & & & & & & & \\
\hline Vanadium, total recoverable & $<10$ & $\mathrm{U} / /$ & $<10$ & & 1 & GE & & & & & & & \\
\hline Zinc, total recoverable & $<10$ & U/I & $<10$ & & 1 & GE & & & & & & & \\
\hline
\end{tabular}

Organic Constituents

\begin{tabular}{|c|c|c|c|c|c|}
\hline Constituents & 1099 & Mod & Filt. & STH & DF \\
\hline Benzene & $<1.0$ & JUนO & $<1.0$ & & 1 \\
\hline Bis(2-ethylhexyl) phthalate & $<10$ & $U / I$ & $<10$ & & 1 \\
\hline Dichloromethane & $<5.0$ & WUIO & $<5.0$ & & 1 \\
\hline Phenols & $<5.0$ & $\mathrm{U} / /$ & $<5.0$ & & 1 \\
\hline Tetrachloroethylene & $<1.0$ & JUЛNO & $<1.0$ & & 1 \\
\hline Trichloroethylene & 1.0 & // & & & 1 \\
\hline Trichlorofluoromethane & $<5.0$ & JU/LO & $<5.0$ & & 1 \\
\hline
\end{tabular}

Unit

ft mst

$\mathrm{pH}$

$\mu$ Sicm

mgl

well volumes

ft msl 
WELL FSB 76B (cont.)

Radioactive Constituents

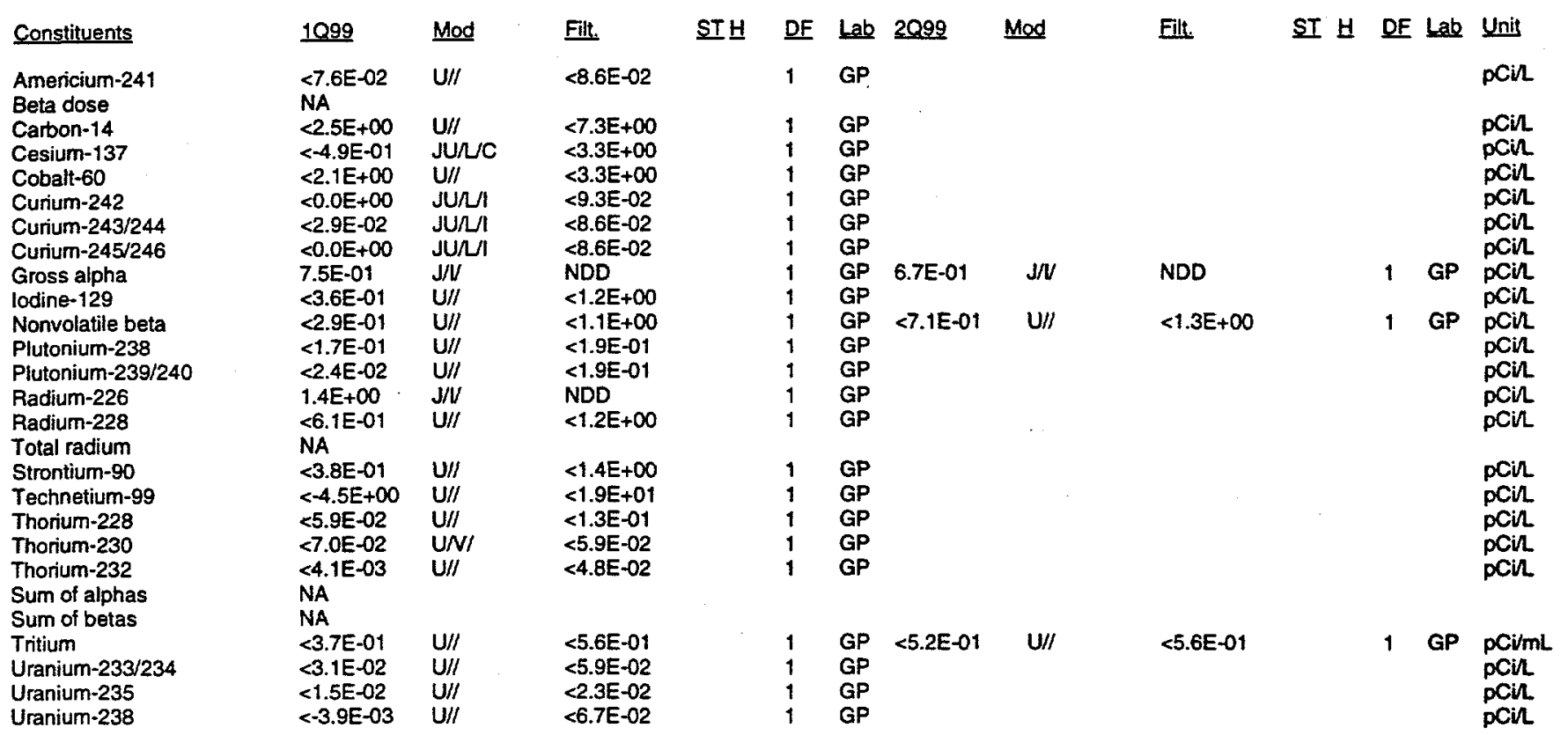

Notes:

- exceeded holding time

= exceeded groundwater protection or monitoring constituent standard (See Appendix A.)

NA - Not applicable. Applies to beta dose and sum of betas if there are no beta-emitting radionuclides above detection limits; to sum of alphas if there are no alphaemitting radionuclides above detection limits; and to total radium if neither radium-226 or radium-228 was above detection limit

UAZ_UTRA - Upper Aquifer Zone of the Upper Three Runs Aquifer; LAZ_UTRA - Lower Aquifer Zone of the Upper Three Runs Aquifer; Gordon - Gordon Aquifer 
WELL FSB 76C

$\begin{array}{lll}\text { SRS Coord. } & \text { Lat/Longitude } \\ \text { N76112.4 } & & 33.277970^{\circ} \mathrm{N} \\ \text { E51396.4 } & 81.679309^{\circ} \mathrm{W}\end{array}$

Screen Zone Elevation

$165.3-154.8 \mathrm{ft} \mathrm{ms} \mid$

01/06/99

SAMPLE DATE

FIELD DATA

Constituents

1099

Water elevation

$\mathrm{pH}$

Sp. conductance

Water temperature

Alkalinity as $\mathrm{CaCO} 3$

Tutbidity

Volumes purged

Sampling code

Synchronous water level

6.0
45

18.9

14

0

4.8

$213.7(03 / 23 / 99)$
Top of Casing

$293.6 \mathrm{ft} \mathrm{msl}$

04/05/99

2099

213.1

5.6

50

21.4

6

2.8

$212.6(06 / 28 / 99)$
Casing Pump

4* PVC S
Screen Zone

LAZ_UTRA

ANALYTICAL DATA

Inorganic Constituents

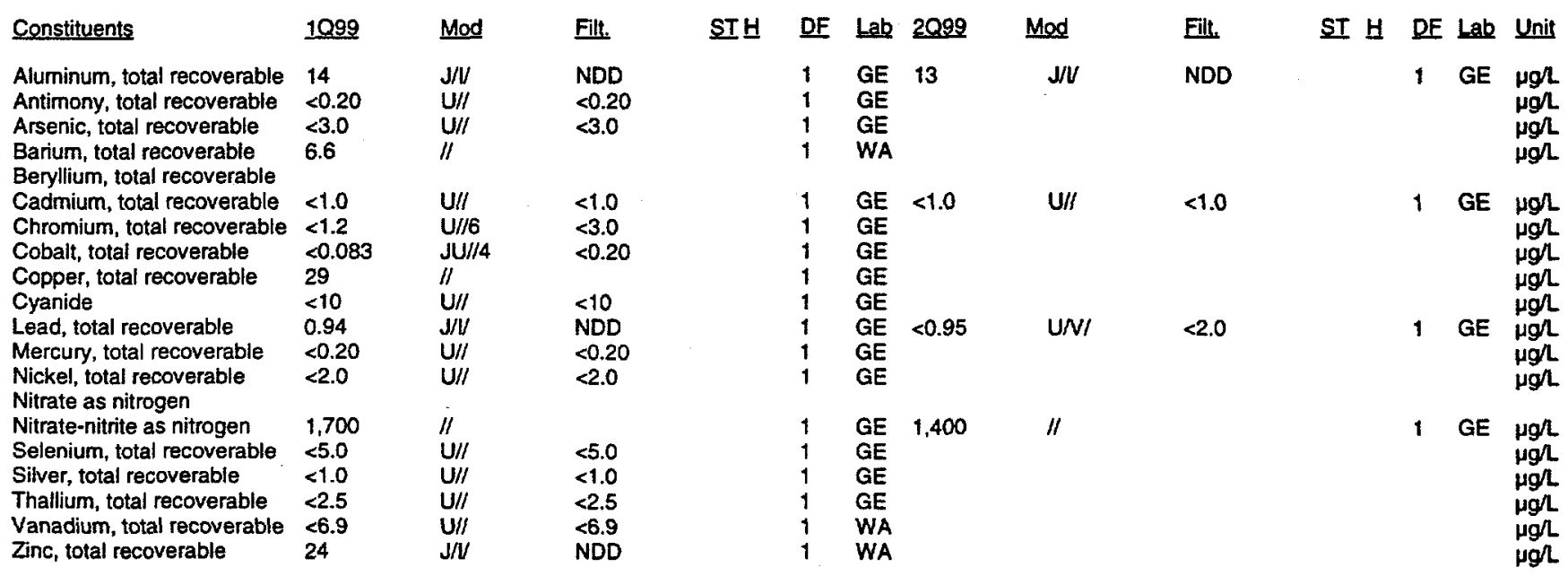

Organic Constituents

\begin{tabular}{llll} 
Constituents & 1099 & Mod & Fill. \\
\cline { 2 - 4 } Benzene & $<1.0$ & JU/UO & $<1.0$ \\
Bis(2-ethylhexyl) phthalate & $<10$ & U// & $<10$ \\
Dichloromethane & $<5.0$ & JU/UO & $<5.0$ \\
Phenols & $<5.0$ & U/I & $<5.0$ \\
Tetrachloroethylene & $<1.0$ & JU/UO & $<1.0$ \\
Trichioroethylene & 2.9 & J/UO & NDD \\
Trichlorofluoromethane & 2.8 & J/LUO & NDD
\end{tabular}

$\begin{array}{ccc}\text { ST브 } & \text { DF } & \text { Lab } \\ 1 & \text { GE } \\ 1 & \text { WA } \\ 1 & \text { GE } \\ 1 & \text { GE } \\ 1 & \text { GE } \\ 1 & \text { GE } \\ 1 & \text { GE }\end{array}$

Unit
tt mst
oH
$\mu S / \mathrm{cm}$
${ }^{\circ} \mathrm{C}$
$\mathrm{mgh}$
$\mathrm{NTU}$
well volumes
ft mst

ft mst 


\section{WELL FSB 76C (cont.)}

\section{Radioactive Constituents}

\begin{tabular}{|c|c|c|c|c|c|c|c|c|c|c|c|c|c|}
\hline Constituents & 1099 & Mod & Eilt. & ST브 & DF & $\underline{\text { Lab }}$ & $\underline{2099}$ & Mod & Filt. & ST $\underline{H}$ & DF & Lab & Unit \\
\hline $\begin{array}{l}\text { Americium-241 } \\
\text { Beta dose }\end{array}$ & $\begin{array}{l}<9.1 E-02 \\
\text { NA }\end{array}$ & $\mathrm{U} / /$ & $<1.0 E-01$ & & 1 & GP & & & & & & & pCin \\
\hline Carbon-14 & $2.0 E+02$ & $R / / 4$ & Rej & & 1 & TM & & & & & & & pCin \\
\hline Cesium-137 & $<4.8 E-01$ & JUЛ/C & $<3.1 E+00$ & & 1 & GP & & & & & & & pCin \\
\hline Cobalt -60 & $<1.4 E-01$ & $\mathrm{U} / I$ & $<3.3 E+00$ & & 1 & GP & & & & & & & pCin \\
\hline Curium-242 & $<0.0 E+00$ & JU几ル & $<1.1 E-01$ & & 1 & GP & & & & & & & pCir \\
\hline Curium-243/244 & $<0.0 E+00$ & JU/II & $<1.0 E-01$ & & 1 & GP & & & & & & & pCin \\
\hline Curium-245/246 & $<3.3 E-02$ & JUЛЛ & $<1.0 E-01$ & & 1 & GP & & & & & & & pCin \\
\hline Gross alpha & $\begin{array}{l}7.6 E-01 \\
\text { - }\end{array}$ & $\mathrm{J} / \mathrm{W}$ & NDD & & $\begin{array}{l}1 \\
1\end{array}$ & GP & $<2.8 E-01$ & $U / I$ & $<6.7 \mathrm{E}-01$ & & 1 & $\mathrm{GP}$ & pCin \\
\hline Iodine-129 & $<-2.2 E-01$ & U/I & $<1.1 E+00$ & & 1 & GP & & & & & & & pCil \\
\hline Nonvolatile beta & $<1.4 E-01$ & U// & $<1.9 E+00$ & & 1 & $T M$ & $<8.8 \mathrm{E}-01$ & Uil & $<1.2 \mathrm{E}+\infty$ & & 1 & GP & pCil \\
\hline Plutonium-238 & 1.9E-01 & $R / 4$ & Rej & & 1 & GP & & & & & & & pCin \\
\hline Plutonium-239/240 & $<0.0 E+00$ & $U / /$ & $<2.6 E-01$ & & 1 & $T M$ & & & & & & & pCin \\
\hline Radium-226 & $2.3 E+00$ & 11 & & & 1 & GP & & & & & & & pCin \\
\hline $\begin{array}{l}\text { Radium-228 } \\
\text { Total radium }\end{array}$ & $\begin{array}{l}1.2 E+\infty \\
2.3 E+\infty\end{array}$ & $\mathrm{J} / \mathrm{W}$ & NDD & & 1 & GP & & & & & & & pCil \\
\hline Strontium-90 & $<-8.0 E-02$ & $\mathrm{U} / /$ & $<1.6 \mathrm{E}+\infty$ & & 1 & TM & & & & & & & pCin \\
\hline Technetium-99 & $<1.4 E+\infty$ & $\mathrm{U} / /$ & $<1.5 E+01$ & & 1 & TM & & & & & & & pCil \\
\hline Thorium-228 & $<1.1 E-02$ & $\mathrm{U} / /$ & $<1.3 E-01$ & & 1 & GP & & & & & & & pCin \\
\hline Thorium-230 & $<3.9 E-02$ & $\mathrm{U} / /$ & $<5.9 E-02$ & & 1 & GP & & & & & & & pCin \\
\hline Thorium-232 & $<0.0 E+00$ & $\mathrm{U} / /$ & $<2.3 E-02$ & & 1 & GP & & & & & & & pCin \\
\hline Sum of alphas & NA & & & & & & & & & & & & \\
\hline Sum of betas & NA & & & & & & & & & & & & \\
\hline Tritium & $2.8 E+\infty 0$ & $/ 1$ & & & 1 & $T M$ & $2.5 E+00$ & $\|$ & & & 1 & GP & pCirmL \\
\hline Uranium-233/234 & $<5.3 E-03$ & $U / /$ & $<9.5 E-02$ & & 1 & GP & & & & & & & pCin \\
\hline Uranium-235 & $<4.3 E-03$ & $\mathrm{U} / /$ & $<6.4 \mathrm{E}-02$ & & 1 & GP & & & & & & & pCin \\
\hline Uranium-238 & $<0.0 \mathrm{E}+00$ & $\mathrm{U} / \mathrm{I}$ & $<2.4 \mathrm{E}-01$ & & 1 & $T M$ & & & & & & & pCIL \\
\hline
\end{tabular}

Notes:

- = exceeded holding time

E = exceeded groundwater protection or monitoring constituent standard (See Appendix A.)

NA - Not applicable. Applies to beta dose and sum of betas if there are no beta-emitting radionuclides above detection limits; to sum of alphas if there are no alphaemitting radionuclides above detection limits; and to total radium if neither radium-226 or radium-228 was above detection limit

UAZ_UTRA - Upper Aquifer Zone of the Upper Three Runs Aquifer, LAZ_UTRA - Lower Aquifer Zone of the Upper Three Runs Aquifer, Gordon - Gordon Aquifer 


\section{WELL FSB 77}

$\begin{array}{ll}\text { SRS Coord. } & \text { Latlongitude } \\ \text { N75129.4 } & 33.274681^{\circ} \\ \text { E50713.1 } & 81.679198 \text { o }\end{array}$

SAMPLE DATE

Screen Zone Elevation

216.4-186.4 ft msl
Top of Casing

$273.3 \mathrm{ft} \mathrm{msl}$
Casing Pump

4" PVC V
Screen Zone

UAZ_UTRA
04/08/99

2099

212.9

3.7

340

20.3

0

2.0

$212.5(06 / 28 / 99)$
Unit

tt msl

pH

$\mu \mathrm{S} / \mathrm{cm}$

${ }^{\circ} \mathrm{C}$

mgh

well volumes

ft msl

Sampling code

6.2

$212.9(03 / 23 / 99)$

ANALYTICAL DATA

Inorganic Constituents

\begin{tabular}{|c|c|c|c|c|c|c|c|c|c|c|c|c|c|}
\hline Constituents & 1099 & Mod & Filt. & $\underline{\text { STH}}$ & DF & Lab & $\underline{2099}$ & Mod & Filt. & ST $\underline{H}$ & DE & Lab & Unit \\
\hline $\begin{array}{l}\text { Aluminum, total recoverable } \\
\text { Antimony, total recoverable } \\
\text { Arsenic, total recoverable } \\
\text { Barium, total recoverable }\end{array}$ & $\begin{array}{l}37,000 \\
<0.20 \\
<60 \\
120\end{array}$ & $\begin{array}{l}/ / \\
\mathrm{u} / / \\
\mathrm{u} / / \\
/ /\end{array}$ & $\begin{array}{l}<0.20 \\
<60\end{array}$ & & $\begin{array}{l}20 \\
1 \\
20 \\
1\end{array}$ & $\begin{array}{l}\text { GE } \\
\text { GE } \\
\text { GE } \\
\text { GE }\end{array}$ & 9,700 & $\mathrm{~J} / \mathrm{K} / \mathrm{I}$ & NDD & & 1 & GE & $\begin{array}{l}\mu g h \\
\mu g h \\
\mu g h \\
\mu g h\end{array}$ \\
\hline Beryllium, total recoverable & & & & & & & & & & & & & \\
\hline $\begin{array}{l}\text { Cadmium, total recoverable } \\
\text { Chromium, total recoverable }\end{array}$ & $\begin{array}{l}9.8 \\
<3.0\end{array}$ & $\mathrm{U} / /$ & $<3.0$ & $\mathbf{a}$ & $\begin{array}{l}1 \\
1\end{array}$ & GE & 4.5 & II. & & & 1 & GE & $\mu g h$ \\
\hline Cobait, total recoverable & 12 & $\|$ & & $\mathbf{0}$ & 1 & GE & & & & & & & pon \\
\hline Copper, total recoverable & 41 & $/ 1$ & & & 1 & GE & & & & & & & \\
\hline Cyanide & $<10$ & $\mathrm{U} / \mathrm{f}$ & $<10$ & & 1 & GE & & & & & & & \\
\hline Lead, total recoverable & 1.4 & $J / V$ & NDD & & 1 & GE & $<20$ & $\mathrm{U} / \prime$ & $<20$ & & 10 & GE & \\
\hline Mercury, total recoverable & 3.7 & 11 & & $\mathbf{\square}$ & 1 & GE & & & & & & & \\
\hline $\begin{array}{l}\text { Nickel, total recoverable } \\
\text { Nitrate as nitrogen }\end{array}$ & 15 & $/ 1$ & & & 1 & GE & & & & & & & \\
\hline Nitrate-nitrite as nitrogen & 63,000 & 11 & & $\mathbf{\square}$ & 25 & GE & 29,000 & $/ /$ & & $\mathbf{\square}$ & 25 & GE & \\
\hline Selenium, total recoverable & $<100$ & $\mathrm{U} / /$ & $<100$ & & 20 & GE & & & & & & & \\
\hline Silver, total recoverable & $<1.0$ & $\mathrm{U} / /$ & $<1.0$ & & 1 & GE & & & & & & & \\
\hline Thallium, total recoverable & 0.30 & $\mathrm{~J} / \mathrm{V}$ & NDD & & 1 & GE & & & & & & & \\
\hline Vanadium, total recoverable & $\begin{array}{l}<10 \\
46\end{array}$ & $\mathrm{U} / 1$ & $<10$ & & 1 & GE & & & & & & & \\
\hline Linc, total recoverable & 46 & & & & 1 & & & & & & & & \\
\hline
\end{tabular}

Organic Constituents

\begin{tabular}{|c|c|c|c|c|c|c|c|c|c|c|}
\hline Constituents & 1099 & Mod & Fill. & STH & $\underline{D F}$ & Lab 2099 & Mod & Filt. & ST H DE Lab & Unit \\
\hline Benzene & $<1.0$ & JUЛO & $<1.0$ & & 1 & GE & & & & $\mu g$ \\
\hline Bis(2-ethylhexyl) phthalate & $<9.9$ & $\mathrm{U} / 1$ & $<9.9$ & & 1 & $\mathrm{GE}$ & & & & $\mu g /$ \\
\hline Dichloromethane & $<5.0$ & JUルO & $<5.0$ & & 1 & GE & & & & $\mu g h$ \\
\hline Phenols & $<5.0$ & $\mathrm{U} / 1$ & $<5.0$ & & $i$ & $\overrightarrow{G E}$ & & & & $\mu g /$ \\
\hline Tetrachloroethylene & $<1.0$ & JU/LO & $<1.0$ & & 1 & $\mathrm{GE}$ & & & & $\mu g /$ \\
\hline Trichloroethylene & $<1.0$ & SUNUO & $<1.0$ & & 1 & GE & & & & $\mu g r$ \\
\hline Trichlorofluoromethane & $<5.0$ & JUん/O & $<5.0$ & & 1 & GE & & & & \\
\hline
\end{tabular}

Notes:

- = exceeded holding time

= exceeded groundwater protection or monitoring constituent standard (See Appendix A.)

NA - Not applicable. Applies to beta dose and sum of betas if there are no beta-emitting radionuclides above detection limits; to sum of alphas if there are no alphaemitting radionuclides above detection limits; and to total radium if neither radium-226 or radium-228 was above detection limit

UAZ_UTRA - Upper Aquifer Zone of the Upper Three Runs Aquifer; LAZ_UTRA - Lower Aquifer Zone of the Upper Three Runs Aquifer, Gordon - Gordon Aquifer 


\section{WELL FSB 77 (cont.)}

Radioactive Constituents

\begin{tabular}{|c|c|c|c|c|c|c|c|c|c|c|c|c|c|}
\hline Constituents & 1099 & Mod & Filt. & STH & $\underline{D F}$ & Lab & 2099 & Mod & Fill. & ST $\underline{H}$ & DF & Lab & Unit \\
\hline Americium-241 & $1.4 E+01$ & $\|$ & & & 1 & GP & & & & & & & pCin \\
\hline Beta dose & 206 & & & च & & & & & & & & & \\
\hline Cabon-14 & $1.2 E+02$ & JUCl & NDD & & 1 & GP & & & & & & & pCi凡 \\
\hline Cesium-137 & $<-9.9 E-01$ & $\mathbf{U} / /$ & $<3.5 E+\infty$ & & 1 & GP & & & & & & & pein \\
\hline Cobalt- 60 & $<5.3 E-01$ & $\mathbf{U} / /$ & $<4.0 E+00$ & & 1 & GP & & & & & & & pcin \\
\hline Curium-242 & $<2.4 E-01$ & $U / /$ & $<2.7 \mathrm{E}-01$ & & 1 & GP & & & & & & & pCin \\
\hline Curium-243/244 & $5.2 E+\infty 0$ & $/ 1$ & & & 1 & GP & & & & & & & pcin \\
\hline Curium-245/246 & $1.1 E+\infty 0$ & $R / / 4$ & Rej & & 1 & GP & & & & & & & pein \\
\hline Gross alpha & $7.2 E+02$ & $\mathrm{~J} / \mathrm{II}$ & NDD & & 1 & GP & $3.8 E+02$ & /I & & $\mathbf{u}$ & 1 & GP & $\mathrm{pCi} \Omega$ \\
\hline lodine-129 & $1.3 E+02$ & $/ 1$ & & $\mathbf{m}$ & 1 & GP & & & & & & & $\mathrm{pCi} \Omega$ \\
\hline Nonvolatile beta & $1.2 E+03$ & $\mathrm{~J} / \mathrm{U}$ & NDD & & 1 & GP & $6.5 E+02$ & $/ 1$ & & $\mathbf{0}$ & 1 & GP & $\mathrm{pCi} \Omega$ \\
\hline Plutonium-238 & $<8.6 E-02$ & $\mathrm{U} / 1$ & $<2.4 E-01$ & & 1 & GP & & & & & & & $\mathrm{pCir}$ \\
\hline Plutonium-239/240 & $<1.7 E-02$ & $U / I$ & $<1.3 E-01$ & & 1 & GP & & & & & & & pCir \\
\hline Radium-226 & $2.0 E+01$ & J/II & NDD & & 1 & GP & & & & & & & pCin \\
\hline $\begin{array}{l}\text { Radium-228 } \\
\text { Total radium }\end{array}$ & $\begin{array}{l}1.1 E+01 \\
1.1 E+01\end{array}$ & 11 & & 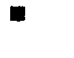 & 1 & GP & & & & & & & $\mathrm{pCi} / \mathrm{L}$ \\
\hline Strontium-90 & $5.9 E+02$ & $\|$ & & E & 1 & GP & & & & & & & pCir \\
\hline Technetium-99 & 9.1E+01 & $\|$ & & $\mathbf{E}$ & 1 & GP & & & & & & & $\mathrm{pCu}$ \\
\hline Thorium-228 & 4.7E-01 & $\mathrm{J} / \mathrm{W}$ & NDD & & 1 & GP & & & & & & & pCin \\
\hline Thorium-230 & $6.3 E-01$ & $\mathrm{~J} / \mathrm{V} /$ & NDD & & 1 & GP & & & & & & & pCin \\
\hline Thorium-232 & $<-2,4 \mathrm{E}-02$ & $U / I$ & $<1.8 E-01$ & & 1 & GP & & & & & & & pCir \\
\hline Sum of alphas & 5.7E+02 & & & 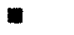 & & & & & & & & & pCin \\
\hline Sum of betas & $8.3 E+02$ & & & $\mathbf{0}$ & & & & & & & & & $\mathrm{pCi}$ \\
\hline Tritium & $2.2 E+03$ & II & & $\mathbf{e}$ & 1 & GP & $7.1 E+02$ & $\| l$ & & $\mathbf{\square}$ & 1 & GP & pCi/mL \\
\hline Uranium-233/234 & $2.5 E+02$ & /I & & 口 & 1 & GP & & & & & & & pCin \\
\hline Uranium-235 & $2.5 E+01$ & /I & & $\mathbf{2}$ & 1 & GP & & & & & & & pCil \\
\hline Uranium-238 & 2.7E+02 & /I & & $\mathbf{n}$ & 1 & GP & & & & & & & pCil \\
\hline
\end{tabular}

Notes:

- exceeded holding time

= exceeded groundwater protection or monitoring constituent standard (See Appendix A.)

NA - Not applicable. Applies to beta dose and sum of betas if there are no beta-emitting radionuclides above detection limits; to sum of alphas if there are no alphaemitting radionuclides above detection limits; and to total radium if neither radium-226 or radium-228 was above detection limit

UAZ_UTRA - Upper Aquifer Zone of the Upper Three Runs Aquifer; LAZ_UTRA - Lower Aquifer Zone of the Upper Three Runs Aquifer, Gordon - Gordon Aquifer 


\section{WELL FSB 78}

$\begin{array}{ll}\text { SRS Coord. } & \text { Lathongitude } \\ \text { N74764.0 } & 33.272978^{\circ} \mathrm{N} \\ \text { E50164.7 } & 81.679932^{\circ} \mathrm{W}\end{array}$

SAMPLE DATE

$01 / 11 / 99$

Screen Zone Elevation

$217.7-187.7 \mathrm{ft} \mathrm{ms}$
Top of Casing

$272.6 \mathrm{ft} \mathrm{msi}$

Casing Pump
4" PVC $v$

Screen Zone

UAZ_UTRA

\section{FIELD DATA}

\section{Constituents}

Water elevation

$\mathrm{pH}$

Sp. conductance

Water temperature

Alkalinity as $\mathrm{CaCO} 3$

Turbidity

Volumes purged

Sampling code

Synchronous water level
1Q99

209.2

3.9

1000

20.3

$208.8(03 / 23 / 99)$
04/07/99

2099
208.6
3.3
990
20.4
0
0
2.8

$207.7(06 / 25 / 99)$
Unit

ft $\mathrm{ms}$

pH

$\mu \mathrm{S} / \mathrm{cm}$

${ }^{\circ} \mathrm{C}$

mgl

well volumes

It msl

\section{ANALYTICAL DATA}

\section{Inorganic Constituents}

\begin{tabular}{|c|c|c|c|c|c|c|c|c|c|c|c|c|c|}
\hline Constituents & 1099 & Mod & Filt. & STH & $\underline{D F}$ & $\underline{L a b}$ & 2099 & Mod & Filt. & ST $\underline{H}$ & DE & Lab & Unit \\
\hline Aluminum, total recoverable & 21,000 & $/ /$ & & & 1 & GE & 22,000 & $\mathrm{~J} / \mathrm{K} / \mathrm{I}$ & NDD & & 1 & GE & $\mu g /$ \\
\hline Antimony, total recoverable & $<0.20$ & U $/ \prime$ & $<0.20$ & & 1 & GE & & & & & & & \\
\hline Arsenic, total recoverable & $<3.0$ & $\mathrm{U} / /$ & $<3.0$ & & 1 & GE & & & & & & & $\mu g h$ \\
\hline $\begin{array}{l}\text { Barium, total recoverable } \\
\text { Beryllium, total recoverable }\end{array}$ & 470 & $\|$ & & & 1 & GE & & & & & & & $\mu \mathrm{g} / \mathrm{L}$ \\
\hline Cadmium, total recoverable & 21 & $\|$ & & $\mathbf{\square}$ & 1 & GE & 18 & $\|$ & & $\mathbf{0}$ & 1 & GE & $\mu g h$ \\
\hline Chromium, total recoverable & 2.0 & $J / V$ & NDD & & 1 & GE & & & & & & & \\
\hline Cobalt, total recoverabie & 13 & $\|$ & & $\square$ & 1 & GE & & & & & & & $g$ \\
\hline Copper, total recoverable & 44 & $/ /$ & & & 1 & GE & & & & & & & $g /$ \\
\hline Cyanide & $<10$ & $\mathrm{U} / /$ & $<10$ & & 1 & GE & & & & & & & \\
\hline Lead, total recoverable & 0.96 & $\mathrm{~J} / \mathrm{l} /$ & NDD & & 1 & GE & $<20$ & $\mathbf{U} / /$ & $<20$ & & 10 & GE & $g / 2$ \\
\hline Mercury, total recoverable & 0.40 & 11 & & & 1 & GE & & & & & & & \\
\hline $\begin{array}{l}\text { Nickel, total recoverable } \\
\text { Nitrate as nitrogen }\end{array}$ & 17 & ll & & & 1 & GE & & & & & & & $\mathbf{L}$ \\
\hline Nitrate-nitrite as nitrogen & 12,000 & $\mathrm{~J} / \mathrm{Q} Q / 1$ & NDD & - & 25 & GE & 120,000 & $\|$ & & - & 50 & GE & gh \\
\hline Selenium, total recoverable & $<5.0$ & $\mathrm{U} / /$ & $<5.0$ & & 1 & GE & & & & & & & \\
\hline Silver, total recoverable & $<1.0$ & U/I & $<1.0$ & & 1 & $\overline{G E}$ & & & & & & & $\mathrm{dg}$ \\
\hline Thallium, total recoverable & 0.37 & $\mathrm{~J} / \mathrm{V}$ & NDD & & 1 & GE & & & & & & & $\mu g h$ \\
\hline Vanadium, total recoverable & $<10$ & $\mathrm{U} / /$ & $<10$ & & 1 & GE & & & & & & & $\mu g /$ \\
\hline Zinc, total recoverable & 97 & $\|$ & & & 1 & GE & & & & & & & \\
\hline \multicolumn{14}{|l|}{ Organic Constituents } \\
\hline & & & & & & & & & & & & & \\
\hline Constituents & 1099 & Mod & Filt. & ST & $\underline{\text { DF }}$ & Lab & 2099 & Mod & Filt. & ST $\mathrm{H}$ & DF & Lab & Unit \\
\hline Benzene & $<1.0$ & JU/L & $<1.0$ & & 1 & GE & & & & & & & $\lg h$ \\
\hline Bis(2-ethylhexyl) phthalate & $<10$ & & $<10$ & & 1 & $\mathrm{GE}$ & & & & & & & gو \\
\hline Dichloromethane & $<5.3$ & JU/L/O8 & $<5.0$ & & 1 & GE & & & & & & & $\mu g /$ \\
\hline Phenols & $<5.0$ & $U / I$ & $<5.0$ & & 1 & GE & & & & & & & Hgh \\
\hline Tetrachloroethylene & $<1.0$ & JU/LOO & $<1.0$ & & 1 & GE & & & & & & & $\mu g / L$ \\
\hline Trichloroethylene & $<1.0$ & JUนUO & $<1.0$ & & 1 & GE & & & & & & & \\
\hline Trichlorofluoromethane & $<5.0$ & JUЛ & $<5.0$ & & 1 & $\overline{G E}$ & & & & & & & \\
\hline
\end{tabular}

Notes:

- exceeded holding time

= exceeded groundwater protection or monitoring constituent standard (See Appendix A.)

NA - Not applicable. Applies to beta dose and sum of betas if there are no beta-emitting radionuclides above detection limits; to sum of alphas if there are no alphaemitting radionuclides above detection limits; and to total radium if neither radium-226 or radium-228 was above detection limit

UAZ_UTRA - Upper Aquifer Zone of the Upper Three Runs Aquifer, LAZ UTRA - Lower Aquifer Zone of the Upper Three Runs Aguifer, Gordon - Gordon Aquifer 
WELLL FSB 78 (cont.)

Radioactive Constituents

\begin{tabular}{|c|c|c|c|c|c|c|c|c|c|c|c|c|c|}
\hline Constituents & 1099 & Mod & Fill: & STH & $\underline{D F}$ & Lab & 2029 & Mod & Filt. & ST $\mathrm{H}$ & DE & Lab & Unit \\
\hline Americium-241 & $2.7 E+01$ & II & & 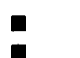 & 1 & GP & & & & & & & pCil \\
\hline $\begin{array}{l}\text { Beta dose } \\
\text { Carbon-14 }\end{array}$ & $1.2 E+02$ & $\mathrm{~J} / \mathrm{Cl}$ & NDD & & 1 & GP & & & & & & & pCin \\
\hline Cesium-137 & $1.9 E+02$ & & & - & 1 & GP & & & & & & & pcin \\
\hline Cobalt-60 & $<1.6 \mathrm{E}+\infty$ & $\mathrm{U} / \mathrm{I}$ & $<4.1 E+\infty$ & & 1 & $G P$ & & & & & & & pCin \\
\hline Curium-242 & $<-7.8 E-04$ & $u / /$ & $<2.4 E-01$ & & 1 & GP & & & & & & & pCin \\
\hline Curium-243/244 & $2.8 \mathrm{E}+01$ & $\|$ & & घ & 1 & GP & & & & & & & pCin \\
\hline Curium-245/246 & $5.8 \mathrm{E}-01$ & $R / / 4$ & Rej & & 1 & GP & & & & & & & pCin \\
\hline Gross alpha & $4.7 E+02$ & $\mathrm{~J} / \mathrm{L}$ & NDD & - & 1 & GP & $6.8 E+02$ & II & & - & 1 & GP & pCin \\
\hline $\begin{array}{l}\text { Iodine-129 } \\
\text { Nonvolatile beta }\end{array}$ & $\begin{array}{l}2.5 E+02 \\
1.6 E+03\end{array}$ & J/I & NDD & & 1 & GP & $1.9 E+03$ & // & & a & 1 & GP & pCin \\
\hline Plutonium-238 & $<8.3 E-02$ & U/I & $<2.2 E-01$ & & 1 & GP & & & & & & & pCin \\
\hline Plutonium-239/240 & $<9.1 E-03$ & $\mathrm{U} / /$ & $<1.2 E-01$ & & 1 & GP & & & & & & & pCin \\
\hline Radium-226 & $1.3 E+01$ & $\mathrm{~J} / \mathrm{L} /$ & NDD & & 1 & $G P$ & & & & & & & pCin \\
\hline Radium-228 & $2.9 E+01$ & 11 & & a & 1 & GP & & & & & & & pCin \\
\hline Total radium & $2.9 E+01$ & & & & & & & & & & & & \\
\hline Strontium-90 & $8.7 E+02$ & $\|$ & & a & 1 & GP & & & & & & & pCin \\
\hline Technetium-99 & $9.1 E+01$ & $\|$ & & a & 1 & GP & & & & & & & pCin \\
\hline Thorium-228 & $1.0 E+00$ & $\mathrm{~J} / U$ & NDD & & 1 & GP & & & & & & & pCin \\
\hline Thorium-230 & $3.0 E-01$ & $\mathrm{~J} / \mathrm{V}$ & NDD & & 1 & GP & & & & & & & pCin \\
\hline Thorium-232 & $<6.6 \mathrm{E}-02$ & UII & $<9.9 E-02$ & & 1 & GP & & & & & & & pCin \\
\hline Sum of alphas & $7.4 E+01$ & & & घ & & & & & & & & & pCin \\
\hline Sum of betas & $1.4 \mathrm{E}+03$ & & & घ & & & & & & & & & pCin \\
\hline Tritium & $3.5 E+03$ & /I & & a & 1 & GP & $3.8 E+03$ & $\|$ & & - & 1 & GP & $\mathrm{pCi} / \mathrm{mL}$ \\
\hline Uranium-233/234 & $1.5 E+02$ & J/KJO & NDD & & 1 & GP & & & & & & & pCin \\
\hline Uranium-235 & $2.0 E+01$ & & & - & 1 & $G P$ & & & & & & & pCin \\
\hline Uranium-238 & $4.8 E+02$ & $\mathrm{~J} / \mathrm{KJO}$ & NDD & & 1 & GP & & & & & & & pCin \\
\hline
\end{tabular}

Notes:

- exceeded holding time

- exceeded groundwater protection or monitoring constituent standard (See Appendix A.)

NA - Not applicable. Applies to beta dose and sum of betas if there are no beta-emitting radionuclides above detection limits; to sum of alphas if there are no alphaemitting radionuclides above detection limits; and to total radium if neither radium-226 or radium-228 was above detection limit

UAZ_UTRA - Upper Aquifer Zone of the Upper Three Runs Aquifer, LAZ_UTRA - Lower Aquifer Zone of the Upper Three Runs Aquifer, Gordon - Gordon Aquiler 


\section{WELL FSB 78A}

$\begin{array}{ll}\text { SRS Goord. } & \text { Lat/Longitude } \\ \text { N74757.7 } & 33.272977^{\circ} \mathrm{N} \\ \text { E50172.8 } & 81.679898^{\circ} \mathrm{W}\end{array}$

SAMPLE DATE

$01 / 07 / 99$

FIELD DATA

Constituents

1099

Water elevation

$\mathrm{pH}$

Sp. conductance

Water temperature

Alkalinity as $\mathrm{CaCO} 3$

Turbidity

Volumes purged

Sampling code

Synchronous water level

ANALYTICAL DATA

Inorganic Constituents

$\begin{array}{lllll}\text { Screen Zone Elevation } & \text { Top of Casing } & \text { Casing } & \text { Pump } & \text { Screen Zone } \\ 37.5-27.0 \mathrm{ft} \mathrm{msl} & 272.6 \mathrm{ft} \mathrm{msl} & 4^{*} \mathrm{PVC} & \mathrm{S} & \text { Gordon }\end{array}$

04/05/99

2099

156.6

6.2

110

20.7

32

3.0

$155.9(06 / 25 / 99)$
Unit

ft msl

pH

$15 / \mathrm{cm}$

${ }^{\circ} \mathrm{C}$

$\mathrm{mgl}$

NTU

well volumes

ft msi

\begin{tabular}{|c|c|c|c|c|c|c|c|c|c|c|c|c|c|}
\hline Constituents & 1099 & Mod & Filt. & STH & $\underline{\text { DF }}$ & Lab & 2099 & Mod & Filt. & ST H & DF & Lab & Unit \\
\hline Aluminum, total recoverable & 14 & $\mathrm{~J} / \mathrm{V}$ & NDD & & 1 & GE & 31 & /I & & & 1 & GE & $\mu g / 2$ \\
\hline Antimony, total recoverable & $<0.20$ & U/I & $<0.20$ & & 1 & GE & & & & & & & jgh \\
\hline Arsenic, total recoverable & $<3.0$ & U/I & $<3.0$ & & 1 & GE & & & & & & & $\mu g / L$ \\
\hline $\begin{array}{l}\text { Barium, total recoverable } \\
\text { Beryllium, total recoverable }\end{array}$ & 22 & 11 & & & 1 & GE & & & & & & & $\mu g /$ \\
\hline Cadmium, total recoverable & $<1.0$ & $\mathrm{u} / /$ & $<1.0$ & & 1 & GE & $<1.0$ & $U / I$ & $<1.0$ & & 1 & GE & $\mu g /$ \\
\hline Chromium, total recoverable & 0.93 & $J / V$ & NDD & & 1 & GE & & & & & & & \\
\hline Cobalt, total recoverable & $<0.14$ & $\mathrm{JU} / / 4$ & $<0.20$ & & 1 & GE & & & & & & & pgh \\
\hline $\begin{array}{l}\text { Copper, total recoverable } \\
\text { Cyanide }\end{array}$ & $\begin{array}{l}0.84 \\
4.6\end{array}$ & $\begin{array}{l}\mathrm{J} / \mathrm{V} \\
\mathrm{J} / \mathrm{IK} / \mathrm{I}\end{array}$ & $\begin{array}{l}\text { NDD } \\
\text { NDD }\end{array}$ & & $\begin{array}{l}1 \\
1\end{array}$ & $\begin{array}{l}\text { GE } \\
\text { GE }\end{array}$ & & & & & & & pron \\
\hline Lead, total recoverable & $<2.0$ & $u / I$ & $<2.0$ & & 1 & GE & $<2.0$ & $\mathbf{U} / /$ & $<2.0$ & & 1 & GE & $\mu g \Omega$ \\
\hline Mercury, total recoverable & $<0.20$ & $U / I$ & $<0.20$ & & 1 & $\mathrm{GE}$ & & & & & & & \\
\hline $\begin{array}{l}\text { Nickel, total recoverable } \\
\text { Nitrate as nitrogen }\end{array}$ & 2.9 & 11 & & & 1 & GE & & & & & & & \\
\hline Nitrate-nitrite as nitrogen & 330 & $\|$ & & & 1 & GE & 700 & // & & & 1 & GE & $\mu g h$ \\
\hline Selenium, total recoverable & $<5.0$ & $\mathrm{u} / /$ & $<5.0$ & & $i$ & GE & & & & & & & \\
\hline Silver, total recoverable & $<0.44$ & $\mathrm{JU} / / 4$ & $<1.0$ & & 1 & GE & & & & & & & $\mu g h$ \\
\hline Thallium, total recoverable & 0.89 & $J / V$ & NDD & & 1 & GE & & & & & & & $\mu g /$ \\
\hline Vanadium, total recoverable & $<10$ & $\mathrm{U} / 1$ & $<10$ & & 1 & GE & & & & & & & Hgl \\
\hline Zinc, total recoverable & $<10$ & $\mathrm{U} / I$ & $<10$ & & 1 & $\overline{\mathrm{GE}}$ & & & & & & & \\
\hline
\end{tabular}

Organic Constituents

\begin{tabular}{|c|c|c|c|c|c|}
\hline Constituents & 1099 & Mod & Filt. & ST브 & DF \\
\hline Benzene & $<1.0$ & $\mathbf{U} / /$ & $<1.0$ & & 1 \\
\hline Bis(2-ethylhexyl) phthalate & $<10$ & $\mathrm{U} / /$ & $<10$ & & 1 \\
\hline Dichloromethane & $<1.6$ & UNI & $<5.0$ & & 1 \\
\hline Phenols & $<5.0$ & $\mathrm{U} / 1$ & $<5.0$ & & 1 \\
\hline Tetrachloroethylene & $<1.0$ & $\mathbf{U} / l$ & $<1.0$ & & 1 \\
\hline Trichloroethyiene & $<1.0$ & $\mathrm{U} / /$ & $<1.0$ & & 1 \\
\hline Trichlorofluoromethane & $<5.0$ & $\mathrm{U} / /$ & $<5.0$ & & 1 \\
\hline
\end{tabular}

Mod Filt.

Filt. ST H DF Lab Unit

$\mu g /$

$\mu g / L$

$\mu g h$

$\mu g h$

$\mu g /$

$\mu g / L$ 
WELL FSB 78A (cont.)

Radioactive Constituents

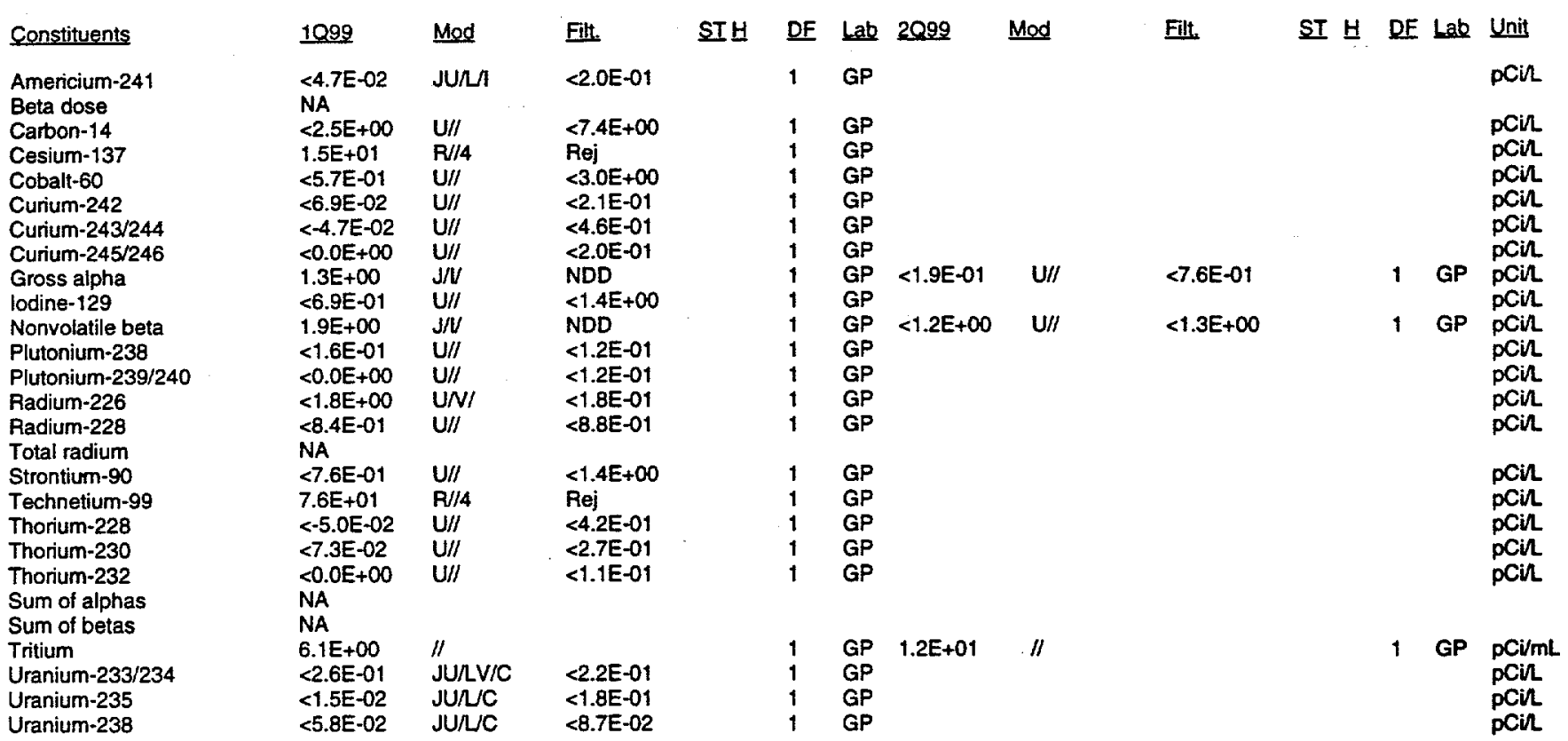

Notes:

= exceeded holding time

= exceeded groundwater protection or monitoring constituent standard (See Appendix A.)

NA - Not applicable. Applies to beta dose and sum of betas if there are no beta-emitting radionuclides above detection limits; to sum of alphas if there are no alphaemitting radionuclides above detection limits; and to total radium if neither radium-226 or radium-228 was above detection limit

UAZ UTRA - Upper Aquifer Zone of the Upper Three Runs Aquiler, LAZ_UTRA - Lower Aquifer Zone of the Upper Three Runs Aquifer, Gordon - Gordon Aquifer 


\section{WELL FSB 78B}

\begin{tabular}{|c|c|}
\hline SRS Coord. & Lat/Longitud \\
\hline $\begin{array}{l}\text { N74765.9 } \\
\text { E50178.8 }\end{array}$ & 81.67989 \\
\hline
\end{tabular}

SAMPLE DATE

Constituents

155.0

7.6

$\mathrm{pH}$

Sp. conductance

Water temperature

Alkalinity as $\mathrm{CaCO} 3$

Turtidity

Volumes purged

Sampling code

Synchronous water level
Screen Zone Elevation

$92.8-82.4 \mathrm{ft} \mathrm{ms}$
Top of Casing

$272.8 \mathrm{ft} \mathrm{msl}$
Casing Pump

4" PVC S
Screen Zone

Gordon
04/15/99

2099
154.8
6.9
240
20.5
69
0
3.5
$154.4(06 / 25 / 99)$

Unis

ft msl

pH

$\mathrm{S} / \mathrm{cm}$

${ }^{\circ} \mathrm{C}$

$\mathrm{mg} /$

NTU

well volumes

ft msl

\section{ANALYTICAL DATA}

Inorganic Constituents

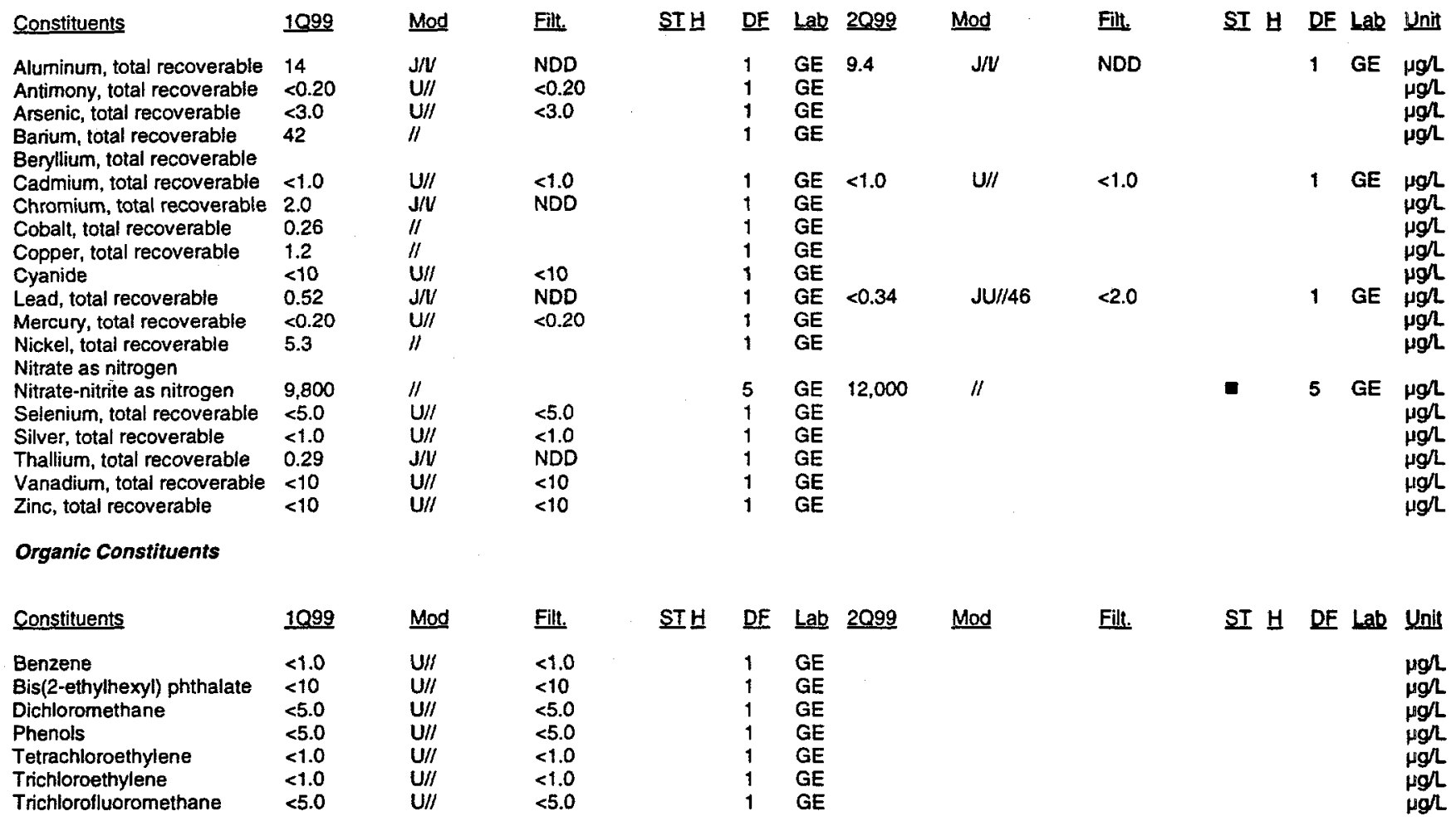

Notes:

- exceeded holding time

E = exceeded groundwater protection or monitoring constituent standard (See Appendix A.)

NA - Not applicable. Applies to beta dose and sum of betas if there are no beta-ernitting radionuclides above detection limits; to sum of alphas if there are no alphaemitting radionuclides above detection limits; and to total radium if neither radium-226 or radium-228 was above detection limit

UAZ UTRA - Upper Aquifer Zone of the Upper Three Runs Aquifer, LAZ UTRA - Lower Aquifer Zone of the Upper Three Runs Aquifer, Gordon - Gordon Aquifer 


\section{WELL FSB 78B (cont.)}

Radioactive Constituents

\begin{tabular}{|c|c|c|c|c|c|c|c|c|c|c|c|c|c|}
\hline Constituents & 1099 & Mod & Filt. & ST브 & DF & Lab & $\underline{2099}$ & Mod & Filt. & $\underline{S T} \underline{H}$ & $\mathrm{DF}$ & Lab & Unit \\
\hline $\begin{array}{l}\text { Americium-241 } \\
\text { Beta dose }\end{array}$ & $\begin{array}{l}<1.6 E-01 \\
\text { NA }\end{array}$ & JU/И & $<2.6 E-01$ & & 1 & GP & & & & & & & pCin \\
\hline Carbon-14 & $<6.6 E+\infty 0$ & $\mathrm{U} / /$ & $<7.4 \mathrm{E}+00$ & & 1 & GP & & & & & & & pCil \\
\hline Cesium-137 & $<4.6 E-01$ & JuILC & $<3.2 E+00$ & & 1 & GP & & & & & & & pCir \\
\hline Cobalt- 60 & $<2.6 E+00$ & U/I & $<3.7 E+00$ & & 1 & GP & & & & & & & $\mathrm{pCu}$ \\
\hline Curium-242 & $<0.0 E+00$ & $\mathrm{U} / 1$ & $<2.7 E-01$ & & 1 & GP & & & & & & & $\mathrm{pCi}$ \\
\hline Curium-243/244 & $<0.0 E+00$ & $\mathrm{U} / \prime$ & $<2.6 \mathrm{E}-01$ & & 1 & GP & & & & & & & $\mathrm{pCi}$ \\
\hline Curium-245/246 & $<8.7 E-02$ & U/I & $<2.6 \mathrm{E}-01$ & & 1 & GP & & & & & & & pCin \\
\hline Gross alpha & $2.6 \mathrm{E}+00$ & $/ 1$ & & & 1 & $\mathrm{GP}$ & $1.6 E+\infty 0$ & $\mathrm{~J} / \mathrm{V}$ & NDD & & 1 & GP & pCin \\
\hline lodine-129 & $1.5 \mathrm{E}+00$ & $\mathrm{~J} / \mathrm{L} /$ & NDD & & 1 & GP & & & & & & & pCin \\
\hline Nonvolatile beta & $3.1 E+\infty$ & /I & & & 1 & GP & $1.5 E+01$ & $\|$ & & & 1 & $\mathrm{TM}$ & pCil \\
\hline Plutonium-238 & $<0.0 \mathrm{E}+00$ & $\mathrm{U} / /$ & $<3.0 E-01$ & & 1 & GP & & & & & & & pCin \\
\hline Plutonium-239/240 & $<-5.1 E-02$ & $\mathrm{U} / 1$ & $<6.3 E-01$ & & 1 & GP & & & & & & & $\mathrm{pCi}$ \\
\hline Radium-226 & $1.1 E+\infty 0$ & $\mathrm{~J} / \mathrm{V}$ & NDD & & 1 & GP & & & & & & & $\mathrm{pCi}$ \\
\hline $\begin{array}{l}\text { Radium-228 } \\
\text { Total radium }\end{array}$ & $1.3 E+\infty$ & $\mathrm{J} / \mathrm{V}$ & NDD & & 1 & GP & & & & & & & pCin \\
\hline Strontium-90 & $<1.9 \mathrm{E}-01$ & $\mathrm{U} / 1$ & $<1.5 E+\infty 0$ & & 1 & GP & & & & & & & $\mathrm{pCin}$ \\
\hline Technetium-99 & 1.1E+02 & $\mathrm{R} / / 4$ & Rej & & 1 & GP & . & & & & & & pCir \\
\hline Thorium-228 & $<7.2 E-02$ & $\mathrm{u} / /$ & $<4.0 E-01$ & & 1 & GP & & & & & & & pcin \\
\hline Thorium-230 & $<2.6 \mathrm{E}-02$ & $\mathrm{U} / /$ & $<2.6 \mathrm{E}-01$ & & 1 & GP & & & & & & & $\mathrm{pCil}$ \\
\hline Thorium-232 & $<2.6 E-02$ & $\mathrm{U} / /$ & $<2.6 \mathrm{E}-01$ & & 1 & GP & & & & & & & pCin \\
\hline Sum of alphas & NA & & & & & & & & & & & & \\
\hline $\begin{array}{l}\text { Sum of betas } \\
\text { Tritium }\end{array}$ & $2.6 E+02$ & $1 /$ & & n & 1 & GP & $29 \mathrm{~F}+02$ & II & & & & 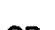 & \\
\hline Uranium-233/234 & $<2.3 E-01$ & JU/L/C & $<2.8 E-01$ & & 1 & GP & & $n$ & & 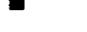 & 1 & $G P$ & pCirmt \\
\hline Uranium-235 & $<7.8 E-02$ & JU/L/C & $<2.4 E-01$ & & $i$ & GP & & & & & & & pCin \\
\hline Uranium-238 & $<8.6 E-02$ & $\mathrm{JU} / \mathrm{L} / \mathrm{C}$ & $<2.1 E-01$ & & 1 & GP & & & & & & & pCir \\
\hline
\end{tabular}




\section{WELL FSB 78C}

\begin{tabular}{|c|c|c|c|c|c|c|}
\hline SRS Coond. & LatLongitude & Screen Zone Elevation & Top of Casing & Casing & Pump & Screen Zone \\
\hline $\begin{array}{l}\text { N74772.5 } \\
E 50170.2\end{array}$ & $\begin{array}{l}33.273006^{\circ} \mathrm{N} \\
81.679934^{\circ} \mathrm{W}\end{array}$ & $151.4-141.6 \mathrm{ft} \mathrm{msl}$ & $273.5 \mathrm{ft} \mathrm{msl}$ & 4" PVC & $v$ & LAZ_UTRA \\
\hline \multicolumn{2}{|c|}{ SAMPLE DATE } & $01 / 13 / 99$ & \multicolumn{2}{|l|}{$04 / 09 / 99$} & & \\
\hline \multicolumn{3}{|l|}{ FIELD DATA } & & & & \\
\hline \multicolumn{2}{|l|}{ Constituents } & 1099 & $\underline{2099}$ & & & Unit \\
\hline \multicolumn{2}{|c|}{$\begin{array}{l}\text { Water elevation } \\
\text { pH } \\
\text { Sp. conductance } \\
\text { Water temperature } \\
\text { Alkalinity as CaCO3 } \\
\text { Turidity } \\
\text { Volumes purged } \\
\text { Sampling code } \\
\text { Synchronous water level }\end{array}$} & $\begin{array}{l}207.9 \\
4.2 \\
2000 \\
20.3 \\
0 \\
1 \\
0.023 \\
\times \\
205.1(03 / 23 / 99)\end{array}$ & $\begin{array}{l}206.3 \\
4.1 \\
2100 \\
20.9 \\
0 \\
2 \\
0.024 \\
X \\
207.4(06 / 25 / 99)\end{array}$ & & & $\begin{array}{l}\text { ft msl } \\
\mathrm{pH} \\
\mu \mathrm{S} / \mathrm{cm} \\
{ }^{\circ} \mathrm{C} \\
\mathrm{mgh} \\
\text { NTU } \\
\text { well volumes } \\
\text { ft msl }\end{array}$ \\
\hline
\end{tabular}

ANALYTICAL DATA

Inorganic Constituents

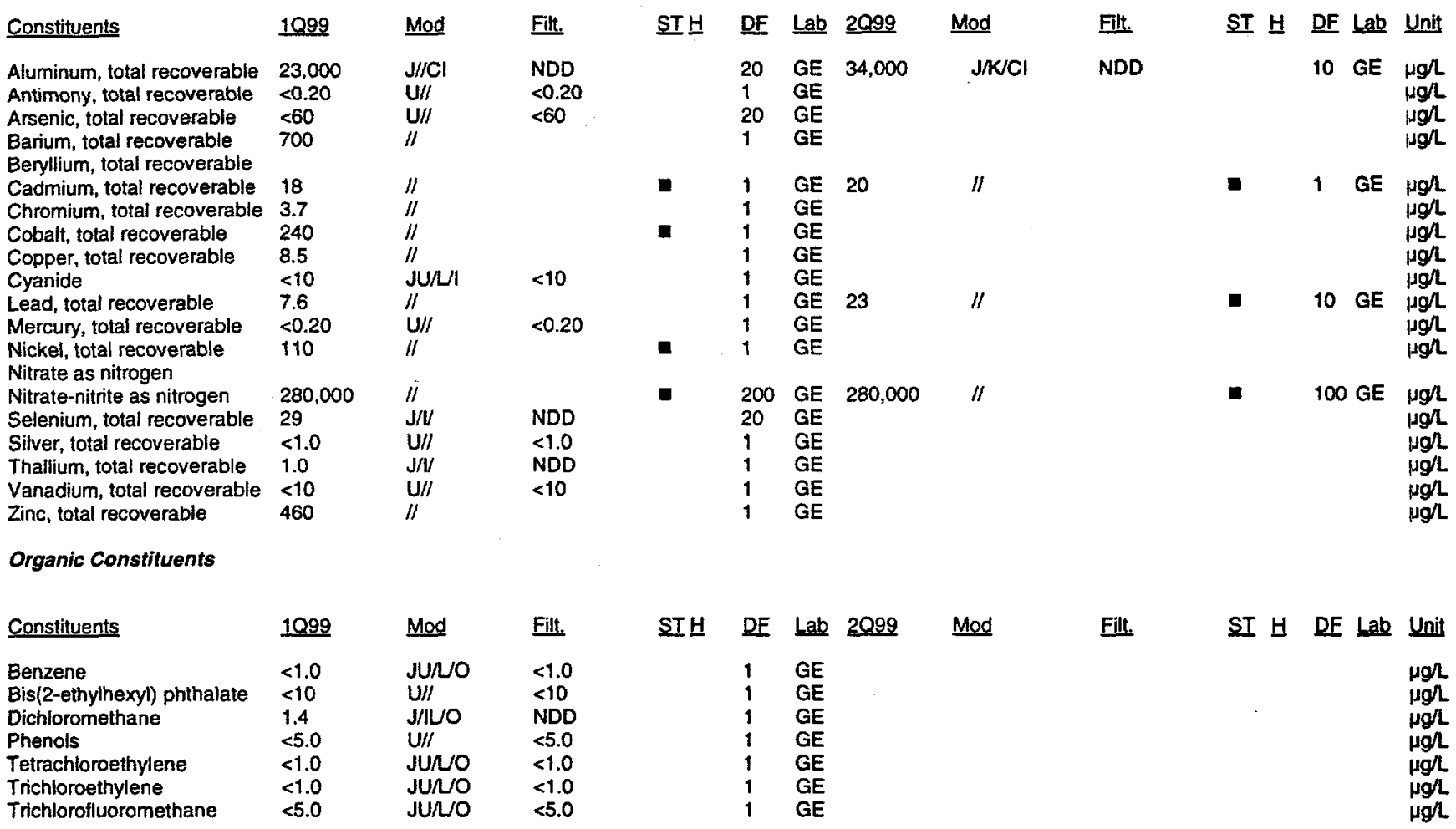

\section{Notes:}

- exceeded holding time

- = exceeded groundwater protection or monitoring constituent standard (See Appendix A.)

NA - Not applicable. Applies to beta dose and sum of betas if there are no beta-emitting radionuclides above detection limits; to sum of alphas if there are no alphaemitting radionuclides above detection limits; and to total radium if neither radium-226 or radium-228 was above detection limit 
WELL FSB $78 C$ (cont.)

Radioactive Constituents

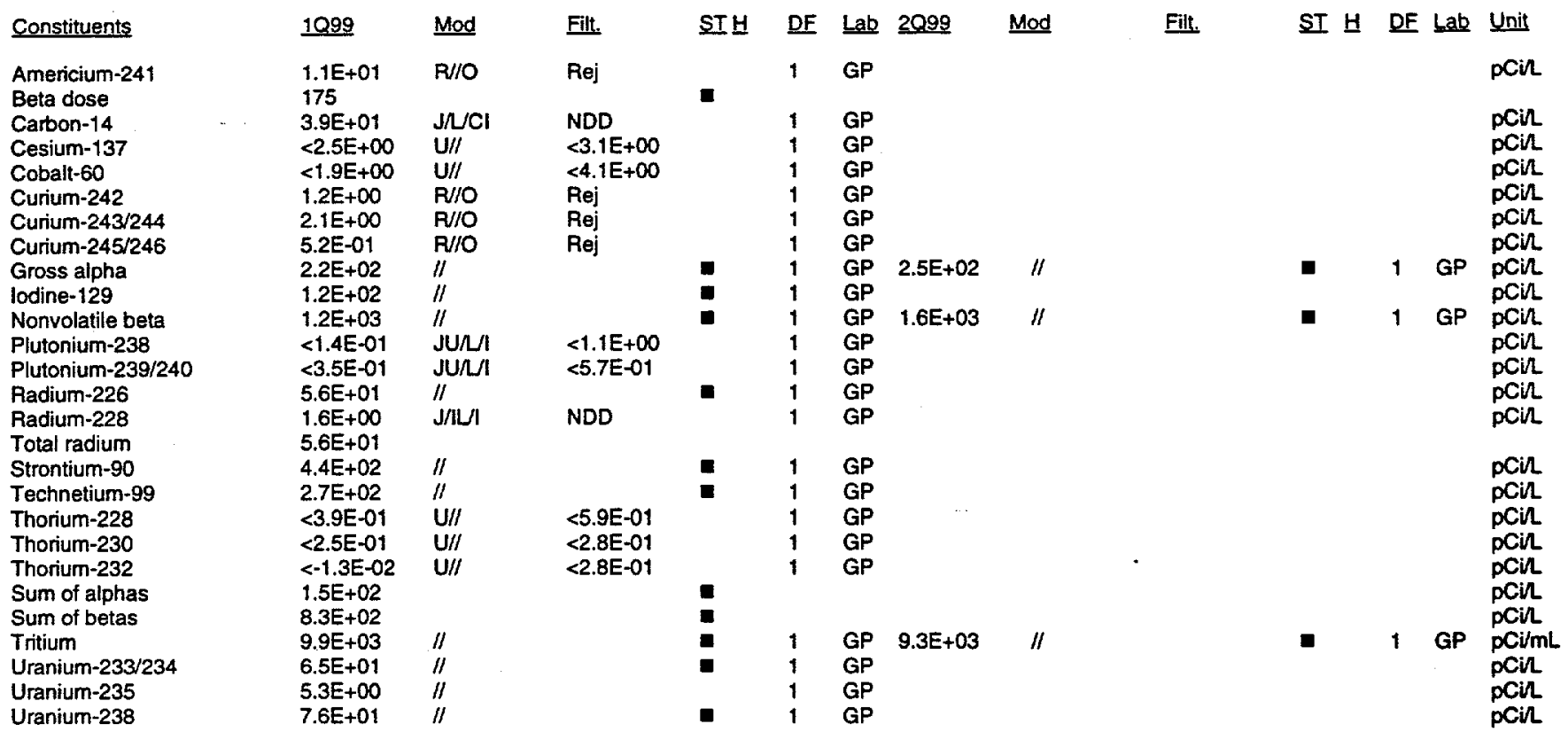


WELL FSB 79

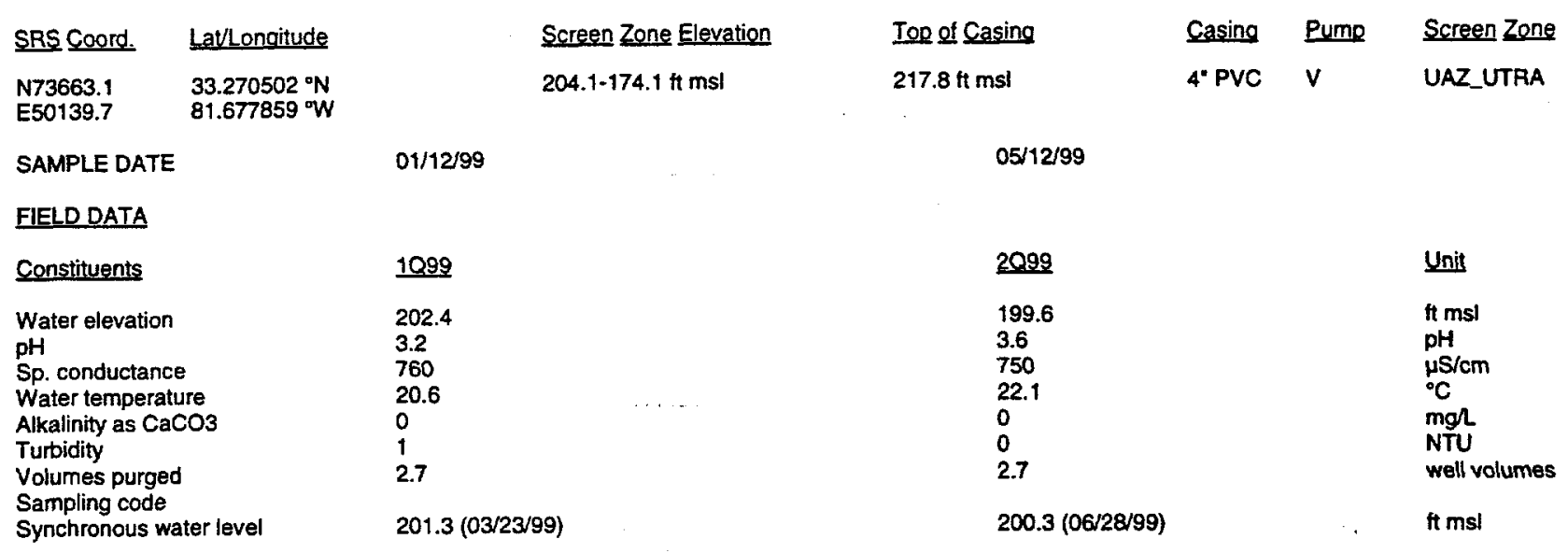

\section{ANALYTICAL DATA}

Inorganic Constituents

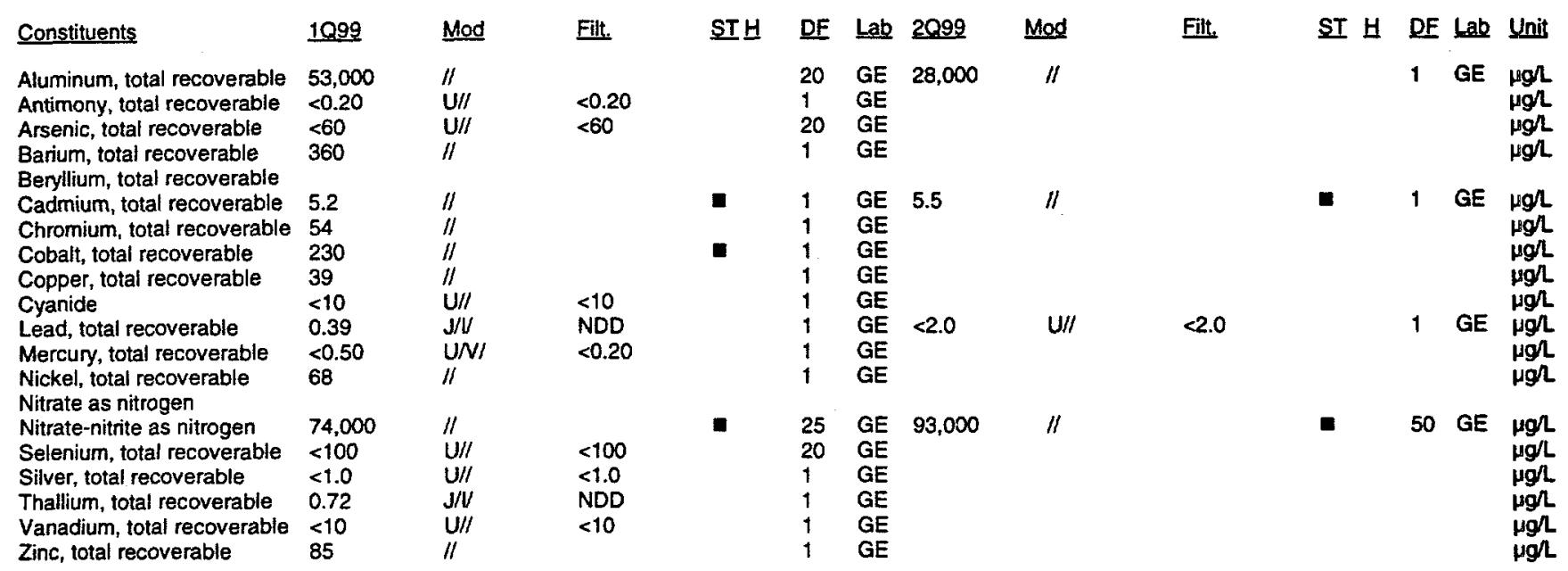

Organic Constituents

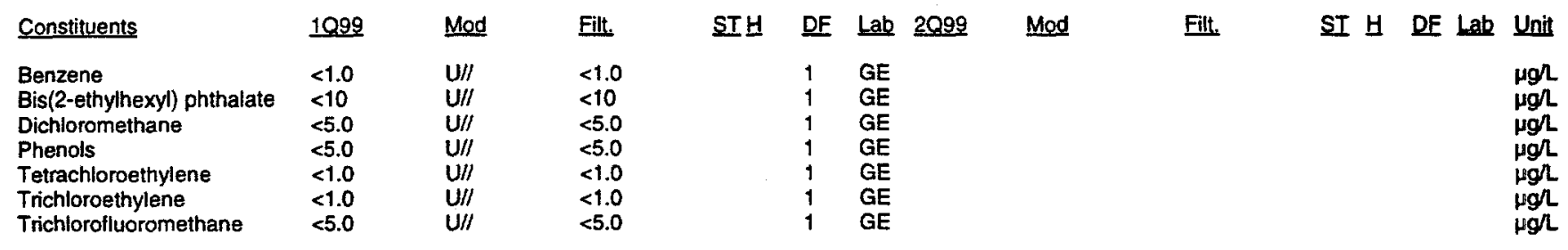

\section{Notes:}

= exceeded holding time

= exceeded groundwater protection or monitoring constituent standard (See Appendix A.)

NA - Not applicable. Applies to beta dose and sum of betas if there are no beta-emitting radionuclides above detection limits; to sum of alphas if there are no alphaemitting radionuclides above detection limits; and to total radium if neither radium-226 or radium-228 was above detection limit

UAZ_UTRA - Upper Aquifer Zone of the Upper Three Runs Aquifer, LAZ_UTRA - Lower Aquifer Zone of the Upper Three Runs Aquifer, Gordon - Gordon Aquifer 
WELL FSB 79 (cont.)

Radioactive Constituents

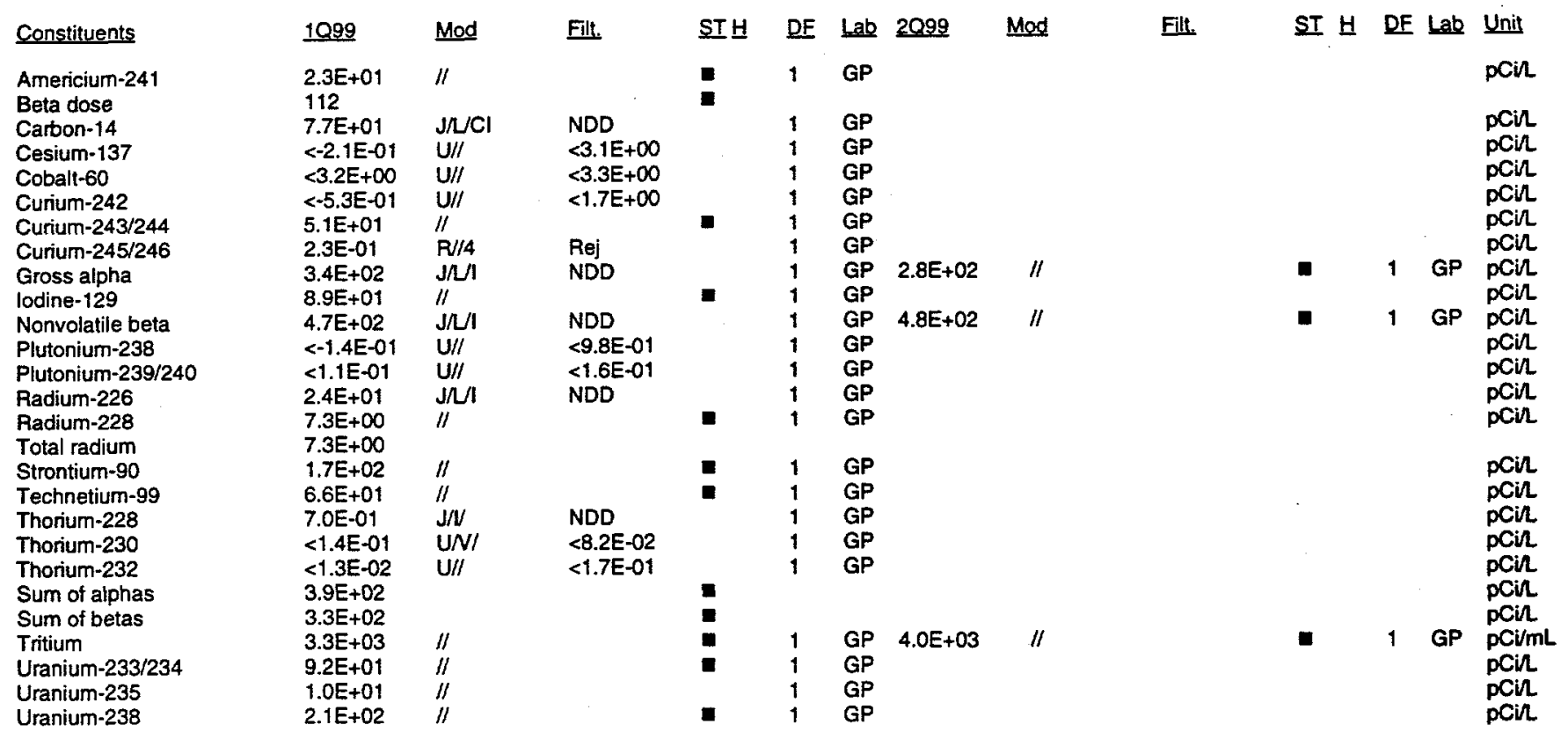

Notes:

- = exceeded holding time

= exceeded groundwater protection or monitoring constituent standard (See Appendix A.)

NA - Not applicable. Applies to beta dose and sum of betas if there are no beta-ernitting radionuclides above detection limits; to sum of alphas if there are no alphaemitting radionuclides above detection limits; and to total radium if neither radium-226 or radium-228 was above detection limit

UAZ_UTRA - Upper Aquifer Zone of the Upper Three Runs Aquifer, LAZ_UTRA - Lower Aquifer Zone of the Upper Three Runs Aquifer, Gordon - Gordon Aquifer 
WELL FSB 79A

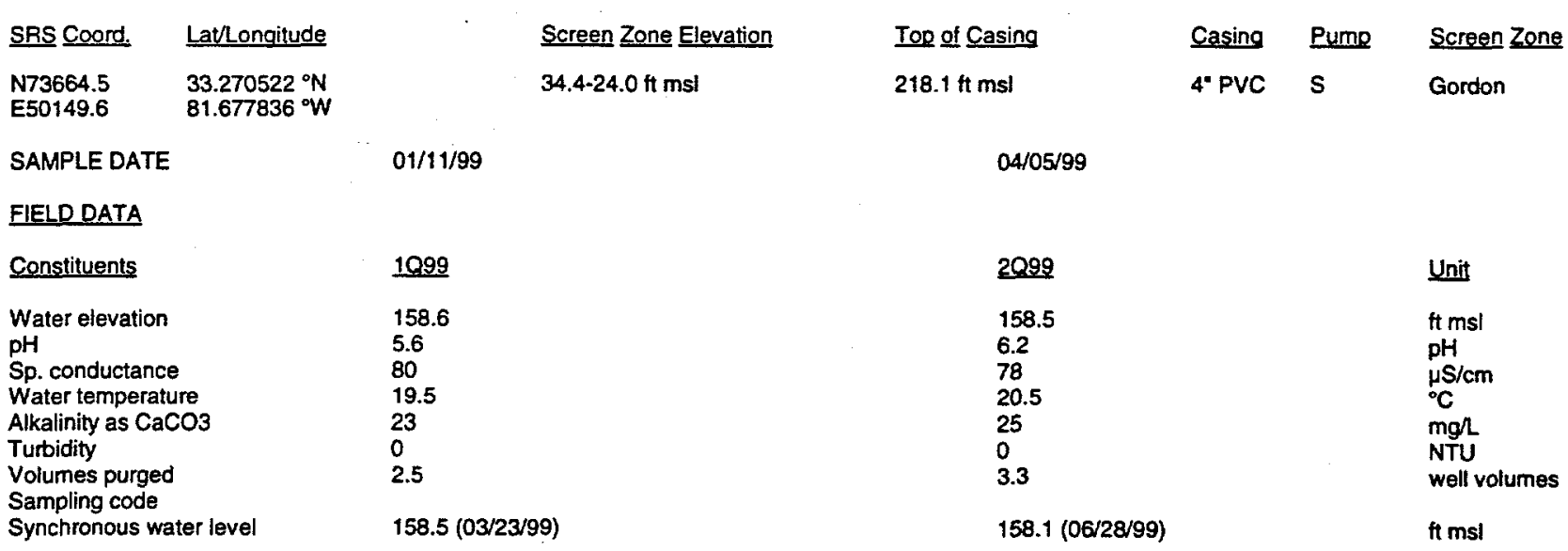

\section{ANALYTICAL DATA}

Inorganic Constituents

\begin{tabular}{|c|c|c|c|c|c|c|c|c|c|c|c|c|c|}
\hline Constituents & 1099 & Mod & Filit. & ST브 & DF & $\underline{\text { Lab }}$ & 2099 & Mod & Filt. & ST H & DF & Lab & Unit \\
\hline $\begin{array}{l}\text { Aluminum, total recoverable } \\
\text { Antimony, total recoverable } \\
\text { Arsenic, total recoverable } \\
\text { Barium, total recoverable } \\
\text { Beryllium, total recoverable }\end{array}$ & $\begin{array}{l}15 \\
<0.20 \\
<3.0 \\
23\end{array}$ & $\begin{array}{l}\mathrm{J} / \mathrm{U} \\
\mathrm{U} / \prime \\
\mathrm{U} / \prime\end{array}$ & $\begin{array}{l}\text { NDD } \\
<0.20 \\
<3.0\end{array}$ & & $\begin{array}{l}1 \\
1 \\
1 \\
1\end{array}$ & $\begin{array}{l}\text { WA } \\
\text { GE } \\
\text { GE } \\
\text { WA }\end{array}$ & $<15$ & $\mathrm{U} / /$ & $<15$ & & 1 & GE & $\begin{array}{l}\mu g h \\
\mu g h \\
\mu g h \\
\mu g h\end{array}$ \\
\hline $\begin{array}{l}\text { Cadmium, total recoverable } \\
\text { Chromium, total recoverable } \\
\text { Cobalt, total recoverable } \\
\text { Copper, total recoverable } \\
\text { Cyanide }\end{array}$ & $\begin{array}{l}<1.0 \\
1.3 \\
<0.048 \\
<0.96 \\
<10\end{array}$ & $\begin{array}{l}\mathrm{U} / / \\
\mathrm{J} / / / \\
\mathrm{JU} / / 4 \\
\mathrm{U} / / 6 \\
\mathrm{U} / /\end{array}$ & $\begin{array}{l}<1.0 \\
\text { NDD } \\
<0.20 \\
<1.0 \\
<10\end{array}$ & & $\begin{array}{l}1 \\
1 \\
1 \\
1 \\
1\end{array}$ & $\begin{array}{l}\text { GE } \\
\text { WA } \\
\text { GE } \\
\text { GE } \\
\text { GE }\end{array}$ & $<1.0$ & U/I & $<1.0$ & & 1 & GE & $\begin{array}{l}\mu g h \\
\mu g h \\
\mu g / l \\
\mu g / \\
\mu g /\end{array}$ \\
\hline $\begin{array}{l}\text { Lead, total recoverable } \\
\text { Mercury, total recoverable } \\
\text { Nickel, total recoverabie } \\
\text { Nitrate as nitrogen }\end{array}$ & $\begin{array}{l}<2.0 \\
<0.20 \\
<2.0\end{array}$ & $\begin{array}{l}U / / \\
U / / \\
U / /\end{array}$ & $\begin{array}{l}<2.0 \\
<0.20 \\
<2.0\end{array}$ & & $\begin{array}{l}1 \\
1 \\
1\end{array}$ & $\begin{array}{l}\text { GE } \\
\text { GE } \\
\text { GE }\end{array}$ & $<2.0$ & $\mathbf{U} / /$ & $<2.0$ & & 1 & GE & $\begin{array}{l}\mu g / L \\
\mu g / L \\
\mu g / L\end{array}$ \\
\hline $\begin{array}{l}\text { Nitrate-nitrite as nitrogen } \\
\text { Selenium, total recoverable } \\
\text { Silver, total recoverable } \\
\text { Thallium, total recoverable } \\
\text { Vanadium, total recoverable } \\
\text { Zinc, total recoverable }\end{array}$ & $\begin{array}{l}1,200 \\
<5.0 \\
<1.0 \\
<0.55 \\
<6.9 \\
<10\end{array}$ & $\begin{array}{l}I / 1 \\
U / / \\
U / / 6 \\
U / I \\
U / I\end{array}$ & $\begin{array}{l}<5.0 \\
<1.0 \\
<2.5 \\
<6.9 \\
<10\end{array}$ & & $\begin{array}{l}1 \\
1 \\
1 \\
1 \\
1 \\
1\end{array}$ & $\begin{array}{l}\text { GE } \\
\text { GE } \\
\text { GE } \\
\text { GE } \\
\text { WA } \\
\text { GE }\end{array}$ & 400 & 11 & & & 1 & GE & $\begin{array}{l}\mu g / L \\
\mu g / L \\
\mu g / L \\
\mu g / L \\
\mu g / l \\
\mu g / l\end{array}$ \\
\hline \multicolumn{14}{|l|}{ Organic Constituents } \\
\hline Constituents & $\underline{1099}$ & Mod & Filt. & STH & $\underline{\text { DF }}$ & Lab & $\underline{2099}$ & Mod & Filt. & SI $\mathrm{H}$ & DF & Lab & Ynit \\
\hline $\begin{array}{l}\text { Benzene } \\
\text { Bis(2-ethylhexyl) phthalate } \\
\text { Dichloromethane } \\
\text { Phenols } \\
\text { Tetrachloroethylene } \\
\text { Trichloroethylene } \\
\text { Trichlorofluoromethane }\end{array}$ & $\begin{array}{l}<1.0 \\
<10 \\
<5.0 \\
<5.0 \\
<1.0 \\
<1.0 \\
<5.0\end{array}$ & $\begin{array}{l}\text { JU/LO } \\
\text { U// } \\
\text { U/I } \\
\text { U// } \\
\text { JUIUO } \\
\text { JUIOO } \\
\text { JU/UO }\end{array}$ & $\begin{array}{l}<1.0 \\
<10 \\
<5.0 \\
<5.0 \\
<1.0 \\
<1.0 \\
<5.0\end{array}$ & & $\begin{array}{l}1 \\
1 \\
1 \\
1 \\
1 \\
1 \\
1\end{array}$ & $\begin{array}{l}\text { GE } \\
\text { GE } \\
\text { WA } \\
\text { GE } \\
\text { GE } \\
\text { GE } \\
\text { GE }\end{array}$ & & & & & & & $\begin{array}{l}\mu g / \\
\mu g / L \\
\mu g / \\
\mu g / L \\
\mu g / L \\
\mu g / L\end{array}$ \\
\hline
\end{tabular}

Notes:

= exceeded holding time

E = exceeded groundwater protection or monitoring constituent standard (See Appendix A.)

NA - Not applicable. Applies to beta dose and sum of betas if there are no beta-emitting radionuclides above detection limits; to sum of alphas if there are no alphaemitting radionuclides above detection limits; and to total radium if neither radium-226 or radium-228 was above detection limit

UAZ UTRA - Upper Aquifer Zone of the Upper Three Runs Aquifer, LAZ UTRA - Lower Aquifer Zone of the Upper Three Runs Aquifer, Gordon - Gordon Aquifer 
WELL FSB 79A (cont.)

Radioactive Constituents

\begin{tabular}{|c|c|c|c|c|c|c|c|c|c|c|c|c|c|}
\hline Constituents & 1099 & Mod & Filt. & ST브 & DF & Lab & 2099 & Mod & Filt. & ST & DF & Lab & Unit \\
\hline $\begin{array}{l}\text { Americium-241 } \\
\text { Beta dose }\end{array}$ & $\begin{array}{l}<4.2 E-02 \\
\mathrm{NA}\end{array}$ & JUルI & $<4.6 E-01$ & & 1 & GP & & & & & & & pCill \\
\hline Carton-14 & $<-1.0 E+\infty$ & $\mathrm{U} / /$ & $<9.4 E+\infty$ & & 1 & GP & & & & & & & pCin \\
\hline Cesium-137 & $<8.1 E-02$ & U/I & $<3.3 E+\infty$ & & 1 & GP & & & & & & & pCin \\
\hline Cobalt-60 & $<9.0 \mathrm{E}-02$ & $\mathrm{U} / /$ & $<4.3 E+\infty 0$ & & 1 & TM & & & & & & & pCin \\
\hline Curium-242 & $<0.0 E+\infty 0$ & $\mathrm{U} / /$ & $<1.6 \mathrm{E}-01$ & & 1 & $\mathrm{TM}$ & & & & & & & pCil \\
\hline Curium-243/244 & $<-1.0 E-02$ & $\mathrm{U} / /$ & $<5.9 E-01$ & & 1 & $\mathrm{TM}$ & & & & & & & pCin \\
\hline Curium-245/246 & $<1,6 E-02$ & U/I & $<1.7 E-01$ & & 1 & GP & & & & & & & pCir \\
\hline Gross alpha & $<-2.4 E-01$ & $\mathrm{U} / /$ & $<1.9 E+\infty$ & & 1 & $T M$ & $<4.2 E-01$ & $\mathrm{U} / /$ & $<6.3 E-01$ & & 1 & GP & pCin \\
\hline lodine-129 & $<2.2 \mathrm{E}-01$ & $\mathrm{U} / /$ & $<1.0 E+\infty$ & & 1 & GP & & & & & & & pCin \\
\hline Nonvolatile beta & $1.6 \mathrm{E}+\infty 0$ & $\mathrm{~J} / \mathrm{IK} / \mathrm{C}$ & NDD & & 1 & GP & $1.6 E+\infty 0$ & $\mathrm{~J} / \mathrm{V}$ & NDD & & 1 & GP & pCin \\
\hline Plutonium-238 & $<3.8 \mathrm{E}-03$ & $\mathrm{U} / /$ & $<1.6 \mathrm{E}-01$ & & 1 & GP & & & & & & & pCin \\
\hline Plutonium-239/240 & $<-5.2 E-03$ & $\mathrm{U} / /$ & $<1.1 E-01$ & & 1 & GP & & & & & & & $\mathrm{pCl}$ \\
\hline Radium-226 & $7.0 \mathrm{E}-01$ & $\mathrm{~J} / \mathrm{V} /$ & NDD & & 1 & GP & & & & & & & peir \\
\hline Radium-228 & $<1.5 \mathrm{E}-01$ & $\mathrm{U} / /$ & $<1.1 E+\infty$ & & 1 & GP & & & & & & & $\mathrm{pCi}$ \\
\hline Total radium & NA & & & & & & & & & & & & \\
\hline Strontium-90 & $<4.0 \mathrm{E}-02$ & $\mathbf{U} / /$ & $<1.4 E+\infty$ & & 1 & $\mathrm{TM}$ & & & & & & & $\mathrm{pCi} \Omega$ \\
\hline Technetium-99 & $<-6.2 E+00$ & $\mathbf{U} / /$ & $<1.4 E+01$ & & 1 & TM & & & & & & & $\mathrm{pCi}$ \\
\hline Thorium-228 & $<3.7 \mathrm{E}-02$ & $\mathbf{u} / /$ & $<1.4 E-01$ & & 1 & GP & & & & & & & pCil \\
\hline Thorium-230 & 6.6E-02 & $\mathrm{J} / \mathrm{L} / \mathrm{I}$ & NDD & & 1 & GP & & & & & & & pCin \\
\hline Thorium-232 & $<0.0 E+00$ & $\mathbf{u} / /$ & $<2.8 E-02$ & & 1 & GP & & & & & & & pCi凡 \\
\hline Sum of alphas & NA & & & & & & & & & & & & \\
\hline Sum of betas & NA & & & & & & & & & & & & \\
\hline Tritium & $1.5 E+01$ & II & & & 1 & $\mathrm{TM}$ & $8.1 E+\infty$ & /I & & & 1 & GP & \\
\hline Uranium-233/234 & $<1.1 E-02$ & $\mathrm{U} / /$ & $<1.4 E-01$ & & 1 & GP & & & & & & & L \\
\hline Uranium-235 & $<-4.1 E-03$ & $U / I$ & $<9.0 E-02$ & & 1 & GP & & & & & & & \\
\hline Uranium-238 & $<1.1 E-02$ & $\mathrm{U} / /$ & $<1.4 E-01$ & & 1 & GP & & & & & & & \\
\hline
\end{tabular}

Notes:

= exceeded holding time

= exceeded groundwater protection or monitoring constituent standard (See Appendix A.)

NA - Not applicable. Applies to beta dose and sum of betas if there are no beta-emitting radionuclides above detection limits; to sum of alphas if there are no alphaemitting radionuclides above detection limits; and to total radium if neither radium-226 or radium-228 was above detection limit

UAZ UTRA - Upper Aquifer Zone of the Upper Three Runs Aquifer, LAZ_UTRA - Lower Aquifer Zone of the Upper Three Runs Aquifer, Gordon - Gordon Aquifer 


\section{WELL FSB 79B}

$\begin{array}{ll}\text { SRS Coord. } & \text { Lat/Longitude } \\ \text { N73666.1 } & 33.270541^{\circ} \mathrm{N} \\ \text { E50159.2 } & 81.677813 \mathrm{~W}\end{array}$

SAMPLE DATE

01/06/99

1099

158.8

6.4

160

18.9

0

2.7

Turbidity

Volumes purged

Sampling code

Synchronous water level
Screen Zone Elevation

91.2-80.7 ft msl

Ton of Casing

$218.2 \mathrm{ft} \mathrm{msl}$

04/05/99

\section{ANALYTICAL DATA}

Inorganic Constituents

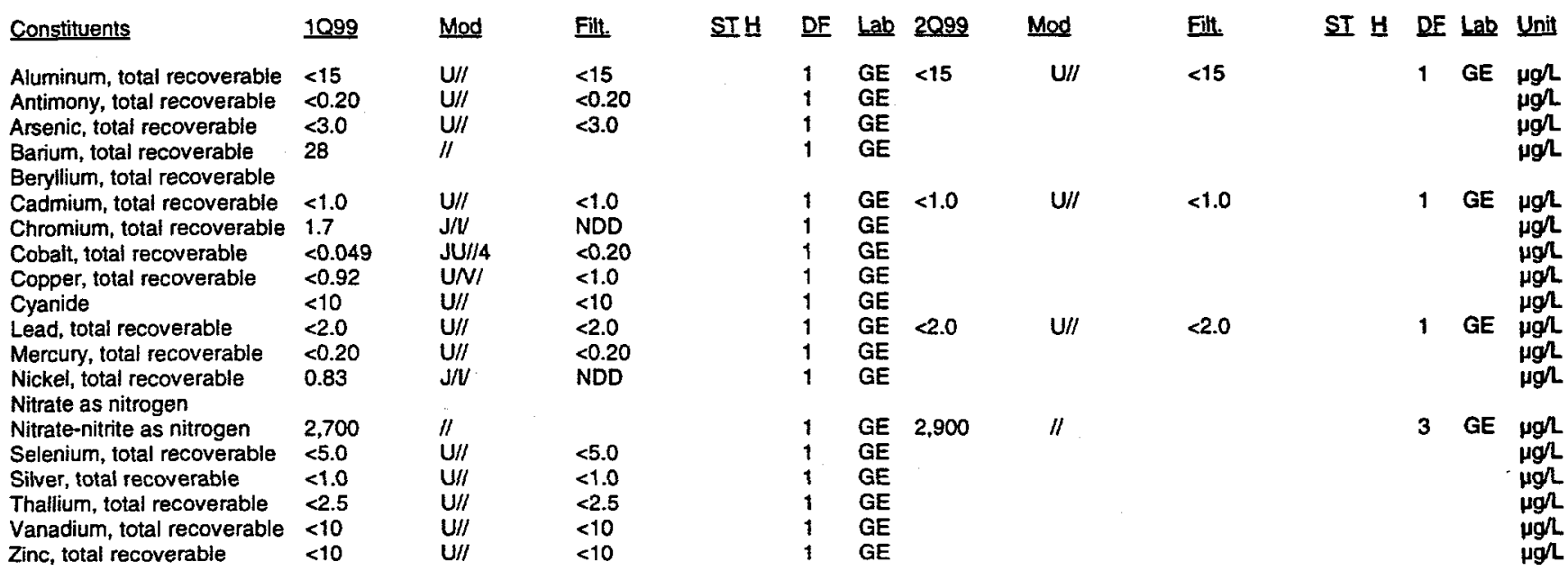

Organic Constituents

\begin{tabular}{llll} 
Constituents & 1099 & Mod & Filt. \\
\cline { 2 - 4 } Benzene & & & \\
Bis(2-ethylhexyl) phthalate & $<9.9$ & JU/UO & $<1.0$ \\
Dichloromethane & $<5.0$ & U// & $<9.9$ \\
Phenols & $<5.0$ & UU// & $<5.0$ \\
Tetrachioroethylene & $<1.0$ & JUNO & $<5.0$ \\
Trichloroethylene & $<1.0$ & JU/O & $<1.0$ \\
Trichlorofluoromethane & $<5.0$ & JUIUO & $<5.0$
\end{tabular}

$\begin{array}{cc}\text { DF } & \text { Lab } \\ 1 & \text { GE } \\ 1 & \text { GE } \\ 1 & \text { GE } \\ 1 & \text { GE } \\ 1 & \text { GE } \\ 1 & \text { GE } \\ 1 & \text { GE }\end{array}$

2099
158.8
6.6
160
20.3
56
0
3.5
$158.3(06 / 28 / 99)$.

Unit

t $\mathrm{ms}$

pH

$\mu S / \mathrm{cm}$

mgh

NTU

well volumes

ft mst
Screen Zone

Gordon 
WELL FSB 79B (cont.)

Radioactive Constituents

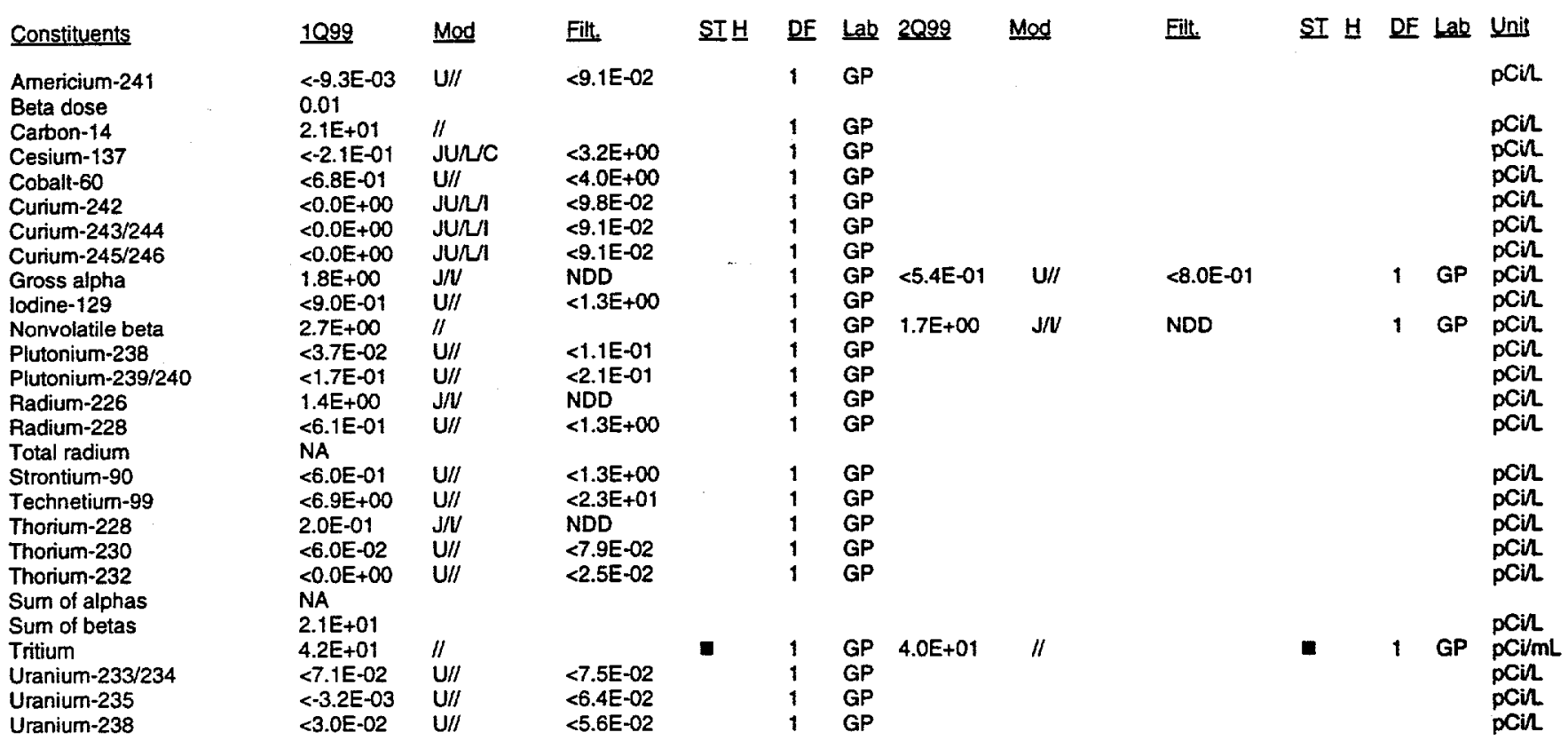

Notes:

- exceeded holding time

= exceeded groundwater protection or monitoring constituent standard (See Appendix A.)

NA - Not applicable. Applies to beta dose and sum of betas if there are no beta-emitting radionuclides above detection limits; to sum of alphas if there are no alphaemitting radionuclides above detection limits; and to total radium if neither radium-226 or radium-228 was above detection limit

UAZ_UTRA - Upper Aquifer Zone of the Upper Three Runs Aquifer, LAZ_UTRA - Lower Aquifer Zone of the Upper Three Runs Aquifer, Gordon - Gordon Aquifer 


\section{WELL FSB 79C}

$\begin{array}{ll}\text { SRS Coord. } & \text { Lathonaitude } \\ \text { N73668.0 } & 33.270565^{\circ} \mathrm{N} \\ \text { E50171.3 } & 81.677785^{\circ} \mathrm{W}\end{array}$

SAMPLE DATE

$01 / 04 / 99$
Screen Zone Elevation

159.6-149.8 ft ms!
Top of Casing

$218.4 \mathrm{ft} \mathrm{mst}$

04/07/99

FIELLD DATA

Constituents

Water elevation

$\mathrm{pH}$

Sp. conductance

Water temperature

Alkalinity as $\mathrm{CaCO} 3$

Turbidity

Volumes purged

Sampling code

Synchronous water level

\section{ANALYTICAL DATA}

Inorganic Constituents
1099

196.6

3.8

1040

17.1

2.3

$196.6(03 / 23 / 99)$
Casing Pump

4: PVC V

Screen Zone

LAZ_UTRA

Unit

ft msl

pH

HS/cm

${ }^{\circ} \mathrm{C}$

$\mathrm{mg} / \mathrm{L}$

NTU

well volumes

ft ms!

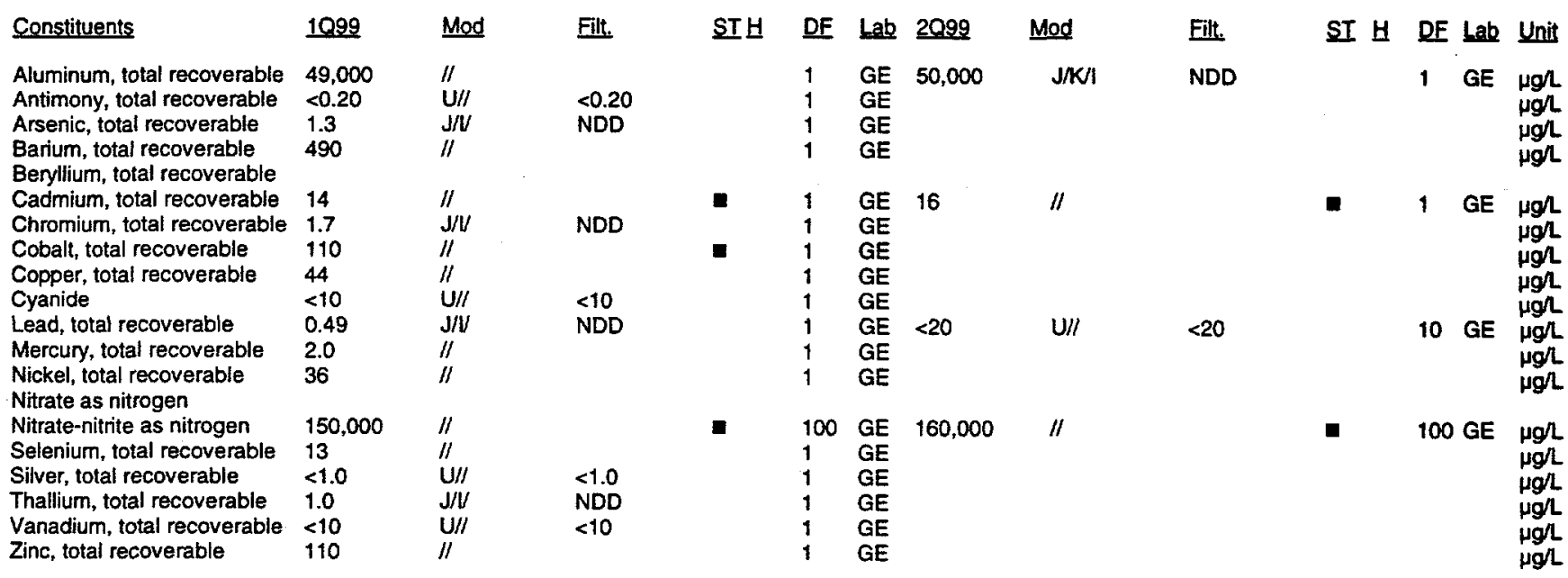

Organic Constituents

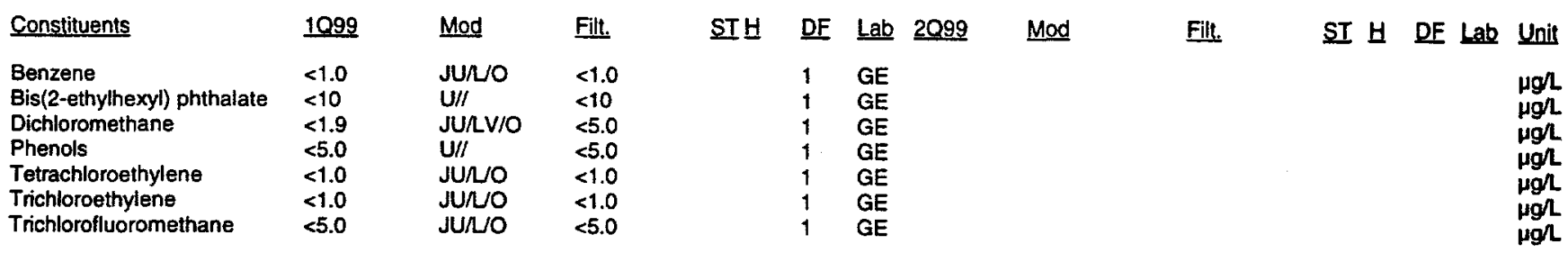

Notes:

- = exceeded holding time

= exceeded groundwater protection or monitoring constituent standard (See Appendix A.)

NA - Not applicable. Applies to beta dose and sum of betas if there are no beta-emitting radionuclides above detection limits; to sum of alphas if there are no alphaemitting radionuclides above delection limits; and to total radium if neither radium-226 or radium-228 was above detection limit

UAZ_UTRA - Upper Aquifer Zone of the Upper Three Runs Aquifer, LAZ UTRA - Lower Aquifer Zone of the Upper Three Runs Aquifer, Gordon - Gordon Aquifer 
WELL FSB 79C (cont.)

Radioactive Constituents

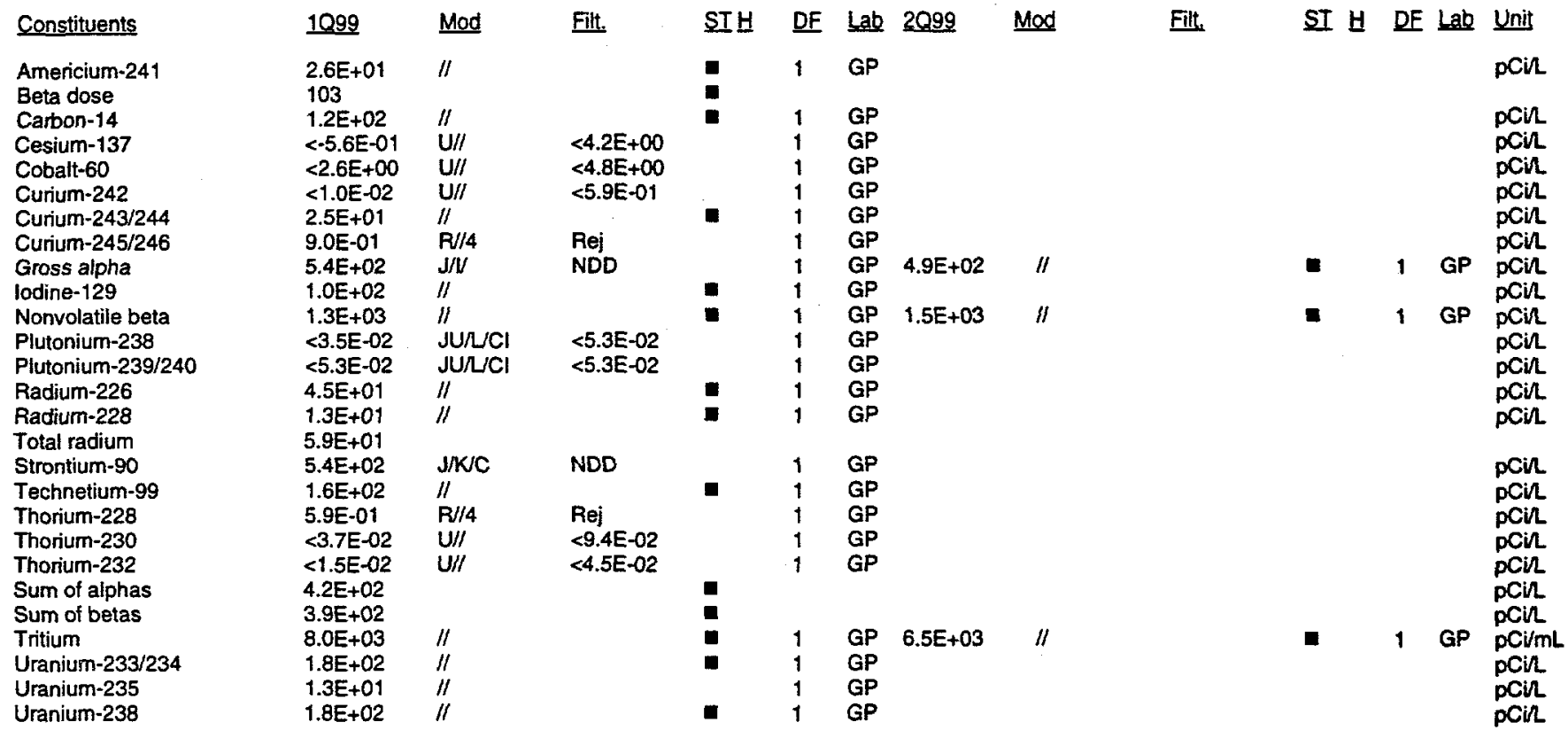

Notes:

- exceeded holding time

- exceeded groundwater protection or monitoring constituent standard (See Appendix A)

NA - Not applicable. Applies to beta dose and sum of betas if there are no beta-emitting radionuclides above detection limits; to sum of alphas if there are no alphaemitting radionuclides above detection limits; and to total radium if neither radium-226 or radium-228 was above detection limit

UAZ UTRA - Upper Aquifer Zone of the Upper Three Runs Aquifer, LAZ UTRA - Lower Aquifer Zone of the Upper Three Runs Aquifer Gordon - Gordon Aquifer 


\section{WELL FSB 87A}

$\begin{array}{ll}\text { SRS Coord. } & \text { Latlongitude } \\ \text { N75601.7 } & 33.274751^{\circ} \mathrm{N} \\ \text { E50115.8 } & 81.681688^{\circ} \mathrm{W}\end{array}$

SAMPLE DATE

1099

Constifuents

Water elevation

$\mathrm{pH}$

Sp. conductance

Water temperature

Alkalinity as $\mathrm{CaCO} 3$

Turbidity

Volumes purged

Sampling code

Synchronous water level

ANALYTICAL DATA

Inorganic Constituents

3.3

$\begin{array}{lllll}\text { Screen Zone Elevation } & \text { Tog of Casing } & \text { Casing } & \text { Pume } & \text { Screen Zone } \\ 43.6-33.1 \mathrm{f} \mathrm{msl} & 287.8 \mathrm{ft} \mathrm{msl} & 4^{*} \mathrm{PVC} & \mathrm{S} & \text { Gordon }\end{array}$

04/06/99

2099

154.4

6.1

92

20.3

2.8

$154.3(03 / 23 / 99)$
$153.8(06 / 25 / 99)$
Unit

ft ms!

pH

$\mathrm{S} / \mathrm{cm}$

NTU

well volumes

ft msi

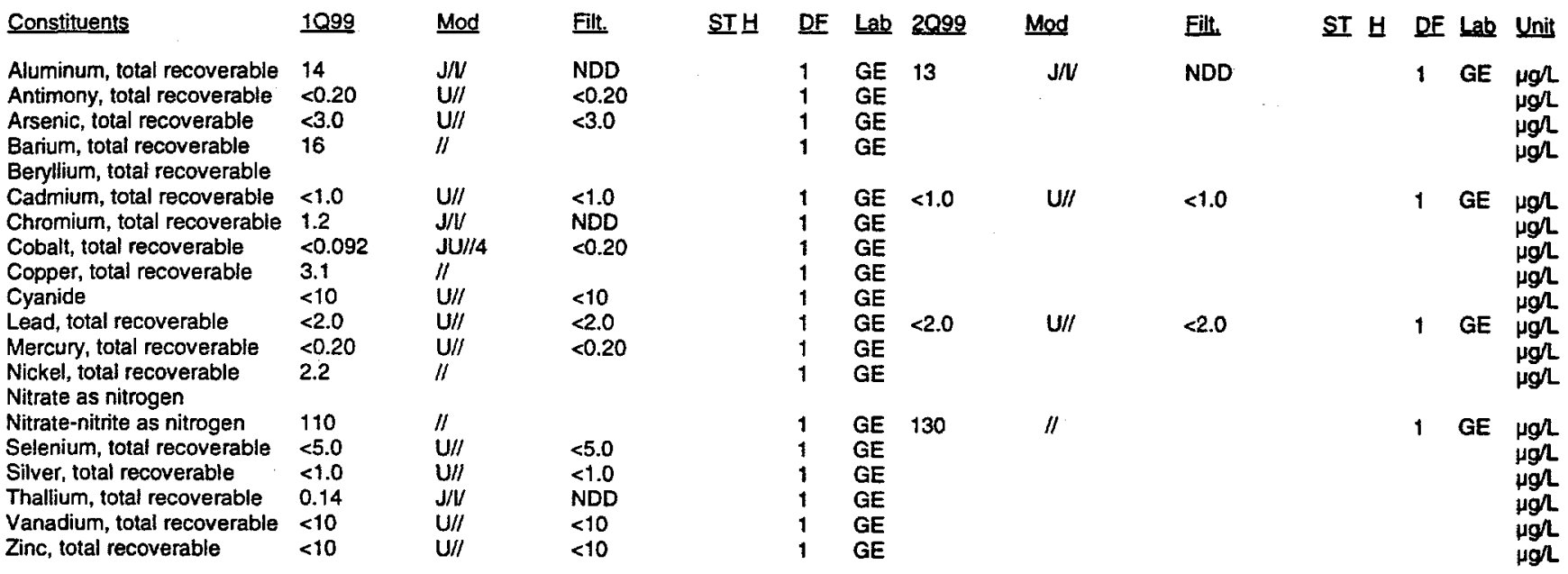

Organic Constituents

\begin{tabular}{|c|c|c|c|c|c|}
\hline Constituents & 1099 & Mod & Filt. & SI & $\mathrm{DF}$ \\
\hline Benzene & $<1.0$ & $\mathrm{U} / /$ & $<1.0$ & & 1 \\
\hline Bis(2-ethylhexyl) phthalate & $<10$ & $\mathrm{U} / /$ & $<10$ & & 1 \\
\hline Dichloromethane & $<5.0$ & $\mathrm{U} / /$ & $<5.0$ & & 1 \\
\hline Phenols & $<5.0$ & $\mathrm{U} / /$ & $<5.0$ & & 1 \\
\hline Tetrachloroethylene & $<1.0$ & $\mathrm{U} / /$ & $<1.0$ & & 1 \\
\hline Trichioroethylene & $<1.0$ & U/I & $<1.0$ & & 1 \\
\hline Trichlorofluoromethane & $<5.0$ & $U / I$ & $<5.0$ & & 1 \\
\hline
\end{tabular}

$\underline{\text { Mod }}$

Filt.

ST H DE Lab Unit

$\mu g h$

$\mu g /$

$\mu g / L$

$\mu g h$

$\mu g /$

$\mu g /$ 


\section{WELL FSB 87 A (cont.)}

Radioactive Constituents

\begin{tabular}{|c|c|c|c|c|c|c|c|c|c|c|c|c|c|}
\hline Constituents & 1099 & Mod & Filt, & STH & $\underline{\text { DF }}$ & $\underline{\text { Lab }}$ & $\underline{2099}$ & Mod & Filt. & ST $H$ & DF & Lab & Unit \\
\hline $\begin{array}{l}\text { Americium-241 } \\
\text { Beta dose }\end{array}$ & $\begin{array}{l}<6.3 E-02 \\
N A\end{array}$ & JU/LII & $<4.3 E-01$ & & 1 & GP & & & & & & & pCin \\
\hline Carbon-14 & $<2.3 E-01$ & $\mathrm{U} / /$ & $<7.4 E+\infty$ & & 1 & GP & & & & & & & pCil \\
\hline Cesium-137 & $<-8.7 E-01$ & JU/L/C & $<4.3 \mathrm{E}+00$ & & 1 & GP & & & & & & & pCin \\
\hline Cobalt-60 & $<-1.3 E+\infty$ & $\mathrm{U} / \prime$ & $<3.9 E+\infty 0$ & & 1 & GP & & & & & & & pcin \\
\hline Curium-242 & $<-2.1 E-02$ & $U / !$ & $<4.5 E-01$ & & 1 & GP & & & & & & & $\mathrm{pCin}$ \\
\hline Curium-243/244 & $<-2.0 E-02$ & $U / /$ & $<4.3 E-01$ & & 1 & GP & & & & & & & pcin \\
\hline Curium-245/246 & $<0.0 E+\infty 0$ & $\mathrm{U} / /$ & $<2.4 \mathrm{E}-01$ & & 1 & GP & & & & & & & pCin \\
\hline Gross alpha & $1.1 E+\infty 0$ & $\mathrm{~J} / \mathrm{J}$ & NDD & & 1 & GP & $1.2 \mathrm{E}+\infty$ & $\mathrm{J} / \mathrm{IKJC}$ & NDD & & 1 & $\mathrm{TM}$ & pCin \\
\hline lodine-129 & $<6.5 \mathrm{E}-01$ & $\mathrm{U} / /$ & $<1.3 E+\infty 0$ & & 1 & GP & & & & & & & pCin \\
\hline Nonvolatile beta & $<1.0 E+00$ & $\mathrm{U} / /$ & $<1.0 \mathrm{E}+00$ & & 1 & GP & 2.7E+00 & $\mathrm{J} / \mathrm{V}$ & NDD & & 1 & GP & pCin \\
\hline Plutonium-238 & $<1.5 E-01$ & $\mathrm{U} / \prime$ & $<1.8 \mathrm{E}-01$ & & 1 & GP & & & & & & & pCin \\
\hline Plutonium-239/240 & $<-1.6 E-04$ & $U / /$ & $<1.2 \mathrm{E}-01$ & & 1 & GP & & & & & & & $\mathrm{pCi} \Omega$ \\
\hline Radium-226 & $2.4 E \div 00$ & 11 & & & 1 & GP & & & & & & & pCin \\
\hline $\begin{array}{l}\text { Radium-228 } \\
\text { Total radium }\end{array}$ & $\begin{array}{l}<9.2 E-01 \\
2.4 E+00\end{array}$ & $\mathrm{U} / /$ & $<1.1 E+00$ & & 1 & GP & & & & & & & $\mathrm{pCi}$ \\
\hline Strontium-90 & $<1.6 \mathrm{E}-01$ & $\mathrm{U} / /$ & $<1.3 E+00$ & & 1 & GP & & & & & & & pCir \\
\hline Technetium-99 & $<2.8 E-01$ & $\mathrm{U} / /$ & $<2.1 E+01$ & & 1 & GP & & & & & & & pCir \\
\hline Thorium-228 & $<2.8 E-02$ & $\mathrm{U} / /$ & $<5.3 E-01$ & & 1 & GP & & & & & & & pCir \\
\hline Thorium-230 & $<4.9 \mathrm{E}-02$ & $\mathrm{U} / /$ & $<2.6 \mathrm{E}-01$ & & 1 & GP & & & & & & & pCir \\
\hline Thorium-232 & $<-2.7 E-02$ & $\mathrm{U} / /$ & $<2.6 \mathrm{E}-01$ & & 1 & GP & & & & & & & pCin \\
\hline Sum of alphas & NA & & & & & & & & & & & & \\
\hline Sum of betas & NA & & & & & & & & & & & & \\
\hline Tritium & $1.5 E+00$ & $/ /$ & & & 1 & GP & $1.6 E+00$ & $\|$ & & & 1 & GP & pCi/mL \\
\hline Uranium-233/234 & $<1.1 E-01$ & JU几C & $<2.0 E-01$ & & 1 & GP & & & & & & & pCin \\
\hline Uranium-235 & $<1.3 E-03$ & JUЛ & $<2.5 E-01$ & & 1 & GP & & & & & & & pCin \\
\hline Uranium-238 & $<1.3 E-03$ & JUЛ & $<2.5 E-01$ & & 1 & GP & & & & & & & pCin \\
\hline
\end{tabular}

Notes:

- exceeded holding time

= exceeded groundwater protection or monitoring constituent standard (See Appendix A.)

NA - Not applicable. Applies to beta dose and sum of betas it there are no beta-emitting radionuclides above detection limits; to sum of alphas if there are no alphaemitting radionuclides above detection limits; and to total radium if neither radium-226 or radium-228 was above detection limit

UAZ UTRA - Upper Aquifer Zone of the Upper Three Runs Aquifer; LAZ_UTAA - Lower Aquifer Zone of the Upper Three Runs Aquifer, Gordon - Gordon Aquifer

F-Area HWMF 


\section{WELL FSB 87B}

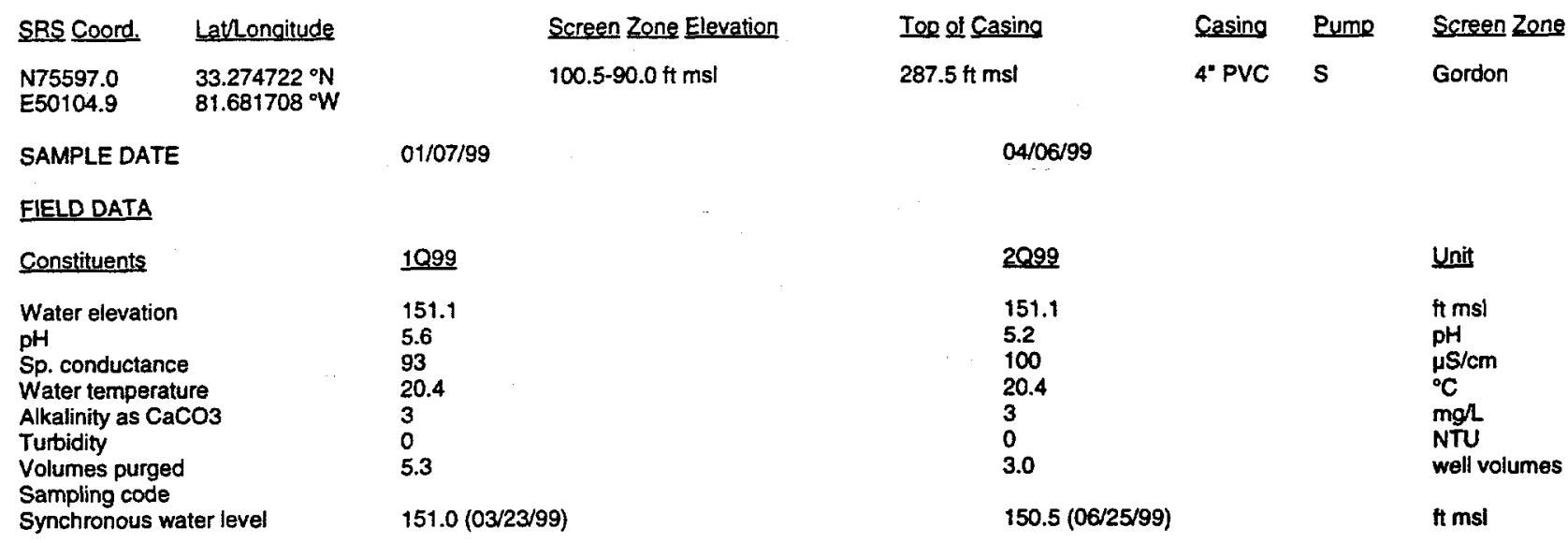

\section{ANALYTICAL DATA}

Inorganic Constituents

\begin{tabular}{|c|c|c|c|c|c|c|c|c|c|c|c|c|c|}
\hline Constituents & 1099 & Mod & Filt. & ST보 & DF & $\underline{\text { Lab }}$ & $2 Q 99$ & Mod & Filt. & ST 브 & DF & Lab & Unit \\
\hline Aluminum, total recoverable & 32 & $/ 1$ & & & 1 & GE & 23 & $\| I$ & & & 1 & $\mathrm{GE}$ & $\mu g /$ \\
\hline Antimony, total recoverable & $<0.20$ & $U / 1$ & $<0.20$ & & 1 & GE & & & & & & & $\mu g h$ \\
\hline Arsenic, total recoverable & $<3.0$ & $\mathrm{U} / \prime$ & $<3.0$ & & 1 & GE & & & & & & & $\mu g /$ \\
\hline $\begin{array}{l}\text { Barium, total recoverable } \\
\text { Beryllium, total recoverable }\end{array}$ & 3.0 & $/ /$ & & & 1 & GE & & & & & & & $\mu g /$ \\
\hline Cadmium, total recoverable & $<1.0$ & $\mathrm{U} / /$ & $<1.0$ & & 1 & GE & $<1.0$ & $U / \prime$ & $<1.0$ & & 1 & GE & $\mu g /$ \\
\hline Chromium, total recoverable & 2.0 & $\mathrm{~J} / \mathrm{L} /$ & NDD & & 1 & GE & & & & & & & \\
\hline $\begin{array}{l}\text { Cobalt, total recoverable } \\
\text { Copper, total recoverable }\end{array}$ & $\begin{array}{l}<0.083 \\
4.7\end{array}$ & $\mathrm{JU}_{/ /}$ & $<0.20$ & & $\begin{array}{l}1 \\
1\end{array}$ & $\begin{array}{l}\text { GE } \\
\text { GE }\end{array}$ & & & & & & & jg/ \\
\hline Cyanide & $<10$ & $\mathrm{U} / 1$ & $<10$ & & 1 & $\mathrm{GE}$ & & & & & & & \\
\hline Lead, total recoverable & 1.5 & $\mathrm{~J} / \mathrm{l} /$ & NDD & & 1 & GE & 1.7 & $\mathrm{~J} / \mathrm{U}$ & NDD & & 1 & GE & $\mu g /$ \\
\hline Mercuny, total recoverable & $<0.20$ & $\mathrm{U} / /$ & $<0.20$ & & 1 & GE & & & & & & & \\
\hline Nickel, total recoverable & 3.2 & // & & & 1 & GE & & & & & & & \\
\hline Nitrate as nitrogen & & $\therefore$ & & & & & & & & & & & \\
\hline Nitrate-nitrite as nitrogen & 8,800 & $/ /$ & & & 5 & GE & 9,900 & 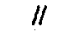 & & & 5 & GE & \\
\hline Selenium, total recoverable & $<5.0$ & $\mathrm{U} / /$ & $<5.0$ & & 1 & GE & & & & & & & \\
\hline Sitver, total recoverable & $<1.0$ & $\mathrm{U} / /$ & $<1.0$ & & 1 & GE & & & & & & & $\mu g h$ \\
\hline Thallium, total recoverable & 0.16 & $\mathrm{~J} / \mathrm{V}$ & NDD & & 1 & GE & & & & & & & $\mu \mathrm{g}$ \\
\hline Vanadium, total recoverable & $<10$ & $\mathrm{U} / /$ & $<10$ & & 1 & GE & & & & & & & \\
\hline Zinc, total recoverable & $<10$ & $\mathrm{U} / l$ & $<10$ & & 1 & GE & & & & & & & \\
\hline
\end{tabular}

Organic constituents

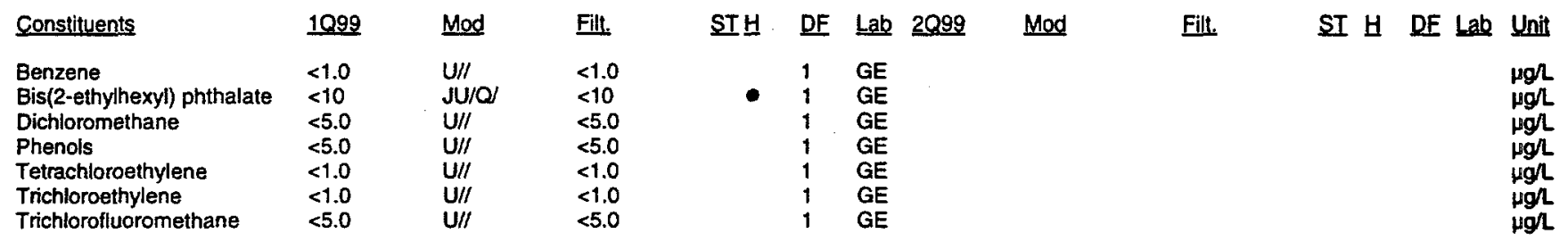

\footnotetext{
Notes:
}

= exceeded holding time

= exceeded groundwater protection or monitoring constituent standard (See Appendix A.)

NA - Not applicable. Applies to beta dose and sum of betas if there are no beta-emitting radionuclides above detection limits; to sum of alphas if there are no alphaernitting radionuclides above detection limits; and to total radium if neither radium-226 or radium-228 was above detection limit

UAZ_UTRA - Upper Aquiter Zone of the Upper Three Runs Aquifer, LAZ_UTRA - Lower Aquifer Zone of the Upper Three Runs Aquifer, Gordon - Gordon Aquifer 


\section{WELL FSB $87 B$ (cont.)}

Radioactive Constituents

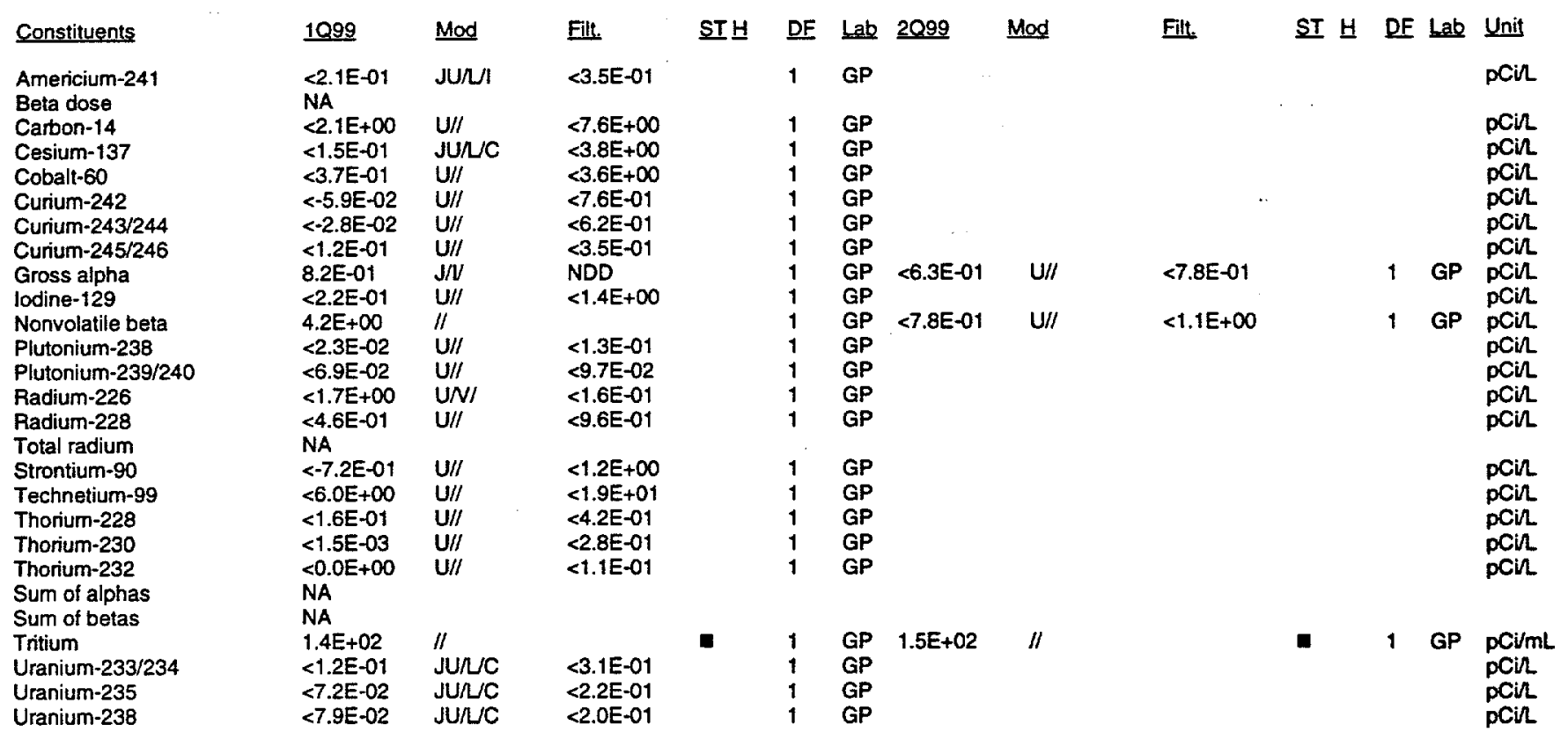

\section{Notes:}

- exceeded holding time

= exceeded groundwater protection or monitoring constituent standard (See Appendix A.)

NA - Not applicable. Applies to beta dose and sum of betas if there are no beta-emitting radionuclides above detection limits; to sum of alphas if there are no alphaemitting radionuclides above detection limits; and to total radium it neither radium-226 or radium-228 was above detection limit

UAZ_UTAA - Upper Aquifer Zone of the Upper Three Runs Aquifer, LAZ UTRA - Lower Aquifer Zone of the Upper Three Runs Aquifer, Gordon - Gordon Aquifer 


\section{WELL FSB 87C}

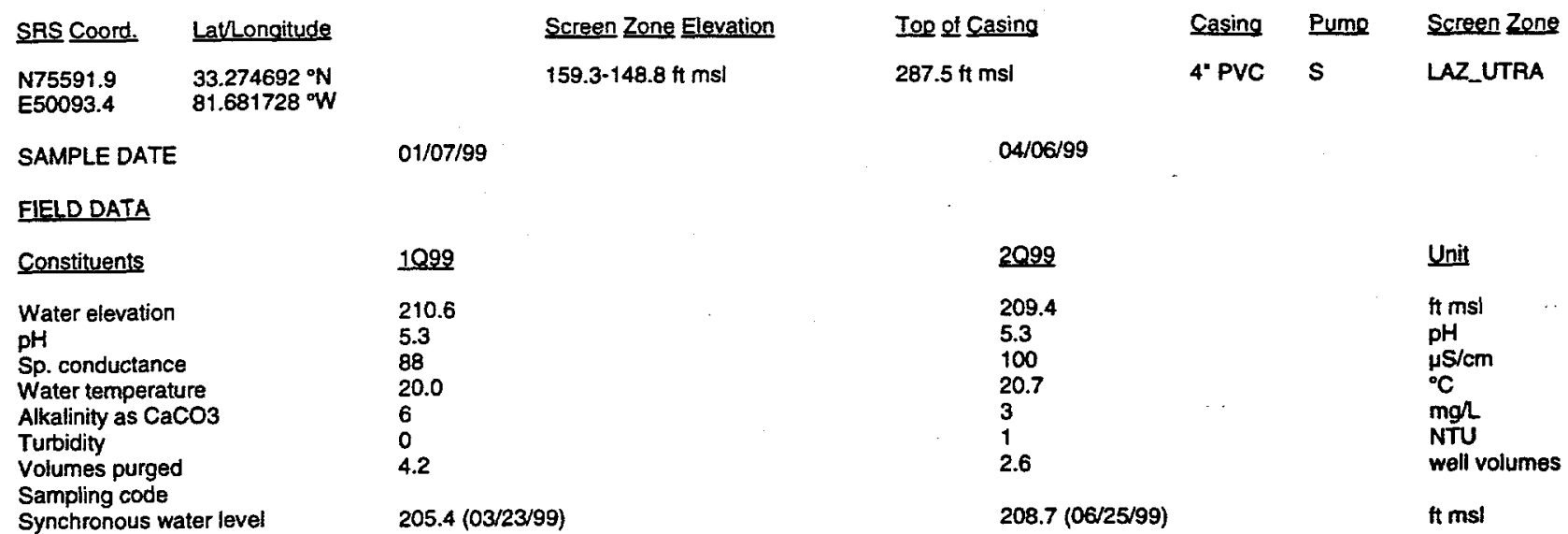

\section{ANALYTICAL DATA}

Inorganic Constituents

\begin{tabular}{|c|c|c|c|c|c|c|c|c|c|c|c|c|c|}
\hline Constituents & 1099 & Mod & Filt. & SIH & $\underline{\mathrm{DF}}$ & Lab & 2099 & Mod & Filt. & ST $\underline{H}$ & DF & Lab & Unit \\
\hline Aluminum, total recoverable & 17 & $/ /$ & & & 1 & GE & 10 & $\mathrm{~J} / \mathrm{N}$ & NDD & & 1 & GE & $\mu g /$ \\
\hline Antimony, total recoverable & $<0.20$ & U/I & $<0.20$ & & 1 & GE & & & & & & & Hgh \\
\hline Arsenic, total recoverable & $<3.0$ & $U / !$ & $<3.0$ & & 1 & GE & & & & & & & $\mu g /$ \\
\hline $\begin{array}{l}\text { Barium, total recoverable } \\
\text { Beryllium, total recoverable }\end{array}$ & 16 & $/ 1$ & & & 1 & GE & & & & & & & $\mu g h$ \\
\hline Cadmium, total recoverable & 0.26 & $\mathrm{~J} / \mathrm{V}$ & NDD & & 1 & GE & $<1.0$ & $\mathrm{U} / \prime$ & $<1.0$ & & 1 & GE & \\
\hline Chromium, total recoverable & 0.82 & $\mathrm{~J} / \mathrm{V}$ & NDD & & 1 & GE & & & & & & & \\
\hline Cobalt, total recoverable & 0.29 & 11 & & & 1 & GE & & & & & & & \\
\hline Copper, total recoverable & 45 & $\|$ & & & 1 & GE & & & & & & & $\mu g /$ \\
\hline Cyanide & 5.4 & $\mathrm{~J} / \mathrm{KK} / \mathrm{I}$ & NDD & & 1 & GE & & & & & & & \\
\hline Lead, total recoverable & 8.1 & $1 /$ & & & 1 & GE & 14 & /I & & & 1 & GE & \\
\hline Mercury, total recoverable & $<0.20$ & $\mathbf{U} / /$ & $<0.20$ & & 1 & GE & & & & & & & \\
\hline $\begin{array}{l}\text { Nickel, total recoverable } \\
\text { Nitrate as nitrogen }\end{array}$ & 4.2 & $/ /$ & & & 1 & GE & & & & & & & \\
\hline Nitrate-nitrite as nitrogen & 8,300 & $\| /$ & & & 5 & GE & 9,700 & /I & & & 5 & GE & \\
\hline Selenium, total recoverable & $<5.0$ & $u / /$ & $<5.0$ & & $t$ & GE & & & & & & & $\mu g$ \\
\hline Silver, total recoverable & $<1.0$ & $\mathbf{u} / /$ & $<1.0$ & & 1 & GE & & & & & & & $\mu g /$ \\
\hline Thallium, total recoverable & $<0.085$ & $\mathrm{JU} / / 4$ & $<2.5$ & & 1 & GE & & & & & & & $g /$ \\
\hline Vanadium, total recoverable & $<10$ & $\mathrm{U} / /$ & $<10$ & & 1 & GE & & & & & & & \\
\hline Zinc, total recoverable & 16 & ll & & & 1 & GE & & & & & & & \\
\hline
\end{tabular}

Organic Constituents

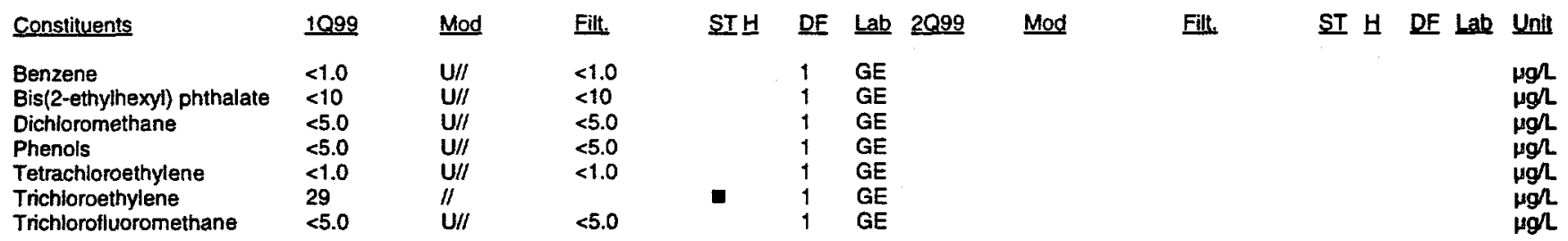

Notes:

- exceeded holding time

= exceeded groundwater protection or monitoring constituent standard (See Appendix A.)

NA - Not applicable. Applies to beta dose and sum of betas if there are no beta-emitting radionuclides above detection limits; to sum of alphas if there are no alphaemitting radionuclides above detection limits; and to total radium if neither radium-226 or radium-228 was above detection limit

UAZ_UTRA - Upper Aquifer Zone of the Upper Three Runs Aquifer, LAZ_UTRA - Lower Aquifer Zone of the Upper Three Runs Aguifer, Gordon - Gordon Aquifer 


\section{WELL FSB 87C (cont.)}

Radioactive Constituents

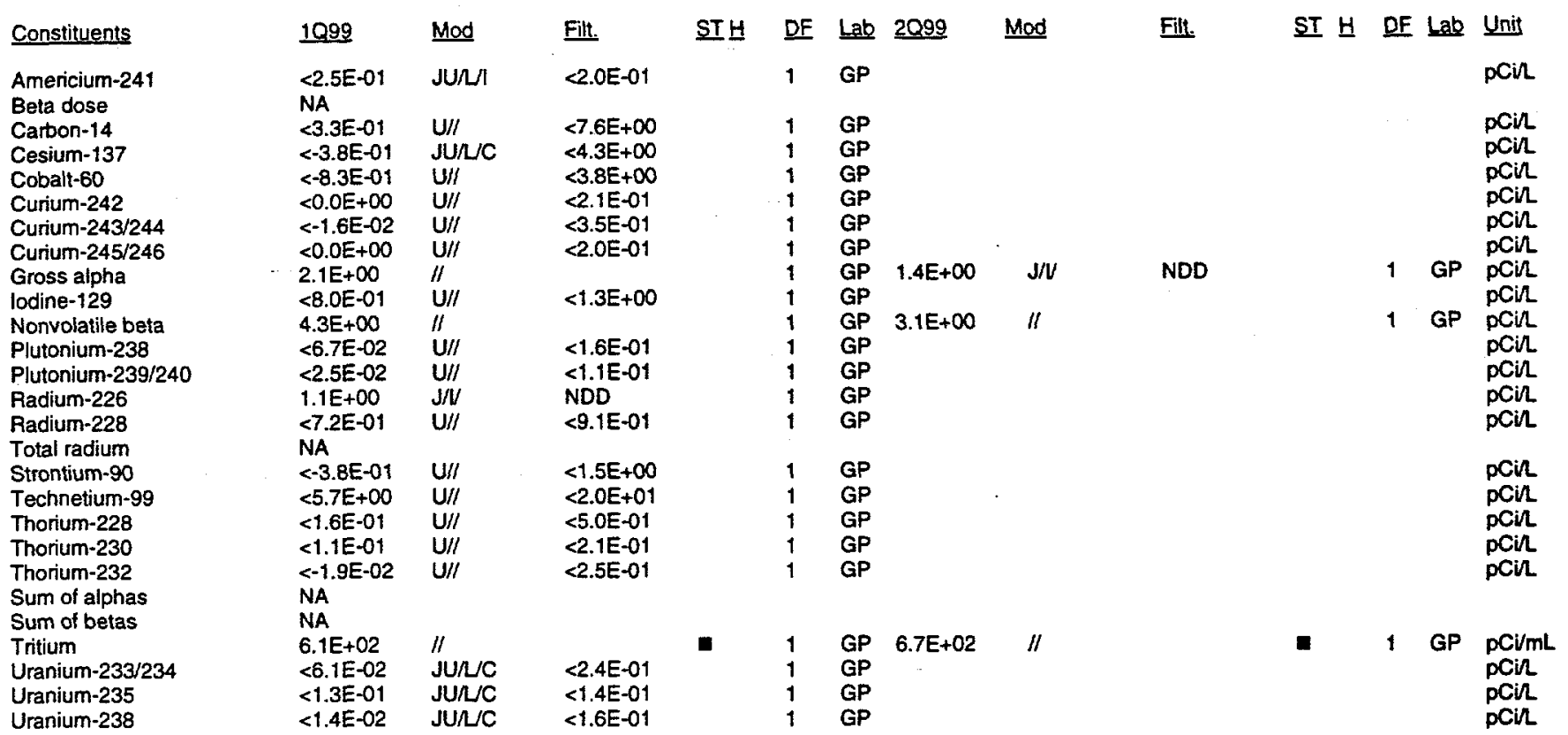

\section{Notes:}

- exceeded holding time

= exceeded groundwater protection or monitoring constituent standard (See Appendix A.)

NA - Not applicable. Applies to beta dose and sum of betas if there are no beta-emitting radionuclides above detection limits; to sum of alphas if there are no alphaemitting radionuclides above detection limits; and to total radium if neither radium-226 or radium-228 was above detection limit

UAZ UTRA - Upper Aquifer Zone of the Upper Three Runs Aquifer; LAZ UTRA - Lower Aquifer Zone of the Upper Three Runs Aquifer, Gordon - Gordon Aquifer 


\section{WELL FSB 87D}

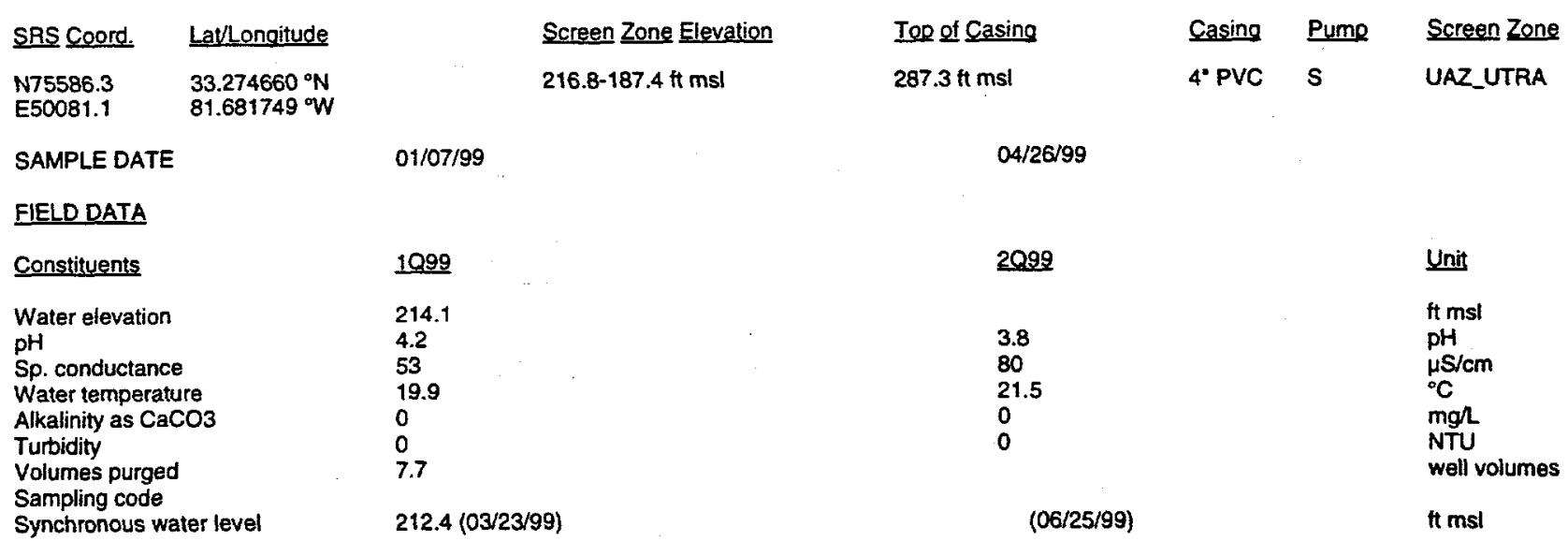

\section{ANALYTICAL DATA}

Inorganic Constituents

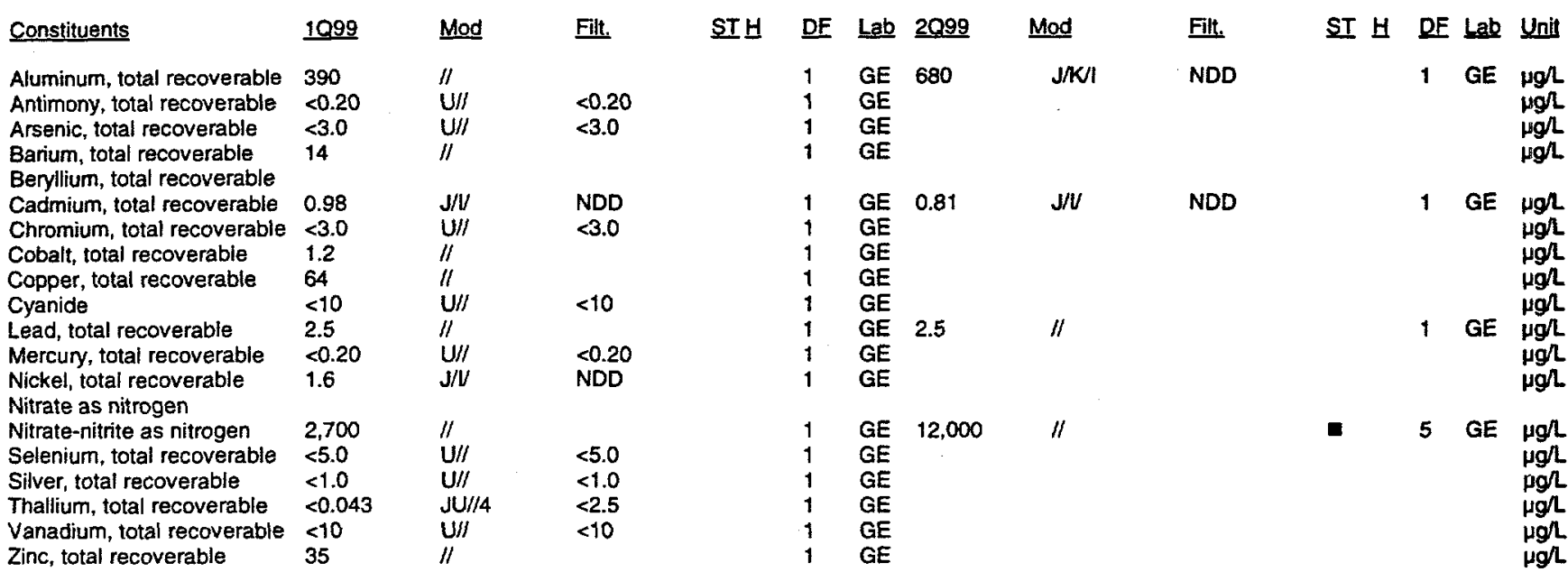

Organic Constituents

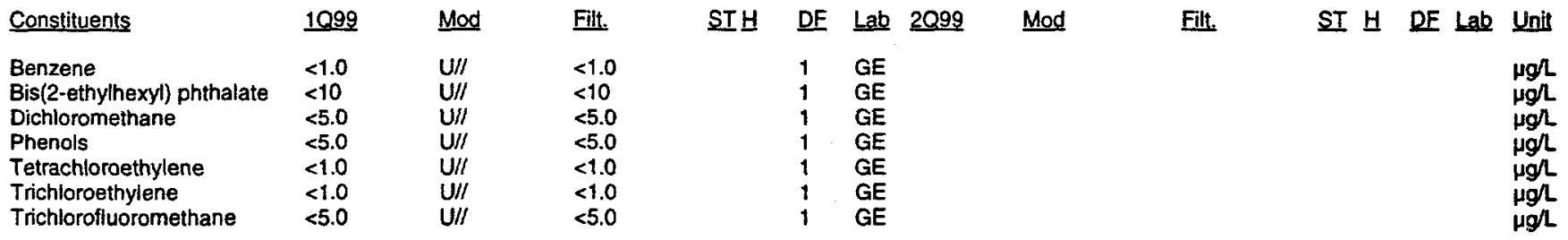

Notes:

- exceeded holding time

= exceeded groundwater protection or monitoring constituent standard (See Appendix A.)

NA - Not applicable. Applies to beta dose and sum of betas if there are no beta-emitting radionuclides above detection limits; to sum of alphas if there are no alphaemitting radionuclides above detection limits; and to total radium if neither radium-226 or radium-228 was above detection limit

UAZ_UTRA - Upper Aquifer Zone of the Upper Three Runs Aquifer, LAZ_UTRA - Lower Aquifer Zone of the Upper Three Runs Aquifer, Gordon - Gordon Aquifer 


\section{WELL FSB 87D (cont.)}

Radioactive Constituents

\begin{tabular}{|c|c|c|c|c|c|c|c|c|c|c|c|c|c|}
\hline Constituents & 1099 & Mod & Filt. & ST브 & DF & Lab & 2099 & Mod & Filt. & ST $\underline{H}$ & DF & Lab & Unit \\
\hline $\begin{array}{l}\text { Americium-241 } \\
\text { Beta dose }\end{array}$ & $\begin{array}{l}<1.4 E-01 \\
0.65\end{array}$ & JU/I & $<2.4 E-01$ & & 1 & GP & & & & & & & pcin \\
\hline Carbon-14 & $<4.0 E+\infty$ & $\mathrm{U} / /$ & $<7.4 E+00$ & & 1 & GP & & & & & & & pCin \\
\hline Cesium-137 & $<-2.3 E+\infty 0$ & JUR & $<3.7 E+\infty$ & & 1 & GP & & & & & & & $\mathrm{pCin}$ \\
\hline Cobalt-60 & $<1.1 \mathrm{E}+\infty$ & UII & $<3.9 E+\infty$ & & $i$ & $\mathrm{GP}$ & & & & & & & pCin \\
\hline Curium-242 & $<0.0 E+00$ & $\mathrm{U} / t$ & $<2.5 E-01$ & & 1 & GP & & & & & & & pCil \\
\hline Curium-243/244 & $<2.4 E-01$ & $U / /$ & $<2.4 E-01$ & & 1 & GP & & & & & & & $\mathrm{pCin}$ \\
\hline Curium-245/246 & $<0.0 E+00$ & $\mathrm{U} / I$ & $<2.4 \mathrm{E}-01$ & & 1 & GP & & & & & & & pCin \\
\hline Gross alpha & $6.5 E+01$ & $\|$ & & 口 & 1 & GP & $8.8 E+01$ & // & & $\mathbf{0}$ & 1 & GP & pCin \\
\hline lodine- 129 & $2.3 E+00$ & $\mathrm{~J} / \mathrm{U}$ & NDD & & 1 & GP & & & & & & & pCin \\
\hline Nonvolatile beta & $3.6 \mathrm{E}+01$ & $\|$ & & & 1 & GP & $5.6 E+01$ & $/ I$ & & - & 1 & GP & pCin \\
\hline Plutonium-238 & $<-3.1 E-02$ & $\mathrm{U} / /$ & $<1.8 E-01$ & & 1 & GP & & & & & & & $\mathrm{pCin}$ \\
\hline Plutonium-239/240 & $<-3.0 E-04$ & $U / /$ & $<1.4 E-01$ & & 1 & GP & & & & & & & pCir \\
\hline Radium-226 & $1.0 \mathrm{E}+00$ & $\mathrm{~J} / \mathrm{V} /$ & NDD & & 1 & GP & & & & & & & pCir \\
\hline Radium-228 & 8.3E-01 & $\mathrm{J} / \mathrm{N}$ & NDD & & 1 & $\mathrm{GP}$ & & & & & & & pCill \\
\hline Total radium & NA & & & & & & & & & & & & \\
\hline Strontium-90 & $5.2 E+\infty 0$ & $/ /$ & & & 1 & GP & & & & & & & pCir \\
\hline Technetium-99 & $<-1.3 E+\infty 0$ & $\mathrm{U} / \mathrm{I}$ & $<2.0 \mathrm{E}+01$ & & 1 & GP & & & & & & & $\mathrm{pCi}$ \\
\hline Thorium-228 & $<3.4 E-02$ & $\mathrm{U} / /$ & $<4.5 E-01$ & & 1 & GP & & & & & & & pCin \\
\hline Thorium-230 & $<1.2 E-01$ & $\mathrm{U} / 1$ & $<2.5 E-01$ & & 1 & $\mathrm{GP}$ & & & & & & & $\mathrm{pCin}$ \\
\hline Thorium-232 & $<2.7 E-02$ & U/I & $<1.9 E-01$ & & 1 & $\mathrm{GP}$ & & & & & & & pCin \\
\hline $\begin{array}{l}\text { Sum of alphas } \\
\text { Sum of betas }\end{array}$ & $\begin{array}{l}6.2 E+01 \\
5.2 E+\infty 0\end{array}$ & & & $\mathbf{0}$ & & & & & & & & & $\begin{array}{l}\text { pCin } \\
\text { pCin }\end{array}$ \\
\hline Tritium & $1.6 E+01$ & /I & & & 1 & GP & $5.8 E+02$ & /I & & $\square$ & 1 & GP & pCi/mL \\
\hline Uranium-233/234 & 1.7E+01 & /I & & $\mathbf{E}$ & 1 & GP & & . & & & & & pCin \\
\hline Uranium-235 & $1.6 E+\infty$ & /1 & & & 1 & GP & & & & & & & pcin \\
\hline Uranium-238 & $4.3 E+01$ & /I & & 旡 & 1 & GP & & & & & & & $\mathrm{Cin}$ \\
\hline
\end{tabular}

Notes:

= exceeded holding time

= exceeded groundwater protection or monitoring constituent standard (See Appendix A.)

NA - Not applicable. Applies to beta dose and sum of betas if there are no beta-emitting radionuclides above detection limits; to sum of aiphas if there are no alphaernitting radionuclides above detection limits; and to total radium if neither radium-226 or radium-228 was above detection limit

UAZ_UTRA - Upper Aquifer Zone of the Upper Three Runs Aquifer; LAZ UTRA - Lower Aquifer Zone of the Upper Three Runs Aquiler, Gordon - Gordon Aquifer 


\section{WELL FSB 88C}

\begin{tabular}{|c|c|}
\hline SRS Coord. & Lat/Long \\
\hline $\begin{array}{l}\text { N75619.4 } \\
\text { E51518.0 }\end{array}$ & $\begin{array}{l}33.277078^{\circ} \\
81.678031^{\circ}\end{array}$ \\
\hline
\end{tabular}

SAMPLE DATE

FIELD DATA

Constituents

Water elevation

$\mathrm{pH}$

Sp. conductance

Water temperature

Alkalinity as $\mathrm{CaCO} 3$

Turbidity

Volumes purged

Sampling code

Synchronous water level
Screen Zone Elevation

168.4-158.4 ft ms

$01 / 11 / 99$
Top of Casing

$283 \mathrm{ft}$ msl

Casing Pume
4" PVC S

Screen Zone

LAZ_UTRA
$04 / 07 / 99$

\begin{tabular}{l}
2099 \\
\hline 213.2 \\
5.0 \\
43 \\
21.0 \\
5 \\
0 \\
4.7
\end{tabular}

$212.3(06 / 28 / 99)$
Unit

ft msl

pH

$\mu \mathrm{S} / \mathrm{cm}$

${ }^{\circ} \mathrm{C}$

mgh

well volumes

ft msi

\section{ANALYTICAL DATA}

Inorganic Constituents

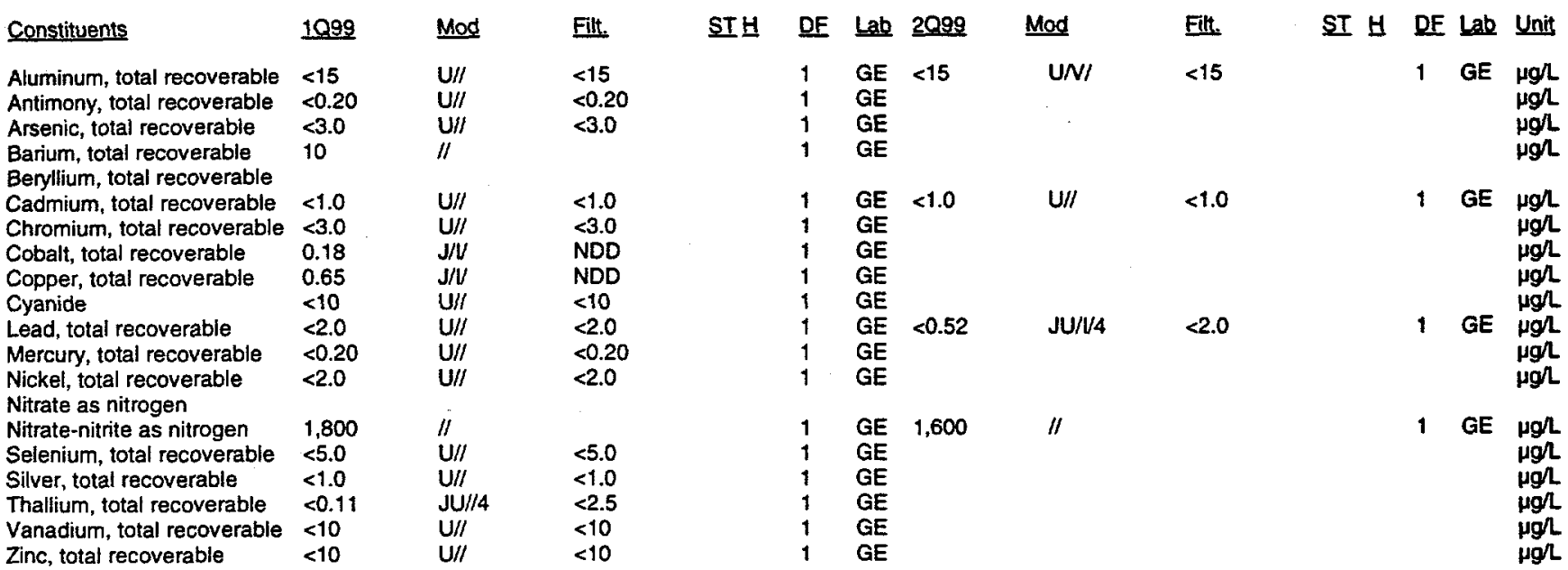

Organic Constituents

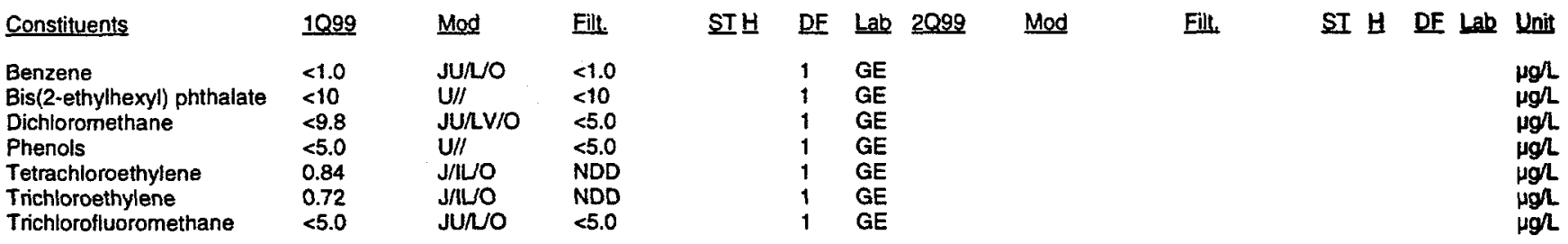

Notes:

- = exceeded holding time

I = exceeded groundwater protection or monitoring constituent standard (See Appendix A.)

NA - Not applicable. Applies to beta dose and sum of betas if there are no beta-emitting radionuclides above detection limits; to sum of alphas if there are no alphaemitting radionuclides above detection limits; and to total radium if neither radium-226 or radium-228 was above detection limit

UAZ UTRA - Upper Aquifer Zone of the Upper Three Runs Aquifer, LAZ UTRA - Lower Aquifer Zone of the Upper Three Runs Aquifer, Gordon - Gordon Aquifer 


\section{WELL FSB 88C (cont.)}

\section{Radioactive Constituents}

\begin{tabular}{|c|c|c|c|c|c|c|c|c|c|c|c|c|c|}
\hline Constituents & 1099 & Mod & Fill. & STH & DF & Lab & $\underline{2099}$ & Mod & Filt. & ST $\underline{H}$ & DF & $L a b$ & Unir \\
\hline $\begin{array}{l}\text { Americium-241 } \\
\text { Beta dose }\end{array}$ & $\begin{array}{l}<1.6 E-02 \\
\text { NA }\end{array}$ & $\mathrm{U} / \mathrm{I}$ & $<7.5 \mathrm{E}-02$ & & 1 & GP & & & & & & & pCin \\
\hline Caron-14 & $<1.6 E+00$ & $u / I$ & $<7.7 E+00$ & & 1 & GP & & & & & & & pCin \\
\hline Cesium-137 & $<-3.9 \mathrm{E}-01$ & UII & $<3.6 E+\infty$ & & 1 & GP & & & & & & & $\mathrm{pCin}$ \\
\hline Cobalt-60 & $<1.5 E+\infty 0$ & $U / I$ & $<4.1 E+\infty 0$ & & 1 & GP & & & & & & & pCin \\
\hline Curium-242 & $<-1.2 E-02$ & $u / \prime$ & $<1.6 \mathrm{E}-01$ & & $t$ & $G P$ & & & & & & & pCin \\
\hline Curium-243/244 & $<2.5 E-02$ & $U / I$ & $<7.5 E-02$ & & 1 & GP & & & & & & & pcir \\
\hline Curium-245/246 & $<0.0 E+\infty$ & $U / I$ & $<7.5 \mathrm{E}-02$ & & 1 & GP & & & & & & & $p \operatorname{Cin}$ \\
\hline $\begin{array}{l}\text { Gross alpha } \\
\text { lodine-129 }\end{array}$ & $\begin{array}{l}7.7 E-01 \\
<1.0 E+\infty 0\end{array}$ & $\begin{array}{l}\mathrm{J} / \mathrm{U} \\
\mathrm{U} / /\end{array}$ & $\begin{array}{l}\text { NDD } \\
<1.3 E+00\end{array}$ & & $\begin{array}{l}1 \\
1\end{array}$ & $\begin{array}{l}\text { GP } \\
\text { GP }\end{array}$ & $9.2 E-01$ & $\mathrm{~J} / \mathrm{V}$ & NDD & & 1 & GP & $\begin{array}{l}p C i n \\
p C i \Omega\end{array}$ \\
\hline Nonvolatile beta & $<6.9 E-01$ & UII & $<1.4 E+\infty$ & & 1 & GP & $<8.4 E-01$ & $U / / I$ & $<9.6 E-01$ & & 1 & GP & pcin \\
\hline Plutonium-238 & $<1.7 E-02$ & UII & $<1.2 \mathrm{E}-01$ & & 1 & GP & & & & & & & $\mathrm{pCin}$ \\
\hline Plutonium-239/240 & $<0.0 E+00$ & $u / I$ & $<6.7 E-02$ & & 1 & GP & & & & & & & $\mathrm{pCi}$ \\
\hline Radium-226 & $3.1 E-01$ & $\mathrm{~J} / \mathrm{V} /$ & NDD & & $i$ & $G P$ & & & & & & & $\mathrm{pCin}$ \\
\hline Radium-228 & $<1.3 E+00$ & UNI & $<1.2 E+\infty$ & & 1 & GP & & & & & & & pcin \\
\hline Total radium & & & & & & & & & & & & & \\
\hline Strontium-90 & $<9.5 E-02$ & U/I & $<1.3 E+00$ & & 1 & GP & & & & & & & pCin \\
\hline Technetium-99 & $<9.4 E-01$ & $u \prime \prime$ & $<2.1 E+01$ & & 1 & GP & & & & & & & pCir \\
\hline Thorium-228 & $1.6 \mathrm{E}-01$ & R/4 & $R e j$ & & 1 & $G P$ & & & & & & & $\mathrm{pCin}$ \\
\hline Thorium-230 & $<4.2 \mathrm{E}-02$ & JULLI & $<6.8 \mathrm{E}-02$ & & $i$ & GP & & & & & & & pCir \\
\hline Thorium-232 & $<0.0 E+\infty$ & JUЛI & $<2.3 E-02$ & & 1 & GP & & & & & & & pCin \\
\hline Sum of alphas & NA & & & & & & & & & & & & \\
\hline Sum of betas & NA & & & & & & & & & & & & \\
\hline Tritium & $1.9 E+01$ & $\|$ & & & 1 & GP & $1.1 E+01$ & "I & & & 1 & GP & $\mathrm{pCi} / \mathrm{ml}$ \\
\hline Uranium-233/234 & $<-3.5 E-02$ & $U / \prime \prime$ & $<2.0 \mathrm{E}-01$ & & 1 & GP & & & & & & & pCin \\
\hline Uranium-235 & $<-2.3 E-02$ & $\mathrm{U} / 1 /$ & $<2.2 E-01$ & & 1 & GP & & & & & & & $\mathrm{pCin}$ \\
\hline Uranium-238 & $<-1.2 E-02$ & U/I & $<1.4 \mathrm{E}-01$ & & $i$ & $G P$ & & & & & & & $\mathrm{pCir}$ \\
\hline
\end{tabular}

\section{Notes:}

= exceeded holding time

- exceeded groundwater protection or monitoring constituent standard (See Appendix A)

NA - Not applicable. Applies to beta dose and sum of betas if there are no beta-emitting radionuclides above detection limits; to sum of alphas if there are no alphaemitting radionuclides above detection limits; and to total radium if neither radium-226 or radium-22B was above detection timit

UAZ_UTRA - Upper Aquifer Zone of the Upper Three Runs Aquifer, LAZ_UTRA - Lower Aquifer Zone of the Upper Three Runs Aquifer, Gordon - Gordon Aquifer 


\section{WELL FSB 88D}

\begin{tabular}{|c|c|}
\hline SRS Coord. & Lat/Longitud \\
\hline $\begin{array}{l}\text { N75621.8 } \\
\text { E51527.0 }\end{array}$ & 81.678012 \\
\hline
\end{tabular}

SAMPLE DATE

$01 / 14 / 99$

Screen Zone Elevation

222.1-202.1 ft msl

FIELD DATA

Constituents

Water elevation

$\mathrm{pH}$

Sp. conductance

Water temperature

Alkalinity as $\mathrm{CaCO} 3$

Turbidity

Volumes purged

Sampling code

Synchronous water level

\section{ANALYTICAL DATA}

Inorganic Constituents

\section{9}

217.7

4.0

240

20.1

0

0.098

$\mathrm{X}$

$217.0(03 / 23 / 99)$
Top of Casing

$282.4 \mathrm{ft}$ msl

04/06/99

2099

216.7

4.0

320

19.9

0

2.0

$216.5(06 / 28 / 99)$
Casing Pump Screen Zone

4" PVC V UAZ_UTRA
Unit

ft msl

pH

$\mu \mathrm{S} / \mathrm{cm}$

${ }^{\circ} \mathrm{C}$

mgl

well volumes

ft msl

Constituents

Aluminum, total recoverable

Antimony, total recoverable

Arsenic, total recoverable

Barium, total recoverable

Beryllium, total recoverable

Cadmium, total recoverable 0.99

Chromium, total recoverable 8.8

Cobalt, total recoverable

Copper, total recoverable

Cyanide

Lead, total recoverable

Mercury, total recoverable

Nickel, total recoverable

Nitrate as nitrogen

Nitrate-nitrite as nitrogen $\quad 33,000$

Selenium, total recoverable $<5.0$

Silver, total recoverable $\quad<1.0$

Thallium, total recoverable $<0.082$

Vanadium, total recoverable $<10$

Zinc, total recoverable

86

Organic Constituents

\begin{tabular}{llll} 
Constifuents & 1099 & Mod & Filt. \\
\cline { 2 - 4 } Benzene & $<1.0$ & JU/O & $<1.0$ \\
Bis(2-ethylhexyl) phthalate & $<9.9$ & U/I & $<9.9$ \\
Dichloromethane & $<1.7$ & JU/LVO & $<5.0$ \\
Phenols & $<5.0$ & U/I & $<5.0$ \\
Tetrachioroethylene & $<1.0$ & JU/O & $<1.0$ \\
Trichloroethylene & $<1.0$ & JU/O & $<1.0$ \\
Trichlorofluoromethane & $<5.0$ & JU/OO & $<5.0$
\end{tabular}

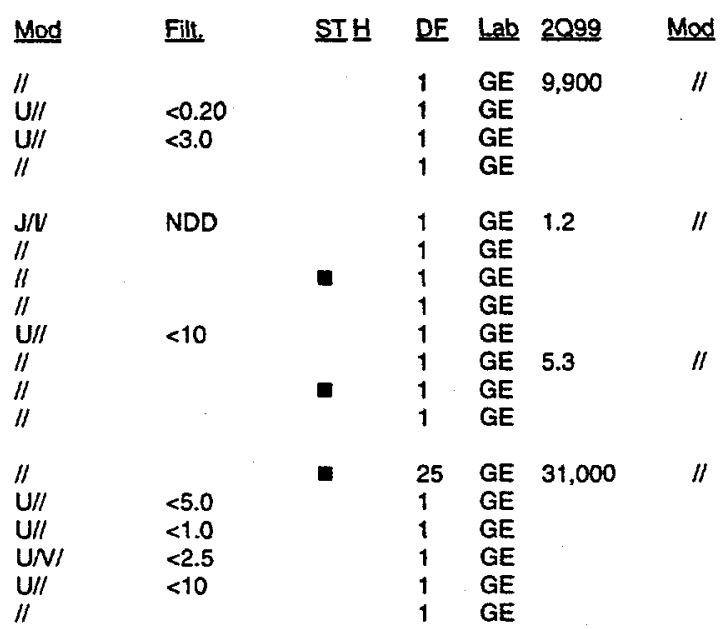

Fit.

ST 브 DF Lab Unit

1 GE $\mu g /$

$\mu g h$

Hgh

1 GE $\mu g /$.

$\mu g / 2$

$\mu g h$

Hg/

$\mu g /$

$\mu g h$

25 GE $\mu g /$

$\mu g / 2$

$\mu g / L$

$\mu g / L$

$\mu g / L$

$\mu g / L$

STH DF Lab $\underline{\underline{2 Q 99}} \quad \underline{\underline{M o d}}$

Filt.

ST H DF Lab Unit

Hg/L

$\mu g h$

$\mu g /$

$\mu g \Omega$

$\mu g / L$

$\mu g h$

Notes:

- = exceeded holding time

= exceeded groundwater protection or monitoring constituent standard (See Appendix A.)

NA - Not applicable. Applies to beta dose and sum of betas if there are no beta-emitting radionuclides above detection limits; to sum of alphas if there are no alphaemitting radionuclides above detection limits; and to total radium if neither radium-226 or radium-228 was above detection limit

UAZ UTRA - Upper Aquiter Zone of the Upper Three Runs Aquifer, LAZ UTRA - Lower Aquiler Zone of the Upper Three Runs Aquifer, Gordon - Gordon Aquifer 


\section{WELL FSB 88D (cont.)}

Radioactive Constituents

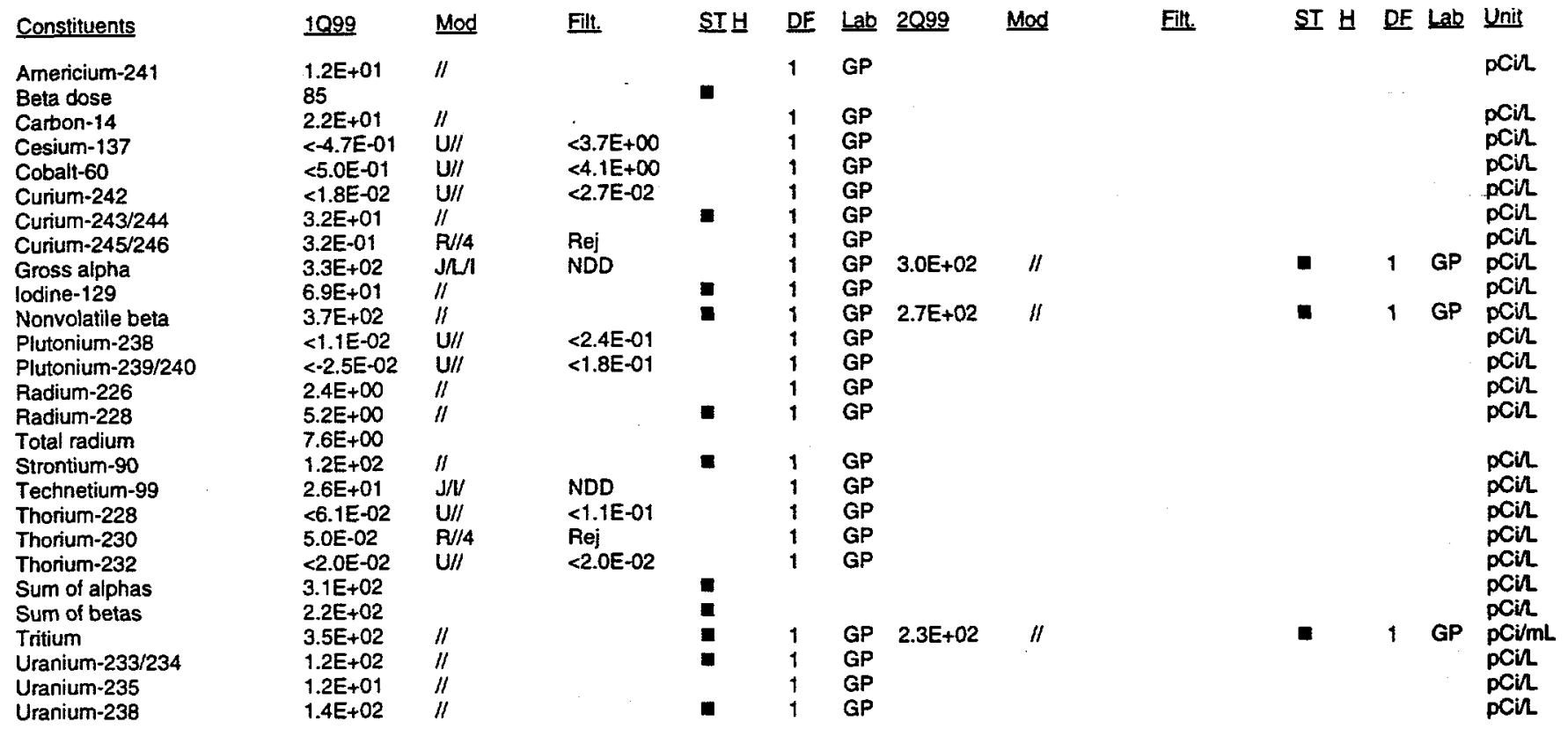

Notes:

- exceeded holding time

= exceeded groundwater protection or monitoring constituent standard (See Appendix A.)

NA - Not applicable. Applies to beta dose and sum of betas it there are no beta-emitting radionuclides above detection limits; to sum of alphas if there are no alphaemitting radionuclides above detection limits; and to total radium if neither radium-226 or radium-228 was above detection limit UAZ_UTRA - Upper Aquifer Zone of the Upper Three Runs Aquifer, LAZ UTRA - Lower Aquifer Zone of the Upper Three Runs Aquifer, Gordon - Gordon Aquifer 


\section{WELL FSB 89C}

\begin{tabular}{|c|c|}
\hline RS Coord. & LatlLongitude \\
\hline & \\
\hline
\end{tabular}

\section{Screen Zone Elevation}

166.1-156.1 ft ms|

01/20/99

\section{Top of Casing}

$281.3 \mathrm{ft} \mathrm{msl}$
Casing Pump

4" PVC S
Screen Zone

LAZ_UTRA
SAMPLE DATE

\section{FIELD DATA}

Constituents

Water elevation

pH

Sp. conductance

Water temperature

Alkalinity as $\mathrm{CaCO} 3$

Turbidity

Volumes purged

Sampling code

Synchronous water level

ANALYTICAL DATA
$04 / 06 / 99$

$\begin{array}{ll}2099 & \text { Unit } \\ 212.3 & \mathrm{ft} \mathrm{msl} \\ 5.4 & \mathrm{pH} \\ 54 & \mu \mathrm{S} / \mathrm{cm} \\ 20.1 & { }^{\circ} \mathrm{C} \\ 8 & \mathrm{mg} / \mathrm{L} \\ 1 & \mathrm{NTU} \\ 3.4 & \text { well volumes } \\ 211.7(06 / 28 / 99) & \mathrm{ft} \mathrm{msl}\end{array}$

Inorganic Constituents

\begin{tabular}{|c|c|c|c|c|c|c|c|c|c|c|c|c|c|}
\hline Constituents & 1099 & Mod & Filt. & ST브 & $\underline{\text { DF }}$ & Lab & $\underline{2099}$ & Mod & Filt. & ST 브 & DF & Lab & Unit \\
\hline Aluminum, total recoverable & $<15$ & $U / /$ & $<15$ & & 1 & GE & $<15$ & $\mathrm{U} / /$ & $<15$ & & 1 & GE & $\mu g / 2$ \\
\hline Antimony, total recoverable & 1.2 & 11 & & & 1 & GE & & & & & & & \\
\hline Arsenic, total recoverable & 1.1 & $\mathrm{~J} / / /$ & NDD & & 1 & GE & & & & & & & \\
\hline $\begin{array}{l}\text { Barium, total recoverable } \\
\text { Beryllium, total recoverable }\end{array}$ & 16 & $/ 1$ & & & 1 & WA & & & & & & & $\mu g h$ \\
\hline Cadmium, total recoverable & 0.49 & $\mathrm{~J} / \mathrm{V}$ & NDD & & 1 & WA & $<1.0$ & U/I & $<1.0$ & & 1 & GE & uod \\
\hline Chromium, total recoverable & 2.3 & $\mathrm{~J} / \mathrm{V}$ & NDD & & 1 & WA & & & & & 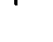 & u & \\
\hline Cobalt, total recoverable & 0.42 & $\|$ & & & 1 & GE & & & & & & & \\
\hline Copper, total recoverable & $<1.7$ & $U / / 6$ & $<1.0$ & & 1 & GE & & & & & & & $\mu g /$ \\
\hline Cyanide & 3.7 & $\mathrm{~J} / \mathrm{V}$ & NDD & & 1 & GE & & & & & & & \\
\hline Lead, total recoverable & 1.2 & $\mathrm{~J} / \mathrm{N}$ & NDD & & 1 & GE & 0.59 & $\mathrm{~J} / \mathrm{V}$ & NDD & & 1 & GE & jon \\
\hline Mercury, total recoverable & $<0.20$ & $\mathrm{U} / /$ & $<0.20$ & & 1 & GE & & & & & & & \\
\hline $\begin{array}{l}\text { Nickel, total recoverable } \\
\text { Nitrate as nitrogen }\end{array}$ & 1.5 & $\mathrm{~J} / \mathrm{V}$ & NDD & & 1 & GE & & & & & & & \\
\hline Nitrate-nitrite as nitrogen & 1,800 & 11 & & & 5 & WA & 1,800 & $/ 1$ & & & 1 & GE & $\mu g / 2$ \\
\hline Selenium, total recoverable & $<5.0$ & $\mathrm{U} / /$ & $<5.0$ & & 1 & GE & & & & & & & \\
\hline Silver, total recoverable & 2.1 & J几I & NDD & & 1 & GE & & & & & & & $\mu g$ \\
\hline Thallium, total recoverable & 1.8 & $J / U$ & NDD & & 1 & GE & & & & & & & $\mu g / L$ \\
\hline Vanadium, total recoverable & $<6.9$ & $\mathrm{U} / /$ & $<6.9$ & & 1 & WA & & & & & & & \\
\hline Zinc, total recoverable & 16 & $\mathrm{~J} / \mathrm{V}$ & NDD & & 1 & WA & & & & & & & \\
\hline
\end{tabular}

Organic Constituents
1099

213.0

5.9

19.

12

2.3

$213.0(03 / 23 / 99)$

\begin{tabular}{|c|c|c|c|c|c|c|c|c|c|c|}
\hline Constituents & 1099 & Mod & Fill. & ST브 & DF & Lab 2099 & Mod & Filt. & ST $H$ DF Lab & Unit \\
\hline $\begin{array}{l}\text { Benzene } \\
\text { Bis(2-ethylhexyl) phthalate } \\
\text { Dichloromethane } \\
\text { Phenols } \\
\text { Tetrachloroethylene } \\
\text { Trichloroethylene } \\
\text { Trichlorofluoromethane }\end{array}$ & $\begin{array}{l}<1.0 \\
<10 \\
<2.2 \\
<5.0 \\
1.1 \\
1.0 \\
3.9\end{array}$ & $\begin{array}{l}\mathrm{U} / / \\
\mathrm{U} / / \\
\mathrm{U} / \mathrm{KV} / \mathrm{O} \\
\mathrm{U} / / \\
\mathrm{J} / \mathrm{K} / \mathrm{O} \\
\mathrm{J} / \mathrm{K} / \mathrm{O} \\
\mathrm{J} / \mathrm{K} / \mathrm{O}\end{array}$ & $\begin{array}{l}<1.0 \\
<10 \\
<5.0 \\
<5.0 \\
\text { NDD } \\
\text { NDD } \\
\text { NDD }\end{array}$ & & $\begin{array}{l}1 \\
1 \\
1 \\
1 \\
1 \\
1 \\
1\end{array}$ & $\begin{array}{l}\text { GE } \\
\text { GE } \\
\text { GE } \\
\text { GE } \\
\text { GE } \\
\text { GE } \\
\text { GE }\end{array}$ & & & & $\begin{array}{l}\mu g h \\
\mu g h / \\
\mu g h \\
\mu g h \\
\mu g h \\
\mu g h \\
\mu h h\end{array}$ \\
\hline
\end{tabular}

Notes:

= exceeded holding time

= exceeded groundwater protection or monitoring constituent standard (See Appendix A.)

NA - Not applicable. Applies to beta dose and sum of betas if there are no beta-emitting radionuclides above detection limits; to sum of alphas if there are no alphaemitting radionuclides above detection limits; and to total radium if neither radium-226 or radium-228 was above detection limit

UAZ UTRA - Upper Aquifer Zone of the Upper Three Runs Aquifer, LAZ UTRA - Lower Aquifer Zone of the Upper Three Runs Aquifer, Gordon - Gordon Aquifer 


\section{WELL FSB 89C (cont.)}

Radioactive Constituents

\begin{tabular}{|c|c|c|c|c|c|c|c|c|c|c|c|c|c|}
\hline Constituents & 1099 & Mod & Filt. & STH & DF & Lab & 2099 & Mod & Filt. & ST $\underline{H}$ & DF & Lab & Unit \\
\hline $\begin{array}{l}\text { Americium-241 } \\
\text { Beta dose }\end{array}$ & $\begin{array}{l}<5.5 E-02 \\
\text { NA }\end{array}$ & $\mathrm{U} / /$ & $<4.8 E-01$ & & 1 & GP & & & & & & & pCin \\
\hline Carbon-14 & $<3.3 E-01$ & U/I & $<7.7 \mathrm{E}+\infty$ & & 1 & GP & & & & & & & pCill \\
\hline Cesium-137 & $<1.4 \mathrm{E}-01$ & $\mathrm{U} / \prime$ & $<3.5 E+\infty$ & & 1 & GP & & & & & & & pCir \\
\hline Cobalt -60 & $<-3.9 E-02$ & $\mathrm{U} / /$ & $<2.7 E+\infty$ & & 1 & GP & & & & & & & pCir \\
\hline Curium-242 & $<-8.7 E-03$ & UII & $<5.4 E-01$ & & $i$ & GP & & & & & & & pCin \\
\hline Curium-243/244 & $<2.0 E-03$ & $U / /$ & $<3.9 E-01$ & & $i$ & GP & & & & & & & pCir \\
\hline Curium-245/246 & $<6.1 E-02$ & $U / I$ & $<1.8 \mathrm{E}-01$ & & 1 & GP & & & & & & & pCin \\
\hline Gross alpha & $<6.1 E-02$ & 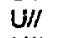 & $<5.8 \mathrm{E}-01$ & & 1 & GP & $<3.9 E-01$ & $U / l$ & $<5.5 E-01$ & & 1 & GP & pCir \\
\hline lodine-129 & $<3.0 E-01$ & $\mathrm{U} / \mathrm{I}$ & $<9.6 E-01$ & & 1 & GP & & & & & & & pCin \\
\hline Nonvolatile beta & $<2.6 E-01$ & $U / I$ & $<1.2 \mathrm{E}+\infty$ & & 1 & GP & $<6.0 E-01$ & $\mathrm{U} / /$ & $<9.7 E-01$ & & 1 & GP & pCiL \\
\hline Plutonium-238 & $<0.0 E+00$ & $\mathrm{U} / /$ & $<5.0 \mathrm{E}-02$ & & 1 & GP & & & & & & & pCil \\
\hline Plutonium-239/240 & $<0.0 E+00$ & $\mathrm{U} / /$ & $<3.1 E-01$ & & 1 & $T M$ & & & " & & & & $\mathrm{pCil}$ \\
\hline Radium-226 & $6.3 E-01$ & $J / U$ & NDD & & 1 & GP & & & & & & & $\mathrm{pCil}$ \\
\hline $\begin{array}{l}\text { Radium-228 } \\
\text { Total radium }\end{array}$ & $\begin{array}{l}<1.7 E-01 \\
\text { NA }\end{array}$ & $U / /$ & $<1.0 E+00$ & & 1 & GP & & & & & & & poil \\
\hline Strontium-90 & $<-2.0 E-02$ & $\mathrm{U} / /$ & $<1.7 E+00$ & & 1 & GP & & & & & & & pCir \\
\hline Technetium-99 & $<-4.9 E-01$ & $\mathrm{U} / /$ & $<2.2 E+01$ & & 1 & GP & & & & & & & $\mathrm{pCin}$ \\
\hline Thorium-228 & $<-2.2 E-02$ & $U / /$ & $<4.4 E-01$ & & 1 & GP & & & & & & & pCin \\
\hline Thorium-230 & $<1.5 E-03$ & UII & $<2.8 E-01$ & & 1 & GP & & & & & & & pCir \\
\hline Thorium-232 & $<-4.0 E-03$ & UII & $<8.7 E-02$ & & $i$ & GP & & & & & & & pCin \\
\hline Sum of alphas & NA & & & & & & & & & & & & \\
\hline Sum of betas & $N A$ & & & & & & & & . & & & & \\
\hline Tritium & $1.4 E+01$ & $\mathrm{~J} / \mathrm{KJC}$ & NDD & & 1 & $T M$ & $1.2 E+01$ & $/ l$ & & & 1 & GP & pCi/mL \\
\hline Uranium-233/234 & 4.3E-01 & $\mathrm{R} / / 4$ & $\operatorname{Rej}$ & & 1 & GP & & & & & & & pCil \\
\hline Uranium-235 & $<0.0 E+\infty$ & $\mathrm{U} / /$ & $<3.0 \mathrm{E}-01$ & & 1 & TM & & & & & & & pCil \\
\hline Uranium-238 & $<3.0 \mathrm{E}-02$ & $\mathrm{U} / /$ & $<8.9 E-02$ & & 1 & GP & & & & & & & pCin \\
\hline
\end{tabular}

\section{Notes:}

- = exceeded holding time

$\mathbf{\square}=$ exceeded groundwater protection or monitoring constituent standard (See Appendix A.)

NA - Not applicable. Applies to beta dose and sum of betas if there are no beta-emitting radionuclides above detection limits; to sum of alphas if there are no alphaemitting radionuclides above detection limits; and to total radium if neither radium-226 or radium-228 was above detection limit

UAZ_UTRA - Upper Aquifer Zone of the Upper Three Runs Aquifer, LAZ_UTRA - Lower Aquifer Zone of the Upper Three Runs Aquifer, Gordon - Gordon Aquifer 
WELL FSB 89D

$\begin{array}{ll}\text { SRS Coord. } & \text { Lathongitude } \\ \text { N75548.3 } & 33.276623^{\circ} \mathrm{N} \\ \text { E51335.8 } & 81.678372^{\circ} \mathrm{W}\end{array}$

SAMPLE DATE
Screen Zone Elevation

$221.9-201.9 \mathrm{ft} \mathrm{ms}$
Iop of Casing

$281.2 \mathrm{ft}$ ms!

Casing Pump
4" PVC V

04/06/99
Unit

216.0

4.0

280

19.5

2

2.3

$215.8(06 / 28 / 99)$

Sp. conductance

Water temperature

Tubidity

Volumes purged

Sampling code

Synchronous water level

3.7

$216.2(03 / 23 / 99)$
Screen Zone

UAZ_UTRA

\section{ANALYTICAL DATA}

Inorganic Constituents

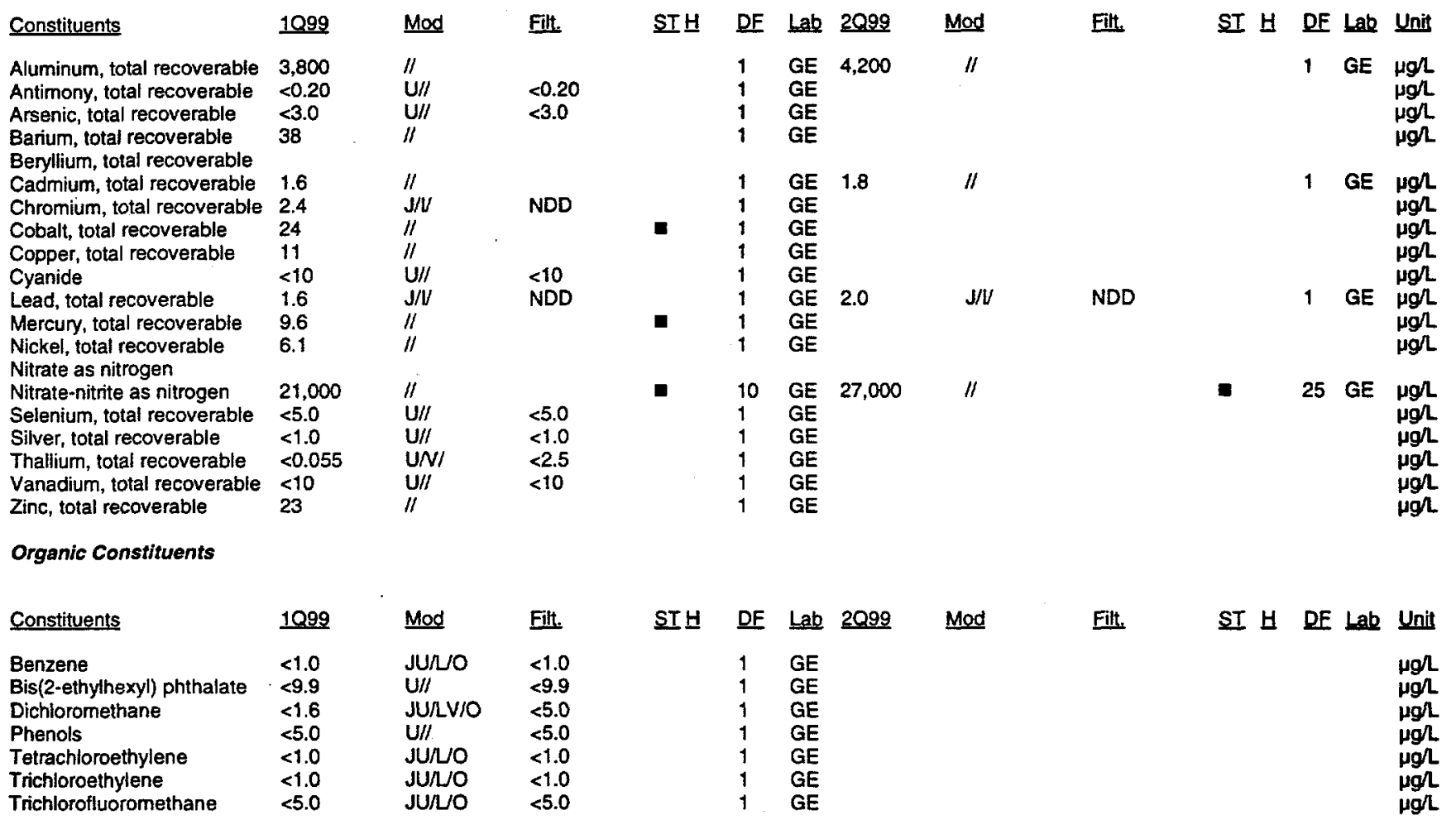

\section{Notes:}

= exceeded holding time

E = exceeded groundwater protection or monitoring constituent standard (See Appendix A.)

NA - Not applicable. Applies to beta dose and sum of betas if there are no beta-emitting radionuclides above detection limits; to sum of alphas if there are no alphaemitting radionuclides above detection limits; and to total radium if neither radium-226 or radium-228 was above detection limit

UAZ_UTRA - Upper Aquifer Zone of the Upper Three Runs Aquifer, LAZ_UTRA - Lower Aquiler Zone of the Upper Three Runs Aquifer, Gordon - Gordon Aquifer

F-Area HWMF 


\section{WELL FSB 89D (cont.)}

Radioactive Constituents

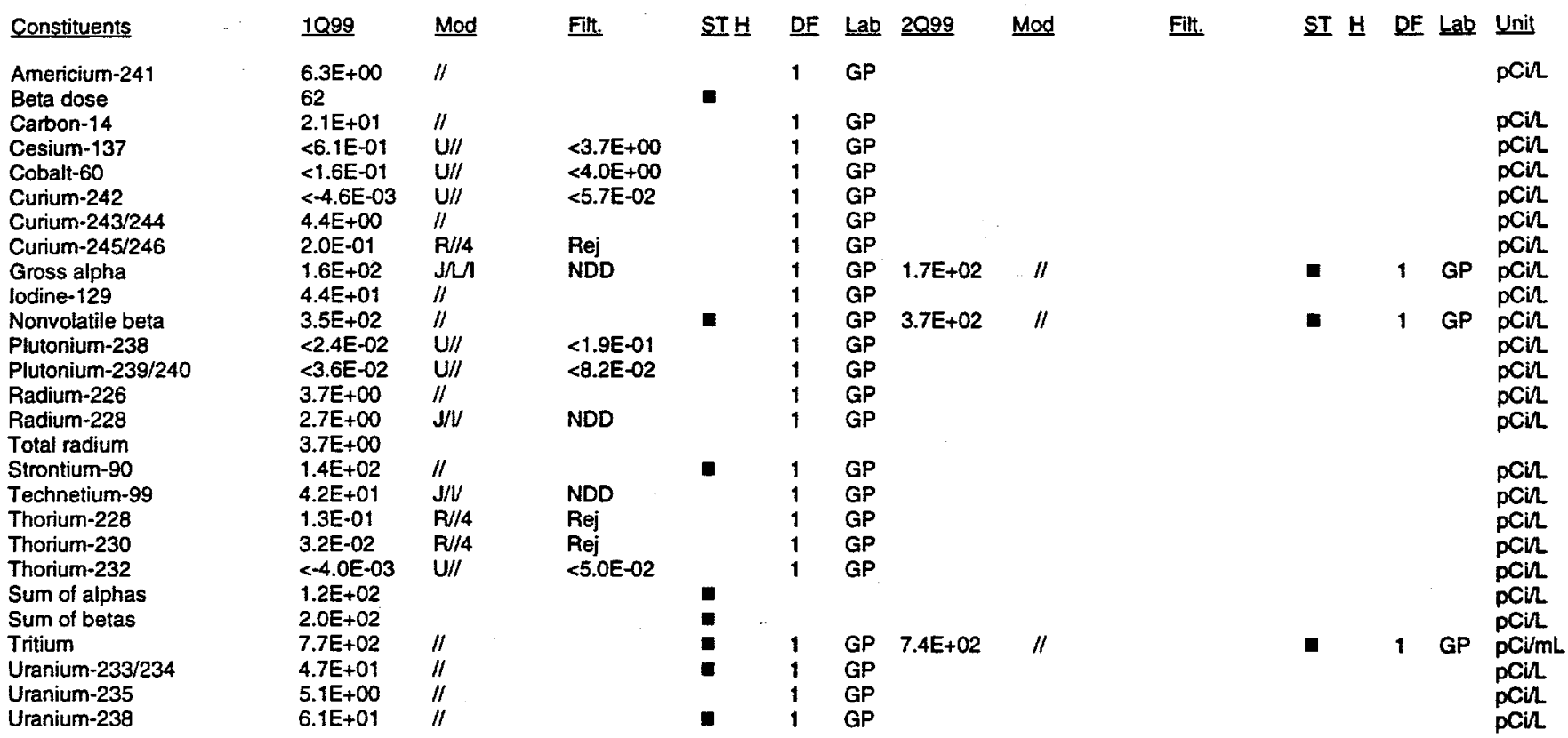

Notes:

- exceeded holding time

= exceeded groundwater protection or monitoring constituent standard (See Appendix A.)

NA - Not applicable. Applies to beta dose and sum of betas if there are no beta-emitting radionuclides above detection limits; to sum of alphas if there are no alphaemitting radionuclides above detection limits; and to total radium if neither radium-226 or radium-228 was above detection limit

UAZ_UTRA - Upper Aquifer Zone of the Upper Three Runs Aquifer, LAZ UTRA - Lower Aquifer Zone of the Upper Three Runs Aquifer, Gordon - Gordon Aquifer 


\section{WELL FSB 90C}

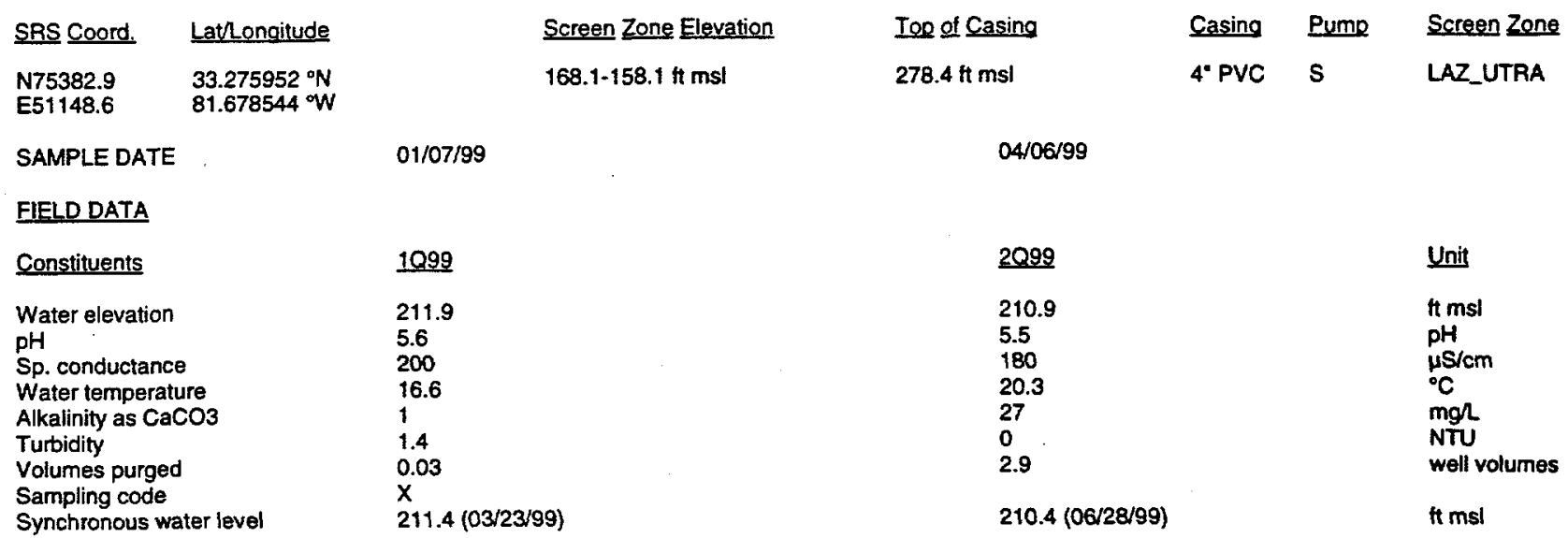

\section{ANALYTICAL DATA}

Inorganic Constituents

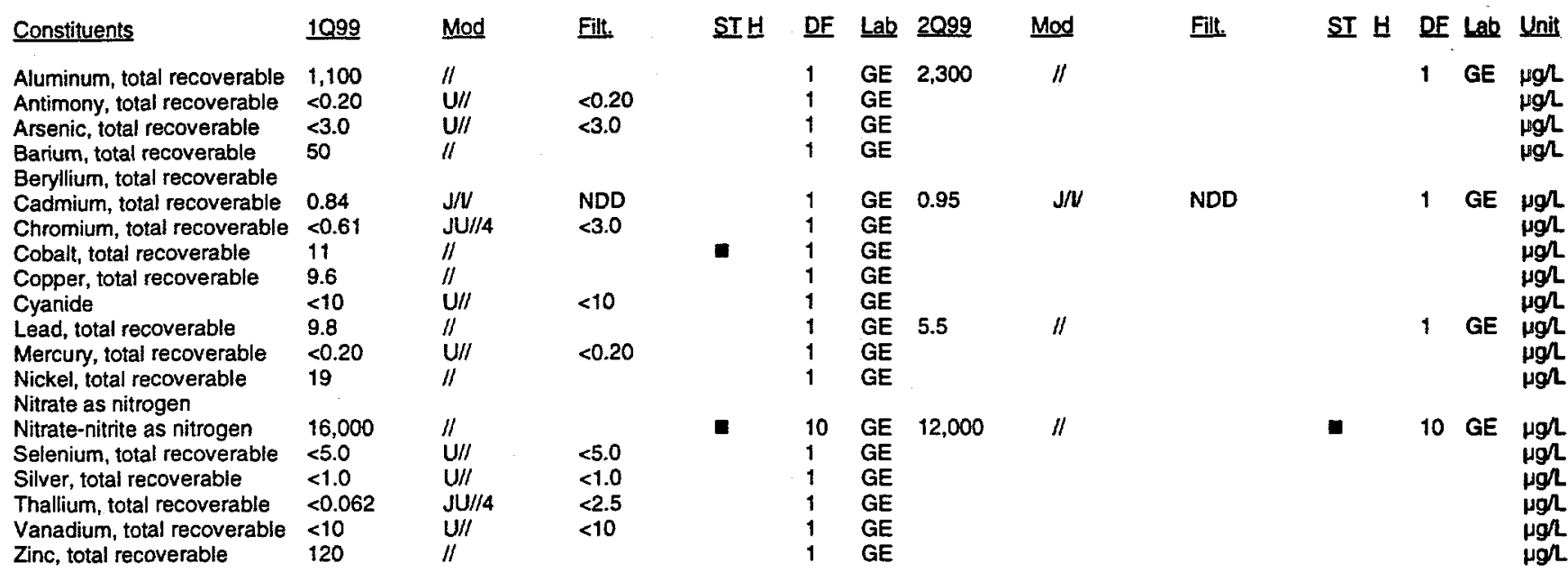

Organic Constituents

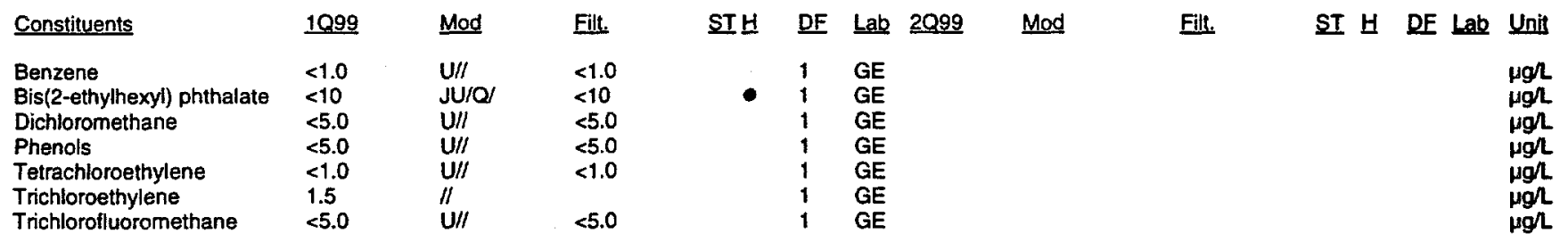

Notes:

= exceeded holding time

- exceeded groundwater protection or monitoring constituent standard (See Appendix A.)

NA - Not applicable. Applies to beta dose and sum of betas if there are no beta-emitting radionuclides above detection limits; to sum of alphas if there are no alpheemitting radionuclides above detection limits; and to total radium if neither radium-226 or radium-228 was above detection limit

UAZ UTRA - Upper Aquifer Zone of the Upper Three Runs Aquifer, LAZ UTRA - Lower Aquifer Zone of the Upper Three Runs Aquifer, Gordon - Gordon Aquifer 


\section{WELL FSB 90C (cont.)}

Radioactive Constituents

\begin{tabular}{|c|c|c|c|c|c|c|c|c|c|c|c|c|c|}
\hline Constituents & 1099 & Mod & Filt. & STH & DF & Lab & 2099 & Mad & Filt. & ST & DF & Lab & Unit \\
\hline & $\begin{array}{l}<6.4 E-03 \\
0.59\end{array}$ & $\mathrm{JU} / \mathrm{L} / \mathrm{I}$ & $<6.2 \mathrm{E}-01$ & & 1 & GP & & & & & & & $\mathrm{pCi} / \mathrm{L}$ \\
\hline $\begin{array}{l}\text { Beta dose } \\
\text { Carbon-14 }\end{array}$ & $\begin{array}{l}0.59 \\
3.0 E+01\end{array}$ & $\| /$ & & & 1 & GP & & & & & & & pCir \\
\hline Cesium-137 & $<1.7 E-01$ & JU/L & $<3.7 E+\infty$ & & 1 & GP & & & & & & & pCin \\
\hline Cobalt-60 & $<-1.1 E+\infty$ & $\mathrm{U} / /$ & $<3.1 E+\infty$ & & 1 & GP & & & & & & & pCin \\
\hline Curium-242 & $<0.0 E+\infty$ & $\mathrm{U} / /$ & $<2.8 E-01$ & & 1 & GP & & & & & & & $\mathrm{pCi}$ \\
\hline Curium-243/244 & $<-4.3 E-02$ & $\mathrm{U} / !$ & $<5.5 E-01$ & & 1 & GP & & & & & & & pCil \\
\hline Curium-245/246 & $<0.0 E+\infty$ & $U / I$ & $<2.7 E-01$ & & 1 & GP & & & & & & & pCin \\
\hline $\begin{array}{l}\text { Gross alpha } \\
\text { lodine-129 }\end{array}$ & $6.9 E+\infty 0$ & $/ 1$ & NDD & & 1 & GP & $3.0 E+\infty 0$ & $\|$ & & & 1 & GP & pCir \\
\hline Nonvolatile beta & $2.1 E+01$ & $/ /$ & IVUU & & 1 & GP & $1.5 E+01$ & /I & & & 1 & GP & pcir \\
\hline Plutonium-238 & $<-8.2 E-03$ & $\mathrm{U} / /$ & $<1.8 E-01$ & & 1 & GP & & & & & & & pCill \\
\hline Plutonium-239/240 & $<-7.7 E-03$ & $\mathrm{U} / /$ & $<9.6 \mathrm{E}-02$ & & 1 & GP & & & & & & & pCir \\
\hline Radium-226 & $1.6 E+\infty 0$ & $/ /$ & & & 1 & GP & & & & & & & pCil \\
\hline $\begin{array}{l}\text { Radium-228 } \\
\text { Total radium }\end{array}$ & $\begin{array}{l}6.6 E-01 \\
1.6 E+00\end{array}$ & $\mathrm{~J} / \mathrm{V}$ & NDD & & 1 & GP & & & & & & & pCin \\
\hline Strontium-90 & $4.6 \mathrm{E}+00$ & 11 & & & 1 & GP & & & & & & & pCin \\
\hline Technetium-99 & $2.8 E+01$ & $\mathrm{~J} / \mathrm{V}$ & NDD & & 1 & GP & & & & & & & $\mathrm{pCi} / \mathrm{L}$ \\
\hline Thorium-228 & $<-8.8 \mathrm{E}-02$ & $\mathrm{U} / /$ & $<4.8 E-01$ & & 1 & GP & & & & & & & pCin \\
\hline Thorium-230 & $<1.5 E-03$ & $\mathrm{U} / /$ & $<2.8 E-01$ & & 1 & GP & & & & & & & pCin \\
\hline Thorium-232 & $<3.7 E-02$ & $U / /$ & $<1.1 E-01$ & & 1 & GP & & & & & & & pCin \\
\hline Sum of alphas & NA & & & & & & & & & & & & \\
\hline Sum of betas & $3.4 E+01$ & & & & & & & & & & & & pCin \\
\hline Tritium & $4.7 E+02$ & $/ 1$ & & $\mathbf{0}$ & 1 & GP & $3.9 E+02$ & II & & $\mathbf{E}$ & 1 & GP & $\mathrm{pCi} / \mathrm{mL}$ \\
\hline Uranium-233/234 & $<1.4 \mathrm{E}-01$ & UЛ/C & $<1.9 E-01$ & & 1 & GP & & & & & & & pcin \\
\hline Uranium-235 & $<1.4 E-02$ & JU/LC & $<1.7 E-01$ & & 1 & GP & & & & & & & pCin \\
\hline Uranium-238 & $<7.5 \mathrm{E}-03$ & JU/LC & $<1.9 E-01$ & & 1 & GP & & & & & & & $\mathrm{pCin}$ \\
\hline
\end{tabular}

- exceeded holding time

= exceeded groundwater protection or monitoring constituent standard (See Appendix A.)

NA - Not applicable. Applies to beta dose and sum of betas if there are no beta-emitting radionuclides above detection limits; to sum of alphas if there are no alphaemitting radionuclides above detection limits; and to total radium if neither radium-226 or radium-228 was above detection limit

UAZ_UTRA - Upper Aquifer Zone of the Upper Three Runs Aquifer, LAZ_UTRA - Lower Aquifer Zone of the Upper Three Runs Aquifer, Gordon - Gordon Aquifer 


\section{WELL FSB 90D}

\begin{tabular}{|c|c|}
\hline SRS Coord. & Lat/Longitude \\
\hline $\begin{array}{l}\text { N75376.9 } \\
\text { E51140.7 }\end{array}$ & $\begin{array}{l}33.27592 \\
81.678553\end{array}$ \\
\hline
\end{tabular}

SAMPLE DATE

$01 / 04 / 99$

Screen Zone Elevation

225.1-205.1 ft ms
Top of Casing

$278.6 \mathrm{ft} \mathrm{mst}$

04/09/99

\section{FIELD DATA}

\section{Constituents}

Water elevation

$\mathrm{pH}$

Sp. conductance

Water temperature

Alkalinity as $\mathrm{CaCO} 3$

Turbidity

Volumes purged

Sampling code

Synchronous water level

\section{ANALYTICAL DATA}

Inorganic Constituents
1029

208.3

4.2

1060

15.7

0

0.7

31

(03/23/99)
2099

3.7

600

18.9

0.021

XD

(06/28/99)
Casing Pump Screen Zone

4" PVC V UAZ_UTRA

\begin{tabular}{|c|c|c|c|c|c|c|c|c|c|c|c|c|c|}
\hline Constituents & $\underline{1099}$ & Mad & Fill: & ST & $\underline{\mathrm{DF}}$ & Lab & $\underline{2099}$ & Mod & Filt. & ST $\underline{H}$ & DF & Lab & Unit \\
\hline Aluminum, total recoverable & 19,000 & $\|$ & & & 1 & GE & 19,000 & $\mathrm{~J} / \mathrm{K} / \mathrm{I}$ & NDD & & 1 & GE & $\mu g / L$ \\
\hline Antimony, total recoverable & $<0.20$ & $\mathrm{U} / / \mathrm{I}$ & $<0.20$ & & 1 & GE & & & & & & & $\mu g / L$ \\
\hline Arsenic, total recoverable & $<3.0$ & UII & $<3.0$ & & 1 & GE & & & & & & & $\mu g h$ \\
\hline Barium, total recoverable & 150 & $\|$ & & & 1 & GE & & & & & & & $\mu g / L$ \\
\hline Beryllium, total recoverable & & & & & & & & & & & & & \\
\hline Cadmium, total recoverable & 8.3 & II & & - & 1 & GE & 7.1 & "I & & घ & 1 & GE & $\mu g / L$ \\
\hline Chromium, total recoverable & 6.3 & 11 & & & 1 & GE & & & & & & & gl \\
\hline Cobalt, total recoverable & 17 & 11 & & - & 1 & GE & & & & & & & $\mu g h$ \\
\hline Copper, total recoverable & 27 & $\|$ & & & 1 & GE & & & & & & & $\mu g / L$ \\
\hline Cyanide & $<10$ & UIII & $<10$ & & 1 & GE & & & & & & & $\mu g / L$ \\
\hline Lead, total recoverable & 14 & $\|$ & & & 1 & GE & 20 & 11 & & - & 1 & GE & $\mu g / L$ \\
\hline Mercury, total recoverable & 17 & $\|$ & & a & 2 & GE & & & & & & & $\mu g^{\prime} L$ \\
\hline Nickel, total recoverable & 18 & $\|$ & & & 1 & GE & & & & & & & $\mu g / L$ \\
\hline Nitrate as nitrogen & & & & & & & & & & & & & \\
\hline Nitrate-nitrite as nitrogen & 74,000 & $/ 1$ & & a & 25 & GE & 64,000 & "ll & & E & 50 & GE & $\mu g / L$ \\
\hline Selenium, total recoverable & $<5.0$ & $\mathrm{U} / \mathrm{I}$ & $<5.0$ & & 1 & GE & & & & & & & \\
\hline Silver, total recoverable & $<1.0$ & $U / I$ & $<1.0$ & & 1 & GE & & & & & & & jgh \\
\hline Thallium, total recoverable & 0.18 & $\mathrm{~J} / \mathrm{V}$ & NDD & & 1 & GE & & & & & & & ugh \\
\hline Vanadium, total recoverable & $<10$ & $U / I$ & $<10$ & & 1 & GE & & & & & & & \\
\hline Zinc, total recoverable & 51 & $\|$ & & & 1 & GE & & & & & & & \\
\hline
\end{tabular}

Organic Constituents

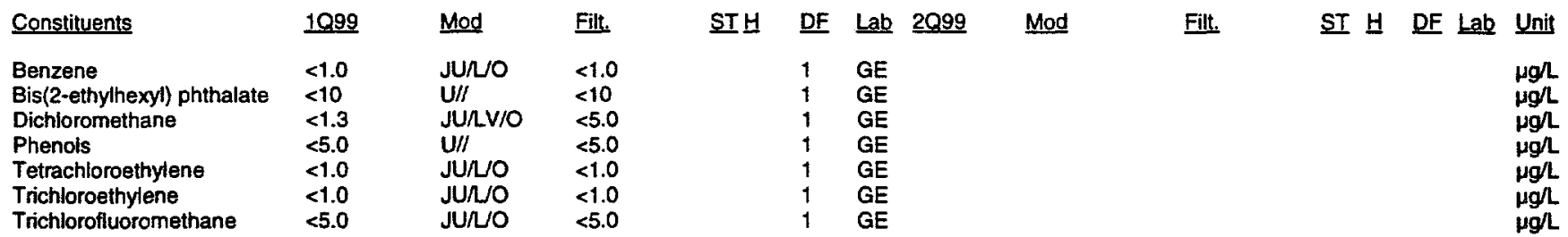

\footnotetext{
Notes:
}

- exceeded holding time

= exceeded groundwater protection or monitoring constituent standard (See Appendix A.)

NA - Not applicable. Applies to beta dose and sum of betas if there are no beta-emitting radionuclides above detection limits; to sum of alphas if there are no alphaemitting radionuclides above detection limits; and to total radium if neither radium-226 or radium-228 was above detection limit UAZ UTRA - Upper Aquifer Zone of the Upper Three Runs Aquifer, LAZ UTRA - Lower Aquifer Zone of the Upper Three Runs Aquifer, Gordon - Gordon Aquifer 


\section{WELL FSB 90D (cont.)}

Radioactive Constituents

\begin{tabular}{|c|c|c|c|c|c|c|c|c|c|c|c|c|c|}
\hline Constituents & 1099 & Mod & Filt. & SI브 & DE & Lab & 2099 & Mod & Filt. & ST $\mathrm{H}$ & DF & Lab & Unit \\
\hline $\begin{array}{l}\text { Americium-241 } \\
\text { Beta dose } \\
\text { Carbon-14 } \\
\text { Cesium-137 } \\
\text { Cobalt-60 } \\
\text { Curium-242 } \\
\text { Curium-243/244 } \\
\text { Curium-245/246 } \\
\text { Gross alpha } \\
\text { lodine-129 } \\
\text { Nonvolatile beta } \\
\text { Plutonium-238 } \\
\text { Plutonium-239/240 } \\
\text { Radium-226 } \\
\text { Radium-228 } \\
\text { Total radium } \\
\text { Strontium-90 } \\
\text { Technetium-99 } \\
\text { Thorium-228 } \\
\text { Thorium-230 } \\
\text { Thorium-232 } \\
\text { Sum of alphas } \\
\text { Sum of betas } \\
\text { Tritium } \\
\text { Uranium-233/234 } \\
\text { Uranium-235 } \\
\text { Uranium-238 }\end{array}$ & $\begin{array}{l}1.6 E+01 \\
205 \\
2.9 E+01 \\
<1.1 E+00 \\
<1.6 E+00 \\
<-1.7 E-01 \\
5.9 E+00 \\
2.8 E-01 \\
5.3 E+02 \\
2.0 E+02 \\
1.3 E+03 \\
2.3 E-01 \\
<3.4 E-02 \\
1.7 E+01 \\
2.4 E+01 \\
4.1 E+01 \\
4.3 E+02 \\
1.2 E+02 \\
4.8 E-01 \\
<7.8 E-03 \\
<-4.2 E-02 \\
4.5 E+02 \\
3.7 E+02 \\
2.2 E+03 \\
2.0 E+02 \\
1.4 E+01 \\
2.2 E+02\end{array}$ & 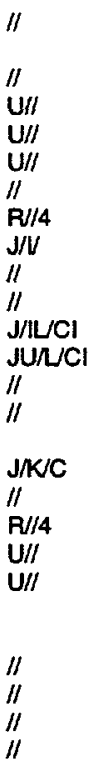 & $\begin{array}{l}<3.7 E+00 \\
<3.6 E+00 \\
<6.4 E-01 \\
\text { Rej } \\
\text { NDD } \\
\text { NDD } \\
<1.0 E-01 \\
\text { NDD } \\
\text { Rej } \\
<1.7 E-01 \\
<2.1 E-01\end{array}$ & $\begin{array}{l}\mathbf{E} \\
\mathbf{a} \\
\mathbf{0} \\
\mathbf{v}\end{array}$ & $\begin{array}{l}1 \\
1 \\
1 \\
1 \\
1 \\
1 \\
1 \\
1 \\
1 \\
1 \\
1 \\
1 \\
1 \\
1 \\
1 \\
1 \\
1 \\
1 \\
1\end{array}$ & $\begin{array}{l}\text { GP } \\
\text { GP } \\
\text { GP } \\
\text { GP } \\
\text { GP } \\
\text { GP } \\
\text { GP } \\
\text { GP } \\
\text { GP } \\
\text { GP } \\
\text { GP } \\
\text { GP } \\
\text { GP } \\
\text { GP } \\
\text { GP } \\
\text { GP } \\
\text { GP } \\
\text { GP } \\
\text { GP }\end{array}$ & $\begin{array}{l}5.7 E+02 \\
1.1 E+03\end{array}$ & $\begin{array}{l}\| \\
\|\end{array}$ & & $=$ & $\begin{array}{l}1 \\
1\end{array}$ & $\begin{array}{l}\text { GP } \\
\text { GP }\end{array}$ & $\begin{array}{l}\text { pCin } \\
\text { pCin } \\
\text { pCin } \\
\text { pCin } \\
\text { pCin } \\
\text { pCin } \\
\text { pCin } \\
\text { pCin } \\
\text { pCin } \\
\text { pCin } \\
\text { pCin } \\
\text { pCin } \\
\text { pCin } \\
\text { pCin } \\
\text { pCin } \\
\text { pCin } \\
\text { pCin } \\
\text { pCin } \\
\text { pCin } \\
\text { pCin } \\
\text { pCin } \\
\text { pCimL } \\
\text { pCin } \\
\text { pCin } \\
\text { pCin }\end{array}$ \\
\hline
\end{tabular}




\section{WELL FSB 91C}

$\begin{array}{lll}\text { SRS Coord. } & \text { Latlongitude } \\ \text { N75213.3 } & & 33.275259^{\circ} \mathrm{N} \\ \text { E50953.5 } & 81.678728^{\circ} \mathrm{W}\end{array}$

SAMPLE DATE

1099

211.8

4.6

120

20.4

1

5
0.024

$\mathrm{X}$

$211.2(03 / 23 / 99)$

Volumes purged

Synchronous water level
Screen Zone Elevation

$159.1-149.1 \mathrm{~h} \mathrm{msl}$

Top of Casing

$279.3 \mathrm{ft} \mathrm{ms}$

04/06/99

2099

211.1

5.4

140

19.1

0.025

$\mathrm{X}$

$210.6(06 / 28 / 99)$
Casing Pump

4: PVC S

Screen Zone

LAZ_UTRA

ANALYTICAL DATA

Inorganic Constituents

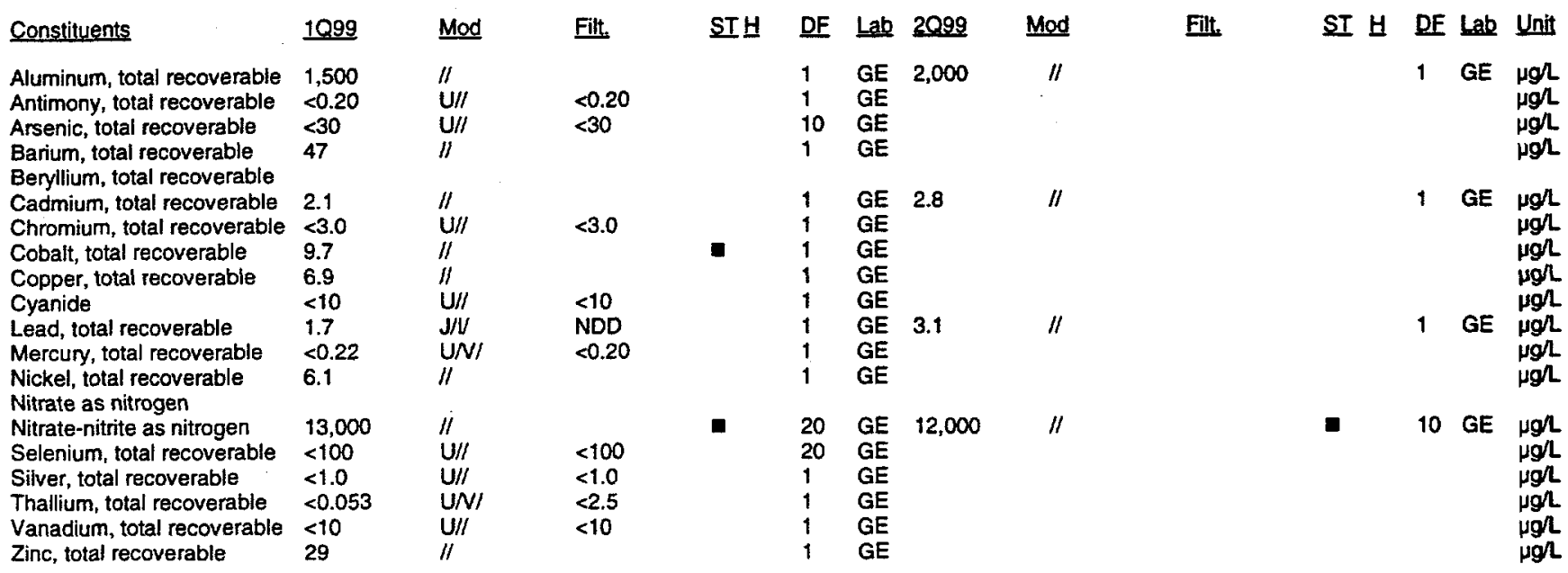

Organic Constituents

\begin{tabular}{|c|c|c|c|c|c|}
\hline Constituents & 1099 & Mod & Filt. & ST H & DF \\
\hline Benzene & $<1.0$ & JUNO & $<1.0$ & & 1 \\
\hline Bis(2-ethylhexyl) phthalate & $<9.9$ & $\mathrm{U} / 1$ & $<9.9$ & & 1 \\
\hline Dichloromethane & $<1.3$ & JUAV/O & $<5.0$ & & 1 \\
\hline Phenols & $<5.0$ & $\mathbf{U} / 1$ & $<5.0$ & & 1 \\
\hline Tetrachloroethylene & $<1.0$ & JUЛO & $<1.0$ & & 1 \\
\hline Trichloroethylene & 1.8 & נח๐O & NDD & & 1 \\
\hline Trichlorofluoromethane & 2.7 & J/L/O & NDD & & 1 \\
\hline
\end{tabular}

Unit

ft msl

$\mathrm{pH}$

${ }^{\mu} \mathrm{C} / \mathrm{cm}$

mgl

NTU

well volumes

th msl

NDD

Notes:

- exceeded holding time

- exceeded groundwater protection or monitoring constituent standard (See Appendix A.)

NA - Not applicable. Applies to beta dose and sum of betas if there are no beta-emitting radionuclides above detection limits; to sum of alphas if there are no alphaemitting radionuclides above detection limits; and to total radium if neither radium-226 or radium-228 was above detection limit

UAZ_UTRA - Upper Aquifer Zone of the Upper Three Runs Aquifer; LAZ_UTRA - Lower Aquifer Zone of the Upper Three Runs Aquifer, Gordon - Gordon Aquiler 
WELL FSB $91 \mathrm{C}$ (cont.)

Radioactive Constituents

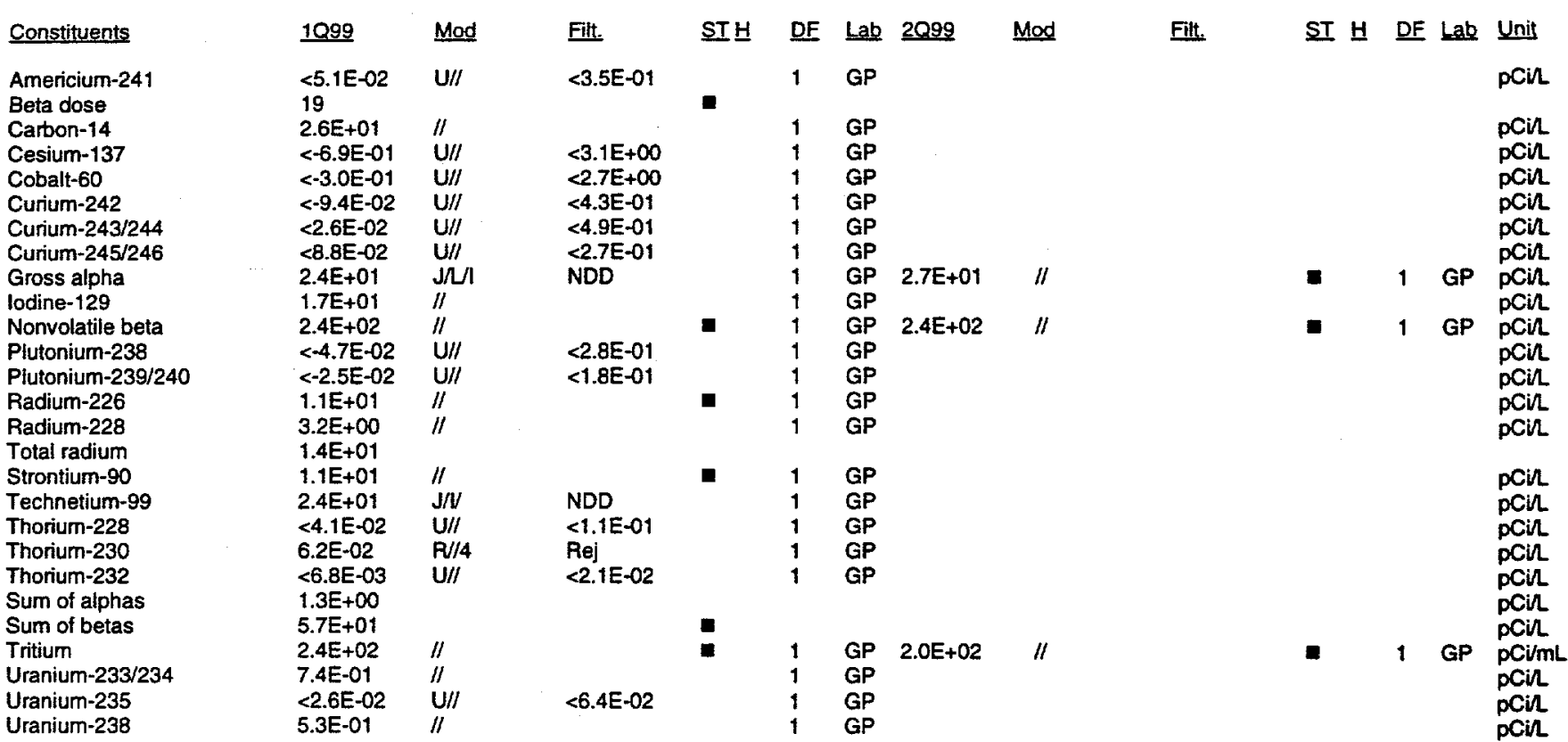

\section{Notes:}

- = exceeded holding time

= exceeded groundwater protection or monitoring constituent standard (See Appendix A.)

NA - Not applicable. Applies to beta dose and sum of betas if there are no beta-emitting radionuclides above detection limits; to sum of alphas if there are no alphaemitting radionuclides above detection limits; and to total radium if neither radium-226 or radium-228 was above detection limit

UAZ_UTRA - Upper Aquifer Zone of the Upper Three Runs Aquifer, LAZ_UTRA - Lower Aquiler Zone of the Upper Three Runs Aquifer, Gordon - Gordon Aquifer 


\section{WELL FSB 91D}

\begin{tabular}{|c|c|}
\hline SRS Coord. & Lat/Longitude \\
\hline $\begin{array}{l}\text { N75207.6 } \\
\text { E50946.6 }\end{array}$ & $\begin{array}{l}33.275235^{\circ} \mathrm{N} \\
81.678735^{\circ} \mathrm{W}\end{array}$ \\
\hline
\end{tabular}

\section{SAMPLE DATE}

1099

214.7

3.2

220

19.8

0

3.3

$213.8(03 / 23 / 99)$

Alkalinity as $\mathrm{CaCO} 3$

Volumes purged

Sampling code

Synchronous water level

\section{Screen Zone Elevation}

220.9-200.9 ft ms

$1 / 14 / 99$
2Q99

213.6

3.9

160

19.8

2.9

$213.5(06 / 28 / 99)$
Casino Pumo

4" PVC V
Screen Zone

UAZ_UTRA
ANALYTICAL DATA

Inorganic Constituents

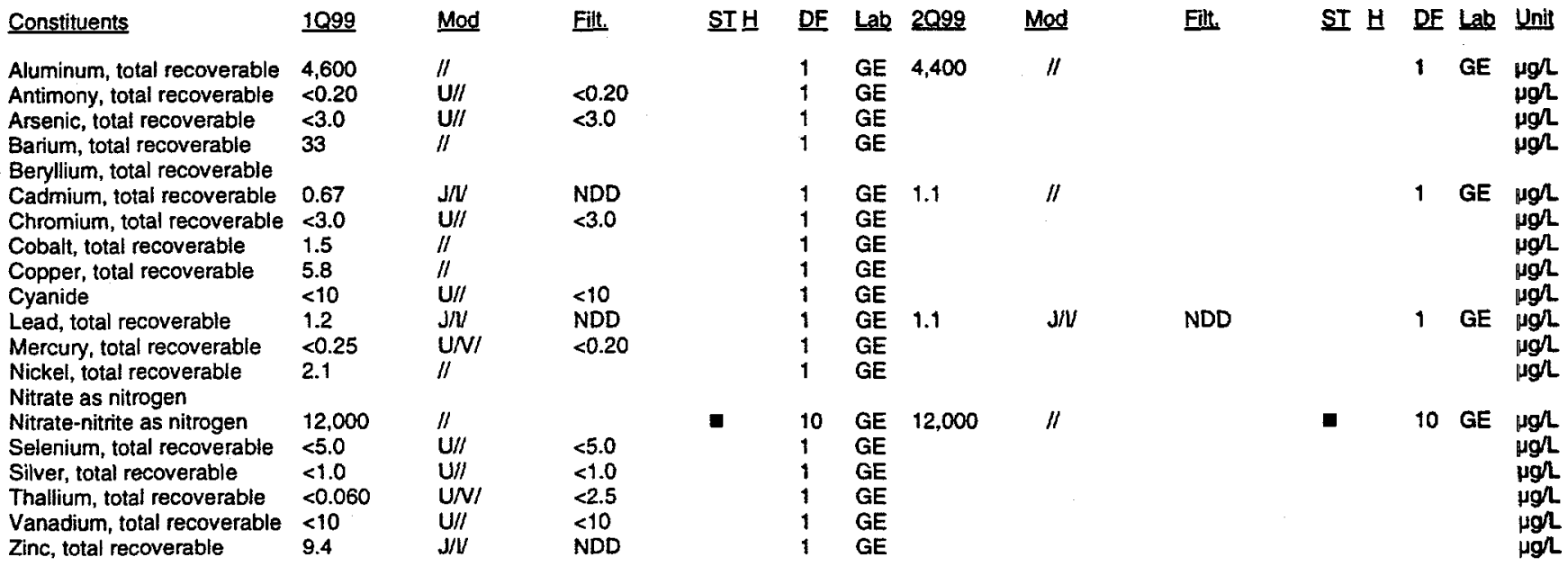

Organic Constituents

\begin{tabular}{llll} 
Constituents & 1099 & Mod & Filt. \\
\cline { 2 - 4 } Benzene & & & \\
Bis(2-ethylhexyl) phthalate & $<9.9$ & JU/lO & $<1.0$ \\
Dichloromethane & $<1.5$ & JUnV/O & $<9.9$ \\
Phenols & $<5.0$ & U/I & $<5.0$ \\
Tetrachloroethylene & $<1.0$ & JU/UO & $<1.0$ \\
Trichloroethylene & $<1.0$ & JUIUO & $<1.0$ \\
Trichlorofluoromethane & $<5.0$ & JUNO & $<5.0$
\end{tabular}

$\begin{array}{ccc}\text { ST브 } & \text { DF } & \text { Lab } \\ 1 & \text { GE } \\ 1 & \text { GE } \\ 1 & \text { GE } \\ 1 & \text { GE } \\ 1 & \text { GE } \\ 1 & \text { GE } \\ 1 & \text { GE }\end{array}$

Unit
tt ms!
$\mathrm{pH}$
$\mu \mathrm{S} / \mathrm{cm}$
${ }^{\circ} \mathrm{C}$
$\mathrm{mg}$
$\mathrm{NTU}$
well volumes
$\mathrm{ft}$ msl

ft msl

Notes:

= exceeded holding time

= exceeded groundwater protection or monitoring constituent standard (See Appendix A.)

NA - Not applicable. Applies to beta dose and sum of betas if there are no beta-emitting radionuclides above detection limits; to sum of alphas if there are no alphaemitting radionuclides above detection limits; and to total radium if neither radium-226 or radium-228 was above detection limit

UAZ_UTRA - Upper Aquifer Zone of the Upper Three Runs Aquifer, LAZ UTRA - Lower Aquifer Zone of the Upper Three Runs Aquifer, Gordon - Gondon Aquifer 


\section{WELL FSB 91D (cont.)}

\section{Radioactive Constituents}

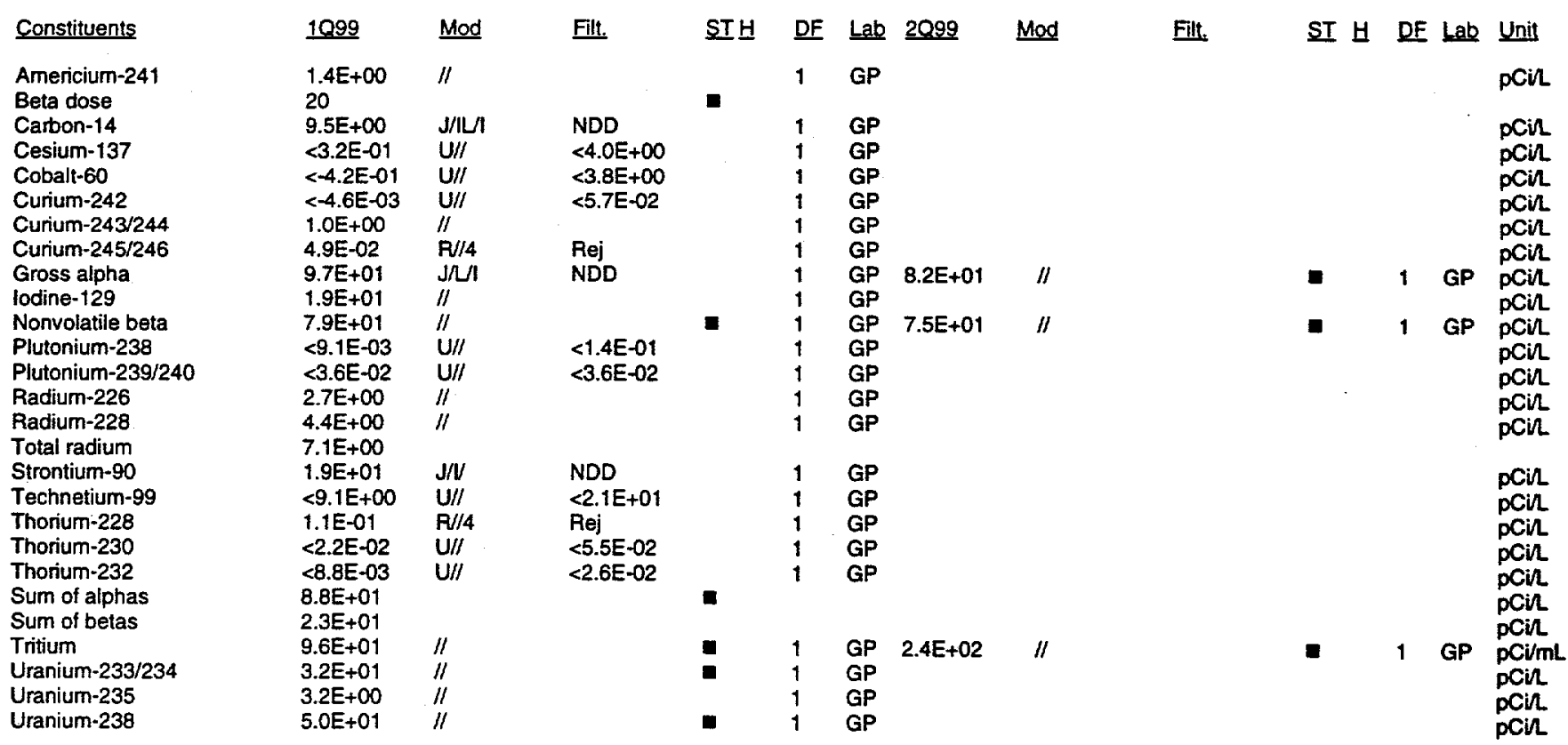

\section{Notes:}

- exceeded holding time

= exceeded groundwater protection or monitoring constituent standard (See Appendix A.)

NA - Not applicable. Applies to beta dose and sum of betas if there are no beta-emitting radionuclides above detection limits; to sum of alphas if there are no alphaemitting radionuclides above detection limits; and to total radium if neither radium-226 or radium-228 was above detection limit

UAZ_UTRA - Upper Aquifer Zone of the Upper Three Runs Aquifer, LAZ_UTRA - Lower Aquifer Zone of the Upper Three Runs Aquifer, Gordon - Gordon Aquifer 


\section{WELL FSB 92C}

\begin{tabular}{|c|c|}
\hline SRS Coord. & LaVLongitude \\
\hline $\begin{array}{l}\text { N75053.2 } \\
\text { E50564.0 }\end{array}$ & 81.679442 \\
\hline
\end{tabular}

SAMPLE DATE

1099

Constituents

Water elevation

pH

Sp. conductance

Water temperature

Alkalinity as $\mathrm{CaCO} 3$

Turbidity

Volumes purged

Sampling code

Synchronous water level

210.6

5.2

260

19.6

5

3

0.024

$209.2(03 / 23 / 99)$

$1 / 14 / 99$
Screen Zone Elevation

$157.6-147.6 \mathrm{ft} \mathrm{ms}$

2099

209.8

5.4

290

20.0

1

1.9

$209.3(06 / 28 / 99)$

04/06/99

\section{Casing Pump}

4" PVC V

LAZ_UTRA

Unit

ft msl

$\mathrm{pH}$

HSIcm

${ }^{\circ} \mathrm{C}$

$\mathrm{mg} /$

NTU

well volumes

ft ms!

\section{ANALYTICAL DATA}

Inorganic Constituents

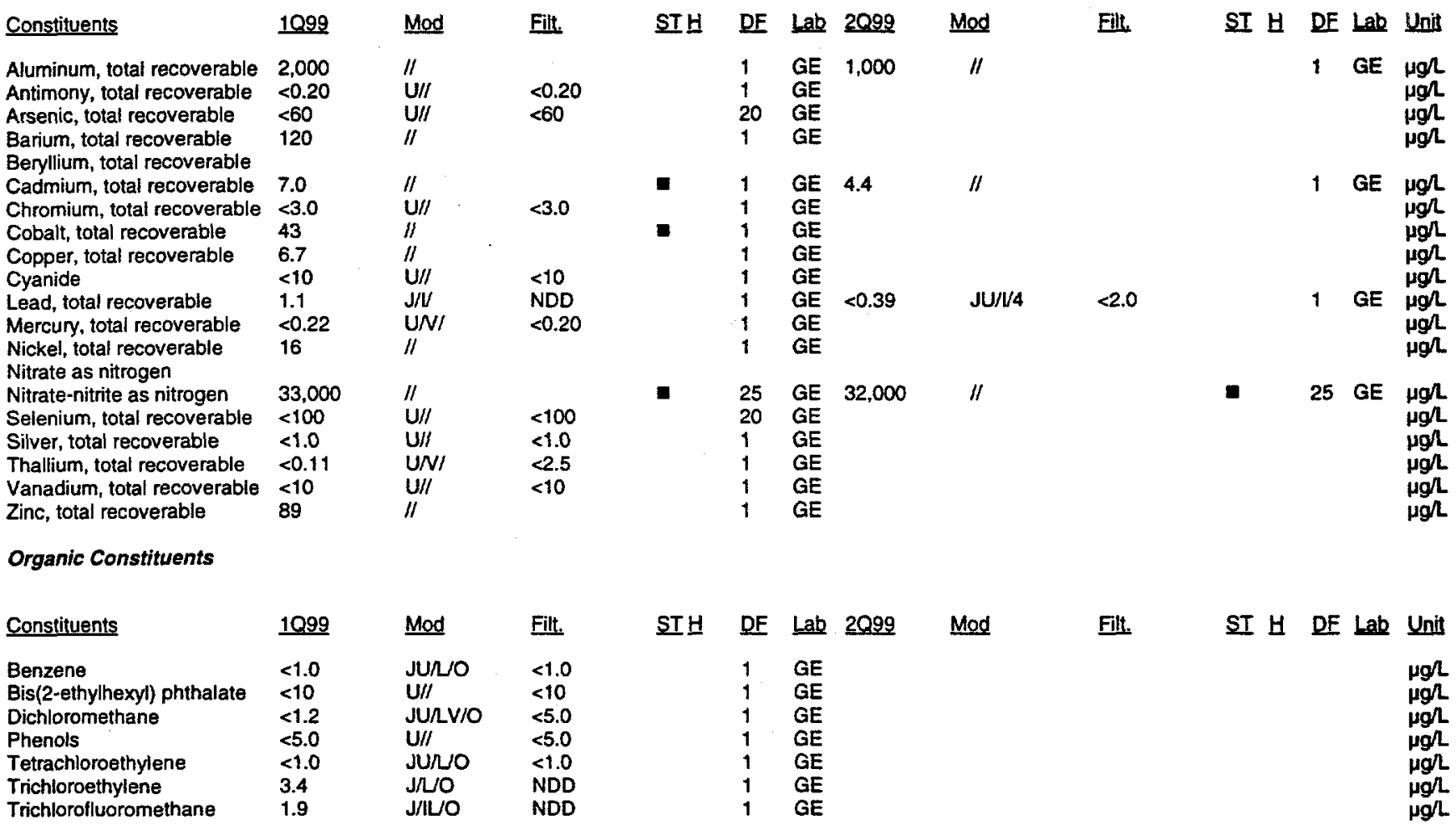

Notes:

- exceeded holding time

= exceeded groundwater protection or monitoring constituent standard (See Appendix A.)

NA - Not applicable. Applies to beta dose and sum of betas if there are no beta-emitting radionuclides above detection limits; to sum of alphas if there are no alphaemitting radionuclides above detection limits; and to total radium if neither radium-226 or radium-228 was above detection limit

UAZ UTRA - Upper Aquifer Zone of the Upper Three Runs Aquifer; LAZ UTRA - Lower Aquifer Zone of the Upper Three Runs Aquifer, Gordon - Gordon Aquifer 


\section{WELL FSB $92 \mathrm{C}$ (cont.)}

Radioactive Constituents

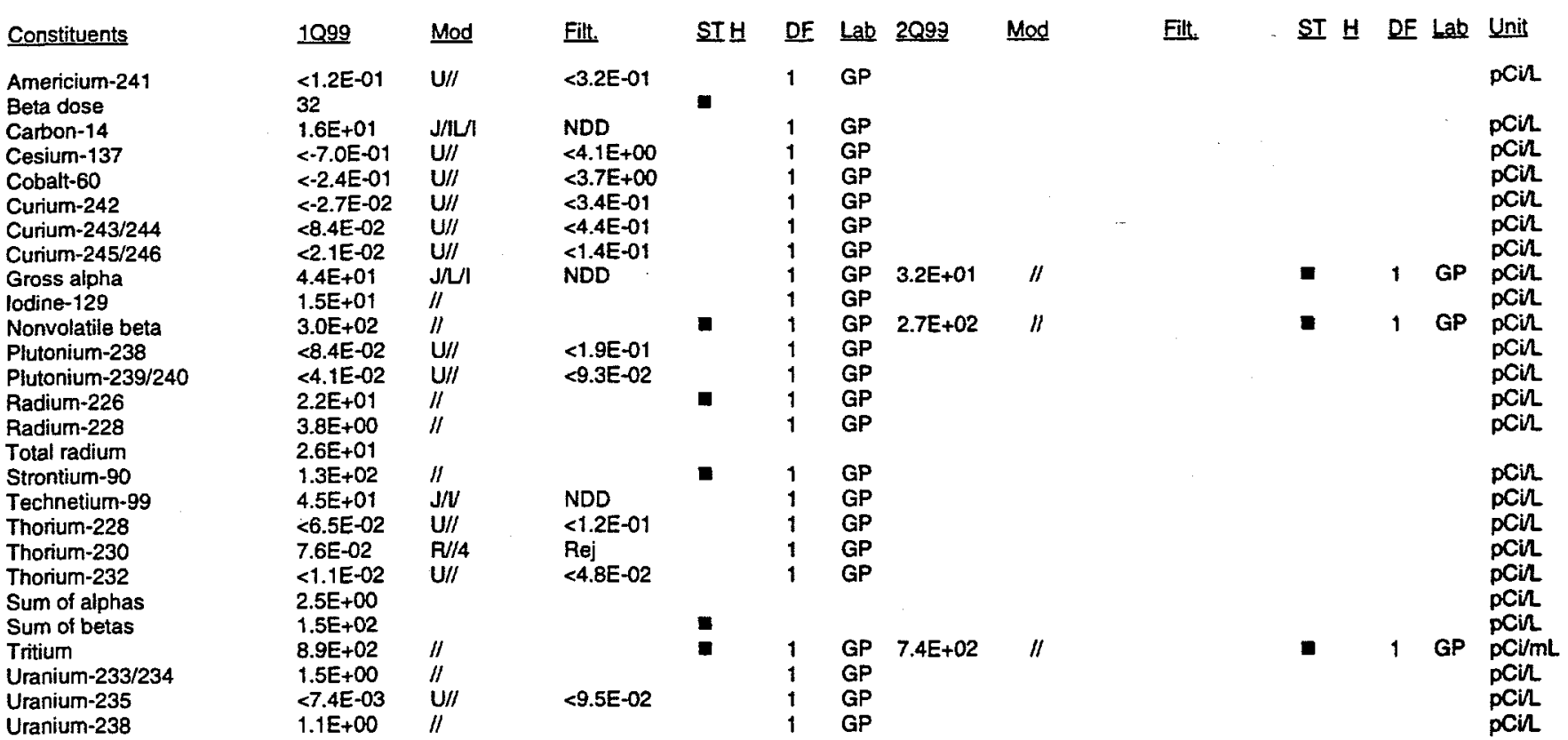

= exceeded holding time

= exceeded groundwater protection or monitoring constituent standard (See Appendix A.)

NA - Not applicable. Applies to beta dose and sum of betas if there are no beta-emitting radionuclides above detection limits; to sum of alphas if there are no alphaemitting radionuclides above detection limits; and to total radium if neither radium-226 or radium-228 was above detection limit

UAZ_UTRA - Upper Aquifer Zone of the Upper Three Runs Aquifer, LAZ_UTRA - Lower Aquifer Zone of the Upper Three Runs Aquifer, Gordon - Gordon Aquifer 


\section{WELL FSB 92D}

\begin{tabular}{|c|c|}
\hline & \\
\hline $\begin{array}{l}N 75045.8 \\
E 50557.6\end{array}$ & $\begin{array}{l}33.2742 \\
81.6794\end{array}$ \\
\hline
\end{tabular}

SAMPLE DATE

\section{FIELD DATA}

Constituents

Water elevation

$\mathrm{pH}$

Sp. conductance

Water temperature

Alkalinity as $\mathrm{CaCO} 3$

Turbidity

Volumes purged

Sampling code

Synchronous water level

\section{ANALYTICAL DATA}

Inorganic Constituents

\section{Screen Zone Elevation}

$221.7-201.7 \mathrm{ft} \mathrm{msl}$

01/04/99

1099

212.9

4.5

695

18.5

0

1
3.4

$212.0(03 / 23 / 99)$

$\begin{array}{lll}\text { Top of Casing } & \text { Casing } & \text { Pump } \\ 275.9 \mathrm{ft} \mathrm{ms} & 4^{*} \mathrm{PVC} & \mathrm{V}\end{array}$

Screen Zone

UAZ_UTRA

04/08/99

2099

212.0

3.6

910

20.1

0

2.4

$211.6(06 / 28 / 99)$
Unit

ft msi

$\mathrm{pH}$

$\mu \mathrm{S} / \mathrm{cm}$

${ }^{\circ} \mathrm{C}$

mgl

well volumes

ft msl

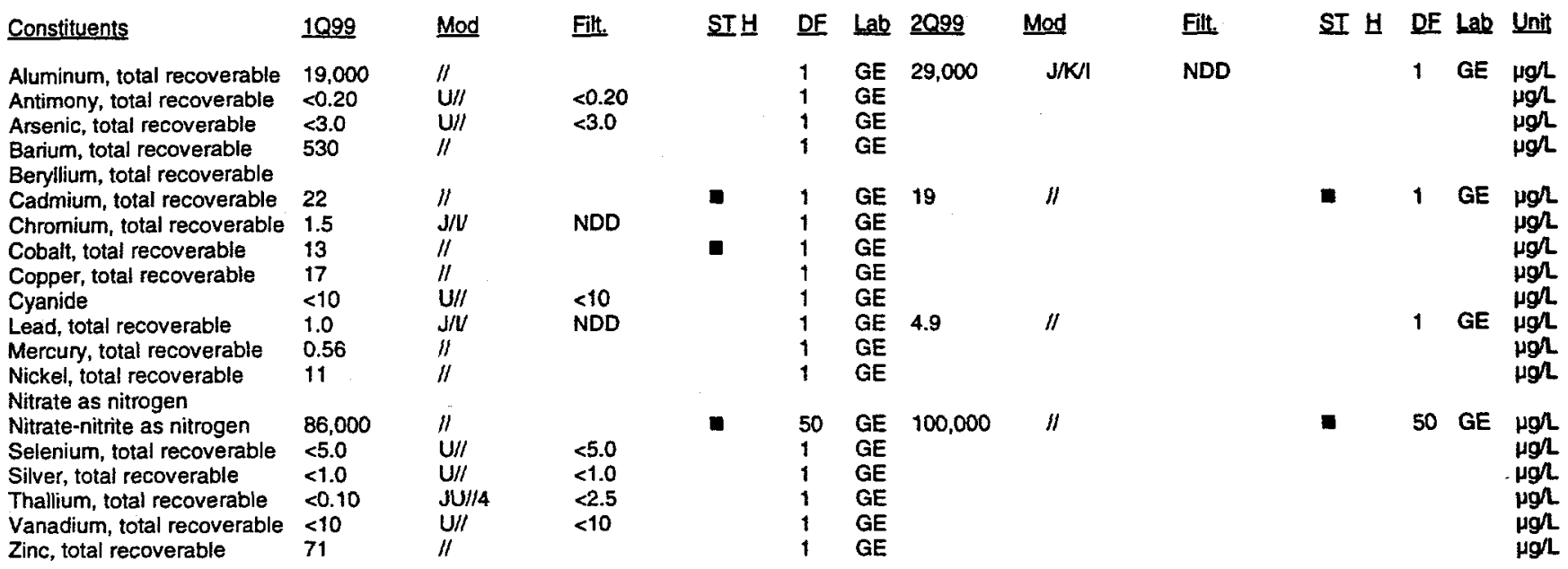

Organic Constituents

$\begin{array}{llll}\text { Constituents } & 1099 & \text { Mod } & \text { Fill. } \\ \text { Benzene } & <1.0 & \text { JUINO } & <1.0 \\ \text { Bis(2-ethylhexyl) phthalate } & <10 & \text { U/I } & <10 \\ \text { Dichloromethane } & <1.7 & \text { JUIVIO } & <5.0 \\ \text { Phenols } & <5.0 & \text { UII } & <5.0 \\ \text { Tetrachloroethylene } & <1.0 & \text { JU/UO } & <1.0 \\ \text { Trichloroethylene } & <1.0 & \text { JU/UO } & <1.0 \\ \text { Trichloroftuoromethane } & <5.0 & \text { JU/O } & <5.0\end{array}$

$\begin{array}{ccc}\text { SIH } & \text { DE } & \text { Lab } \\ 1 & \mathrm{GE} \\ 1 & \mathrm{GE} \\ 1 & \mathrm{GE} \\ 1 & \mathrm{GE} \\ 1 & \mathrm{GE} \\ 1 & \mathrm{GE} \\ 1 & \mathrm{GE}\end{array}$

Filt.

ST $\forall$ DF Lab Unit

$\mu g h$

$\mu g h$

$\mu g h$

$\mu g /$

$\mu g /$

$\mu g h$

$\mu g /$ 


\section{WELL FSB 92D (cont.)}

Radioactive Constituents

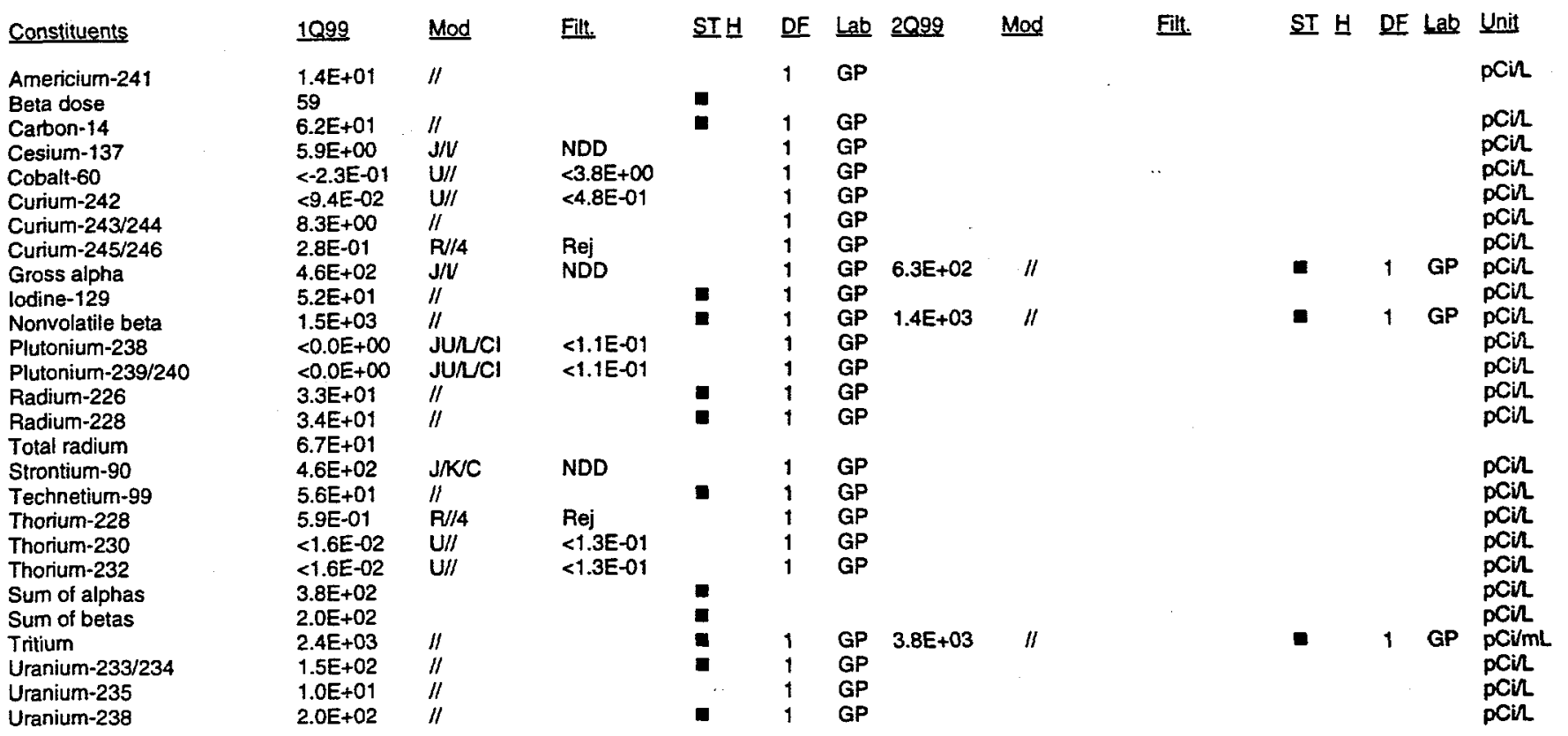

Notes:

= exceeded holding time

= exceeded groundwater protection or monitoring constituent standard (See Appendix A.)

NA - Not applicable. Applies to beta dose and sum of betas if there are no beta-emitting radionuclides above detection limits; to sum of alphas if there are no alphaemitting radionuclides above detection limits; and to total radium if neither radium-226 or radium-228 was above detection limit

UAZ_UTRA - Upper Aquifer Zone of the Upper Three Runs Aquifer, LAZ_UTRA - Lower Aquifer Zone of the Upper Three Runs Aquifer, Gordon - Gordon Aquifer 


\section{WELL FSB 93C}

$\begin{array}{ll}\text { SRS Coord. } & \text { Lat/Longitude } \\ \text { N74897.3 } & 33.273752 \text { ०N } \\ \text { E50458.3 } & 81.679418 \text { W }\end{array}$

\section{Screen Zone Elevation}

152.0-142.0 ft ms

$01 / 12 / 99$

\section{Top of Casing}

$276.2 \mathrm{ft} \mathrm{ms}$

Casing Pump
4" PVC

04/06/99

\section{SAMPLE DATE}

1099

\section{Constituents}

Water elevation

pH

Sp. conductance

Water temperature

Alkalinity as $\mathrm{CaCO} 3$

Turbidity

Volumes purged

Sampling code

Synchronous water level

\section{ANALYTICAL DATA}

Inorganic Constituents
209.7

4.7

270

19.5

0

5.2

$207.3(03 / 23 / 99)$
2099

208.7

4.4

280

20.4

1

4.7

$208.4(06 / 28 / 99)$
Screen Zone

LAZ_UTRA

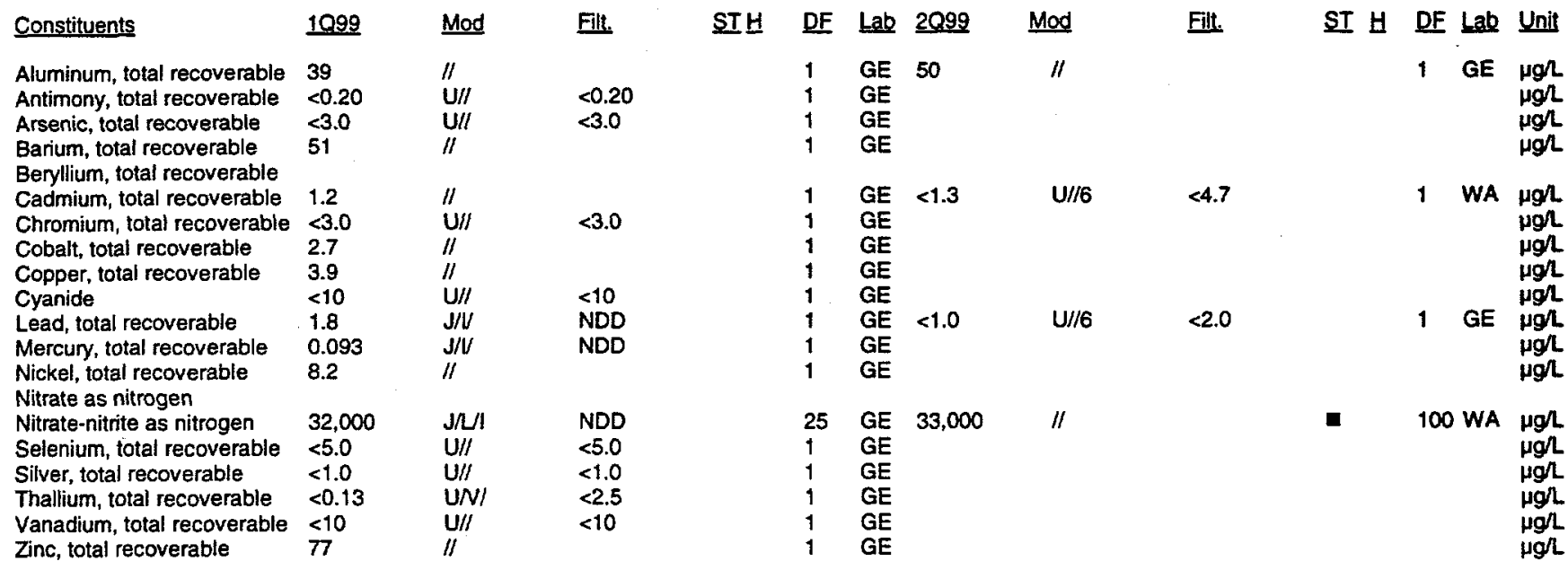

Organic Constituents

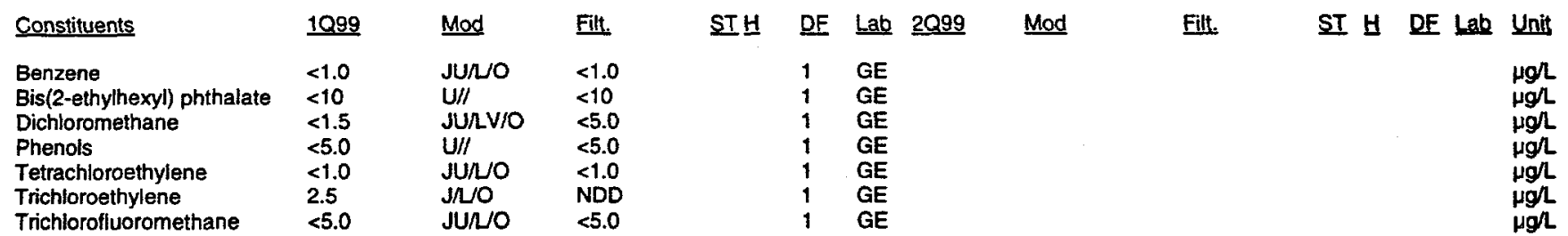

Notes:

= exceeded holding time

= exceeded groundwater protection or monitoring constituent standard (See Appendix A.)

NA - Not applicable. Applies to beta dose and sum of betas if there are no beta-emitting radionuclides above detection limits; to sum of alphas if there are no alpha emitting radionuclides above detection limits; and to total radium if neither radium-226 or radium-228 was above detection limit

UAZ UTRA - Upoer Aquifer Zone of the Upper Three Runs Aquifer, LAZ UTRA - Lower Aquifer Zone of the Upper Three Runs Aquifer, Gordon - Gordon Aquifer 
WELL FSB 93C (cont.)

Radioactive Constituents

\begin{tabular}{|c|c|c|c|c|c|c|c|c|c|c|c|c|c|}
\hline Constituents & 1099 & Mod & Filt. & ST브 & DF & $\underline{\text { Lab }}$ & $\underline{2099}$ & Mod & Fill. & SI $\mathrm{H}$ & DF & Lab & Unit \\
\hline Americium-241 & $<2.2 E-01$ & JU/LI & $<2.5 E-01$ & & 1. & GP & & & & & & & pCin \\
\hline Beta dose & & & & a & & & & & & & & & \\
\hline Carbon-14 & $1.3 E+01$ & $\mathrm{~J} / N$ & NDD & & 1 & GP & & & & & & & pCin \\
\hline Cesium-137 & $<-1.9 E+\infty$ & JUחI & $<3.4 E+\infty 0$ & & 1 & & & & & & & & $\begin{array}{l}\text { PCUL } \\
\text { DCin }\end{array}$ \\
\hline $\begin{array}{l}\text { Cobalt-60 } \\
\text { Curium-24? }\end{array}$ & $\begin{array}{l}<5.1 E-01 \\
<-1,4 E-01\end{array}$ & $U / 1$ & $<3.4 E+\infty 0$ & & 1 & $\begin{array}{l}\text { GP } \\
\text { GP }\end{array}$ & & & & & & & pCir \\
\hline $\begin{array}{l}\text { Curium-242 } \\
\text { Curium-243/244 }\end{array}$ & $\begin{array}{l}<-1.4 \mathrm{E}-01 \\
<4.4 \mathrm{E}-02\end{array}$ & $\begin{array}{l}\text { JUILC } \\
\text { JURC }\end{array}$ & $\begin{array}{l}<3.4 \mathrm{E}-01 \\
<4.3 \mathrm{E}-01\end{array}$ & & 1 & GP & & & & & & & pCir \\
\hline Curium-245/246 & $<-3.0 \mathrm{OE}-02$ & JU/LC & $<1.8 \mathrm{E}-01$ & & $i$ & $G P$ & & & & & & & pCin \\
\hline Gross alpha & $2.6 E+00$ & "I & & & 1 & GP & $8.8 E+\infty$ & /I & & & 1 & GP & poin \\
\hline ladine-129 & $8.8 E+00$ & /I & & & 1 & GP & & & & & & & pCin \\
\hline Nonvolatile beta & $6.4 E+01$ & $J / K / C$ & NDD & & 1 & GP & $7.9 E+01$ & $\|$ & & = & 1 & TM & pcin \\
\hline Plutonium-238 & $<1.5 \mathrm{E}-02$ & $u / /$ & $<4.6 \mathrm{E}-02$ & & 1 & GP & & & & & & & pCin \\
\hline Plutonium-239/240 & $<3.8 \mathrm{E}-02$ & $\mathrm{U} / /$ & $<9.5 E-02$ & & 1 & $G P$ & & & & & & & pCin \\
\hline $\begin{array}{l}\text { Radium-226 } \\
\text { Reth }\end{array}$ & $2.7 E+\infty 0$ & $\|$ & & & 1 & $G P$ & & & & & & & pcin \\
\hline Radium-228 & $<1.5 \mathrm{E}+\infty$ & JU/LVI & $<8.8 E-01$ & & 1 & $G P$ & & & & & & & pCin \\
\hline Total radium & $2.7 \mathrm{E}+00$ & & & & & & & & & & & & \\
\hline Strontium-90 & $2.3 E+01$ & $\|$ & & ט & 1 & GP & & & & & & & pCin \\
\hline Technetium-99 & $3.0 \mathrm{E}+01$ & $\mathrm{~J} / \mathrm{N}$ & NDD & & 1 & GP & & & & & & & pcin \\
\hline Thorium-228 & $<2.1 \mathrm{E}-02$ & $\mathrm{u} / /$ & $<2.4 \mathrm{E}-01$ & & 1 & GP & & & & & & & pCin \\
\hline Thorium-230 & $<6.4 \mathrm{E}-02$ & $\mathrm{U} / \mathrm{I}$ & $<8.9 E-02$ & & 1 & GP & & & & & & & pCir \\
\hline Thorium-232 & $<-1.4 \mathrm{E}-04$ & $\mathrm{U} / /$ & $<1.1 E-01$ & & 1 & GP & & & & & & & pCin \\
\hline Sum of alphas & & & & & & & & & & & & & \\
\hline Sum of betas & $3.1 E+01$ & & & & & & & & & & & & pCil \\
\hline Tritium & $1.0 \mathrm{E}+03$ & $/ /$ & & [ & 1 & GP & $9.4 \mathrm{E}+02$ & $\|$ & & - & 1 & GP & pCivmL \\
\hline Uranium-233/234 & $<3.4 \mathrm{E}-02$ & $\mathrm{U} / /$ & $<1.0 \mathrm{E}-01$ & & 1 & GP & & & & & & & pCil \\
\hline Uranium-235 & $<-1.4 \mathrm{E}-02$ & $\mathrm{U} / \mathrm{I}$ & $<1.1 \mathrm{E}-01$ & & 1 & GP & & & & & & & pCin \\
\hline Uranium-238 & $<2.6 \mathrm{E}-02$ & $U / I$ & $<7.9 \mathrm{E}-02$ & & 1 & GP & & & & & & & pcin \\
\hline
\end{tabular}

Notes:

= exceeded holding time

= exceeded groundwater protection or monitoring constituent standard (See Appendix A.)

NA - Not applicable. Applies to beta dose and sum of betas if there are no beta-emitting radionuclides above detection limits; to sum of alphas if there are no alphaemitting radionuclides above detection limits; and to total radium if neither radium-226 or radium-228 was above detection limit

UAZ_UTRA - Upper Aquifer Zone of the Upper Three Runs Aquifer, LAZ UTRA - Lower Aquifer Zone of the Upper Three Runs Aquifer, Gordon - Gordon Aquifer 


\section{WELL FSB 93D}

$\begin{array}{ll}\text { SRS Coord. } & \text { Lat/Longitud } \\ \text { N74888.5 } & 33.273723^{\circ} \\ \text { E50452.4 } & 81.679416^{\circ}\end{array}$

SAMPLE DATE
Screen Zone Elevation

$217.9-197.9 \mathrm{ft} \mathrm{msl}$
Top of Casing

$276.1 \mathrm{ft}$ msl

$\begin{array}{ll}\text { Casing Pump } & \text { P. PVC V }\end{array}$

Casing Pump

Screen Zone
UAZ_UTRA
$04 / 12 / 99$

2099

210.4

3.9
660

660

0

1

0.12

209.9 (06/28/99)
Unit

ft msl

pH

$\mu S / \mathrm{cm}$

${ }^{\circ} \mathrm{C}$

$\mathrm{mg} / \mathrm{L}$

NTU

well volumes

ft msl
0.12

$210.6(03 / 23 / 99)$

\section{ANALYTICAL DATA}

Inorganic Constituents

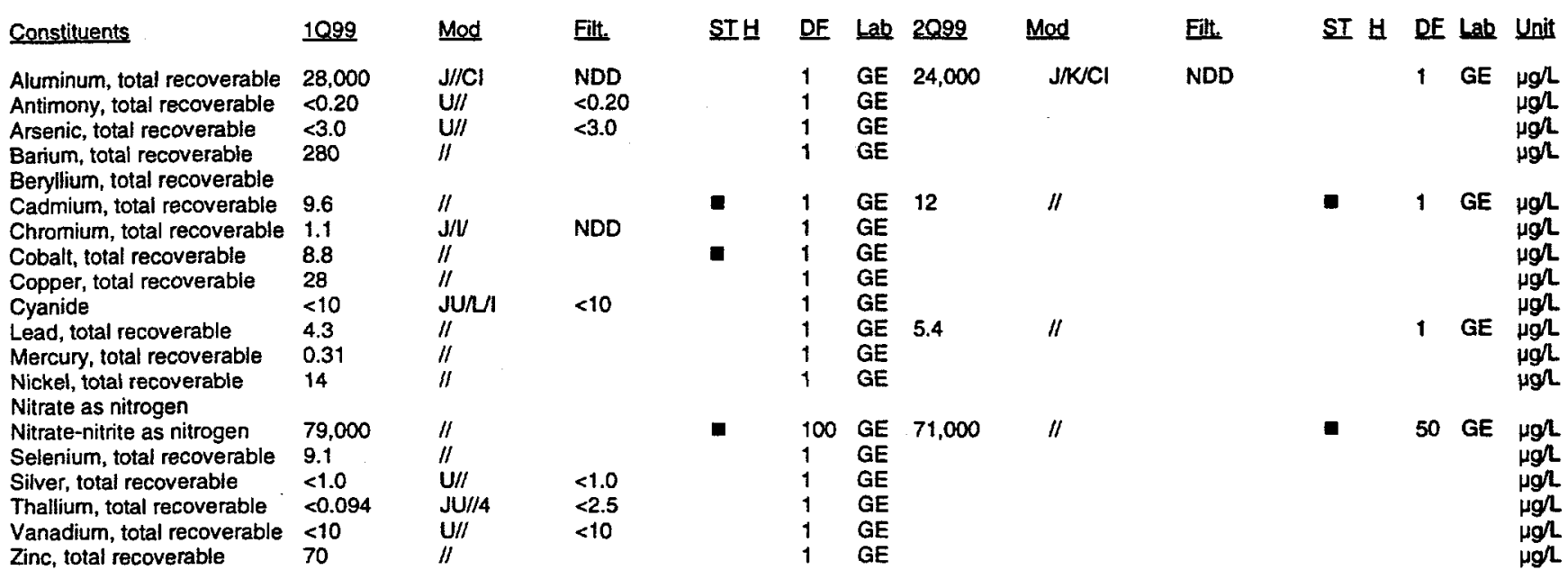

Organic Constituents

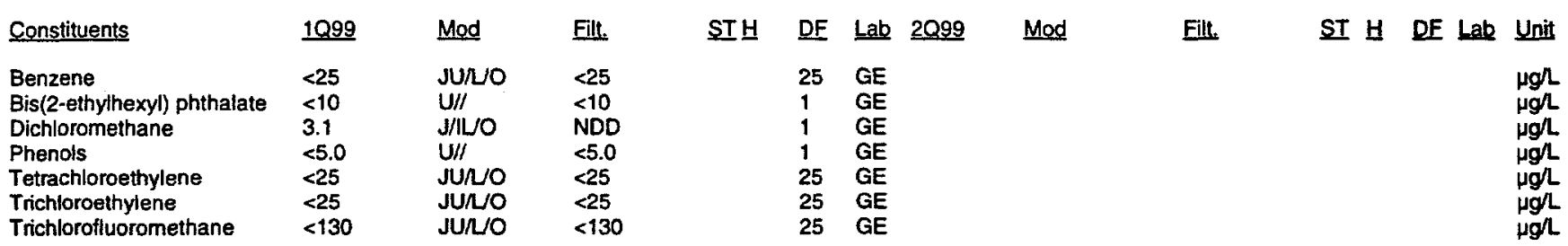

Notes:

- exceeded holding time

- = exceeded groundwater protection or monitoring constituent standard (See Appendix A.)

NA - Not applicable. Applies to beta dose and sum of betas if there are no beta-emitting radionuclides above detection limits; to sum of alphas if there are no alphaemitting radionuclides above detection limits; and to total radium if neither radium-226 or radium-228 was above detection limit

UAZ UTRA - Upper Aquifer Zone of the Upper Three Runs Aquifer, LAZ UTRA - Lower Aquifer Zone of the Upper Three Runs Aquifer, Gordon - Gordon Aquifer 


\section{WELL FSB 93D (cont.)}

Radioactive Constituents

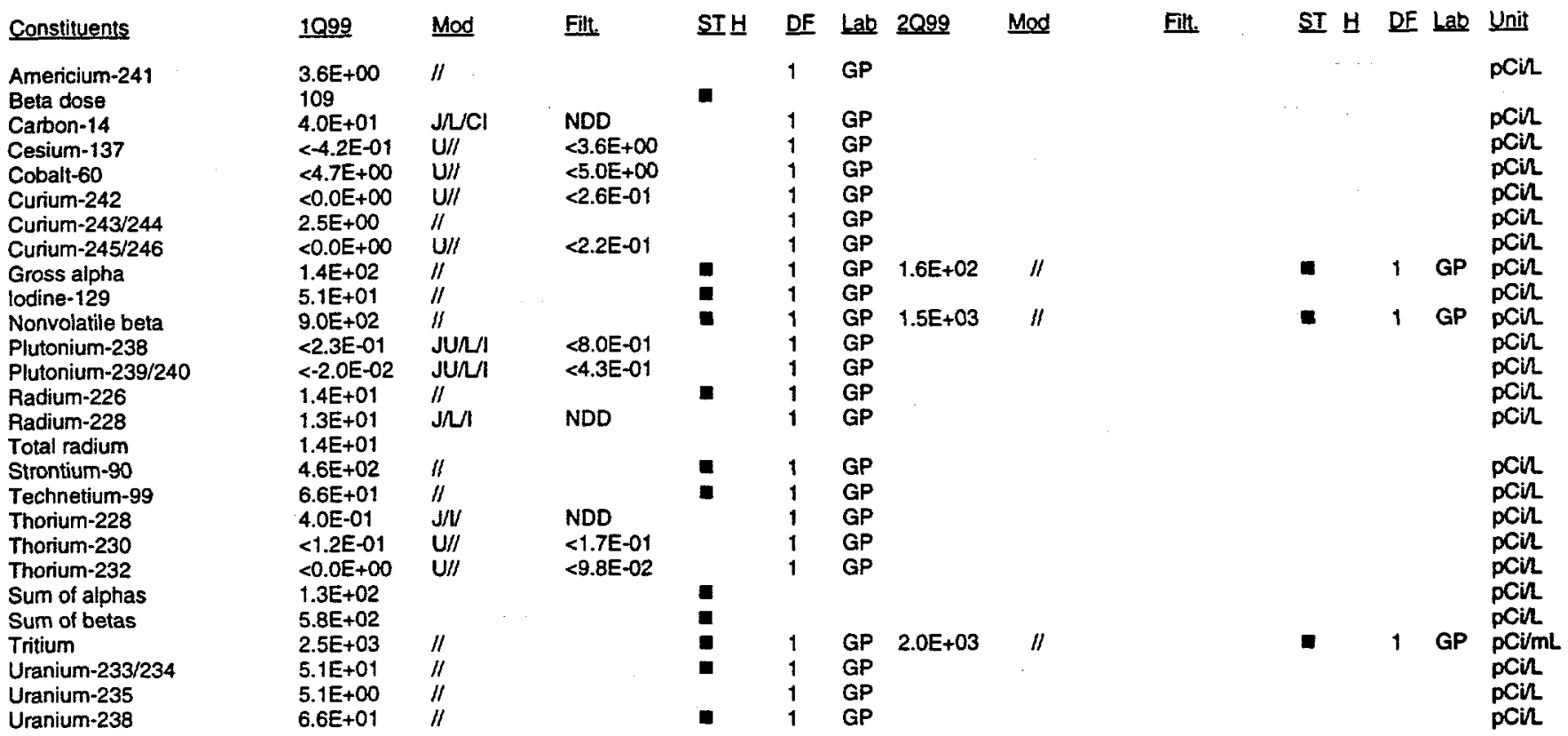

Notes:

- exceeded holding time

E = exceeded groundwater protection or monitoring constituent standard (See Appendix A.)

NA - Not applicable. Applies to beta dose and sum of betas if there are no beta-emitting radionuclides above detection limits; to sum of alphas if there are no alphaemitting radionuclides above detection limits; and to total radium if neither radium-226 or radium-228 was above detection limit

UAZ_UTRA - Upper Aquifer Zone of the Upper Three Runs Aquifer, LAZ_UTRA - Lower Aquifer Zone of the Upper Three Runs Aquifer, Gordon - Gordon Aquifer

F-Area HWMF 
WELL FSB 94C

\begin{tabular}{|c|c|c|c|c|c|c|}
\hline SRS Coord. & Latlongitude & Screen Zone Elevation & Tog of Casing & Casing & Pumo & Screen Zone \\
\hline $\begin{array}{l}\text { N748869.0 } \\
\text { E50180.0 }\end{array}$ & $\begin{array}{l}33.273235^{\circ} \mathrm{N} \\
81.680096^{\circ} \mathrm{W}\end{array}$ & $149.8-139.8 \mathrm{ft} \mathrm{msl}$ & $281.1 \mathrm{ft} \mathrm{msl}$ & 4" PVC & $v$ & LAZ UTRA \\
\hline SAMPLE DA & & $01 / 13 / 99$ & $04 / 09 / 99$ & & & \\
\hline \multicolumn{7}{|l|}{ FIELD DATA } \\
\hline \multicolumn{2}{|l|}{ Constituents } & 1099 & 2099 & & & Unit \\
\hline \multicolumn{2}{|c|}{$\begin{array}{l}\text { Water elevation } \\
\text { pH } \\
\text { Sp. conductance } \\
\text { Water temperature } \\
\text { Alkalinity as } \mathrm{CaCO} \\
\text { Turbidity } \\
\text { Volumes purged } \\
\text { Sampling code } \\
\text { Synchronous water level }\end{array}$} & $\begin{array}{l}208.6 \\
3.8 \\
2000 \\
20.3 \\
0 \\
2 \\
0.022 \\
x \\
206.5(03 / 23 / 99)\end{array}$ & $\begin{array}{l}206.5 \\
4.1 \\
2200 \\
20.1 \\
0 \\
1 \\
0.023 \\
x \\
207.3(06 / 25 / 99)\end{array}$ & & & $\begin{array}{l}\mathrm{ft} \mathrm{msl} \\
\mathrm{pH} \\
\mu \mathrm{S} / \mathrm{cm} \\
{ }^{\circ} \mathrm{C} \\
\mathrm{mgh} \\
\text { NTU } \\
\text { well volumes } \\
\mathrm{ft} \mathrm{msl}\end{array}$ \\
\hline
\end{tabular}

ANALYTICAL DATA

Inorganic Constituents

\begin{tabular}{|c|c|c|c|c|c|c|c|c|c|c|c|c|c|}
\hline Constituents & 1099 & Mod & Fill. & STH & $\underline{\mathrm{DF}}$ & Lab & 2099 & Mod & Fill. & SI $H$ & DE & Lab & Unit \\
\hline Aluminum, total recoverable & 79,000 & $\mathrm{~J} / / \mathrm{Cl}$ & NDD & & 20 & GE & 84,000 & $\mathrm{~J} / \mathrm{KCCl}$ & NDD & & 10 & GE & \\
\hline Antimony, total recoverable & $<0.20$ & $\mathbf{U} / \prime$ & $<0.20$ & & 1 & GE & & & & & & & \\
\hline $\begin{array}{l}\text { Arsenic, total recoverable } \\
\text { Barium, total recoverable }\end{array}$ & $\begin{array}{l}<60 \\
760\end{array}$ & $\mathrm{U} / / /$ & $<60$ & & $\begin{array}{l}20 \\
1\end{array}$ & $\begin{array}{l}\text { GE } \\
\text { GE }\end{array}$ & & & & & & & \\
\hline $\begin{array}{l}\text { Barium, total recoverable } \\
\text { Beryllium, total recoverable }\end{array}$ & $\begin{array}{l}760 \\
20\end{array}$ & $\begin{array}{l}\prime \prime \\
\prime \prime\end{array}$ & & & 1 & GE & & & & & & & \\
\hline Cadmium, total recoverable & 38 & " & & m & 1 & GE & 36 & /I & & - & 1 & GE & \\
\hline Chromium, total recoverable & $<3.0$ & $U / I$ & $<3.0$ & & 1 & $\overrightarrow{G E}$ & & & & & & & \\
\hline Cobalt, total recoverable & 630 & II & & $\mathbf{m}$ & 1 & GE & & & & & & & \\
\hline Copper, total recoverable & 65 & $" 1$ & & & 1 & GE & & & & & & & \\
\hline Cyanide & $<10$ & JU/I & $<10$ & & 1 & GE & & & & & & & \\
\hline Lead, total recoverable & 14 & $\mathrm{~J} / \mathrm{V} / \mathrm{H}$ & NDD & & 20 & GE & 35 & 11 & & - & 10 & GE & \\
\hline Mercury, total recoverable & $<0.20$ & $\mathrm{U} / \mathrm{I}$ & $<0.20$ & & 1 & GE & & & & & & & \\
\hline $\begin{array}{l}\text { Nickel, total recoverable } \\
\text { Nitrate as nitrogen }\end{array}$ & 170 & $/ 1$ & & च & 1 & GE & & & & & & & \\
\hline Nitrate-nitrite as nitrogen & 320,000 & 11 & & m & 200 & GE & 320,000 & $\|$ & & - & & GE & \\
\hline $\begin{array}{l}\text { Selenium, total recoverable } \\
\text { Silver, total recoverable }\end{array}$ & $\begin{array}{l}28 \\
<10\end{array}$ & $\mathrm{~J} / / / \prime$ & NDD & & 20 & GE & & & & & & & \\
\hline Thallium, total recoverable & 27 & $\mathrm{~J} / \mathrm{J} / \mathrm{i}$ & $\begin{array}{l}<1.0 \\
\text { NDD }\end{array}$ & & $\begin{array}{l}1 \\
20\end{array}$ & $\begin{array}{l}\text { GE } \\
\text { GE }\end{array}$ & & & & & & & \\
\hline Vanadium, total recoverable & $<10$ & $\mathrm{U} / /$ & $<10$ & & 1 & GE & & & & & & & \\
\hline Zinc, total recoverable & 350 & // & & & 1 & GE & & & & & & & \\
\hline
\end{tabular}

Organic Constituents

\begin{tabular}{|c|c|c|c|c|c|c|c|c|c|c|c|}
\hline Constituents & 1099 & Mod & Filt. & ST브 & DF & Lab $\underline{2099}$ & Mod & Fill. & SI $H$ & DF Lab & Unit \\
\hline $\begin{array}{l}\text { Benzene } \\
\text { Bis(2-ethylhexyl) phthalate } \\
\text { Dichloromethane } \\
\text { Phenols } \\
\text { Tetrachloroethylene } \\
\text { Trichloroethylene } \\
\text { Trichlorofluoromethane }\end{array}$ & $\begin{array}{l}<1.0 \\
<11 \\
2.5 \\
<5.0 \\
<1.0 \\
<1.0 \\
<5.0\end{array}$ & $\begin{array}{l}\text { JU/NO } \\
\text { U/I } \\
\text { J/ILOO } \\
\text { U/I } \\
\text { JULOO } \\
\text { JUROO } \\
\text { JURO }\end{array}$ & $\begin{array}{l}<1.0 \\
<11 \\
N D D \\
<5.0 \\
<1.0 \\
<1.0 \\
<5.0\end{array}$ & & $\begin{array}{l}1 \\
1 \\
1 \\
1 \\
1 \\
1 \\
1\end{array}$ & $\begin{array}{l}\text { GE } \\
\text { GE } \\
\text { GE } \\
\text { GE } \\
\text { GE } \\
\text { GE. } \\
\text { GE }\end{array}$ & & & & & $\begin{array}{l}\mu g h \\
\mu g h \\
\mu g / L \\
\mu g h \\
\mu g / L \\
\mu g h \\
\mu g / L\end{array}$ \\
\hline
\end{tabular}




\section{WELL FSB 94C (cont.)}

Radioactive Constituents

\begin{tabular}{|c|c|c|c|c|c|c|c|c|c|c|c|c|c|}
\hline Constituents & 1099 & Mod & Filt. & ST브 & DF & Lab & 2099 & Mod & Filt. & ST $\underline{H}$ & DF & Lab & Unit \\
\hline $\begin{array}{l}\text { Americium-241 } \\
\text { Beta dose }\end{array}$ & $\begin{array}{l}2.2 E+00 \\
255\end{array}$ & $\mathrm{~J} / \mathrm{V}$ & NDD & $\mathbf{a}$ & 1 & GP & & & & & & & pCin \\
\hline $\begin{array}{l}\text { Carbon-14 } \\
\text { Cesium-137 }\end{array}$ & $\begin{array}{l}6.7 E+01 \\
<2.1 E-01\end{array}$ & $\begin{array}{l}\mathrm{J} / \mathrm{N} / \mathrm{Cl} \\
\mathrm{U} / /\end{array}$ & $\begin{array}{l}\text { NDD } \\
<4.4 E+00\end{array}$ & & $\begin{array}{l}1 \\
1\end{array}$ & $\begin{array}{l}\text { GP } \\
\text { GP }\end{array}$ & & & & & & & $\begin{array}{l}\mathrm{pCin} \\
\mathrm{pCin}\end{array}$ \\
\hline Cobalt- 60 & $<-1.1 E+\infty$ & WII & $<3.9 E+00$ & & 1 & GP & & & & & & & pCil \\
\hline Curium-242 & $<1.6 \mathrm{E}-01$ & $\mathrm{U} / /$ & $<4.9 E-01$ & & 1 & GP & & & & & & & pCin \\
\hline $\begin{array}{l}\text { Curium-243/244 } \\
\text { Curium-245/246 }\end{array}$ & $\begin{array}{l}<7.1 E-01 \\
<1.4 E-01\end{array}$ & $\begin{array}{l}\mathrm{U} / / \\
\mathrm{U} / /\end{array}$ & $\begin{array}{l}<1.0 E+00 \\
<4.2 E-01\end{array}$ & & $\begin{array}{l}1 \\
1\end{array}$ & $\begin{array}{l}\text { GP } \\
\text { GP }\end{array}$ & & & & & & & $\begin{array}{l}\text { pCin } \\
\text { pCin }\end{array}$ \\
\hline $\begin{array}{l}\text { Gross alpha } \\
\text { lodine-129 }\end{array}$ & $\begin{array}{l}2.0 E+02 \\
1.4 E+02\end{array}$ & & & a & $\begin{array}{l}1 \\
1\end{array}$ & $\begin{array}{l}\text { GP } \\
\text { GP }\end{array}$ & $1.5 E+02$ & II & & $\mathbf{\square}$ & 1 & GP & pCin \\
\hline Nonvolatile beta & 2.1E+03 & $\|$ & & 口 & 1 & GP & $2.4 E+03$ & $\| /$ & & E & 1 & GP & pcin \\
\hline Plutonium-238 & 8.3E-01 & $\mathrm{R} / / 4$ & Rej & & 1 & GP & & & & & & & pein \\
\hline Plutonium-239/240 & $<4.2 E-01$ & JUルI & $<4.2 E-01$ & & 1 & GP & & & & & & & pCin \\
\hline Radium-226 & $7.5 E+01$ & & & a & 1 & GP & & & & & & & pCin \\
\hline Radium-228 & $1.8 E+01$ & $\mathrm{~J} / \mathrm{L} / \mathrm{I}$ & NDD & & 1 & GP & & & & & & & pCin \\
\hline Total radium & $7.5 E+01$ & & & & & & & & & & & & \\
\hline Strontium-90 & $9.2 E+02$ & $/ 1$ & & $\mathbf{\square}$ & 1 & GP & & & & & & & pCin \\
\hline Technetium-99 & $2.7 E+02$ & $\|$ & & $\mathbf{\square}$ & 1 & GP & & & & & & & pCin \\
\hline Thorium-228 & 7.2E-01 & $/ /$ & & & 1 & GP & & & & & & & pCin \\
\hline Thorium-230 & $<5.1 E-02$ & $U / /$ & $<6.4 \mathrm{E}-02$ & & 1 & GP & & & & & & & pCin \\
\hline Thorium-232 & $<-8.1 E-03$ & $\mathrm{U} / \prime$ & $<6.4 E-02$ & & 1 & GP & & & & & & & pCin \\
\hline Sum of alphas & $1.0 E+02$ & & & $\mathbf{0}$ & & & & & & & & & pCil \\
\hline Sum of betas & $1.3 E+03$ & & & 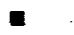 & & & & & & & & & pCin \\
\hline Tritium & $1.4 E+04$ & /I & & $\mathbf{z}$ & 1 & GP & 1.2E+04 & $\|$ & & $\boldsymbol{\varepsilon}$ & 1 & GP & $\mathrm{pCi} / \mathrm{mi}$ \\
\hline Uranium-233/234 & $5.1 E+01$ & $\|$ & & $\mathbf{a}$ & 1 & GP & & & & & & & pCill \\
\hline Uranium-235 & $3.2 E+00$ & $1 /$ & & & 1 & GP & & & & & & & pCin \\
\hline Uranium-238 & $4.9 E+01$ & $\|$ & & 룰 & 1 & GP & & & & & & & pcin \\
\hline
\end{tabular}

Notes:

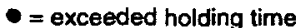

= exceeded groundwater protection or monitoring constituent standard (See Appendix A.)

NA - Not applicable. Applies to beta dose and sum of betas if there are no beta-emitting radionuclides above detection limits; to sum of alphas if there are no alpheemitting radionuclides above detection limits; and to total radium if neither radium-226 or radium-228 was above detection limit

UAZ_UTRA - Upper Aquifer Zone of the Upper Three Runs Aquifer, LAZ_UTRA - Lower Aquifer Zone of the Upper Three Runs Aquifer, Gordon - Gordon Aquifer

F-Area HWMF

First and Second Quarter 1999 
WELL FSB 94DR

$\begin{array}{ll}\text { SRS Coord. } & \text { Lat/Longitude } \\ \text { N74869.1 } & 33.273207^{\circ} \mathrm{N} \\ \text { E50162.9 } & \mathbf{8 1 . 6 8 0 1 4 1 ^ { \circ } \mathrm { W }}\end{array}$

SAMPLE DATE

1099

210.2

4.4

410

19.0

0

0.3

3.7

$209.7(03 / 23 / 99)$
Screen Zone Elevation

203.4-183.3 ft msl
Top of Casing

$280.5 \mathrm{ft} \mathrm{ms}$
Cașing Pump

Screen Zone

UAZ_UTRA
04/07/99

2099

209.6

3.4

440

20.7

2.9

$208.8(06 / 25 / 99)$
Unit

tt $\mathrm{msl}$

pH

$\mu \mathrm{S} / \mathrm{cm}$

'C

well volumes

$\mathrm{ft} \mathrm{msl}$

\section{ANALYTICAL DATA}

Inorganic Constituents

Constituents

Aluminum, total recoverable Antimony, total recoverable

Arsenic, total recoverable

Barium, total recoverable

Beryllium, total recoverable

Cadmium, total recoverable 2.2

Chromium, total recoverable 1.3

Cobalt, total recoverable

Copper, total recoverable

Cyanide

Lead, total recoverable

Mercury, total recoverable

Nickel, total recoverable

Nitrate as nitrogen

Nitrate-nitrite as nitrogen $\quad 50,000$

Selenium, total recoverable $<5.0$

Silver, total recoverable $<1.0$

Thallium, total recoverable $\quad 0.22$

Vanadium, total recoverabje $<10$

Zinc, total recoverable

45

Organic Constituents

Constituents
Benzene
Bis(2-ethylhexyl) phthalate
Dichloromethane
Phenols
Tetrachloroethylene
Trichloroethylene
Trichlorofluoromethane

1099
$<1.0$
$<10$
$<5.0$
$<5.0$
$<1.0$
5.6
$<5.0$

JUIO

$\mathrm{JU} / \mathrm{Q}$

JUINO

UtI

JU/L

JUO

$\mathrm{JU} / \mathrm{LO}$

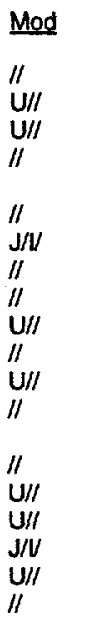

$<0.20$

$<3.0$

NDD

$<10$

$<0.20$

$<5.0$

$<1.0$

NDD

$<10$
ST브.

DF Lab $2099 \quad$ Mod

GE $\quad 13,000$

GE

GE

GE 1.8

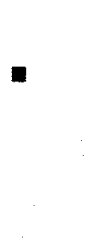

GE

GE
GE
GE
GE
GE
GE
GE

$\begin{array}{ll}25 & \mathrm{GE} \\ 1 & \mathrm{GE} \\ 1 & \mathrm{GE} \\ 1 & \mathrm{GE}\end{array}$

1 GE
$\mathbf{J} / \mathbf{K} /$

Fili.

NDD

45,000

GE

GE

1.8

$<1.6 \quad$ UNI $\quad<2.0$

ST H DF Lab Unit

1 GE $\mu \mathrm{g} /$

$\mu g / 2$

$1 \mathrm{GE} \mu \mathrm{g} / \mathrm{L}$

$\mu g / 2$

$\mu g / 2$

$\mu g h$

$\mu g / L$

1 GE $\mu g /$

$\mu g / L$

$100 \mathrm{GE} \mu \mathrm{gh}$

ugl

$\mu g h$

$\mu g /$

Hg/L

$\begin{array}{lrrr}\text { Filt. } & \text { STH } & \text { DF } & \text { Lab } \\ <1.0 & & 1 & \text { GE } \\ <10 & \bullet & 1 & \text { GE } \\ <5.0 & & 1 & \text { GE } \\ <5.0 & & 1 & \text { GE } \\ <1.0 & 1 & \text { GE } \\ \text { NDD } & & 1 & \text { GE } \\ <5.0 & & 1 & \text { GE }\end{array}$

Mod

Filt.

ST $H$ DF Lab Unit

$\mu g /$

$\mu g /$

$\mu g / 2$

$\mu g /$

$\mu g / 2$

$\mu g /$

jugh

Notes:

- = exceeded holding time

= exceeded groundwater protection or monitoring constituent standard (See Appendix A.)

NA - Not applicable. Applies to beta dose and sum of betas if there are no beta-emitting radionuclides above detection limits; to sum of alphas if there are no alphaemitting radionuclides above detection limits; and to total radium if neither radium-226 or radium-228 was above detection limit

UAZ UTRA - Upper Aquifer Zone of the Upper Three Runs Aquiter, LAZ UTRA - Lower Aquiter Zone of the Upper Three Runs Aquifer, Gordon - Gondon Aquifer 


\section{WELL FSB 94DR (cont.)}

Radioactive Constituents

\begin{tabular}{|c|c|c|c|c|c|c|c|c|c|c|c|c|c|}
\hline Constituents & 1099 & Mod & Fill. & ST브 & DF & Lab & 2099 & Mad & Filt. & ST 브 & DF & Lab & Unit \\
\hline $\begin{array}{l}\text { Americium-241 } \\
\text { Beta dose }\end{array}$ & $\begin{array}{l}2.0 E+01 \\
40\end{array}$ & $\|$ & & 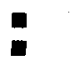 & 1 & GP & & & & & & & $\mathrm{pCi} / \mathrm{L}$ \\
\hline Carbon-14 & $3.4 E+01$ & $\|$ & & & 1 & GP & & & & & & & pCil \\
\hline Cesium-137 & $9.6 E+01$ & $\|$ & & E & 1 & GP & & & & & & & pCil \\
\hline Cobalt-60 & $<6.2 \mathrm{E}-01$ & $U / I$ & $<3.8 E+\infty$ & & 1 & GP & & & & & & & pCin \\
\hline Curium-242 & $<-1,6 E-01$ & $\mathrm{U} / /$ & $<1.1 E+\infty$ & & 1 & GP & & & & & & & pCin \\
\hline Curium-243/244 & $2.9 E+01$ & /I & & $\mathbf{7}$ & 1 & GP & & & & & & & pCil \\
\hline Curium-245/246 & $6.2 E-01$ & $R / / 4$ & Rej & & 1 & GP & & & & & & & pCin \\
\hline $\begin{array}{l}\text { Gross alpha } \\
\text { lodine-129 }\end{array}$ & $\begin{array}{l}9.2 E+02 \\
3.5 E+01\end{array}$ & $\begin{array}{l}\mathrm{J} / \mathrm{W} \\
/ /\end{array}$ & NDD & & 1 & $\begin{array}{l}\text { GP } \\
\text { GP }\end{array}$ & $5.2 E+02$ & $\|$ & & 口 & 1 & GP & $\begin{array}{l}\text { pCin } \\
\text { pCil }\end{array}$ \\
\hline Nonvolatile beta & $7.9 \mathrm{E}+02$ & $\mathrm{~J} / \mathrm{U}$ & NDD & & $i$ & $\mathrm{GP}$ & 5.1Ë+02 & II & & - & 1 & GP & pCin \\
\hline Plutonium-238 & $<0.0 \mathrm{E}+\infty$ & JUЛ & $<1.4 E-01$ & & 1 & GP & & & & & & & pCil \\
\hline Plutonium-239/240 & $<4.6 \mathrm{E}-02$ & JUR/CI & $<1.4 E-01$ & & 1 & GP & & & & & & & pCin \\
\hline Radium-226 & $5.4 E+\infty 0$ & /I & & $\mathbf{0}$ & 1 & GP & & & & & & & pCin \\
\hline $\begin{array}{l}\text { Radium-228 } \\
\text { Total radium }\end{array}$ & $\begin{array}{l}2.4 E+01 \\
3.0 E+01\end{array}$ & // & & $\square$ & 1 & GP & & & & & & & $\mathrm{pCi}$ \\
\hline Strontium-90 & $1.4 \mathrm{E}+02$ & $\mathrm{~J} / \mathrm{K} / \mathrm{C}$ & NDD & & 1 & GP & & & & & & & pCin \\
\hline Technetium-99 & $5.3 E+01$ & /I & & $\mathbf{a}$ & 1 & GP & & & & & & & pCir \\
\hline Thorium-228 & $7.2 E-01$ & $R / 4$ & Rej & & 1 & GP & & & & & & & pCin \\
\hline Thorium-230 & $<-4.0 E-04$ & $\mathrm{U} / /$ & $<1.9 E-01$ & & 1 & GP & & & & & & & pCint \\
\hline Thorium-232 & $<-1.0 \mathrm{E}-02$ & $\mathrm{U} / \mathrm{I}$ & $<1.3 E-01$ & & 1 & GP & & & & & & & pCin \\
\hline Sum of alphas & $6.7 E+02$ & & & $\mathbf{0}$ & & & & & & & & & pCin \\
\hline Sum of betas & $2.4 E+02$ & & & $\boldsymbol{\square}$ & & & & & & & & & pCiL \\
\hline Tritium & $2.5 E+03$ & $\|$ & & $\mathbf{\square}$ & 1 & GP & $1.9 E+03$ & $\|$ & & घ & 1 & GP & pCi/ml \\
\hline Uranium-233/234 & $1.9 E+02$ & $/ 1$ & & $\mathbf{n}$ & 1 & GP & & & & & & & $\mathrm{pCil}$ \\
\hline Uranium-235 & $1.8 E+01$ & /l & & $\mathbf{0}$ & 1 & GP & & & & & & & pCin \\
\hline Uranium-238 & 4.2E+02 & $/ /$ & & $\square$ & 1 & GP & & & & & & & pCin \\
\hline
\end{tabular}

- exceeded holding time

= exceeded groundwater protection or monitoring constituent standard (See Appendix A.)

NA - Not applicable. Applies to beta dose and sum of betas if there are no beta-emitting radionuclides above detection limits; to sum of alphas if there are no alphaemitting radionuclides above detection limits; and to total radium if neither radium-226 or radium-228 was above detection limit

UAZ UTRA - Upper Aquifer Zone of the Upper Three Runs Aquifer, LAZ UTRA - Lower Aquifer Zone of the Upper Three Runs Aquifer, Gordon - Gordon Aquifer 


\section{WELLL FSB 95CR}

$\begin{array}{ll}\text { SRS Coord. } & \text { Latlongitude } \\ \text { N75001.9 } & 33.273215^{\circ} \mathrm{N}\end{array}$

$81.680860^{\circ} \mathrm{W}$

SAMPLE DATE

Screen Zone Elevation

$161.9-151.9 \mathrm{tt} \mathrm{ms}$
Too of Casing

$284 \mathrm{ft} \mathrm{msl}$
Casing Pump

4'PVC S
Screen Zone

LAZ_UTRA

\section{FIELD DATA}

Constituents

Water elevation

pH

Sp. conductance

Water temperature

Alkalinity as $\mathrm{CaCO} 3$

Turbidity

Volumes purged

Sampling code

Synchronous water level

\section{ANALYTICAL DATA}

Inorganic Constituents

\section{9}

208.2

3.0

2000

20.6

0

2.5

$205.7(03 / 23 / 99)$
04/07/99

2099

206.4

3.7

2000

20.6

0

2.8

$206.8(06 / 25 / 99)$
Unit

tt ms!

pH

$\mu \mathrm{S} / \mathrm{cm}$

${ }^{\circ} \mathrm{C}$

$\mathrm{mgh}$

NTU

well volumes

ft msl

\begin{tabular}{|c|c|c|c|c|c|c|c|c|c|c|c|c|c|}
\hline Constituents & 1099 & Mod & Filt. & SIH & DF & Lab & 2099 & Mod & Filt. & ST $\mathrm{H}$ & DF & Lab & Unit \\
\hline Aluminum, total recoverable & 65,000 & /I & & & 1 & $\mathrm{GE}$ & 66,000 & $\mathrm{~J} / \mathrm{K} / \mathrm{I}$ & NDD & & 1 & GE & $\mu g /$ \\
\hline Antimony, total recoverable & $<0.20$ & $\mathrm{U} / /$ & $<0.20$ & & 1 & GE & & & & & & & pgl \\
\hline Arsenic, total recoverable & 1.8 & $\mathrm{~J} / \mathrm{V}$ & NDD & & 1 & GE & & & & & & & $\mu g /$ \\
\hline Barium, total recoverable & 970 & $/ /$ & & & 1 & GE & & & & & & & Hgh \\
\hline Beryllium, total recoverable & 14 & $/ /$ & & & 1 & GE & & & & & & & \\
\hline Cadmium, total recoverable & 17 & $\|$ & & $\mathbf{0}$ & 1 & GE & 20 & $\|$ & & $\mathbf{\square}$ & 1 & GE & Wh \\
\hline Chromium, total recoverable & 1.4 & $\mathrm{~J} / \mathrm{N}$ & NDD & & 1 & $\overrightarrow{G E}$ & & & & & & & \\
\hline Cobalt, total recoverable & 450 & $/ 1$ & & $\mathbf{a}$ & 1 & GE & & & & & & & $\mu g h$ \\
\hline Copper, total recoverable & 54 & 11 & & & 1 & $\overrightarrow{\mathrm{GE}}$ & & & & & & & $\mu g /$ \\
\hline Cyanide & $<10$ & $\mathrm{U} / /$ & $<10$ & & 1 & GE & & & & & & & \\
\hline Lead, total recoverable & 0.87 & $\mathrm{~J} / \mathrm{L} /$ & NDD & & 1 & GE & $<20$ & $\mathrm{U} / \prime$ & $<20$ & & 10 & GE & \\
\hline Mercury, total recoverable & 1.1 & $1 /$ & & & 1 & GE & & & & & & & \\
\hline Nickel, total recoverable & 99 & $\|$ & & & 1 & GE & & & & & & & $\mu g /$ \\
\hline Nitrate as nitrogen & & & & & & & & & & & & & \\
\hline Nitrate-nitrite as nitrogen & 270,000 & $\|$ & & $\mathbf{m}$ & 100 & GE & 270,000 & II & & $\mathbf{a}$ & 125 & GE & $n$ \\
\hline Selenium, total recoverable & 7.0 & $1 /$ & & & 1 & GE & & & & & & & \\
\hline Silver, total recoverable & $<1.0$ & $\mathbf{U} / /$ & $<1.0$ & & 1 & GE & & & & & & & \\
\hline Thallium, total recoverable & 1.1 & $J N$ & NDD & & 1 & GE & & & & & & & \\
\hline Vanadium, total recoverable & $<10$ & $\mathrm{U} / /$ & $<10$ & & 1 & GE & & & & & & & \\
\hline Zinc, total recoverable & 310 & $/ /$ & & & 1 & GE & & & & & & & \\
\hline \multicolumn{14}{|l|}{ Organic Constituents } \\
\hline Constituents & 1099 & Mod & Filt. & ST브 & $\underline{\mathrm{DF}}$ & Lab & 2099 & Mod & Filt. & ST $H$ & DF & Lab & Unit \\
\hline Benzene & $<1.0$ & JU/L/O & $<1.0$ & & 1 & GE & & & & & & & \\
\hline Bis(2-ethylhexyl) phthalate & $<10$ & & $<10$ & & 1 & GE & & & & & & & \\
\hline Dichloromethane & $<5.0$ & JUนO & $<5.0$ & & 1 & GE & & & & & & & gl \\
\hline Phenols & $<5.0$ & $\mathrm{U} / /$ & $<5.0$ & & 1 & GE & & & & & & & $g /$ \\
\hline Tetrachloroethylene & $<1.0$ & JUNRO & $<1.0$ & & 1 & $\mathrm{GE}$ & & & & & & & ugl \\
\hline Trichloroethylene & $<1.0$ & JUNOO & $<1.0$ & & 1 & GE & & & & & & & $\mu g /$ \\
\hline Trichlorofluoromethane & $<5.0$ & JU/LO & $<5.0$ & & 1 & GE & & & & & & & \\
\hline
\end{tabular}

Notes:

= exceeded holding time

= exceeded groundwater protection or monitoring constituent standard (See Appendix A.)

NA - Not applicable. Applies to beta dose and sum of betas if there are no beta-emitting radionuclides above detection limits; to sum of atphas if there are no alphaemitting radionuclides above detection limits; and to total radium if neither radium-226 or radium-228 was above detection limit

UAZ_UTRA - Upper Aquifer Zone of the Upper Three Runs Aquifer, LAZ UTRA - Lower Aquifer Zone of the Upper Three Runs Aquifer, Gordon - Gordon Aquifer 
WELL FSB 95CR (cont.)

Radioactive Constituents

\begin{tabular}{|c|c|c|c|c|c|c|c|c|c|c|c|c|c|}
\hline Constituents & 1099 & Mod & Filt. & SI브 & $\underline{D F}$ & Lab & 2099 & Mod & Fill. & $\underline{S T} \underline{H}$ & DE & Lab & Unit \\
\hline $\begin{array}{l}\text { Americium-241 } \\
\text { Beta dose }\end{array}$ & $\begin{array}{l}2.0 E+01 \\
116\end{array}$ & "I & & 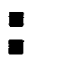 & 1 & GP & & & & & & & poir \\
\hline Carbon-14 & $9.0 \mathrm{E}+01$ & $\|$ & & घ & 1 & GP & & & & & & & pcin \\
\hline Cesium-137 & $<-9.9 \mathrm{E}-01$ & $\mathrm{u} / \prime$ & $<4.5 E+00$ & & 1 & GP & & & & & & & pcin \\
\hline Cobalt -60 & $<-2.6 \mathrm{E}-01$ & $\mathrm{U} / I$ & $<3.7 \mathrm{E}+00$ & & 1 & GP & & & & & & & pCin \\
\hline Curium-242 & $<-4.6 \mathrm{E}-01$ & $u / \prime$ & $<1.0 E+00$ & & 1 & GP & & & & & & & pCin \\
\hline $\begin{array}{l}\text { Curium-243/244 } \\
\text { Curium-245/246 }\end{array}$ & $\begin{array}{l}2.9 \mathrm{E}+01 \\
7.5 \mathrm{E}-01\end{array}$ & & Rej & च & 1 & $\begin{array}{l}\text { GP } \\
\text { GP }\end{array}$ & & & & & & & $\begin{array}{l}\text { pCin } \\
\text { pCin }\end{array}$ \\
\hline $\begin{array}{l}\text { Curium-245/246 } \\
\text { Gross alpha }\end{array}$ & $\begin{array}{l}7.5 E-01 \\
1.2 E+03\end{array}$ & $\begin{array}{l}\mathrm{R} / 4 \\
\mathrm{~J} / \mathrm{I}\end{array}$ & $\begin{array}{l}\text { Rej } \\
\text { NDD }\end{array}$ & & $\frac{1}{1}$ & GP & $8.6 E+02$ & $/ I$ & & a & 1 & GP & pCir. \\
\hline lodine-129 & $1.1 E+02$ & 11 & & घ & 1 & GP & & & & & & & pCin \\
\hline Nonvolatile beta & $2.1 E+03$ & $\|$ & & - & 1 & GP & $1.9 E+03$ & II & & - & 1 & GP & pCin \\
\hline Plutonium-238 & $<8.6 \mathrm{E}-02$ & JUACI & $<2.1 E-01$ & & 1 & GP & & & & & & & $\mathrm{pCin}$ \\
\hline Plutonium-239/240 & $<4.6 \mathrm{E}-02$ & JU/UCI & $<1.4 E-01$ & & 1 & GP & & & & & & & pCin \\
\hline Radium-226 & $1.1 E+02$ & $/ 1$ & & 口 & 1 & GP & & & & & & & pcin \\
\hline Radium-228 & $2.7 E+01$ & /I & & - & 1 & GP & & & & & & & pCin \\
\hline Total radium & $1.4 E+02$ & & & & & & & & & & & & \\
\hline Strontium-90 & $5.2 E+02$ & $\mathrm{~J} / \mathrm{KJC}$ & NDD & & 1 & GP & & & & & & & pCin \\
\hline Technetium-99 & $2.8 E+02$ & 11 & & - & 1 & $G$ & & & & & & & DCin \\
\hline $\begin{array}{l}\text { Thorium-228 } \\
\text { Thorium-230 }\end{array}$ & 6.4E-01 & $\begin{array}{l}\mathrm{R} / / 4 \\
\mathrm{U} / /\end{array}$ & Rej & & 1 & GP & & & & & & & PCll \\
\hline $\begin{array}{l}\text { Thorium-230 } \\
\text { Thorium-232 }\end{array}$ & $\begin{array}{l}<5.8 \mathrm{E}-02 \\
<2.5 \mathrm{E}-02\end{array}$ & $u / / 7$ & $\begin{array}{l}<1.0 E=-01 \\
<1.0 E-01\end{array}$ & & $i$ & GP & & & & & & & $\mathrm{pCin}$ \\
\hline Sum of alphas & $9.3 E+02$ & & & a & & & & & & & & & pCir \\
\hline $\begin{array}{l}\text { Sum of betas } \\
\text { Tritium }\end{array}$ & $5.1 E+02$ & & & E & & & & & & & & & pCil \\
\hline Tritium & $1.1 E+04$ & 11 & & 口 & 1 & GP & $9.4 E+03$ & $\|$ & & a & 1 & GP & pCi/mL \\
\hline Uranium-233/234 & $4.0 \mathrm{E}+02$ & /I & & - & 1 & GP & & & & & & & pCin \\
\hline Uranium-235 & $3.1 E+01$ & /I & & a & 1 & GP & & & & & & & pCin \\
\hline Uranium-238 & $4.5 E+02$ & /I & & - & 1 & GP & & & & & & & pCir \\
\hline
\end{tabular}

Notes:

- =xceeded holding time

= exceeded groundwater protection or monitoring constituent standard (See Appendix A.)

NA - Not applicable. Applies to beta dose and sum of betas if there are no beta-emitting radionuclides above detection limits; to sum of alphas if there are no alphaemitting radionuclides above detection limits; and to total radium if neither radium-226 or radium-228 was above detection limit

UAZ UTRA - Upper Aquifer Zone of the Upper Three Runs Aquifer, LAZ_UTRA - Lower Aquifer Zone of the Upper Three Runs Aquifer, Gordon - Gordon Aquifer 


\section{WELL FSB 95DR}

$\begin{array}{ll}\text { SRS Coord. } & \text { Lat/Longitude } \\ \text { N74991.7 } & 33.273206^{\circ} \mathrm{N} \\ \text { E49996.0 } & 81.680818^{\circ} \mathrm{W}\end{array}$

SAMPLE DATE
Screen Zone Elevation

207.0-187.0 ft ms
Iop 의 Casing

$284.1 \mathrm{ft} \mathrm{ms}$

\section{Casing Pump}

4"PVC S
Screen Zone

UAZ_UTRA
04/07/99

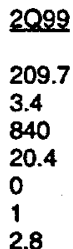

$209.0(06 / 25 / 99)$
Unit

tt msl

$\mathrm{pH}$

$\mu \mathrm{Sicm}$

${ }^{\circ} \mathrm{C}$

mgl

NTU

well volumes

ft ms!
$209.8(03 / 23 / 99)$

\section{ANALYTICAL DATA}

Inorganic Constituents

\begin{tabular}{|c|c|c|c|c|c|c|c|c|c|c|c|c|c|}
\hline Constituents & 1099 & Mod & Filt. & STH & DF & Lab & 2099 & Mod & Filt. & ST $\mathbf{H}$ & DF & Lab & Unis \\
\hline Aluminum, total recoverable & 29,000 & 11 & & & 1 & GE & 29,000 & $\mathrm{~J} / \mathrm{K} /$ & NDD & & 1 & GE & $\mu g \lambda$ \\
\hline Antimony, total recoverable & $<0.23$ & $\mathrm{JU} / / 4$ & $<0.89$ & & $i$ & $\mathrm{GE}$ & & & & & & & \\
\hline Arsenic, total recoverable & $<3.0$ & U// & $<3.0$ & & 1 & GE & & & & & & & \\
\hline $\begin{array}{l}\text { Barium, total recoverable } \\
\text { Beryliium, total recoverable }\end{array}$ & 290 & $/ /$ & & & 1 & GE & & & & & & & \\
\hline Cadmium, total recoverable & 2.5 & $/ 1$ & & & 1 & GE & 1.6 & $/ 1$ & & & 1 & GE & $\mu g /$ \\
\hline Chromium, total recoverable & 4.1 & 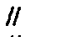 & & & 1 & GE & & & & & & & \\
\hline Cobalt, total recoverable & 11 & $/ 1$ & & $\mathbf{a}$ & 1 & GE & & & & & & & \\
\hline Copper, total recoverable & 100 & $/ 1$ & & & 1 & $\overline{\mathrm{GE}}$ & & & & & & & \\
\hline Cyanide & $<10$ & $U / /$ & $<10$ & & 1 & GE & & & & & & & \\
\hline Lead, total recoverable & 15 & /I & & & 1 & GE & 14 & 11 & & & 1 & GE & \\
\hline Mercury, total recoverable & 1.5 & 11 & & & 1 & GE & & & & & & & \\
\hline $\begin{array}{l}\text { Nickel, total recoverable } \\
\text { Nitrate as nitrogen }\end{array}$ & 16 & 11 & & & 1 & $\overline{G E}$ & & & & & & & $\mu g h$ \\
\hline Nitrate-nitrite as nitrogen & 97,000 & 11 & & $\mathbf{\square}$ & 50 & GE & 82,000 & $\|$ & & $\mathbf{n}$ & 50 & GE & $\mu g /$ \\
\hline Selenium, total recoverable & $<5.0$ & $\mathrm{U} / /$ & $<5.0$ & & 1 & GE & & & & & & & \\
\hline Sitver, total recoverable & $<1.0$ & $\mathrm{U} / /$ & $<1.0$ & & 1 & GE & & & & & & & \\
\hline Thallium, total recoverable & 0.28 & $\mathrm{~J} / \mathrm{N}$ & NDD & & 1 & GE & & & & & & & $\mu g h$ \\
\hline $\begin{array}{l}\text { Vanadium, total recoverable } \\
\text { Zinc, total recoverable }\end{array}$ & $\begin{array}{l}<10 \\
85\end{array}$ & $\mathrm{U} / /$ & $<10$ & & $\begin{array}{l}1 \\
1\end{array}$ & $\begin{array}{l}\text { GE } \\
\text { GE }\end{array}$ & & & & & & & $g / l$ \\
\hline
\end{tabular}

Organic Constituents

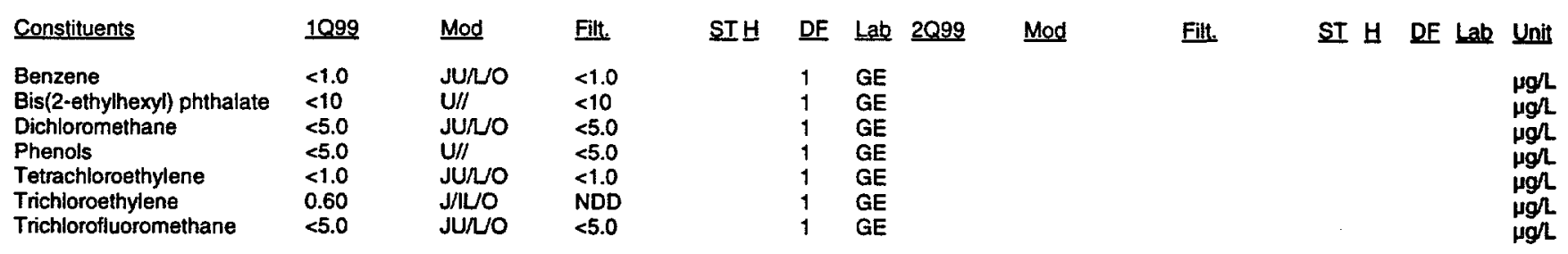

Notes:

- = exceeded holding time

- exceeded groundwater protection or monitoring constituent standard (See Appendix A.)

NA - Not applicable. Applies to beta dose and sum of betas if there are no beta-emitting radionuclides above detection limits; to sum of alphas if there are no alphaemitting radionuclides above detection limits; and to total radium if neither radium-226 or radium-228 was above detection limit

UAZ UTRA - Upper Aquifer Zone of the Upper Three Runs Aquifer, LAZ UTRA - Lower Aquifer Zone of the Upper Three Runs Aquiter, Gordon - Gordon Aquifer 
WELL FSB 95DR (cont.)

Radioactive Constituents

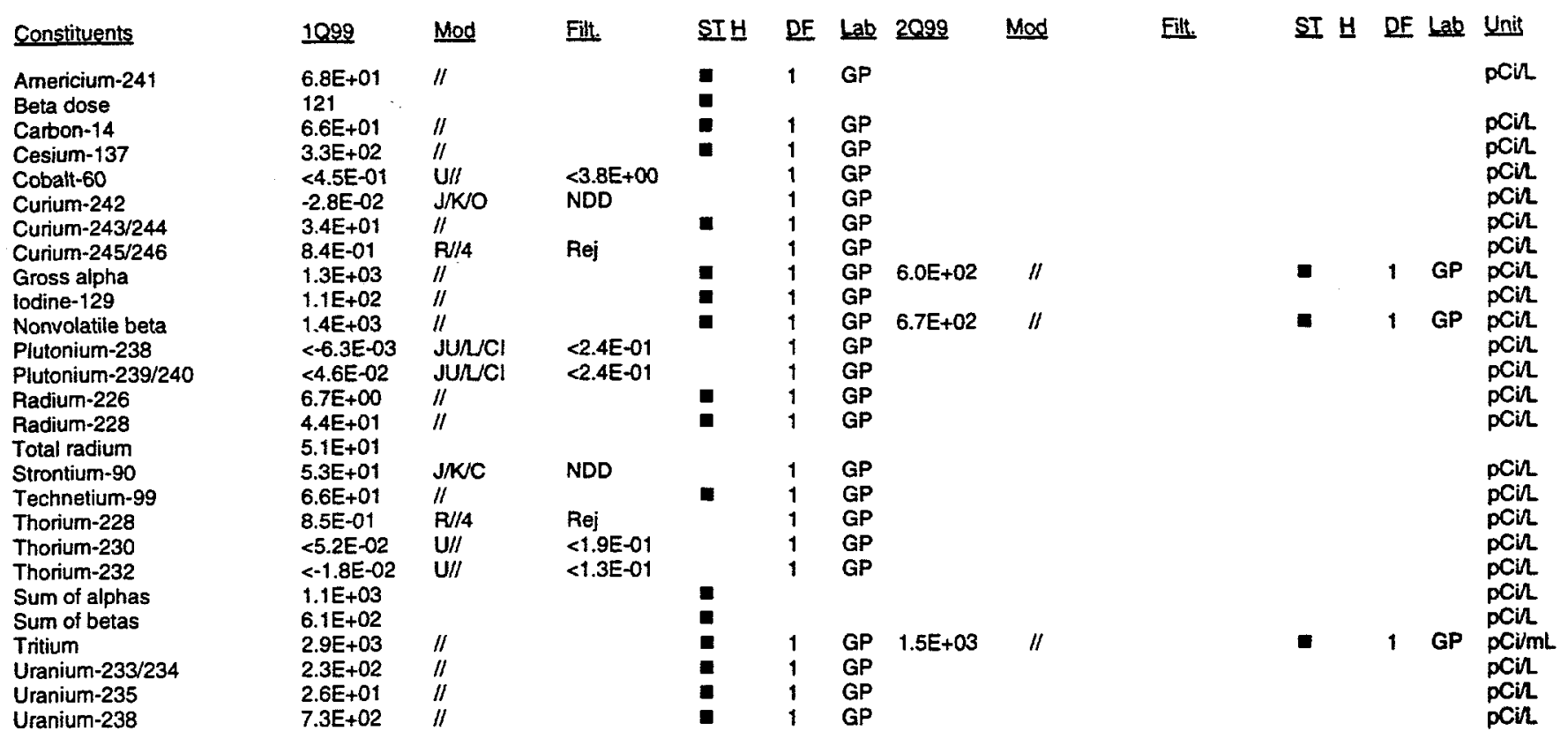

Notes:

- = exceeded holding time

E = exceeded groundwater protection or monitoring constituent standard (See Appendix A.)

NA - Not applicable. Applies to beta dose and sum of betas if there are no beta-emitting radionuclides above detection limits; to sum of alphas if there are no alphaemitting radionuclides above detection limits; and to total radium if neither radium-226 or radium-228 was above detection limit

UAZ_UTRA - Upper Aquifer Zone of the Upper Three Runs Aquifer, LAZ_UTRA - Lower Aquifer Zone of the Upper Three Runs Aquifer, Gordon - Gordon Aquifer 


\section{WELL FSB 96AR}

\begin{tabular}{|c|c|}
\hline SAS coord. & Lat/Longitude \\
\hline $\begin{array}{l}N 74914.9 \\
\text { E49746.6 }\end{array}$ & $\begin{array}{l}33.27262 \\
81.68132\end{array}$ \\
\hline
\end{tabular}

Screen Zone Elevation

89.0-79.0 ft msl

$01 / 08 / 99$

\section{Top of Casing}

$281.2 \mathrm{ft} \mathrm{ms}$
Casing Pump

4'PVC S
Screen Zone

Gordon
SAMPLE DATE

1099

Constituents

Water elevation

$\mathrm{pH}$

$\mathrm{Sp}$. conductance

Water temperature

Alkalinity as $\mathrm{CaCO} 3$

Turbidity

Volumes purged

Sampling code

Synchronous water level

ANALYTICAL DATA

Inorganic Constituents
04/07/99

2099
153.4
6.6
170
21.2
58
0
2.9

$152.9(06 / 25 / 99)$
Unit

tt msi

pH

$\mathrm{SS} / \mathrm{cm}$

mgl

well volumes

tt msl

\begin{tabular}{|c|c|c|c|c|c|c|c|c|c|c|c|c|c|}
\hline Constituents & 1099 & Mod & Filt. & ȘT & $\underline{\text { DF }}$ & Lab & $\underline{2099}$ & Mod & Filt. & ST H & DF & Lab & Unit \\
\hline Aluminum, total recoverable & $<15$ & $u / I$ & $<15$ & & 1 & GE & $<23$ & UNI & $<15$ & & 1 & GE & $\mu g /$ \\
\hline Antimony, total recoverable & $<0.20$ & $\mathrm{U} / /$ & $<0.20$ & & 1 & GE & & & & & & & \\
\hline Arsenic, total recoverable & $<3.0$ & $U / I$ & $<3.0$ & & 1 & GE & & & & & & & $\mu g h$ \\
\hline $\begin{array}{l}\text { Barium, total recoverable } \\
\text { Beryllium, total recoverable }\end{array}$ & 37 & $/ /$ & & & 1 & GE & & & & & & & $\mu g /$ \\
\hline Cadmium, total recoverable & $<1.0$ & $\mathrm{U} / /$ & $<1.0$ & & 1 & GE & $<1.0$ & $u^{\prime \prime}$ & $<1.0$ & & 1 & GE & $\mu g /$ \\
\hline Chromium, total recoverable & 1.6 & $J W$ & NDD & & 1 & $\overline{G E}$ & & & & & & & \\
\hline Cobalt, total recoverable & $<0.12$ & UN/ & $<0.20$ & & 1 & GE & & & & & & & \\
\hline Copper, total recoverable & 1.7 & $\|$ & & & 1 & GE & & & & & & & \\
\hline Cyanide & $<10$ & $\mathrm{U} / /$ & $<10$ & & 1 & GE & & & & & & & \\
\hline Lead, total recoverable & 0.31 & $\mathrm{~J} / \mathrm{N}$ & NDD & & 1 & GE & $<2.0$ & $\mathbf{U} / /$ & $<2.0$ & & 1 & GE & \\
\hline Mercury, total recoverable & $<0.20$ & $\mathbf{U N} /$ & $<0.20$ & & 1 & GE & & & & & & & \\
\hline $\begin{array}{l}\text { Nickel, total recoverable } \\
\text { Nitrate as nitrogen }\end{array}$ & 2.8 & 11 & & & 1 & GE & & & & & & & \\
\hline Nitrate-nitrite as nitrogen & 1,200 & $\|$ & & & 1 & GE & 1,600 & $\|$ & & & 1 & GE & \\
\hline Selenium, total recoverable & $<5.0$ & $\mathrm{u} / /$ & $<5.0$ & & 1 & GE & & & & & & & \\
\hline Silver, total recoverable & $<0.39$ & $J U / / 4$ & $<1.0$ & & 1 & GE & & & & & & & \\
\hline Thallium, total recoverable & $<0.72$ & $\mathrm{UNI}$ & $<2.5$ & & 1 & GE & & & & & & & \\
\hline Vanadium, total recoverable & $<10$ & $\mathrm{U} / 1$ & $<10$ & & 1 & GE & & & & & & & \\
\hline Zinc, total recoverable & $<10$ & $\mathbf{U} / /$ & $<10$ & & 1 & GE & & & & & & & \\
\hline
\end{tabular}

Organic Constituents

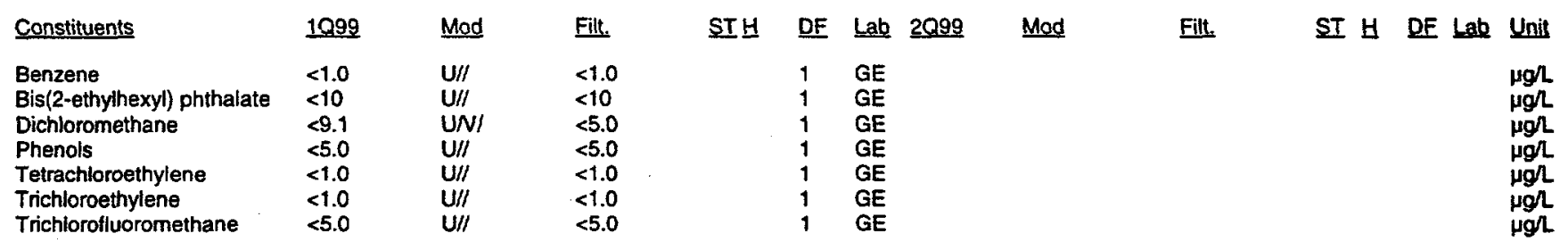

Notes:

- = exceeded holding time

= exceeded groundwater protection or monitoring constituent standard (See Appendix A.)

NA - Not applicable. Applies to beta dose and sum of betas if there are no beta-emitting radionuclides above detection limits; to sum of alphas if there are no alpha* emitting radionuclides above detection limits; and to total radium if neither radium-226 or radium-228 was above detection limit

UAZ_UTRA - Upper Aquifer Zone of the Upper Three Runs Aquifer, LAZ_UTRA - Lower Aquifer Zone of the Upper Three Runs Aquifer, Gordon - Gordon Aquifer 


\section{WELL FSB 96AR (cont.)}

Radioactive Constituents

\begin{tabular}{|c|c|c|c|c|c|c|c|c|c|c|c|c|c|}
\hline Constituents & 1099 & Mod & Fill. & ST브. & $\underline{D F}$ & Lab & 2099 & Mad & Filt. & ST $H$ & DF & Lab & Unit \\
\hline $\begin{array}{l}\text { Americium-241 } \\
\text { Beta dose }\end{array}$ & $\begin{array}{l}<6.6 \mathrm{E}-03 \\
0.01\end{array}$ & JU/LV/ & $<1.5 E-01$ & & 1 & GP & & & & & & & pCin \\
\hline Carbon-14 & $2.6 E+01$ & $\| 1$ & & & 1 & GP & & & & & & & poin \\
\hline Cesium-137 & $<2.2 E+\infty 0$ & $\mathrm{U} / 1$ & $<3.8 E+\infty 0$ & & 1 & GP & & & & & & & pCin \\
\hline Cobalt -60 & $<8.0 E-01$ & $\mathrm{U} / !$ & $<4.3 E+\infty 0$ & & 1 & GP & & & & & & & pCin \\
\hline Curium-242 & $<0.0 \mathrm{E}+00$ & $\mathbf{U} / /$ & $<4.9 \mathrm{E}-02$ & & 1 & GP & & & & & & & pCin \\
\hline Curium-243/244 & $1.3 E+\infty 0$ & 11 & & & 1 & GP & & & & & & & pCin \\
\hline Curium-245/246 & 2.3E-01 & $J / V$ & NDD & & 1 & GP & & & & & & & $\mathrm{pCin}$ \\
\hline Gross alpha & $<6.5 E-01$ & $\mathrm{U} / /$ & $<8.8 E-01$ & & 1 & GP & $9.2 E=01$ & $\mathrm{~J} / \mathrm{V}$ & NDD & & 1 & GP & pCin \\
\hline lodine-129 & $<6.2 E-01$ & $\mathrm{U} / /$ & $<8.4 E-01$ & & 1 & GP & & & & & & & $\mathrm{pCin}$ \\
\hline Nonvolatile beta & $1.5 E+\infty$ & $\mathrm{J} / \mathrm{V}$ & NDD & & 1 & GP & $2.0 E+00$ & $\mathrm{~J} / \mathrm{I} /$ & NDD & & 1 & GP & pCin \\
\hline Plutonium-238 & $<-8.8 E-02$ & $\mathrm{U} / /$ & $<2.9 E-01$ & & 1 & GP & & & & & & & pCin \\
\hline Plutonium-239/240 & $<0.0 \mathrm{E}+00$ & $\mathrm{U} / 1$ & $<4.7 E-02$ & & 1 & GP & & & & & & & pCin \\
\hline Radium-226 & 9.1E-01 & $\mathrm{J} / \mathrm{W}$ & NDD & & 1 & GP & & & & & & & pCin \\
\hline Radium-228 & $<7.0 \mathrm{E}-01$ & $U / /$ & $<1.0 E+\infty$ & & 1 & GP & & & & & & & pCin \\
\hline Total radium & NA & & & & & & & & & & & & \\
\hline Strontium-90 & $<-2.2 E-01$ & $\mathbf{U} / /$ & $<1.3 E+\infty 0$ & & 1 & GP & & & & & & & pCin \\
\hline Technetium-99 & $<-1.8 E+01$ & $\mathbf{U} / \prime$ & $<4.9 E+01$ & & 1 & GP & & & & & & & pCir \\
\hline Thorium-228 & $2.0 \mathrm{E}-01$ & $\mathrm{R} / \mathrm{4}$ & Rej & & 1 & GP & & & & & & & pCin \\
\hline Thorium-230 & 7.5E-02 & $R / 4$ & Rej & & 1 & GP & & & & & & & $\mathrm{pCin}$ \\
\hline Thorium-232 & $<1.9 E-02$ & $\mathrm{U} / /$ & $<2.8 E-02$ & & 1 & GP & & & & & & & pCin \\
\hline Sum of alphas & $1.3 E+00$ & & & & & & & & & & & & pCn \\
\hline Sum of betas & $2.6 \mathrm{E}+01$ & & & & & & & & & & & & pCil \\
\hline Tritium & 1.7E+01 & $1 /$ & & & 1 & GP & 2.3E+01 & $\|$ & & $\boldsymbol{\square}$ & 1 & GP & $\mathrm{pCi} / \mathrm{m}$ \\
\hline Uranium-233/234 & $<2.3 E-02$ & $\mathbf{U} / /$ & $<3.0 E-01$ & & 1 & GP & & & & & & & pCin \\
\hline Uranium-235 & $<-2.5 E-02$ & U/l & $<2.7 E-01$ & & 1 & GP & & & & & & & pCin \\
\hline Uranium-238 & $<-6.1 E-02$ & $\mathrm{U} / \mathrm{I}$ & $<2.5 E-01$ & & 1 & GP & & & & & & & pCin \\
\hline
\end{tabular}

= exceeded groundwater protection or monitoring constituent standard (See Appendix A.)

NA - Not applicable. Applies to beta dose and sum of betas if there are no beta-emitting radionuclides above detection limits; to sum of alphas if there are no alphaemitting radionuclides above detection limits; and to total radium if neither radium-226 or radium-228 was above detection limit

UAZ_UTRA - Upper Aquifer Zone of the Upper Three Runs Aquifer, LAZ_UTRA - Lower Aquifer Zone of the Upper Three Runs Aquifer, Gordon - Gordon Aquifer 
WELL FSB 97A

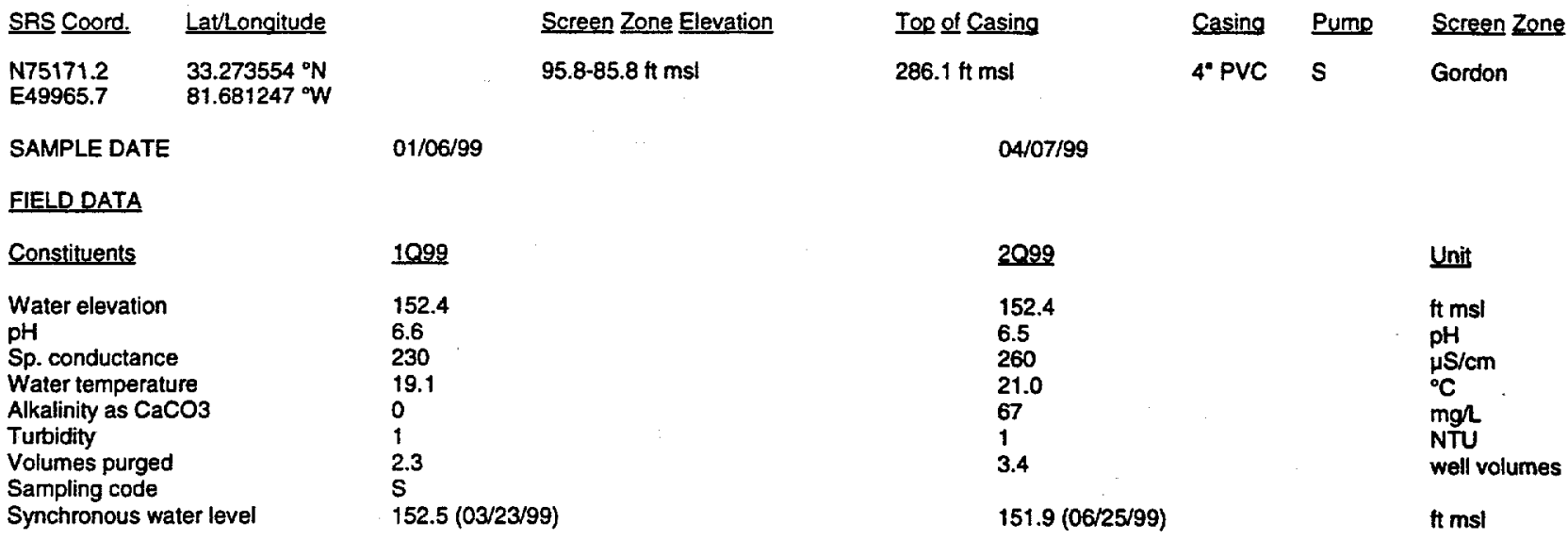

ANALYTICAL DATA

Inorganic Constituents

\begin{tabular}{|c|c|c|c|c|c|c|c|c|c|c|c|c|c|}
\hline Constituents & 1099 & Mod & Filt. & STH & DF & Lab & 2099 & Mod & Filt. & ST H & DF & Lab & Unif \\
\hline $\begin{array}{l}\text { Aluminum, total recoverable } \\
\text { Antimony, total recoverable } \\
\text { Arsenic, total recoverable } \\
\text { Barium, total recoverable } \\
\text { Beryllium, total recoverable }\end{array}$ & $\begin{array}{l}11 \\
<0.20 \\
<3.0 \\
39\end{array}$ & $\begin{array}{l}\mathrm{J} / \mathrm{U} \\
\mathrm{U} / / \\
\mathrm{U} / / \\
/ I\end{array}$ & $\begin{array}{l}\text { NDD } \\
<0.20 \\
<3.0\end{array}$ & & $\begin{array}{l}1 \\
1 \\
1 \\
1\end{array}$ & $\begin{array}{l}\text { GE } \\
\text { GE } \\
\text { GE } \\
\text { GE }\end{array}$ & $<12$ & UNI & $<15$ & & 1 & GE & $\begin{array}{l}\mu g h \\
\mu g h \\
\mu g h \\
\mu g h\end{array}$ \\
\hline $\begin{array}{l}\text { Cadmium, total recoverable } \\
\text { Chromium, total recoverable } \\
\text { Cobalt, total recoverable } \\
\text { Copper, total recoverable } \\
\text { Cyanide }\end{array}$ & $\begin{array}{l}<1.0 \\
4.0 \\
<0.078 \\
<0.86 \\
<10\end{array}$ & $\begin{array}{l}\mathrm{U} / / \\
/ / \\
\mathrm{U} / / 4 \\
\mathrm{U} / \mathrm{W} / \\
\mathrm{U} / /\end{array}$ & $\begin{array}{l}<1.0 \\
<0.20 \\
<1.0 \\
<10\end{array}$ & & $\begin{array}{l}1 \\
1 \\
1 \\
1 \\
1\end{array}$ & $\begin{array}{l}\text { GE } \\
\text { GE } \\
\text { GE } \\
\text { GE } \\
\text { GE }\end{array}$ & $<1.0$ & $\mathrm{U} / /$ & $<1,0$ & & 1 & GE & $\begin{array}{l}\mu g h \\
\mu g h \\
\mu g h \\
\mu g h \\
\text { uoh }\end{array}$ \\
\hline $\begin{array}{l}\text { Lead, total recoverable } \\
\text { Mercury, total recoverable } \\
\text { Nickel, total recoverable } \\
\text { Nitrate as nitrogen }\end{array}$ & $\begin{array}{l}0.36 \\
<0.20 \\
1.7\end{array}$ & $\begin{array}{l}\mathrm{J} / \mathrm{U} \\
\mathrm{U} / / \\
\mathrm{J} / \mathrm{U}\end{array}$ & $\begin{array}{l}\text { NDD } \\
<0.20 \\
\text { NDD }\end{array}$ & & $\begin{array}{l}1 \\
1 \\
1\end{array}$ & $\begin{array}{l}\text { GE } \\
\text { GE } \\
\text { GE }\end{array}$ & $<0.34$ & $\mathrm{JU} / \mathrm{/} / 4$ & $<2.0$ & & 1 & $\mathrm{GE}$ & $\begin{array}{l}\mu g / 2 \\
\mu g / L \\
\mu g / L\end{array}$ \\
\hline $\begin{array}{l}\text { Nitrate-nitrite as nitrogen } \\
\text { Selenium, total recoverable } \\
\text { Silver, total recoverable } \\
\text { Thallium, total recoverable } \\
\text { Vanadium, total recoverable } \\
\text { Zinc, total recoverable }\end{array}$ & $\begin{array}{l}13,000 \\
<5.0 \\
<1.0 \\
<0.023 \\
<10 \\
<10\end{array}$ & $\begin{array}{l}\prime \prime \\
U / / \\
U / / \\
J U / / 4 \\
U / / \\
U / /\end{array}$ & $\begin{array}{l}<5.0 \\
<1.0 \\
<2.5 \\
<10 \\
<10\end{array}$ & 0 & $\begin{array}{l}5 \\
1 \\
1 \\
1 \\
1 \\
1\end{array}$ & $\begin{array}{l}\text { GE } \\
\text { GE } \\
\text { GE } \\
\text { GE } \\
\text { GE } \\
\text { GE }\end{array}$ & 13,000 & $/ 1$ & & - & 25 & GE & $\begin{array}{l}\mu g h \\
\mu g / L \\
\mu g / L \\
\mu g h \\
\mu g h\end{array}$ \\
\hline
\end{tabular}

Organic Constituents

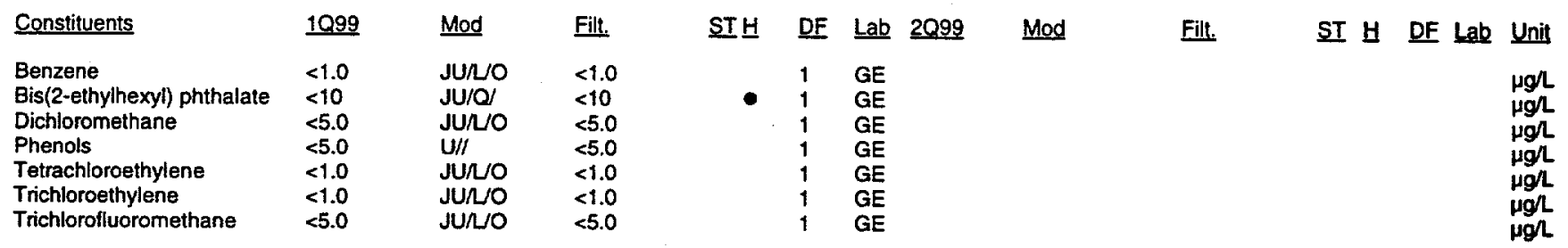

Notes:

- = exceeded holding time

= exceeded groundwater protection or monitoring constituent standard (See Appendix A.)

NA - Not applicable. Applies to beta dose and sum of betas if there are no beta-emitting radionuclides above detection limits; to sum of alphas if there are no alphaemitting radionuclides above detection limits; and to total radium if neither radium-226 or radium-228 was above detection limit

UAZ_UTRA - Upper Aquifer Zone of the Upper Three Runs Aquifer, LAZ UTRA - Lower Aquifer Zone of the Upper Three Runs Aquifer, Gordon - Gordon Aquifer 
WELL FSB $97 A$ (cont.)

Radioactive Constituents

\begin{tabular}{|c|c|c|c|c|c|c|c|c|c|c|c|c|c|}
\hline Constituents & 1099 & Mod & Filt. & SI브 & $\underline{D F}$ & Lab & $\underline{2099}$ & Mod & Filt. & ST 브 & DF & $L a b$ & Unit \\
\hline $\begin{array}{l}\text { Americium-241 } \\
\text { Beta dose }\end{array}$ & $\begin{array}{l}<5.8 E-02 \\
\text { NA }\end{array}$ & $\mathrm{U} / \mathrm{I}$ & $<1.0 E-01$ & & 1 & GP & & & & & & & $\mathrm{pCin}$ \\
\hline Carban-14 & $1.4 E+01$ & $\mathrm{~J} / \mathrm{V}$ & NDD & & 1 & GP & & & & & & & pCin \\
\hline Cesium-137 & $<-3.7 E-01$ & JU/C & $<3.4 \mathrm{E}+\infty$ & & 1 & GP & & & & & & & pCin \\
\hline Cobalt-60 & $<-4.2 E-01$ & $\mathrm{U} / \mathrm{I}$ & $<3.0 E+00$ & & 1 & GP & & & & & & & pCin \\
\hline Curium-242 & $<1.8 \mathrm{E}-02$ & JU/I & $<2.3 E-01$ & & 1 & GP & & & & & & & pCin \\
\hline Curium-243/244 & $<5.0 \mathrm{E}-02$ & JU/I & $<2.1 E-01$ & & 1 & GP & & & & & & & pcin \\
\hline Curium-245/246 & $<0.0 E+00$ & JU/LI & $<1.0 E-01$ & & 1 & GP & & & & & & & $p C i n$ \\
\hline $\begin{array}{l}\text { Gross alpha } \\
\text { lodine-129 }\end{array}$ & $4.0 \mathrm{E}+\infty 0$ & II & & & 1 & GP & $2.9 E+\infty 0$ & $\|$ & & & 1 & GP & $\mathrm{pCin}$ \\
\hline $\begin{array}{l}\text { lodine-129 } \\
\text { Nonvolatile beta }\end{array}$ & $1.2 E+00$ & $J / U$ & NDD & & 1 & GP & & & & & & & pCin \\
\hline $\begin{array}{l}\text { Nonvolatile beta } \\
\text { Plutonium-238 }\end{array}$ & $\begin{array}{l}4.3 \mathrm{E}+\infty 0 \\
<9.8 E-02\end{array}$ & $\mathrm{u} / 1$ & & & 1 & GP & $2.7 E+00$ & $\|$ & & & 1 & GP & pCin \\
\hline $\begin{array}{l}\text { Plutonium-238 } \\
\text { Plutonium-239/240 }\end{array}$ & $\begin{array}{l}<9.8 E-02 \\
<0.0 E+\infty 0\end{array}$ & UII & $\begin{array}{l}<9.8 \mathrm{E}-02 \\
<9.8 \mathrm{E}-02\end{array}$ & & $\begin{array}{l}1 \\
1\end{array}$ & $\begin{array}{l}\text { GP } \\
G P\end{array}$ & & & & & & & $p \operatorname{Cin}$ \\
\hline Radium-226 & $1.9 E+01$ & $" 1$ & & - & $i$ & GP & & & & & & & $\begin{array}{l}\mathrm{pC} \Omega \\
\mathrm{pC} \Omega\end{array}$ \\
\hline $\begin{array}{l}\text { Radium-228 } \\
\text { Total radium }\end{array}$ & $\begin{array}{l}1.2 E+\infty \\
1.9 E+01\end{array}$ & $\mathrm{~J} / \mathrm{V}$ & NDD & & 1 & GP & & & & & & & $\mathrm{pCin}$ \\
\hline Strontium-90 & $<2.3 E-01$ & $\mathrm{U} / \mathrm{I}$ & $<1.3 \mathrm{E}+\infty 0$ & & 1 & GP & & & & & & & pCin \\
\hline Technetium-99 & $<1.9 E+01$ & U/I & $<2.0 E+01$ & & 1 & GP & & & & & & & pCin \\
\hline Thorium-228 & $<3.3 E-02$ & UII & $<1.3 \mathrm{E}-01$ & & 1 & GP & & & & & & & pCin \\
\hline Thorium-230 & $<6.6 \mathrm{E}-02$ & UNI & $<2.2 E-02$ & & 1 & GP & & & & & & & pCir \\
\hline $\begin{array}{l}\text { Thorium-232 } \\
\text { Sum of alphas }\end{array}$ & $\begin{array}{l}<3.8 E-03 \\
N A\end{array}$ & $\mathrm{U} / /$ & $<4.6 \mathrm{E}-02$ & & 1 & GP & & & & & & & $\operatorname{pcin}$ \\
\hline $\begin{array}{l}\text { Sum of alphas } \\
\text { Sum of betas }\end{array}$ & & & & & & & & & & & & & \\
\hline Tritium & $3.5 E+02$ & 11 & & E & 1 & GP & $4.0 E+02$ & "I & & - & 1 & GP & $\mathrm{pCV} / \mathrm{mL}$ \\
\hline Uranium-233/234 & $1.5 \mathrm{E}-01$ & $\mathrm{~J} / \mathrm{N}$ & NDD & & 1 & GP & & & & & & & pCin \\
\hline Uranium-235 & $<6.4 \mathrm{E}-04$ & UII & $<6.6 \mathrm{E}-02$ & & 1 & GP & & & & & & & pCil \\
\hline Uranium-238 & $9.1 E-02$ & $\mathrm{~J} / \mathrm{V}$ & NDD & & 1 & GP & & & & & & & pCin \\
\hline
\end{tabular}

Notes:

- exceeded hoiding time

- = exceeded groundwater protection or monitoring constituent standard (See Appendix A.)

NA - Not applicable. Applies to beta dose and sum of betas if there are no beta-emitting radionuclides above detection limits; to sum of alphas if there are no alpheemitting radionuclides above detection limits; and to total radium if neither radium-226 or radium-228 was above detection limit

UAZ_UTAA - Upper Aquifer Zone of the Upper Three Runs Aquifer, LAZ_UTRA - Lower Aquifer Zone of the Upper Three Runs Aquifer, Gordon - Gordon Aquifer 


\section{WELL FSB 97C}

$\begin{array}{ll}\text { SRS Coord. } & \text { Lat/Longitude } \\ \text { N75179.6 } & 33.273580^{\circ} \mathrm{N} \\ \text { E49970.6 } & 81.681250^{\circ} \mathrm{W}\end{array}$

SAMPLE DATE

$01 / 11 / 99$

Screen Zone Elevation

$153.8-143.8 \mathrm{ft} \mathrm{ms}$

1099

Constituents

Water elevation

$\mathrm{pH}$

Sp. conductance

Water temperature

Alkalinity as $\mathrm{CaCO} 3$

Turbidity

Volumes purged

Sampling code

Synchronous water leve

\section{ANALYTICAL DATA}

Inorganic Constituents
209.2

4.2

820

22.2

0

0.023

$\mathrm{X}$

$207.9(03 / 23 / 99)$
Top of Casing

$286.1 \mathrm{ft} \mathrm{ms}$

$04 / 05 / 99$

$\begin{array}{lll}\text { Casing Pump } & \text { Screen Zone } \\ \text { 4" PVC } & V & \text { LAZ_UTRA }\end{array}$

Unit

ft msl

pH

uSicm

${ }^{\circ} \mathrm{C}$

mgl

well volumes

1.9

$207.9(06 / 25 / 99)$

$\mathrm{ft} \mathrm{msl}$

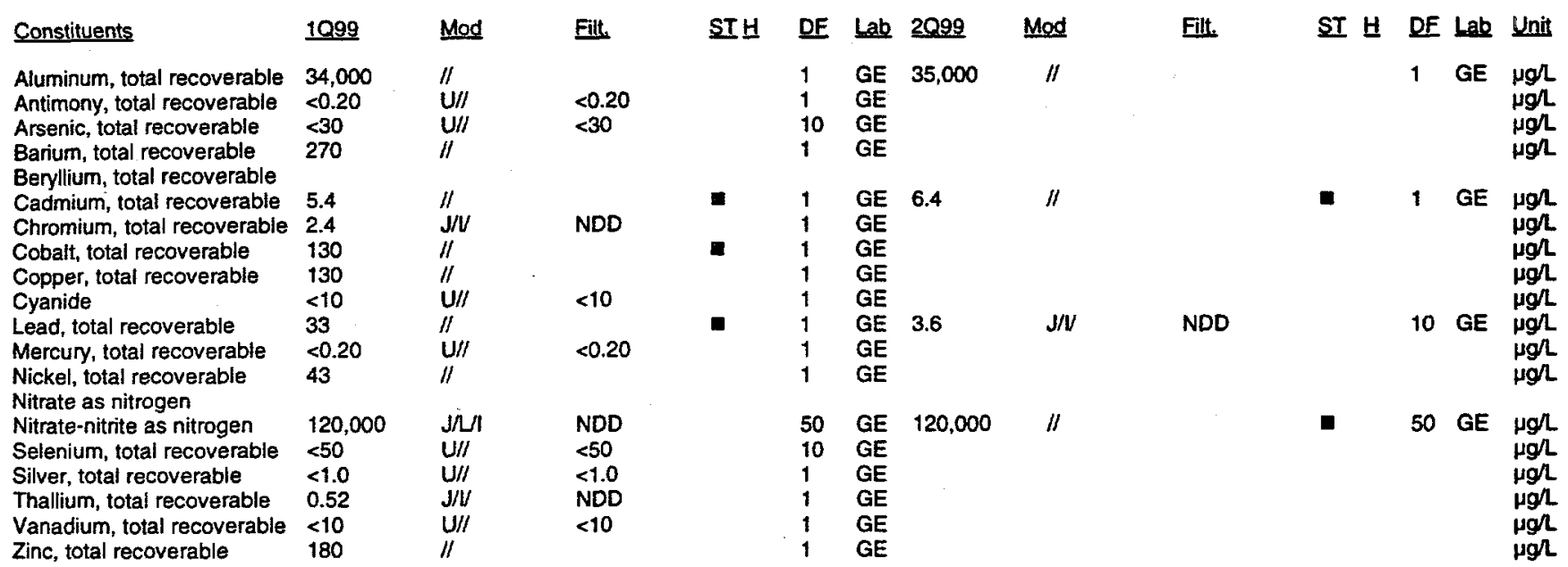

Organic Constituents

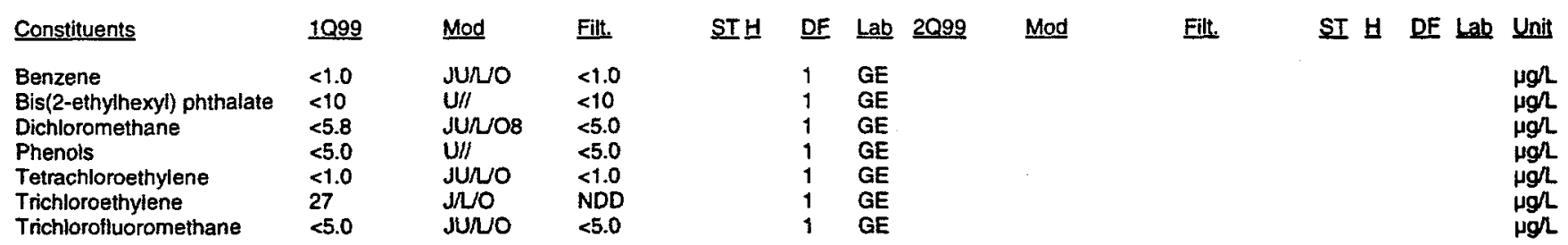

Notes:

- exceeded holding time

= exceeded groundwater protection or monitoring constituent standard (See Appendix A.)

NA - Not applicable. Applies to beta dose and sum of betas if there are no beta-emitting radionuclides above detection limits; to sum of alphas if there are no alphaemitting radionuclides above detection limits; and to total radium if neither radium-226 or radium-228 was above detection limit

UAZ_UTRA - Upper Aquifer Zone of the Upper Three Runs Aquifer; LAZ UTRA - Lower Aquifer Zone of the Upper Three Runs Aquiter, Gordon - Gordon Aquifer 
WELL. FSB $97 \mathrm{C}$ (cont.)

Radioactive Constituents

\begin{tabular}{|c|c|c|c|c|c|c|c|c|c|c|c|c|c|}
\hline Constituents & 1099 & Mod & Fill. & ST브 & $\underline{\mathrm{DF}}$ & Lab & 2099 & Mod & Filt. & SI $H$ & DF & Lab & Unit \\
\hline $\begin{array}{l}\text { Americium-241 } \\
\text { Beta dose }\end{array}$ & $\begin{array}{l}1.2 E+01 \\
63\end{array}$ & $\|$ & & 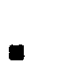 & 1 & GP & & & & & & & pCin \\
\hline Carbon-14 & $4.2 E+01$ & $\mathrm{~J} / \mathrm{Cl}$ & NDD & & 1 & GP & & & & & & & pCin \\
\hline Cesium-137 & $7.4 E+\infty 0$ & $\mathrm{R} / \mathrm{4}$ & & & 1 & GP & & & & & & & pCin \\
\hline Cobalt -60 & $<1.1 E+00$ & $\mathrm{U} / /$ & $<4.5 E+\infty 0$ & & 1 & GP & & & & & & & pein \\
\hline Curium-242 & $<-1.8 E-02$ & $\mathrm{U} / \mathrm{I}$ & $<1.9 E-01$ & & 1 & GP & & & & & & & pCin \\
\hline Curium-243/244 & $7.7 E+\infty 0$ & $/ 1$ & & & 1 & GP & & & & & & & pCin \\
\hline Curium-245/246 & $7.9 E-01$ & $\mathrm{R} / / 4$ & $\mathbf{R e j}_{\mathbf{j}}$ & & 1 & GP & & & & & & & pCin \\
\hline $\begin{array}{l}\text { Gross alpha } \\
\text { lodine-129 }\end{array}$ & $\begin{array}{l}3.9 E+02 \\
4.0 E+01\end{array}$ & J/I & NDD & & $\begin{array}{l}1 \\
1\end{array}$ & $\begin{array}{l}\text { GP } \\
\text { GP }\end{array}$ & $5.0 E+02$ & $\mathrm{~J} / \mathrm{K} \mathbf{I}$ & NDD & & 1 & GP & $\begin{array}{l}\text { pCin } \\
\text { pCir }\end{array}$ \\
\hline Nonvolatile beta & $4.5 E+02$ & $\mathrm{~J} / \mathrm{N} /$ & NDD & & 1 & GP & $5.8 E+02$ & $\mathrm{~J} / \mathrm{K} /$ & NDD & & 1 & GP & pCir \\
\hline Plutonium-238 & $<6.6 \mathrm{E}-02$ & U/I & $<1.8 E-01$ & & 1 & GP & & & & & & & pCin \\
\hline Plutonium-239/240 & $<4.1 E-02$ & $\mathrm{U} / /$ & $<1.0 E-01$ & & 1 & GP & & & & & & & pCil \\
\hline Radium-226 & $2.5 E+01$ & $\mathrm{~J} / \mathrm{\Omega} / \mathrm{I}$ & NDD & & 1 & GP & & & & & & & pCir \\
\hline Radium-228 & $1.4 E+01$ & $/ 1$ & & $\mathbf{E}$ & 1 & GP & & & & & & & pCin \\
\hline Total radium & $1.4 E+01$ & & & & & & & & & & & & \\
\hline Strontium-90 & $1.6 \mathrm{E}+02$ & $/ /$ & & $\mathbf{0}$ & 1 & GP & & & & & & & pCin \\
\hline Technetium-99 & $7.7 E+01$ & $/ 1$ & & $\mathbf{a}$ & 1 & GP & & & & & & & pCin \\
\hline Thorium-228 & $6.5 E-0 t$ & $\mathrm{~J} / \mathrm{V}$ & NDD & & 1 & GP & & & . & & & & $\mathrm{pCin}$ \\
\hline Thorium-230 & 5.0E-01 & $\mathrm{J} / \mathrm{V}$ & NDD & & 1 & GP & & & & & & & pCir \\
\hline Thorium-232 & $<-1.5 E-02$ & $\mathrm{U} / 1$ & $<1.8 E-01$ & & 1 & GP & & & & & & & pcin. \\
\hline Sum of alphas & $3.4 E+02$ & & & $\mathbf{0}$ & & & & & & & & & pCin \\
\hline Sum of betas & $2.9 E+02$ & & & 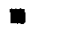 & & & & & & & & & pCin \\
\hline Tritium & 3.7E+03 & $\|$ & & $\mathbf{E}$ & 1 & GP & $4.1 E+03$ & $/ 1$ & & $\mathbf{\square}$ & 1 & GP & pCiml \\
\hline Uranium-233/234 & $1.4 E+02$ & $/ 1$ & & $\mathbf{0}$ & 1 & GP & & & & & & & pcin \\
\hline Uranium-235 & $1.4 E+01$ & $\mathrm{~J} / \mathrm{K} / \mathrm{O}$ & NDD & & 1 & GP & & & & & & & pCin \\
\hline Uranium-238 & $1.8 E+02$ & & & $\mathbf{\square}$ & 1 & GP & & & & & & & pCin. \\
\hline
\end{tabular}

Notes:

- exceeded holding time

= exceeded groundwater protection or monitoring constituent standard (See Appendix A.)

NA - Not applicable. Applies to beta dose and sum of betas if there are no beta-emitting radionuclides above detection limits; to sum of alphas if there are no alphaemitting radionuclides above detection limits; and to total radium if neither radium-226 or radium-228 was above detection limit

UAZ_UTRA - Upper Aquifer Zone of the Upper Three Runs Aquifer, LAZ UTRA - Lower Aquifer Zone of the Upper Three Runs Aquifer, Gordon - Gordon Aquifer 


\section{WELL FSB 97D}

$\begin{array}{lll}\text { SRS Coord. } & \text { LatlLongitude } \\ \text { N75188.9 } & & 33.273609^{\circ} \mathrm{N} \\ \text { E49975.5 } & 81.681255^{\circ} \mathrm{W}\end{array}$

SAMPLE DATE

$01 / 11 / 99$

Screen Zone Elevation

$216.9-196.9 \mathrm{ft} \mathrm{ms}$

1099

211.7

4.4

400

19.9

4

0.21

$\times$

$210.9(03 / 23 / 99)$
Top of Casing

$286 \mathrm{ft} \mathrm{mst}$

$04 / 07 / 99$

2099

210.8

3.8

510

20.5

0.11

$\times$

$210.3(06 / 25 / 99)$

\section{Casing Pump}

4" PVC V
Screen Zone

UAZ_UTRA

Sampling code

Synchronous water level

ANALYTICAL DATA

Inorganic Constituents

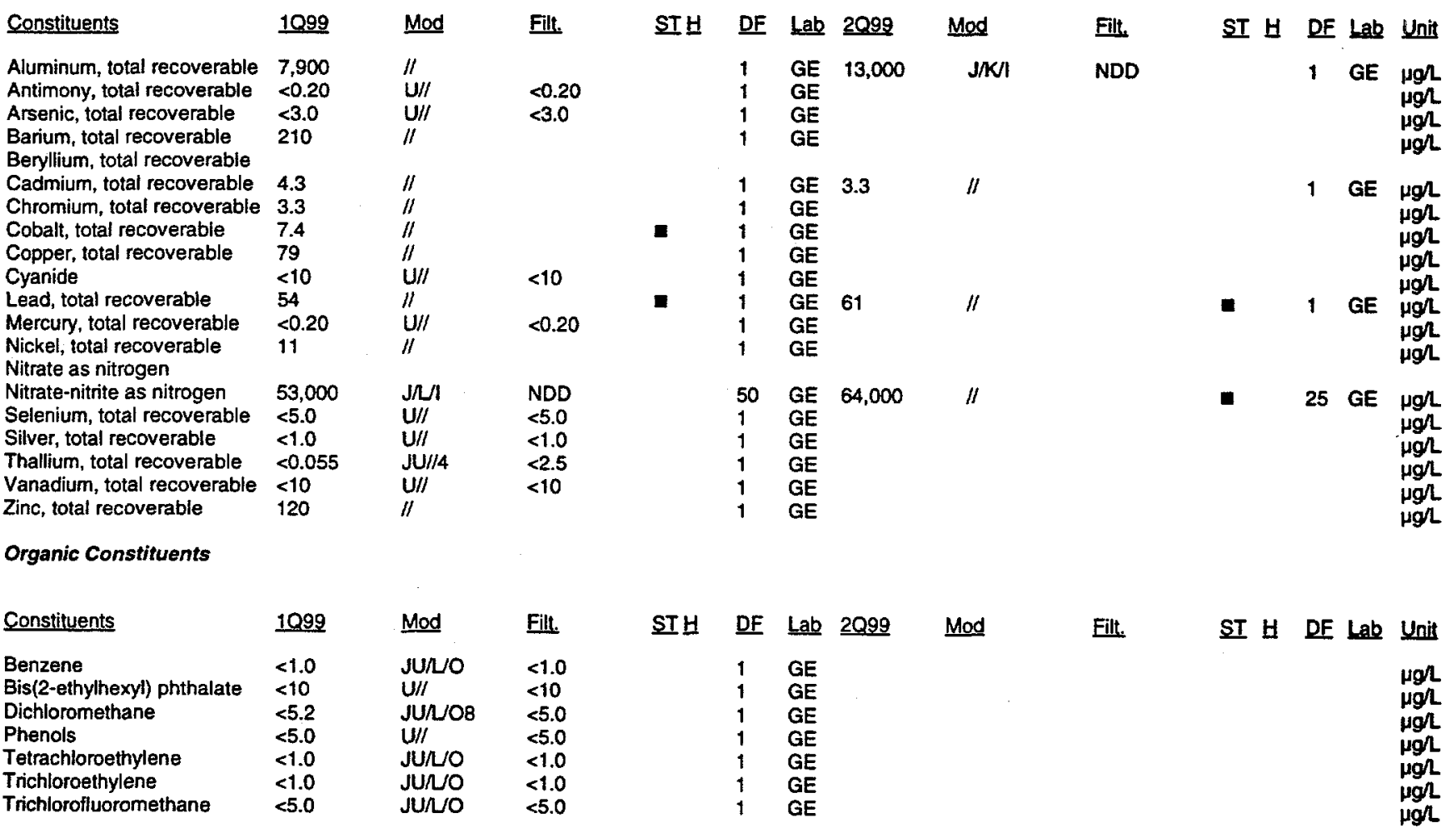

Notes:

- exceeded holding time

= exceeded groundwater protection or monitoring constituent standard (See Appendix A.)

NA - Not applicable. Applies to beta dose and sum of betas if there are no beta-emitting radionuclides above detection limits; to sum of alphas if there are no alphaemitting radionuclides above detection limits; and to total radium if neither radium-226 or radium-228 was above detection limit

UAZ UTRA - Upper Aquifer Zone of the Upper Three Runs Aquifer, LAZ_UTRA - Lower Aquifer Zone of the Upper Three Runs Aquifer, Gordon - Gordon Aquifer 
WELL FSB 97D (cont.)

Radioactive Constituents

\begin{tabular}{|c|c|c|c|c|c|c|c|c|c|c|c|c|c|}
\hline Constituents & 1099 & Mod & Filt. & ST브 & DF & Lab & $\underline{2099}$ & Mod & Filt. & ST $H$ & DF & Lab & Unit \\
\hline Americium-241 & $\begin{array}{l}1.4 E+01 \\
56\end{array}$ & /I & & $\mathbf{q}$ & 1 & GP & & & & & & & pCil \\
\hline $\begin{array}{l}\text { Beta dose } \\
\text { Carbon-14 }\end{array}$ & $\begin{array}{l}56 \\
3.8 E+01\end{array}$ & $\mathrm{~J} / \mathrm{Cl}$ & NDD & & 1 & GP & & & & & & & oCil \\
\hline Cesium-137 & $<3.1 E+00$ & $\mathrm{U} / /$ & $<4.6 \mathrm{E}+00$ & & 1 & GP & & & & & & & pCin \\
\hline Cobalt-60 & $<8.5 E-01$ & $\mathrm{U} / /$ & $<3.8 E+\infty 0$ & & 1 & GP & & & & & & & pCin \\
\hline Curium-242 & $<4.9 E-02$ & $\mathrm{U} / /$ & $<1.6 \mathrm{E}-01$ & & 1 & GP & & & & & & & pCin \\
\hline Curium-243/244 & $1.2 \mathrm{E}+01$ & 11 & & & 1 & GP & & & & & & & pCin \\
\hline Curium-245/246 & 3.1E-01 & $R / / 4$ & Rei & & 1 & GP & & & & & & & pCin \\
\hline $\begin{array}{l}\text { Gross alpha } \\
\text { lodine- } 129\end{array}$ & $\begin{array}{l}5.8 E+02 \\
4.0 E+01\end{array}$ & $\begin{array}{l}\mathrm{J} / \mathrm{I} \\
\mathrm{l}\end{array}$ & NDD & & $\begin{array}{l}1 \\
1\end{array}$ & $\begin{array}{l}\text { GP } \\
\text { GP }\end{array}$ & $4.8 E+02$ & 11 & & = & 1 & GP & $\begin{array}{l}\text { pCin } \\
\text { pCin }\end{array}$ \\
\hline Nonvolatile beta & $4.9 E+02$ & J/UI & NDD & & 1 & GP & $4.8 E+02$ & II & & E & 1 & GP & $\mathrm{pCi} / \mathrm{L}$ \\
\hline Plutonium-238 & $<1.4 E-01$ & $\mathrm{U} / /$ & $<2.3 E-01$ & & 1 & GP & & & & & & & pCill \\
\hline Plutonium-239/240 & $<-8.6 E-03$ & U/l & $<1.1 E-01$ & & 1 & GP & & & & & & & $\mathrm{pCi}$ \\
\hline Radium-226 & 1.2E+01 & $\mathrm{J} / \mathrm{L} / \mathrm{I}$ & NDD & & 1 & GP & & & & & & & pCil \\
\hline Radium-228 & $1.1 E+01$ & 11 & & E & 1 & GP & & & & & & & $\mathrm{pCi} / \mathrm{L}$ \\
\hline Total radium & $1.1 E+01$ & & & & & & & & & & & & \\
\hline Strontium-90 & $1.1 E+02$ & 11 & & a & 1 & GP & & & & & & & pCin \\
\hline Technetium-99 & $3.2 E+01$ & $\mathrm{~J} / \mathrm{V}$ & NDD & & 1 & GP & & & & & & & $\mathrm{pCin}$ \\
\hline Thorium-228 & $4.2 E-01$ & $\mathrm{~J} / \mathrm{V} /$ & NDD & & 1 & GP & & & & & & & pCin \\
\hline Thorium-230 & $<1.4 E-01$ & $\mathrm{U} / /$ & $<2.2 E-01$ & & 1 & GP & & & & & & & $\mathrm{pCi} /$ \\
\hline Thorium-232 & $<0.0 E+00$ & $\mathbf{U} / /$ & $<7.5 E-02$ & & 1 & GP & & & & & & & pCil \\
\hline Sum of alphas & $5.9 E+02$ & & & $\mathbf{z}$ & & & & & & & & & pCil \\
\hline Sum of betas & $1.6 E+02$ & & & ט & & & & & & & & & pcil \\
\hline Tritium & $1.3 E+03$ & 11 & & $\mathbf{v}$ & 1 & GP & $1.4 E+03$ & /1 & & $\mathbf{\square}$ & 1 & GP & pCi/mL \\
\hline Uranium-233/234 & $1.6 E+02$ & $\|$ & & $\mathbf{\square}$ & 1 & GP & & & & & & & $\mathrm{Cin}$ \\
\hline Uranium-235 & $1.9 E+01$ & $\|$ & & 口 & 1 & GP & & & & & & & pCin \\
\hline Uranium-238 & $3.9 E+02$ & /I & & $\mathbf{E}$ & 1 & GP & & & & & & & $\mathrm{Cin}$ \\
\hline
\end{tabular}

Notes:

- exceeded holding time

= exceeded groundwater protection or monitoring constituent standard (See Appendix A)

NA - Not applicable. Applies to beta dose and sum of betas if there are no beta-emitting radionuclides above detection limits; to sum of alphas if there are no alphaemitting radionuclides above detection limits; and to total radium if neither radium-226 or radium-228 was above detection limit

UAZ UTRA - Upper Aquifer Zone of the Upper Three Runs Aquifer, LAZ UTRA - Lower Aquifer Zone of the Upper Three Runs Aquifer Gordon - Gordon Aquifer

F-Area HWMF

C-81

First and Second Quarter 1999 


\section{WELL FSB 98AR}

$\begin{array}{ll}\text { SRS Coord. } & \text { LatlLongitude } \\ \text { N75362.0 } & 33.274204^{\circ} \mathrm{N} \\ \text { E50105.8 } & 81.681249^{\circ} \mathrm{W}\end{array}$

SAMPLE DATE
Screen Zone Elevation

92.1-82.1 $\mathrm{ft} \mathrm{ms}$
Top of Casing

$284 \mathrm{ft}$ msl
Casing Pumo

4" PVC
Screen Zone

Gordon
$04 / 15 / 99$

\begin{tabular}{l}
2099 \\
\hline 151.7 \\
6.8 \\
160 \\
20.5 \\
61 \\
1 \\
3.1
\end{tabular}

3.1

$151.2(06 / 25 / 99)$
Unit

It msl

pH

$\mathrm{HS} / \mathrm{cm}$

${ }^{\circ} \mathrm{C}$

mgh

NTU

well volumes

ft msl

\section{ANALYTICAL DATA}

Inorganic Constituents

Constituents

Aluminum, total recoverable Antimony, total recoverable Arsenic, total recoverable

Barium, total recoverable

Beryllium, total recoverable

Cadmium, total recoverable $<1.0$

Chromium, total recoverable 2.0

Cobalt, total recoverable

Copper, total recoverable

Cyanide

Lead, total recoverable

Mercury, total recoverable

Nickel, total recoverable

Nitrate as nitrogen

Nitrate-nitrite as nitrogen

Selenium, total recoverable

Silver, total recoverable

Thallium, total recoverable

Vanadium, total recoverable

1099

$<0.20$

$<3.0$

$<1.0$
2.0

$<0.078$

0.77

$<10$

1.2

3,500

$<5.0$

$<1.0$

$<0.35$

Zinc, total recoverabie

$<10$

Organic Constituents

\begin{tabular}{llll} 
Constituents & 1099 & Mod & Filt. \\
\cline { 2 - 4 } Benzene & $<1.0$ & $\mathrm{U} / /$ & $<1.0$ \\
Bis(2-ethylhexyl) phthalate & $<9.9$ & $\mathrm{U} / \prime$ & $<9.9$ \\
Dichloromethane & $<7.1$ & $\mathrm{U} / /$ & $<5.0$ \\
Phenols & $<5.0$ & $\mathrm{U} / /$ & $<5.0$ \\
Tetrachloroethylene & $<1.0$ & $\mathrm{U} / /$ & $<1.0$ \\
Trichloroethylene & $<1.0$ & $\mathrm{U} / 1$ & $<1.0$ \\
Trichiorofluoromethane & $<5.0$ & $\mathrm{U} / /$ & $<5.0$
\end{tabular}

Trichiorofluoromethane

\begin{tabular}{|c|c|}
\hline Mod & Fill. \\
\hline \multicolumn{2}{|l|}{ /I } \\
\hline $\mathrm{U} / \mathrm{I}$ & $<0.20$ \\
\hline$U / I$ & $<3.0$ \\
\hline \multicolumn{2}{|l|}{ /I } \\
\hline $\mathrm{U} / /$ & $<1.0$ \\
\hline$J / V$ & NDD \\
\hline UNI & $<0.20$ \\
\hline $\mathrm{J} / \mathrm{U}$ & NDD \\
\hline $\mathrm{U} / /$ & $<10$ \\
\hline U/I & $<2.0$ \\
\hline UNI & $<0.20$ \\
\hline $\mathrm{J} / N$ & NDD \\
\hline \multicolumn{2}{|l|}{ /I } \\
\hline $\mathrm{U} / /$ & $<5.0$ \\
\hline $\mathrm{U} / /$ & $<1.0$ \\
\hline UNI & $<2.5$ \\
\hline $\mathrm{U} / /$ & $<10$ \\
\hline $\mathrm{U} / /$ & $<10$ \\
\hline
\end{tabular}

ST

$\begin{array}{ll}\text { DF } & \text { La } \\ 1 & G \\ 1 & G \\ 1 & G\end{array}$

$$
\text { Lab } 2099
$$

Mod

$\mathrm{U} / 1$

GE

1 GE

1 GE

$1 \mathrm{GE}$

1 GE

1 GE

$<2.0 \quad U / 1 \quad<2.0$

$<1.0$

U/I

GE

$\begin{array}{ll}2 & \mathrm{GE} \\ 1 & \mathrm{GE} \\ 1 & \mathrm{GE}\end{array}$

3,400

GE

GE

1 GE
ST 브 Lab Unit

1 GE $\mu g /$

jgh

$\mu g h$

$\mu g h$

1 GE $\mu g /$

rgl

$\mu g h$

ugl

1 GE

ugh

$2 \mathrm{GE} \mu g / L$

$\mu g /$

$\mu g / 2$

$\mu g / 2$

$\mu g / 2$

$\mu g /$.

STI브

$\begin{array}{ll}\text { DF } & \text { Lab } \\ 1 & \text { GE } \\ 1 & \text { GE } \\ 1 & \text { GE } \\ 1 & \text { GE } \\ 1 & \text { GE } \\ 1 & \text { GE } \\ 1 & \text { GE }\end{array}$

Filt.

ST H DF Lab Unit

$\mu g /$

pgl

$\mu g /$

$\mu g / 2$

$\mu g /$

$\mu g h$.

ugl

Notes:

- exceeded holding time

- = exceeded groundwater protection or monitoring constituent standard (See Appendix A.)

NA - Not applicable. Applies to beta dose and sum of betas if there are no beta-emiting radionuclides above detection limits; to sum of alphas if there are no alphaemitting radionuclides above detection limits; and to total radium if neither radium-226 or radium-228 was above detection limit

UAZ_UTRA - Upper Aquifer Zone of the Upper Three Runs Aquifer, LAZ UTRA - Lower Aquifer Zone of the Upper Three Runs Aquifer, Gordon - Gordon Aquifer 


\section{WELL FSB 98AR (cont.)}

Radioactive Constituents

\begin{tabular}{|c|c|c|c|c|c|c|c|c|c|c|c|c|c|}
\hline Constituents & 1099 & Mod & Filt. & ST브 & $\underline{D F}$ & LLab & 2099 & Mod & Filt. & ST 브 & DF & Lab & Unit \\
\hline $\begin{array}{l}\text { Americium-241 } \\
\text { Beta dose }\end{array}$ & $\begin{array}{l}<-9.3 E-03 \\
\text { NA }\end{array}$ & JU/LV/I & $<6.0 \mathrm{E}-02$ & & 1 & GP & & & & & & & $\mathrm{pCi}$ \\
\hline Carbon-14 & $<2.4 E+\infty$ & $\mathrm{U} / /$ & $<7.2 E+\infty$ & & 1 & GP & & & & & & & pCil \\
\hline Cesium-137 & $<-1.9 \mathrm{E}-01$ & $\mathrm{U} / /$ & $<3.0 \mathrm{E}+00$ & & 1 & GP & & & & & & & pCin \\
\hline Cobalt-60 & $<-4.3 E-01$ & $U / I$ & $<3.5 E+00$ & & 1 & GP & & & & & & & pCin \\
\hline Curium-242 & $<0.0 E+\infty$ & $U / /$ & $<6.2 \mathrm{E}-02$ & & 1 & GP & & & & & & & $\mathrm{pCi} / \mathrm{L}$ \\
\hline Curium-243/244 & $<0.0 \mathrm{E}+00$ & $U / I$ & $<6.0 \mathrm{E}-02$ & & 1 & GP & & & & & & & pCin \\
\hline Curium-245/246 & $<2.0 \mathrm{E}-02$ & $U / /$ & $<6.0 \mathrm{E}-02$ & & 1 & GP & & & & & & & pCil \\
\hline Gross alpha & $<4.1 E-01$ & $U / I$ & $<7.9 E-01$ & & 1 & GP & $1.6 E+\infty$ & $\mathrm{J} / \mathrm{KK} / \mathrm{C}$ & NDD & & 1 & TM & $\mathrm{pCin}$ \\
\hline Nonvolatile beta & $2.6 \mathrm{E}+\infty 0$ & $\begin{array}{l}\mathrm{R} / \mathrm{4} \\
\mathrm{J} / \mathrm{J}\end{array}$ & $\begin{array}{l}\text { Rej } \\
\text { NDD }\end{array}$ & & 1 & GP & $5.8 E+\infty$ & /I & & & 1 & $\mathrm{TM}$ & pCin \\
\hline Plutonium-238 & $<1.0 \mathrm{E}-01$ & $\mathrm{U} / /$ & $<2.0 E-01$ & & 1 & $\mathrm{GP}$ & & & & & & & pCin \\
\hline Plutonium-239/240 & $<7.2 E-03$ & $\mathrm{U} / \%$ & $<9.1 E-02$ & & 1 & GP & & & & & & & $\mathrm{pCir}$ \\
\hline Radium-226 & 4.3E-01 & $\mathrm{J} / \mathrm{V}$ & NDD & & 1 & GP & & & & & & & $\mathrm{pCin}$ \\
\hline Radium-228 & $<4.0 E-01$ & $\mathrm{U} / I$ & $<1.1 E+00$ & & 1 & GP & & & & & & & pCin \\
\hline Total radium & NA & & & & & & & & & & & & \\
\hline Strontium-90 & $<-2.7 E-01$ & $\mathrm{U} / /$ & $<1.2 E+00$ & & 1 & GP & & & & & & & $\mathrm{pCi} /$ \\
\hline Technetium-99 & $<9.3 E+\infty$ & $U / /$ & $<1.8 E+01$ & & 1 & GP & & & & & & & $\mathrm{pCin}$ \\
\hline Thorium-228 & 2.5E-01 & $\mathrm{J} / \mathrm{V}$ & NDD & & 1 & GP & & & & & & & pCil \\
\hline Thorium-230 & $<3.9 \mathrm{E}-02$ & $\mathrm{U} / /$ & $<7.0 E-02$ & & 1 & GP & & & & & & & pCir \\
\hline Thorium-232 & $<0.0 E+\infty$ & $\mathrm{U} / /$ & $<3.3 E-02$ & & 1 & GP & & & & & & & pCill \\
\hline Sum of alphas & NA & & & & & & & & & & & & \\
\hline Sum of betas & NA & & & & & & & & & & & & \\
\hline Tritium & $5.2 E+01$ & $\|$ & & 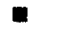 & 1 & GP & 4.7E+01 & $H$ & & $\mathbf{n}$ & 1 & GP & pCiml \\
\hline Uranium-233/234 & $<1.9 E-02$ & $\mathrm{U} / /$ & $<3.5 E-01$ & & 1 & GP & & & & & & & pCin \\
\hline Uranium-235 & $<-9.3 E-05$ & $U / /$ & $<7.1 E-02$ & & 1 & GP & & & & & & & pCin \\
\hline Uranium-238 & $<4.5 \mathrm{E}-03$ & $\mathrm{U} / /$ & $<8.1 \mathrm{E}-02$ & & 1 & GP & & & & & & & $\mathrm{pCil}$ \\
\hline
\end{tabular}

Notes:

- exceeded holding time

- = exceeded groundwater protection or monitoring constituent standard (See Appendix A.)

NA - Not applicable. Applies to beta dose and sum of betas if there are no beta-emitting radionuclides above detection limits; to sum of alphas if there are no alphaemitting radionuclides above detection limits; and to total radium if neither radium-226 or radium-228 was above detection limit

UAZ UTRA - Upper Aquifer Zone of the Upper Three Runs Aquifer: LAZ UTRA - Lower Aquifer Zone of the Upper Three Runs Aquiter, Gordon - Gordon Aquifer 


\section{WELL FSB 98C}

$\begin{array}{ll}\text { SRS Coord. } & \text { Lat/Longitude } \\ \text { N75381.2 } & \\ \text { E50116.5 } & 33.274264^{\circ} \mathrm{N} \\ & 81.681258^{\circ} \mathrm{W}\end{array}$

SAMPLE DATE

$01 / 11 / 99$

Screen Zone Elevation

158.4-148.4 $\mathrm{ft} \mathrm{msl}$
Top of Casing

$284.5 \mathrm{ft} \mathrm{ms}$
Casing

4" PVC
Screen Zone

LAZ_UTRA

$05 / 12 / 99$

\section{FIELD DATA}

Constiluents

Water elevation

$\mathrm{pH}$

Sp. conductance

Water temperature

Alkalinity as $\mathrm{CaCO} 3$

Turbidity

Volumes purged

Sampling code

Synchronous water level

\section{ANALYTICAL DATA}

1099

210.1

3.2

660

20.1

2.9

$206.1(03 / 23 / 99)$
2099

209.8

3.4

22.4
0

0

2.7

$208.7(06 / 25 / 99)$
Unit

ft mst

pH

$\mathrm{S} / \mathrm{cm}$

${ }^{\circ} \mathrm{C}$

mgll

well volumes

ft msl

Inorganic Constituents

Constituents

Aluminum, total recoverable Antimony, total recoverable Arsenic, total recoverable

Barium, total recoverable

Beryllium, total recoverable

Cadmium, total recoverable $\mathbf{5 . 5}$

Chromium, total recoverable 0.71

Cobalt, total recoverable

Copper, total recoverable

Cyanide

Lead, total recoverable

Mercury, total recoverable

Nickel, total recoverable

Nitrate as nitrogen

Nitrate-nitrite as nitrogen $\quad 65,000$

Selenium, total recoverable $<50$

Silver, total recoverable

Thallium, total recoverable 0.46

Vanadium, total recoverable $<10$

Zinc, total recoverable

1099

Mod

/l

16,000
$<0.20$

$<30$

130
27

$<10$

0.38

$<0.20$

21

$<50$

0.46
$<10$

71

Organic Constituents

Constituents
Benzene
Bis(2-ethylhexyl) phthalate
Dichloromethane
Phenols
Tetrachloroethylene
Trichloroethylene
Trichlorofluoromethane

1099
$<1.0$
$<10$
$<4.2$
$<5.0$
$<1.0$
54
$<5.0$

II

"II

U/I

$\mathrm{J} / \mathrm{V}$

II

$\mathrm{U} / / /$

$\mathrm{J} / \mathrm{V}$

U/I

$\begin{array}{ll}\text { Mod } & \text { Filt. } \\ \text { JUAO } & <1.0 \\ \text { U/I } & <10 \\ \text { JU/UO8 } & <5.0 \\ \text { U/I } & <5.0 \\ \text { JUINO } & <1.0 \\ \text { JUNO } & \text { NDD } \\ \text { JUAO } & <5.0\end{array}$

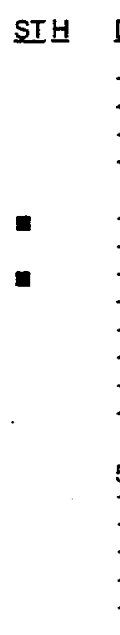

$\begin{array}{llll}\text { DF } & \text { Lab } & 2099 & \text { Mod } \\ 1 & \text { GE } & 16,000 & / 1 \\ 1 & \text { GE } & & \\ 10 & \text { GE } & \\ 1 & \text { GE } & & \end{array}$

Mod

$<0.20$

UII $\quad<30$

JN N NDD

$\mathrm{J} / \mathrm{L} / \mathrm{I}$

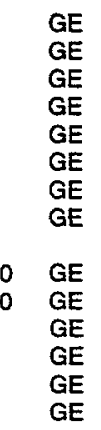

STH $\begin{array}{cc}\text { DF } & \text { Lab } \\ 1 & \mathrm{GE} \\ 1 & \mathrm{GE} \\ 1 & \mathrm{GE} \\ 1 & \mathrm{GE} \\ 1 & \mathrm{GE} \\ 1 & \mathrm{GE} \\ 1 & \mathrm{GE}\end{array}$

$6.2 \quad 1$

$<2.0 \quad U$

59,000

NDD

$<1.0$

NDD
Filt.

ST H DF Lab Unit

1 GE $\mu g /$

igl

$\mu g /$

$<2.0$
$2099 \quad$ Mod

Filt.

SI H DE Lab Unit

Hgh.

pgh

$\mu g h$.

pgl.

pgl

ugh

ugh.

Notes:

- exceeded holding time

= exceeded groundwater protection or monitoring constituent standard (See Appendix A.)

NA - Not applicable. Applies to beta dose and sum of betas if there are no beta-emitting radionuclides above detection limits; to sum of alphas if there are no alphaemitting radionuclides above detection limits; and to total radium if neither radium-226 or radium-228 was above detection limit

UAZ_UTRA - Upper Aquifer Zone of the Upper Three Runs Aquifer, LAZ UTRA - Lower Aquifer Zone of the Upper Three Runs Aquifer, Gordon - Gordon Aquifer 


\section{WELL FSB 98C (cont.)}

Radioactive Constituents

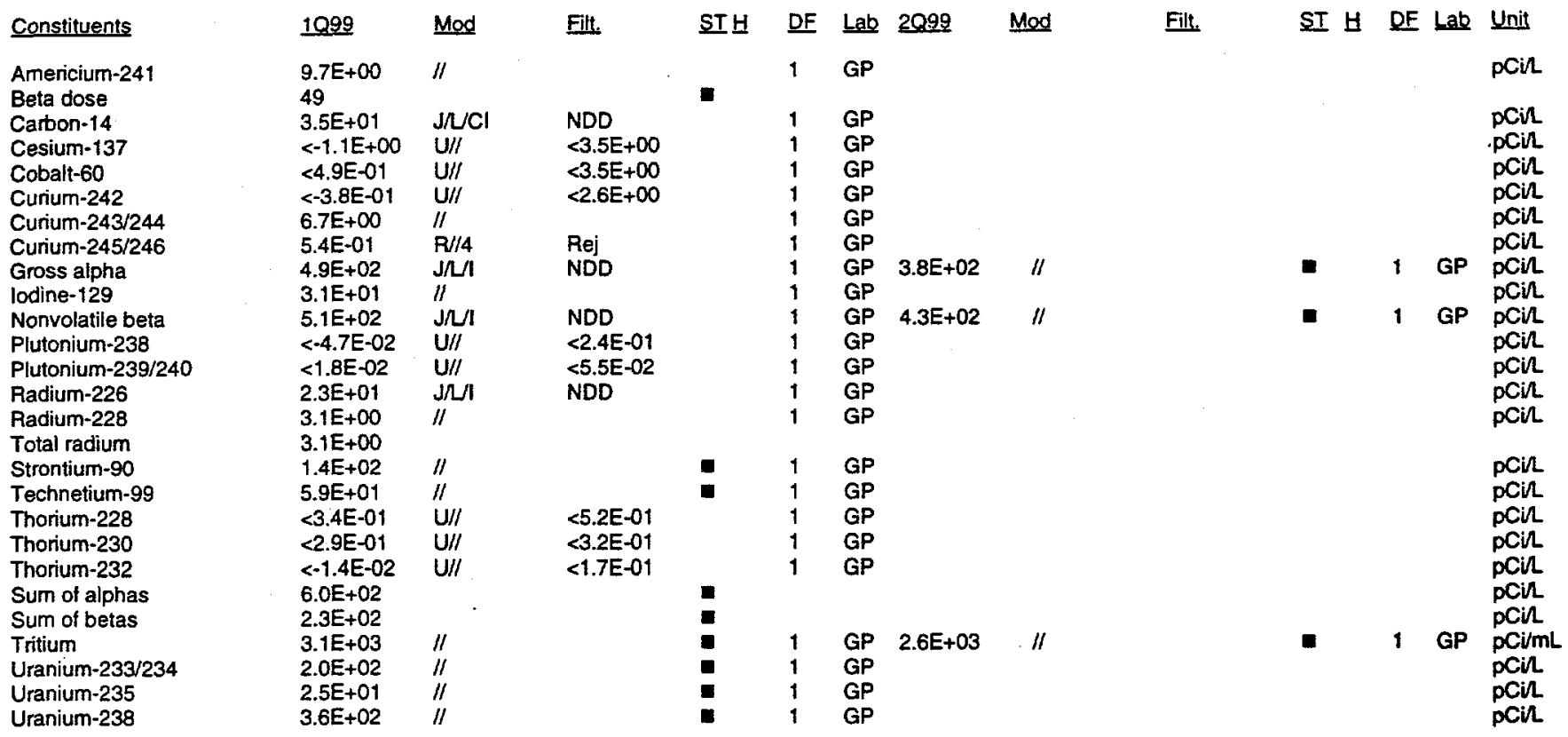

Notes:

- = exceeded holding time

= exceeded groundwater protection or monitoring constituent standard (See Appendix A.)

NA - Not applicable. Applies to beta dose and sum of betas if there are no beta-emitting radionuclides above detection limits; to sum of alphas if there are no alphaemitting radionuclides above detection limits; and to total radium if neither radium-226 or radium-22B was above detection limit

UAZ_UTRA - Upper Aquifer Zone of the Upper Three Runs Aquifer, LAZ_UTRA - Lower Aquifer Zone of the Upper Three Runs Aquifer, Gordon - Gordon Aquifer

F-Area HWMF 
WELL FSB 98D

$\begin{array}{ll}\text { SRS Coord. } & \text { LatlLongitude } \\ \text { N75371.9 } & 33.274235^{\circ} \mathrm{N} \\ \text { E50111.6 } & 81.681253^{\circ} \mathrm{W}\end{array}$

SAMPLE DATE
Screen Zone Elevation

220.3-200.3 ft ms!

Top of Casing

$284.5 \mathrm{ft} \mathrm{msl}$

05/04/99

\section{EIELD DATA}

Constituents

Water elevation

$\mathrm{pH}$

Sp. canductance

Water temperature

Alkalinity as $\mathrm{CaCO} 3$

Turbidity

Volumes purged

Sampling code

Synchronous water level

\section{ANALYTICAL DATA}

Inorganic Constituents
1099

212.9

3.6

670

19.1

0

0.12

$\mathrm{X}$

209.5 (03/23/99) $\underline{2099}$

212.7

6.0

20.0

46

0.12

$\mathrm{x}$

$211.8(06 / 25 / 99)$
Casing Pump

4. PVC V
Screen Zone

UAZ UTRA

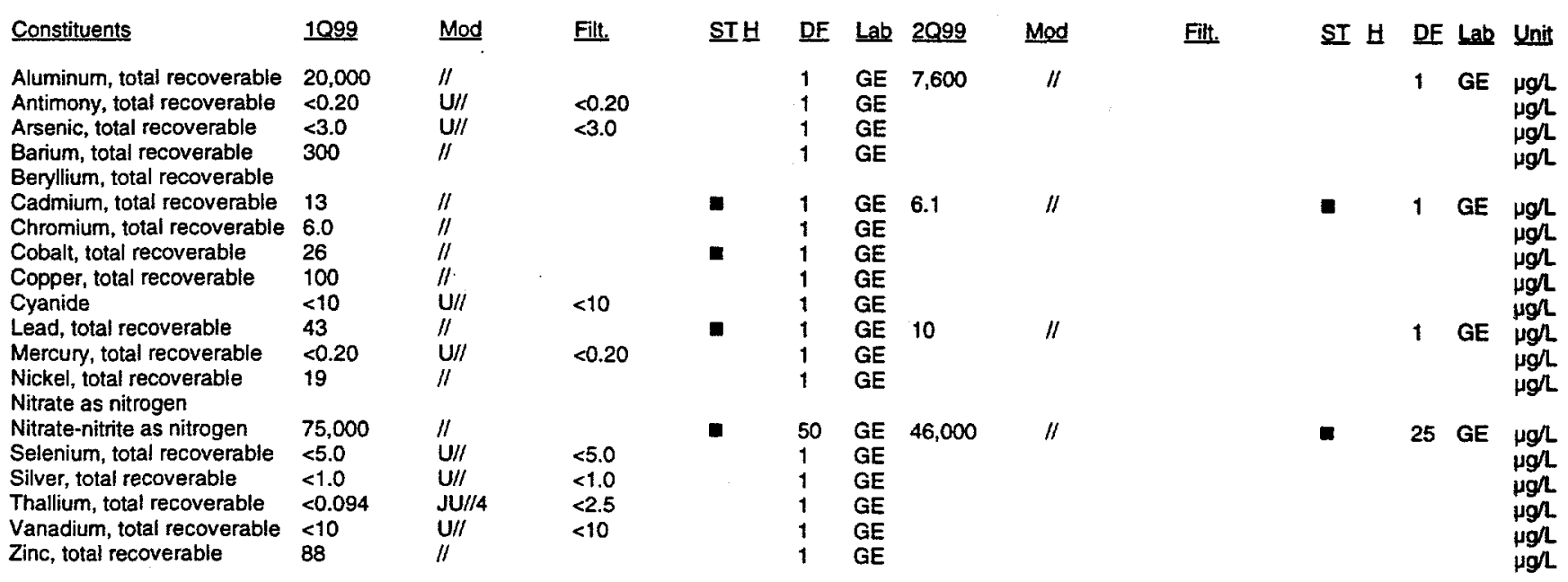

Organic Constituents

\begin{tabular}{|c|c|c|c|c|c|c|c|c|c|c|}
\hline Constituents & 1099 & Mod & Filt. & ST & DF & $\underline{\text { Lab } \quad 2099}$ & Mod & Fill. & DF Lab & Unit \\
\hline Benzene & $<1.0$ & JU/LO & $<1.0$ & & 1 & GE & & & & \\
\hline Bis(2-ethylhexyl) phthalate & $<10$ & JU/Q & $<10$ & - & 1 & $\overline{G E}$ & & & & \\
\hline Dichloromethane & $<5.0$ & JUルO & $<5.0$ & & 1 & GE & & & & \\
\hline Phenols & $<5.0$ & & $<5.0$ & & 1 & $\overline{G E}$ & & & & \\
\hline Tetrachloroethylene & $<1.0$ & JUR/O & $<1.0$ & & 1 & GE & & & & \\
\hline Trichloroethylene & $<1.0$ & JUนOO & $<1.0$ & & 1 & GE & & & & \\
\hline Trichlorofluoromethane & $<5.0$ & JUL/O & $<5.0$ & & 1 & $\mathrm{GE}$ & & & & \\
\hline
\end{tabular}

Notes:

= exceeded holding time

- exceeded groundwater protection or monitoring constituent standard (See Appendix A.)

NA - Not applicable. Applies to beta dose and sum of betas if there are no beta-emitting radionuclides above detection limits; to sum of alphas if there are no alphaemitting radionuclides above detection limits; and to total radium if neither radium-226 or radium-228 was above detection limit

UAZ_UTRA - Upper Aquifer Zone of the Upper Three Runs Aquifer, LAZ UTRA - Lower Aquifer Zone ol the Upper Three Runs Aquifer, Gordon - Gordon Aquifer 
WELL FSB 98D (cont.)

Radioactive Constituents

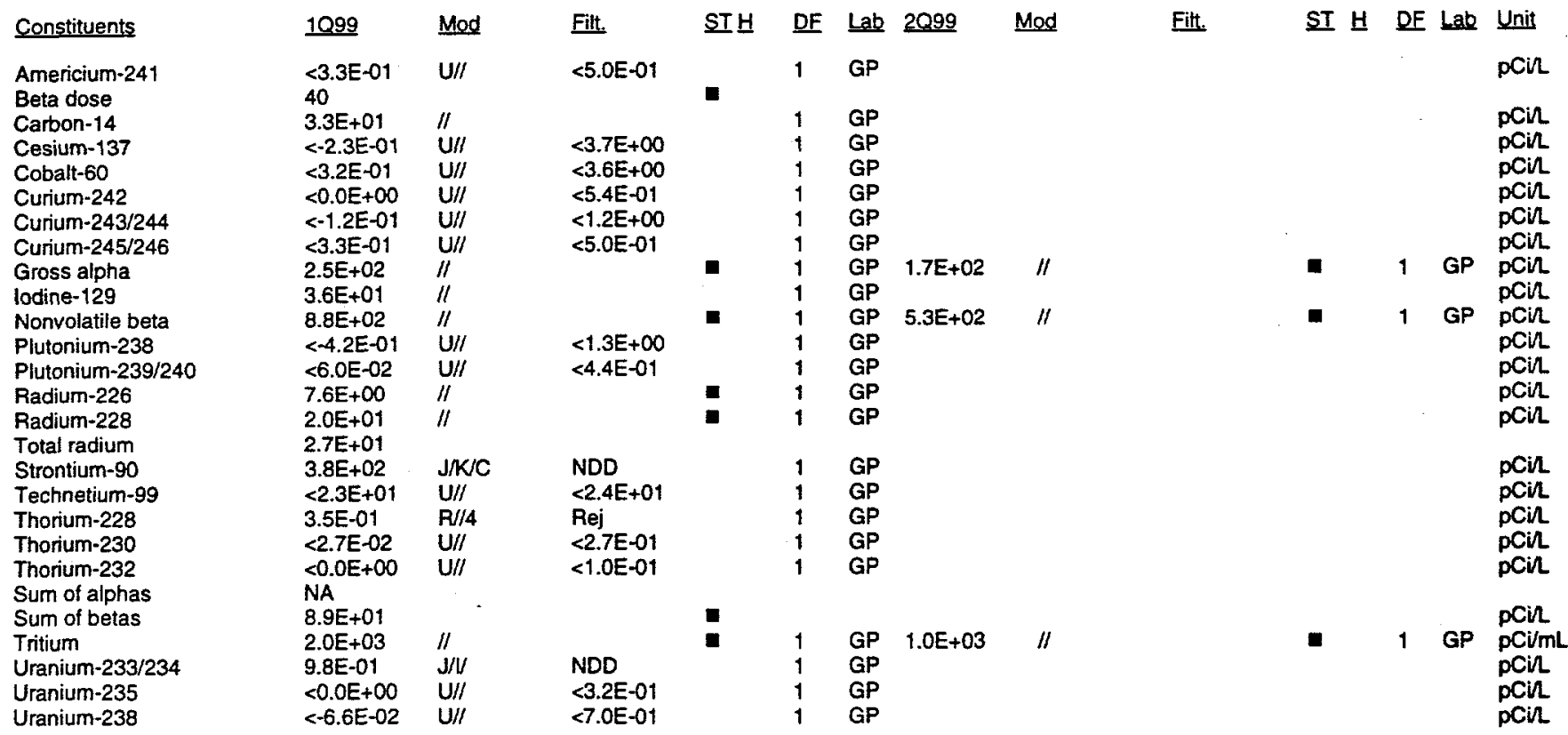




\section{WELL FSB 99A}

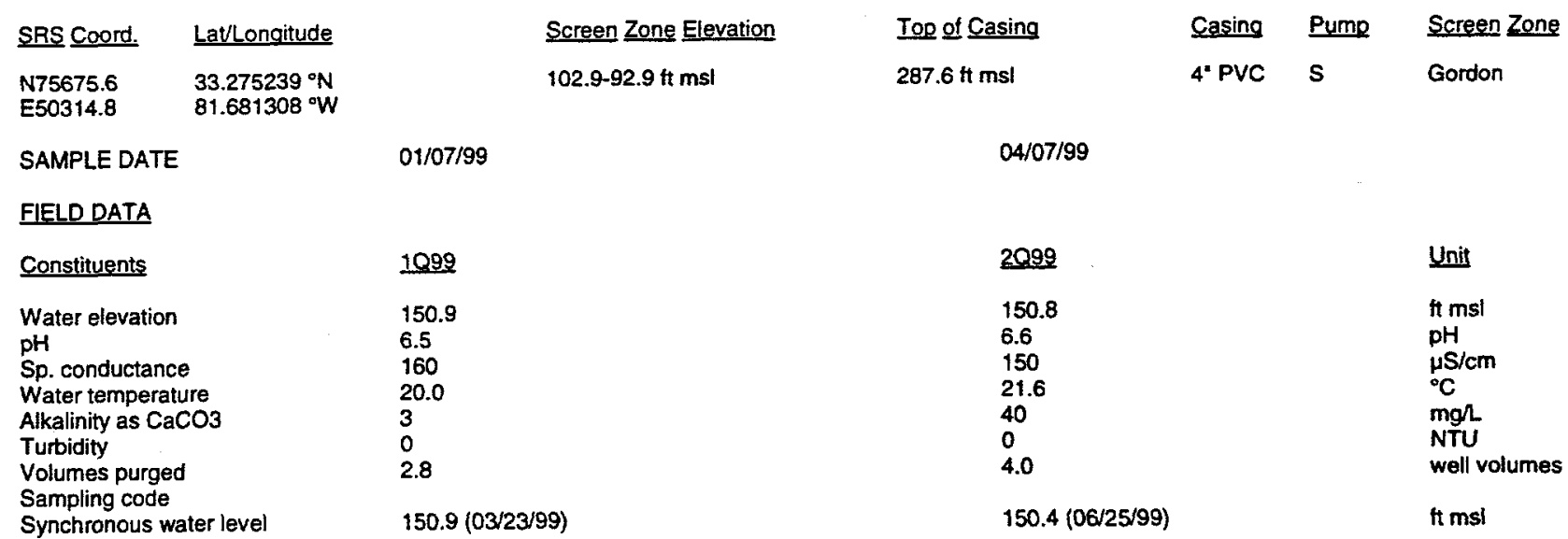

ANALYTICAL DATA

Inorganic Constituents

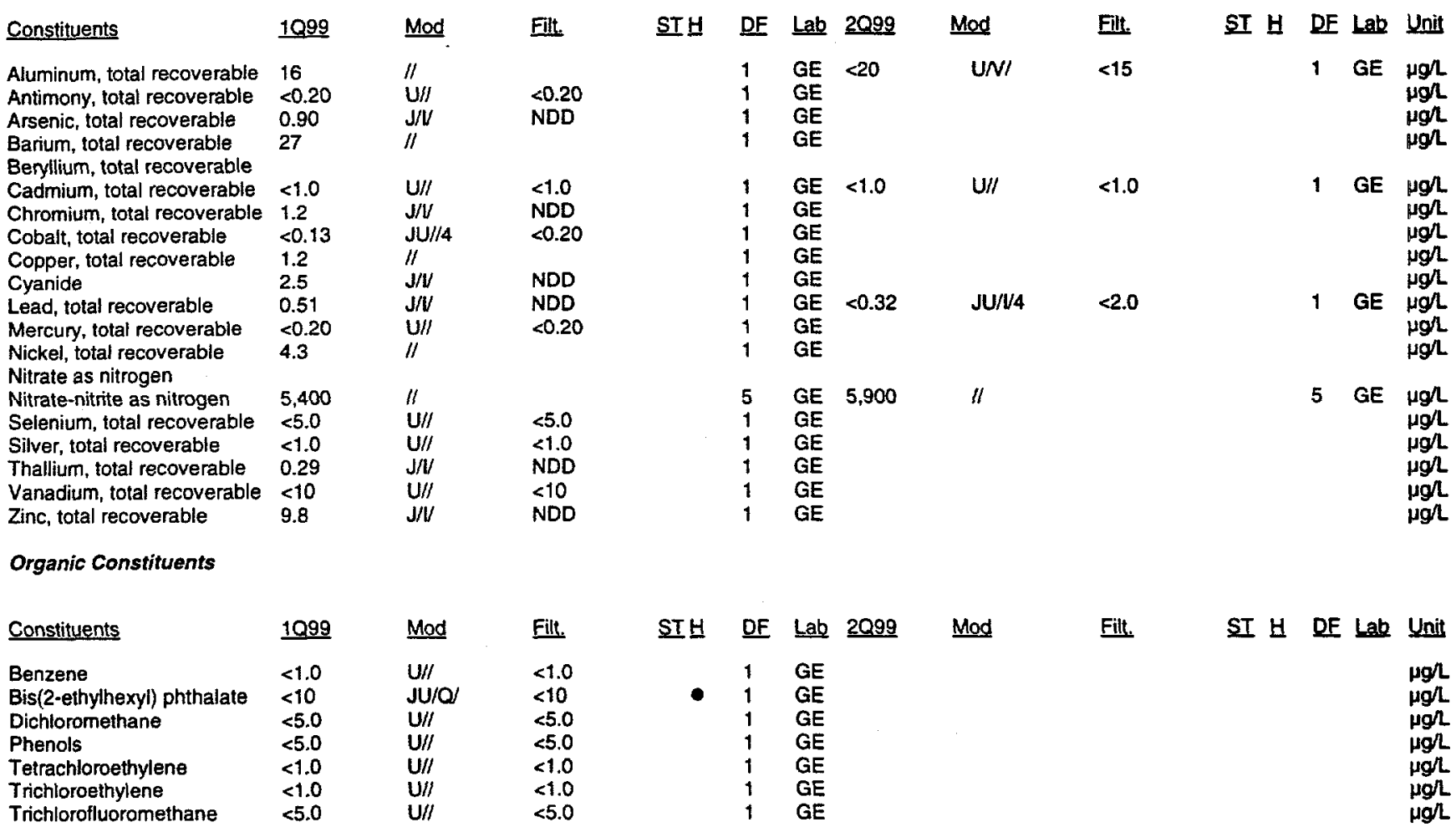

Notes:

- exceeded holding time

- = exceeded groundwater protection or monitoring constituent standard (See Appendix A.)

NA - Not applicable. Applies to beta dose and sum of betas if there are no beta-emitting radionuclides above detection limits; to sum of alphas if there are no alphaemitting radionuclides above detection limits; and to total radium if neither radium-226 or radium-228 was above detection limit

UAZ_UTRA - Upper Aquifer Zone of the Upper Three Runs Aquifer; LAZ_UTRA - Lower Aquifer Zone of the Upper Three Runs Aquifer, Gordon - Gordon Aquifer 


\section{WELL FSB 99A (cont.)}

Radioactive Constituents

\begin{tabular}{|c|c|c|c|c|c|c|c|c|c|c|c|c|c|}
\hline Constituents & 1099 & Mod & Filt. & ST브 & $\underline{D F}$ & Lab & $\underline{2099}$ & Mod & Filt. & ST $\underline{H}$ & DF & Lab & Unit \\
\hline Americium-241 & $\begin{array}{l}<-3.8 E-02 \\
N A\end{array}$ & JUルI & $<4.2 E-01$ & & 1 & GP & & & & & & & pCin \\
\hline $\begin{array}{l}\text { Beta dose } \\
\text { Carbon-14 }\end{array}$ & $9.8 \mathrm{E}+00$ & $\mathrm{~J} / \mathrm{V}$ & NDD & & 1 & GP & & & & & & & pCin \\
\hline Cesium-137 & $<-9.2 E-01$ & JU/C & $<3.3 E+\infty$ & & 1 & GP & & & & & & & pCir \\
\hline Cobalt- 60 & $<5.9 E-01$ & $\mathrm{U} / 1$ & $<3.5 E+\infty$ & & 1 & GP & & & & & & & pCin \\
\hline Curium-242 & $<-4.0 E-02$ & $\mathbf{U} / \prime$ & $<5.2 E-01$ & & 1 & GP & & & & & & & $\mathrm{pCi} / \mathrm{L}$ \\
\hline Curium-243/244 & $<-3.8 E-02$ & U/I & $<5.0 E-01$ & & 1 & GP & & & & & & & pCin \\
\hline Curium-245/246 & $<0.0 \mathrm{E}+00$ & U// & $<2.4 E-01$ & & 1 & GP & & & & & & & pCin \\
\hline Gross alpha & $\begin{array}{l}1.3 E+00 \\
<8.8 E-02\end{array}$ & $\begin{array}{l}\mathrm{J} / \mathrm{V} \\
\mathrm{U} / /\end{array}$ & $\begin{array}{l}\text { NDD } \\
<7.2 E-01\end{array}$ & & $\begin{array}{l}1 \\
1\end{array}$ & $\begin{array}{l}\text { GP } \\
\text { GP }\end{array}$ & $1.1 E+\infty$ & $\mathrm{J} / \mathrm{V}$ & NDD & & 1 & GP & $\begin{array}{l}\text { pCin } \\
\text { pCin }\end{array}$ \\
\hline Nonvolatile beta & $2.5 E+00$ & /I & & & 1 & GP & $1.7 E+\infty$ & $/ 1$ & & & 1 & GP & pCir \\
\hline Plutonium-238 & $<-7.0 E-02$ & $\mathrm{U} / \mathrm{\prime}$ & $<2.6 E-01$ & & 1 & GP & & & & & & & $\mathrm{pCi}$ \\
\hline Plutonium-239/240 & $<1.7 E-02$ & UII & $<5.2 E-02$ & & 1 & GP & & & & & & & pCin \\
\hline Radium-226 & 5.3E-01 & $\mathrm{J} / \mathrm{V}$ & NDD & & 1 & GP & & & & & & & pCin \\
\hline Radium-228 & $\begin{array}{l}9.1 \text { E-01 } \\
\text { NA }\end{array}$ & $\mathrm{J} / \mathrm{N}$ & NDD & & 1 & GP & & & & & & & $\mathrm{pCi}$ \\
\hline Strontium-90 & $<9.4 E-02$ & $\mathrm{U} / /$ & $<1.5 E+\infty$ & & 1 & GP & & & & & & & pCir \\
\hline Technetium-99 & $<1.4 E+01$ & $\mathrm{U} / 1$ & $<2.2 \mathrm{E}+01$ & & 1 & GP & & & & & & & pCir \\
\hline Thorium-228 & $<-1.3 E-02$ & $\mathrm{U} / /$ & $<4.2 E-01$ & & 1 & GP & & & & & & & pCin \\
\hline Thorium-230 & 2.2E-01 & $\mathrm{R} / / 4$ & Rej & & 1 & GP & & & & & & & pCir \\
\hline Thorium-232 & $<-3.4 E-02$ & $\mathrm{U} / \prime$ & $<2.7 E-01$ & & 1 & GP & & & & & & & pCir \\
\hline Sum of alphas & NA & & & & & & & & & & & & \\
\hline Sum of betas & NA & & & & & & & & & & & & \\
\hline Tritium & $1.6 E+02$ & $/ /$ & & $\mathbf{E}$ & 1 & GP & 1.7E+02 & $\|$ & & 口 & 1 & GP & $\mathrm{pCi} / \mathrm{m}$ \\
\hline Uranium-233/234 & $<1.9 E-01$ & JU/C & $<2.4 E-01$ & & 1 & GP & & & & & & & pCil \\
\hline Uranium-235 & $<4.0 E-02$ & JUAC & $<2.2 E-01$ & & 1 & GP & & & & & & & pCin \\
\hline Uranium-238 & $<8.8 \mathrm{E}-03$ & JU/C & $<2.2 E-01$ & & 1 & GP & & & & & & & pCin \\
\hline
\end{tabular}

= exceeded holding time

= exceeded groundwater protection or monitoring constituent standard (See Appendix A.)

NA - Not applicable. Applies to beta dose and sum of betas it there are no beta-emitting radionuclides above detection limits; to sum of alphas if there are no alphaemitting radionuclides above detection limits; and to total radium if neither radium-226 or radium-228 was above detection limit

UAZ UTRA - Upper Aquifer Zone of the Upper Three Runs Aquifer, LAZ UTRA - Lower Aquifer Zone of the Upper Three Runs Aquifer, Gordon - Gordon Aquifer 


\section{WELL FSB 99C}

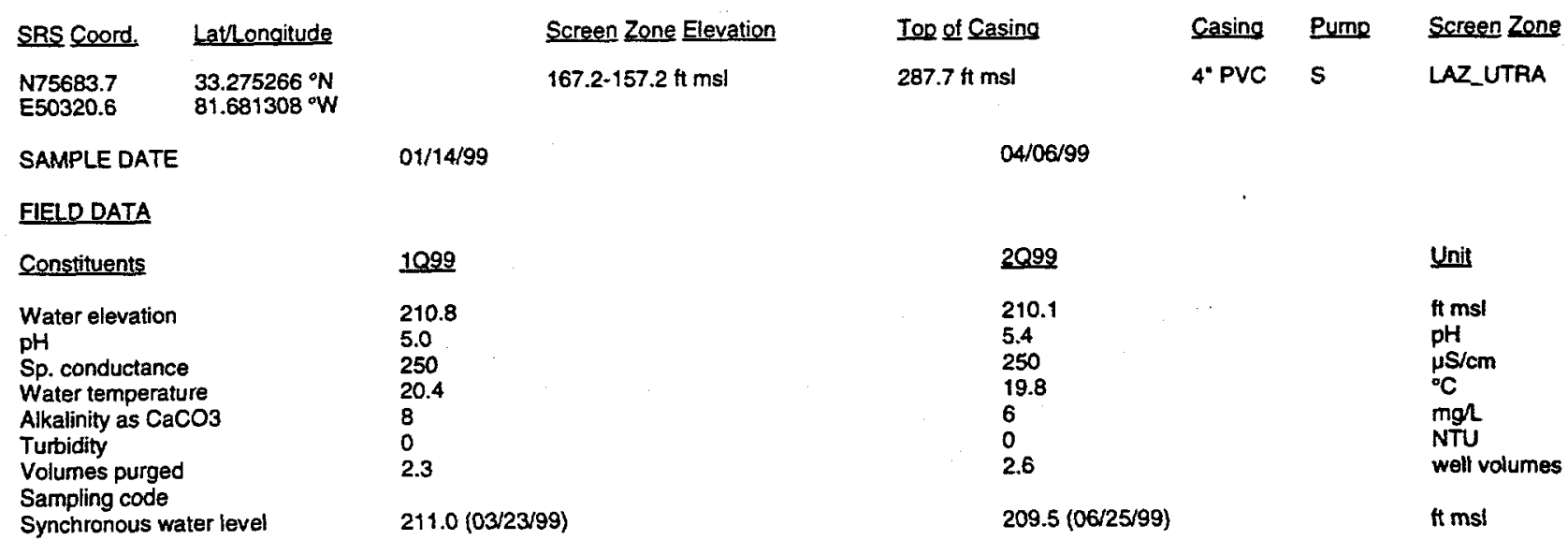

\section{ANALYTICAL DATA}

Inorganic Constituents

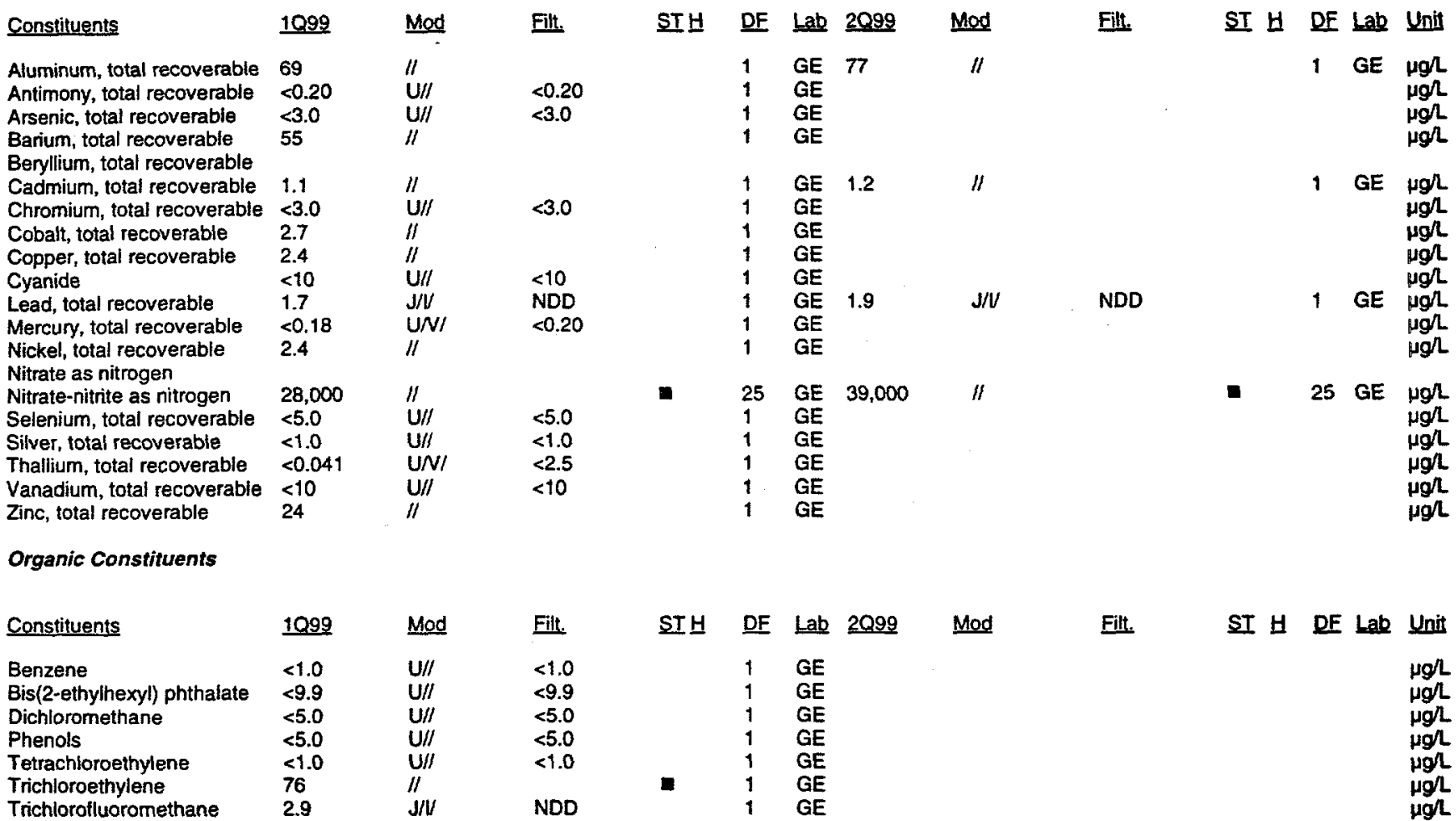

Notes:

- exceeded holding time

- exceeded groundwater protection or monitoring constituent standard (See Appendix A.)

NA - Not applicable. Applies to beta dose and sum of betas if there are no beta-emitting radionuclides above detection limits; to sum of alphas if there are no alphaemitting radionuclides above detection limits; and to total radium if neither radium-226 or radium-228 was above detection limit 
WELL FSB 99C (cont.)

Radioactive Constituents

\begin{tabular}{|c|c|c|c|c|c|c|c|c|c|c|c|c|c|}
\hline Constituents & 1099 & Mod & Fill. & ST브 & DF & Lab & $\underline{2099}$ & Mod & Filt. & ST $\mathrm{H}$ & DF & Lab & Unit \\
\hline $\begin{array}{l}\text { Americium-241 } \\
\text { Beta dose }\end{array}$ & $\begin{array}{l}<2.2 E-02 \\
14\end{array}$ & $\mathrm{U} / \prime$ & $<1.7 E-01$ & a & 1 & GP & & & & & & & $\mathrm{pCil}$ \\
\hline Carbon-14 & $8.6 E+\infty$ & $\mathrm{J} / \mathrm{L} / \mathrm{I}$ & NDD & & 1 & GP & & & & & & & pCin \\
\hline Cesium-137 & $<-1.2 \mathrm{E}+\infty$ & $U I I I$ & $<3.5 E+\infty$ & & 1 & GP & & & & & & & $\mathrm{pCil}$ \\
\hline Cobalt-60 & $<4.1 E-01$ & UII & $<3.6 E+\infty$ & & 1 & GP & & & & & & & pCin \\
\hline Curium-242 & $<4.9 E-02$ & $\mathrm{u} / 1 /$ & $<1.6 E-01$ & & 1 & GP & & & & & & & $\mathrm{pCin}$ \\
\hline Curium-243/244 & $<-7.8 \mathrm{E}-03$ & $\mathrm{U} / \mathrm{I}$ & $<1.5 E-01$ & & 1 & GP & & & & & & & pCin \\
\hline Curium-245/246 & $<0.0 E+\infty 0$ & $\mathrm{U} / /$ & $<4.4 E-02$ & & 1 & GP & & & & & & & $\mathrm{pCin}$ \\
\hline Gross alpha & $2.5 \mathrm{E}+01$ & J几I & NDD & & 1 & GP & $2.5 E+01$ & "I & & - & 1 & GP & pCin \\
\hline lodine-129 & 1.2E+01 & II & & & 1 & GP & & & & & & & pCil \\
\hline Nonvolatile beta & $6.3 \mathrm{E}+01$ & $\|$ & & घ & 1 & GP & $6.8 \mathrm{E}+01$ & $\|$ & & - & 1 & GP & $\mathrm{pCin}$ \\
\hline Plutonium-238 & $<2.2 E-01$ & $\mathrm{U} / /$ & $<4.0 E-01$ & & 1 & GP & & & & & & & $\mathrm{pCin}$ \\
\hline Plutonium-239/240 & $<1.8 \mathrm{E}-02$ & $\mathrm{U} / 1$ & $<2.1 E-01$ & & 1 & GP & & & & & & & $\mathrm{pCin}$ \\
\hline Radium-226 & $5.5 \mathrm{E}+00$ & $\|$ & & ש & 1 & GP & & & & & & & $p \mathrm{Cin}$ \\
\hline Padium-228 & $1.8 \mathrm{E}+00$ & $\mathrm{~J} / \mathrm{V}$ & NDD & & 1 & $G P$ & & & & & & & $\mathrm{pCin}$ \\
\hline Total radium & $5.5 \mathrm{E}+00$ & & & & & & & & & & & & \\
\hline Strontium-90 & $1.3 E+01$ & "I & & ש & 1 & GP & & & & & & & $\mathrm{pCi} / \mathrm{L}$ \\
\hline Technetium-99 & $5.5 E+01$ & $/ 1$ & & a & 1 & GP & & & & & & & pCin \\
\hline Thorium-228 & $<6.7 \mathrm{E}-02$ & $u / \prime$ & $<1.3 E-01$ & & 1 & GP & & & & & & & pCin \\
\hline Thorium-230 & 4.2E-02 & $\mathbf{R} / 4$ & Rej & & 1 & GP & & & & & & & pCin \\
\hline Thorium-232 & $<8.3 E-03$ & U/I & $<2.5 E-02$ & & 1 & GP & & & & & & & pcin \\
\hline Sum of alphas & $1.3 E+01$ & & & & & & & & & & & & $\mathrm{PCi}$ \\
\hline Sum of betas & $7.9 E+01$ & & & घ & & & & & & & & & pCin \\
\hline Tritium & $9.1 E+02$ & 11 & & घ & 1 & GP & $7.9 \mathrm{E}+02$ & $\|$ & & $\mathbf{a}$ & 1 & GP & $\mathrm{pCi} / \mathrm{mL}$ \\
\hline Uranium-233/234 & $5.8 E+00$ & "I & & & 1 & GP & & & & & & & pCin \\
\hline Uranium-235 & 8.7E-01 & II & & & 1 & GP & & & & & & & pCin \\
\hline Uranium-238 & $6.6 E+00$ & /I & & & 1 & GP & & & & & & & pCir \\
\hline
\end{tabular}

Notes:

= exceeded holding time

= exceeded groundwater protection or monitoring constituent standard (See Appendix A.)

NA - Not applicable. Applies to beta dose and sum of betas if there are no beta-emitting radionuclides above detection limits; to sum of alphas if there are no alphaemitting radionuclides above detection limits; and to total radium if neither radium-226 or radium-228 was above detection limit

UAZ_UTRA - Upper Aquifer Zone of the Upper Three Runs Aquifer, LAZ UTRA - Lower Aquifer Zone of the Upper Three Runs Aquifer, Gordon - Gordon Aquifer 


\section{WELL FSB 99D}

$\begin{array}{ll}\text { SRS Coord. } & \text { Lathongitude } \\ \text { N75691.7 } & 33.275294^{\circ} \\ \text { E50326.9 } & 81.681307^{\circ}\end{array}$

SAMPLE DATE

Screen Zone Elevation

218.1-198.1 ft ms

1099

Constituents

Water elevation

$\mathrm{pH}$

Sp. conductance

Water temperature

Alkalinity as $\mathrm{CaCO}$

Turbidity

Volumes purged

Sampling code

Synchronous water level

\section{ANALYTICAL DATA}

Inorganic Constituents

Antimony, total recoverable $<0.20$

Arsenic, total recoverable $\quad<3.0$

Barium, total recoverable $\quad 7.7$

Beryllium, total recoverable

Cadmium, total recoverable $<1.0$

Chromium, total recoverable $<3.0$

Cobalt, total recoverable

Copper, total recoverable

Cyanide

Lead, total recoverable

Mercury, total recoverable

Nickel, total recoverable

Nitrate as nitrogen

Nitrate-nitrite as nitrogen $\quad 1,400$

Selenium, total recoverable $<5.0$

Silver, total recoverable $\quad<1.0$

Thallium, total recoverable $<2.5$

Vanadium, total recoverable $<10$

Zinc, total recoverable

26
Top of Casing

$287.6 \mathrm{ft} \mathrm{msl}$
Casing Pump

4" PVC S
Screen Zone

UAZ_UTRA
04/07/99

2099

213.8

4.7

34

22.3

0

5.2

$213.4(06 / 25 / 99)$
Unil

ft $\mathrm{msi}$

pH

$\mu S / \mathrm{cm}$

${ }^{\circ} \mathrm{C}$

mgl

NTU

well volumes

ft ms!

Organic Constituents

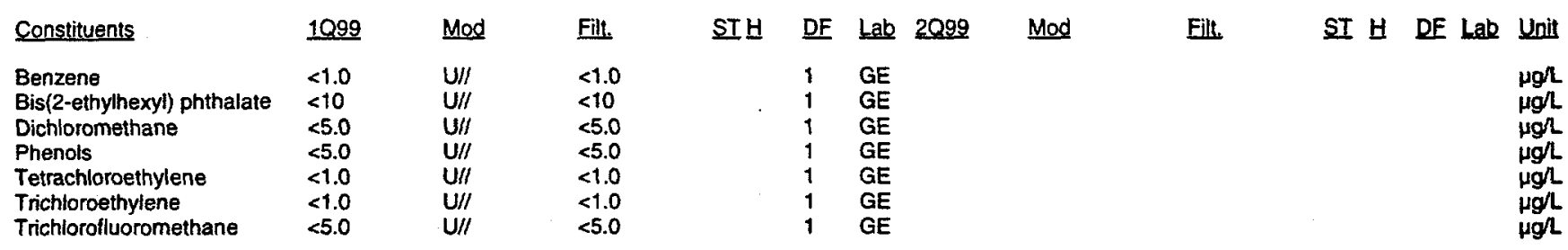

Notes:

- exceeded holding time

= exceeded groundwater protection or monitoring constituent standard (See Appendix A.)

NA - Not applicable. Applies to beta dose and sum of betas if there are no beta-emitting radionuclides above detection limits; to sum of alphas if there are no alphaemitting radionuclides above detection limits; and to total radium if neither radium-226 or radium-228 was above detection limit

UAZ_UTRA - Upper Aquifer Zone of the Upper Three Runs Aquifer; LAZ UTRA - Lower Aquifer Zone of the Upper Three Runs Aquifer, Gordon - Gordon Aquifer 


\section{WELL FSB 99D (cont.)}

Radioactive Constituents

\begin{tabular}{|c|c|c|c|c|c|c|c|c|c|c|c|c|c|}
\hline Constituents & 1099 & Mod & Filt. & SI & DF & Lab & 2099 & Mod & Filt. & ST $H$ & DF & Lab & Unit \\
\hline $\begin{array}{l}\text { Americium-241 } \\
\text { Beta dose }\end{array}$ & $\begin{array}{l}<2.7 E-02 \\
\text { NA }\end{array}$ & JU/ル & $<3.1 E-01$ & & 1 & GP & & & & & & & pCin \\
\hline Carbon-14 & $<3.2 \mathrm{E}+\infty$ & $U / /$ & $<7.3 E+\infty 0$ & & 1 & GP & & & & & & & pCin \\
\hline Cesium-137 & $<-8.6 E-01$ & JU/LC & $<3.6 E+\infty 0$ & & 1 & $\mathrm{GP}$ & & & & & & & pCin \\
\hline Cobalt -60 & $<1.3 E+00$ & $\mathrm{U} / /$ & $<3.8 E+\infty 0$ & & 1 & GP & & & & & & & pCin \\
\hline Curium-242 & $<-1.5 E-02$ & $\mathrm{U} / /$ & $<3.3 E-01$ & & 1 & GP & & & & & & & pCin \\
\hline Curium-243/244 & $<-1.4 E-02$ & $\mathrm{U} / /$ & $<3.1 E-01$ & & 1 & GP & & & & & & & pCin \\
\hline Curium-245/246 & $<0.0 E+\infty 0$ & U/I & $<1.8 \mathrm{E}-01$ & & 1 & GP & & & & & & & pCin \\
\hline Gross alpha & $9.4 E+\infty 0$ & 11 & & & 1 & GP & $8.8 E+\infty$ & $\|$ & & & 1 & GP & pCil \\
\hline lodine-129 & $<2.0 E-01$ & $U / I$ & $<1.1 E+\infty$ & & 1 & GP & & & & & & & $p C i$ \\
\hline Nonvolatile beta & $8.8 E+\infty$ & $/ 1$ & & & 1 & GP & $1.0 E+01$ & $\|$ & & & 1 & GP & pCil \\
\hline Plutanium-238 & $<1.8 \mathrm{E}-02$ & $U / I$ & $<5.4 E-02$ & & 1 & GP & & & & & & & pCin \\
\hline Plutonium-239/240 & $<3.9 \mathrm{E}-02$ & $U / /$ & $<9.9 E-02$ & & 1 & GP & & & & & & & pCin \\
\hline Radium-226 & $6.6 E-01$ & $\mathrm{~J} / \mathrm{V}$ & NDD & & 1 & GP & & & & & & & pCill \\
\hline Radium-228 & $<6.0 \mathrm{E}-01$ & $\mathrm{U} / /$ & $<9.3 E-01$ & & 1 & GP & & & & & & & pCil \\
\hline Total radium & NA & & & & & & & & & & & & \\
\hline Strontium-90 & 2. $1 E \div 00$ & $\mathrm{~J} / \mathrm{N}$ & NDD & & 1 & GP & & & & & & & pCil \\
\hline Technetium-99 & $<1.9 \mathrm{E}+00$ & $\mathrm{U} / /$ & $<2.1 E+01$ & & 1 & GP & & & & & & & pCin \\
\hline Thorium-228 & $<-6.0 E-02$ & $\mathrm{U} / /$ & $<4.0 E-01$ & & 1 & GP & & & & & & & $\mathrm{pCi}$ \\
\hline Thorium-230 & $1.6 \mathrm{E}-01$ & $R / / 4$ & Rej & & 1 & GP & & & & & & & peir \\
\hline & $<1.7 \mathrm{E}-02$ & $\mathrm{U} / /$ & $<2.0 E-01$ & & 1 & GP & & & & & & & pCin \\
\hline $\begin{array}{l}\text { Sum of alphas } \\
\text { Sum of betas }\end{array}$ & $\begin{array}{l}\text { NA } \\
\text { NA }\end{array}$ & & & & & & & & & & & & \\
\hline Tritium & 1.7E+01 & $1 /$ & & & 1 & GP & $7.7 E+01$ & $\|$ & & $\mathbf{a}$ & 1 & GP & $\mathrm{pCi} / \mathrm{mL}$ \\
\hline Uranium-233/234 & $2.0 E+\infty 0$ & $\mathrm{~J} / \mathrm{LC}$ & NDD & & 1 & GP & & & & & & & pCin \\
\hline Uranium-235 & 4.5E-01 & $\mathrm{J} / \mathrm{IL/C}$ & NDD & & 1 & GP & & & & & & & pCil \\
\hline Uranium-238 & $5.3 E+\infty 0$ & $\mathrm{~J} / \mathrm{L} / \mathrm{C}$ & NDD & & 1 & GP & & & & & & & $\mathrm{pCi}$ \\
\hline
\end{tabular}

Notes:

- exceeded holding time

= exceeded groundwater protection or monitoring constituent standard (See Appendix A.)

NA - Not applicable. Applies to beta dose and sum of betas if there are no beta-emitting radionuclides above detection limits; to sum of alphas if there are no alphaemitting radionuclides above detection limits; and to total radium if neither radium-226 or radium-228 was above detection limit

UAZ_UTRA - Upper Aquifer Zone of the Upper Three Runs Aquifer, LAZ_UTRA - Lower Aquifer Zone of the Upper Three Runs Aquifer; Gordon - Gordon Aquifer 


\section{WELL FSB100A}

\begin{tabular}{|c|c|}
\hline SRS Coord. & Lat/Longitude \\
\hline E50958.4 & 81.67933 \\
\hline
\end{tabular}

SAMPLE DATE

01/08/99

Screen Zone Elevation

$105.8-95.8 \mathrm{ft} \mathrm{ms}$
Top of Casing

$286 \mathrm{ft} \mathrm{ms}$
Casing Pump

4" PVC S
Screen Zone

Gordon

\section{FIELLD DATA}

\section{Constituents}

Water elevation

pH

Sp. conductance

Water temperature

Alkalinity as $\mathrm{CaCO} 3$

Turbidity

Volumes purged

Sampling code

Synchronous water level

\section{9}

151.9

6.8

160

19.9

70

5.5

$151.8(03 / 23 / 99)$

04/07/99

2099

151.8

6.5
140

22.1

44

5.0

$151.4(06 / 28 / 99)$
Unit

ft ms!

pH

$\mu \mathrm{S} / \mathrm{cm}$

${ }^{\circ} \mathrm{C}$

mgl

NTU

well volumes

tt msl

\section{ANALYTICAL DATA}

Inorganic Constituents

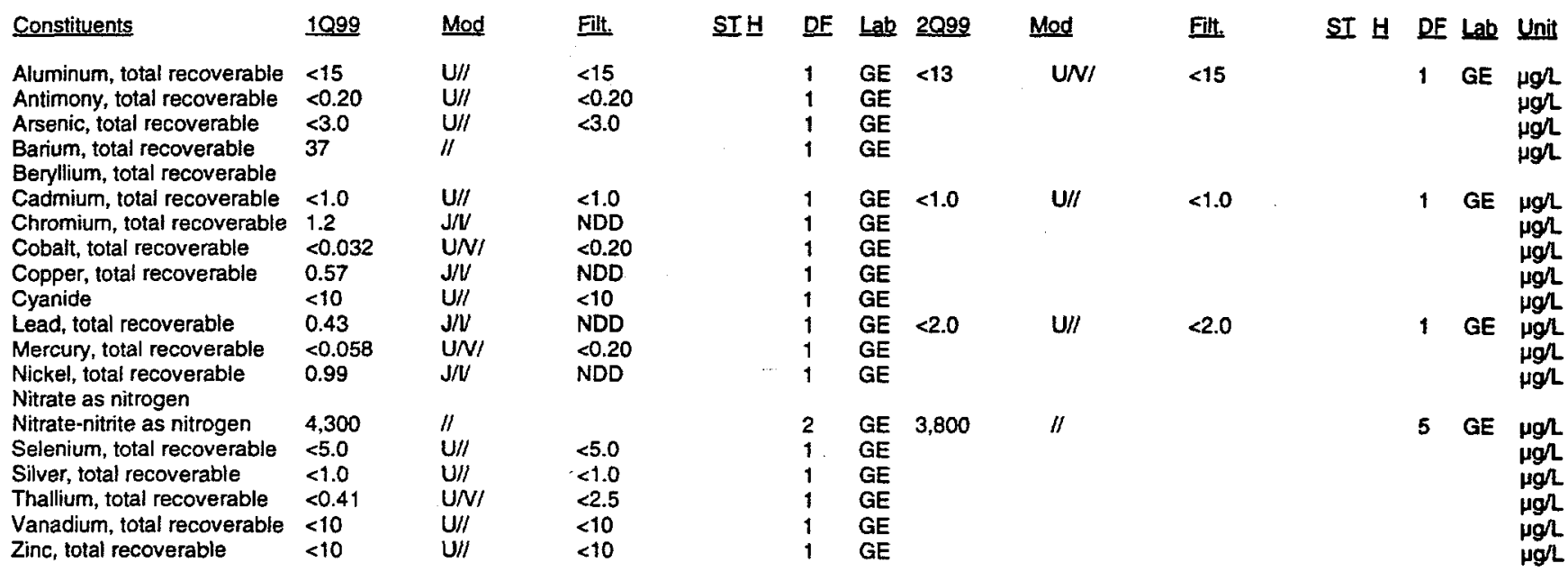

Organic Constituents

\begin{tabular}{llll} 
Constituents & 1099 & Mod & Filt. \\
\cline { 2 - 4 } Benzene & & & \\
Bis(2-ethylhexyl) phthalate & $<1.0$ & $\mathrm{U} / /$ & $<1.0$ \\
Dichloromethane & $<7.8$ & $\mathrm{U} / /$ & $<10$ \\
Phenols & $<5.0$ & $\mathrm{U} / /$ & $<5.0$ \\
Tetrachloroethylene & $<1.0$ & $\mathrm{U} / /$ & $<5.0$ \\
Trichloroethylene & $<1.0$ & $\mathrm{U} / /$ & $<1.0$ \\
Trichlorofluoromethane & $<5.0$ & $\mathrm{U} / /$ & $<1.0$ \\
& & & $<5.0$
\end{tabular}

STH $\quad \begin{array}{ll}\text { DF } & \text { Lab } \\ 1 & \text { GE } \\ 1 & \text { GE } \\ 1 & \text { GE } \\ 1 & \text { GE } \\ 1 & \text { GE } \\ 1 & \text { GE } \\ 1 & \text { GE }\end{array}$

Fill.

ST H DF Lab Unir

ugh

ugh

$\mu \mathrm{gh}$

$\mu g / 2$

$\mu g /$

pgh

$\mu g h$

\section{Notes:}

- = exceeded holding time

= exceeded groundwater protection or monitoring constituent standard (See Appendix A.)

NA - Not applicable. Applies to beta dose and sum of betas if there are no beta-emitting radionuclides above detection limits; to sum of alphas if there are no alphaemitting radionuclides above detection limits; and to total radium if neither radium-226 or radium-228 was above detection limit

UAZ_UTRA - Upper Aquifer Zone of the Upper Three Runs Aquifer; LAZ_UTRA - Lower Aquifer Zone of the Upper Three Runs Aquifer, Gordon - Gordon Aquifer 


\section{WELL FSB100A (cont.)}

Radioactive Constituents

\begin{tabular}{|c|c|c|c|c|c|c|c|c|c|c|c|c|c|}
\hline Constituents & 1099 & Mod & Filt. & ST브 & DF & Lab & 2099 & Mod & Filt. & ST 브 & DF & $\mathrm{Lab}$ & Unit \\
\hline $\begin{array}{l}\text { Americium-241 } \\
\text { Beta dose }\end{array}$ & $\begin{array}{l}<1.8 E-02 \\
\mathrm{NA}\end{array}$ & JUんV/I & $<1.2 E-01$ & & 1 & GP & & & & & & & $\mathrm{pCi}$ \\
\hline Carbon-14 & $9.9 E+\infty 0$ & $\mathrm{~J} / \mathrm{V}$ & NDD & & 1 & GP & & & & & & & $\mathrm{pCi}$ \\
\hline Cesium-137 & $<2.4 E-0.4$ & $U / I$ & $<3.2 E+00$ & & 1 & GP & & & & & & & pCin \\
\hline Cobalt-60 & $<-4.2 E-02$ & $\mathrm{U} / \mathrm{I}$ & $<3.0 \mathrm{E}+00$ & & 1 & GP & & & & & & & pCin \\
\hline Curium-242 & $<0.0 E+\infty$ & $U / I$ & $<5.7 E-02$ & & 1 & GP & & & & & & & pCin \\
\hline Curium-243/244 & $<-1.8 E-04$ & $\mathbf{U} / /$ & $<1.4 \mathrm{E}-01$ & & 1 & GP & & & & & & & pCin \\
\hline Curium-245/246 & $<5.5 \mathrm{E}-02$ & $\mathrm{U} / /$ & $<5.5 E-02$ & & 1 & GP & & & & & & & $\mathrm{pCin}$ \\
\hline $\begin{array}{l}\text { Gross alpha } \\
\text { lodine-129 }\end{array}$ & $\begin{array}{l}<8.3 E-01 \\
<1.4 E+\infty\end{array}$ & $\begin{array}{l}U / / \\
U N /\end{array}$ & $\begin{array}{l}<8.4 E-01 \\
<1.0 E+00\end{array}$ & & 1 & $\begin{array}{l}\text { GP } \\
\text { GP }\end{array}$ & $1.4 \mathrm{E}+\infty$ & $\mathrm{J} / \mathrm{V}$ & NDD & & 1 & GP & $\begin{array}{l}\text { pCin } \\
\text { pCin }\end{array}$ \\
\hline Nonvolatile beta & $1.9 E+00$ & $\mathrm{~J} / \mathrm{V}$ & NDD & & 1 & GP & 2.1E+CO & $J / V$ & NDD & & 1 & GP & poin \\
\hline Plutonium-238 & $<1.4 E-02$ & $U / l$ & $<2.7 E-01$ & & 1 & GP & & & & & & & pcil \\
\hline Plutonium-239/240 & $<-3.1 E-04$ & $\mathrm{U} / /$ & $<1.5 E-01$ & & 1 & GP & & & & & & & pCil \\
\hline Radium-226 & 8.OE-01 & $\mathrm{J} / \mathrm{V}$ & NDD & & 1 & GP & & & & & & & pCin \\
\hline Radium-228 & $<5.2 E-01$ & $\mathrm{U} / /$ & $<8.8 E-01$ & & 1 & GP & & & & & & & pCin \\
\hline Total radium & NA & & & & & & & & & & & & \\
\hline Strontium-90 & $<4.9 E-01$ & $\mathrm{U} / /$ & $<1.1 E+\infty$ & & 1 & GP & & & & & & & pCin \\
\hline Technetium-99 & $<7.7 E+\infty$ & $\mathrm{U} / /$ & $<2.0 E+01$ & & 1 & GP & & & & & & & pCin \\
\hline Thorium-228 & $<3.4 E-02$ & $\mathrm{U} / /$ & $<1.7 E-01$ & & 1 & GP & & & & & & & pCin \\
\hline Thorium-230 & $<4.0 \mathrm{E}-02$ & $\mathrm{U} / /$ & $<3.0 E-02$ & & 1 & GP & & & & & & & pCin \\
\hline Thorium-232 & $<-5.1 E-03$ & $\mathrm{U} / /$ & $<6.3 E-02$ & & 1 & GP & & & & & & & pCil \\
\hline Sum of alphas & NA & & & & & & & & & & & & \\
\hline Sum of betas & NA & & & & & & & & & & & & \\
\hline Tritium & $7.5 E+01$ & $/ 1$ & & $\mathbf{0}$ & 1 & GP & $6.8 E+01$ & $/ 1$ & & - & 1 & GP & $\mathrm{pCi} / \mathrm{mL}$ \\
\hline Uranium-233/234 & $1.4 E-01$ & $R / / 4$ & Rej & & 1 & GP & & & & & & & pCin \\
\hline Uranium-235 & $<-4.7 E-03$ & $\mathrm{U} / 1$ & $<8.0 E-02$ & & 1 & GP & & & & & & & pCin \\
\hline Uranium-238 & $<4.1 E-02$ & $\mathbf{U} / /$ & $<8.0 E-02$ & & 1 & GP & & & & & & & pCil \\
\hline
\end{tabular}




\section{WELL FSB101A}

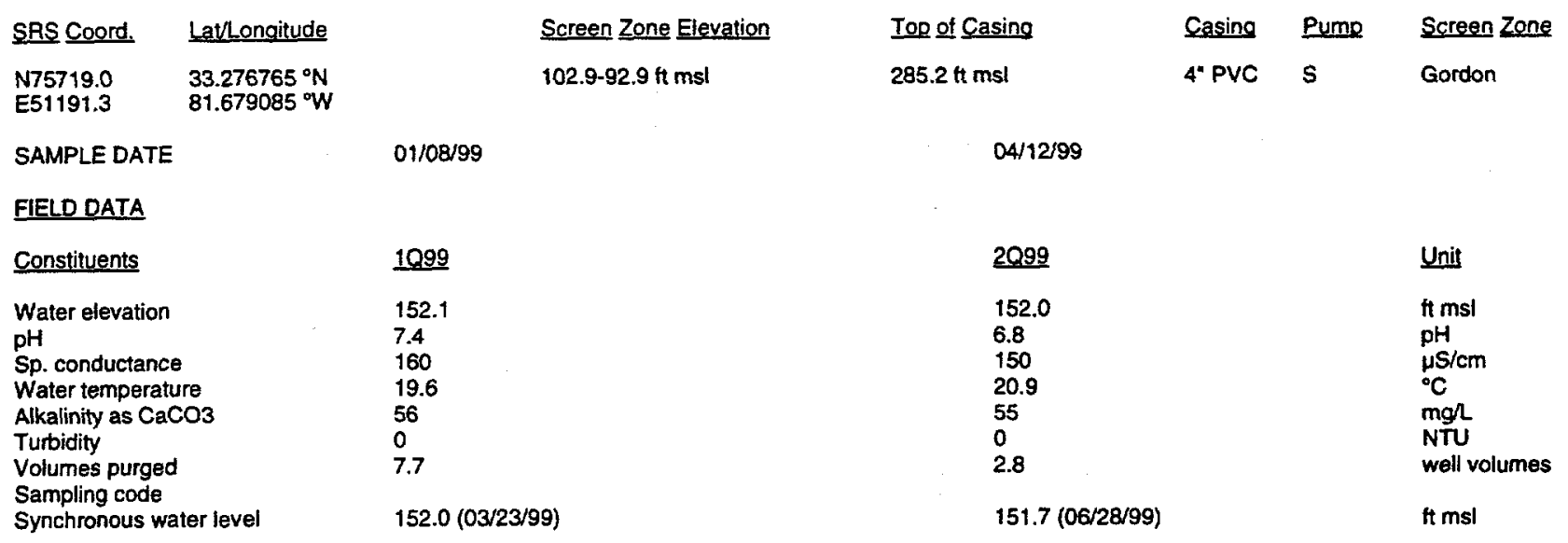

\section{ANALYTICAL DATA}

Inorganic Constituents

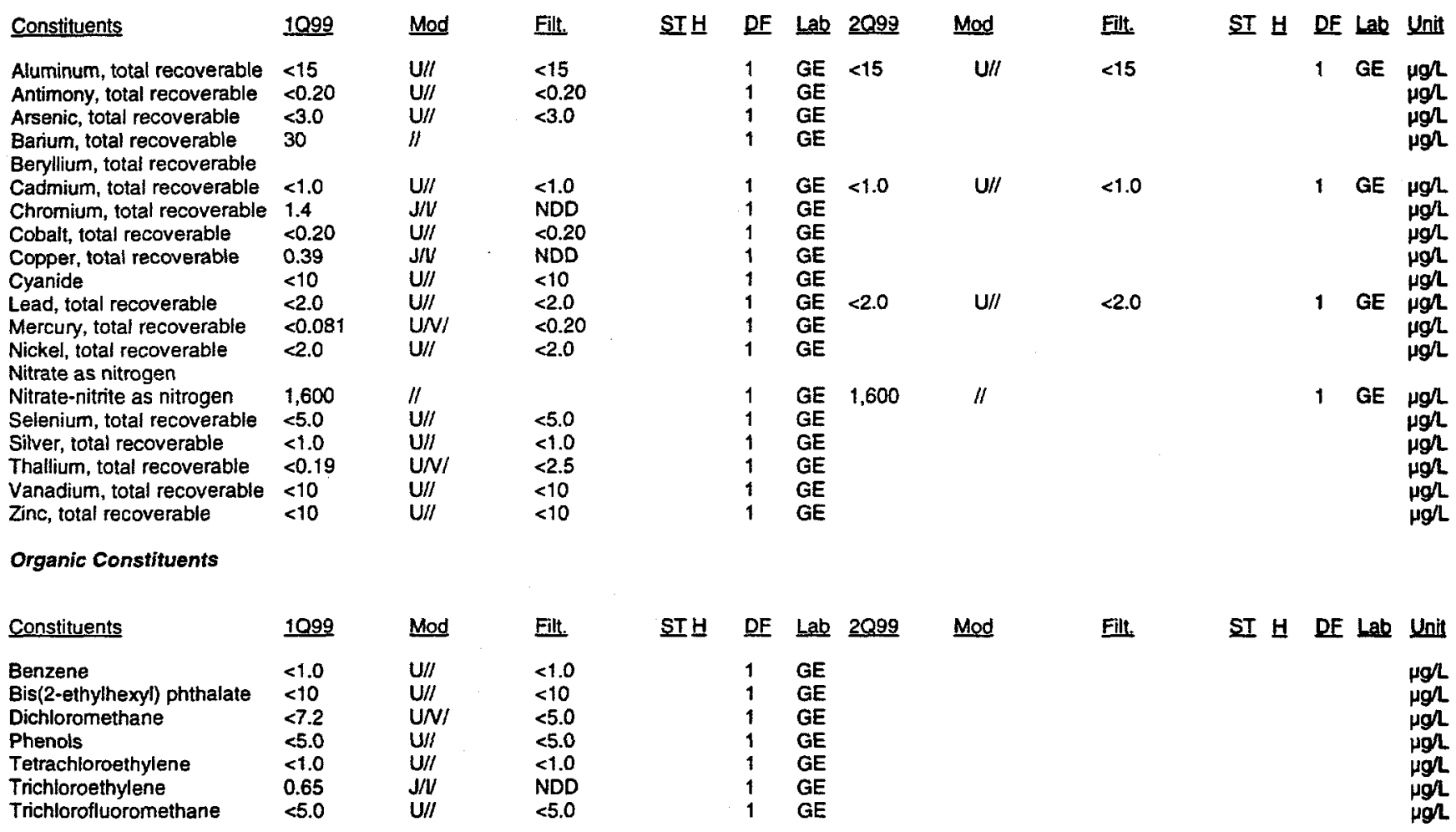

Notes:

- exceeded holding time

- exceeded groundwater protection or monitoring constituent standard (See Appendix A.)

NA - Not applicable. Applies to beta dose and sum of betas if there are no beta-emitting radionuclides above detection limits; to sum of alphas if there are no alphaemitting radionuclides above detection limits; and to total radium if neither radium-226 or radium-228 was above detection limit

UAZ UTRA - Upper Aquiler Zone of the Upper Three Runs Aquifer; LAZ UTRA - Lower Aquifer Zone of the Upper Three Runs Aquifer, Gordon - Gordon Aquifer 


\section{WELL FSB101A (cont.)}

Radioactive Constituents

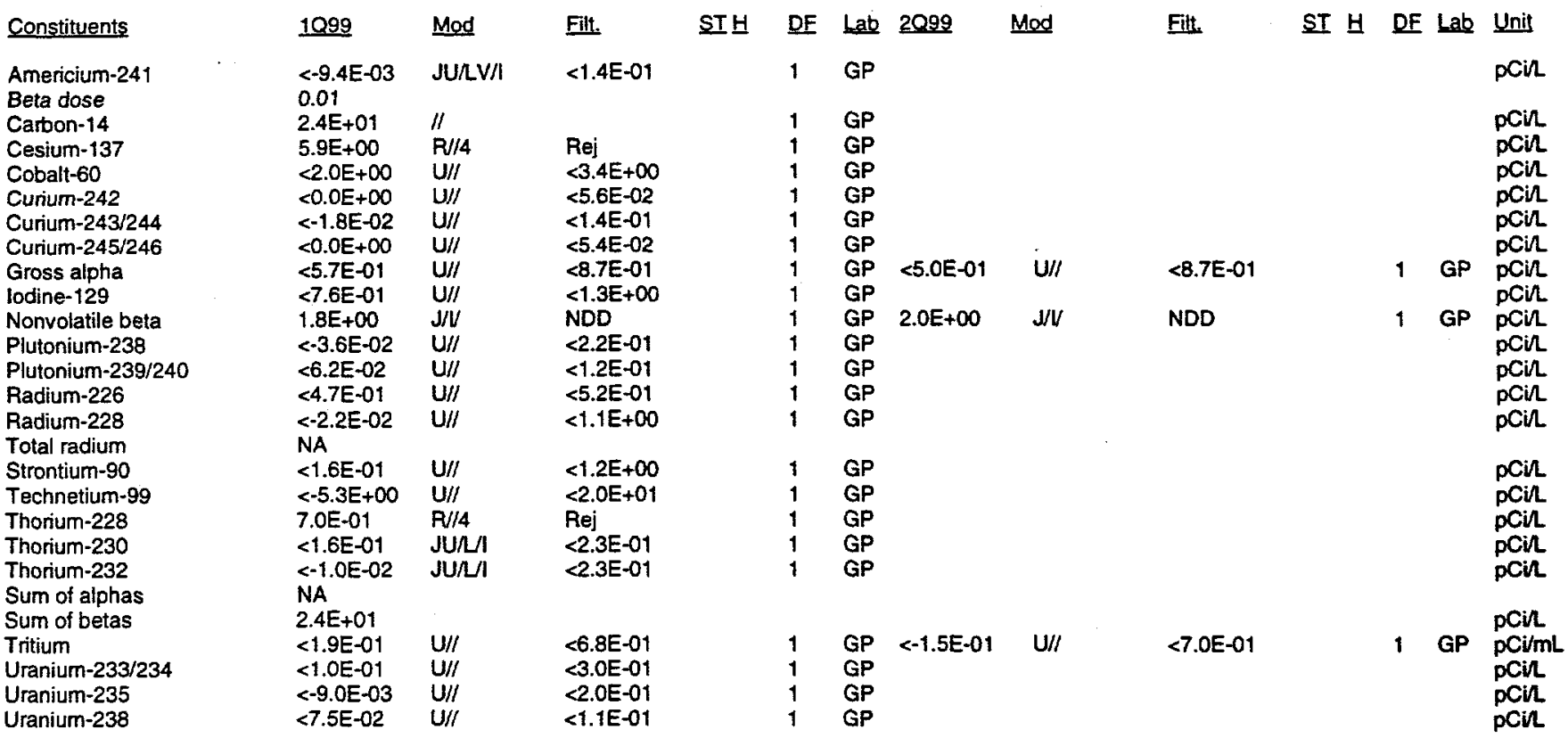

\section{Notes:}

- =xceeded holding time

E = exceeded groundwater protection or monitoring constituent standard (See Appendix A)

NA - Not applicable. Applies to beta dose and sum of betas if there are no bela-emitting radionuclides above detection limits; to sum of alphas if there are no alphaemitting radionuclides above detection limits; and to total radium if neither radium-226 or radium-228 was above detection limit

UAZ UTRA - Upper Aquifer Zone of the Upper Three Runs Aquifer, LAZ UTRA - Lower Aquifer Zone of the Upper Three Runs Aquifer Gordon - Gordon Aguifer 


\section{WELL FSB102C}

$\begin{array}{ll}\text { SRS Coond. } & \text { Latllongitude } \\ \text { N73582.9 } & 33.271459^{\circ} \mathrm{N} \\ \text { E50834.8 } & 81.675873^{\circ} \mathrm{W}\end{array}$

SAMPLE DATE

Screen Zone Elevation

$155.9-145.9 \mathrm{ft} \mathrm{ms}$
Top of Casing

$201.1 \mathrm{ft} \mathrm{msl}$

Casing Pump
4'PVC V

Screen Zone

LAZ_UTRA

\section{FIELD DATA}

\section{Constituents}

Water elevation

Sp. conductance

Water temperature

Alkalinity as $\mathrm{CaCO} 3$

Turbidity

Volumes purged

Sampling code

Synchronous water level
1099

195.3

4.4

200

18.5

0

4.2

$195.2(03 / 23 / 99)$
04/06/99

\begin{tabular}{l}
2099 \\
\hline \\
195.2 \\
4.8 \\
200 \\
17.7 \\
0 \\
0 \\
2.2
\end{tabular}

$194.8(06 / 28 / 99)$
Unit

tt ms!

$\mathrm{pH}$

$\mu S / \mathrm{cm}$

${ }^{\circ} \mathrm{C}$

mgl

well volumes

ti msi

\section{ANALYTICAL DATA}

\section{Inorganic Constituents}

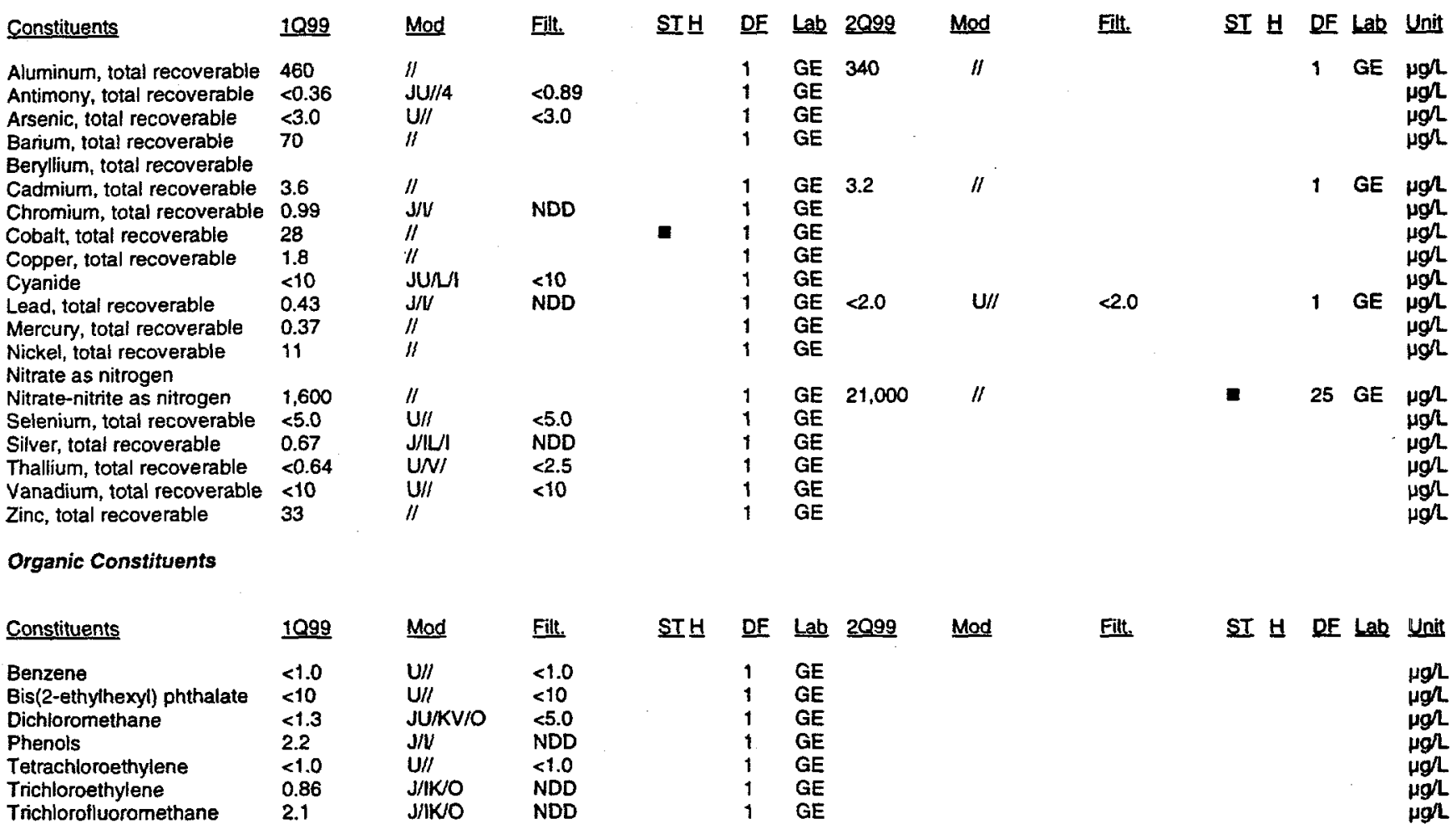

\section{Notes:}

- exceeded holding time

E = exceeded groundwater protection or monitoring constituent standard (See Appendix A.)

NA - Not applicable. Applies to beta dose and sum of betas if there are no beta-emitting radionuclides above detection limits; to sum of alphas if there are no alphaemitting radionuclides above detection limits; and to total radium if neither radium-226 or radium-228 was above detection limit

UAZ_UTRA - Upper Aquifer Zone of the Upper Three Runs Aquifer, LAZ_UTRA - Lower Aquifer Zone of the Upper Three Runs Aquifer, Gordon - Gordon Aquiter 


\section{WELL FSB102C (cont.)}

Radioactive Consitituents

\begin{tabular}{|c|c|c|c|c|c|c|c|c|c|c|c|c|c|}
\hline Constituents & 1099 & Mod & Filt. & STH & $\underline{D F}$ & $\underline{L a b}$ & $\underline{2099}$ & Mod & Fill. & SI $\underline{H}$ & $\underline{\mathrm{DF}}$ & Lab & Unit \\
\hline Americium-241 & $<2.1 E-01$ & $\mathrm{U} / /$ & $<5.2 \mathrm{E}-01$ & & 1 & GP & & & & & & & pCir \\
\hline Beta dose & 12 & & & 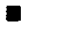 & & & & & & & & & \\
\hline Carbon-14 & $3.0 E+01$ & $\|$ & & & 1 & GP & & & & & & & $\mathrm{pCin}$ \\
\hline Cesium-137 & $<-6.8 \mathrm{E}-01$ & $\mathrm{U} / /$ & $<4.1 E+\infty 0$ & & 1 & GP & & & & & & & pCin \\
\hline Cobalt- 60 & $<-1.7 E+\infty$ & $\mathrm{U} / /$ & $<4.1 E+\infty 0$ & & 1 & GP & & & & & & & pCin \\
\hline Curium-242 & $<-1.2 \mathrm{E}-02$ & $\mathrm{U} / I$ & $<7.2 E-01$ & & 1 & GP & & & & & & & pCin \\
\hline Curium-243/244 & $<-8.2 E-02$ & $\mathrm{U} / /$ & $<5.5 E-01$ & & 1 & GP & & & & & & & pCir \\
\hline Curium-245/246 & $<0.0 E+00$ & $\mathrm{U} / 1$ & $<2.0 \mathrm{E}-01$ & & 1 & GP & & & & & & & $\mathrm{pCin}$ \\
\hline Gross alpha & $4.9 E+00$ & II & & & 1 & GP & $4.5 E+\infty 0$ & $\|$ & & & 1 & GP & pCin \\
\hline Jodine-129 & $2.5 E+01$ & $\mathrm{~J} / \mathrm{K} / \mathrm{C}$ & NDD & & 1 & GP & & & & & & & pCil \\
\hline Nonvolatile beta & $2.5 E+02$ & $/ 1$ & & - & 1 & GP & $2.2 E+02$ & $/ 1$ & & $\mathbf{\square}$ & 1 & GP & pCin \\
\hline Plutonium-238 & $<4.9 E-02$ & $\mathrm{U} / /$ & $<4.9 E-02$ & & 1 & GP & & & & & & & pCin \\
\hline Plutonium-239/240 & $9.8 E-02$ & $R / / 4$ & Rej & & 1 & GP & & & & & & & pCill \\
\hline Radium-226 & $3.3 E+00$ & $/ 1$ & & & 1 & GP & & & & & & & pCil \\
\hline Radium-228 & $2.0 E+00$ & $\mathrm{~J} / \mathrm{N}$ & NDD & & 1 & GP & & & & & & & pCin \\
\hline Total radium & $3.3 E+\infty 0$ & & & & & & & & & & & & \\
\hline Strontium-90 & $1.0 E+02$ & 11 & & $n$ & 1 & GP & & & & & & & pCin \\
\hline Technetium-99 & 4.1E+01 & $\mathrm{J} / \mathrm{N}$ & NDD & & 1 & GP & & & & & & & pCin \\
\hline Thorium-228 & $<8.8 E-02$ & $\mathrm{U} / /$ & $<3.6 E-01$ & & 1 & GP & & & & & & & pCil \\
\hline Thorium-230 & $<2.8 E-02$ & $\mathrm{U} / /$ & $<2.1 E-01$ & & 1 & GP & & & & & & & pCin \\
\hline Thorium-232 & $<-1.3 E-02$ & $\mathrm{U} / /$ & $<1.7 \mathrm{E}-01$ & & 1 & GP & & & & & & & $\mathrm{pCi} \Omega$ \\
\hline Sum of alphas & NA & & & & & & & & & & & & \\
\hline Sum of betas & $1.3 E+02$ & & & $\mathbf{\square}$ & & & & & & & & & pCin \\
\hline Tritium & 4.4E+02 & $/ 1$ & & $\mathbf{E}$ & 1 & GP & $4.3 E+02$ & $/ /$ & & n & 1 & GP & $\mathrm{pCi} / \mathrm{mL}$ \\
\hline Uranium-233/234 & 2.7E-01 & $\mathrm{J} / \mathrm{N}$ & NDD & & 1 & GP & & & & & & & pCir \\
\hline Uranium-235 & $<2.3 E-02$ & $\mathrm{U} / \prime$ & $<1.6 E-01$ & & 1 & GP & & & & & & & pCil \\
\hline Uranium-238 & $1.8 \mathrm{E}-01$ & $\mathrm{~J} / \mathrm{V}$ & NDD & & 1 & GP & & & & & & & $\mathrm{pCi}$ \\
\hline
\end{tabular}

Notes:

- exceeded holding time

= exceeded groundwater protection or monitoring constituent standard (See Appendix A.)

NA - Not applicable. Applies to beta dose and sum of betas if there are no beta-emitting radionuclides above detection limits; to sum of alphas if there are no alphaemitting radionuclides above detection limits; and to total radium if neither radium-226 or radium-228 was above detection limit

UAZ_UTRA - Upper Aquifer Zone of the Upper Three Runs Aquifer, LAZ_UTRA - Lower Aquifer Zone of the Upper Three Runs Aquifer, Gordon - Gordon Aquifer 


\section{WELL FSB103C}

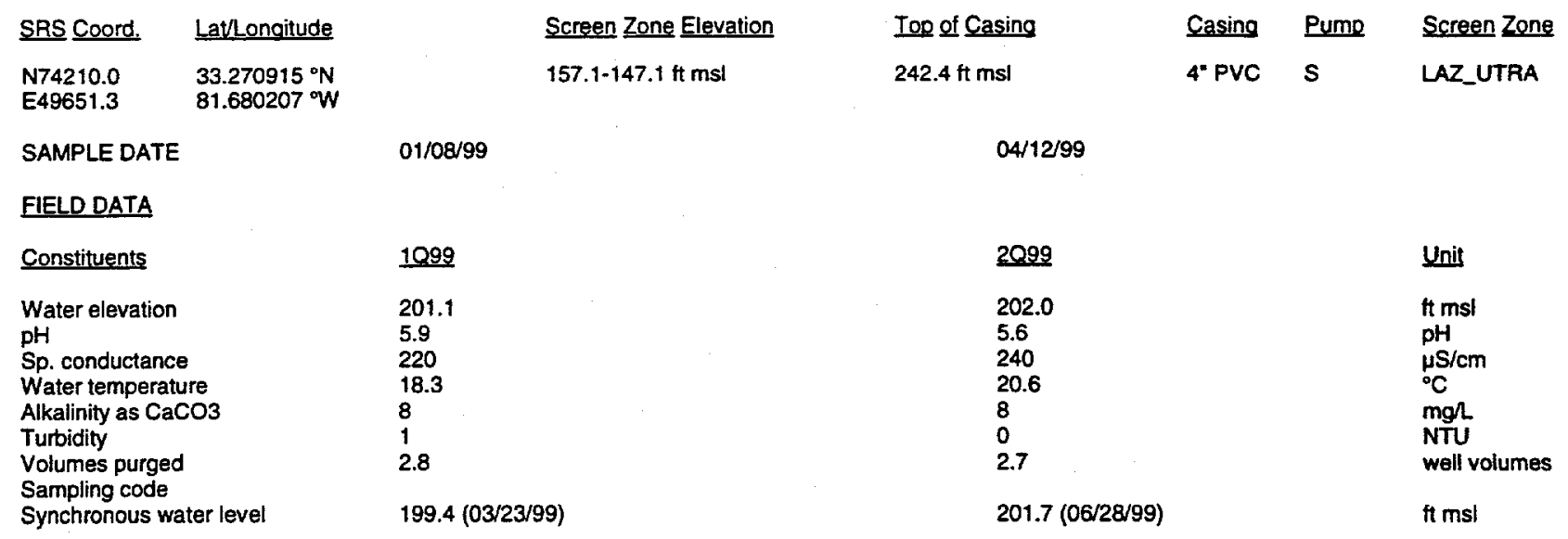

ANALYTICAL DATA

Inorganic Constituents

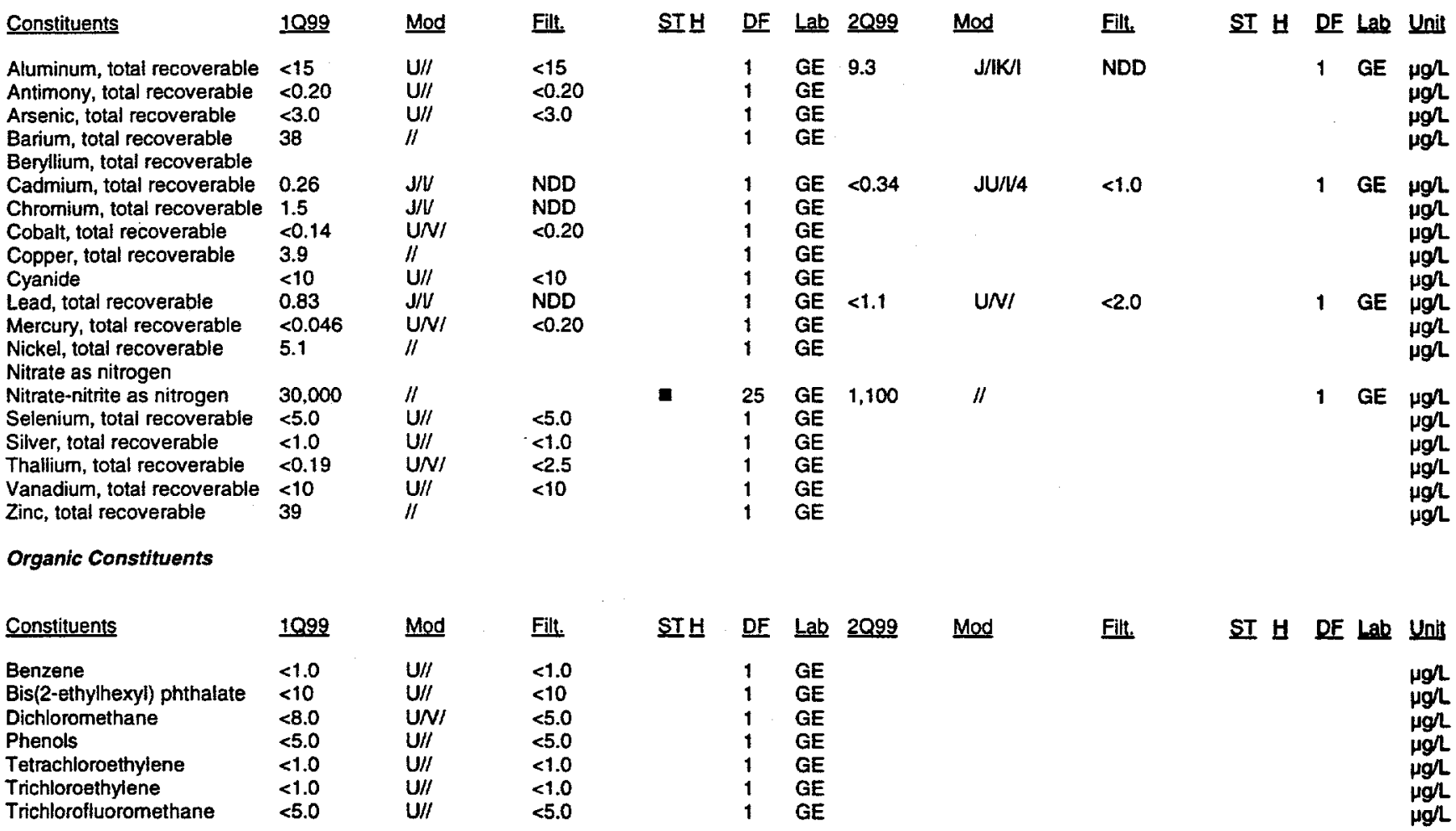

Notes:

= exceeded holding time

= exceeded groundwater protection or monitoring constituent standard (See Appendix A.)

NA - Not applicable. Applies to beta dose and sum of betas if there are no beta-emitting radionuclides above detection limits; to sum of alphas if there are no alphaemitting radionuclides above detection limits; and to total radium if neither radium-226 or radium-228 was above detection limit

UAZ UTRA - Upper Aquifer Zone of the Upper Three Runs Aquifer; LAZ UTRA - Lower Aquiler Zone of the Upper Three Runs Aquifer, Gordon - Gordon Aquifer 
WELL FSB103C (cont.)

Radioactive Constituents

\begin{tabular}{|c|c|c|c|c|c|c|c|c|c|c|c|c|c|}
\hline Constituents & 1099 & Mod & Filt. & ST브 & $\mathrm{DF}$ & Lab & 2099 & Mod & Filt. & ST $\mathrm{H}$ & DF & Lab & Unit \\
\hline $\begin{array}{l}\text { Americium-241 } \\
\text { Beta dose }\end{array}$ & $\begin{array}{l}7.0 E-02 \\
0.69\end{array}$ & $R / / 4$ & Rej & & 1 & $G P$ & & & & & & & $\mathrm{pCi} / \mathrm{L}$ \\
\hline Carbon-14 & $1.4 E+01$ & $\mathrm{~J} / \mathrm{N}$ & NDD & & 1 & GP & & & & & & & pCir \\
\hline Cesium-137 & $1.3 E+01$ & $R / / 4$ & Rej & & 1 & GP & & & & & & & $\mathrm{pCi}$ \\
\hline Cobalt- 60 & $<5.5 \mathrm{E}-01$ & $\mathrm{U} / /$ & $<2.9 E+00$ & & 1 & GP & & & & & & & pCil \\
\hline Curium-242 & $<0.0 E+\infty 0$ & $U / I$ & $<4.1 E-02$ & & 1 & GP & & & & & & & pCin \\
\hline Curium-243/244 & $<1.3 \mathrm{E}-02$ & U/I & $<4.0 \mathrm{E}-02$ & & 1 & GP & & & & & & & pCin \\
\hline Curium-245/246 & $<1.3 E-02$ & $\mathrm{U} / /$ & $<4.0 E-02$ & & 1 & GP & & & & & & & $\mathrm{pCin}$ \\
\hline Gross alpha & $<7.7 E-01$ & $\mathrm{U} / 1$ & $<8.4 E-01$ & & 1 & GP & $<5.8 E=01$ & $\mathrm{U} / I$ & $<7.3 E-01$ & & 1 & GP & pCin \\
\hline lodine-129 & $<2.5 E+00$ & $U N I$ & $<8.2 E-01$ & & 1 & GP & & & & & & & $\mathrm{pCin}$ \\
\hline Nonvolatile beta & $7.8 \mathrm{E}+\infty$ & 11 & & & 1 & GP & $1.7 E+01$ & $\|$ & & & 1 & GP & pCin \\
\hline Plutonium-238 & $<8.2 \mathrm{E}-02$ & $\mathrm{U} / I$ & $<1.9 E-01$ & $\cdots$ & 1 & GP & & & & & & & pCin \\
\hline Plutonium-239/240 & $<-7.7 \mathrm{E}-03$ & $\mathbf{U} / !$ & $<1.3 E-01$ & & 1 & GP & & & & & & & pCin \\
\hline Radium-226 & 7.4E-01 & $\mathrm{J} / \mathrm{V}$ & NDD & & 1 & GP & & & & & & & $\mathrm{pCi}$ \\
\hline Radium-228 & $3.2 E+\infty$ & $/ /$ & & & $t$ & GP & & & & & & & $\mathrm{pCi} /$ \\
\hline Total radium & $3.2 E+\infty$ & & & & & & & & & & & & \\
\hline Strontium-90 & $<-2.4 E-01$ & $\mathbf{U} / !$ & $<1.2 E+00$ & & 1 & GP & & & & & & & pCin \\
\hline Technetium-99 & $4.9 E+01$ & $\|$ & & & 1 & GP & & & & & & & pCin \\
\hline Thorium-228 & $<3.3 E-01$ & JUЛ/ & $<4.6 E-01$ & & 1 & GP & & & & & & & pCir \\
\hline Thorium-230 & 2.1E-01 & $\mathrm{R} / / 4$ & Rej & & 1 & GP & & & & & & & pein \\
\hline Thorium-232 & $<-8.7 E-03$ & JUルI & $<1.9 E-01$ & & 1 & GP & & & & & & & pCin \\
\hline Sum of alphas & NA & & & & & & & & & & & & \\
\hline Sum of betas & $5.2 E+01$ & & & a & & & & & & & & & pCin \\
\hline Tritium & $6.3 E+02$ & 11 & & $\boldsymbol{\square}$ & 1 & GP & $5.8 \mathrm{E}+02$ & 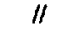 & & $\mathbf{\square}$ & 1 & GP & pCims \\
\hline Uranium-233/234 & $<4.1 E-02$ & $\mathrm{U} / /$ & $<1.2 \mathrm{E}-01$ & & 1 & GP & & & & & & & pCin \\
\hline Uranium-235 & $<-4.0 E-02$ & $\mathrm{U} / /$ & $<3.1 E-01$ & & 1 & GP & & & & & & & pCin \\
\hline Uranium-238 & $<7.3 E-02$ & $\mathrm{u} / /$ & $<2.2 E-01$ & & 1 & GP & & & & & & & pcin \\
\hline
\end{tabular}

= exceeded groundwater protection or monitoring constituent standard (See Appendix A.)

NA - Not applicable. Applies to beta dose and sum of betas if there are no beta-emitting radionuclides above detection limits; to sum of alphas if there are no alphaemitting radionuclides above detection limits; and to total radium if neither radium-226 or radium-228 was above detection limit

UAZ UTRA - Upper Aquifer Zone of the Upper Three Runs Aquifer, LAZ UTRA - Lower Aquifer Zone of the Upper Three Runs Aquifer, Gordon - Gordon Aquifer 


\section{WELL FSB104C}

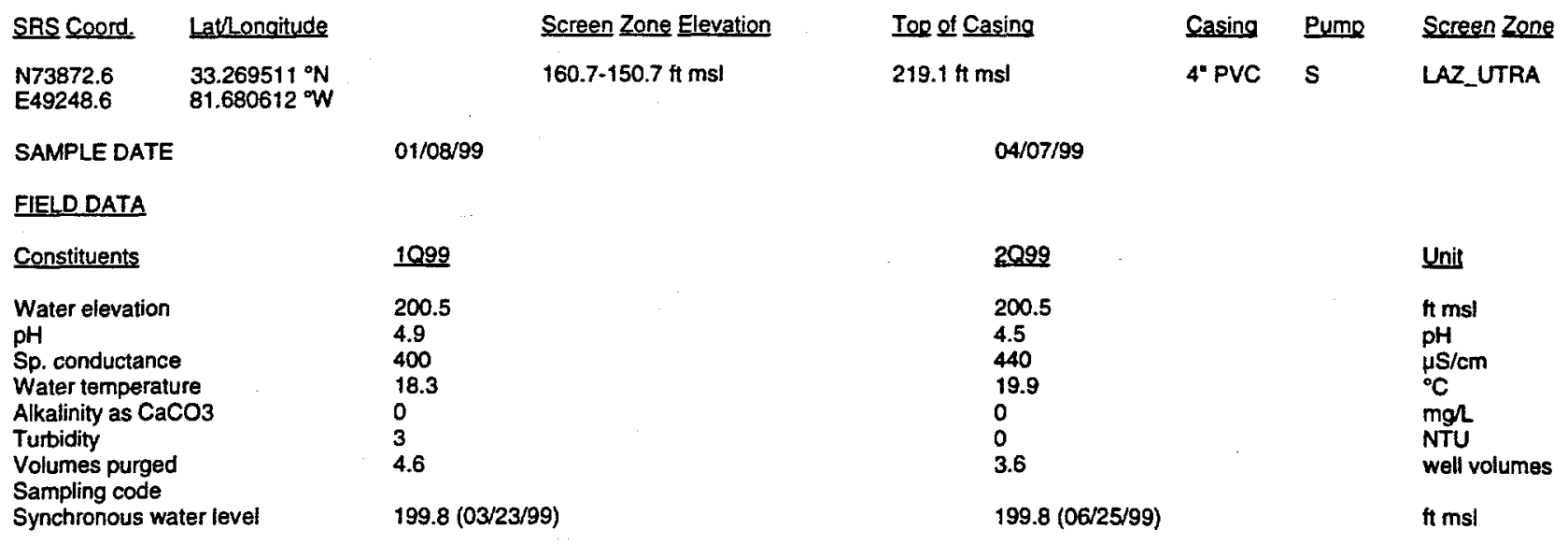

ANALYTICAL DATA

Inorganic Constituents

\begin{tabular}{|c|c|c|c|c|c|c|c|c|c|c|c|c|c|}
\hline Constituents & 1099 & Mod & Filt. & STH & $\underline{\text { DF }}$ & Lab & $\underline{2099}$ & Mod & Fill. & ST 브 & DF & Lab & Unit \\
\hline Aluminum, total recoverable & 52 & $/ 1$ & & & 1 & $\mathrm{GE}$ & $<53$ & UN! & $<15$ & & 1 & GE & nol \\
\hline Antimony, total recoverabie & $<0.20$ & $U / I$ & $<0.20$ & & 1 & GE & & & & & & & $\mu g h$ \\
\hline Arsenic, total recoverable & $<3.0$ & $U_{\prime \prime}^{\prime \prime}$ & $<3.0$ & & 1 & GE & & & & & & & $\mu g /$ \\
\hline $\begin{array}{l}\text { Barium, total recoverable } \\
\text { Beryllium, total recoverable }\end{array}$ & 88 & & & & 1 & GE & & & & & & & $\mu g /$ \\
\hline Cadmium, total recoverable & 0.87 & $J / W$ & NDD & & 1 & GE & $<0.73$ & $\mathrm{JU} / \mathrm{N} / \mathbf{4}$ & $<1.0$ & & 1 & GE & $\mu g /$ \\
\hline Chromium, total recoverable & $<0.64$ & $\mathrm{JU} / / 4$ & $<3.0$ & & 1 & GE & & & & & & & $\mu g h$ \\
\hline Cobalt, total recoverable & 0.54 & & & & 1 & GE & & & & & & & \\
\hline Copper, total recoverable & 4.0 & & & & 1 & GE & & & & & & & gro \\
\hline Cyanide & $<10$ & $\mathrm{U} / /$ & $<10$ & & 1 & GE & & & & & & & $19 h$ \\
\hline $\begin{array}{l}\text { Lead, total recoverable } \\
\text { Mercury, total recoverable }\end{array}$ & $\begin{array}{l}0.77 \\
<0.065\end{array}$ & $\begin{array}{l}\mathrm{J} / \mathrm{V} \\
\mathrm{U} / \mathrm{N}\end{array}$ & $\begin{array}{l}\text { NDD } \\
<0.20\end{array}$ & & $\begin{array}{l}1 \\
1\end{array}$ & GE & 0.61 & $\mathrm{~J} / \mathrm{W}$ & NDD & & 1 & GE & $\mu g / 2$ \\
\hline $\begin{array}{l}\text { Nickel, total recoverable } \\
\text { Nitrate as nitrogen }\end{array}$ & & $/ 1$ & & & 1 & GE & & & & & & & \\
\hline Nitrate-nitrite as nitrogen & 52,000 & $\|$ & & $\mathbf{a}$ & 25 & GE & 50,000 & $\|$ & & $\mathbf{a}$ & 25 & GE & $\mu g / L$ \\
\hline Selenium, total recoverable & $<5.0$ & $\mathrm{U} / /$ & $<5.0$ & & 1 & GE & & & & & & & $\mu g /$. \\
\hline Silver, total recoverable & $<1.0$ & U/l & $<1.0$ & & 1 & GE & & & & & & & 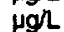 \\
\hline Thallium, total recoverable & $<0.21$ & UN/ & $<2.5$ & & 1 & GE & & & & & & & $\mathrm{Jgh}$ \\
\hline Vanadium, total recoverable & $<10$ & $U / /$ & $<10$ & & 1 & GE & & & & & & & gh/ \\
\hline Zinc, total recoverable & 49 & 11 & & & 1 & GE & & & & & & & \\
\hline
\end{tabular}

Organic Constituents

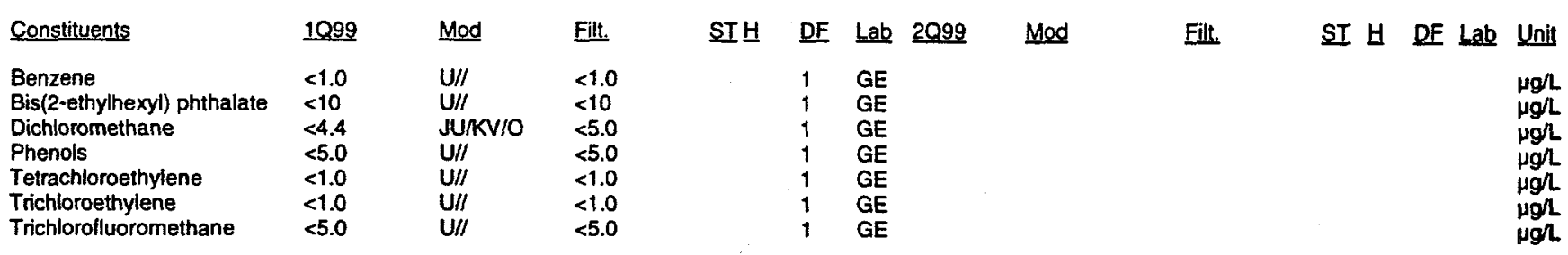

Notes:

- exceeded holding time

= exceeded groundwater protection or monitoring constituent standard (See Appendix A.)

NA - Not applicable. Applies to beta dose and sum of betas if there are no beta-emitting radionuclides above detection limits; to sum of alphas if there are no alphaemitting radionuclides above detection limits; and to total radium if neither radium-226 or radium-228 was above detection limit

UAZ UTRA - Upper Aquifer Zone of the Upper Three Runs Aquifer; LAZ UTRA - Lower Aquifer Zone of the Upper Three Runs Aquiler, Gordon - Gordon Aquifer 
WELL FSB104C (cont.)

Radioactive Constituents

\begin{tabular}{|c|c|c|c|c|c|c|c|c|c|c|c|c|c|}
\hline Constituents & 1099 & Mod & Filt. & STH & DF & Lab & 2099 & Mod & Filt. & ST H & DF & Lat & Unit \\
\hline $\begin{array}{l}\text { Americium-241 } \\
\text { Beta dose }\end{array}$ & $\begin{array}{l}<5.2 \mathrm{E}-04 \\
0.11\end{array}$ & $\mathrm{U} / \mathrm{I}$ & $<2.8 \mathrm{E}-01$ & & 1 & GP & & & & & & & pCin \\
\hline Carbon-14 & $3.1 E+01$ & $\|$ & & & 1 & GP & & & & & & & $\mathrm{pCin}$ \\
\hline Cesium-137 & $<7.6 E-01$ & $U / I$ & $<5.1 E+\infty 0$ & & 1 & GP & -. & & & & & & $\mathrm{pCin}$ \\
\hline Cobalt-60 & $<-1.9 E+\infty$ & $\mathrm{U} / \prime$ & $<4.0 E+00$ & & 1 & GP & & & & & & & pCin \\
\hline Curium-242 & $<6.7 E-02$ & U// & $<2.2 E-01$ & & 9 & GP & & & & & & & pCin \\
\hline Curium-243/244 & $<3.2 E-02$ & $\mathrm{U} / \mathrm{I}$ & $<1.4 E-01$ & & 1 & GP & & & & & & & $\mathrm{pCin}$ \\
\hline Curium-245/246 & $<2.1 E-02$ & U/I & $<6.4 E-02$ & & 1 & GP & & & & & & & $p \operatorname{cin}$ \\
\hline Gross alpha & $3.4 E+\infty$ & $\|$ & & & 1 & GP & $1.7 E+00$ & $\mathrm{~J} / \mathrm{V}$ & NDD & & 1 & GP & pCin \\
\hline lodine-129 & $<7.3 E+00$ & UN/ & $<3.0 \mathrm{E}+00$ & & 1 & GP & & & & & & & pCin \\
\hline Nonvolatile beta & $2.3 E+01$ & "I & & & 1 & GP & $3.0 E+01$ & "I & & & 1 & GP & pCin \\
\hline Plutonium-238 & $<-3.8 \mathrm{E}-02$ & $U / I$ & $<2.2 \mathrm{E}-01$ & & 1 & GP & & & & & & & $p \operatorname{cin}$ \\
\hline Plutonium-239/240 & $<-2.2 \mathrm{E}-02$ & $\mathrm{U} / /$ & $<1.3 \mathrm{E}-01$ & & 1 & GP & & & & & & & p cin \\
\hline Radium-226 & $9.0 E-01$ & $\mathrm{~J} / \mathrm{V}$ & NDD & & 1 & GP & & & & & & & pCir \\
\hline $\begin{array}{l}\text { Radium-228 } \\
\text { Total radium }\end{array}$ & $\begin{array}{l}<5.7 E-02 \\
N A\end{array}$ & U/I & $<1.1 E+\infty$ & & 1 & GP & & & & & & & $\mathrm{pCi \Omega}$ \\
\hline Strontium-90 & $<1.4 \mathrm{E}-01$ & UII & $<9.0 E-01$ & & 1 & GP & & & & & & & pCin \\
\hline Technetium-99 & $8.4 E+01$ & $\|$ & & च & 1 & GP & & & & & & & $p C i n$ \\
\hline Thorium-228 & $7.2 \mathrm{E}-01$ & $\mathrm{R} / \mathrm{4}$ & Rej & & 1 & GP & & & & & & & pCir \\
\hline Thorium-230 & $<-9.0 E-03$ & JU/LI & $<2.0 E-01$ & & 1 & GP & & & & & & & $\mathrm{pCi} \Omega$ \\
\hline $\begin{array}{l}\text { Thorium-232 } \\
\text { Sum of }\end{array}$ & $<-2.7 E-02$ & JUMII & $<2.6 E-01$ & & 1 & GP & & & & & & & pCin \\
\hline $\begin{array}{l}\text { Sum of alphas } \\
\text { Sum of betas }\end{array}$ & $1.1 E+02$ & & & a & & & & & & & & & pCin \\
\hline Tritium & $1.1 E+03$ & "I & & - & 1 & GP & $1.0 E+03$ & $1 /$ & & 匹 & 1 & GP & $\mathrm{pCi} / \mathrm{mL}$ \\
\hline Uranium-233/234 & $<7.0 \mathrm{E}-02$ & $\mathrm{U} / 1$ & $<2.9 E-01$ & & 1 & GP & & & & & & & pCin \\
\hline Uranium-235 & $<7.0 \mathrm{E}-02$ & $\mathrm{U} / \prime$ & $<2.9 \mathrm{E}-01$ & & 1 & GP & & & & & & & $\mathrm{pCin}$ \\
\hline Uranium-238 & $<3.5 \mathrm{E}-02$ & $\mathrm{U} / \prime \prime$ & $<2.4 \mathrm{E}-01$ & & 1 & GP & & & & & & & pCin \\
\hline
\end{tabular}

E = exceeded groundwater protection or monitoring constituent standard (See Appendix A.)

NA - Not applicable. Applies to beta dose and sum of betas if there are no beta-emitting radionuclides above detection limits; to sum of alphas if there are no alphaemitting radionuclides above detection limits; and to total radium if neither radium-226 or radium-228 was above detection limit

UAZ_UTRA - Upper Aquifer Zone of the Upper Three Runs Aquifer, LAZ UTRA - Lower Aquifer Zone of the Upper Three Runs Aquifer, Gordon - Gordon Aquifer 


\section{WELL FSB104D}

$\begin{array}{ll}\text { SRS Coord. } & \text { Lat/Longitude } \\ \text { N73865.2 } & 33.269506^{\circ} \mathrm{N} \\ \text { E49255.4 } & 81.680579 \text { W }\end{array}$

SAMPLE DATE
Screen Zone Elevation

210.4-190.4 ft msl
Top of Casing

$219.2 \mathrm{ft} \mathrm{ms}$
Casing Pumo

4" PVC V
Screen Zone

UAZ_UTRA
$05 / 04 / 99$
Unit

$\mathrm{ft} \mathrm{msl}$

pH

$\mu \mathrm{S} / \mathrm{cm}$

${ }^{\circ} \mathrm{C}$

mgl

well volumes

ft ms

Volumes purged

Synchronous water level

$204.3(03 / 23 / 99)$

ANALYTICAL DATA

Inorganic Constituents

Constituents

Aluminum, total recoverable Antimony, total recoverable

Arsenic, total recoverable

Barium, total recoverable

Beryllium, total recoverable

Cadmium, total recoverable 3.1

Chromium, total recoverable 1.7

Cobalt, total recoverable

Copper, total recoverable

Cyanide

Lead, total recoverable

Mercury, total recoverable

Nickel, total tecoverable

Nitrate as nitrogen

Nitrate-nitrite as nitrogen

Selenium, total recoverable

Silver, total recoverable

Thallium, total recoverable

Vanadium, total recoverable

Zinc, total recoverable

1099
9,900
$<0.20$
$<3.0$
66

3.1
1.7
13
18
$<10$
$<2.0$
$<0.20$
6.5

24,000
$<5.0$
$<1.0$
$<0.094$
$<10$
22

Mod

II

U/I

U/I

II

$$
\text { II }
$$

J

II

U//

U/t

$\mathrm{U} / /$

II

U/I/

U/I

JU//4

U/I

Organic Constituents

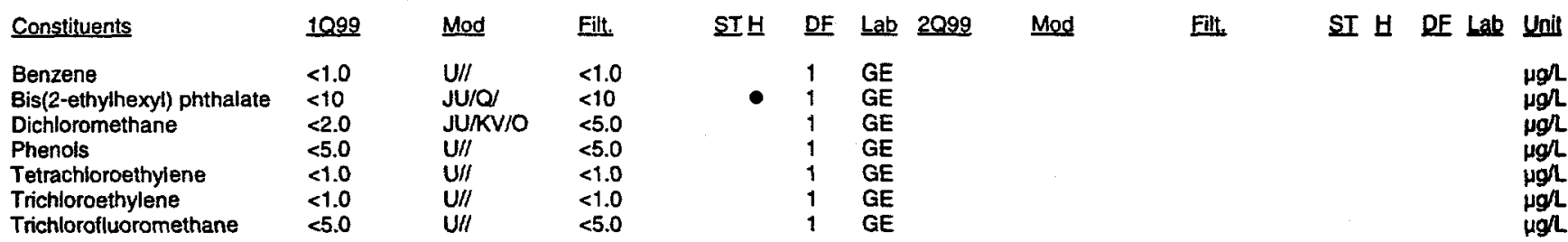

Notes:

- exceeded holding time

I = exceeded groundwater protection or monitoring constituent standard (See Appendix A.)

NA - Not applicable. Applies to beta dose and sum of betas if there are no beta-emitting radionuclides above detection limits; to sum of alphas if there are no alphaemitting radionuclides above detection limits; and to total radium if neither radium-226 or radium-228 was above detection timit

UAZ UTRA - Upper Aquifer Zone of the Upper Three Runs Aquifer, LAZ UTRA - Lower Aquifer Zone of the Upper Three Runs Aquifer, Gordon - Gordon Aquifer 
WELL FSB104D (cont.)

Radioactive Constituents

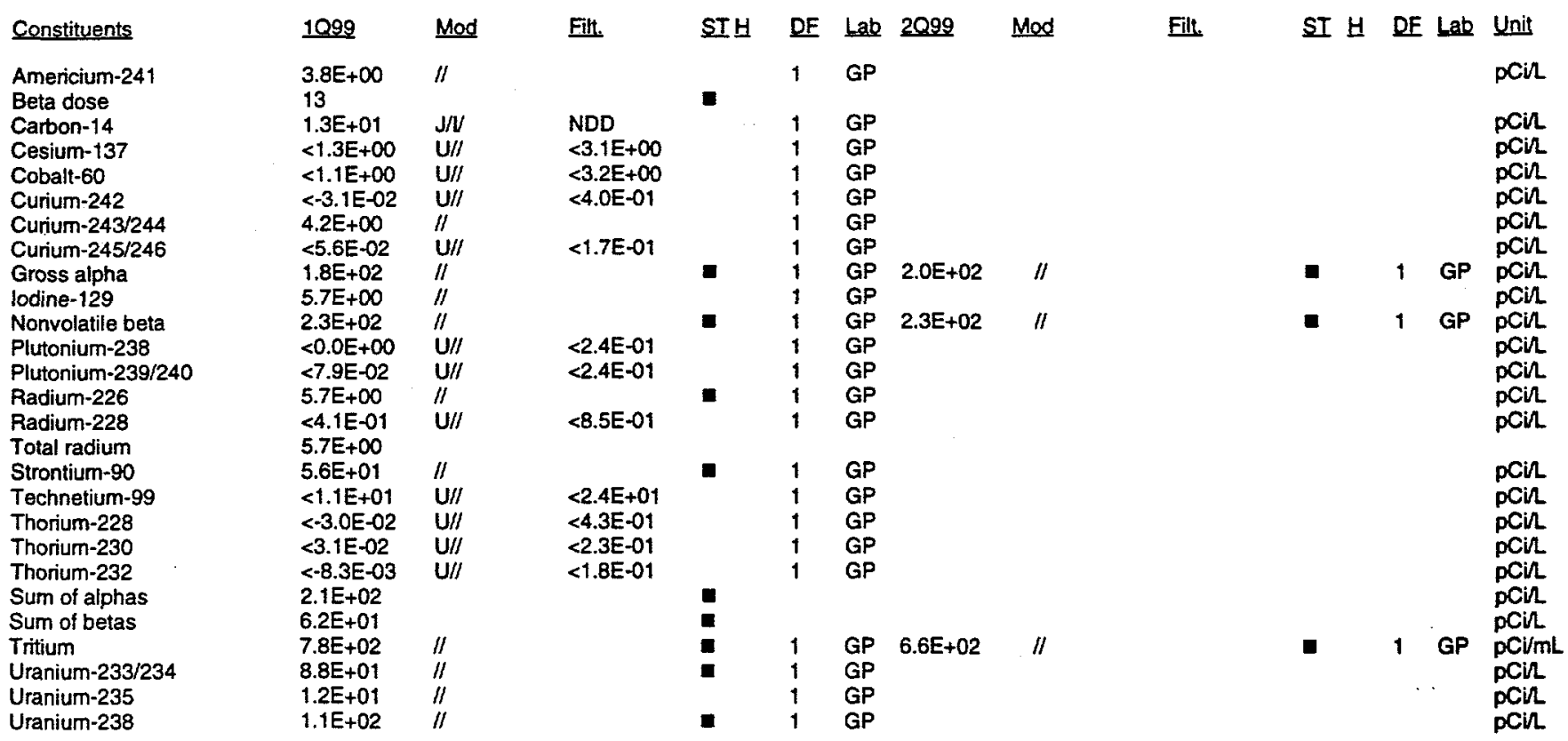

Notes:

= exceeded holding time

- = exceeded groundwater protection or monitoring constituent standard (See Appendix A.)

NA - Not applicable. Applies to beta dose and sum of betas if there are no beta-emitting radionuclides above detection limits; to sum of alphas if there are no alphaemitting radionuclides above delection limits; and to total radium if neither radium-226 or radium-228 was above detection limit

UAZ UTRA - Upper Aquifer Zone of the Upper Three Runs Aquifer, LAZ UTRA - Lower Aquifer Zone of the Upper Three Runs Aquifer, Gordon - Gordon Aquifer

F-Area HWMF

C-105

First and Second Quarter 1999 


\section{WELL FSB105C}

$\begin{array}{ll}\text { SRS Coord. } & \text { Latlonoitude } \\ \text { N75234.2 } & 33.273468^{\circ} \mathrm{N} \\ \text { E49828.0 } & 81.681732^{\circ} \mathrm{W}\end{array}$

SAMPLE DATE

\section{FIELD DATA}

Constituents

1099

Water elevation

$\mathrm{pH}$

Sp. conductance

Water temperature

Alkalinity as $\mathrm{CaCO}$

Turbidity

Volumes purged

Sampling code

Synchronous water leve
Screen Zone Elevation

$151.5-141.5 \mathrm{ft} \mathrm{ms}$
Top of Casing

285.8 it $\mathrm{ms}$ !
Casing Pumo

4" PVC V
Screen Zone

LAZ_UTRA

$\begin{array}{ll}20.99 & \text { Unit } \\ 208.0 & \\ 3.5 & \mathrm{tt} \text { mst } \\ 1100 & \mathrm{pH} \\ 20.3 & \mathrm{\mu S} / \mathrm{cm} \\ 0 & { }^{\circ} \mathrm{C} \\ 0.3 & \mathrm{mgh} \\ 2.2 & \mathrm{NTU} \\ 207.2(06 / 25 / 99) & \text { well volumes }\end{array}$

ANALYTICAL DATA

Inorganic Constituents
208.6

3.9

1000

20.6

0

2.4

$206.6(03 / 23 / 99)$

\begin{tabular}{|c|c|c|c|c|c|c|c|c|c|c|c|c|c|}
\hline Constituents & 1099 & $\underline{\text { Mod }}$ & Filt. & STH & $\underline{\text { DF }}$ & $\underline{\text { Lab }}$ & $2 Q 99$ & Mod & Filt. & ST 브 & DF & Lab & Unit \\
\hline $\begin{array}{l}\text { Aluminum, total recoverable } \\
\text { Antimony, total recoverable } \\
\text { Arsenic, total recoverable } \\
\text { Barium, total recoverable } \\
\text { Beryllium, total recoverable }\end{array}$ & $\begin{array}{l}34,000 \\
<0.29 \\
<60 \\
380\end{array}$ & $\begin{array}{l}/ / \\
J U / / 4 \\
U / / \\
/ /\end{array}$ & $\begin{array}{l}<0.89 \\
<60\end{array}$ & & $\begin{array}{l}20 \\
1 \\
20 \\
1\end{array}$ & $\begin{array}{l}\mathrm{GE} \\
\mathrm{GE} \\
\mathrm{GE} \\
\mathrm{GE}\end{array}$ & 38,000 & $/ /$ & & & 1 & GE & $\begin{array}{l}\mu g / \\
\mu g h \\
\mu g / L \\
\mu g / 2\end{array}$ \\
\hline Cadmium, total recoverable & 13 & $/ /$ & & $\mathbf{n}$ & 1 & GE & 15 & /I & & $\mathbf{a}$ & 1 & GE & $\mu g /$ \\
\hline Chromium, total recoverable & 1.1 & $\mathrm{~J} / 1 /$ & NDD & & 1 & GE & & & & & & & \\
\hline $\begin{array}{l}\text { Cobalt, total recoverable } \\
\text { Copper, total recoverable }\end{array}$ & $\begin{array}{l}290 \\
47\end{array}$ & & & $\mathbf{a}$ & $\begin{array}{l}1 \\
1\end{array}$ & $\begin{array}{l}\text { GE } \\
\text { GE }\end{array}$ & & & & & & & ugh \\
\hline Cyanide & $<10$ & $\mathrm{U} / /$ & $<10$ & & 1 & GE & & & & & & & \\
\hline Lead, total recoverable & 0.40 & $\mathrm{~J} / \mathrm{V}$ & NDD & & 1 & GE & $<20$ & U/I & $<20$ & & 10 & GE & ugh \\
\hline $\begin{array}{l}\text { Mercury, total recoverable } \\
\text { Nickel, total recoverabie } \\
\text { Nitrate as nitrogen }\end{array}$ & $\begin{array}{l}<0.35 \\
44\end{array}$ & $\mathrm{UN}_{/ /}$ & $<0.20$ & & $\begin{array}{l}1 \\
1\end{array}$ & $\begin{array}{l}\text { GE } \\
\text { GE }\end{array}$ & & & & & & & $\mu g h$ \\
\hline Nitrate-nitrite as nitrogen & 140,000 & 11 & & : & 50 & GE & 130,000 & $\|$ & & $\mathbf{n}$ & 50 & GE & $\mu g /$ \\
\hline Selenium, total recoverable & $<100$ & $\mathrm{U} / /$ & $<100$ & & 20 & GE & & & & & & & $\mu g /$ \\
\hline Silver, total recoverable & $<1.0$ & $\mathrm{U} / /$ & $<1.0$ & & 1 & GE & & & & & & & $\mu g h$ \\
\hline Thallium, total recoverable & 0.53 & $\mathrm{~J} / \mathrm{V}$ & NDD & & 1 & GE & & & & & & & \\
\hline $\begin{array}{l}\text { Vanadium, total recoverable } \\
\text { Zinc, total recoverable }\end{array}$ & $\begin{array}{l}<10 \\
130\end{array}$ & $\begin{array}{l}\text { U/I } \\
\| /\end{array}$ & $<10$ & & $\begin{array}{l}1 \\
1\end{array}$ & GE & & & & & & & \\
\hline
\end{tabular}

Organic Constituents

\begin{tabular}{|c|c|c|c|c|c|c|c|c|c|c|}
\hline Constituents & 1099 & Mod & Filt. & ST브 & $\underline{\text { DF }}$ & Lab 2099 & Mod & Filt. & SI $H$ DF Lab & Unit \\
\hline Benzene & $<1.0$ & $U / /$ & $<1.0$ & & 1 & GE & & & & $\mu \sigma^{\prime}$ \\
\hline Bis(2-ethylhexyl) phthalate & $<10$ & $\mathbf{U} / !$ & $<10$ & & 1 & GE & & & & $\mu g / L$ \\
\hline Dichloromethane & $<5.0$ & $\mathrm{U} / \prime$ & $<5.0$ & & 1 & GE & & & & $\mu g /$ \\
\hline Phenols & $<5.0$ & $\mathbf{U} / /$ & $<5.0$ & & 1 & GE & & & & $\mu g /$ \\
\hline Tetrachloroethylene & $<1.0$ & $\mathrm{U} / /$ & $<1.0$ & & 1 & GE & & & & $\mu g / L$ \\
\hline Trichloroethylene & 17 & 11 & & $\mathbf{n}$ & 1 & GE & & & & $\mu g h$ \\
\hline Trichlorofluoromethane & $<5.0$ & $u / /$ & $<5.0$ & & 1 & GE & & & & \\
\hline
\end{tabular}

- exceeded holding time

= exceeded groundwater protection or monitoring constituent standard (See Appendix A.)

NA - Not applicable. Applies to beta dose and sum of betas if there are no beta-emitting radionuclides above detection limits; to sum of alphas if there are no alphaemitting radionuclides above detection limits; and to total radium if neither radium-226 or radium-228 was above detection limit

UAZ_UTRA - Upper Aquifer Zone of the Upper Three Runs Aquifer, LAZ UTRA - Lower Aquifer Zone of the Upper Three Runs Aquifer, Gordon - Gordon Aquifer 


\section{WELL FSB105C (cont.)}

Radioactive Constituents

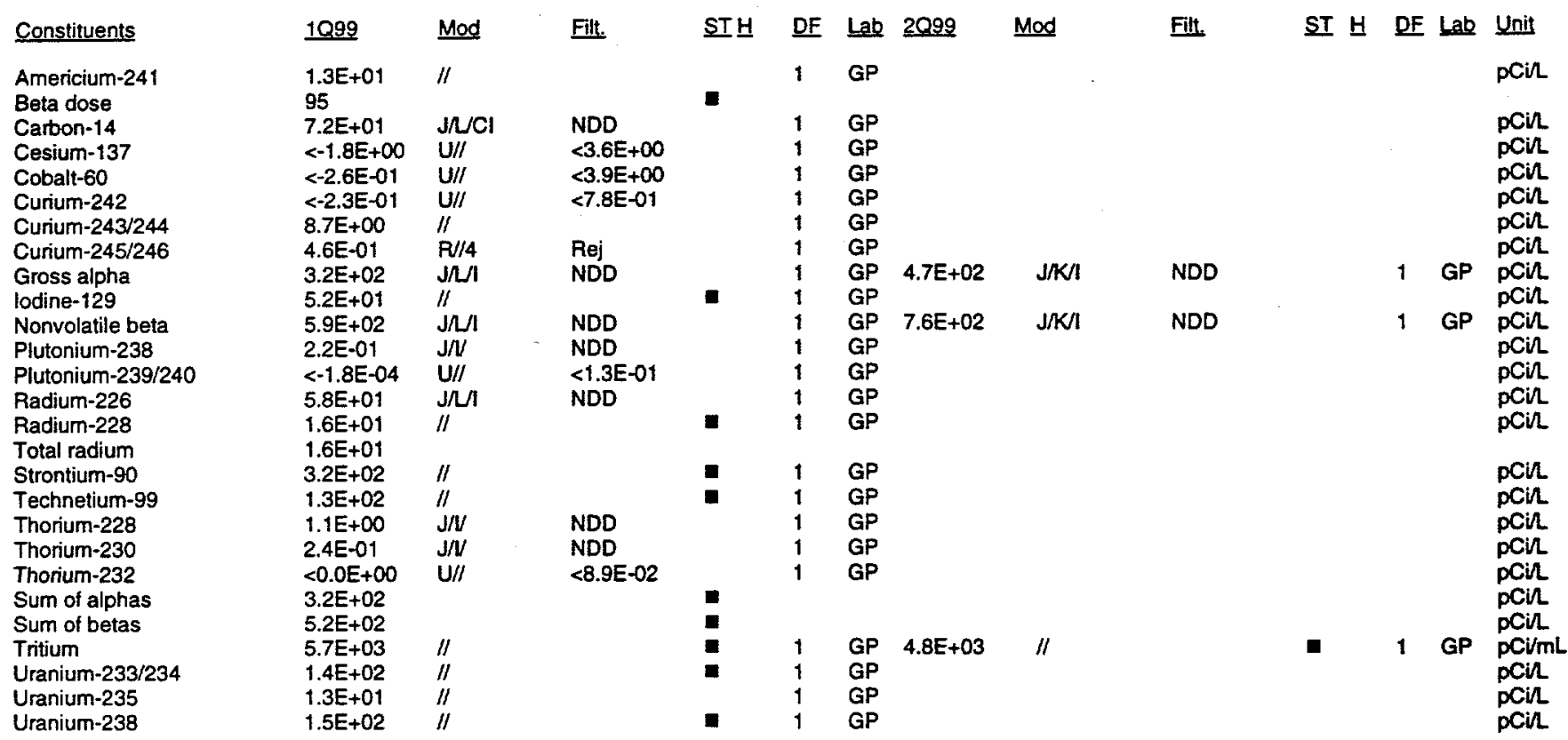

Notes:

- exceeded holding time

D = exceeded groundwater protection or monitoring constituent standard (See Appendix A.)

NA - Not applicable. Applies to beta dose and sum of betas if there are no beta-emitting radionuclides above detection limits; to sum of alphas if there are no alpheemitting radionuclides above detection limits; and to total radium if neither radium-226 or radium-228 was above detection limit

UAZ_UTRA - Upper Aquifer Zone of the Upper Three Runs Aquifer, LAZ UTRA - Lower Aquifer Zone of the Upper Three Runs Aquifer, Gordon - Gordon Aquiler

F-Area HWMF

C-107

First and Second Quarter 1999 
WELL FSB105DR

$\begin{array}{lll}\text { SRS Coord. } & \text { Latlongitude } \\ \text { N75258.1 } & & 33.273542 \text { oN } \\ \text { E49841.0 } & 81.681744 \text { o }\end{array}$

SAMPLE DATE

1099

211.6

3.7

85

20.3

0

14

3.2

$210.8(03 / 23 / 99)$
Screen Zone Elevation

208.6-188.5 $\mathrm{ft} \mathrm{ms}$
Top of Casing

$285.6 \mathrm{ft} \mathrm{msl}$
Casing Pump

4" PVC V
Screen Zone

UAZ_UTRA
04/06/99

2099
210.7
4.2
68
19.9
0
1
2.5
$210.0(06 / 25 / 99)$

Unit

ft ms!

$\mathrm{pH}$

$\mu$ Sicm

${ }^{\circ} \mathrm{C}$

mgh

NTU

well volumes

ft msl

\section{ANALYTICAL DATA}

Inorganic Constituents

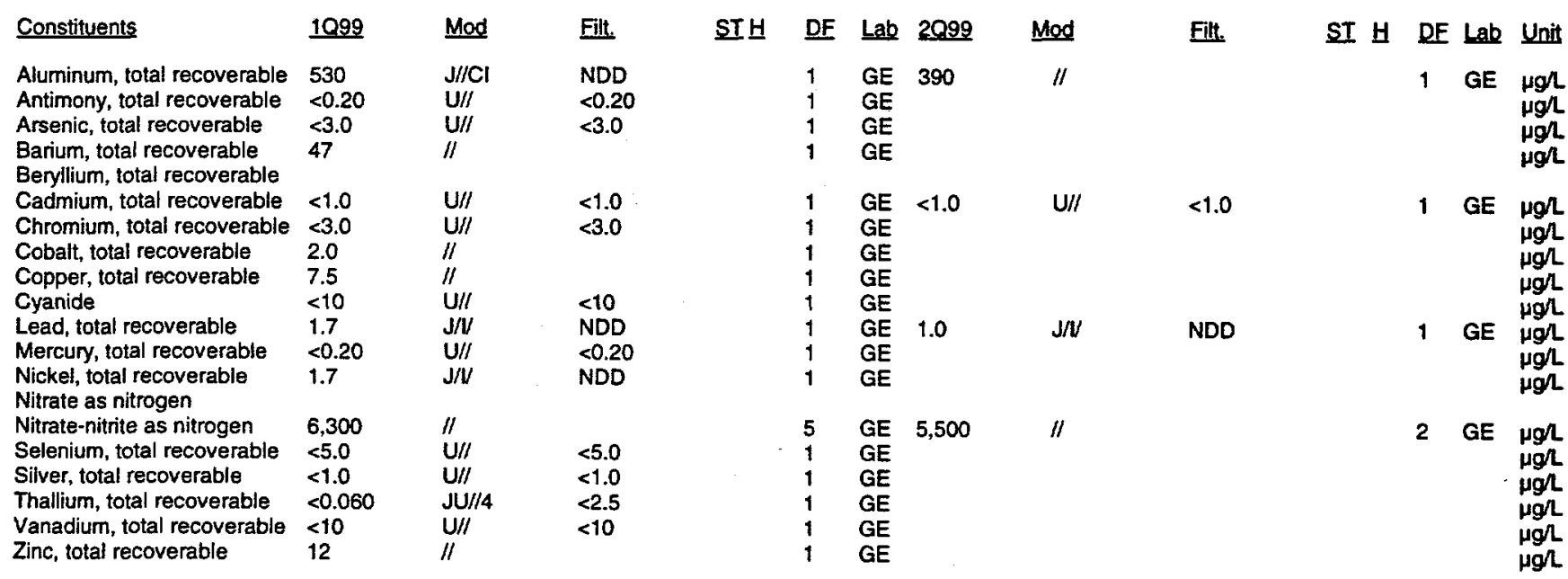

Organic Constituents

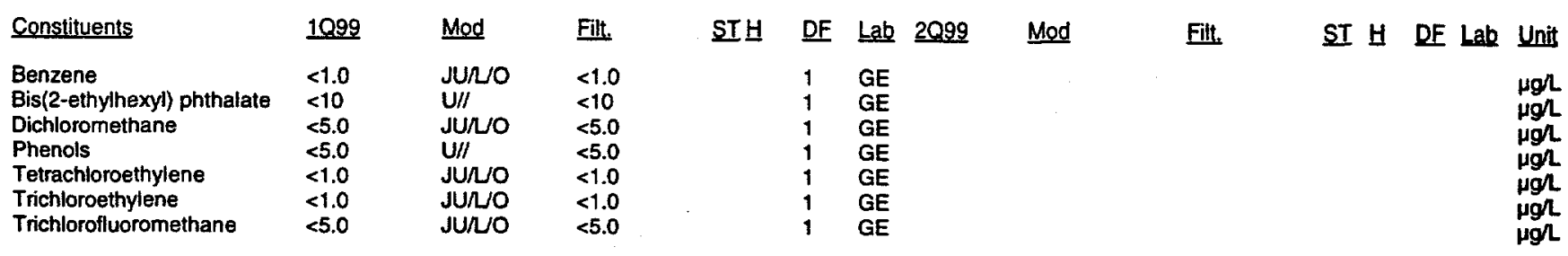

Notes:

$\checkmark=$ exceeded holding time

E = exceeded groundwater protection or monitoring constituent standard (See Appendix A.)

NA - Not applicable. Applies to beta dose and sum of betas if there are no beta-emitting radionuclides above detection limits; to sum of alphas if there are no alphaemitting radionuclides above detection limits; and to total radium if neither radium-226 or radium-228 was above detection limit

UAZ_UTRA - Upper Aquifer Zone of the Upper Three Runs Aquifer, LAZ_UTRA - Lower Aquifer Zone of the Upper Three Runs Aquifer, Gordon - Gordon Aquifer 


\section{WELL FSB105DR (cont.)}

Radioactive Constituents

\begin{tabular}{|c|c|c|c|c|c|c|c|c|c|c|c|c|c|}
\hline Constituents & 1099 & Mod & Filt. & STH & $\underline{D F}$ & Lab & 2099 & Mod & Filt. & ST $\underline{H}$ & DF & Lab & Unit \\
\hline $\begin{array}{l}\text { Americium-241 } \\
\text { Beta dose }\end{array}$ & $\begin{array}{l}<3.8 E-01 \\
13\end{array}$ & $\mathrm{U} / /$ & $<4.0 E-01$ & E & 1 & GP & & & & & & & pCin \\
\hline Carbon-14 & $\begin{array}{l}9.9 E+\infty 0 \\
<7.8 E-01\end{array}$ & $\mathrm{~J} / \mathrm{V}$ & NDD & & 1 & GP & & & & & & & pcin \\
\hline $\begin{array}{l}\text { Cesium-137 } \\
\text { Cobalt-60 }\end{array}$ & $<4.7 E-01$ & $\mathrm{U} / \mathrm{I}$ & $\begin{array}{l}<3.7 E+\infty \\
<4.4 E+\infty\end{array}$ & & $\begin{array}{l}1 \\
1\end{array}$ & $\begin{array}{l}\text { GP } \\
\text { GP }\end{array}$ & & & & & & & $\begin{array}{l}\text { pCir } \\
\text { pCir }\end{array}$ \\
\hline Curium-242 & $<-4.2 E-02$ & $\mathrm{U} / /$ & $<3.3 E-01$ & & 1 & GP & & & & & & & pCil \\
\hline $\begin{array}{l}\text { Curium-243/244 } \\
\text { Curium-245/246 }\end{array}$ & $\begin{array}{l}<9.1 E-02 \\
<0.0 F+00\end{array}$ & $\mathrm{U} / \prime$ & $<6.0 E-01$ & & 1 & $G P$ & & & & & & & pCin \\
\hline $\begin{array}{l}\text { Cunium-245/246 } \\
\text { Gross alpha }\end{array}$ & $6.4 E+01$ & Un & $<1.2<-01$ & 口 & $\begin{array}{l}1 \\
1\end{array}$ & $\begin{array}{l}\text { GP } \\
\text { GP }\end{array}$ & $5.9 E+01$ & $\|$ & & 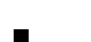 & 1 & $G P$ & pCin \\
\hline Jodine-129 & $1.2 E+01$ & /I & & & 1 & $G P$ & & $"$ & & & I & or & pCin \\
\hline Nonvolatile beta & $4.8 E+01$ & $\| 1$ & & & 1 & GP & $3.2 E+01$ & $\|$ & & & 1 & GP & pCin \\
\hline Plutonium-238 & $<5.1 E-02$ & $\mathrm{U} / /$ & $<1.1 E-01$ & & 1 & GP & & & & & & & pCil \\
\hline $\begin{array}{l}\text { Plutonium-239/240 } \\
\text { Radium-226 }\end{array}$ & $\begin{array}{l}<-4.8 E-03 \\
3.2 E+00\end{array}$ & $\mathrm{U} / /$ & $<8.2 E-02$ & & 1 & $\begin{array}{l}\text { GP } \\
\text { GP }\end{array}$ & & & & & & & pCin \\
\hline Radium-228 & $1.9 \mathrm{E}+\infty 0$ & $\mathrm{~J} / \mathrm{V}$ & NDD & & $i$ & GP & & & & & & & $\begin{array}{l}\text { pcin } \\
\text { pCin }\end{array}$ \\
\hline Total radium & $3.2 E+\infty 0$ & & & & & & & & & & & & \\
\hline Strontium-90 & $4.4 \mathrm{E}+\infty$ & $/ 1$ & & & 1 & GP & & & & & & & pCin \\
\hline Technetium-99 & $<8.3 E+00$ & $U / /$ & $<1.9 E+01$ & & 1 & GP & & & & & & & pCin \\
\hline Thorium-228 & $<1.7 E-01$ & $U / /$ & $<4.1 E-01$ & & 1 & GP & & & & & & & pcin \\
\hline Thorium-230 & $<5.4 E-02$ & $\mathrm{U} / I$ & $<1.6 \mathrm{E}-01$ & & 1 & GP & & & & & & & pCin \\
\hline Thorium-232 & $<2.3 E-02$ & $\mathrm{U} / /$ & $<1.6 E-01$ & & 1 & GP & & & & & & & pCin \\
\hline $\begin{array}{l}\text { Sum of alphas } \\
\text { Sum of betas }\end{array}$ & $\begin{array}{l}\text { 8.1E+01 } \\
1.6 E+01\end{array}$ & & & $\mathbf{E}$ & & & & & & & & & $\begin{array}{l}\text { pCin } \\
\text { pCin }\end{array}$ \\
\hline Tritium & $7.7 E+01$ & $/ 1$ & & a & 1 & GP & $5.1 E+01$ & /I & & $\mathbf{E}$ & 1 & GP & pCi/mL \\
\hline Uranium-233/234 & $2.1 E+01$ & /I & & $\mathbf{n}$ & 1 & GP & & & & & & & pCin \\
\hline Uranium-235 & $2.3 E+00$ & $/ 1$ & & & 1 & GP & & & & & & & $\mathrm{pCin}$ \\
\hline Uranium-238 & $5.7 E+01$ & $/ /$ & & $\mathbf{0}$ & 1 & GP & & & & & & & $\mathrm{pCin}$ \\
\hline
\end{tabular}

Notes;

- exceeded holding time

D = exceeded groundwater protection or monitoring constituent standard (See Appendix A.)

NA - Not applicable. Applies to beta dose and sum of betas if there are no beta-emitting radionuclides above detection limits; to sum of alphas if there are no alphaemitting radionuclides above detection limits; and to total radium if neither radium-226 or radium-228 was above detection limit

UAZ_UTAA - Upper Aquifer Zone of the Upper Three Runs Aquifer, LAZ UTRA - Lower Aquifer Zone of the Upper Three Runs Aquifer, Gordon - Gordon Aquifer 


\section{WELL FSB106C}

$\begin{array}{ll}\text { SRS Coord. } & \text { Latllongitude } \\ \text { N74190.1 } & 33.272503^{\circ} \mathrm{N} \\ \text { E50651.3 } & 81.677536^{\circ} \mathrm{W}\end{array}$

SAMPLE DATE

\section{FIELD DATA}

Constituents

Water elevation

$\mathrm{pH}$

Sp. conductance

Water temperature

Alkalinity as $\mathrm{CaCO} 3$

Turbidity

Volumes purged

Sampling code

Synchronous water level

\section{ANALYTICAL DATA}

Inorganic Constituents
Screen Zone Elevation

$166.0-156.0 \mathrm{ft} \mathrm{msl}$

01/20/99
2099

201.1

5.3

470

18.4

5

2.5

$200.3(06 / 28 / 99)$
Casing Pumo

4"PVC V
Screen Zone

LAZ_UTRA

04/06/99

5.1

800

2

2.7

$201.0(03 / 23 / 99)$
Unit

It msl

$\mathrm{pH}$

$\mu \mathrm{S} / \mathrm{cm}$

${ }^{\circ} \mathrm{C}$

mgl

well volumes

$\mathrm{ft} \mathrm{msl}$

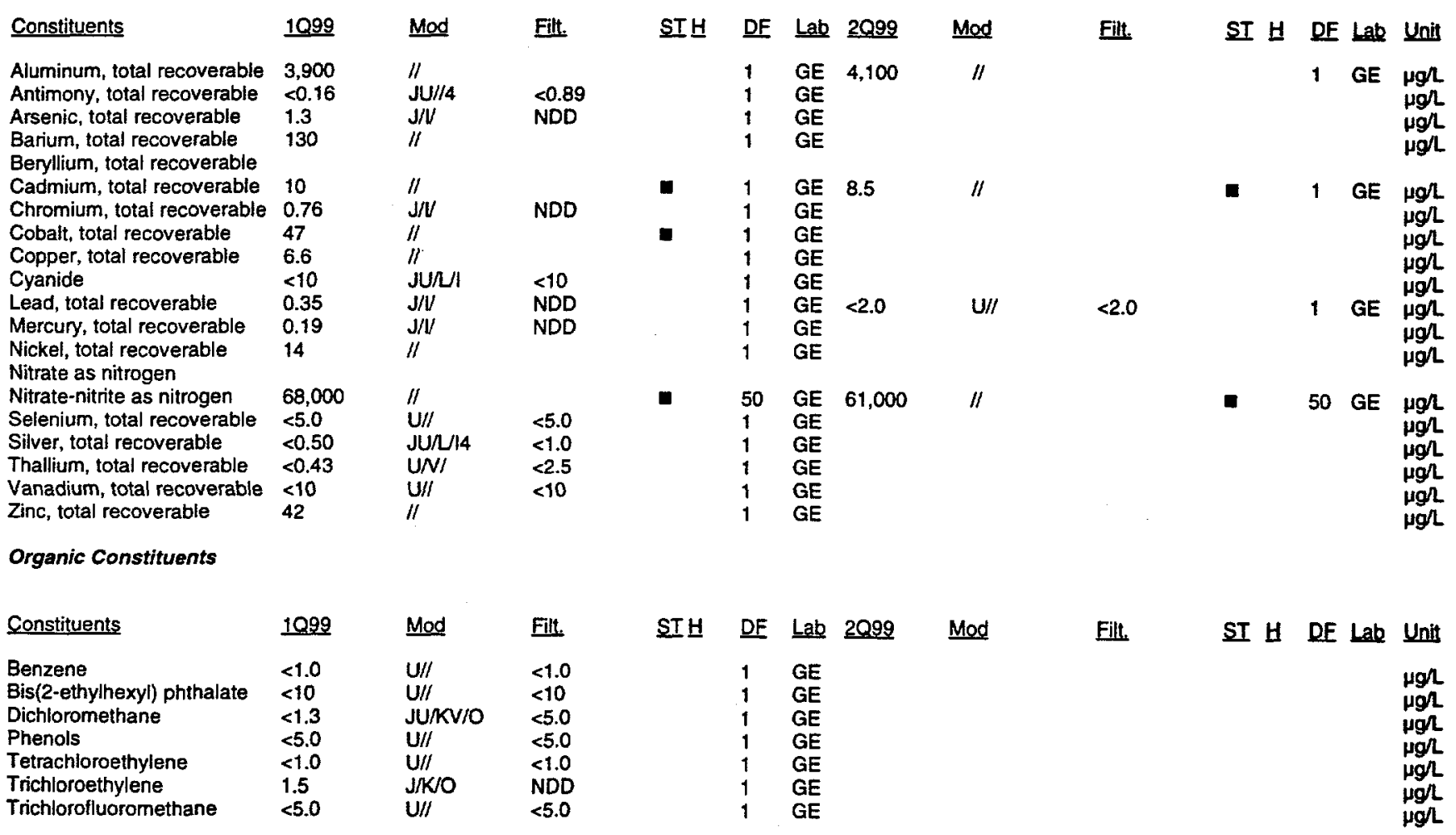

Notes:

- exceeded holding time

= exceeded groundwater protection or monitoring constituent standard (See Appendix A.)

NA - Not applicable. Applies to beta dose and sum of betas if there are no beta-emitting radionuclides above detection limits; to sum of alphas if there are no alphaemitting radionuclides above detection limits; and to total radium if neither radium-226 or radium-228 was above detection limit

UAZ_UTRA - Upper Aquifer Zone of the Upper Three Runs Aquifer; LAZ_UTRA - Lower Aquifer Zone of the Upper Three Runs Aquifer, Gordon - Gordon Aquifer 
WELL FSB106C (cont.)

Radioactive Constituents

\begin{tabular}{|c|c|c|c|c|c|c|c|c|c|c|c|c|c|}
\hline Constituents & 1099 & $\underline{\text { Mod }}$ & Fill. & ST브 & DF & $\underline{\text { Lab }}$ & 2099 & Mod & Fill. & ST $H$ & DE & Lab & Unit \\
\hline $\begin{array}{l}\text { Americium-241 } \\
\text { Beta dose }\end{array}$ & $\begin{array}{l}<1.8 E-01 \\
31\end{array}$ & $u / I$ & $<2.7 E-01$ & E & 1 & GP & & & & & & & pCir \\
\hline Carbon-14 & $1.1 E+02$ & "I & & $\mathbf{m}$ & 1 & GP & & & & & & & pCin \\
\hline Cesium-137 & $<1.3 E+\infty 0$ & $U / I$ & $<3.9 E+\infty 0$ & & 1 & GP & & & & & & & pCil \\
\hline Cobalt-60 & $<1.9 \mathrm{E}+\infty 0$ & $\mathrm{U} / \prime$ & $<4.9 E+00$ & & 1 & GP & & & & & & & pCin \\
\hline Curium-242 & $<0.0 E+00$ & U/I & $<2.5 \mathrm{E}-01$ & & 1 & GP & & & & & & & pCin \\
\hline Curium-243/244 & $<0.0 E+\infty 0$ & $u \prime \prime \prime$ & $<2.2 \mathrm{E}-01$ & & 1 & GP & & & & & & & pCin \\
\hline Curium-245/246 & $<0.0 E+00$ & $\mathrm{U} / \prime$ & $<2.7 E-01$ & & 1 & GP & & & & & & & pCin \\
\hline Gross alpha & 2.5E+01 & "I & & च & 1 & GP & $4.6 E+01$ & $\|$ & & $=$ & 1 & GP & pCin \\
\hline lodine-129 & $7.1 E+01$ & $\mathrm{~J} / \mathrm{K} / \mathrm{C}$ & NDD & & 1 & GP & & & & & & & pCin \\
\hline Nonvolatile beta & $5.6 E+02$ & 11 & & 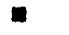 & 1 & GP & $5.6 \mathrm{E}+02$ & II & & a & 1 & GP & pCin \\
\hline Plutonium-238 & $<7.8 \mathrm{E}-03$ & $\mathrm{U} / \prime$ & $<9.9 E-02$ & & 1 & GP & & & & & & & pCir \\
\hline Plutonium-239/240 & $<0.0 E+00$ & $\mathrm{U} / \prime \prime$ & $<4.7 E-02$ & & 1 & GP & & & & & & & $\mathrm{pCin}$ \\
\hline Radium-226 & $1.8 E+01$ & 11 & & a & 1 & GP & & & & & & & pCir \\
\hline Radium-228 & $\begin{array}{l}<2.3 E-01 \\
18 E+01\end{array}$ & $\mathrm{U} / \mathrm{I}$ & $<8.9 E-01$ & & 1 & GP & & & & & & & $\mathrm{pCin}$ \\
\hline $\begin{array}{l}\text { Total radium } \\
\text { Strontium-90 }\end{array}$ & $\begin{array}{l}1.8 E+01 \\
2.5 E+02\end{array}$ & II & & च & 1 & GP & & & & & & & pCin \\
\hline Technetium-99 & $1.1 E+02$ & $\|$ & & - & 1 & GP & & & & & & & $\mathrm{pCin}$ \\
\hline Thorium-228 & $<6.7 \mathrm{E}-02$ & U/I & $<4.1 E-01$ & & 1 & GP & & & & & & & pCin \\
\hline Thorium-230 & $<9.0 \mathrm{E}-02$ & $\mathrm{U} / /$ & $<1,6 \mathrm{E}-01$ & & 1 & GP & & & & & & & pCin \\
\hline Thorium-232 & $<2.0 \mathrm{E}-02$ & U/I & $<1,4 E-01$ & & 1 & GP & & & & & & & pCin \\
\hline Sum of alphas & NA & & & & & & & & & & & & \\
\hline Sum of betas & $4.7 E+02$ & & & 문 & & & & & & & & & pCin \\
\hline Tritium & $1.1 E+03$ & 11 & & - & 1 & GP & $1.0 E+03$ & 11 & & $=$ & 1 & GP & pCi/mL \\
\hline Uranium-233/234 & $3.9 E-01$ & $\mathrm{~J} / N$ & NDD & & 1 & GP & & & & & & & pCin \\
\hline Uranium-235 & $<1.0 \mathrm{E}-01$ & $\mathrm{U} / \mathrm{I}$ & $<1.9 \mathrm{E}-01$ & & 1 & GP & & & & & & & pCin \\
\hline Uranium-238 & $5.5 E-01$ & $\mathrm{~J} / \mathrm{V}$ & NDD & & 1 & GP & & & & & & & pCin \\
\hline
\end{tabular}

Notes:

- = exceeded holding time

= exceeded groundwater protection or monitoring constituent standard (See Appendix A.)

NA - Not applicable. Applies to beta dose and sum of betas if there are no beta-emitting radionuclides above detection limits; to sum of alphas if there are no alphaemitting radionuclides above detection limits; and to total radium if neither radium-226 or radium-228 was above detection limit

UAZ_UTRA - Upper Aquifer Zone of the Upper Three Runs Aquifer, LAZ_UTRA - Lower Aquifer Zone of the Upper Three Runs Aquifer; Gordon - Gordon Aquifer 


\section{WELL FSB106D}

$\begin{array}{ll}\text { SRS Coord. } & \text { Latlonaitude } \\ \text { N74193.0 } & 33.272485^{\circ} \\ \text { E50636.8 } & 81.677580^{\circ} \mathrm{W}\end{array}$

SAMPLE DATE

$01 / 12 / 99$

Screen Zone Elevation

222.9-202.9 ft ms
Top of Casing

$234.9 \mathrm{ft} \mathrm{msi}$
Casing Pump

4" PVC S
Screen Zone

UAZ_UTRA
04/26/99

2099

6.2

80

21.2

33

$\mathrm{X}$

(06/28/99)
Unit

ft msi

pH

uS/cm

${ }^{\circ} \mathrm{C}$

NTU

fi msl

Sampling code
Synchronous water level

$\mathrm{XN}$

$205.5(03 / 23 / 99)$

ANALYTICAL DATA

Inorganic Constituents

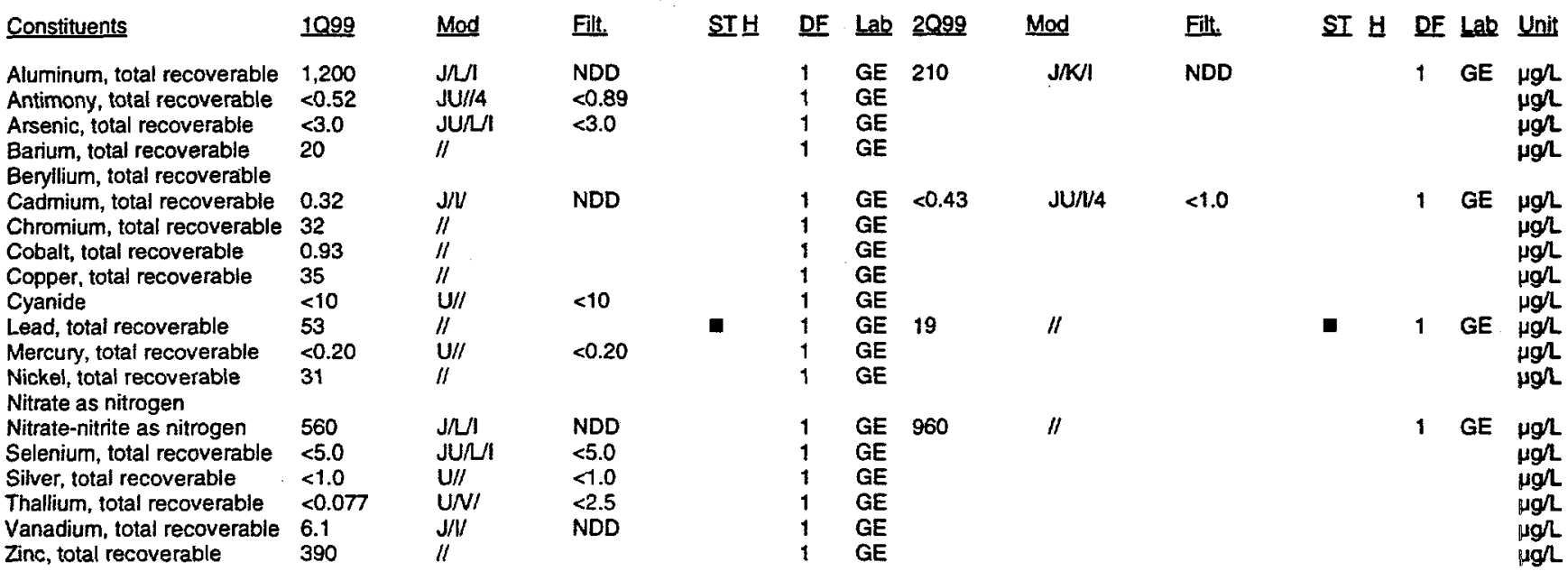

Organic Constituents

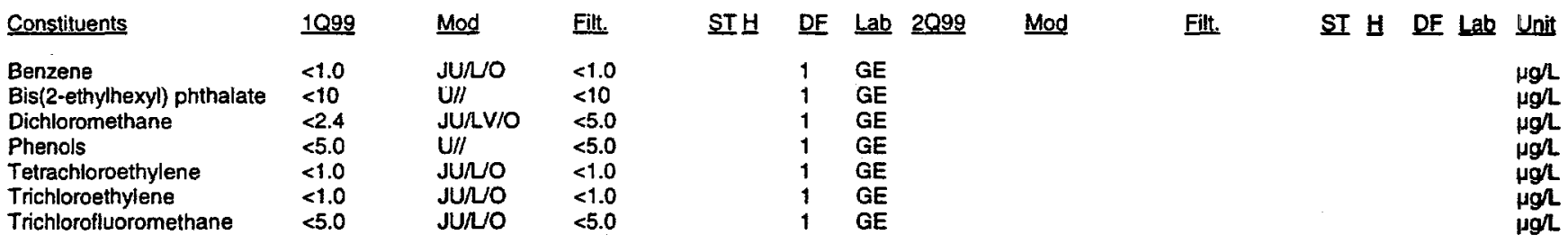

= exceeded holding time

NA - Not applicable. Applies to beta dose and sum of betas if there are no beta-emitting radionuclides above detection limits; to sum of alphas if there are no alphaemitting radionuclides above detection limits; and to total radium if neither radium-226 or radium-228 was above detection limit

UAZ_UTAA - Upper Aquifer Zone of the Upper Three Runs Aquifer, LAZ_UTRA - Lower Aquifer Zone of the Upper Three Runs Aquifer, Gordon - Gordon Aquifer 


\section{WELL FSB106D (cont.)}

Radioactive Constituents

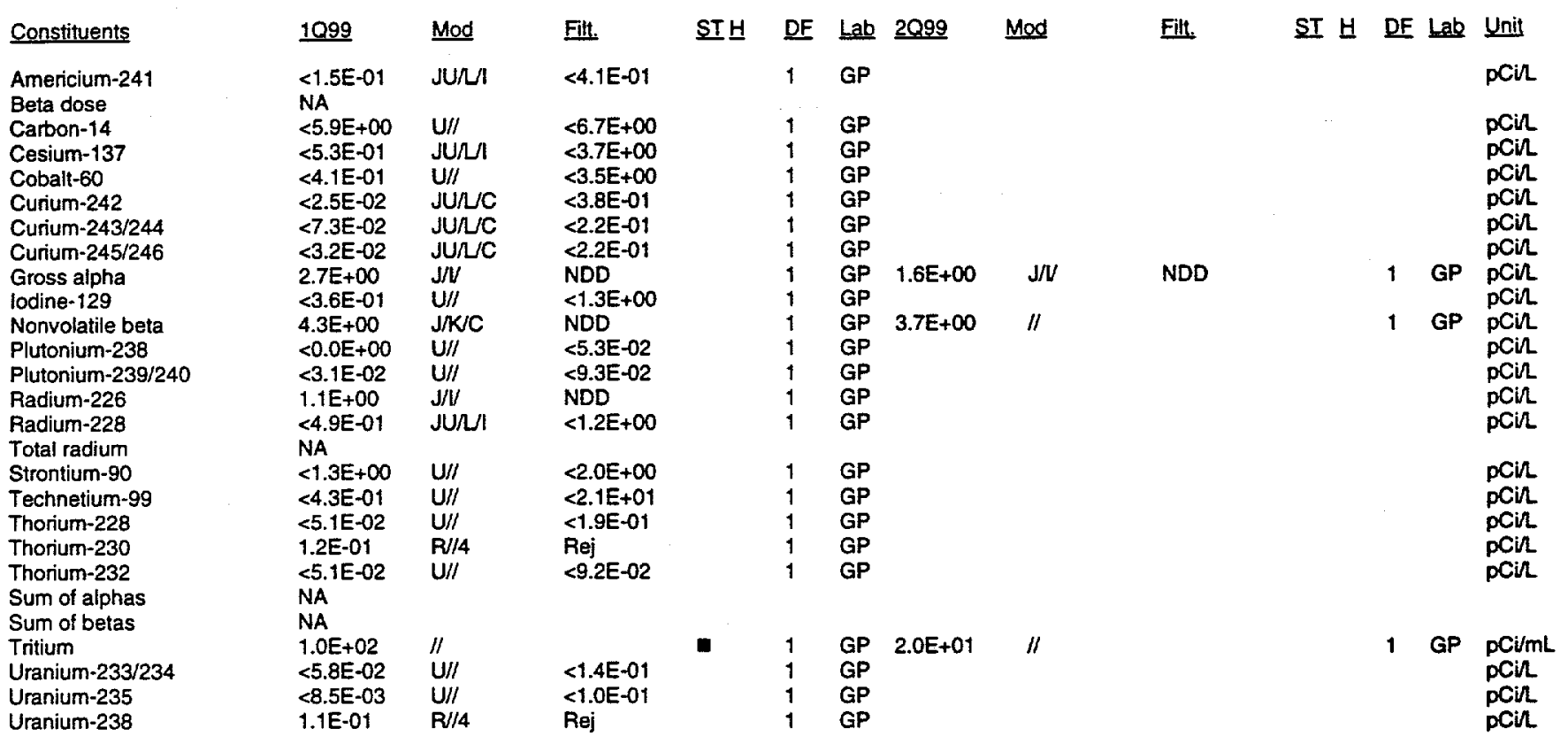

Notes:

= exceeded holding time

= exceeded groundwater protection or monitoring constituent standard (See Appendix A.)

NA - Not applicable. Applies to beta dose and sum of betas if there are no beta-emitting radionuclides above detection limits; to sum of alphas if there are no alpheemitting radionuclides above detection limits; and to total radium if neither radium-226 or radium-228 was above detection limit

UAZ_UTRA - Upper Aquifer Zone of the Upper Three Runs Aquifer, LAZ_UTRA - Lower Aquifer Zone of the Upper Three Runs Aquifer, Gordon - Gordon Aquifer 


\section{WELL FSB107C}

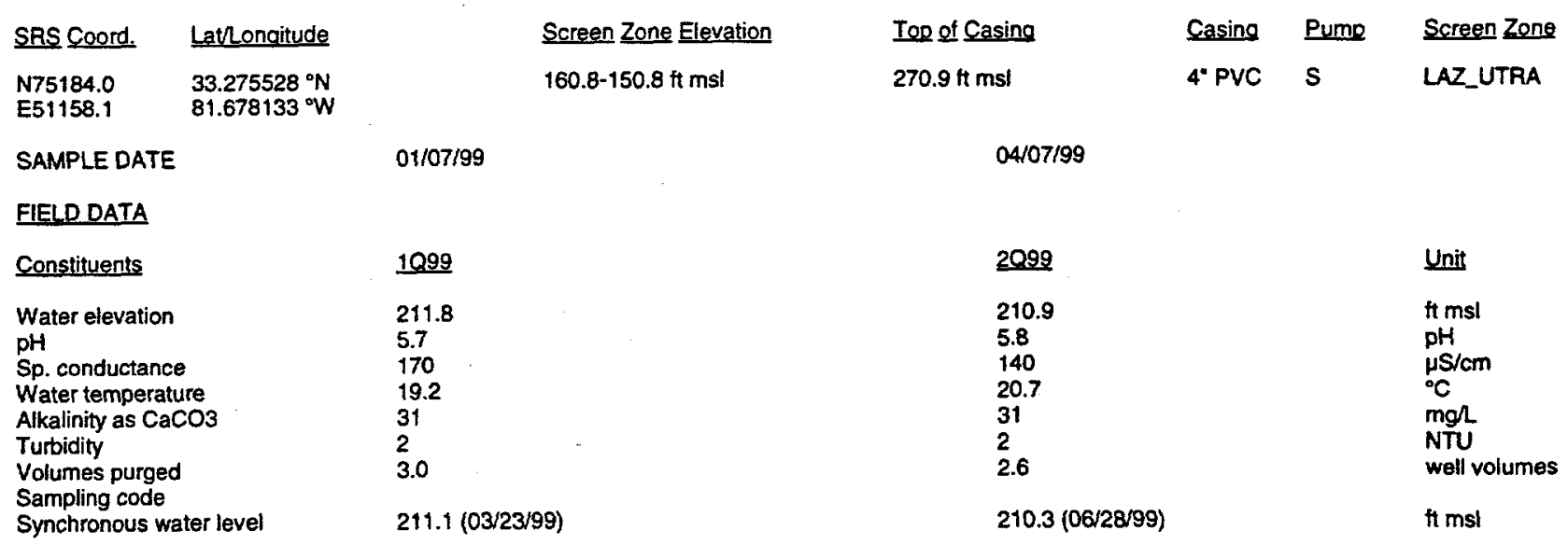

ANALYTICAL DATA

Inorganic Constituents

\begin{tabular}{|c|c|c|c|c|c|c|c|c|c|c|c|c|c|}
\hline Constituents & 1099 & Mod & Filt. & STH & DF & $\underline{\text { Lab }}$ & 2099 & Mod & Filt. & ST 브 & DF & Lab & Unit \\
\hline Aluminum, total recoverable & 50 & II & & & 1 & GE & $<56$ & UNI & $<15$ & & 1 & GE & $\mu g h$ \\
\hline Antimony, total recoverable & $<0.20$ & $\mathrm{u} / \prime$ & $<0.20$ & & 1 & GE & & & & & & & $\mu g^{\prime}$ \\
\hline $\begin{array}{l}\text { Arsenic, total recoverable } \\
\text { Barium, total recoverable }\end{array}$ & $\begin{array}{l}<3.0 \\
40\end{array}$ & $\begin{array}{l}U^{\prime} / / \\
\end{array}$ & $<3.0$ & & $\begin{array}{l}1 \\
1\end{array}$ & $\begin{array}{l}\text { GE } \\
\text { GE }\end{array}$ & & & & & & & $\begin{array}{l}\mu g / \\
\mu g h\end{array}$ \\
\hline Beryllium, total recoverable & & & & & & & & & & & & & \\
\hline Cadmium, total recoverable & 0.84 & $\mathrm{~J} / \mathrm{V}$ & NDD & & 1 & GE & $<0.29$ & $J U / / 4$ & $<1.0$ & & 1 & GE & $\mu g /$ \\
\hline Chromium, total recoverable & $\begin{array}{l}1.2 \\
0.34\end{array}$ & $\mathrm{~J} / \mathrm{V}$ & & & $\begin{array}{l}1 \\
1\end{array}$ & GE & & & & & & & ugh \\
\hline $\begin{array}{l}\text { Cobalt, total recoverable } \\
\text { Copper, total recoverable }\end{array}$ & 1.3 & $/ /$ & & & 1 & GE & & & & & & & $\mu g h$ \\
\hline Cyanide & 2.2 & $\mathrm{~J} / \mathrm{V}$ & NDD & & 1 & GE & & & & & & & \\
\hline Lead, total recoverable & 0.47 & $\mathrm{~J} / \mathrm{V}$ & NDD & & 1 & GE & $<2.0$ & $U / /$ & $<2.0$ & & 1 & GE & \\
\hline Mercury, total recoverable & 0.067 & $\mathrm{~J} / \mathrm{V}$ & NDD & & 1 & $\mathrm{GE}$ & & & & & & & \\
\hline Nickel, total recoverable & 4.7 & $/ /$ & & & 1 & GE & & & & & & & $\mu g$ \\
\hline Nitrate as nitrogen & & & & & & & & & & & & & \\
\hline Nitrate-nitrite as nitrogen & 9,700 & $/ 1$ & & & 5 & GE & 7,500 & $\|$ & & & 5 & GE & \\
\hline Selenium, total recoverable & $<5.0$ & $U / /$ & $<5.0$ & & 1 & GE & & & & & & & \\
\hline Silver, total recoverable & $<1.0$ & $\mathrm{U} / /$ & $<1.0$ & & 1 & GE & & & & & & & $\mu g$ \\
\hline Thallium, total recoverable & $<0.072$ & $\mathrm{JU} / / 4$ & $<2.5$ & & 1 & GE & & & & & & & ng \\
\hline Vanadium, total recoverable & $<10$ & $\mathrm{U} / 1$ & $<10$ & & 1 & GE & & & & & & & $\mu \mathrm{g}$ \\
\hline Zinc, total recoverable & 22 & $/ 1$ & & & 1 & GE & & & & & & & \\
\hline
\end{tabular}

Organic Constituents

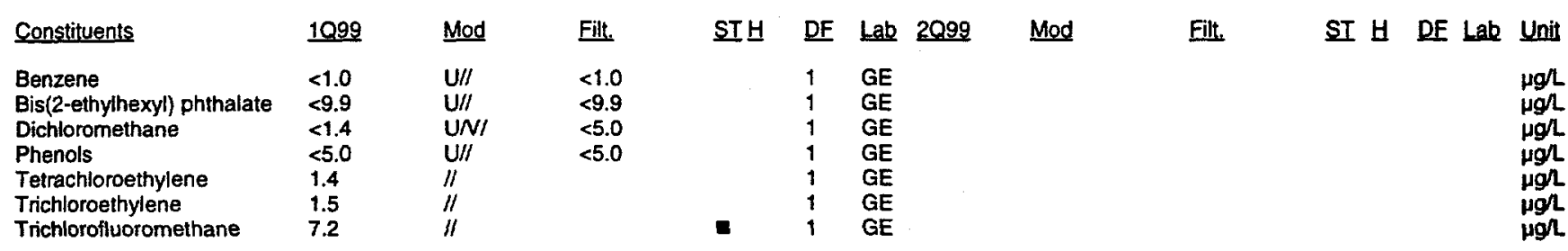

Notes:

- = exceeded holding time

= exceeded groundwater protection or monitoring constituent standard (See Appendix A.)

NA - Not applicable. Applies to beta dose and sum of betas if there are no beta-emitting radionuclides above detection limits; to sum of alphas if there are no alphaemitting radionuclides above detection limits; and to total radium if neither radium-226 or radium-228 was above detection limit

UAZ UTRA - Upper Aquifer Zone of the Upper Three Runs Aquifer; LAZ UTRA - Lower Aquifer Zone of the Upper Three Runs Aquifer, Gordon - Gordon Aquifer 


\section{WELL FSB107C (cont.)}

Radioactive Constituents

\begin{tabular}{|c|c|c|c|c|c|c|c|c|c|c|c|c|c|}
\hline Constituents & 1099 & Mod & Filt. & ST브 & DE & Lab & 2099 & Mod & Filt. & ST $\underline{H}$ & DF & Lab & Unit \\
\hline $\begin{array}{l}\text { Americium-241 } \\
\text { Beta dose }\end{array}$ & $\begin{array}{l}<3.9 E-02 \\
1.9\end{array}$ & $U / /$ & $<8.7 E-02$ & च & 1 & GP & & & & & & & pCin \\
\hline Carbon-14 & $9.9 \mathrm{E}+\infty 0$ & $\mathrm{~J} / \mathrm{V}$ & NDD & & 1 & GP & & & & & & & pCin \\
\hline Cesium- 137 & $<-9.7 E-02$ & Jun/C & $<3.5 E+\infty 0$ & & 1 & GP & & & & & & & pcin \\
\hline Cobalt-60 & $\angle 4.9 E-01$ & $U / I$ & $<4.0 E+00$ & & 1 & GP & & & & & & & pCin \\
\hline Curium-242 & $<0.0 \mathrm{E}+00$ & $U / I$ & $<4.5 E-02$ & & 1 & GP & & & & & & & pCin \\
\hline Curium-243/244 & $<2.1 E-02$ & $\mathrm{U} / \mathrm{I}$ & $<8.7 E-02$ & & 1 & GP & & & & & & & $\mathrm{pCin}$ \\
\hline Curium-245/246 & $<0.0 E+\infty 0$ & $U / I$ & $<4.1 E-02$ & & 1 & GP & & & & & & & pCin \\
\hline Gross alpha & $3.7 E+00$ & 11 & & & 1 & GP & $1.6 E+\infty$ & $\mathrm{J} / \mathrm{V}$ & NDD & & 1 & GP & pCin \\
\hline lodine-129 & $2.3 E+\infty 0$ & $\mathrm{~J} / \mathrm{J}$ & NDD & & 1 & GP & & & & & & & pCin \\
\hline Nonvolatile beta & $4.0 E+01$ & 11 & & & 1 & GP & 3.tE+01 & $\|$ & & & 1 & GP & $\mathrm{pCin}$ \\
\hline Plutonium-238 & 2.2E-01 & $\mathrm{R} / / 4$ & Rej & & 1 & GP & & & & & & & $\mathrm{pCi}$ \\
\hline Plutonium-239/240 & $<1.7 E-02$ & U/I & $<2.6 E-02$ & & 1 & GP & & & & & & & $\mathrm{pCi}$ \\
\hline Radium-226 & $1.1 E+00$ & $\mathrm{~J} / \mathrm{V}$ & NDD & & 1 & GP & & & & & & & pCin \\
\hline $\begin{array}{l}\text { Radium-228 } \\
\text { Total radium }\end{array}$ & $\begin{array}{l}<4.2 E-01 \\
N A\end{array}$ & $\mathrm{U} / /$ & $<4.3 E-01$ & & 1 & GP & & & & & & & $\mathrm{pCi} /$ \\
\hline Strontium-90 & $1.5 E+01$ & $/ 1$ & & घ & 1 & GP & & & & & & & pCin \\
\hline Technetium-99 & $<1.6 \mathrm{E}+01$ & $\mathbf{U} / /$ & $<1.9 E+01$ & & 1 & GP & & & & & & & $\mathrm{pCi} \Omega$ \\
\hline Thorium-228 & $<2.9 E-01$ & $\mathrm{U} / 1$ & $<3.0 \mathrm{E}-01$ & & 1 & GP & & & & & & & pCil \\
\hline Thorium-230 & $<1.3 E-01$ & $U / I$ & $<2.1 E-01$ & & 1 & GP & & & & & & & pCir \\
\hline $\begin{array}{l}\text { Thorium-232 } \\
\text { Sum of alphas }\end{array}$ & $\begin{array}{l}<0.0 E+00 \\
N A\end{array}$ & $\mathrm{U} / \prime$ & $<5.6 \mathrm{E}-02$ & & 1 & GP & & & & & & & pCir \\
\hline Sum of betas & $1.5 E+01$ & & & & & & & & & & & & pCil \\
\hline Tritium & $1.3 E+02$ & $/ 1$ & & $\mathbf{c}$ & 1 & GP & $1.0 E+02$ & $\|$ & & $\mathbf{\square}$ & 1 & GP & $\mathrm{pCi} / \mathrm{ml}$ \\
\hline Uranium-233/234 & 2.2E-01 & $\mathrm{J} / \mathrm{V}$ & NDD & & 1 & GP & & & & & & & pcin \\
\hline Uranium-235 & $<-3.0 \mathrm{E}-02$ & $\mathrm{U} / /$ & $<1.4 \mathrm{E}-01$ & & 1 & GP & & & & & & & pCin \\
\hline Uranium-238 & $<8.9 \mathrm{E}-02$ & $\mathrm{U} / /$ & $<1.1 E-01$ & & 1 & GP & & & & & & & pCil \\
\hline
\end{tabular}

Notes:

= exceeded holding time

= exceeded groundwater protection or monitoring constituent standard (See Appendix A.)

NA - Not applicable. Applies to beta dose and sum of betas it there are no beta-emitting radionuclides above detection limits; to sum of alphas if there are no alpha. emitting radionuclides above detection limits; and to total radium if neither radium-226 or radium-228 was above detection limit

UAZ_UTRA - Upper Aquiter Zone of the Upper Three Runs Aquifer, LAZ UTRA - Lower Aquifer Zone of the Upper Three Runs Aquifer, Gordon - Gordon Aquifer 


\section{WELL FSB107D}

$\begin{array}{ll}\text { SPS Coord. } & \text { Latlongitude } \\ \text { N75177.2 } & 33.275499^{\circ} \\ \text { E51149.8 } & 81.678141^{\circ}\end{array}$

SAMPLE DATE

02/02/99

Screen Zone Elevation

$220.9-200.9 \mathrm{ft} \mathrm{ms})$

FIELDD DATA

Constituents

Water elevation

$\mathrm{pH}$

Sp. conductance

Water temperature

Alkalinity as $\mathrm{CaCO}$

Turbidity

Volumes purged

Sampling code

Synchronous water level

$\begin{array}{llll}\text { Top of Casing } & \text { Casing } & \text { Pump } & \text { Screen Zone } \\ 271 \mathrm{ft} \mathrm{msl} & 4^{*} \text { PVC } & V & \text { UAZ_UTRA }\end{array}$

$05 / 04 / 99$

$\begin{array}{ll}2099 & \text { Unit } \\ 214.6 & \\ 3.7 & \mathrm{tt} \mathrm{msl} \\ 270 & \mathrm{pH} \\ 19.3 & \mu \mathrm{S} / \mathrm{cm} \\ 0 & { }^{\circ} \mathrm{C} \\ 0 & \mathrm{mgl} \\ 2.4 & \mathrm{NTU} \\ 213.9(06 / 28 / 99) & \text { well volumes } \\ & \text { ft msl }\end{array}$

\section{ANALYTICAL DATA}

Inorganic Constituents

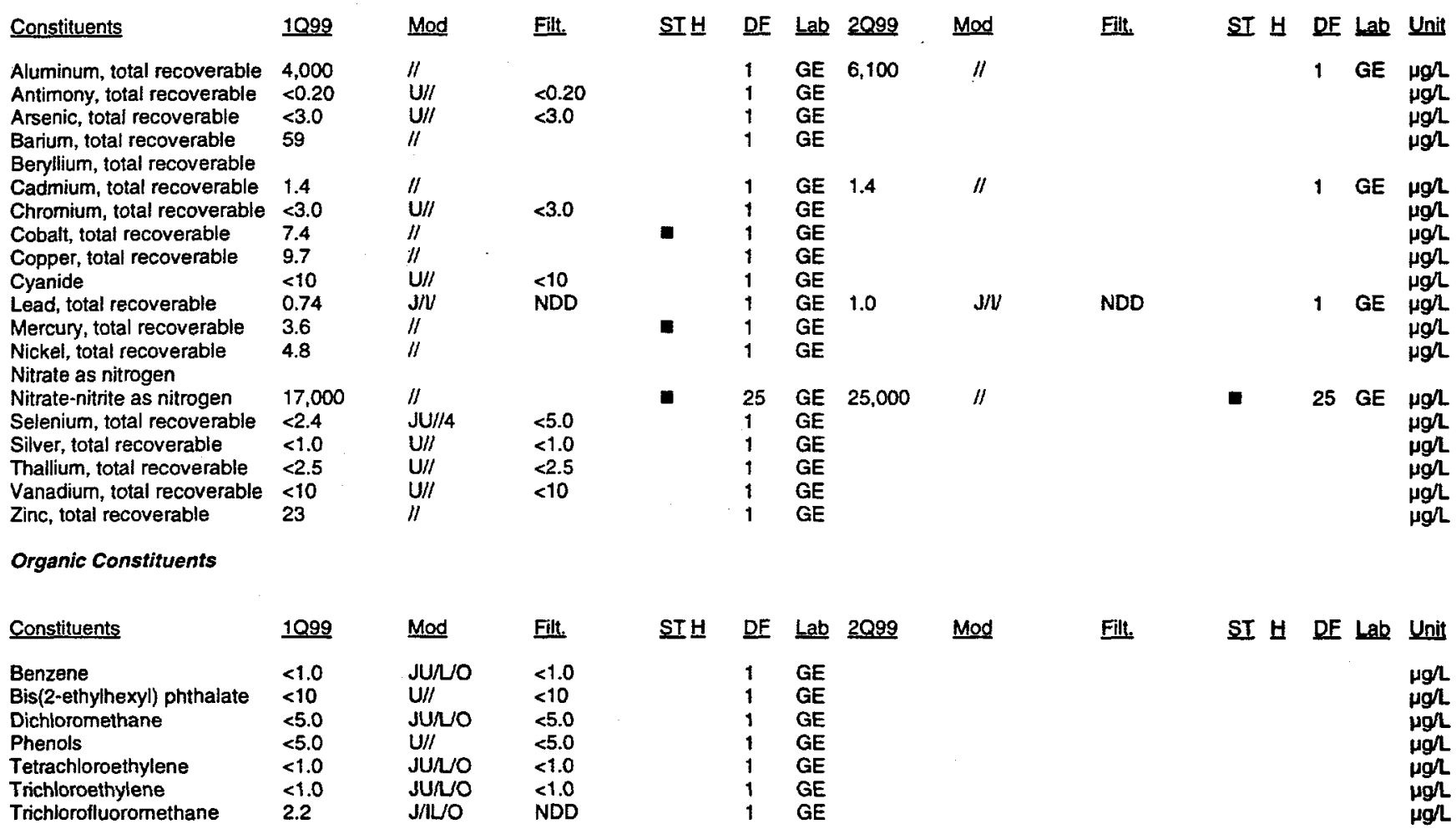

Notes:

- =xceeded holding time

I = exceeded groundwater protection or monitoring constituent standard (See Appendix A.)

NA - Not applicable. Applies to beta dose and sum of betas if there are no beta-emitting radionuclides above detection limits; to sum of alphas if there are no alphaemitting radionuclides above detection limits; and to total radium if neither radium-226 or radium-228 was above detection limit

UAZ_UTRA - Upper Aquifer Zone of the Upper Three Runs Aquifer, LAZ UTRA - Lower Aquifer Zone of the Upper Three Runs Aquifer, Gordon - Gordon Aquifer 


\section{WELL FSB107D (cont.)}

Radioactive Constituents

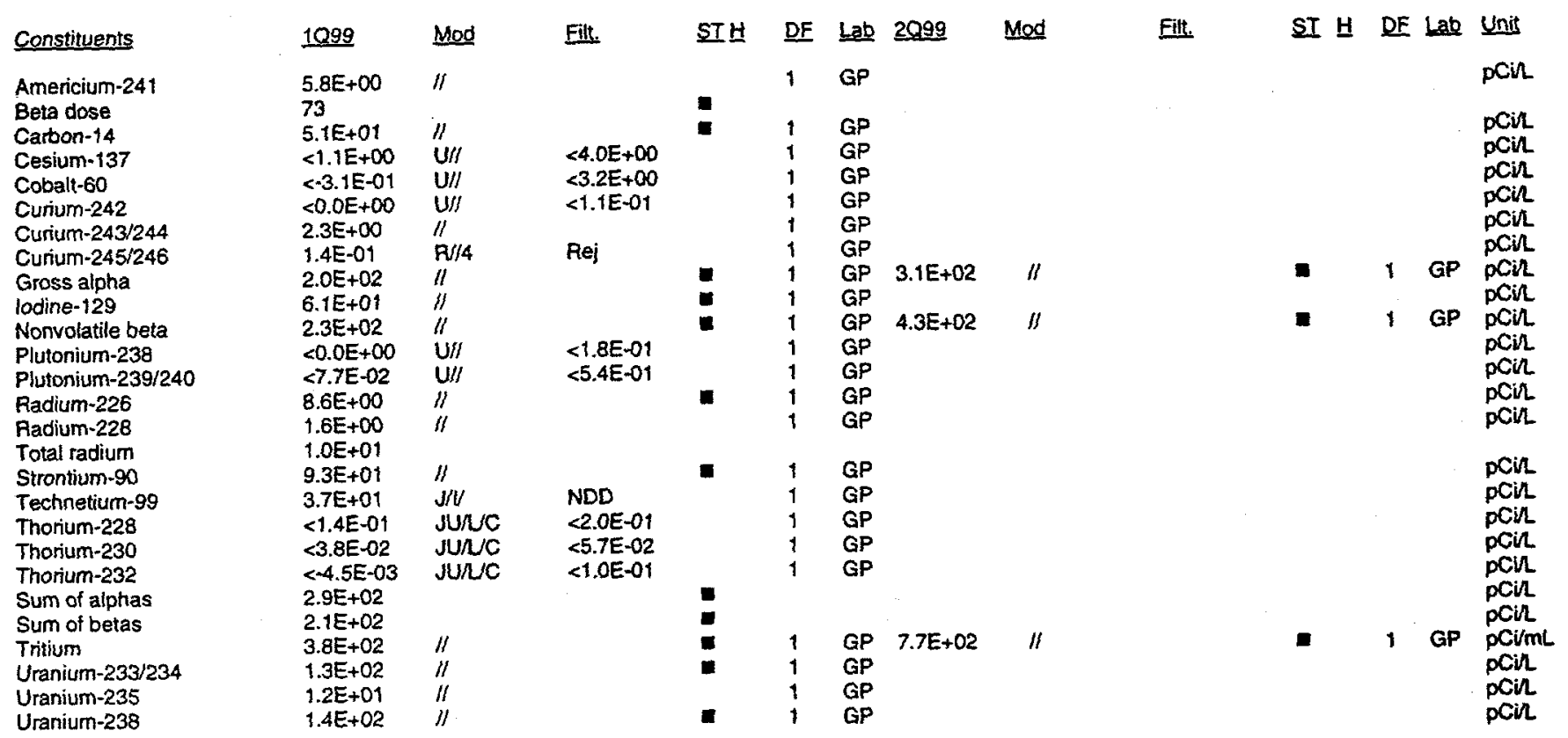

Notes:

- exceeded holding time

= exceeded groundwater protection or monitoring constituent standard (See Appendix A)

NA - Not applicable. Applies to beta dase and sum of betas if there are no beta-mitting radionuclides above detection limits; to sum of alphas if there are no alpheemitting radionuclides above detection limits; and to total radium if neither radium-226 or radium-228 was above detection limit

UAZ UTRA - Upper Aquifer Zone of the Upper Three Runs Aquifer, LAZ UTRA - Lower Aquiter Zone of the Upper Three Runs Aquifer. Gordon - Gordon Aquifer

F-Area HWMF

C-117

First and Second Quarter 1999 


\section{WELL FSB108D}

$\begin{array}{ll}\text { SRS Goord. } & \text { Lathongitude } \\ \text { N76260.7 } & 33.277883^{\circ} \\ \text { E51142.3 } & 81.680266^{\circ} \mathrm{W}\end{array}$

SAMPLE DATE

Screen Zone Elevation

223.8-203.8 $\mathrm{ft} \mathrm{ms}$
Top of Casing

$298 \mathrm{ft} \mathrm{msl}$

$\begin{array}{ll}\text { Casing Pump } & \text { S } \\ \text { 4" PVC } \mathrm{S}\end{array}$

Screen Zone

UAZ_UTRA

$04 / 12 / 99$

\section{FIELD DATA}

Constituents

Water elevation

$\mathrm{PH}$

Sp. conductance

Water temperature

Alkalinity as $\mathrm{CaCO} 3$

Turbidity

Voiumes purged

Sampling code

Synchronous water level

1099

219.6

5.6

38

21.3

3

2.8

$218.6(03 / 23 / 99)$
2099

218.5

5.0

31

20.8

4

3

0.10

$x$

$218.2(06 / 25 / 99)$
Unit

fi msl

pH

$\mu \mathrm{S} / \mathrm{cm}$

${ }^{\circ} \mathrm{C}$

mgl

well volumes

tt msi

\section{ANALYTICAL DATA}

Inorganic Constituents

\begin{tabular}{|c|c|c|c|c|c|c|c|c|c|c|c|c|c|}
\hline Constituents & 1099 & Mod & Filt. & STH & DF & Lab & 2099 & Mod & Filt. & ST H & DF & Lab & Unit \\
\hline Aluminum, total recoverable & 14 & $\mathrm{~J} / \mathrm{V}$ & NDD & & 1 & GE & 69 & $\mathrm{~J} / \mathrm{K} / \mathrm{I}$ & NDD & & 1 & GE & $\mu g /$ \\
\hline Antimony, total recoverable & $<0.20$ & $\mathrm{U} / I$ & $<0.20$ & & 1 & GE & & & & & & & $\mu \mathrm{gh}$ \\
\hline Arsenic, total recoverable & $<3.0$ & $\mathrm{U} / /$ & $<3.0$ & & 1 & GE & & & & & & & $\mu g /$ \\
\hline $\begin{array}{l}\text { Barium, total recoverable } \\
\text { Beryllium, total recoverable }\end{array}$ & 9.5 & $/ /$ & & & 1 & GE & & & & & & & $\mu g /$ \\
\hline Cadmium, total recoverable & $<1.0$ & $\mathrm{U} / \prime$ & $<1.0$ & & 1 & GE & $<1.0$ & UII & $<1.0$ & & 1 & GE & $\mu g h$ \\
\hline Chromium, total recoverable & $<3.0$ & $\mathrm{U} / \prime$ & $<3.0$ & & 1 & GE & & & & & & & \\
\hline Cobalt, total recoverable & 0.16 & $\mathrm{~J} / \mathrm{V}$ & NDD & & 1 & GE & & & & & & & $\mu g /$ \\
\hline Copper, total recoverable & 5.4 & $\|$ & & & 1 & GE & & & & & & & $\mu g h$ \\
\hline Cyanide & $<10$ & $\mathrm{U} / /$ & $<10$ & & 1 & GE & & & & & & & $\mu g h$ \\
\hline Lead, total recoverable & 4.4 & $/ /$ & & & 1 & GE & 8.9 & $\|$ & & & 1 & GE & $\mu g /$ \\
\hline Mercury, total recoverable & $<0.20$ & $U / /$ & $<0.20$ & & 1 & GE & & & & & & & $\mu g / L$ \\
\hline $\begin{array}{l}\text { Nickel, total recoverable } \\
\text { Nitrate as nitrogen }\end{array}$ & 1.9 & $\mathrm{~J} / \mathrm{U}$ & NDD & & 1 & GE & & & & & & & \\
\hline Nitrate-nitrite as nitrogen & 1,200 & $/ / 1$ & & & 1 & GE & 1,100 & $/ /$ & & & 1 & GE & $\mu g$ \\
\hline Selenium, total recoverable & $<5.0$ & $\mathrm{U} / /$ & $<5.0$ & & $i$ & $\overline{\mathrm{GE}}$ & & & & & & & \\
\hline Silver, total recoverable & $<1.0$ & $\mathrm{U} / \mathrm{I}$ & $<1.0$ & & 1 & GE & & & & & & , & ugl \\
\hline Thallium, total recoverable & $<0.051$ & $\mathrm{JU} / / 4$ & $<2.5$ & & 1 & GE & & & & & & & L \\
\hline Vanadium, total recoverable & $<10$ & $\mathbf{U} / \prime$ & $<10$ & & 1 & GE & & & & & & & $\mu g /$ \\
\hline Zinc, total recoverable & 27 & $\| l$ & & & 1 & GE & & & & & & & \\
\hline \multicolumn{14}{|l|}{ Organic Constituents } \\
\hline Constituents & 1099 & Mod & Filt. & ST브 & DF & $\underline{\text { Lab }}$ & 2099 & Mod & Filt. & ST $\mathrm{H}$ & DE & Lab & Unit \\
\hline Benzene & $<1.0$ & $\mathrm{JU} / \mathrm{L} / \mathrm{O}$ & $<1.0$ & & 1 & GE & & & & & & & $\mu g$ \\
\hline Bis(2-ethylhexyl) phthalate & $<10$ & $\mathrm{v} \prime \prime$ & $<10$ & & 1 & GE & & & & & & & \\
\hline Dichloromethane & $<10$ & JUAVIO & $<5.0$ & & 1 & GE & & & & & & & $\mu g h$ \\
\hline Phenols & $<5.0$ & $\mathrm{U} / /$ & $<5.0$ & & 1 & $\overline{G E}$ & & & & & & & $\mu g h$ \\
\hline Tetrachloroethylene & $<1.0$ & JU/L/O & $<1.0$ & & 1 & GE & & & & & & & $\mu g /$ \\
\hline Trichloroethylene & $<1.0$ & JU/LO & $<1.0$ & & 1 & GE & & & & & & & \\
\hline Trichlorofluoromethane & $<5.0$ & JU/LO & $<5.0$ & & 1 & GE & & & & & & & \\
\hline
\end{tabular}

Notes:

- exceeded holding time

= exceeded groundwater protection or monitoring constituent standard (See Appendix A.)

NA - Not applicable. Applies to beta dose and sum of betas if there are no beta-emitting radionuclides above detection limits; to sum of alphas if there are no alphaemitting radionuclides above detection limits; and to total radium if neither radium-226 or radium-228 was above detection limit

UAZ_UTRA - Upper Aquifer Zone of the Upper Three Runs Aquifer, LAZ_UTRA - Lower Aquifer Zone of the Upper Three Runs Aquifer, Gordon - Gordon Aquifer 


\section{WELL FSB108D (cont.)}

\section{Radioactive Constituents}

\begin{tabular}{|c|c|c|c|c|c|c|c|c|c|c|c|c|c|}
\hline Constituents & 1099 & Mod & Filt. & ST브 & DF & Lab & $\underline{2099}$ & Mod & Filt. & SI 브 & DF & Lab & Unit \\
\hline $\begin{array}{l}\text { Americium-241 } \\
\text { Beta dose }\end{array}$ & $\begin{array}{l}<4.1 E-02 \\
\text { NA }\end{array}$ & $\mathrm{U} / /$ & $<7.6 \mathrm{E}-02$ & & 1 & GP & & & & & & & pCin \\
\hline Carbon-14 & $<-4.3 E-01$ & $U / I$ & $<7.7 E+00$ & & 1 & GP & & & & & & & pCin \\
\hline Cesium-137 & $6.6 E+\infty 0$ & $R / / 4$ & Rej & & 1 & GP & & & & & & & pCin \\
\hline $\begin{array}{l}\text { Cobalt-60 } \\
\text { Curium-242 }\end{array}$ & $\begin{array}{l}<-3.4 E-01 \\
<0.0 E+\infty 0\end{array}$ & $\begin{array}{l}\mathrm{u} / / \\
\mathrm{u} / /\end{array}$ & $<3.3 E+\infty 0$ & & 1 & GP & & & & & & & pCin \\
\hline Curium-243/244 & $<-3.2 \mathrm{E}-02$ & $\mathrm{U} / 1$ & $\begin{array}{l}<8.1 E-02 \\
<2.1 E-01\end{array}$ & & $\begin{array}{l}1 \\
1\end{array}$ & $\begin{array}{l}\text { GP } \\
\text { GP }\end{array}$ & & & & & & & $\begin{array}{l}\text { pCin } \\
\text { pCir }\end{array}$ \\
\hline Curium-245/246 & $<0.0 E+\infty 0$ & $\mathrm{U} / /$ & $<7.6 E-02$ & & 1 & GP & & & & & & & pCir \\
\hline $\begin{array}{l}\text { Gross alpha } \\
\text { lodine-129 }\end{array}$ & $\begin{array}{l}<7.2 E-01 \\
<3.4 E-01\end{array}$ & $\begin{array}{l}\mathrm{U} / / \\
\mathrm{U} / /\end{array}$ & $\begin{array}{l}<9.2 E-01 \\
<1.1 E+\infty 0\end{array}$ & & $\begin{array}{l}1 \\
1\end{array}$ & $\begin{array}{l}\text { GP } \\
\text { GP }\end{array}$ & $1.2 E+\infty$ & $\mathrm{J} / \mathrm{V}$ & NDD & & 1 & GP & pCin \\
\hline Nonvolatile beta & $<-2.0 E-01$ & $\mathrm{U} / 1$ & $<1.4 E+\infty$ & & 1 & $\mathrm{GP}$ & $<1.2 E+\infty$ & $u / l$ & $<1.2 E+\infty$ & & 1 & GP & $\begin{array}{l}\text { pCin } \\
\text { pCin }\end{array}$ \\
\hline Plutonium-238 & $<0.0 \mathrm{E}+\infty 0$ & $\mathrm{U} / /$ & $<6.9 E-02$ & & 1 & GP & & & & & & & pCil \\
\hline Plutonium-239/240 & $<2.3 E-02$ & $\mathrm{U} / 1$ & $<6.9 E-02$ & & 1 & GP & & & & & & & pCill \\
\hline Radium-226 & $<3.6 E-01$ & $\mathrm{U} / 1$ & $<6.2 E-01$ & & 1 & GP & & & & & & & pCir \\
\hline $\begin{array}{l}\text { Radium-228 } \\
\text { Total radium }\end{array}$ & $\begin{array}{l}<3.4 E-01 \\
N A\end{array}$ & $U / !$ & $<1.2 \mathrm{E}+00$ & & 1 & GP & & & & & & & pCil \\
\hline Strontium-90 & $<5.7 E-01$ & $\mathrm{U} / /$ & $<1.5 E+\infty$ & & 1 & GP & & & & & & & pCin \\
\hline Technetium-99 & $<1.1 E+00$ & U/I & $<1.8 E+01$ & & 1 & GP & & & & & & & poin \\
\hline Thorium-228 & $1.6 \mathrm{E}-01$ & $R / 4$ & Rej & & 1 & GP & & & & & & & pCir \\
\hline Thorium-230 & $6.0 \mathrm{E}-02$ & $\mathrm{~J} / \mathrm{L} / \mathrm{I}$ & NDD & & 1 & GP & & & & & & & pCin \\
\hline Thorium-232 & $<0.0 E+00$ & JUAI & $<2.4 E-02$ & & 1 & GP & & & & & & & pCin \\
\hline Sum of atphas & NA & & & & & & & & & & & & \\
\hline Sum of betas & NA & & & & & & & & & & & & \\
\hline Tritium & $1.1 E+01$ & 11 & & & 1 & GP & 1.1E+01 & $\|$ & & & 1 & GP & $\mathrm{pCi} / \mathrm{ml}$ \\
\hline Uranium-233/234 & $<9.3 E-03$ & U/I & $<1.7 E-01$ & & 1 & GP & & & & & & & pCin \\
\hline Uranium-235 & $<-1.9 E-02$ & $\mathrm{U} / /$ & $<1.5 \mathrm{E}-01$ & & 1 & GP & & & & & & & pCin \\
\hline Uranium-238 & $<5.7 \mathrm{E}-02$ & $\mathrm{U} / /$ & $<5.7 \mathrm{E}-02$ & & 1 & GP & & & & & & & pCin \\
\hline
\end{tabular}




\section{WELL FSB109D}

$\begin{array}{ll}\text { SRS Coord. } & \text { Latl.ongitude } \\ \text { N75855.9 } & 33.275921^{\circ} \mathrm{N} \\ \text { E50488.6 } & 81.681200^{\circ} \mathrm{W}\end{array}$

SAMPLE DATE

$01 / 11 / 99$

Screen Zone Elevation

225.8-205.8 tt ms

\section{FIELD DATA}

\section{Constituents}

Water elevation

$\mathrm{pH}$

Sp. conductance

Water temperature

Alkalinity as $\mathrm{CaCO} 3$

Turbidity

Volumes purged

Sampling code

Synchronous water level
1099

215.9

6.0

50

20.1

5

6.2

$214.8(03 / 23 / 99)$
Ton of Casing

$293.1 \mathrm{ft}$ msl

Casing Pumo
4*PVC S

Screen Zone

UAZ_UTRA

$04 / 07 / 99$

2099

214.6

5.4

32

22.6

3

4.1

$214.2(06 / 25 / 99)$
Unit

ft msl pH

$\mu \mathrm{Sicm}$

${ }^{\circ} \mathrm{C}$

mgh

well volumes

ft mst

ANALYTICAL DATA

Inorganic Constituents

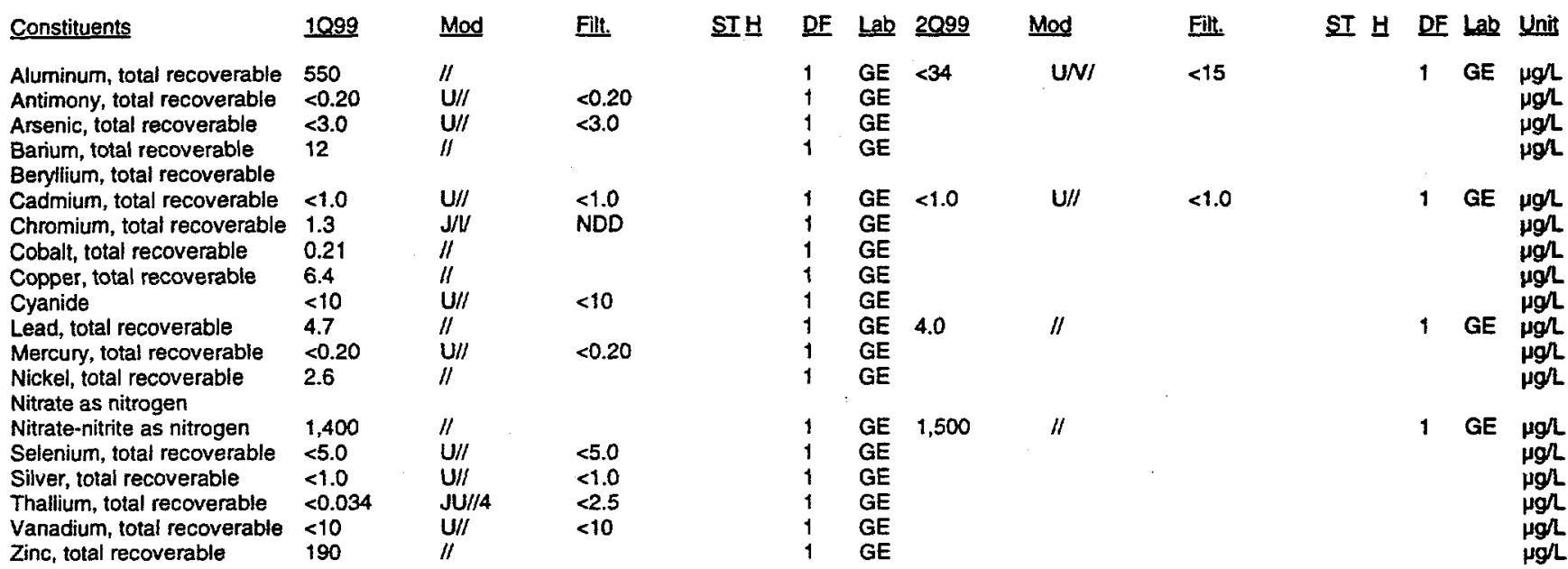

Organic Constituents

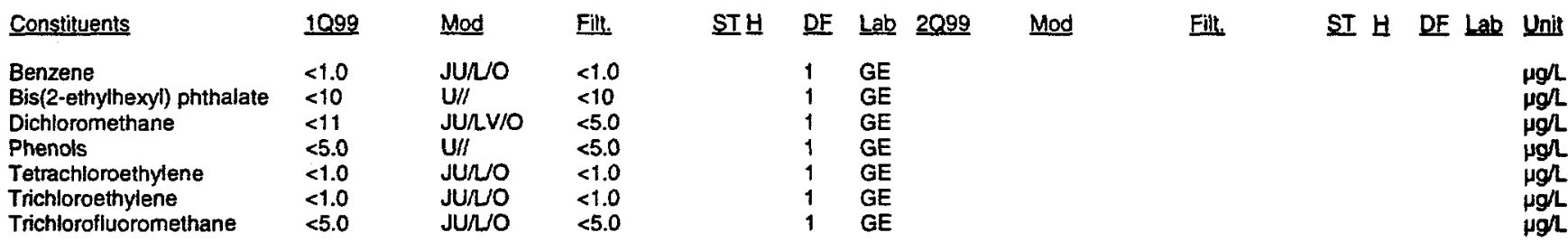

Notes:

- = exceeded holding time

- = exceeded groundwater protection or monitoring constituent standard (See Appendix A.)

NA - Not applicable. Applies to beta dose and sum of betas if there are no beta-emitting radionuclides above detection limits; to sum of alphas if there are no alphaemitting radionuclides above detection limits; and to total radium if neither radium-226 or radium-228 was above detection limit

UAZ UTRA - Upper Aquifer Zone of the Upper Three Runs Aquifer, LAZ UTRA - Lower Aquifer Zone of the Upper Three Runs Aquifer, Gordon - Gordon Aquifer

F-Area HWMF

C-120

First and Second Quarter 1999 


\section{WELL FSB109D (cont.)}

Radioactive Constituents

\begin{tabular}{|c|c|c|c|c|c|c|c|c|c|c|c|c|c|}
\hline Constituents & 1099 & Mod & Filt. & STH & DF & Lab & $\underline{2099}$ & Mod & Filt. & ST $H$ & $\mathrm{DF}$ & Lab & Unit \\
\hline $\begin{array}{l}\text { Americium-241 } \\
\text { Beta dose }\end{array}$ & $\begin{array}{l}<4.3 E-02 \\
\text { NA }\end{array}$ & $\mathrm{U} / /$ & $<7.9 E-02$ & & 1 & GP & & & & & & & $\mathrm{pCi} \Omega$ \\
\hline Carbon-14 & $<-2.3 E+\infty$ & $\mathrm{U} / /$ & $<7.5 E+00$ & & 1 & GP & & & & & & & pCin \\
\hline Cesium-137 & $<3.3 E-01$ & $U / l$ & $<3.5 E+00$ & & 1 & GP & & & & & & & pCin \\
\hline Cobalt -60 & $<-1.6 \mathrm{E}+\infty$ & $\mathrm{U} / /$ & $<3.2 E+00$ & & 1 & GP & & & & & & & pCin \\
\hline Curium-242 & & $\begin{array}{l}U / / \\
\mathrm{U} / /\end{array}$ & $\begin{array}{l}<8.4 E-02 \\
<1.6 E-01\end{array}$ & & $\begin{array}{l}1 \\
1\end{array}$ & $\begin{array}{l}\text { GP } \\
\text { GP }\end{array}$ & & & & & & & $\begin{array}{l}\text { pCil } \\
\text { pCil }\end{array}$ \\
\hline $\begin{array}{l}\text { Curium-243/244 } \\
\text { Curium-245/246 }\end{array}$ & $\begin{array}{l}<-1.1 E-02 \\
<2.6 E-02\end{array}$ & $\mathrm{U} / /$ & $<7.9 \mathrm{E}-02$ & & 1 & GP & & & & & & & pCin \\
\hline Gross alpha & $<7.6 E-01$ & $\mathrm{U} / /$ & $<9.0 \mathrm{E}-01$ & & 1 & GP & $7.4 E-01$ & $\mathrm{~J} / \mathrm{U}$ & NDD & & 1 & GP & pCin \\
\hline lodine-129 & $<6.6 E-01$ & $\mathrm{U} / /$ & $<1.3 \mathrm{E}+00$ & & 1 & GP & & & & & & & pCin \\
\hline Nonvolatile beta & $1.7 E+\infty 0$ & $\mathrm{~J} / \mathrm{V}$ & NDD & & 1 & GP & $1.3 E+\infty$ & $\mathrm{J} / \mathrm{V}$ & NDD & & 1 & GP & $\mathrm{pCi} / \mathrm{L}$ \\
\hline Plutonium-238 & $<1.1 E-02$ & $\mathrm{U} / \mathrm{I}$ & $<1.3 E-01$ & & 1 & GP & & & & & & & pCin \\
\hline Plutonium-239/240 & $<0.0 E+00$ & $\mathrm{U} / \prime$ & $<6.2 \mathrm{E}-02$ & & 1 & GP & & & & & & & pCin \\
\hline Radium-226 & $<4.1 E-01$ & $\mathrm{U} / /$ & $<4.8 E-01$ & & 1 & GP & & & & & & & pCin \\
\hline Radium-228 & $<2.7 E-01$ & $\mathrm{U} / /$ & $<1.1 E+\infty$ & & 1 & GP & & & & & & & pCin \\
\hline Total radium & NA & & & & & & & & & & & & \\
\hline Strontium-90 & $<4.1 E-01$ & $\mathrm{U} / /$ & $<1.4 E+00$ & & 1 & GP & & & & & & & pCin \\
\hline Technetium-99 & $<-1.3 E+00$ & $\mathrm{U} / /$ & $<1.9 \mathrm{E}+01$ & & 1 & GP & & & & & & & pCin \\
\hline Thorium-228 & $1.5 E-01$ & $\mathrm{R} / / 4$ & Rej & & 1 & GP & & & & & & & pCin \\
\hline Thorium-230 & 6.3E-02 & J/UI & NDD & & 1 & GP & & & & & & & pCin \\
\hline Thorium-232 & $<1.6 E-02$ & JUЛЛ & $<2.4 E-02$ & & 1 & GP & & & & & & & pCin \\
\hline Sum of alphas & NA & & & & & & & & & & & & \\
\hline Sum of betas & NA & & & & & & & & & & & & \\
\hline Tritium & $1.0 E+01$ & 11 & & & 1 & GP & $9.1 E+\infty 0$ & $/ 1$ & & & 1 & GP & pCi/m \\
\hline Uranium-233/234 & $<4.6 \mathrm{E}-02$ & $U / /$ & $<6.9 E-02$ & & 1 & GP & & & & & & & pCin \\
\hline Uranium-235 & $<0.0 \mathrm{E}+00$ & $\mathrm{U} / /$ & $<6.9 \mathrm{E}-02$ & & 1 & GP & & & & & & & pCin \\
\hline Uranium-238 & $<-2.3 E-02$ & $\mathrm{U} / \mathrm{I}$ & $<1.8 E-01$ & & 1 & GP & & & & & & & pCin \\
\hline
\end{tabular}

Notes:

= exceeded holding time

- exceeded groundwater protection or monitoring constituent standard (See Appendix A.)

NA - Not applicable. Applies to beta dose and sum of betas if there are no beta-emitting radionuclides above detection limits; to sum of alphas if there are no alphaemitting radionuclides above detection limits; and to total radium if neither radium-226 or radium-228 was above detection limit UAZ_UTRA - Upper Aquifer Zone of the Upper Three Runs Aquifer, LAZ UTRA - Lower Aquifer Zone of the Upper Three Runs Aquifer, Gordon - Gordon Aquifer 


\section{WELL FSB110C}

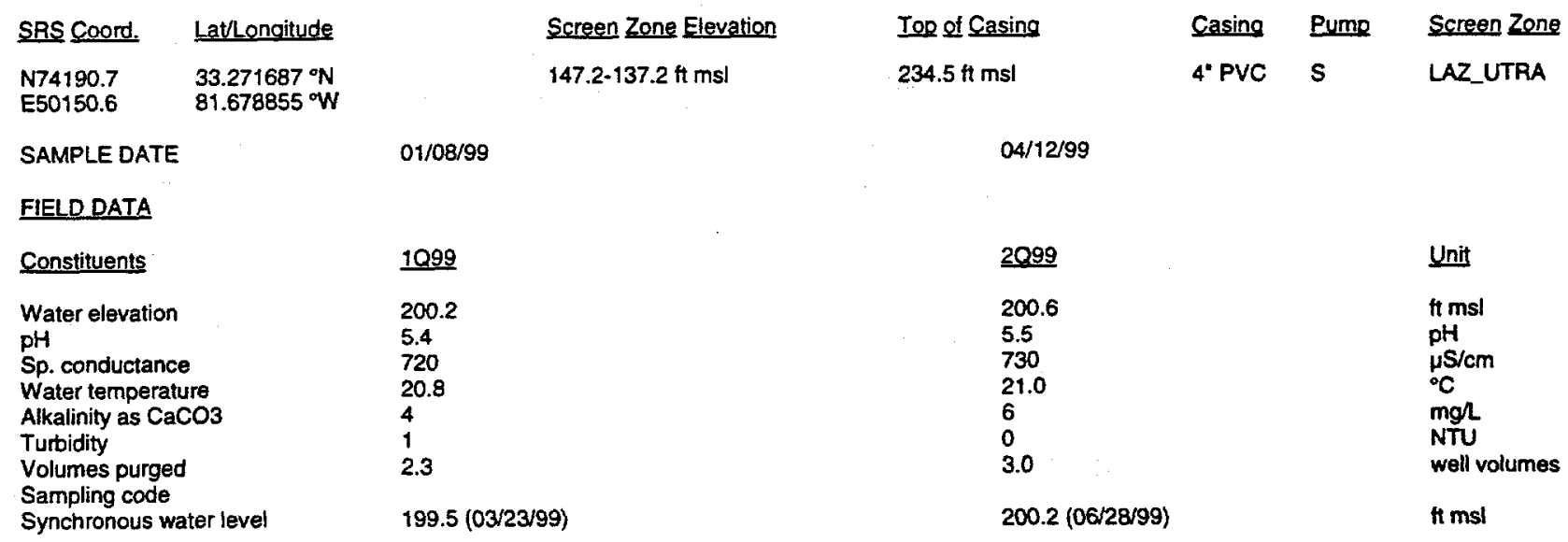

\section{ANALYTICAL DATA}

Inorganic Constituents

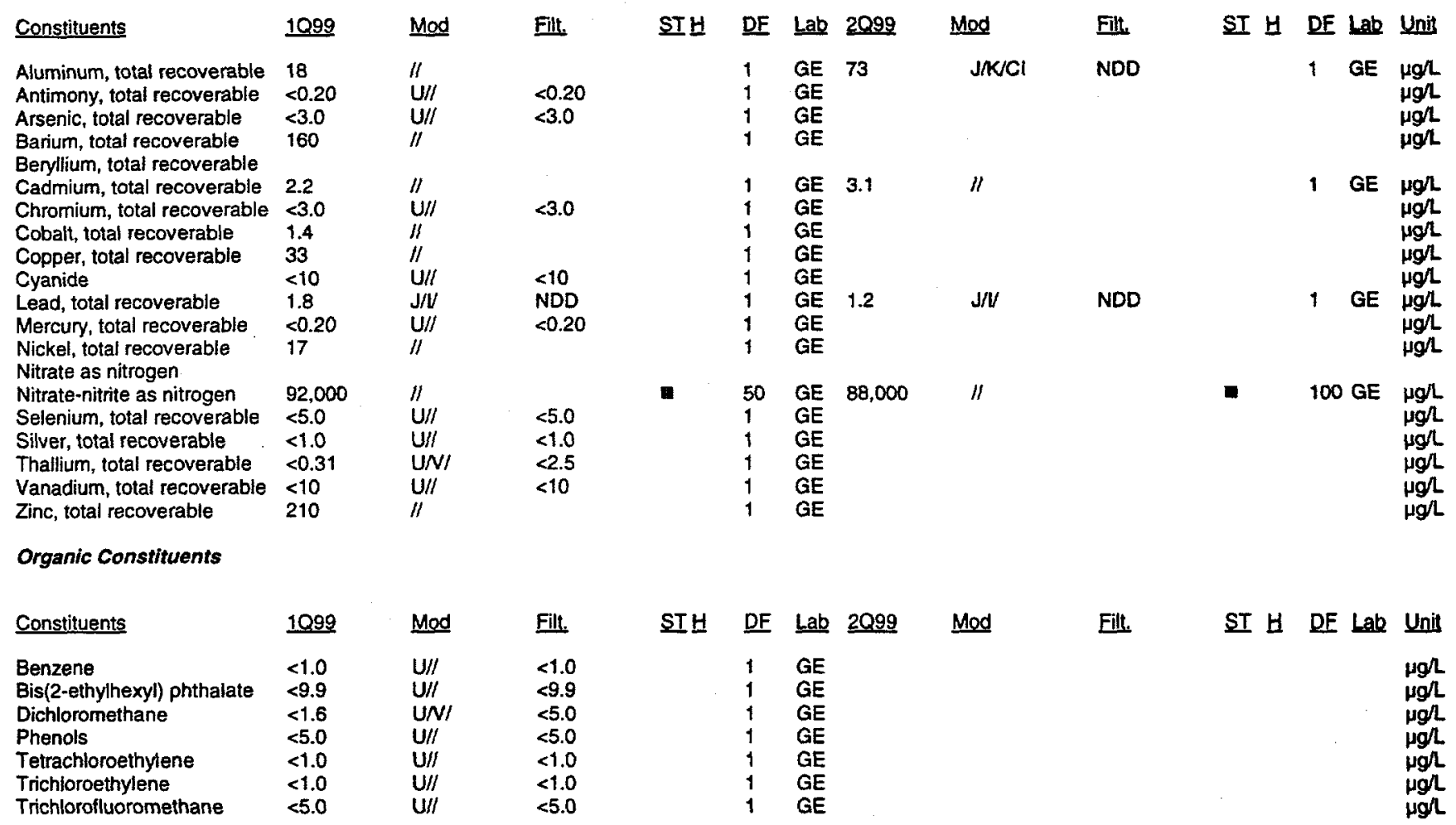

Notes:

- exceeded holding time

E = exceeded groundwater protection or monitoring constituent standard (See Appendix A.)

NA - Not applicable. Applies to beta dose and sum of betas if there are no beta-emitting radionuclides above detection limits; to sum of alphas if there are no alphaemitting radionuclides above detection limits; and to total radium if neither radium-226 or radium-228 was above detection limit

UAZ UTRA - Upper Aquifer Zone of the Upper Three Runs Aquifer, LAZ_UTRA - Lower Aquifer Zone of the Upper Three Runs Aquifer, Gordon - Gordon Aquifer 
WELL FSB110C (cont.)

Radioactive Constituents

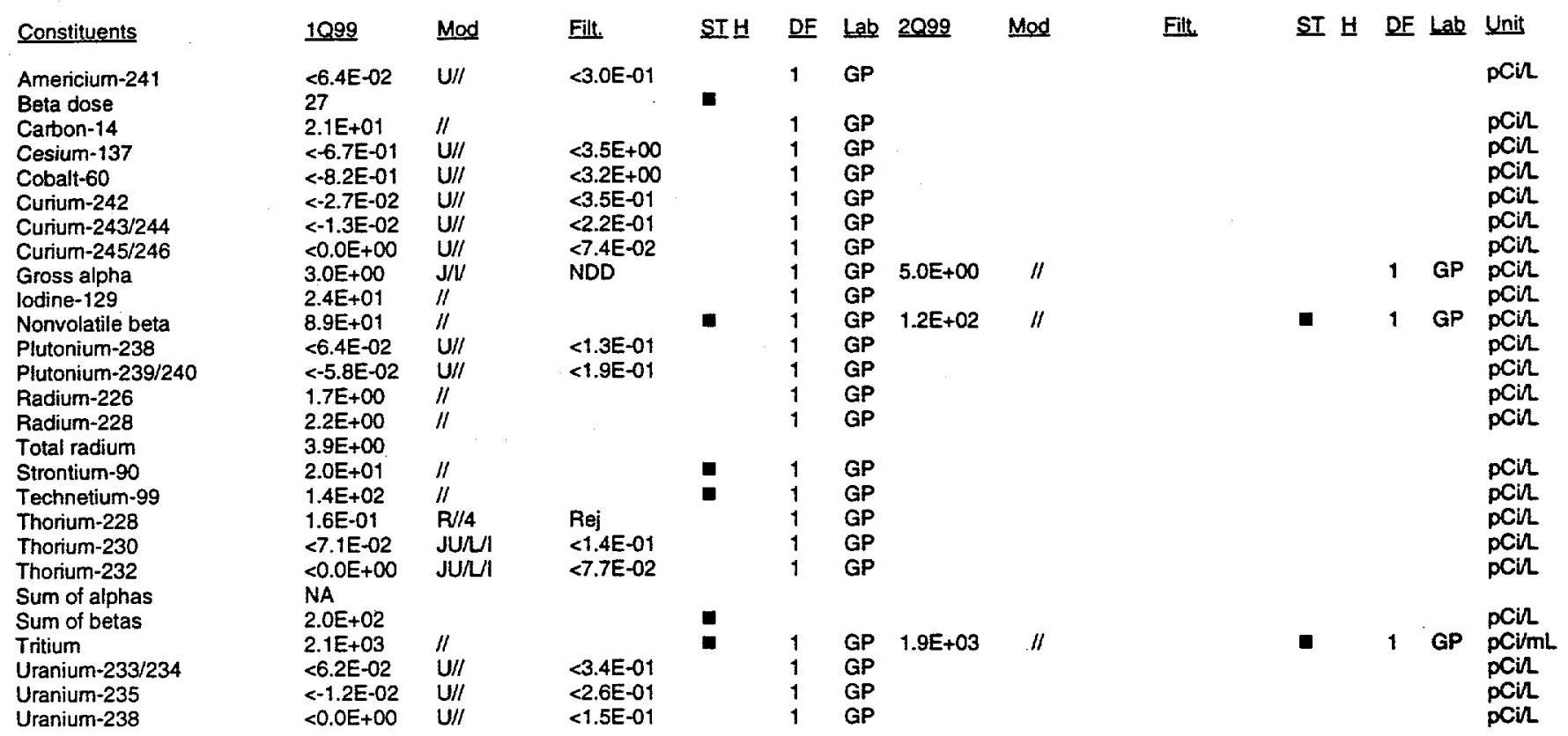

Notes:

= exceeded holding time

= exceeded groundwater protection or monitoring constituent standard (See Appendix A.)

NA - Not applicable. Applies to beta dose and sum of betas if there are no beta-emitting radionuclides above detection limits; to sum of alphas if there are no alphe. emitting radionuclides above detection limits; and to total radium if neither radium-226 or radium-228 was above detection limit

UAZ UTRA - Upper Aquifer Zone of the Upper Three Runs Aquifer, LAZ_UTRA - Lower Aquifer Zone of the Upper Three Runs Aquifer, Gordon - Gordon Aquifer 


\section{WELL FSB110D}

$\begin{array}{lll}\text { SRS Coord. } & & \text { Lat/Longitude } \\ \text { N74193.3 } & & 33.271678^{\circ} \mathrm{N} \\ \text { E50141.6 } & 81.678884^{\circ} \mathrm{W}\end{array}$

SAMPLE DATE

01/12/99

Screen Zone Elevation

211.1-191.1 ft ms

1099

Constituents

Water elevation

pH

Sp. conductance

Water temperature

Alkalinity as $\mathrm{CaCO} 3$

Turidity

Volumes purged

Sampling code

Synchronous water level

205.0

3.1

480

19.8

0

4.4

$205.0(03 / 23 / 99)$
Top of Casing

$234.5 \mathrm{ft}$ msl
Casing Pump

4" PVC V
Screen Zone

UAZ_UTRA
04/05/99

2099

205.0

3.6

390

20.3

0

2.3

$203.8(06 / 28 / 99)$
Unit

ft msl

$\mathrm{pH}$

$\mu \mathrm{S} / \mathrm{cm}$

${ }^{\circ} \mathrm{C}$

mgll

well volumes

ft $\mathrm{msl}$

\section{ANALYTICAL DATA}

Inorganic Constituents

\begin{tabular}{|c|c|c|c|c|c|c|c|c|c|c|c|c|c|}
\hline Constituents & 1099 & Mod & Filt. & ST & $\underline{D F}$ & Lab & $\underline{2 Q 99}$ & Mod & Filt. & ST $\mathrm{H}$ & DF & Lab & Unit \\
\hline Aluminum, total recoverable & 14,000 & $/ /$ & & & 1 & GE & 9,500 & /I & & & 1 & GE & $\mu g h$ \\
\hline Antimony, total recoverable & $<0.20$ & $\mathrm{U} / /$ & $<0.20$ & & 1 & GE & & & & & & & \\
\hline Arsenic, total recoverable & $<3.0$ & $\mathrm{U} / /$ & $<3.0$ & & 1 & GE & & & & & & & \\
\hline $\begin{array}{l}\text { Barium, total recoverable } \\
\text { Bentlium, total recoverable }\end{array}$ & 140 & $/ /$ & & & 1 & GE & & & & & & & \\
\hline Cadmium, total recoverable & 2.6 & $/ /$ & & & 1 & GE & 1.7 & $/ /$ & & & 1 & GE & Igh \\
\hline Chromium, total recoverable & 1.3 & $\mathrm{~J} / \mathrm{U}$ & NDD & & 1 & GE & & & & & & & \\
\hline Cobalt, total recoverable & 5.3 & /I & & - & 1 & GE & & & & & & & \\
\hline Copper, total recoverable & 21 & $/ /$ & & & 1 & GE & & & & & & & \\
\hline Cyanide & $<10$ & $\mathbf{U} / /$ & $<10$ & & 1 & GE & & & & & & & 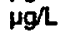 \\
\hline Lead, total recoverable & 0.34 & $\mathrm{~J} N$ & NDD & & 1 & GE & $<0.55$ & UNI & $<2.0$ & & 1 & GE & \\
\hline Mercury, total recoverable & $<0.087$ & UNI & $<0.20$ & & 1 & GE & & & & & & & \\
\hline Nickel, total recoverable & 9.9 & $/ 1$ & & & 1 & GE & & & & & & & \\
\hline Nitrate as nitrogen & & & & 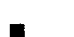 & & & & & & & & & \\
\hline & 62,000 & & & $\mathbf{D}$ & 50 & GE & 60,000 & II & & 口 & 25 & GE & 5 \\
\hline $\begin{array}{l}\text { Selenium, total recoverable } \\
\text { Silver, total recoverable }\end{array}$ & $\begin{array}{l}<5.0 \\
<1.0\end{array}$ & $\begin{array}{l}\mathrm{U} / \prime \\
\mathrm{U} / \prime\end{array}$ & $\begin{array}{l}<5.0 \\
<1.0\end{array}$ & & $\begin{array}{l}1 \\
1\end{array}$ & $\begin{array}{l}\mathrm{GE} \\
\mathrm{GE}\end{array}$ & & & & & & & \\
\hline $\begin{array}{l}\text { Silver, total recoverable } \\
\text { Thallium, total recoverable }\end{array}$ & $<0.13$ & UN/ & $<2.5$ & & 1 & GE & & & & & & & \\
\hline Vanadium, total recoverable & $<10$ & $\mathrm{U} / /$ & $<10$ & & 1 & $\mathrm{GE}$ & & & & & & & \\
\hline Zinc, total recoverable & 46 & II & & & 1 & GE & & & & & & & \\
\hline
\end{tabular}

Organic Constituents

$\begin{array}{lllllll}\text { Constituents } & 1099 & \text { Mod } & \text { Filt. } & \text { ST H } & \text { DF } & \text { Lab } \\ \text { Benzene } & <1.0 & \text { JU/L/O } & <1.0 & & 1 & \text { GE } \\ \text { Bis(2-ethylhexyl) phthalate } & <9.9 & \text { U/I } & <9.9 & & 1 & \text { GE } \\ \text { Dichloromethane } & <5.0 & \text { JU/UN } & <5.0 & & 1 & \text { GE } \\ \text { Phenols } & <5.0 & \text { U/I } & <5.0 & & 1 & \text { GE } \\ \text { Tetrachloroethylene } & <1.0 & \text { JU/L/O } & <1.0 & & 1 & \text { GE } \\ \text { Trichloroethylene } & <1.0 & \text { JU/U/O } & <1.0 & & 1 & \text { GE } \\ \text { Trichlorofluoromethane } & <5.0 & \text { JU/U/O } & <5.0 & & 1 & \text { GE }\end{array}$

Filt.

SI H DE Lab Unit

$\mu \mathrm{g} / \mathrm{L}$

$\mu \mathrm{g} / \mathrm{L}$

$\mu g h$

ugh

$\mu g /$ L

ngh

$\mu \mathrm{g}$.

Notes:

- exceeded holding time

= exceeded groundwater protection or monitoring constituent standard (See Appendix A.)

NA - Not applicable. Applies to beta dose and sum of betas if there are no beta-emitting radionuclides above detection limits; to sum of alphas if there are no alphaemitting radionuclides above detection limits; and to total radium if neither radium-226 or radium-228 was above detection limit

UAZ UTRA - Upper Aquifer Zone of the Upper Three Runs Aquifer, LAZ UTRA - Lower Aquifer Zone of the Upper Three Runs Aquifer, Gordon - Gordon Aquifer 


\section{WELL FSB110D (cont.)}

Radioactive Constituents

\begin{tabular}{|c|c|c|c|c|c|c|c|c|c|c|c|c|c|}
\hline Constituents & 1099 & Mod & Filt. & STH & DF & Lab & 2099 & Mod & Filt. & ST H & DF & Lab & Unit \\
\hline $\begin{array}{l}\text { Americium-241 } \\
\text { Beta dose }\end{array}$ & $\begin{array}{l}1.0 E+01 \\
420\end{array}$ & $\|$ & & च & 1 & GP & & & & & & & pCill \\
\hline Carbon-14 & $8.2 E+01$ & $\mathrm{~J} / \mathrm{Cl}$ & NDD & & 1 & GP & & & & & & & pCin \\
\hline Cesium-137 & $<-1.9 E+00$ & $\mathrm{U} / /$ & $<3.1 E+\infty 0$ & & 1 & GP & & & & & & & pein \\
\hline Cobalt -60 & $<-8.3 E-01$ & $\mathrm{U} / \mathrm{J}$ & $<3.3 E+00$ & & 1 & GP & & & & & & & pCin \\
\hline Curium-242 & $<1.0 \mathrm{E}-01$ & $U / /$ & $<1.9 \mathrm{E}-01$ & & 1 & GP & & & & & & & pCin \\
\hline Curium-243/244 & $1.5 E+01$ & /I & & v & 1 & GP & & & & & & & pCil \\
\hline Curium-245/246 & 4.5E-01 & $\mathrm{R} / 4$ & Rej & & 1 & GP & & & & & & & pCin \\
\hline $\begin{array}{l}\text { Gross alpha } \\
\text { lodine- } 129\end{array}$ & $\begin{array}{l}4.7 E+02 \\
4.0 E+02\end{array}$ & $\mathrm{~J} / \mathrm{I}$ & NDD & 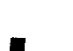 & 1 & $\begin{array}{l}\text { GP } \\
G P\end{array}$ & $3.1 E+02$ & $\mathrm{~J} / \mathrm{K} / \mathrm{I}$ & NDD & & 1 & GP & pCin \\
\hline Nonvolatile beta & $5.2 E+02$ & J/II & NDD & & 1 & GP & 3.1E+02 & $\mathrm{J} / \mathrm{K} / \mathbf{I}$ & NDD & & 1 & GP & pCin \\
\hline Plutonium-238 & $<1.5 \mathrm{E}-02$ & $u / 1$ & $<4.5 E-02$ & & 1 & GP & & & & & & & pCil \\
\hline Plutonium-239/240 & $<0.0 E+00$ & $\mathrm{U} / /$ & $<4.5 E-02$ & & 1 & GP & & & & & & & pCir \\
\hline Radium-226 & $1.5 E+01$ & $\mathrm{~J} / \mathrm{L} / \mathrm{I}$ & NDD & & 1 & GP & & & & & & & pCin \\
\hline $\begin{array}{l}\text { Radium-228 } \\
\text { Total radium }\end{array}$ & $1.1 E+01$ & $/ 1$ & & 口 & 1 & GP & & & & & & & pCil \\
\hline Strontium-90 & $1.5 E+02$ & $\|$ & & $\mathbf{0}$ & 1 & GP & & & & & & & oCil \\
\hline Technetium-99 & $1.0 \mathrm{E}+02$ & $\|$ & & 口 & 1 & GP & & & & & & & pCir \\
\hline Thorium-228 & $<4.3 E-01$ & $\mathrm{U} / /$ & $<4.4 E-01$ & & 1 & GP & & & & & & & pCin \\
\hline Thorium-230 & 3.6E-01 & $\mathrm{J} / \mathrm{N}$ & NDD & & 1 & GP & & & & & & & $\mathrm{pCi}$ \\
\hline Thorium-232 & $<5.4 E-02$ & $\mathrm{U} / /$ & $<8.0 E-02$ & & 1 & GP & & & & & & & pCin \\
\hline Sum of alphas & $6.9 E+02$ & & & $\boldsymbol{B}$ & & & & & & & & & pCir \\
\hline Sum of betas & $6.7 E+02$ & & & $\mathbf{n}$ & & & & & & & & & $\mathrm{pCil}$ \\
\hline Tritium & $1.9 E+03$ & // & & $\mathbf{n}$ & 1 & GP & $2.5 E+03$ & $/ /$ & & $\mathbf{0}$ & 1 & GP & pCi/mL \\
\hline Uranium-233/234 & $1.7 E+02$ & // & & $\mathbf{n}$ & 1 & GP & & & & & & & pCir \\
\hline Uranium-235 & $1.5 E+01$ & 11 & & $\mathbf{\square}$ & 1 & GP & & & & & & & pCin \\
\hline Uranium-238 & $4.9 E+02$ & /l & & $\mathbf{\square}$ & 1 & GP & & & & & & & $\mathrm{pCi}$ \\
\hline
\end{tabular}

Notes:

- exceeded holding time

a = exceeded groundwater protection or monitoring constituent standard (See Appendix A.)

NA - Not applicable. Applies to beta dose and sum of betas if there are no beta-emitting radionuclides above detection limits; to sum of alphas if there are no alphaemitting radionuclides above detection limits; and to total radium if neither radium-226 or radium-228 was above detection limit

UAZ_UTRA - Upper Aquifer Zone of the Upper Three Runs Aquifer, LAZ. UTRA - Lower Aquifer Zone of the Upper Three Runs Aquifer, Gordon - Gordon Aquifer 


\section{WELL FSB111C}

$\begin{array}{ll}\text { SRS Coord. } & \text { Lat/Longitude } \\ \text { N75383.3 } & 33.276569^{\circ} \mathrm{N} \\ \text { E51526.3 } & 81.677550^{\circ} \mathrm{W}\end{array}$

$01 / 07 / 99$

Screen Zone Elevation

$169.0-159.0 \mathrm{ft} \mathrm{ms}$
Top of Cassing

$276.3 \mathrm{ft} \mathrm{ms}$
Casing Pump

4" PVC S
Screen Zone

LAZ_UTRA
SAMPLE DATE

FIELLD DATA

Constituents

1099

Water elevation

$\mathrm{pH}$

Sp. conductance

Water temperature

Alkalinity as $\mathrm{CaCO} 3$

Turbidity

Volumes purged

Sampling code

Synchronous water level

\section{ANALYTICAL DATA}

Inorganic Constituents

\section{$04 / 05 / 99$}

213.6
4.6
56
19.3
10
0
3.4
$213.0(03 / 23 / 99)$

Unit

ft mst

$\mathrm{pH}$

$\mu$ Sicm

${ }^{\circ} \mathrm{C}$

mgh

well volumes

2.8

$211.9(06 / 28 / 99)$

ft msl

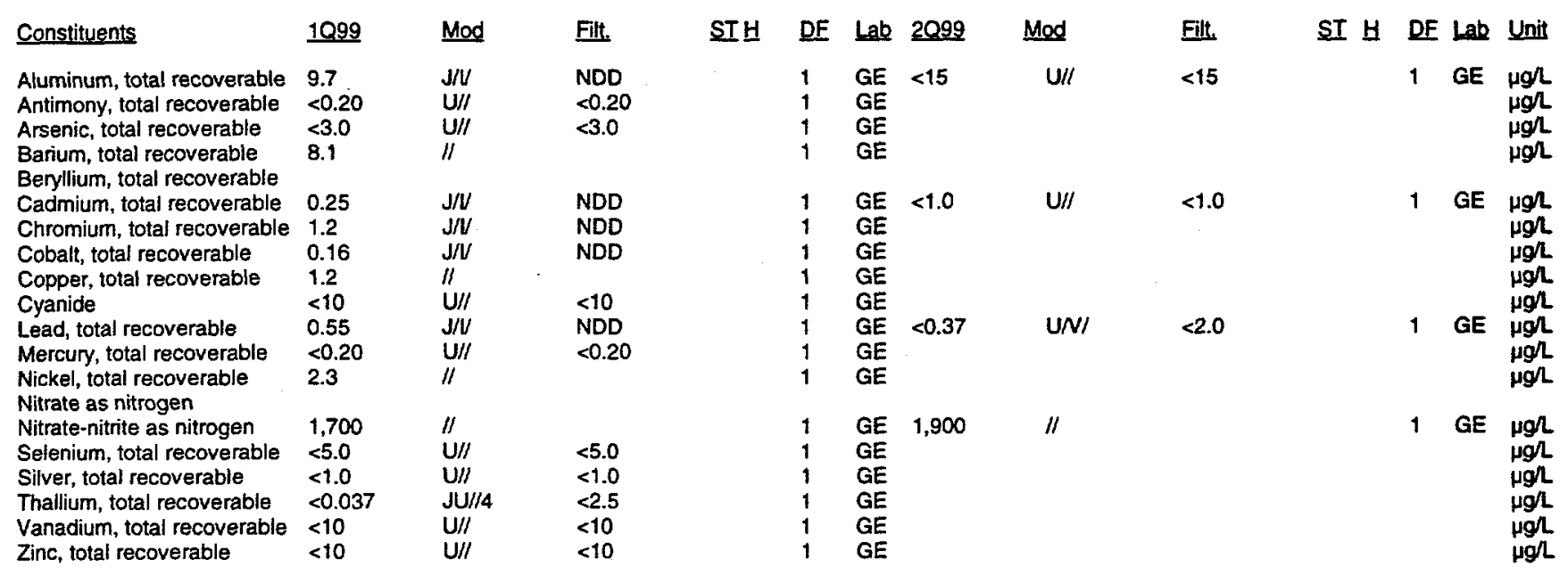

Organic Constituents

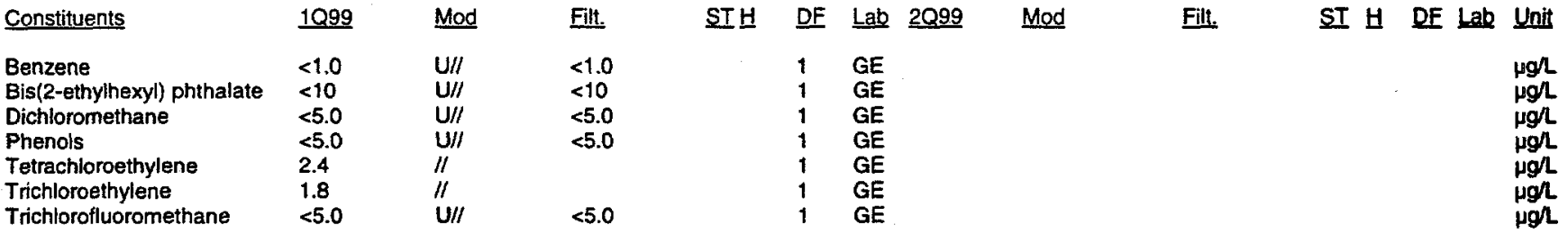

Notes:

- exceeded holding time

= exceeded groundwater protection or monitoring constituent standard (See Appendix A.)

NA - Not applicable. Applies to beta dose and sum of betas if there are no beta-emitting radionuclides above detection limits; to sum of alphas if there are no alphaemitting radionuclides above detection limits; and to total radium if neither radium-226 or radium-228 was above detection limit

UAZ UTRA - Upper Aquifer Zone of the Upper Three Runs Aquifer, LAZ UTRA - Lower Aguifer Zone of the Upper Three Runs Aquifer, Gordon - Gordon Aquifer 


\section{WELL FSB111C (cont.)}

Radioactive Constituents

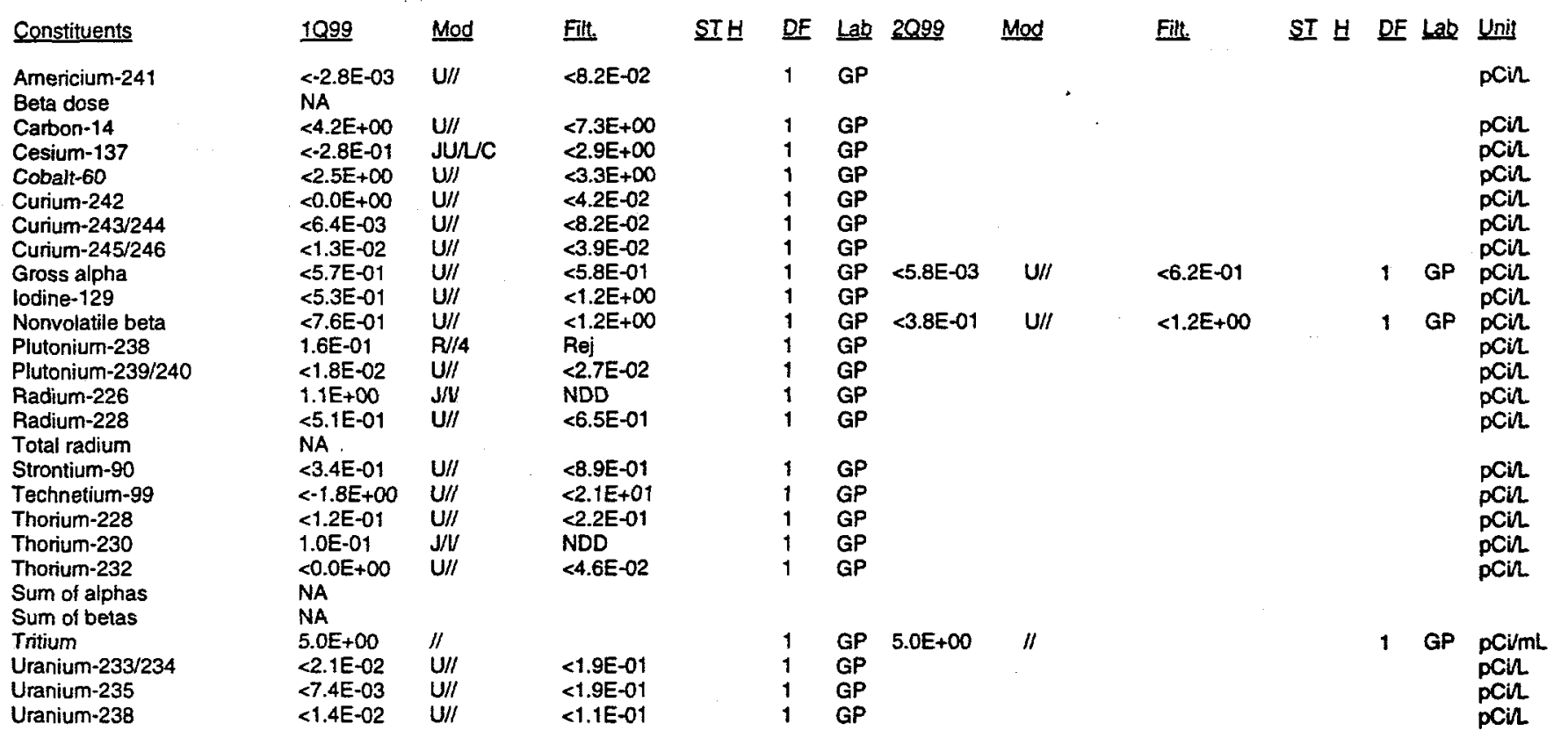




\section{WELL FSB111D}

\begin{tabular}{|c|c|}
\hline SRS Coord. & Lat/Longitud \\
\hline $\begin{array}{l}\text { N75382.9 } \\
\text { E51515.9 }\end{array}$ & 1677 \\
\hline
\end{tabular}

SAMPLE DATE

1099

216.8

4.6

48

17.2

12

8.10

$215.9(03 / 23 / 99)$

Sp. conductance

Alkalinity as $\mathrm{CaCO} 3$

Turbidity

Volumes purged

Sampling code
Screen Zone Elevation

$221.7-201.7 \mathrm{ft} \mathrm{msl}$

Synchronous water level

ANALYTICAL DATA

Inorganic Constituents

$\begin{array}{llll}\text { Top of Casing } & \text { Casing } & \text { Pump } & \text { Screen Zone } \\ 276.6 \mathrm{ft} \mathrm{msi} & 4^{\prime} \text { PVC } & S & \text { UAZ_UTRA }\end{array}$

04/05/99

2099

215.8

5.0
44

20.4

0

2.6

$215.5(06 / 28 / 99)$
Unit

tt ms!

$\mathrm{pH}$

uSicm

$m g \Omega$

NTU

well volumes

ft msi

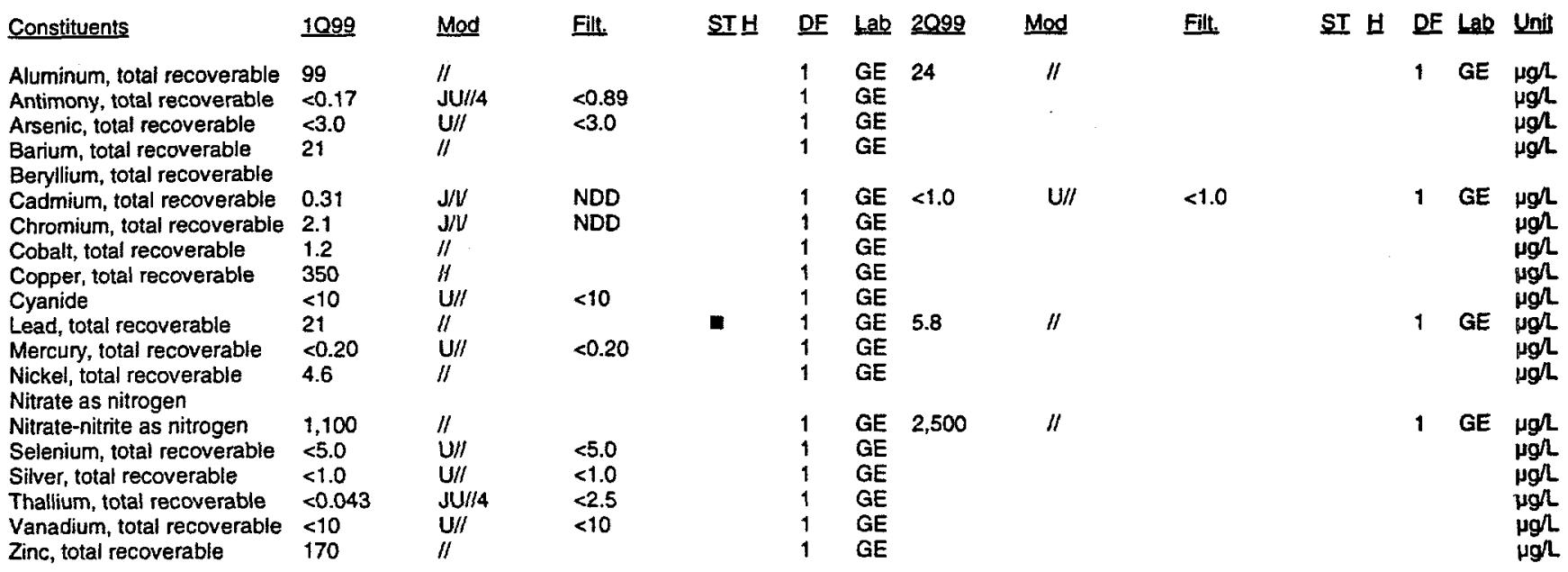

Organic Constituents

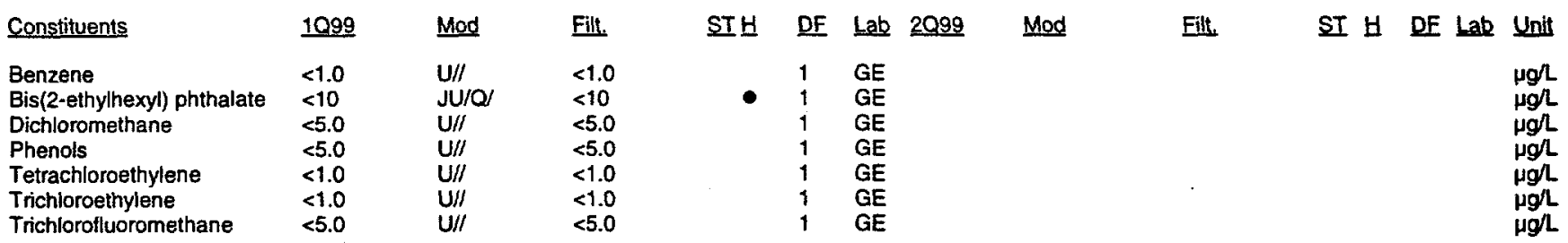

Notes:

- exceeded holding time

= exceeded groundwater protection or monitoring constituent standard (See Appendix A.)

NA - Not applicable. Applies to beta dose and sum of betas it there are no beta-emitting radionuclides above detection limits; to sum of alphas if there are no alphaemitting radionuclides above detection limits; and to total radium if neither radium-226 or radium-228 was above detection limit

UAZ UTRA - Upper Aquifer Zone of the Upper Three Runs Aquifer, LAZ UTRA - Lower Aquifer Zone of the Upper Three Runs Aquifer, Gordon - Gordon Aquifer

F-Area HWMF

C-128

First and Second Quarter 1999 
WELL FSB111D (cont.)

Radioactive Constituents

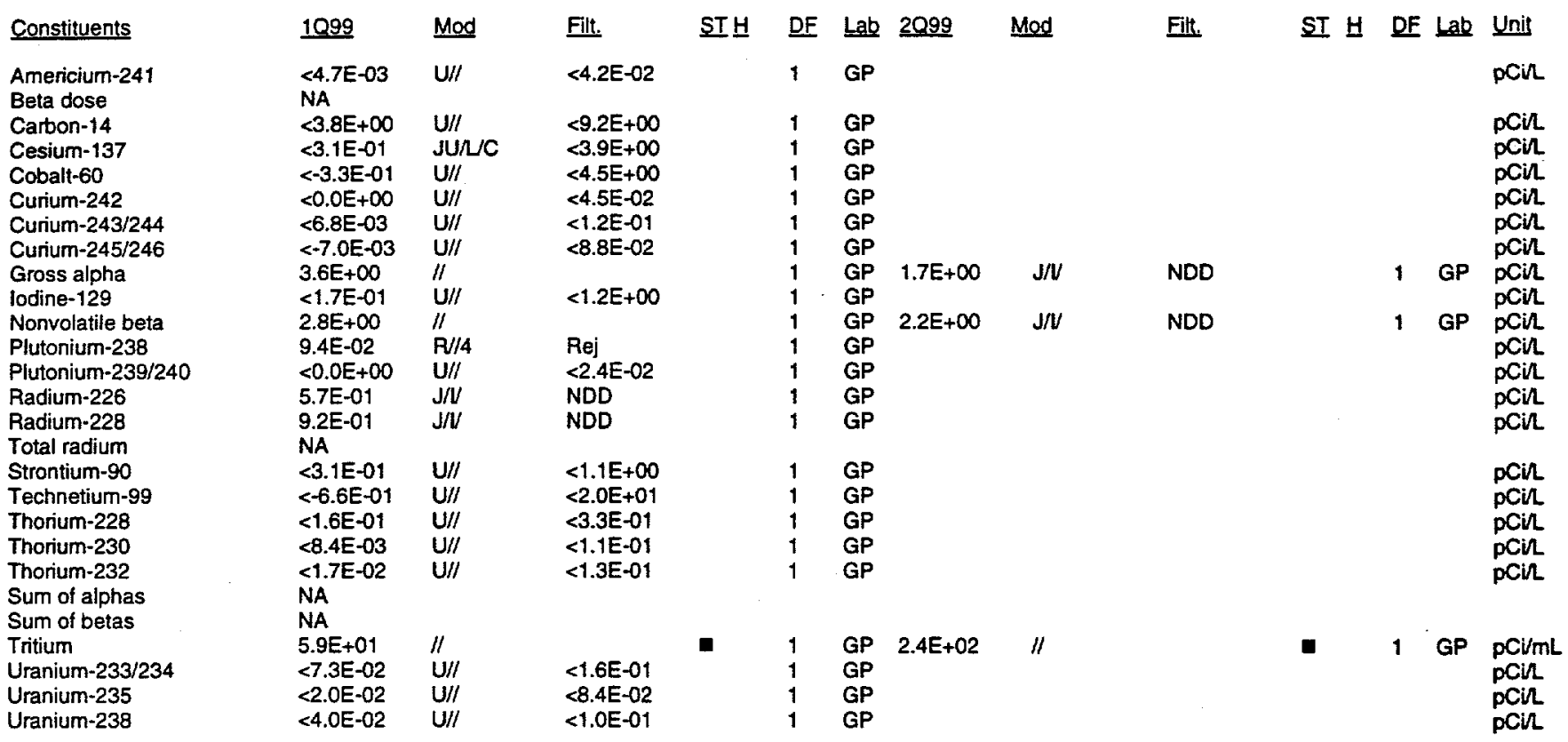

Notes:

- exceeded holding time

= exceeded groundwater protection or monitoring constituent standard (See Appendix A.)

NA - Not applicable. Applies to beta dose and sum of betas if there are no beta-emitting radionuclides above detection limits; to sum of alphas if there are no alphaemitting radionuclides above detection limits; and to total radium if neither radium-226 or radium-228 was above detection limit

UAZ_UTRA - Upper Aquifer Zone of the Upper Three Runs Aquifer, LAZ UTRA - Lower Aquifer Zone of the Upper Three Runs Aquiter, Gordon - Gordon Aquifer

F-Area HWMF

C-129

First and Second Quarter 1999 


\section{WELL FSB112A}

$\begin{array}{ll}\text { SRS Coord. } & \text { Latlongitude } \\ \text { N74231.4 } & 33.269588^{\circ} \mathrm{N} \\ \text { E48809.1 } & 81.682466^{\circ} \mathrm{W}\end{array}$

SAMPLE DATE
Screen Zone Elevation

91.0-81.0 ft msl
Top of Casing

$229.1 \mathrm{ft} \mathrm{msl}$
Casing Pump

4" PVC S
Screen Zone

Gordon
$04 / 12 / 99$

2099
153.5
6.5
150
19.9
37
0
2.7

$152.9(06 / 25 / 99)$
Unit

ft msi

$\mathrm{pH}$

$\mu \mathrm{S} / \mathrm{cm}$

mgh

well volumes

ft msl
$153.5(03 / 23 / 99)$

\section{ANALYTICAL DATA}

Inorganic Constituents

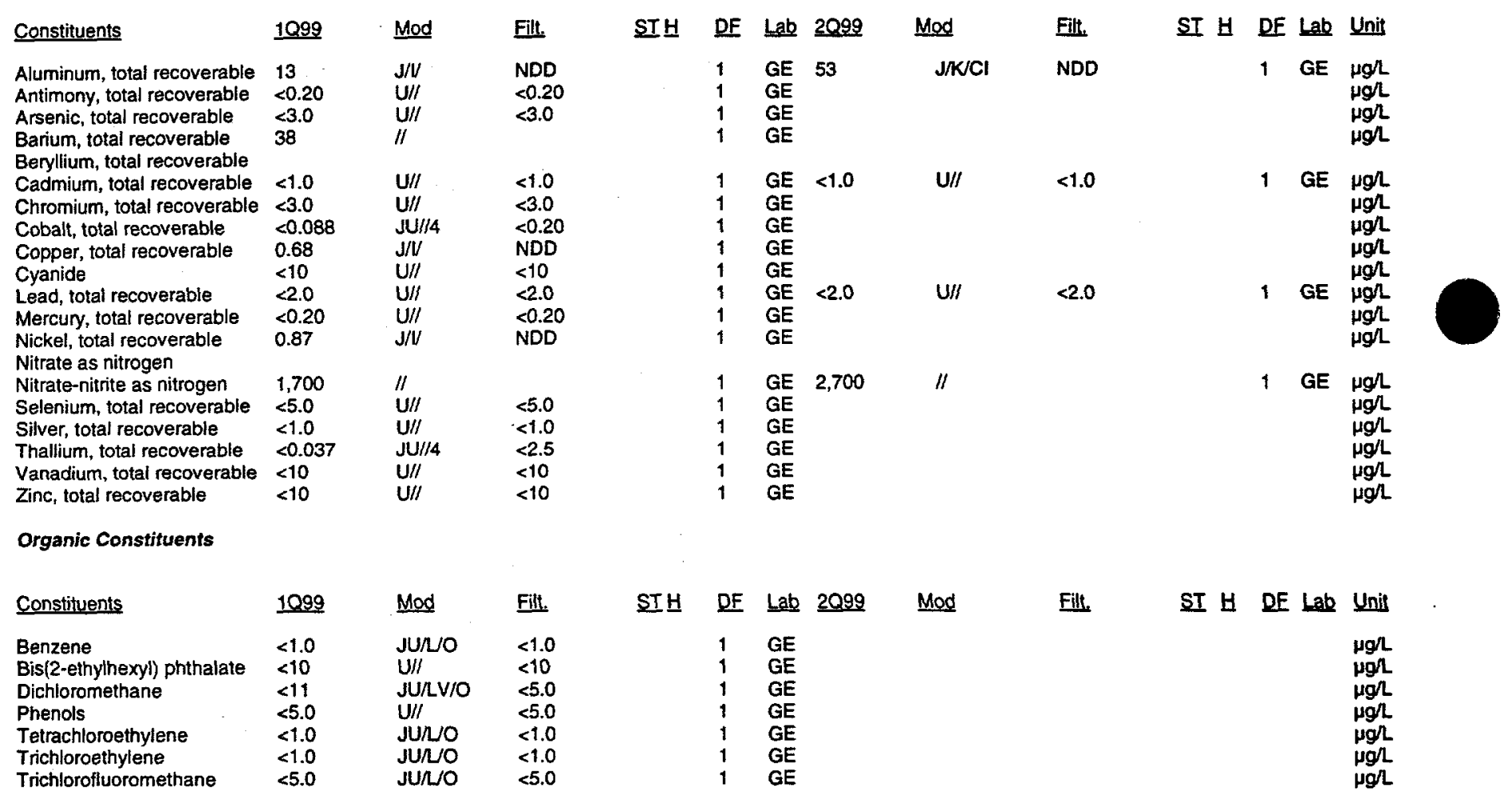

\section{Notes:}

= exceeded holding time

= exceeded groundwater protection or monitoring constituent standard (See Appendix A.)

NA - Not applicable. Applies to beta dose and sum of betas if there are no beta-emitting radionuclides above detection limits; to sum of alphas if there are no alphaemitting radionuclides above detection limits; and to total radium if neither radium-226 or radium-228 was above detection limit

UAZ UTRA - Upper Aquifer Zone of the Upper Three Runs Aquifer; LAZ_UTRA - Lower Aquifer Zone of the Upper Three Runs Aquiter, Gordon - Gondon Aquifer 


\section{WELL FSB112A (cont.)}

Radioactive Constituents

\begin{tabular}{|c|c|c|c|c|c|c|c|c|c|c|c|c|c|}
\hline Constituents & 1099 & Mod & Filt. & SI H & DF & Lab & $\underline{2099}$ & Mod & Filt. & ST $\mathrm{H}$ & DF & Lab & Unit \\
\hline $\begin{array}{l}\text { Americium-241 } \\
\text { Beta dose }\end{array}$ & $\begin{array}{l}<-3.6 \mathrm{E}-02 \\
0.34\end{array}$ & $\mathrm{U} / /$ & $<2.4 E-01$ & & 1 & GP & & & & & & & $\mathrm{pCin}$ \\
\hline Cabon-14 & $<-1.3 E+\infty 0$ & $\mathrm{U} / /$ & $<7.7 E+\infty$ & & 1 & GP & & & & & & & pCin \\
\hline Cesium-137 & $<2.4 E+00$ & $\mathrm{U} / l$ & $<3.9 E+\infty$ & & 1 & GP & & & & & & & pCin \\
\hline Cobalt -60 & $<1.3 E+\infty 0$ & $\mathrm{U} / /$ & $<4.1 E+\infty$ & & 1 & GP & & & & & & & pCin \\
\hline Curium-242 & $<-1.1 E-02$ & $\mathrm{U} / /$ & $<1.6 \mathrm{E}-01$ & & 1 & GP & & & & & & & $\mathrm{pCin}$ \\
\hline Curium-243/244 & $<-2.0 E-02$ & $\mathrm{U} / /$ & $<1.8 E-01$ & & 1 & GP & & & & & & & pCin \\
\hline Curium-245/246 & $<0.0 \mathrm{E}+\infty$ & $U / /$ & $<7.2 E-02$ & & 1 & GP & & & & & & & pCin \\
\hline Gross alpha & $<4.2 E-01$ & $\mathrm{U} / /$ & $<6.5 \mathrm{E}-01$ & & 1 & GP & 9.9E-01 & $\mathrm{J} / \mathrm{V}$ & NDD & & 1 & GP & pCin \\
\hline lodine-129 & $<6.9 \mathrm{E}-01$ & $U / I$ & $<1.0 E+\infty$ & & 1 & GP & & & & & & & pCil \\
\hline Nonvolatile beta & $8.3 E+\infty$ & 11 & & & 1 & GP & $1.3 E+01$ & $\|$ & & & 1 & GP & PCin \\
\hline Plutonium-238 & $<5.4 E-02$ & $\mathrm{U} / /$ & $<8.1 E-02$ & & 1 & GP & & & & & & & PCin \\
\hline Plutonium-239/240 & $<0.0 \mathrm{E}+00$ & $\mathrm{U} / /$ & $<8.1 E-02$ & & 1 & GP & & & & & & & PCin \\
\hline Radium-226 & $<4.7 E-01$ & $\mathrm{U} / /$ & $<4.8 E-01$ & & 1 & GP & & & & & & & pCin \\
\hline $\begin{array}{l}\text { Radium-228 } \\
\text { Total radium }\end{array}$ & $\begin{array}{l}<-2.6 E-02 \\
\text { NA }\end{array}$ & $\mathrm{U} / /$ & $<1.2 E+00$ & & 1 & GP & & & & & & & pCin \\
\hline Strontium-90 & $2.7 E+\infty$ & /I & & & 1 & GP & & & & & & & pCin \\
\hline Technetium-99 & $<1.1 E+00$ & $U / /$ & $<1.9 \mathrm{E}+01$ & & 1 & GP & & & & & & & pCin \\
\hline Thorium-228 & $1.5 E-01$ & $\mathrm{R} / / 4$ & Rej & & 1 & GP & & & & & & & pCin \\
\hline Thorium-230 & $7.6 \mathrm{E}-02$ & J/ILI & NDD & & 1 & GP & & & & & & & pCil \\
\hline Thorium-232 & $<2.3 E-02$ & JU/L/I & $<2.3 E-02$ & & 1 & GP & & & & & & & pCin \\
\hline Sum of alphas & NA & & & & & & & & & & & & \\
\hline Sum of betas & 2.7E+00 & & & & & & & & & & & & in \\
\hline Tritium & $4.2 E+01$ & /I & & $\mathbf{a}$ & 1 & GP & $7.4 E+01$ & $\|$ & & $\mathbf{\square}$ & 1 & GP & \\
\hline Uranium-233/234 & $<4.3 E-02$ & $\mathrm{U} / /$ & $<6.5 E-02$ & & 1 & GP & & & & & & & \\
\hline Uranium-235 & $<0.0 E+00$ & $\mathrm{U} / /$ & $<6.5 E-02$ & & 1 & GP & & & & & & & Cir \\
\hline Uranium-238 & $<2.2 E-02$ & $\mathrm{U} / /$ & $<1.7 E-01$ & & 1 & GP & & & & & & & $\mathrm{pCi}$ \\
\hline
\end{tabular}

Notes:

- exceeded holding time

- = exceeded groundwater protection or monitoring constituent standard (See Appendix A.)

NA - Not applicable. Applies to beta dose and sum of betas if there are no beta-emitting radionuclides above detection limits; to sum of alphas if there are no alphaemitting radionuclides above detection limits; and to total radium if neither radium-226 or radium-228 was above detection limit

UAZ UTRA - Upper Aquifer Zone of the Upper Three Runs Aquifer, LAZ UTRA - Lower Aquifer Zone of the Upper Three Runs Aquifer, Gordon - Gordon Aquiler 


\section{WELL FSB112C}

$\begin{array}{ll}\text { SRS Coord. } & \text { Lat/Longitude } \\ \text { N74227.5 } & 33.269556^{\circ} \mathrm{N} \\ \text { E48794.8 } & 81.682496^{\circ} \mathrm{W}\end{array}$

SAMPLE DATE

$556^{\circ} \mathrm{N}$

Screen Zone Elevation

139.1-129.1 $\mathrm{ft} \mathrm{ms!}$

FIELD DATA

Constituents

Water elevation

pH

Sp. conductance

Water temperature

Alkalinity as $\mathrm{CaCO} 3$

Tubidity

Volumes purged

Sampling code

Synchronous water level

\section{ANALYTICAL DATA}

Inorganic Constituents

Constituents

Aluminum, total recoverable

Antimony, total recoverable

Arsenic, total recoverable

Barium, total recoverable

Beryllium, total recoverable

Cadmium, total recoverable 48

Chromium, total recoverable 0.91

Cobalt, total recoverable

Copper, total recoverable

Cyanide

Lead, total recoverable

Mercury, total recoverable

Nickel, total recoverable

Nitrate as nitrogen

Nitrate-nitrite as nitrogen $\quad 240,000$

Selenium, total recoverable

Silver, total recoverable

Thallium, total recoverable

Vanadium, total recoverable

Zinc, total recoverable

1099
28,000
$<0.20$
$<60$
790

48
0.91
300
25
$<10$
$<40$
0.43
95
240,000
$<100$
$<1.0$
24
$<10$
220

$\mathrm{J} / \mathrm{Cl}$

$\mathrm{U} / \mathrm{U} \quad<0.20$

$U / I \quad<60$

II

$\mathrm{J} /$

/I

JULI

$\mathrm{U} / /$

/I

II

U/I

UII

$\mathrm{J} / \mathrm{V}$

U//

Organic Constituents

\begin{tabular}{llll} 
Constituents & 1099 & Mod & Fitt. \\
\cline { 2 - 4 } Benzene & $<1.0$ & JU/UO & $<1.0$ \\
Bis(2-ethylhexyl) phthalate & $<10$ & U/I & $<10$ \\
Dichloromethane & 1.4 & J/U/O & NDD \\
Phenols & $<5.0$ & U/I & $<5.0$ \\
Tetrachloroethylene & $<1.0$ & JU/NO & $<1.0$ \\
Trichloroethylene & $<1.0$ & JU/O & $<1.0$ \\
Trichlorofluoromethane & $<5.0$ & JURO & $<5.0$
\end{tabular}

Trichlorofluoromethane

JULO
Top of Casing

04/09/99

$\begin{array}{lll}\text { Casing } & \text { Pumo } & \text { Screen Zone } \\ \text { 4" PVC } & S & \text { LAZ_UTRA }\end{array}$

AZ UTRA

Unit

tt msl

pH

$\mathrm{HS} / \mathrm{cm}$

${ }^{\circ} \mathrm{C}$

mgl

well volumes

ft msi

NDD

ST브

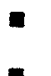

$<100$

$<1.0$

$<10$

$200.3(06 / 25 / 99)$

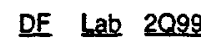

Mod

$\mathrm{J} / \mathrm{KJCl}$

20 GE

$20 \mathrm{GE}$

$\begin{array}{ll}1 & \mathrm{GE} \\ 1 & \mathrm{GE} \\ 1 & \mathrm{GE} \\ 1 & \mathrm{GE} \\ 1 & \mathrm{GE} \\ 20 & \mathrm{GE} \\ 1 & \mathrm{GE} \\ 1 & \mathrm{GE}\end{array}$

$<20$

U/I

$\begin{array}{ll}100 & \mathrm{GE} \\ 20 \mathrm{GE} & \end{array}$

Filt.

NDD

$<20$

$220,000 \quad / I$

1 GE

1 GE

1 GE
ST $H$ DF Lab Unit

$10 \mathrm{GE} \mu g \Omega$

$\mu g h$

ugh

- 1 GE

$\mu g /$

$\mu \mathrm{g}$ L

$\mu g \Omega$

$\mu g h$

$10 \mathrm{GE} \mu g / \mathrm{L}$

$\mu g h$

$\mu g / \mathrm{L}$

- $100 \mathrm{GE} \mu g \mathrm{~L}$

$\mu g h$

$\mu g h$

$\mu \mathrm{g} / \mathrm{L}$

$\mu \mathrm{gh}$

$\mu g \Omega$

$\begin{array}{lll}\text { SIH } & \text { LF } & \text { Lab } \\ 1 & \text { GE } \\ 1 & \text { GE } \\ 1 & \text { GE } \\ 1 & \text { GE } \\ 1 & \text { GE } \\ 1 & \text { GE } \\ 1 & \text { GE }\end{array}$

Fill.

ST $H$ DF Lab Unit

$\mu g h$

$\mu \mathrm{gh}$

$\mu g /$

$\mu g /$

$\mu \mathrm{gh}$

ugh

Notes:

- = exceeded holding time

= exceeded groundwater protection or monitoring constituent standard (See Appendix A.)

NA - Not applicable. Applies to beta dose and sum of betas if there are no beta-emitting radionuclides above detection limits; to sum of alphas if there are no alphaemitting radionuclides above detection limits; and to total radium il neither radium-226 or radium-228 was above detection limit

UAZ UTRA - Upper Aquifer Zone of the Upper Three Runs Aquiter, LAZ UTRA - Lower Aquifer Zone of the Upper Three Runs Aquifer, Gordon - Gordon Aguifer 
WELL FSB112C (cont.)

Radioactive Constituents

\begin{tabular}{|c|c|c|c|c|c|c|c|c|c|c|c|c|c|}
\hline Constituents & 1099 & Mod & Filt. & STH & DF & Lab & 2099 & Mod & Fill. & ST $H$ & DF & Lab & Unit \\
\hline $\begin{array}{l}\text { Americium-241 } \\
\text { Beta dose }\end{array}$ & $\begin{array}{l}4.3 E+01 \\
207\end{array}$ & $/ /$ & & $\mathbf{0}$ & 1 & GP & & & & & & & pCil \\
\hline Carbon-14 & $8.9 E+\infty 0$ & $\mathrm{~J} / \mathrm{L} / \mathrm{Cl}$ & NDD & & 1 & GP & & & & & & & pCin \\
\hline Cesium-137 & $<-8.0 E-01$ & $\mathrm{U} / /$ & $<4.1 E+\infty$ & & 1 & GP & & & & & & & pCin \\
\hline Cobalt -60 & $<2.7 E+\infty$ & $\mathrm{U} / /$ & $<5.3 E+\infty 0$ & & 1 & $G P$ & & & & & & & poin \\
\hline Curium-242 & $<5.7 E-01$ & $\mathrm{u} / \prime$ & $<4.3 E-01$ & & 1 & GP & & & & & & & pCin \\
\hline Curium-243/244 & $1.5 E+\infty 0$ & $\mathrm{~J} / \mathrm{V}$ & NDD & & 1 & GP & & & & & & & pCin \\
\hline Curium-245/246 & $2.5 E+\infty 0$ & $R / 4$ & Rej & & 1 & GP & & & & & & & pCill \\
\hline Gross alpha & $2.1 E+02$ & $\|$ & & $=$ & 1 & GP & $1.8 \mathrm{E}+02$ & $/ /$ & & $\mathbf{a}$ & 1 & GP & pCil \\
\hline lodine-129 & $1.1 E+02$ & $\|$ & & $\mathbf{n}$ & 1 & GP & & & & & & & pCin \\
\hline Nonvolatile beta & $1,8 E+03$ & 11 & & $\mathbf{a}$ & 1 & GP & $1.9 E+03$ & $\|$ & & च & 1 & GP & pCir \\
\hline Plutonium-238 & $<2.9 E-01$ & JU/1 & $<3.4 E-01$ & & 1 & GP & & & & & & & pCin \\
\hline Plutonium-239/240 & $<2.9 E-02$ & JU/A & $<2.1 E-01$ & & 1 & GP & & & & & & & pCir \\
\hline Radium-226 & $1.3 E+02$ & $\|$ & & a & 1 & GP & & & & & & & pCil \\
\hline Radium-228 & $<-1.5 E+\infty$ & JU/L/ & $<1.5 E+00$ & & 1 & GP & & & & & & & pCin \\
\hline Total radium & $1.3 E+02$ & & & & & & & & & & & & \\
\hline Strontium-90 & 7.7E+02 & $\|$ & & $\mathbf{a}$ & 1 & GP & & & & & & & pCin \\
\hline Technetium-99 & $3.5 E+02$ & $/ 1$ & & $\boldsymbol{m}$ & 1 & GP & & & & & & & pCir \\
\hline Thorium-228 & $9.4 \mathrm{E}-01$ & $\mathrm{~J} / \mathrm{V}$ & NDD & & 1 & GP & & & & & & & pCil \\
\hline Thorium-230 & $<-8.5 E-03$ & $\mathrm{U} / /$ & $<1.9 E-01$ & & 1 & GP & & & & & & & pCin \\
\hline Thorium-232 & $<0.0 E+00$ & $U / /$ & $<1.1 E-01$ & & 1 & GP & & & & & & & pCir \\
\hline Sum of alphas & $\begin{array}{l}5.0 E+01 \\
1.2 E+03\end{array}$ & & & 8 & & & & & & & & & pCil \\
\hline $\begin{array}{l}\text { Sum of belas } \\
\text { Tritium }\end{array}$ & $8.0 E+03$ & /I & & 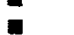 & 1 & GP & & & & & & & pCin \\
\hline $\begin{array}{l}\text { Tritium } \\
\text { Uranium-233/234 }\end{array}$ & $4.1 E+00$ & $\|$ & & & 1 & GP & $6.7 E+03$ & 11 & & 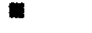 & 1 & GP & pCimt \\
\hline $\begin{array}{l}\text { Uranium-233/234 } \\
\text { Uranium-235 }\end{array}$ & $<4,5 E-01$ & $\mathrm{u} / 1$ & & & 1 & GP & & & & & & & pCil \\
\hline $\begin{array}{l}\text { Uranium-235 } \\
\text { Uranium-238 }\end{array}$ & $\begin{array}{l}<4.5 E-U 1 \\
3.3 E+00\end{array}$ & Un & $<5.1 E-01$ & & 1 & GP & & & & & & & $\begin{array}{l}\text { pCin } \\
\text { pCin }\end{array}$ \\
\hline
\end{tabular}

Notes:

- exceeded holding time

I = exceeded groundwater protection or monitoring constituent standard (See Appendix A)

NA - Not applicable. Applies to beta dose and sum of betas if there are no beta-emitting radionuclides above detection timits; to sum of alphas if there are no alphaemitting radionuclides above detection limits; and to total radium if neither radium-226 or radium-228 was above detection limit

UAZ UTRA - Upper Aquifer Zone of the Upper Three Runs Aquifer; LAZ_UTRA - Lower Aquifer Zone of the Upper Three Runs Aquifer, Gordon - Gordon Aquifer

F-Area HWMF

C-133

First and Second Quarter 1999 
WELL FSB112D

$\begin{array}{ll}\text { SRS Coord. } & \text { Lat/longitude } \\ \text { N74223.7 } & 33.269523^{\circ} \mathrm{N} \\ \text { E48780.0 } & 81.682527^{\circ} \mathrm{W}\end{array}$

SAMPLE DATE

Screen Zone Elevation

$208.9-188.9 \mathrm{ft} \mathrm{msl}$
Top of Casing

$229.6 \mathrm{ft} \mathrm{msl}$
Casing Pump

4. PVC 5
Screen Zone

UAZ_UTRA
04/12/99

2099
205.1
4.0
220
18.7
0
1
3.0
$203.8(06 / 25 / 99)$

Unit
$\mathrm{ftmsl}$
$\mathrm{pH}$
$\mathrm{HS} / \mathrm{cm}$
${ }^{\circ} \mathrm{C}$
$\mathrm{mgh}$
well volumes
$\mathrm{ft} \mathrm{msl}$

\section{FIELD DATA}

Constituents

1099

Water elevation

$\mathrm{pH}$

Sp. conductance

Water temperature

Alkalinity as $\mathrm{CaCO} 3$

Turbidity

Volumes purged

Sampling code

Synchronous water level

205.6

3.3

140

4.3

205.4 (03/23/99)

\section{ANALYTICAL DATA}

Inorganic Constituents

\begin{tabular}{|c|c|c|c|c|c|c|c|c|c|c|c|c|c|}
\hline Constituents & 1099 & Mod & Filt. & STH & $\underline{\mathrm{DF}}$ & Lab & 2099 & Mod & Filt. & ST $\mathrm{H}$ & DF & Lab & Unit \\
\hline Aluminum, total recoverable & 4,500 & 11 & & & 1 & GE & 7,500 & $\mathrm{~J} / \mathrm{K} / \mathrm{I}$ & NDD & & 1 & GE & \\
\hline Antimony, total recoverable & $<0.20$ & $\mathrm{U} / /$ & $<0.20$ & & 1 & GE & & & & & & & \\
\hline Arsenic, total recoverable & $<3.0$ & $\mathrm{U} / /$ & $<3.0$ & & 1 & GE & & & & & & & \\
\hline $\begin{array}{l}\text { Barium, total recoverable } \\
\text { Beryllium, total recoverable }\end{array}$ & 180 & $/ /$ & & & 1 & GE & & & & & & & \\
\hline Cadmium, total recoverable & 1.8 & 11 & & & 1 & GE & 3.6 & $\|$ & & & 1 & GE & \\
\hline Chromium, total recoverable & 0.99 & $\mathrm{~J} / \mathrm{U}$ & NDD & & 1 & GE & & & & & & & \\
\hline $\begin{array}{l}\text { Cobalt, total recoverable } \\
\text { Copper, total recoverable }\end{array}$ & & & & $\mathbf{a}$ & $\begin{array}{l}1 \\
1\end{array}$ & $\begin{array}{l}\text { GE } \\
\text { GE }\end{array}$ & & & & & & & \\
\hline Cyanide & $<10$ & JU/I & $<10$ & & 1 & $\mathrm{GE}$ & & & & & & & \\
\hline Lead, total recoverable & 2.2 & $/ 1$ & & & 1 & GE & 2.5 & II & & & 1 & GE & \\
\hline Mercury, total recoverable & $<0.20$ & $U / /$ & $<0.20$ & & 1 & GE & & & & & & & \\
\hline $\begin{array}{l}\text { Nickel, total recoverable } \\
\text { Nitrate as nitrogen }\end{array}$ & 4.9 & $\|$ & & & 1 & GE & & & & & & & \\
\hline Nitrate-nitrite as nitrogen & 12,000 & $1 /$ & & $\mathbf{D}$ & 25 & GE & 20,000 & 11 & & 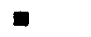 & 25 & GE & \\
\hline Selenium, total recoverable & 2.8 & $\mathrm{~J} / W$ & NDD & & 1 & GE & & & & & & & \\
\hline Silver, total recoverable & $<0.42$ & JU/ル14 & $<1.0$ & & 1 & GE & & & & & & & \\
\hline Thallium, total recoverable & $<0.23$ & UN/ & $<2.5$ & & 1 & GE & & & & & & & \\
\hline Vanadium, total recoverable & $<10$ & $\mathrm{U} / /$ & $<10$ & & 1 & GE & & & & & & & \\
\hline Zinc, total recoverable & 39 & $/ /$ & & & 1 & $G E$ & & & & & & & \\
\hline
\end{tabular}

Organic Constituents

$\begin{array}{llll}\text { Constituents } & 1099 & \text { Mod } & \text { Filt. } \\ \text { Benzene } & <1.0 & \mathrm{U} / \prime & <1.0 \\ \text { Bis(2-ethylhexyl) phthalate } & <10 & \mathrm{U} / \prime & <10 \\ \text { Dichloromethane } & <1.3 & \mathrm{JU} / \mathrm{KV} / \mathrm{O} & <5.0 \\ \text { Phenols } & <5.0 & \mathrm{U} / \prime & <5.0 \\ \text { Tetrachloroethylene } & <1.0 & \mathrm{U} / \prime & <1.0 \\ \text { Trichloroethylene } & <1.0 & \mathrm{U} / \prime & <1.0 \\ \text { Trichlorofluoromethane } & <5.0 & \mathrm{U} / \prime & <5.0\end{array}$

$\begin{array}{ccc}\text { ST브 } & \text { DF } & \text { Lab } \\ 1 & \text { GE } \\ 1 & \text { GE } \\ 1 & \text { GE } \\ 1 & \text { GE } \\ 1 & \text { GE } \\ 1 & \text { GE } \\ 1 & \text { GE }\end{array}$

Fill.

ST H DF Lab Unit

$\mu g h$

$\mu g$ L

$\mu g h$

$\mu \mathrm{g} / \mathrm{L}$

$\mu g h$

$\mu g h$

$\mu g h$

Notes:

- = exceeded holding time

- exceeded groundwater protection or monitoring constituent standard (See Appendix A.)

NA - Not applicable. Applies to beta dose and sum of betas if there are no beta-emitting radionuclides above detection limits; to sum of alphas if there are no aiphaemitting radionuclides above detection limits; and to total radium if neither radium-226 or radium-228 was above detection limit

UAZ_UTRA - Upper Aquifer Zone of the Upper Three Runs Aquifer, LAZ_UTRA - Lower Aquifer Zone of the Upper Three Runs Aquifer, Gordon - Gordon Aquifer 


\section{WELL FSB112D (cont.)}

Radioactive Constituents

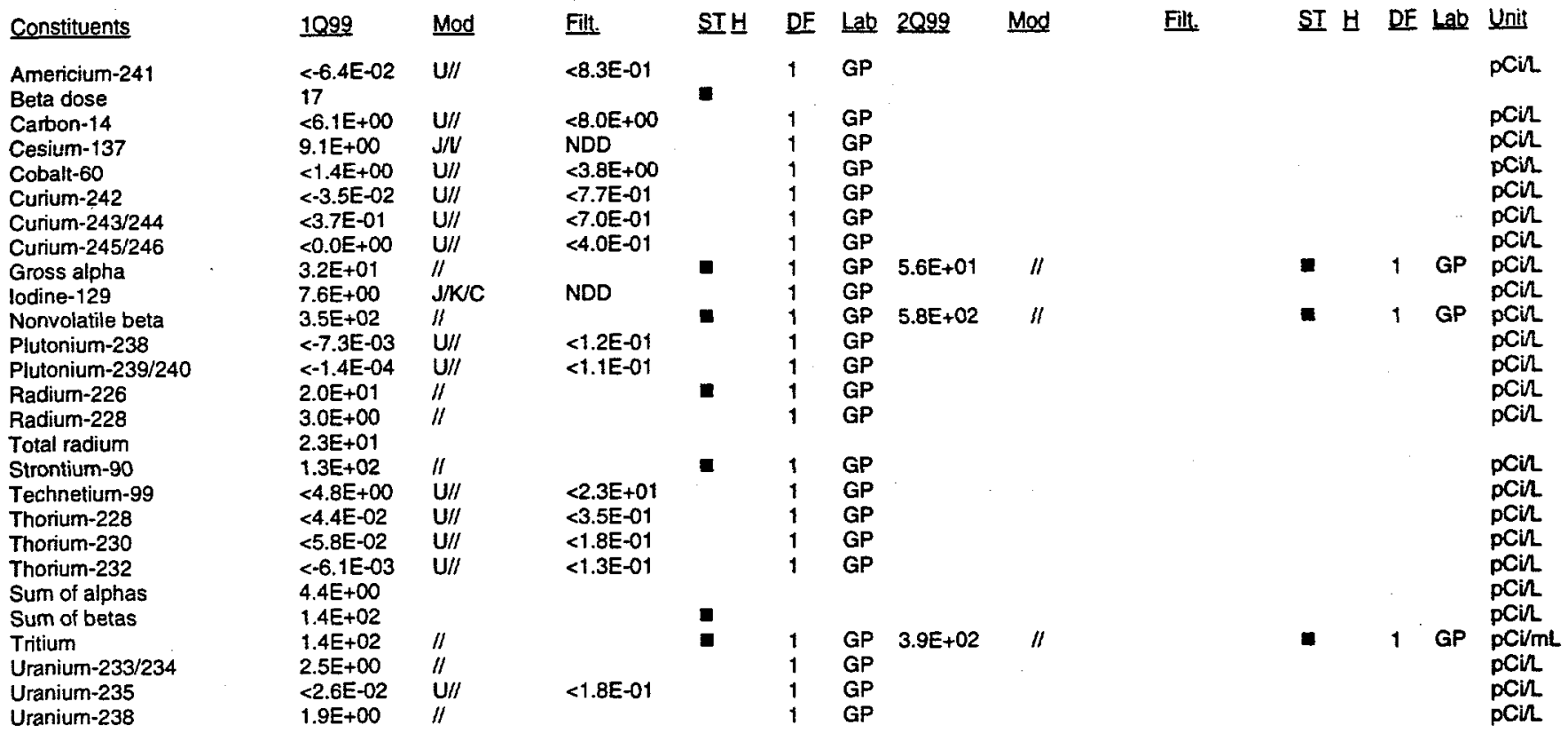


WELL FSB113A

\begin{tabular}{|c|c|}
\hline SRS Coord. & Lat/Longitude \\
\hline $\begin{array}{l}\text { N74167.5 } \\
\text { E51068.1 }\end{array}$ & $\begin{array}{l}33.273133^{\circ} \mathrm{N} \\
81.676395 \mathrm{on}\end{array}$ \\
\hline
\end{tabular}

SAMPLE DATE

Screen Zone Elevation

91.3-81.0 ft ms
Too of Casing

$223.2 \mathrm{ft} \mathrm{msl}$
Casing

Pumo

4* PVC S
Screen Zone

Gordon
04/05/99

2099
159.6
11.4
2200
19.7
473
0
0.019
$\times$
$159.8(06 / 28 / 99)$

Unit

t ms!

$\mathrm{pH}$

us/em

${ }^{\circ} \mathrm{C}$

mgl

well volumes

0.09

$160.5(03 / 23 / 99)$

ft ms!

\section{ANALYTICAL DATA}

Inorganic Constituents

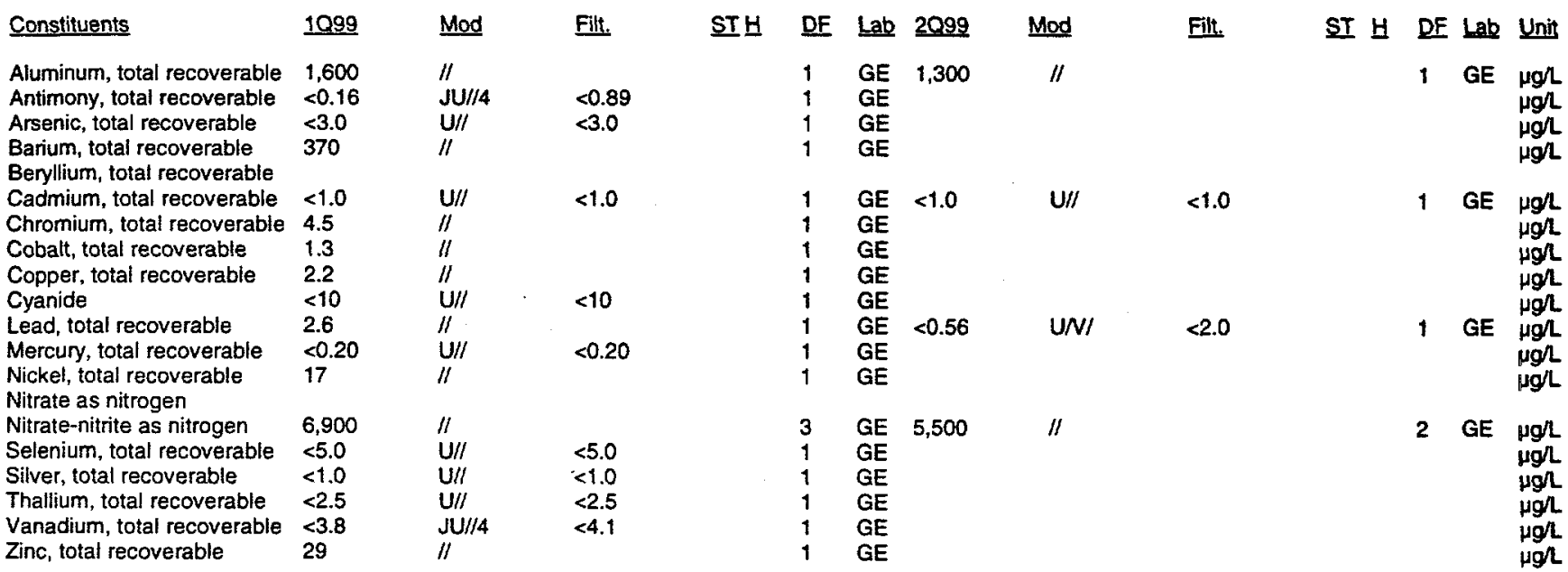

Organic Constituents

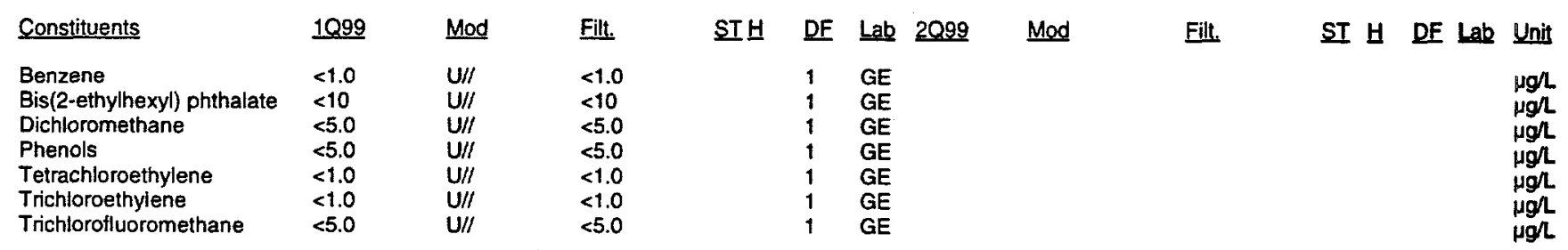

Notes:

- = exceeded holding time

= exceeded groundwater protection or monitoring constituent standard (See Appendix A.)

NA - Not applicable. Applies to beta dose and sum of betas if there are no beta-emitting radionuclides above detection limits; to sum of alphas if there are no alphaemitting radionuclides above detection limits; and to total radium if neither radium-226 or radium-228 was above detection limit

UAZ_UTRA - Upper Aquiler Zone of the Upper Three Runs Aquifer, LAZ UTRA - Lower Aquifer Zone of the Upper Three Runs Aquifer, Gordon - Gordon Aquiter 


\section{WELL FSB113A (cont.)}

Radiaactive Constituents

Constituents
Americium-241
Beta dose
Carbon-14
Cesium-137
Cobalt-60
Curium-242
Curium-243/244
Curium-245/246
Gross alpha
lodine-129
Nonvolatile beta
Plutonium-238
Plutonium-239/240
Radium-226
Radium-228
Total radium
Strontium-90
Technetium-99
Thorium-228
Thorium-230
Thorium-232
Sum of alphas
Sum of betas
Tritium
Uranium-233/234
Uranium-235
Uranium-238

\begin{tabular}{ll}
1099 & Mod \\
\hline$<5.0 E-03$ & $U / /$ \\
NA & \\
$<3.9 E+00$ & $U / /$ \\
$1.3 E+01$ & $\mathrm{R} / 4$ \\
$<6.3 \mathrm{E}-01$ & $\mathrm{U} / /$ \\
$<0.0 \mathrm{E}+00$ & $\mathrm{U} / /$ \\
$<-1.4 \mathrm{E}-04$ & $\mathrm{U} / /$ \\
$<0.0 \mathrm{E}+00$ & $\mathrm{U} / /$ \\
$5.4 \mathrm{E}+00$ & $/ /$ \\
$<9.5 \mathrm{E}-01$ & $\mathrm{U} / /$ \\
$7.1 \mathrm{E}+00$ & $/ /$ \\
$<1.3 \mathrm{E}-02$ & $\mathrm{U} / /$ \\
$<8.5 \mathrm{E}-03$ & $\mathrm{U} / /$ \\
$2.6 \mathrm{E}+00$ & $I /$ \\
$7.8 \mathrm{E}-01$ & $\mathrm{~J} / \mathrm{I}$ \\
$2.6 \mathrm{E}+00$ & \\
$<2.7 \mathrm{E}-02$ & $\mathrm{U} / /$ \\
$<1.1 \mathrm{E}+01$ & $\mathrm{U} / /$ \\
$<9.8 \mathrm{E}-02$ & $\mathrm{U} / /$ \\
$<8.4 \mathrm{E}-02$ & $\mathrm{U} / /$ \\
$<-7.1 \mathrm{E}-03$ & $\mathrm{U} / /$ \\
$\mathrm{NA}$ & \\
NA & \\
$1.7 \mathrm{E}+02$ & $/ /$ \\
$<-2.2 \mathrm{E}-02$ & $\mathrm{U} / /$ \\
$<-2.2 \mathrm{E}-02$ & $\mathrm{U} / /$ \\
$<1.4 \mathrm{E}-02$ & $\mathrm{U} / /$
\end{tabular}

Filt.
$<4.3 E-02$
$<7.5 E+00$
Rej
$<2.8 E+00$
$<4.6 E-02$
$<1.1 E-01$
$<4.3 E-02$
$<1.2 E+00$
$<1.0 E-01$
$<8.3 E-02$
$N D D$
$<1.0 E+00$
$<1.9 E+01$
$<2.8 E-01$
$<1.1 E-01$
$<8.8 E-02$

SI H

DF

1

GP

1 GP

1 GP

1 GP

1 GP

1 GP

1 GP

$1 G P$

1 GP

1 GP

1 GP

1 GP

$1.9 \mathrm{E}+\infty \mathrm{J} / \mathrm{V}$

$4.0 \mathrm{E}+\infty$

NDD

1 GP

1 GP

1 GP

$\begin{array}{ll}1 & \text { GP } \\ 1 & \text { GP }\end{array}$

口

1.1E+02 //

Filt.

$\begin{array}{ll}1 & \text { GP } \\ 1 & \text { GP } \\ 1 & \text { GP }\end{array}$

ST $H$ DE Lab Unit

pCin

pcin

pCin

pCir

pCir

pCin

pCil

1 GP pCir

$1 \mathrm{GP}{ }_{\mathrm{pCi}}^{\mathrm{PCi}}$

pCir

pCin

pCill

pCir

pCin

pCir

pCir

pCil

pcil

pCil

$<15 E-01$

pCi/mL

pCir

pCir

1 GP

pCir

Notes:

- exceeded holding time

E = exceeded groundwater protection or monitoring constituent standard (See Appendix A.)

NA - Not applicable. Applies to beta dose and sum of betas if there are no beta-emitting radionuclides above detection limits; to sum of alphas if there are no alphaemitting radionuclides above detection limits; and to total radium if neither radium-226 or radium-228 was above detection limit

UAZ_UTRA - Upper Aquifer Zone of the Upper Three Runs Aquifer, LAZ_UTRA - Lower Aquifer Zone of the Upper Three Runs Aquifer, Gordon - Gordon Aquiler

F-Area HWMF

C-137

First and Second Quarter 1999 


\section{WELL FSB113C}

SRS Coord. Latlongitude

N74160.7 $33.273144^{\circ} \mathrm{N}$

E51084.2 $81.676339^{\circ} \mathrm{W}$

SAMPLE DATE

Screen Zone Elevation

$164.0-154.0 \mathrm{ft} \mathrm{ms!}$

Top of Casing

$222.9 \mathrm{ft} \mathrm{msl}$

04/05/99

Constituents

Water elevation

$\mathrm{pH}$

Sp. conductance

Water temperature

Alkalinity as $\mathrm{CaCO} 3$

Turbidity

Volumes purged

Sampling code

Synchronous water level

\section{ANALYTICAL DATA}

Inorganic Constituents
1099

Aluminum, total recoverable 1,000

Antimony, total recoverat

Arsenic, total recoverable

Barium, total recoverable

Beryllium, total recoverable

Cadmium, total recoverable 1.3

Chromium, total recoverable 1.3

Cobalt total recoverable $\quad 6.2$

Copper, total recoverable

Cyanide

Lead, total recoverable

Nickel, total recoverable 15

Nitrate as nitrogen

Nitrate-nitrite as nitrogen $\quad 5,600$

Selenium total recoverable $<5.0$

Silver, total recoverable $<1.0$

Thallium, total recoverable 0.14

Vanadium total recoverable $<10$

Zinc, total recoverable

120

Organic Constituents

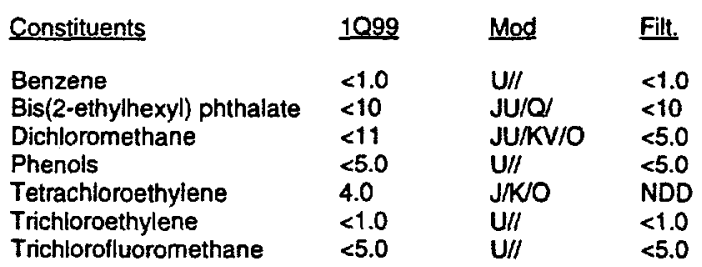

1099

$\begin{array}{ll}2099 & \text { Unit } \\ 202.4 & \\ 6.6 & \mathrm{tt} \mathrm{msl} \\ 130 & \mathrm{pH} \\ 21.4 & \mu \mathrm{SS} / \mathrm{cm} \\ 27 & { }^{\circ} \mathrm{C} \\ 1 & \mathrm{mgh} \\ 2.3 & \mathrm{NTU} \\ 201.6(06 / 28 / 99) & \text { well volumes } \\ & \text { tt msl }\end{array}$

$202.3(03 / 23 / 99)$
Casing Pump

4* PVC S

Screen Zone

LAZ_UTRA

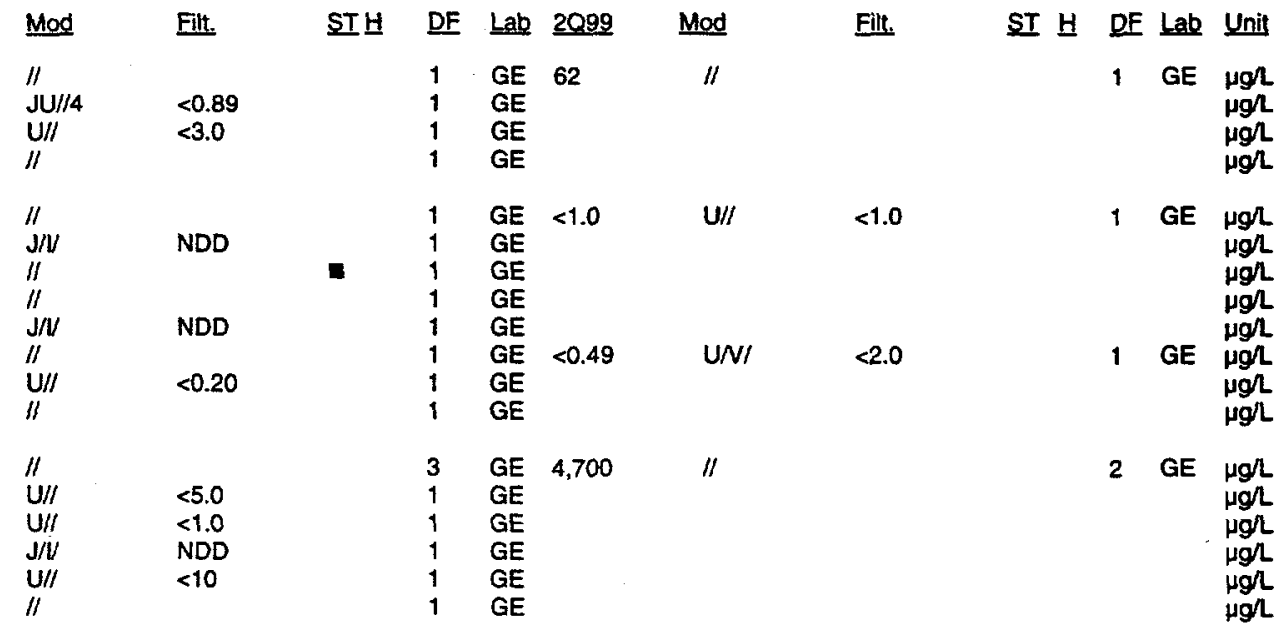

Notes:

- = exceeded holding time

- exceeded groundwater protection or monitoring constituent standard (See Appendix A)

NA - Not applicable. Applies to beta dose and sum of betas if there are no beta-mitting radionuclides above detection limits; to sum of alphas if there are no alphaemitting radionuclides above detection limits; and to total radium if neither radium-226 or radium-228 was above delection limit

UAZ_UTRA - Upper Aquiter Zone of the Upper Three Runs Aquiler, LAZ UTRA - Lower Aquifer Zone of the Upper Three Runs Aquifer, Gordon - Gordon Aquifer 
WELL FSB113C (cont.)

Radioactive Constituents

\begin{tabular}{|c|c|c|c|c|c|c|c|c|c|c|c|c|c|}
\hline Constituents & 1099 & Mod & Filt. & ST브 & DF & Lab & 2099 & Mod & Filt. & ST $H$ & DF & Lab & Unit \\
\hline $\begin{array}{l}\text { Americium-241 } \\
\text { Beta dose }\end{array}$ & $\begin{array}{l}<-1.7 E-03 \\
\text { NA }\end{array}$ & $U / /$ & $<9.6 \mathrm{E}-02$ & & 1 & GP & & & & & & & pCin \\
\hline Carbon-14 & $<2.1 E+\infty$ & $\mathrm{U} / /$ & $<7.3 \mathrm{E}+\infty$ & & 1 & GP & & & & & & & pCin \\
\hline Cesium-137 & $<8.8 E-01$ & $\mathrm{U} / /$ & $<4.2 E+\infty 0$ & & 1 & GP & & & & & & & pCin \\
\hline Cobalt -60 & $<-8.9 E-01$ & $\mathrm{U} / \prime$ & $<4.1 E+00$ & & 1 & GP & & & & & & & pCin \\
\hline Curium-242 & $<-8.3 E-03$ & $\mathrm{U} / /$ & $<1.0 E-01$ & & 1 & GP & & & & & & . & pCin \\
\hline Curium-243/244 & $<-7.7 E-03$ & $\mathrm{U} / /$ & $<9.6 \mathrm{E}-02$ & & 1 & GP & & & . & & & & pCil \\
\hline Curium-245/246 & $<7.6 \mathrm{E}-03$ & $\mathrm{U} / /$ & $<9.6 E-02$ & & $i$ & GP & & & & & & & pCin \\
\hline Gross alpha & $1.7 E+\infty$ & $\mathrm{JN}$ & NDD & & 1 & GP & $<2.5 E-01$ & $U / I$ & $<7.9 E-01$ & & 1 & GP & peil \\
\hline lodine-129 & $<1.2 \mathrm{E}-01$ & $\mathrm{U} / I$ & $<1.3 E+\infty 0$ & & 1 & GP & & & & & & & pCin \\
\hline Nonvolatile beta & $2.5 E+00$ & $\mathrm{~J} / \mathrm{V}$ & NDD & & 1 & GP & $1.7 E+\infty 0$ & $\mathrm{~J} / \mathrm{V}$ & NDD & & 1 & GP & pCin \\
\hline Plutonium-238 & $1.5 E-01$ & $\mathrm{R} / / 4$ & Rej & & 1 & GP & 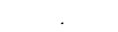 & & & & & & $\mathrm{pCil}$ \\
\hline Plutonium-239/240 & $<2.1 \mathrm{E}-02$ & UII & $<7.3 E-02$ & & 1 & GP & & & & & & & pCil \\
\hline Radium-226 & $1.1 E+00$ & $1 /$ & & & 1 & GP & & & & & & & pCir \\
\hline $\begin{array}{l}\text { Radium-228 } \\
\text { Total radium }\end{array}$ & $\begin{array}{l}<5.7 E-01 \\
1.1 E+00\end{array}$ & $\mathrm{u} / /$ & $<7.5 E-01$ & & 1 & GP & & & & & & & pCill \\
\hline Strontium-90 & $<-1.0 E-01$ & $\mathrm{U} / /$ & $<1.0 \mathrm{E}+\infty$ & & 1 & GP & & & & & & & pCin \\
\hline Technetium-99 & $<9.4 E+\infty$ & $\mathrm{U} / /$ & $<1.9 E+01$ & & 1 & GP & & & & & & & pCir \\
\hline Thorium-228 & $<1.9 E-01$ & $\mathrm{U} / \mathrm{I}$ & $<2.7 E-01$ & & 1 & GP & & & & & & & pCin \\
\hline Thorium-230 & $<1.2 \mathrm{E}-01$ & $\mathrm{U} / \mathrm{I}$ & $<1.3 E-01$ & & 1 & GP & & & & & & & pCin \\
\hline Thorium-232 & $<8.5 E-03$ & $\mathrm{U} / /$ & $<1.1 E-01$ & & 1 & GP & & & & & & & $\mathrm{pCi} / \mathrm{L}$ \\
\hline Sum of alphas & & & & & & & & & & & & & \\
\hline Sum of betas & NA & & & & & & & & & & & & \\
\hline Tritium & $6.5 E+01$ & $1 /$ & & $\boldsymbol{0}$ & $i$ & GP & $5.3 E+01$ & $/ /$ & & $\mathbf{0}$ & 1 & GP & pCiml \\
\hline Uranium-233/234 & $<7.0 E-02$ & $\mathrm{U} / /$ & $<1.4 E=01$ & & 1 & GP & & & & & & & pCin \\
\hline Uranium-235 & $<-1.6 \mathrm{E}-04$ & $\mathrm{U} / \prime$ & $<1.2 \mathrm{E}-01$ & & 1 & GP & & & & & & & pCin \\
\hline Uranium-238 & $<3.9 E-02$ & UII & $<1.4 \mathrm{E}-01$ & & 1 & GP & & & & & & & pCin \\
\hline
\end{tabular}

Notes:

- exceeded holding time

I = exceeded groundwater protection or monitoring constituent standard (See Appendix A.)

NA - Not applicable. Applies to beta dose and sum of betas if there are no beta-emitting radionuclides above detection limits; to sum of alphas if there are no alphaemitting radionuclides above detection limits; and to total radium if neither radium-226 or radium-228 was above detection limit

UAZ_UTRA - Upper Aquifer Zone of the Upper Three Runs Aquifer, LAZ_UTRA - Lower Aquifer Zone of the Upper Three Runs Aquifer, Gordon - Gordon Aquifer

F-Area HWMF

C-139

First and Second Quarter 1999 
WELL FSB113D

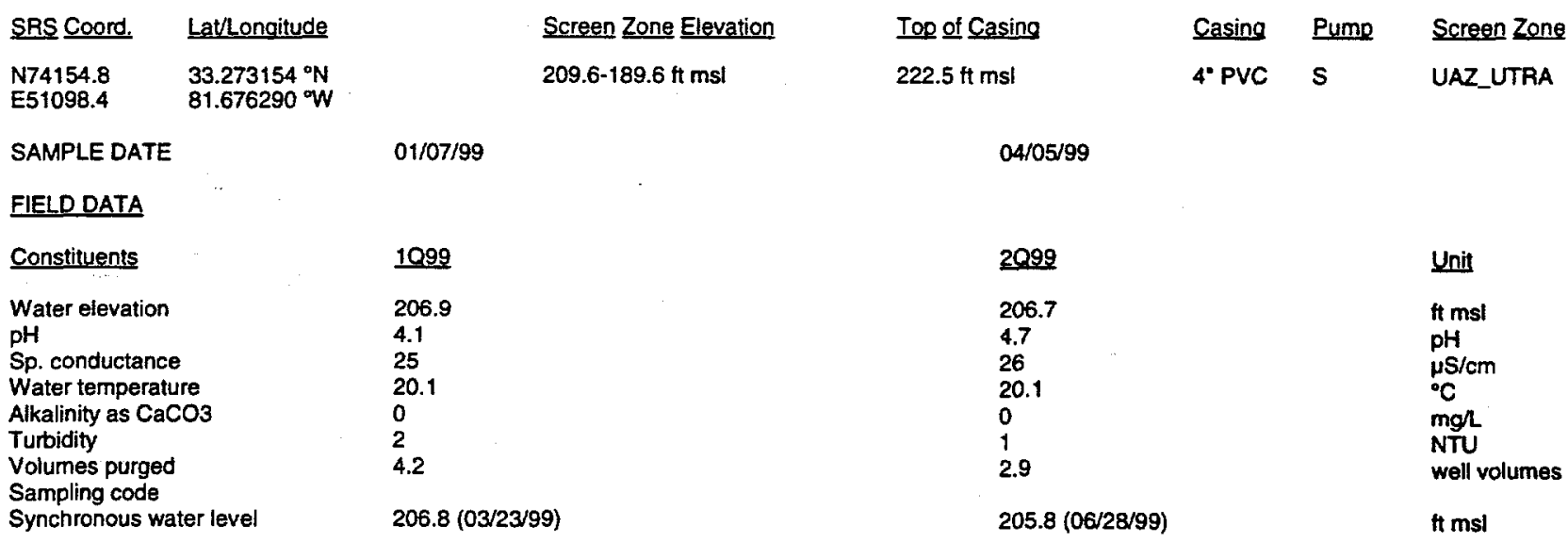

\section{ANALYTICAL DATA}

Inorganic Constituents

\begin{tabular}{|c|c|c|c|c|c|c|c|c|c|c|c|c|c|}
\hline Constituents & 1099 & Mod & Filt. & STH & DF & Lab & 2099 & Mod & Filt. & ST $H$ & DE & Lab & Unit \\
\hline Aluminum, total recoverable & 47 & $/ 1$ & & & 1 & GE & 38 & $/ /$ & & & 1 & GE & $\mu g /$ \\
\hline Antimony, total recoverable & $<0.20$ & $\mathrm{U} / /$ & $<0.20$ & & 1 & GE & & & & & & & ugh \\
\hline Arsenic, total recoverable & $<3.0$ & $\mathrm{U} / \prime$ & $<3.0$ & & 1 & GE & & & & & & & $\mu g /$ \\
\hline $\begin{array}{l}\text { Barium, total recoverable } \\
\text { Beryllium, total recoverable }\end{array}$ & 5.0 & $/ 1$ & & & 1 & GE & & & & & & & $\mu g / 2$ \\
\hline Cadmium, total recoverable & $<1.0$ & $\mathrm{u} / /$ & $<1.0$ & & 1 & GE & $<1.0$ & $\mathrm{U} / /$ & $<1.0$ & & 1 & GE & $\mu g / L$ \\
\hline Chromium, total recoverable & 1.2 & $\mathrm{~J} / \mathrm{V}$ & NDD & & 1 & GE & & & & & & & $\mu g / L$ \\
\hline Cobalt, total recoverable & 0.31 & $\|$ & & & 1 & GE & & & & & & & $\mu g h$ \\
\hline $\begin{array}{l}\text { Copper, total recoverable } \\
\text { Cyanide }\end{array}$ & 13 & $\|$ & & & 1 & GE & & & & & & & $\mu g /$ \\
\hline Cyanide & 3.2 & $\mathrm{~J} / \mathrm{N}$ & NDD & & 1 & GE & & & & & & & $\mu g /$ \\
\hline Lead, total recoverable & 1.1 & $\mathrm{~J} / \mathrm{V}$ & NDD & & 1 & GE & $<0.92$ & UNI & $<2.0$ & & 1 & GE & $\mu g /$ \\
\hline Mercury, total recoverable & $<0.20$ & $U / I$ & $<0.20$ & & 1 & GE & & & & & & & \\
\hline $\begin{array}{l}\text { Nickel, total recoverable } \\
\text { Nitrate as nitrogen }\end{array}$ & 1.6 & $\mathrm{~J} / \mathrm{V} /$ & NDD & & 1 & GE & & & & & & & \\
\hline Nitrate-nitrite as nitrogen & 630 & $/ 1$ & & & 1 & GE & 610 & /l & & & 1 & GE & $\mu g h$ \\
\hline Selenium, total recoverable & $<5.0$ & $U / /$ & $<5.0$ & & 1 & GE & & & & & & & jgh \\
\hline Silver, total recoverable & $<1.0$ & $\mathrm{U} / /$ & $<1.0$ & & 1 & GE & & & & & & & Hg \\
\hline Thallium, total recoverable & $<2.5$ & $\mathrm{U} / /$ & $<2.5$ & & 1 & GE & & & & & & & $\mu g h$ \\
\hline Vanadium, total recoverable & $<10$ & $U / /$ & $<10$ & & 1 & $\mathrm{GE}$ & & & & & & & gh \\
\hline Zinc, total recoverable & $<10$ & $\mathrm{U} / /$ & $<10$ & & 1 & $\overline{\mathrm{GE}}$ & & & & & & & \\
\hline
\end{tabular}

Organic Constituents

\begin{tabular}{|c|c|c|c|c|c|c|c|c|c|c|c|}
\hline Constituents & 1099 & Mod & Filt. & ST보 & $\underline{\mathrm{DF}}$ & Lab 2099 & Mod & Fill. & ST H & DF Lab & Unit \\
\hline $\begin{array}{l}\text { Benzene } \\
\text { Bis(2-ethylhexyl) phthalate } \\
\text { Dichloromethane } \\
\text { Phenols } \\
\text { Tetrachloroethylene } \\
\text { Trichloroethylene } \\
\text { Trichlorofluoromethane }\end{array}$ & $\begin{array}{l}<1.0 \\
<10 \\
<12 \\
<5.0 \\
<1.0 \\
<1.0 \\
<5.0\end{array}$ & $\begin{array}{l}U / / \\
U / I \\
J / K V / O \\
U / / \\
U / I \\
U / I \\
U / I\end{array}$ & $\begin{array}{l}<1.0 \\
<10 \\
<5.0 \\
<5.0 \\
<1.0 \\
<1.0 \\
<5.0\end{array}$ & & $\begin{array}{l}1 \\
1 \\
1 \\
1 \\
1 \\
1 \\
1\end{array}$ & $\begin{array}{l}\mathrm{GE} \\
\mathrm{GE} \\
\mathrm{GE} \\
\mathrm{GE} \\
\mathrm{GE} \\
\mathrm{GE} \\
\mathrm{GE}\end{array}$ & & & & & $\begin{array}{l}\mu g / \mathrm{L} \\
\mu g h \\
\mu g h \\
\mu g h \\
\mu g h \\
\mu g h \\
\mu g h\end{array}$ \\
\hline
\end{tabular}

Notes:

- exceeded holding time

= exceeded groundwater protection or monitoring constituent standard (See Appendix A.)

NA - Not applicable. Applies to beta dose and sum of betas if there are no beta-emitting radionuclides above detection limits; to sum of alphas if there are no alphaemitting radionuclides above detection limits; and to total radium if neither radium-226 or radium-228 was above detection limit

UAZ UTRA - Upper Aquifer Zone of the Upper Three Runs Aquifer, LAZ UTRA - Lower Aquifer Zone of the Upper Three Runs Aquiter, Gordon - Gordon Aquifer
F-Area HWMF
C-140
First and Second Quarter 1999 
WELL FSB113D (cont.)

Radioactive Constituents

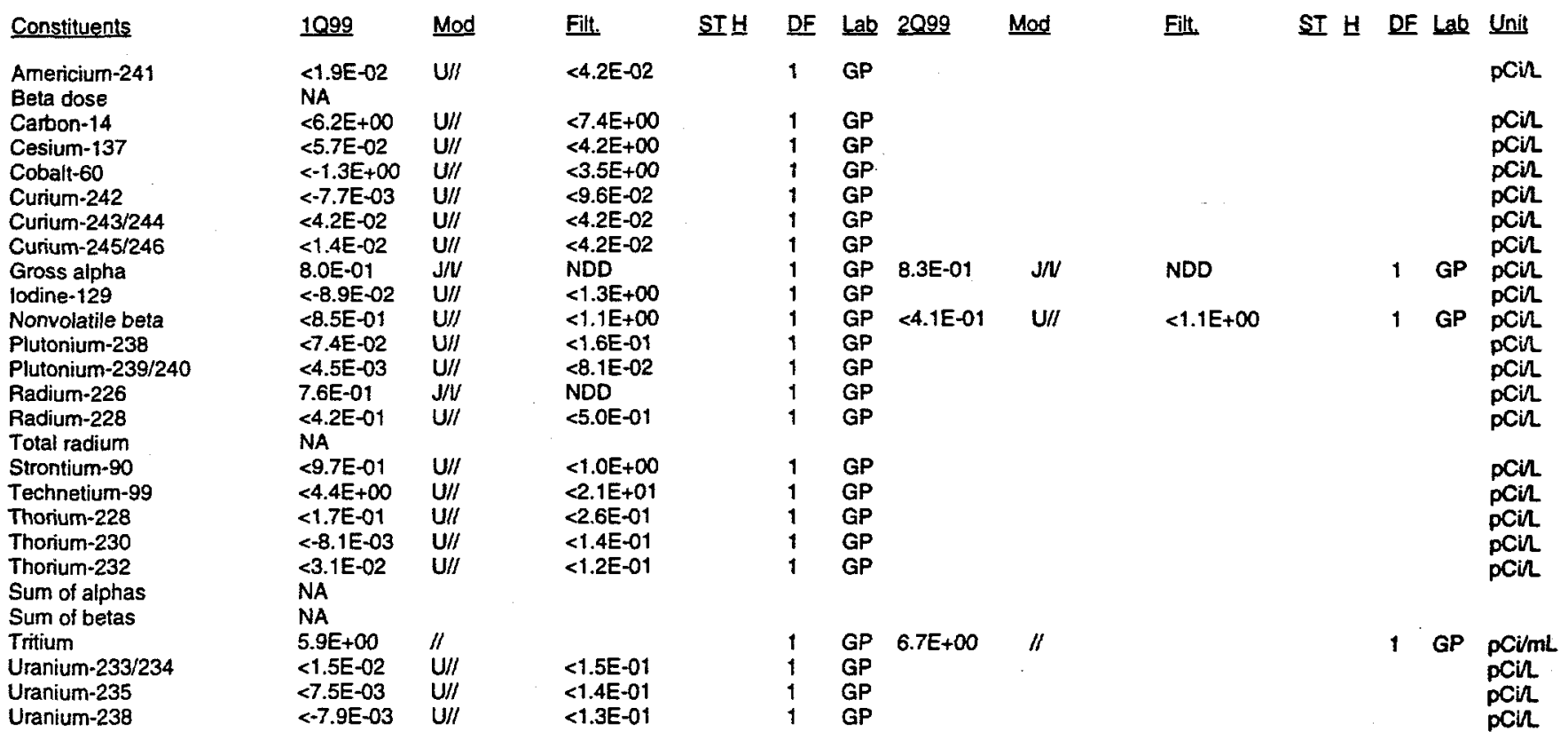

- = exceeded holding time

- exceeded groundwater protection or monitoring constituent standard (See Appendix A.)

NA - Not applicable. Applies to beta dose and sum of betas if there are no beta-emitting radionuclides above detection limits; to sum of alphas if there are no alphaemitting radionuclides above detection limits; and to total radium if neither radium-226 or radium-228 was above detection limit UAZ UTRA - Upper Aquifer Zone of the Upper Three Runs Aquifer, LAZ. UTRA - Lower Aquifer Zone of the Upper Three Runs Aquifer, Gordon - Gordon Aquifer 


\section{WELL FSB114A}

$\begin{array}{ll}\text { SRS Coord. } & \text { Latllongitude } \\ \text { N75297.4 } & \\ \text { E52046.6 } & 81.277229^{\circ} \mathrm{N} \\ & 81.676014^{\circ} \mathrm{W}\end{array}$

SAMPLE DATE
Screen Zone Elevation

$105.0-95.2 \mathrm{ft} \mathrm{mst}$
Top of Casing

$252 \mathrm{ft} \mathrm{msl}$

\section{Casing Pump}

4" PVC S
Screen Zone

Gordon
$01 / 07 / 99$

$04 / 05 / 99$

2099

155.8

7.3

180

21.9

64

2.7

$155.8(03 / 23 / 99)$
Unit

it ms!

pH

jS/cm

${ }^{\circ} \mathrm{C}$

mgh

well volumes

ft msi
Synchronous water level

$155.4(06 / 28 / 99)$

\section{ANALYTICAL DATA}

Inorganic Constituents

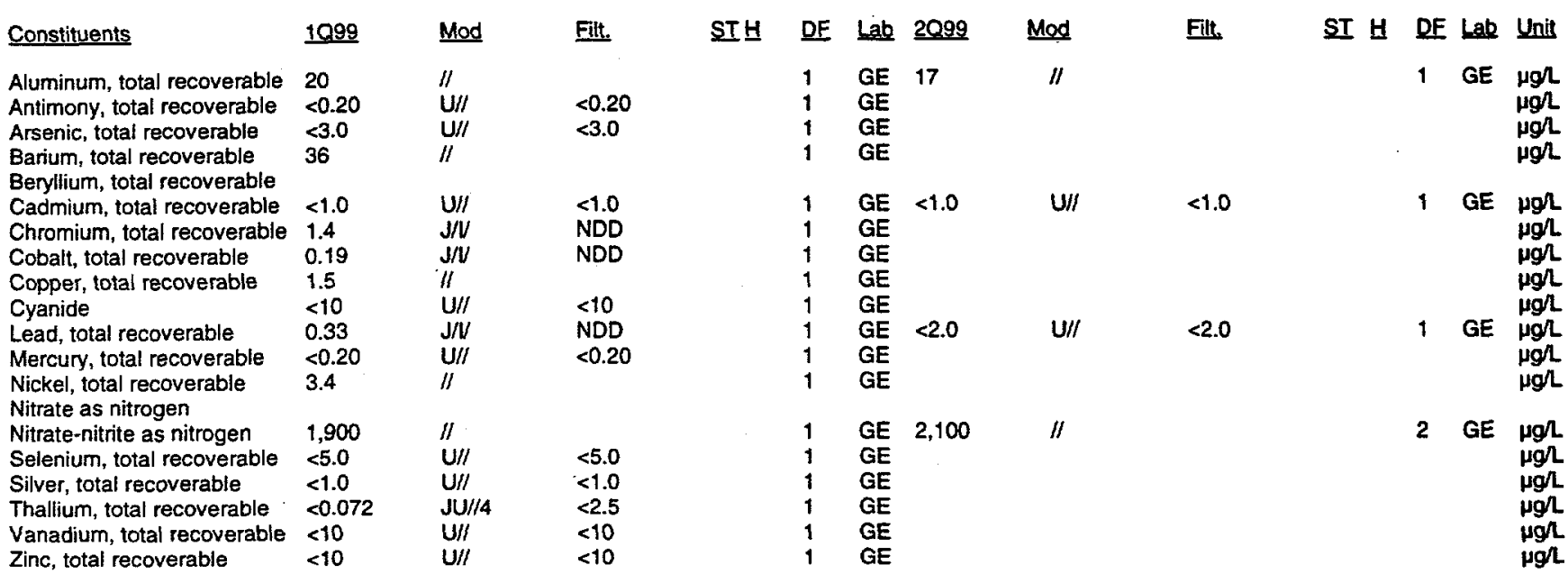

Organic Constituents

\begin{tabular}{|c|c|c|c|c|c|}
\hline Constituents & 1099 & Mod & Filt. & ST브 & DF \\
\hline Benzene & $<1.0$ & $\mathrm{U} / /$ & $<1.0$ & & 1 \\
\hline Bis(2-ethylhexyl) phthalate & $<10$ & $\mathrm{U} / /$ & $<10$ & & 1 \\
\hline Dichloromethane & $<11$ & UNI & $<5.0$ & & 1 \\
\hline Phenois & $<5.0$ & $\mathbf{U} / \prime$ & $<5.0$ & & 1 \\
\hline Tetrachloroethylene & $<1.0$ & $\mathrm{U} / /$ & $<1.0$ & & 1 \\
\hline Trichloroethylene & 1.6 & $\|$ & & & 1 \\
\hline Trichlorofluoromethane & $<5.0$ & $\mathrm{u} / /$ & $<5.0$ & & 1 \\
\hline
\end{tabular}

Filt.

ST H DE Lab Unit

$\mu g \Omega$

$\mu g \Omega$

$\mu g h$

$\mu g h$

pgl

$\mu \mathrm{g} L$

$\mu g h$

\section{Notes:}

- = exceeded holding time

- = exceeded groundwater protection or monitoring constituent standard (See Appendix A.)

NA - Not applicable. Applies to beta dose and sum of betas if there are no beta-emitting radionuclides above detection limits; to sum of alphas if there are no alphaemitting radionuclides above detection limits; and to total radium if neither radium-226 or radium-228 was above detection limit

UAZ_UTRA - Upper Aquifer Zone of the Upper Three Runs Aquifer, LAZ_UTRA - Lower Aquifer Zone of the Upper Three Runs Aquifer, Gordon - Gordon Aquifer 


\section{WELL FSB114A (cont.)}

Radioactive Constituents

\begin{tabular}{|c|c|c|c|c|c|c|c|c|c|c|c|c|c|}
\hline Constituents & 1099 & Mod & Filt. & STH & DF & Lab & 2099 & Mod & Filt. & ST $\underline{H}$ & DE & Lab & Unit \\
\hline $\begin{array}{l}\text { Americium-241 } \\
\text { Beta dose }\end{array}$ & $\begin{array}{l}<1.2 E-02 \\
N A\end{array}$ & $\mathbf{U} / /$ & $<3.2 E-02$ & & 1 & GP & & & & & & & pCin \\
\hline Carbon-14 & $<-3.1 E+\infty 0$ & $\mathbf{U} / \prime$ & $<7.2 E+00$ & & 1 & GP & & & & & & & pCin \\
\hline $\begin{array}{l}\text { Cesium-137 } \\
\text { Cobalt-60 }\end{array}$ & $\begin{array}{l}<4.5 E-01 \\
<1.3 E+00\end{array}$ & $\begin{array}{l}U / I \\
U / /\end{array}$ & $\begin{array}{l}<4.3 E+00 \\
<4.2 E+00\end{array}$ & & $\begin{array}{l}1 \\
1\end{array}$ & $\begin{array}{l}\text { GP } \\
\text { GP }\end{array}$ & & & & $\cdot$ & & & pCin \\
\hline Curium-242 & $<0.0 E+00$ & $\mathrm{U} / /$ & $<3.5 E-02$ & & 1 & GP & & & & & & & $\begin{array}{l}\text { pCin } \\
\text { pCin }\end{array}$ \\
\hline Curium-243/244 & $<-5.5 E-03$ & $U / I$ & $<9.4 E-02$ & & 1 & GP & & & & & & & pCil \\
\hline Curium-245/246 & $<0.0 E+00$ & $\mathrm{U} / /$ & $<3.2 \mathrm{E}-02$ & & 1 & GP & & & & & & & pCir \\
\hline $\begin{array}{l}\text { Gross alpha } \\
\text { lodine-129 }\end{array}$ & $<2.3 E-01$ & $\mathrm{U} / /$ & $<9.2 \mathrm{E}-01$ & & 1 & GP & $<5.6 E-01$ & $\mathrm{U} / /$ & $<9.3 E-01$ & & 1 & GP & $\mathrm{pCil}$ \\
\hline $\begin{array}{l}\text { lodine-129 } \\
\text { Nonvolatile beta }\end{array}$ & $\begin{array}{l}<2.7 E-01 \\
<5.3 E-01\end{array}$ & $\begin{array}{l}\mathrm{U} / / \\
\mathrm{U} / /\end{array}$ & & & 1 & GP & & & & & & & pCil \\
\hline & $1.9 E-01$ & $\mathrm{R} / / \mathrm{l}$ & $\begin{array}{l}<1.5 \mathrm{E}+00 \\
\text { Rej }\end{array}$ & & 1 & GP & $1.2 E+\infty$ & $\mathrm{J} / \mathrm{L}$ & NDD & & 1 & GP & pCin \\
\hline $\begin{array}{l}\text { Plutonium-238 } \\
\text { Plutonium-239/240 }\end{array}$ & $<3.5 \mathrm{E}-03$ & $\mathrm{U} / /$ & $\begin{array}{l}\text { Rej } \\
<4.5 E-02\end{array}$ & & $\begin{array}{l}1 \\
1\end{array}$ & $\begin{array}{l}\text { GP. } \\
\text { GP }\end{array}$ & & & & & & & $\begin{array}{l}\text { pCin } \\
\text { pCin }\end{array}$ \\
\hline Radium-226 & 4.7E-01 & $\mathrm{J} / \mathrm{V}$ & NDD & & 1 & GP & & & & & & & pCiL \\
\hline $\begin{array}{l}\text { Radium-228 } \\
\text { Total radium }\end{array}$ & $\begin{array}{l}<5.5 E-01 \\
\text { NA }\end{array}$ & $\mathrm{U} / /$ & $<5.9 E-01$ & & 1 & GP & & & & & & & $\mathrm{pCi} L$ \\
\hline Strontium-90 & $<2.2 E-01$ & $\mathrm{U} / I$ & $<1.1 E+\infty$ & & 1 & GP & & & & & & & $\mathrm{pCi}$ \\
\hline Technetium-99 & $<-4.6 E-01$ & $\mathrm{U} / /$ & $<1.9 E+01$ & & 1 & GP & & & & & & & $\mathrm{p} \mathrm{Ci} / \mathrm{L}$ \\
\hline Thorium-228 & 2.4E-01 & $\mathrm{J} / \mathrm{V}$ & NDD & & 1 & GP & & & & & & & $\mathrm{pCi}$ \\
\hline Thorium-230 & $<7.2 \mathrm{E}-02$ & $\mathrm{U} / /$ & $<1.4 E-01$ & & 1 & GP & & & & & & & pCin \\
\hline $\begin{array}{l}\text { Thorium-232 } \\
\text { Sum of alphas }\end{array}$ & $\begin{array}{l}<-9.2 E-03 \\
\text { NA }\end{array}$ & $\mathrm{u} / 1$ & $<1.1 E-01$ & & 1 & GP & & & & & & & pCir \\
\hline Sum of betas & NA & & & & & & & & & & & & \\
\hline Tritium & $9.2 E-01$ & $\mathrm{~J} / \mathrm{V}$ & NDD & & 1 & GP & 8.4E-01 & $J / / V$ & NDD & & 1 & GP & pCi/mL \\
\hline Uranium-233/234 & $<3.8 \mathrm{E}-02$ & $\mathrm{U} / /$ & $<1.6 E-01$ & & 1 & $\mathrm{GP}$ & & & & & & & pCin \\
\hline Uranium-235 & $<2.3 E-02$ & $\mathrm{U} / /$ & $<9.8 E-02$ & & 1 & GP & & & & & & & pCin \\
\hline Uranium-238 & $<2.3 E-02$ & $\mathrm{U} / /$ & $<9.8 E-02$ & & 1 & GP & & & & & & & pCin \\
\hline
\end{tabular}

- = exceeded holding time

= exceeded groundwater protection or monitoring constituent standard (See Appendix A.)

NA - Not applicable. Applies to beta dose and sum of betas if there are no beta-emitting radionuclides above detection limits; to sum of alphas if there are no alphaemitting radionuclides above detection limits; and to total radium if neither radium-226 or radium-228 was above detection limit

UAZ_UTRA - Upper Aquifer Zone of the Upper Three Runs Aquifer, LAZ_UTRA - Lower Aquifer Zone of the Upper Three Runs Aquifer, Gordon - Gordon Aquifer 


\section{WELL FSB114C}

$\begin{array}{ll}\text { SRS Coord. } & \text { Lathongitude } \\ \text { N75288.5 } & 33.277188^{\circ} \mathrm{N} \\ \text { E52033.8 } & 81.676030^{\circ} \mathrm{W}\end{array}$

SAMPLE DATE

1099

214.4

5.6

58

19.9

Sp. conductance

Alkalinity as $\mathrm{CaCO} 3$

Turbidity

Volumes purged

Sampling code

ANALYTICAL DATA

Inorganic Constituents
Synchronous water level

\section{Screen Zone Elevation}

$168.0-158.0 \mathrm{ft} \mathrm{ms}$

$1107 / 99$
$213.9(03 / 23 / 99)$

\section{Ioo of Casing}

$252.2 \mathrm{ft} \mathrm{ms}$

04/05/99

$\underline{2099}$

213.4

5.3

49

21.1

5

3.4

$212.8(06 / 28 / 99)$

\section{Casing Pump}

4" PVC S
Szreen Zone

LAZ_UTRA
Unit

ft ms!

$\mathrm{pH}$

HSicm

${ }^{\circ} \mathrm{C}$

$\mathrm{mgl}$

NTU

well volumes

ft msl

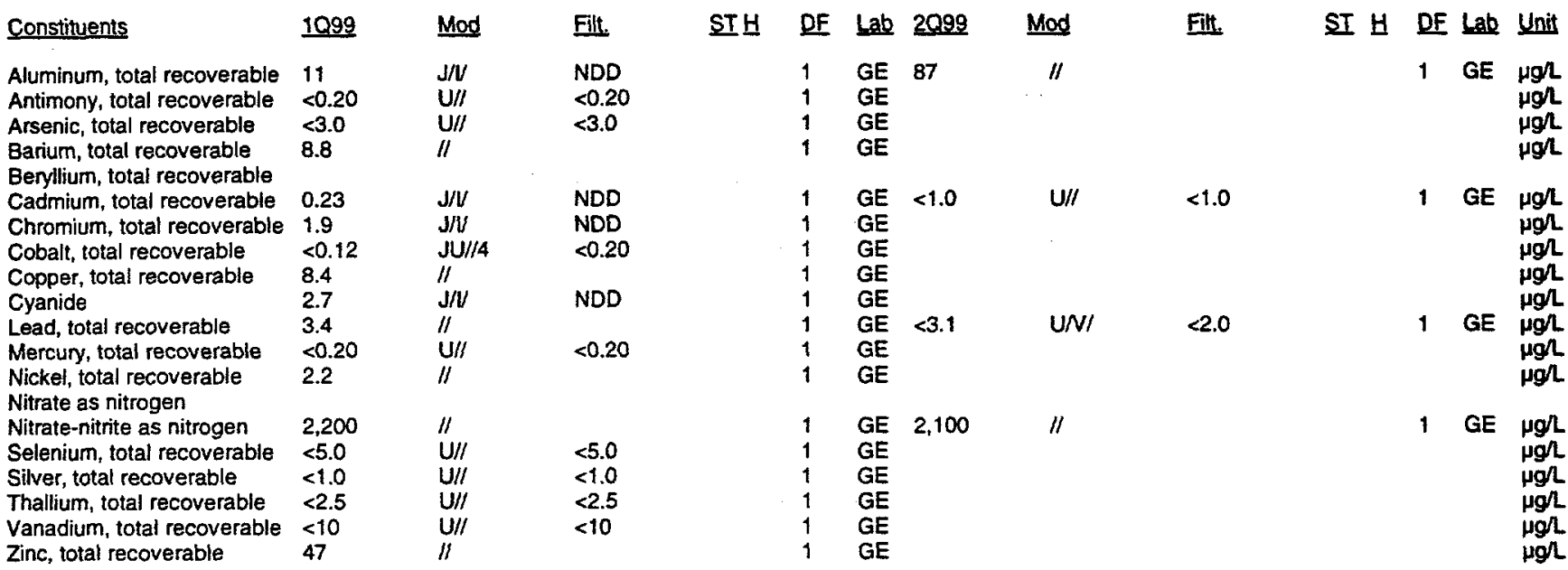

Organic Constituents

\begin{tabular}{|c|c|c|c|c|c|}
\hline Constituents & 1099 & Mod & Filt & STH & DF \\
\hline Benzene & $<1.0$ & $\mathrm{U} / I$ & $<1.0$ & & 1 \\
\hline Bis(2-ethylhexyl) phthalate & $<10$ & uII & $<10$ & & $i$ \\
\hline Dichloromethane & $<10$ & $\mathrm{U} N /$ & $<5.0$ & & 1 \\
\hline Phenols & $<5.0$ & $\mathrm{U} / \mathrm{I}$ & $<5.0$ & & 1 \\
\hline Tetrachloroethylene & $<1.0$ & $\mathrm{U} / \prime$ & $<1.0$ & & 1 \\
\hline Trichloroethylene & 0.76 & $\mathrm{~J} / \mathrm{V}$ & NDD & & 1 \\
\hline Trichlorofluoromethane & $<5.0$ & $\mathrm{U} / \prime$ & $<5.0$ & & 1 \\
\hline
\end{tabular}

Fill. ST H DE Lab Unit

wor

pgr

pgh

pgr

$\mu g /$

$\mu g / \mathrm{L}$

$\mu g h$

Notes:

= exceeded holding time

= exceeded groundwater protection or monitoring constituent standard (See Appendix A.)

NA - Not applicable. Applies to beta dose and sum of betas if there are no beta-emitting radionuclides above detection limits; to sum of alphas if there are no alphaemitting radionuclides above detection limits; and to total radium if neither radium-226 or radium-228 was above detection limit

UAZ_UTRA - Upper Aquifer Zone of the Upper Three Runs Aquifer, LAZ UTRA - Lower Aquifer Zone of the Upper Three Runs Aquifer, Gordon - Gordon Aquifer 
WELL FSB114C (cont.)

Radioactive Constituents

\begin{tabular}{|c|c|c|c|c|c|c|c|c|c|c|c|c|c|}
\hline Constituents & 1099 & Mod & Filt. & ST브 & $\underline{D F}$ & Lab & 2099 & Mod & Filt. & ST $\mathbf{H}$ & DE & Lab & Unit \\
\hline $\begin{array}{l}\text { Americium-241 } \\
\text { Beta dose }\end{array}$ & $\begin{array}{l}<1.6 \mathrm{E}-02 \\
\text { NA }\end{array}$ & $\mathrm{U} / /$ & $<3.8 E-02$ & & 1 & GP & & & & & & & $p C i \Omega$ \\
\hline Carbon-14 & $<-2.5 E+\infty$ & $\mathrm{U} / /$ & $<7.3 E+\infty 0$ & & 1 & GP & & & & & & & pCin \\
\hline Cesium-137 & $<-1.2 E+\infty 0$ & $\mathrm{u} / /$ & $<3.5 E+\infty 0$ & & 1 & GP & & & & & & & pCir \\
\hline Cobalt-60 & $<-5.7 E-01$ & $\mathbf{U} / /$ & $<3.6 E+\infty 0$ & & 1 & GP & & & & & & & pCin \\
\hline Curium-242 & $<0.0 E+00$ & $U / !$ & $<4.1 E-02$ & & 1 & GP & & & & & & & pCin \\
\hline Curium-243/244 & $<6.3 E-03$ & $\mathrm{U} / /$ & $<8.0 E-02$ & & 1 & GP & & & & & & & pCin \\
\hline Curium-245/246 & $<0.0 \mathrm{E}+\infty$ & $\mathrm{U} / \mathrm{I}$ & $<3.8 E-02$ & & 1 & GP & & & & & & & pCil \\
\hline $\begin{array}{l}\text { Gross alpha } \\
\text { Jodine- } 129\end{array}$ & $\begin{array}{l}9.0 E-01 \\
<1.6 E-01\end{array}$ & $\begin{array}{l}\mathrm{J} / \mathrm{V} \\
\mathrm{U} / /\end{array}$ & NDD & & $\begin{array}{l}1 \\
1\end{array}$ & $\begin{array}{l}\text { GP } \\
\text { GP }\end{array}$ & $1.2 E+\infty$ & $J / V$ & NDD & & 1 & GP & $\begin{array}{l}\mathrm{pCil} \\
\mathrm{pCin}\end{array}$ \\
\hline Nonvolatile beta & $3.7 E+00$ & $/ /$ & $<8 . / E-01$ & & 1 & GP & $<1.1 E+\infty$ & $\mathrm{U} / \mathrm{I}$ & $<1.2 E+\infty$ & & 1 & GP & pCin \\
\hline Plutonium-238 & $<1,1 E-01$ & $\mathrm{U} / /$ & $<1.1 E-01$ & & 1 & GP & & & & & & Gr & poin \\
\hline Plutonium-239/240 & $<7.8 E-03$ & $\mathrm{U} / /$ & $<7.6 E-02$ & & 1 & GP & & & & & & & pCin \\
\hline Radium-226 & $4.0 \mathrm{E}-01$ & $\mathrm{~J} / \mathrm{V}$ & NDD & & 1 & GP & & $\ldots$. & & & & & pCin \\
\hline $\begin{array}{l}\text { Radium-228 } \\
\text { Total radium }\end{array}$ & $\begin{array}{l}<2.8 E-01 \\
N A\end{array}$ & $\mathrm{U} / /$ & $<1.2 \mathrm{E}+\infty$ & & 1 & GP & & $\cdots$ & & & & & pCir \\
\hline Strontium-90 & $<4.3 E-01$ & $\mathrm{U} / /$ & $<1.1 E+00$ & & 1 & GP & & & & & & & pCin \\
\hline Technetium-99 & $<-6.4 E-01$ & $\mathrm{U} / /$ & $<1.9 E+01$ & & 1 & GP & & & & & & & pCir \\
\hline Thorium-228 & $3.6 E-01$ & $\mathrm{~J} / \mathrm{V}$ & NDD & & 1 & GP & & & & & & & pin \\
\hline Thorium-230 & $1.5 E-01$ & $R / / 4$ & Rej & & 1 & GP & & & & & & & $\mathrm{pCin}$ \\
\hline Thorium-232 & $\begin{array}{l}<-8.7 E-03 \\
N A\end{array}$ & $U / /$ & $<1.5 E-01$ & & 1 & GP & & & & & & & pCir \\
\hline $\begin{array}{l}\text { Sum of alphas } \\
\text { Sum of betas }\end{array}$ & $\begin{array}{l}\text { NA } \\
\text { NA }\end{array}$ & & & & & & & & & & & & \\
\hline Tritium & $4.0 E+\infty 0$ & $\|$ & & & 1 & GP & $2.6 E+\infty$ & $\| l$ & & & 1 & GP & $\mathrm{pCi} / \mathrm{mL}$ \\
\hline Uranium-233/234 & $<4.4 E-02$ & $\mathrm{U} / /$ & $<4.4 E-02$ & & 1 & GP & & & & & & & $\mathrm{pCi}$ \\
\hline Uranium-235 & $<2.2 \mathrm{E}-02$ & $\mathrm{U} / /$ & $<9.2 E-02$ & & 1 & GP & & & & & & & $\mathrm{pCin}$ \\
\hline Uranium-238 & $<4.3 E-02$ & $\mathrm{U} / /$ & $<1.1 E-01$ & & 1 & GP & & & & & & & \\
\hline
\end{tabular}

Notes:

- exceeded hoiding time

E = exceeded groundwater protection or monitoring constituent standard (See Appendix A.)

NA - Not applicable. Applies to beta dose and sum of betas if there are no beta-emitting radionuclides above detection limits; to sum of alphas if there are no alphaemitting radionuclides above detection limits; and to total radium if neither radium-226 or radium-228 was above detection limit

UAZ_UTRA - Upper Aquifer Zone of the Upper Three Runs Aquifer; LAZ_UTAA - Lower Aquifer Zone of the Upper Three Runs Aquifer, Gordon - Gordon Aquifer 
WELL FSB114D

$\begin{array}{ll}\text { SRS Coord. } & \text { Lat/Longitud } \\ \text { N75278.6 } & 33.277141^{\circ} \\ \text { E52018.6 } & 81.676051^{\circ}\end{array}$

SAMPLE DATE

Screen Zone Elevation

$217.8-197.7 \mathrm{ft} \mathrm{msl}$

Top of Casing

Casing Pump

4" PVC S

Screen Zone

$252.2 \mathrm{ft} \mathrm{msl}$

04/05/99

1099

217.4

4.3
47

19.7

4.6

$216.8(03 / 23 / 99)$ $\underline{2099}$

215.7

4.7

46

20.2

0

4.6

$216.2(06 / 28 / 99)$
Unit

tt msl

$\mathrm{pH}$

$\mu \mathrm{S} / \mathrm{cm}$

${ }^{\circ} \mathrm{C}$

mgl

well volumes

$\mathrm{ft}$ msl

\section{ANALYTICAL DATA}

Inorganic Constituents

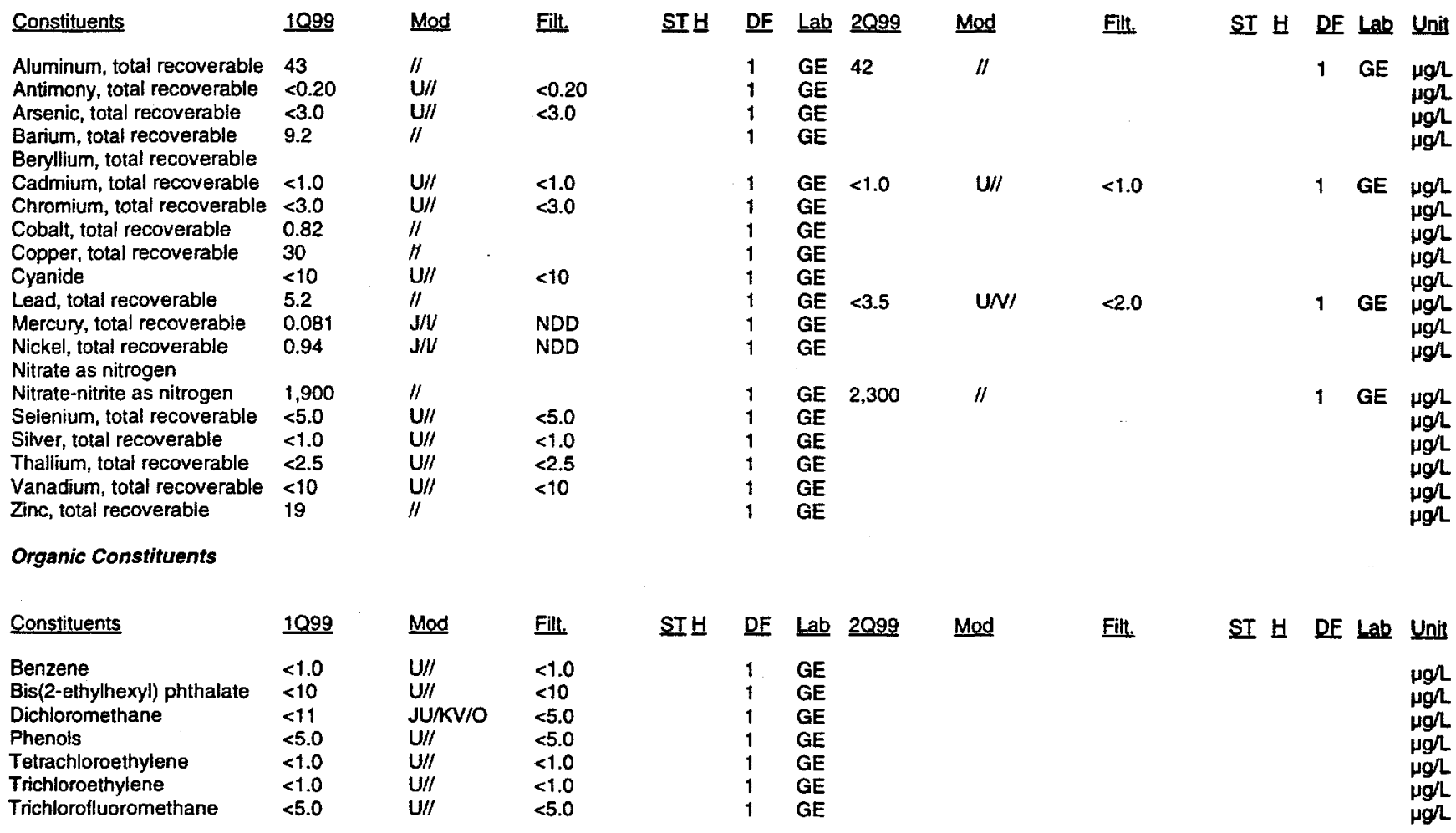

Notes:

= exceeded holding time

w = exceeded groundwater protection or monitoring constituent standard (See Appendix A.)

NA - Not applicable. Applies to beta dose and sum of betas if there are no beta-emitting radionuclides above detection limits; to sum of alphas if there are no alphaemitting radionuclides above detection limits; and to total radium if neither radium-226 or radium-228 was above detection limit

UAZ_UTRA - Upper Aquifer Zone of the Upper Three Runs Aquifer, LAZ_UTRA - Lower Aquifer Zone of the Upper Three Runs Aquifer, Gordon - Gordon Aquifer 


\section{WELL FSB114D (cont.)}

Radioactive Constituents

\begin{tabular}{|c|c|c|c|c|c|c|c|c|c|c|c|c|c|}
\hline Constituents & 1099 & Mod & Filt. & ST브 & $\underline{D F}$ & $\underline{L a b}$ & 2099 & Mod & Filt. & ST $\underline{\text { H }}$ & DF & Lab & Unit \\
\hline $\begin{array}{l}\text { Americium-241 } \\
\text { Beta dose }\end{array}$ & $\begin{array}{l}<1.5 E-02 \\
N A\end{array}$ & $\mathrm{U} / /$ & $<3.7$ E-02 & & 1 & GP & & & & & & & pCin \\
\hline Carbon-14 & $<5.5 \mathrm{E}-01$ & $\mathrm{U} / /$ & $<7.2 E+\infty 0$ & & 1 & GP & & & & & & & $\mathrm{pCi}$ \\
\hline Cesium-137 & $<-1.5 E+00$ & $\mathrm{U} / I$ & $<4.1 E+00$ & & 1 & GP & & & & & & & pcir \\
\hline Cobalt- 60 & $<-1.2 E+\infty 0$ & $\mathrm{U} / \prime$ & $<4.0 \mathrm{E}+00$ & & 1 & GP & & & & & & & pCir \\
\hline Cunium-242 & $<-5.9 E-03$ & $\mathrm{U} / /$ & $<7.4 \mathrm{E}-02$ & & 1 & GP & & & & & & & pCil \\
\hline Curium-243/244 & $<0.0 \mathrm{E}+00$ & $\mathrm{U} / 1$ & $<3.7 E-02$ & & 1 & GP & & & & & & & $\mathrm{pCin}$ \\
\hline Curium-245/246 & $<1.2 E-02$ & $\mathrm{U} / 1$ & $<3.7 E-02$ & & 1 & GP & & & & & & & pCin \\
\hline Gross alpha & $1.5 E+\infty 0$ & $\mathrm{~J} / \mathrm{V}$ & NDD & & 1 & GP & $1.7 E+\infty 0$ & $\mathrm{~J} / \mathrm{V}$ & NDD & & 1 & GP & pCil \\
\hline lodine-129 & $<-3.3 E-01$ & $\mathrm{U} / 1$ & $<1.2 E+\infty 0$ & & 1 & GP & & & & & & & $\mathrm{pCi} / \mathrm{L}$ \\
\hline Nonvolatile beta & $<1.5 E+\infty$ & $\mathrm{U} / /$ & $<1.9 E+\infty 0$ & & 1 & GP & $1.2 E+\infty$ & $\mathrm{J} / \mathrm{V}$ & NDD & & 1 & GP & $\mathrm{pCi} / \mathrm{L}$ \\
\hline Plutonium-238 & $1.4 E-01$ & $R / / 4$ & Rej & & 1 & GP & & & & & & & $\mathrm{pCi}$ \\
\hline Plutonium-239/240 & $<0.0 E+\infty 0$ & $\mathrm{U} / 1$ & $<2.4 E-02$ & & 1 & GP & & & & & & & $\mathrm{pCi}$ \\
\hline Radium-226 & $1.5 E+00$ & $\|$ & & & 1 & GP & & & & & & & $\mathrm{pCi}$ \\
\hline $\begin{array}{l}\text { Radium-228 } \\
\text { Total radium }\end{array}$ & $\begin{array}{l}<4.0 E-01 \\
1.5 E+\infty 0\end{array}$ & $U / /$ & $<9.3 E-01$ & & 1 & GP & & & & & & & $\mathrm{pCi}$ \\
\hline Strontium-90 & $<-3.7 E-02$ & $\mathbf{U} / /$ & $<1.3 E+\infty$ & & 1 & GP & & & & & & & pCin \\
\hline Technetium-99 & $<-1.3 E+\infty$ & $\mathrm{U} / !$ & $<1.8 E+01$ & & 1 & GP & & & & & & & pCin \\
\hline Thorium-228 & $<1.1 E-01$ & $\mathbf{u} / !$ & $<1.9 \mathrm{E}-01$ & & 1 & GP & & & & & & & pCil \\
\hline Thorium-230 & $1.2 E-01$ & $\mathrm{~F} / / 4$ & Rej & & 1 & GP & & & & & & & pCir \\
\hline Thorium-232 & $<3.6 \mathrm{E}-02$ & $\mathbf{U} / /$ & $<9.1 E-02$ & & 1 & GP & & & & & & & $\mathrm{pCin}$ \\
\hline Sum of alphas & NA & & & & & & & & & & & & \\
\hline Sum of betas & NA & & & & & & & & & & & & \\
\hline Tritium & $7.2 E+\infty 0$ & 11 & & & 1 & GP & $6.1 E+\infty$ & $\|$ & & & 1 & GP & $\mathrm{pCi} / \mathrm{mL}$ \\
\hline Uranium-233/234 & $<5.5 E-02$ & $U / I$ & $<1.4 E-01$ & & 1 & $\overrightarrow{G P}$ & & & & & & & pCin \\
\hline Uranium-235 & $<-1.6 E-04$ & $\mathrm{U} / /$ & $<1.2 E-01$ & & $i$ & GP & & & & & & & pCin \\
\hline Uranium-238 & $<-8.2 E-03$ & $U / I$ & $<1.4 E-01$ & & 1 & GP & & & & & & & poin \\
\hline
\end{tabular}

Notes:

- =xceeded holding time

= exceeded groundwater protection or monitoring constituent standard (See Appendix A.)

NA - Not applicable. Applies to beta dose and sum of betas if there are no beta-emitting radionuclides above detection limits; to sum of alphas if there are no alphaemitting radionuclides above detection limits; and to total radium if neither radium-226 or radium-228 was above detection limit

UAZ UTRA - Upper Aquifer Zone of the Upper Three Runs Aquifer, LAZ UTRA - Lower Aquifer Zone of the Upper Three Runs Aquifer, Gordon - Gordon Aquifer 


\section{WELL FSB115C}

SRS Coord. Latlongitud

N72515.5 $\quad 33.267306^{\circ} \mathrm{N}$

E49736.0 $81.676692^{\circ} \mathrm{W}$

SAMPLE DATE
Screen Zone Elevation

$173.8-163.8 \mathrm{ft} \mathrm{msl}$
Ton of Casing

$207.8 \mathrm{ft} \mathrm{msi}$

Casing Pumo
4. PVC

Screen Zone
LAZ_UTRA
$04 / 06 / 99$

$\underline{2099}$
184.5
5.9
19
18.1
1
1
0.074
$x$
$184.3(06 / 28 / 99)$

Unit

th ms

$\mathrm{pH}$

$\mu S / \mathrm{cm}$

$\mathrm{mgh}$

NTU

well volumes

ft mst

\section{ANALYTICAL DATA}

Inorganic Constituents

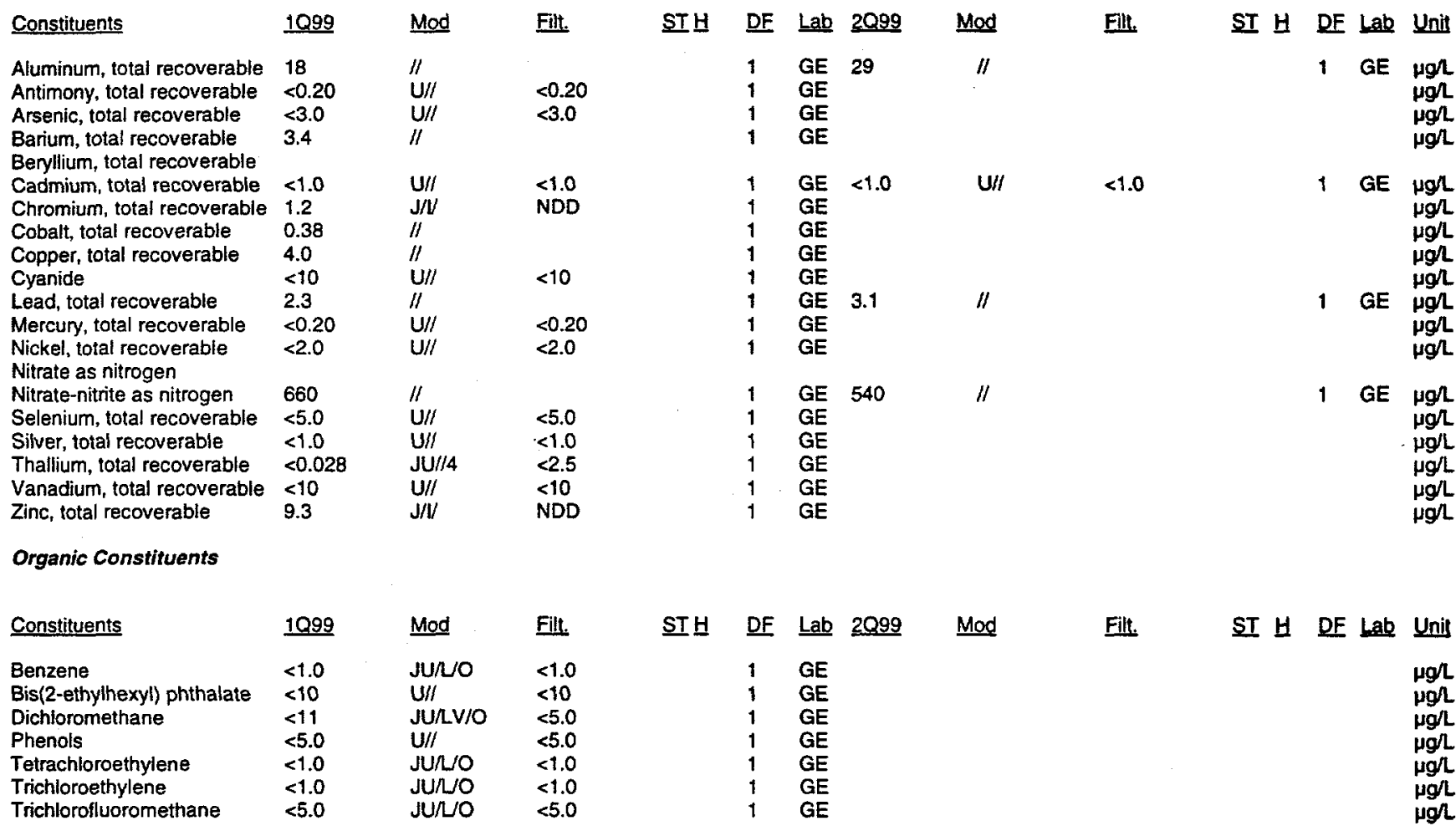

Notes:

- exceeded holding time

= exceeded groundwater protection or monitoring constituent standard (See Appendix A.)

NA - Not applicable. Applies to beta dose and sum of betas if there are no beta-emitting radionuclides above detection limits; to sum of alphas if there are no alphaemitting radionuclides above detection limits; and to total radium if neither radium-226 or radium-228 was above detection limit 
WELL FSB115C (cont.)

Radioactive Constituents

\begin{tabular}{|c|c|c|c|c|c|c|c|c|c|c|c|c|c|}
\hline Constituents & 1099 & Mod & Filt. & ST브 & DF & Lab & 2899 & Mod & Filt. & ST $\underline{H}$ & DE & Lab & Unit \\
\hline $\begin{array}{l}\text { Americium-241 } \\
\text { Beta dose }\end{array}$ & $\begin{array}{l}<4.2 E-02 \\
N A\end{array}$ & $\mathbf{U} / \prime$ & $<2.4 E-01$ & & 1 & GP & & & & & & & pcin \\
\hline Carbon-14 & $<-4.1 E+\infty$ & $\mathrm{U} / /$ & $<7.4 E+00$ & & 1 & GP & & & & & & & pein \\
\hline Cesium-137 & $<1.4 E+00$ & UII & $<3.8 E+\infty 0$ & & 1 & GP & & & & & & & poir \\
\hline Cobalt-60 & $<3.1 E+00$ & $\mathrm{u} / /$ & $<3.4 E+\infty 0$ & & 1 & GP & & & & & & & pCin \\
\hline Curium-242 & $<1.8 \mathrm{E}-02$ & u/I & $<1.9 \mathrm{E}-01$ & & 1 & GP & & & & & & & pCin \\
\hline Curium-243/244 & $<2.9 E-02$ & $\mathrm{u} / \mathrm{I}$ & $<8.8 E-02$ & & 1 & GP & & & & & & & pCir \\
\hline Curium-245/246 & $<0.0 \mathrm{E}+00$ & $\mathrm{u} / \mathrm{I}$ & $<8.8 E-02$ & & 1 & GP & & & & & & & pCin \\
\hline Gross alpha & $<1.4 E-01$ & UII & $<6.2 E-01$ & & 1 & GP & $<3.1 E-01$ & $\mathrm{U} / / /$ & $<5.6 \mathrm{E}-01$ & & 1 & GP & pCin \\
\hline lodine-129 & $<2.8 \mathrm{E}-01$ & U/I & $<8.9 E-01$ & & 1 & GP & & & & & & & pCin \\
\hline Nonvolatile beta & $<1.5 \mathrm{E}-01$ & $\mathrm{U} / /$ & $<1.2 \mathrm{E}+\infty 0$ & & 1 & GP & $<7.1 E-01$ & $U / /$ & $<1.0 E+\infty 0$ & & 1 & GP & pCin \\
\hline Plutonium-238 & 1.9E-01 & $R / / 4$ & Rej & & 1 & GP & & & & & & & pCir \\
\hline Plutonium-239/240 & $<2.1 E-02$ & u/I & $<1.4 \mathrm{E}-01$ & & 1 & GP & & & & & & & pCil \\
\hline Radium-226 & $<1.5 E-01$ & U/I & $<6.0 E-01$ & & 1 & GP & & & & & & & $\mathrm{pcin}$ \\
\hline Radium-228 & $<-1.3 E-01$ & $\mathrm{U} / /$ & $<1.4 \mathrm{E}+\infty$ & & 1 & GP & & & & & & & pCir \\
\hline Total radium & NA & & & & & & & & & & & & \\
\hline Strontium-90 & $<-2.0 \mathrm{E}-01$ & $\mathrm{U} / \mathrm{I}$ & $<1.3 E+00$ & & 1 & GP & & & & & & & PCin \\
\hline Technetium-99 & $<-2.1 E+\infty$ & U/I & $<1.8 E+01$ & & 1 & GP & & & & & & & pCin \\
\hline Thorium-228 & 2.1E-01 & $\mathrm{R} / / 4$ & Rej & & 1 & GP & & & & & & & pCin \\
\hline Thorium-230 & $6.0 \mathrm{E}-02$ & $\mathrm{R} / \mathrm{4}$ & & & 1 & GP & & & & & & & pCir \\
\hline Thorium-232 & $<4.2 E-03$ & JUนA & $<5.4 E-02$ & & 1 & GP & & & & & & & pCir \\
\hline Sum of alphas & NA & & & & & & & & & & & & \\
\hline Sum of betas & NA & & & & & & & & & & & & \\
\hline Tritium & $6.7 E+\infty 0$ & $/ /$ & & & 1 & GP & $7.1 E+\infty 0$ & 11 & & & 1 & GP & $\mathrm{pCi} / \mathrm{mL}$ \\
\hline Uranium-233/234 & $<4.8 E-02$ & $\mathrm{u} / \mathrm{I}$ & $<1.2 E-01$ & & 1 & GP & & & & & & & pCin \\
\hline Uranium-235 & $<1.9 E-02$ & U/I & $<5.7 E-02$ & & 1 & GP & & & & & & & pcin \\
\hline Uranium-238 & $<7.6$ E-02 & $u / /$ & $<5.7 E-02$ & & 1 & GP & & & & & & & $\mathrm{PCil}$ \\
\hline
\end{tabular}

Notes:

$\bullet=$ exceeded holding time

= exceeded groundwater protection or monitoring constituent standard (See Appendix A.)

NA - Not applicable. Applies to beta dose and sum of betas if there are no beta-emitting radionuclides above detection limits; to sum of alphas it there are no alphaemitting radionuclides above detection limits; and to total radium if neither radium-226 or radium-228 was above detection limit

UAZ_UTRA - Upper Aquifer Zone of the Upper Three Runs Aquifer, LAZ_UTRA - Lower Aquifer Zone of the Upper Three Runs Aquifer, Gordon - Gordon Aquifer 


\section{WELL FSB115D}

$\begin{array}{ll}\text { SRS Coord. } & \text { Lavlongitude } \\ \text { N72504.3 } & 33.267268^{\circ} \mathrm{N} \\ \text { E49728.3 } & 81.676691^{\circ} \mathrm{W}\end{array}$

SAMPLE DATE

Screen Zone Elevation

$192.5-182.5 \mathrm{ft} \mathrm{ms}$
Top of Casing

$208.5 \mathrm{ft} \mathrm{msl}$
Casing Pump

4" PVC S
Screen Zone

UAZ_UTRA
04/06/99

2099
191.2
4.9
15
22.0
0
1
2.6

$190.5(06 / 28 / 99)$
Unit

ft msl

pH

$\mu \mathrm{S} / \mathrm{cm}$

${ }^{\circ} \mathrm{C}$

$\mathrm{mgh}$

NTU

well volumes

$f t$ msl

Synchronous water level

$191.2(03 / 23 / 99)$

\section{ANALYTICAL DATA}

Inorganic Constituents

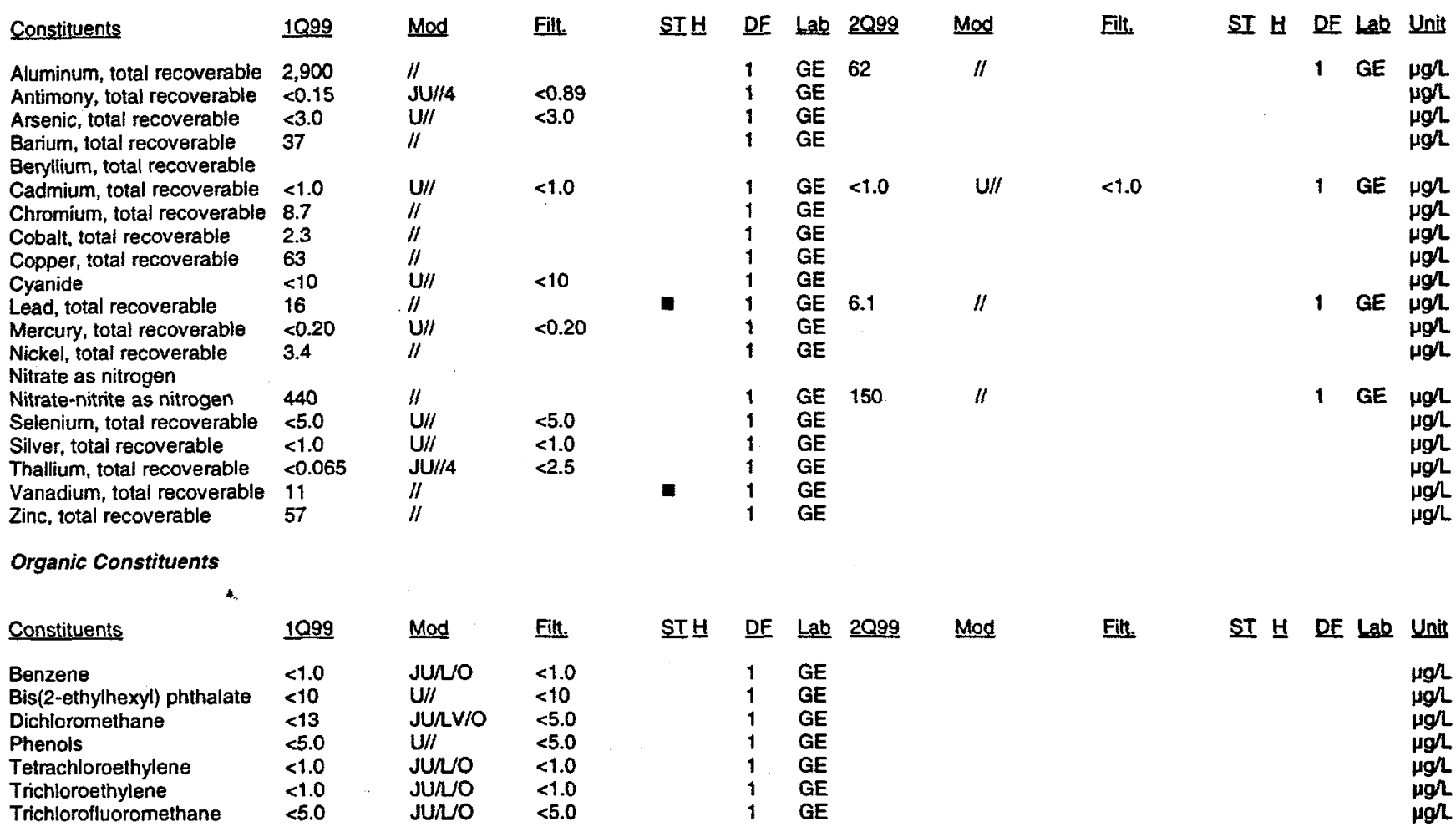

Notes:

- exceeded holding time

= exceeded groundwater protection or monitoring constituent standard (See Appendix A)

NA - Not applicable. Applies to beta dose and sum of betas if there are no beta-emitting radionuclides above detection limits; to sum of alphas if there are no alphaemitting radionuclides above detection limits; and to total radium if neither radium-226 or radium-228 was above detection limit

UAZ UTRA - Upper Aquifer Zone of the Upper Three Runs Aquifer; LAZ UTRA - Lower Aquifer Zone of the Upper Three Runs Aquifer, Gordon - Gordon Aquifer 


\section{WELL FSB115D (cont.)}

Radioactive Constituents

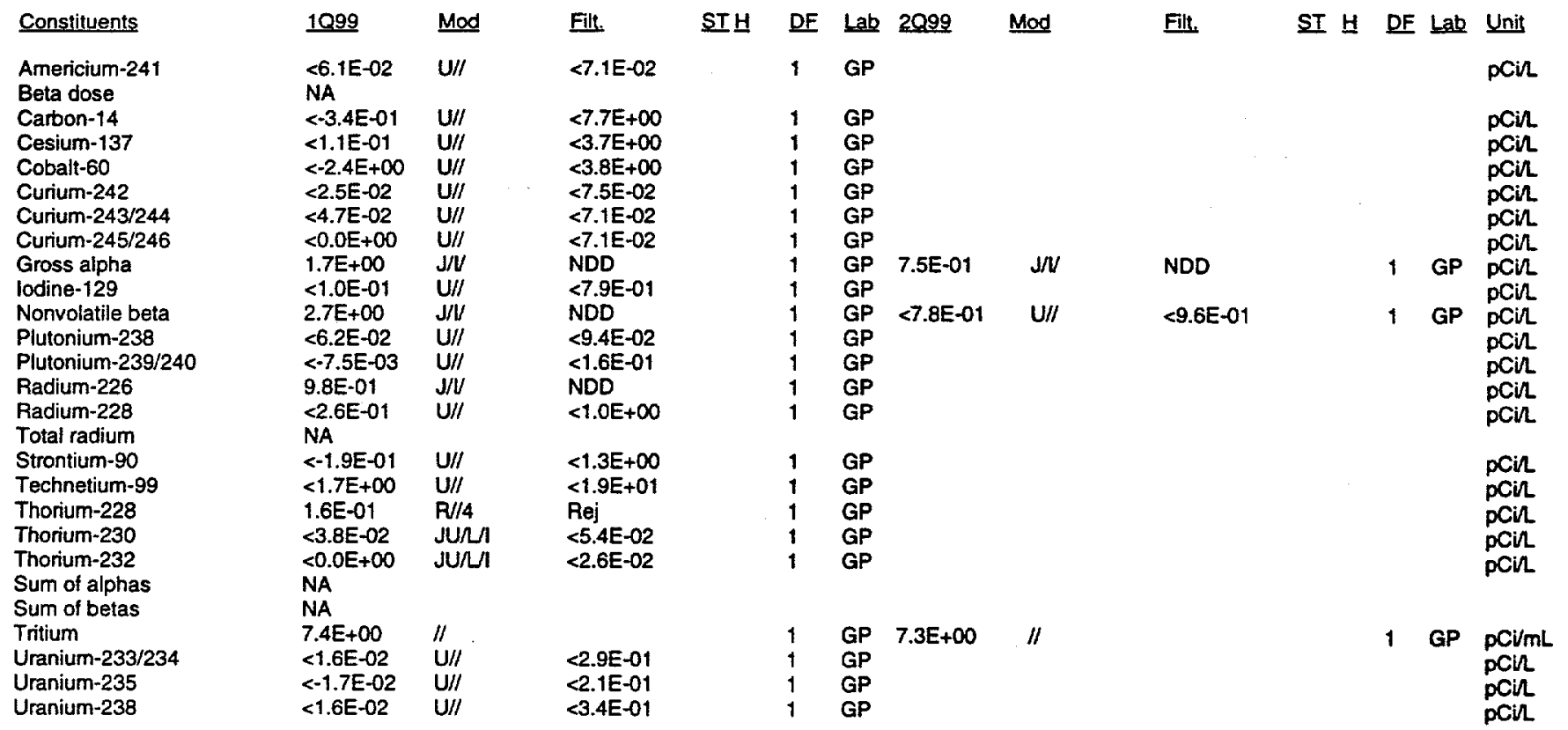

Notes:

= exceeded holding time

a = exceeded groundwater protection or monitoring constituent standard (See Appendix A.)

NA - Not applicable. Applies to beta dose and sum of betas if there are no beta-emitting radionuclides above detection limits; to sum of alphas if there are no alphaemitting radionuclides above detection limits; and to total radium if neither radium-226 or radium-228 was above detection limit

UAZ_UTRA - Upper Aquifer Zone of the Upper Three Runs Aquifer, LAZ UTRA - Lower Aquifer Zone of the Upper Three Runs Aquifer, Gordon - Gordon Aquifer 
WELL FSB116C

$\begin{array}{ll}\text { SRS Coord. } & \text { Lat/Longitude } \\ \text { N72725.5 } & 33.269255^{\circ} \mathrm{N} \\ \text { E50645.9 } & \mathbf{8 1 . 6 7 4 7 0 5 ^ { \circ } \mathrm { W }}\end{array}$

SAMPLE DATE

1099

189.7

4.9

20

17.7

1

4.0

$189.5(03 / 23 / 99)$

Turbidity

Volumes purged

Sampling code

Synchronous water leve
Screen Zone Elevation

170.5-160.5 ft ms!

Top of Casing

$202.5 \mathrm{ft} \mathrm{ms}$

04/06/99

$\underline{2099}$

189.5

4.8

21

19.0

2

3.8

$189.0(06 / 28 / 99)$
Casing Pumo

4" PVC S
Screen Zone

LAZ_UTRA

ANALYTICAL DATA

Inorganic Constituents

Constituents

Aluminum, total recoverable

Antimony, total recoverable $<0.24$

Arsenic, total recoverable $<3.0$

Barium, total recoverable $\quad 4.9$

Beryllium, total recoverable

Cadmium, total recoverable $<1.0$

Chromium, total recoverable 4.2

Cobait, total recoverable $\quad 0.84$

Copper, total recoverable $\quad 7.3$

Cyanide

Lead, total recoverable $\quad 1.6$

Mercury, total recoverable $\quad<0.20$

Nickel, total recoverable $\quad 3.3$

Nitrate as nitrogen

Nitrate-nitrite as nitrogen $\quad 1,100$

Selenium, total recoverable $<50$

Sitver, total recoverable $<1.0$

Thallium, total recoverable $<0.032$

Vanadium, total recoverable $<10$

Zinc, total recoverable

$\begin{array}{ll}\text { Mod } & \text { Filt. } \\ J / & \\ \text { JU//4 } & <0.89 \\ \mathrm{U} / / & <3.0 \\ / / & \\ \mathrm{U} / / & <1.0 \\ I / & \\ I / & \\ I / & \\ \mathrm{U} / / & <10 \\ \mathrm{~J} / \mathrm{V} & \mathrm{ND0} \\ \mathrm{U} / / & <0.20 \\ / / & \\ / / & \\ \mathrm{U} / / & <5.0 \\ \mathrm{U} / / & <1.0 \\ \mathrm{JU} / / 4 & <2.5 \\ \mathrm{U} / / & <10 \\ \mathrm{U} / / & <10\end{array}$

STH

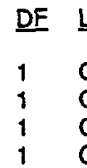

$\begin{array}{ll}\text { Lab } & 2 Q \\ \text { GE } & 70 \\ \text { GE } & \\ \text { GE } & \\ \text { GE } & \end{array}$

$\underline{\text { Mod }}$

1 GE

1 GE

1 GE

1 GE

1 GE

1 GE

1 GE

1 GE

1 GE

1 GE

1 GE
Unit

t $\mathrm{ms}$

$\mathrm{pH}$

HS/cm

mgll

NTU

well volumes

ft msl

Organic Constituents

\begin{tabular}{llll} 
Constituents & 1099 & Mod & Filt. \\
\cline { 2 - 4 } Benzene & $<1.0$ & JU/UO & $<1.0$ \\
Bis(2-ethylhexyl) phthalate & $<10$ & U/I & $<10$ \\
Dichloromethane & $<14$ & JU/LV/O & $<5.0$ \\
Phenols & $<5.0$ & U/I & $<5.0$ \\
Tetrachloroethylene & $<1.0$ & JU/NO & $<1.0$ \\
Trichloroethylene & $<1.0$ & JU/UO & $<1.0$ \\
Trichlorofluoromethane & $<5.0$ & JU/UO & $<5.0$
\end{tabular}

Fill.

ST H DF Lab Unir

1 GE $\mu g /$

ugl

$\mu g /$

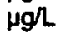

1 GE $\mu g^{\prime} L$

$\mu g h$

ugl

$\mu \mathrm{gl}$

2.4

1 GE

Hgh

$\mu g /$

$\mu \mathrm{gl}$

1. GE $\mu g /$

$\mu g h$

$\mu g h$

$\mu g / L$

$\mu g / L$

$\mu g / 2$

Trichlorofluoromethane

$<5.0$

$\underline{2099 \quad \text { Mod }}$

Filt.

ST H DF Lab Unit

$\mu g /$

$\mu g / 2$

$\mu \mathrm{g} / \mathrm{h}$

$\mu g h$

$\mu g / 2$

$\mu g / 2$

$\mu g h$

Notes:

= exceeded holding time

E = exceeded groundwater protection or monitoring constituent standard (See Appendix A.)

NA - Not applicable. Applies to beta dose and sum of betas if there are no beta-emitting radionuclides above detection limits; to sum of alphas if there are no alphaemitting radionuclides above detection limits; and to total radium if neither radium-226 or radium-228 was above detection limit

UAZ UTRA - Upper Aquifer Zone of the Upper Three Runs Aquifer, LAZ UTRA - Lower Aquifer Zone of the Upper Three Runs Aquifer, Gordon - Gordon Aquiter
F-Area HWMF
C-152
First and Second Quarter 1999 


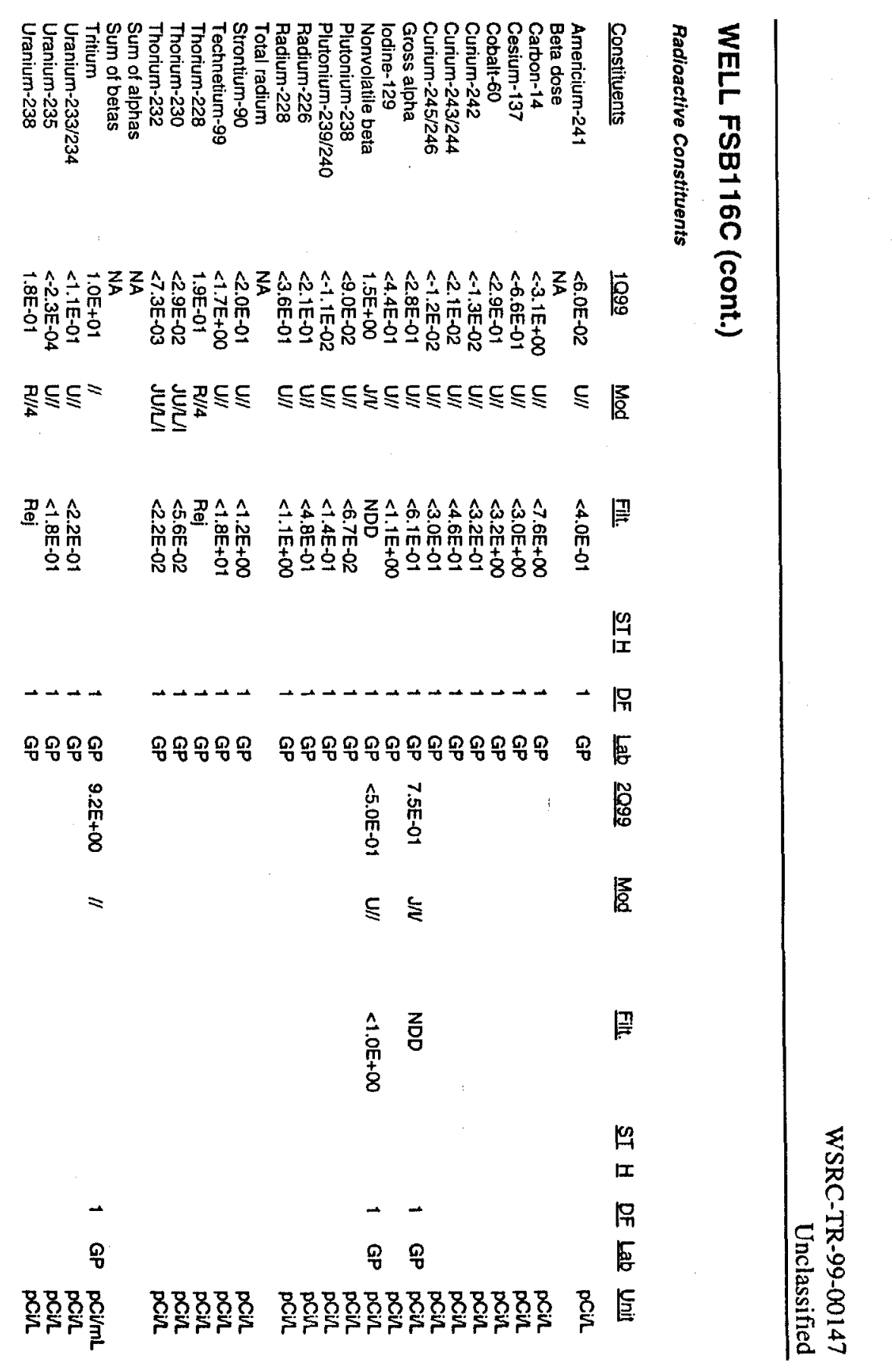




\section{WELL FSB116D}

$\begin{array}{ll}\text { SRS Coord. } & \text { Latllongitude } \\ \text { N72727.4 } & 33.269233^{\circ} \mathrm{N} \\ \text { E50629.7 } & 81.674751^{\circ} \mathrm{W}\end{array}$

SAMPLE DATE

\section{FIELD DATA}

Constituents

Water elevation

$\mathrm{pH}$

Sp. conductance

Water temperature

Alkalinity as $\mathrm{CaCO} 3$

Turbidity

Volumes purged

Sampling code

Synchronous water level

\section{ANALYTICAL DATA}

Inorganic Constituents
Screen Zone Elevation

$196.4-186.4 \mathrm{ft} \mathrm{ms}$

$01 / 11 / 99$
Top of Casing

$202.9 \mathrm{ft} \mathrm{msl}$

\section{Casing Pumo}

4' PVC S
Screen Zone

UAZ_UTRA
04/06/99

$\underline{2099}$

191.7

5.2

27

25.8

2

14

X

$191.3(06 / 28 / 99)$
Unit

tt ms

$\mathrm{pH}$

$\mu \mathrm{S} / \mathrm{cm}$

${ }^{\circ} \mathrm{C}$

mgr

well volumes

$\mathrm{ft} \mathrm{ms}$

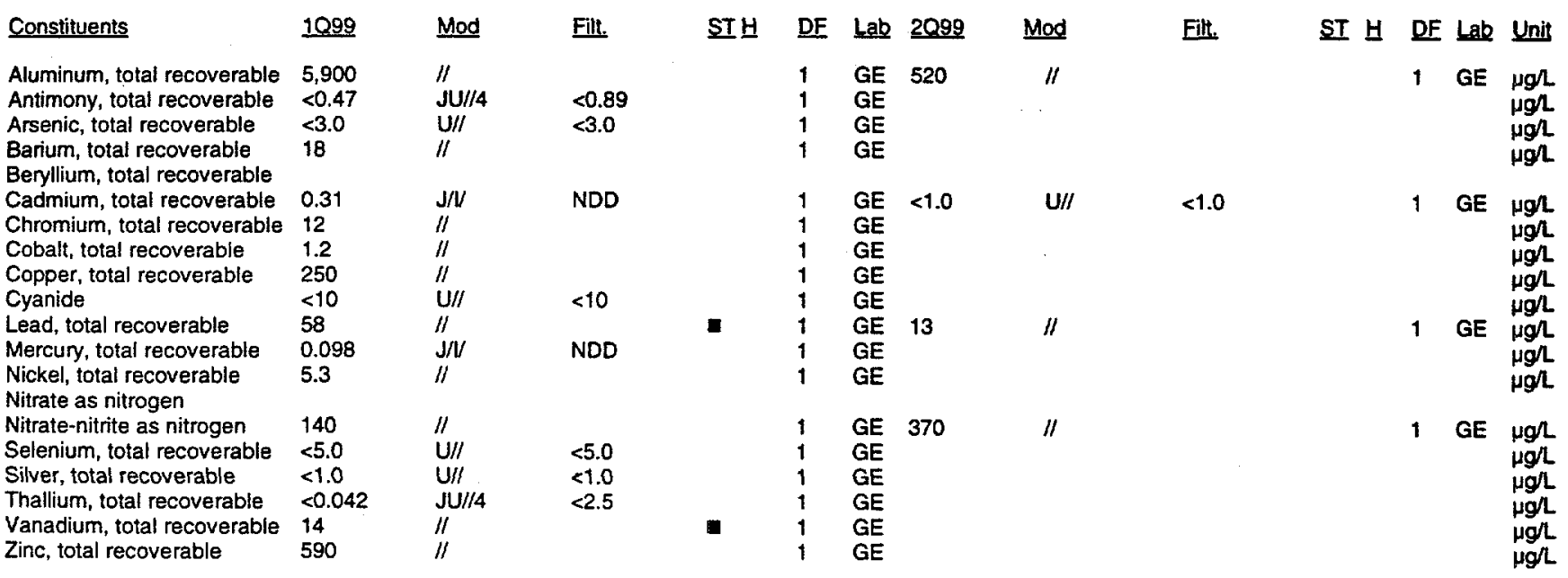

Organic Constituents

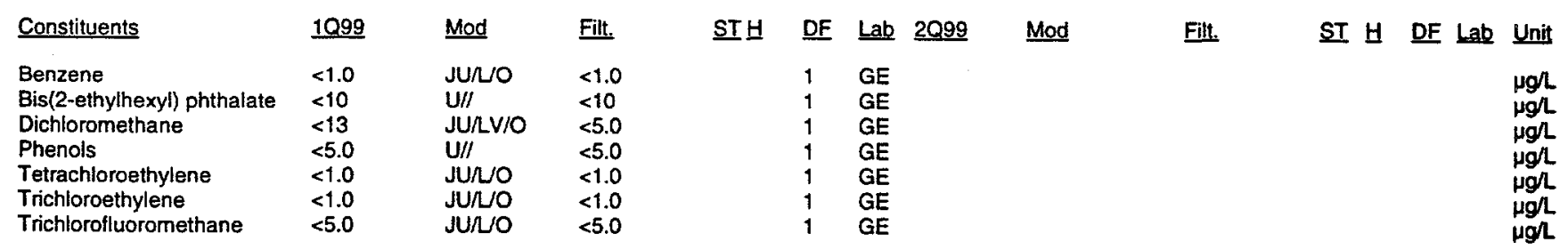

Notes:

- exceeded holding time

= exceeded groundwater protection or monitoring constituent standard (See Appendix A.)

NA - Not applicable. Applies to beta dose and sum of betas if there are no beta-emitting radionuclides above detection limits; to sum of alphas if there are no alphaemitting radionuclides above detection limits; and to total radium if neither radium-226 or radium-228 was above detection limit

UAZ_UTRA - Upper Aquiter Zone of the Upper Three Runs Aquifer, LAZ_UTRA - Lower Aquifer Zone of the Upper Three Runs Aquiler, Gordon - Gordon Aquifer 
WELL FSB116D (cont.)

Radioactive Constituents

\begin{tabular}{|c|c|c|c|c|c|c|c|c|c|c|c|c|c|}
\hline Constituents & 1099 & Mod & Fill. & STH & $\underline{D F}$ & $\underline{L a b}$ & $\underline{2099}$ & Mod & Filt. & ST $\underline{H}$ & DF & Lab & Unit \\
\hline $\begin{array}{l}\text { Americium-241 } \\
\text { Beta dose }\end{array}$ & $\begin{array}{l}<1.6 \mathrm{E}-01 \\
\mathrm{NA}\end{array}$ & $\mathrm{U} / /$ & $<4.6 \mathrm{E}-01$ & & 1 & GP & & & & & & & pcin \\
\hline Carbon-14 & $<1.2 E+00$ & $\mathrm{U} / /$ & $<7.5 E+00$ & 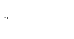 & 1 & GP & & & & & & & pCin \\
\hline Cesium-137 & $<2.3 E+00$ & $\mathrm{U} / /$ & $<3.8 E+\infty$ & & 1 & GP & & & & & & & pCin \\
\hline Cobalt-60 & $<7.2 E-01$ & $\mathrm{U} / \mathrm{I}$ & $<3.9 E+\infty$ & & 1 & GP & & & & & & & pCin \\
\hline Curium-242 & $<-3.2 E-02$ & $\mathrm{U} / /$ & $<4.0 E-01$ & & 1 & GP & & & & & & & pCin \\
\hline Curium-243/244 & $<-2.7 \mathrm{E}-02$ & $\mathrm{U} / /$ & $<7.3 E-01$ & & 1 & GP & & & & & & & $\mathrm{pCi}$ \\
\hline Curium-245/246 & $<0.0 E+\infty$ & $U / /$ & $<9.2 \mathrm{E}-02$ & & 1 & GP & & & & & & & $\mathrm{pCin}$ \\
\hline Gross alpha & $3.0 E+\infty 0$ & 11 & & & 1 & GP & $6.8 E-01$ & $\mathrm{~J} / \mathrm{V}$ & NDD & & 1 & GP & pCil \\
\hline lodine-129 & $<6.3 E-01$ & $\mathrm{U} / /$ & $<1.2 \mathrm{E}+\infty$ & & 1 & GP & & & & & & & $\mathrm{pCin}$ \\
\hline Nonvolatile beta & $5.3 E+\infty$ & $\|$ & & & 1 & GP & $<8.2 E-01$ & $U / I$ & $<1.2 E+\infty$ & & 1 & GP & pCin \\
\hline Plutonium-238 & $<1.8 \mathrm{E}-02$ & $U / I$ & $<1.3 E-01$ & & 1 & GP & & & & & & & pCin \\
\hline Plutonium-239/240 & $<-5.7 E-03$ & $\mathrm{U} / /$ & $<1.2 E-01$ & & 1 & GP & & & & & & & pCill \\
\hline Radium-226 & $<2.4 \mathrm{E}-01$ & $\mathrm{U} / /$ & $<4.5 E-01$ & & 1 & GP & & & & & & & $\mathrm{pCin}$ \\
\hline Radium-228 & $<1.9 E-01$ & $U / /$ & $<1.0 E+00$ & & 1 & GP & & & & & & & pCin \\
\hline Total radium & NA & & & & & & & & & & & & \\
\hline Strontium-90 & $<-4.0 \mathrm{E}-02$ & $\mathrm{U} / \mathrm{H}$ & $<1.0 E+00$ & & 1 & GP & & & & & & & pCin \\
\hline Technetium-99 & $<-1.2 E+\infty$ & $\mathrm{U} / /$ & $<2.0 E+01$ & & 1 & GP & & & & & & & pCin \\
\hline Thorium-228 & $<3.2 E-02$ & $\mathrm{U} / /$ & $<2.4 E-01$ & & 1 & GP & & & & & & & $\mathrm{pCi} / \mathrm{L}$ \\
\hline Thorium-230 & $<7.1 E-02$ & $U / I$ & $<1.0 E-01$ & & 1 & GP & & & & & & & pCin \\
\hline Thorium-232 & $<1.6 E-02$ & $\mathrm{U} / /$ & $<1.2 E-01$ & & 1 & GP & & & & & & & pCin \\
\hline Sum of alphas & NA & & & & & & & & & & & & \\
\hline Sum of betas & NA & & & & & & & & & & & & \\
\hline Tritium & $8.4 E+00$ & $\|$ & & & 1 & GP & $9.5 E+00$ & $/ /$ & & & 1 & GP & $\mathrm{pCi} / \mathrm{mL}$ \\
\hline Uranium-233/234 & $<1.0 E-01$ & $U / /$ & $<2.3 E-01$ & & 1 & GP & & & & & & & pCin \\
\hline Uranium-235 & $<1.1 E-02$ & $U / /$ & $<1.4 \mathrm{E}-01$ & & 1 & GP & & & & & & & $\mathrm{pCin}$ \\
\hline Uranium-238 & 1.6E-01 & $R / 4$ & $\mathrm{Rej}$ & & 1 & GP & & & & & & & $\rho C i / L$ \\
\hline
\end{tabular}

Notes:

- exceeded holding time

- exceeded groundwater protection or monitoring constituent standard (See Appendix A)

NA - Not applicable. Applies to beta dose and sum of betas if there are no beta-emitting radionuclides above detection limits; to sum of alphas if there are no alphaemitting radionuclides above detection limits; and to total radium if neither radium-226 or radium-228 was above detection limit

UAZ UTRA - Upper Aquifer Zone of the Upper Three Runs Aquifer, LAZ UTRA - Lower Aquifer Zone of the Upper Three Runs Aquifer, Gordon - Gordon Aquiler 


\section{WELL FSB117D}

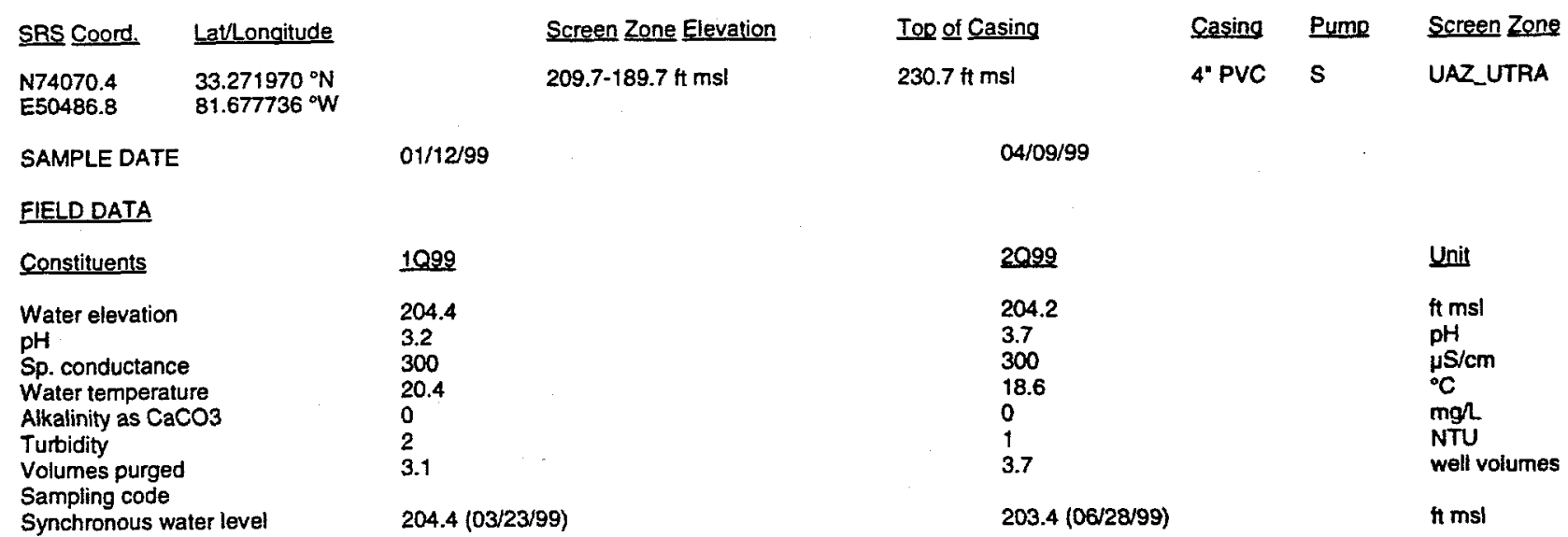

\section{ANALYTICAL DATA}

Inorganic Constituents

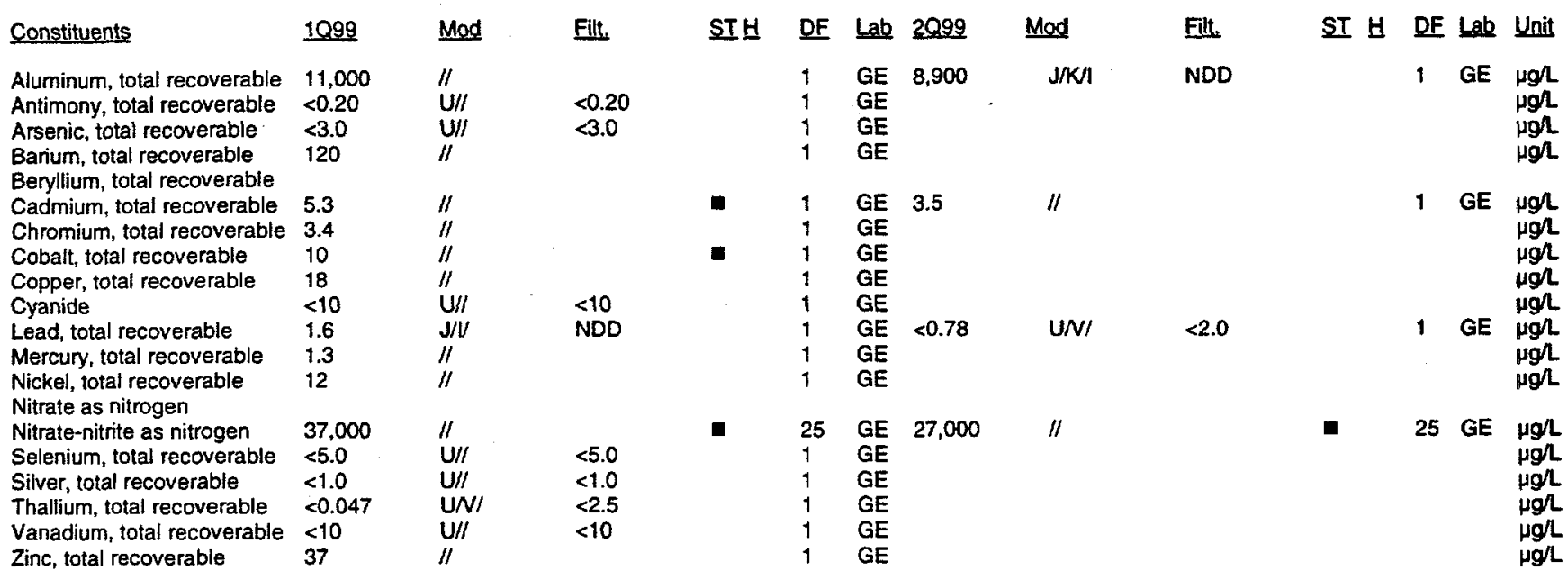

Organic Constituents

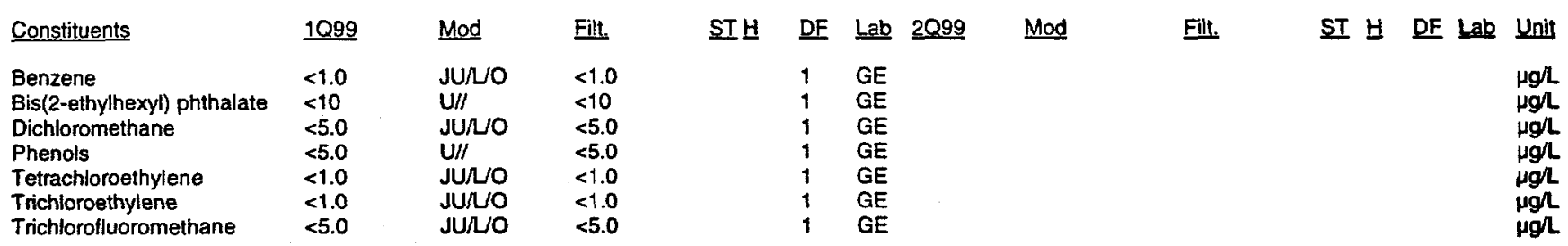

Notes:

- = exceeded holding time

= exceeded groundwater protection or monitoring constituent standard (See Appendix A.)

NA - Not applicable. Applies to beta dose and sum of betas if there are no beta-emitting radionuclides above detection limits; to sum of alphas if there are no alphaemitting radionuclides above detection limits; and to total radium if neither radium-226 or radium-228 was above detection limit

UAZ_UTRA - Upper Aquifer Zone of the Upper Three Runs Aquifer, LAZ. UTRA - Lower Aquifer Zone of the Upper Three Runs Aquifer, Gordon - Gordon Aquifer 
WELL FSB117D (cont.)

Radioactive Constituents

\begin{tabular}{|c|c|c|c|c|c|c|c|c|c|c|c|c|c|}
\hline Constituents & 1099 & Mod & Filt. & STH & DF & Lab & 2099 & Mod & Filt. & ST $\underline{H}$ & $\mathrm{DF}$ & Lab & Unit \\
\hline Americium-241 & $5.0 \mathrm{E}+00$ & $\|$ & & & 1 & GP & & & & & & & pCir \\
\hline Beta dose & & & & $\mathbf{\square}$ & & & & & & & & & \\
\hline $\begin{array}{l}\text { Carbon-14 } \\
\text { Cecium-137 }\end{array}$ & $5.0 \mathrm{E}+01$ & $\mathrm{~J} / \mathrm{L} / \mathrm{Cl}$ & NDD & & 1 & GP & & & & & & & pCin \\
\hline Cesium-137 & $<3.7 E-01$ & $U / I$ & $<3.3 E+00$ & & 1 & GP & & & & & & & pCin \\
\hline Cobalt-60 & $<2.1 E-01$ & $\mathbf{U} / /$ & $<3.2 E+00$ & & 1 & GP & & & & & & & pCin \\
\hline Curium-242 & $<1.5 E-02$ & $U / / I$ & $<1.8 E-01$ & & 1 & GP & & & & & & & pCin \\
\hline Curium-243/244 & $1.5 E+\infty 0$ & $" 1$ & & & 1 & GP & & & & & & & pcin \\
\hline Curium-245/246 & 2.3E-01 & $\mathrm{R} / 4$ & Rej & & 1 & GP & & & & & & & pCin \\
\hline $\begin{array}{l}\text { Gross alpha } \\
\text { Jodine-129 }\end{array}$ & $5.2 E+02$ & $\mathrm{~J} / \mathrm{L} / \mathrm{I}$ & NDD & $=$ & 1 & GP & $5.2 E+02$ & 11 & & घ & 1 & GP & pein \\
\hline $\begin{array}{l}\text { lodine-129 } \\
\text { Nonvolatile beta }\end{array}$ & $\begin{array}{l}7.5 E+01 \\
6.4 E+02\end{array}$ & J/1 & NDD & 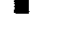 & 1 & $\begin{array}{l}\text { GP } \\
\text { GP }\end{array}$ & $5.5 \mathrm{E}+02$ & 11 & & 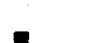 & 1 & GP & pcin \\
\hline $\begin{array}{l}\text { Nonvolatile beta } \\
\text { Plutonium-238 }\end{array}$ & $\begin{array}{l}6.4 E+02 \\
<2.6 E-02\end{array}$ & $u / \prime$ & $<1.5 E-01$ & & $\uparrow$ & $\mathrm{GP}$ & $0.0 E+U L$ & 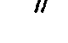 & & 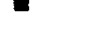 & 1 & G & pCir \\
\hline Plutonium-239/240 & $<1.8 E-02$ & $\mathrm{U} / /$ & $<5.3 E-02$ & & 1 & GP & & & & & & & $\mathrm{pCin}$ \\
\hline Radium-226 & $1.0 E+01$ & $\mathrm{~J} / \mathrm{I}$ & NDD & & 1 & GP & & & & & & & pCin \\
\hline Radium-228 & 4.3E+ $+\infty$ & $\|$ & & & 1 & GP & & & & & & & pCin \\
\hline Total radium & $4.3 E+00$ & & & & & & & & & & & & \\
\hline Strontium-90 & $2.4 E+02$ & $\|$ & & E & 1 & GP & & & & & & & pCin \\
\hline Technetium-99 & $6.1 E+01$ & $" n$ & & \pm & 1 & GP & & & & & & & PCil \\
\hline Thorium-228 & 6.0E-01 & $\mathrm{J} / \mathrm{V}$ & NDD & & 1 & GP & & & & & & & pCin \\
\hline Thorium-230 & $<2.0 E-01$ & $\mathrm{U} / \mathrm{I}$ & $<2.5 \mathrm{E}-01$ & & 1 & GP & & & & & & & $\mathrm{pCin}$ \\
\hline Thorium-232 & $<3.3 E-02$ & $\mathrm{u} / /$ & $<9.9 E-02$ & & 1 & GP & & & & & & & pCin \\
\hline Sum of alphas & $5.6 E+02$ & & & E & & & & & & & & & pCin \\
\hline Sum of betas & $3.8 \mathrm{E}+02$ & .. & & a & & & & & & & & & $\mathrm{pCin}$ \\
\hline Tritium & 1.4E+03 & $\|$ & & $=$ & 1 & $G P$ & $6.3 E+02$ & $\|$ & & a & 1 & GP & pCi/mL \\
\hline Uranium-233/234 & $2.6 E+02$ & /I & & च & 1 & GP & & & & & & & pCin \\
\hline Uranium-235 & $3.6 E+01$ & 11 & & E & 1 & GP & & & & & & & pCin \\
\hline Uranium-238 & $2.7 E+02$ & $\|$ & & a & 1 & GP & & & & & & & pCin \\
\hline
\end{tabular}

Notes:

- exceeded holding time

- exceeded groundwater protection or monitoring constituent standard (See Appendix A.)

NA - Not applicable. Applies to beta dose and sum of betas if there are no beta-emitting radionuclides above detection limits; to sum of alphas if there are no alphaemitting radionuclides above detection limits; and to total radium if neither radium-226 or radium-228 was above detection limit

UAZ UTRA - Upper Aquifer Zone of the Upper Three Runs Aquifer, LAZ UTRA - Lower Aquifer Zone of the Upper Three Runs Aquifer, Gordon - Gordon Aquifer

F-Area HWMF

C-157

First and Second Quarter 1999 


\section{WELL FSB118D}

$\begin{array}{ll}\text { SRS Coord } & \text { Lathongitude } \\ \text { N74697.9 } & 33.274646^{\circ} \mathrm{N} \\ \text { E51276.3 } & 81.676877^{\circ} \mathrm{W}\end{array}$

SAMPLE DATE

$01 / 11 / 99$

Screen Zone Elevation

211.3-191.3 ft ms
Top of Casing

$243.3 \mathrm{ft} \mathrm{ms}$

Casing Pump

4" PVC S
Screen Zone

UAZ_UTRA

\section{FIELD DATA}

\section{Constituents}

Water elevation

pH

Sp. conductance

Water temperature

Alkalinity as $\mathrm{CaCO} 3$

Turbidity

Volumes purged

Sampling code

Synchronous water level

\section{ANALYTICAL DATA}

Inorganic Constituents

04/07/99

\section{9}

211.7

4.7

28

18.2

8.1

$211.4(03 / 23 / 99)$
211.2

4.6

28

20.0

0

6.3

$210.7(06 / 28 / 99)$
Unit

tt msl

pH

$\mu \mathrm{S} / \mathrm{cm}$

${ }^{\circ} \mathrm{C}$

$\mathrm{mgh}$

NTU

well volumes

ft msl

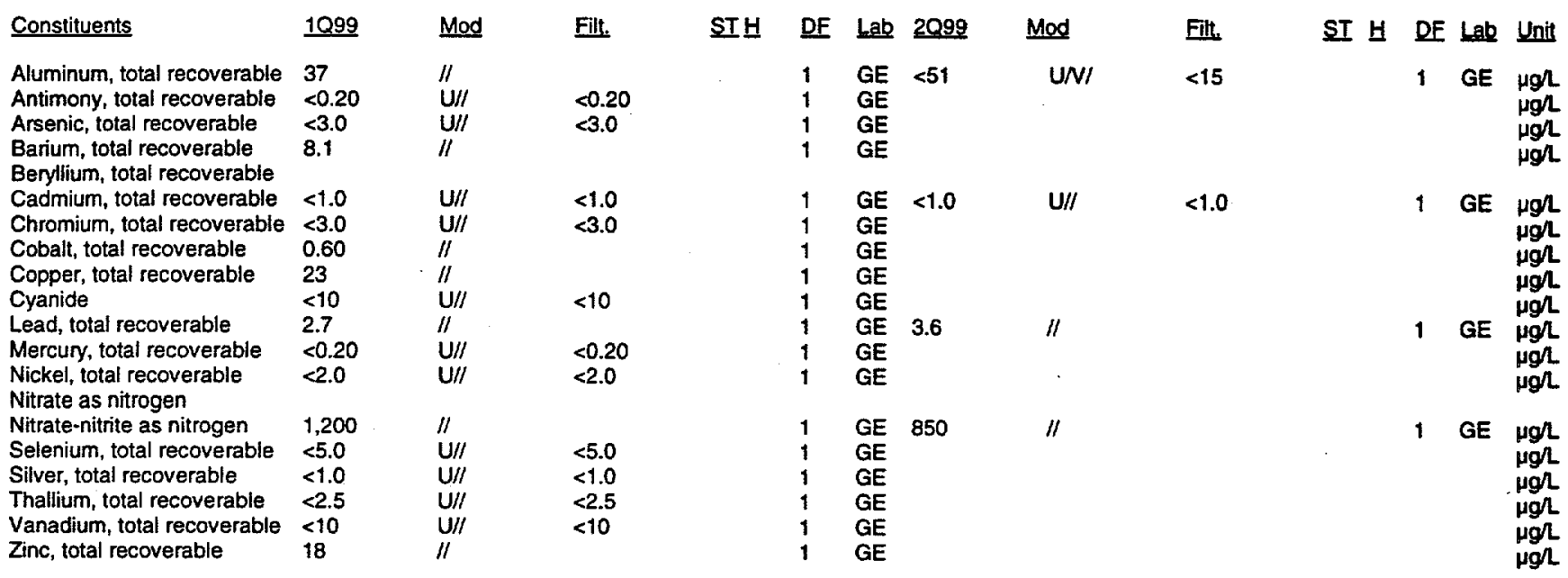

Organic Constituents

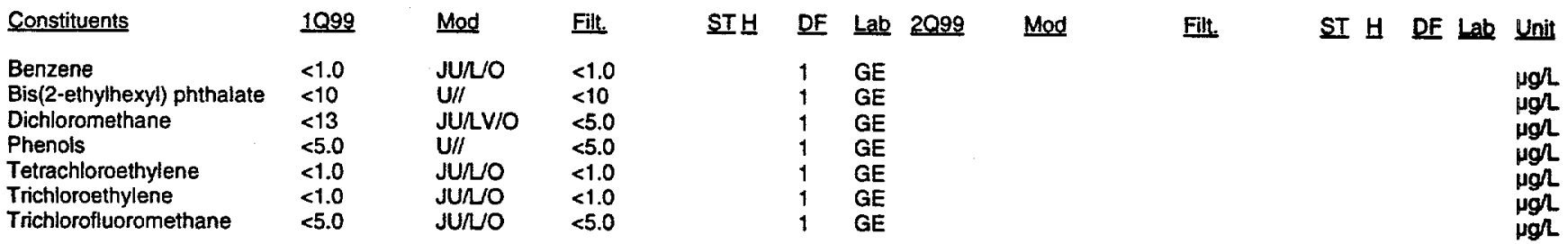

Notes:

- exceeded hoiding time

= exceeded groundwater protection or monitoring constituent standard (See Appendix A.)

NA - Not applicable. Applies to beta dose and sum of betas if there are no beta-emitting radionuclides above detection limits; to sum of alphas if there are no alphaemitting radionuclides above detection limits; and to total radium if neither radium-226 or radium-228 was above detection limit

UAZ_UTRA - Upper Aquifer Zone of the Upper Three Runs Aquifer, LAZ UTRA - Lower Aquifer Zone of the Upper Three Runs Aquifer, Gordon - Gordon Aquifer 


\section{WELL FSB118D (cont.)}

Radioactive Constituents

\begin{tabular}{|c|c|c|c|c|c|c|c|c|c|c|c|c|c|}
\hline Constituents & 1099 & Mod & Filt. & ST브 & DF & $\underline{L a b}$ & 2099 & Mod & Filt. & ST $H$ & DF & Lab & Unit \\
\hline $\begin{array}{l}\text { Americium-241 } \\
\text { Beta dose }\end{array}$ & $\begin{array}{l}<2.5 E-02 \\
N A\end{array}$ & $\mathrm{U} / I$ & $<4.9 E-01$ & & 1 & GP & & & & & & & pCin \\
\hline Carbon-14 & $<-1.0 E+\infty$ & $u^{\prime \prime}$ & $<7.4 E+\infty 0$ & & 1 & GP & & & & & & & $\mathrm{pCi} / \mathrm{L}$ \\
\hline Cesium-137 & $<2.9 \mathrm{E}-01$ & $U \prime \prime$ & $<3.6 E+00$ & & 1 & $\mathrm{GP}$ & & & & & & & pein \\
\hline Cobalt -60 & $<2.9 E-01$ & $\mathrm{U} / /$ & $<4.1 E+\infty$ & & 1 & GP & & & & & & & pCin \\
\hline Curium-242 & $<-8.9 E-02$ & $\mathrm{U} / I$ & $<4.1 E-01$ & & 1 & GP & & & & & & & peir \\
\hline Curium-243/244 & $<4.7 E-01$ & U/I & $<6.3 E-01$ & & 1 & GP & & & & & & & pCin \\
\hline Curium-245/246 & $<-1.3 \mathrm{E}-02$ & $\mathrm{U} / \mathrm{I}$ & $<1.8 E-01$ & & $i$ & GP & & & & & & & pCin \\
\hline Gross alpha & $1.2 E+00$ & $\mathrm{~J} / \mathrm{V}$ & NDD & & 1 & $G P$ & $1.3 E+\infty$ & $\mathrm{J} / \mathrm{N}$ & NDD & & 1 & GP & pCin \\
\hline $\begin{array}{l}\text { lodine- } 129 \\
\text { Nonvolatile beta }\end{array}$ & $\begin{array}{l}<6.1 E-01 \\
1.5 E+00\end{array}$ & $\mathrm{~d} / \mathrm{U}$ & $\begin{array}{l}<8.2 E-01 \\
N D D\end{array}$ & & 1 & $G P$ & $1.3 E+\infty$ & $\mathrm{J} / \mathrm{V}$ & NDD & & 1 & GP & pCil \\
\hline Plutonium-238 & 3.1E-01 & $\mathrm{J} / \mathrm{V}$ & NDD & & 1 & GP & & & & & & & $\mathrm{pCu}$ \\
\hline Plutonium-239/240 & $<8.7 E-02$ & $\mathrm{U} / /$ & $<1.5 E-01$ & & 1 & GP & & & & & & & pCin \\
\hline Radium-226 & $1.5 E+00$ & $\mathrm{~J} / \mathrm{V}$ & NDD & & 1 & GP & & & & & & & $\mathrm{pCi} / \mathrm{L}$ \\
\hline $\begin{array}{l}\text { Radium-228 } \\
\text { Total radium }\end{array}$ & $\begin{array}{l}<9.8 \mathrm{E}-01 \\
\mathrm{NA}\end{array}$ & $U / l$ & $<1.4 E+\infty$ & & 1 & GP & & & & & & & $\mathrm{pCi} / \mathrm{L}$ \\
\hline Strontium-90 & $<-9.8 \mathrm{E}-02$ & $\mathrm{U} / /$ & $<1.5 E+\infty 0$ & & 1 & $\mathrm{GP}$ & & & & & & & pcin \\
\hline Technetium-99 & $<1.3 E+\infty 0$ & $\mathrm{U} / /$ & $<1.9 E+01$ & & 1 & GP & & & & & & & $\mathrm{pCin}$ \\
\hline Thorium-228 & 1.3E-01 & $\mathrm{R} / / 4$ & $\mathrm{Rej}$ & & 1 & $\overrightarrow{G P}$ & & & & & & & pCin \\
\hline Thorium-230 & $3.8 E-02$ & J/IL/I & NDD & & 1 & $\mathrm{GP}$ & & & & & & & $\mathrm{pCi} /$ \\
\hline Thorium-232 & $<-3.8 E-03$ & JU/L/ & $<4.8 E-02$ & & 1 & GP & & & & & & & pCin \\
\hline Sum of alphas & NA & & & & & & & & & & & & \\
\hline Sum of betas & $\begin{array}{l}\text { NA } \\
6.9 E+00\end{array}$ & & & & & & & & & & & & \\
\hline Tritium & $6.9 E+00$ & $1 /$ & & & 1 & GP & $6.6 E+00$ & / & & & 1 & GP & pCi/m \\
\hline Uranium-233/234 & $<5.4 \mathrm{E}-02$ & $U / I$ & $<2.6 E-01$ & & 1 & GP & & & & & & & \\
\hline Uranium-235 & $<2.2 E-02$ & $\mathrm{U} / \mathrm{H}$ & $<6.5 E-02$ & & 1 & GP & & & & & & & pcin \\
\hline Uranium-238 & $<7.6 E-02$ & $\mathrm{U} / \mathrm{I}$ & $<1.4 E-01$ & & 1 & GP & & & & & & & \\
\hline
\end{tabular}




\section{WELL FSB119D}

$\begin{array}{ll}\text { SRS Coord. } & \text { LatlLongitude } \\ \text { N74599.7 } & 33.273326^{\circ} \mathrm{N} \\ \text { E50600.6 } & \text { B1.678465 } \mathrm{W}\end{array}$

SAMPLE DATE

$\begin{array}{lllll}\text { Screen Zone Elevation } & \text { Top of Casing } & \text { Casing } & \text { Pump } & \text { Screen Zone } \\ 213.1-193.1 \mathrm{ft} \mathrm{msl} & 254.1 \mathrm{ft} \mathrm{msl} & 4^{*} \text { PVC } & \text { S } & \text { UAZ_UTRA }\end{array}$

04/09/99

2099
207.7

3.6

900

19.2

19.2
121

121
0.10

$\mathrm{X}$

$206.8(06 / 28 / 99)$

Unit

tt ms!

pH

$\mu \mathrm{Sicm}$

${ }^{\circ} \mathrm{C}$

mgt

$\mathrm{x}$

$207.9(03 / 23 / 99)$ well volumes ft msl

\section{ANALYTICAL DATA}

Inorganic Constituents

Constituents

Aluminum, total recoverable 40,000

Antimony, total recoverable $<0.20$

Arsenic, total recoverable $<60$

Barium, total recoverabie 290

Beryllium, total recoverable

Cadmium, total recoverable 6.8

Chromium, total recoverable 7.1

Cobalt, total recoverable

Copper, total recoverable

Cyanide

Lead, total recoverable

Mercury, total recoverable

Nickel, total recoverable 28

Nitrate as nitrogen

Nitrate-nitrite as nitrogen $\quad 100,000$

Selenium, total recoverable $<100$

Silver, total recoverable $<1.0$

Thallium, total recoverable $\quad 0.30$

Vanadium, total recoverable $<10$

Zinc, total recoverable

$<10$
79

\begin{tabular}{|c|c|c|c|c|c|c|}
\hline Mod & Filt. & ST브 & DF & Lab & 2099 & Mod \\
\hline $\begin{array}{l}\mathrm{J} / / \mathrm{Cl} \\
\mathrm{U} / / \\
\mathrm{U} / / \\
/ /\end{array}$ & $\begin{array}{l}\text { NDD } \\
<0.20 \\
<60\end{array}$ & & $\begin{array}{l}20 \\
1 \\
20 \\
1\end{array}$ & $\begin{array}{l}\text { GE } \\
\text { GE } \\
\text { GE } \\
\text { GE }\end{array}$ & 35,000 & $\mathrm{~J} / \mathrm{K} /$ \\
\hline $\begin{array}{l}\| 1 \\
11 \\
1 / \\
1 /\end{array}$ & & 口 & $\begin{array}{l}1 \\
1 \\
1 \\
1\end{array}$ & $\begin{array}{l}\text { GE } \\
\text { GE } \\
\text { GE } \\
\text { GE }\end{array}$ & 7.5 & $\|$ \\
\hline $\begin{array}{l}\text { JU// } \\
\| / \\
/ 1 \\
/ 1\end{array}$ & $<10$ & & $\begin{array}{l}1 \\
1 \\
1 \\
1\end{array}$ & $\begin{array}{l}\mathrm{GE} \\
\mathrm{GE} \\
\mathrm{GE} \\
\mathrm{GE}\end{array}$ & 30 & $/ /$ \\
\hline $\begin{array}{l}/ l \\
\mathrm{U} / / \\
\mathrm{U} / \prime \\
\mathrm{J} / \mathrm{\prime} \\
\mathrm{U} / \prime \\
/ /\end{array}$ & $\begin{array}{l}<100 \\
<1.0 \\
\text { NDD } \\
<10\end{array}$ & घ & $\begin{array}{l}100 \\
20 \\
1 \\
1 \\
1 \\
1\end{array}$ & $\begin{array}{l}\mathrm{GE} \\
\mathrm{GE} \\
\mathrm{GE} \\
\mathrm{GE} \\
\mathrm{GE} \\
\mathrm{GE}\end{array}$ & 100,000 & $\|$ \\
\hline
\end{tabular}

Organic Constituents

\begin{tabular}{llll} 
Constituents & 1099 & Mod & Filt. \\
\cline { 2 - 4 } Benzene & $<1.0$ & JU/NO & $<1.0$ \\
Bis(2-ethylhexyl) phthalate & $<9.9$ & U/I & $<9.9$ \\
Dichloromethane & 3.8 & J/IUO & NDD \\
Phenols & $<5.0$ & U/I & $<5.0$ \\
Tetrachloroethylene & $<1.0$ & JU/NO & $<1.0$ \\
Trichloroethylene & $<1.0$ & JU/UO & $<1.0$ \\
Trichlorofiuoromethane & $<5.0$ & JU/UO & $<5.0$
\end{tabular}

Filt.

NDD

\section{ST보}

$\begin{array}{ll}\text { DF } & \text { Lab } \\ 1 & \text { GE } \\ 1 & \text { GE } \\ 1 & \text { GE } \\ 1 & \text { GE } \\ 1 & \text { GE } \\ 1 & \text { GE } \\ 1 & \text { GE }\end{array}$

Mod

Fill.

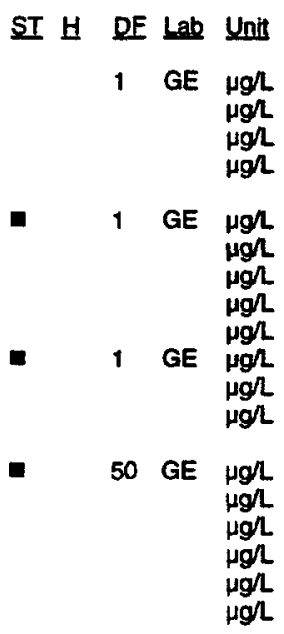

SI H DE Lab Unit

$\mu g h$

$\mu \mathrm{g} / \mathrm{L}$

$\mu g / L$

$\mu g /$

$\mu g /$

$\mu g /$

Notes:

= exceeded holding time

- exceeded groundwater protection or monitoring constituent standard (See Appendix A.)

NA - Not applicable. Applies to beta dose and sum of betas if there are no beta-emitting radionuclides above detection limits; to sum of atphas if there are no alphaemitting radionuclides above detection limits; and to total radium if neither radium-226 or radium-228 was above detection limit

UAZ UTRA - Upper Aquifer Zone of the Upper Three Runs Aquifer, LAZ UTRA - Lower Aquifer Zone of the Upper Three Runs Aquifer, Gordon - Gordon Aquifer 
WELL FSB119D (cont.)

Radioactive Constituents

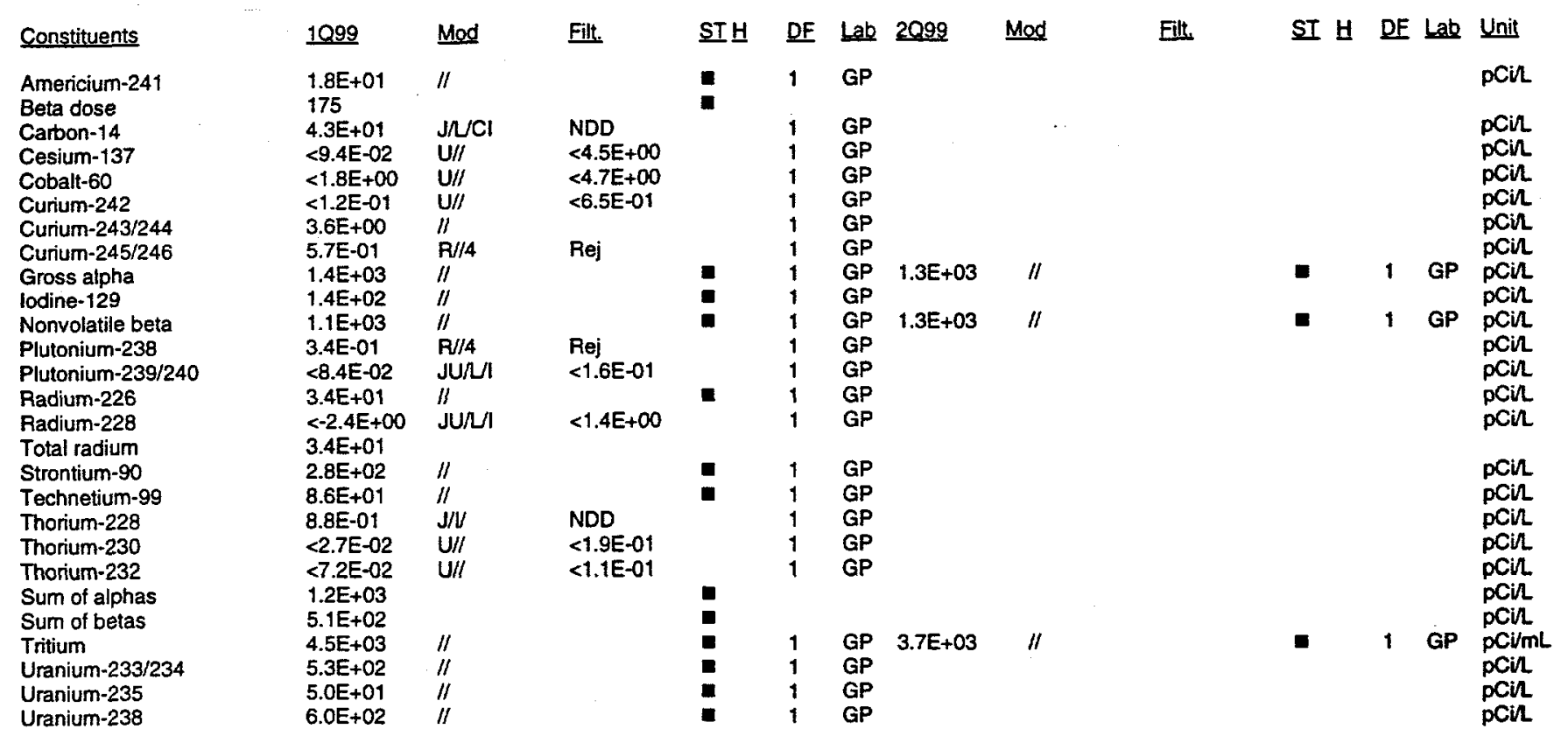




\section{WELL FSB120A}

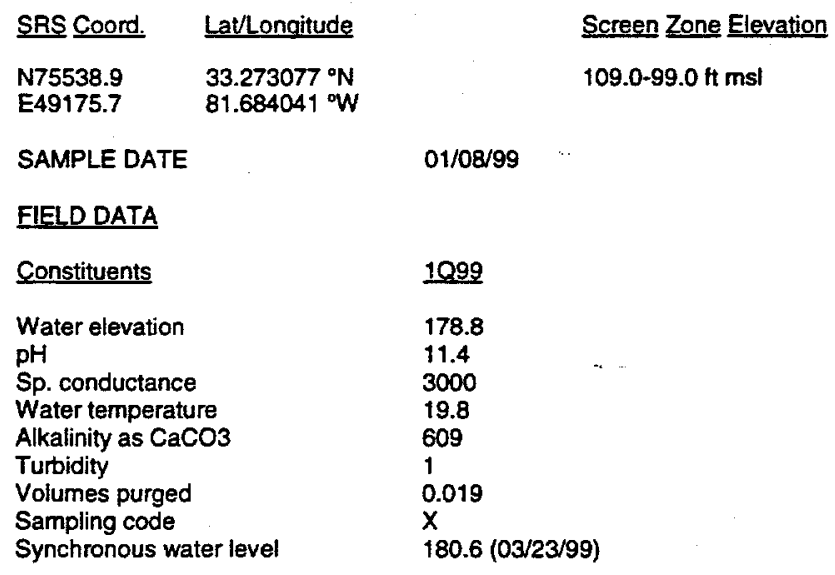

$\begin{array}{llll}\text { Top of Casing } & \text { Casing } & \text { Pume } & \text { Screen Zone } \\ 280.1 \mathrm{ft} \mathrm{msl} & 4^{*} \text { PVC } & \text { S } & \text { Gordon }\end{array}$

04/06/99

$\begin{array}{ll}2099 & \text { Unit } \\ 179.7 & \\ 11.5 & \mathrm{tt} \mathrm{msi} \\ 2400 & \mathrm{pH} \\ 18.4 & \mathrm{HS} / \mathrm{cm} \\ 461 & { }^{\circ} \mathrm{C} \\ 0 & \mathrm{mgh} \\ 0.019 & \mathrm{NTU} \\ \mathrm{X} & \text { well volumes } \\ 164.1(06 / 25 / 99) & \\ & \text { ft msl }\end{array}$

\section{ANALYTICAL DATA}

Inorganic Constituents

\begin{tabular}{|c|c|c|c|c|c|c|c|c|c|c|c|c|c|}
\hline Constifuents & 1099 & Mod & Filt. & STH & DE & Lab & 2099 & Mod & Fill & SI H & DE & Lab & Unit \\
\hline $\begin{array}{l}\text { Aluminum, total recoverable } \\
\text { Antimony, total recoverable } \\
\text { Arsenic, total recoverable } \\
\text { Barium, total recoverable } \\
\text { Benyllium, total recoverable }\end{array}$ & $\begin{array}{l}1,800 \\
<0.14 \\
<3.0 \\
98\end{array}$ & $\begin{array}{l}\text { JU//4 } \\
\mathrm{U} / / \\
\| /\end{array}$ & $\begin{array}{l}<0.89 \\
<3.0\end{array}$ & & $\begin{array}{l}1 \\
1 \\
1 \\
1\end{array}$ & $\begin{array}{l}\mathrm{GE} \\
\mathrm{GE} \\
\mathrm{GE} \\
\mathrm{GE}\end{array}$ & 1,200 & $\|$ & & & 1 & GE & $\begin{array}{l}\mu g h \\
\mu g h \\
\mu g h \\
\mu g h\end{array}$ \\
\hline Cadmium, total recoverable & $<1.0$ & $\mathrm{U} / \mathrm{I}$ & $<1.0$ & & 1 & GE & $<1.0$ & $\mathrm{U} / \mathrm{I}$ & $<1.0$ & & 1 & GE & $\mu g / 2$ \\
\hline $\begin{array}{l}\text { Chromium, total recoverable } \\
\text { Cobalt, total recoverable }\end{array}$ & $\begin{array}{l}1.9 \\
0.48\end{array}$ & $\mathrm{~J} / N$ & NDD & & $\begin{array}{l}1 \\
1\end{array}$ & $\begin{array}{l}\text { GE } \\
\text { GE }\end{array}$ & & & & & & & $n$ \\
\hline Copper, total recoverable & 0.85 & $\mathrm{~J} / \mathrm{V}$ & NDD & & 1 & GE & & & & & & & $\begin{array}{l}\mu g / 2 \\
\mu g /\end{array}$ \\
\hline Cyanide & $<10$ & $U / I$ & $<10$ & & 1 & $\mathrm{GE}$ & & & & & & & $\mu g \Omega$ \\
\hline $\begin{array}{l}\text { Lead, total recoverable } \\
\text { Mercury, total recoverable }\end{array}$ & $\begin{array}{l}1.5 \\
<0.042\end{array}$ & $\begin{array}{l}\mathrm{J} / \mathrm{V} \\
\mathrm{U} N \mathrm{I}\end{array}$ & $\begin{array}{l}\text { NDD } \\
<0.20\end{array}$ & & $\begin{array}{l}1 \\
1\end{array}$ & $\begin{array}{l}G E \\
G E\end{array}$ & 2.5 & "I & & & 1 & GE & $\mu g / 2$ \\
\hline $\begin{array}{l}\text { Nickel, total recoverable } \\
\text { Nitrate as nitrogen }\end{array}$ & 4.1 & 11 & & & $i$ & $\overline{G E}$ & & & & & & & \\
\hline Nitrate-nitrite as nitrogen & 18,000 & 11 & & - & 10 & GE & 26,000 & /I & & घ & 25 & GE & \\
\hline $\begin{array}{l}\text { Selenium, total recoverable } \\
\text { Silver, total recoverable }\end{array}$ & $\begin{array}{l}<5.0 \\
<1.0\end{array}$ & $\begin{array}{l}\mathrm{U} / / \\
\mathrm{U} / /\end{array}$ & $\begin{array}{l}<5.0 \\
<1.0\end{array}$ & & $\begin{array}{l}1 \\
1\end{array}$ & GE & & & & & & & \\
\hline Thallium, total recoverable & $<0.083$ & UN/ & $<2.5$ & & 1 & GE & & & & & & & \\
\hline Vanadium, total recoverable & $<10$ & $\mathrm{U} / \mathrm{I}$ & $<10$ & & 1 & GE & & & & & & & \\
\hline Zinc, total recoverable & $<10$ & $\mathrm{u} / \mathrm{I}$ & $<10$ & & 1 & GE & & & & & & & \\
\hline
\end{tabular}

Organic Constituents

\begin{tabular}{|c|c|c|c|c|c|c|c|c|c|c|c|}
\hline Constituents & 1099 & Mod & Filt. & $\underline{\text { STH}}$ & DE & Lab 2099 & Mod & Fill. & SI $\mathrm{H}$ & DE Lab & Unil \\
\hline $\begin{array}{l}\text { Benzene } \\
\text { Bis(2-ethylhexyl) phthaiate } \\
\text { Dichloromethane } \\
\text { Phenols } \\
\text { Tetrachloroethylene } \\
\text { Trichloroethylene } \\
\text { Trichlorofluoromethane }\end{array}$ & $\begin{array}{l}<1.0 \\
<9.9 \\
<2.2 \\
<5.0 \\
<1.0 \\
1.4 \\
<5.0\end{array}$ & $\begin{array}{l}U / I \\
U / I \\
U N I \\
U / I \\
U / I \\
I / \\
U / I\end{array}$ & $\begin{array}{l}<1.0 \\
<9.9 \\
<5.0 \\
<5.0 \\
<1.0\end{array}$ & & $\begin{array}{l}1 \\
1 \\
1 \\
1 \\
1 \\
1 \\
1\end{array}$ & $\begin{array}{l}\mathrm{GE} \\
\mathrm{GE} \\
\mathrm{GE} \\
\mathrm{GE} \\
\mathrm{GE} \\
\mathrm{GE} \\
\mathrm{GE}\end{array}$ & & & & & $\begin{array}{l}\mu g h \\
\mu g h \\
\mu g h \\
\mu g / h \\
\mu g h \\
\mu g / h \\
\mu g h\end{array}$ \\
\hline
\end{tabular}

Notes:

= exceeded holding time

= exceeded groundwater protection or monitoring constituent standard (See Appendix A.)

NA - Not applicable. Applies to beta dose and sum of betas if there are no beta-emitting radionuclides above detection limits; to sum of alphas if there are no alphaemitting radionuclides above detection limits; and to total radium if neither radium-226 or radium-228 was above detection limit

UAZ UTRA - Upper Aquifer Zone of the Upper Three Runs Aquifer, LAZ_UTRA - Lower Aquifer Zone of the Upper Three Runs Aquifer, Gordon - Gordon Aquifer 


\section{WELL FSB120A (cont.)}

Radioactive Constituents

\begin{tabular}{|c|c|c|c|c|c|c|c|c|c|c|c|c|c|}
\hline Constituents & 1099 & Mod & Filt. & ST브 & DF & Lab & 2099 & Mod & Filt. & ST $H$ & DF & Lab & Unit \\
\hline $\begin{array}{l}\text { Americium-241 } \\
\text { Beta dose }\end{array}$ & $\begin{array}{l}<-1.9 E-02 \\
8.5\end{array}$ & $\mathrm{U} / /$ & $<2.2 E-01$ & $\mathbf{E}$ & 1 & GP & & & & & & & pCin \\
\hline Carbon-14 & $<4.7 E+\infty$ & $\mathrm{U} / /$ & $<7.3 E+\infty$ & & 1 & GP & & & & & & & pCin \\
\hline Cesium-137 & $<-9.2 E-03$ & $\mathbf{U} / \prime$ & $<3.2 E+\infty$ & & 1 & GP & & & & & & & pCin \\
\hline Cobalt-60 & $5.6 \mathrm{E}+\infty$ & $R / / 4$ & $R e j$ & & 1 & GP & & & & & & & pCin \\
\hline Curium-242 & $<-1.8 \mathrm{E}-02$ & $\mathrm{U} / /$ & $<1.7 E-01$ & & 1 & GP & & & & & & & pCin \\
\hline Curium-243/244 & $<-1.7 E-02$ & $\mathbf{U} / /$ & $<1.3 E-01$ & & 1 & GP & & & & & & & pCir \\
\hline Curium-245/246 & $<0.0 E+00$ & $\mathrm{U} / /$ & $<5.1 E-02$ & & 1 & GP & & & & & & & $\mathrm{pCi} / \mathrm{L}$ \\
\hline $\begin{array}{l}\text { Gross alpha } \\
\text { lodine-129 }\end{array}$ & $\begin{array}{l}5.8 \mathrm{E}+\infty \\
7.4 \mathrm{E}+\infty\end{array}$ & 11 & & & $\begin{array}{l}1 \\
1\end{array}$ & $\begin{array}{l}\text { GP } \\
\text { GP }\end{array}$ & $4.4 E+00$ & $\|$ & & & 1 & GP & $\begin{array}{l}\text { poin } \\
\text { pCin }\end{array}$ \\
\hline Nonvolatile beta & $2.7 E+01$ & 11 & & & 1 & GP & 2.3E+01 & $\|$ & & & 1 & GP & pCir \\
\hline Plutonium-238 & $<-8.0 E-03$ & $\mathbf{U} / /$ & $<2.0 E-01$ & & 1 & GP & & & & & & & pCin \\
\hline Plutonium-239/240 & $<1.4 \mathrm{E}-02$ & $\mathrm{U} / I$ & $<4.3 E-02$ & & 1 & GP & & & & & & & pCin \\
\hline Radium-226 & $2.4 E+\infty 0$ & 11 & & & 1 & GP & & & & & & & pCin \\
\hline $\begin{array}{l}\text { Radium-228 } \\
\text { Total radium }\end{array}$ & $\begin{array}{l}<7.8 E-01 \\
2.4 E+00\end{array}$ & $\mathrm{U} / /$ & $<9.0 E-01$ & & 1 & GP & & & & & & & pCin \\
\hline Strontium-90 & $9.0 E+00$ & /I & & [ & 1 & GP & & & & & & & pCin \\
\hline Technetium-99 & $3.1 E+01$ & $\mathrm{~J} / \mathrm{W}$ & NDD & & 1 & GP & & & & & & & pcin \\
\hline Thorium-228 & 5.0E-01 & $R / / 4$ & Rej & & 1 & GP & & & & & & & pCir \\
\hline Thorium-230 & $<9.7 E-02$ & JU/UI & $<1.9 E-01$ & & 1 & GP & & & & & & & pCill \\
\hline Thorium-232 & $<-8.4 E-03$ & JURI & $<1.9 E-01$ & & 1 & GP & & & & & & & pCin \\
\hline Sum of alphas & NA & & & & & & & & & & & & \\
\hline Sum of betas & $1.6 E+01$ & & & & & & & & & & & & pCill \\
\hline Tritium & $4.2 E+02$ & 11 & & $\mathbf{z}$ & 1 & GP & $5.6 E+02$ & $/ /$ & & $\mathbf{0}$ & 1 & GP & pCirmi \\
\hline Uranium-233/234 & $<7.0 \mathrm{E}-03$ & $\mathrm{U} / /$ & $<8.3 E-01$ & & 1 & GP & & & & & & & pCir \\
\hline Uranium-235 & $<3.1 E-01$ & $\mathrm{U} / /$ & $<5.5 E-01$ & & 1 & GP & & & & & & & pCin \\
\hline Uranium-238 & $<4.6 \mathrm{E}-02$ & $\mathrm{U} / /$ & $<5.5 \mathrm{E}-01$ & & 1 & GP & & & & & & & pCin \\
\hline
\end{tabular}

\section{Notes:}

= exceeded holding time

= exceeded groundwater protection or monitoring constituent standard (See Appendix A.)

NA - Not applicable. Applies to beta dose and sum of betas if there are no beta-emitting radionuclides above detection limits; to sum of alphas if there are no alphaemitting radionuclides above detection limits; and to total radium if neither radium-226 or radium-228 was above detection limit

UAZ_UTRA - Upper Aquifer Zone of the Upper Three Runs Aquifer, LAZ UTRA - Lower Aquifer Zone of the Upper Three Runs Aquifer, Gordon - Gordon Aquifer 


\section{WELL FSB120C}

$\begin{array}{ll}\text { SRS Coord. } & \text { Lathonoitud } \\ \text { N75549.8 } & 33.273094^{\circ} \\ \text { E49171.1 } & 81.684074^{\circ}\end{array}$

SAMPLE DATE

$01 / 08 / 99$

Screen Zone Elevation

$160.7-150.7 \mathrm{ft} \mathrm{ms}$
Top of Casing

$279.7 \mathrm{ft} \mathrm{msl}$
Casing Pump

4: PVC S
Screen Zone

LAZ_UTRA
04/06/99

2099

206.1

6.2

280

19.6

11

2.9

$205.3(06 / 25 / 99)$
Unit

ft msl

pH

$\mu \mathrm{S} / \mathrm{cm}$

${ }^{\circ} \mathrm{C}$

NTU

well volumes

ft msi

Synchronous water level

$205.8(03 / 23 / 99)$

ANALYTICAL DATA

Inorganic Constituents

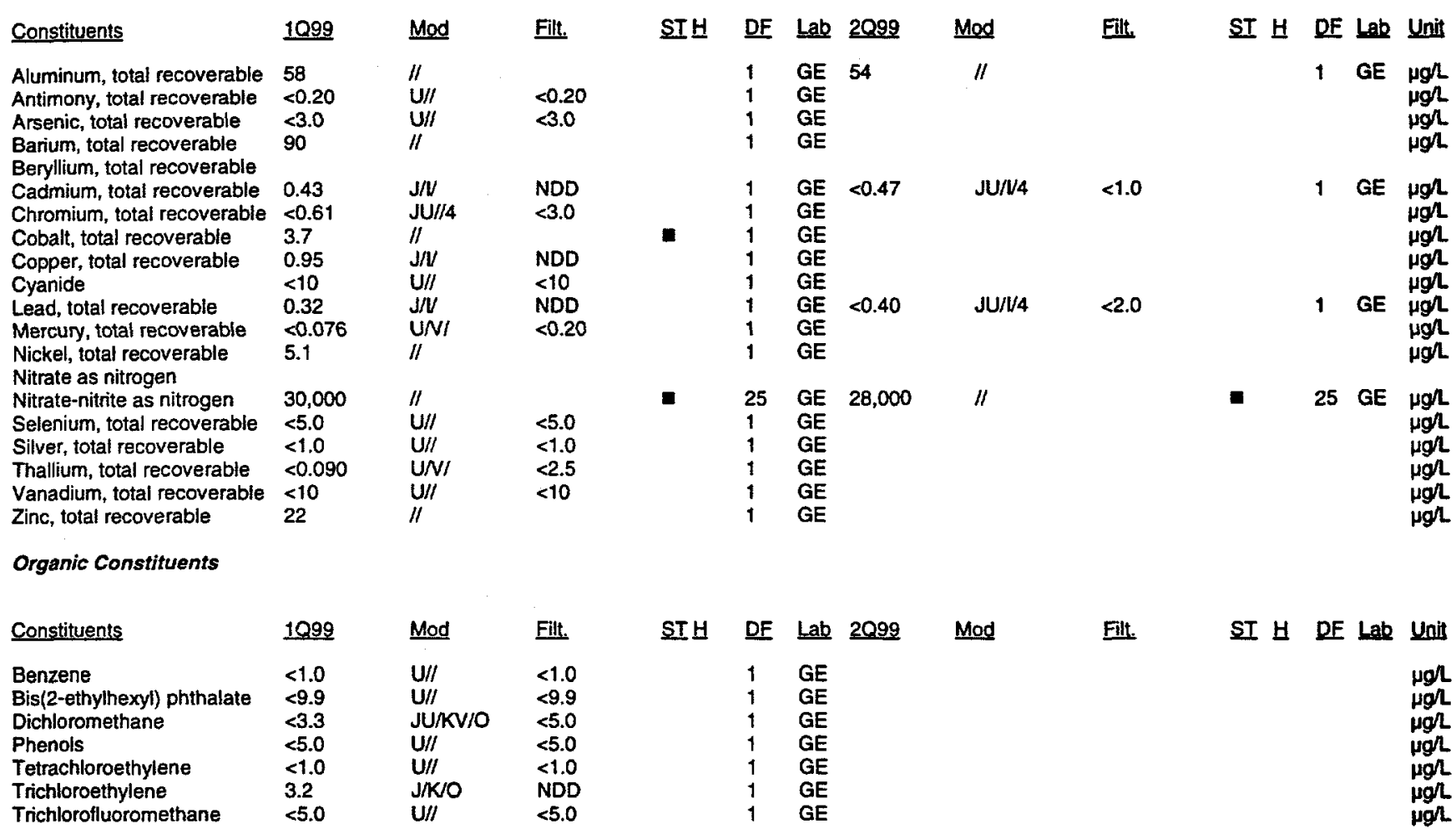

Notes:

- = exceeded holding time

- exceeded groundwater protection or monitoring constituent standard (See Appendix A.)

NA - Not applicable. Applies to beta dose and sum of betas if there are no beta-emitting radionuclides above detection limits; to sum of alphas if there are no alphaemitting radionuclides above detection limits; and to total radium if neither radium-226 or radium-228 was above detection limit

UAZ_UTRA - Upper Aquifer Zone of the Upper Three Runs Aquifer, LAZ_UTRA - Lower Aquifer Zone of the Upper Three Runs Aquifer, Gordon - Gordon Aquifer 


\section{WELL FSB120C (cont.)}

Radioactive Constituents

\begin{tabular}{|c|c|c|c|c|c|c|c|c|c|c|c|c|c|}
\hline Constituents & 1099 & Mod & Filt. & SI브 & DF & Lab & $\underline{2099}$ & Mod & Filt. & ST $H$ & DF & Lab & Unit \\
\hline $\begin{array}{l}\text { Americium-241 } \\
\text { Beta dose }\end{array}$ & $\begin{array}{l}<-6.1 E-02 \\
7.9\end{array}$ & $\mathrm{U} / /$ & $<3.2 \mathrm{E}-01$ & a & 1 & GP & & & & & & & pCin \\
\hline Carbon-14 & $2.1 E+01$ & 11 & & & 1 & GP & & & & & & & pCil \\
\hline Cesium-137 & $<-1.3 E+\infty$ & $\mathrm{U} / /$ & $<3.6 E+\infty$ & & 1 & GP & & & & & & & pCin \\
\hline Cobalt-60 & $<-1.7 E+\infty$ & $\mathrm{U} / /$ & $<3.6 \mathrm{E}+\infty$ & & 1 & GP & & & & & & & pCin \\
\hline Curium-242 & $<-9.7 E-02$ & $\mathrm{U} / /$ & $<2.8 E-01$ & & 1 & GP & & & & & & & pCin \\
\hline Curium-243/244 & $<0.0 E+\infty 0$ & $\mathrm{U} / \mathrm{I}$ & $<6.1 \mathrm{E}-02$ & & 1 & GP & & & & & & & pCin \\
\hline Cunium-245/246 & $<2.0 E-02$ & $U / !$ & $<6.1 E+02$ & & 1 & GP & & & & & & & pCin \\
\hline $\begin{array}{l}\text { Gross alpha } \\
\text { lodine-129 }\end{array}$ & $\begin{array}{l}1.2 E+01 \\
7.9 E+\infty\end{array}$ & & & & $\begin{array}{l}1 \\
1\end{array}$ & $\begin{array}{l}\text { GP } \\
\text { GP }\end{array}$ & 2.1E+01 & $/ 1$ & & 0 & 1 & GP & $\mathrm{pCin}$ \\
\hline Nonvolatile beta & $3.2 E+01$ & $\|$ & & & $i$ & GP & $3.6 E+01$ & $\|$ & & & 1 & GP & pCiL \\
\hline Plutonium-238 & $<-1.6 \mathrm{E}-02$ & $\mathrm{U} / /$ & $<2.2 E-01$ & & 1 & GP & & & & & & & pCir \\
\hline Plutonium-239/240 & $<-7.7 E-03$ & $\mathrm{u} / /$ & $<9.6 E-02$ & & 1 & GP & & & & & & & pCin \\
\hline Radium-226 & $6.3 E+\infty 0$ & $\|$ & & $\mathbf{\square}$ & 1 & GP & & & & & & & $\mathrm{pCin}$ \\
\hline Radium-228 & 1.7E+00 & $\mathrm{J} / \mathrm{V}$ & NDD & & 1 & GP & & & & & & & pCil \\
\hline Total radium & $6.3 E+00$ & & & & & & & & & & & & \\
\hline Strontium-90 & $3.2 E+00$ & $J / V$ & NDD & & 1 & GP & & & & - & & & pCir \\
\hline Technetium-99 & 4.1E+01 & $J / V$ & NDD & & 1 & GP & & & & & & & pcin \\
\hline Thorium-228 & $<1.7 E-01$ & JU/月 & $<4.6 E-01$ & & 1 & GP & & & & & & & pCin \\
\hline Thorium-230 & $<3.9 E-02$ & JU/M & $<2.8 E-01$ & & 1 & GP & & & & & & & pein \\
\hline $\begin{array}{l}\text { Thorium-232 } \\
\text { Sum of alphas }\end{array}$ & $\begin{array}{l}<-9.0 \mathrm{E}-03 \\
\mathrm{NA}\end{array}$ & JU/UI & $<2.0 E-01$ & & 1 & GP & & & & & & & $\mathrm{pCil}$ \\
\hline Sum of betas & $2.8 E+01$ & & & & & & & & & & & & pCin \\
\hline Tritium & $7.5 E+02$ & 11 & & $\boldsymbol{\square}$ & 1 & GP & $6.6 \mathrm{E}+02$ & $\|$ & & $\mathbf{a}$ & 1 & GP & pCi/mL \\
\hline Uranium-233/234 & $<7.4 E-02$ & $\mathrm{U} / /$ & $<2.2 E-01$ & & 1 & GP & & & & & & & pCin \\
\hline Uranium-235 & $<-3.0 E-02$ & $\mathrm{U} / /$ & $<2.9 E-01$ & & 1 & GP & & & & & & & pCin \\
\hline Uranium-238 & $<2.0 \mathrm{E}-01$ & $\mathrm{U} / /$ & $<2.2 \mathrm{E}-01$ & & 1 & GP & & & & & & & $\mathrm{pCn}$ \\
\hline
\end{tabular}

\section{Notes:}

- exceeded holding time

- exceeded groundwater protection or monitoring constituent standard (See Appendix A.)

NA - Not applicable. Applies to beta dose and sum of betas if there are no beta-emitting radionuclides above detection limits; to sum of alphas if there are no alphaemitting radionuclides above detection limits; and to total radium if neither radium-226 or radium-228 was above detection limit

UAZ UTAA - Upper Aquifer Zone of the Upper Three Runs Aquifer, LAZ_UTRA - Lower Aquifer Zone of the Upper Three Runs Aquifer, Gordon - Gordon Aquifer 


\section{WELL FSB120D}

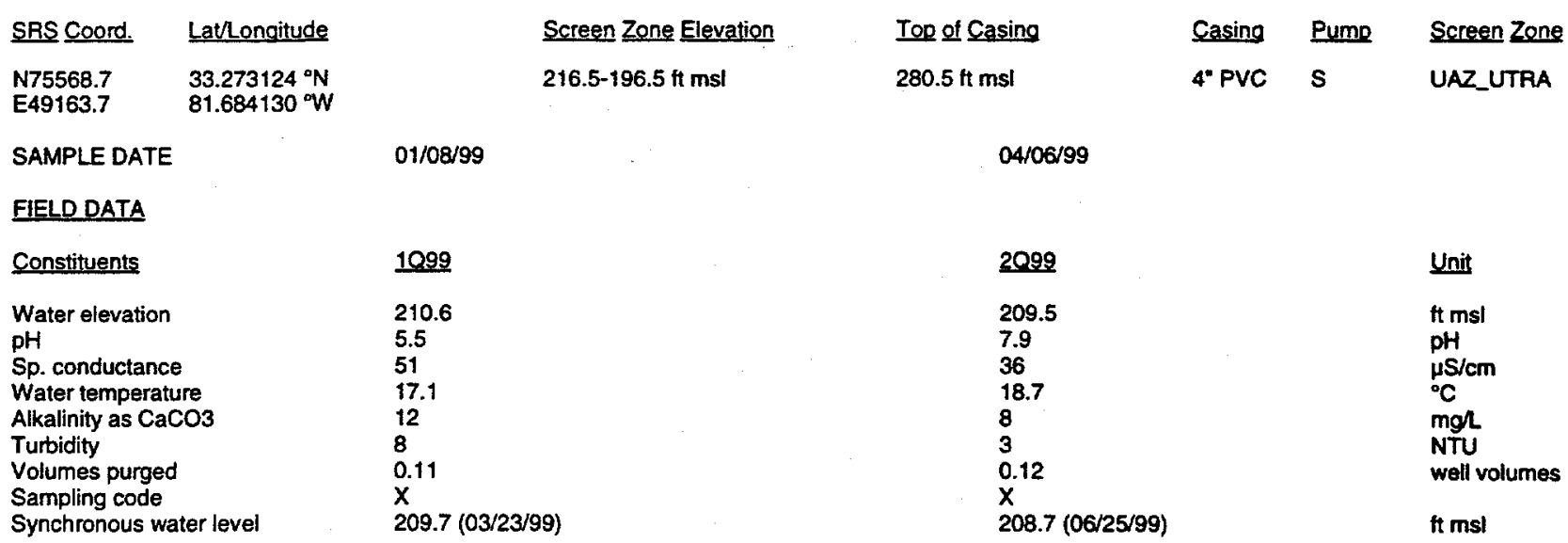

ANALYTICAL DATA

Inorganic Constituents

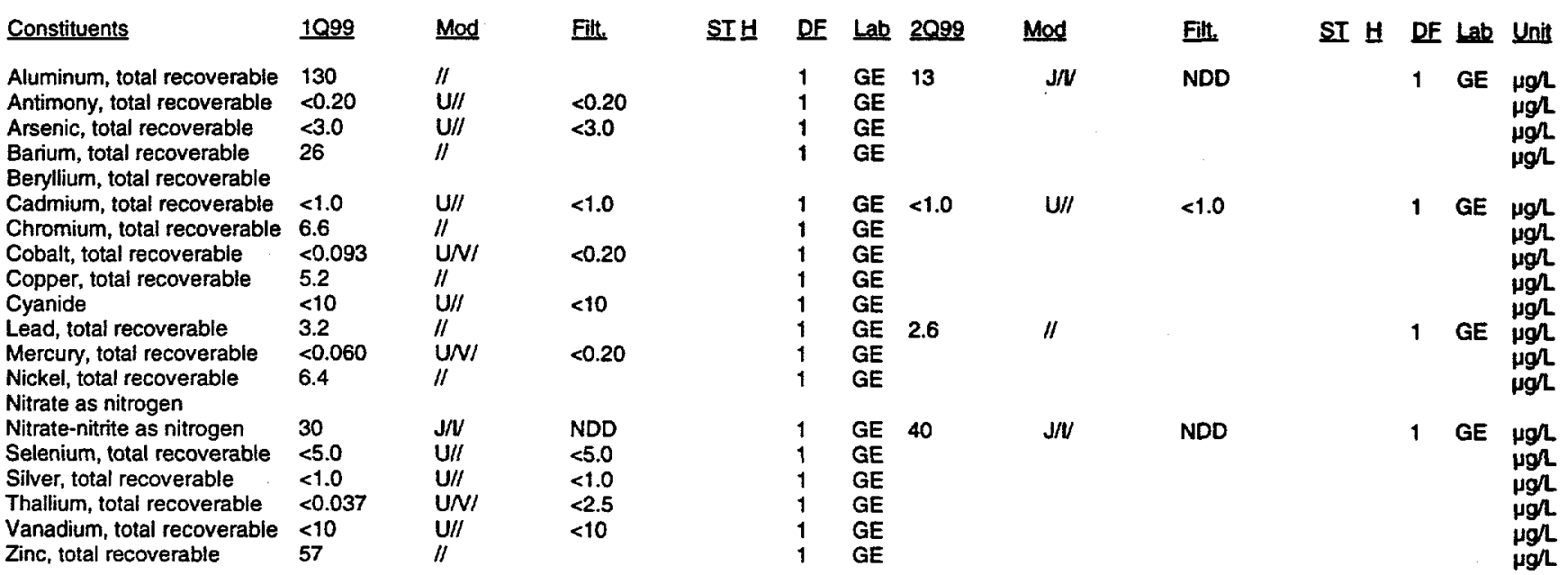

Organic Constituents

\begin{tabular}{|c|c|c|c|c|c|c|c|c|c|c|c|}
\hline Constituents & 1099 & Mod & Filt. & STH & DF & Lab & 2099 & Mod & Filt. & ST H DF Lab & Unit \\
\hline $\begin{array}{l}\text { Benzene } \\
\text { Bis(2-ethylhexyl) phthalate } \\
\text { Dichloromethane } \\
\text { Phenols } \\
\text { Tetrachloroethylene } \\
\text { Trichloroethylene } \\
\text { Trichlorofluoromethane }\end{array}$ & $\begin{array}{l}<1.0 \\
<10 \\
<5.0 \\
<5.0 \\
<1.0 \\
<1.0 \\
<5.0\end{array}$ & $\begin{array}{l}\text { JU/LQ/O } \\
\text { U/I } \\
\text { JU/LQ/O } \\
\text { U/I } \\
\text { JU/LQ/O } \\
\text { JU/LQ/O } \\
\text { JU/L/O }\end{array}$ & $\begin{array}{l}<1.0 \\
<10 \\
<5.0 \\
<5.0 \\
<1.0 \\
<1.0 \\
<5.0\end{array}$ & - & $\begin{array}{l}1 \\
1 \\
1 \\
1 \\
1 \\
1 \\
1\end{array}$ & $\begin{array}{l}\text { GE } \\
\text { GE } \\
\text { GE } \\
\text { GE } \\
\text { GE } \\
\text { GE } \\
\text { GE }\end{array}$ & & & & & $\begin{array}{l}\mu g / \\
\mu g / \\
\mu g / \\
\mu g / \\
\mu g / \\
\mu g /\end{array}$ \\
\hline
\end{tabular}

Notes:

- = exceeded holding time

= exceeded groundwater protection or monitoring constituent standard (See Appendix A.)

NA - Not applicable. Applies to beta dose and sum of betas if there are no beta-emitting radionuclides above detection limits; to sum of alphas if there are no alphaemitting radionuclides above detection limits; and to total radium if neither radium-226 or radium-228 was above detection limit

UAZ_UTRA - Upper Aquifer Zone of the Upper Three Runs Aquifer, LAZ_UTRA - Lower Aquifer Zone of the Upper Three Runs Aquifer, Gordon - Gordon Aquifer 


\section{WELL FSB120D (cont.)}

Radioactive Constituents

\begin{tabular}{|c|c|c|c|c|c|c|c|c|c|c|c|c|c|}
\hline Constituents & 1099 & Mod & Filt & STH & DF & Lab & 2099 & Mod & Filt. & ST $H$ & DF & Lab & Unit \\
\hline $\begin{array}{l}\text { Americium-241 } \\
\text { Beta dose }\end{array}$ & $\begin{array}{l}<B .5 E-02 \\
N A\end{array}$ & $\mathrm{U} / /$ & $<2.8 E-01$ & & 1 & GP & & & & & & & pCil \\
\hline Carbon-14 & $<-2.8 E+\infty 0$ & $\mathrm{U} / /$ & $<7.1 E+\infty 0$ & & 1 & GP & & & & & & & pCin \\
\hline Cesium-137 & $<-2.7 E-01$ & $\mathrm{U} / /$ & $<4.0 E+\infty 0$ & & 1 & GP & & $\cdot$ & & & & & pCin \\
\hline Cobalt -60 & $<1.5 E-01$ & $\mathrm{u} / /$ & $<3.9 E+\infty 0$ & & 1 & GP & & & & & & & pCin \\
\hline & $\begin{array}{l}<-7.3 E-02 \\
<-28 E-02\end{array}$ & $\mathrm{U} / /$ & $<4.0 E-01$ & & 1 & $\begin{array}{l}\text { GP } \\
\text { GP }\end{array}$ & & & & & & & $\begin{array}{l}\text { pCin } \\
\text { pCin }\end{array}$ \\
\hline $\begin{array}{l}\text { Curium-243/244 } \\
\text { Curium-245/246 }\end{array}$ & $\begin{array}{l}<-2.8 E-U 2 \\
<5.4 E-02\end{array}$ & $\mathrm{U} / 1$ & $<8.2 E-02$ & & 1 & GP & & & & & & & pCuL \\
\hline $\begin{array}{l}\text { Gross alpha } \\
\text { lodine-129 }\end{array}$ & $\begin{array}{l}8.2 E-01 \\
<3.0 E-01\end{array}$ & $\begin{array}{l}\mathrm{J} / \mathrm{V} \\
\mathrm{U} / /\end{array}$ & $\begin{array}{l}\text { NDD } \\
<7.9 E-01\end{array}$ & & $\begin{array}{l}1 \\
1\end{array}$ & $\begin{array}{l}\text { GP } \\
\text { GP }\end{array}$ & $9.2 E-01$ & $\mathrm{~J} / \mathrm{V}$ & NDD & & 1 & GP & $\begin{array}{l}\text { pCin } \\
\text { pCin }\end{array}$ \\
\hline Nonvolatile beta & $<8.0 E-01$ & $\mathrm{U} / /$ & $<1.2 E+\infty 0$ & & 1 & GP & $<9.2 E-01$ & $\mathrm{U} / /$ & $<1.2 E+\infty$ & & 1 & GP & pCil \\
\hline Plutonium-238 & $<-2.2 E-02$ & U/I & $<2.2 E-01$ & & 1 & GP & & & & & & & pCir \\
\hline Plutonium-239/240 & $<-1.4 \mathrm{E}-02$ & $\mathrm{U} / /$ & $<1.1 E-01$ & & 1 & GP & & & & & & & pCin \\
\hline Radium-226 & $6.9 E-01$ & $\mathrm{~J} / \mathrm{V}$ & NDD & & 1 & GP & & & & & & & pCin \\
\hline $\begin{array}{l}\text { Radium-228 } \\
\text { Total radium }\end{array}$ & $\begin{array}{l}<3.6 E-01 \\
N A\end{array}$ & $\mathbf{U} / /$ & $<9.0 \mathrm{E}-01$ & & 1 & GP & & & & & & & pCin \\
\hline Strontium-90 & $<5.0 E-02$ & $\mathrm{U} / /$ & $<1.4 \mathrm{E}+00$ & & 1 & GP & & & & & & & pCin \\
\hline Technetium-99 & $<-2.0 \mathrm{E}+00$ & & $<1.5 E+01$ & & 1 & GP & & & & & & & pcin \\
\hline Thorium-228 & $<2.7 E-01$ & JU/Л & $<3.1 E-01$ & & 1 & GP & & & & & & & $\mathrm{pCi} / \mathrm{L}$ \\
\hline Thorium-230 & $<5.3 E-02$ & JUЛЛ & $<2.2 \mathrm{E}-01$ & & 1 & GP & & & & & & & pCin \\
\hline Thonium-232 & $<2.6 E-02$ & แบน & $<1.8 \mathrm{E}-01$ & & 1 & GP & & & & & & & pcin \\
\hline Sum of alphas & NA & & & & & & & & & & & & \\
\hline Sum of betas & NA & & & & & & & & & & & & \\
\hline Tritium & 1.7E+01 & 11 & & & 1 & GP & $1.8 \mathrm{E}+01$ & $\| 1$ & & & 1 & GP & pCi/mL \\
\hline Uranium-233/234 & $<-6.2 E-03$ & $\mathrm{U} / /$ & $<1.3 E-01$ & & 1 & GP & & & & & & & pCin \\
\hline Uranium-235 & $<1.5 E-02$ & $\mathrm{U} / /$ & $<4.6 E-02$ & & 1 & GP & & & & & & & pCin \\
\hline Uranium-238 & $<-1.1 E-02$ & $\mathrm{U} / /$ & $<1.0 E-01$ & & 1 & GP & & & & & & & $\mathrm{pCi} / \mathrm{L}$ \\
\hline
\end{tabular}

Notes:

- exceeded holding time

= exceeded groundwater protection or monitoring constituent standard (See Appendix A.)

NA - Not applicable. Applies to beta dose and sum of betas if there are no beta-emitting radionuclides above detection limits; to sum of alphas if there are no alphaemitting radionuclides above detection limits; and to total radium if neither radium-226 or radium-228 was above detection limit

UAZ_UTRA - Upper Aquifer Zone of the Upper Three Runs Aquifer, LAZ_UTRA - Lower Aquifer Zone of the Upper Three Runs Aquifer, Gordon - Gordon Aquifer 


\section{WELL FSB121C}

$\begin{array}{ll}\text { SRS Coord. } & \text { Lathongitude } \\ \text { N75155.7 } & 33.270985^{\circ} \mathrm{N} \\ \text { E48413.1 } & 81.685304^{\circ} \mathrm{W}\end{array}$

SAMPLE DATE

Screen Zone Elevation

158.4-148.4 $\mathrm{ft} \mathrm{ms}$

1099

Constituents

204.9

5.4

Sp. conductance

Water temperature

Alkalinity as $\mathrm{CaCO} 3$

Turbidity

Volumes purged

Sampling code

Synchronous water leve

ANALYTICAL DATA

Inorganic Constituents

\section{Constituents}

Aluminum, total recoverable

Antimony, total recoverable $<0.20$

Arsenic, total recoverable $<3.0$

Barium, total recoverable

Beryllium, total recoverable

Cadmium, total recoverable $<1.0$

Chromium, total recoverable 1.5

Cobalt, total recoverable

Copper, total recoverable

Cyanide

Lead, total recoverable

Mercury, total recoverable $\quad<0.20$

Nickel, total recoverable $\quad 3.8$

Nitrate as nitrogen

Nitrate-nitrite as nitrogen $\quad 3,100$

Selenium, total recoverable $<5.0$

Silver, total recoverable $<1.0$

Thallium total recoverable $<0.23$

Vanadium, total recoverable

Zinc, total recoverable

$<6.9$

38

Organic Constituents

Constituents
Benzene
Bis(2-ethylhexyl) phthalate
Dichloromethane
Phenols
Tetrachloroethylene
Trichloroethylene
Trichlorofluoromethane

1099
$<1.0$
$<10$
5.6
$<5.0$
$<1.0$
1.4
$<5.0$

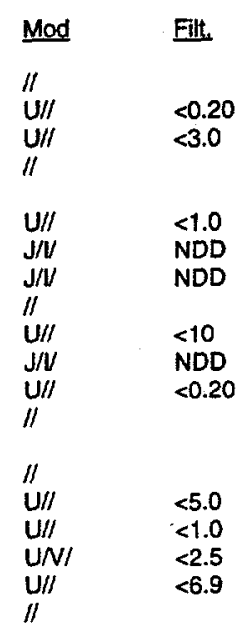

$\begin{array}{ll}\text { Mod } & \text { Filt. } \\ \text { JU/NO } & <1.0 \\ \text { U/I } & <10 \\ \text { J/UO8 } & \text { NDD } \\ \text { U/I } & <5.0 \\ \text { JUNO } & <1.0 \\ \text { J/N } & \text { NDD } \\ \text { JUIOO } & <5.0\end{array}$

Top of Casing

$256.5 \mathrm{ft} \mathrm{ms}$

$04 / 2 / 99$

2099
204.0
5.6
49
20.5
7
0
3.7
$203.2(06 / 25 / 99)$

Unit

It mst

$\mathrm{pH}$

$\mu$ Sicm

${ }^{\circ} \mathrm{C}$

mgh

well volumes

ft msl
Q99 Mod

.8

.0

10

20

$204.1(03 / 23 / 99)$

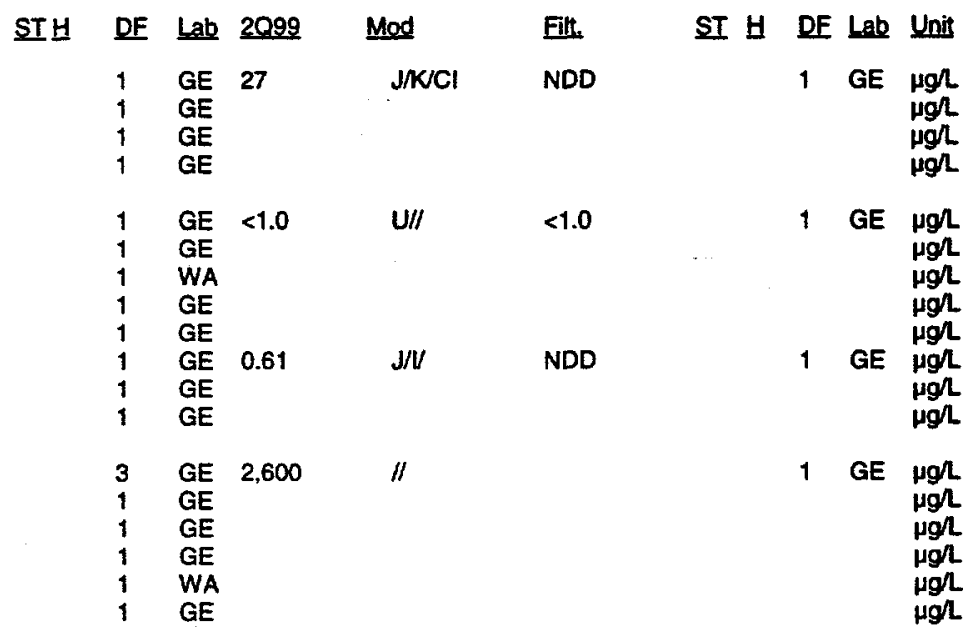

$\begin{array}{rll}\text { STH } & \text { DF } & \text { Lab } \\ 1 & \text { GE } \\ 1 & \text { GE } \\ 1 & \text { GE } \\ 1 & \text { GE } \\ 1 & \text { GE } \\ 1 & \text { WA } \\ 1 & \text { GE }\end{array}$

Filt.

SI H DF Lab Unit

$\mu g / L$

$\mu g /$

$\mu g h$.

$\mu g / L$

$\mu g / L$

$\mu g /$

$\mu g h$

Notes:

- exceeded holding time

= exceeded groundwater protection or monitoring constituent standard (See Appendix A.)

NA - Not applicable. Applies to beta dose and sum of betas if there are no beta-emitting radionuclides above detection limits; to sum of alphas if there are no alphaemitting radionuclides above detection limits; and to total radium if neither radium-226 or radium-228 was above detection limit

UAZ UTRA - Upper Aquifer Zone of the Upper Three Runs Aquifer, LAZ UTRA - Lower Aquifer Zone of the Upper Three Runs Aquifer, Gordon - Gordon Aquifer 
WELL FSB121C (cont.)

Radioactive Constituents

\begin{tabular}{|c|c|c|c|c|c|c|c|c|c|c|c|c|c|}
\hline Constituents & 1099 & Mod & Filt. & ST브 & DF & Lab & 2099 & Mod & Filt. & ST $\mathrm{H}$ & $\mathrm{DF}$ & Lab & Unit \\
\hline $\begin{array}{l}\text { Americium-241 } \\
\text { Beta dose }\end{array}$ & $\begin{array}{l}<-4.9 E-02 \\
N A\end{array}$ & $\mathrm{u} / /$ & $<3.6 \mathrm{E}-01$ & & 1 & GP & & & & & & & pCin \\
\hline Cabon-14 & $<8.0 E-01$ & $\mathrm{U} / /$ & $<7.6 E+00$ & & 1 & GP & & & & & & & pCin \\
\hline Cesium- 137 & $1.4 E+01$ & $R / / 4$ & & & 1 & $\overrightarrow{G P}$ & & & & & & & pCir \\
\hline Cobalt -60 & $<1.9 E-01$ & $\mathrm{U} / /$ & $<2.9 E+00$ & & 1 & GP & & & & & & & pCin \\
\hline Curium-242 & $<-2.7 E-02$ & $\mathrm{JU} / \mathrm{L} / \mathrm{C}$ & $<3.6 \mathrm{E}-01$ & & 1 & GP & & & & & & & pCin \\
\hline Curium-243/244 & $<-9.0 E-03$ & JUЛ & $<2.0 E-01$ & & 1 & GP & & & & & & & pCin \\
\hline Curium-245/246 & $<-9.0 E-03$ & JU/C & $<2.0 E-01$ & & 1 & GP & & & & & & & pCin \\
\hline Gross alpha & $1.8 \mathrm{E}+\infty 0$ & JN & NDD & & 1 & $T M$ & $1.6 E+\infty 0$ & $\mathrm{~J} / \mathrm{U}$ & NDD & & 1 & GP & pCin \\
\hline lodine-129 & $1.1 E+\infty$ & $\mathrm{J} / \mathrm{/} /$ & NDD & & 1 & GP & & & & & & & pCin \\
\hline Nonvolatile beta & $9.6 E+\infty 0$ & $\|$ & & & 1 & TM & 4.7E+00 & $/ /$ & & & 1 & GP & pCin \\
\hline Plutonium-238 & 2.2E-01 & $\mathrm{R} / / 4$ & Rej & & 1 & GP & & & & & & & pCin \\
\hline Plutonium-239/240 & $<-5.5 E-03$ & $U / /$ & $<1.2 E-01$ & & 1 & GP & & & & & & & pCin \\
\hline Radium-226 & $2.3 E+\infty 0$ & /1 & & & 1 & GP & & & & & & & pCin \\
\hline $\begin{array}{l}\text { Radium-228 } \\
\text { Total radium }\end{array}$ & $\begin{array}{l}<8.2 E-01 \\
2.3 E+00\end{array}$ & $\mathrm{U} / /$ & $<9.3 E-01$ & & 1 & GP & & & & & & & pcin \\
\hline Strontium-90 & $<-1.5 E-01$ & $\mathrm{U} / /$ & $<1.5 \mathrm{E}+00$ & & 1 & $\mathrm{TM}$ & & & & & & & pCin \\
\hline Technetium-99 & $<2.0 E+\infty$ & $\mathrm{U} / /$ & $<1.9 E+01$ & & 1 & GP & & & & & & & pcil \\
\hline Thorium-228 & 2.2E-01 & $\mathrm{R} / / 4$ & Rej & & 1 & GP & & & & & & & pCil \\
\hline Thorium-230 & 5.2E-02 & $\mathrm{J} / \mathrm{V}$ & NDD & & 1 & GP & & & & & & & pCir \\
\hline Thorium-232 & 4.9E-01 & $\mathrm{J} / \mathrm{W}$ & NDD & & 1 & $\mathrm{TM}$ & & & & & & & pCin \\
\hline Sum of alphas & NA & & & & & & & & & & & & \\
\hline Sum of betas & NA & & & & & & & & & & & & \\
\hline Tritium & $1.2 \mathrm{E}+02$ & $\|$ & & 口 & 1 & $T M$ & $1.0 E+02$ & $\|$ & & $\mathbf{a}$ & 1 & GP & $\mathrm{pCi} / \mathrm{mL}$ \\
\hline Uranium-233/234 & $<-5.9 E-04$ & $\mathrm{U} / /$ & $<2.2 E-01$ & & 1 & GP & & & & & & & pCin \\
\hline Uranium-235 & $<-1.0 E-02$ & $\mathrm{U} / /$ & $<2.6 E-01$ & & 1 & $\mathrm{TM}$ & & & & & & & pCin \\
\hline Uranium-238 & $<1.0 \mathrm{E}-02$ & $\mathrm{U} / /$ & $<3.0 \mathrm{E}-01$ & & 1 & TM & & & & & & & pCin \\
\hline
\end{tabular}

- exceeded groundwater protection or monitoring constituent standard (See Appendix A.)

NA - Not applicable. Applies to beta dose and sum of betas if there are no beta-emitting radionuclides above detection limits; to sum of alphas if there are no alphaemitting radionuclides above detection limits; and to total radium if neither radium-226 or radium-228 was above detection limit

UAZ UTAA - Upper Aquifer Zone of the Upper Three Runs Aquifer, LAZ UTRA - Lower Aquifer Zone of the Upper Three Runs Aquifer, Gordon - Gordon Aquifer 
WELL FSB121DR

$\begin{array}{ll}\text { SRS Coord. } & \text { Lanhongitud } \\ \text { N75151.9 } & 33.271004^{\circ} \\ \text { E48429.7 } & 81.685253^{\circ} \mathrm{W}\end{array}$

SAMPLE DATE

Screen Zone Elevation

$211.3-191.3 \mathrm{ft} \mathrm{msl}$
Top of Casing

$255.5 \mathrm{th} \mathrm{ms}$
Casing Pume

4* PVC S
Screen Zone

UAZ_UTRA

$04 / 07 / 99$

\section{FIELD DATA}

\section{Constituents}

Water elevation

$\mathrm{pH}$

Sp. conductance

Water temperature

Alkalinity as $\mathrm{CaCO} 3$

Turbidity

Volumes purged

Sampling code

Synchronous water level
1099

207.9

3.9

34

17.7

0

0.092

$\mathrm{X}$

$207.2(03 / 23 / 99)$
2099

207.1

4.4

20.0

0

2

0.097

$\mathrm{x}$

$206.0(06 / 25 / 99)$
Unit

tt ms!

$\mathrm{pH}$

1 Sicm

${ }^{\circ} \mathrm{C}$

mgl

well volumes

ft msl

\section{ANALYTICAL DATA}

Inorganic Constituents

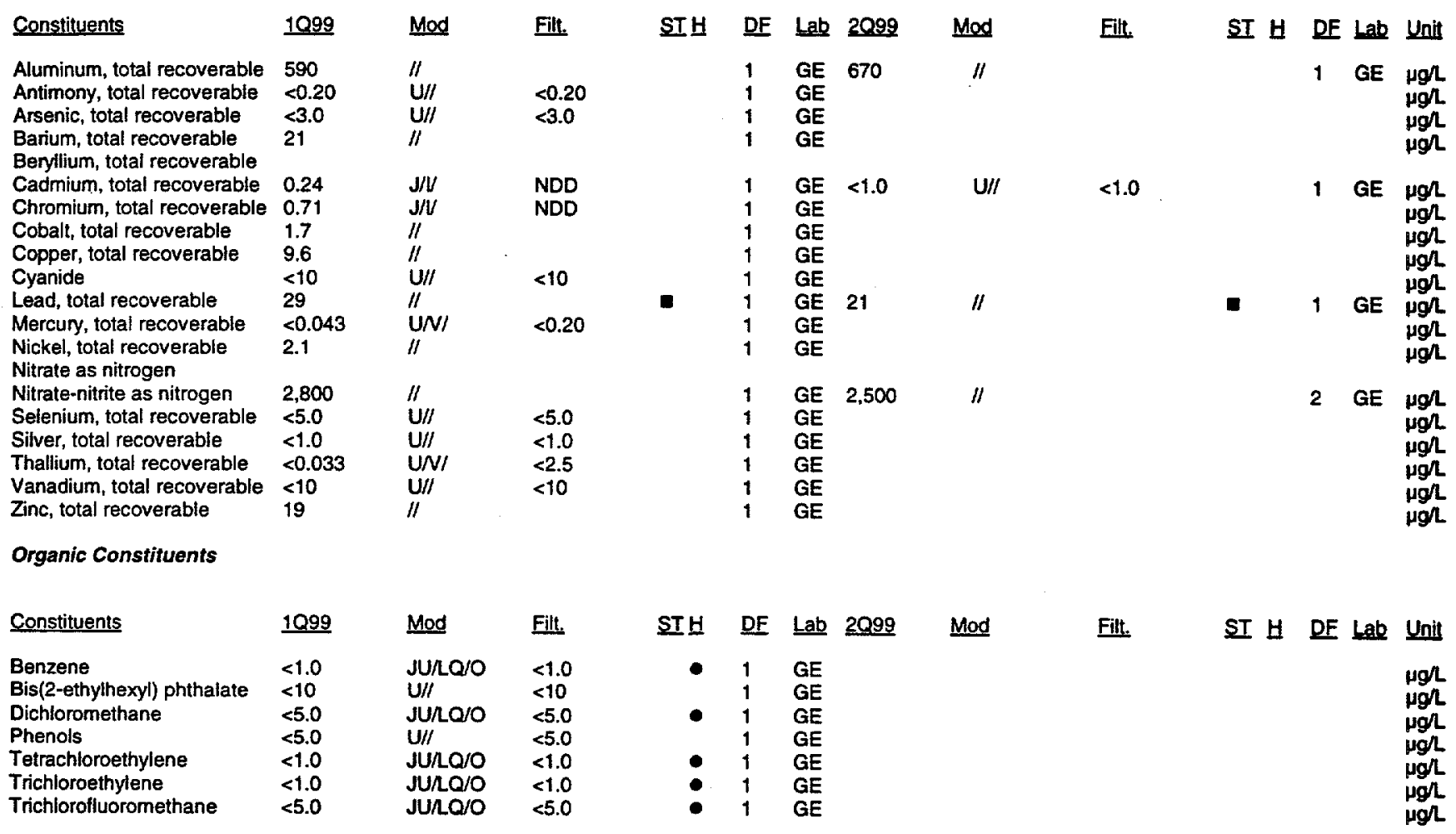

Notes:

= exceeded holding time

= exceeded groundwater protection or monitoring constituent standard (See Appendix A.)

NA - Not applicable. Applies to beta dose and sum of betas if there are no beta-emitting radionuclides above detection limits; to sum of alphas if there are no alphaemitting radionuclides above detection limits; and to total radium if neither radium-226 or radium-228 was above detection limit

UAZ UTRA - Upper Aquifer Zone of the Upper Three Runs Aquifer, LAZ UTRA - Lower Aquifer Zone of the Upper Three Runs Aquifer, Gordon - Gordon Aquifer 
WELL FSB121DR (cont.)

\section{Radioactive Constituents}

\begin{tabular}{|c|c|c|c|c|c|c|c|c|c|c|c|c|c|}
\hline Constituents & 1099 & Mod & Fill. & ST브 & $\underline{\mathrm{DF}}$ & $\underline{\text { Lab }}$ & 2099 & Mod & Filt. & ST $H$ & DE & Lab & Unif \\
\hline $\begin{array}{l}\text { Americium-241 } \\
\text { Beta dose }\end{array}$ & $\begin{array}{l}<-3.6 \mathrm{E}-03 \\
1.0\end{array}$ & $u / /$ & $<9.6 \mathrm{E}-01$ & - & 1 & GP & & & & & & & pCir \\
\hline Carbon-14 & $<5.0 E+\infty$ & $\mathrm{U} / /$ & $<7.4 E+\infty$ & & 1 & GP & & & & & & & pCir \\
\hline Cesium-137 & $<-2.0 E+\infty$ & $U / /$ & $<2.9 E+\infty 0$ & & 1 & GP & & & & & & & pCin \\
\hline Cobalt-60 & $<1.3 E-01$ & $U / /$ & $<3.5 \mathrm{E}+\infty$ & & $i$ & $G P$ & & & & & & & pCin \\
\hline Curium-242 & $<8.7 E-01$ & $U / /$ & $<8.8 E-01$ & & 1 & GP & & & & & & & pcin \\
\hline Curium-243/244 & $<6.7 E-01$ & $\mathrm{U} / /$ & $<1.4 E+\infty$ & & 1 & GP & & & & & & & pcin \\
\hline Curium-245/246 & $<-7.2 E-02$ & $\mathrm{U} / /$ & $<5.5 \mathrm{E}-01$ & & $i$ & GP & & & & & & & pcin \\
\hline Gross alpha & $9.2 \mathrm{E}+\infty$ & $\|$ & & & 1 & GP & $8.1 E+\infty$ & $\|$ & & & 1 & GP & pain \\
\hline lodine-129 & $<8.7 E-01$ & $U / I$ & $<1.2 \mathrm{E}+00$ & & 1 & $G P$ & & & & & & & pCiL \\
\hline Nonvolatile beta & $2.9 \mathrm{E}+01$ & /1 & & & 1 & GP & $3.2 \mathrm{E}+01$ & $\|$ & & & 1 & GP & pCin \\
\hline Plutonium-238 & $<-8.8 E-03$ & $\mathrm{U} / \mathrm{I}$ & $<2.5 \mathrm{E}-01$ & & 1 & GP & & & & & & & pcin \\
\hline Plutonium-239/240 & $<1.5 \mathrm{E}-02$ & UII & $<1.5 \mathrm{E}-01$ & & 1 & GP & & & & & & & pcin \\
\hline Radium-226 & $3.0 E+\infty 0$ & $\|$ & & & 1 & GP & & & & & & & pCin \\
\hline Radium-228 & $1.2 \mathrm{E}+00$ & $\mathrm{~J} / \mathrm{U}$ & NDD & & 1 & GP & & & & & & & pCin \\
\hline Total radium & $3.0 \mathrm{E}+00$ & & & & & & & & & & & & \\
\hline Strontium-90 & $8.1 E+00$ & $1 /$ & & $\mathbf{E}$ & 1 & GP & & & & & & & pCin \\
\hline Technetium-99 & $<2.2 E+\infty$ & $\mathrm{u} / /$ & $<1.9 E+01$ & & 1 & GP & & & & & & & pCin \\
\hline Thorium-228 & $<1.1 \mathrm{E}-01$ & JU/LI & $<4.8 E-01$ & & 1 & GP & & & & & & & $\mathrm{pCin}$ \\
\hline Thorium-230 & $<-1.9 E-02$ & JU/UI & $<2.5 \mathrm{E}-01$ & & 1 & $G P$ & & & & & & & pCir \\
\hline Thorium-232 & $<0.0 E+\infty$ & JUกI & $<1.2 E-01$ & & 1 & GP & & & & & & & pCir \\
\hline Sum of alphas & NA & & & & & & & & & & & & \\
\hline Sum of betas & $8.1 E+\infty$ & & & & & & & & & & & & pCil \\
\hline Tritium & $1.5 \mathrm{E}+01$ & $\|$ & & & 1 & GP & $1.2 \mathrm{E}+01$ & $\|$ & & & 1 & GP & pCirmL \\
\hline Uranium-233/234 & $1.2 E+\infty$ & $R / / 4$ & Rej & & $i$ & GP & & & & & & & $\mathrm{pCil}$ \\
\hline Uranium-235 & $<9.4 E-02$ & $\mathrm{U} / \mathrm{I}$ & $<2.8 E-01$ & & $i$ & GP & & & & & & & $\mathrm{pCi}$ \\
\hline Uranium-238 & $1.0 \mathrm{E}+\infty$ & $R / / 4$ & Rej & & 1 & GP & & & & & & & $\mathrm{pCin}$ \\
\hline
\end{tabular}

\section{Notes:}

= exceeded holding time

9 = exceeded groundwater protection or monitoring constituent standard (See Appendix A)

NA - Not applicable. Applies to beta dose and sum of betas if there are no beta-emitting radionuclides above detection limits; to sum of alphas if there are no alphaemitting radionuclides above detection limits; and to total radium if neither radium-226 or radium-228 was above detection limit

UAZ_UTRA - Upper Aquifer Zone of the Upper Three Runs Aquifer, LAZ_UTRA - Lower Aquiler Zone of the Upper Three Runs Aquifer, Gordon - Gordon Aquifer 


\section{WELL FSB122C}

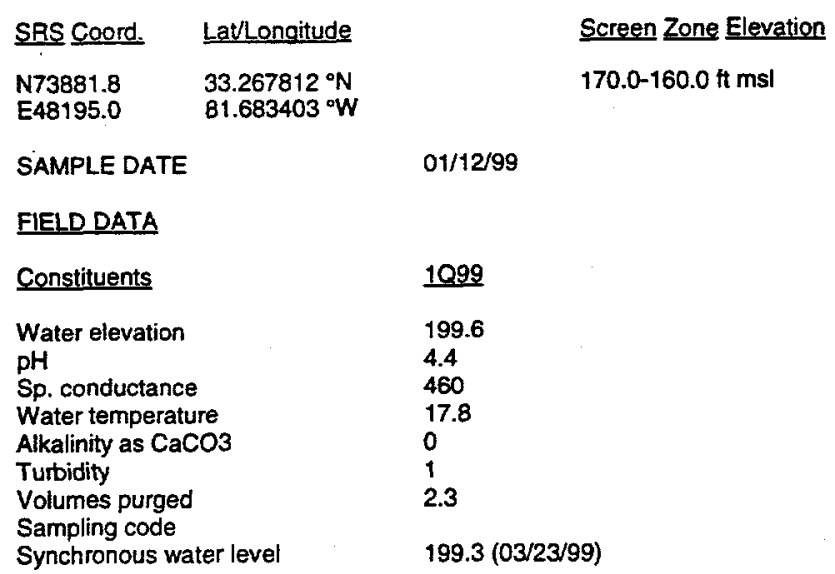

$\begin{array}{llll}\text { Top of Casing } & \text { Casing } & \text { Pump } & \text { Screen Zone } \\ 218 \mathrm{ft} \mathrm{ms} \text { l } & 4^{*} \text { PVC } & \text { S } & \text { LAZ_UTRA }\end{array}$

$04 / 07 / 99$

$\begin{array}{ll}2099 & \text { Unit } \\ 199.2 & \mathrm{ft} \text { msl } \\ 4.2 & \mathrm{pH} \\ 460 & \mu \mathrm{SS} / \mathrm{cm} \\ 19.2 & { }^{\circ} \mathrm{C} \\ 0 & \mathrm{mgh} \\ 2 & \mathrm{NTU} \\ 4.6 & \text { well volumes } \\ 198.0(06 / 25 / 99) & \mathrm{ft} \mathrm{ms!}\end{array}$

\section{ANALYTICAL DATA}

Inorganic Constituents

\begin{tabular}{|c|c|c|c|c|c|c|c|c|c|c|c|c|c|}
\hline Constituents & 1099 & Mod & Filt. & STH & DF & Lab & $\underline{2099}$ & Mod & Filt. & ST H & DE & Lab & Unit \\
\hline Aluminum, total recoverable & 720 & $\|$ & & & 1 & GE & 730 & $\|$ & & & 1 & GE & $\mu g$ \\
\hline Antimony, total recoverable & $<0.20$ & $\mathrm{U} / \prime$ & $<0.20$ & & 1 & GE & & & & & & & \\
\hline Arsenic, total recoverable & $<15$ & $\mathrm{U} / /$ & $<15$ & & 5 & GE & & & & & & & \\
\hline Barium, total recoverable & 130 & $\|$ & & & 1 & GE & & & & & & & \\
\hline Cadmium, total recoverable & 1.2 & // & & & 1 & GE & 1.0 & $/ 1$ & & & 1 & GE & L \\
\hline Chromium, total recoverable & 0.92 & $\mathrm{~J} / \mathrm{L}$ & NDD & & 1 & $\overline{G E}$ & & & & & & & \\
\hline Cobalt, total recoverable & 16 & $1 /$ & & $\mathbf{E}$ & 1 & GE & & & & & & & \\
\hline Copper, total recoverable & 1.5 & $\|$ & & & 1 & GE & & & & & & & \\
\hline Cyanide & $<10$ & JUЛ/ & $<10$ & & 1 & GE & & & & & & & \\
\hline Lead, total recoverable & $<2.0$ & $U / I$ & $<2.0$ & & 1 & GE & 0.68 & $\mathbf{J} / \mathbf{V}$ & NDD & & 1 & GE & \\
\hline Mercury, total recoverable & $<0.20$ & $\mathrm{U} / \mathrm{f}$ & $<0.20$ & & 1 & GE & & & & & & & \\
\hline $\begin{array}{l}\text { Nickel, total recoverable } \\
\text { Nitrate as nitrogen }\end{array}$ & 15 & $\|$ & & & 1 & GE & & & & & & & \\
\hline Nitrate-nitrite as nitrogen & 57,000 & $/ 1$ & & 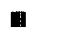 & 25 & GE & 54,000 & $\|$ & & $\mathbf{\square}$ & 25 & GE & \\
\hline Selenium, total recoverable & $<25$ & $\mathrm{u} / /$ & $<25$ & & 5 & $\overline{\mathrm{GE}}$ & & & & & & & \\
\hline Silver, total recoverable & $<1.0$ & $\mathrm{U} / \mathrm{I}$ & $<1.0$ & & 1 & $\overline{G E}$ & & & & & & & \\
\hline Thallium, total recoverable & 0.35 & $\mathrm{~J} / \mathrm{W}$ & NDD & & 1 & GE & & & & & & & \\
\hline Vanadium, total recoverable & $<10$ & $U / I$ & $<10$ & & 1 & $\mathrm{GE}$ & & & & & & & \\
\hline Zinc, total recoverable & 79 & $/ 1$ & & & 1 & GE & & & & & & & \\
\hline
\end{tabular}

Organic Constituents

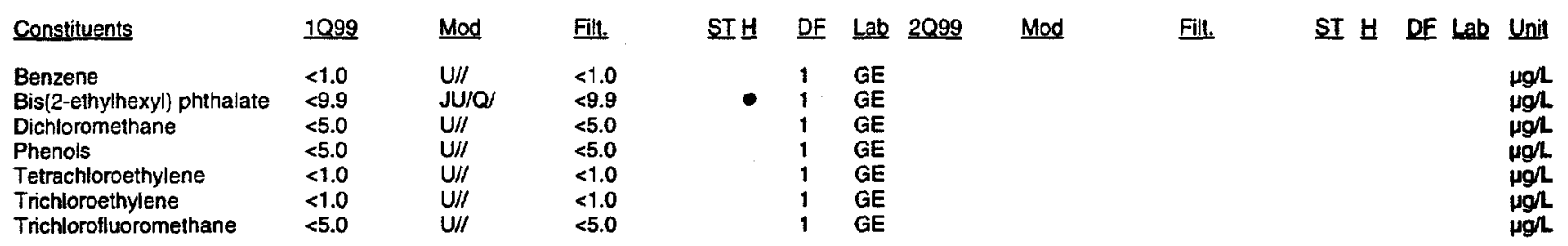

\footnotetext{
Notes:
}

- exceeded holding time

= exceeded groundwater protection or monitoring constituent standard (See Appendix A.)

NA - Not applicable. Applies to beta dose and sum of betas if there are no beta-emitting radionuclides above detection limits; to sum of alphas if there are no alphaemitting radionuclides above detection limits; and to total radium if neither radium-226 or radium-228 was above detection limit

UAZ_UTRA - Upper Aquifer Zone of the Upper Three Runs Aquifer, LAZ UTRA - Lower Aguifer Zone of the Upper Three Runs Aquifer, Gordon - Gordon Aquifer 
WELL FSB122C (cont.)

Radioactive Constituents

\begin{tabular}{|c|c|c|c|c|c|c|c|c|c|c|c|c|c|}
\hline Constifuents & $\underline{1099}$ & Mod & Filt. & SIH & DF & Lab & $\underline{2099}$ & Mod & Filt. & SI $H$ & DE & Lab & Unit \\
\hline Americium-241 & $<0.0 E+\infty 0$ & $\mathbf{U} / /$ & $<7.4 E-01$ & & 1 & GP & & & & & & & pCin \\
\hline Beta dose & 12 & & & ש & & & & & & & & & \\
\hline Carbon-14 & $1.4 E+01$ & $\mathrm{~J} / \mathrm{IUCl}$ & NDD & & 1 & GP & & & & & & & $\mathrm{pCin}$ \\
\hline Cesium-137 & $<8.1 E-01$ & & $<3.5 \mathrm{E}+\infty$ & & 1 & GP & . & & & & & & pcin \\
\hline Cobalt -60 & $<-1.4 E+\infty$ & $\mathrm{U} / /$ & $<2.8 E+\infty 0$ & & 1 & GP & & & & & & & pCin \\
\hline Curium-242 & $<0.0 E+\infty$ & $\mathrm{U} / t$ & $<8.5 E-01$ & & 1 & GP & & & & & & & pCil \\
\hline Curium-243/244 & $<1.9 E-01$ & $\mathrm{U} / /$ & $<1.3 E+\infty 0$ & & 1 & GP & & & & & & & pCin \\
\hline Curium-245/246 & $<4.9 \mathrm{E}-01$ & $\mathrm{U} / /$ & $<7.4 E-01$ & & 1 & GP & & & & & & & pCir \\
\hline Gross alpha & $\begin{array}{l}2.8 \mathrm{E}+01 \\
1.2 \mathrm{E}+01\end{array}$ & $\mathrm{~J} / \mathrm{I}$ & NDD & & 1 & GP & $1.6 E+01$ & $\|$ & & $\mathbf{n}$ & 1 & GP & pcir \\
\hline Nonvolatile beta & $5.9 \mathrm{E}+01$ & $\mathrm{~J} / \mathrm{n}$ & NDD & & 1 & GP & $6.8 E+01$ & $/ /$ & & $\mathbf{\square}$ & 1 & GP & pCir \\
\hline Plutonium-238 & $<5.3 E-01$ & JUR/ & $<1.1 E+\infty 0$ & & $i$ & GP & & & & & & & pCin \\
\hline Plutonium-239/240 & $<1.6 E-01$ & JU/I & $<4.8 \mathrm{E}-01$ & & 1 & GP & & & & & & & pCin \\
\hline Radium-226 & $1.1 E+01$ & & & - & 1 & GP & & & & & & & pCin \\
\hline Radium-228 & $<-5.0 E-01$ & JULI & $<1.3 E+00$ & & 1 & GP & & & & & & & pCin \\
\hline Total radium & $1.1 E+01$ & & & & & & & & & & & & \\
\hline Strontium-90 & $<5.1 E-01$ & $\mathrm{u} / /$ & $<2.0 E+00$ & & 1 & GP & & & & & & & pein. \\
\hline Technetium-99 & $1.3 E+02$ & 11 & & $\boldsymbol{\Xi}$ & 1 & GP & & & & & & & $\mathrm{pCin}$ \\
\hline Thorium-228 & $<6.2 E-02$ & $\mathrm{U} / /$ & $<3.7 E-01$ & & 1 & GP & & & & & & & pCin \\
\hline Thorium-230 & $<7.3 E-02$ & U/I & $<2.2 \mathrm{E}-01$ & & 1 & GP & & & & & & & poin \\
\hline Thorium-232 & $<0.0 E+00$ & $\mathrm{u} / /$ & $<9.7 E-02$ & & 1 & GP & & & & & & & pCin \\
\hline Sum of alphas & NA & & & & & & & & & & & & م: \\
\hline Sum of betas & $1.5 E+02$ & & & $=$ & & & & & & & & & pCir \\
\hline Tritium & $1.6 E+03$ & $\|$ & & $\mathbf{0}$ & 1 & GP & $1.4 E+03$ & $\|$ & & $\mathbf{0}$ & 1 & GP & pCi/mL \\
\hline Uranium-233/234 & $<5.0 E-01$ & UN/ & $<3.4 E-01$ & & 1 & GP & & & & & & & pCin \\
\hline Uranium-235 & $<4.9 E-01$ & UN/ & $<1.5 E-01$ & & 1 & GP & & & & & & & $\mathrm{pCin}$ \\
\hline Uranium-238 & $<1.5 E-01$ & $\mathrm{U} / /$ & $<1.5 E-01$ & & 1 & GP & & & & & & & pcil \\
\hline
\end{tabular}

Notes:

- exceeded holding time

- exceeded groundwater protection or monitoring constituent standard (See Appendix A.)

NA - Not applicable. Applies to beta dose and sum of betas if there are no beta-emitting radionuclides above detection limits; to sum of alphas if there are no alphaemitting radionuclides above detection limits; and to total radium if neither radium-226 or radium-228 was above detection limit

UAZ UTRA - Upper Aquiler Zone of the Upper Three Runs Aquifer, LAZ UTRA - Lower Aquifer Zone of the Upper Three Runs Aquifer, Gordon - Gordon Aquifer

F-Area HWMF

C-173

First and Second Quarter 1999 


\section{WELL FSB122D}

$\begin{array}{ll}\text { SRS Coord. } & \text { Lat/Longitud } \\ \text { N73865.5 } & 33.267787 \text { o } \\ \text { E48201.7 } & 81.683354 \text { \% }\end{array}$

SAMPLE DATE

Screen Zone Elevation

206.6-186.6 ft msi
Ton of Casing

$217.6 \mathrm{ft} \mathrm{ms}$
Casing Pumo

4" PVC S
Screen Zone

UAZ_UTRA

04/07/99

\section{FIELD DATA}

Constituents

Water elevation

$\mathrm{pH}$

Sp. conductance

Water temperature

Alkalinity as $\mathrm{CaCO} 3$

Turbidity

Volumes purged

Sampling code

Synchronous water level

203.0

4.1

210

18.6

0

7.5

$202.8(03 / 23 / 99)$
2099

202.7

4.5

180

19.3

0

4.3

$201.3(06 / 25 / 99)$
Unit

tt msl

pH

$\mu \mathrm{S} / \mathrm{cm}$

${ }^{\circ} \mathrm{C}$

mgl

NTU

well volumes

tt msl

\section{ANALYTICAL DATA}

Inorganic Constituents

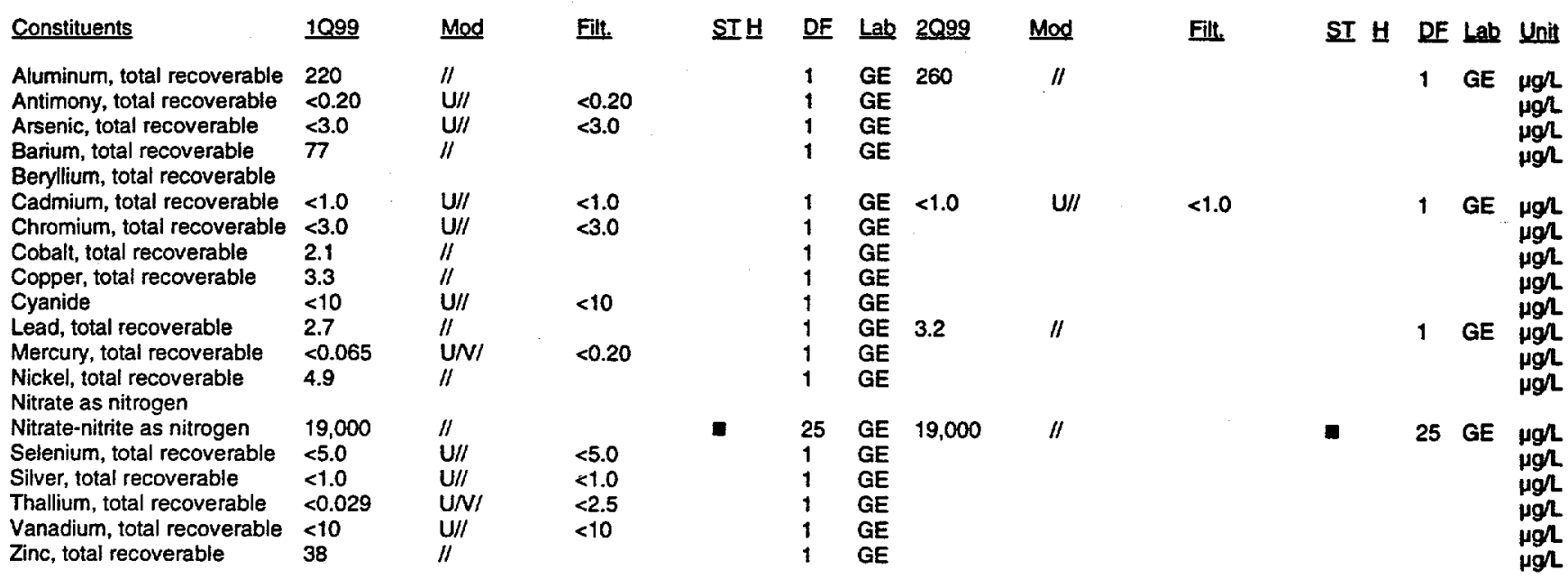

Organic Constituents

\begin{tabular}{|c|c|c|c|c|c|c|c|c|c|c|}
\hline Constituents & 1099 & Mod & Filt. & ST브 & DF & Lab 2099 & Mod & Filt. & ST $H$ DE Lab & Unit \\
\hline $\begin{array}{l}\text { Benzene } \\
\text { Bis(2-ethylhexyl) phthalate } \\
\text { Dichloromethane } \\
\text { Phenols } \\
\text { Tetrachloroethylene } \\
\text { Trichloroethylene } \\
\text { Trichlorofluoromethane }\end{array}$ & $\begin{array}{l}<1.0 \\
<10 \\
<5.0 \\
<5.0 \\
<1.0 \\
<1.0 \\
<5.0\end{array}$ & $\begin{array}{l}\mathrm{JU/Q} / \\
\mathrm{U} / / \\
\mathrm{JU} / \mathrm{Q} / \\
\mathrm{U} / / \\
\mathrm{JU} / \mathrm{Q} / \\
\mathrm{JU/Q} / \\
\mathrm{JU/Q}\end{array}$ & $\begin{array}{l}<1.0 \\
<10 \\
<5.0 \\
<5.0 \\
<1.0 \\
<1.0 \\
<5.0\end{array}$ & • & $\begin{array}{l}1 \\
1 \\
1 \\
1 \\
1 \\
1 \\
1\end{array}$ & $\begin{array}{l}\mathrm{GE} \\
\mathrm{GE} \\
\mathrm{GE} \\
\mathrm{GE} \\
\mathrm{GE} \\
\mathrm{GE} \\
\mathrm{GE}\end{array}$ & & & & $\begin{array}{l}\mu g h \\
\mu g h \\
\mu g h \\
\mu g h \\
\mu g h \\
\mu g h \\
\mu g h\end{array}$ \\
\hline
\end{tabular}

- = exceeded groundwater protection or monitoring constituent standard (See Appendix A.)

NA - Not applicable. Applies to beta dose and sum of betas if there are no beta-emitting radionuclides above detection limits; to sum of alphas if there are no alphaemitting radionuclides above detection limits; and to total radium if neither radium-226 or radium-228 was above detection limit

UAZ_UTRA - Upper Aquifer Zone of the Upper Three Runs Aquifer, LAZ UTRA - Lower Aquifer Zone of the Upper Three Runs Aquifer, Gordon - Gordon Aquifer 


\section{WELL FSB122D (cont.)}

Radioactive Constituents

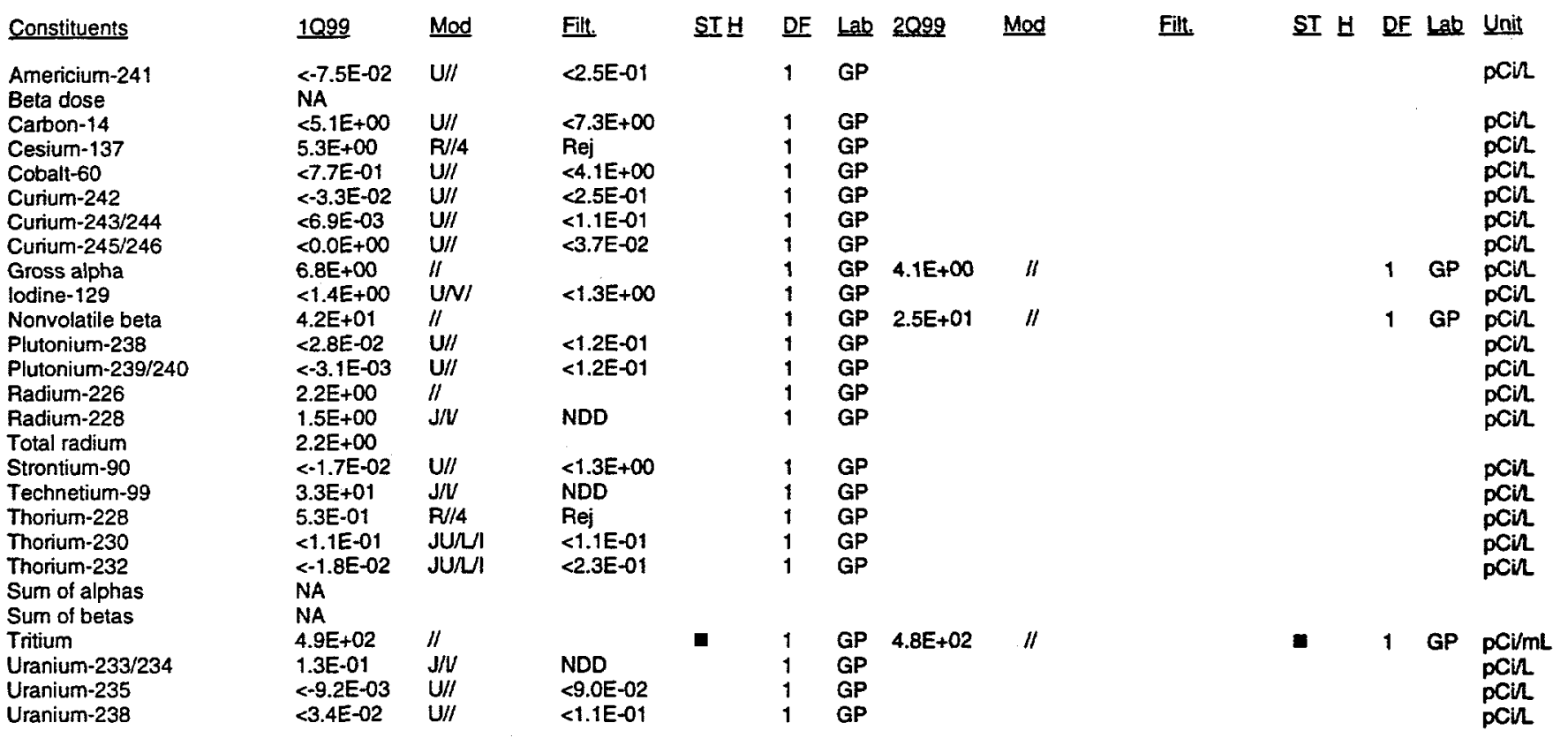

= exceeded groundwater protection or monitoring constituent standard (See Appendix A.)

NA - Not applicable. Applies to beta dose and sum of betas if there are no beta-emitting radionuclides above detection limits; to sum of alphas if there are no alphaemitting radionuclides above detection limits; and to total radium if neither radium-226 or radium-228 was above detection limit

UAZ_UTRA - Upper Aquifer Zone of the Upper Three Runs Aquifer; LAZ_UTRA - Lower Aquifer Zone of the Upper Three Runs Aquifer, Gordon - Gordon Aquifer 


\section{WELL FSB123C}

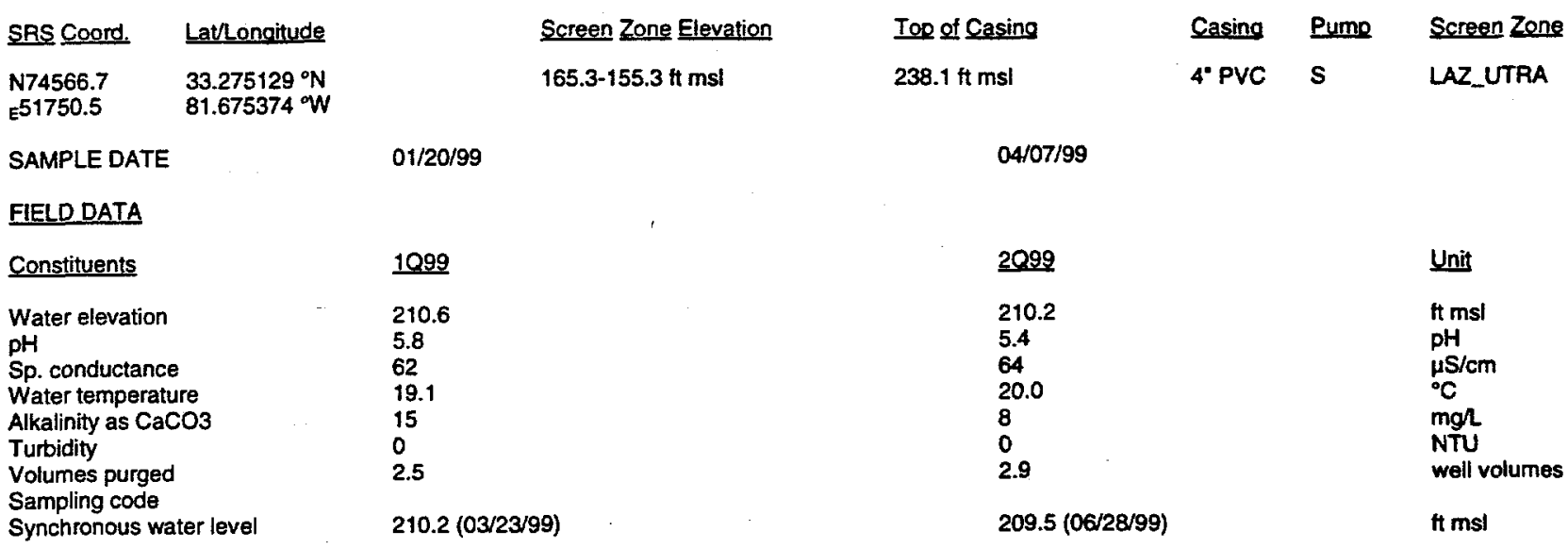

\section{ANALYTICAL DATA}

Inorganic Constituents

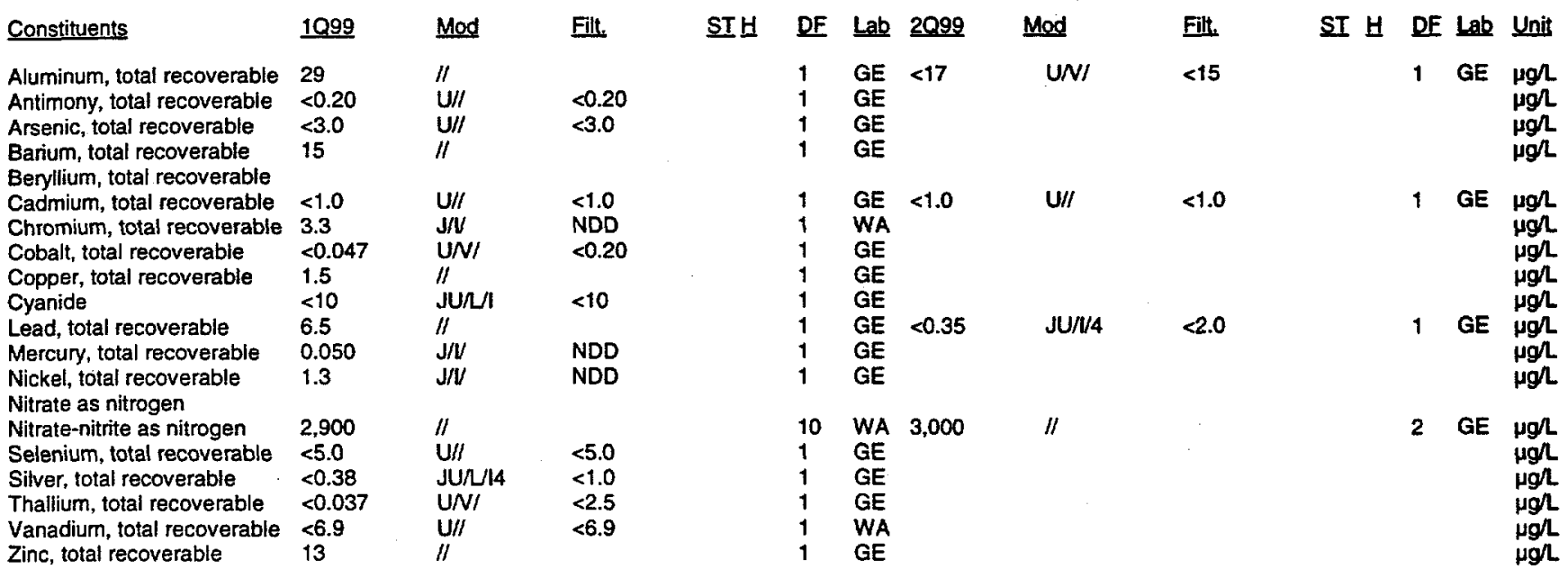

Organic Constituents

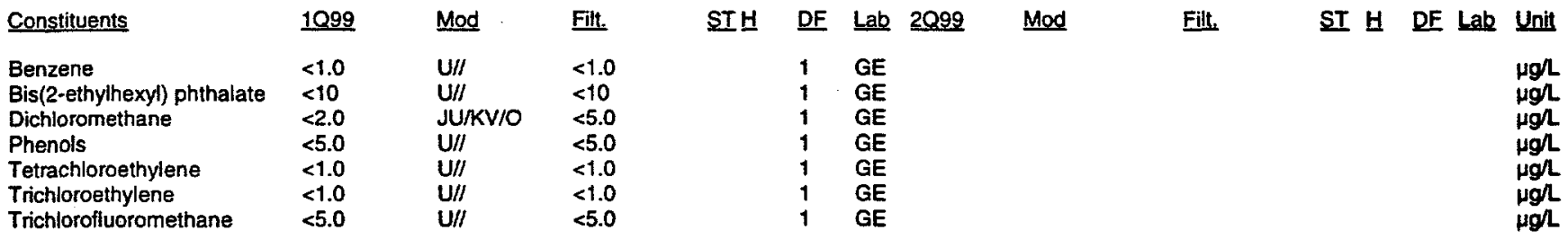

Notes:

= exceeded holding time

- exceeded groundwater protection or monitoring constituent standard (See Appendix A.)

NA - Not applicable. Applies to beta dose and sum of betas if there are no beta-emitting radionuclides above delection limits; to sum of alphas if there are no alphaemitting radionuclides above detection limits; and to total radium if neither radium-226 or radium-228 was above detection limit

UAZ UTRA - Upper Aquifer Zone of the Upper Three Runs Aquifer, LAZ UTRA - Lower Aquifer Zone of the Upper Three Runs Aquifer, Gordon - Gordon Aquifer 


\section{WELL FSB123C (cont.)}

Radioactive Constituents

\begin{tabular}{|c|c|c|c|c|c|c|c|c|c|c|c|c|c|}
\hline Constituents & 1099 & Mod & Fill. & ST븐 & DF & Lab & $\underline{2099}$ & Mod & Filt. & ST $\underline{H}$ & $\mathrm{DF}$ & Lab & Unit \\
\hline $\begin{array}{l}\text { Americium-241 } \\
\text { Beta dose }\end{array}$ & $\begin{array}{l}<6.5 E-02 \\
\text { NA }\end{array}$ & $\mathrm{U} / \prime$ & $<4.4 E-01$ & & 1 & GP & & & & & & & pCil \\
\hline Carbon-14 & $<1.8 E+\infty$ & $\mathrm{U} / /$ & $<9.4 E+\infty$ & & 1 & GP & & & & & & & pCin \\
\hline Cesium-137 & $<-4.0 E-01$ & $\mathbf{U} / /$ & $<4.1 E+\infty 0$ & & 1 & GP & & & & & & & pCin \\
\hline Cobalt-60 & $<-7.4 E-01$ & $\mathrm{U} / 1$ & $<3.4 \mathrm{E}+\infty 0$ & & 1 & GP & & & & & & & pCin \\
\hline Curium-242 & $<0.0 E+\infty 0$ & $\mathrm{U} / /$ & $<2.3 E-01$ & & 1 & GP & & & & & & & pCin \\
\hline Curium-243/244 & $<-3.0 \mathrm{E}-02$ & U/I & $<5.1 E-01$ & & 1 & $\mathrm{TM}$ & & & & & & & $\mathrm{pCi} / \mathrm{L}$ \\
\hline Curium-245/246 & $<0.0 E+00$ & $\mathrm{U} / /$ & $<2.2 \mathrm{E}-01$ & & 1 & GP & & & & & & & pCil \\
\hline $\begin{array}{l}\text { Gross alpha } \\
\text { lodine-129 }\end{array}$ & $\begin{array}{l}<8.7 E-02 \\
<1.7 E-01\end{array}$ & $\begin{array}{l}U / / \\
U / /\end{array}$ & $\begin{array}{l}<5.9 E-01 \\
<9.8 E-01\end{array}$ & & $\begin{array}{l}1 \\
1\end{array}$ & $\begin{array}{l}\text { GP } \\
\text { GP }\end{array}$ & $<3.9 E-01$ & $\mathrm{U} / /$ & $<5.1 E-01$ & & 1 & GP & $\begin{array}{l}\text { pCin } \\
\text { pCin }\end{array}$ \\
\hline Nonvolatile beta & $1.2 E+\infty 0$ & $\mathrm{~J} / \mathrm{V}$ & NDD & & 1 & GP & $1.2 E+\infty 0$ & $\mathrm{~J} / \mathrm{V}$ & NDD & & 1 & GP & $\mathrm{pCin}$ \\
\hline Plutonium-238 & $6.8 \mathrm{E}-01$ & $\mathrm{R} / / \mathrm{O}$ & Rej & & 1 & TM & & & & & & & $\mathrm{pCi}$ \\
\hline Plutonium-239/240 & 2.3E-01 & R/O & Rej & & 1 & TM & & & & & & & $\mathrm{pCin}$ \\
\hline Radium-226 & $<3.2 E-01$ & UN' & $<2.9 \mathrm{E}-01$ & & 1 & TM & & & & & & & pcin \\
\hline $\begin{array}{l}\text { Radium-228 } \\
\text { Total radium }\end{array}$ & $\begin{array}{l}<5.6 E-01 \\
N A\end{array}$ & $\mathrm{U} / /$ & $<1.3 E+00$ & & 1 & GP & & & & & & & pCin \\
\hline Strontium-90 & $<-4.4 E-01$ & $\mathrm{U} / /$ & $<1.4 E+00$ & & 1 & GP & & & & & & & pCin \\
\hline Technetium-99 & $<4.0 \mathrm{E}+00$ & $U / /$ & $<1.3 E+01$ & & 1 & TM & & & & & & & pCin \\
\hline Thorium-228 & $<-1.1 E-02$ & $U / /$ & $<4.4 E-01$ & & 1 & GP & & & & & & & pcin \\
\hline Thorium-230 & $<7.9 E-02$ & $U / I$ & $<3.1 E-01$ & & 1 & GP & & & & & & & pein \\
\hline $\begin{array}{l}\text { Thorium-232 } \\
\text { Sum of alphas } \\
\text { Sum of betas }\end{array}$ & $\begin{array}{l}<-8.1 E-03 \\
\text { NA } \\
\text { NA }\end{array}$ & $\mathrm{U} / /$ & $<1.8 E-01$ & & 1 & GP & & & & & & & $\mathrm{pCi} / \mathrm{L}$ \\
\hline Tritium & $2.6 E+\infty$ & $\mathrm{J} / \mathrm{K} / \mathrm{C}$ & NDD & & 1 & TM & $2.5 E+\infty$ & $\|$ & & & 1 & $\mathbf{G P}$ & pCi/mL \\
\hline Uranium-233/234 & $<9.1 E-02$ & $\mathrm{U} / /$ & $<2.3 E-01$ & & 1 & GP & & & & & & & pcin \\
\hline Uranium-235 & $<-7.2 \mathrm{E}-03$ & $\mathrm{U} / /$ & $<1.6 \mathrm{E}-01$ & & 1 & GP & & & & & & & pCir \\
\hline Uranium-238 & $<1.5 E-02$ & $\mathrm{U} / /$ & $<1.9 E-01$ & & 1 & GP & & & & & & & pCiL \\
\hline
\end{tabular}

\section{Notes:}

- exceeded holding time

= exceeded groundwater protection or monitoring constituent standard (See Appendix A.)

NA - Not applicable. Applies to beta dose and sum of betas if there are no beta-emitting radionuclides above detection limits; to sum of alphas if there are no alphaemitting radionuclides above detection limits; and to total radium if neither radium-226 or radium-228 was above detection limit

UAZ_UTRA - Upper Aquifer Zone of the Upper Three Runs Aquifer, LAZ, UTRA - Lower Aquifer Zone of the Upper Three Runs Aquifer, Gordon - Gordon Aquifer 


\section{WELL FSB123D}

\begin{tabular}{|c|c|}
\hline SRS Coord. & Latlongitude \\
\hline $\begin{array}{l}\text { N74562.7 } \\
\text { E51734.8 }\end{array}$ & $\begin{array}{l}33.275095^{\circ} \mathrm{N} \\
81.675407^{\circ} \mathrm{W}\end{array}$ \\
\hline
\end{tabular}

SAMPLE DATE

Screen Zone Elevation

$214.1-194.1 \mathrm{ft} \mathrm{msl}$

$01 / 11 / 99$

1099

211.8

4.3

40

19.4

0

3.1

$211.6(03 / 23 / 99)$
Top 의 Casing

$238.1 \mathrm{f} \mathrm{ms}$

04/07/99
FIELD DATA

Water elevation

Sp. conductance

Water temperature

Alkalinity

Volumes purged

Sampling code

Synchronous water level

ANALYTICAL DATA

Inorganic Constituents

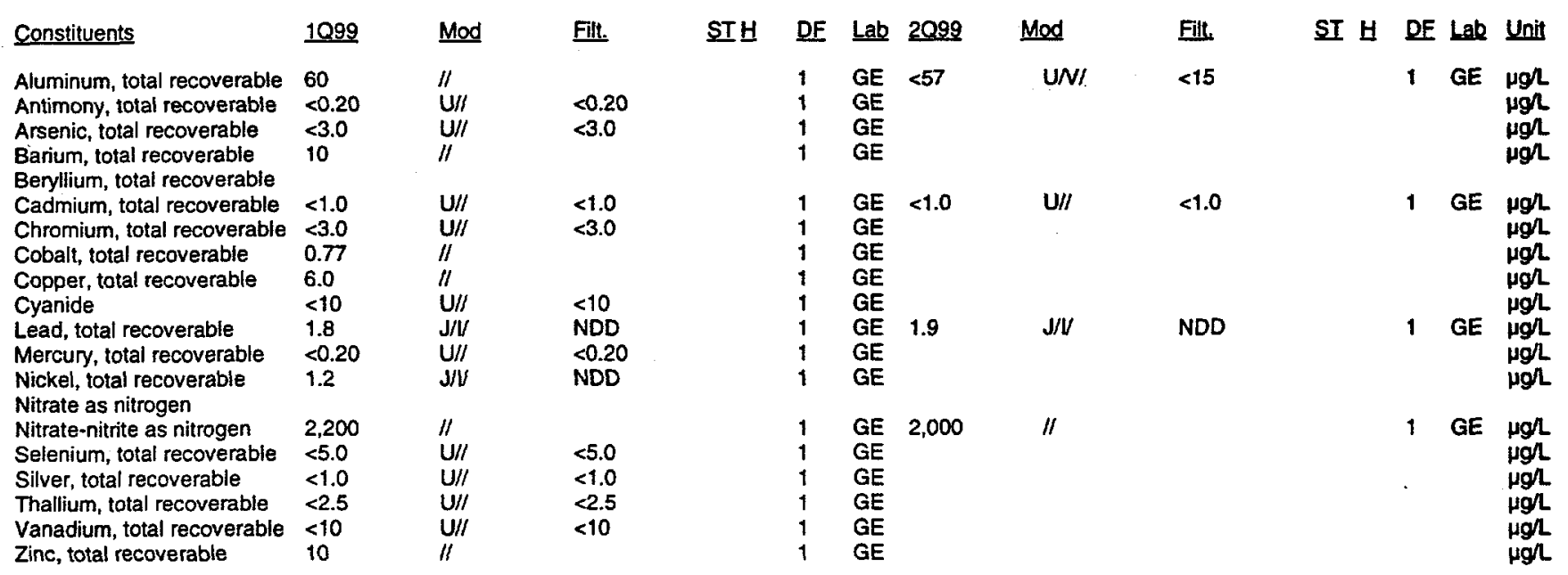

Organic Constituents

\begin{tabular}{|c|c|c|c|c|c|c|c|c|c|c|}
\hline Constituents & 1099 & Mod & Filt. & STH & DF & Lab 2099 & Mod & Fill. & ST H DF Lab & Unit \\
\hline $\begin{array}{l}\text { Benzene } \\
\text { Bis(2-ethylhexyl) phthalate } \\
\text { Dichloromethane } \\
\text { Phenols } \\
\text { Tetrachloroethylene } \\
\text { Trichloroethylene } \\
\text { Trichlorofluoromethane }\end{array}$ & $\begin{array}{l}<1.0 \\
<10 \\
<13 \\
<5.0 \\
<1.0 \\
<1.0 \\
<5.0\end{array}$ & $\begin{array}{l}\text { JU//O } \\
\text { U/I } \\
\text { JU/LVOO } \\
\text { U// } \\
\text { JU/OO } \\
\text { JUR/O } \\
\text { JUR/O }\end{array}$ & $\begin{array}{l}<1.0 \\
<10 \\
<5.0 \\
<5.0 \\
<1.0 \\
<1.0 \\
<5.0\end{array}$ & & $\begin{array}{l}1 \\
1 \\
1 \\
1 \\
1 \\
1 \\
1\end{array}$ & $\begin{array}{l}\text { GE } \\
\text { GE } \\
\text { GE } \\
\text { GE } \\
\text { GE } \\
\text { GE } \\
\text { GE }\end{array}$ & & & & $\begin{array}{l}\mu g / 2 \\
\mu g h \\
\mu g / 2 \\
\mu g / \\
\mu g h \\
\mu g h \\
\mu g /\end{array}$ \\
\hline
\end{tabular}

Notes:

- = exceeded holding time

= exceeded groundwater protection or monitoring constituent standard (See Appendix A.)

NA - Not applicable. Applies to beta dose and sum of betas if there are no beta-emitting radionuclides above detection limits; to sum of alphas if there are no alphaemitting radionuclides above detection limits; and to total radium if neither radium-226 or radium-228 was above detection limit

UAZ_UTRA - Upper Aquifer Zone of the Upper Three Runs Aquifer, LAZ_UTRA - Lower Aguifer Zone of the Upper Three Runs Aquifer, Gordon - Gordon Aquifer

F-Area HWMF

C-178

First and Second Quarter 1999 
WELL FSB123D (cont.)

Radioactive Constituents

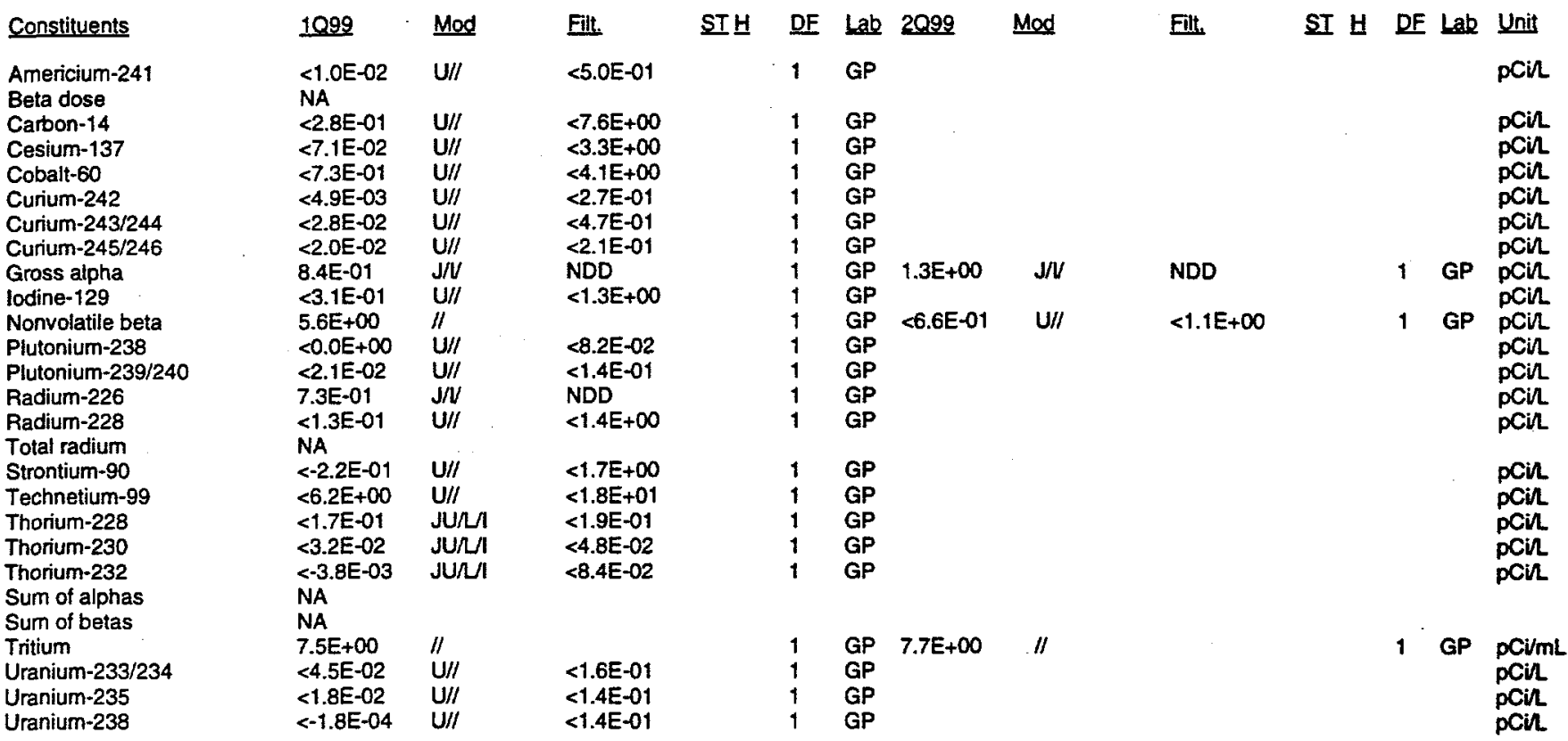

- = exceeded groundwater protection or monitoring constituent standard (See Appendix A.)

NA - Not applicable. Applies to beta dose and sum of betas if there are no beta-emitting radionuclides above detection limits; to sum of alphas if there are no alphaemitting radionuclides above detection limits; and to total radium if neither radium-226 or radium-228 was above detection limit 
WELL FSL 1D

\begin{tabular}{|c|c|c|c|c|c|c|}
\hline SRS Coord. & Lat/Longitude & Screen Zone Elevation & Too of Casing & Casing & Pump & Screen Zone \\
\hline $\begin{array}{l}\text { N79063.1 } \\
\text { E52992.5 }\end{array}$ & $\begin{array}{l}33.287100^{\circ} \mathrm{N} \\
81.680840^{\circ} \mathrm{W}\end{array}$ & $228.6-208.5 \mathrm{ft} \mathrm{msl}$ & $310.8 \mathrm{ft} \mathrm{msl}$ & $2^{*}$ PVC & v & UAZ_UTRA \\
\hline \multicolumn{2}{|c|}{ SAMPLE DATE } & $01 / 26 / 99$ & $04 / 28 / 99$ & & & \\
\hline \multicolumn{7}{|l|}{ FIELD DATA } \\
\hline \multicolumn{2}{|l|}{ Constituents } & 1099 & $\underline{2099}$ & & & Unit \\
\hline \multicolumn{2}{|c|}{$\begin{array}{l}\text { Water elevation } \\
\text { pH } \\
\text { Sp. conductance } \\
\text { Water temperature } \\
\text { Alkalinity as } \mathrm{CaCO} 3 \\
\text { Turbidity } \\
\text { Volumes purged } \\
\text { Sampling code } \\
\text { Synchronous water level }\end{array}$} & $\begin{array}{l}224.7 \\
4.0 \\
120 \\
20.0 \\
0 \\
9.3 \\
0.38 \\
\times \\
224.3(03 / 30 / 99)\end{array}$ & $\begin{array}{l}224.3 \\
4.0 \\
60 \\
22.2 \\
0 \\
8 \\
0.39 \\
X N \\
223.9(06 / 30 / 99)\end{array}$ & & & $\begin{array}{l}\mathrm{t} \mathrm{msl} \\
\mathrm{pH} \\
\mu \mathrm{S} / \mathrm{cm} \\
{ }^{\circ} \mathrm{C} \\
\mathrm{mgh} \\
\mathrm{NTU} \\
\text { well volumes } \\
\mathrm{ft} \mathrm{msl}\end{array}$ \\
\hline
\end{tabular}

ANALYTICAL DATA

Inorganic Constituents

\begin{tabular}{|c|c|c|c|c|c|c|c|c|c|c|c|c|c|}
\hline Constituents & 1099 & Mod & Filt. & ST브 & DF & $\underline{\text { Lab }}$ & $\underline{2099}$ & Mod & Fill. & $\underline{\text { ST }}$ 브 & DF & Lab & Unit \\
\hline $\begin{array}{l}\text { Aluminum, total recoverable } \\
\text { Antimony, total recoverable } \\
\text { Arsenic, total recoverable } \\
\text { Barium, total recoverable } \\
\text { Beryllium, total recoverable }\end{array}$ & $\begin{array}{l}910 \\
<0.18 \\
<3.0 \\
22\end{array}$ & $\begin{array}{l}\mathrm{J} / \mathrm{I} \\
\mathrm{U} N / \\
\mathrm{U} / / \\
/ /\end{array}$ & $\begin{array}{l}\text { NDD } \\
<0.20 \\
<3.0\end{array}$ & & $\begin{array}{l}1 \\
1 \\
1 \\
1\end{array}$ & $\begin{array}{l}\text { GE } \\
\text { GE } \\
\text { GE } \\
\text { GE }\end{array}$ & 770 & $\|$ & & & 1 & GE & \\
\hline & $\begin{array}{l}1.0 \\
4.1\end{array}$ & "I & & & 1 & GE & 1.3 & $\|$ & & & 1 & GE & \\
\hline $\begin{array}{l}\text { Chromium, total recoverable } \\
\text { Cobalt, total recoverable }\end{array}$ & $\begin{array}{l}4.1 \\
5.6\end{array}$ & $\|$ & & a & $\begin{array}{l}1 \\
1\end{array}$ & $\begin{array}{l}\text { GE } \\
\text { GE }\end{array}$ & & & & & & & \\
\hline Copper, total recoverable & 14 & "I & & & 1 & $\overline{G E}$ & & & & & & & \\
\hline Cyanide & $<10$ & $\mathrm{u} / \mathrm{\prime}$ & $<10$ & & 1 & GE & & & & & & & \\
\hline $\begin{array}{l}\text { Lead, total recoverable } \\
\text { Mercury, total recoverable }\end{array}$ & $\begin{array}{l}40 \\
0.042\end{array}$ & $\begin{array}{l}1 / \\
\mathrm{J} / \mathrm{l}\end{array}$ & NDD & 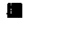 & 1 & $\begin{array}{l}\text { GE } \\
\text { GE }\end{array}$ & 68 & $/ /$ & & = & 1 & GE & \\
\hline $\begin{array}{l}\text { Nickel, total recoverable } \\
\text { Nitrate as nitrogen }\end{array}$ & 7.4 & $/ 1$ & 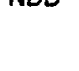 & & 1 & GE & & & & & & & \\
\hline Nitrate-nitrite as nitrogen & 11,000 & 11 & & a & 25 & GE & 6,000 & $/ 1$ & & & 3 & GE & \\
\hline $\begin{array}{l}\text { Selenium, total recoverable } \\
\text { Silver, total recoverable }\end{array}$ & $\begin{array}{l}<5.0 \\
<1.0\end{array}$ & $\begin{array}{l}U / / \\
U / I\end{array}$ & $\begin{array}{r}<5.0 \\
<1.0\end{array}$ & & $\begin{array}{l}1 \\
1\end{array}$ & $\begin{array}{l}\mathrm{GE} \\
\mathrm{GE}\end{array}$ & & & & & & & \\
\hline Thallium, total recoverable & 0.32 & 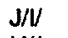 & NDD & & 1 & GE & & & & & & & \\
\hline Vanadium, total recoverable & $<10$ & U// & $<10$ & & 1 & GE & & & & & & & \\
\hline Zinc, total recoverable & 31 & "I & & & 1 & GE & & & & & & & \\
\hline
\end{tabular}

Organic Constituents

\begin{tabular}{|c|c|c|c|c|c|c|c|c|c|c|}
\hline Constituents & $\underline{1099}$ & Mod & Filt. & 오브 & DF & Lab 2099 & Mod & Fill. & DF Lab & Unit \\
\hline $\begin{array}{l}\text { Benzene } \\
\text { Bis(2-ethylhexyl) phthalate } \\
\text { Dichloromethane } \\
\text { Phenols } \\
\text { Tetrachloroethylene } \\
\text { Trichloroethylene } \\
\text { Trichlorofluoromethane }\end{array}$ & $\begin{array}{l}<1.0 \\
<10 \\
<5.0 \\
<5.0 \\
<1.0 \\
2.7 \\
12\end{array}$ & $\begin{array}{l}\text { JU/LO } \\
\text { UII } \\
\text { JU/LO } \\
\text { UII } \\
\text { JU/LO } \\
\text { J/LO } \\
\text { J/OO }\end{array}$ & $\begin{array}{l}<1.0 \\
<10 \\
<5.0 \\
<5.0 \\
<1.0 \\
\text { NDD } \\
\text { NDD }\end{array}$ & & $\begin{array}{l}1 \\
1 \\
1 \\
1 \\
1 \\
1 \\
1 \\
1\end{array}$ & $\begin{array}{l}\mathrm{GE} \\
\mathrm{GE} \\
\mathrm{GE} \\
\mathrm{GE} \\
\mathrm{GE} \\
\mathrm{GE} \\
\mathrm{GE}\end{array}$ & & & & $\begin{array}{l}\mu g / L \\
\mu g / L \\
\mu g / L \\
\mu g / L \\
\mu g / L \\
\mu g / L \\
\mu g / L\end{array}$ \\
\hline
\end{tabular}


WELL FSL 1D (cont.)

Radioactive Constituents

\begin{tabular}{|c|c|c|c|c|c|c|c|c|c|c|c|c|c|}
\hline Constituents & 1099 & Mod & Fill. & ST브 & DF & Lab & 2099 & Mod & Filt. & ST H & DF & Lab & Unit \\
\hline $\begin{array}{l}\text { Americium-241 } \\
\text { Beta dose }\end{array}$ & $\begin{array}{l}<1.3 E-01 \\
\text { NA }\end{array}$ & $\mathrm{U} / \mathrm{I}$ & $<5.7 E-01$ & & 1 & GP & & & & & & & $\mathrm{pCin}$ \\
\hline Carbon-14 & $<-2.9 E-01$ & $\mathrm{u} / /$ & $<9.7 E+\infty 0$ & & 1 & GP & & & & & & & pCin \\
\hline Cesium-137 & $<3.2 E-01$ & JU/I & $<3.7 E+\infty$ & & 1 & GP & & & & & & & pCin \\
\hline Cobalt -60 & $<-4.8 E-01$ & $\mathrm{U} / 1$ & $<3.7 E+\infty 0$ & & 1 & GP & & & & & & & pCin \\
\hline Curium-242 & $<-6.5 E-02$ & $\mathrm{U} / 1$ & $<5.1 E-01$ & & 1 & GP & & & & & & & pCir \\
\hline Curium-243/244 & $<-1.3 E-02$ & $\mathrm{U} / 1$ & $<5.1 E-01$ & & 1 & GP & & & & & & & pCin \\
\hline Curium-245/246 & $<0.0 E+\infty 0$ & $\mathrm{U} / I$ & $<1.9 E-01$ & & 1 & GP & & & & & & & pCin \\
\hline Gross alpha & $1.3 E+01$ & 11 & & & 1 & GP & $7.1 E+\infty$ & $\|$ & & & 1 & GP & pCin \\
\hline lodine-129 & $2.9 \mathrm{E}+00$ & $\mathrm{~J} / \mathrm{V}$ & NDD & & 1 & GP & & & & & & & pCir \\
\hline Nonvolatile beta & $1.0 \mathrm{E}+01$ & 11 & & & 1 & GP & $5.9 E+\infty 0$ & $\|$ & & & 1 & GP & pCin \\
\hline Plutonium-238 & $<0.0 E+\infty$ & $\mathrm{u} / /$ & $<1.1 E-01$ & & 1 & GP & & & & & & & $\mathrm{pCin}$ \\
\hline Plutonium-239/240 & $<-4.4 E-02$ & $U / I$ & $<3.0 E-01$ & & 1 & GP & & & & & & & $\mathrm{pCin}$ \\
\hline Radium-226 & $3.9 \mathrm{E}+00$ & & & & $i$ & $\overrightarrow{G P}$ & & & & & & & pCir \\
\hline Radium-228 & $<8.7 E-01$ & JURV/I & $<8.6 \mathrm{E}-01$ & & 1 & GP & & & & & & & $\mathrm{pCin}$ \\
\hline Total radium & $3.9 E+\infty$ & & & & & & & & & & & & \\
\hline Strontium-90 & $<7.4 E-01$ & $U / \prime$ & $<1.4 E+00$ & & 1 & GP & & & & & & & pCin \\
\hline Technetium-99 & $<5.1 E+\infty 0$ & UII & $<2.2 E+01$ & & 1 & GP & & & & & & & $\mathrm{pCin}$ \\
\hline Thorium-228 & $<1.0 E-01$ & $u / I$ & $<4.3 E-01$ & & 1 & GP & & & & & & & $\mathrm{pCin}$ \\
\hline Thorium-230 & $<2.7 E-02$ & u/I & $<2.7 E-01$ & & 1 & GP & & & & & & & pCin \\
\hline Thorium-232 & $<0.0 E+\infty 0$ & $\mathrm{U} / /$ & $<1.0 E-01$ & & 1 & GP & & & & & & & pCiL \\
\hline Sum of alphas & NA & & & & & & & & & & & & \\
\hline Sum of betas & & & & & & & & & & & & & \\
\hline Tritium & $1.9 E+02$ & $\|$ & & घ & 1 & GP & $8.3 E+01$ & $\|$ & & a & 1 & GP & $\mathrm{pCi} / \mathrm{mL}$ \\
\hline Uranium-233/234 & $<1.5 E-01$ & U/I & $<2.7 E-01$ & & 1 & GP & & & & & & & pCin \\
\hline Uranium-235 & $<5.5 E-02$ & $\mathrm{U} / /$ & $<3.0 E-01$ & & 1 & GP & & & & & & & pCir \\
\hline Uranium-238 & $<7.8 E-02$ & $\mathrm{u} / 1$ & $<3.5 \mathrm{E}-01$ & & 1 & GP & & & & & & & pCir \\
\hline
\end{tabular}

Notes:

= exceeded holding time

E = exceeded groundwater protection or monitoring constituent standard (See Appendix A.)

NA - Not applicable. Applies to beta dose and sum of betas if there are no beta-emitting radionuclides above detection limits; to sum of alphas if there are no alphaemitting radionuclides above detection limits; and to total radium if neither radium-226 or radium-228 was above detection limit

UAZ UTRA - Upper Aquifer Zone of the Upper Three Runs Aquifer. LAZ_UTRA - Lower Aquifer Zone of the Upper Three Runs Aquifer, Gordon - Gordon Aquifer 
WELL FSL 2D

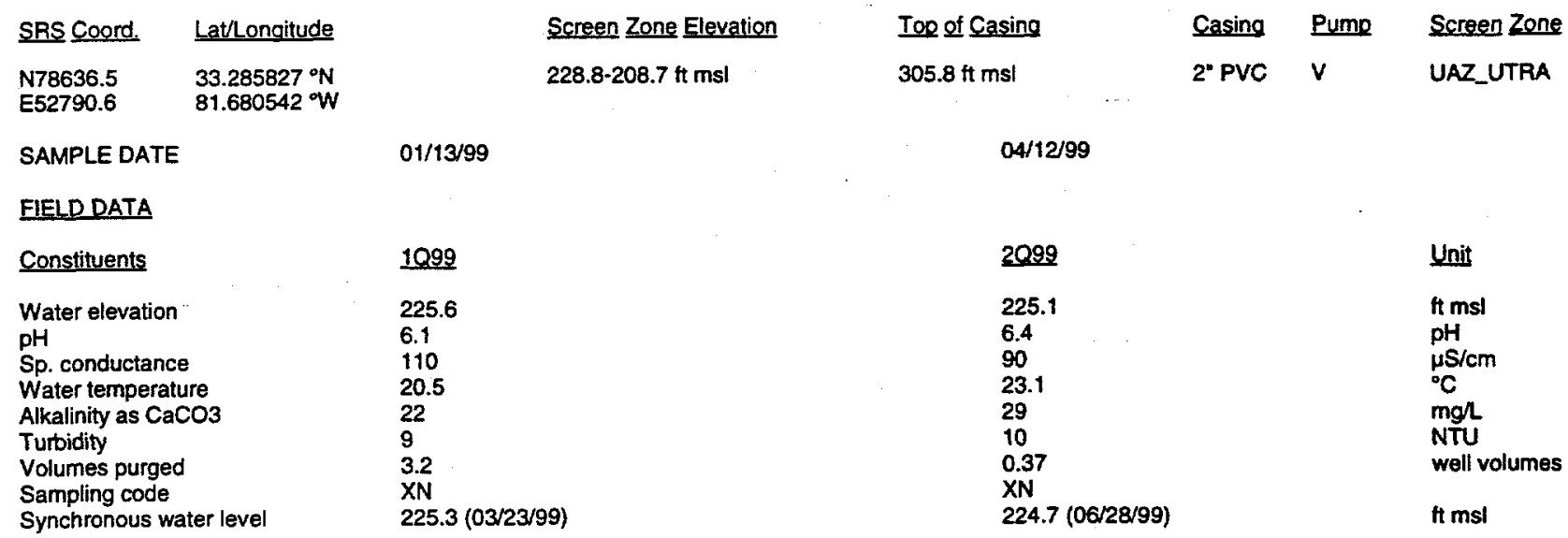

\section{ANALYTICAL DATA}

Inorganic Constituents

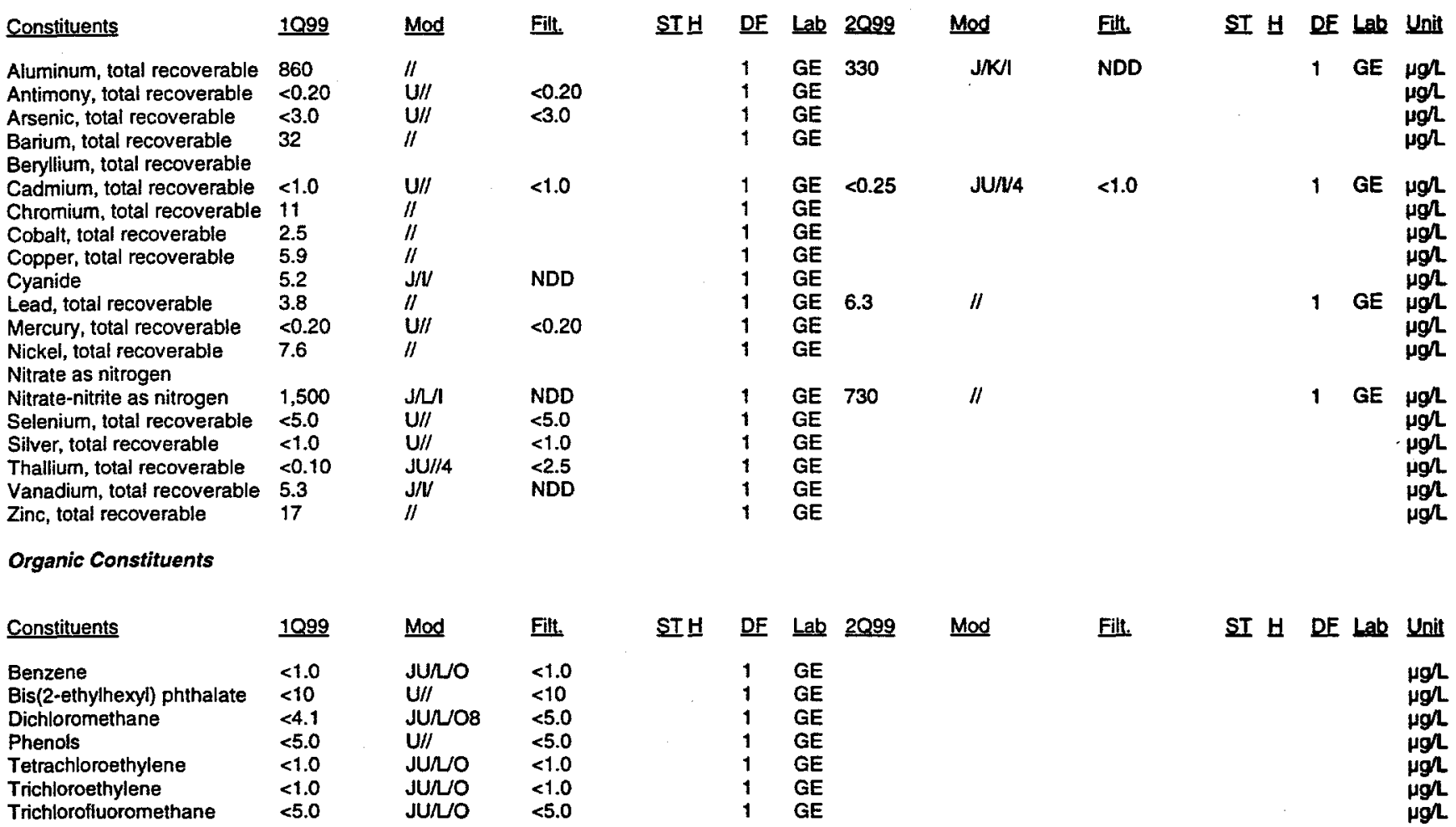

Notes:

= exceeded holding time

- exceeded groundwater protection or monitoring constituent standard (See Appendix A.)

NA - Not applicable. Applies to beta dose and sum of betas if there are no beta-emitting radionuclides above detection limits; to sum of alphas if there are no alpheemitting radionuclides above detection limits; and to total radium if neither radium-226 or radium-228 was above detection limit

UAZ_UTRA - Upper Aquifer Zone of the Upper Three Runs Aquifer, LAZ UTRA - Lower Aquifer Zone of the Upper Three Runs Aquifer, Gordon - Gordon Aquiler 


\section{WELL FSL 2D (cont.)}

Radioactive Constituents

\begin{tabular}{|c|c|c|c|c|c|c|c|c|}
\hline Constituents & 1099 & Mod & Filt. & ST브 & $\underline{D F}$ & Lab & $\underline{2099}$ & Mod \\
\hline $\begin{array}{l}\text { Americium-241 } \\
\text { Beta dose }\end{array}$ & $\begin{array}{l}<1.6 E-02 \\
\text { NA }\end{array}$ & $\mathrm{U} / /$ & $<1.0 E-01$ & & 1 & GP & & \\
\hline Carbon-14 & $<5.5 E+\infty$ & $\mathrm{U} / /$ & $<9.3 E+\infty 0$ & & 1 & GP & & \\
\hline Cesium-137 & $6.5 E+00$ & $\mathrm{R} / / 4$ & $R e j$ & & 1 & GP & & \\
\hline Cobalt -60 & $<2.1 E+00$ & $\mathrm{U} / /$ & $<4.1 \mathrm{E}+00$ & & 1 & GP & & \\
\hline Curium-242 & $<0.0 \bar{E}+\infty 0$ & $U / I$ & $<5.4 E-02$ & & 1 & GP & & \\
\hline Curium-243/244 & $<8.0 E-02$ & $\mathrm{U} / I$ & $<1.2 E-01$ & & 1 & GP & & \\
\hline Curium-245/246 & $<0.0 E+00$ & $\mathrm{U} / /$ & $<4.8 E-02$ & & 1 & GP & & \\
\hline Gross alpha & $2.6 E+\infty 0$ & $1 /$ & & & 1 & GP & $4.8 E+\infty$ & /I \\
\hline lodine-129 & $<1.9 E-01$ & $\mathrm{U} / /$ & $<9.6 \mathrm{E}-01$ & & 1 & GP & & \\
\hline Nonvolatile beta & $6.8 E+00$ & $1 /$ & & & 1 & GP & $6.4 E+00$ & II \\
\hline Plutonium-238 & $<-8.1 E-03$ & $U / /$ & $<1.5 E-01$ & & 1 & GP & & \\
\hline Plutonium-239/240 & $<1.5 E-02$ & $U / I$ & $<9.0 E-02$ & & 1 & GP & & \\
\hline Radium-226 & $9.6 \mathrm{E}-01$ & & NDD & & 1 & GP & & \\
\hline $\begin{array}{l}\text { Radium-228 } \\
\text { Total radium }\end{array}$ & $\begin{array}{l}<1.0 \mathrm{E}+00 \\
\text { NA }\end{array}$ & JU/ & $<1.1 E+00$ & & 1 & GP & & \\
\hline Strontium-90 & $1.8 E+00$ & $\mathrm{~J} / \mathrm{N}$ & NDD & & 1 & GP & & \\
\hline Technetium-99 & $<-3.9 E+00$ & $\mathrm{U} / \mathrm{I}$ & $<2.4 E+01$ & & 1 & GP & & \\
\hline Thorium-228 & $<6.6 E-03$ & $U / I$ & $<1.3 E-01$ & & 1 & GP & & \\
\hline Thorium-230 & $<5.4 \mathrm{E}-02$ & $\mathrm{U} / /$ & $<8.4 \mathrm{E}-02$ & & 1 & GP & & \\
\hline Thorium-232 & $<-3.8 E-03$ & $\mathrm{U} / /$ & $<7.5 E-02$ & & 1 & GP & & \\
\hline Sum of alphas & NA & & & & & & & \\
\hline Sum of betas & NA & & & & & & & \\
\hline Tritium & $9.4 E+\infty 0$ & $\|$ & & & 1 & GP & $6.7 E+\infty$ & /l \\
\hline Uranium-233/234 & $<2.7 E-02$ & $\mathrm{U} / /$ & $<6.8 E-02$ & & 1 & GP & & \\
\hline Uranium-235 & $<1.3 E-02$ & $\mathrm{U} / /$ & $<5.6 \mathrm{E}-02$ & & 1 & GP & & \\
\hline Uranium-238 & $<4.0 \mathrm{E}-02$ & $\mathrm{U} / /$ & $<5.6 \mathrm{E}-02$ & & 1 & GP & & \\
\hline
\end{tabular}

Filt. ST H DF Lab Unit

pCin

pCin

pCir

pCin

pCin

pCin

pCin

1 GP pCil

pCin

1 GP $\mathrm{pCl}$

pCir

pCin

pCin

pein

pCin

pCir

pCir

pCin

pCin

1 GP pCiml

pCil

pcir

pcin

Notes:

= exceeded holding time

= exceeded groundwater protection or monitoring constituent standard (See Appendix A.)

NA - Not applicable. Applies to beta dose and sum of betas if there are no beta-emitting radionuclides above detection limits; to sum of alphas if there are no alphaemiting radionuclides above detection limits; and to total radium if neither radium-226 or radium-228 was above detection limil

UAZ UTRA - Upper Aquifer Zone of the Upper Three Runs Aquifer, LAZ_UTRA - Lower Aquifer Zone of the Upper Three Runs Aquiler, Gordon - Gordon Aquifer 


\section{WELL FSL 3D}

$\begin{array}{ll}\text { SRS Coord } & \text { LatlLongitude } \\ \text { N77765.2 } & 33.283369^{\circ} \mathrm{N} \\ \text { E52465.2 } & 81.679706^{\circ} \mathrm{W}\end{array}$

SAMPLE DATE $81.679706^{\circ} \mathrm{W}$

\section{FIELD DATA}

Constituents

Water elevation

$\mathrm{pH}$

Sp. conductance

Water temperature

Alkalinity as $\mathrm{CaCO} 3$

Turbidity

Volumes purged

Sampling code

Synchronous water level

Screen Zone Elevation

$226.0-205.9 \mathrm{ft} \mathrm{msl}$

$01 / 12 / 99$

$\begin{array}{llll}\text { Top of Casing } & \text { Casing } & \text { Pums } & \text { Screen Zone } \\ 302 \mathrm{ft} \mathrm{ms} & \text { 2" PVC } & V & \text { UAZ_UTRA }\end{array}$

$04 / 12 / 99$

$\begin{array}{ll}2099 & \text { Unit } \\ 222.9 & \mathrm{tt} \mathrm{msl} \\ 6.2 & \mathrm{pH} \\ 80 & \mu \mathrm{S} / \mathrm{cm} \\ 28.7 & { }^{\circ} \mathrm{C} \\ 26 & \mathrm{mgh} \\ 6 & \mathrm{NTU} \\ 5.8 & \text { well volumes } \\ 222.3(06 / 28 / 99) & \mathrm{ft} \mathrm{msl}\end{array}$

\section{ANALYTICAL DATA}

1099

223.6

8.0

98

22.0

4

7.6

$223.1(03 / 23 / 99)$

Inorganic Constituents

\begin{tabular}{|c|c|c|c|c|c|c|c|c|c|c|c|c|c|}
\hline Constituents & 1099 & Mod & Filt. & ST뵤 & $\underline{\text { DF }}$ & Lab & 2099 & Mod & Filt. & SI $\mathrm{H}$ & DF & Lab & Unit \\
\hline $\begin{array}{l}\text { Aluminum, total recoverable } \\
\text { Antimony, total recoverable } \\
\text { Arsenic, total recoverable } \\
\text { Barium, total recoverable } \\
\text { Beryllium, total recoverable }\end{array}$ & $\begin{array}{l}990 \\
<0.33 \\
2.0 \\
120\end{array}$ & $\begin{array}{l}\mathrm{J} / \mathrm{L} / 1 \\
\mathrm{JU} / / 4 \\
\mathrm{~J} / \mathrm{LL} / \\
/ /\end{array}$ & $\begin{array}{l}\text { NDD } \\
<0.89 \\
\text { NDD }\end{array}$ & & $\begin{array}{l}1 \\
1 \\
1 \\
1\end{array}$ & $\begin{array}{l}\text { GE } \\
\text { GE } \\
\text { GE } \\
\text { GE }\end{array}$ & 130 & $J / K / I$ & NDD & & 1 & GE & $\begin{array}{l}\mu g / L \\
\mu g h \\
\mu g / L \\
\mu g / L\end{array}$ \\
\hline $\begin{array}{l}\text { Cadmium, total recoverable } \\
\text { Chromium, total recoverable } \\
\text { Cobalt, total recoverable } \\
\text { Copper, total recoverable } \\
\text { Cyanide }\end{array}$ & $\begin{array}{l}<1.0 \\
9.4 \\
1.2 \\
3.7 \\
<10\end{array}$ & $\begin{array}{l}\mathrm{U} / / \\
/ 1 \\
/ I \\
I I \\
\mathrm{U} / /\end{array}$ & $\begin{array}{l}<1.0 \\
<10\end{array}$ & & $\begin{array}{l}1 \\
1 \\
1 \\
1 \\
1\end{array}$ & $\begin{array}{l}\text { GE } \\
\text { GE } \\
\text { GE } \\
\text { GE } \\
\text { GE }\end{array}$ & $<1.0$ & $\mathrm{U} / \prime$ & $<1.0$ & & 1 & GE & $\begin{array}{l}\mu g h \\
\mu g h \\
\mu g h \\
\mu g h \\
\mu \alpha h\end{array}$ \\
\hline $\begin{array}{l}\text { Lead, total recoverable } \\
\text { Mercury, total recoverable } \\
\text { Nickel, total recoverable } \\
\text { Nitrate as nitrogen }\end{array}$ & $\begin{array}{l}1.9 \\
<0.20 \\
5.0\end{array}$ & $\begin{array}{l}\mathrm{J} / \mathrm{V} \\
\mathrm{U} / / \\
/ /\end{array}$ & $\begin{array}{l}\text { NDD } \\
<0.20\end{array}$ & & $\begin{array}{l}1 \\
1 \\
1\end{array}$ & $\begin{array}{l}\mathrm{GE} \\
\mathrm{GE} \\
\mathrm{GE}\end{array}$ & $<1.8$ & UNI & $<2.0$ & & 1 & GE & $\begin{array}{l}\mu g / L \\
\mu g / L \\
\mu g / L\end{array}$ \\
\hline $\begin{array}{l}\text { Nitrate-nitrite as nitrogen } \\
\text { Selenium, total recoverable } \\
\text { Silver, total recoverable } \\
\text { Thallium, total recoverable } \\
\text { Vanadium, total recoverable } \\
\text { Zinc, total recoverable }\end{array}$ & $\begin{array}{l}3,000 \\
<5.0 \\
<1.0 \\
<0.12 \\
7.2 \\
11\end{array}$ & $\begin{array}{l}\text { J/UI } \\
\text { JU/II } \\
\text { U/I } \\
\text { UN/ } \\
\text { J/I } \\
I /\end{array}$ & $\begin{array}{l}\text { NDD } \\
<5.0 \\
<1.0 \\
<2.5 \\
\text { NDD }\end{array}$ & & $\begin{array}{l}2 \\
1 \\
1 \\
1 \\
1 \\
1\end{array}$ & $\begin{array}{l}\text { GE } \\
\text { GE } \\
\text { GE } \\
\text { GE } \\
\text { GE } \\
\text { GE }\end{array}$ & 2,500 & $\|$ & & & 1 & GE & $\begin{array}{l}\mu g h \\
\mu g h \\
\mu g h \\
\mu g h \\
\mu g h \\
\mu g h\end{array}$ \\
\hline \multicolumn{14}{|l|}{ Organic Constituents } \\
\hline Constituents & 1099 & Mod & Fill. & SI & DE & Lab & $\underline{2099}$ & Mod & Filt. & ST H & $\underline{\mathrm{DF}}$ & Lab & \\
\hline $\begin{array}{l}\text { Benzene } \\
\text { Bis(2-ethylhexyl) phthalate } \\
\text { Dichloromethane } \\
\text { Phenols } \\
\text { Tetrachloroethylene } \\
\text { Trichloroethylene } \\
\text { Trichlorolluoromethane }\end{array}$ & $\begin{array}{l}<1.0 \\
<11 \\
<5.0 \\
<5.0 \\
<1.0 \\
1.7 \\
14\end{array}$ & $\begin{array}{l}\text { JU/LO } \\
\text { U// } \\
\text { JU/LO } \\
\text { U// } \\
\text { JU/LO } \\
\text { J/OO } \\
\text { J/OO }\end{array}$ & $\begin{array}{l}<1.0 \\
<11 \\
<5.0 \\
<5.0 \\
<1.0 \\
\text { NDD } \\
\text { NDD }\end{array}$ & & $\begin{array}{l}1 \\
1 \\
1 \\
1 \\
1 \\
1 \\
1\end{array}$ & $\begin{array}{l}\text { GE } \\
\text { GE } \\
\text { GE } \\
\text { GE } \\
\text { GE } \\
\text { GE } \\
\text { GE }\end{array}$ & & & & & & & $\begin{array}{l}\mu g h \\
\mu g h \\
\mu g h \\
\mu g h \\
\mu g / l \\
\mu g / L \\
\mu g / L\end{array}$ \\
\hline
\end{tabular}

$\mu g h$
$\mu g h$
$\mu g h$
$\mu g h$
$\mu g h$
$\mu g h$
$\mu g h$

Notes:

- exceeded holding time

- exceeded groundwater protection or monitoring constituent standard (See Appendix A.)

NA - Not applicable. Applies to beta dose and sum of betas if there are no beta-emitting radionuclides above detection limits; to sum of alphas if there are no alphaemitting radionuclides above detection limits; and to total radium if neither radium-226 or radium-22B was above detection limit

UAZ_UTRA - Upper Aquifer Zone of the Upper Three Runs Aquifer, LAZ UTRA - Lower Aquifer Zone of the Upper Three Runs Aguifer, Gordon - Gordon Aquifer 
WELL FSL 3D (cont.)

Radioactive Constituents

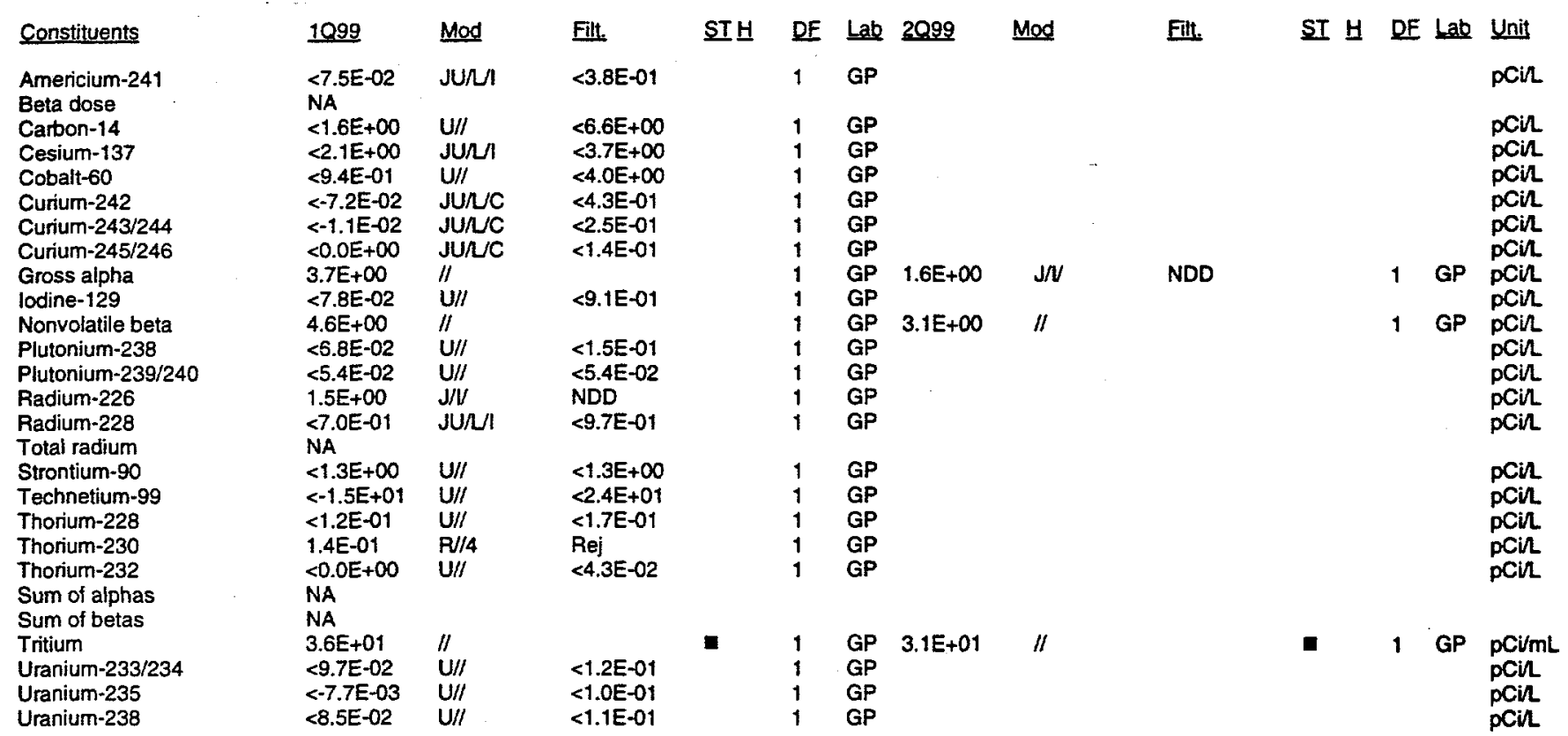

NA - Not applicable. Applies to beta dose and sum of betas if there are no beta-emitting radionuclides above detection limits; to sum of alphas if there are no alphaemitting radionuclides above detection limits; and to total radium if neither radium-226 or radium-228 was above detection limit 
WELL FSL 4D

$\begin{array}{lll}\text { SRS Coord. } & \text { Lat/Longitude } \\ \text { N77452.4 } & & 33.282294^{\circ} \mathrm{N} \\ \text { E52230.4 } & 81.679717^{\circ} \mathrm{W}\end{array}$

SAMPLE DATE
Screen Zone Elevation

224.1-204.0 ft ms
Top 의 Casing

$294.1 \mathrm{ft} \mathrm{msl}$
Casing Pume
2" PVC V
Screen Zone

UAZ_UTRA
$04 / 12 / 99$

2099
217.9
4.5
40
23.7
4
2
0.44
$X N$
$216.6(06 / 28 / 99)$

Unit

ft msi $\mathrm{pH}$ $\mu S / c m$ ${ }^{\circ} \mathrm{C}$ mis well volumes $\mathrm{ft} \mathrm{msl}$
1099

218.1

39

22.1

3

1.7

$217.5(03 / 23 / 99)$
Volumes purged

Synchronous water level

\section{ANALYTICAL DATA}

Inorganic Constituents

\begin{tabular}{|c|c|c|c|c|c|c|c|c|c|c|c|c|c|}
\hline Constituents & 1099 & $\underline{\text { Mod }}$ & Filt. & $\underline{\mathbf{S T}} \underline{\mathbf{H}}$ & $\underline{\mathrm{DF}}$ & $\underline{\text { Lab }}$ & 2099 & Mod & Filt. & ST H & DF & Lab & Unit \\
\hline $\begin{array}{l}\text { Aluminum, total recoverable } \\
\text { Antimony, total recoverable } \\
\text { Arsenic, total recoverable } \\
\text { Barium, total recoverable } \\
\text { Beryllium, total recoverable }\end{array}$ & $\begin{array}{l}150 \\
<0.20 \\
<3.0 \\
8.6\end{array}$ & $\begin{array}{l}\mathrm{J} / \mathrm{U} / \\
\mathrm{U} / / \\
\mathrm{JU} / \mathrm{I}\end{array}$ & $\begin{array}{l}\text { NDD } \\
<0.20 \\
<3.0\end{array}$ & & $\begin{array}{l}1 \\
1 \\
1 \\
1\end{array}$ & $\begin{array}{l}\text { GE } \\
\text { GE } \\
\text { GE } \\
\text { GE }\end{array}$ & 160 & $\mathrm{~J} / \mathrm{K} / \mathrm{I}$ & NDD & & 1 & GE & $\begin{array}{l}\mu g h \\
\mu g h \\
\mu g h \\
\mu g h \\
\mu g\end{array}$ \\
\hline Cadmium, total recoverable & 0.39 & $\mathrm{~J} / \mathrm{V}$ & NDD & & 1 & GE & $<1.0$ & $\mathrm{U} / /$ & $<1.0$ & & 1 & GE & \\
\hline Chromium, total recoverable & 5.5 & $\|$ & & & $i$ & GE & & & & & & & \\
\hline Cobalt, total recoverable & 1.2 & /I & & & 1 & GE & & & & & & & \\
\hline Copper, total recoverable & 8.1 & /I & & & 1 & $\mathrm{GE}$ & & & & & & & \\
\hline Cyanide & 2.2 & $\mathrm{~J} / \mathrm{V}$ & NDD & & 1 & GE & & & & & & & \\
\hline Lead, total recoverable & 21 & $/ 1$ & & च & 1 & GE & 13 & /I & & & 1 & GE & \\
\hline Mercury, total recoverable & $<0.20$ & $\mathrm{U} / \mathrm{I}$ & $<0.20$ & & 1 & GE & & & & & & & \\
\hline $\begin{array}{l}\text { Nickel, total recoverable } \\
\text { Nitrate as nitrogen }\end{array}$ & 3.1 & $/ /$ & & & 1 & GE & & & & & & & \\
\hline Nitrate-nitrite as nitrogen & 2,200 & $\mathrm{~J} / \mathrm{L} /$ & NDD & & 1 & GE & 1,800 & $\|$ & & & 1 & GE & \\
\hline Selenium, total recoverable & $<5.0$ & JU/UI & $<5.0$ & & 1 & GE & & & & & & & \\
\hline Silver, total recoverable & $<1.0$ & $\mathrm{U} / \mathrm{I}$ & $<1.0$ & & 1 & $\mathrm{GE}$ & & & & & & & \\
\hline Thallium, total recoverable & $<0.071$ & UNI & $<2.5$ & & 1 & GE & & & & & & & \\
\hline Vanadium, total recoverable & $<10$ & $\mathrm{U} / /$ & $<10$ & & 1 & $G E$ & & & & & & & \\
\hline Zinc, total recoverable & 10 & $\|$ & & & 1 & GE & & & & & & & \\
\hline
\end{tabular}

Organic Constituents

\begin{tabular}{|c|c|c|c|c|c|c|c|c|c|c|}
\hline Constituents & 1099 & Mod & Fill. & ST브 & DF & Lab 2099 & Mod & Filt. & ST $H$ DE Lab & Unit \\
\hline $\begin{array}{l}\text { Benzene } \\
\text { Bis\{2-ethylhexyl) phthalate } \\
\text { Dichloromethane } \\
\text { Phenols } \\
\text { Tetrachloroethylene } \\
\text { Trichloroethylene } \\
\text { Trichlorofluoromethane }\end{array}$ & $\begin{array}{l}<1.0 \\
<10 \\
<5.0 \\
<5.0 \\
<1.0 \\
<1.0 \\
3.2\end{array}$ & $\begin{array}{l}J U / L O \\
\text { U/I } \\
\text { JU/LO } \\
\text { UII } \\
\text { JURO } \\
\text { JU/UO } \\
\text { J/LUO }\end{array}$ & $\begin{array}{l}<1.0 \\
<10 \\
<5.0 \\
<5.0 \\
<1.0 \\
<1.0 \\
\text { NDD }\end{array}$ & & $\begin{array}{l}1 \\
1 \\
1 \\
1 \\
1 \\
1 \\
1\end{array}$ & $\begin{array}{l}\mathrm{GE} \\
\mathrm{GE} \\
\mathrm{GE} \\
\mathrm{GE} \\
\mathrm{GE} \\
\mathrm{GE} \\
\mathrm{GE}\end{array}$ & & & & $\begin{array}{l}\mu g \\
\mu g \\
\mu g \\
\mu g \\
\mu g \\
\mu g\end{array}$ \\
\hline
\end{tabular}

Notes:

- exceeded holding time

- exceeded groundwater protection or monitoring constituent standard (See Appendix A.)

NA - Not applicable. Applies to beta dose and sum of betas if there are no bela-emitting radionuclides above detection limits; to sum of alphas if there are no alpheemitting radionuclides above detection limits; and to total radium if neither radium-226 or radium-228 was above detection limit 


\section{WELL FSL 4D (cont.)}

Radioactive Constituents

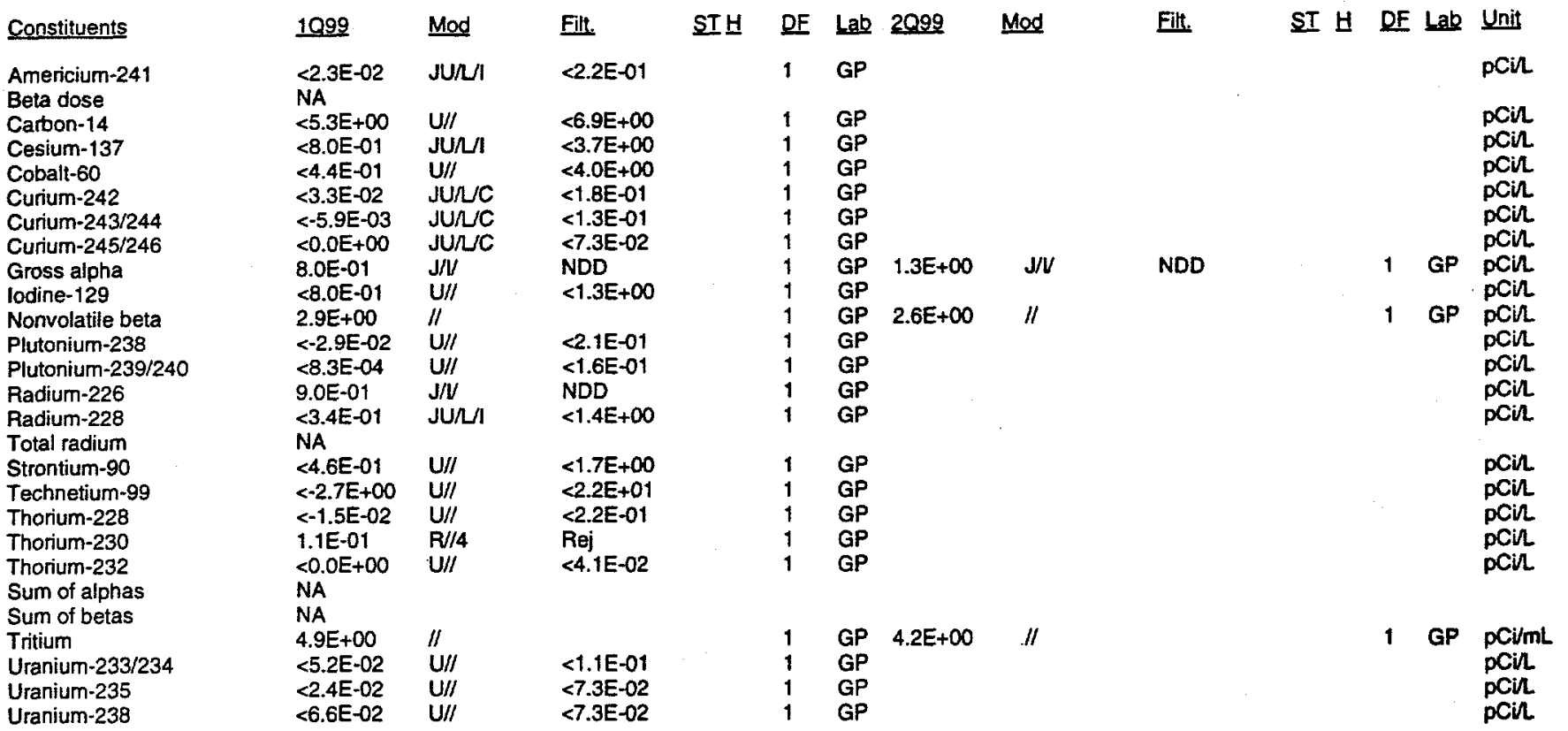




\section{WELL FSL 5D}

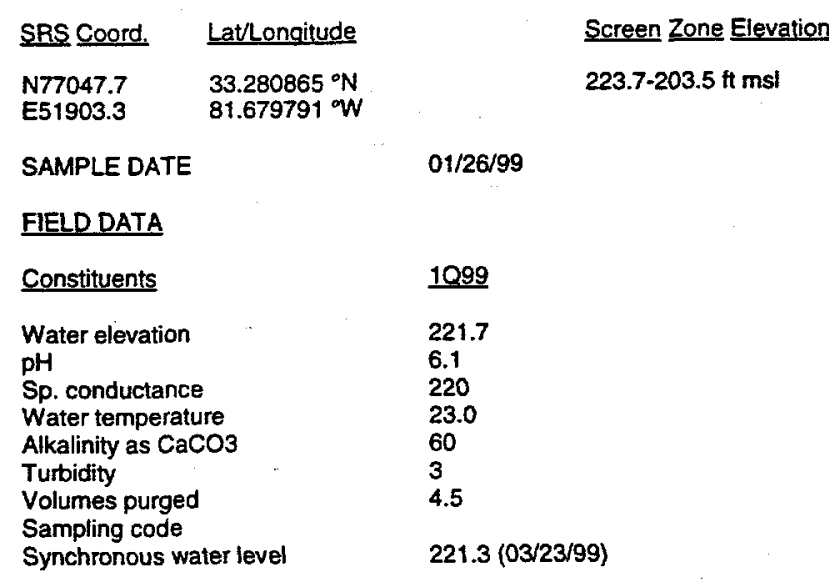

$\begin{array}{llll}\text { Top of Casing } & \text { Casing } & \text { Pump } & \text { Screen Zone } \\ 291.8 \mathrm{ft} \mathrm{msl} & 2^{*} \text { PVC } & V & \text { UAZ_UTRA }\end{array}$

$04 / 12 / 99$

2099
221.0
6.0
190
22.8
35
1
2.4

$220.5(06 / 28 / 99)$
Unit

ft msl

pH

$\mu \mathrm{S} / \mathrm{cm}$

${ }^{\circ} \mathrm{C}$

mgh

NTU

well volumes

tt msl

\section{ANALYTICAL DATA}

Inorganic Constituents

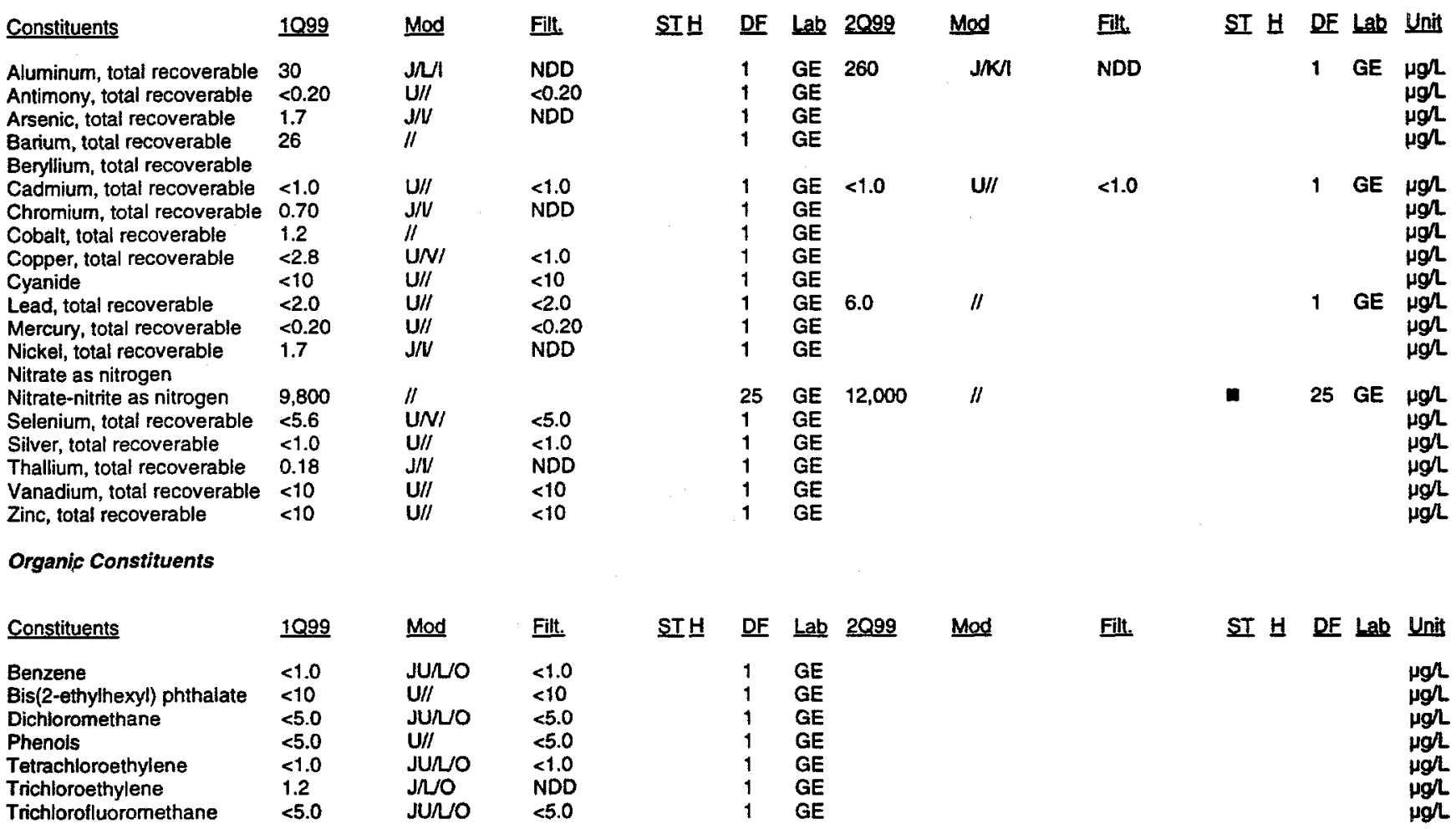

Notes:

- exceeded holding time

E = exceeded groundwater protection or monitoring constituent standard (See Appendix A.)

NA - Not applicable. Applies to beta dose and sum of betas if there are no beta-emitting radionuclides above detection limits; to sum of alphas if there are no alphaemitting radionuclides above detection limits; and to total radium if neither radium-226 or radium-228 was above detection limit

UAZ_UTRA - Upper Aquifer Zone of the Upper Three Runs Aquifer, LAZ UTRA - Lower Aquifer Zone of the Upper Three Runs Aquifer, Gordon - Gordon Aquifer 


\section{WELL FSL 5D (cont.)}

\section{Radioactive Constituents}

\begin{tabular}{|c|c|c|c|c|c|c|c|c|c|c|c|c|c|}
\hline Constituents & $\underline{1099}$ & Mod & Filt. & ST브 & DE & Lab & $\underline{2099}$ & Mod & Fill. & SI H & DE & Lab & Unit \\
\hline Americium-241 & $\begin{array}{l}<5.3 E-03 \\
2.2\end{array}$ & $\mathrm{U} / \mathrm{I}$ & $<6.2 \mathrm{E}-01$ & $=$ & 1 & GP & & & & & & & pCin \\
\hline Carbon-14 & $<8.7 \mathrm{E}+\infty 0$ & $U / /$ & $<1.1 E+01$ & & 1 & GP & & & & & & & pCin \\
\hline Cesium-137 & $7.3 E+\infty 0$ & $R / / 4$ & & & 1 & GP & & & & & & & $p \operatorname{cin}$ \\
\hline Cobalt-60 & $<5.3 E-02$ & $\mathrm{U} / /$ & $<2.9 E+00$ & & 1 & GP & & & & & & & pCin \\
\hline Curium-242 & $<9.3 \mathrm{E}-02$ & $U / /$ & $<6.3 E-01$ & & 1 & GP & & & & & & & pCin \\
\hline Curium-243/244 & $<-6.4 \mathrm{E}-02$ & $\mathrm{U} / I$ & $<5.0 \mathrm{E}-01$ & & 1 & GP & & & & & & & pCin \\
\hline Curium-245/246 & $<3.4 E-02$ & $\mathrm{U} / \mathrm{I}$ & $<4.1 E-01$ & & 1 & GP & & & & & & & pCir \\
\hline Gross alpha & 1.2E+01 & 11 & & & 1 & $G P$ & $6.4 E+\infty 0$ & $\|$ & & & 1 & GP & pCin \\
\hline lodine-129 & $3.0 E+00$ & $\mathrm{~J} / \mathrm{V}$ & NDD & & 1 & GP & & & & & & & pCil \\
\hline Nonvolatile beta & $7.5 E+01$ & 11 & & - & 1 & GP & $7.7 E+01$ & /I & & ש & 1 & GP & pCil \\
\hline Plutonium-238 & $<4.7 \mathrm{E}-02$ & $U / I$ & $<2.6 \mathrm{E}-01$ & & 1 & GP & & & & & & & pCir \\
\hline Plutonium-239/240 & $<1.6 E-02$ & & $<1.9 E-01$ & & 1 & GP & & & & & & & pCis \\
\hline Radium-226 & $3.4 E+\infty 0$ & & & & 1 & GP & & & & & & & pCin \\
\hline Radium-228 & $<1.5 \mathrm{E}+00$ & JURV/I & $<9.6 E-01$ & & 1 & GP & & & & & & & pCil \\
\hline Total radium & $3.4 E+\infty$ & & & & & & & & & & & & \\
\hline Strontium-90 & $1.1 E+01$ & /I & & a & 1 & GP & & & & & & & pCin \\
\hline Technetium-99 & $7.4 E+02$ & $\|$ & & = & 1 & GP & & & & & & & pCir \\
\hline Thorium-228 & $<-2.8 E-02$ & U/I & $<5.8 E-01$ & & 1 & GP & & & & & & & pCin \\
\hline Thorium-230 & $<9.6 \mathrm{E}-02$ & $\mathrm{U} / /$ & $<2.4 E-01$ & & 1 & GP & & & & & & & pCir \\
\hline Thorium-232 & $<6.7 E-02$ & $U / /$ & $<2.0 \mathrm{E}-01$ & & 1 & GP & & & & & & & pCir \\
\hline Sum of alphas & $\begin{array}{l}\text { NA } \\
75 E+02\end{array}$ & & & च & & & & & & & & & \\
\hline $\begin{array}{l}\text { Sum of betas } \\
\text { Tritium }\end{array}$ & $8.3 E+01$ & /I & & 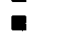 & 1 & GP & $1.5 \mathrm{E}+02$ & 11 & & च & 1 & GP & pCilmt \\
\hline Uranium-233/234 & $<9.3 E-02$ & $\mathrm{u} / /$ & $<3.2 E-01$ & & $i$ & GP & & & & & & & $\mathrm{pCin}$ \\
\hline Uranium-235 & $<1.7 \mathrm{E}-02$ & $\mathbf{U} / /$ & $<2.0 E-01$ & & 1 & GP & & & & & & & pCin \\
\hline Uranium-238 & $<-1.6 \mathrm{E}-02$ & $\mathrm{U} / \mathrm{I}$ & $<2.0 E-01$ & & 1 & GP & & & & & & & pCin \\
\hline
\end{tabular}


WELL FSL 6D

$\begin{array}{lll}\text { SRS Coord. } & \text { Lat/Longitude } \\ \text { N76733.1 } & & 33.279883^{\circ} \mathrm{N} \\ \text { E51727.9 } & 81.6796422^{\circ}\end{array}$

SAMPLE DATE

FIELD DATA

Constituents

Water elevation

$\mathrm{pH}$

Sp. conductance

Water temperature

Alkalinity as $\mathrm{CaCO} 3$

Turbidity

Volumes purged

Sampling code

Synchronous water level

Sccreen Zone Elevation

$222.1-202.1 \mathrm{ft} \mathrm{ms}$

$01 / 12 / 99$
Too 의 Casing

$286.2 \mathrm{ft}$ ms!

\section{Casing Pume}

2" PVC V
Screen Zone

UAZ UTRA
$04 / 12 / 99$

2099
220.2
4.8
140
23.1
0
1
7.1
$219.7(06 / 28 / 99)$

Unit

ft msl

$\mathrm{pH}$

$\mu \mathrm{S} / \mathrm{cm}$

${ }^{\circ} \mathrm{C}$

mgl

well volumes

ft msl

\section{ANALYTICAL DATA}

Inorganic Constituents

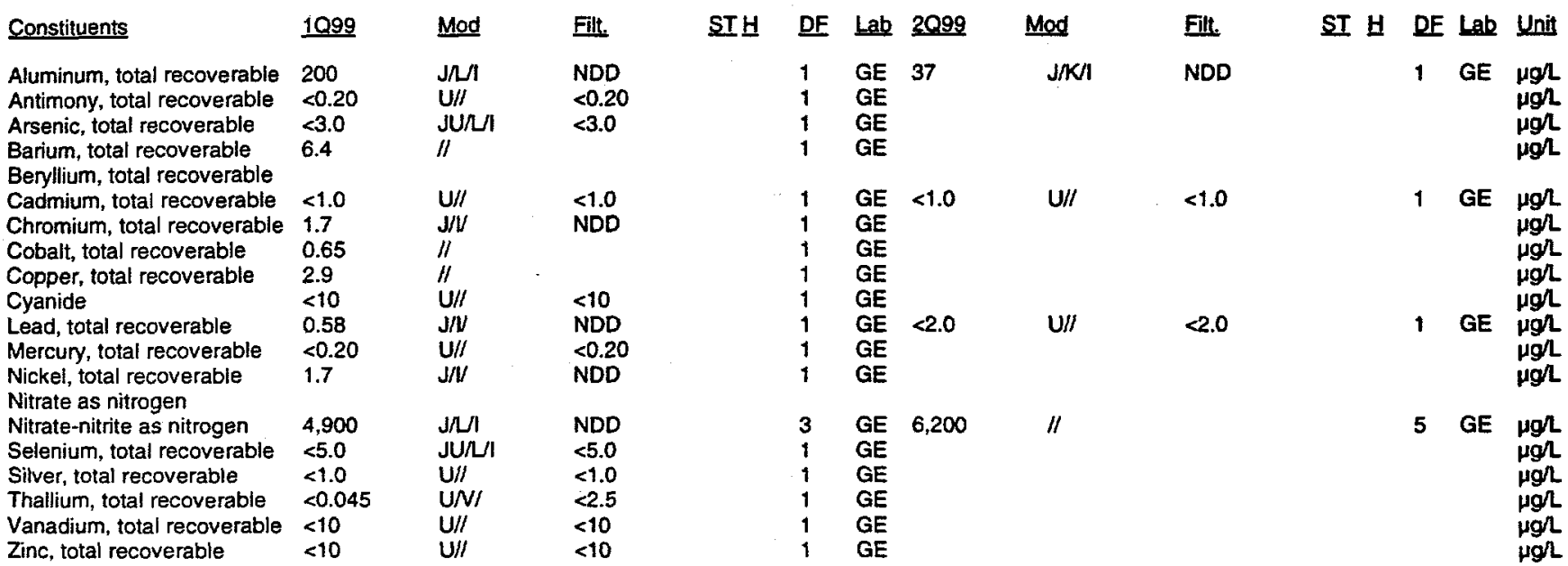

Organic Constituents

\begin{tabular}{|c|c|c|c|c|c|c|c|c|c|c|}
\hline Constituents & 1099 & Mod & Filt. & STH & DE & Lab 2099 & Mod & Filt. & SI H DE Lab & Unit \\
\hline Benzene & $<1.0$ & JUルO & $<1.0$ & & 1 & GE & & & & $\mu g / 2$ \\
\hline Bis(2-ethythexyl) phthalate & $<10$ & $\mathbf{v} / /$ & $<10$ & & 1 & GE & & & & $\mu g /$ \\
\hline Dichloromethane & $<2.2$ & JURVIO & $<5.0$ & & 1 & GE & & & & $\mu g h$ \\
\hline Phenols & $<5.0$ & $\mathrm{u} / /$ & $<5.0$ & & 1 & GE & & & & $\mu g / L$ \\
\hline Tetrachloroethylene & $<1.0$ & JU/LO & $<1.0$ & & 1 & GE & & & & $\mu g h$. \\
\hline Trichloroethylene & 2.4 & $\|$ & & & 1 & GE & & & & $\mu g h$ \\
\hline Trichlorofluoromethane & 3.4 & J/IL/O & NDD & & 1 & GE & & & & $\mu g / 2$ \\
\hline
\end{tabular}

Notes:

- = exceeded holding time

$\square$ = exceeded groundwater protection or monitoring constituent standard (See Appendix A.)

NA - Not applicable. Applies to beta dose and sum of betas if there are no beta-emitting radionuclides above detection limits; to sum of alphas if there are no alphaemitting radionuclides above detection limits; and to total radium if neither radium-226 or radium-228 was above detection limit

UAZ_UTRA - Upper Aquifer Zone of the Upper Three Runs Aquifer, LAZ_UTRA - Lower Aquifer Zone of the Upper Three Runs Aquifer, Gordon - Gordon Aquifer 
WELL FSL 6D (cont.)

Radioactive Constituents

\begin{tabular}{|c|c|c|c|c|c|c|c|c|c|c|c|c|c|}
\hline Constituents & 1099 & Mod & Filt. & ST브 & $\underline{\mathrm{DF}}$ & Lab & 2099 & Mod & Filt. & ST H & DF & Lab & Unit \\
\hline Americium-241 & $\begin{array}{l}<1.8 E-02 \\
50\end{array}$ & JUAI & $<3.2 E-01$ & 口 & 1 & GP & & & & & & & pCir \\
\hline Carbon-14 & $1.1 E+01$ & $\mathrm{~J} / \mathrm{V}$ & NDD & & 1 & GP & & & & & & & pCin \\
\hline Cesium-137 & $<-5.6 E-01$ & JU/II & $<4.2 E+\infty$ & & 1 & GP & & & & & & & pCin \\
\hline Cobalt- 60 & $<1.5 E+\infty$ & $\mathrm{U} / /$ & $<3.8 E+00$ & & 1 & GP & & & & & & & pCin \\
\hline Curium-242 & $<2.8 \mathrm{E}-03$ & JUAC & $<3.3 E-01$ & & 1 & GP & & & & & & & pCin \\
\hline Curium-243/244 & $<-7.9 E-03$ & JU/C & $<1.7 E-01$ & & 1 & GP & & & & & & & $\mathrm{pCil}$ \\
\hline Curium-245/246 & $<0.0 E+00$ & JU/U/C & <1.1E-01 & & 1 & GP & & & & & & & pCin \\
\hline Gross alpha & $4.5 E+00$ & $/ 1$ & & & 1 & GP & $1.8 E+\infty$ & $\mathrm{J} / \mathrm{V}$ & NDD & & 1 & GP & $\mathrm{pCir}$ \\
\hline lodine-129 & $5.0 \mathrm{E}+01$ & $\|$ & & E & 1 & GP & & & & & & & $\mathrm{pCi} /$ \\
\hline Nonvolatile beta & $1.7 E+01$ & $/ 1$ & & & 1 & GP & $9.4 \mathrm{E}+00$ & $\|$ & & & 1 & GP & pCin \\
\hline Plutonium-238 & 5.0E-02 & $\mathrm{R} / / \mathrm{l}$ & Rej & & 1 & GP & & & & & & & pCin \\
\hline Plutonium-239/240 & $<-3.2 E-04$ & $\mathrm{U} / /$ & $<2.5 E-01$ & & 1 & GP & & & & & & & $\mathrm{pCin}$ \\
\hline Radium-226 & 4.1E+00 & $/ /$ & & & 1 & GP & & & & & & & pCin \\
\hline Radium-228 & $<9.0 E-01$ & JU/LV/I & $<9.0 E-01$ & & 1 & GP & & & & & & & $\mathrm{pCin}$ \\
\hline $\begin{array}{l}\text { Total radium } \\
\text { Strontium-90 }\end{array}$ & $\begin{array}{l}4.1 E+\infty \\
<1.3 E+\infty 0\end{array}$ & $\mathrm{U} / \mathrm{I}$ & $<1.4 E+00$ & & 1 & GP & & & & & & & pCin \\
\hline Technetium-99 & $3.6 E+01$ & $\mathrm{~J} / \mathrm{V}$ & NDD & & $i$ & GP & & & & & & & pCin \\
\hline Thorium-228 & $<9.7 E-02$ & $\mathrm{U} / \mathrm{I}$ & $<2.5 E-01$ & & 1 & GP & & & & & & & $\mathrm{pCin}$ \\
\hline Thorium-230 & $<9.6 E-02$ & $\mathrm{U} / /$ & $<1.2 E-01$ & & 1 & GP & & & & & & & pCin \\
\hline Thorium-232 & $<4.8 E-02$ & $\mathrm{U} / /$ & $<4.8 E-02$ & & 1 & GP & & & & & & & pCin \\
\hline Sum of alphas & NA & & & & & & & . & & & & & \\
\hline Sum of betas & $5.0 E+01$ & & & $\mathbf{\square}$ & & & & & & & & & pCin \\
\hline Tritium & $6.6 E+01$ & $/ /$ & & n. & 1 & GP & $9.1 E+01$ & $1 /$ & & 口 & 1 & GP & $\mathrm{pCi} / \mathrm{mL}$ \\
\hline Uranium-233/234 & $2.5 E-01$ & $\mathrm{~J} / \mathrm{V}$ & NDD & & 1 & GP & & & & & & & poin \\
\hline Uranium-235 & $<1.2 E-02$ & $\mathrm{U} / /$ & $<8.5 E-02$ & & 1 & GP & & & & & & & pCir \\
\hline Uranium-238 & 2.6E-01 & $\mathrm{J} / \mathrm{V}$ & NDD & & 1 & GP & & & & & & & pCin \\
\hline
\end{tabular}

NA - Not applicable. Applies to beta dose and sum of betas if there are no beta-emitting radionuclides above detection limits; to sum of alphas if there are no alphaemitting radionuclides above detection limits; and to total radium if neither radium-226 or radium-228 was above detection timit 
WELL FSL 7D

$\begin{array}{ll}\text { SRS Coord. } & \text { Latllongitude } \\ \text { N76327.8 } & 33.278592^{\circ} \mathrm{N} \\ \text { E51485.6 } & 81.679492^{\circ} \mathrm{W}\end{array}$

SAMPLE DATE

$01 / 12 / 99$

Screen Zone Elevation

219.6-199.5 ft ms
Top of Casing

$287.6 \mathrm{ft} \mathrm{msl}$ $\begin{array}{ll}\text { Casing Pump } & \\ \text { 2" PVC } & V\end{array}$
Screen Zane

UAZ_UTRA

\section{FIELD DATA}

\section{Constituents}

Water elevation

$\mathrm{PH}$

Sp. conductance

Water temperature

Alkalinity as $\mathrm{CaCO} 3$

Turbidity

Volumes purged

Sampling code

Synchronous water level
1099

219.9

4.3

360

18.8

0

0.30

$\mathrm{X}$

219.0 (03/23/99)
04/07/99

2099

218.9

3.8

340

21.8

0

3

0.31

$\mathrm{X}$

218.5 (06/28/99)
Unit

tt msI

pH

$\mu \mathrm{S} / \mathrm{cm}$

${ }^{\circ} \mathrm{C}$

mgh

well volumes

ft $\mathrm{ms}$

\section{ANALYTICAL DATA}

Inorganic Constituents

\begin{tabular}{|c|c|c|c|c|c|c|c|c|c|c|c|c|c|}
\hline Constituents & 1099 & Mod & Filt. & STH & DF & Lab & 2099 & Mod & Filt. & ST $H$ & DF & Lab & Unit \\
\hline $\begin{array}{l}\text { Aluminum, total recoverable } \\
\text { Antimony, total recoverable } \\
\text { Arsenic, total recoverable } \\
\text { Barium, total recoverable } \\
\text { Beryllium, total recoverable }\end{array}$ & $\begin{array}{l}2,400 \\
<0.20 \\
<15 \\
99\end{array}$ & $\begin{array}{l}\mathrm{J} / \mathrm{U} / \\
\mathrm{U} / / \\
\mathrm{JU} / \mathrm{L} / \\
/ /\end{array}$ & $\begin{array}{l}\text { NDD } \\
<0.20 \\
<15\end{array}$ & & $\begin{array}{l}1 \\
1 \\
5 \\
1\end{array}$ & $\begin{array}{l}\mathrm{GE} \\
\mathrm{GE} \\
\mathrm{GE} \\
\mathrm{GE}\end{array}$ & 3,000 & $\|$ & & & $\mathbf{1}$ & GE & $\begin{array}{l}\mu g h \\
\mu g / \\
\mu g / \\
\mu g h\end{array}$ \\
\hline $\begin{array}{l}\text { Cadmium, total recoverable } \\
\text { Chromium, total recoverable }\end{array}$ & $\begin{array}{l}14 \\
2.1\end{array}$ & $J / V$ & NDD & $\mathbf{z}$ & $\begin{array}{l}1 \\
1\end{array}$ & $\begin{array}{l}\text { GE } \\
\text { GE }\end{array}$ & 16 & $\|$ & & $\mathbf{n}$ & 1 & GE & ngh \\
\hline Cobalt, total recoverable & 3.0 & $\|$ & TYUD & a & 1 & GE & & & . & & & & HoL \\
\hline Copper, total recoverable & 8.7 & /I & & & 1 & GE & & & & & & & \\
\hline Cyanide & $<10$ & $\mathrm{U} / \mathrm{I}$ & $<10$ & & 1 & GE & & & & & & & \\
\hline Lead, total recoverable & 1.7 & $\mathrm{~J} / \mathrm{U}$ & NDD & & 1 & GE & 6.3 & $\mathrm{~J} / \mathrm{N}$ & NDD & & 10 & GE & ugh \\
\hline Mercury, total recoverable & $<0.20$ & $\mathrm{U} / \prime$ & $<0.20$ & & 1 & GE & & & & & & & \\
\hline $\begin{array}{l}\text { Nickel, total recoverable } \\
\text { Nitrate as nitrogen }\end{array}$ & 6.3 & $1 /$ & & & 1 & GE & & & & & & & \\
\hline Nitrate-nitrite as nitrogen & 45,000 & J/UI & NDD & & 25 & GE & 42,000 & $\|$ & & $\mathbf{0}$ & 25 & GE & \\
\hline Selenium, total recoverable & $<25$ & JU/L/ & $<25$ & & 5 & GE & & & & & & & \\
\hline Silver, total recoverable & $<1.0$ & $\mathrm{U} / 1$ & $<1.0$ & & 1 & GE & & & & & & . & \\
\hline Thallium, total recoverable & $<0.054$ & UNI & $<2.5$ & & 1 & GE & & & & & & & Igh \\
\hline $\begin{array}{l}\text { Vanadium, total recoverable } \\
\text { Zinc, total recoverable }\end{array}$ & $\begin{array}{l}<10 \\
18\end{array}$ & $\mathrm{u} / /$ & $<10$ & & $\begin{array}{l}1 \\
1\end{array}$ & $\begin{array}{l}\text { GE } \\
\text { GE }\end{array}$ & & & & & & & $\mu g / 2$ \\
\hline \multicolumn{14}{|l|}{ Organic Constituents } \\
\hline Constituents & 1099 & Mod & Fill. & STH & DF & Lab & 2099 & Mod & Filt. & SI H & DF & Lab & \\
\hline Benzene & $<1.0$ & JU/ & $<1.0$ & & 1 & GE & & & & & & & \\
\hline Bis(2-ethylhexyl) phthalate & $<10$ & $\mathrm{U} / /$ & $<10$ & & 1 & GE & & & & & & & \\
\hline Dichloromethane & $<1.9$ & JURVIO & $<5.0$ & & 1 & GE & & & & & & & \\
\hline Phenols & $<5.0$ & $\mathrm{U} / /$ & $<5.0$ & & 1 & GE & & & & & & & \\
\hline Tetrachloroethylene & $<1.0$ & JU/LO & $<1.0$ & & 1 & $\overline{\mathrm{GE}}$ & & & & & & & \\
\hline Trichloroethylene & $<1.0$ & JU/LO & $<1.0$ & & 1 & GE & & & & & & & \\
\hline Trichlorofluoromethane & $<5.0$ & JU/U/O & $<5.0$ & & 1 & GE & & & & & & & \\
\hline
\end{tabular}

Notes:

- = exceeded holding time

= exceeded groundwater protection or monitoring constituent standard (See Appendix A.)

NA - Not applicable. Applies to beta dose and sum of betas if there are no beta-emitting radionuclides above detection limits; to sum of alphas if there are no aipheemitting radionuclides above detection limits; and to total radium if neither radium-226 or radium-228 was above detection limit

UAZ_UTRA - Upper Aquifer Zone of the Upper Three Runs Aquifer, LAZ_UTRA - Lower Aquifer Zone of the Upper Three Runs Aquifer, Gordon - Gordon Aquifer 
WELL FSL 7D (cont.)

Radioactive Constituents

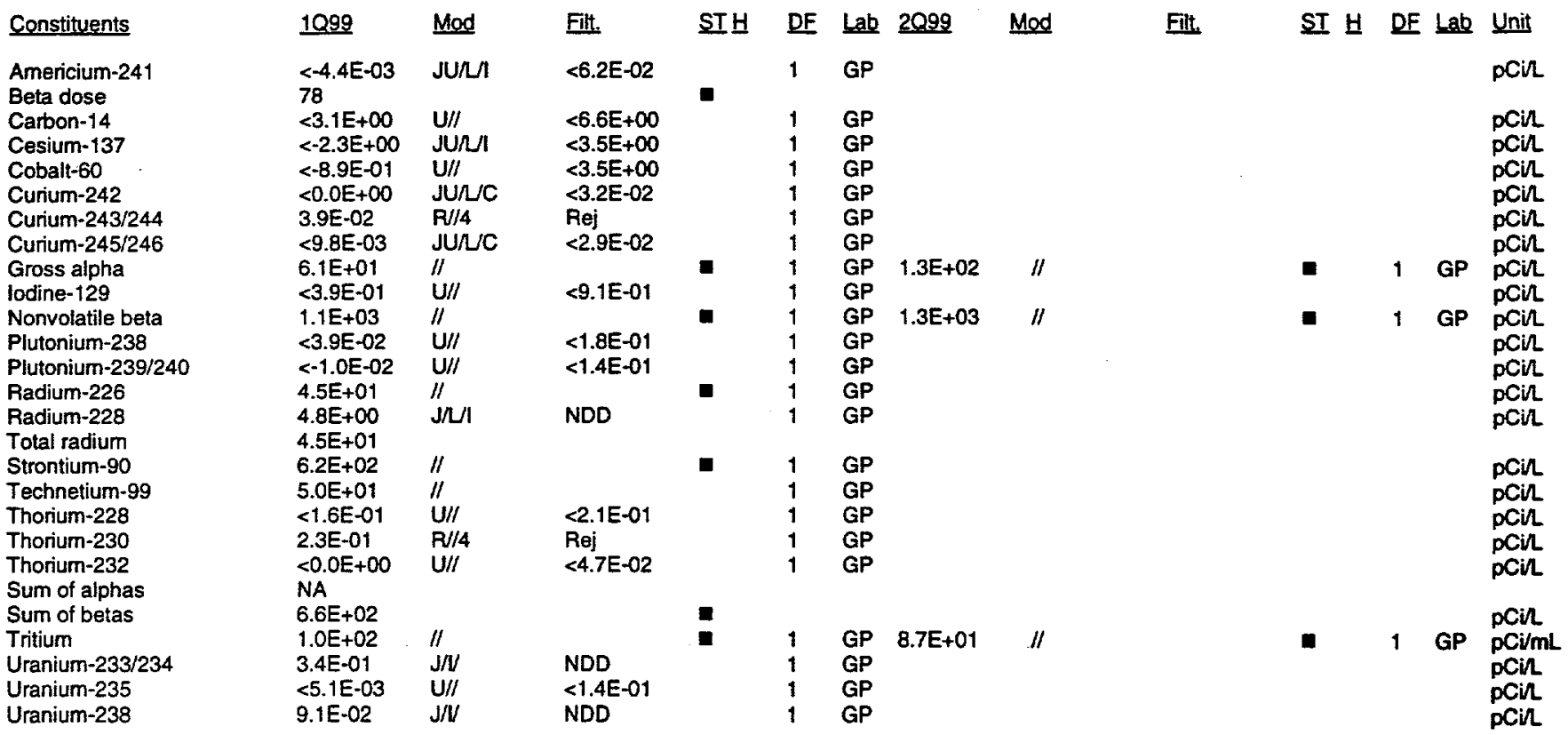

NA - Not applicable. Applies to beta dose and sum of betas if there are no beta-emitting radionuclides above detection limits; to sum of alphas if there are no alphaemitting radionuclides above detection limits; and to total radium if neither radium-226 or radium-228 was above detection limit 
WELL FSL 8D

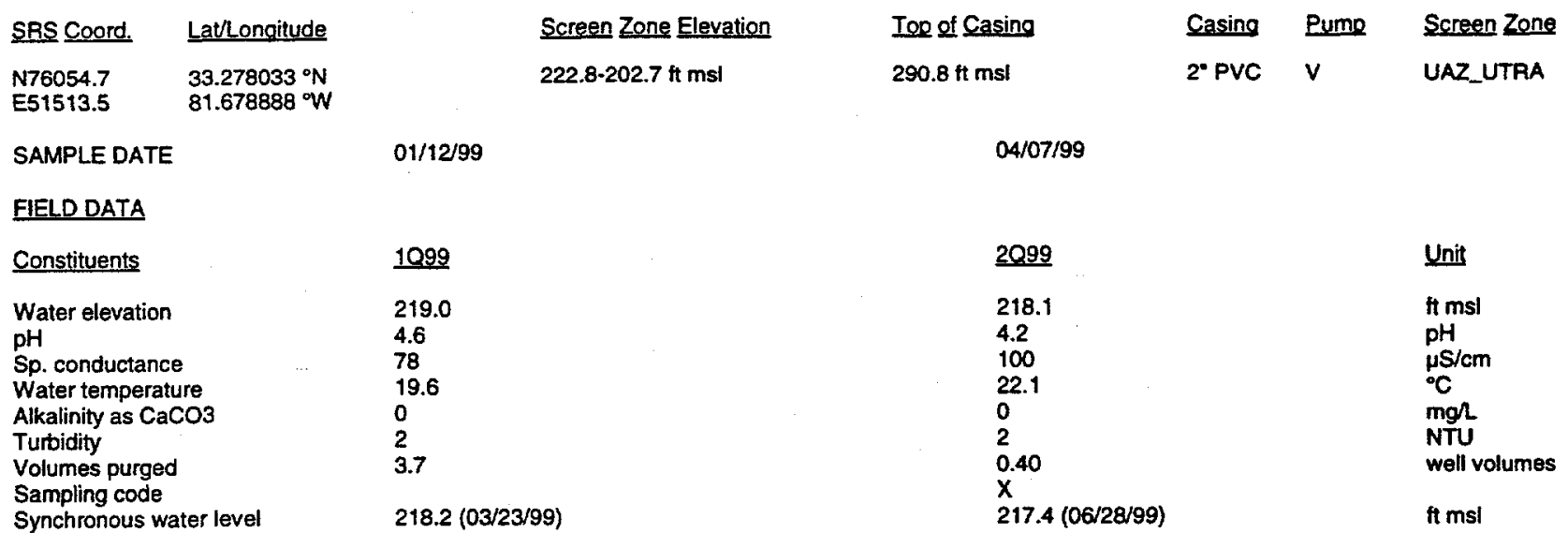

ANALYTICAL DATA

Inorganic Constituents

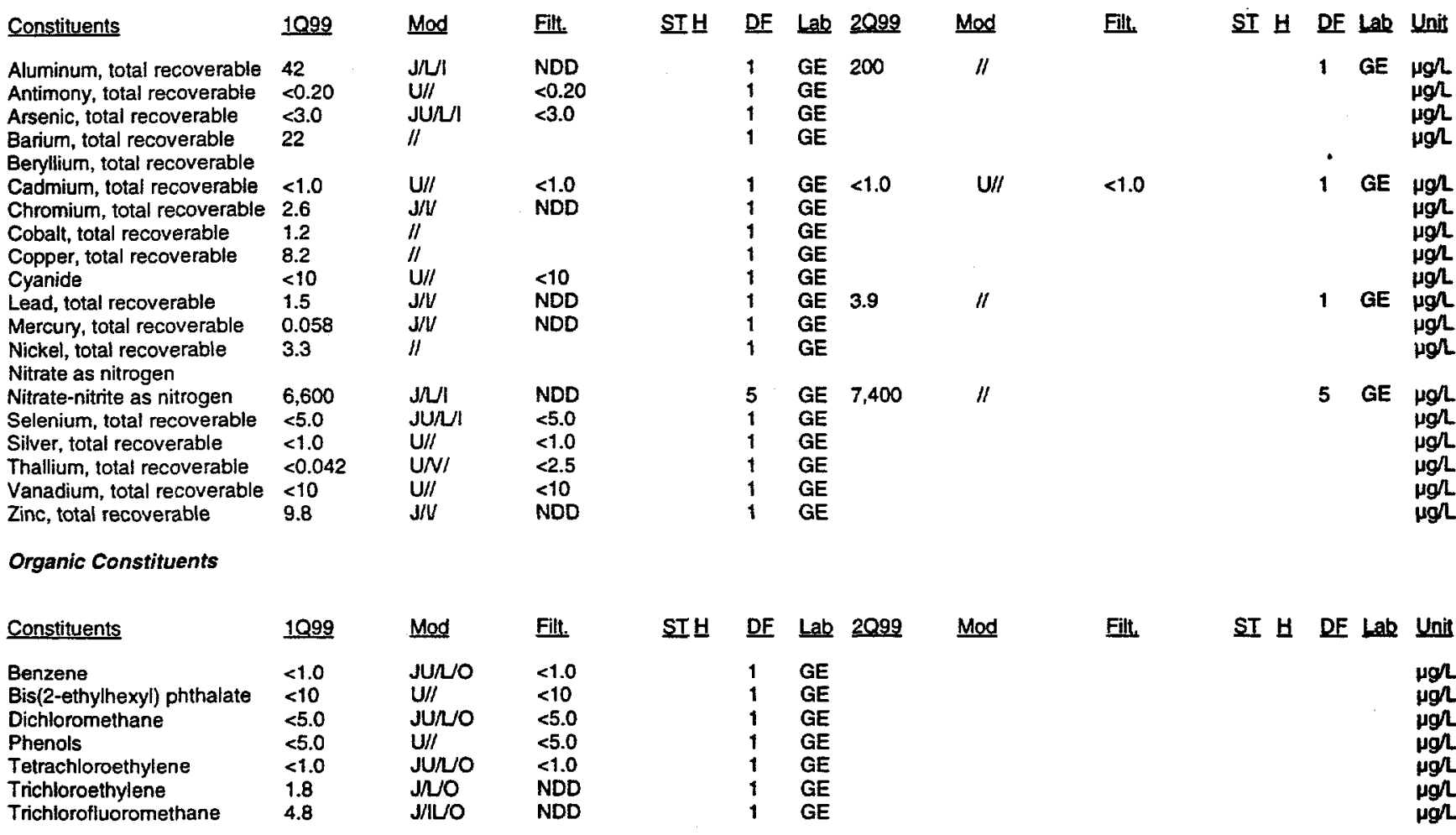

$\mu g h$

$\mu g / 2$

$\mu g / 2$

$\mu g / 2$

$\mu g / 2$

Notes:

- exceeded holding time

= exceeded groundwater protection or monitoring constituent standard (See Appendix A.)

NA - Not applicable. Applies to beta dose and sum of betas if there are no beta-emitting radionuclides above detection limits; to sum of alphas if there are no alphaemitting radionuclides above detection limits; and to total radium if neither radium-226 or radium-228 was above detection limit

UAZ UTRA - Upper Aquifer Zone of the Upper Three Runs Aquifer, LAZ UTRA - Lower Aquifer Zone of the Upper Three Runs Aquifer, Gordon - Gordon Aquifer 
WELL FSL 8D (cont.)

Radioactive Constituents

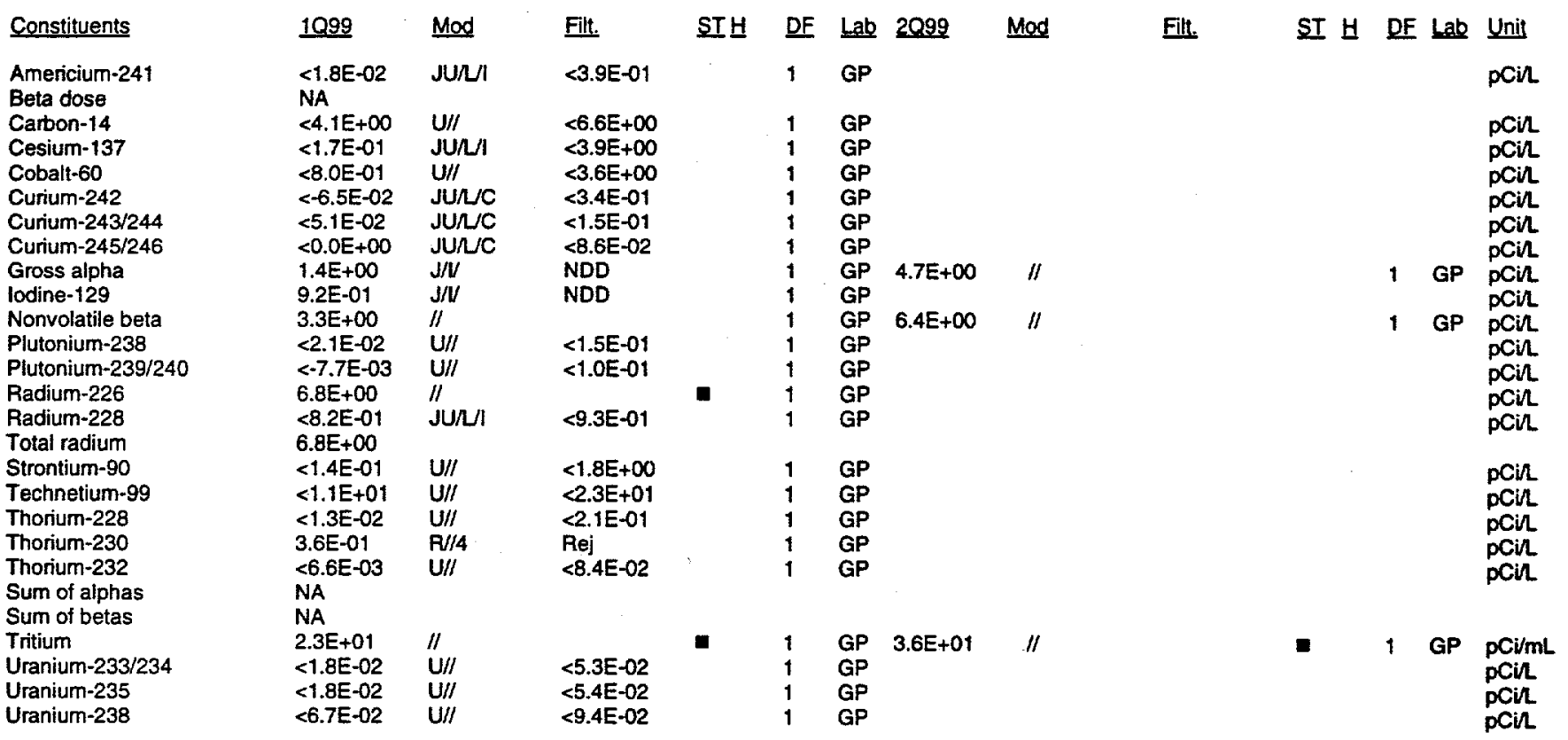

- exceeded holding time

= exceeded groundwater protection or monitoring constituent standard (See Appendix A.)

NA - Not applicable. Applies to beta dose and sum of betas if there are no beta-emitting radionuclides above detection limits; to sum of alphas if there are no alphaemitting radionuclides above detection limits; and to total radium if neither radium-226 or radium-228 was above detection limit

UAZ_UTRA - Upper Aquifer Zone of the Upper Three Runs Aquifer, LAZ_UTRA - Lower Aquifer Zone of the Upper Three Runs Aquifer, Gordon - Gordon Aquifer 


\section{WELL FSL 9D}

$\begin{array}{ll}\text { SRS Coord. } & \text { Latllongitude } \\ \text { N75768.4 } & 33.277450^{\circ} \mathrm{N} \\ \text { E } 51543.9 & 81.678252^{\circ} \mathrm{W}\end{array}$

SAMPLE DATE

Screen Zone Elevation

221.5-201.4 ft ms
Top of Casing

$285.9 \mathrm{ft} \mathrm{msl}$
Casing Pump

2. PVC V
Screen Zone

UAZ_UTRA
$04 / 07 / 99$

2099
216.9
3.8
340
23.9
0
9
0.39
$\times$
216.5

$216.5(06 / 28 / 99)$
Unit

ft msl $\mathrm{pH}$ uSicm ${ }^{\circ} \mathrm{C}$ mgh NTU well volumes ft msl

Synchronous water level

$217.1(03 / 23 / 99)$

ANALYTICAL DATA

Inorganic Consfituents

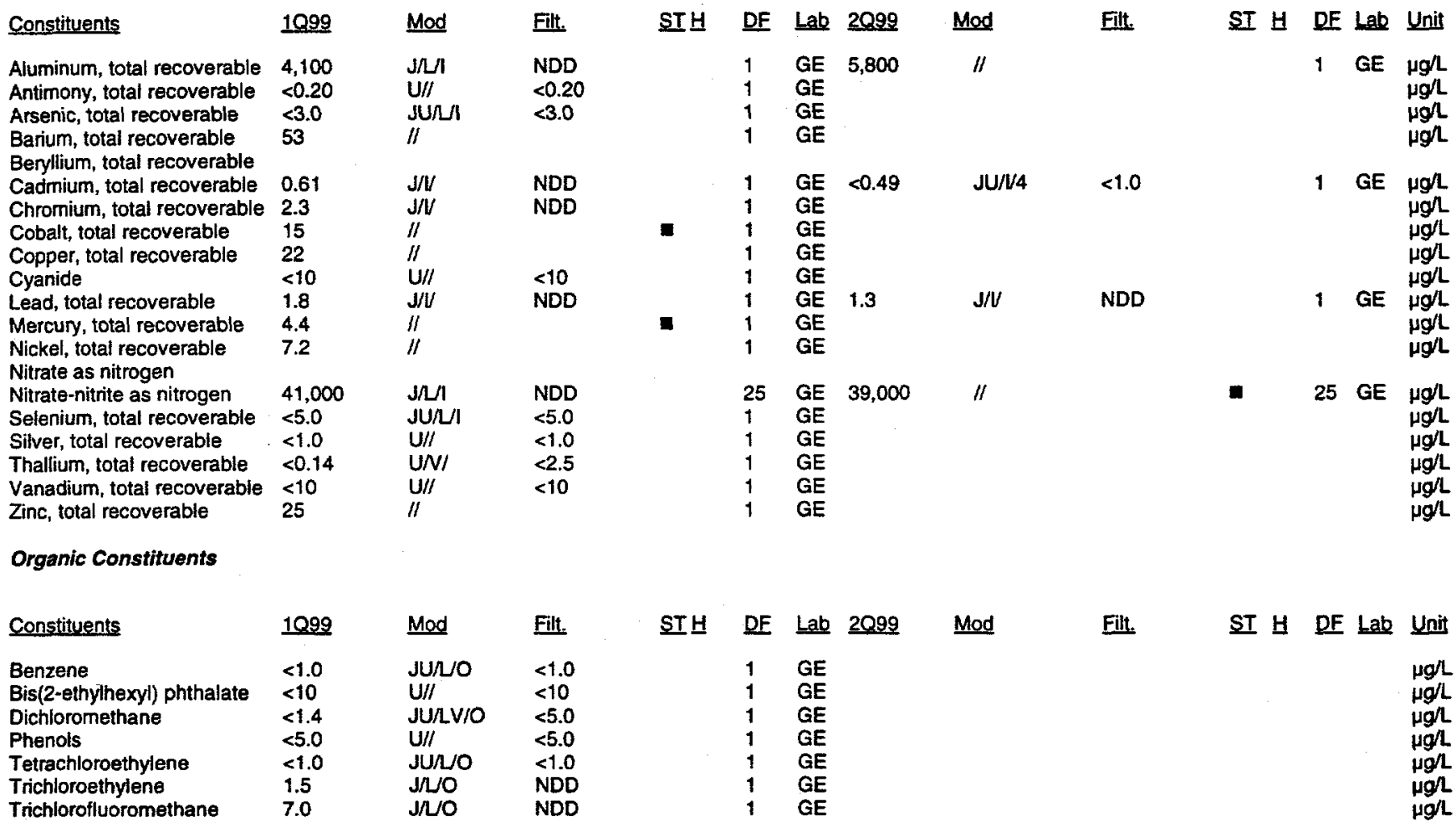

Notes:

- exceeded holding time

= exceeded groundwater protection or monitoring constituent standard (See Appendix A.)

NA - Not applicable. Applies to beta dose and sum of betas if there are no beta-emitting radionuclides above detection limits; to sum of alphas if there are no alphaemitting radionuclides above detection fimits; and to total radium if neither radium-226 or radium-228 was above detection limit

UAZ UTRA - Upper Aquifer Zone of the Upper Three Runs Aquifer; LAZ UTRA - Lower Aquifer Zone of the Upper Three Runs Aquifer, Gordon - Gordon Aguifer 


\section{WELL FSL 9D (cont.)}

Radioactive Constituents

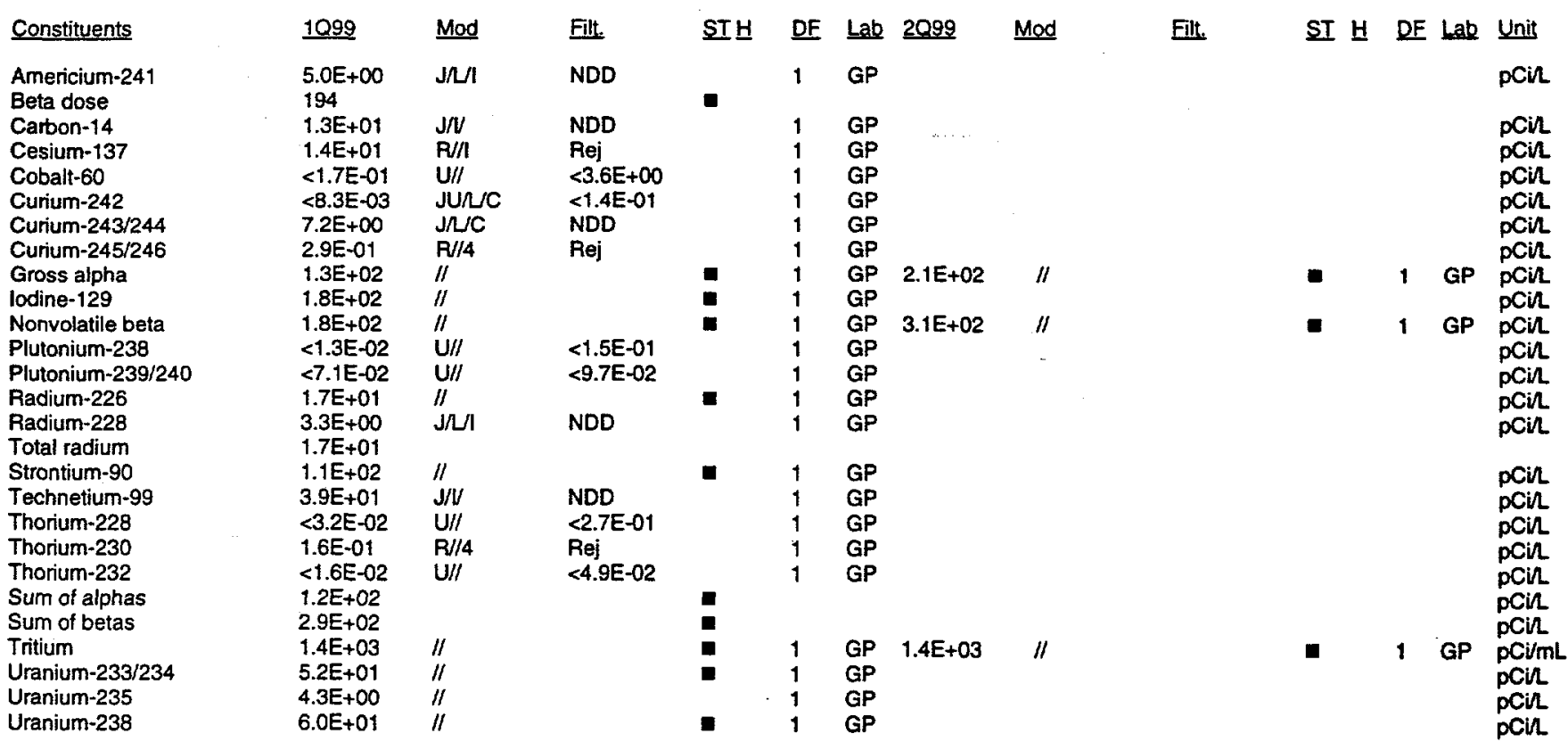

\section{Notes:}

- exceeded holding time

= exceeded groundwater protection or monitoring constituent standard (See Appendix A.)

NA - Not applicable. Applies to beta dose and sum of betas it there are no beta-emitting radionuclides above detection limits; to sum of alphas if there are no alphaemitting radionuclides above detection limits; and to total radium if neither radium-226 or radium-228 was above detection limit

UAZ_UTRA - Upper Aquifer Zone of the Upper Three Runs Aquifer, LAZ_UTRA - Lower Aguifer Zone of the Upper Three Runs Aguifer, Gordon - Gordon Aguifer 


\section{WELL HSB 85A}

$\begin{array}{lll}\text { SRS Coord. } & & \text { Lat/Longitude } \\ \text { N73791.9 } & & 33.285152^{\circ} \mathrm{N} \\ \text { E58943.4 } & 81.654930^{\circ} \mathrm{W}\end{array}$

SAMPLE DATE

$01 / 13 / 99$

FIELDDATA

Constituents

Water elevation

pH

Sp. conductance

Water temperature

Alkalinity as $\mathrm{CaCO} 3$

Turbidity

Volumes purged

Sampling code

Synchronous water level
Screen Zone Elevation

$71.1-61.1 \mathrm{ft} \mathrm{msl}$

Too of Casing

$294.4 \mathrm{ft} \mathrm{msl}$

$04 / 21 / 99$
Casing Pump

4" PVC
Screen Zone

Gordon

\section{ANALYTICAL DATA}

Inorganic Constituents

\begin{tabular}{|c|c|c|c|c|c|c|c|c|c|c|c|c|c|}
\hline Constituents & 1099 & Mod & Filt. & STH & DF & Lab & 2099 & Mod & Fill. & S드 브 & DE & Lab & Unit \\
\hline $\begin{array}{l}\text { Aluminum, total recoverable } \\
\text { Antimony, total recoverable } \\
\text { Arsenic, total recoverable } \\
\text { Barium, total recoverable } \\
\text { Berylium, total recoverable }\end{array}$ & $\begin{array}{l}<15 \\
<0.20 \\
<3.0 \\
29\end{array}$ & $\begin{array}{l}U / I \\
U / I \\
U / I \\
I /\end{array}$ & $\begin{array}{l}<15 \\
<0.20 \\
<3.0\end{array}$ & & $\begin{array}{l}1 \\
1 \\
1 \\
1\end{array}$ & $\begin{array}{l}\mathrm{GE} \\
\mathrm{GE} \\
\mathrm{GE} \\
\mathrm{GE}\end{array}$ & $<15$ & $\mathrm{U} / /$ & $<15$ & & 1 & GE & $\begin{array}{l}\mu g / L \\
\mu g / \\
\mu g h \\
\mu g h\end{array}$ \\
\hline $\begin{array}{l}\text { Cadmium, total recoverable } \\
\text { Chromium, total recoverable } \\
\text { Cobalt, total recoverable } \\
\text { Copper, total recoverable } \\
\text { Cyanide }\end{array}$ & $\begin{array}{l}<1.0 \\
1.4 \\
<0.11 \\
5.4 \\
<10\end{array}$ & $\begin{array}{l}\mathrm{U} / / \\
\mathrm{J} / / \\
\mathrm{JU} / / 4 \\
l / \\
\mathrm{U} / /\end{array}$ & $\begin{array}{l}<1.0 \\
\text { NDD } \\
<0.20\end{array}$ & & $\begin{array}{l}1 \\
1 \\
1 \\
1 \\
1\end{array}$ & $\begin{array}{l}\text { GE } \\
\text { GE } \\
\text { GE } \\
\text { GE } \\
\text { GE }\end{array}$ & $<0.33$ & $\mathrm{JU} / \mathrm{N} / 4$ & $<1.0$ & & 1 & GE & $\begin{array}{l}\mu g / \\
\mu g h \\
\mu g / L \\
\mu g h\end{array}$ \\
\hline $\begin{array}{l}\text { Cyanide } \\
\text { Lead, total recoverable }\end{array}$ & 0.61 & $\begin{array}{l}\mathrm{U} / / \\
\mathrm{J} / \mathrm{I}\end{array}$ & $\begin{array}{l}<10 \\
\text { NDD }\end{array}$ & & $\begin{array}{l}1 \\
1\end{array}$ & GE & 0.63 & $\mathrm{~J} / \mathrm{V}$ & NDD & & 1 & GE & $\begin{array}{l}\mu g / \\
\mu g / L\end{array}$ \\
\hline $\begin{array}{l}\text { Mercury, total recoverable } \\
\text { Nickel, total recoverable }\end{array}$ & $\begin{array}{l}<0.20 \\
1.9\end{array}$ & $\begin{array}{l}U / I \\
\mathrm{~J} / \mathrm{I}\end{array}$ & $\begin{array}{l}<0.20 \\
\text { NDD }\end{array}$ & & $\begin{array}{l}1 \\
1\end{array}$ & $\begin{array}{l}\text { GE } \\
\text { GE }\end{array}$ & $<0.20$ & $u / /$ & $<0.20$ & & 1 & GE & $\operatorname{lgg}^{\mu} / 2$ \\
\hline $\begin{array}{l}\text { Nitrate as nitrogen } \\
\text { Nitrate-nitrite as nitrogen }\end{array}$ & 200 & $\mathrm{~J} / \mathrm{L} / \mathrm{I}$ & NDD & & 1 & GE & 30 & $\mathrm{~J} / \mathrm{V}$ & NDD & & 1 & GE & $\mu g / L$ \\
\hline Selenium, total recoverable & $<5.0$ & $\mathrm{U} / /$ & $<5.0$ & & 1 & GE & & & & & & & $\mu g h$ \\
\hline Silver, total recoverable & $<1.0$ & $U / /$ & $<1.0$ & & 1 & GE & & & & & & & \\
\hline Thallium, total recoverable & $<0.023$ & $\mathrm{JU} / / 4$ & $<2.5$ & & 1 & GE & & & & & & & \\
\hline $\begin{array}{l}\text { Vanadium, total recoverable } \\
\text { Zinc, total recoverable }\end{array}$ & $\begin{array}{l}<10 \\
<10\end{array}$ & $\begin{array}{l}\mathrm{U} / / \\
\mathrm{U} / /\end{array}$ & $\begin{array}{l}<10 \\
<10\end{array}$ & & $\begin{array}{l}1 \\
1\end{array}$ & $\begin{array}{l}\text { GE } \\
\text { GE }\end{array}$ & & & & & & & \\
\hline Organic Constituents & & & & & & & & & & & & & \\
\hline Constituents & 1099 & Mod & Filt. & ST보 & DF & Lab & $\underline{2099}$ & Mod & Fill. & SI H & DF & Lab & Unit \\
\hline Benzene & $<1.0$ & JU/NO & $<1.0$ & & 1 & GE & & & & & & & pgl \\
\hline Bis(2-ethylhexyl) phthalate & $<10$ & & $<10$ & & 1 & GE & & & & & & & $g / 2$ \\
\hline $\begin{array}{l}\text { Dichloromethane } \\
\text { Phenols }\end{array}$ & $\begin{array}{l}<3.8 \\
<5.0\end{array}$ & $\begin{array}{l}\text { JU/NOB } \\
\text { U/I }\end{array}$ & $\begin{array}{l}<5.0 \\
<5.0\end{array}$ & & $\begin{array}{l}1 \\
1\end{array}$ & $\begin{array}{l}\text { GE } \\
\text { GE }\end{array}$ & & & & & & & Hgh \\
\hline Tetrachloroethylene & $<1.0$ & JU/UO & $<1.0$ & & 1 & GE & & & & & & & \\
\hline Trichloroethylene & $<1.0$ & JU/UO & $<1.0$ & & 1 & GE & & & & & & & \\
\hline Trichlorofluoromethane & $<5.0$ & JU/ & $<5.0$ & & 1 & & & & & & & & \\
\hline
\end{tabular}

Notes:

- exceeded holding time

- exceeded groundwater protection or monitoring constituent standard (See Appendix A.)

NA - Not applicable. Applies to beta dose and sum of betas if there are no beta-emitting radionuclides above detection limits; to sum of alphas if there are no alphaemitting radionuclides above detection limits; and to total radium if neither radium-226 or radium-228 was above detection limit

UAZ_UTRA - Upper Aquifer Zone of the Upper Three Runs Aquifer, LAZ UTRA - Lower Aquifer Zone of the Upper Three Runs Aquifer, Gordon - Gordon Aquifer 


\section{WELL HSB 85A (cont.)}

Radioactive Constituents

\begin{tabular}{|c|c|c|c|c|c|c|c|c|c|c|c|c|c|}
\hline Constituents & 1099 & Mod & Filt. & STH & $\underline{\mathrm{DF}}$ & Lab & 2099 & Mod & Filt. & ST $H$ & DF & Lab & Unit \\
\hline $\begin{array}{l}\text { Americium-241 } \\
\text { Beta dose }\end{array}$ & $\begin{array}{l}<2.3 E-02 \\
N A\end{array}$ & $\mathrm{U} / \mathrm{I}$ & $<1.8 E-01$ & & 1 & GP & & & & & & & $\mathrm{pCin}$ \\
\hline Carbon-14 & $<1.2 E+\infty$ & $u / I$ & $<6.7 E+\infty 0$ & & 1 & GP & & & & & & & pCil \\
\hline Cesium-137 & $<-2.2 E+00$ & $\mathbf{U} / \prime$ & $<4.5 E+\infty 0$ & & 1 & GP &. & & & & & & pCin \\
\hline Cobalt 60 & $<1.7 E+\infty$ & $\mathbf{U} / I$ & $<5.9 E+\infty 0$ & & 1 & GP & & & & & & & pcin \\
\hline Curium-242 & $<-1.3 E-02$ & $\mathbf{U} / \prime$ & $<1.7 E-01$ & & 1 & GP & & & & & & & pCir \\
\hline Cunium-243/244 & $<2.4 E-02$ & $\mathrm{u} / /$ & $<7.1 E-02$ & & 1 & GP & & & & & & & pCir \\
\hline Curium-245/246 & $<-1.2 E-02$ & $\mathrm{U} / /$ & $<1.5 E-01$ & & $i$ & $\mathrm{GP}$ & & & & & & & $\mathrm{pCi} / \mathrm{L}$ \\
\hline $\begin{array}{l}\text { Gross alpha } \\
\text { lodine- } 129\end{array}$ & $\begin{array}{l}<1.3 E-01 \\
<6.1 E-02\end{array}$ & $\begin{array}{l}u / \prime \\
u / \prime\end{array}$ & $\begin{array}{l}<8.0 E-01 \\
<8.1 E-01\end{array}$ & & $\begin{array}{l}1 \\
1\end{array}$ & $\begin{array}{l}\text { GP } \\
\text { GP }\end{array}$ & $3.9 E+\infty$ & $\|$ & & & 1 & GP & pCill \\
\hline Nonvolatile beta & $1.3 E+\infty$ & $\mathrm{J} / \mathrm{V}$ & NDD & & 1 & GP & $9.2 E+02$ & $\|$ & & $\mathbf{a}$ & 1 & GP & pCin \\
\hline Plutonium-238 & $<7.4 \mathrm{E}-02$ & $\mathbf{U} / I$ & $<1.0 E-01$ & & 1 & GP & & & & & & & pCiL \\
\hline Plutonium-239/240 & $<4.8 E-02$ & $\mathrm{U} / /$ & $<1.0 \mathrm{E}-01$ & & 1 & GP & & & & & & & pCin \\
\hline Radium-226 & $7.4 E-01$ & $J / V$ & NDD & & 1 & GP & & & & & & & pCin \\
\hline $\begin{array}{l}\text { Radium-228 } \\
\text { Total radium }\end{array}$ & $\begin{array}{l}<4.0 E-01 \\
N A\end{array}$ & JURルI & $<1.0 E+00$ & & $i$ & GP & & & & & & & pcin \\
\hline Strontium-90 & $<8.2 E-01$ & $u / \prime$ & $<1.4 E+00$ & & 1 & GP & & & & & & & pCin \\
\hline Technetium-99 & $<-8.6 E+\infty 0$ & $\mathrm{U} / /$ & $<2.0 E+01$ & & 1 & GP & & & & & & & pcin \\
\hline Thorium-228 & $<2.0 \mathrm{E}-02$ & $\mathrm{U} / /$ & $<1.2 \mathrm{E}-01$ & & 1 & $\overrightarrow{\mathrm{GP}}$ & & & & & & & peil \\
\hline Thorium-230 & $<3.6 \mathrm{E}-02$ & $U / /$ & $<5.1 E-02$ & & 1 & GP & & & & & & & pCin \\
\hline $\begin{array}{l}\text { Thorium-232 } \\
\text { Sum of alphas } \\
\text { Sum of betas }\end{array}$ & $\begin{array}{l}<8.0 E-03 \\
\text { NA } \\
\text { NA }\end{array}$ & $\mathrm{U} / /$ & $<2.4 E-02$ & & 1 & $\overrightarrow{G P}$ & & & & & & & pCir \\
\hline Tritium & $<-6.1 E-02$ & $\mathrm{U} / /$ & $<6.2 E-01$ & & 1 & GP & $<-2.5 E-01$ & $U / /$ & $<6.5 E-01$ & & 1 & GP & $\mathrm{pCi} / \mathrm{mL}$ \\
\hline Uranium-233/234 & $5.0 E-02$ & $R / 14$ & $\mathbf{R e j}$ & & 1 & GP & & & & & & & pCin \\
\hline Uranium-235 & $<1.3 E-02$ & $\mathrm{U} / /$ & $<5.3 E-02$ & & 1 & GP & & & & & & & pein \\
\hline Uranium-238 & $<1.3 E-02$ & $\mathrm{U} / /$ & $<5.3 E-02$ & & 1 & GP & & & & & & & pein \\
\hline
\end{tabular}

Notes:

= exceeded holding time

- exceeded groundwater protection or monitoring constituent standard (See Appendix A.)

NA - Not applicable. Applies to beta dose and sum of betas if there are no beta-emitting radionuclides above detection limits; to sum of alphas if there are no atpha. emitting radionuclides above detection limits; and to total radium if neither radium-226 or radium-228 was above detection limit

UAZ_UTRA - Upper Aquifer Zone of the Upper Three Runs Aquifer, LAZ UTRA - Lower Aquifer Zone of the Upper Three Runs Aquifer, Gordon - Gordon Aquifer 
THIS PAGE WAS LEFT BLANK INTENTIONALLY. 
WSRC-TR-99-00147

Unclassified

APPENDIX D: TIME SERIES PLOTS 
THIS PAGE WAS LEFT BLANK INTENTIONALLY. 


\section{Cadmium Concentrations \\ Well Cluster FSB 79}

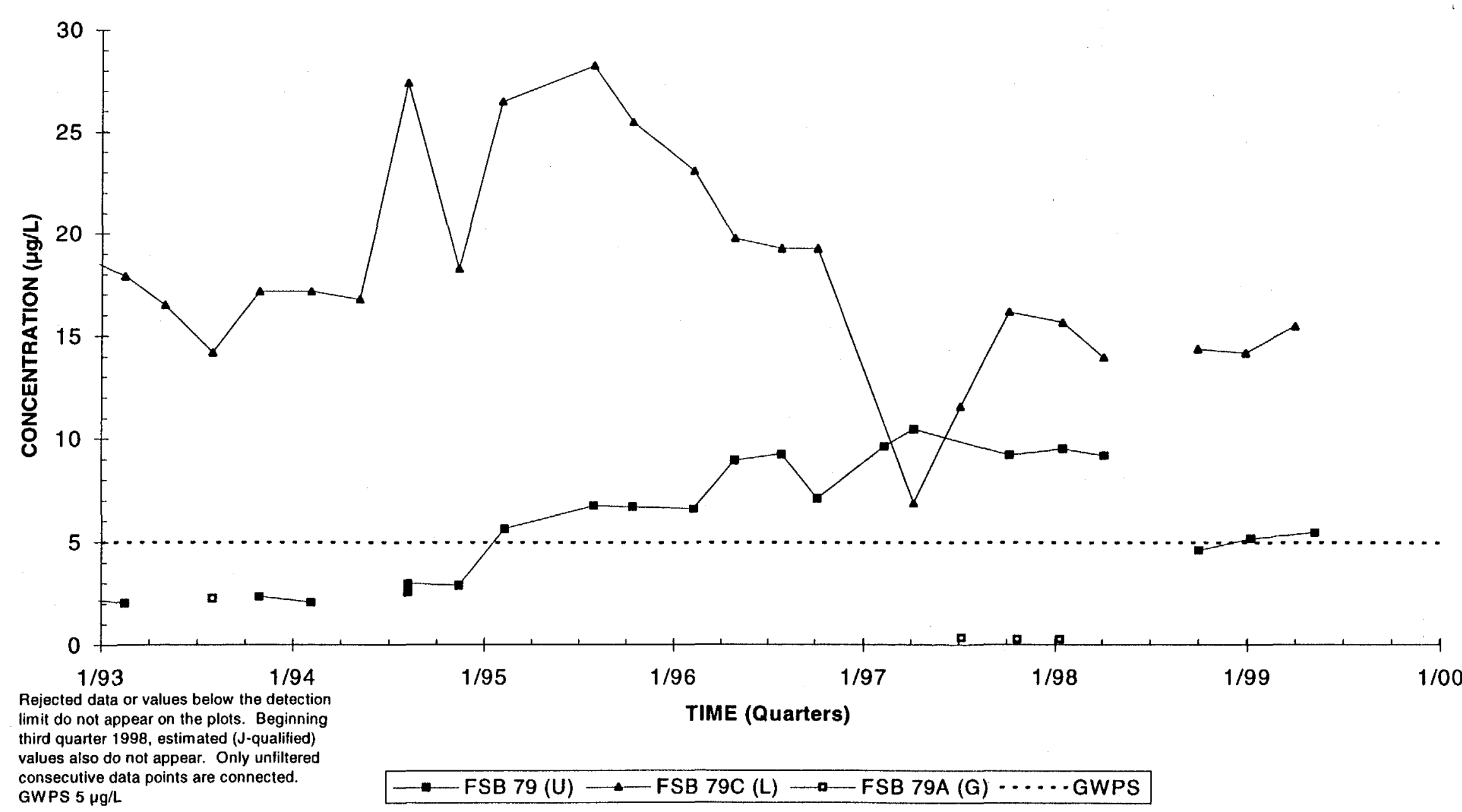

$U=$ Upper Aquifer Zone of the Upper Three Runs Aquifer, $L=$ Lower Aquifer Zone of the Upper Three Runs Aquifer, $G=$ Gordon Aquiler 


\section{Cadmium Concentrations \\ Well FSB 91D}

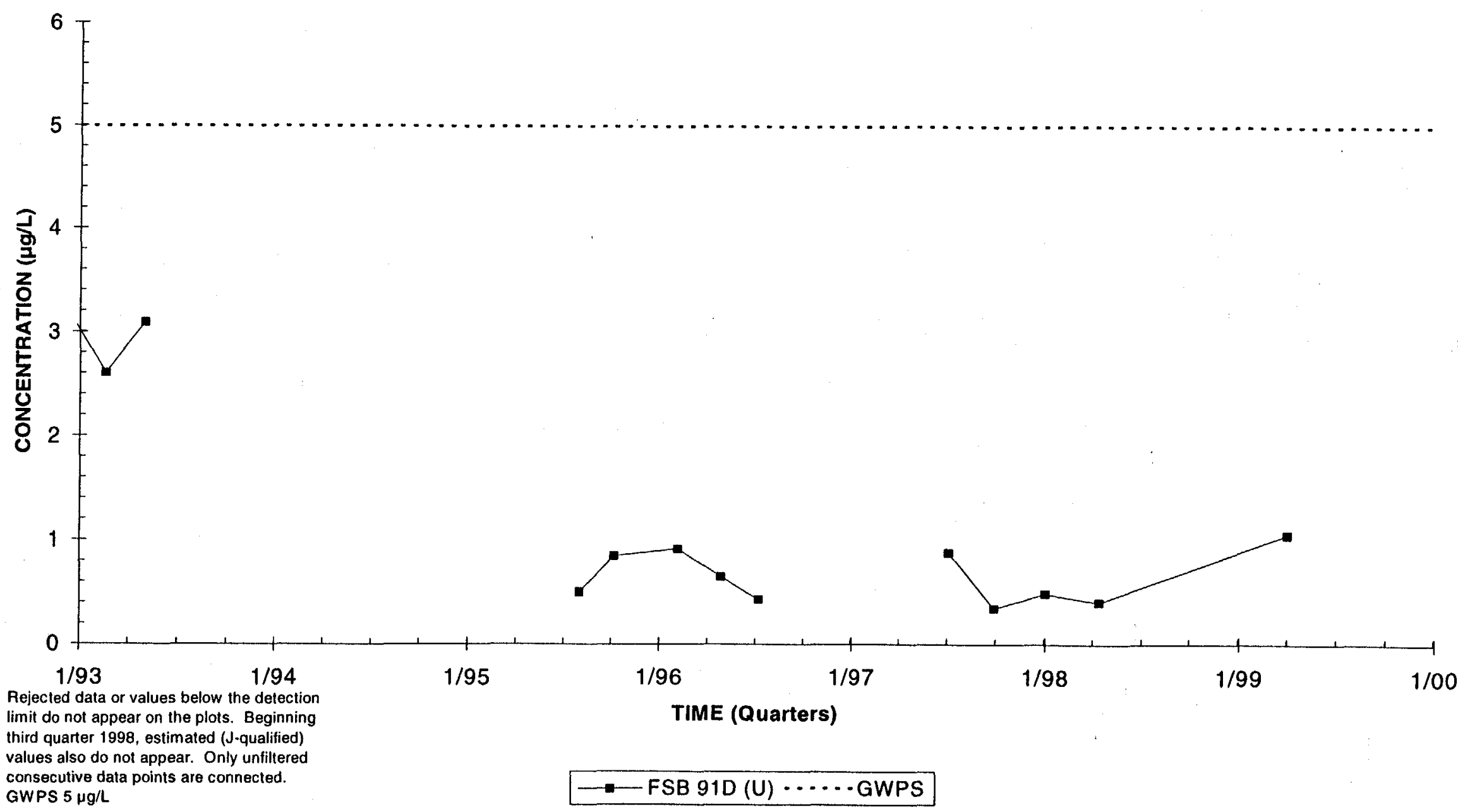

$U=$ Upper Aquifer Zone of the Upper Three Auns Aquifer, $L=$ Lower Aquifer Zone of the Upper Three Runs Aquiler, $G=$ Gordon Aquifer 


\section{Cadmium Concentrations \\ Well FSB 93D}

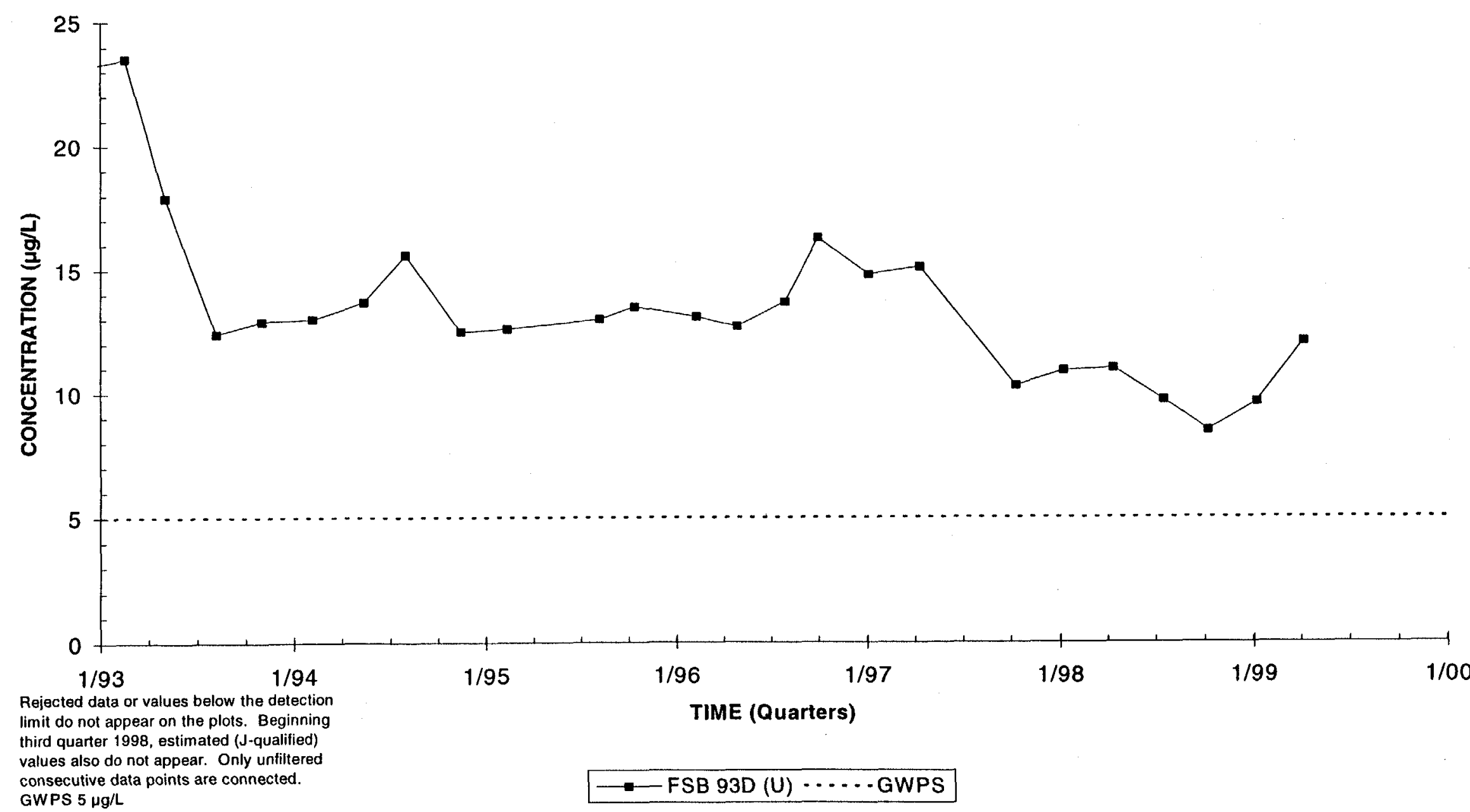

$U=$ Upper Aquifer Zone of the Upper Three Runs Aquiler; $L=$ Lower Aquifer Zone of the Upper Three Runs Aquifer; $G=$ Gordon Aquifer 


\section{Cadmium Concentrations Well FSB 95CR}

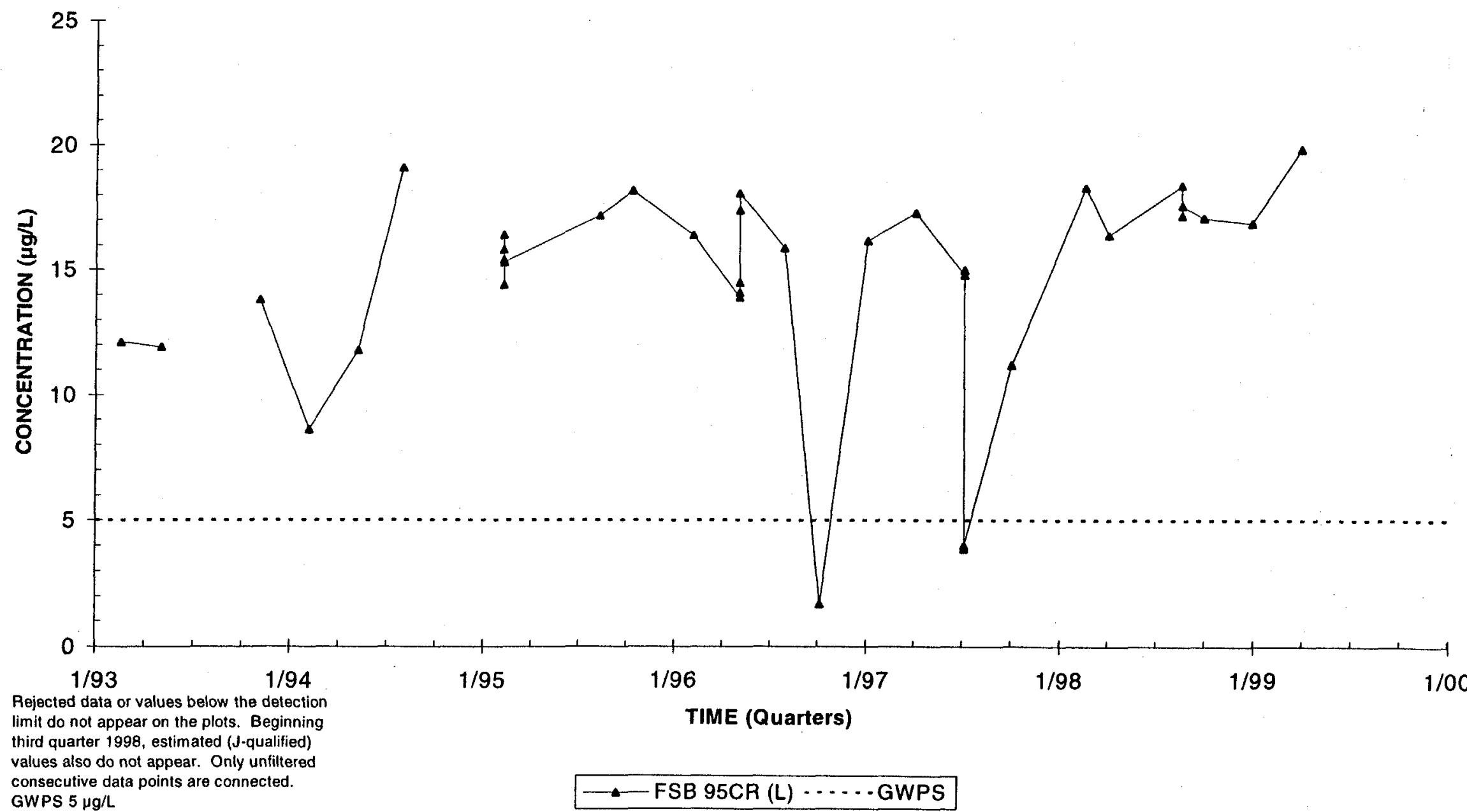

$U=$ Upper Aquifer Zone of the Upper Three Runs Aquifer, $L=$ Lower Aquifer Zone of the Upper Three Runs Aquiler, $G=$ Gordon Aquifer 


\section{Cadmium Concentrations \\ Well FSB102C}

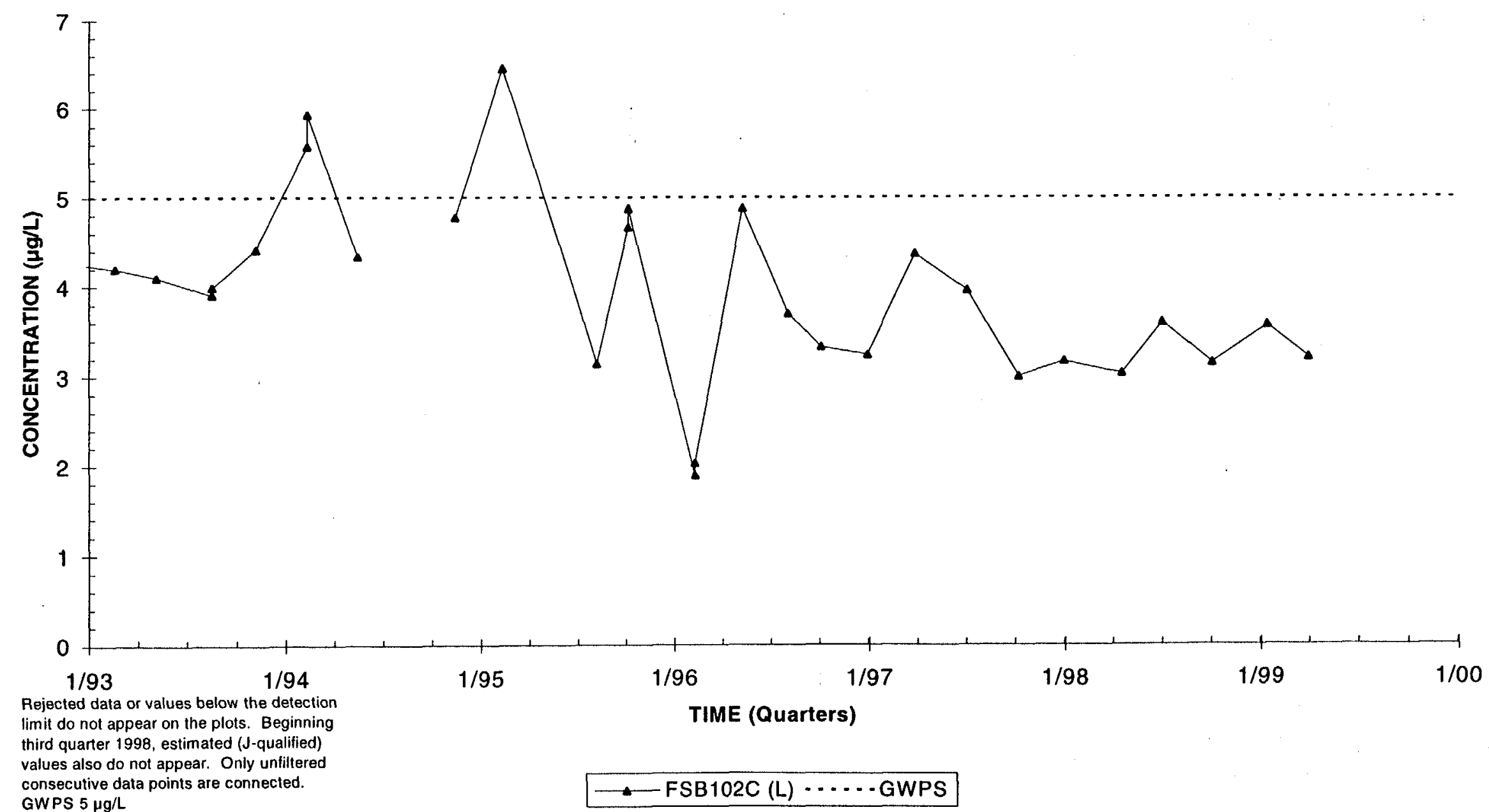

$U=$ Upper Aquifer Zone of the Upper Three Runs Aquifer, $L=$ Lower Aquifer Zone of the Upper Three Runs Aquifer, $G=$ Gordon Aquifer 


\section{Cadmium Concentrations}

Well Cluster FSB104

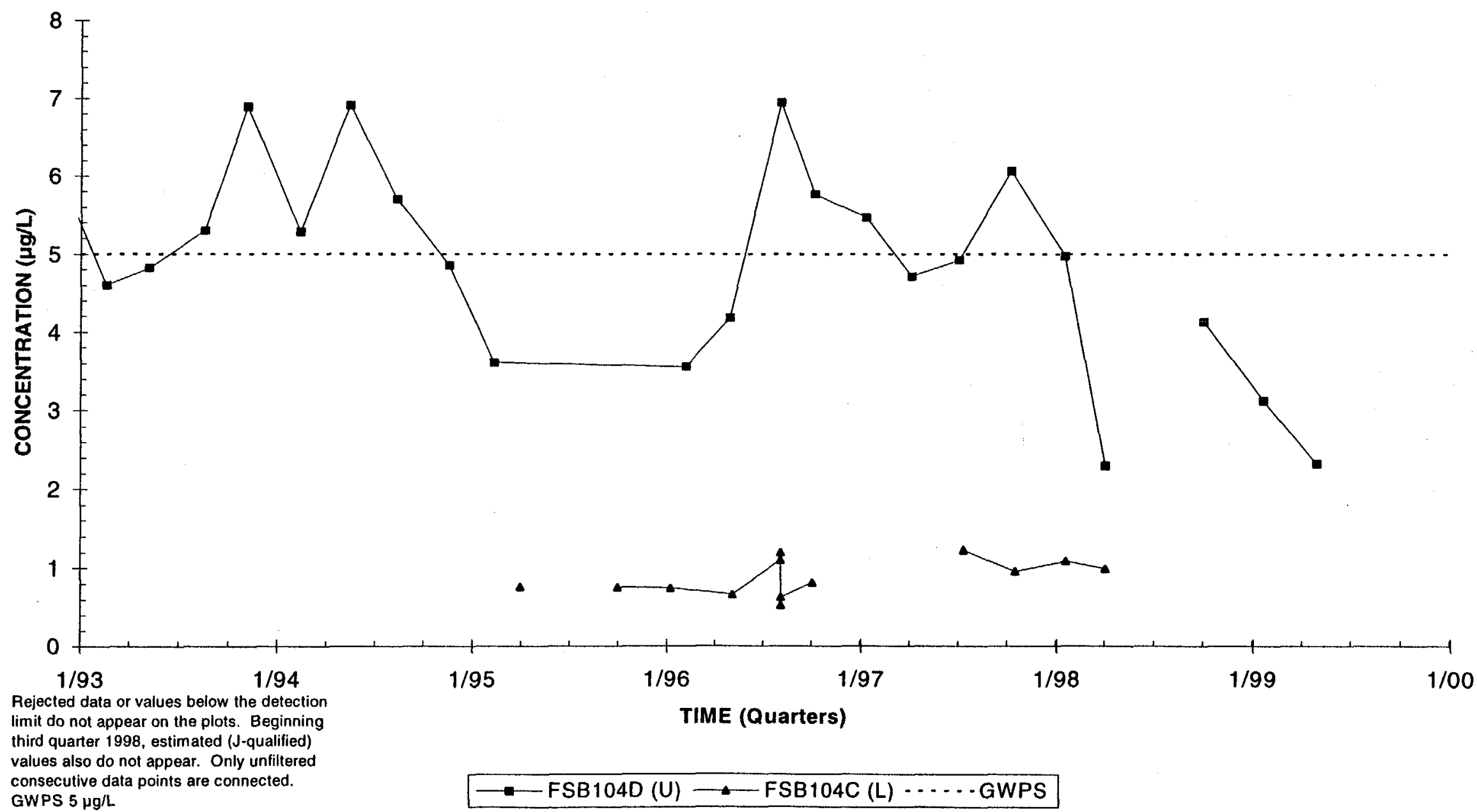

$\mathrm{U}=$ Upper Aquiler Zone of the Upper Three Runs Aquiler, $\mathrm{L}=$ Lower Aquiler Zone of the Upper Three Runs Aquiler, $\mathrm{G}=$ Gordon Aquifer 


\section{Cadmium Concentrations \\ Well Cluster FSB106}

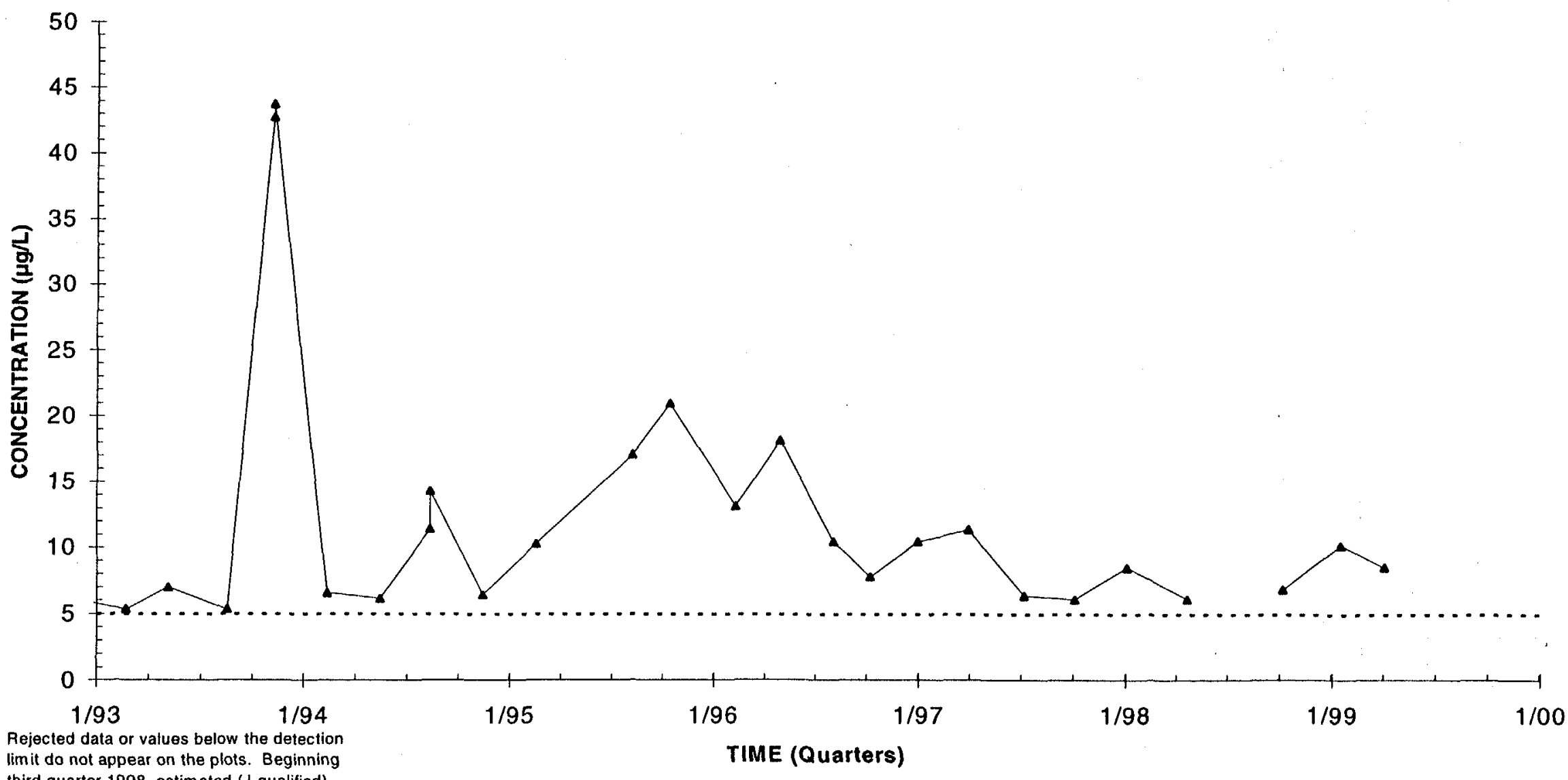

(J)

consecutive data points are connected.

$\rightarrow$ FSB106D (U) $\longrightarrow$ FSB106C (L) $\ldots \ldots$ GWPS 


\section{Cadmium Concentrations Well FSB112C}

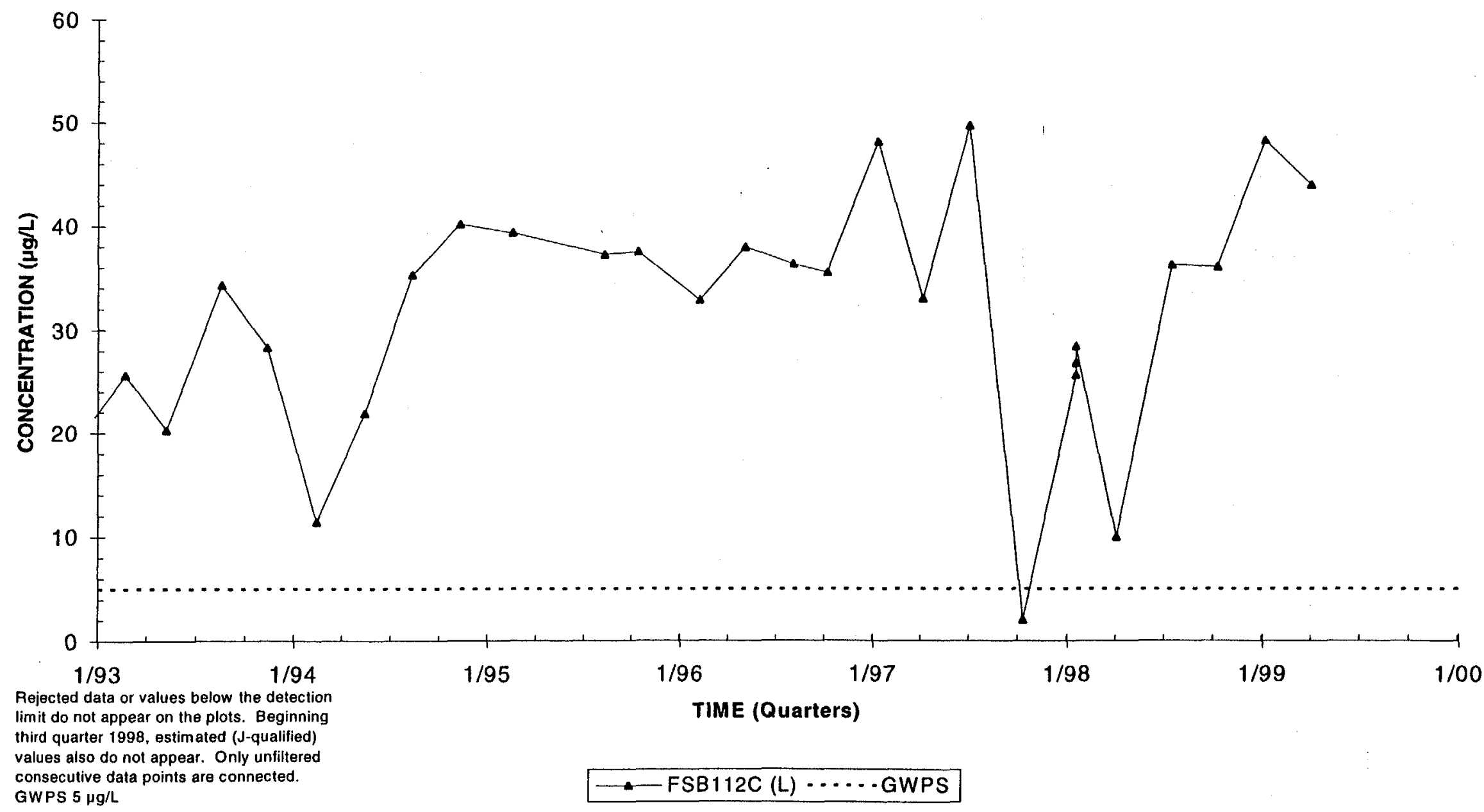

$\mathrm{U}=$ Upper Aquiter Zone of the Upper Three Runs Aquifer, $\mathrm{L}=$ Lower Aquifer Zone of the Upper Three Runs Aquifer, $\mathrm{G}=$ Gordon Aquiter 

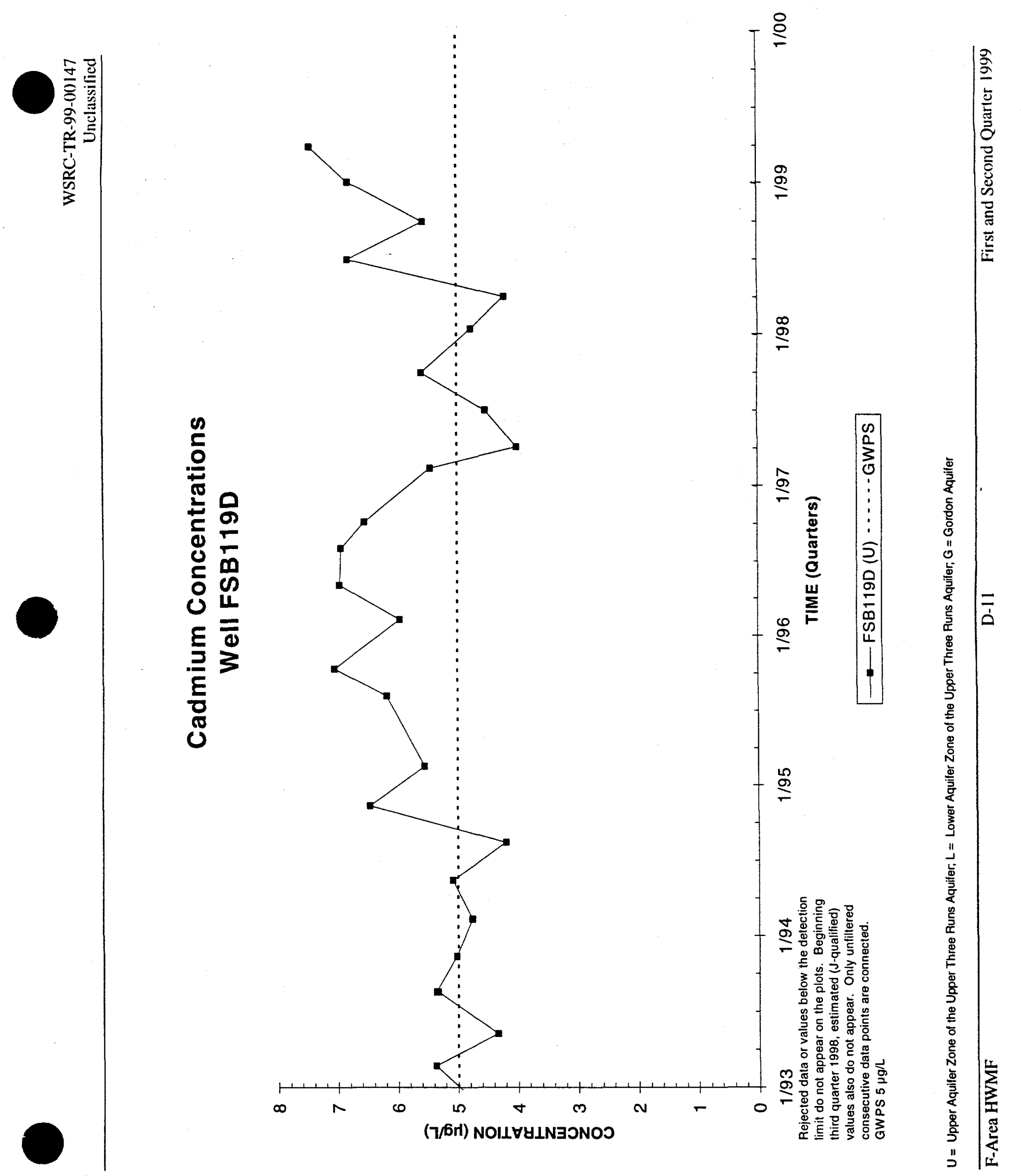


\section{Lead Concentrations \\ Well FSB 76}

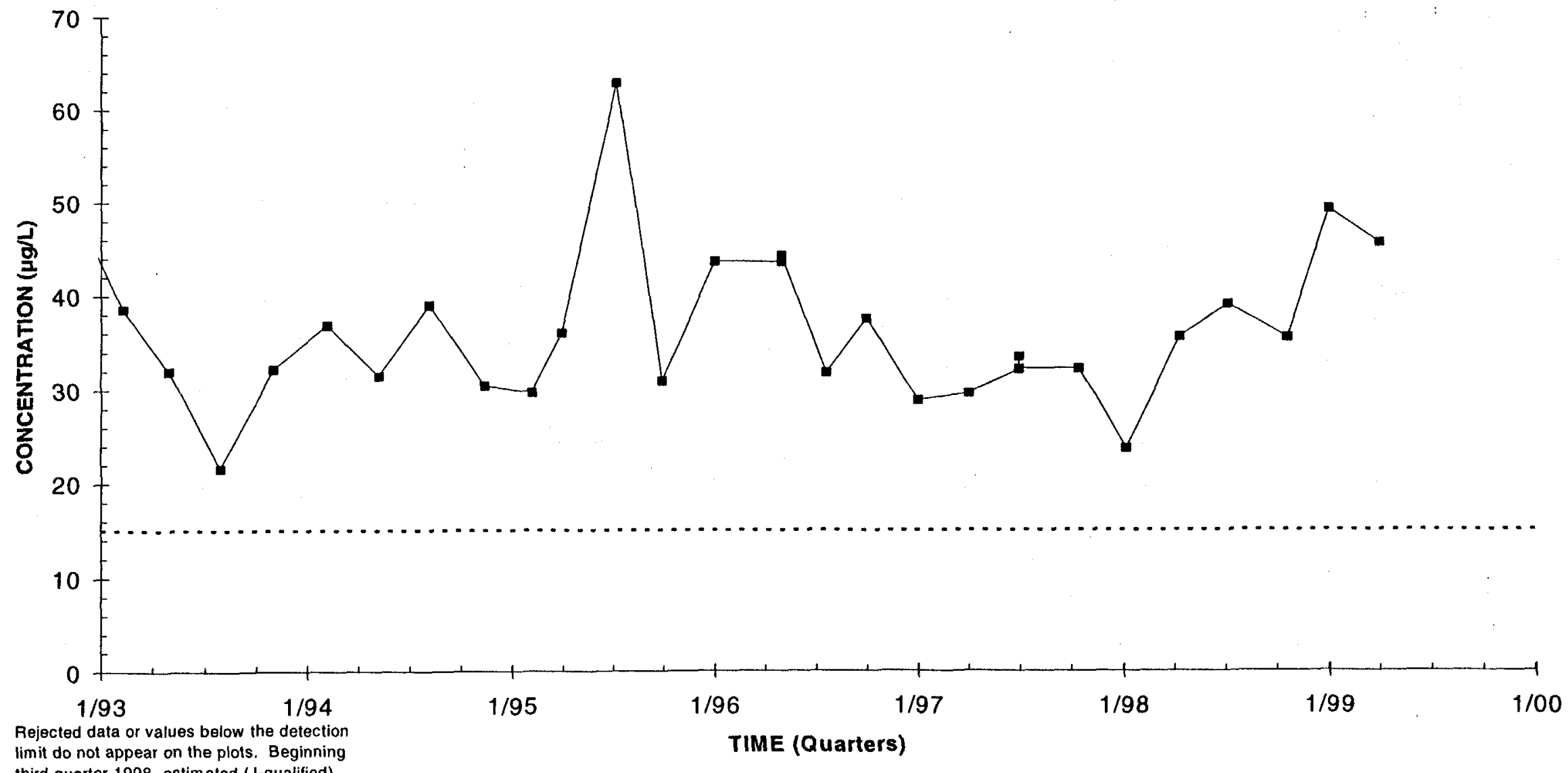

third quarter 1998, estimated (J-qualified)

values also do not appear. Only unfiltered

GWPS $15 \mu \mathrm{g} / \mathrm{L}$

GWPS

$U=$ Upper Aquifer Zone of the Upper Three Runs Aquifer, $L=$ Lower Aquiler Zone of the Up̀per Three Runs Aquifer, $G=$ Gordon Aquiler

F-Area HWMF




\section{Lead Concentrations Well FSB 77}

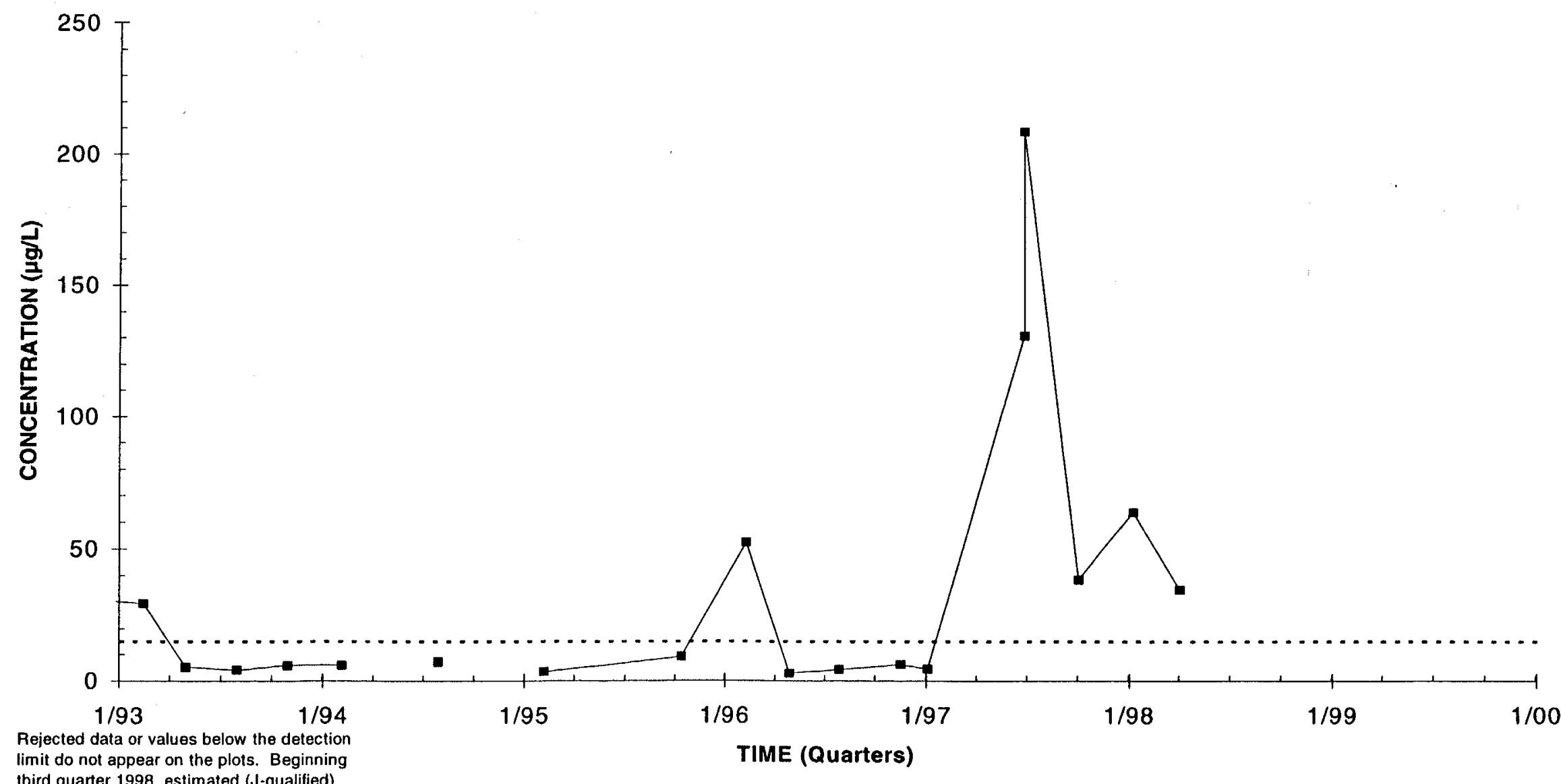

pear on the plots. Beg

Third quarter 1998, estimated (J-qualified)

consecutive data points are connected.

- FSB $77(\mathrm{U}) \cdots \cdots$ GWPS

GWPS $15 \mu \mathrm{g} / \mathrm{L}$

$\mathrm{U}=$ Upper Aquiler Zone of the Upper Three Runs Aquifer, $\mathrm{L}=$ Lower Aquifer Zone of the Upper Three Runs Aquifer, $G=$ Gordon Aquifer 


\section{Lead Concentrations \\ Well Cluster FSB 78}

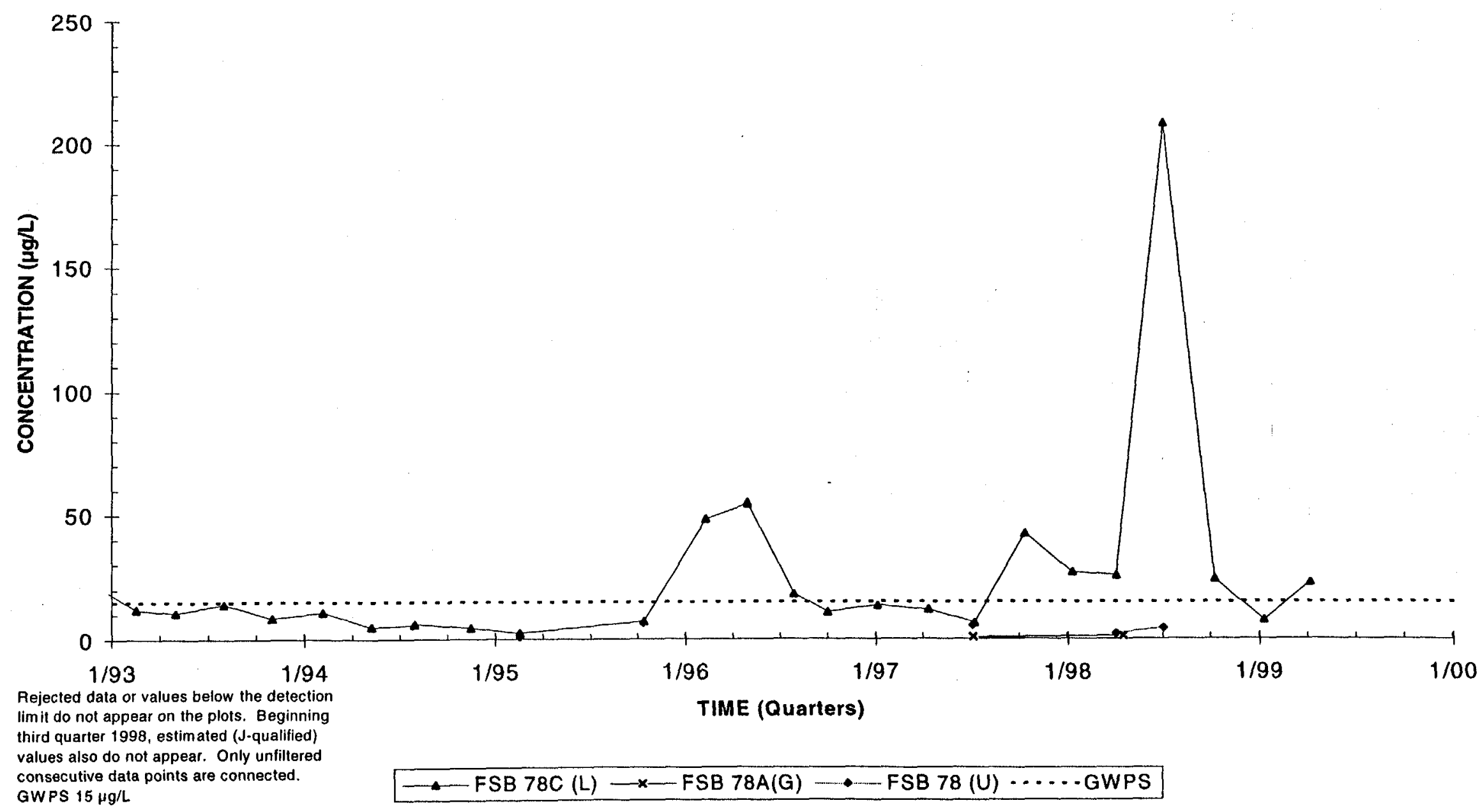

$U=$ Upper Aquiler Zone of the Upper Three Runs Aquiter, $L=$ Lower Aquiler Zone of the Upper Three Runs Aquiler, $G=G$ Gordon Aquiler 


\section{Lead Concentrations}

Well FSB 90D

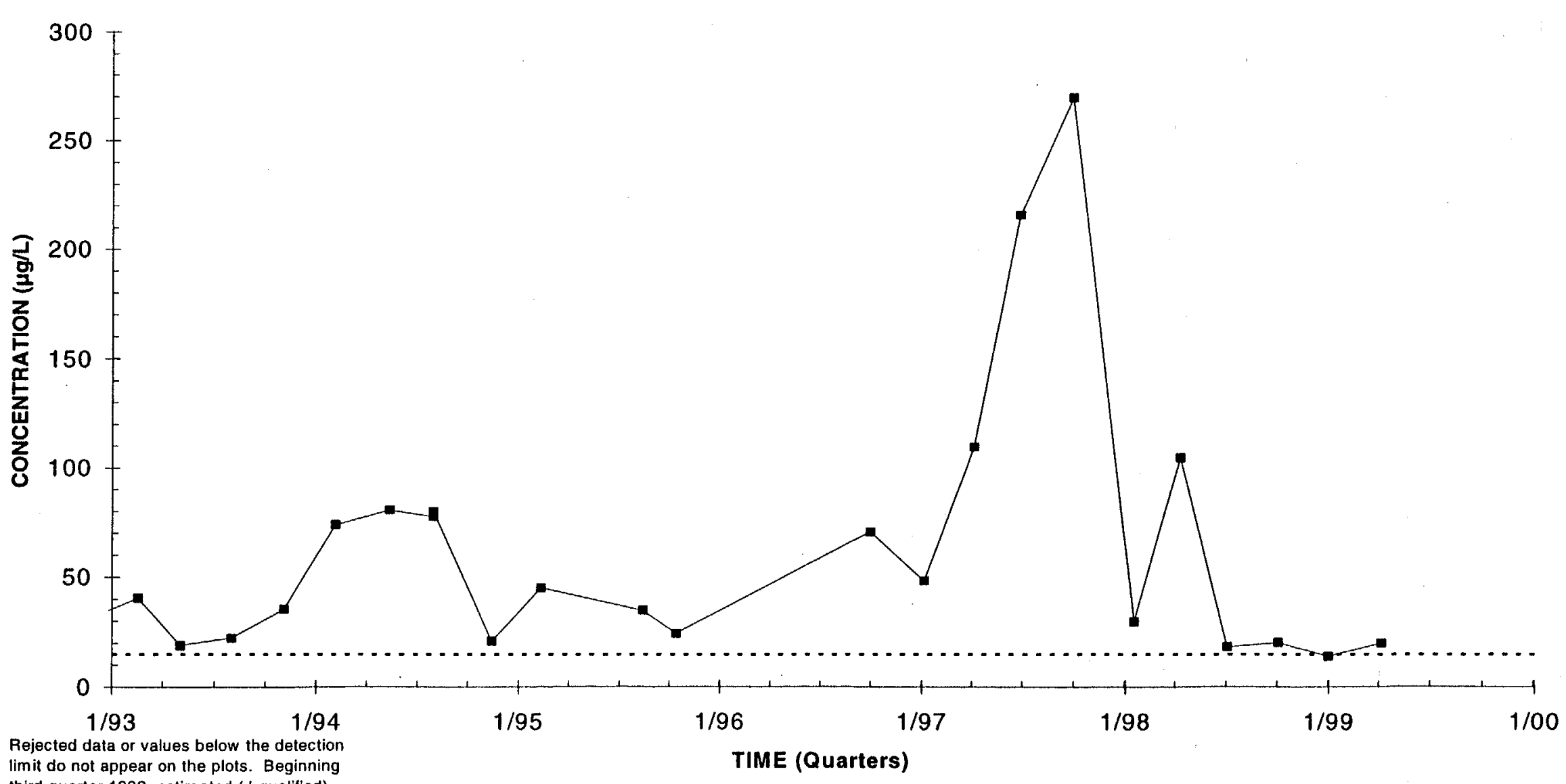

third quarter 1998, estimated ( $\mathrm{J}$-qualified)

values also do not appear. Only unfilte

GWPS $15 \mu \mathrm{g} / \mathrm{h}$ 


\section{Lead Concentrations \\ Well Cluster FSB 94}

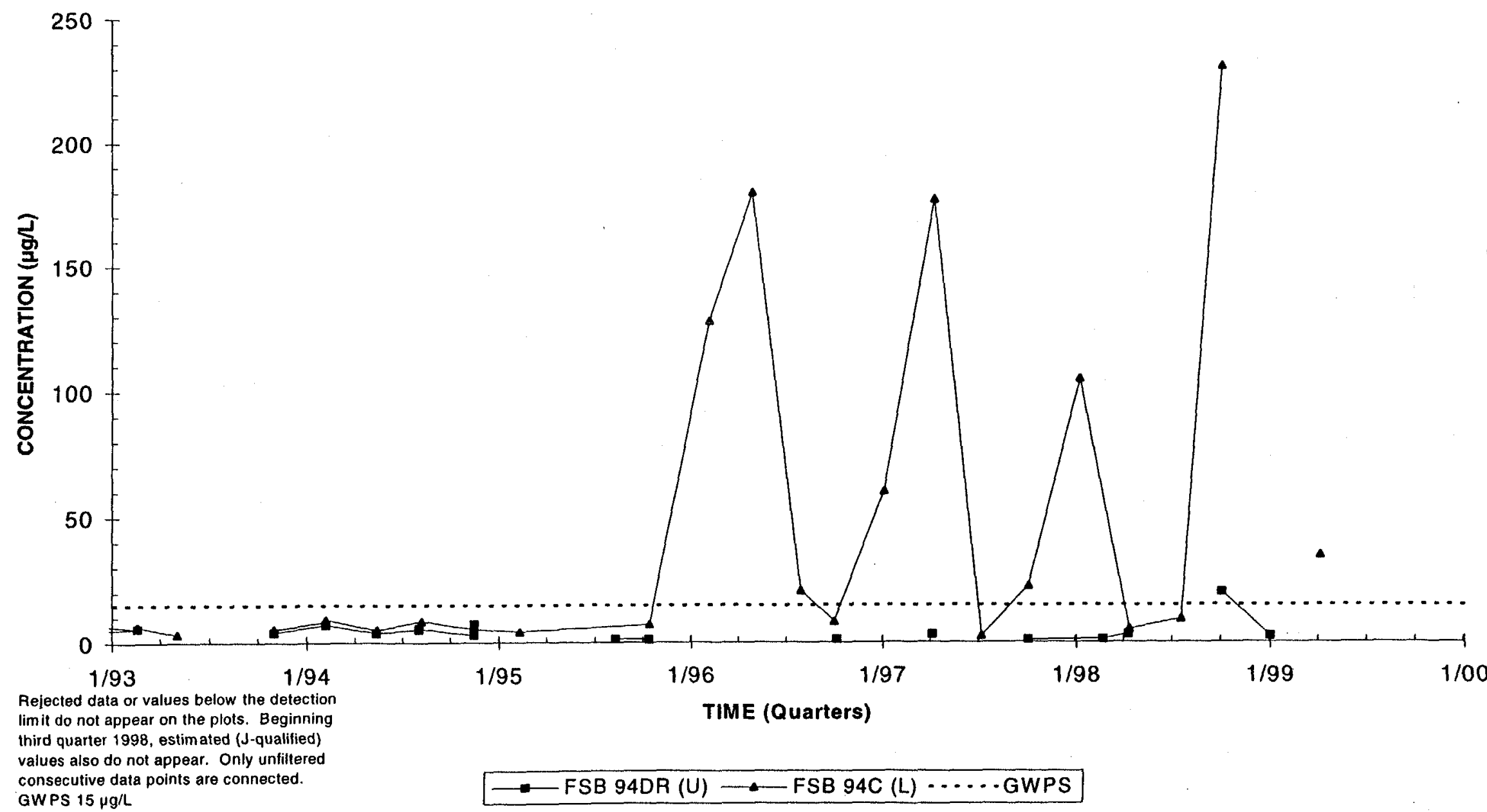

$\mathrm{U}=$ Upper Aquiler Zone of the Upper Three Runs Aquifer, $\mathrm{L}=$ Lower Aquiler Zone of the Upper Three Runs Aquifer, $G=$ Gordon Aquiler 


\section{Lead Concentrations \\ Well FSB 95DR}

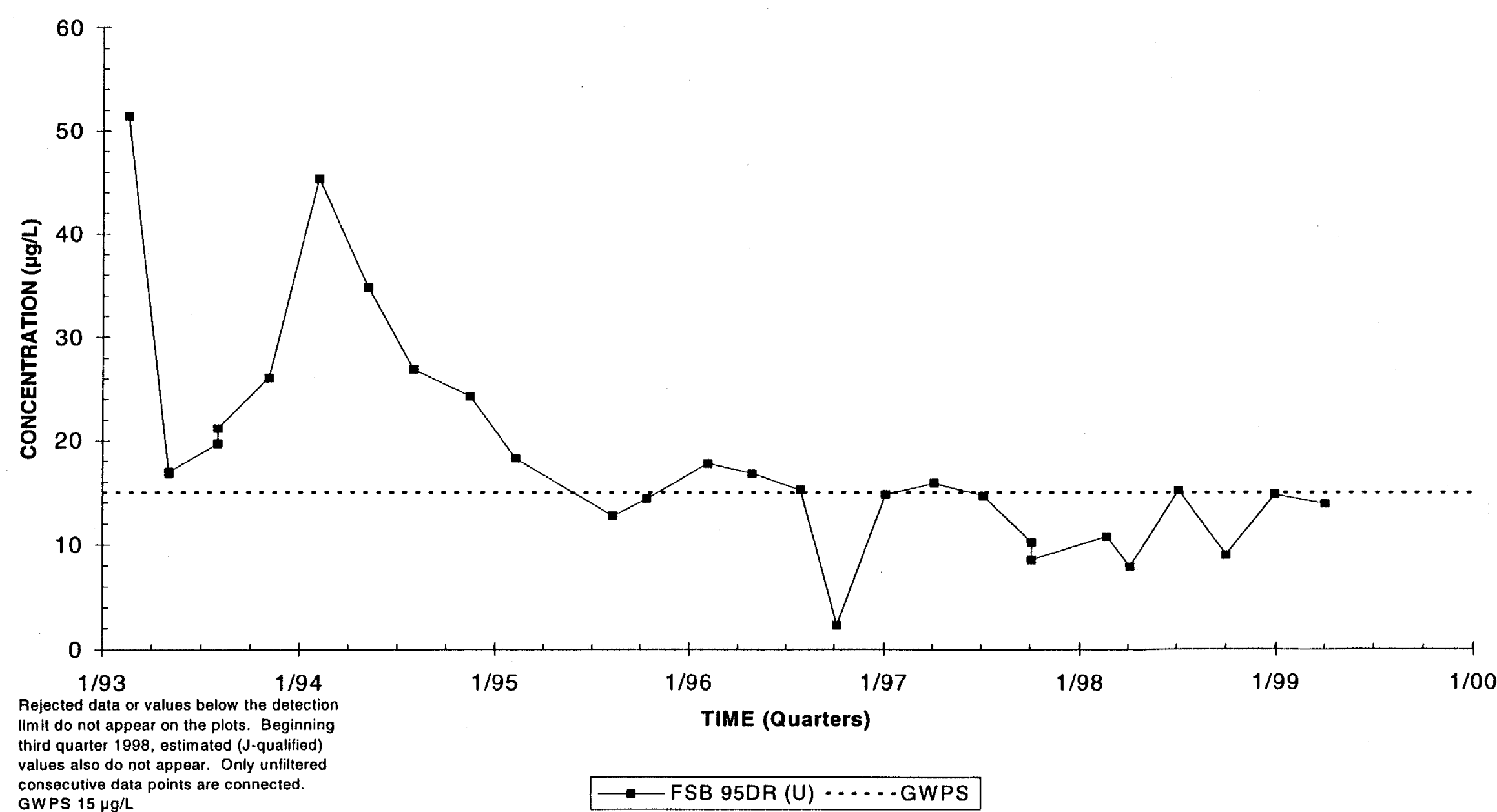

$U=$ Upper Aquifer Zone of the Upper Three Runs Aquifer, $L=$ Lower Aquifer Zone of the Upper Three Runs Aquifer, $G=$ Gordon Aquifer 


\section{Lead Concentrations \\ Well Cluster FSB 97}

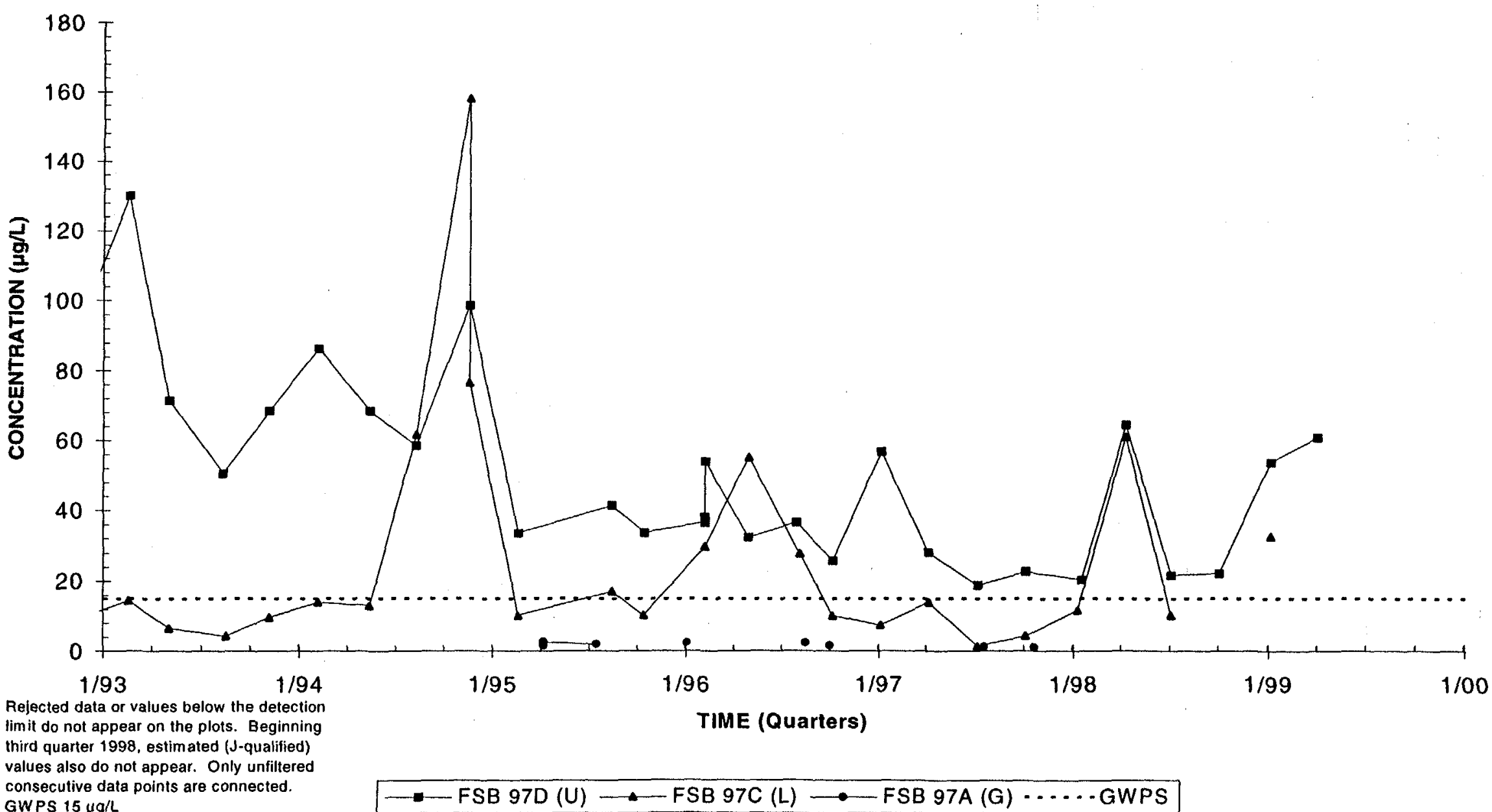

$U=$ Upper Aquifer Zone of the Upper Three Runs Aquifer, $L=$ Lower Aquifer Zone of the Upper Three Runs Aquiler; $G=$ Gordon Aquiler

F-Area HWMF




\section{Lead Concentrations Well FSB108D}

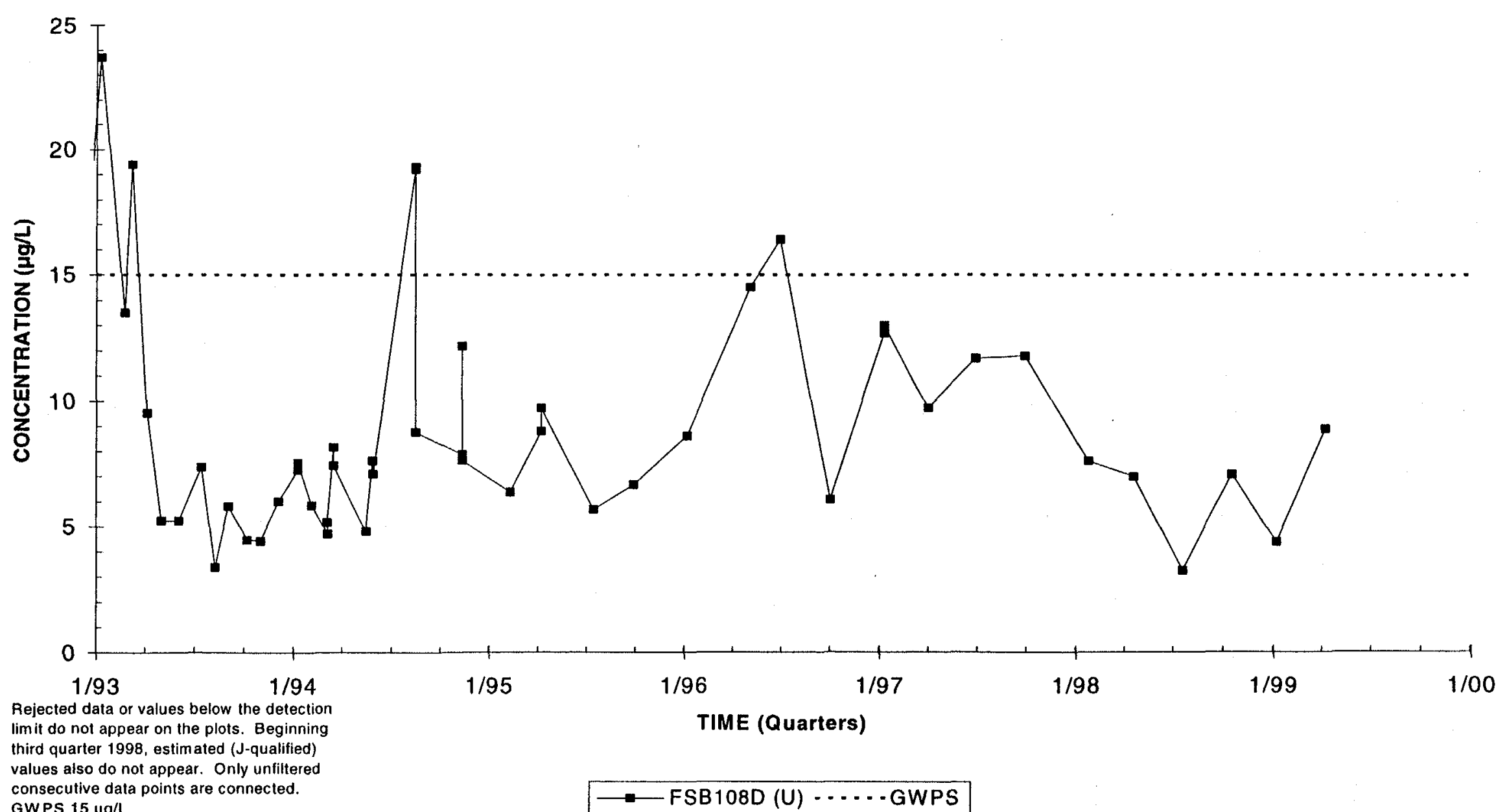

$U=$ Upper Aquifer Zone of the Upper Three Auns Aquiler, $L=$ Lower Aquifer Zone of the Upper Three Runs Aquiler, $G=$ Gordon Aquifer 


\section{Lead Concentrations \\ Well FSB119D}

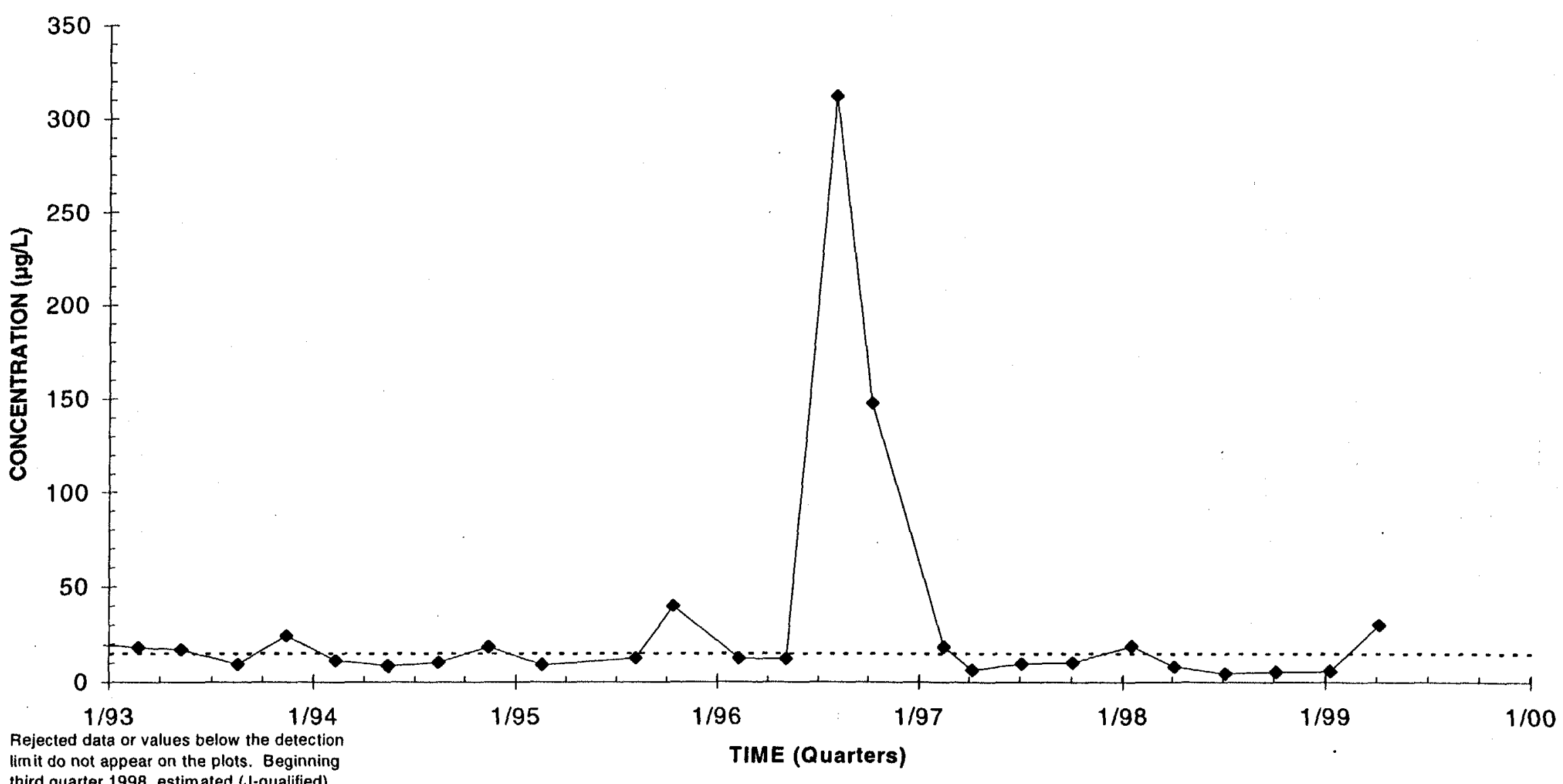

Hit do not appear on the plots. Beginning

values also do not appear. Only unfiltered

consecutive data points are connected.

GWPS $15 \mu \mathrm{g} / \mathrm{L}$

$\because$ FSB119D (U) $\ldots \ldots$ GWPS

$U=$ Upper Aquifer Zone of the Upper Three Runs Aquifer, $L=$ Lower Aquifer Zone of the Upper Three Runs Aquifer, $G=$ Gordon Aquiler 


\section{Tritium Activities Well Cluster FSB 79}

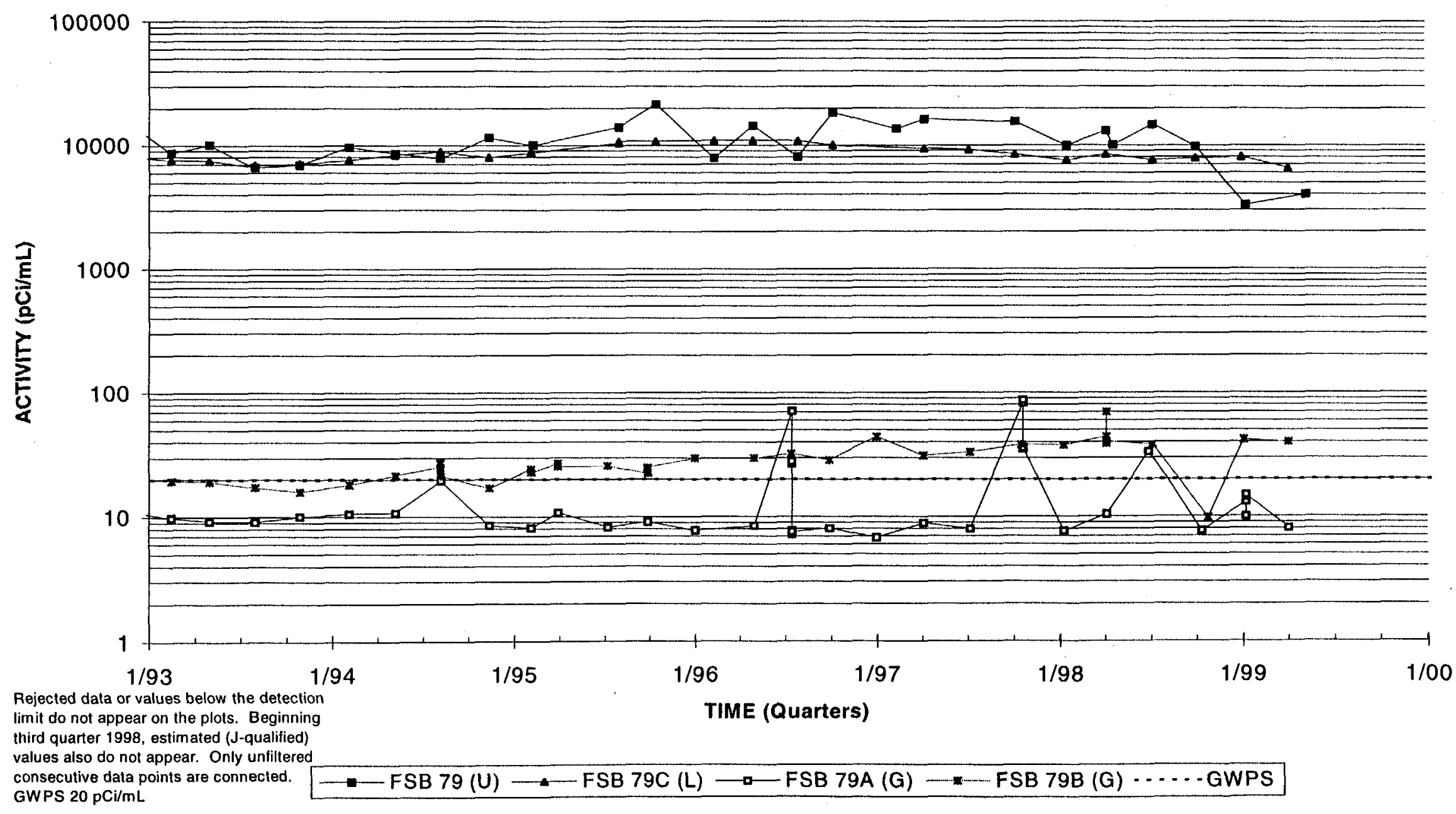

$U=$ Upper Aquiler Zone of the Upper Three Runs Aquifer, $L=$ Lower Aquifer Zone of the Upper Three Runs Aquiler; $G=$ Gordon Aquiler 


\section{Tritium Activities Well Cluster FSB 94}

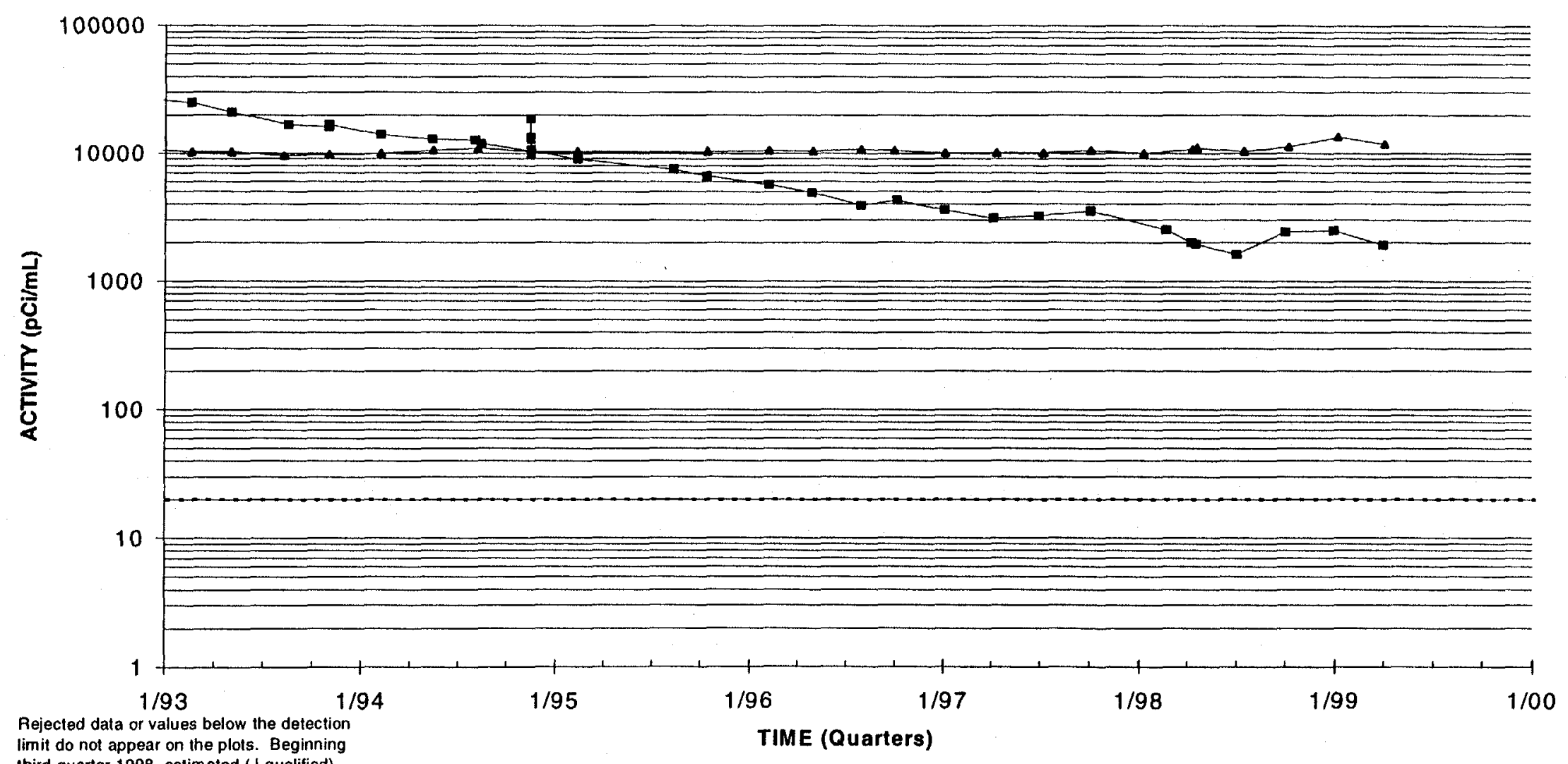

limit do not appear on the plots. Beginning

third quarter 1998, estimated (J-qualified)

values also do not appear. Only unfittered

consecutive data points are connected.

$\longrightarrow$ FSB 94DR (U) $\multimap$ FSB 94C (L) ..... GWPS 


\section{Tritium Activities}

\section{Well Cluster FSB 97}

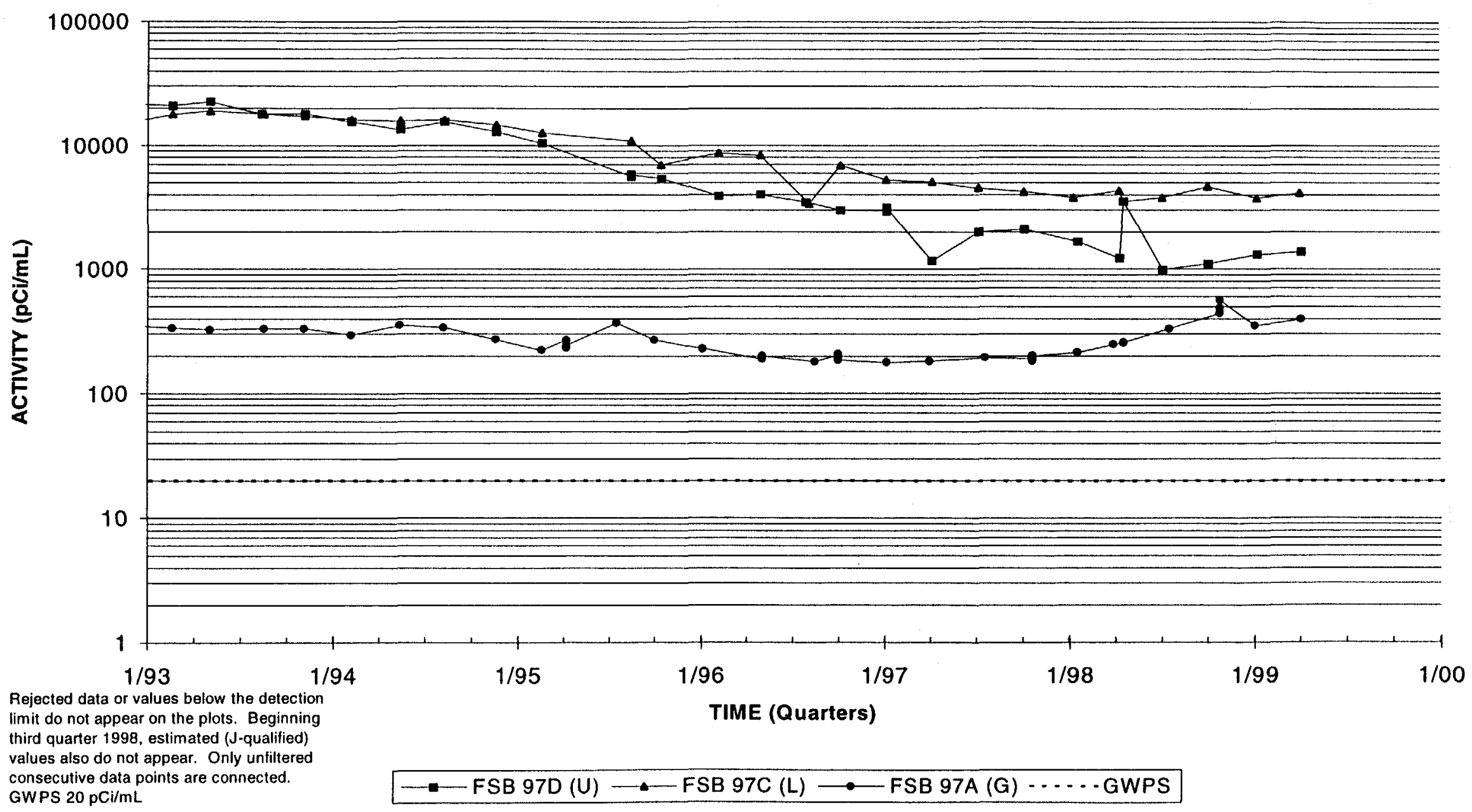

$\mathrm{U}=$ Upper Aquifer Zone of the Upper Three Runs Aquiter, $\mathrm{L}=$ Lower Aquifer Zone of the Upper Three Runs Aquifer; $\mathrm{G}=$ Gordon Aquifer 


\section{Tritium Activities \\ Well Cluster FSB 99}

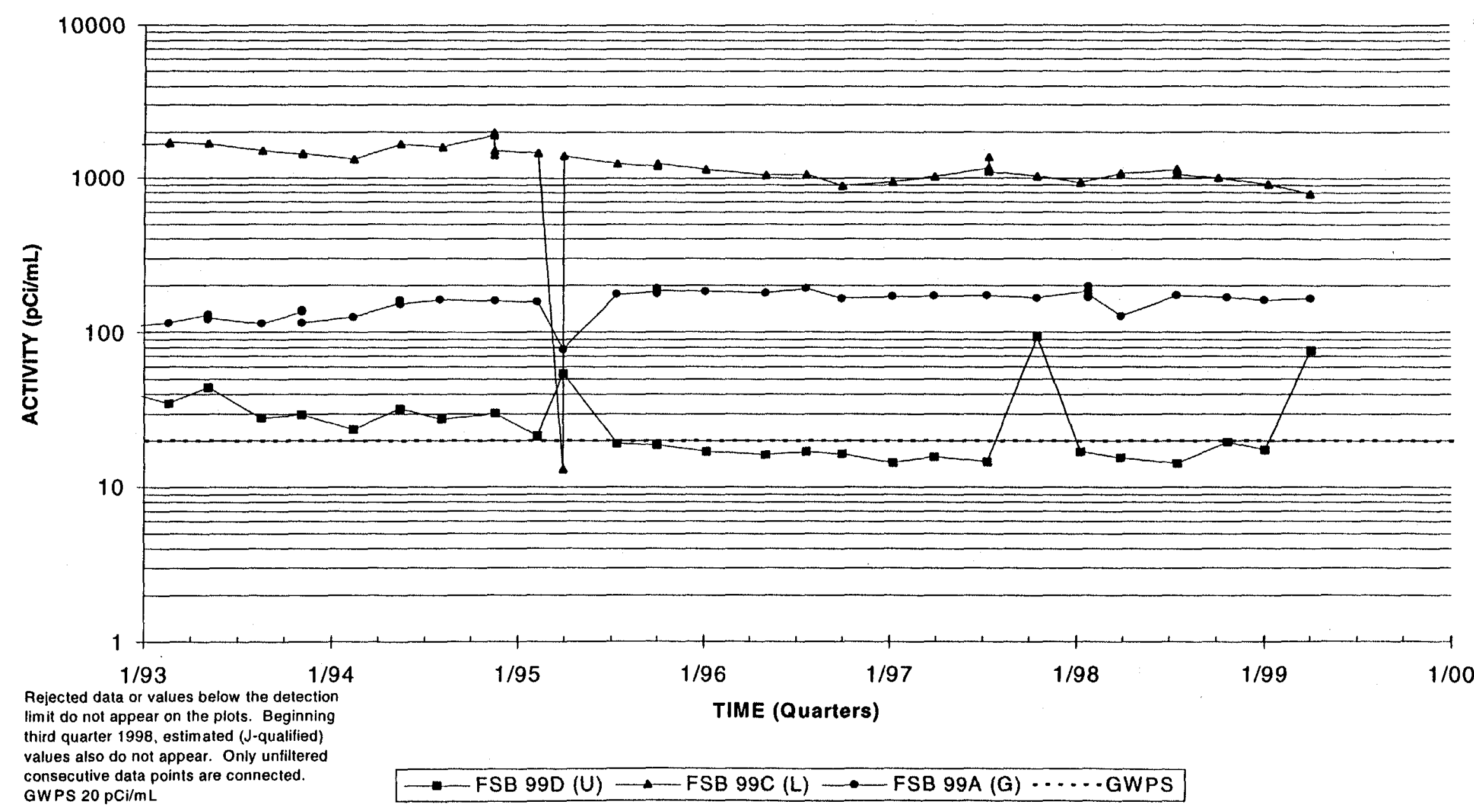

$U=$ Upper Aquiler Zone of the Upper Three Runs Aquiler, $L=$ Lower Aquiler Zone of the Upper Three Runs Aquifer, $G=$ Gordon Aquiler 


\section{Tritium Activities \\ Well FSB102C}

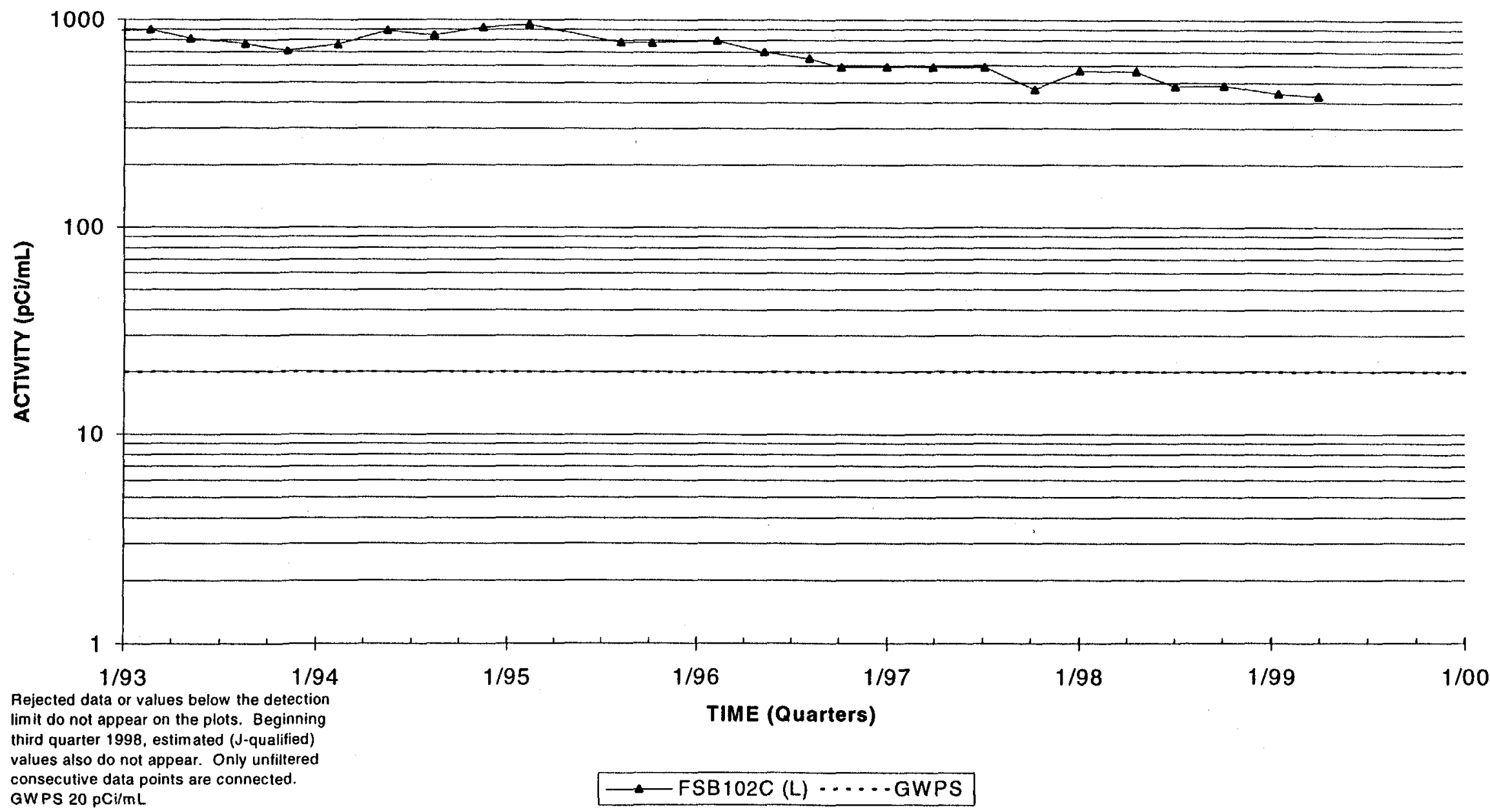

$U=$ Upper Aquifer Zone of the Upper Three Runs Aquifer, $L=$ Lower Aquifer Zone of the Upper Three Runs Aquifer, $G=$ Gordon Aquifer 


\section{Tritium Activities Well Cluster FSB107}

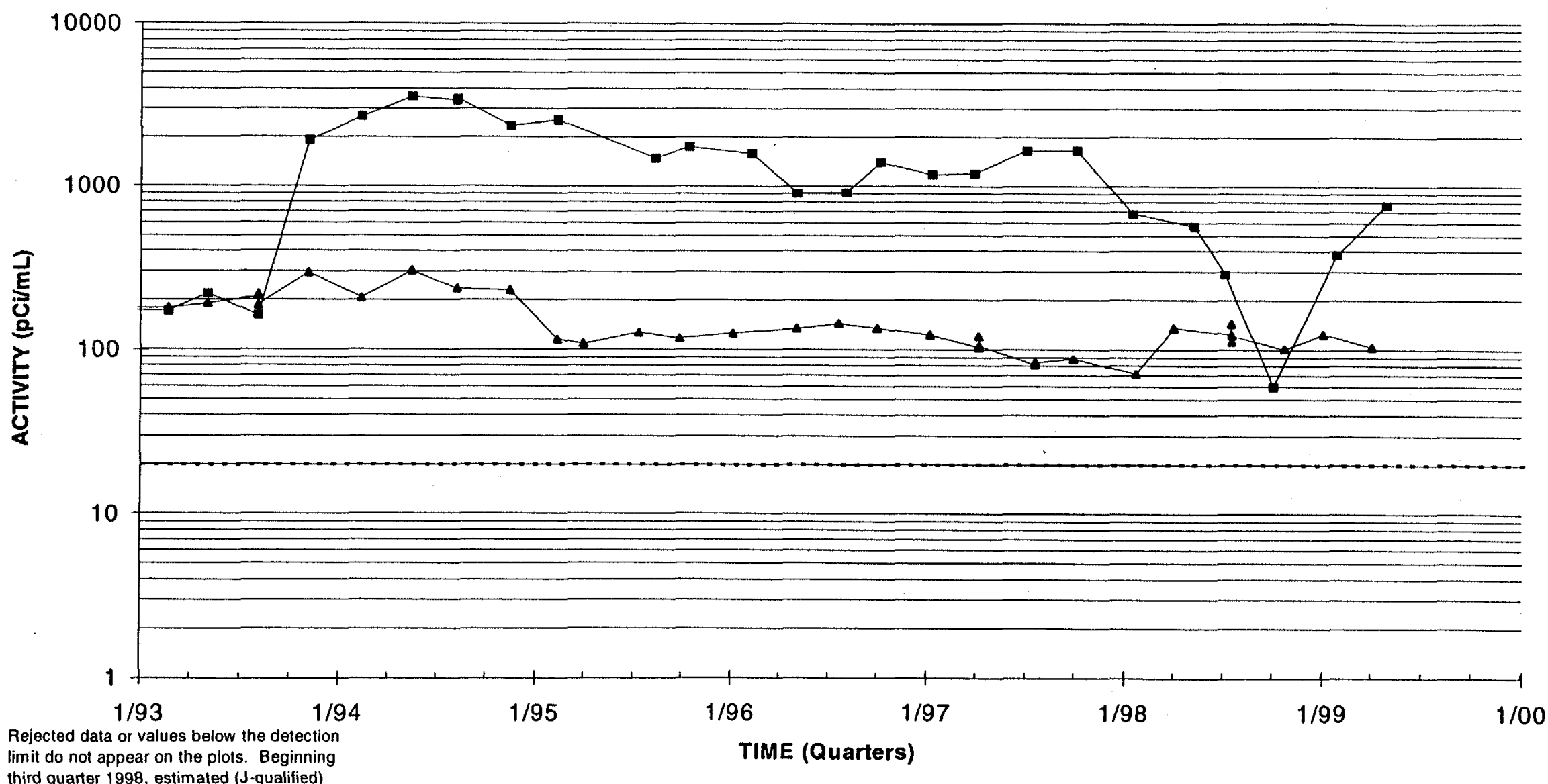

hird quar appear on the plots. Beginning

values also do not appear. Only unfiltered

consecutive data points are connected.

GWPS $20 \mathrm{pCl} / \mathrm{mL}$

$\longrightarrow$ FSB107D $(U) \multimap$ FSB107C (L) $\cdots \cdots$. GWPS

$U=$ Upper Aquiler Zone of the Upper Three Runs Aquifer, $L=$ Lower Aquifer Zone of the Upper Three Auns Aquifer, $G=$ Gordon Aquifer 


\section{Tritium Activities \\ Well FSB108D}

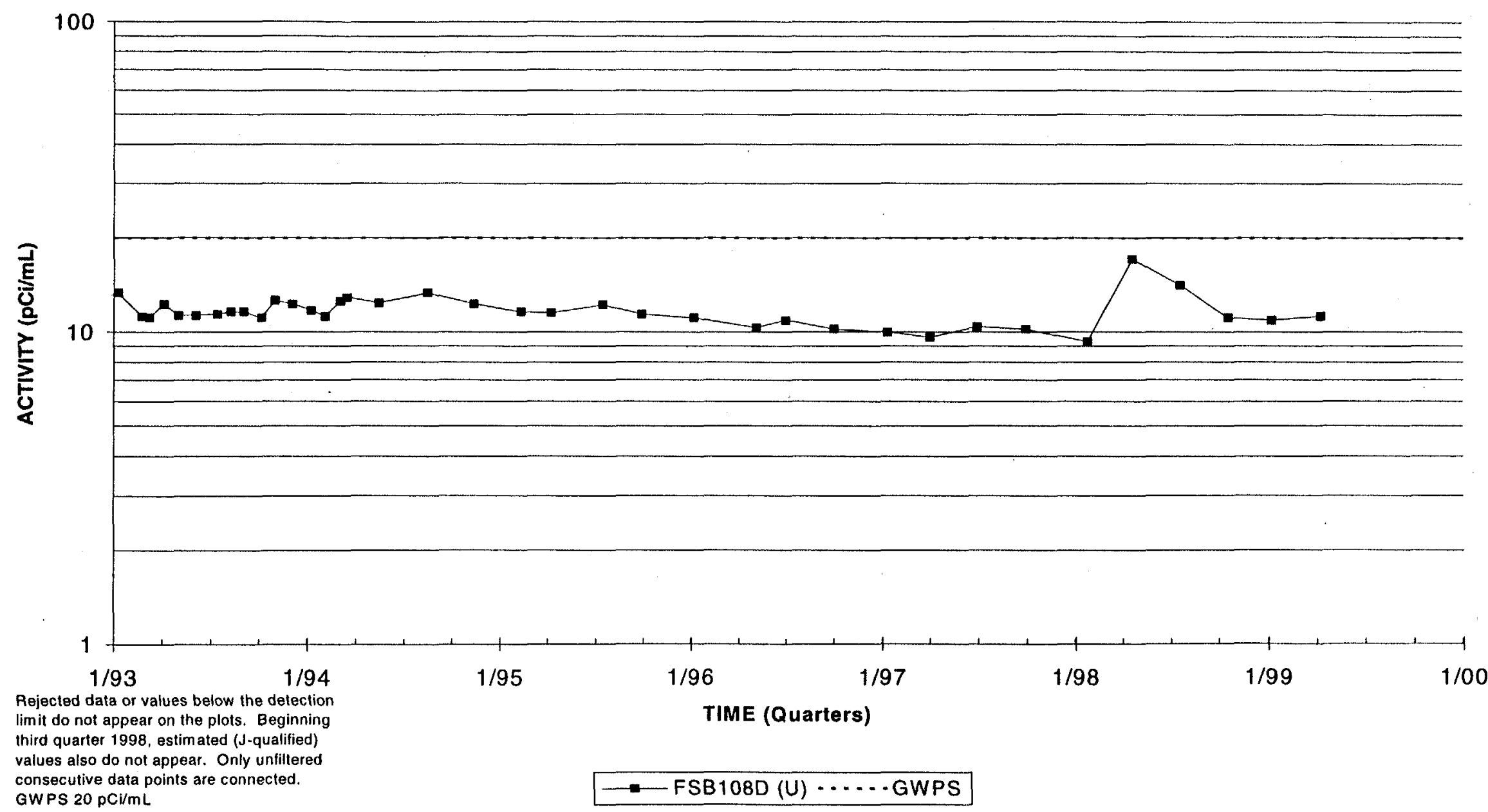

$\mathrm{U}=$ Upper Aquifer Zone of the Upper Three Runs Aquiler, $\mathrm{L}=$ Lower Aquifer Zone of the Upper Three Runs Aquiler, $\mathrm{G}=$ Gordon Aquifer 


\section{Tritium Activities \\ Well Cluster FSB110}

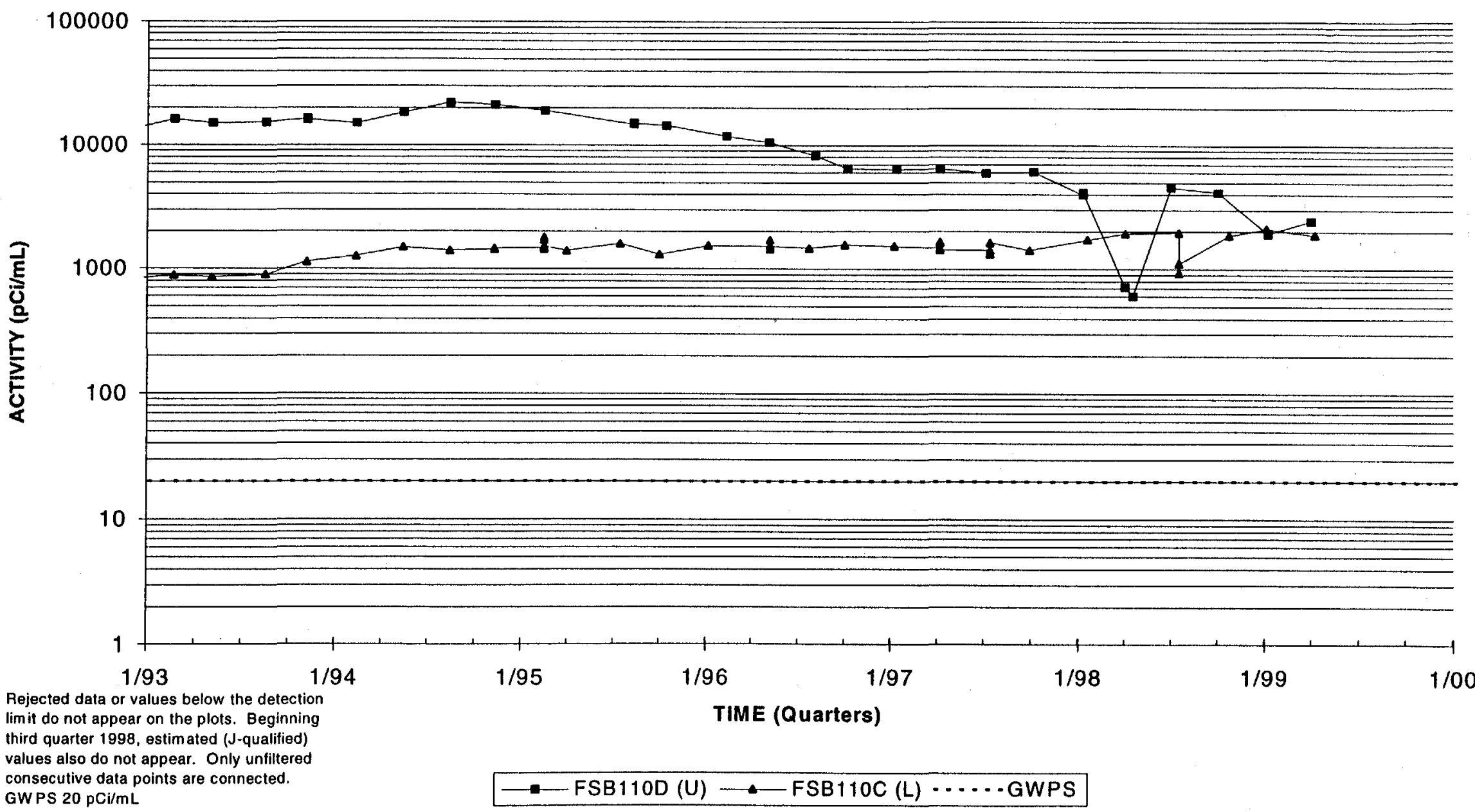

$\mathrm{U}=$ Upper Aquifer Zone of the Upper Three Runs Aquifer, $\mathrm{L}=$ Lower Aquifer Zone of the Upper Three Runs Aquifer, $\mathrm{G}=$ Gordon Aquiler 


\section{Tritium Activities \\ Well Cluster FSB120}

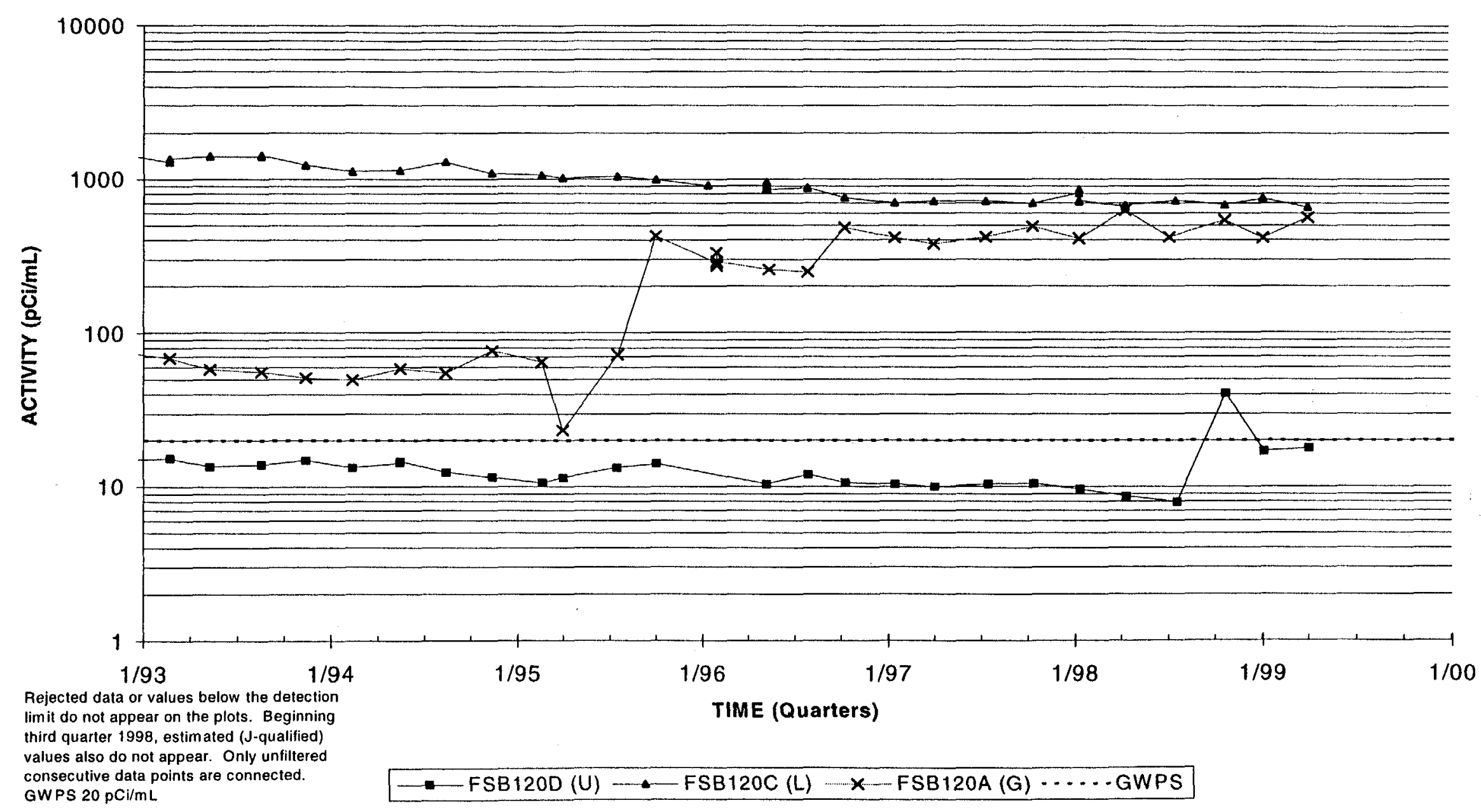

$U=$ Upper Aquifer Zone of the Upper Three Runs Aquifer, $L=$ Lower Aquifer Zone of the Upper Three Runs Aquifer; $G=$ Gordon Aquifer 


\section{Tritium Activities \\ Well Cluster FSB122}

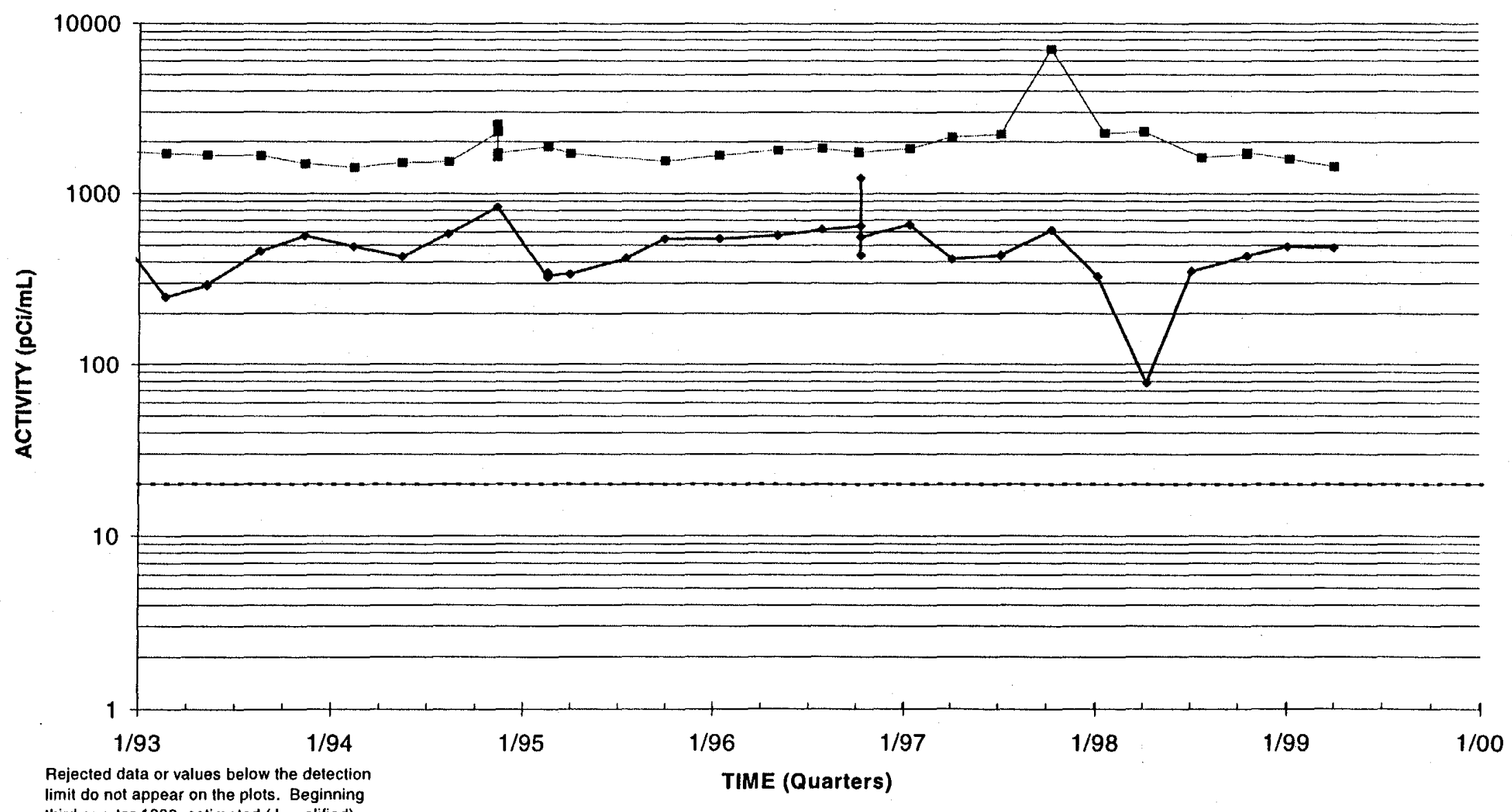

limit do not appear on the plots. Beginning

third quarter 1998, estimated (J-qualified)

values also do not appear. Only unfiltered

consecutive data points are connected.

$\longrightarrow$ FSB 122D (U) $\longrightarrow$ FSB122C (L) .....GWPS

GWPS $20 \mathrm{pCi} / \mathrm{mL}$

$U=$ Upper Aquiler Zone of the Upper Three Runs Aquifer, $L=$ Lower Aquifer Zone of the Upper Three Runs Aquifer, $G=$ Gordon Aquiler 


\section{Nitrate Concentrations \\ Well Cluster FSB 87}

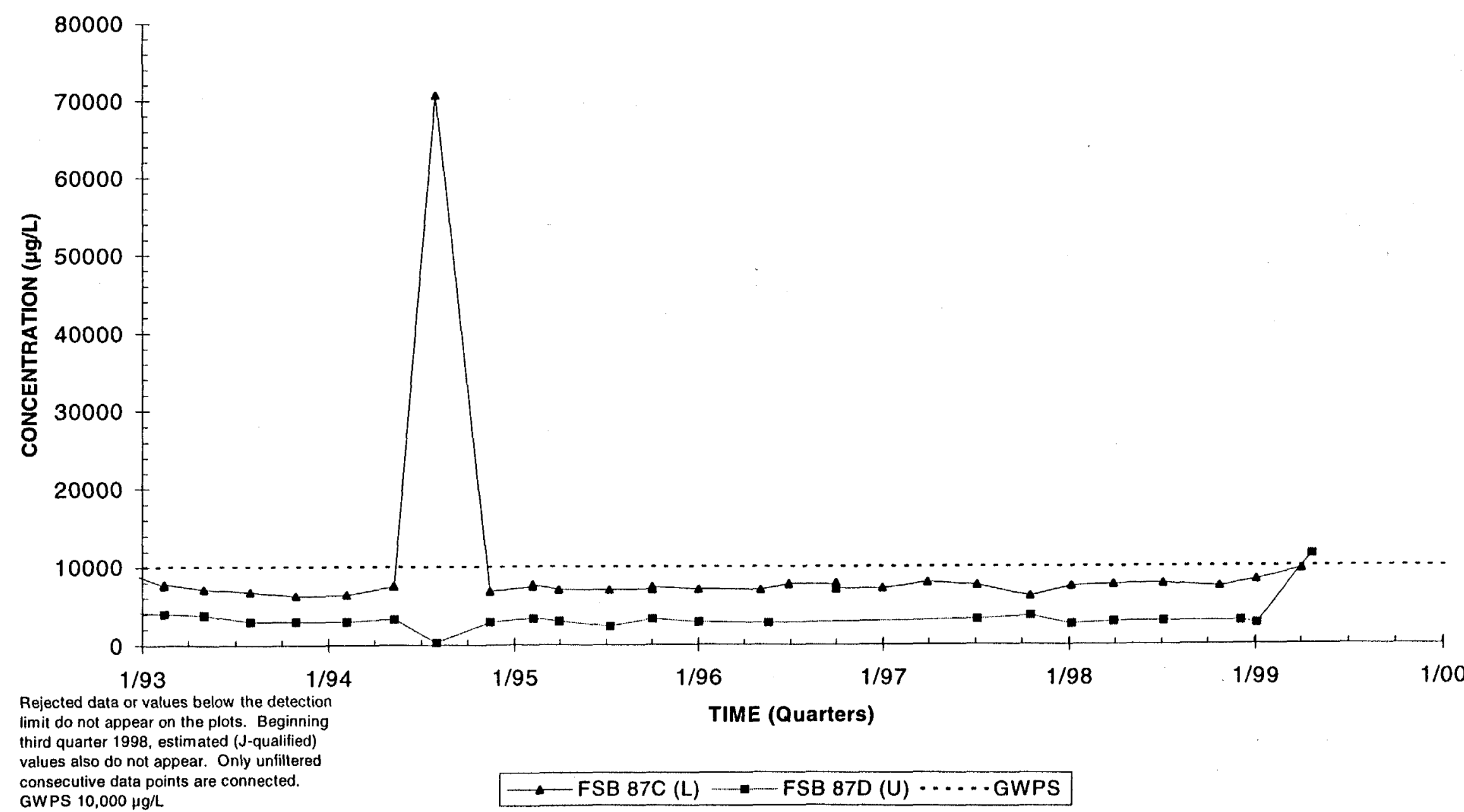

$U=$ Upper Aquifer Zone of the Upper Three Runs Aquifer, $L=$ Lower Aquiler Zone of the Upper Three Runs Aquiler, $G=$ Gordon Aquifer 


\section{Nitrate Concentrations \\ Well FSB 88D}

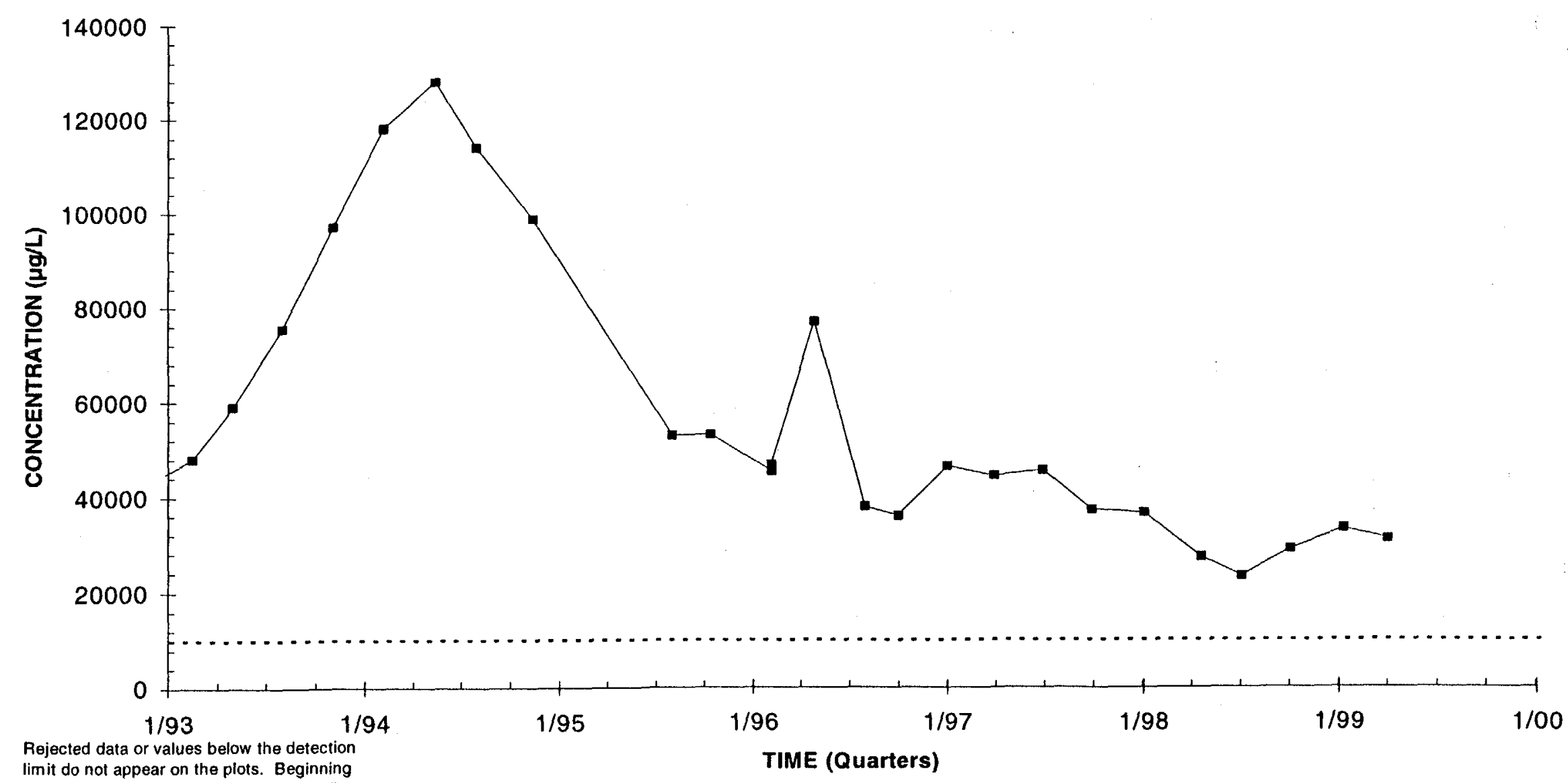

limit do not appear on the plots. Beginning

third quarter 1998, estimated (J-qualified)

values also do not appear. Only unfittered

consecutive data points are connected.

GWPS $10,000 \mu \mathrm{g} / \mathrm{h}$

$\mathrm{U}=$ Upper Aquiler Zone of the Upper Three Runs Aquifer, $\mathrm{L}=$ Lower Aquifer Zone of the Upper Three Runs Aquifer, $\mathrm{G}=$ Gordon Aquifer 


\section{Nitrate Concentrations \\ Well FSB 91D}

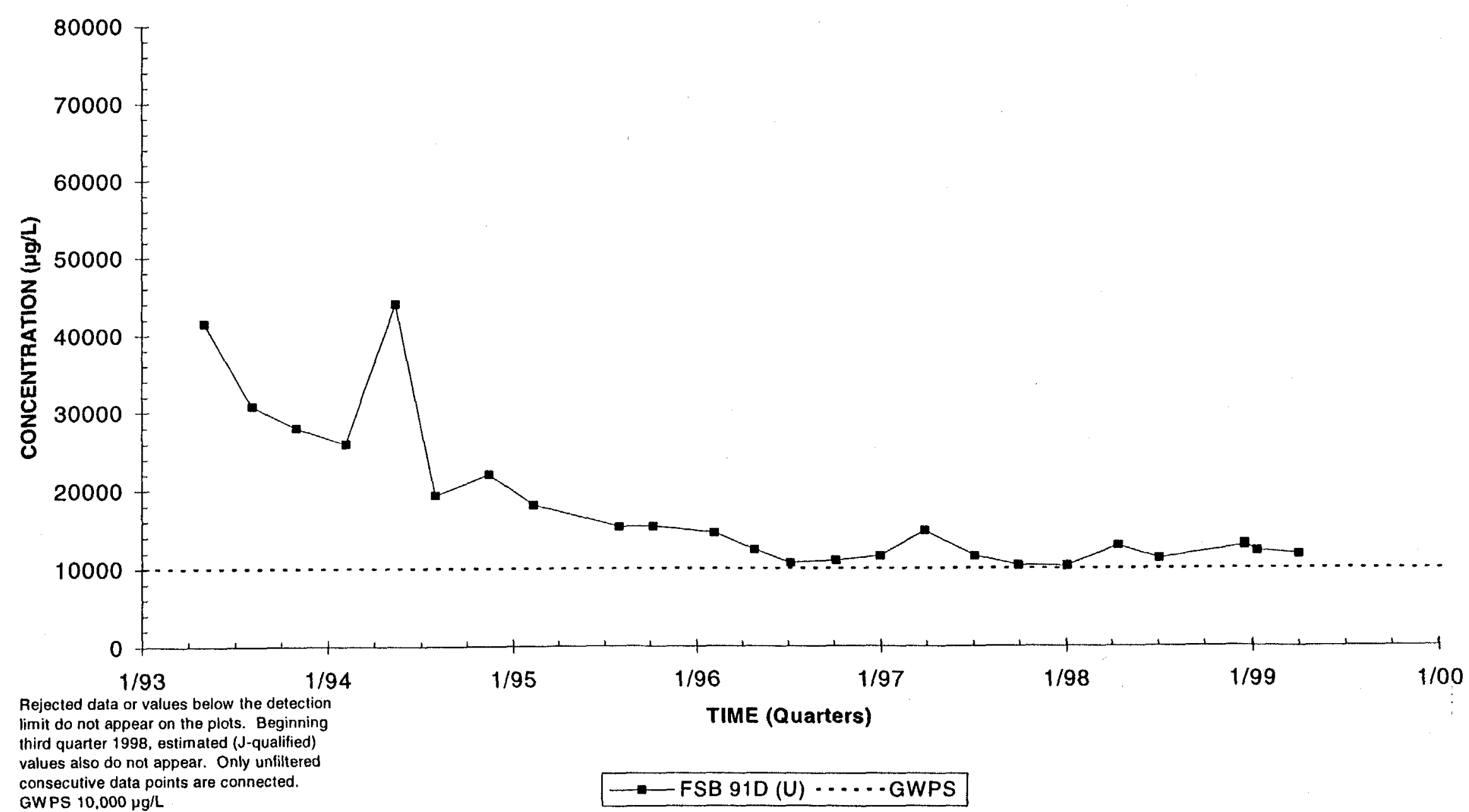

$U=$ Upper Aquifer Zone of the Upper Three Runs Aquifer, $L=$ Lower Aquiler Zone of the Upper Three Runs Aquifer; $G=$ Gordon Aquifer 


\section{Nitrate Concentrations \\ Well FSB102C}

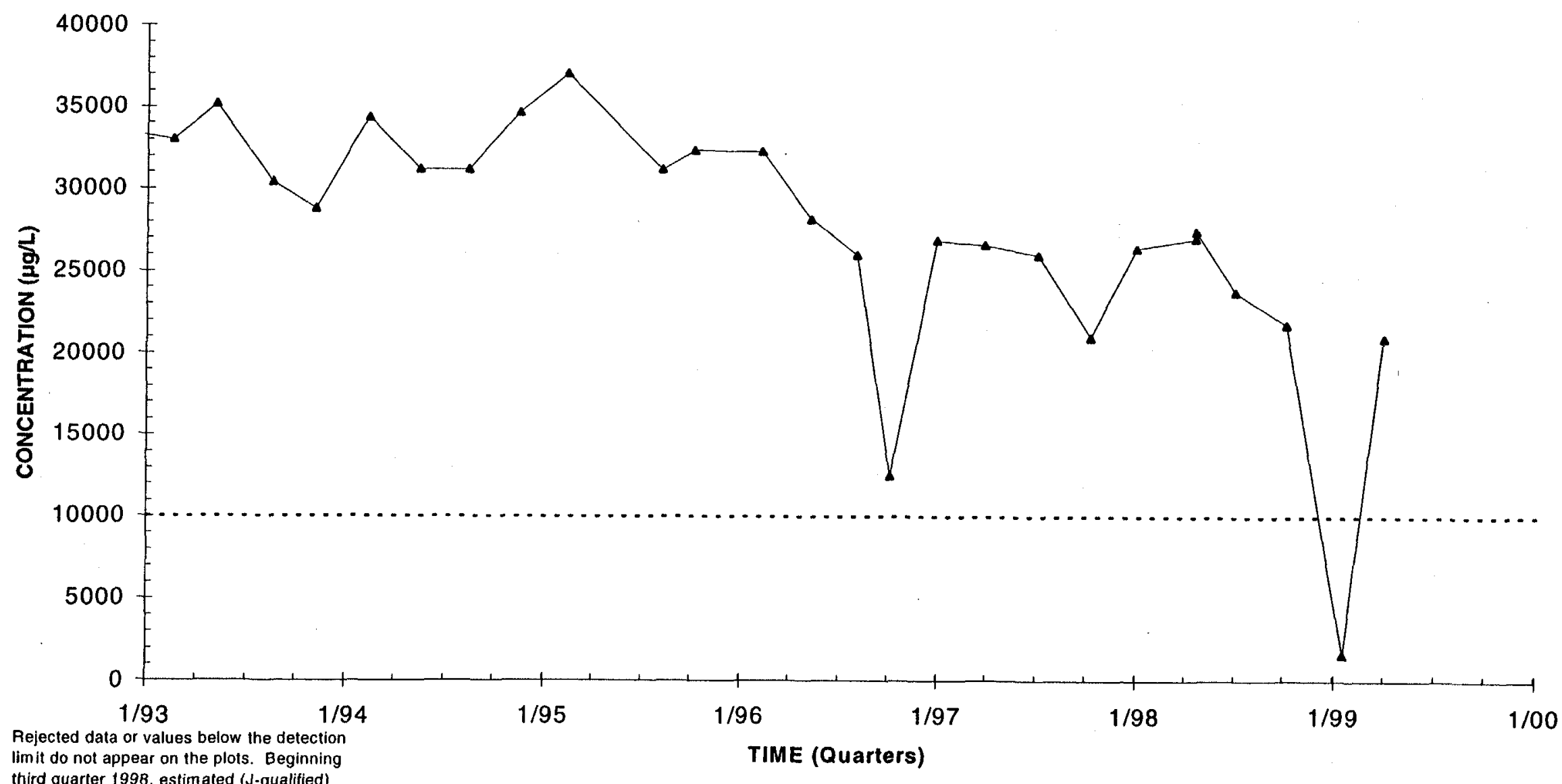

third quarter 199B, estime

values also do not appear. Only unfiltered

consecutive data points are connected.

FSB102C (L) $\cdots$. . GWPS

$\mathrm{U}=$ Upper Aquiler Zone of the Upper Three Runs Aquifer, $\mathrm{L}=$ Lower Aquifer Zone of the Upper Three Runs Aquifer, $\mathrm{G}=$ Gordon Aquifer 


\section{Nitrate Concentrations \\ Well Cluster FSB104}

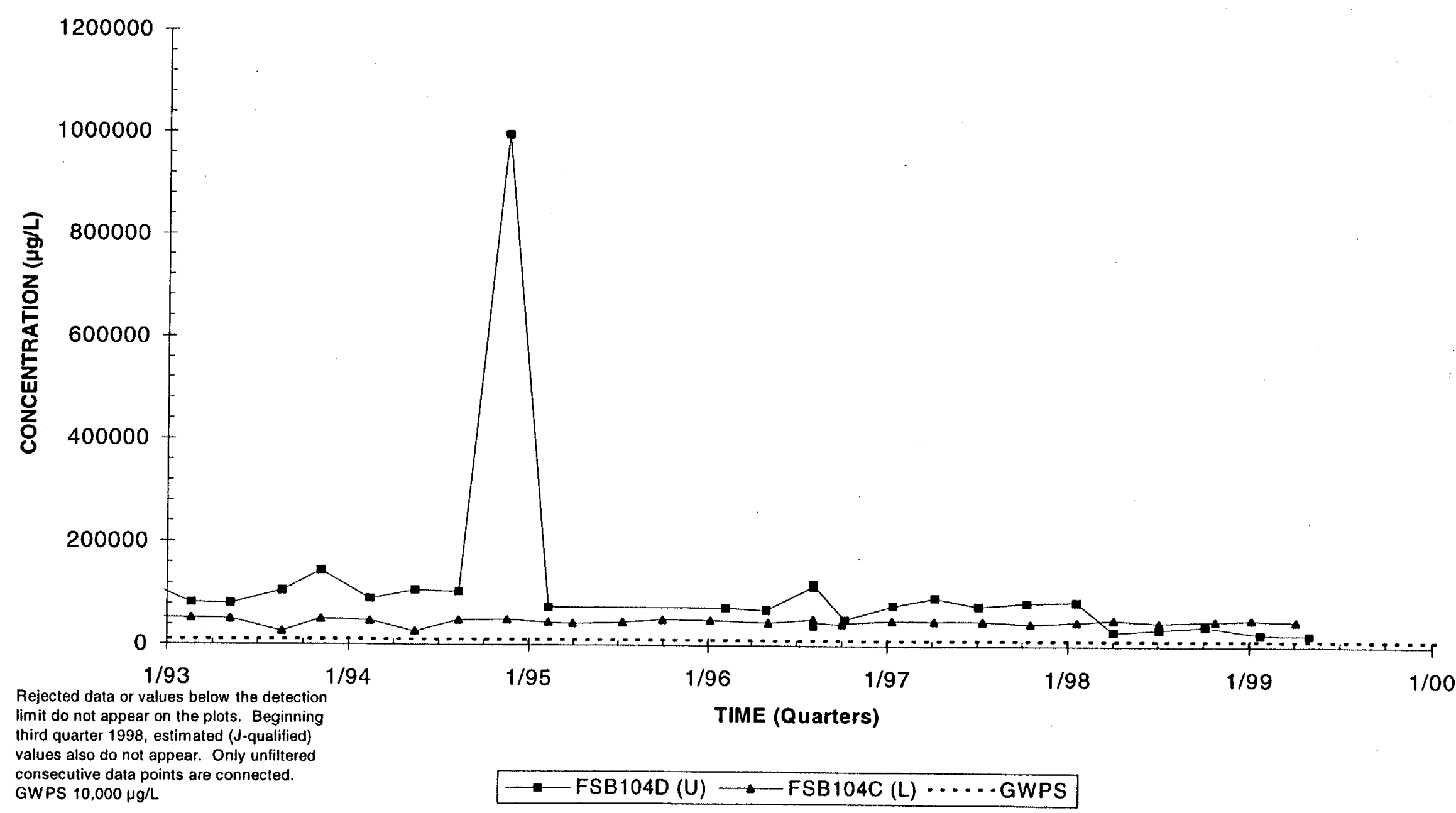

$U=$ Upper Aquiler Zone of the Upper Three Runs Aquifer, $L=$ Lower Aquifer Zone of the Upper Three Runs Aquifer, $G=$ Gordon Aquiler 


\section{Nitrate Concentrations \\ Well FSB110D}

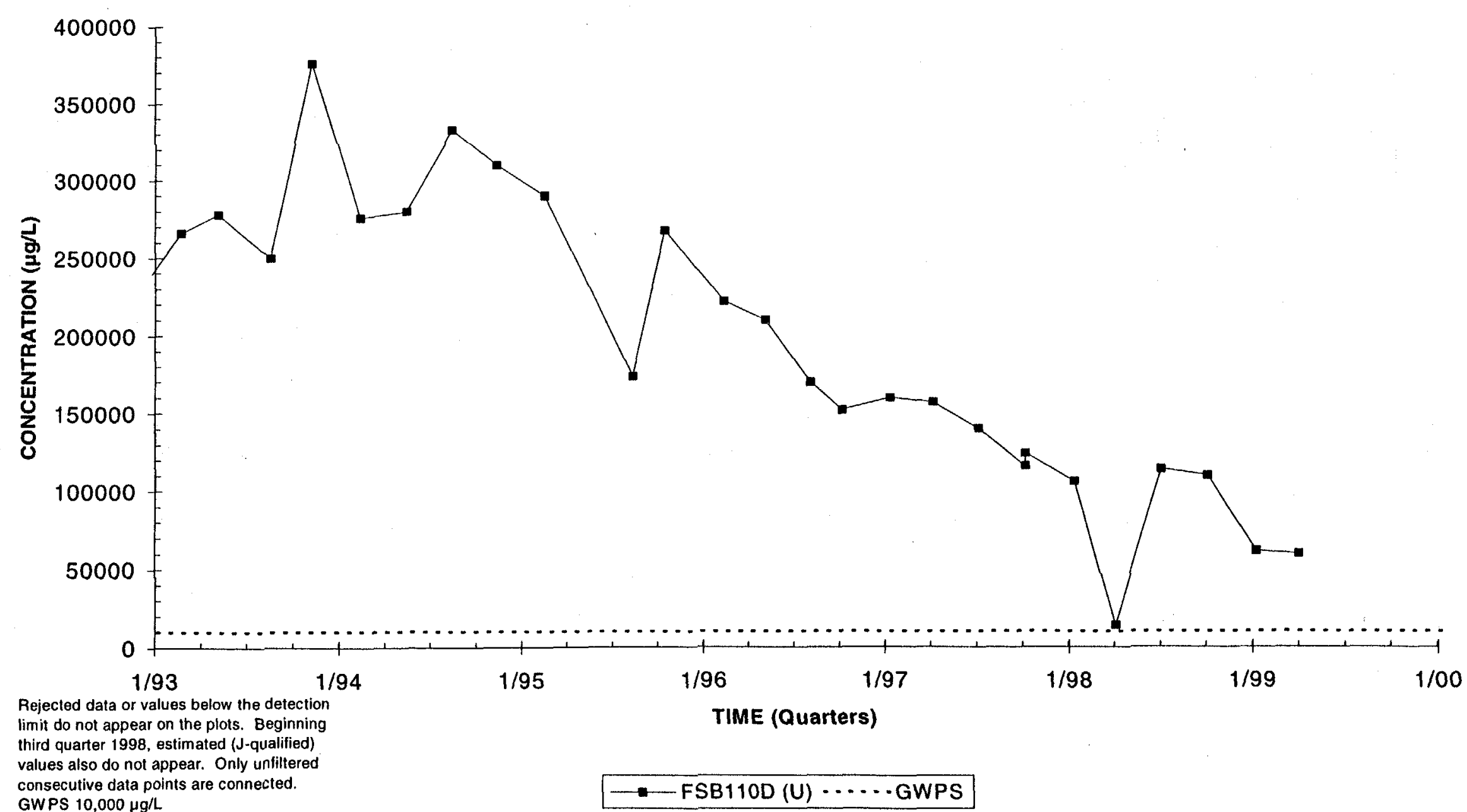

$\mathrm{U}=$ Upper Aquifer Zone of the Upper Three Runs Aquifer, $\mathrm{L}=$ Lower Aquifer Zone of the Upper Three Runs Aquifer, $\mathrm{G}=$ Gordon Aquiler

F-Area HWMF




\section{Nitrate Concentrations \\ Well FSB117D}

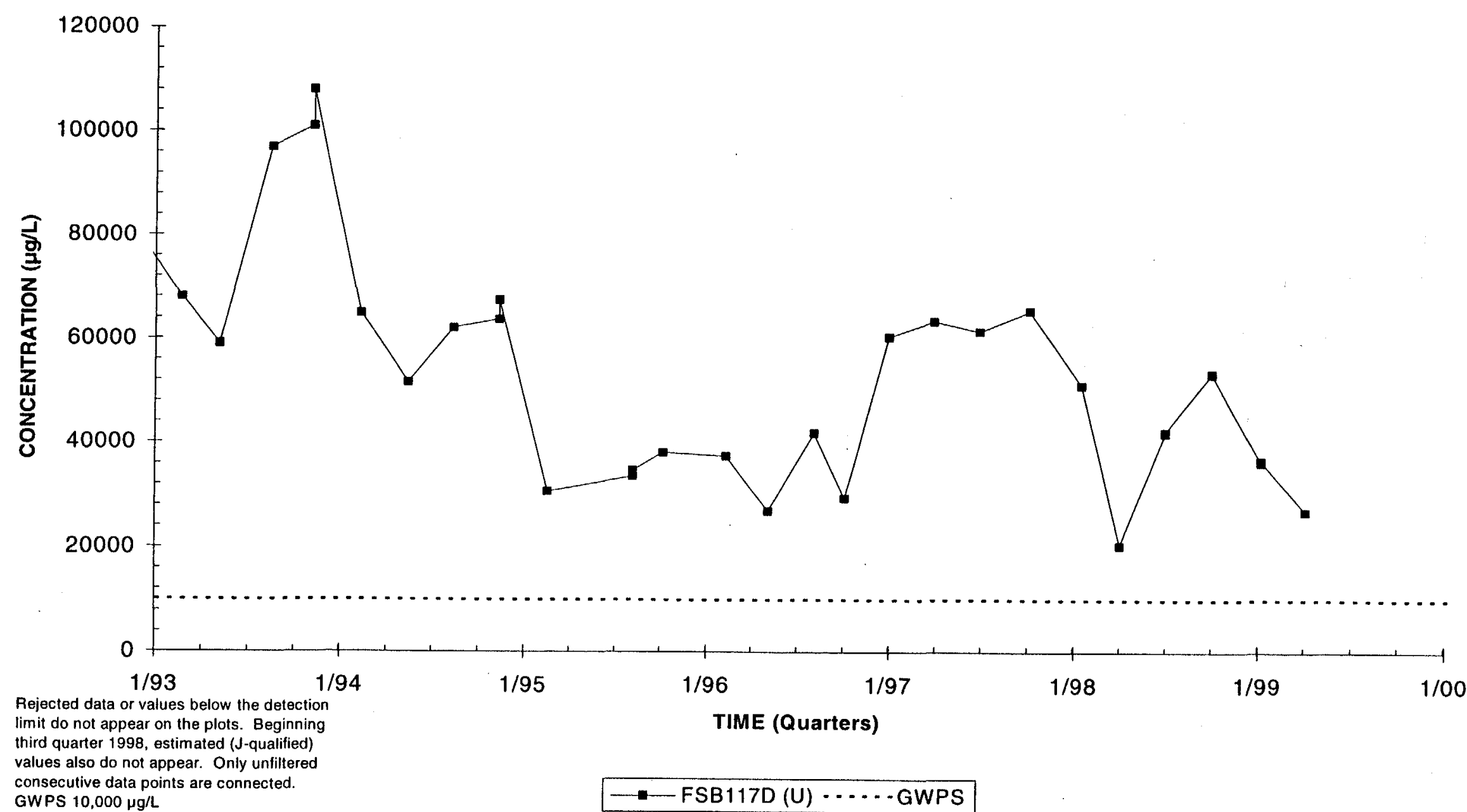

$\mathrm{U}=$ Upper Aquifer Zone of the Upper Three Runs Aquifer, $\mathrm{L}=$ Lower Aquifer Zone of the Upper Three Runs Aquiler, G = Gordon Aquifer 


\section{Nitrate Concentrations \\ Well FSB119D}

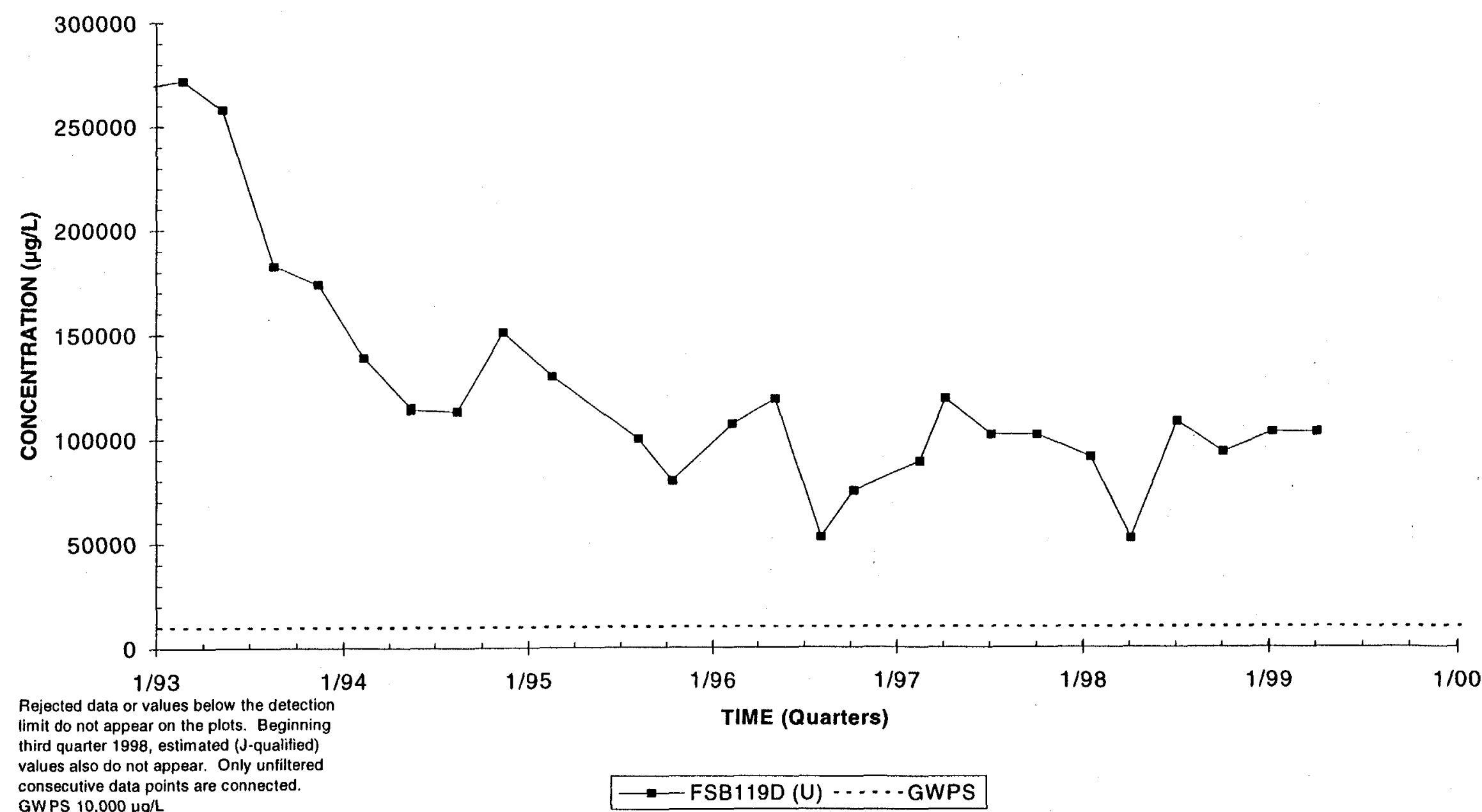

$U=$ Upper Aquiler Zone of the Upper Three Runs Aquifer, $L=L$ Lower Aquifer Zone of the Upper Three Runs Aquifer; $G=$ Gordon Aquifer 


\section{Nitrate Concentrations \\ Well Cluster FSB120}

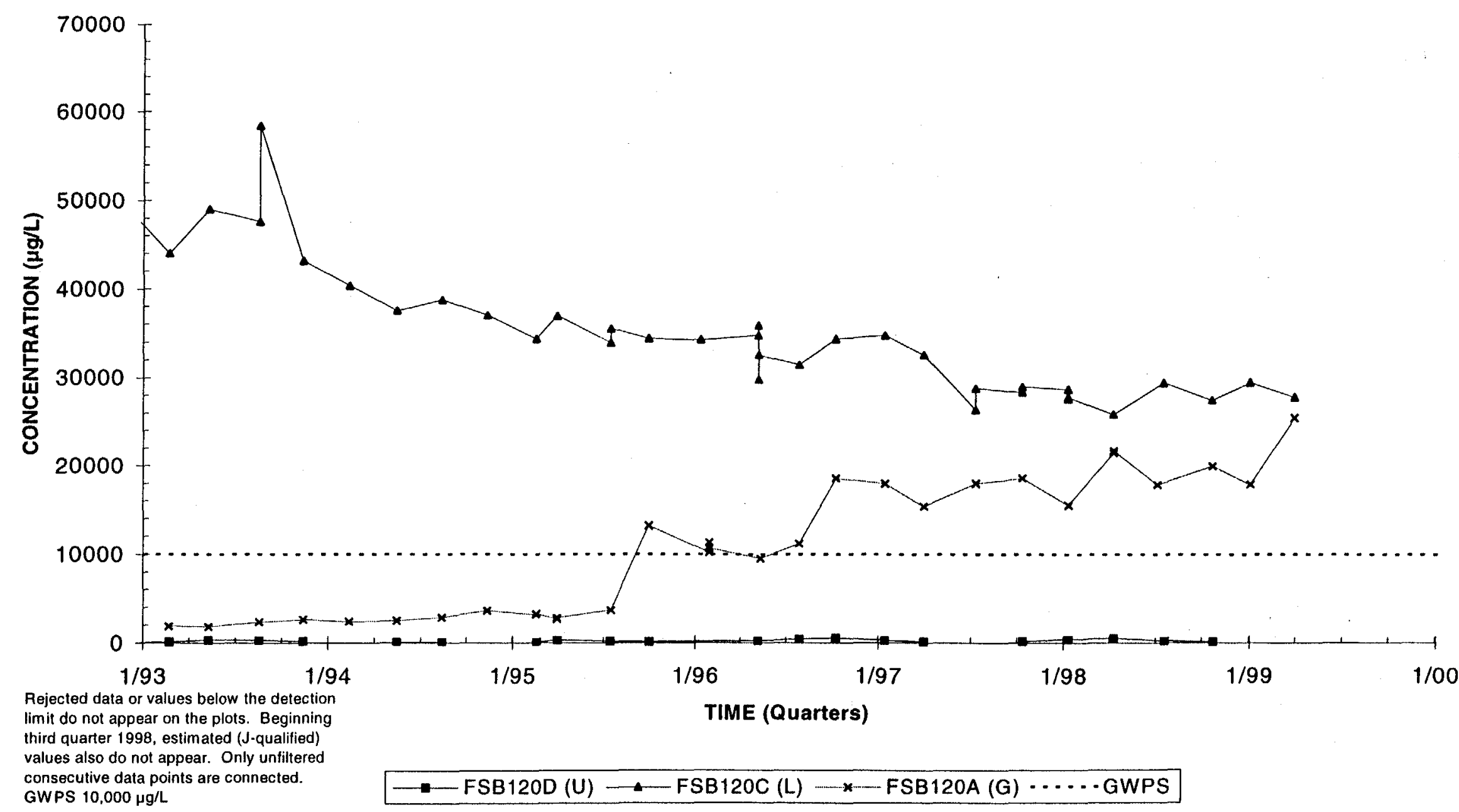

$U=$ Upper Aquifer Zone of the Upper Three Runs Aquifer; $L=$ Lower Aquifer Zone of the Upper Three Runs Aquifer, $G=$ Gordon Aquifer 


\section{Nitrate Concentrations \\ Well Cluster FSB122}

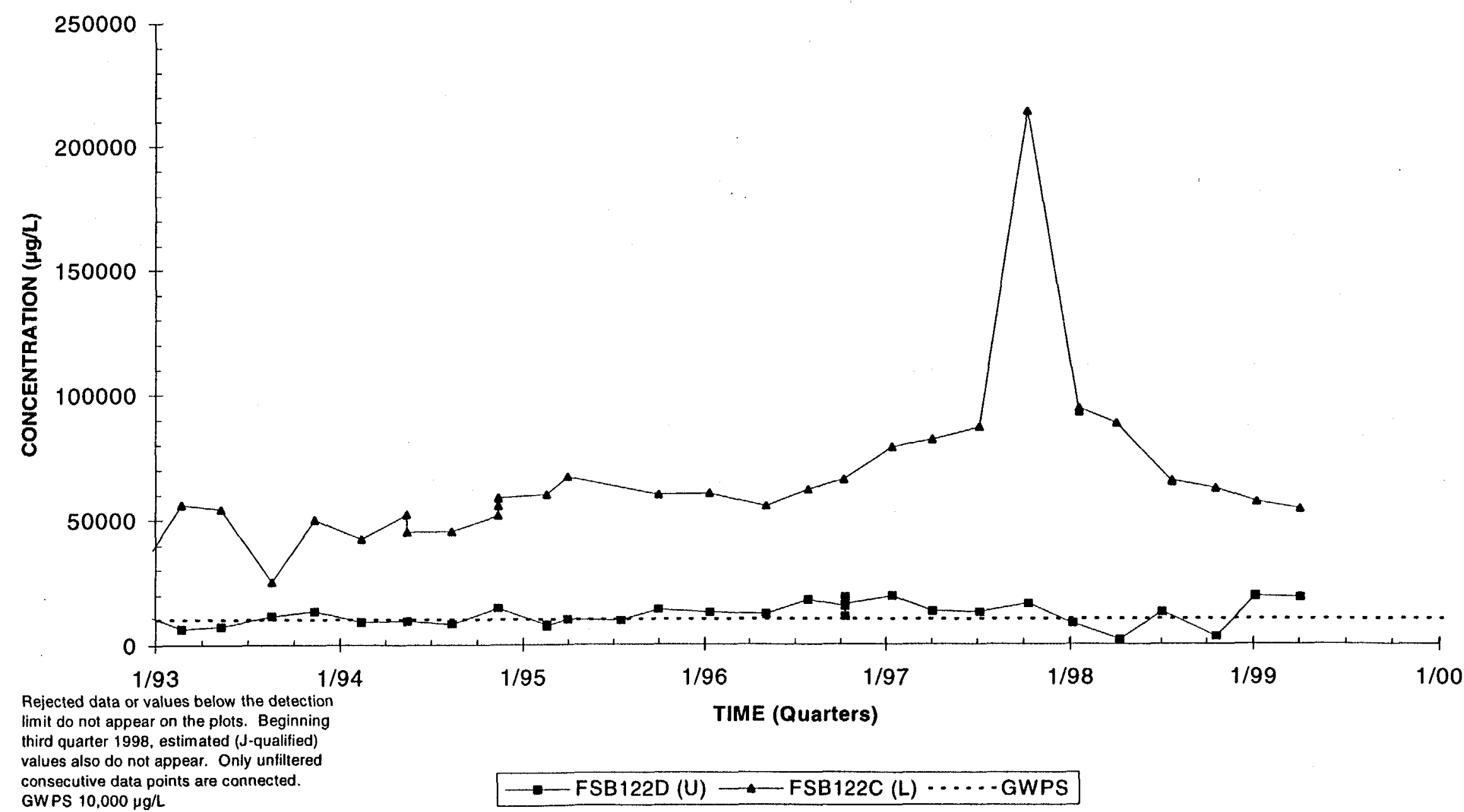

$U=$ Upper Aquifer Zone of the Upper Three Runs Aquifer, L = Lower Aquiler Zone of the Upper Three Runs Aquifer, $G=$ Gordon Aquifer 


\section{Uranium-233/234 Activities \\ Well FSB 77}

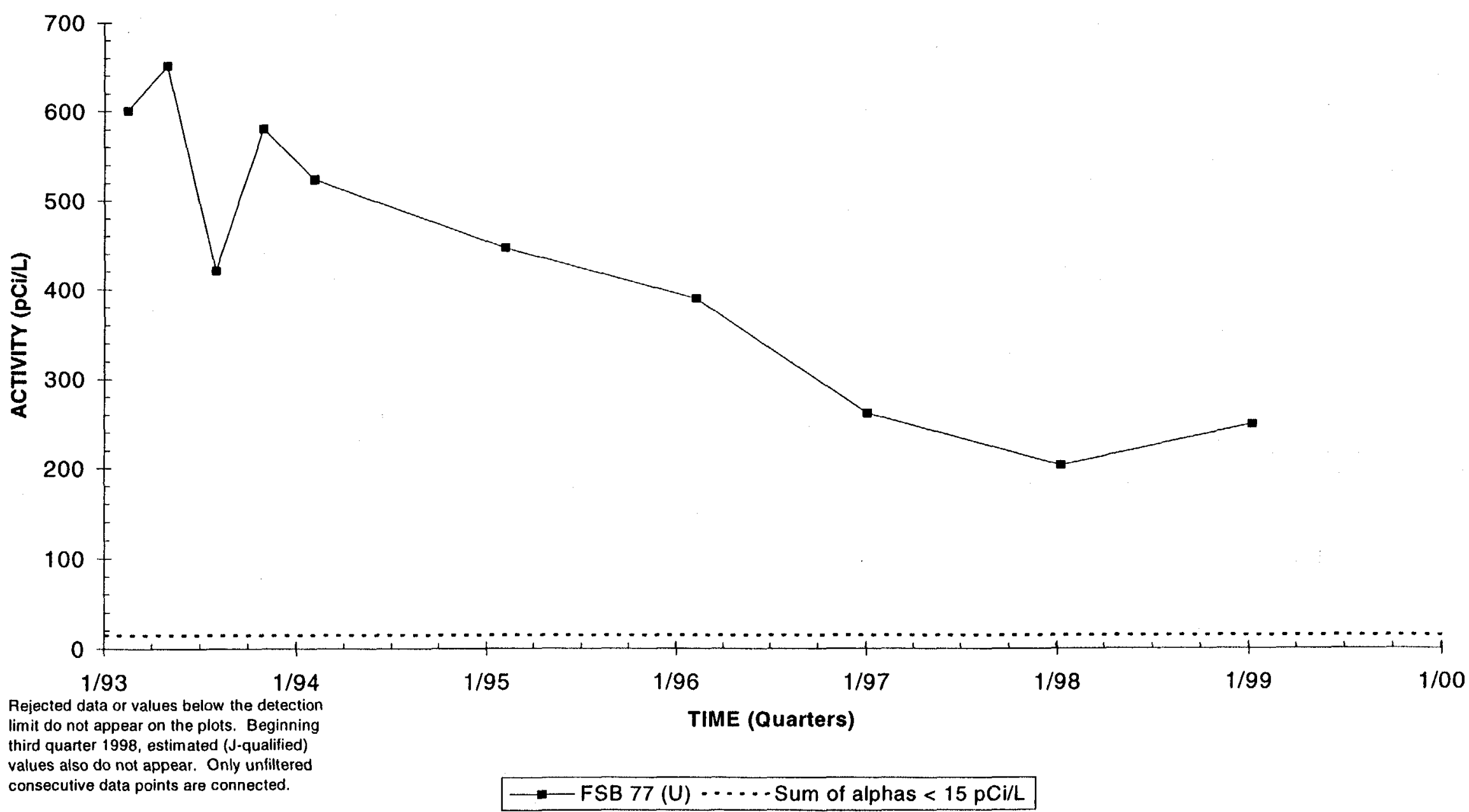

$U=$ Upper Aquifer Zone of the Upper Three Runs Aquifer, $L=$ Lower Aquifer Zone of the Upper Three Runs Aquifer; $G=$ Gordon Aquifer 


\section{Uranium-233/234 Activities Well Cluster FSB 79}

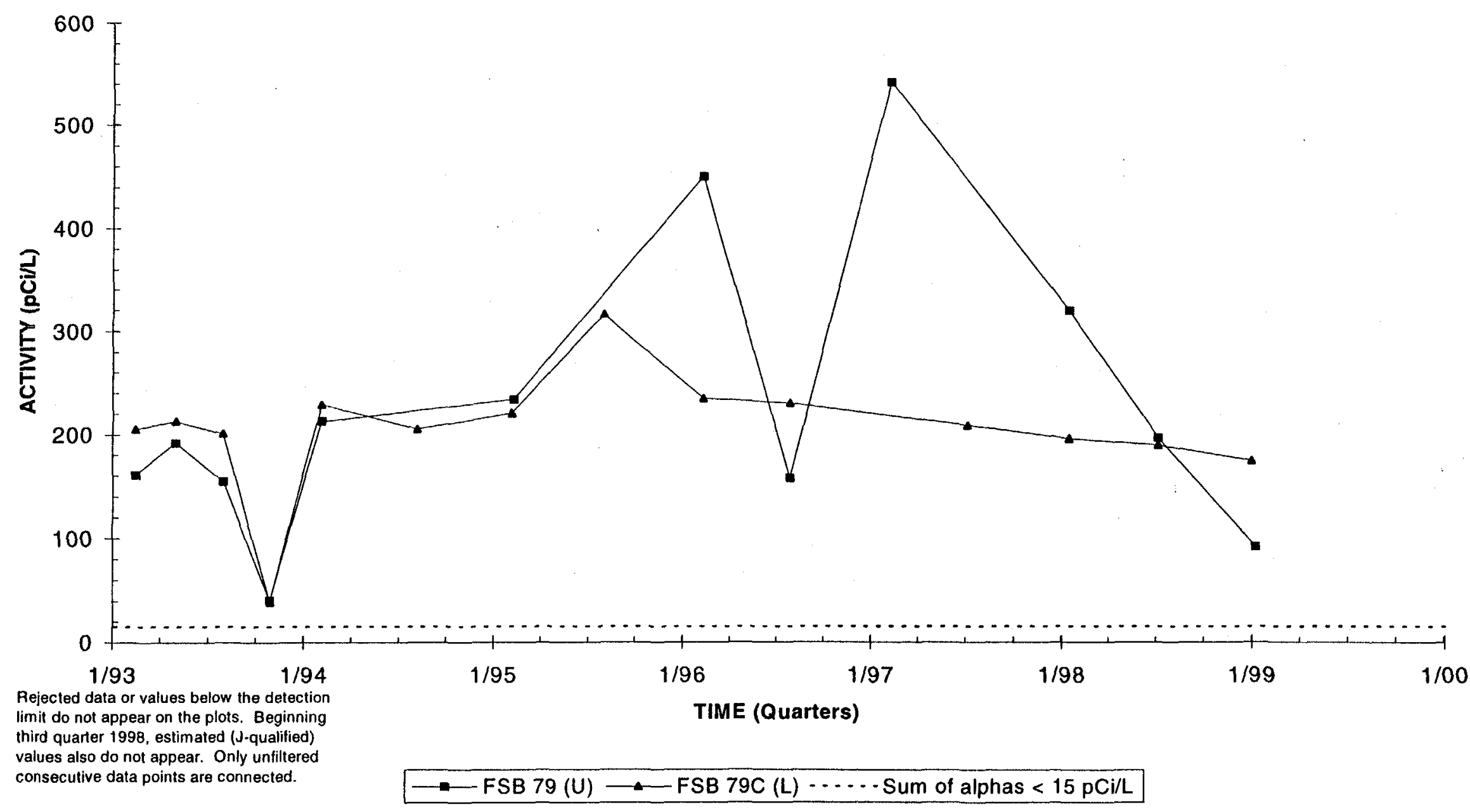

$U=$ Upper Aquifer Zone of the Upper Three Runs Aquifer, $L=$ Lower Aquifer Zone of the Upper Three Runs Aquifer; $G=$ Gordon Aquifer 


\section{Uranium-233/234 Activities \\ Well Cluster FSB 88}

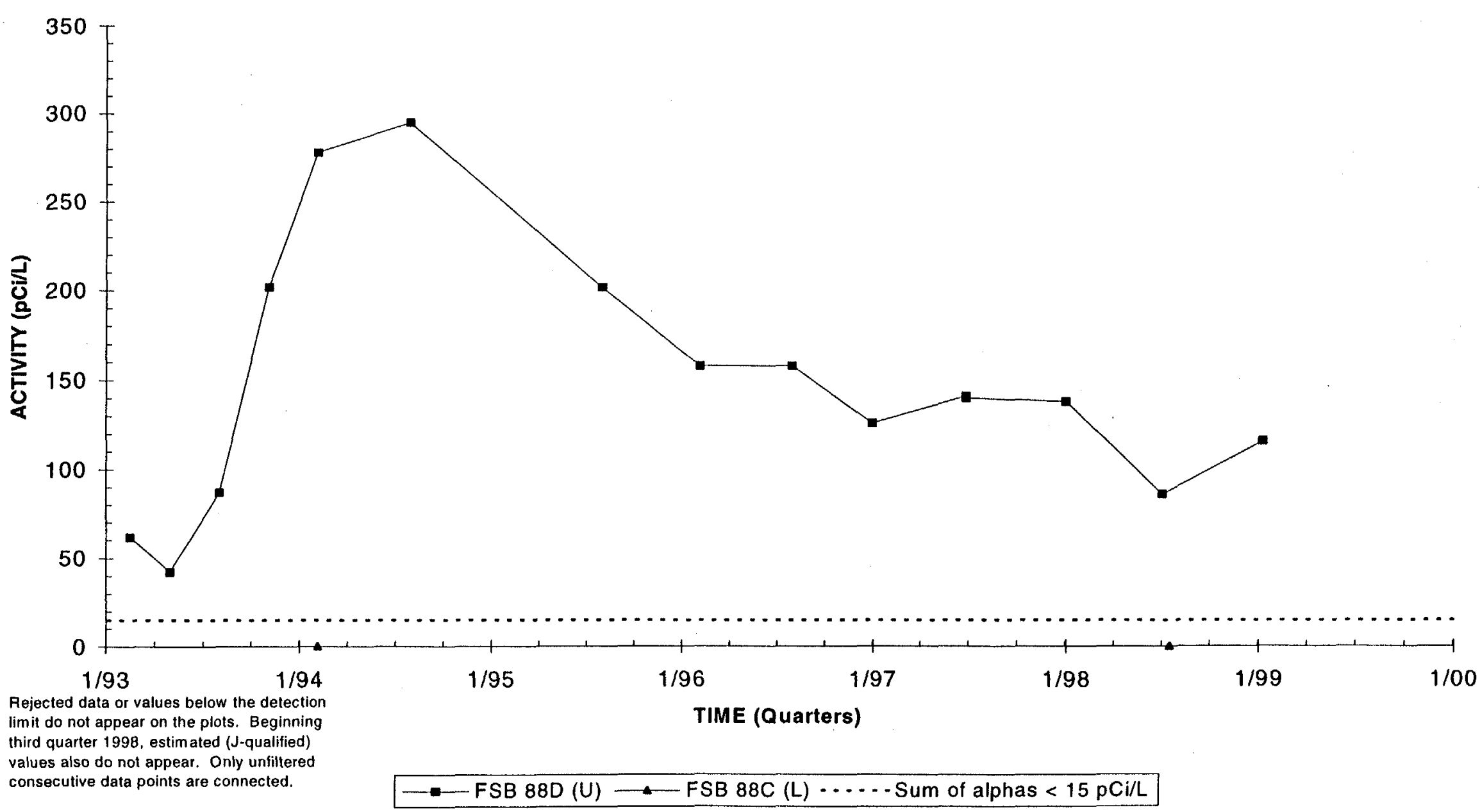

$\mathrm{U}=$ Upper Aquifer Zone of the Upper Three Runs Aquifer, $\mathrm{L}=$ Lower Aquifer Zone of the Upper Three Runs Aquifer; $G=$ Gordon Aquifer 


\section{Uranium-233/234 Activities \\ Well Cluster FSB 94}

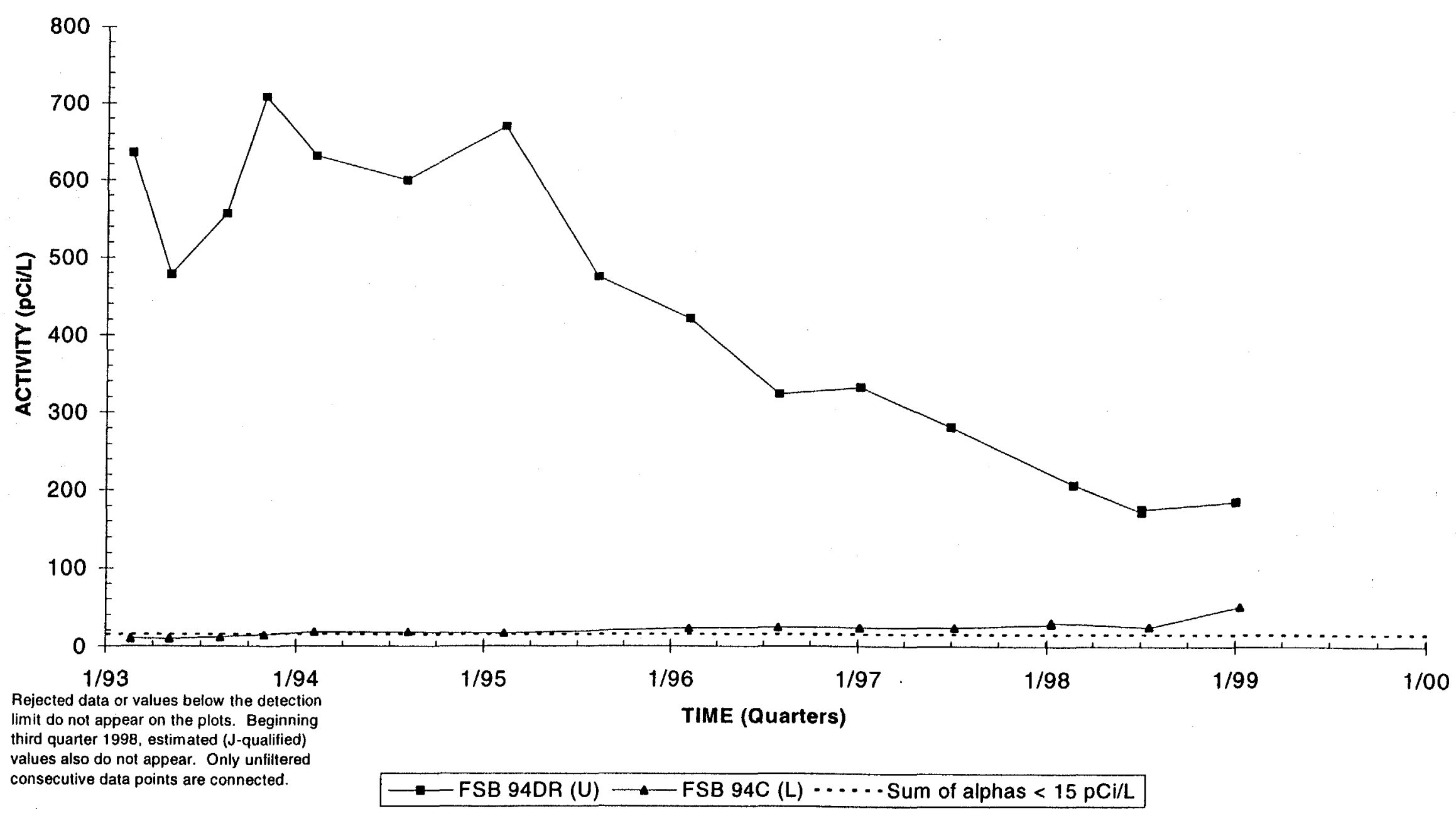

$U=$ Upper Aquifer Zone of the Upper Three Runs Aquiter, $L=$ Lower Aquifer Zone of the Upper Three Runs Aquifer, G = Gordon Aquifer 


\section{Uranium-233/234 Activities Well Cluster FSB 95}

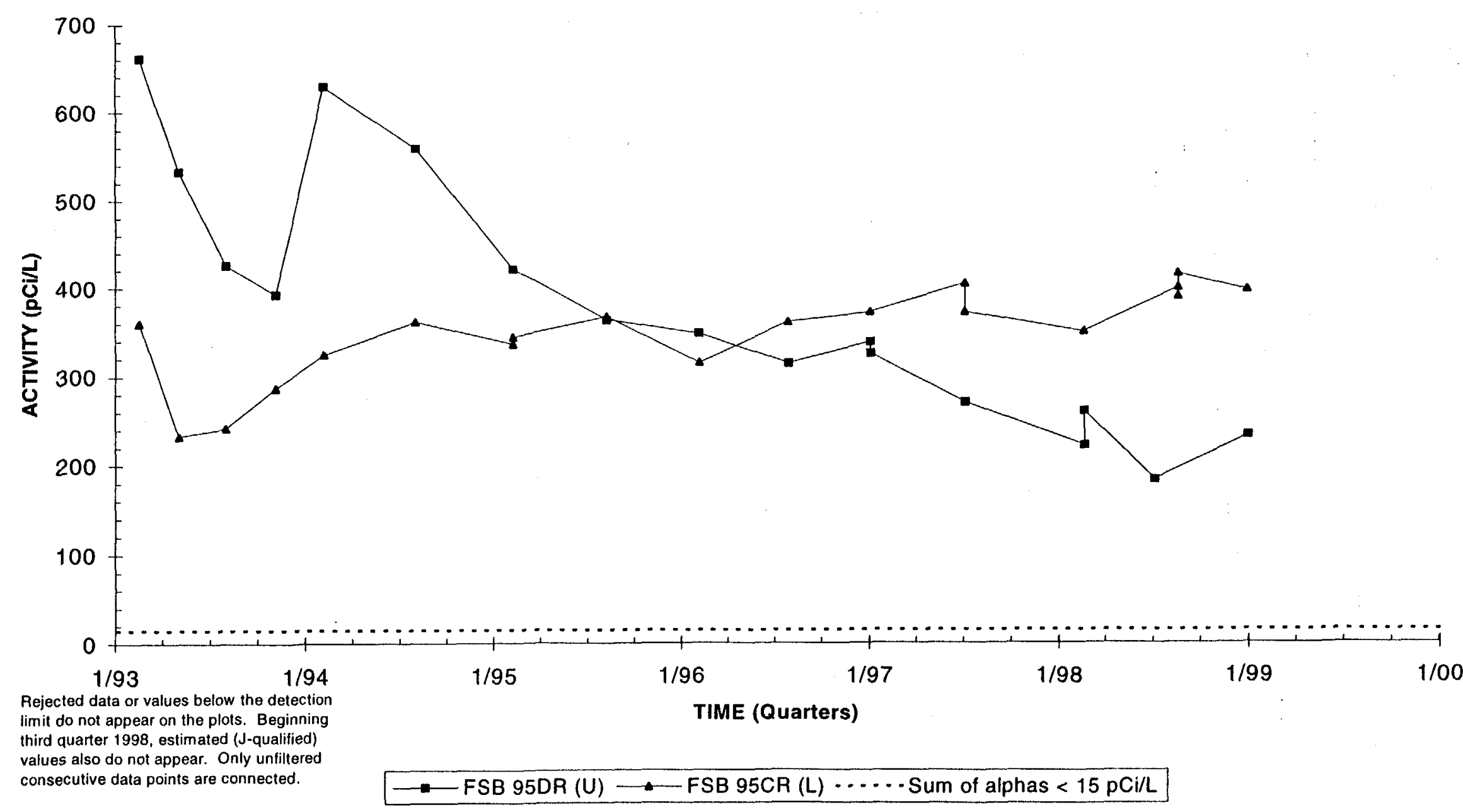

$\mathrm{U}=$ Upper Aquiter Zone of the Upper Three Runs Aquiler, $\mathrm{L}=$ Lower Aquiler Zone of the Upper Three Runs Aquiler; $G=$ Gordon Aquifer 


\section{Uranium-233/234 Activities Well Cluster FSB 97}

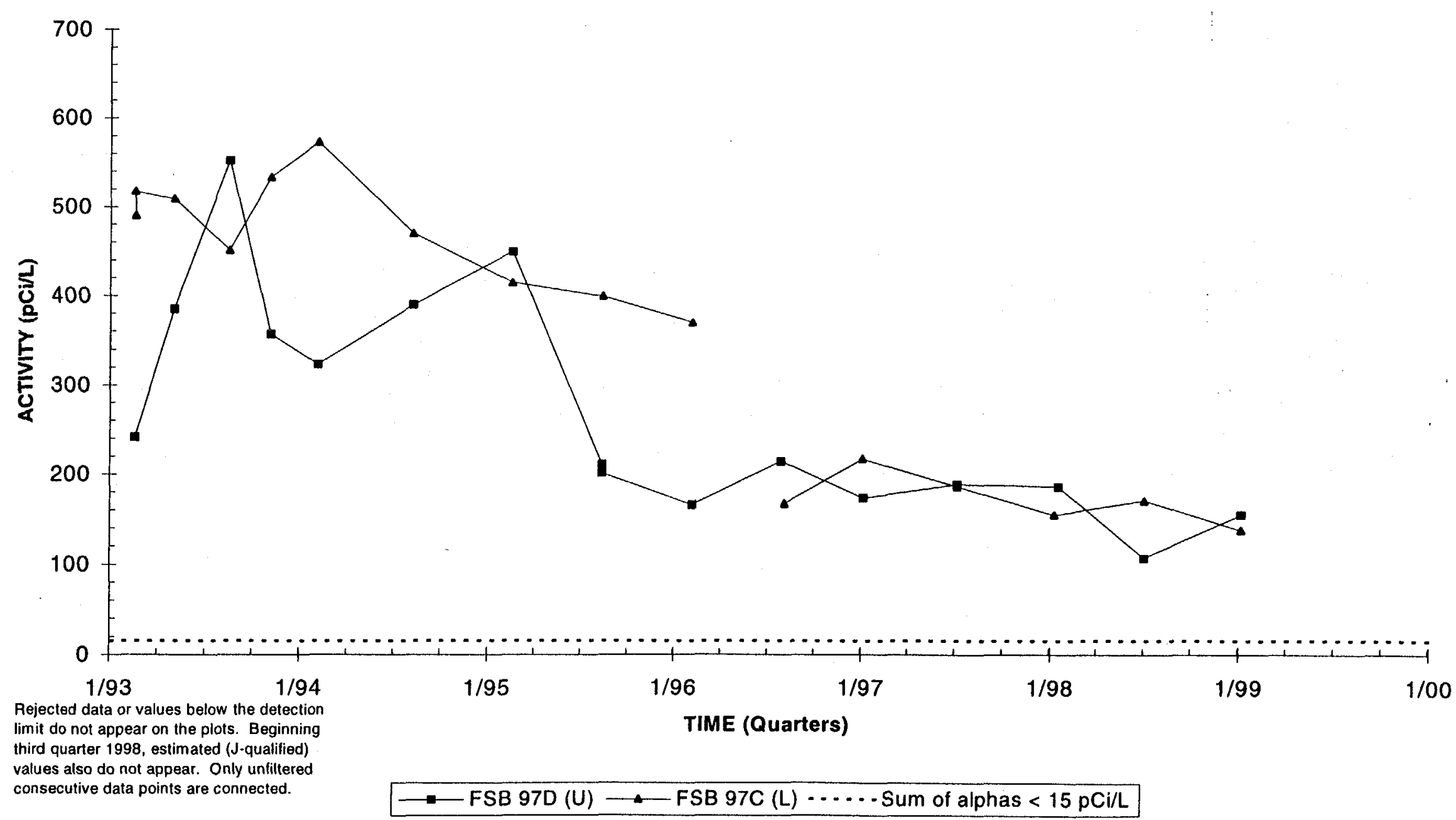

$U=$ Upper Aquifer Zone of the Upper Three Runs Aquifer; $L=$ Lower Aquifer Zone of the Upper Three Runs Aquifer; $G=$ Gordon Aquifer 


\section{Uranium-233/234 Activities \\ Well Cluster FSB 98}

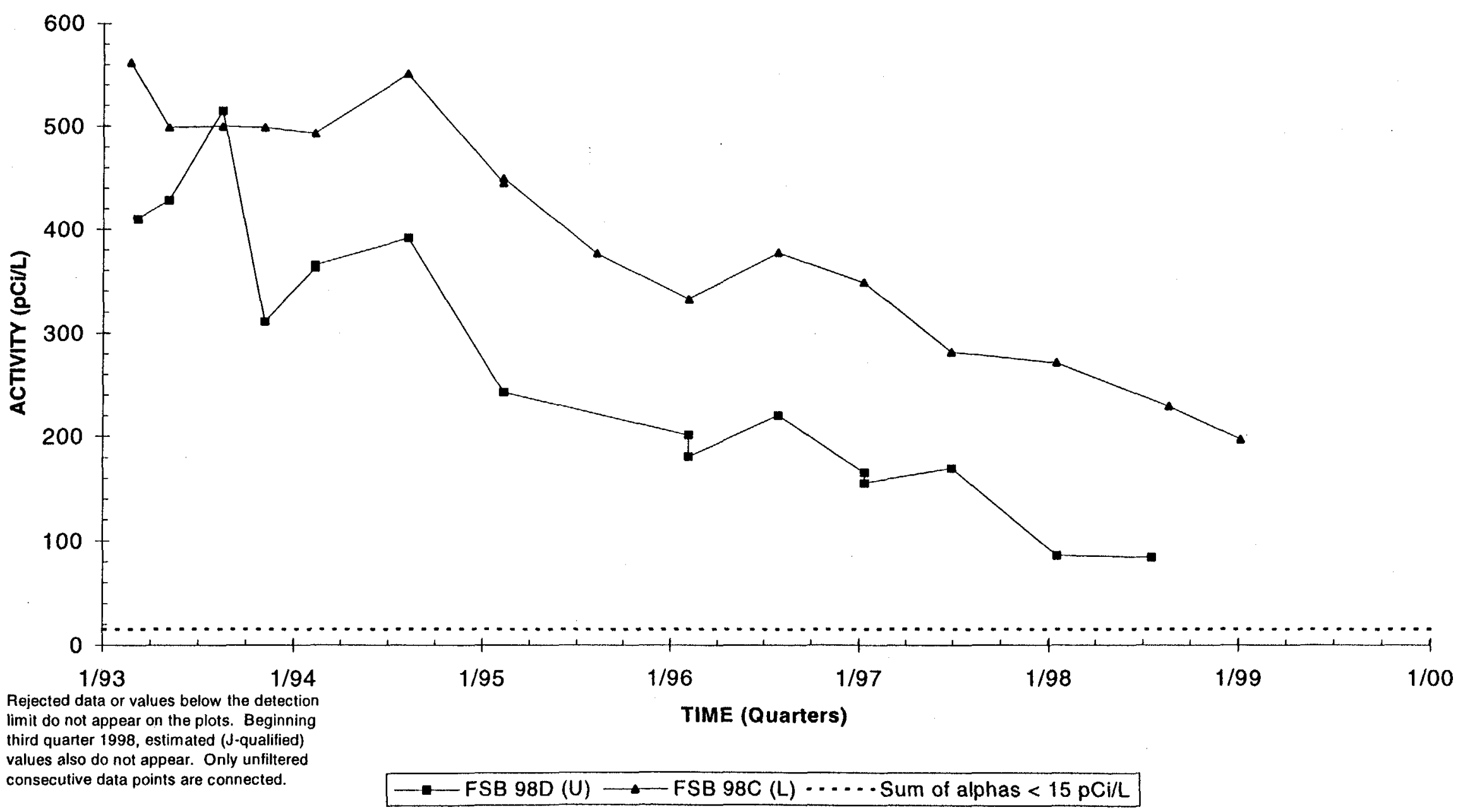

$U=$ Upper Aquifer Zone of the Upper Three Runs Aquifer, $L=$ Lower Aquifer Zone of the Upper Three Runs Aquiler; $G=$ Gordon Aquifer 


\section{Uranium-233/234 Activities}

Well Cluster FSB104

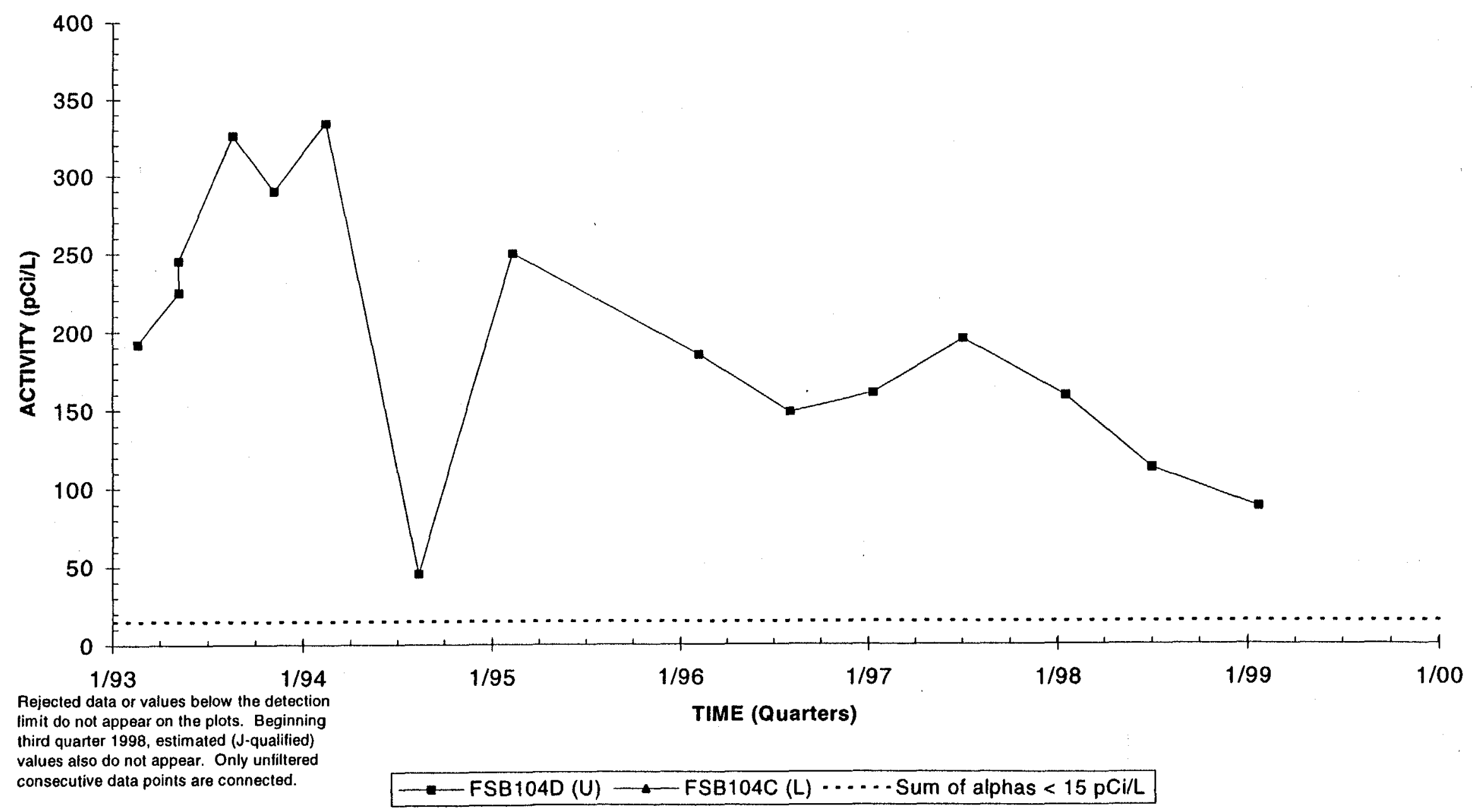

$U=$ Upper Aquifer Zone of the Upper Three Runs Aquifer, $L=$ Lower Aquiler Zone of the Upper Three Runs Aquifer, $G=$ Gordon Aquiler 


\section{Uranium-233/234 Activities \\ Well Cluster FSB107}

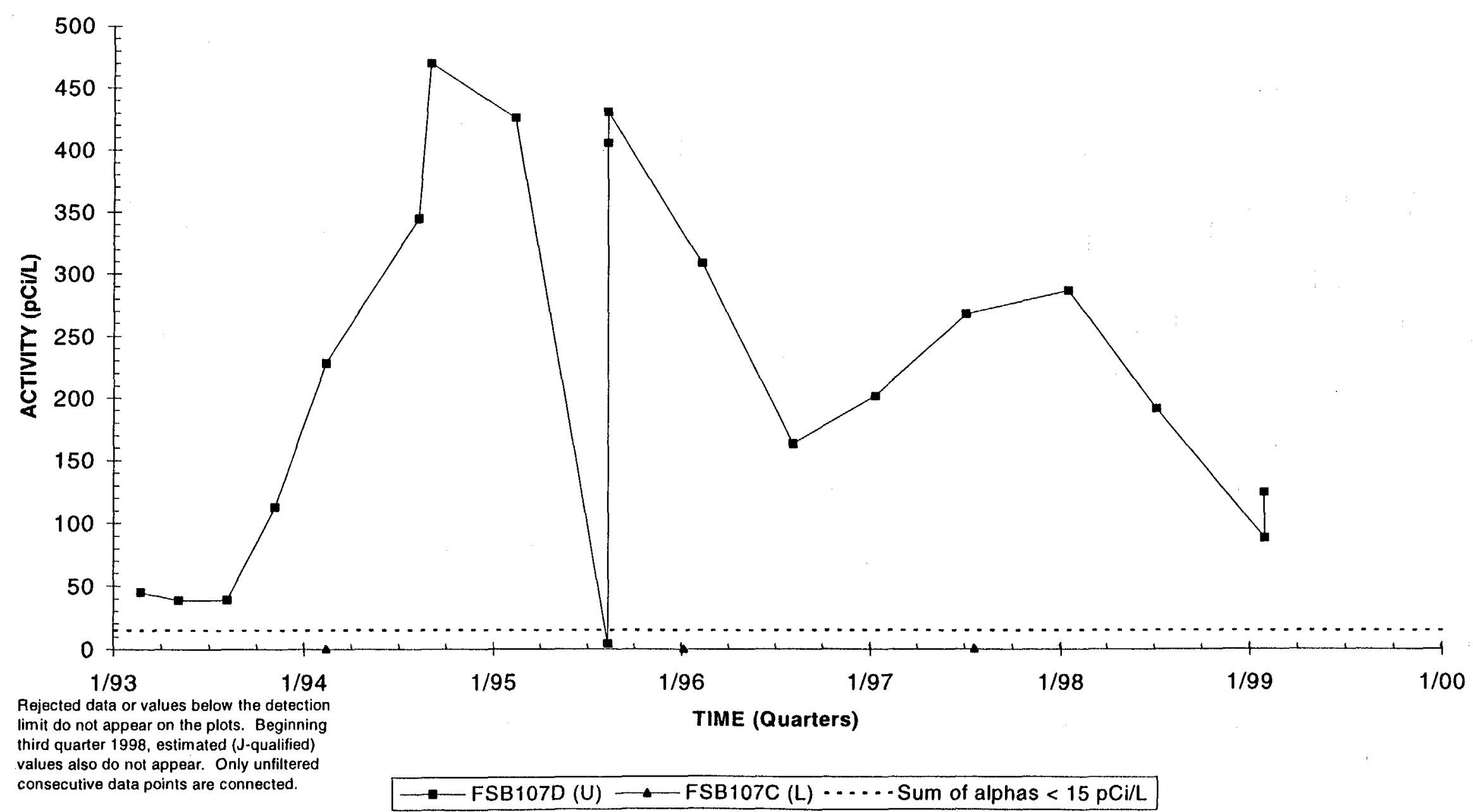

$U=$ Upper Aquifer Zone of the Upper Three Runs Aquifer, $L=$ Lower Aquifer Zone of the Upper Three Runs Aquifer, $G=$ Gordon Aquifer 


\section{Uranium-238 Activities}

Well FSB 77

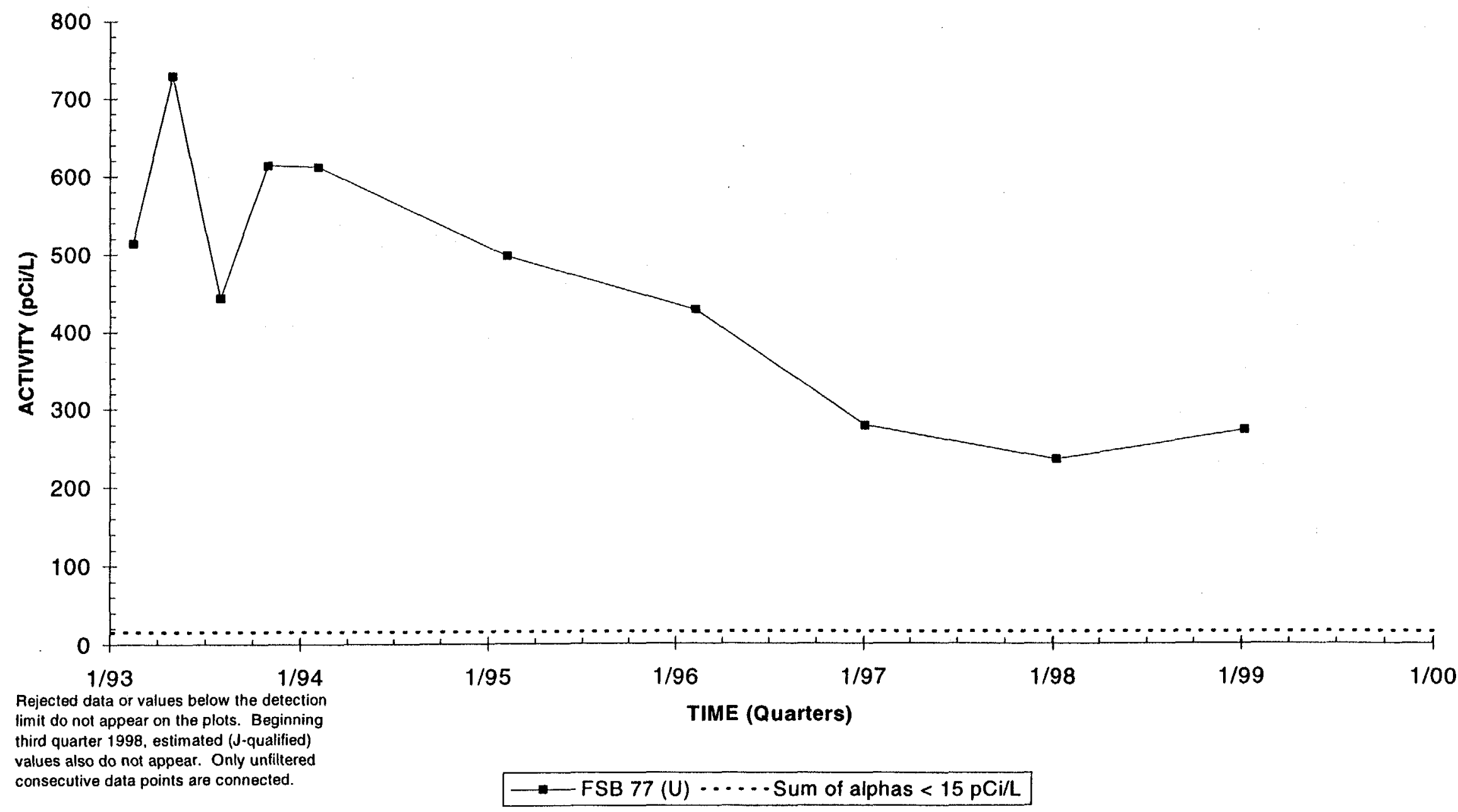

$U=$ Upper Aquifer Zone of the Upper Three Runs Aquifer, $L=$ Lower Aquifer Zone of the Upper Three Runs Aquifer; $G=$ Gordon Aquifer 


\section{Uranium-238 Activities \\ Well Cluster FSB 79}

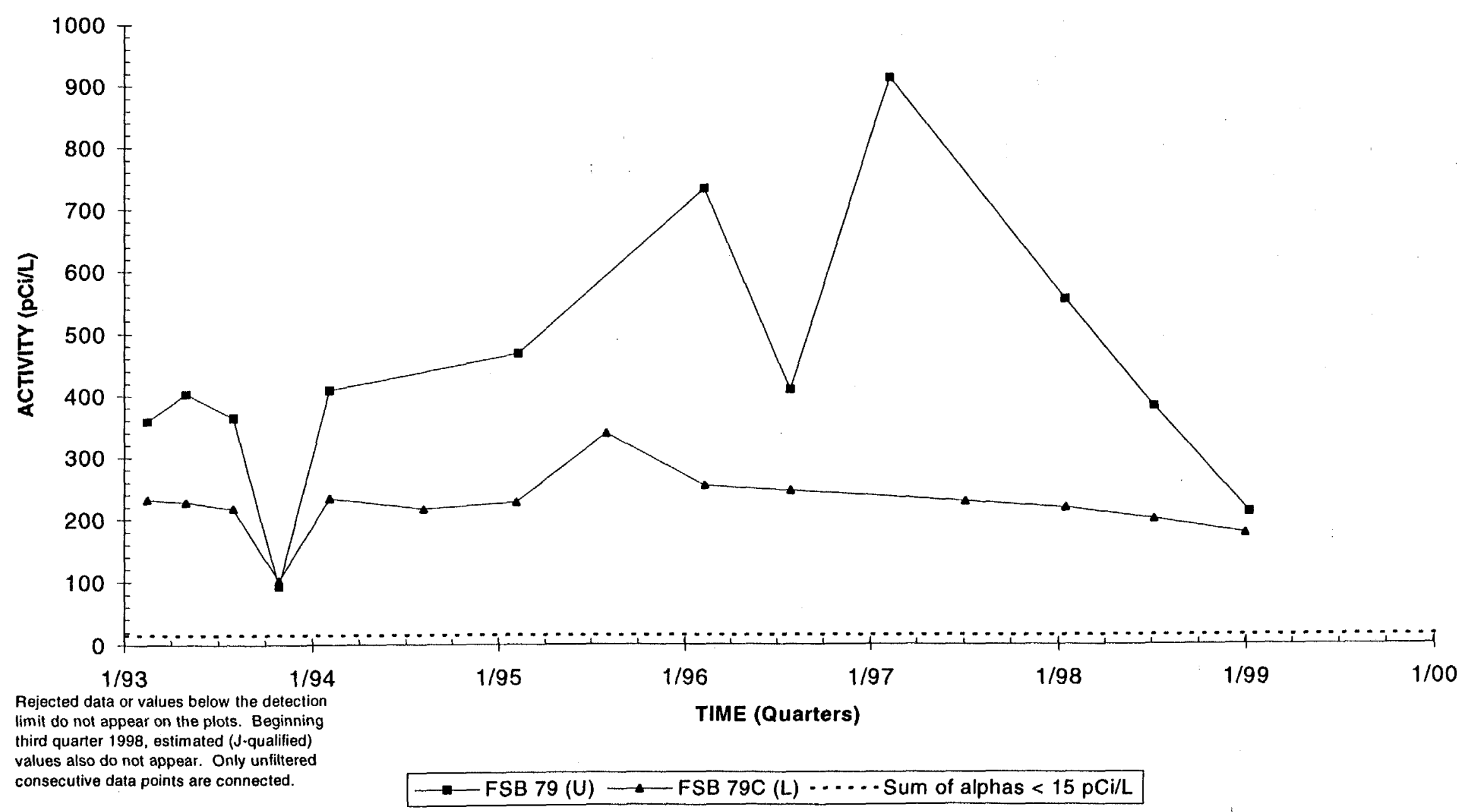

$U=$ Upper Aquifer Zone of the Upper Three Runs Aquifer, $L=$ Lower Aquifer Zone of the Upper Three Runs Aquifer; $G=$ Gordon Aquifer 


\section{Uranium-238 Activities \\ Well Cluster FSB 88}

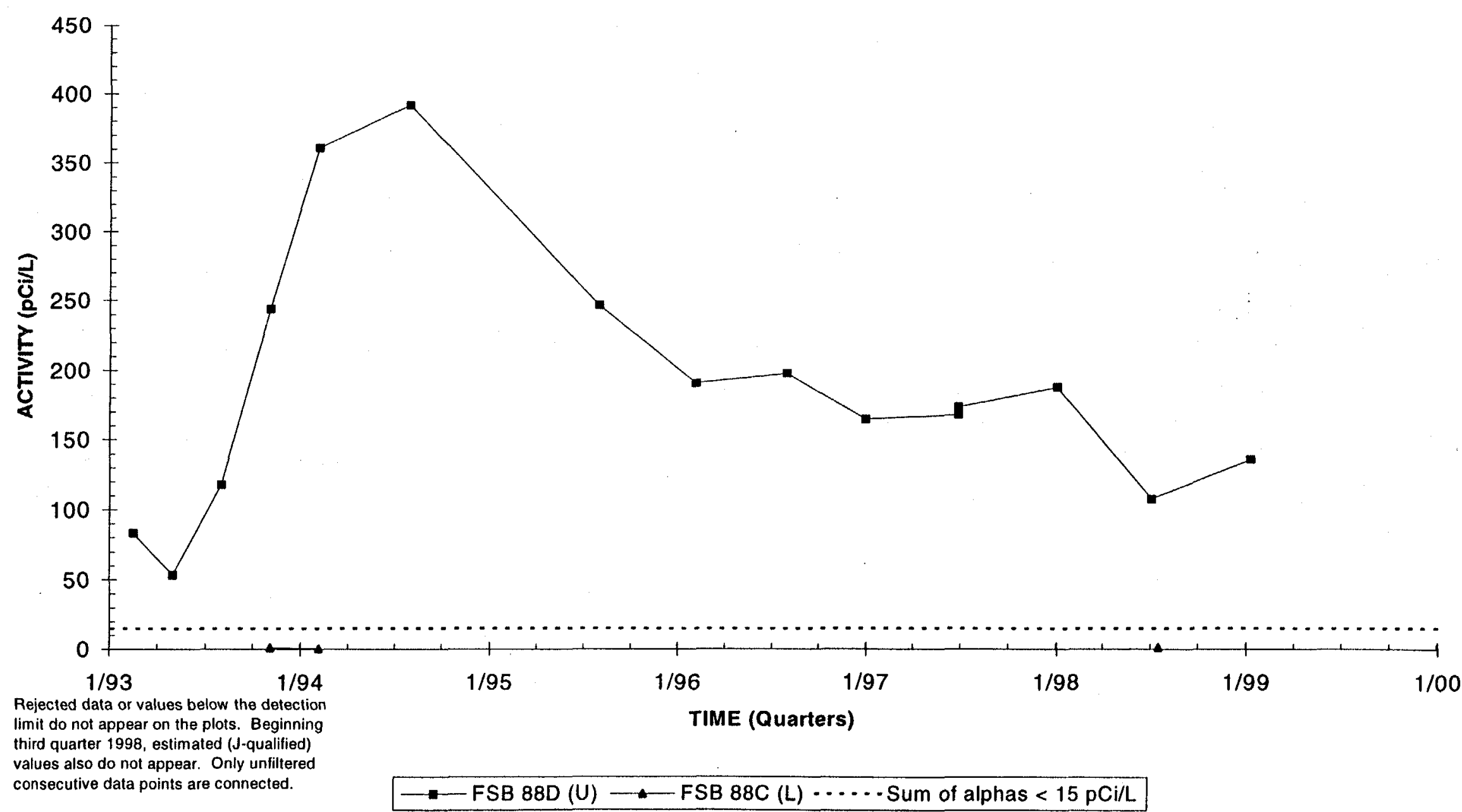

$U=$ Upper Aquifer Zone of the Upper Three Runs Aquifer, $L=$ Lower Aquifer Zone of the Upper Three Runs Aquifer, $G$ = Gordon Aquifer

\section{F-Area HWMF}




\section{Uranium-238 Activities \\ Well Cluster FSB 94}

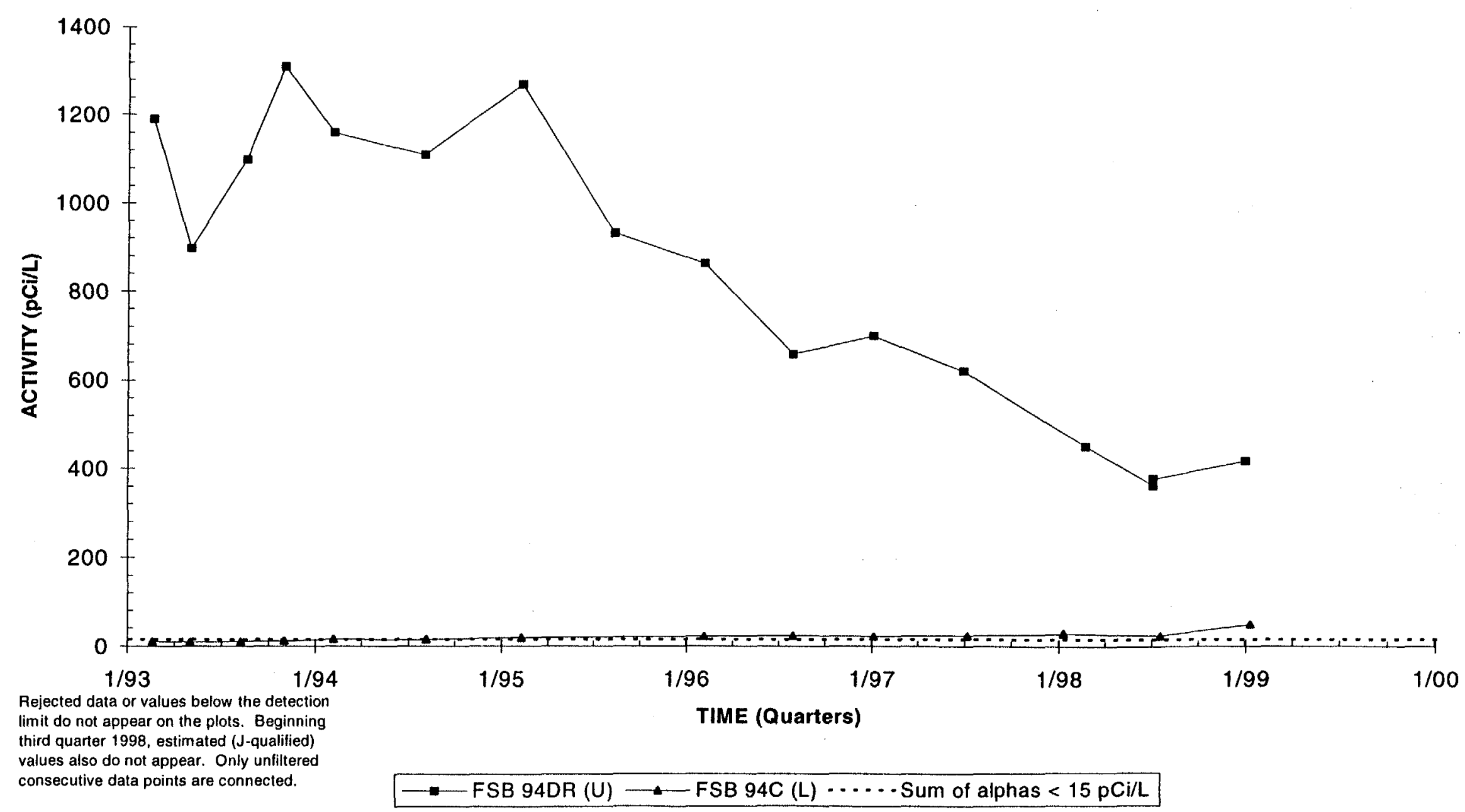

$U=$ Upper Aquiler Zone of the Upper Three Runs Aquiler, $L=$ Lower Aquifer Zone of the Upper Three Runs Aquifer; $G=$ Gordon Aquiler 


\section{Uranium-238 Activities}

Well Cluster FSB 95

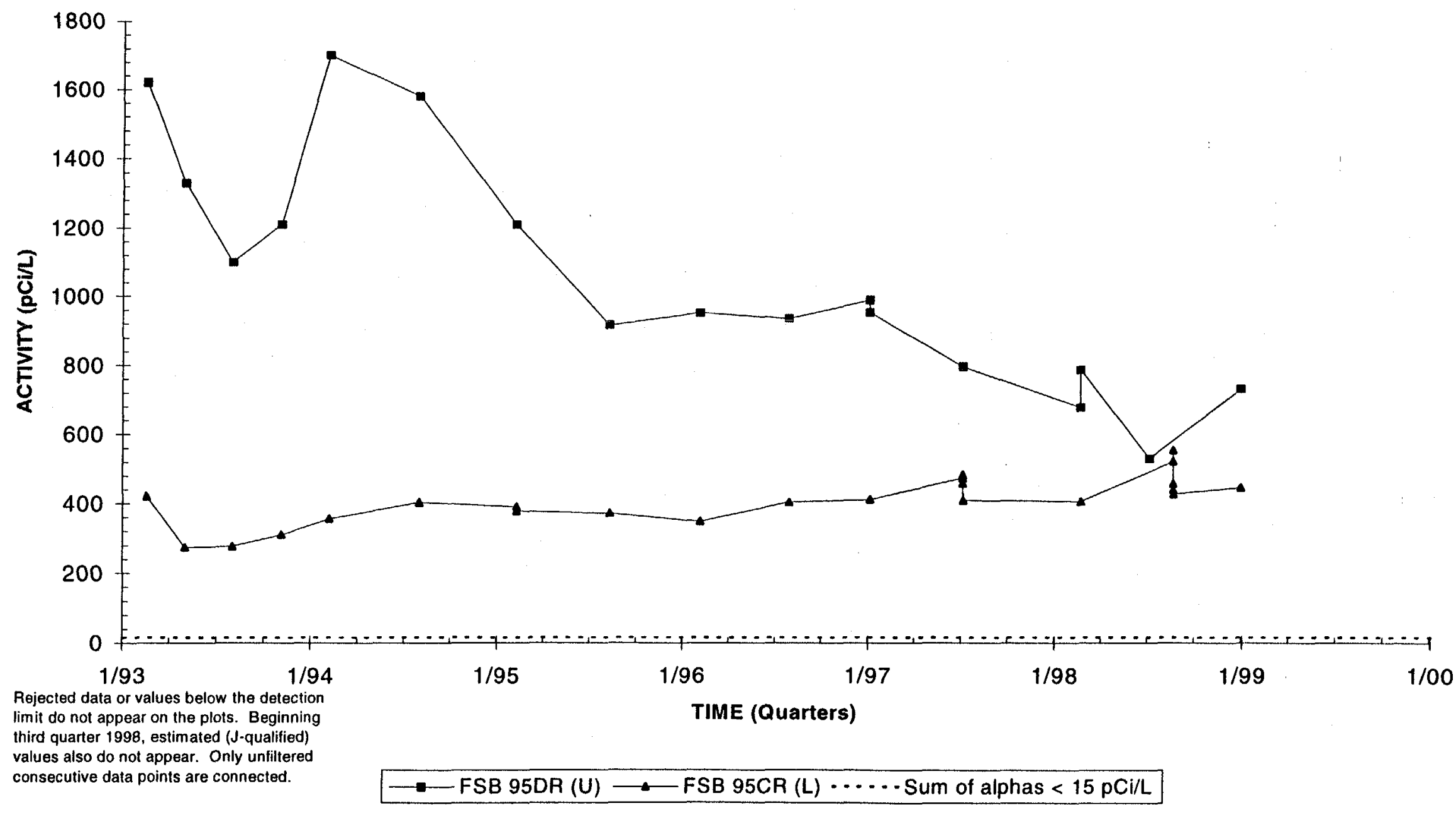

U = Upper Aquifer Zone of the Upper Three Runs Aquifer, $L=$ Lower Aquifer Zone of the Upper Three Runs Aquifer, $G=$ Gordon Aquifer 


\section{Uranium-238 Activities \\ Well Cluster FSB 97}

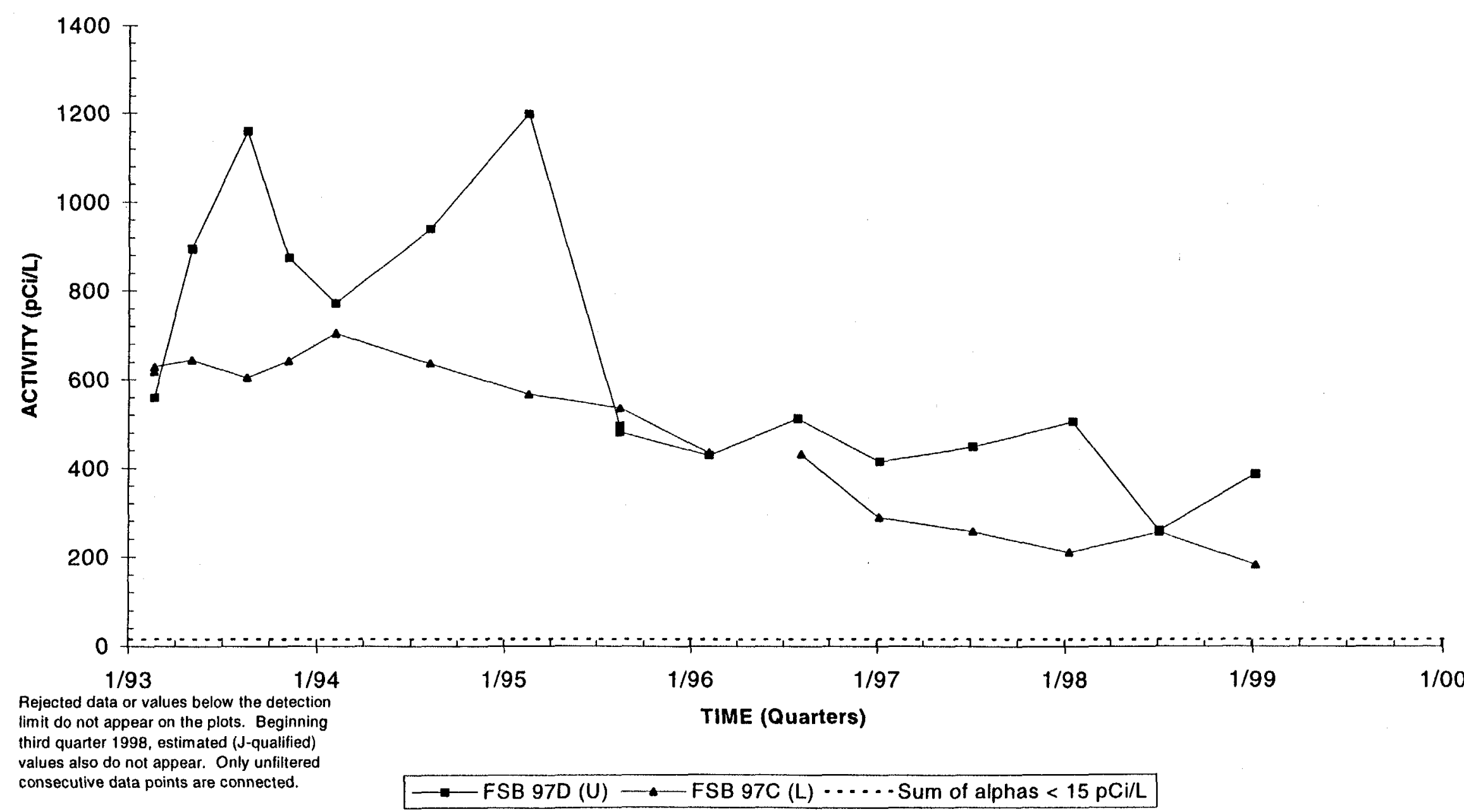

$U=$ Upper Aquifer Zone of the Upper Three Runs Aquifer, $L=$ Lower Aquifer Zone of the Upper Three Runs Aquifer; $G=$ Gordon Aquifer 


\section{Uranium-238 Activities \\ Well Cluster FSB 98}

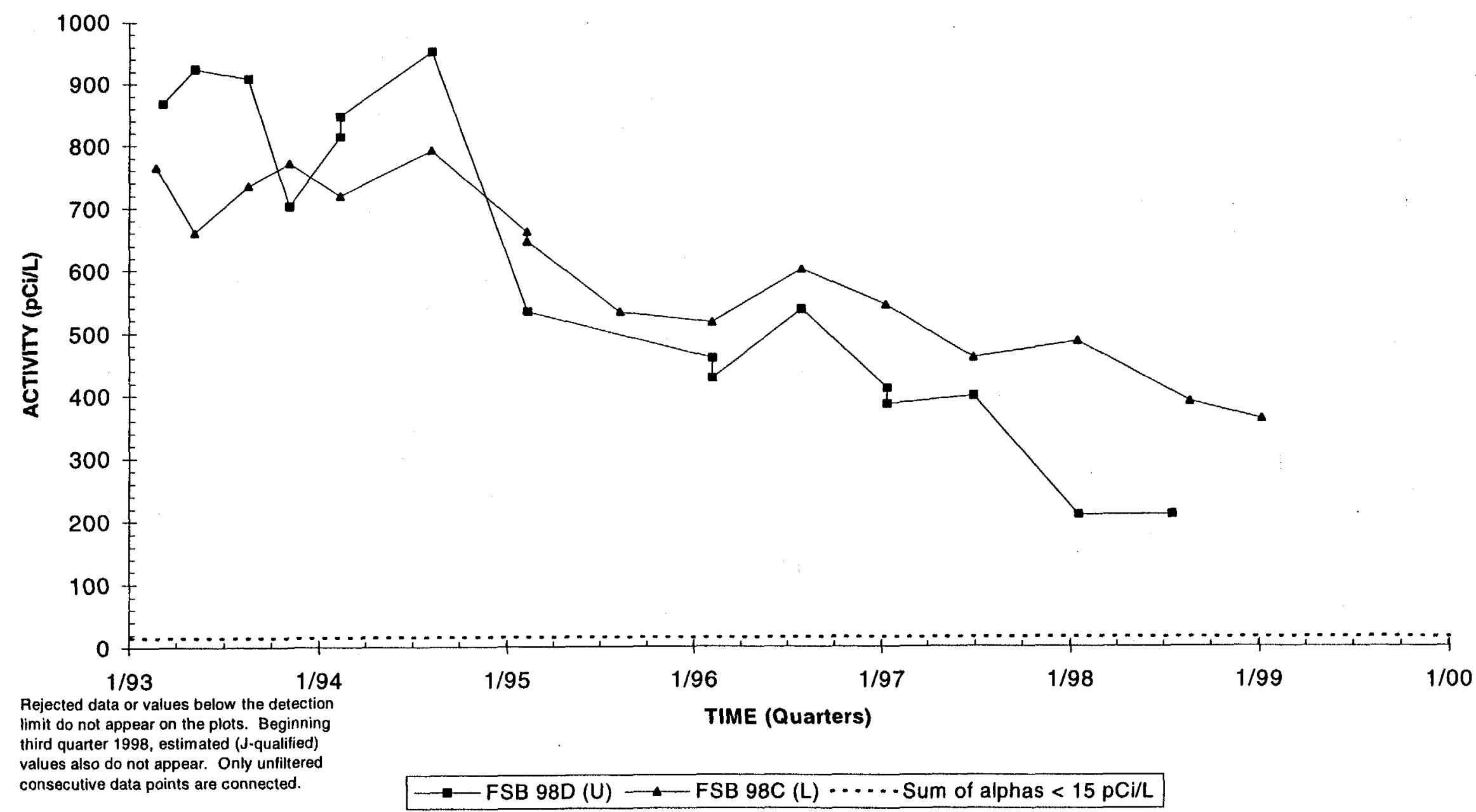

$\mathrm{U}=$ Upper Aquifer Zone of the Upper Three Runs Aquifer, $\mathrm{L}=$ Lower Aquiler Zone of the Upper Three Runs Aquifer; $G=$ Gordon Aquifer 


\section{Uranium-238 Activities \\ Well Cluster FSB104}

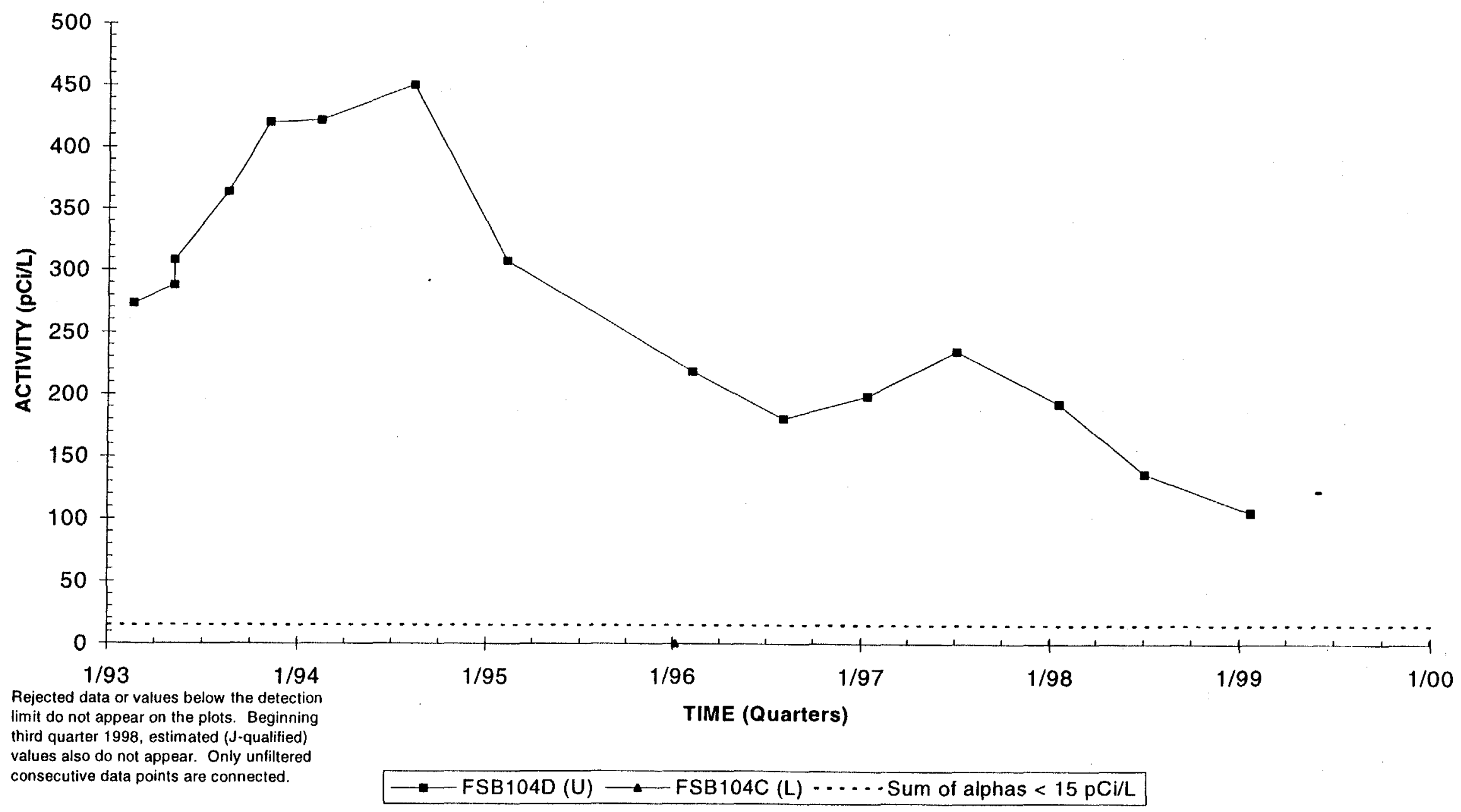

$U=$ Upper Aquifer Zone of the Upper Three Runs Aquifer, $\mathrm{L}=$ Lower Aquifer Zone of the Upper Three Runs Aquiter; $\mathrm{G}=\mathrm{Gordon}$ Aquifer 


\section{Uranium-238 Activities}

Well Cluster FSB107

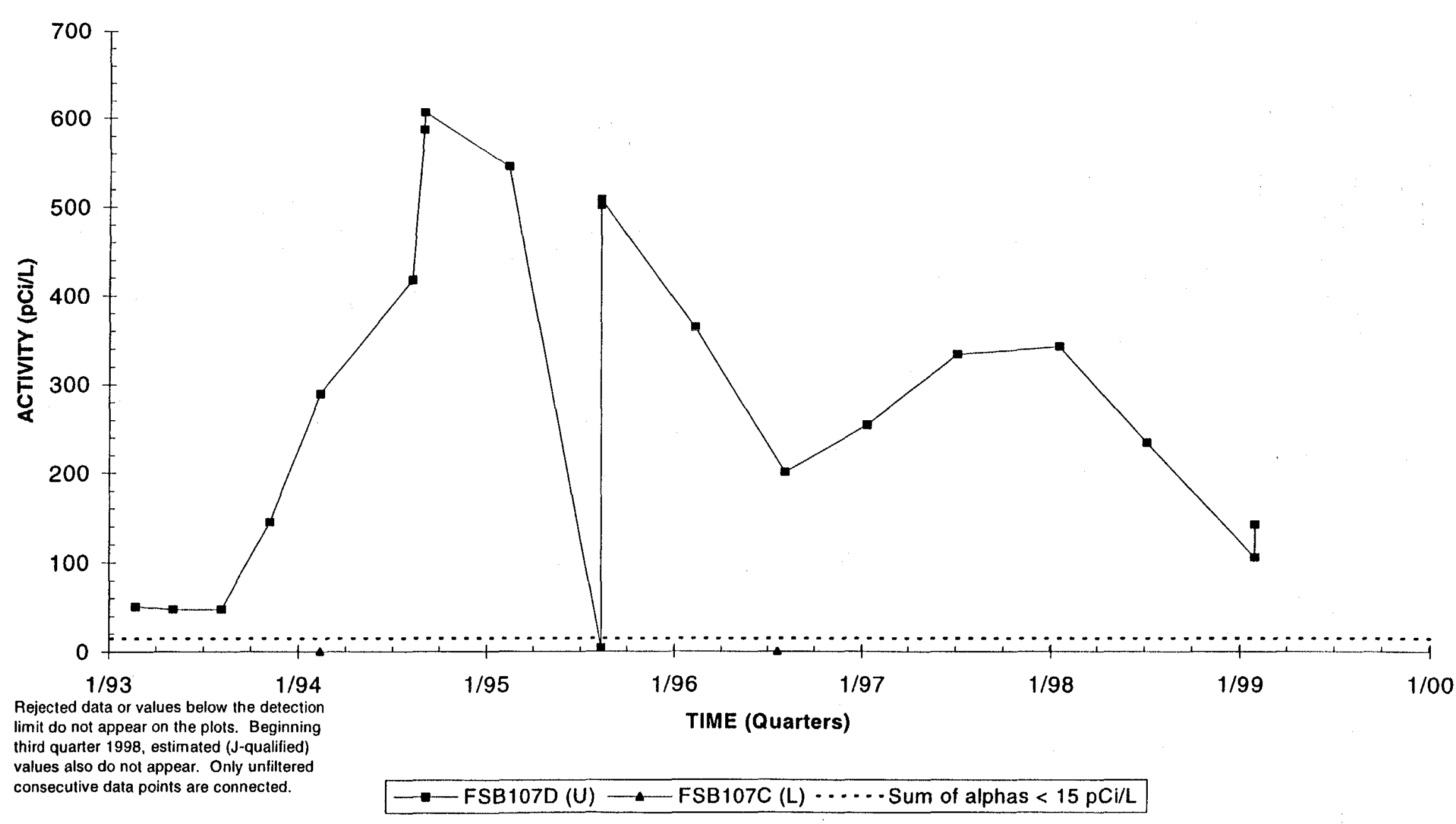

$U=$ Upper Aquifer Zone of the Upper Three Runs Aquifer, $L=$ Lower Aquifer Zone of the Upper Three Runs Aquifer, $G=$ Gordon Aquifer

F-Area HWMF D-58




\section{Strontium-90 Activities \\ Well Cluster FSB 78}

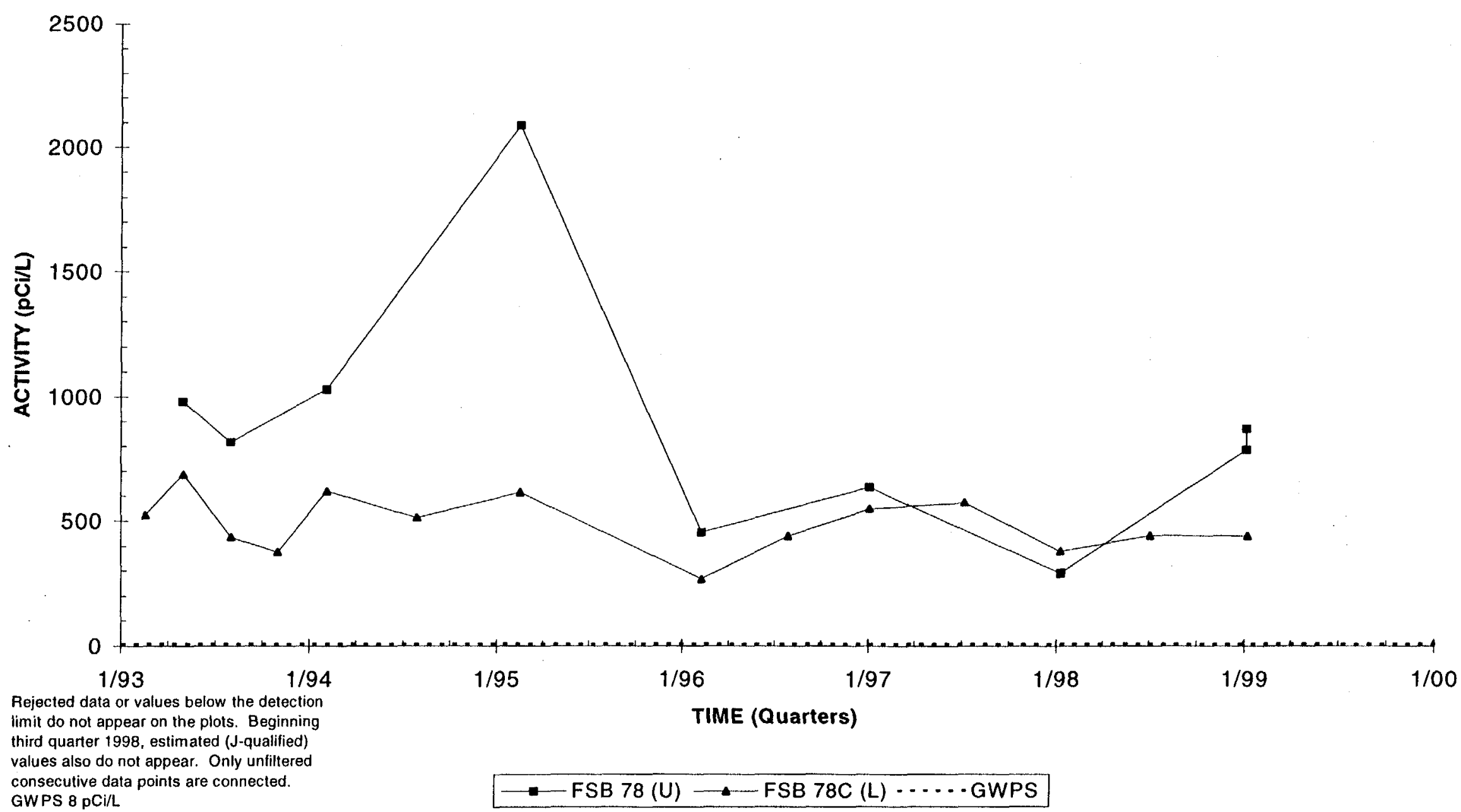

$U=$ Upper Aquifer Zone of the Upper Three Runs Aquifer, $L=$ Lower Aquifer Zone of the Upper Three Runs Aquifer, $G=$ Gordon Aquifer 


\section{Strontium-90 Activities \\ Well Cluster FSB 90}

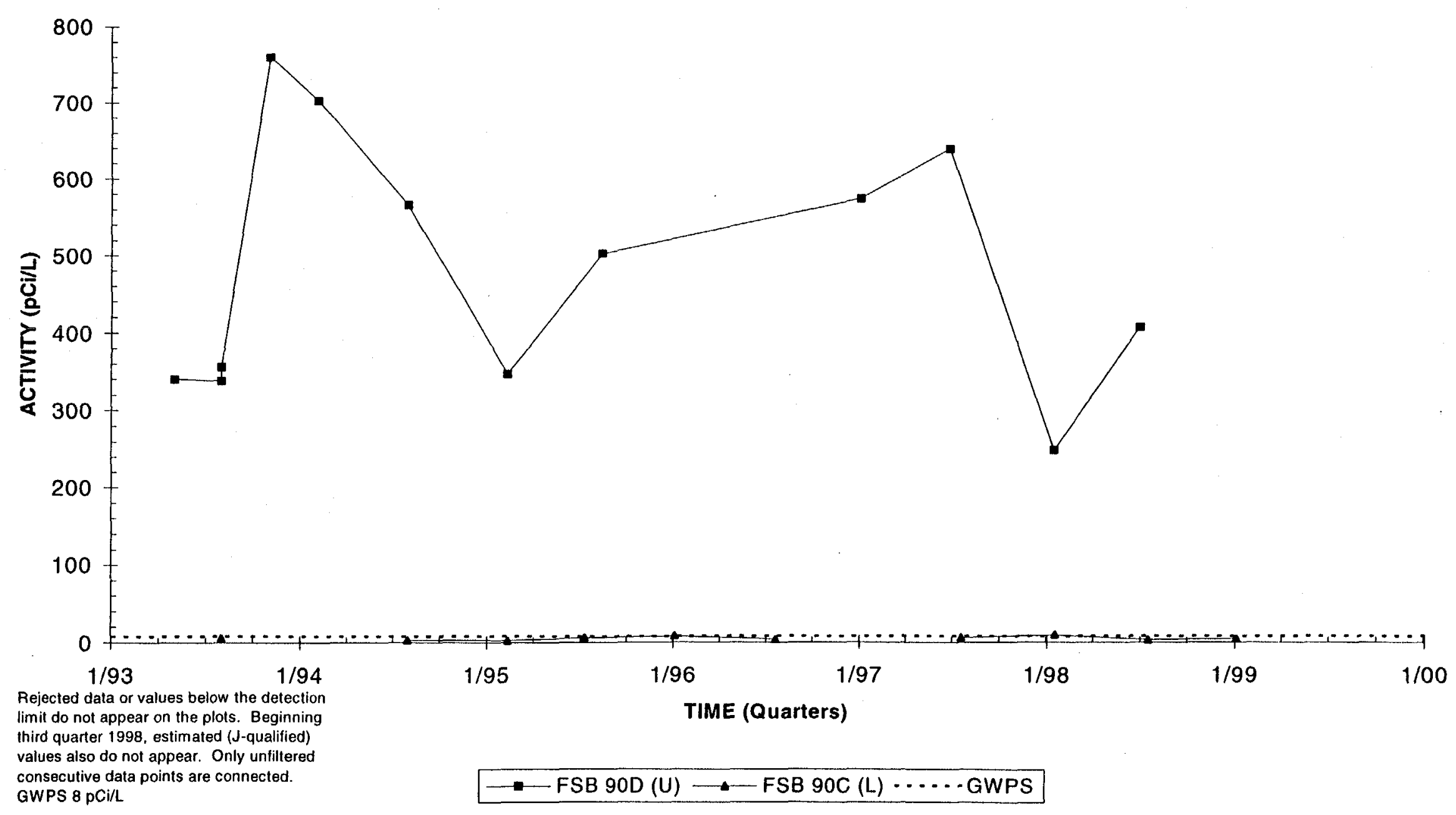

$U=$ Upper Aquifer Zone of the Upper Three Runs Aquifer; $L=$ Lower Aquifer Zone of the Upper Three Runs Aquifer; $G=$ Gordon Aquifer 


\section{Strontium-90 Activities}

Well Cluster FSB 92

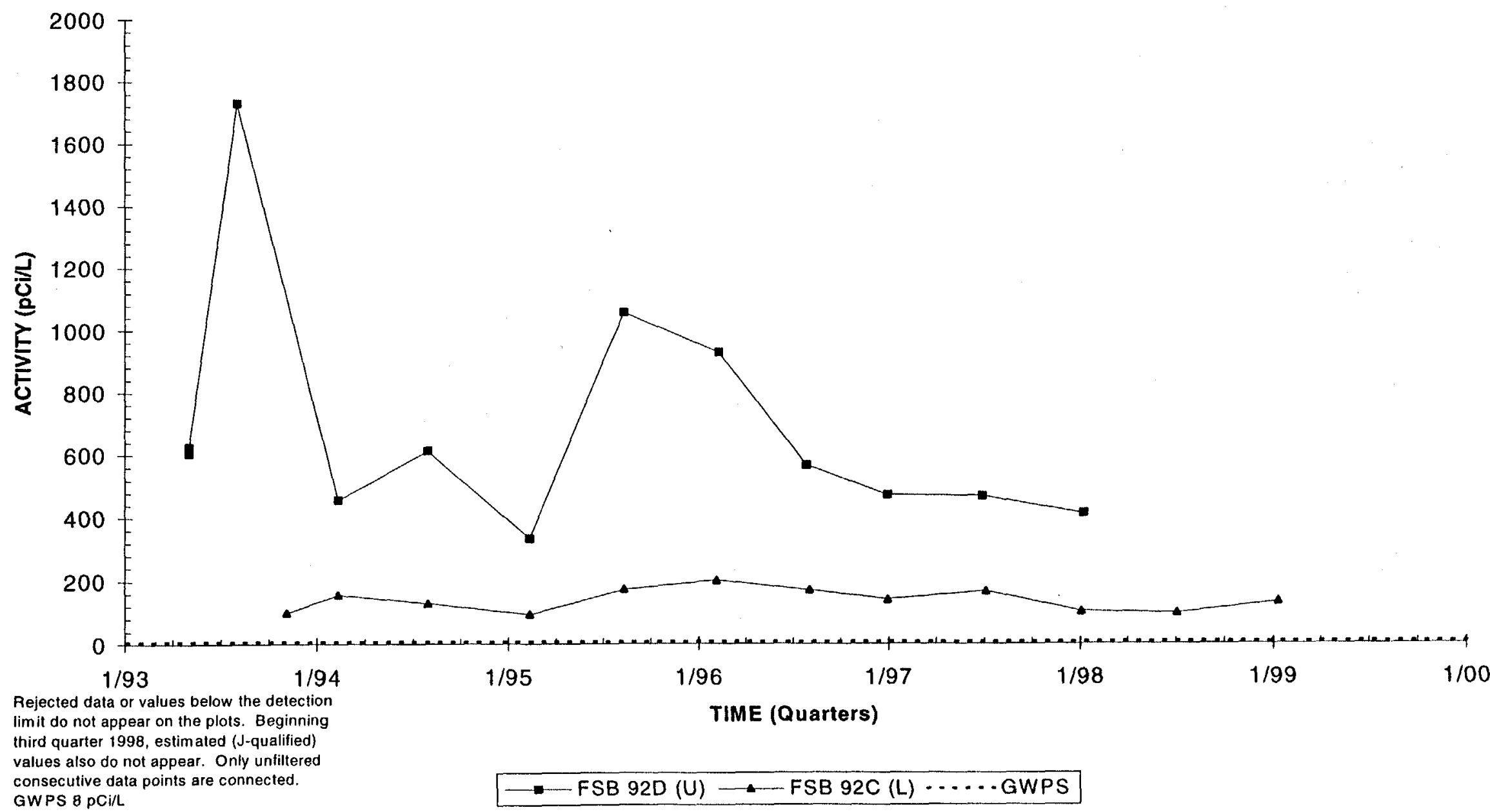

$\mathrm{U}=$ Upper Aquifer Zone of the Upper Three Runs Aquifer; $\mathrm{L}=$ Lower Aquifer Zone of the Upper Three Runs Aquifer; $\mathrm{G}=$ Gordon Aquifer 


\section{Strontium-90 Activities \\ Well Cluster FSB 94}

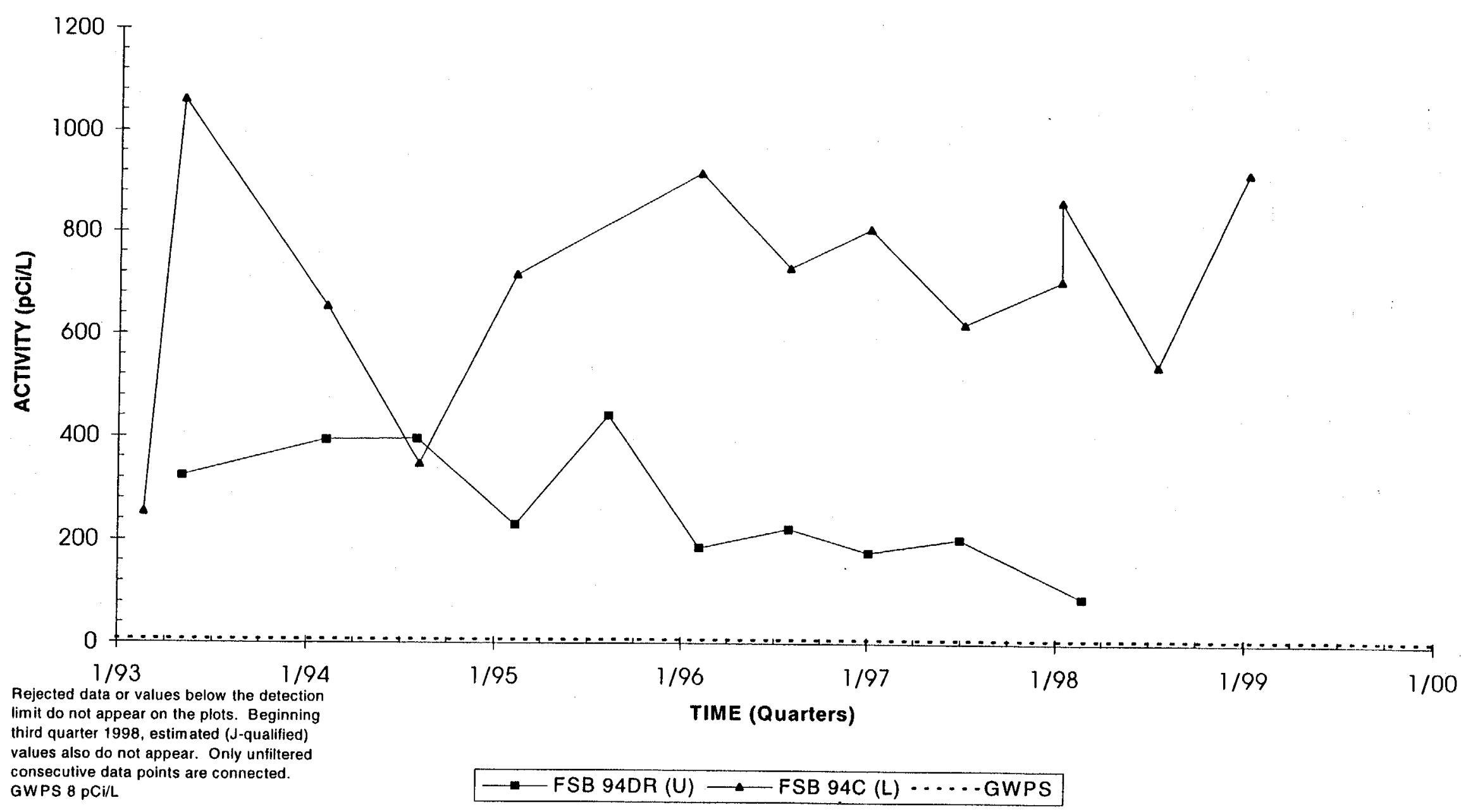

$U=$ Upper Aquifer Zone of the Upper Three Runs Aquifer; $L=$ Lower Aquifer Zone of the Upper Three Runs Aquifer; $\mathbf{G}=$ Gordon Aquifer 


\section{Strontium-90 Activities \\ Well Cluster FSB 95}

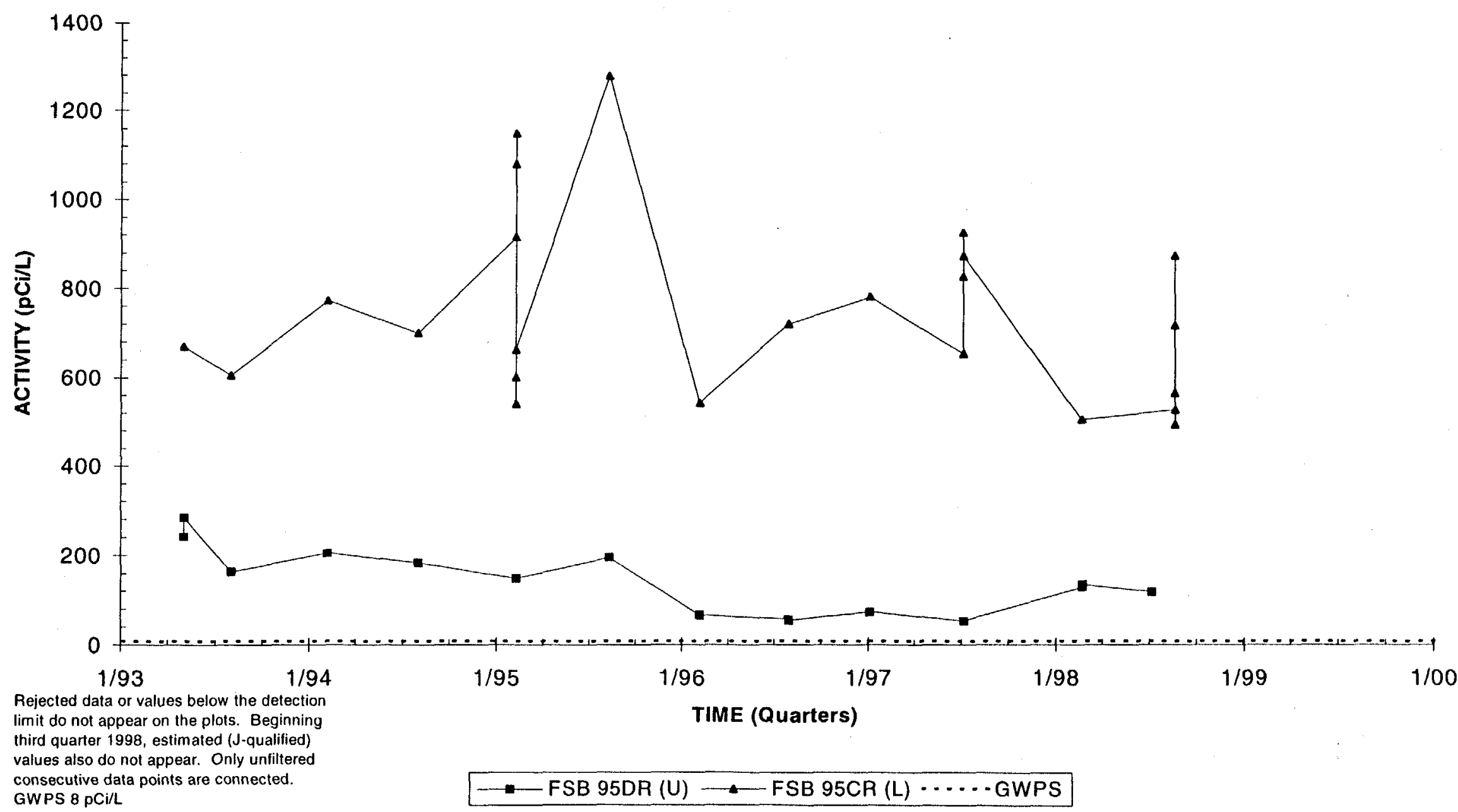

$U=$ Upper Aquifer Zone of the Upper Three Runs Aquifer, $L=$ Lower Aquifer Zone of the Upper Three Runs Aquifer; $G=$ Gordon Aquifer 


\section{Strontium-90 Activities \\ Well Cluster FSB 97}

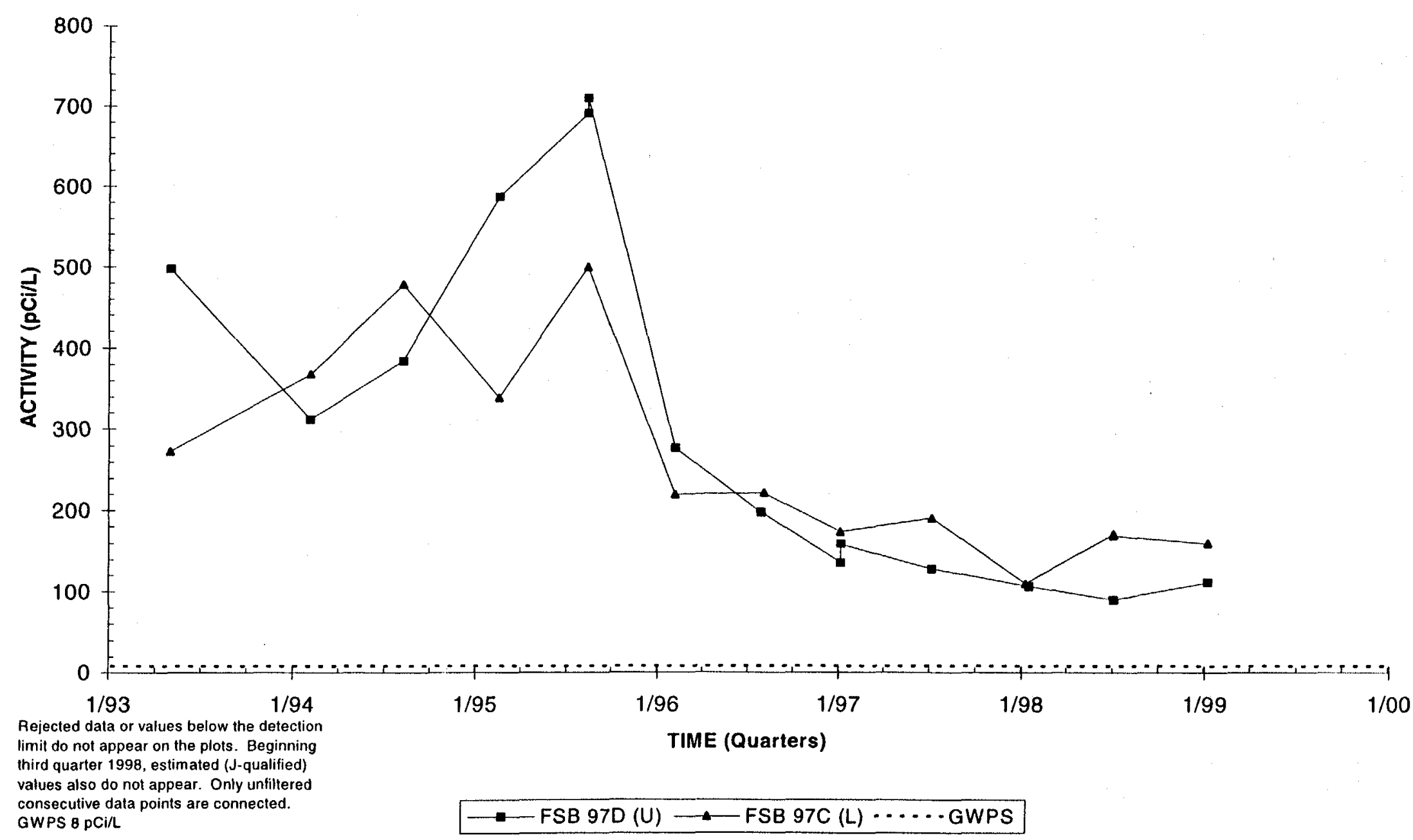

$U=$ Upper Aquifer Zone of the Upper Three Runs Aquifer, $\mathbf{L}=$ Lower Aquifer Zone of the Upper Three Runs Aquiler; $G=$ Gordon Aquifer 


\section{Strontium-90 Activities \\ Well Cluster FSB 98}

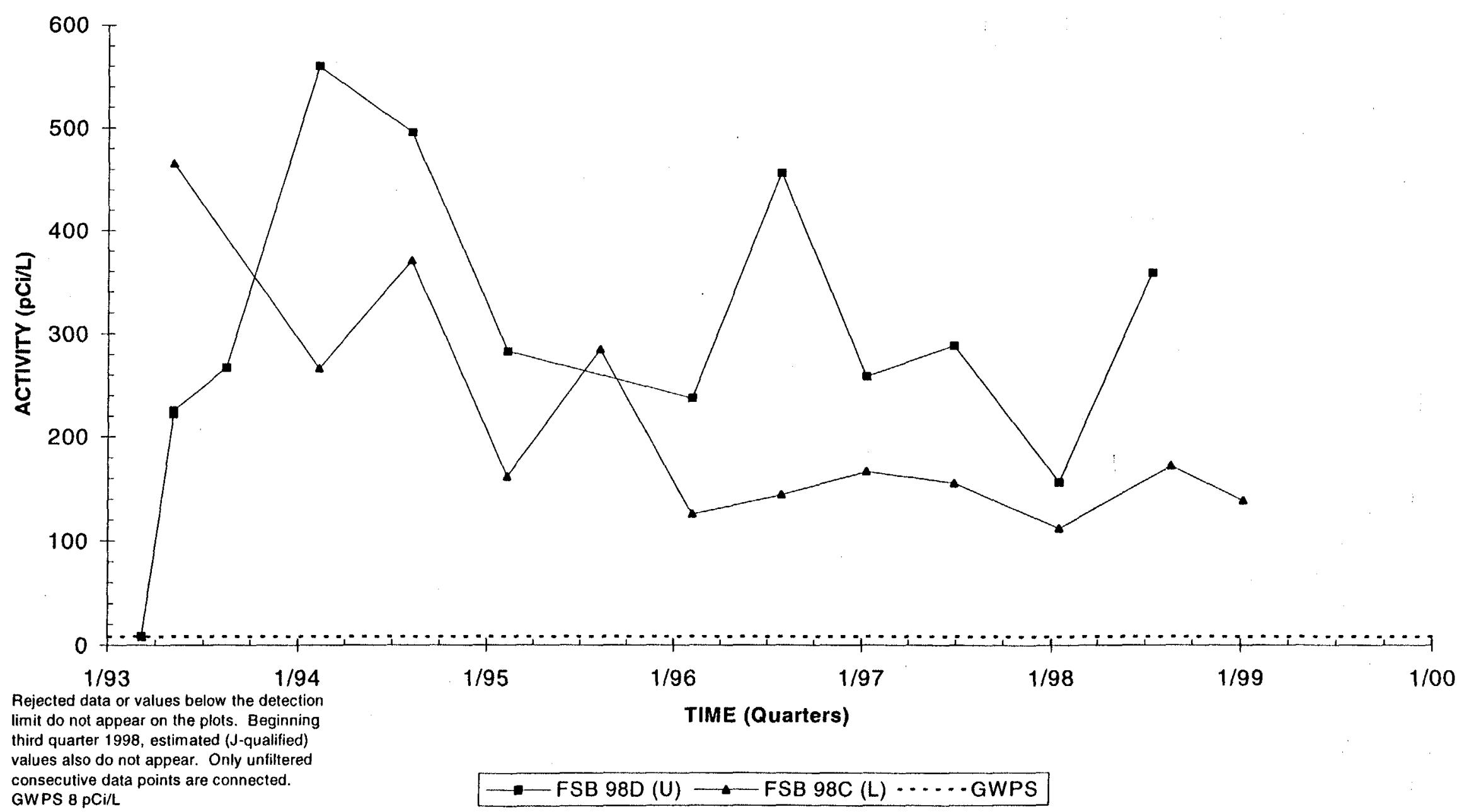

$U=$ Upper Aquifer Zone of the Upper Three Runs Aquiler; $L=$ Lower Aquifer Zone of the Upper Three Runs Aquiler; $G=$ Gordon Aquifer 


\section{Strontium-90 Activities \\ Well FSB102C}

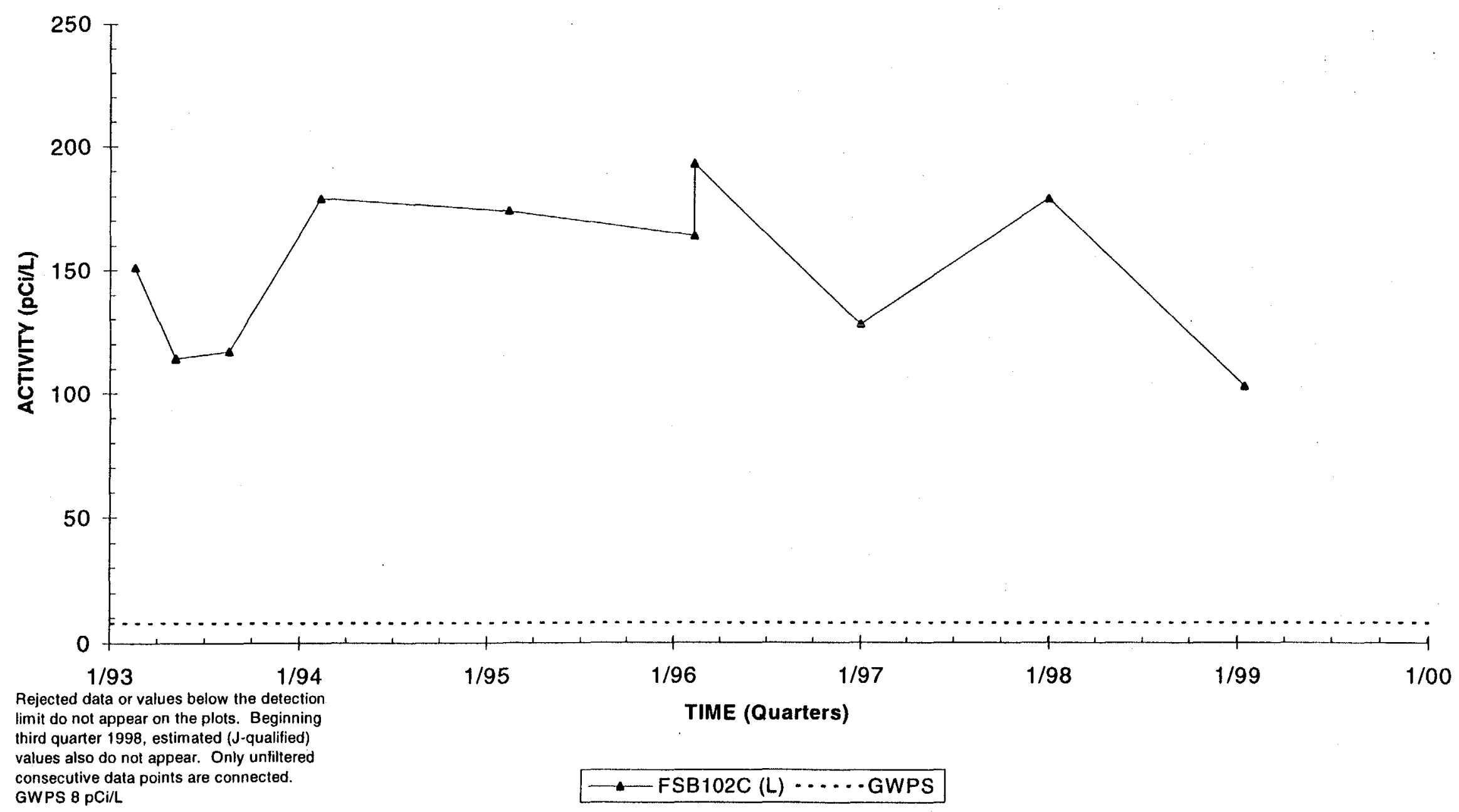

$\mathrm{U}=$ Upper Aquifer Zone of the Upper Three Runs Aquiter, $\mathrm{L}=\mathrm{L}$ Lower Aquifer Zone of the Upper Three Runs Aquifer, $\mathrm{G}=$ Gordon Aquifer 


\section{Strontium-90 Activities \\ Well Cluster FSB110}

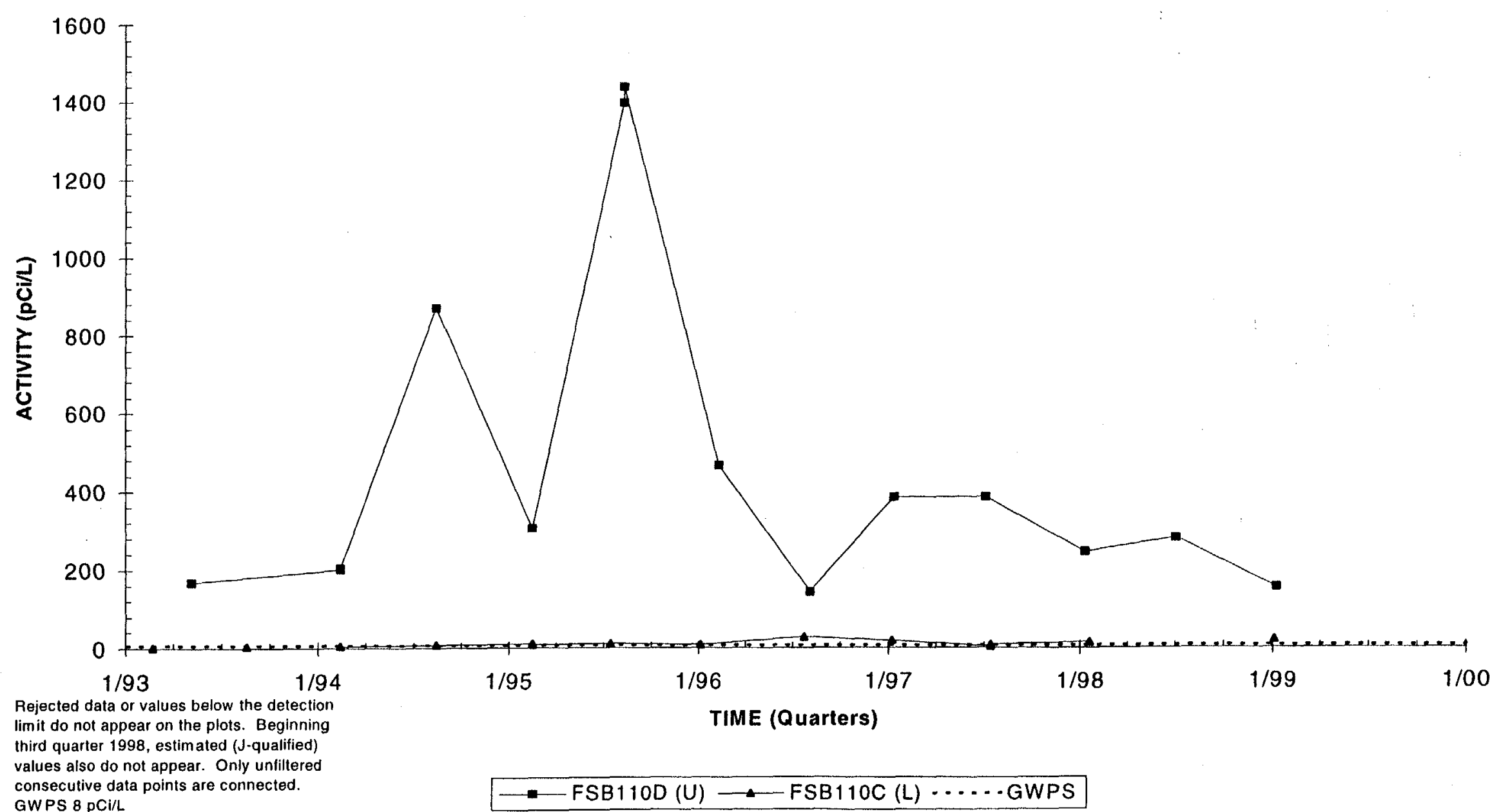

$\mathrm{U}=$ Upper Aquifer Zone of the Upper Three Runs Aquifer, $\mathrm{L}=$ Lower Aquifer Zone of the Upper Three Runs Aquifer; $\mathrm{G}=\mathrm{Gordon}$ Aquifer 


\section{Strontium-90 Activities \\ Well Cluster FSB112}

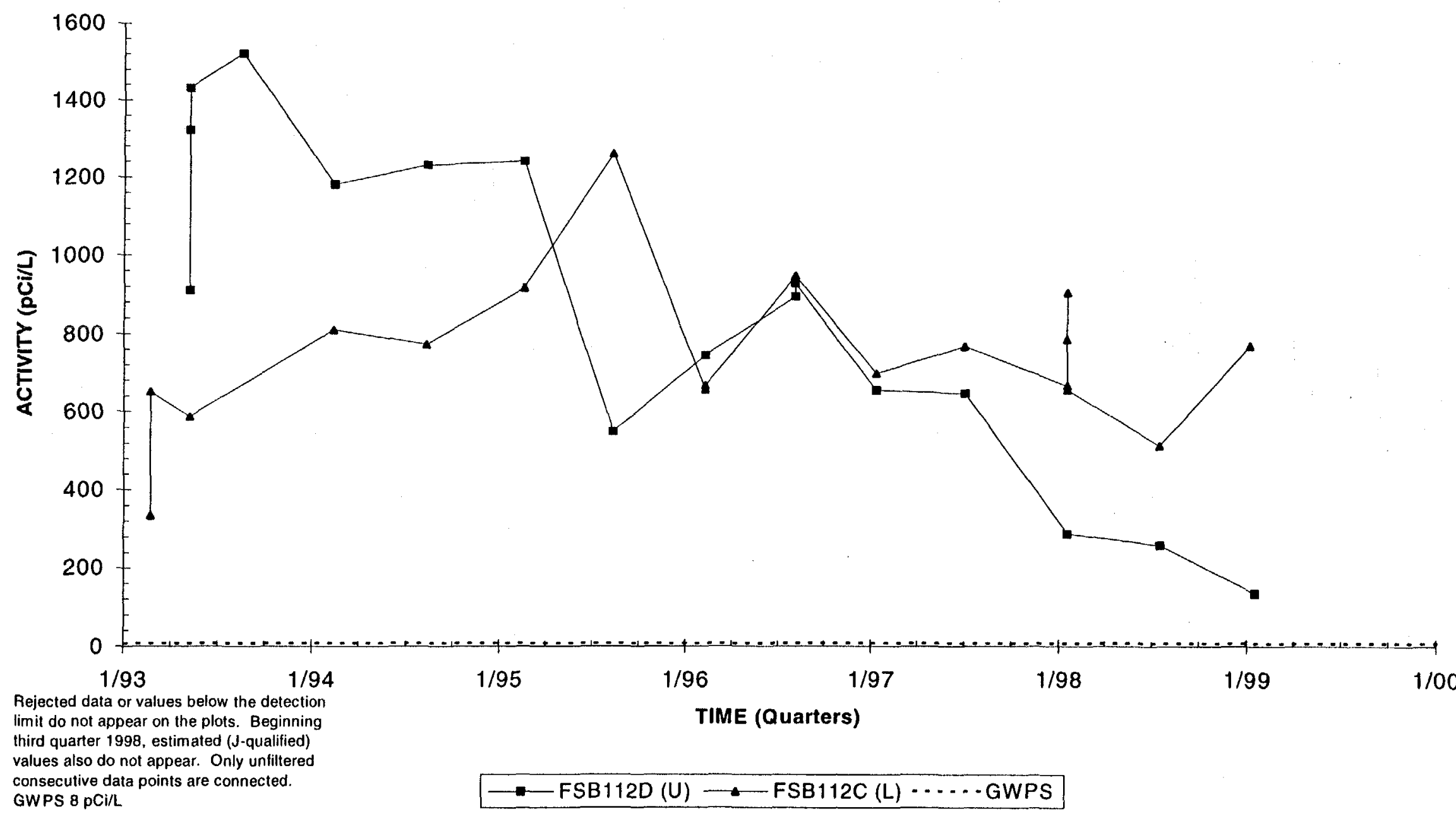

$U=$ Upper Aquifer Zone of the Upper Three Runs Aquifer; $L=$ Lower Aquiler Zone of the Upper Three Runs Aquifer; $G=$ Gordon Aquifer 


\section{Technetium-99 Activities \\ Well Cluster FSB 78}

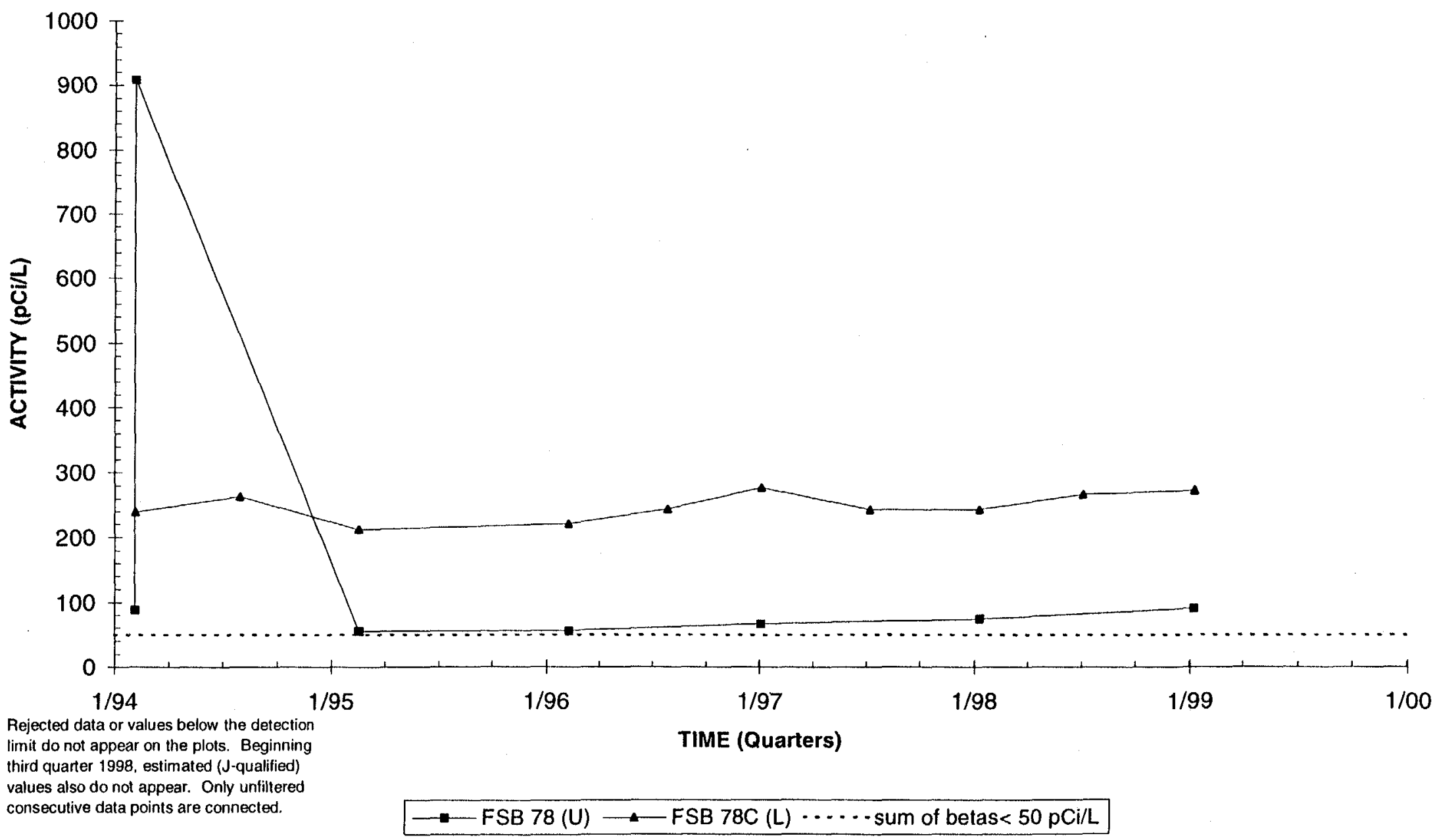

Note: The time-series plots for technetium-99 begin with 1994 data because results during 1993 were anomalously high.

$U=$ Upper Aquifer Zone of the Upper Three Runs Aquifer, $L=$ Lower Aquifer Zone of the Upper Three Runs Aquifer, $G=$ Gordon Aquifer 


\section{Technetium-99 Activities \\ Well Cluster FSB 90}

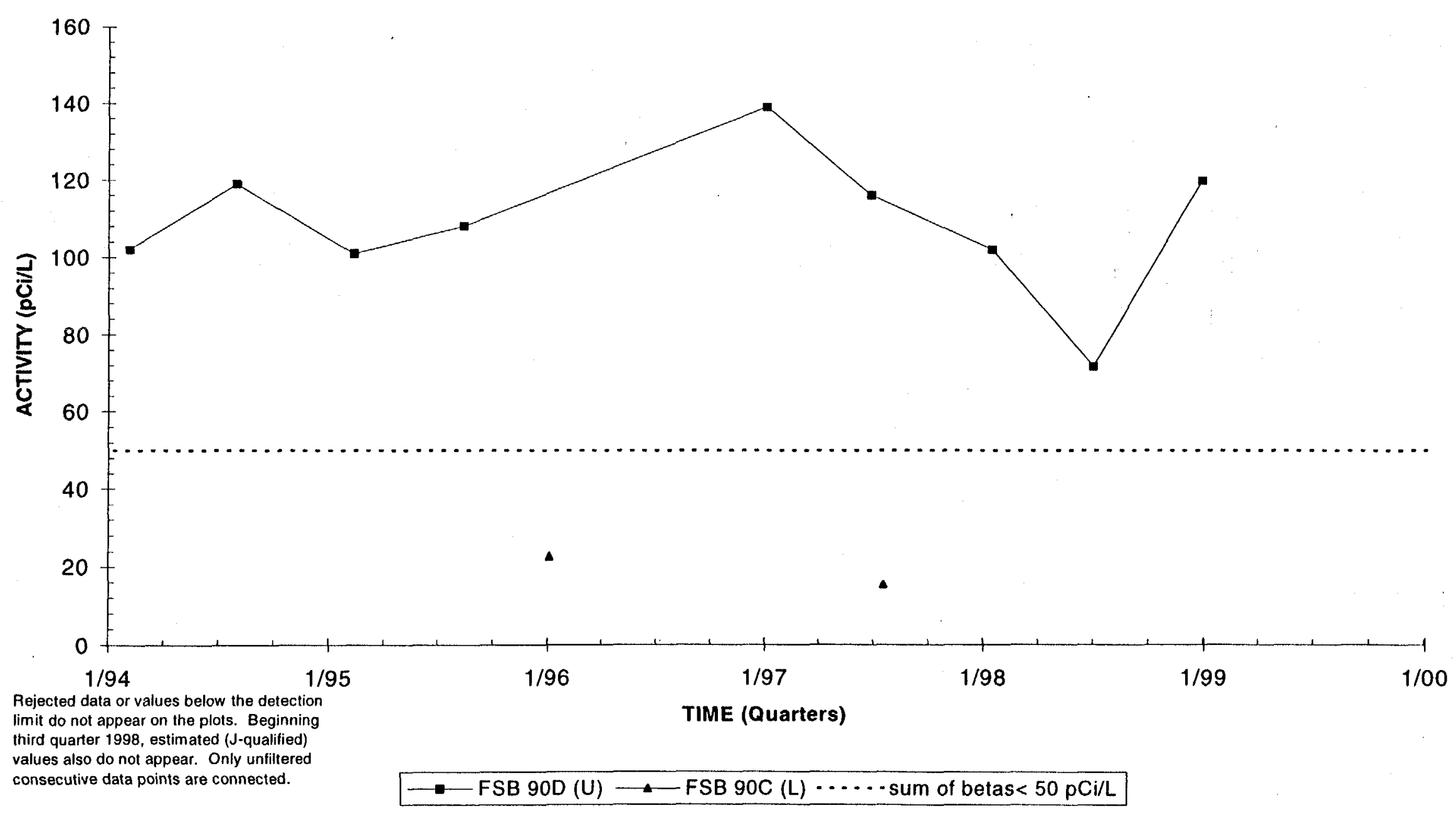

Note: The time-series plots for technetium-99 begin with 1994 data because results during 1993 were anomalously high.

$\mathbf{U}=$ Upper Aquifer Zone of the Upper Three Runs Aquifer; $\mathbf{L}=$ Lower Aquifer Zone of the Upper Three Runs Aquiler; $\mathbf{G}=$ Gordon Aquifer 


\section{Technetium-99 Activities \\ Well Cluster FSB 92}

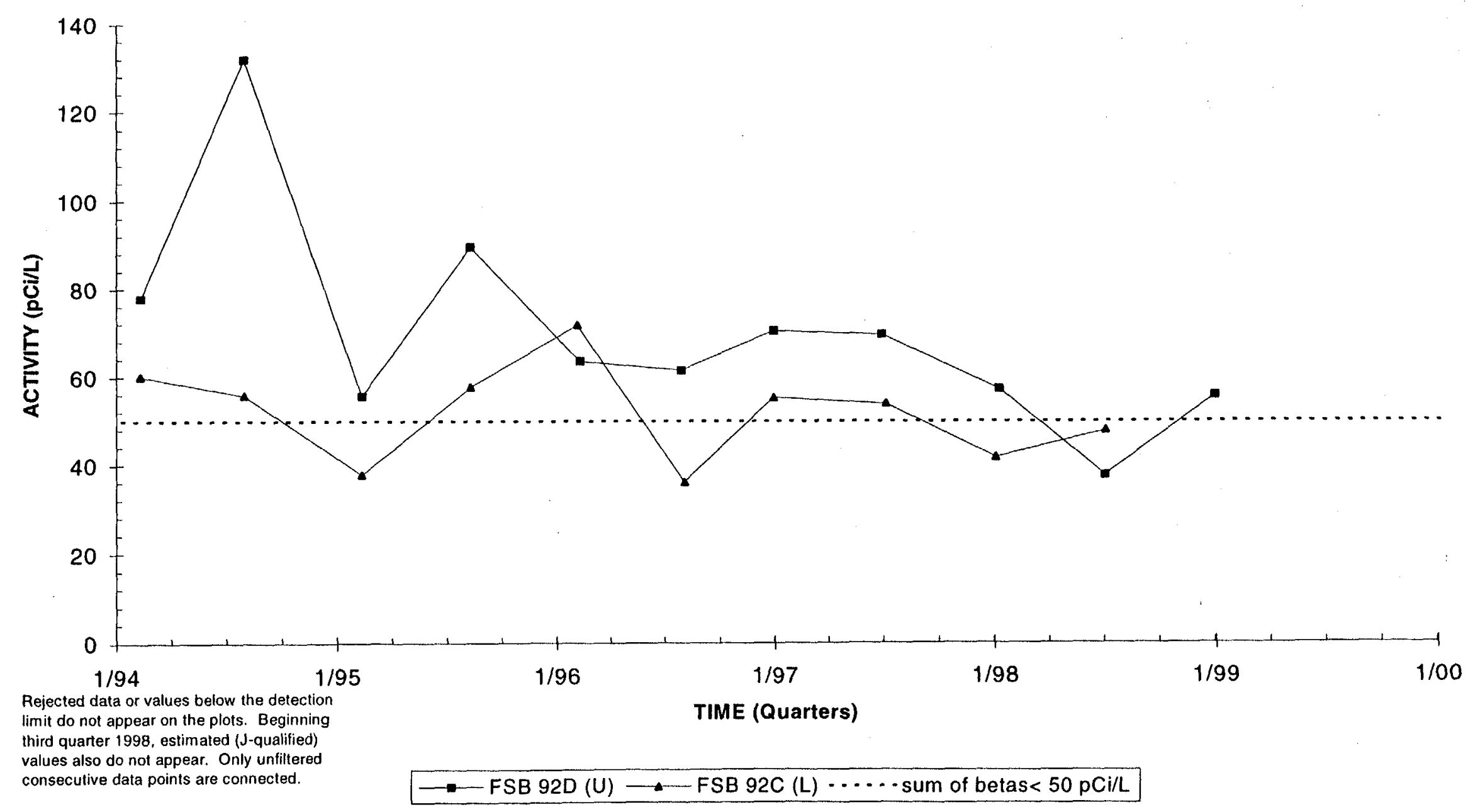

Note: The time-series plots for technetium-99 begin with 1994 data because results during 1993 were anomalously high.

$U=$ Upper Aquifer Zone of the Upper Three Runs Aquifer, $L=$ Lower Aquifer Zone of the Upper Three Runs Aquifer, $G=$ Gordon Aquifer 


\section{Technetium-99 Activities \\ Well Cluster FSB 94}

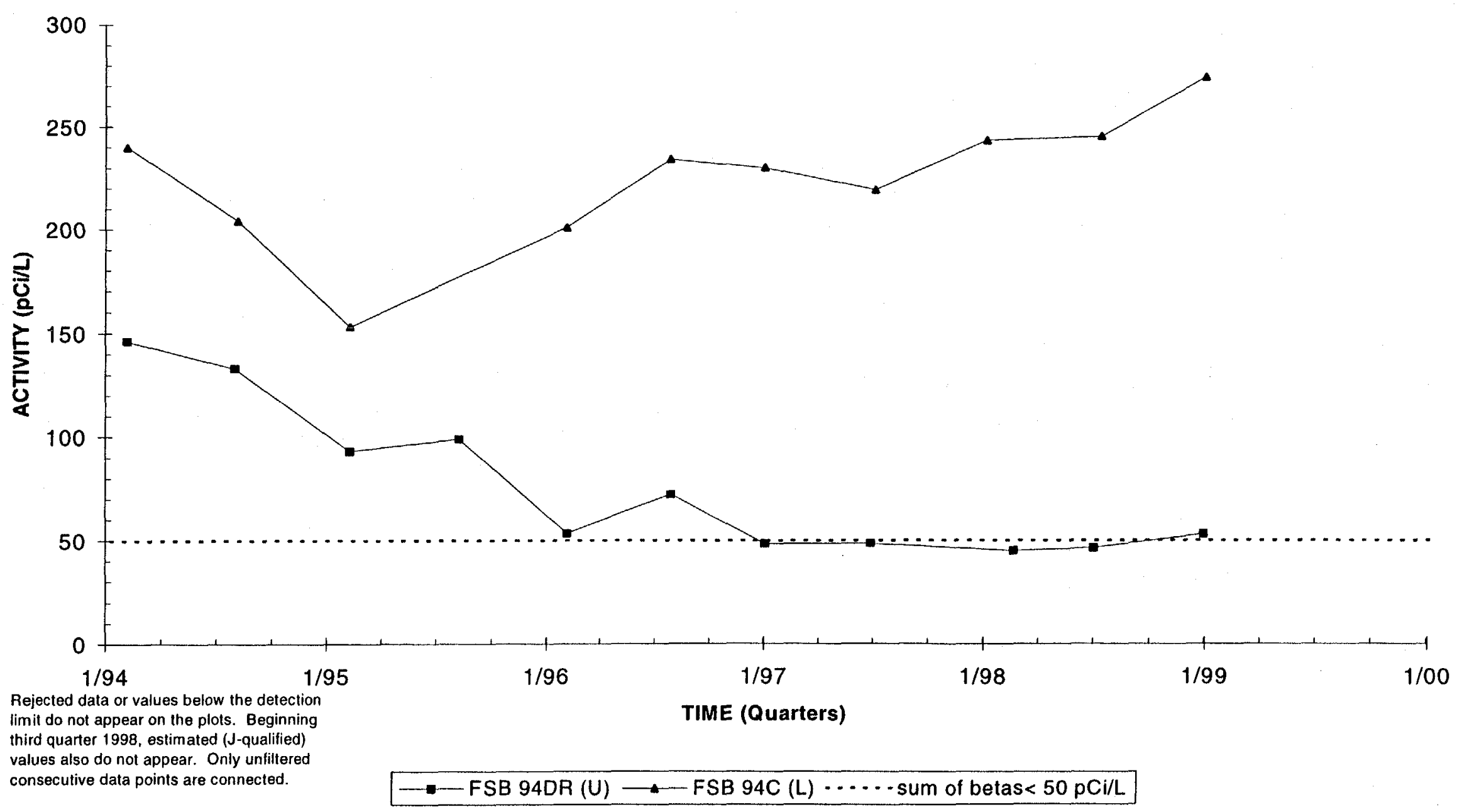

Note: The time-series plots for technetium-99 begin with 1994 data because results during 1993 were anomalously high.

$U=$ Upper Aquiler Zone of the Upper Three Runs Aquifer, $L=$ Lower Aquifer Zone of the Upper Three Runs Aquifer; $G=$ Gordon Aquiler

\begin{tabular}{ll}
\hline F-Area HWMF & D-72
\end{tabular}




\section{Technetium-99 Activities \\ Well Cluster FSB 95}

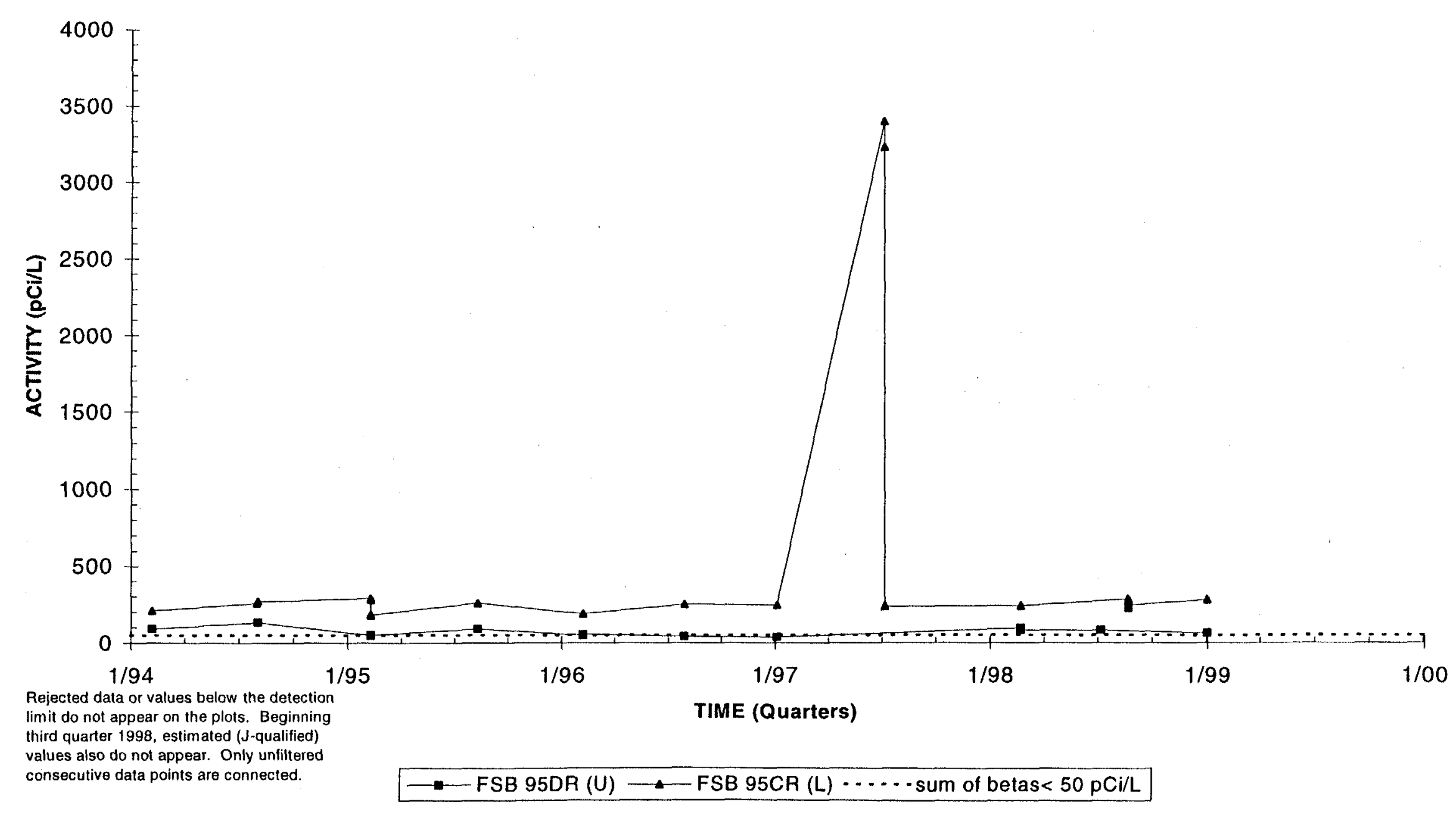

Note: The time-series plots for technetium-99 begin with 1994 data because results during 1993 were anomalously high.

$U=$ Upper Aquifer Zone of the Upper Three Runs Aquiler, $L=$ Lower Aquifer Zone of the Upper Three Runs Aquiler, $G=$ Gordon Aquifer 


\section{Technetium-99 Activities \\ Well Cluster FSB 97}

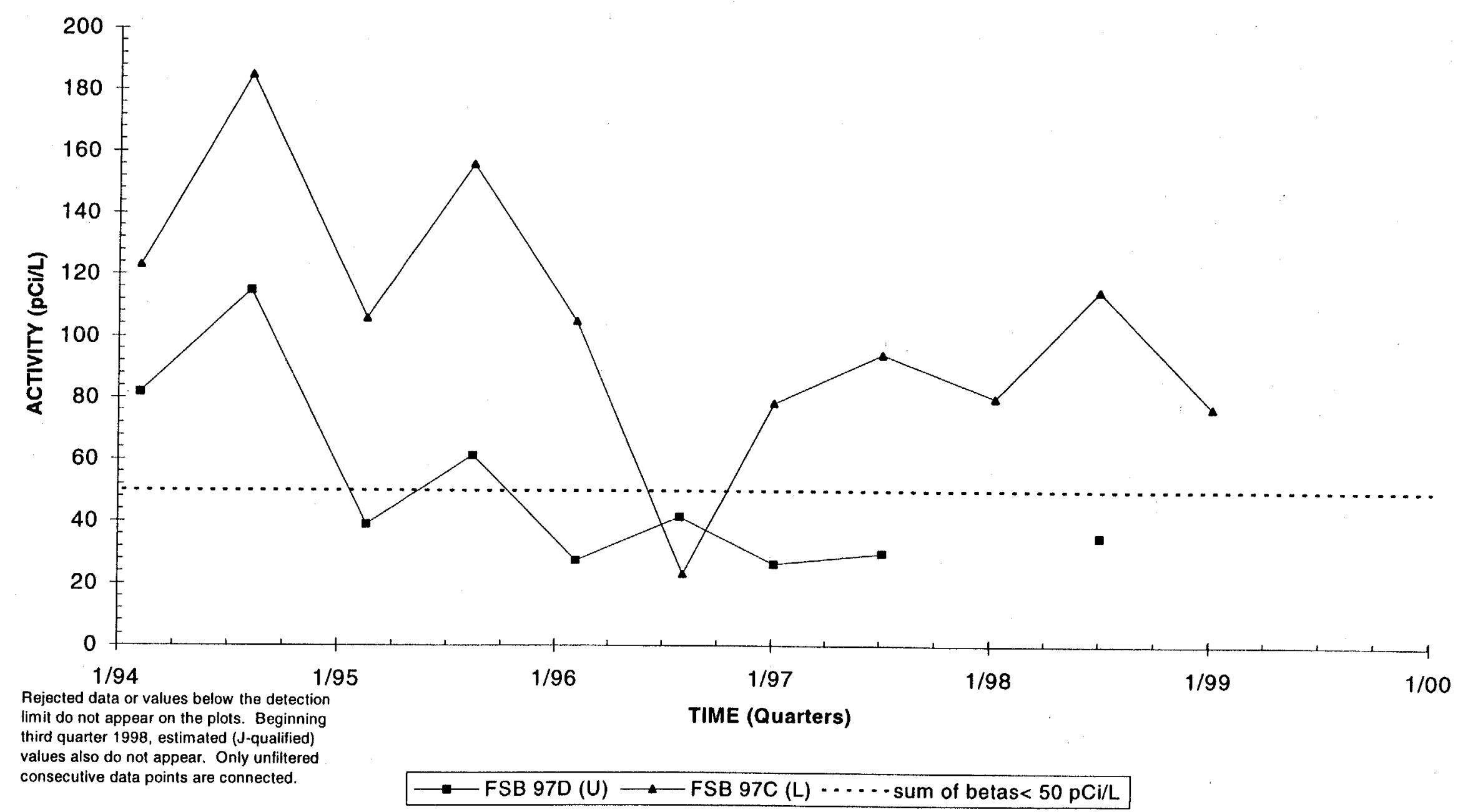

Note: The time-series plots for technetium-99 begin with 1994 data because results during 1993 were anomalously high.

$U=$ Upper Aquifer Zone of the Upper Three Runs Aquiler, $L=$ Lower Aquifer Zone of the Upper Three Runs Aquifer; $G=$ Gordon Aquiler 


\section{Technetium-99 Activities \\ Well Cluster FSB 98}

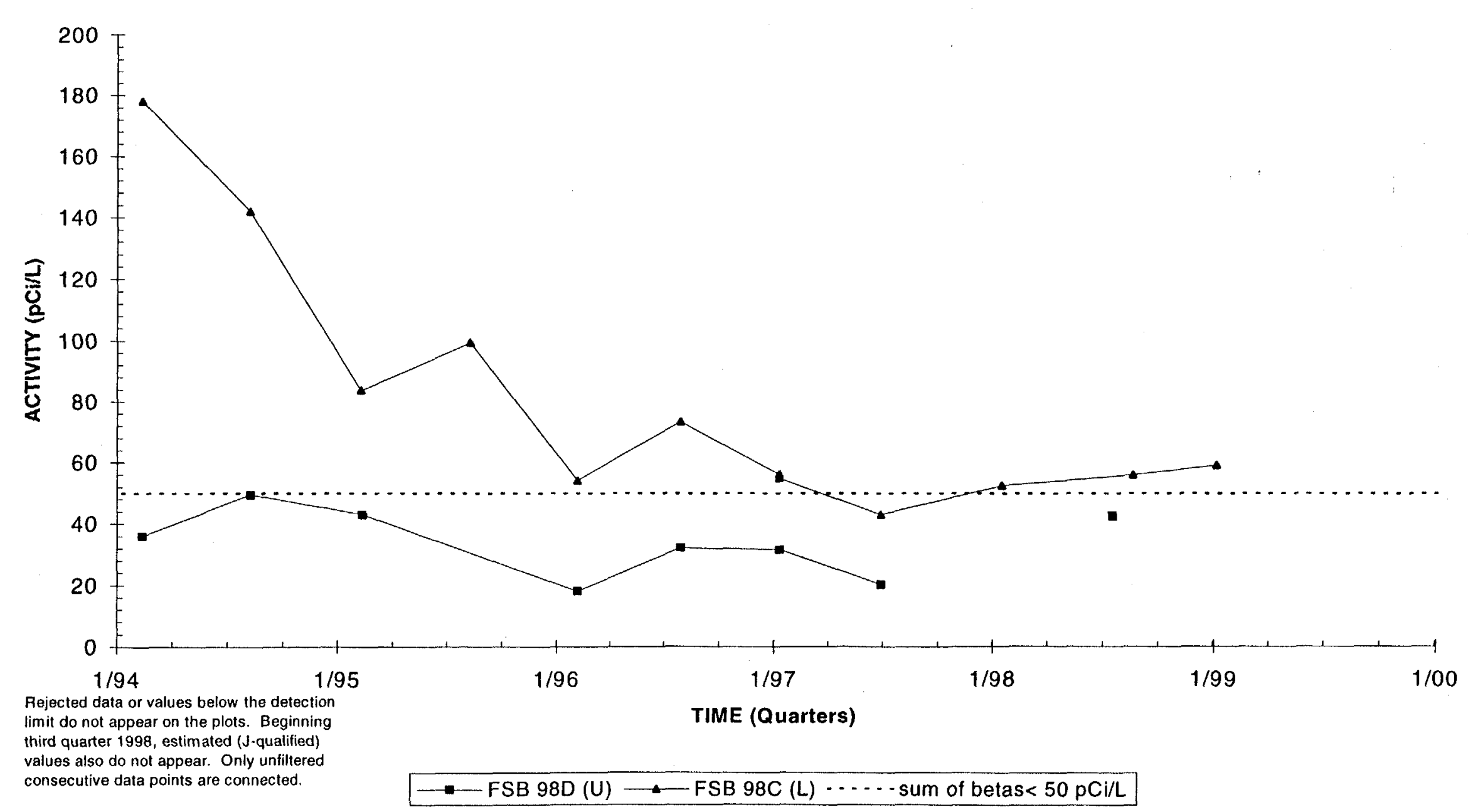

Note: The time-series plots for technetium-99 begin with 1994 data because results during 1993 were anomalously high.

$\mathrm{U}=$ Upper Aquifer Zone of the Upper Three Runs Aquifer; $\mathrm{L}=$ Lower Aquifer Zone of the Upper Three Runs Aquifer, $\mathrm{G}=$ Gordon Aquifer 


\section{Technetium-99 Activities \\ Well FSB102C}

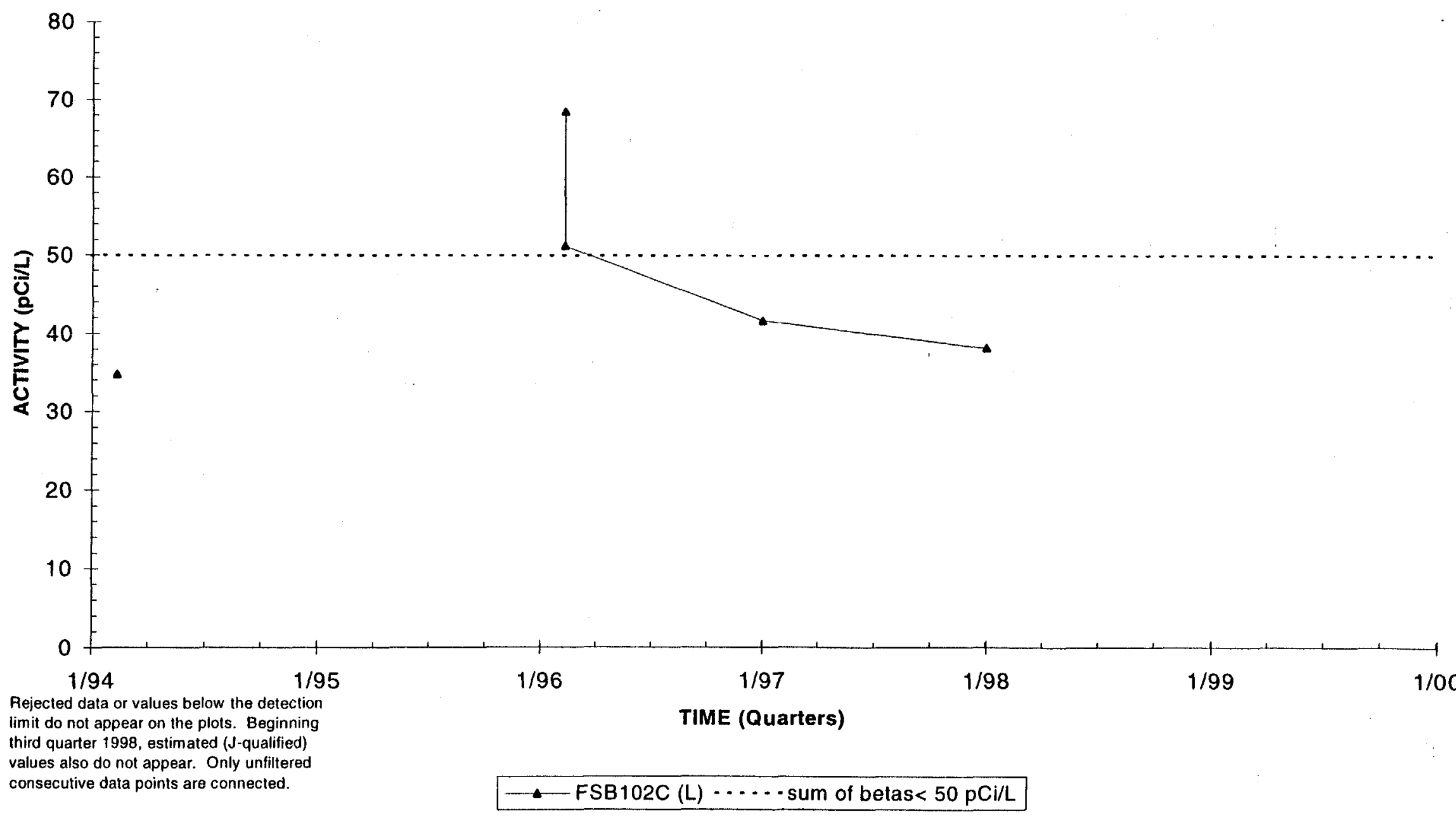

Note: The time-series plots for technetium-99 begin with 1994 data because results during 1993 were anomalously high.

$U=$ Upper Aquifer Zone of the Upper Three Runs Aquifer; $L=$ Lower Aquifer Zone of the Upper Three Runs Aquifer, $G=$ Gordon Aquifer 


\section{Technetium-99 Activities \\ Well Cluster FSB110}

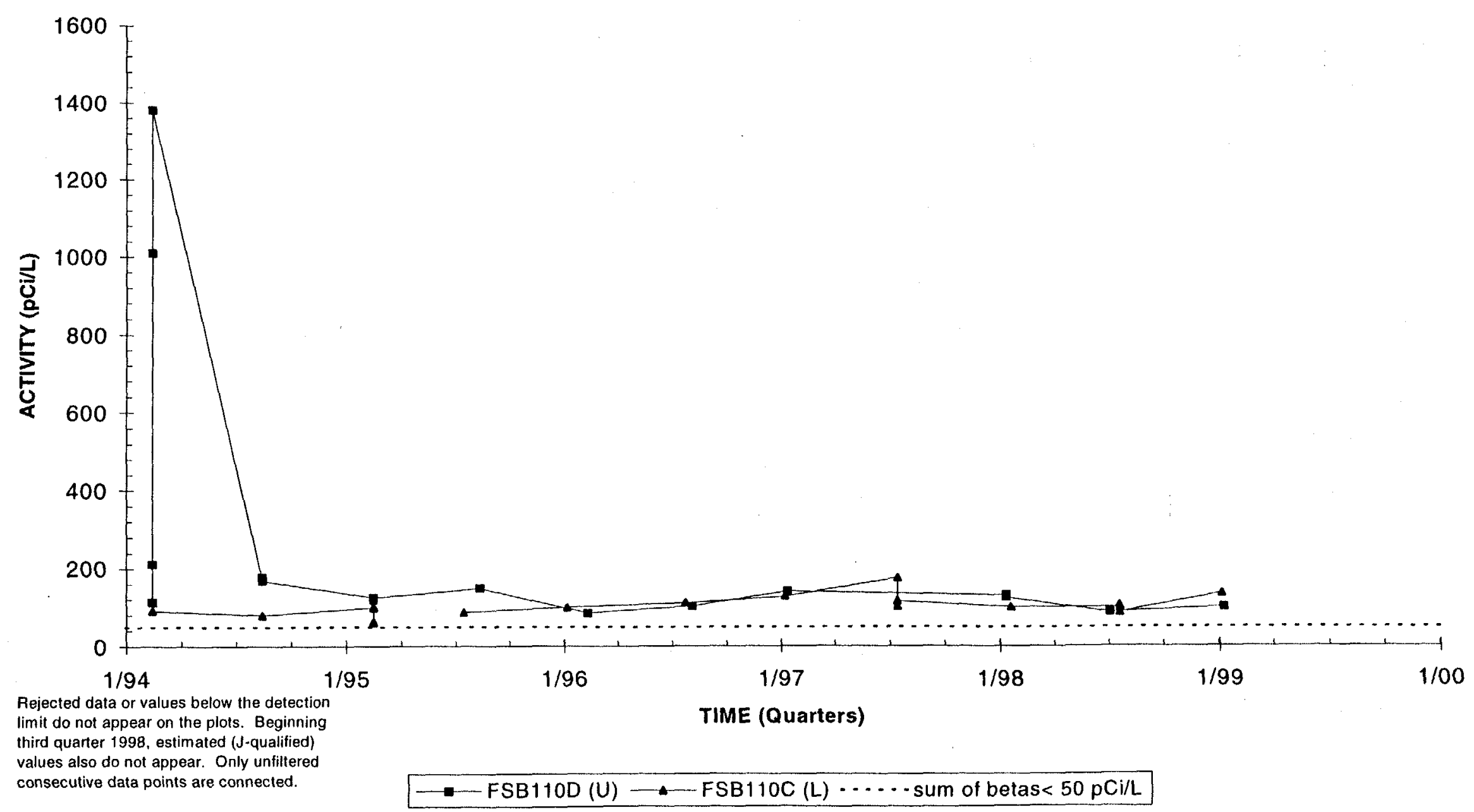

Note: The time-series plots for technetium-99 begin with 1994 data because results during 1993 were anomalously high.

$U=$ Upper Aquifer Zone of the Upper Three Runs Aquifer, $\mathrm{L}=$ Lower Aquiter Zone of the Upper Three Runs Aquifer, $\mathrm{G}=$ Gordon Aquifer 


\section{Technetium-99 Activities \\ Well Cluster FSB112}

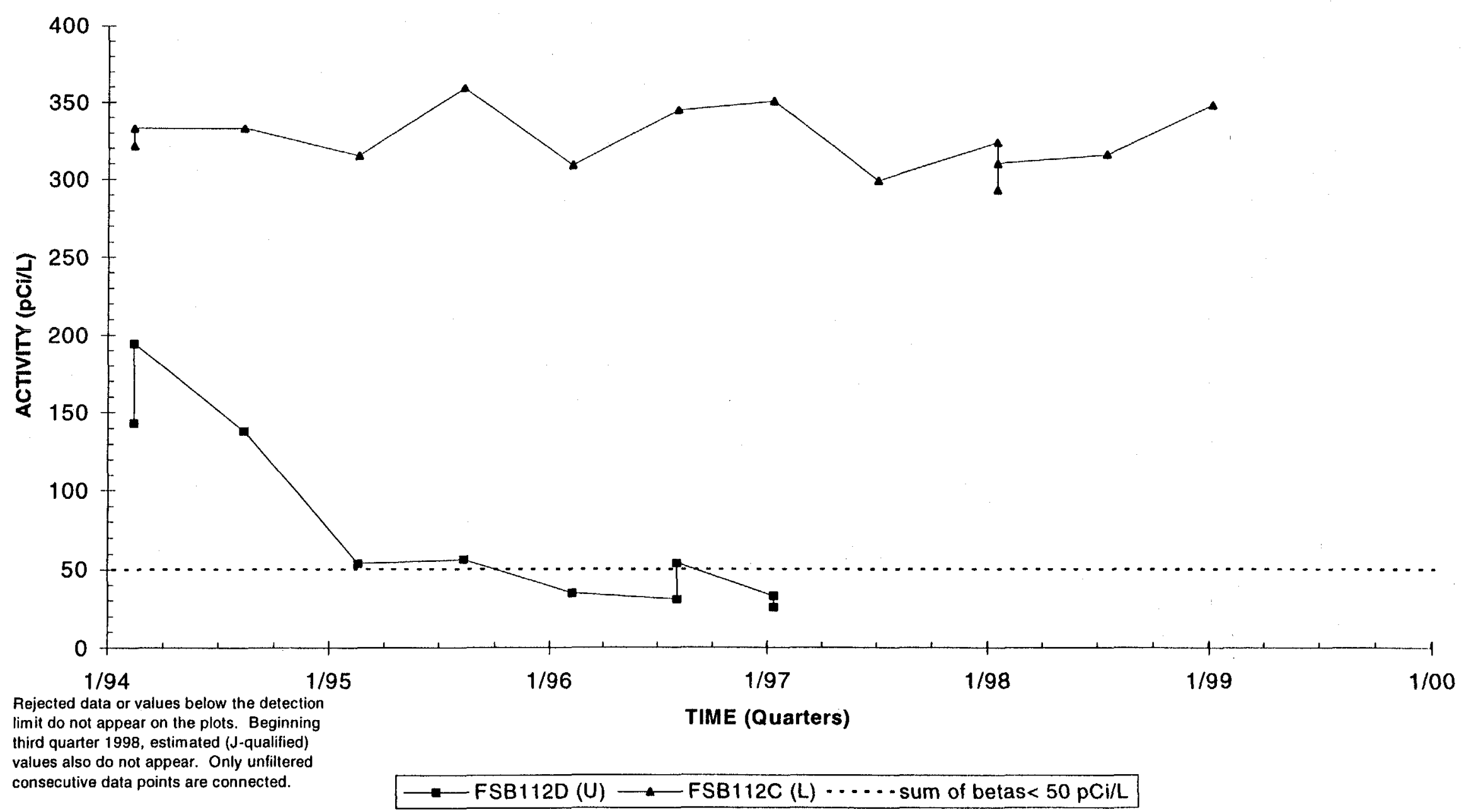

Note: The time-series plots for technetium-99 begin with 1994 data because results during 1993 were anomalously high.

$\mathrm{U}=$ Upper Aquifer Zone of the Upper Three Runs Aquiler, $\mathrm{L}=$ Lower Aquifer Zone of the Upper Three Runs Aquifer, $\mathrm{G}=$ Gordon Aquiler 


\section{Gross Alpha Activities}

Well FSB 77

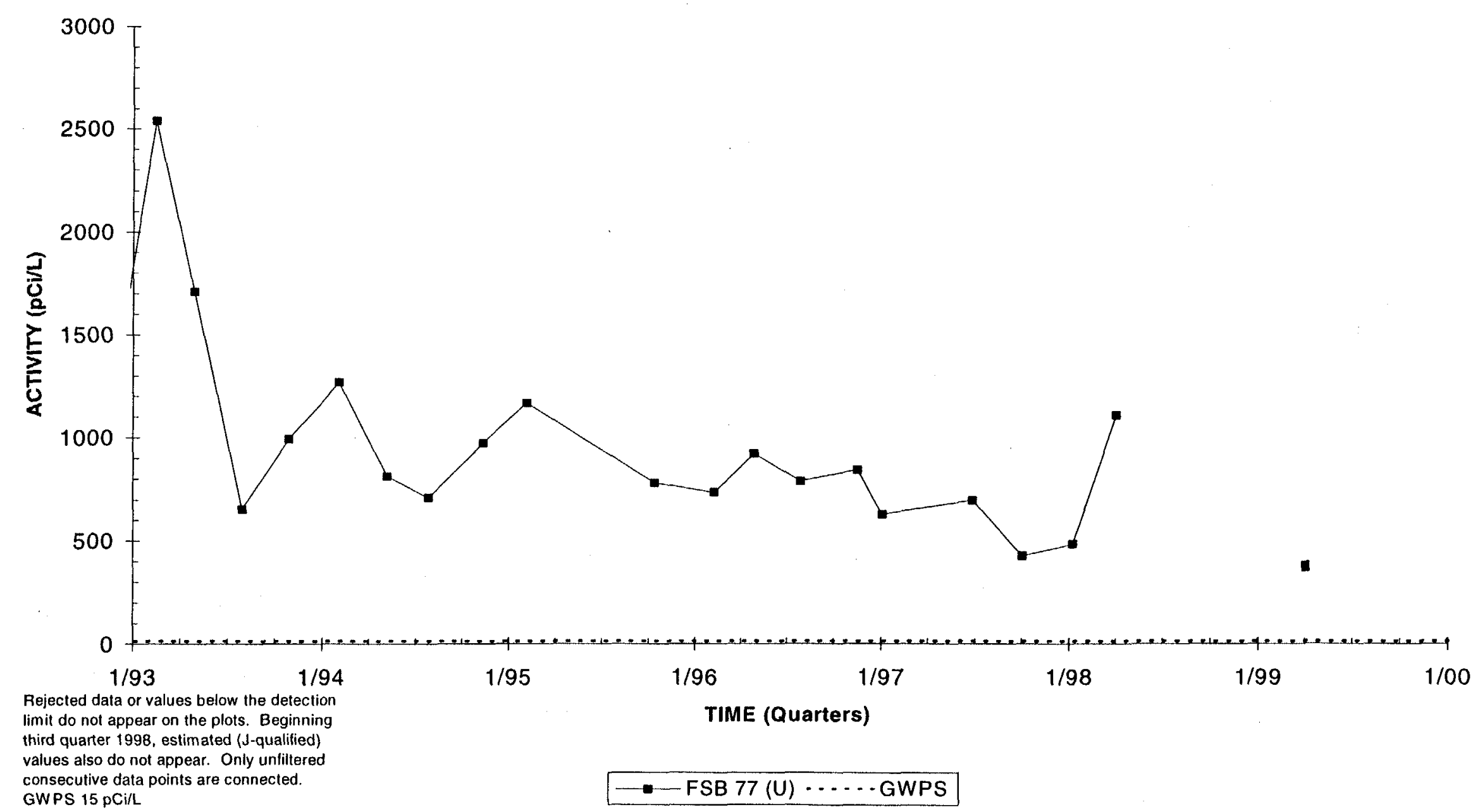

$\mathrm{U}=$ Upper Aquifer Zone of the Upper Three Runs Aquifer; $\mathrm{L}=$ Lower Aquifer Zone of the Upper Three Runs Aquiler; $\mathrm{G}=$ Gordon Aquifer 


\section{Gross Alpha Activities \\ Well Cluster FSB 79}

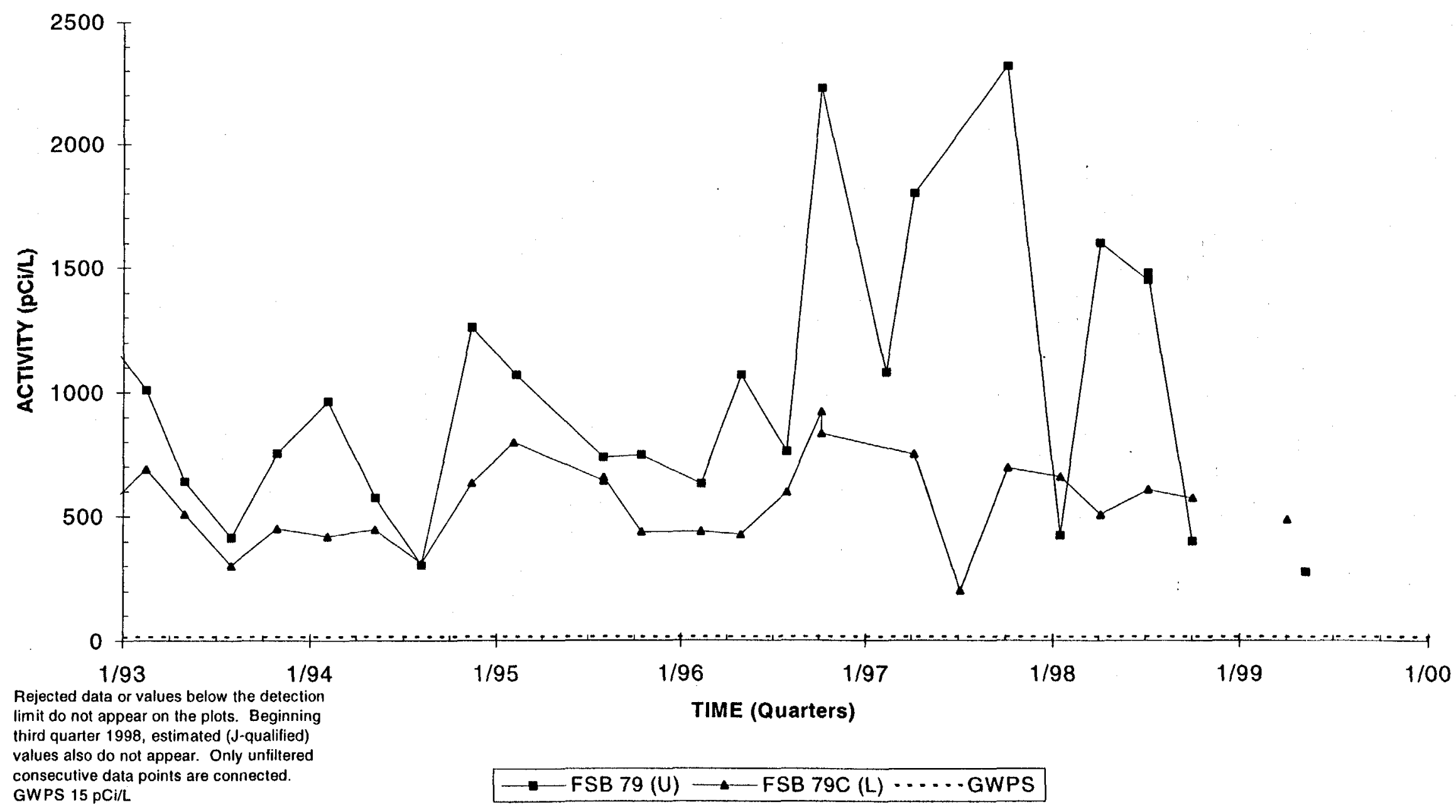

$\mathrm{U}=$ Upper Aquiler Zone of the Upper Three Runs Aquifer, $\mathrm{L}=$ Lower Aquifer Zone of the Upper Three Runs Aquifer, $G=G$ Gordon Aquifer 


\section{Gross Alpha Activities \\ Well Cluster FSB 88}

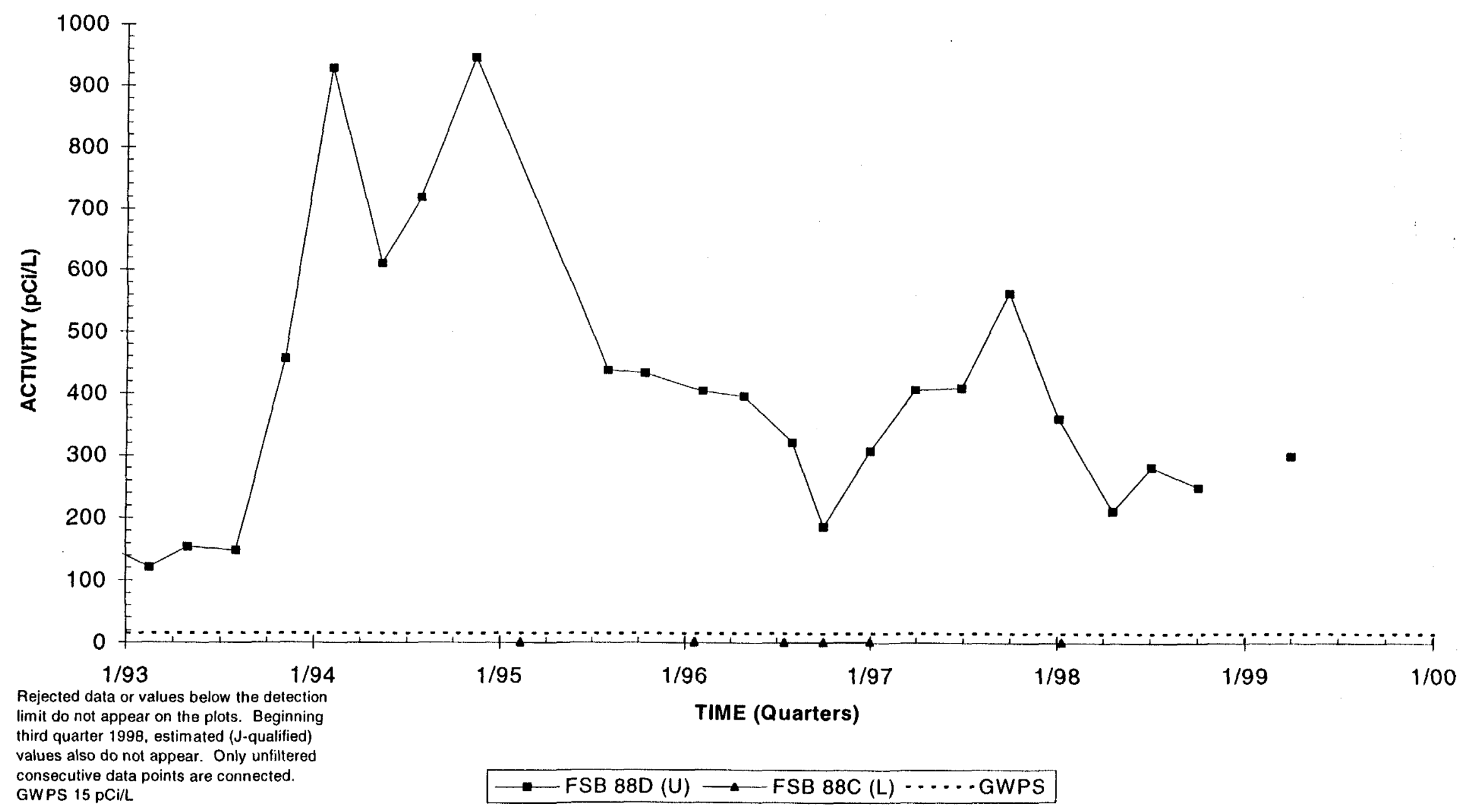

$\mathrm{U}=$ Upper Aquifer Zone of the Upper Three Runs Aquifer, $\mathrm{L}=$ Lower Aquifer Zone of the Upper Three Runs Aquifer; $G$ = Gordon Aquifer 


\section{Gross Alpha Activities \\ Well Cluster FSB 94}

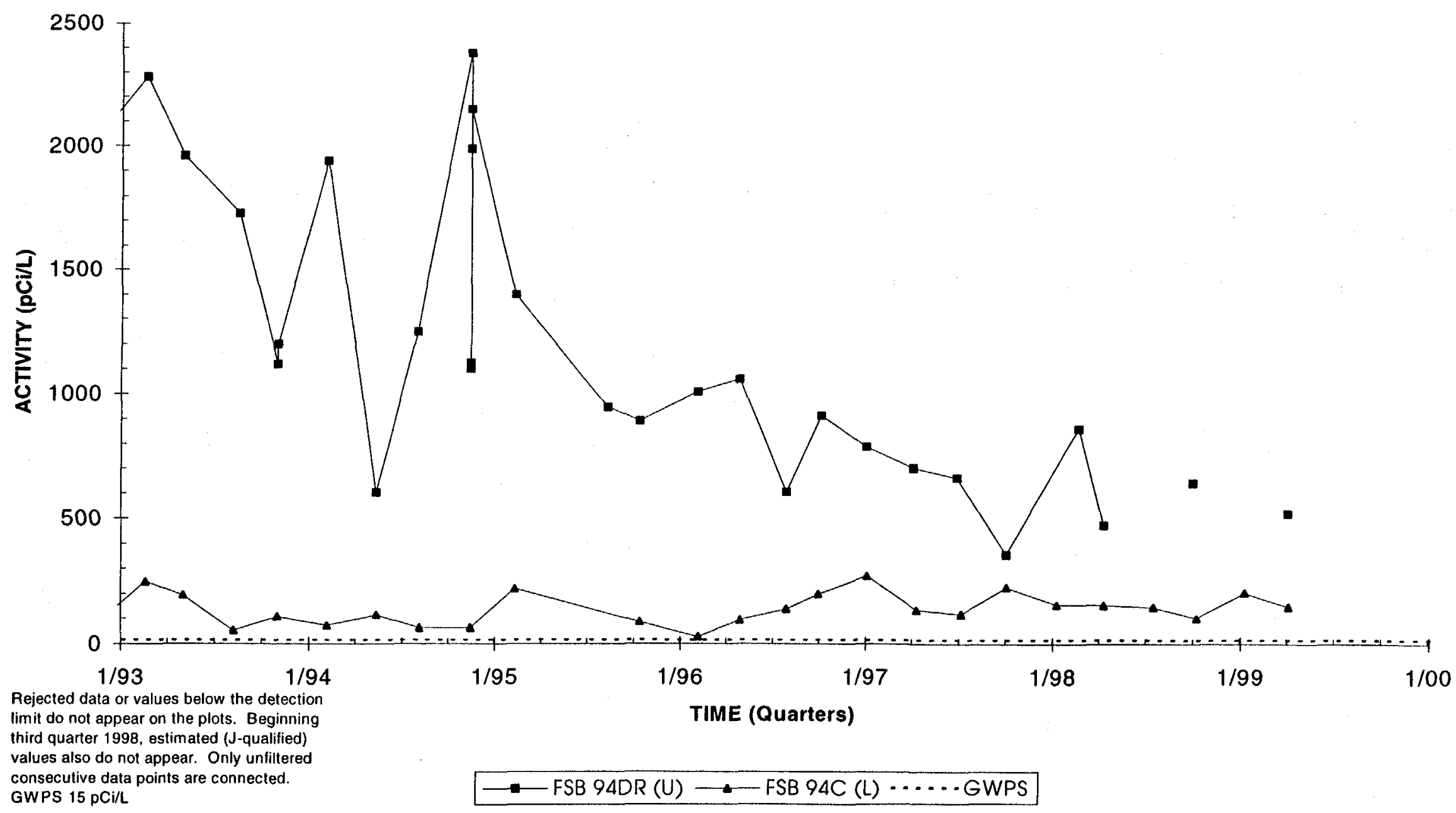

$U=$ Upper Aquifer Zone of the Upper Three Runs Aquifer, $L=$ Lower Aquifer Zone of the Upper Three Runs Aquiler; $G=$ Gordon Aquifer 


\section{Gross Alpha Activities \\ Well Cluster FSB 95}

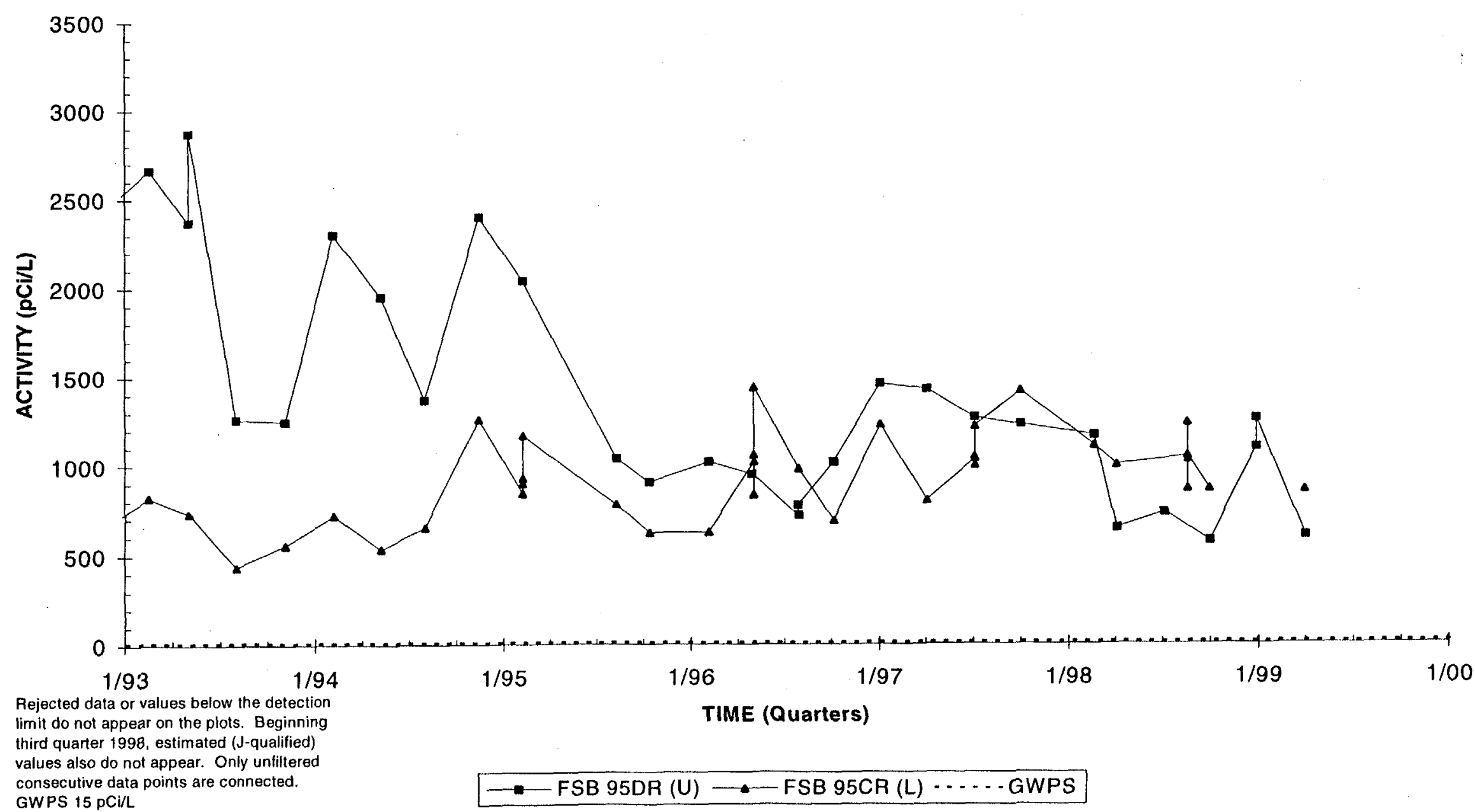

$\mathrm{U}=$ Upper Aquifer Zone of the Upper Three Runs Aquifer; $\mathrm{L}=$ Lower Aquifer Zone of the Upper Three Runs Aquifer; $\mathrm{G}=\mathrm{Gordon}$ Aquifer 


\section{Gross Alpha Activities \\ Well Cluster FSB 97}

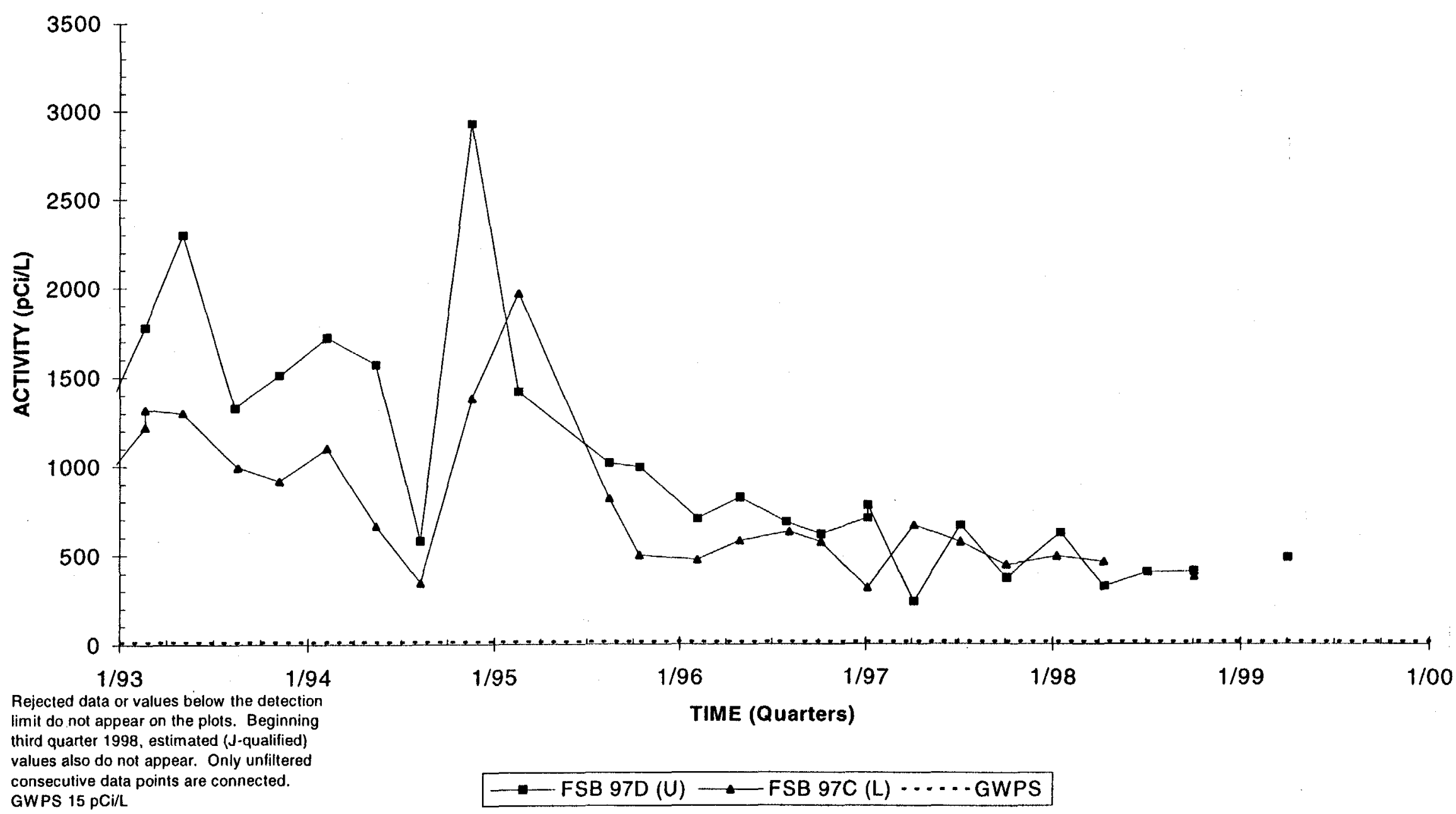

$\mathbf{U}=$ Upper Aquifer Zone of the Upper Three Runs Aquifer, $\mathbf{L}=$ Lower Aquifer Zone of the Upper Three Runs Aquiler, $G=$ Gordon Aquifer 


\section{Gross Alpha Activities \\ Well Cluster FSB 98}

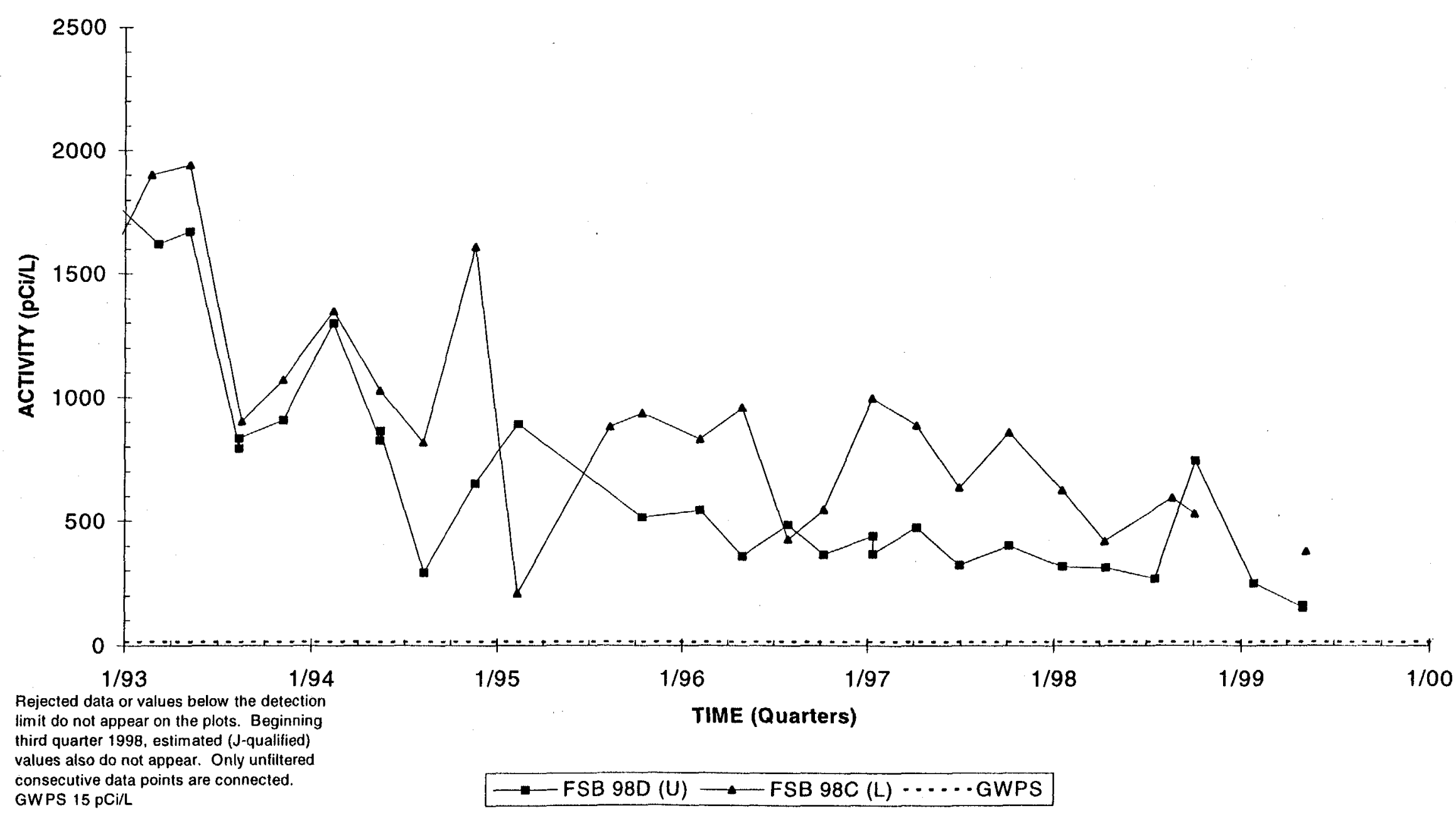

$U=$ Upper Aquifer Zone of the Upper Three Runs Aquifer, $L=$ Lower Aquifer Zone of the Upper Three Runs Aquifer; $G=$ Gordon Aquifer 


\section{Gross Alpha Activities \\ Well Cluster FSB104}

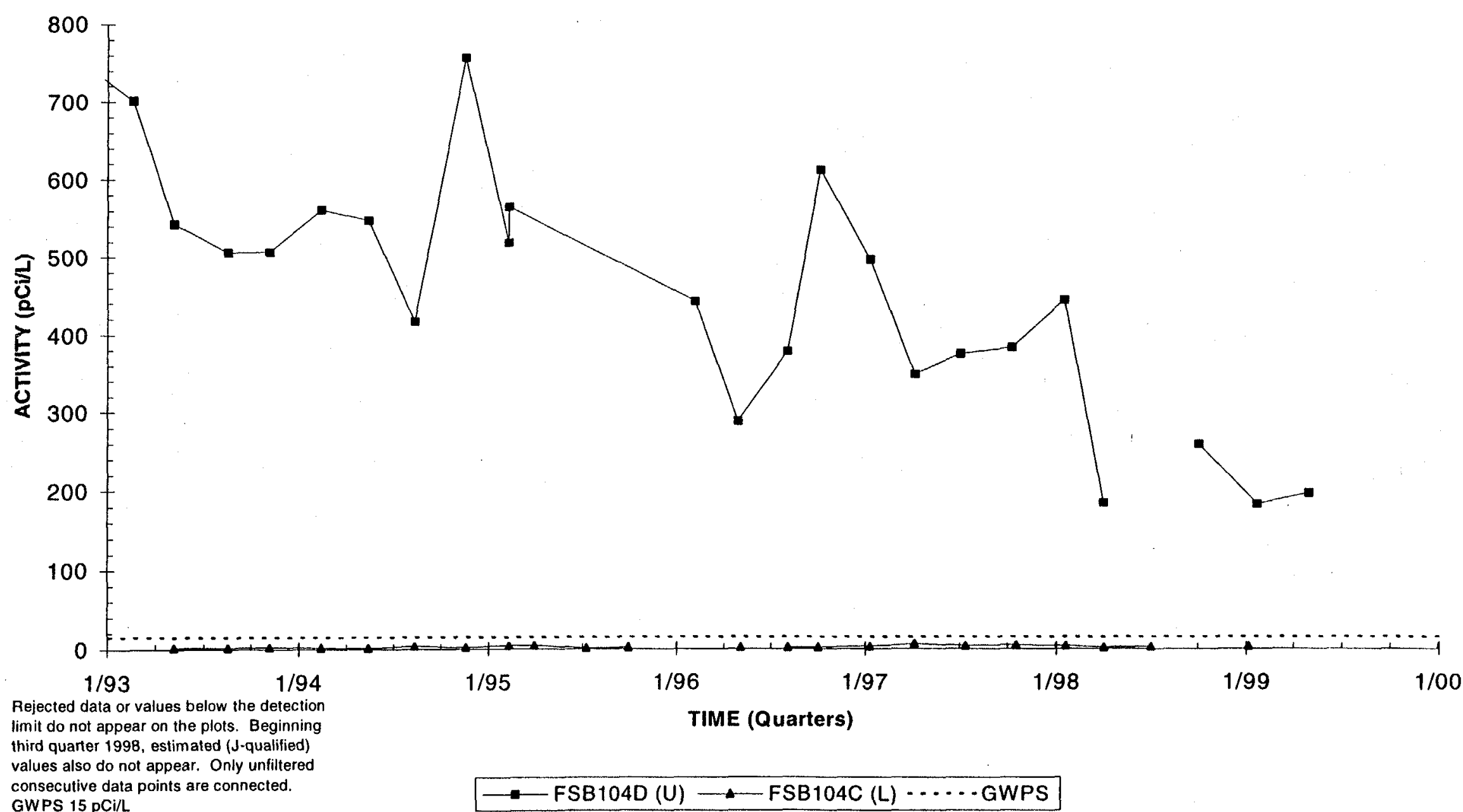

$\mathrm{U}=$ Upper Aquiler Zone of the Upper Three Runs Aquifer, $\mathrm{L}=$ Lower Aquifer Zone of the Upper Three Runs Aquifer; $\mathrm{G}=$ Gordon Aquiler

F-Area HWMF D-86




\section{Gross Alpha Activities \\ Well Cluster FSB107}

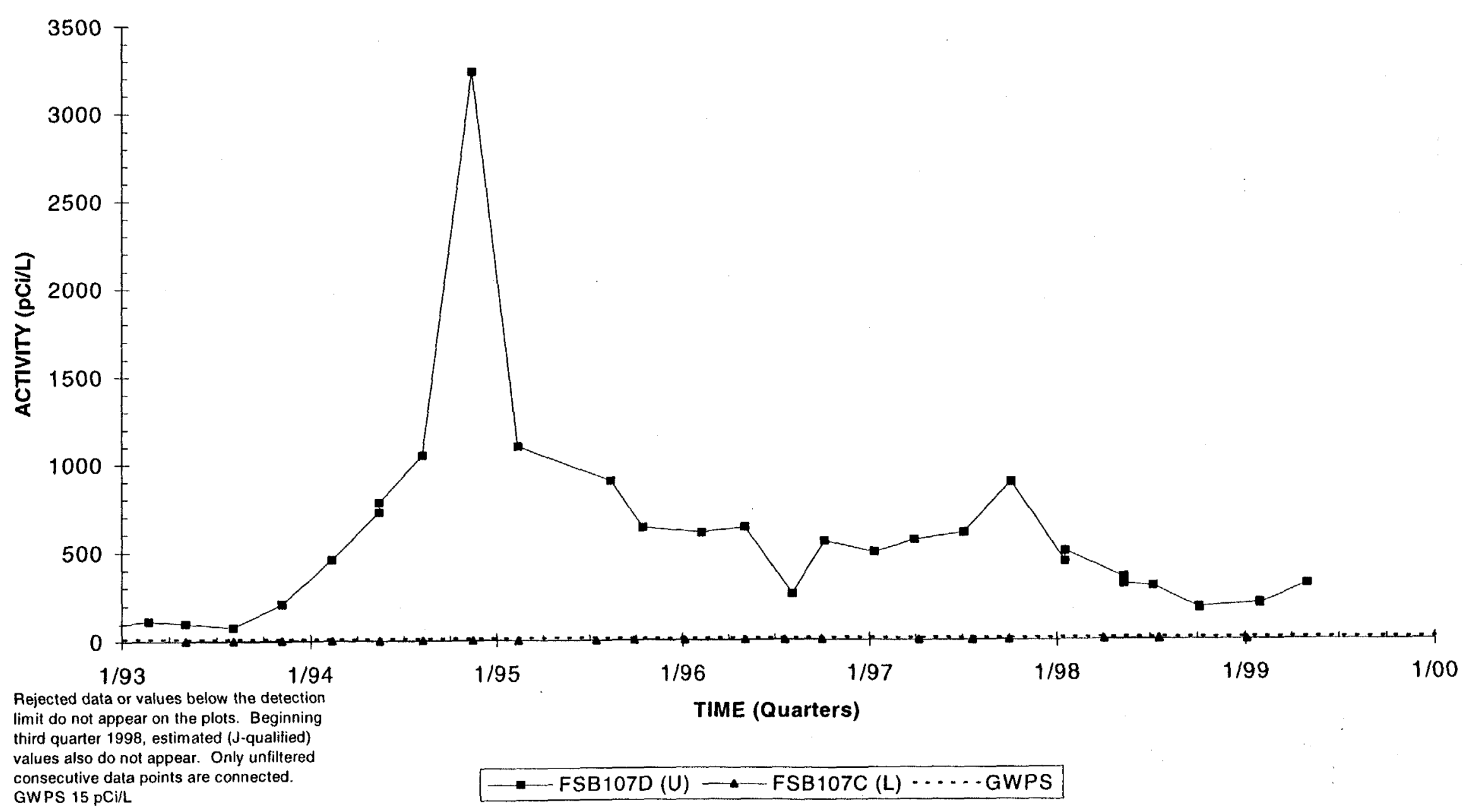

$U=$ Upper Aquifer Zone of the Upper Three Runs Aquifer, $L=$ Lower Aquifer Zone of the Upper Three Runs Aquifer; $G=$ Gordon Aquifer 
Nonvolatile Beta Activities

Well Cluster FSB 78

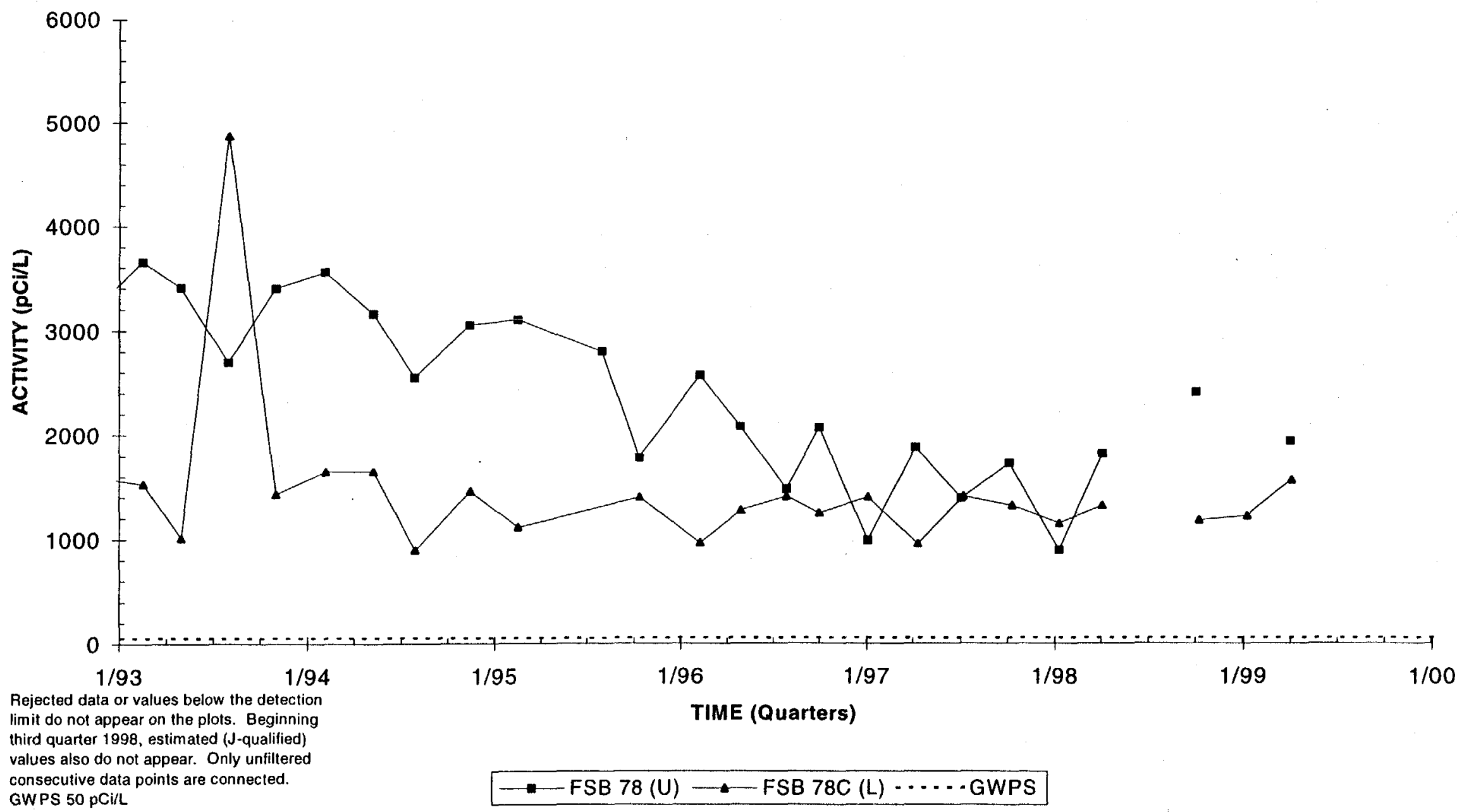

$U=$ Upper Aquifer Zone of the Upper Three Runs Aquifer; $L=$ Lower Aquifer Zone of the Upper Three Runs Aquiler, $G=$ Gordon Aquifer 


\section{Nonvolatile Beta Activities}

Well Cluster FSB 90

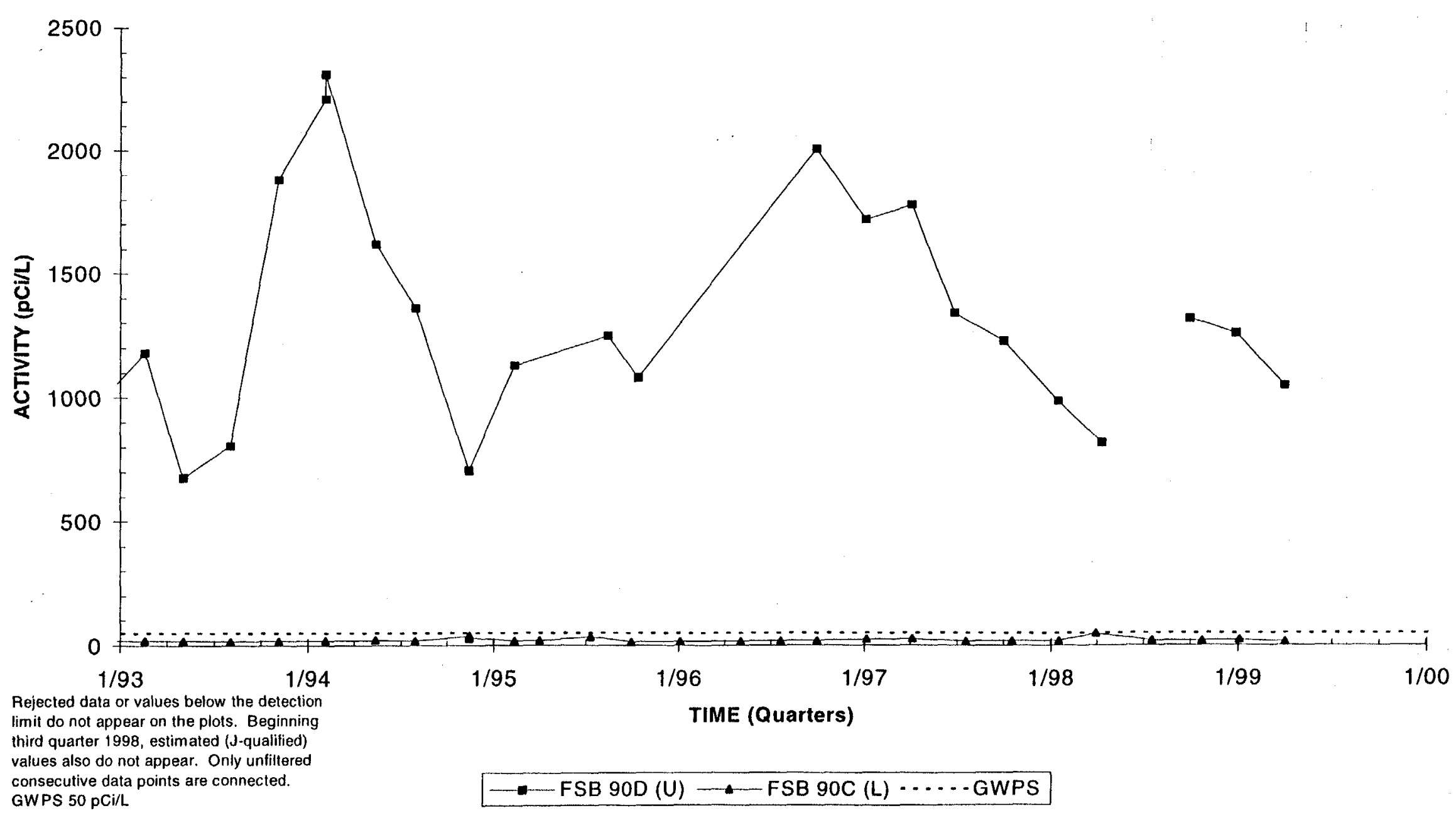

$U=$ Upper Aquiler Zone of the Upper Three Runs Aquifer, $L=$ Lower Aquifer Zone of the Upper Three Runs Aquifer; $G=$ Gordon Aquiler 


\section{Nonvolatile Beta Activities \\ Well Cluster FSB 92}

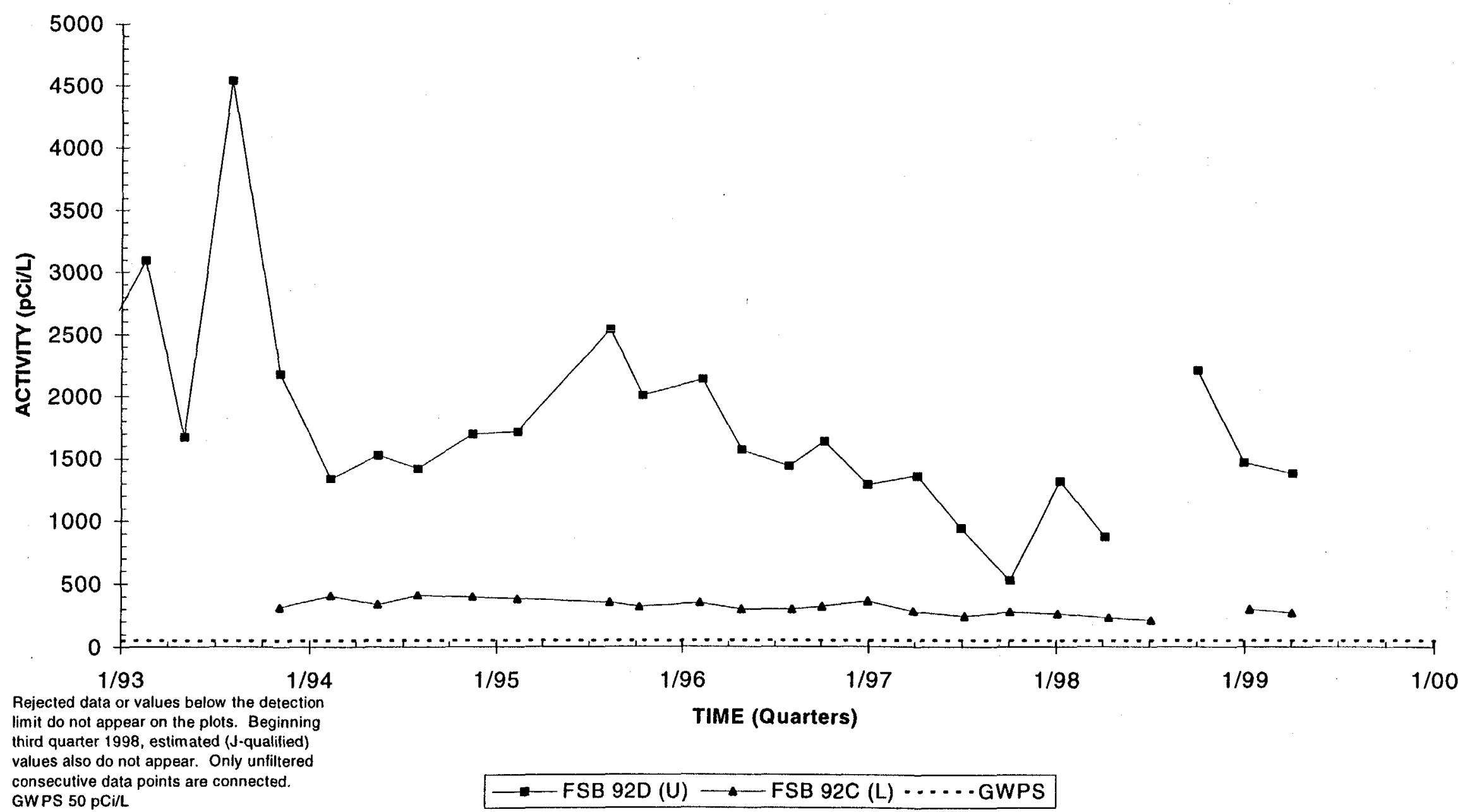

$U=$ Upper Aquifer Zone of the Upper Three Runs Aquifer, $L=$ Lower Aquifer Zone of the Upper Three Runs Aquifer; $G=$ Gordon Aquifer

\begin{tabular}{ll}
\hline F-Area HWMF & D-90
\end{tabular}




\section{Nonvolatile Beta Activities}

Well Cluster FSB 94

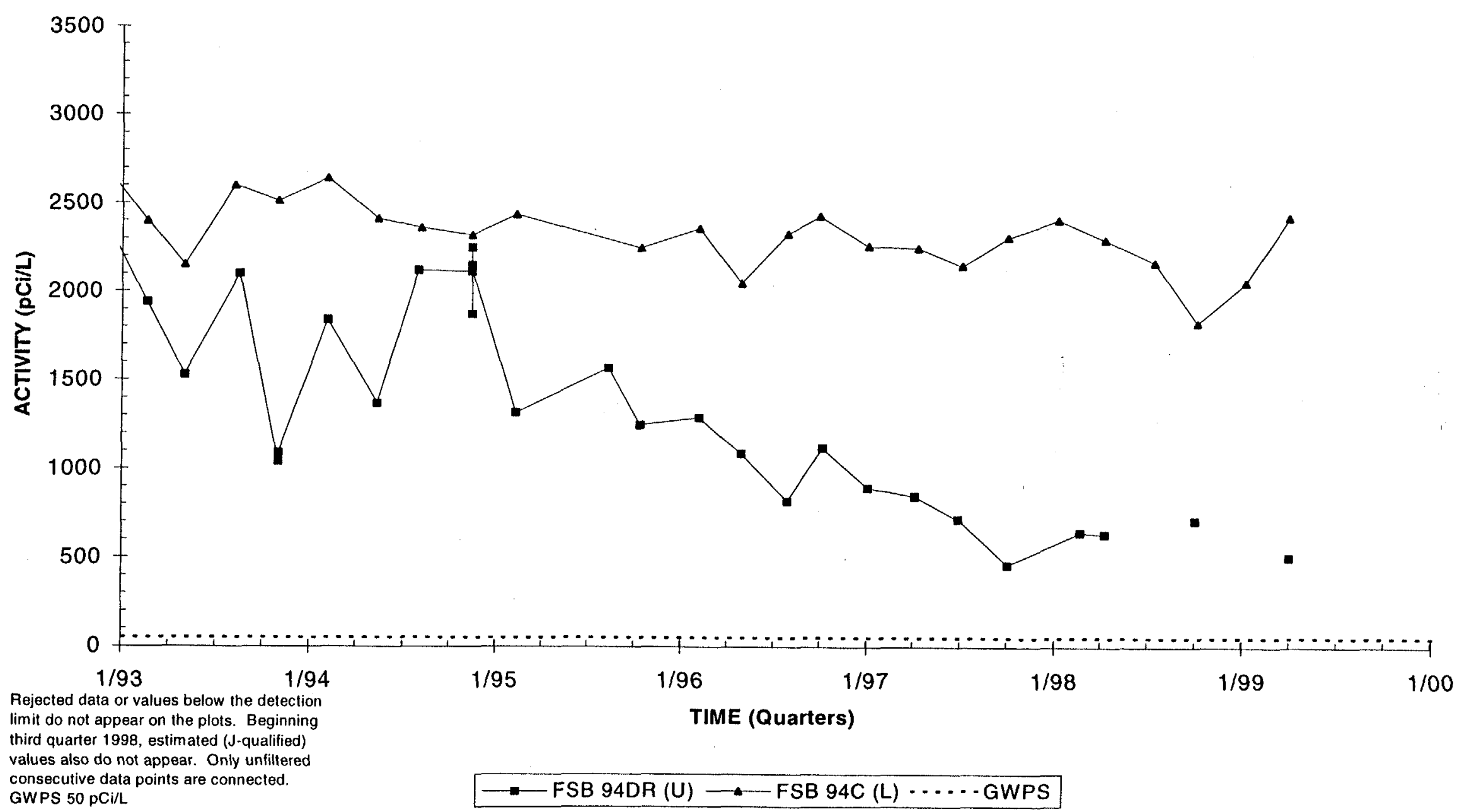

U = Upper Aquifer Zone of the Upper Three Runs Aquifer; $L=$ Lower Aquiter Zone of the Upper Three Runs Aquiler, $G=$ Gordon Aquifer 


\section{Nonvolatile Beta Activities \\ Well Cluster FSB 95}

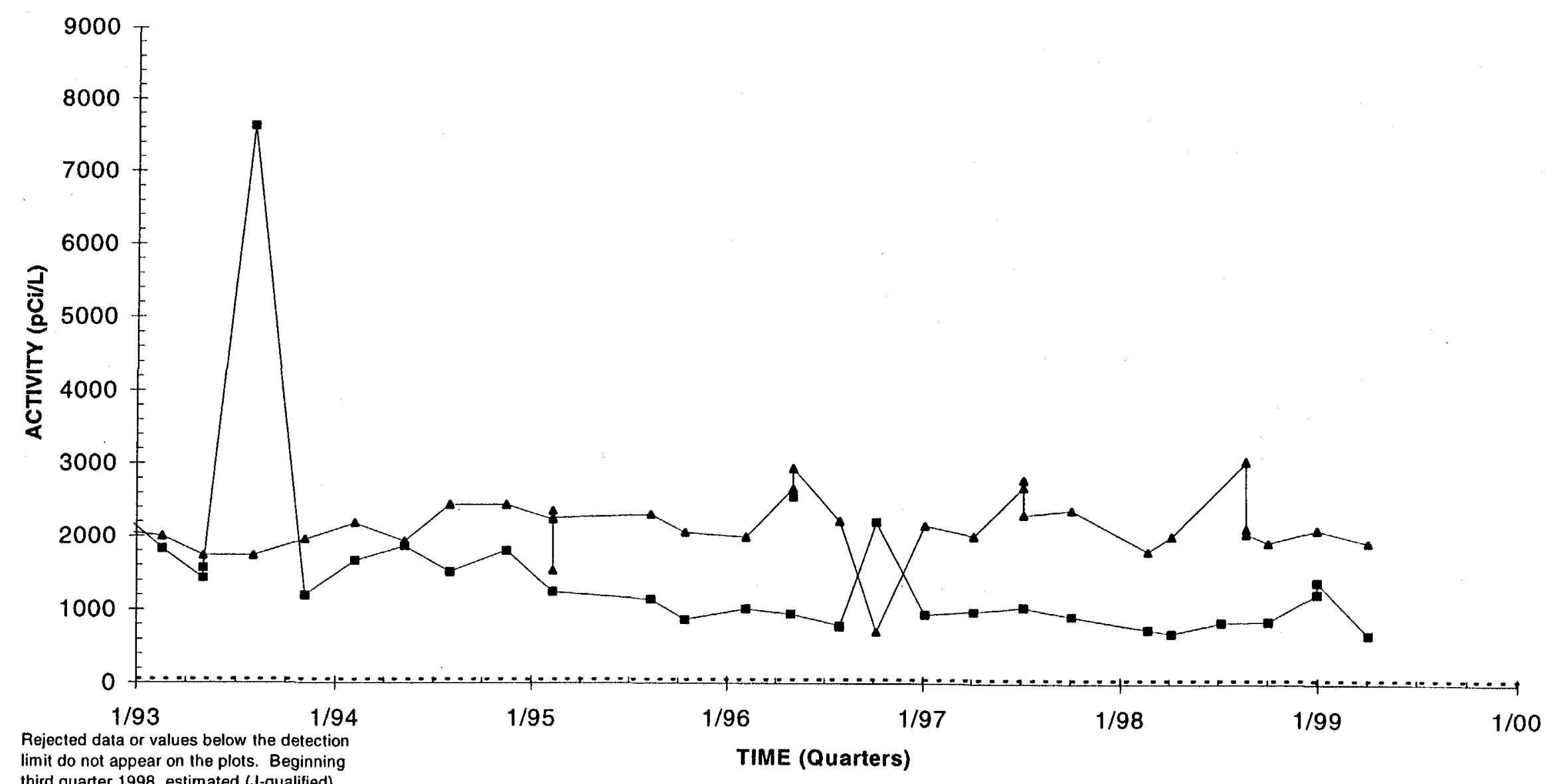

values also do not appear. Only unfiltered

consecutive data points are connected.

GWPS $50 \mathrm{pCi} / \mathrm{L}$ 


\section{Nonvolatile Beta Activities}

Well Cluster FSB 97

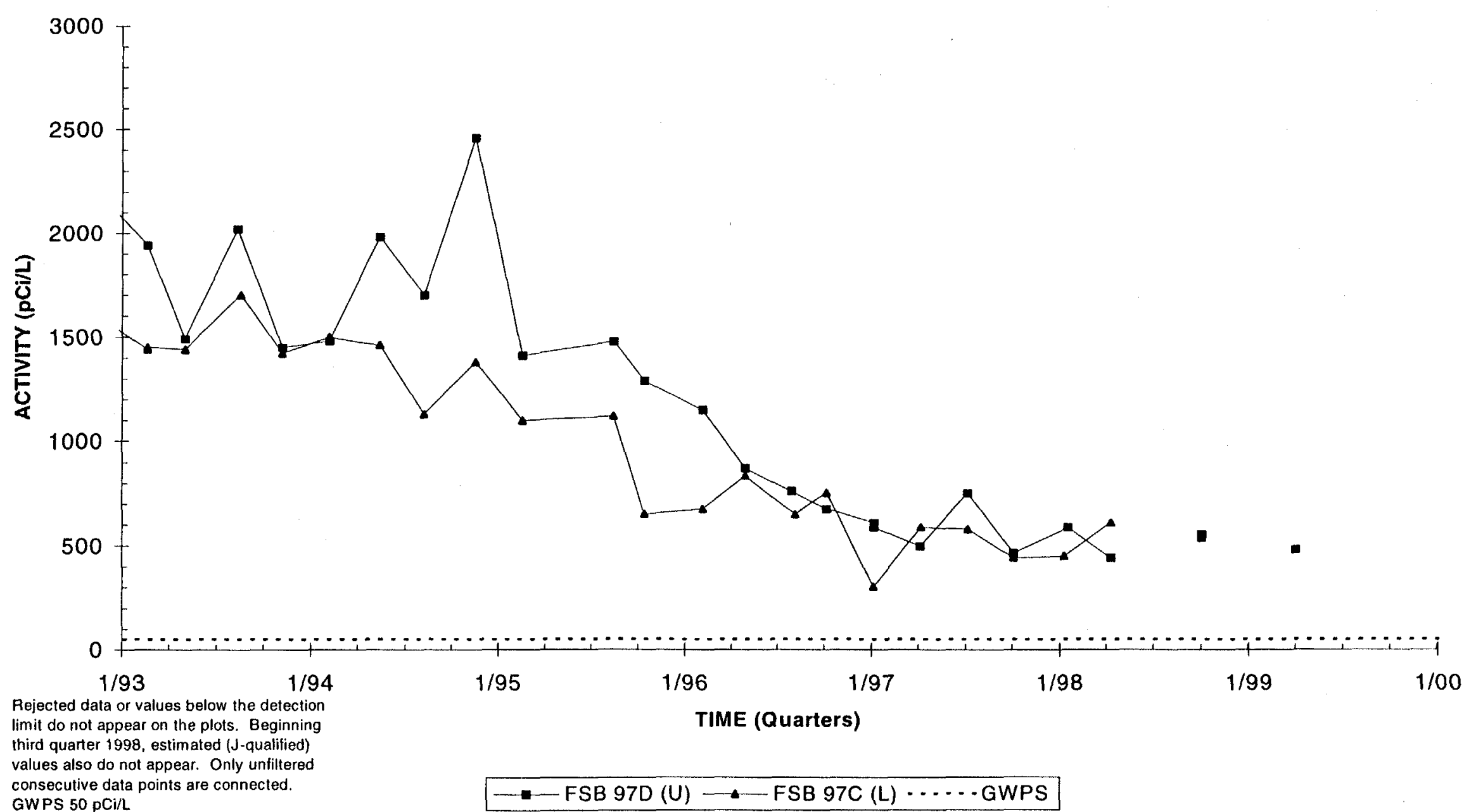

$\mathrm{U}=$ Upper Aquiler Zone of the Upper Three Runs Aquifer; $\mathrm{L}=$ Lower Aquiler Zone of the Upper Three Runs Aquifer; $\mathrm{G}=$ Gordon Aquifer 


\section{Nonvolatile Beta Activities \\ Well Cluster FSB 98}

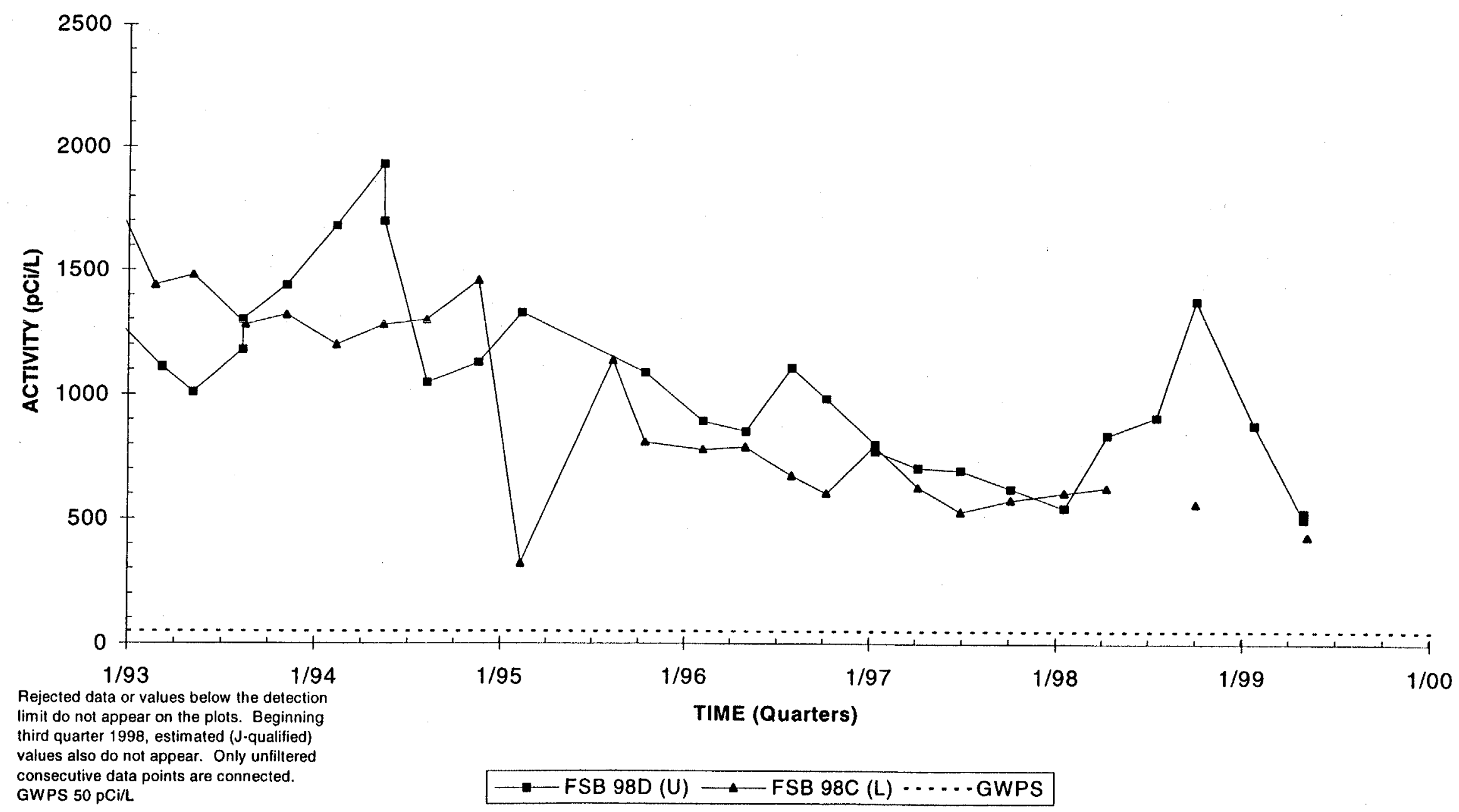

$U=$ Upper Aquifer Zone of the Upper Three Runs Aquifer, $L=$ Lower Aquifer Zone of the Upper Three Runs Aquifer; $G=$ Gordon Aquifer 


\section{Nonvolatile Beta Activities \\ Well FSB102C}

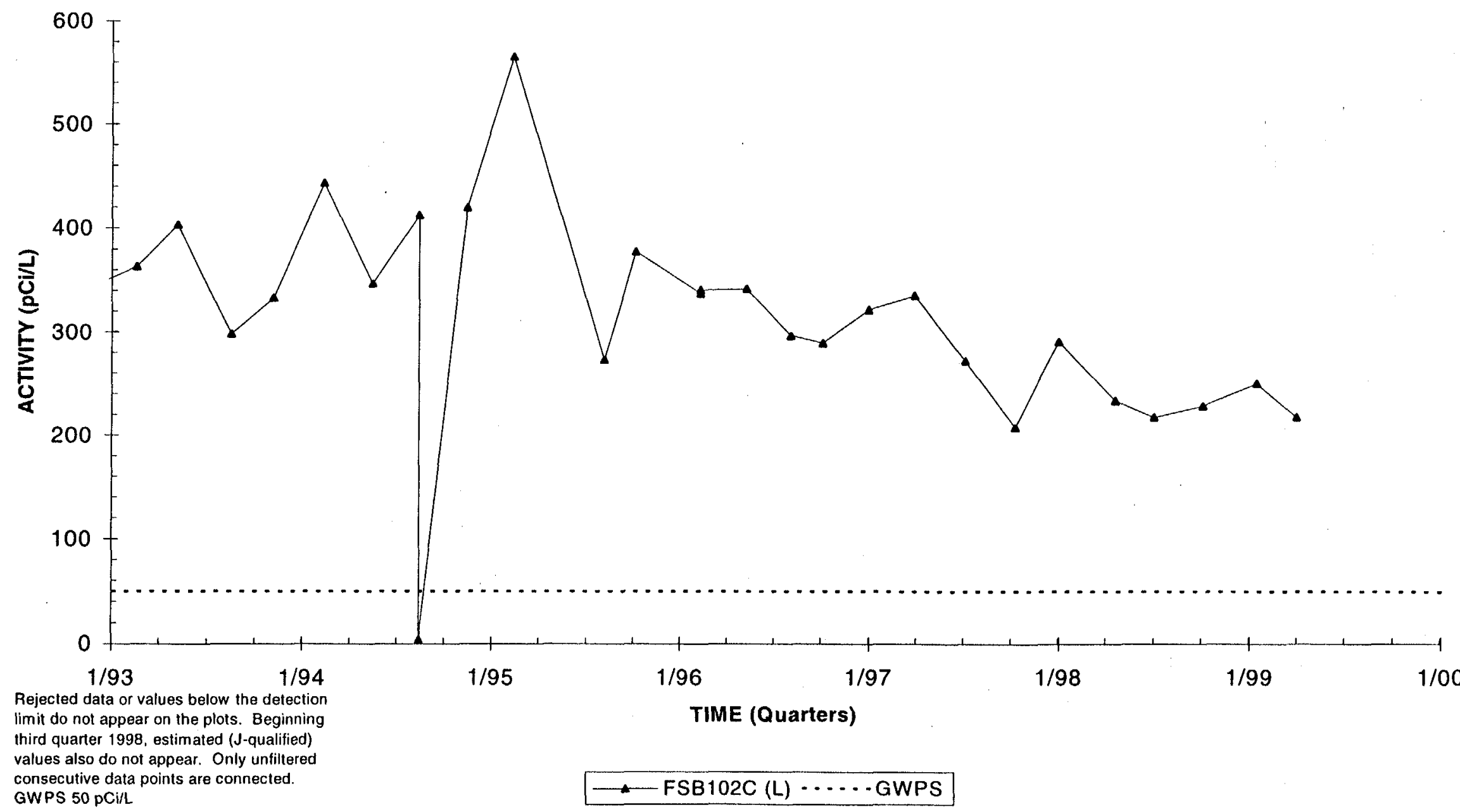

$U=$ Upper Aquifer Zone of the Upper Three Runs Aquifer, $\mathrm{L}=$ Lower Aquifer Zone of the Upper Three Runs Aquifer; $\mathrm{G}=$ Gordon Aquifer 


\section{Nonvolatile Beta Activities}

Well Cluster FSB110

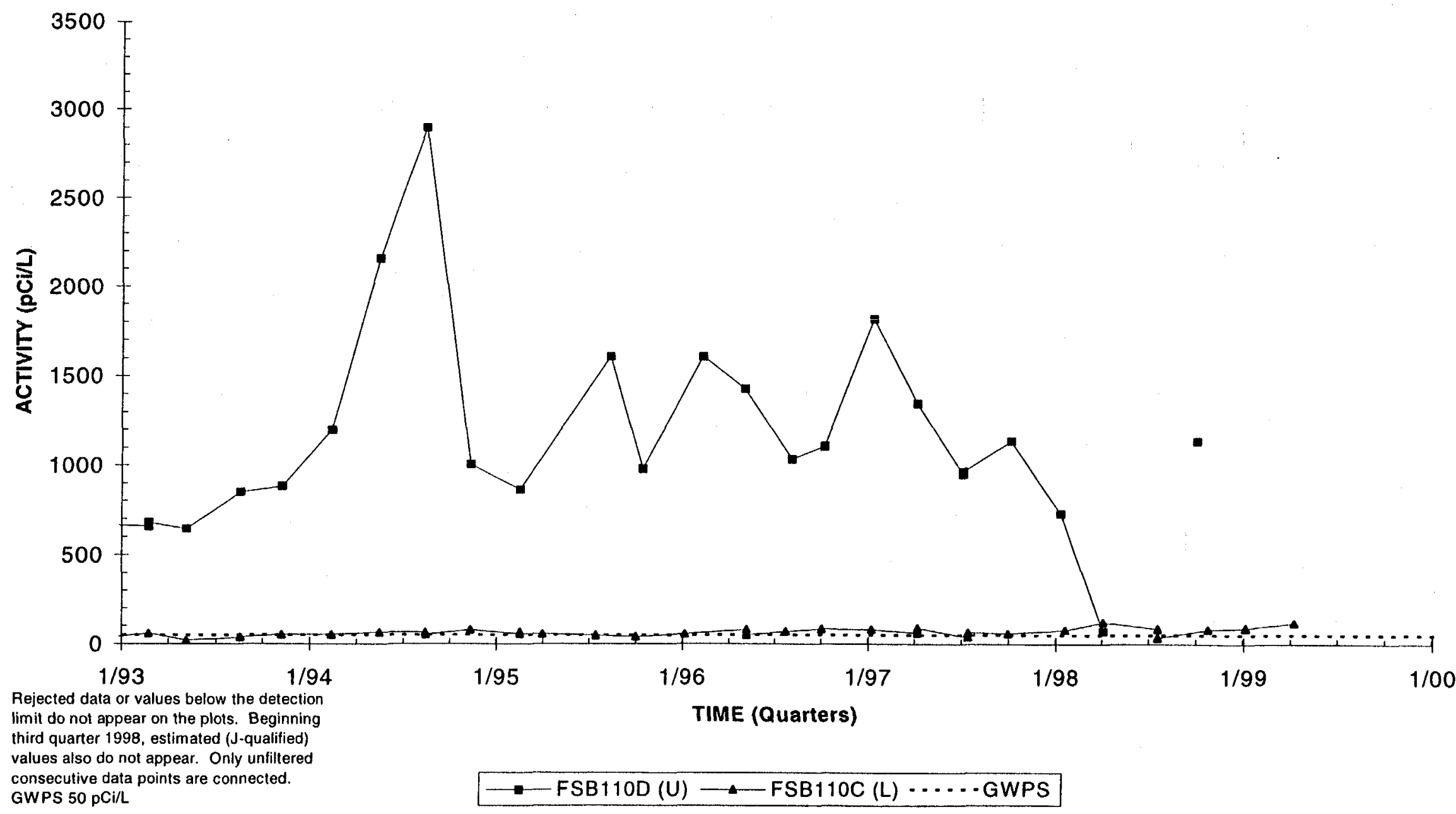

$U=$ Upper Aquifer Zone of the Upper Three Runs Aquiler, $\mathrm{L}=$ Lower Aquifer Zone of the Upper Three Runs Aquifer, $\mathrm{G}=$ Gordon Aquifer 


\section{Nonvolatile Beta Activities \\ Well Cluster FSB112}

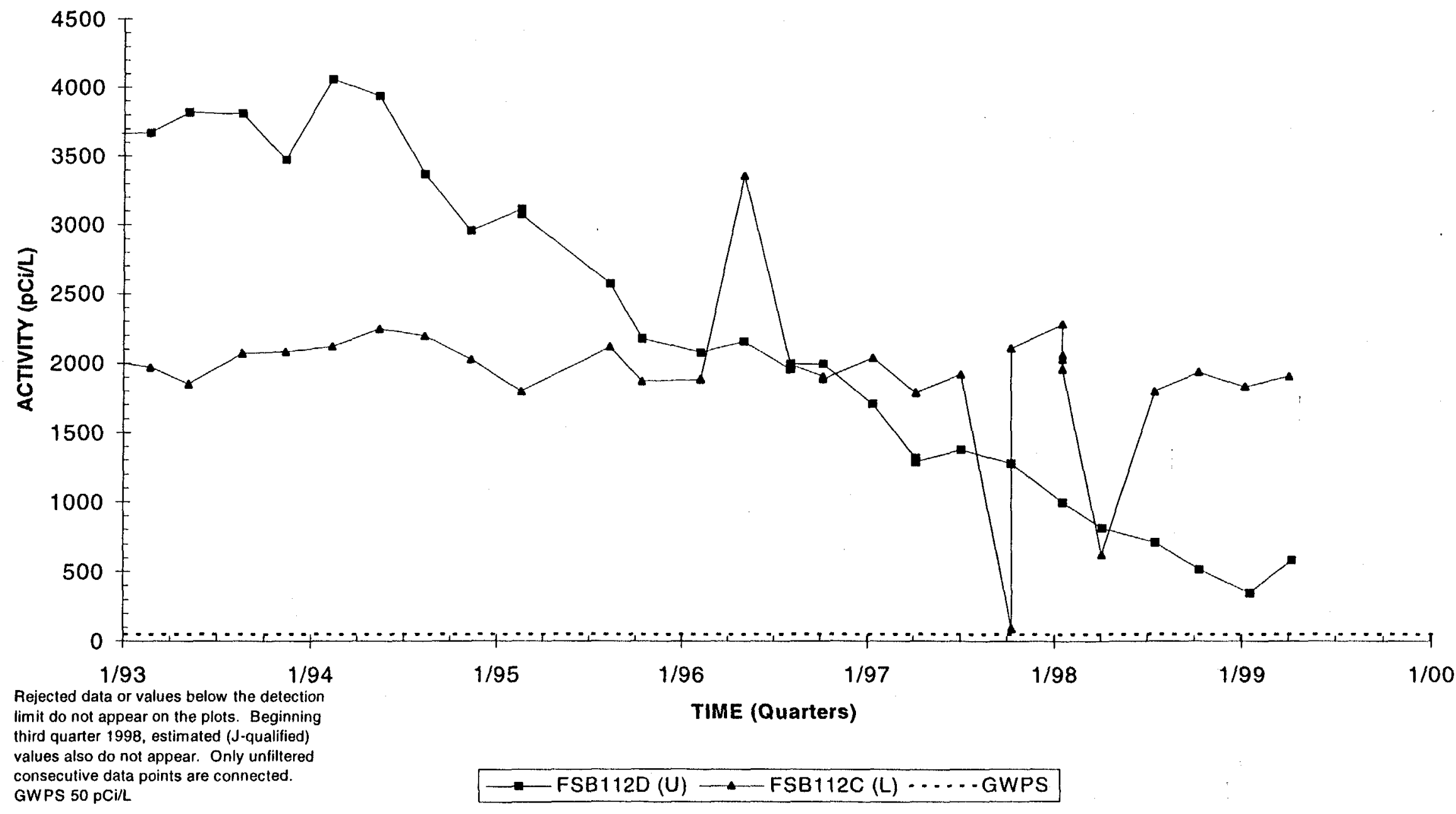

$\mathrm{U}=$ Upper Aquiler Zone of the Upper Three Runs Aquiter, $\mathrm{L}=$ Lower Aquifer Zone of the Upper Three Auns Aquifer; $\mathrm{G}=\mathrm{Gordon}$ Aquifer 
WSRC-TR-99-00147

Unclassified

THIS PAGE WAS LEFT BLANK INTENTIONALLY. 


\section{APPENDIX E: HYDROGRAPHS}


WSRC-TR-99-00147

Unclassified

THIS PAGE WAS LEFT BLANK INTENTIONALLY. 


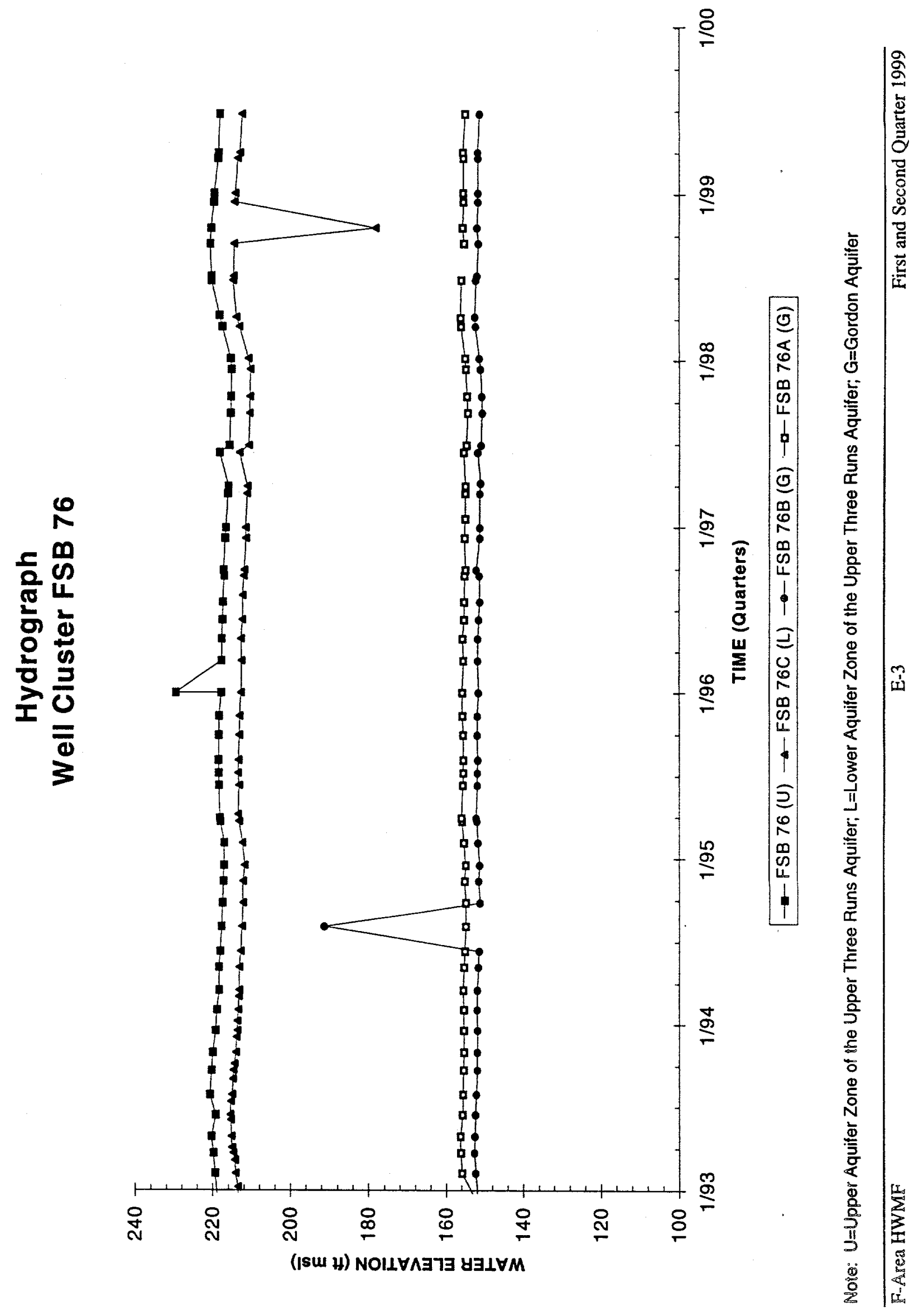




\section{Hydrograph}

Well FSB 77

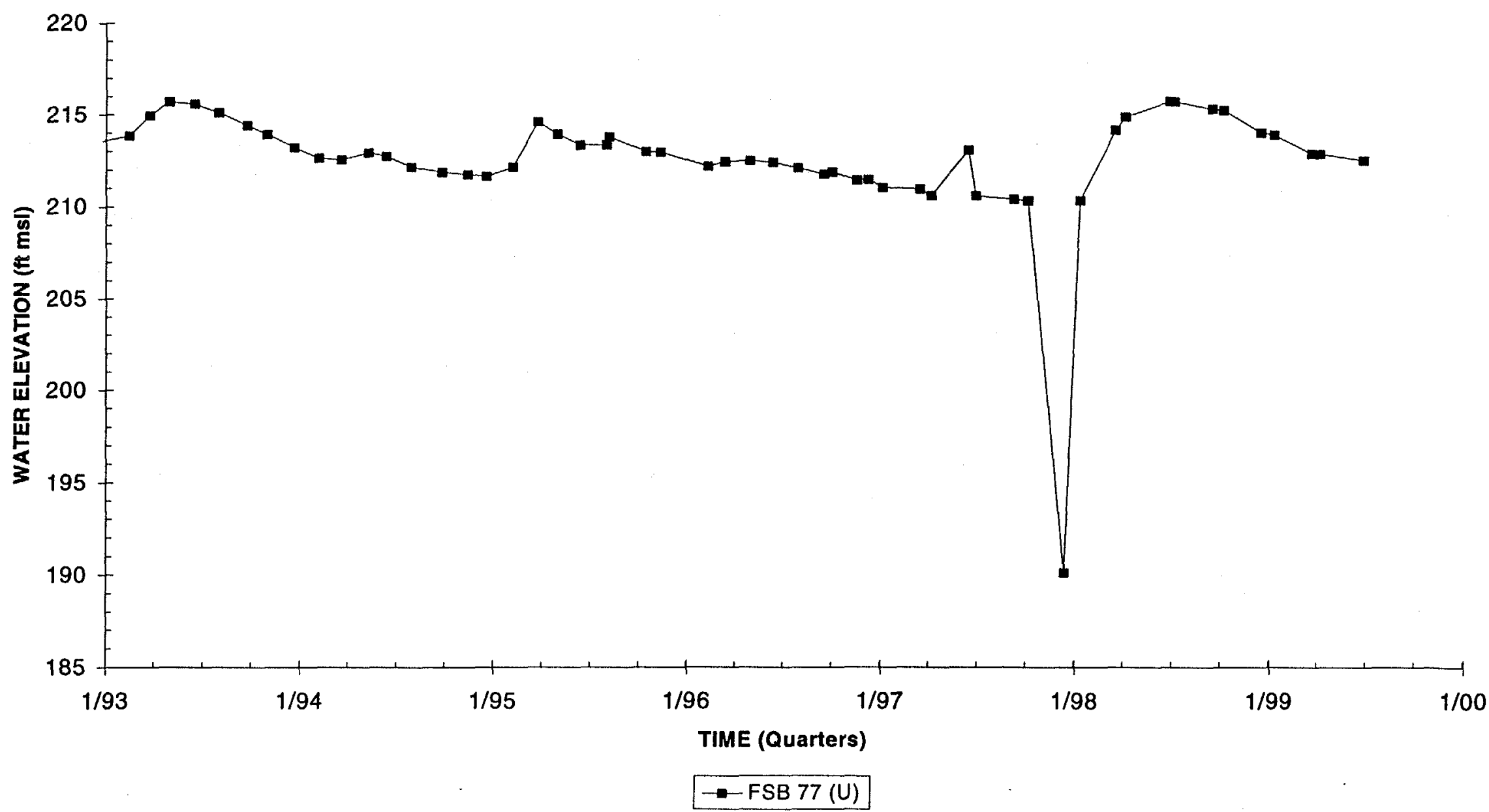

Note: $U=$ Upper Aquifer Zone of the Upper Three Runs Aquifer; L=Lower Aquifer Zone of the Upper Three Runs Aquifer; G=Gordon Aquifer 


\section{Hydrograph \\ Well Cluster FSB 78}

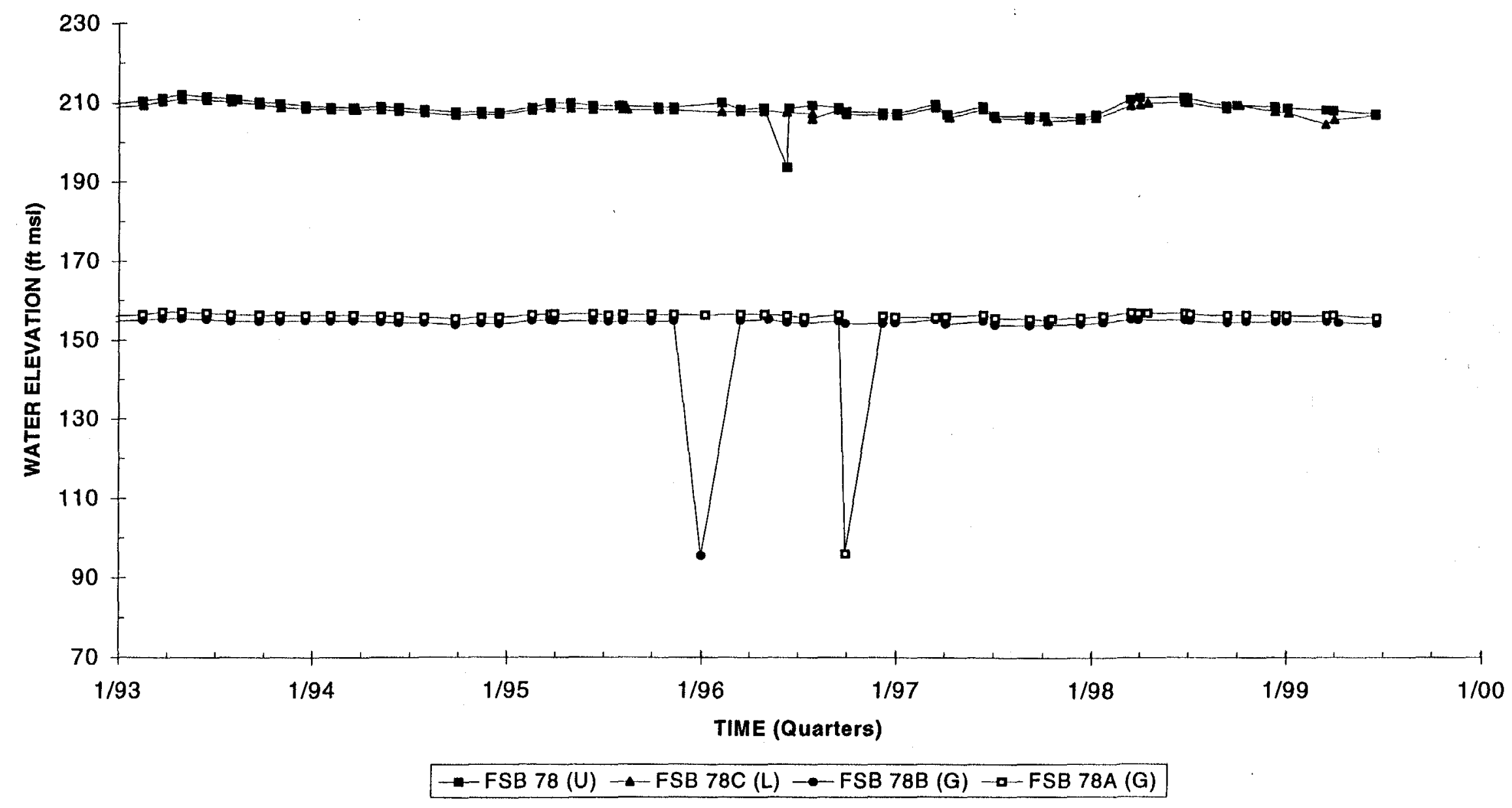

Note: $U=U p p e r$ Aquiter Zone of the Upper Three Runs Aquifer; L=Lower Aquifer Zone of the Upper Three Runs Aquifer; G=Gordon Aquifer 


\section{Hydrograph \\ Well Cluster FSB 79}

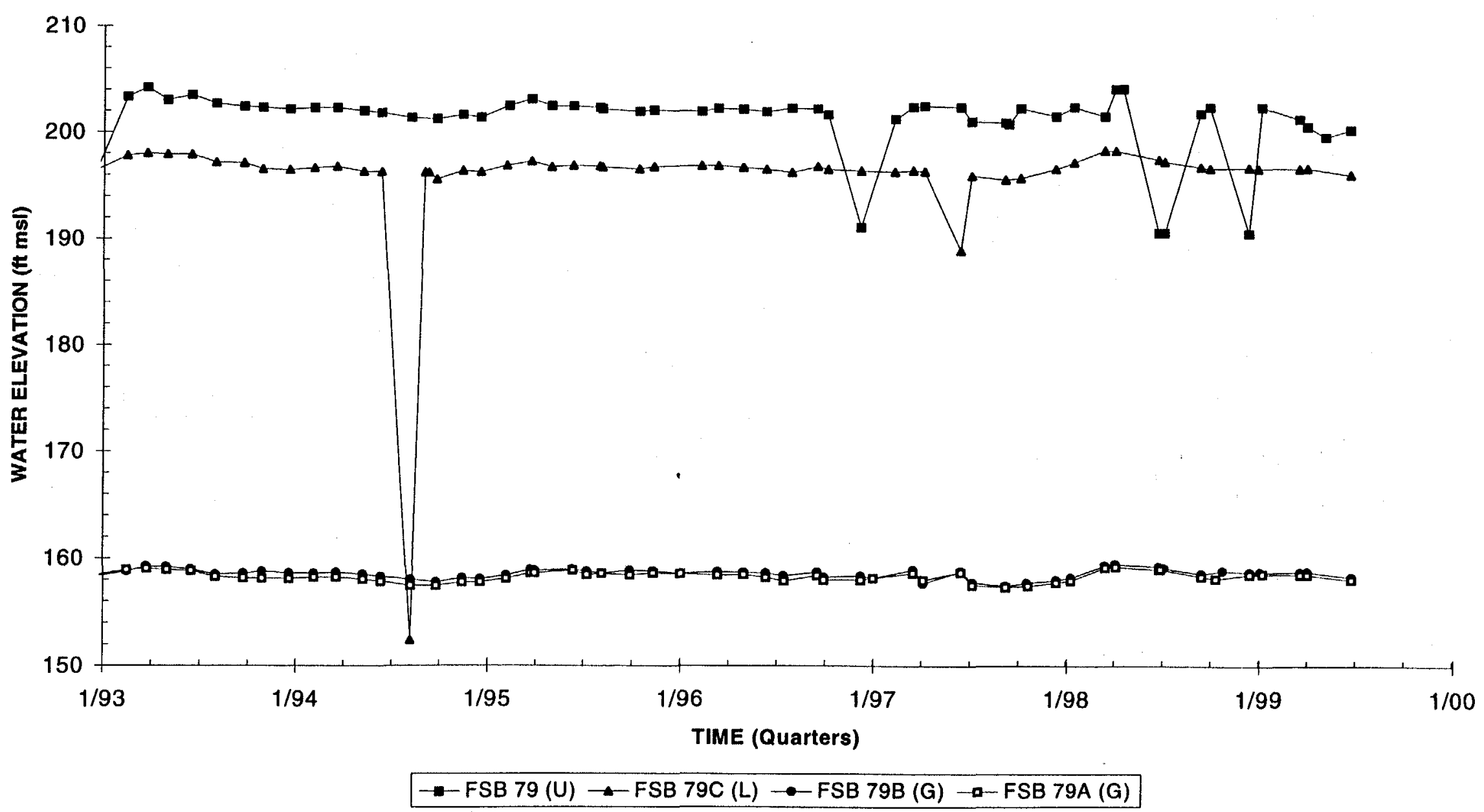

Note: U=Upper Aquifer Zone of the Upper Three Runs Aquifer; L=Lower Aquifer Zone of the Upper Three Runs Aquifer; G=Gordon Aquifer 


\section{Hydrograph \\ Well Cluster FSB 87}

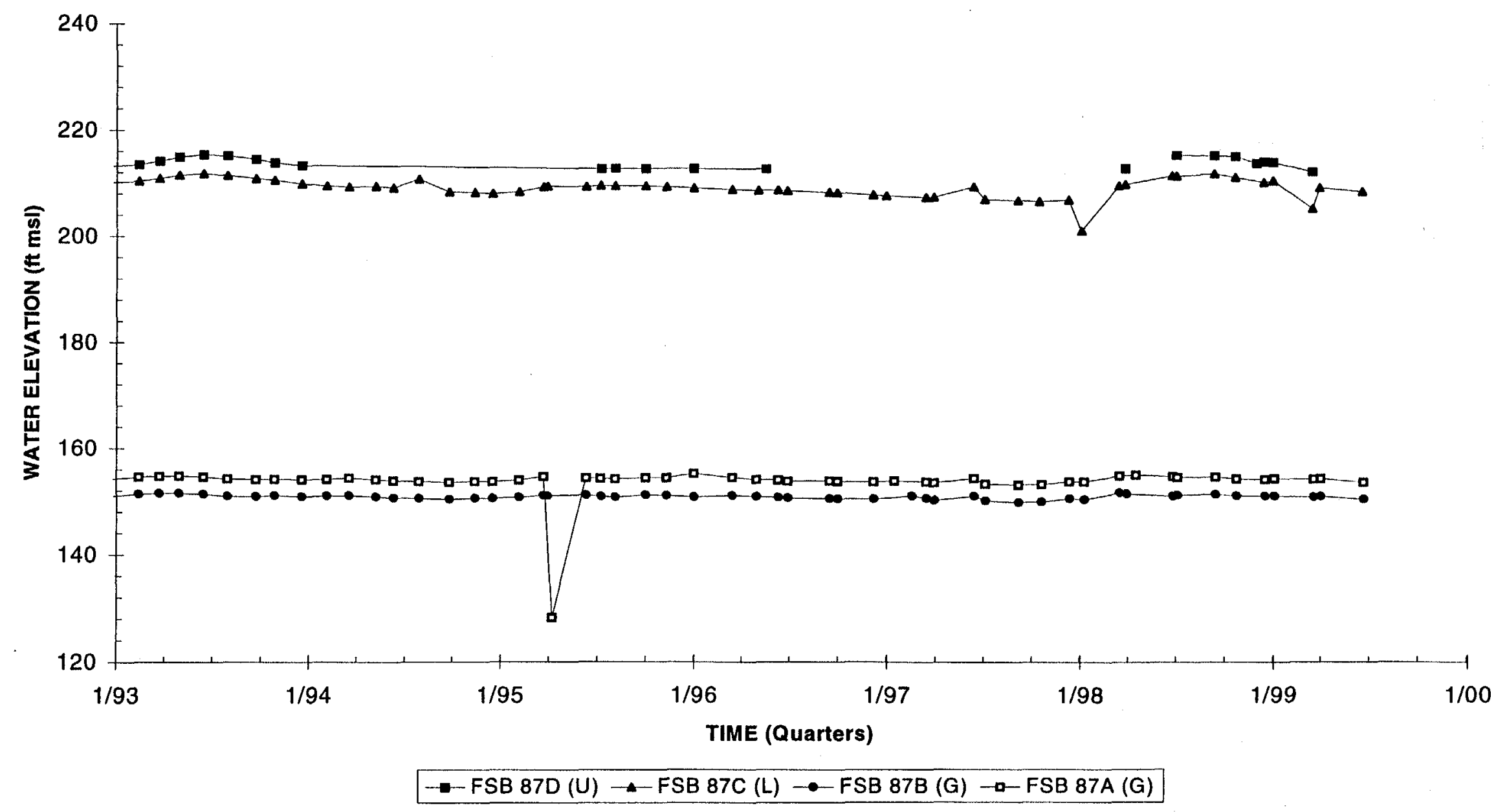

Note: U=Upper Aquifer Zone of the Upper Three Runs Aquifer; L=Lower Aquifer Zone of the Upper Three Runs Aquifer; G=Gordon Aquifer 


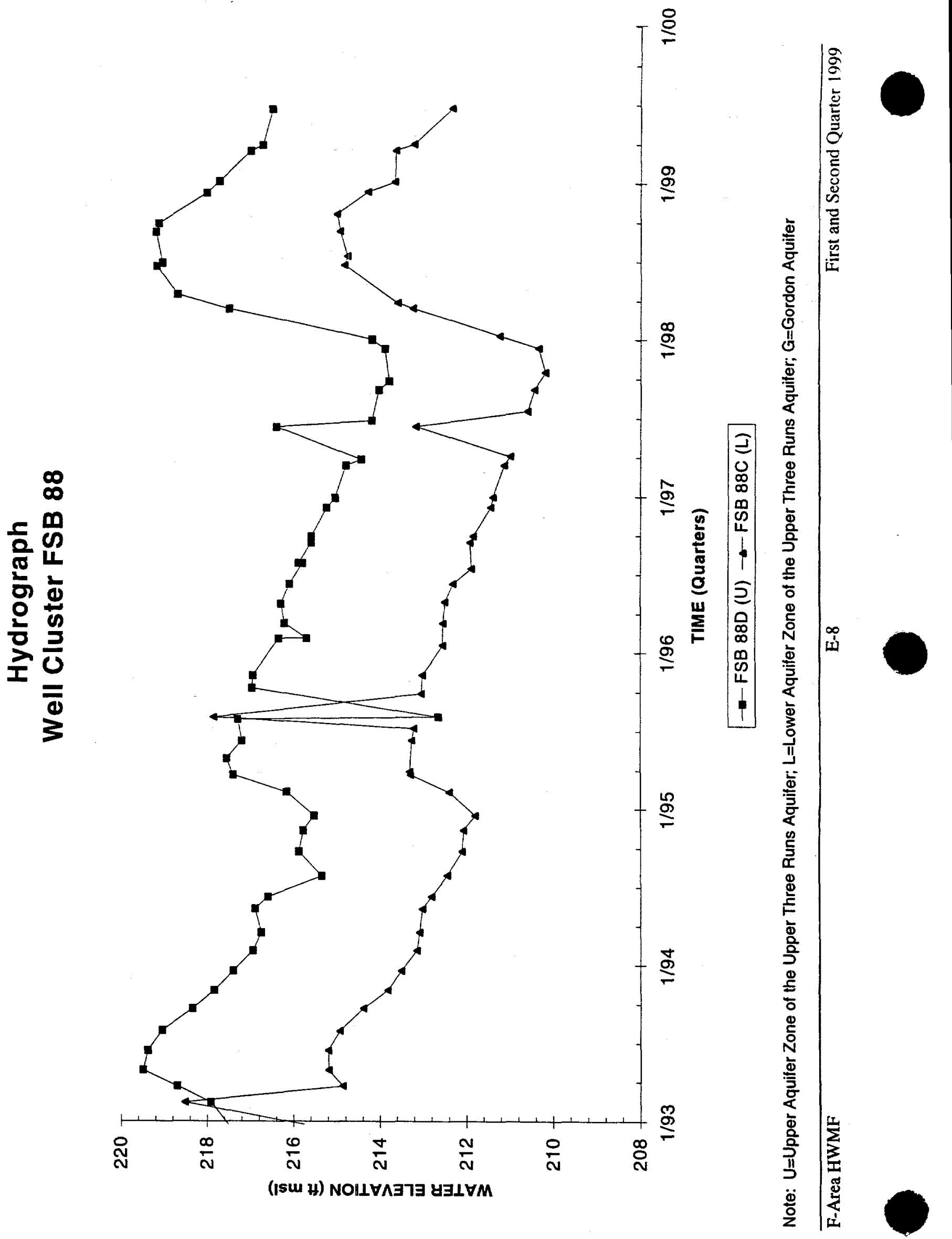



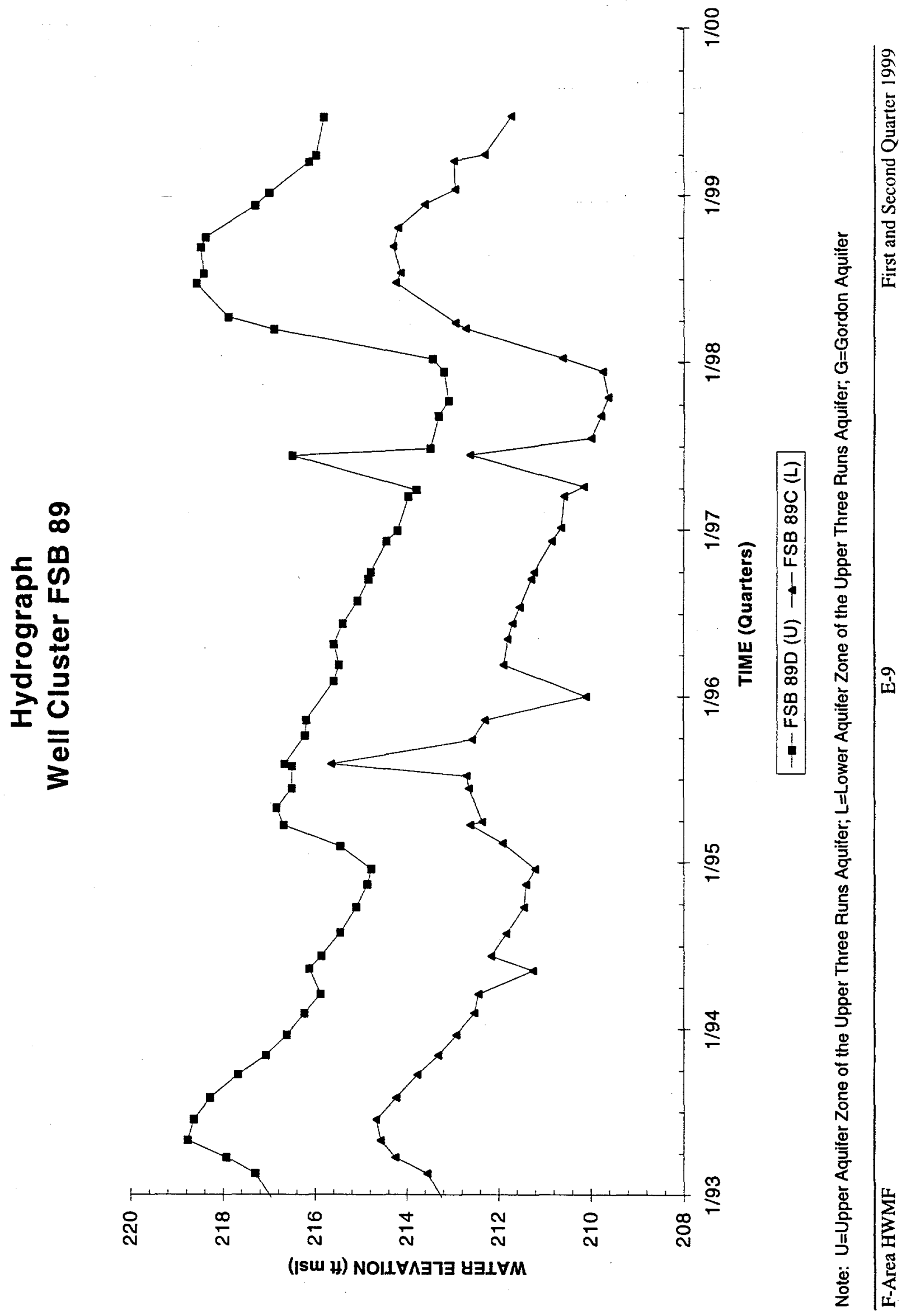


\section{Hydrograph \\ Well Cluster FSB 90}

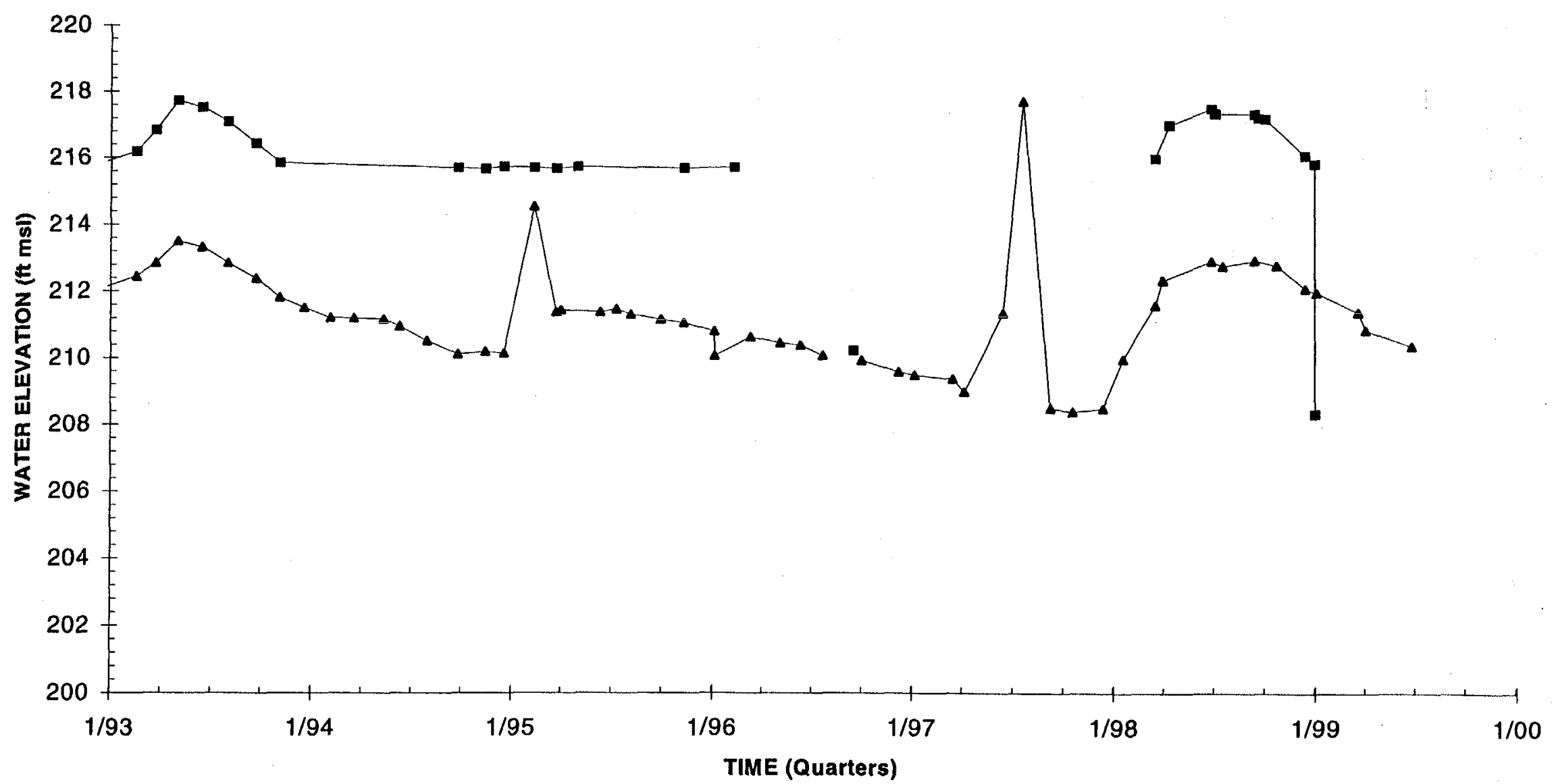

$\rightarrow$ FSB $900(\mathrm{U}) \multimap$ FSB $90 \mathrm{C}(\mathrm{L})$

Note: U=Upper Aquifer Zone of the Upper Three Runs Aquifer; L=Lower Aquifer Zone of the Upper Three Runs Aquifer; G=Gordon Aquifer 


\section{Hydrograph \\ Well Cluster FSB 91}

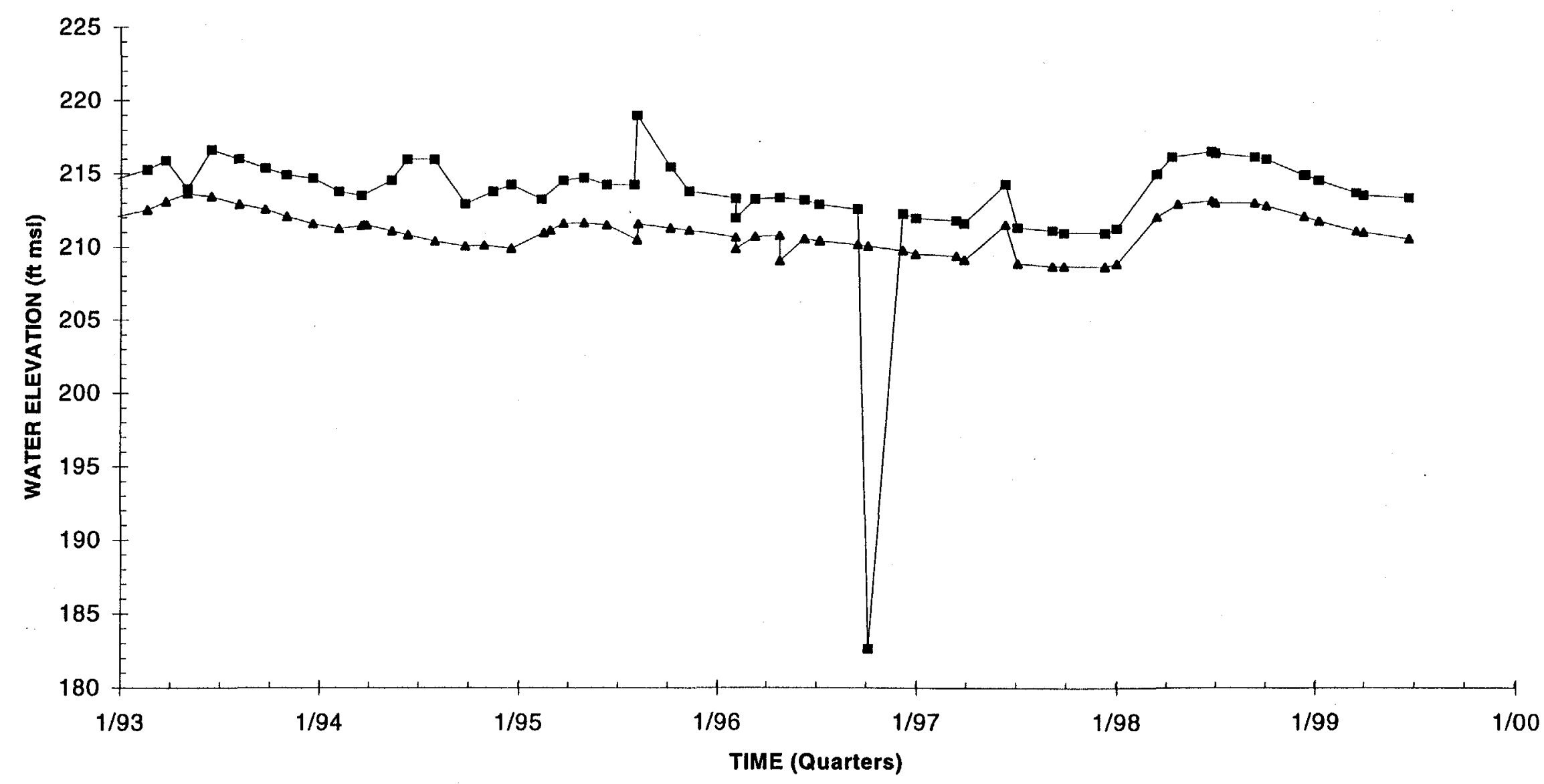

$\rightarrow-F S B 91 D(U) \multimap$ FSB $91 C(L)$

Note: U=Upper Aquifer Zone of the Upper Three Runs Aquifer; L=Lower Aquifer Zone of the Upper Three Runs Aquifer; G=Gordon Aquifer 


\section{Hydrograph \\ Well Cluster FSB 92}

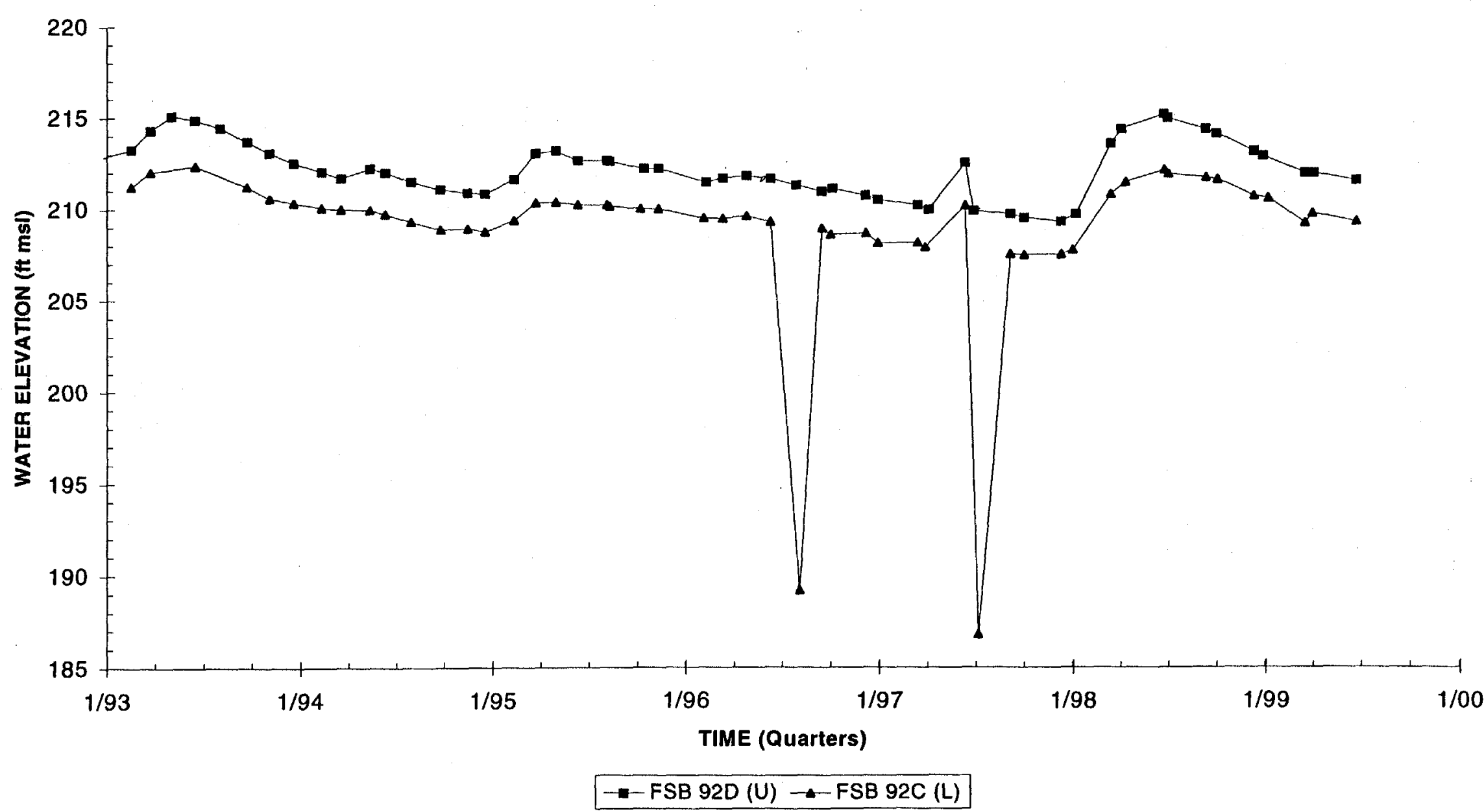

Note: U=Upper Aquifer Zone of the Upper Three Runs Aquifer; L=Lower Aquiter Zone of the Upper Three Runs Aquifer; G=Gordon Aquifer 


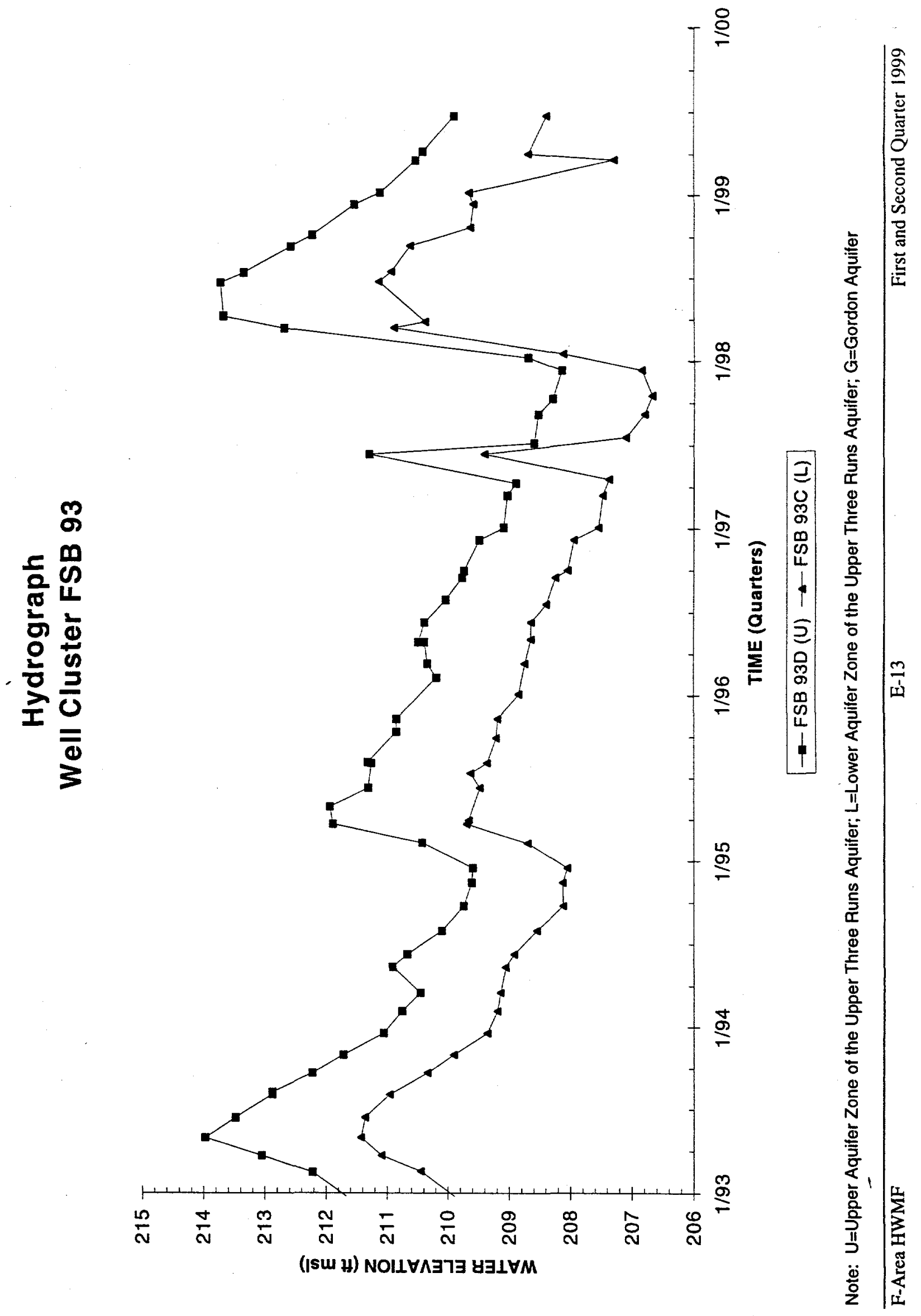




\section{Hydrograph \\ Well Cluster FSB 94}

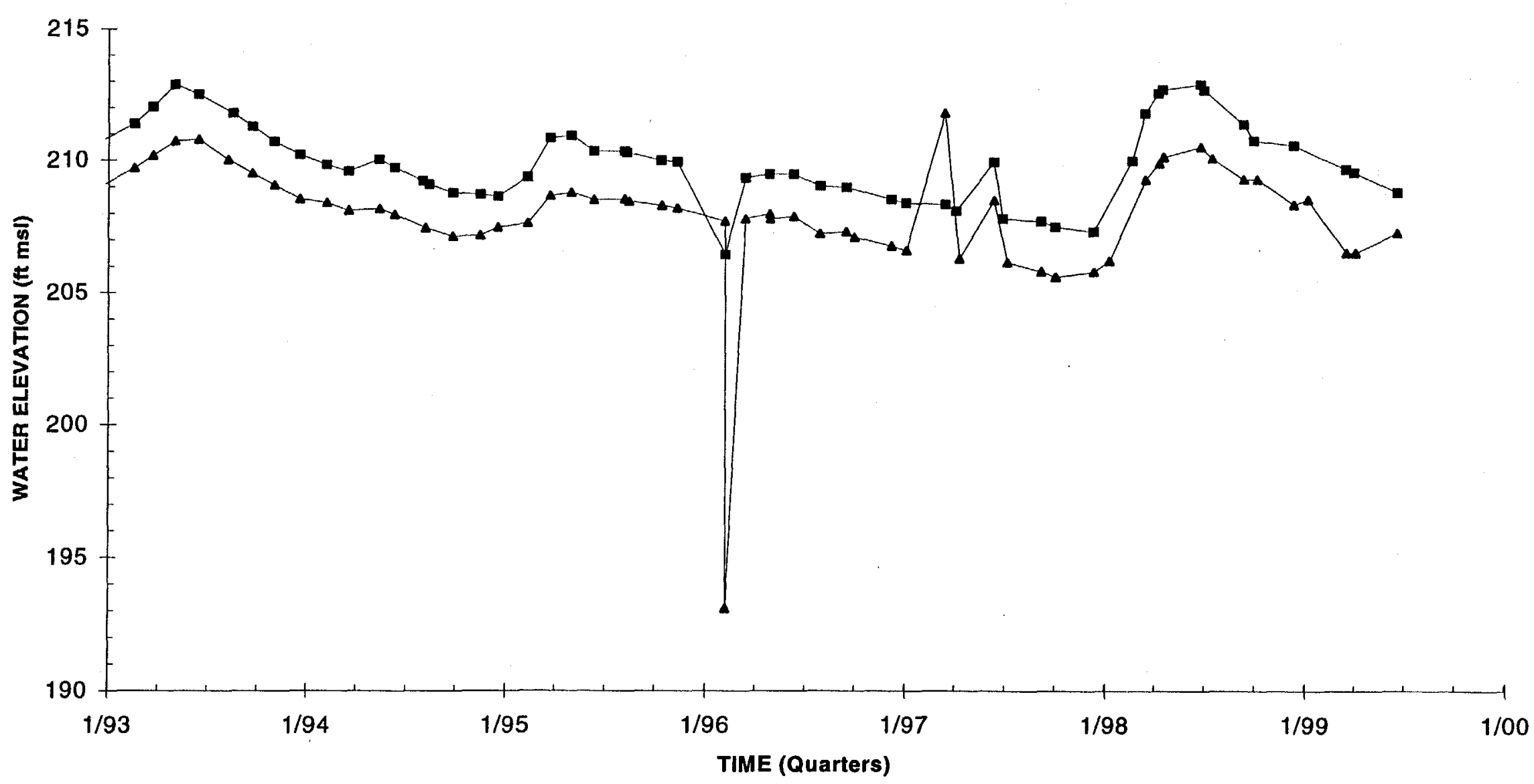

$$
\because \text { FSB 94DR (U) } \multimap \text { FSB 94C (L) }
$$

Note: U=Upper Aquifer Zone of the Upper Three Runs Aquifer; L=Lower Aquifer Zone of the Upper Three Runs Aquifer; G=Gordon Aquifer 


\section{Hydrograph \\ Well Cluster FSB 95}

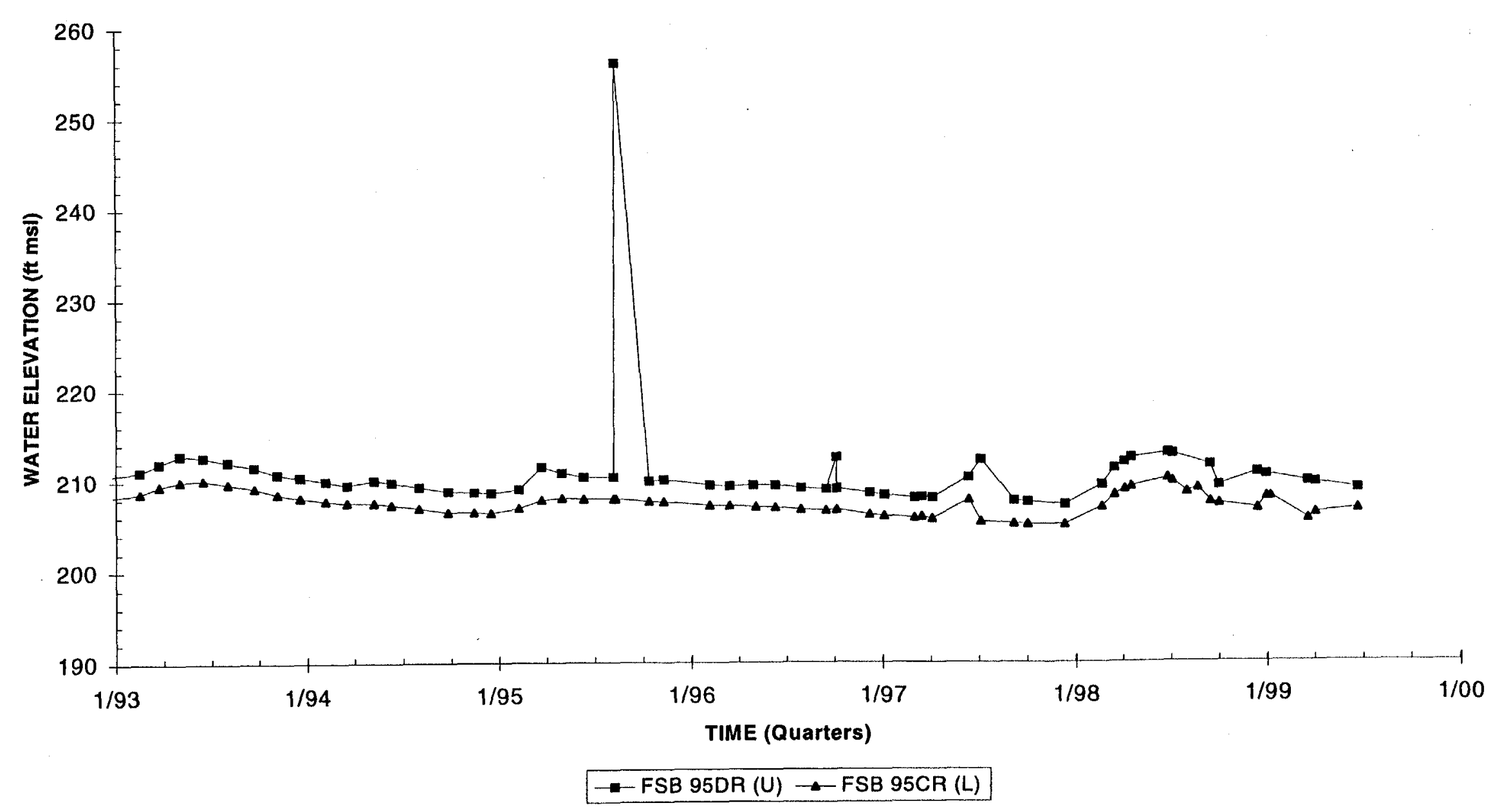

Note: U=Upper Aquifer Zone of the Upper Three Runs Aquifer; L=Lower Aquifer Zone of the Upper Three Runs Aquifer; G=Gordon Aquifer 


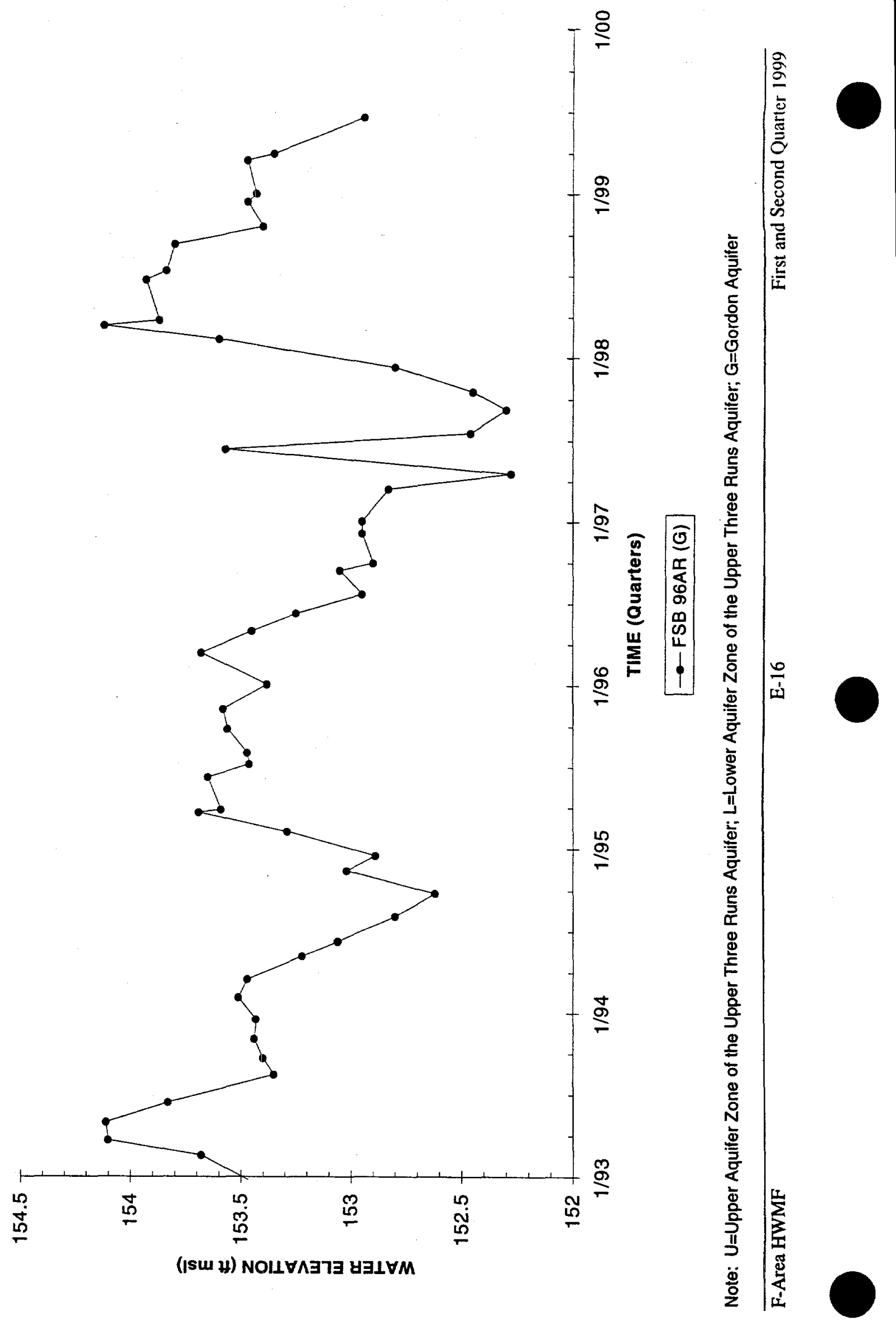




\section{Hydrograph \\ Well Cluster FSB 97}

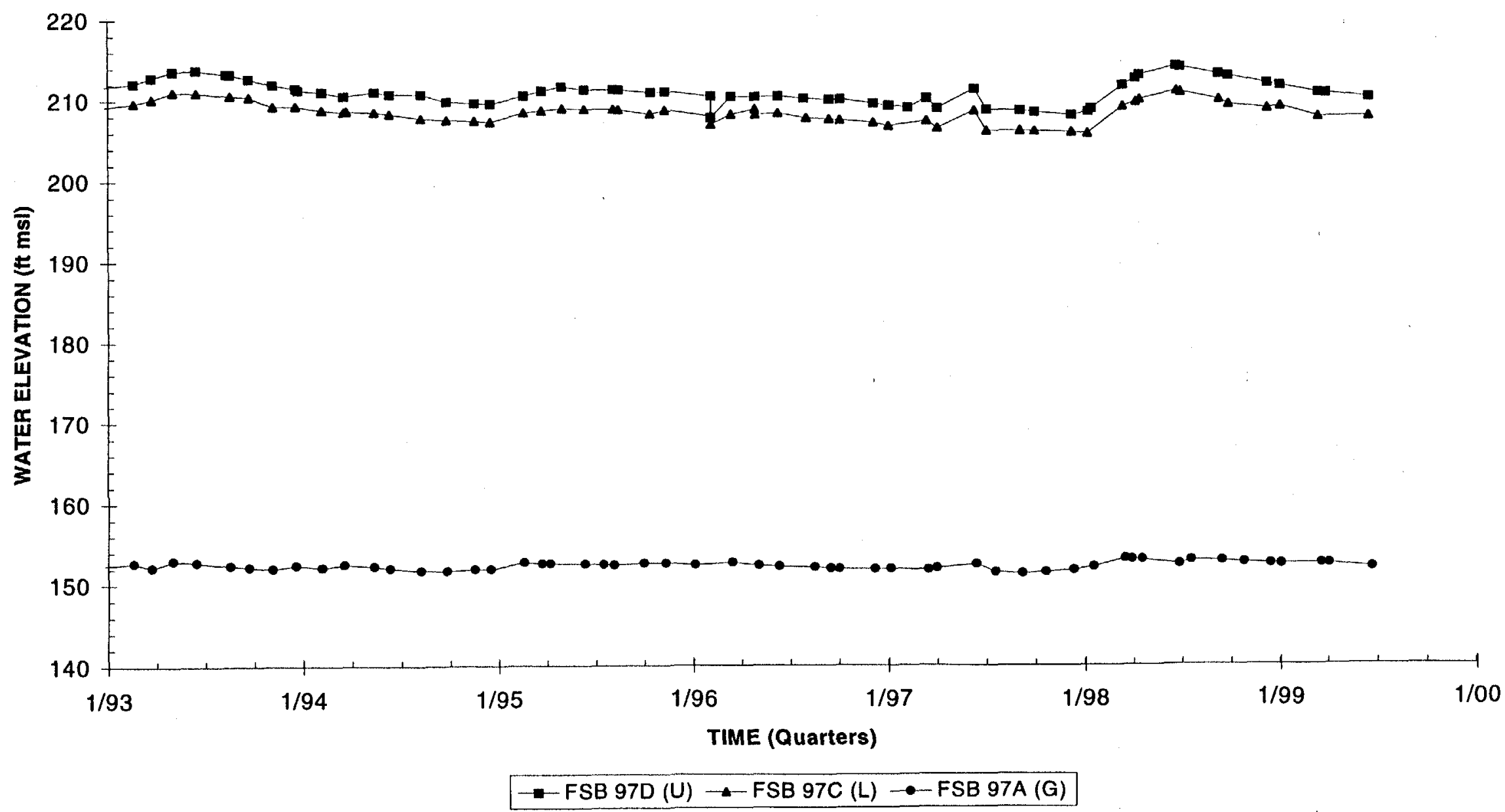

Note: U=Upper Aquifer Zone of the Upper Three Runs Aquifer; L=Lower Aquifer Zone of the Upper Three Runs Aquifer; G=Gordon Aquifer 


\section{Hydrograph \\ Well Cluster FSB 98}

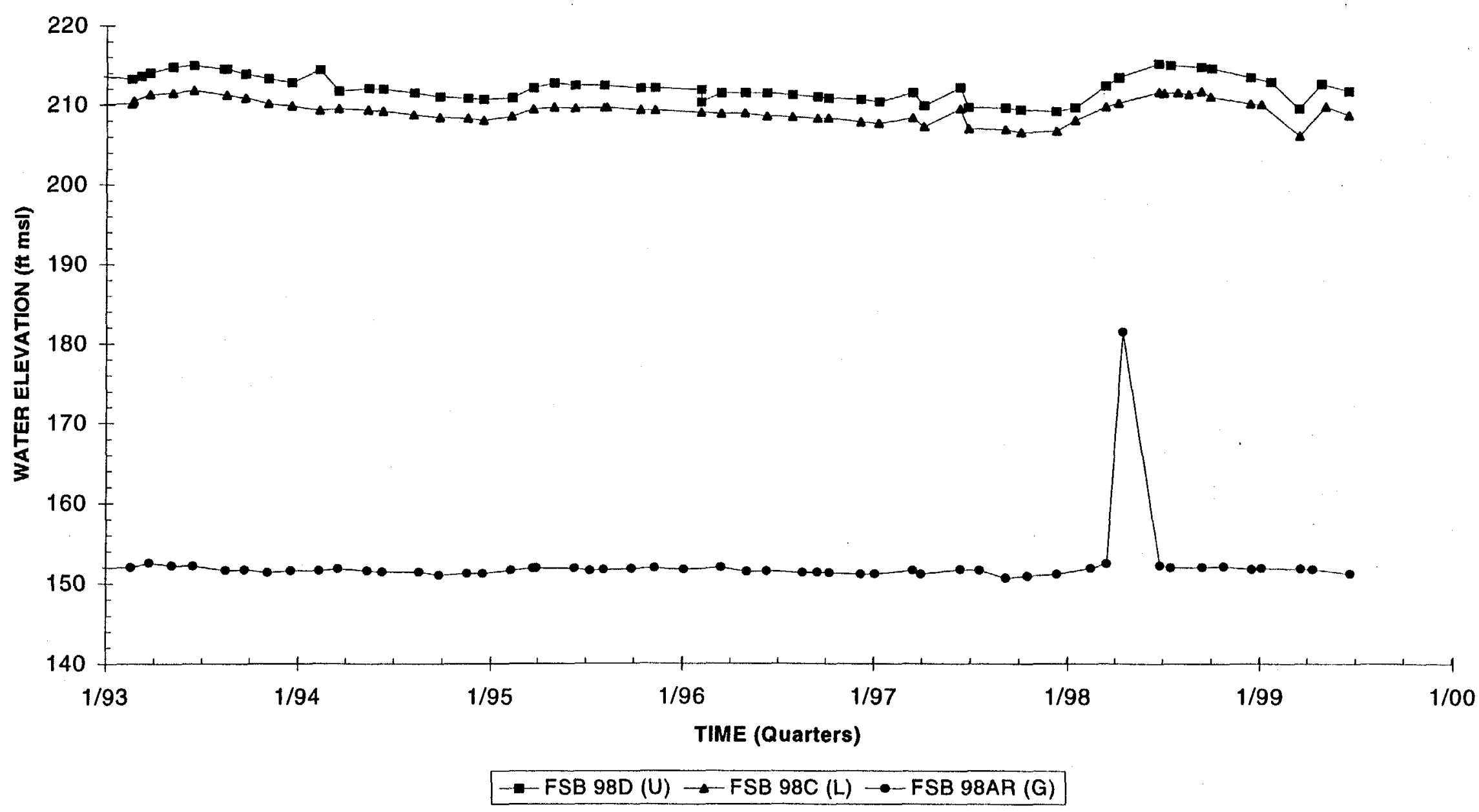

Note: U=Upper Aquifer Zone of the Upper Three Runs Aquifer; L=Lower Aquifer Zone of the Upper Three Runs Aquifer; G=Gordon Aquifer 


\section{Hydrograph \\ Well Cluster FSB 99}

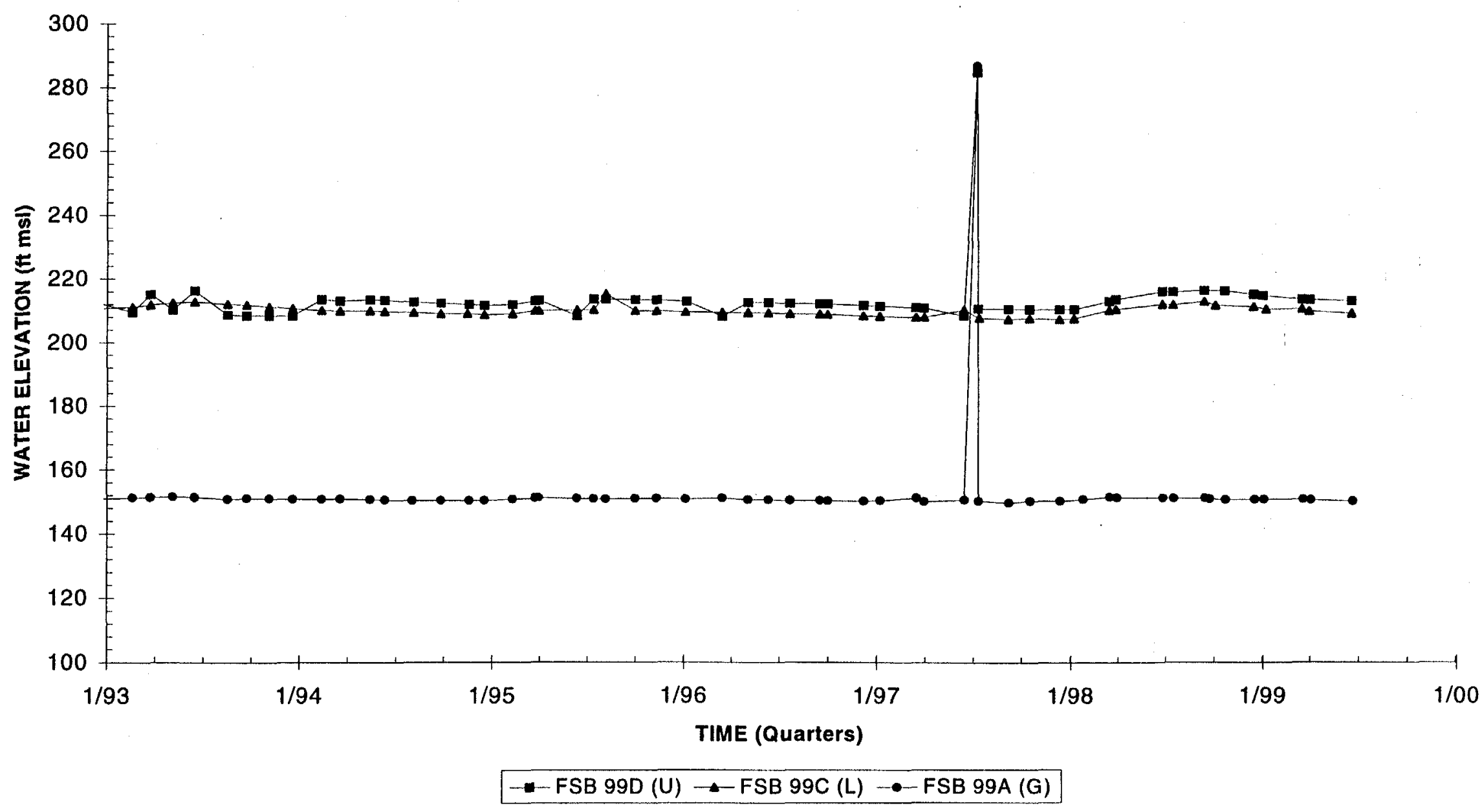

Note: $U=$ Upper Aquifer Zone of the Upper Three Runs Aquifer; L=Lower Aquifer Zone of the Upper Three Runs Aquifer; G=Gordon Aquifer 


\section{Hydrograph \\ Well FSB100A}

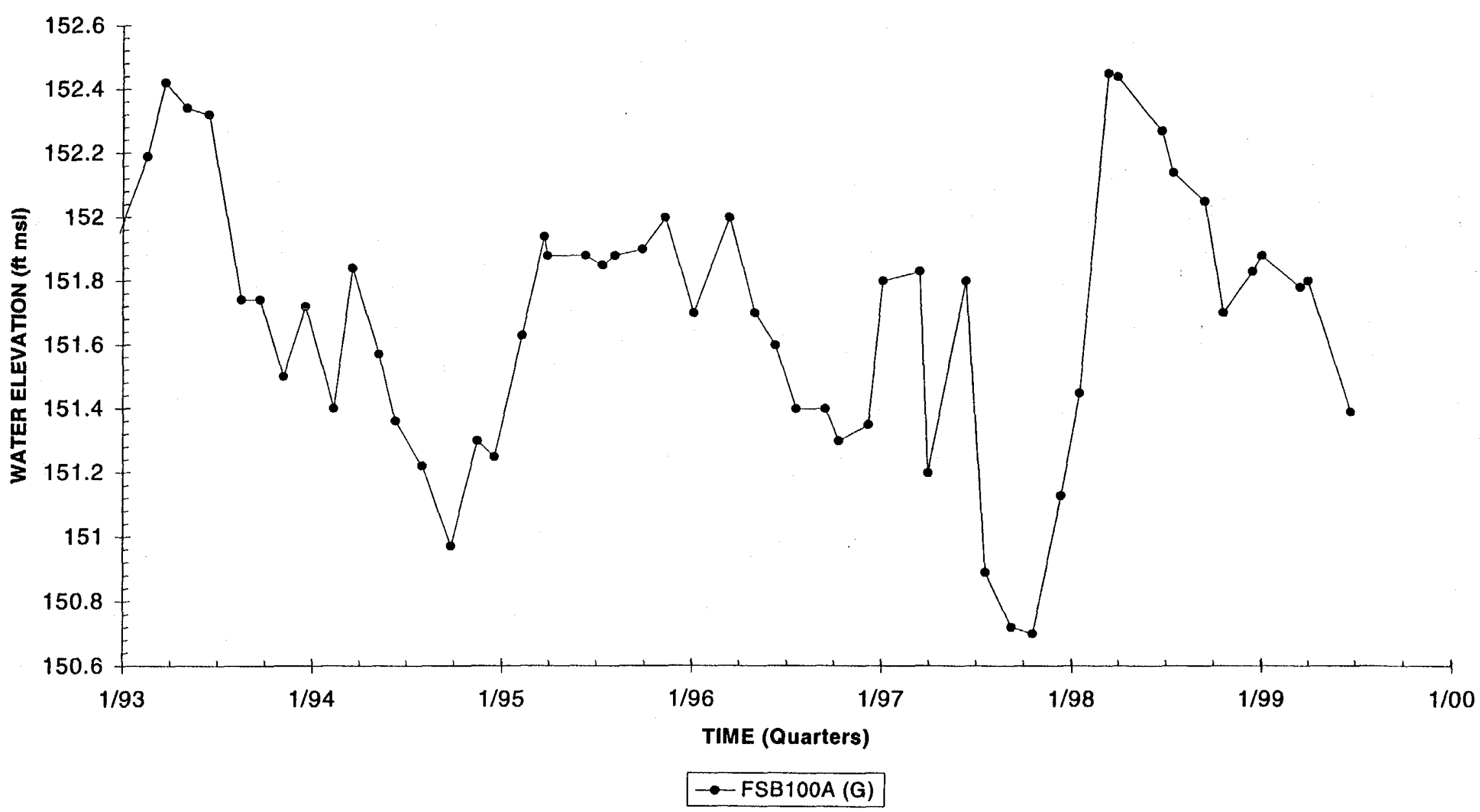

Note: U=Upper Aquifer Zone of the Upper Three Runs Aquifer; L=Lower Aquifer Zone of the Upper Three Runs Aquifer; G=Gordon Aquifer 


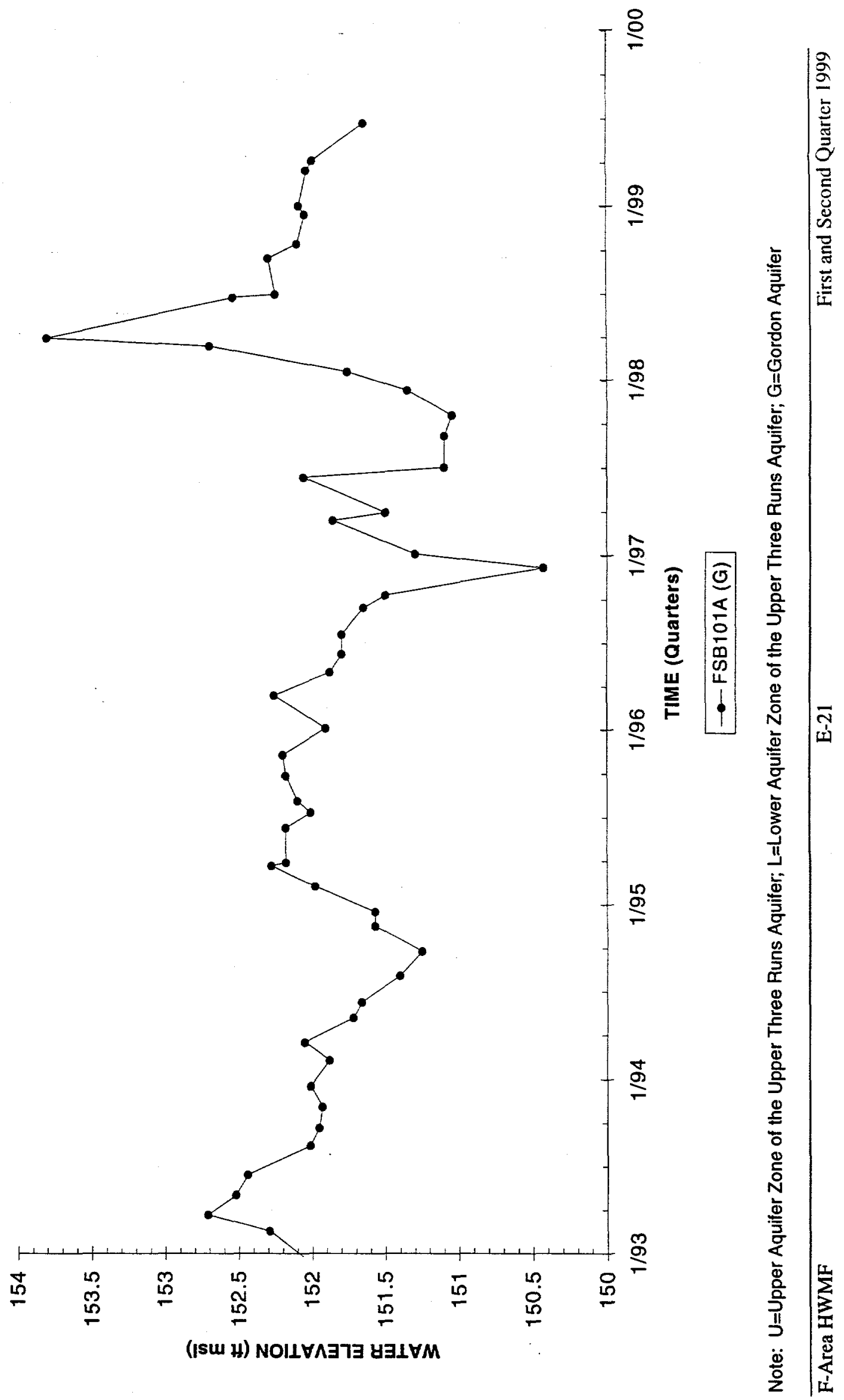



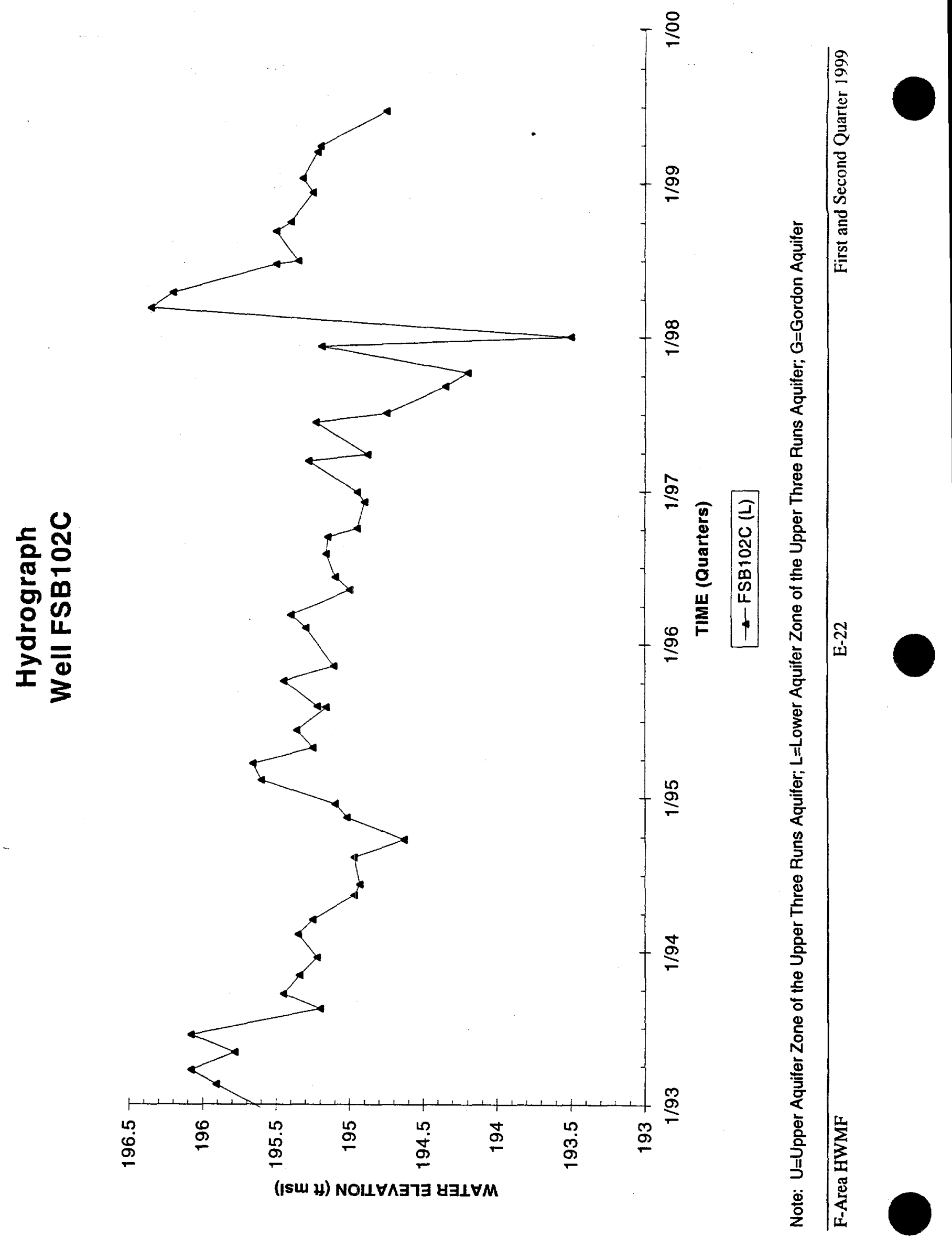


\section{Hydrograph \\ Well FSB103C}

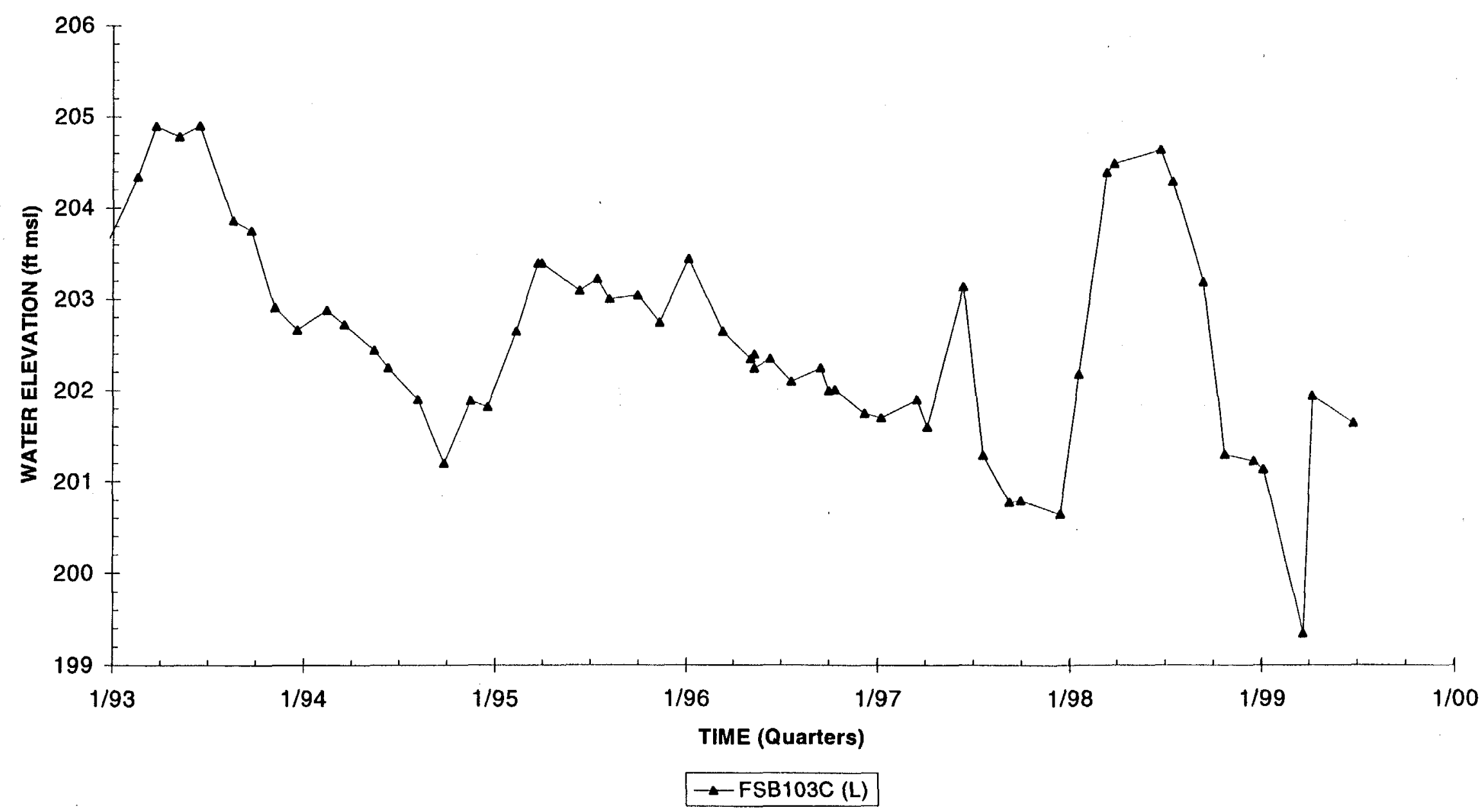

Note: U=Upper Aquifer Zone of the Upper Three Runs Aquifer; L=Lower Aquifer Zone of the Upper Three Runs Aquifer; G=Gordon Aquifer 

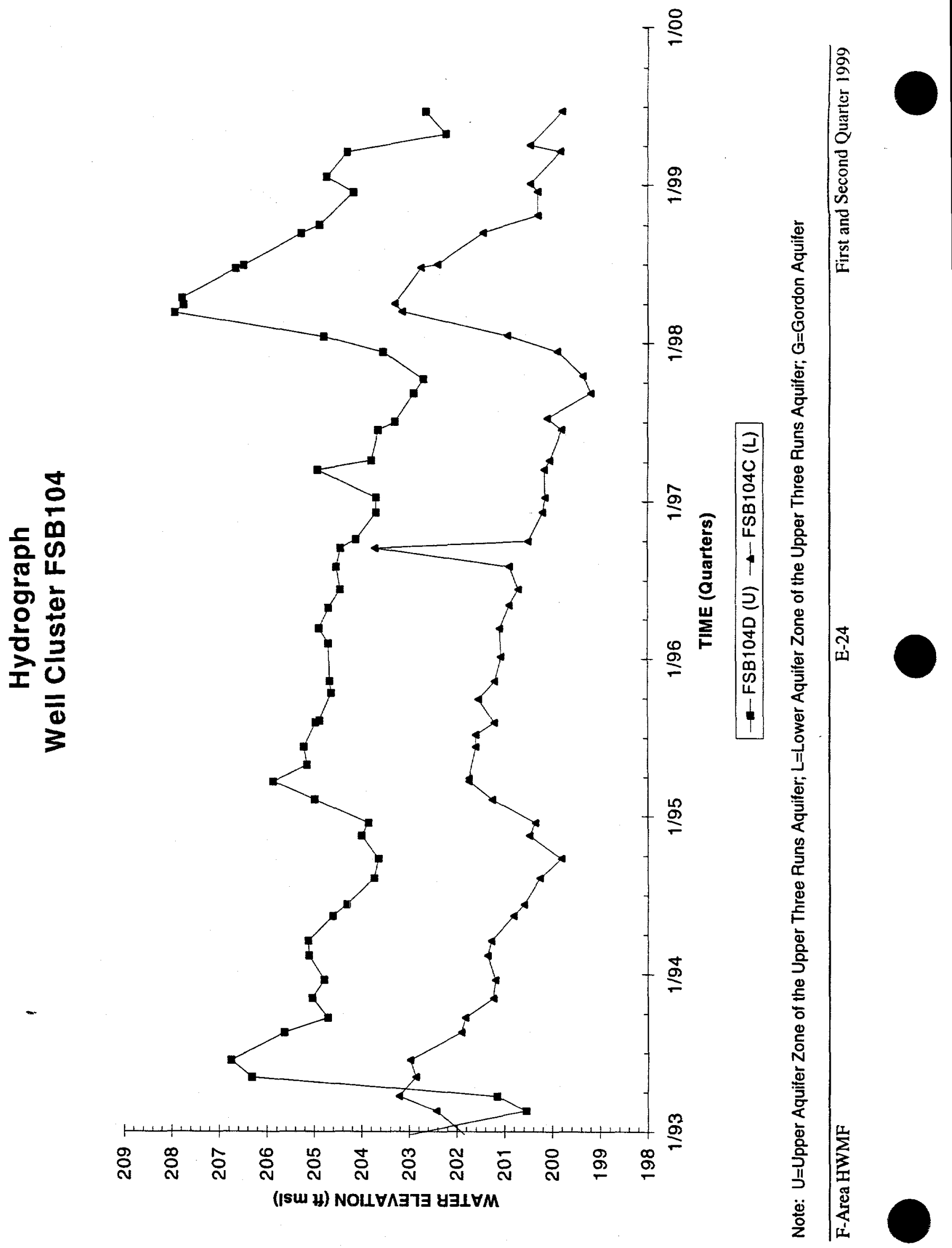


\section{Hydrograph \\ Well Cluster FSB105}

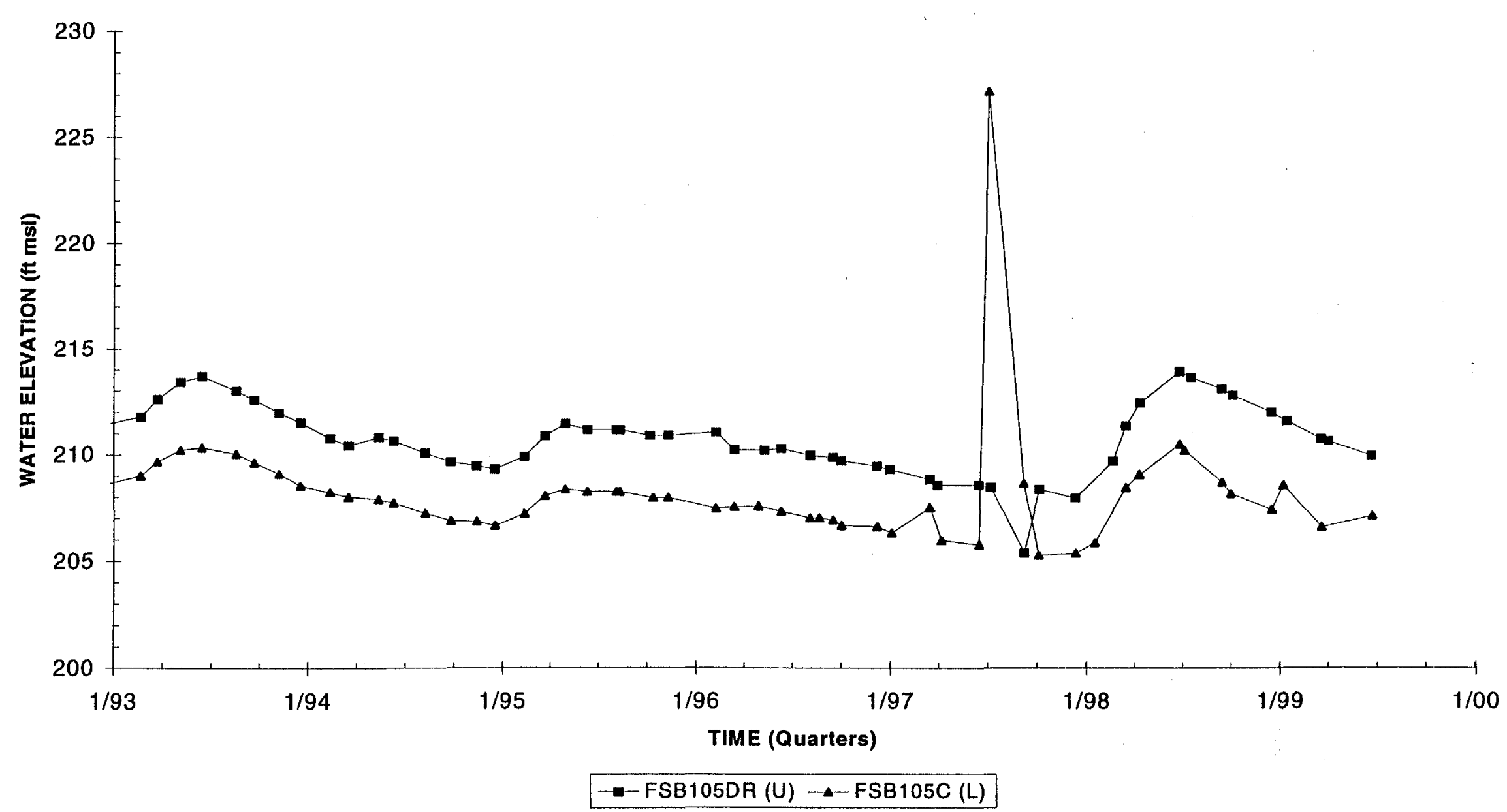

Note: U=Upper Aquifer Zone of the Upper Three Runs Aquifer; L=Lower Aquifer Zone of the Upper Three Runs Aquifer; G=Gordon Aquifer 


\section{Hydrograph \\ Well Cluster FSB106}

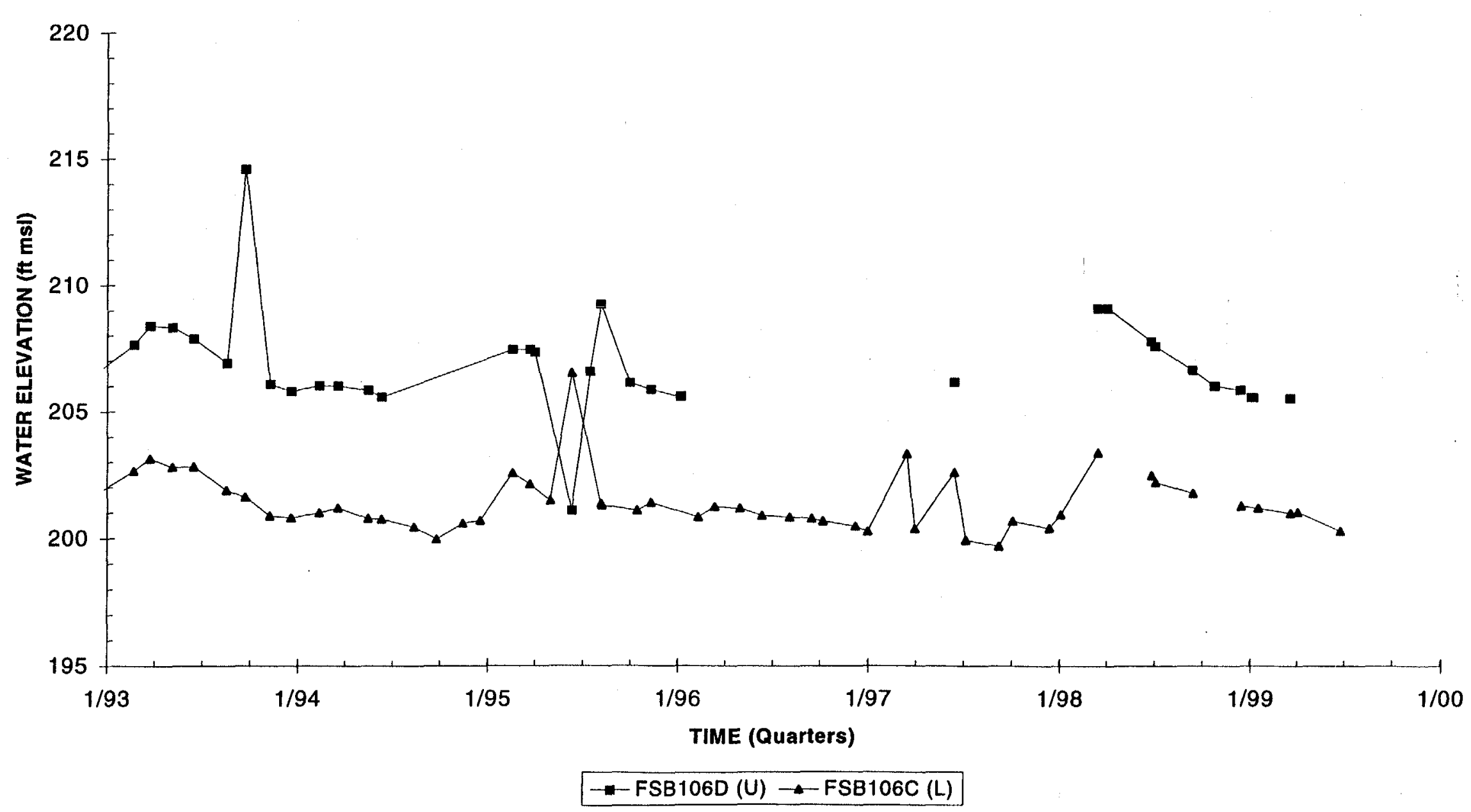

Note: U=Upper Aquifer Zone of the Upper Three Runs Aquifer; L=Lower Aquifer Zone of the Upper Three Runs Aquifer; G=Gordon Aquifer 


\section{Hydrograph \\ Well Cluster FSB107}

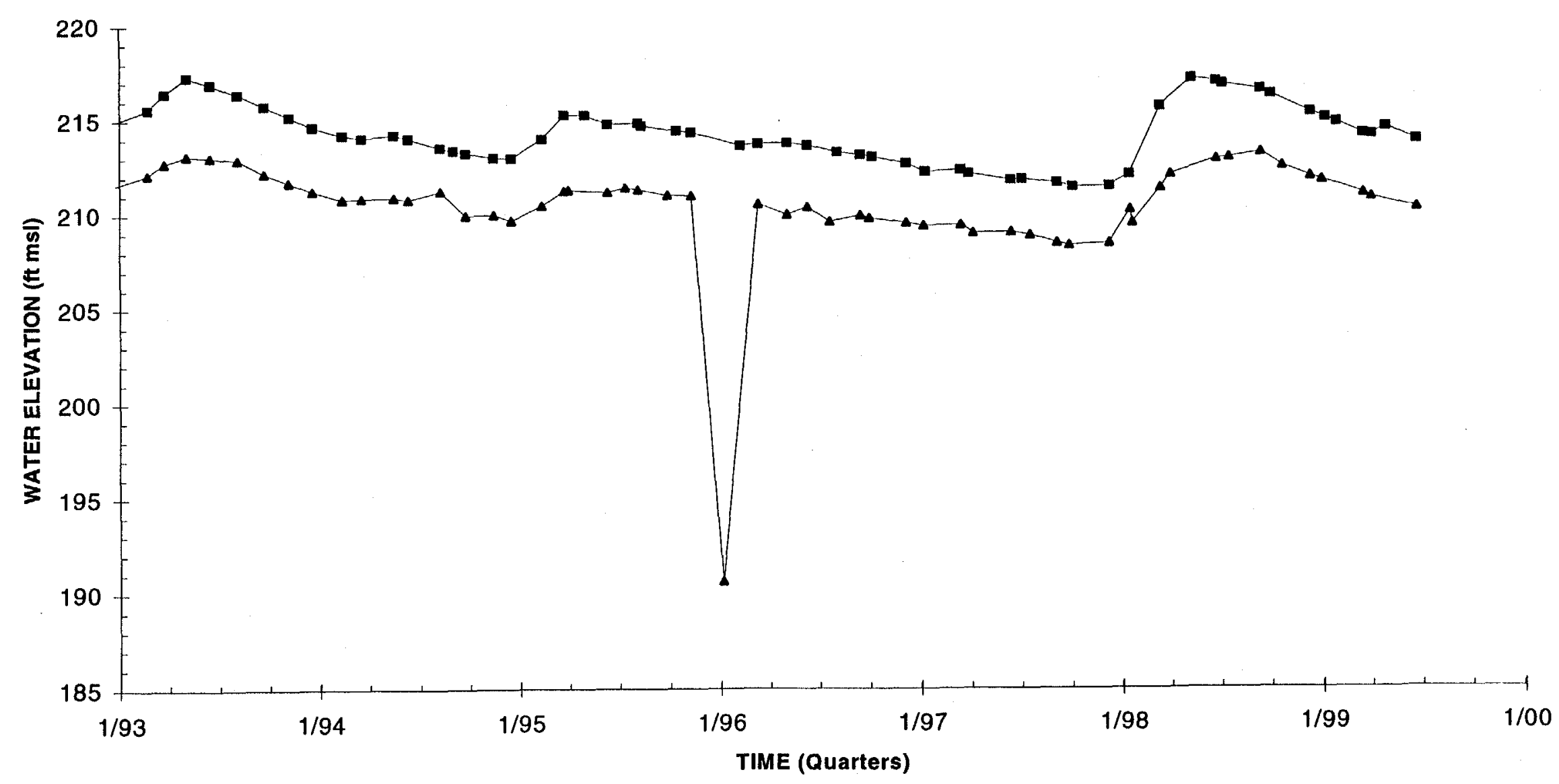

FSB107D (U) $\rightarrow-$ FSB107C (L)

Note: U=Upper Aquifer Zone of the Upper Three Runs Aquifer; L=Lower Aquifer Zone of the Upper Three Runs Aquifer; G=Gordon Aquifer 


\section{Hydrograph \\ Well FSB108D}

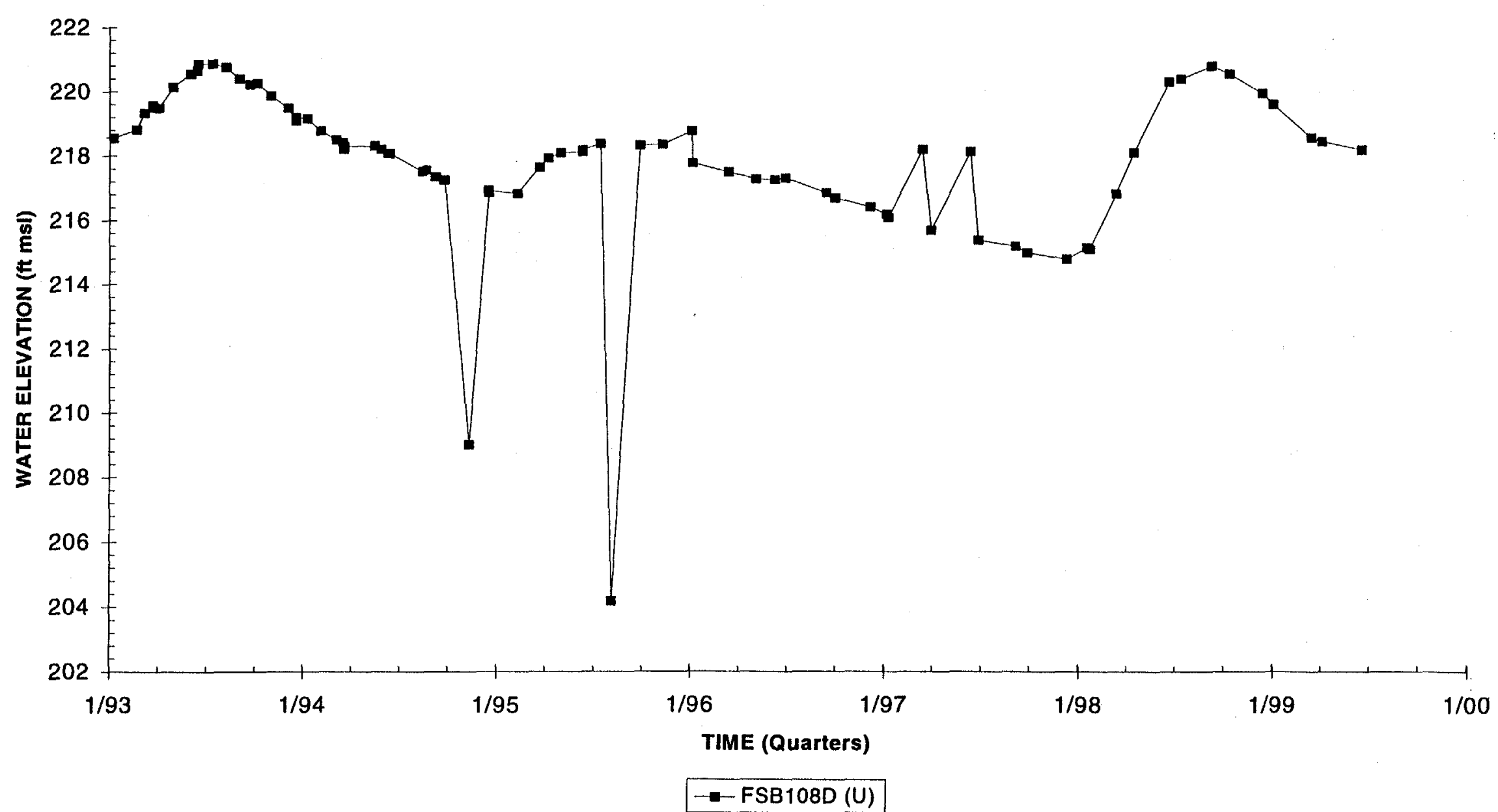

Note: U=Upper Aquifer Zone of the Upper Three Runs Aquifer; L=Lower Aquifer Zone of the Upper Three Runs Aquifer; G=Gordon Aquifer 


\section{Hydrograph \\ Well FSB109D}

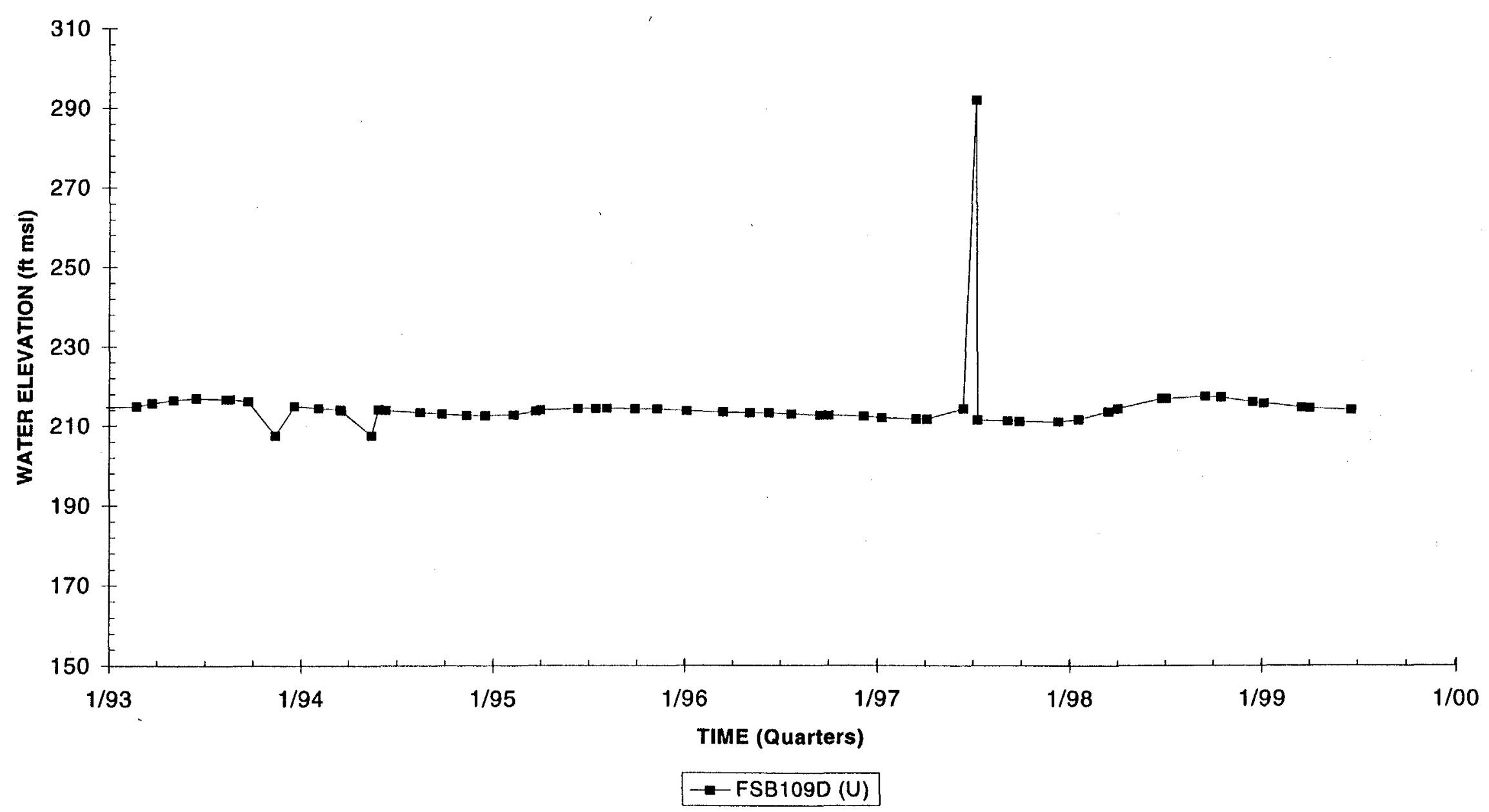

Note: U=Upper Aquifer Zone of the Upper Three Runs Aquifer; L=Lower Aquifer Zone of the Upper Three Runs Aquifer; G=Gordon Aquifer 


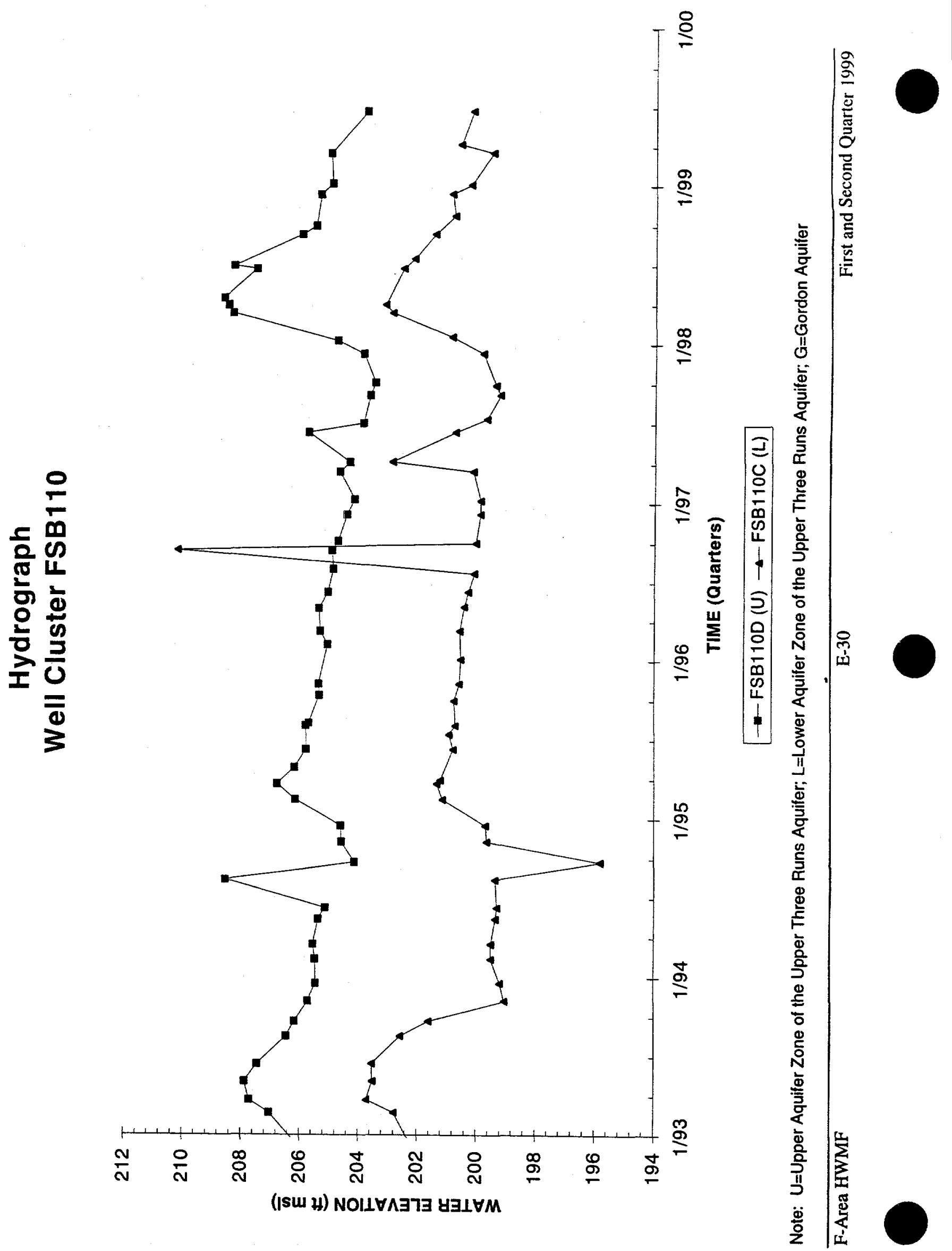




\section{Hydrograph \\ Well Cluster FSB111}

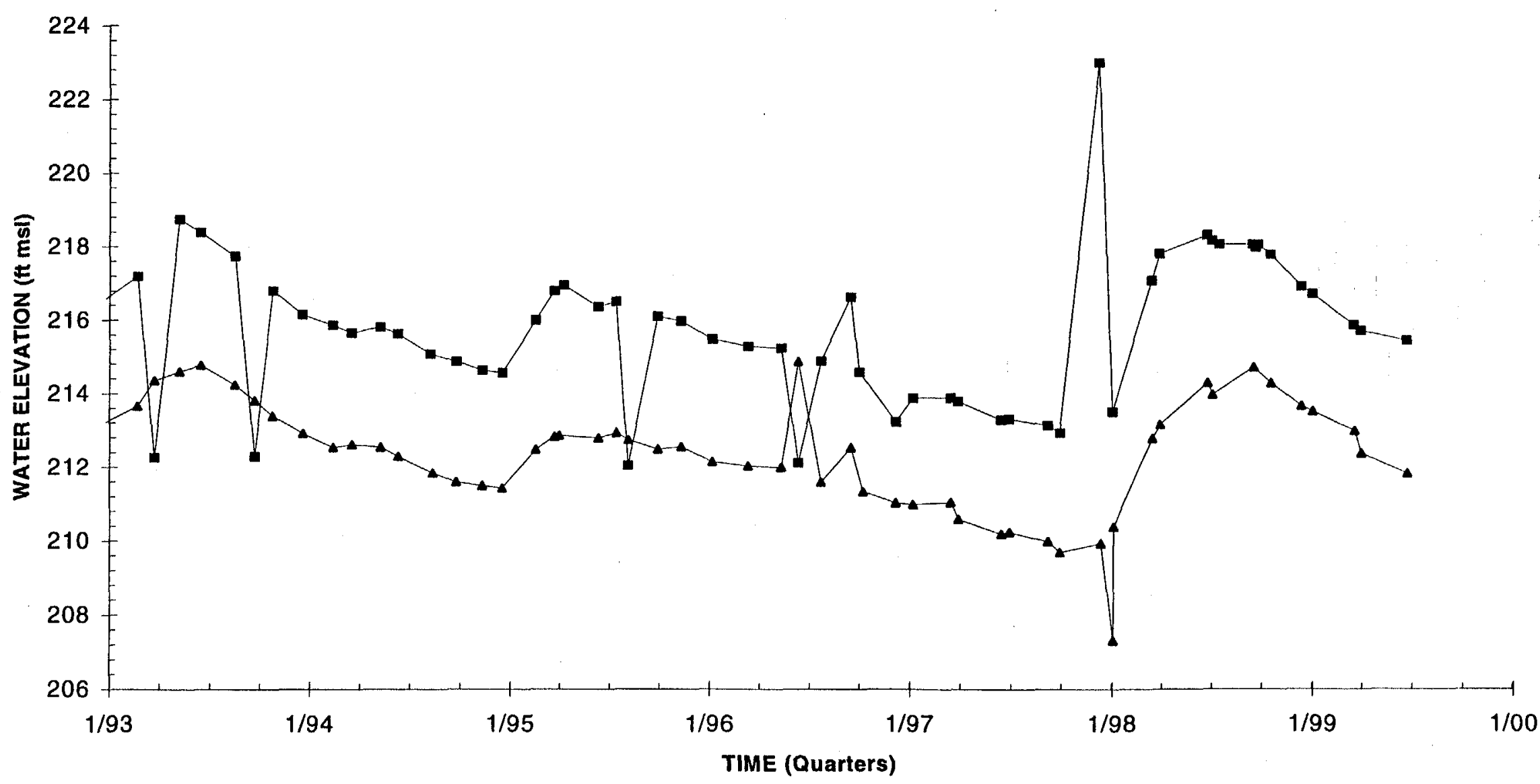

$-F S B 111 D(U) \rightarrow F S B 111 C(L)$

Note: U=Upper Aquifer Zone of the Upper Three Runs Aquifer; L=Lower Aquifer Zone of the Upper Three Runs Aquifer; G=Gordon Aquifer 


\section{Hydrograph \\ Well Cluster FSB112}

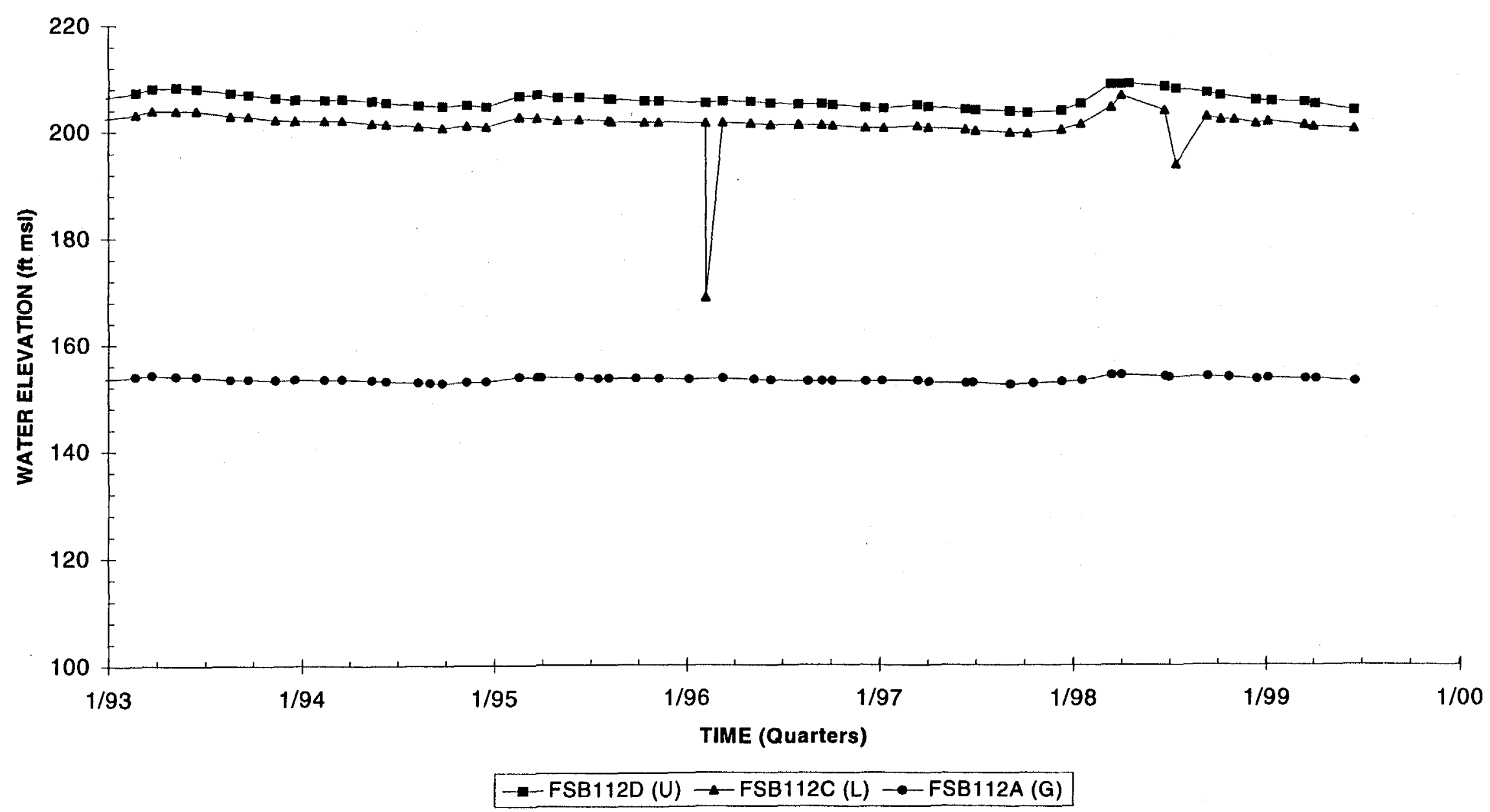

Note: U=Upper Aquifer Zone of the Upper Three Runs Aquifer; L=Lower Aquifer Zone of the Upper Three Runs Aquifer; G=Gordon Aquifer 


\section{Hydrograph \\ Well Cluster FSB113}

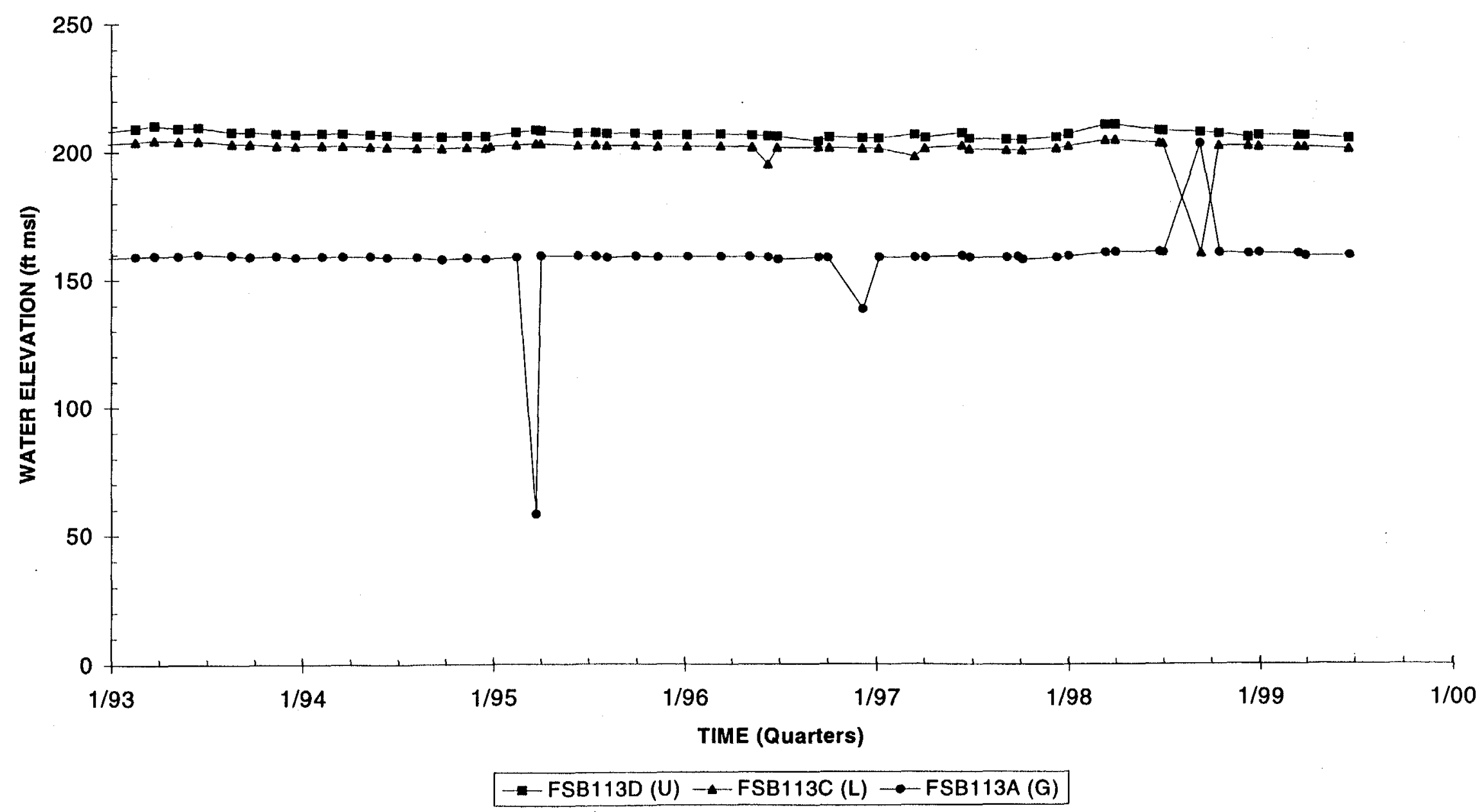

Note: U=Upper Aquifer Zone of the Upper Three Runs Aquifer; L=Lower Aquifer Zone of the Upper Three Runs Aquifer; G=Gordon Aquifer 


\section{Hydrograph \\ Well Cluster FSB114}

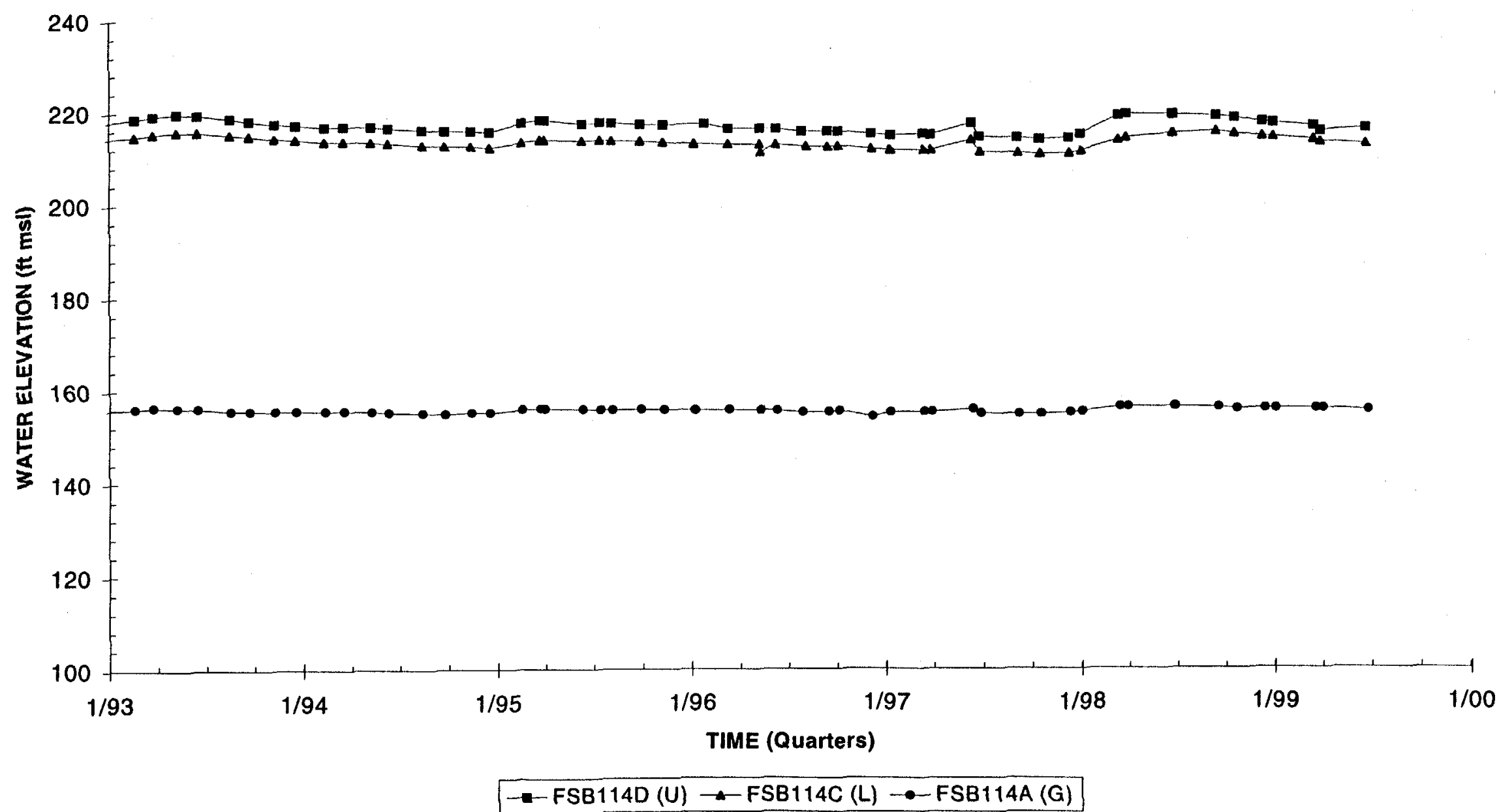

Note: U=Upper Aquifer Zone of the Upper Three Runs Aquifer; L=Lower Aquifer Zone of the Upper Three Runs Aquifer; G=Gordon Aquifer 


\section{Hydrograph \\ Well Cluster FSB115}

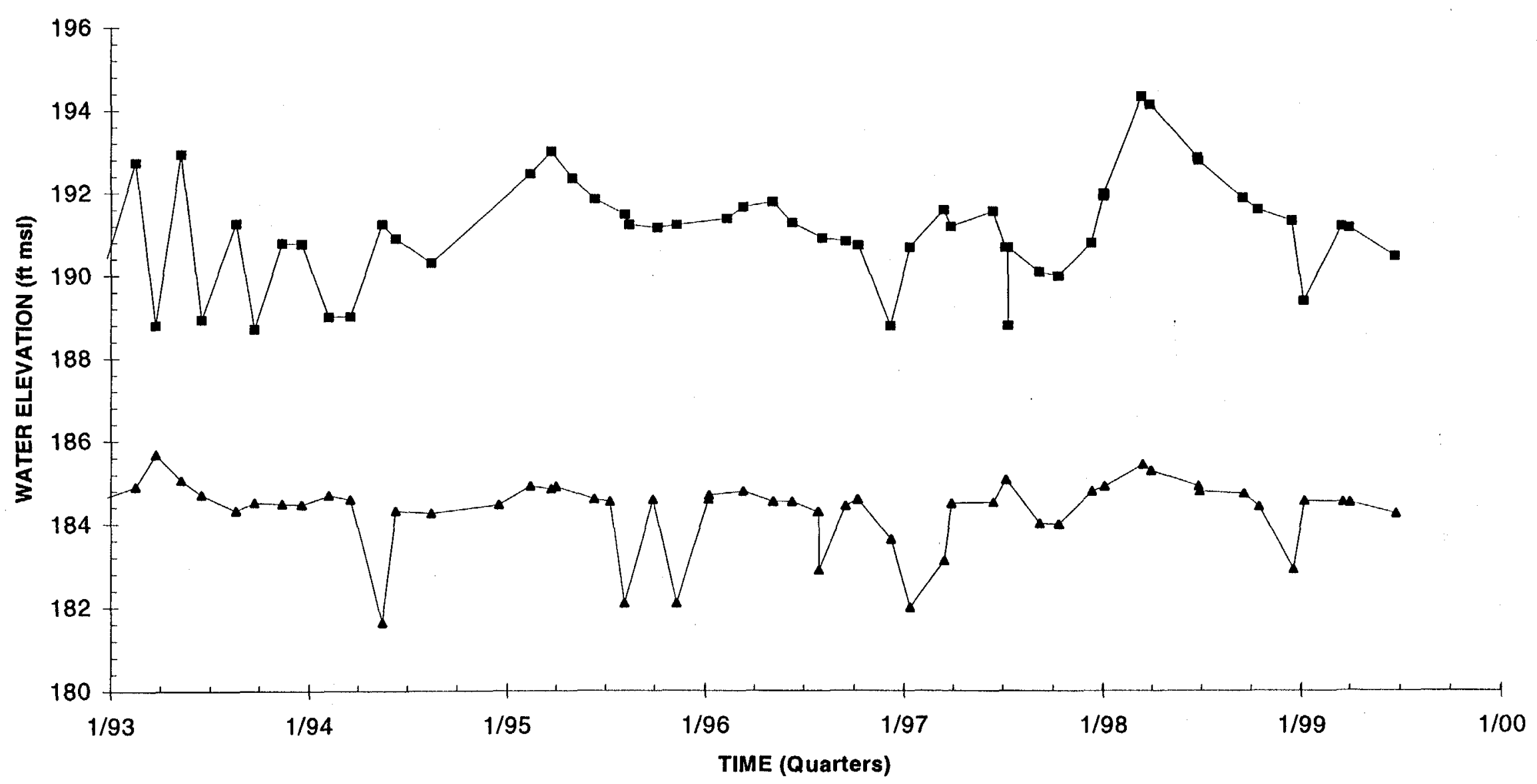

$\rightarrow-F S B 115 D(U) \rightarrow$ FSB115C (L)

Note: $U=U p p e r$ Aquifer Zone of the Upper Three Runs Aquifer; L=Lower Aquifer Zone of the Upper Three Runs Aquifer; G=Gordon Aquifer 


\section{Hydrograph \\ Well Cluster FSB116}

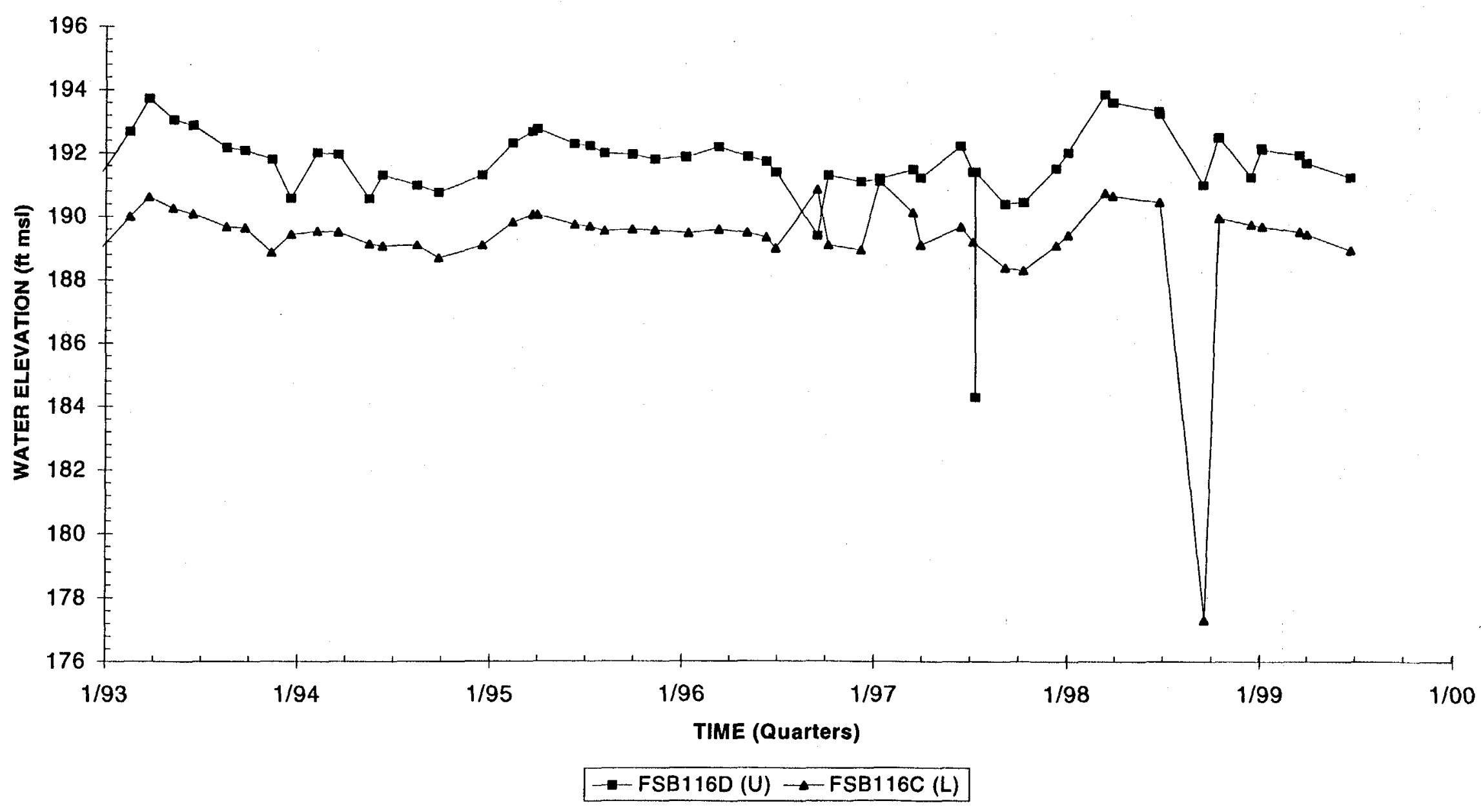

Note: U=Upper Aquifer Zone of the Upper Three Runs Aquifer; L=Lower Aquifer Zone of the Upper Three Runs Aquifer; G=Gordon Aquifer 


\section{Hydrograph \\ Well FSB117D}

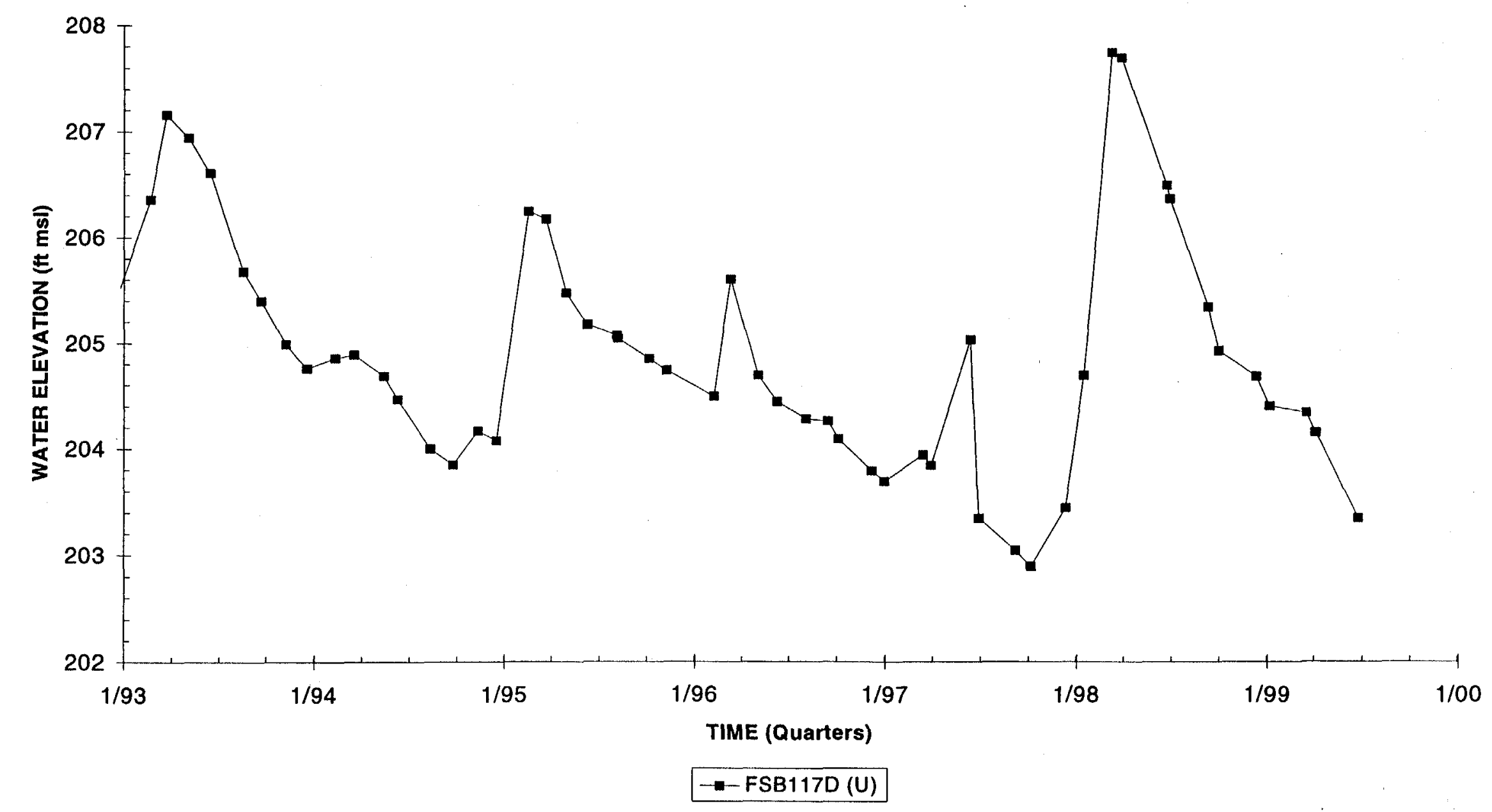

Note: U=Upper Aquifer Zone of the Upper Three Runs Aquifer; L=Lower Aquifer Zone of the Upper Three Runs Aquifer; G=Gordon Aquifer 


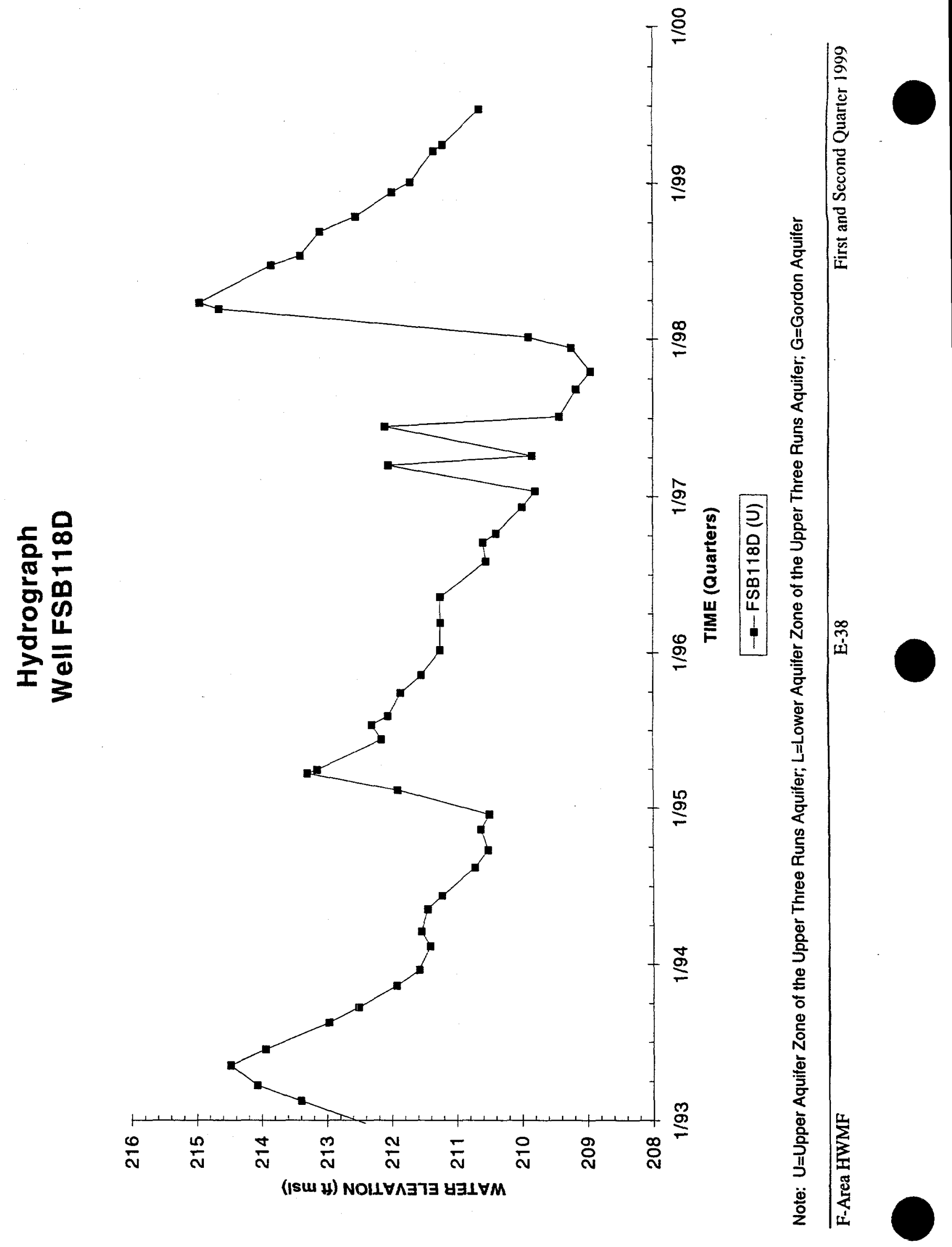

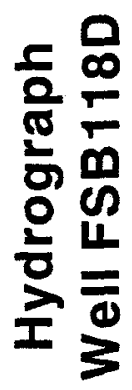




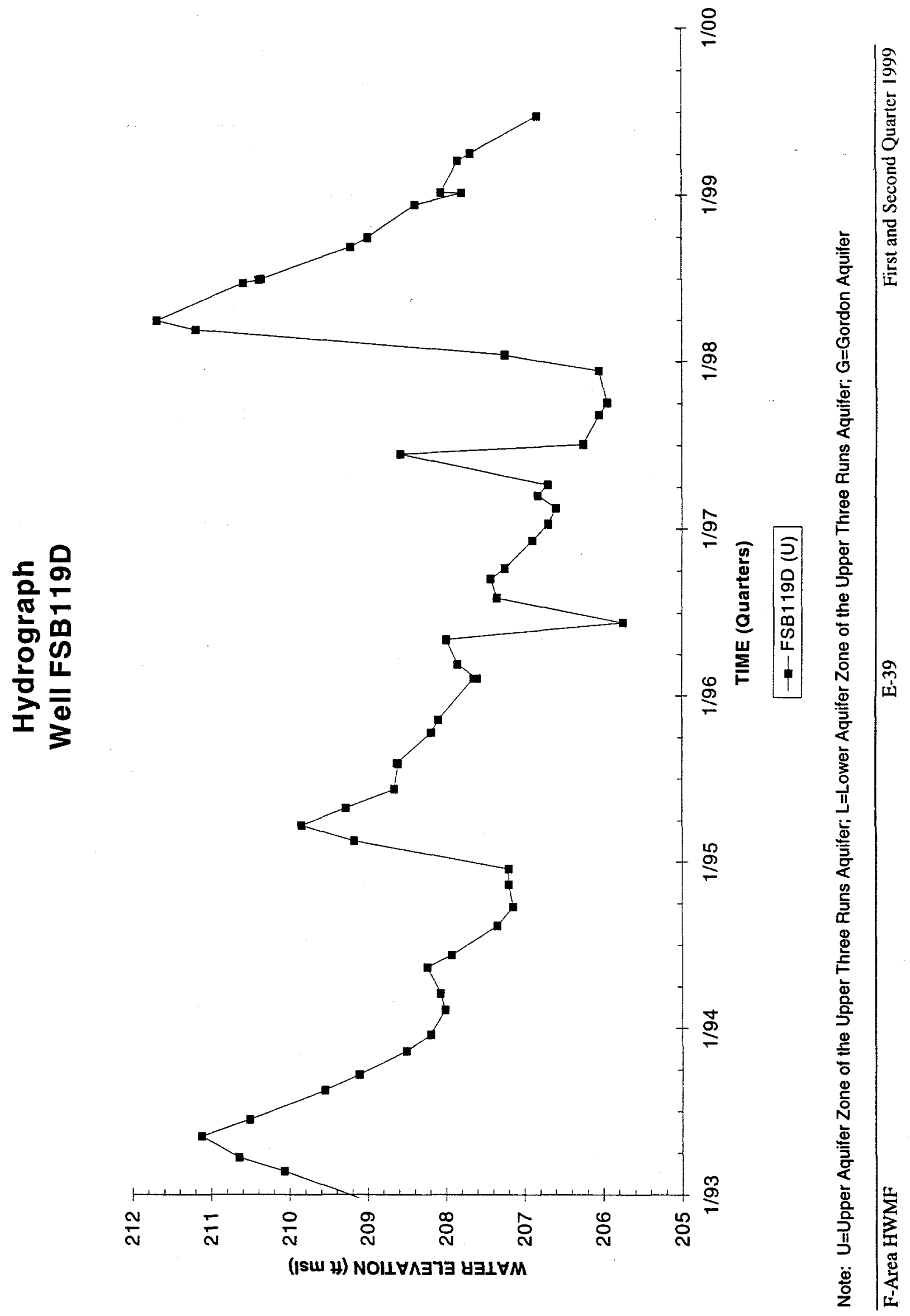




\section{Hydrograph \\ Well Cluster FSB120}

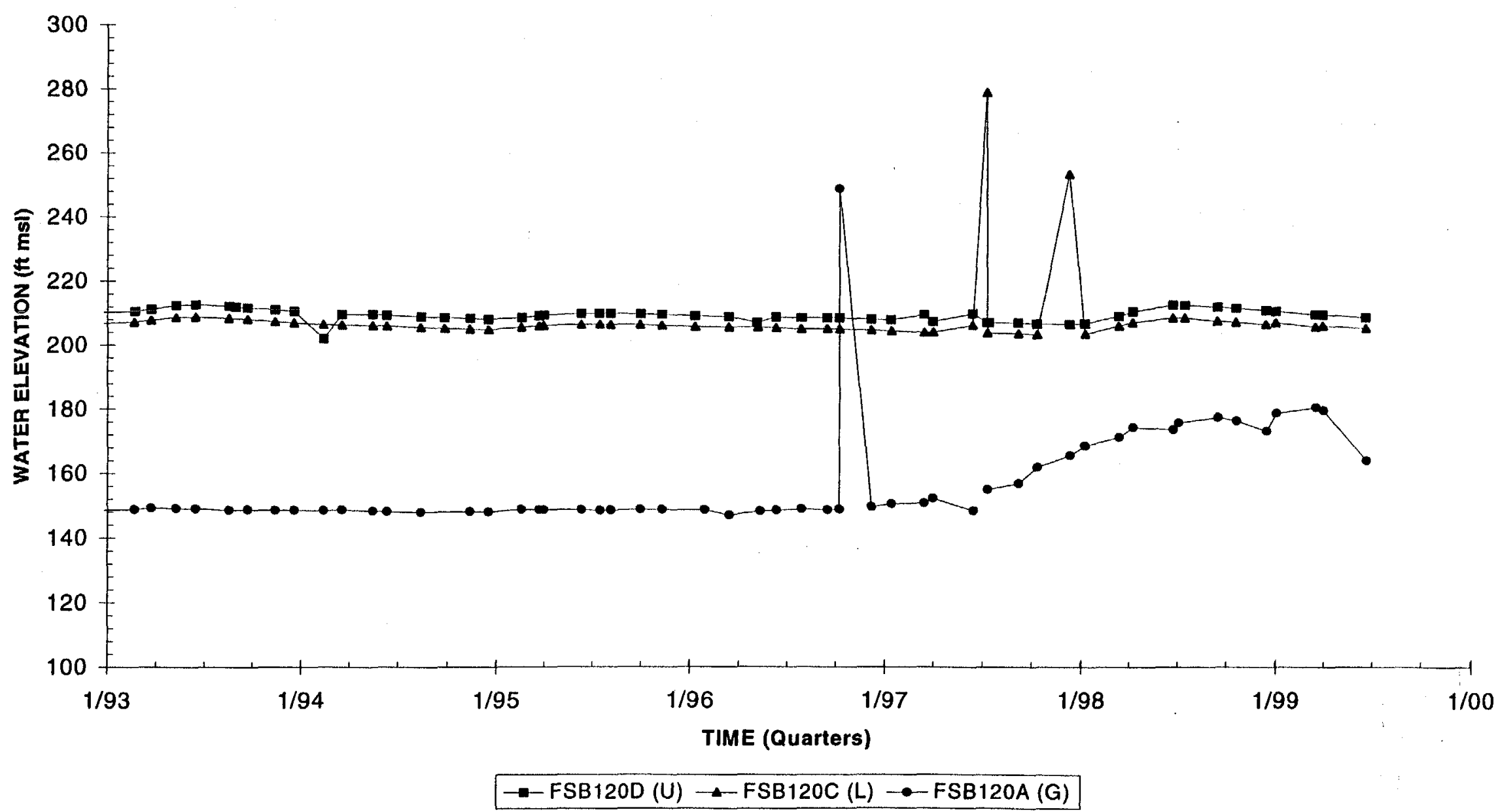

Note: U=Upper Aquifer Zone of the Upper Three Runs Aquifer; L=Lower Aquifer Zone of the Upper Three Runs Aquifer; G=Gordon Aquifer 


\section{Hydrograph \\ Well Cluster FSB121}

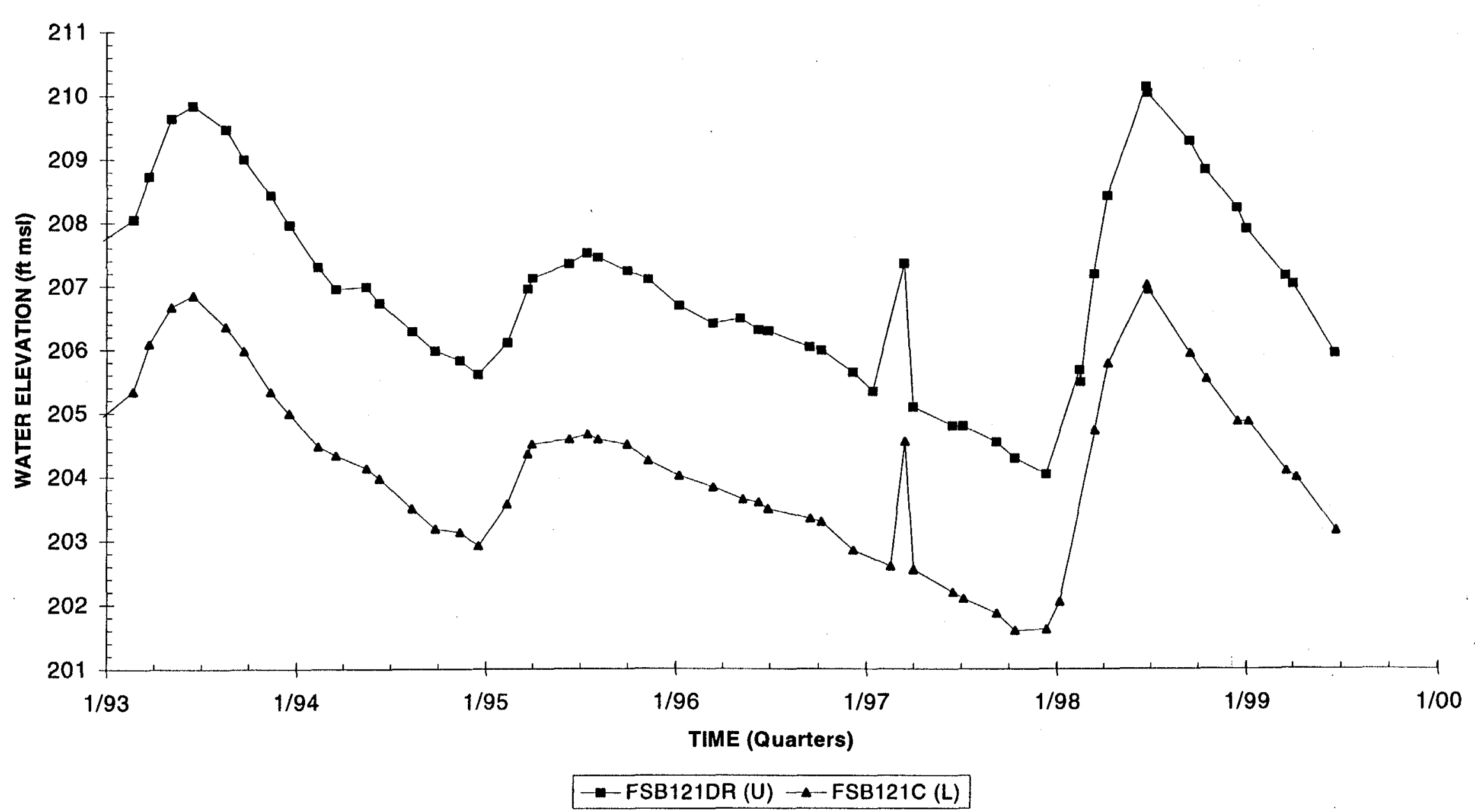

Note: U=Upper Aquifer Zone of the Upper Three Runs Aquifer; L=Lower Aquifer Zone of the Upper Three Runs Aquifer; G=Gordon Aquifer 


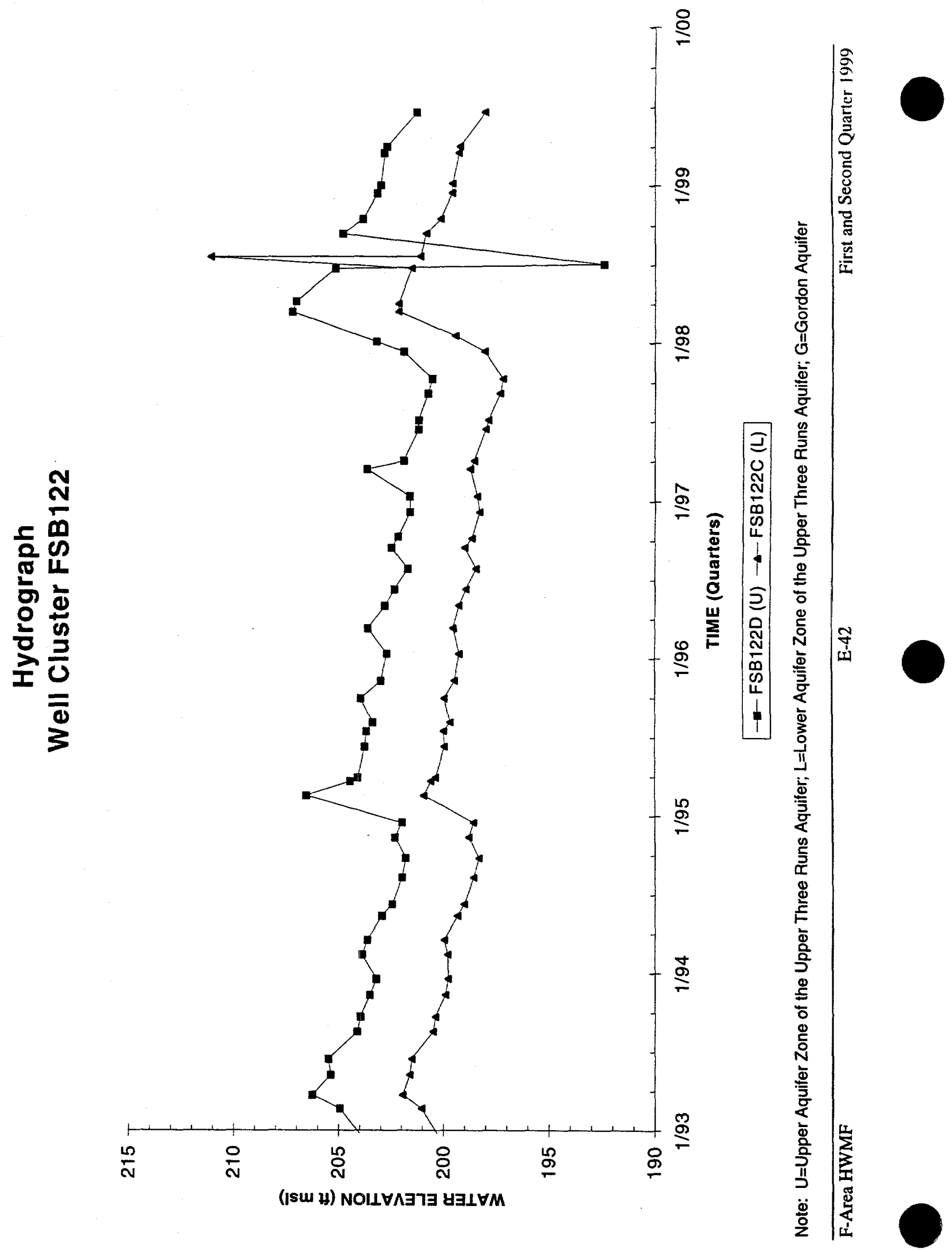




\section{Hydrograph \\ Well Cluster FSB123}

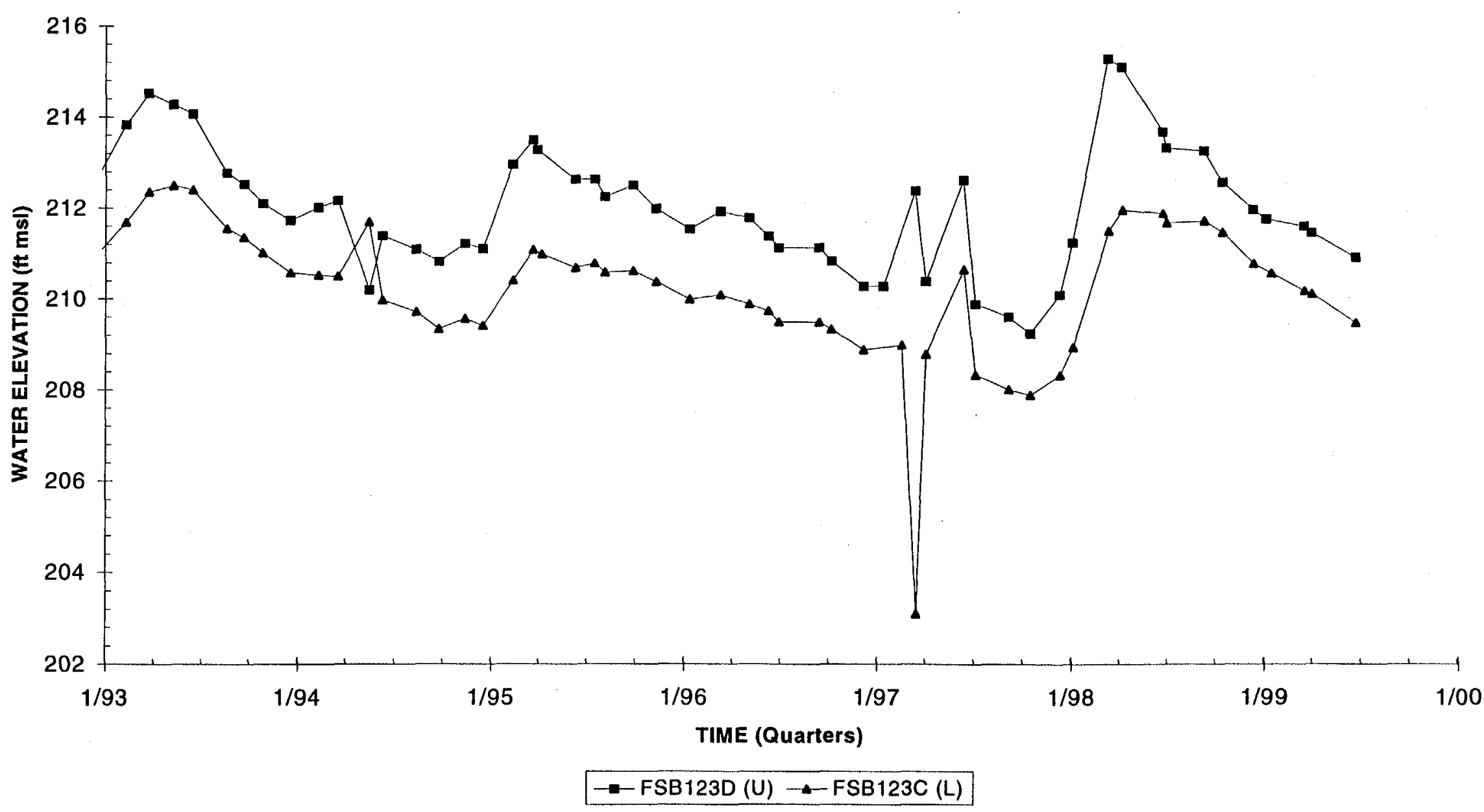

Note: U=Upper Aquifer Zone of the Upper Three Runs Aquifer; L=Lower Aquifer Zone of the Upper Three Auns Aquifer; G=Gordon Aquifer 


\section{Hydrograph}

Well FSL 1D

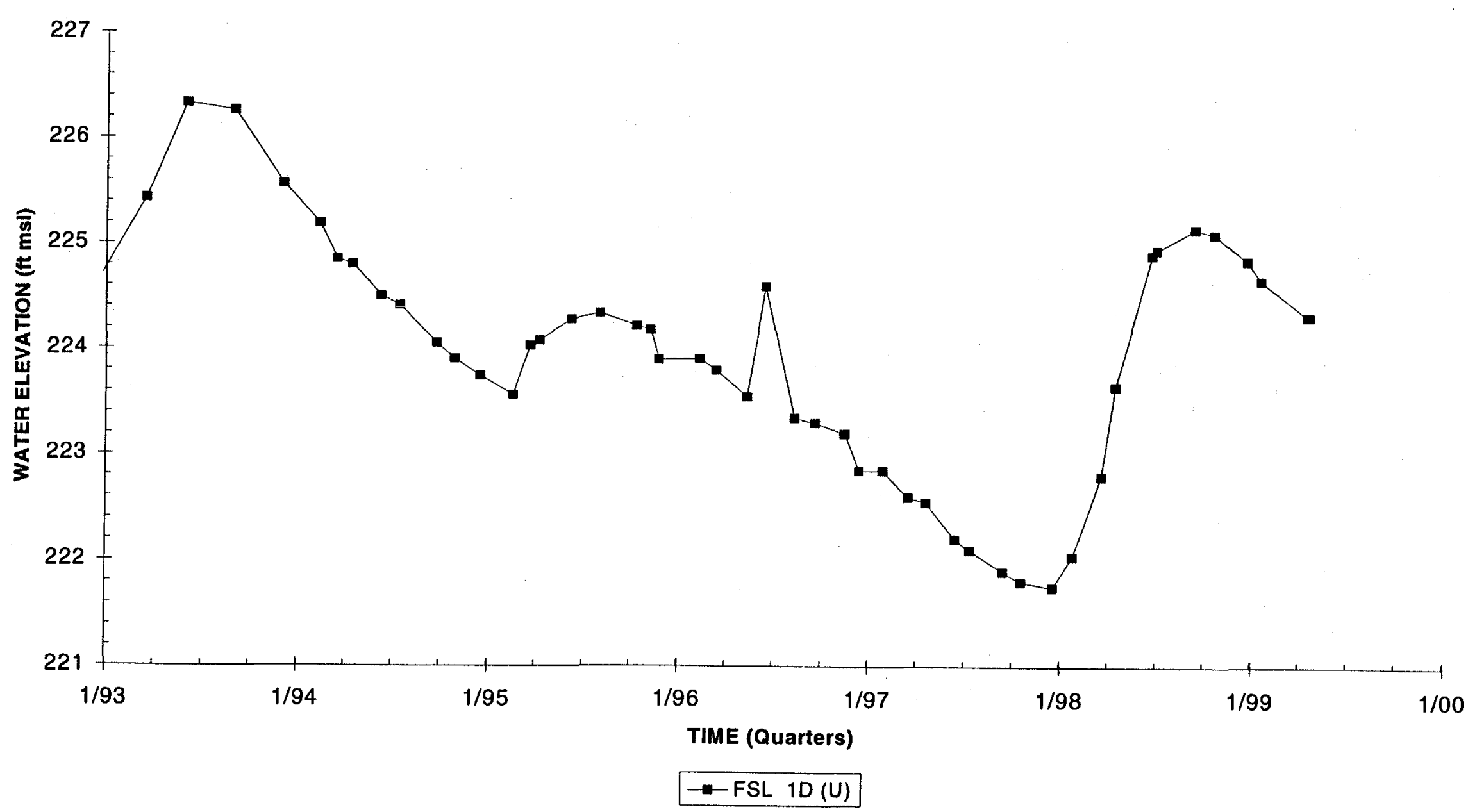

Note: U=Upper Aquifer Zone of the Upper Three Runs Aquifer; L=Lower Aquifer Zone of the Upper Three Runs Aquifer; G=Gordon Aquifer 


\section{Hydrograph \\ Well FSL 2D}

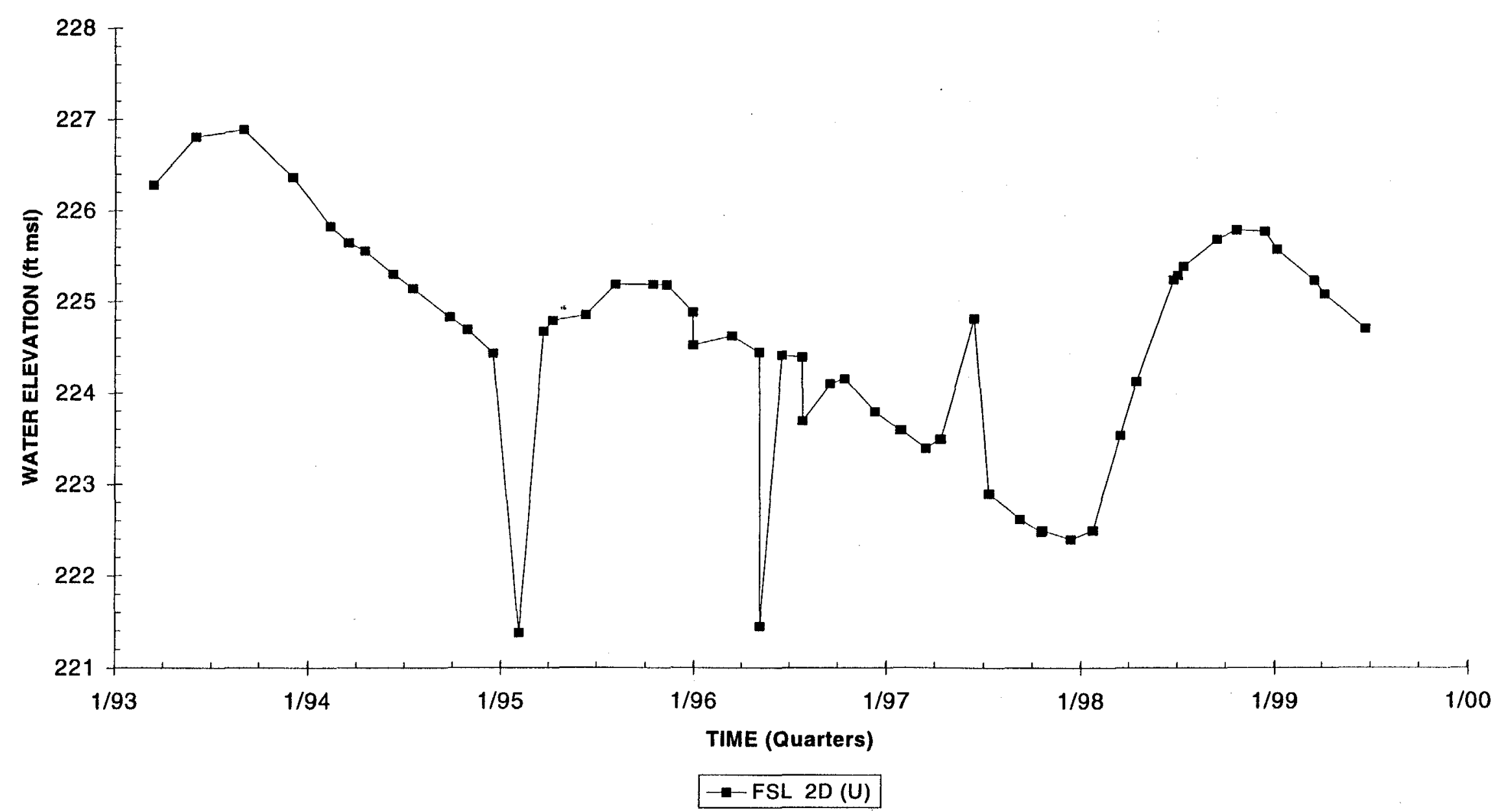

Note: U=Upper Aquifer Zone of the Upper Three Runs Aquifer; L=Lower Aquifer Zone of the Upper Three Runs Aquifer; G=Gordon Aquifer 

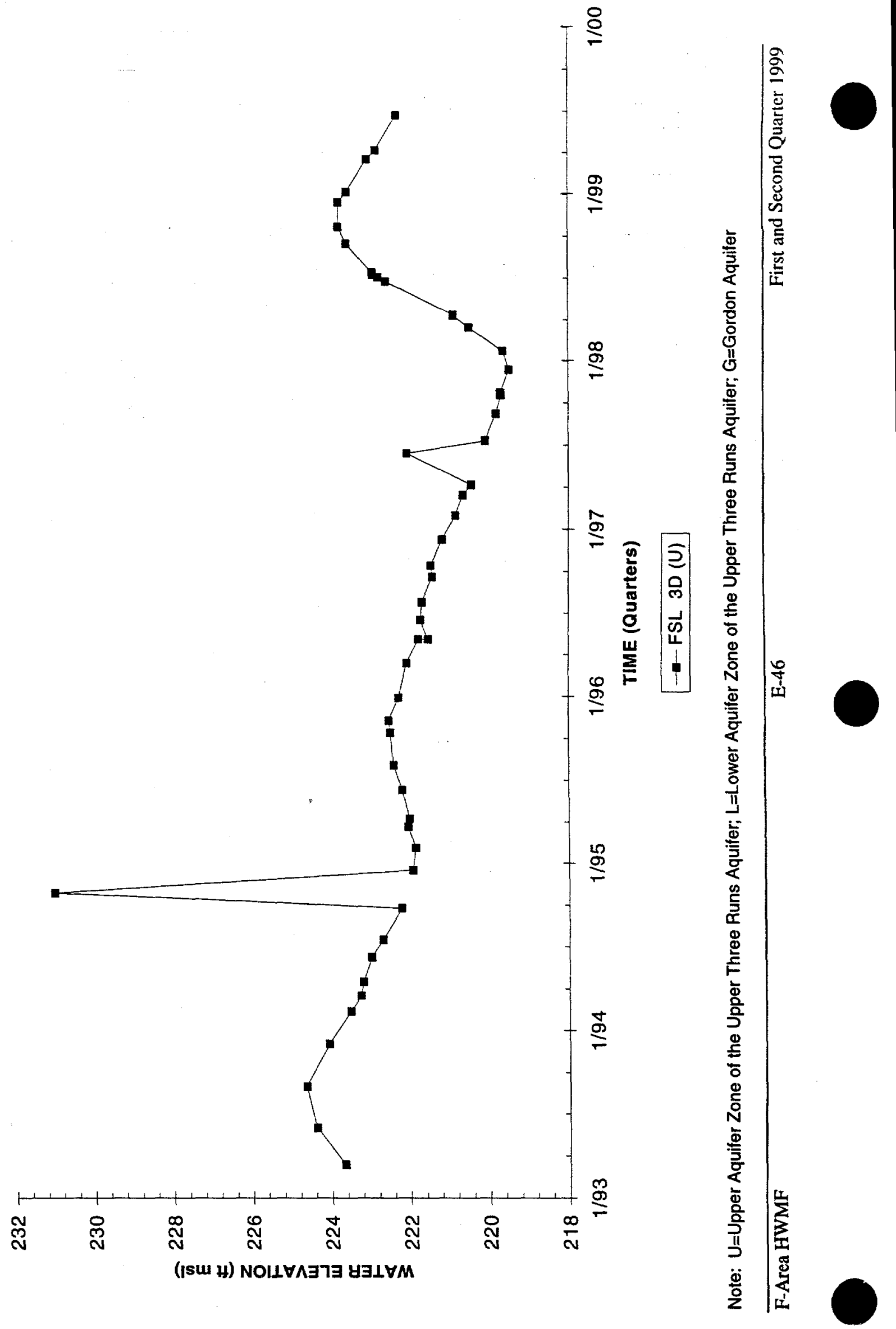


\section{Hydrograph \\ Well FSL 4D}

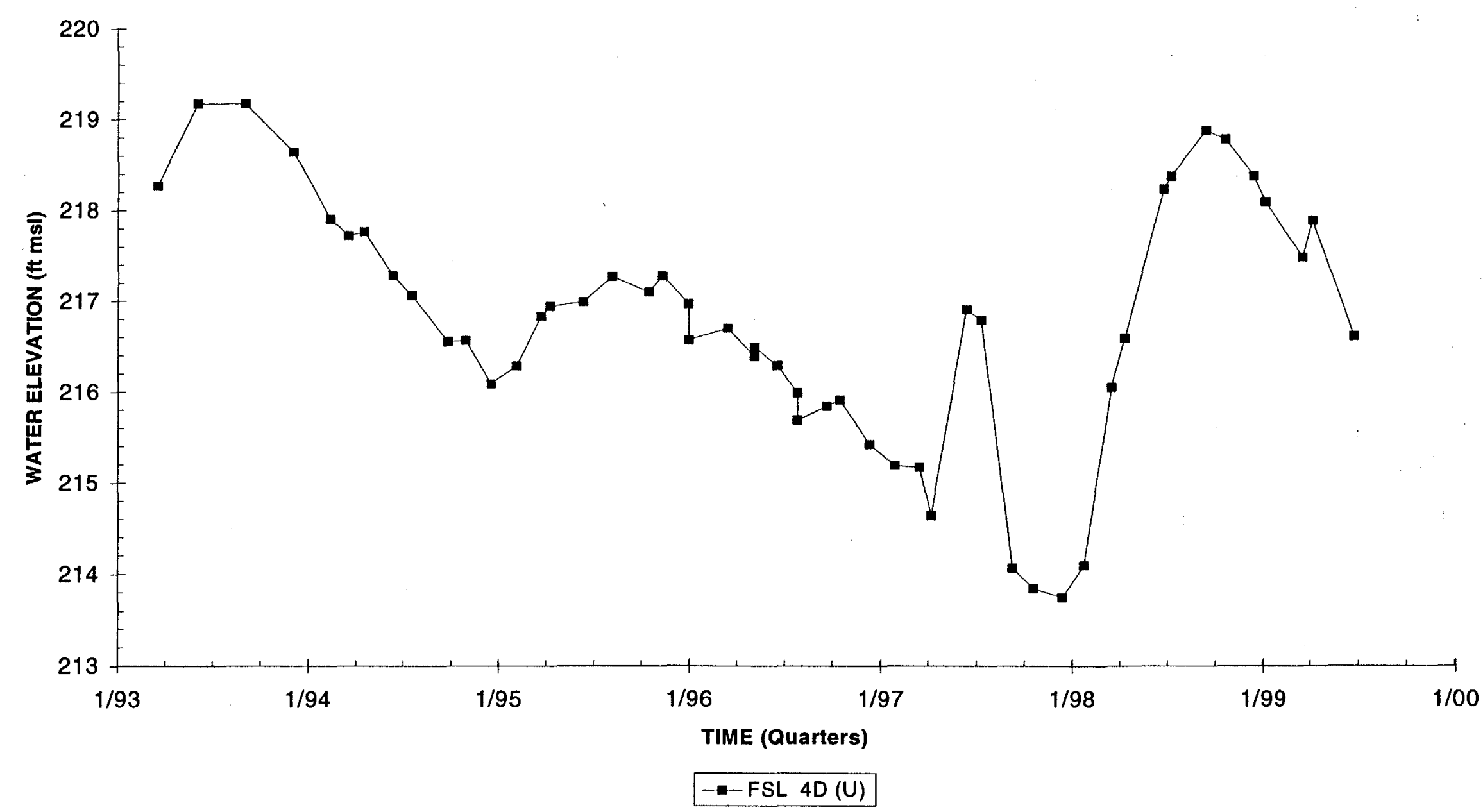

Note: U=Upper Aquifer Zone of the Upper Three Runs Aquifer; L=Lower Aquifer Zone of the Upper Three Runs Aquifer; G=Gordon Aquifer 


\section{Hydrograph}

Well FSL 5D

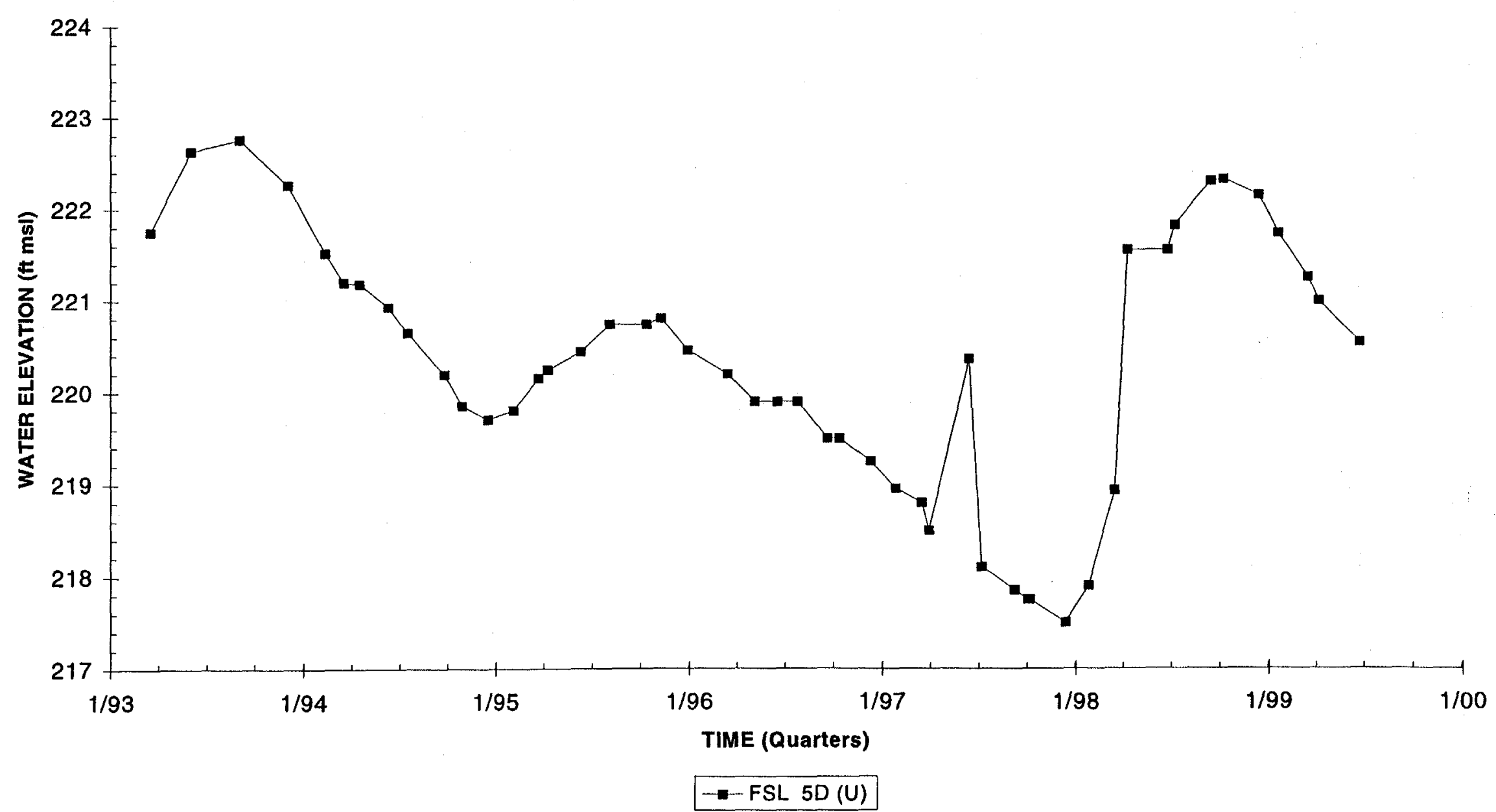

Note: U=Upper Aquifer Zone of the Upper Three Runs Aquifer; L=Lower Aquifer Zone of the Upper Three Runs Aquifer; G=Gordon Aquifer 

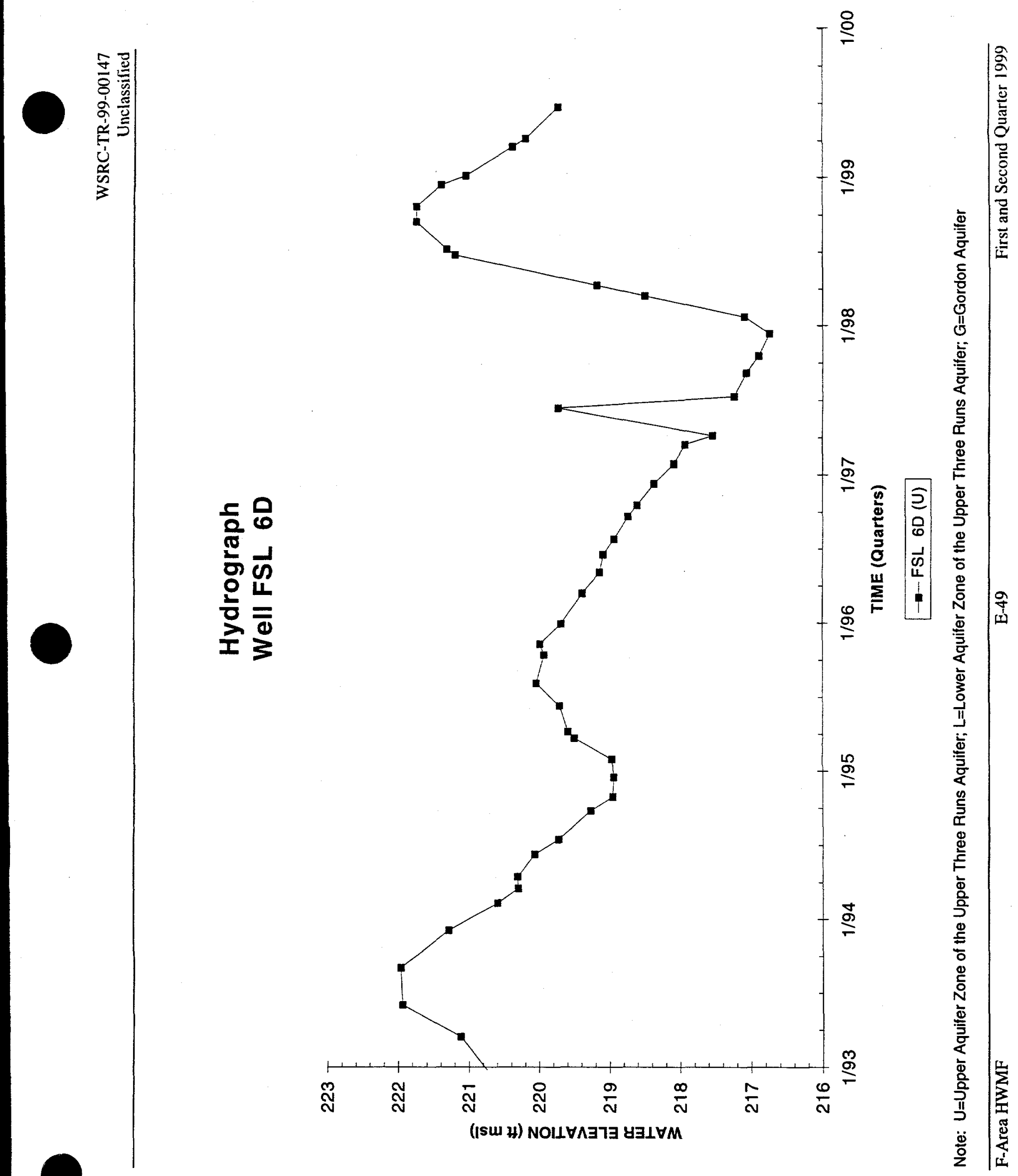


\section{Hydrograph}

Well FSL 7D

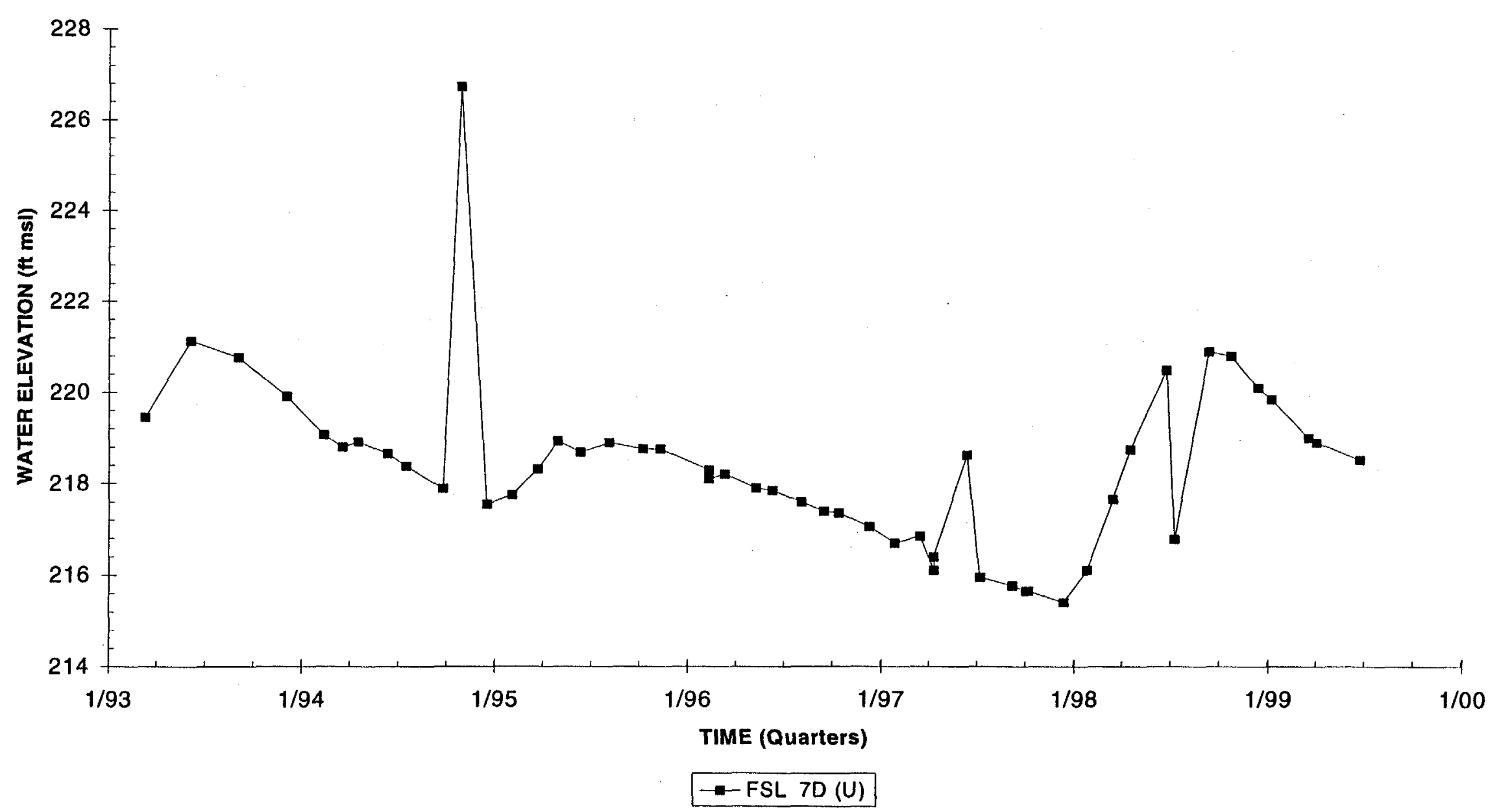

Note: $U=$ Upper Aquifer Zone of the Upper Three Runs Aquifer; L=Lower Aquifer Zone of the Upper Three Runs Aquifer; G=Gordon Aquifer 


\section{Hydrograph \\ Well FSL 8D}

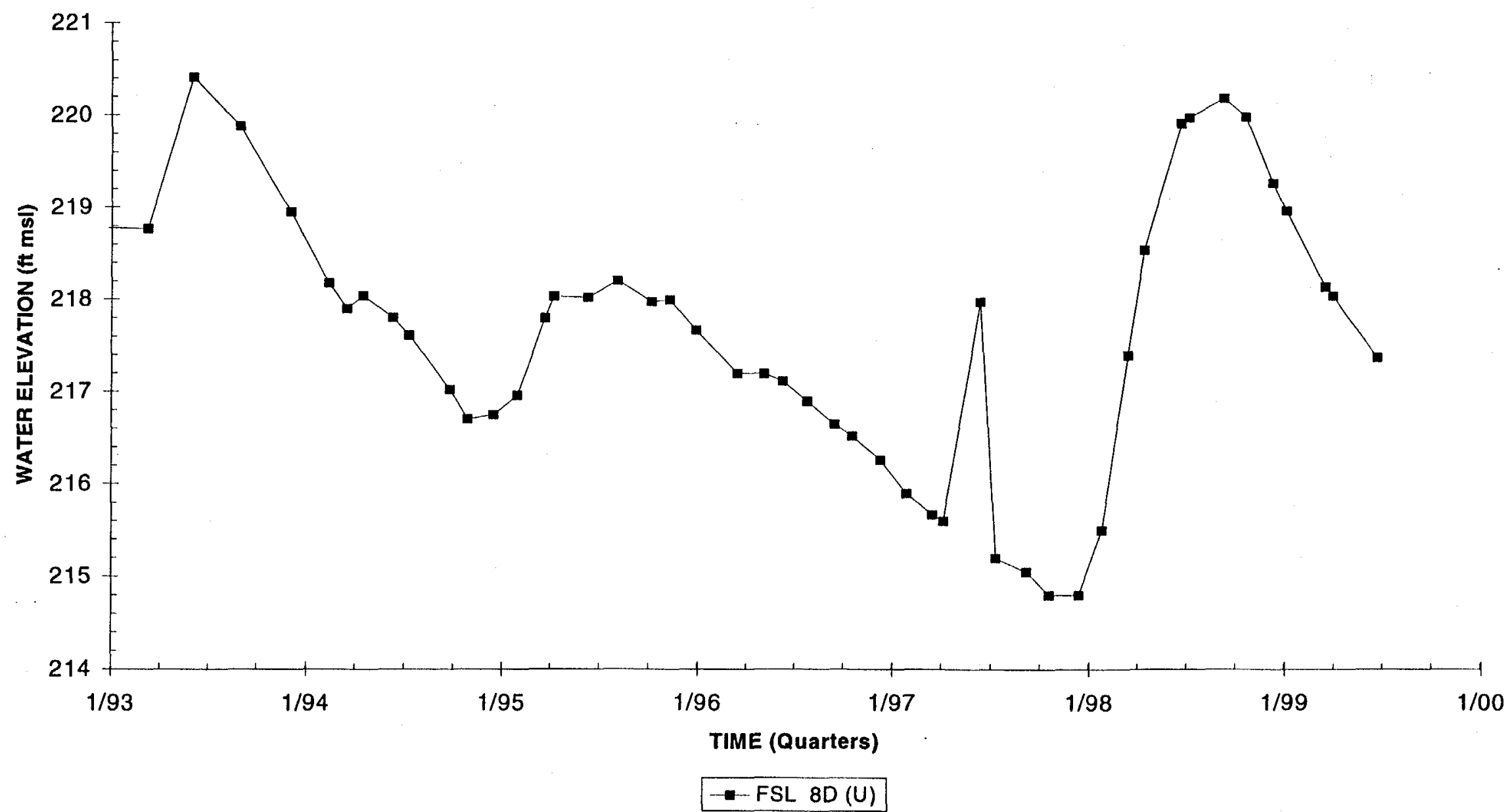

Note: U=Upper Aquifer Zone of the Upper Three Runs Aquifer; L=Lower Aquifer Zone of the Upper Three Runs Aquifer; G=Gordon Aquifer 


\section{Hydrograph}

Well FSL 9D

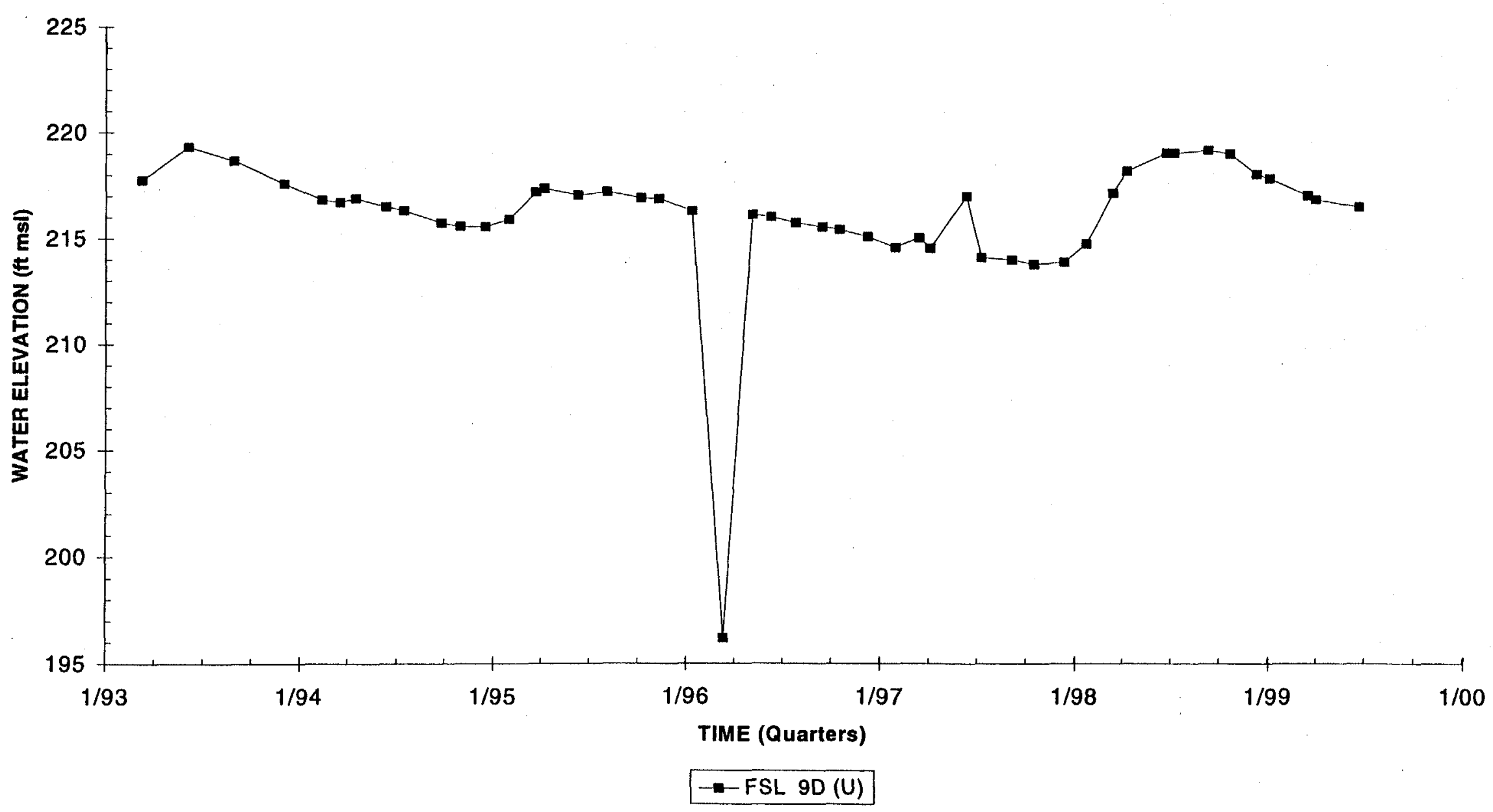

Note: U=Upper Aquifer Zone of the Upper Three Runs Aquifer; L=Lower Aquifer Zone of the Upper Three Runs Aquifer; G=Gordon Aquifer 


\section{Hydrograph}

Well HSB 85A

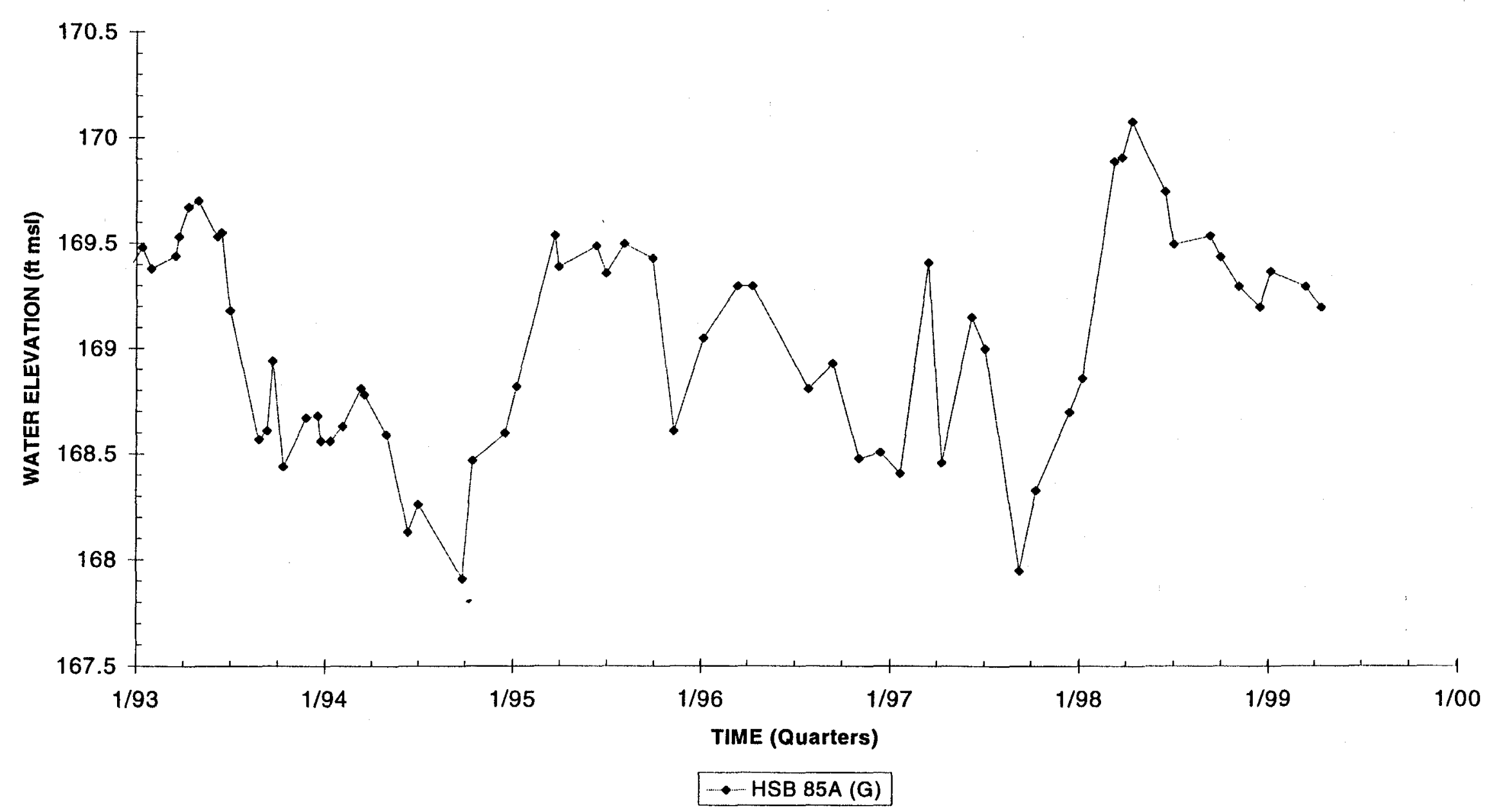

Note: U=Upper Aquifer Zone of the Upper Three Runs Aquifer; L=Lower Aquifer Zone of the Upper Three Runs Aquifer; G=Gordon Aquifer 
THIS PAGE WAS LEFT BLANK INTENTIONALLY. 
APPENDIX F: UIC SAMPLING RESULTS 
F-Area WTU UIC Sample Results: January-June 1999

\begin{tabular}{|c|c|c|c|c|c|c|c|c|}
\hline UIC Permitted Constituents & & Reg & Jan-99 & Feb-99 & Mar-99 & Apr-99 & May-99 & Jun-99 \\
\hline Constituent & Unit & Limit & Result & Result & Result & Result & Result & Result \\
\hline \multicolumn{9}{|l|}{ Section I, INORGANICS } \\
\hline Antimony & $\mu \mathrm{g} / \mathrm{L}$ & 6 & $<0.2$ & $<0.2$ & $<0.2$ & $<0.2$ & $<2$ & $<2$ \\
\hline Arsenic & $\mu \mathrm{g} / \mathrm{L}$ & 50 & $<3$ & $<3$ & $<3$ & $<3$ & $<3$ & $<3$ \\
\hline Barium & $\mu g / L$ & 2000 & 14.80 & 14.20 & 4.93 & 8.84 & 6.88 & 6.74 \\
\hline Cadmium & $\mu \mathrm{g} / \mathrm{L}$ & 5 & $<1$ & $<1$ & $<1$ & $<1$ & $<1$ & $<1$ \\
\hline Chromium & $\mu \mathrm{g} / \mathrm{L}$ & 100 & $<3$ & $<3$ & $<3$ & $<3$ & $<3$ & 3.42 \\
\hline Cobalt & $\mu g / L$ & 140 & 3.54 & 7.52 & 2.00 & 3.57 & 2.81 & 2.50 \\
\hline Copper & $\mu \mathrm{g} / \mathrm{L}$ & 1300 & 1.94 & 6.96 & 5.02 & 2.52 & 3.28 & 1.68 \\
\hline Cyanide & $\mu \mathrm{g} / \mathrm{L}$ & 200000 & $<10$ & $<10$ & $<10$ & $<10$ & $<10$ & $<10$ \\
\hline Lead & $\mu g / L$ & 50 & $<2$ & $<2$ & $<2$ & $<2$ & $<2$ & $<2$ \\
\hline Mercury & $\mu \mathrm{g} / \mathrm{L}$ & 2 & $<0.2$ & $<0.2$ & $<0.2$ & 0.24 & $<0.2$ & 0.28 \\
\hline Nickel & $\mu g / L$ & 100 & 1.78 & 2.33 & $<2$ & $<2$ & $<2$ & 3.47 \\
\hline Selenium & $\mu \mathrm{g} / \mathrm{L}$ & 50 & $<5$ & $<5$ & $<5$ & $<5$ & $<5$ & $<5$ \\
\hline Silver & $\mu g / L$ & 50 & $<1$ & $<1$ & $<1$ & $<1$ & $<1$ & $<1$ \\
\hline Thallium & $\mu \mathrm{g} / \mathrm{L}$ & 2 & $<2.5$ & $<2.5$ & $<2.5$ & $<2.5$ & $<0.5$ & $<0.5$ \\
\hline Vanadium & $\mu g / L$ & 49 & $<2$ & $<2$ & $<10$ & 4100 & $<10$ & $<10$ \\
\hline Zinc & $\mu g / L$ & 5000 & $<5$ & $<5$ & $<10$ & 26.90 & $<10$ & $<10$ \\
\hline \multicolumn{9}{|l|}{ Section II, ORGANICS } \\
\hline Benzene & $\mu \mathrm{g} / \mathrm{L}$ & 5 & $<1$ & $<1$ & $<1$ & $<1$ & $<1$ & $<1$. \\
\hline B2EHP & $\mu \mathrm{g} / \mathrm{L}$ & 140 & $<10.8$ & $<10$ & $<10$ & $<9.9$ & $<11.1$ & $<10$ \\
\hline Methylene Chloride & $\mu \mathrm{g} / \mathrm{L}$ & 5 & $<5$ & $<5$ & $<5$ & $<5$ & $<5$ & $<5$ \\
\hline Phenol & $\mu \mathrm{g} / \mathrm{L}$ & 10 & $<5$ & $<5$ & $<5$ & $<5$ & $<5$ & $<5$ \\
\hline Tetrachloroethylene & $\mu \mathrm{g} / \mathrm{L}$ & 5 & $<1$ & $<1$ & $<1$ & $<1$ & $<1$ & $<1$ \\
\hline Trichloroethylene & $\mu \mathrm{g} / \mathrm{L}$ & 5 & $<1$ & $<1$ & $<1$ & $<1$ & $<1$ & $<1$ \\
\hline Trichlorofluoromethane & $\mu \mathrm{g} / \mathrm{L}$ & 100 & $<5$ & $<5$ & $<5$ & $<1$ & $<5$ & $<5$ \\
\hline
\end{tabular}


F-Area WTU UIC Sample Results: January-June 1999, cont.

\begin{tabular}{|c|c|c|c|c|c|c|c|c|}
\hline UIC Permitted Constituents & & Reg & Jan-99 & Feb-99 & Mar-99 & Apr-99 & May-99 & Jun-99 \\
\hline Constituent & Unit & Limit & Result & Result & Result & Result & Result & Result \\
\hline \multicolumn{9}{|l|}{ Section III, RADIONUCLIDES } \\
\hline Americium-241 & $\mathrm{pCi} / \mathrm{L}$ & $<15$ & $<1.096$ & $<2.934$ & $<2.624$ & 1.73 & $<1.54$ & $<2.11$ \\
\hline Cesium-137 & $\mathrm{pCi} / \mathrm{L}$ & $<50$ & $<7.41$ & $<7.35$ & $<7.7$ & $<5.71$ & $<7.42$ & $<8.03$ \\
\hline Curium-242 & $\mathrm{pCi} / \mathrm{L}$ & $<15$ & $<0.587$ & $<2.332$ & $<2.098$ & $<0.553$ & $<0.944$ & $<0.952$ \\
\hline Curium-243/244 & $\mathrm{pCi} / \mathrm{L}$ & $<15$ & $<0.81$ & $<4.3$ & $<0.929$ & $<1.96$ & $<2.1$ & $<2.51$ \\
\hline Curium-246 & $\mathrm{pCi} / \mathrm{L}$ & $<15$ & $<0.286$ & $<1.198$ & $<0.878$ & $<1.03$ & $<0.378$ & $<1.3$ \\
\hline Carbon-14 & $\mathrm{pCi} / \mathrm{L}$ & $<150$ & $<50$ & $<22.04$ & $<39.2$ & 61.90 & $<23.6$ & 18.80 \\
\hline Cobalt-60 & $\mathrm{pCi} / \mathrm{L}$ & $<50$ & $<5.21$ & $<7.68$ & $<8.96$ & $<6.47$ & $<8.1$ & $<7.71$ \\
\hline lodine-129 & $\mathrm{pCi} / \mathrm{L}$ & $<60$ & 10000 & 45.80 & 36.00 & 39.30 & 6.99 & 36.90 \\
\hline Plutonium-238 & $\mathrm{pCi} / \mathrm{L}$ & $<15$ & $<0.448$ & $<0.678$ & $<1.512$ & $<1.4$ & $<6.33$ & $<0.321$ \\
\hline Plutonium-239/240 & $\mathrm{pCi} / \mathrm{L}$ & $<15$ & $<0.254$ & $<0.549$ & $<0.953$ & $<1.76$ & $<1.86$ & $<0.321$ \\
\hline Radium-226 & $\mathrm{pCi} / \mathrm{L}$ & TR $<25$ & $<1.307$ & $<2.145$ & $<2.403$ & $<1.45$ & $<2.25$ & 1.29 \\
\hline Radium-228 & $\mathrm{pCi} / \mathrm{L}$ & TR $<25$ & $<2.696$ & $<1.897$ & $<1.994$ & $<1.79$ & $<1.87$ & $<1.97$ \\
\hline Strontium-90 & $\mathrm{pCi} / \mathrm{L}$ & $<170$ & 5.38 & 4.75 & $<2.682$ & 3.65 & 5.49 & $<6.85$ \\
\hline Technetium-99 & $\mathrm{pCi} / \mathrm{L}$ & $<170$ & $<44.4$ & 16.00 & $<12.16$ & 12.20 & $<11.3$ & 27.10 \\
\hline Thorium-228 & $\mathrm{pCi} / \mathrm{L}$ & $<15$ & $<2.34$ & $<2.418$ & $<2.586$ & $<1.92$ & $<8.45$ & $<1.74$ \\
\hline Thorium-230 & $\mathrm{pCi} / \mathrm{L}$ & $<15$ & $<1.148$ & $<1.133$ & $<2.057$ & $<0.781$ & $<3.67$ & $<0.707$ \\
\hline Thorium-232 & $\mathrm{pCi} / \mathrm{L}$ & $<15$ & $<0.222$ & $<1.062$ & $<1.752$ & $<0.467$ & $<0.918$ & $<0.429$ \\
\hline Uranium-233/234 & $\mathrm{pCi} / \mathrm{L}$ & $<25$ & 3.18 & 4.57 & 3.78 & $<6.1$ & $<5.89$ & 18.00 \\
\hline Uranium-235 & $\mathrm{pCi} / \mathrm{L}$ & $<15$ & $<0.819$ & $<1.739$ & $<0.966$ & 9.43 & $<3.81$ & 2.17 \\
\hline Uranium-238 & $\mathrm{pCi} / \mathrm{L}$ & $<50$ & 6.21 & 9.27 & 4.00 & 6.41 & $<4.97$ & 31.90 \\
\hline
\end{tabular}




\section{F-Area WTU UIC Sample Results: January 1999}

\begin{tabular}{|c|c|c|c|c|c|c|c|c|}
\hline \multicolumn{2}{|c|}{ UIC Permitted Constituents } & \multirow{2}{*}{\begin{tabular}{|l|} 
Reg \\
Limit
\end{tabular}} & \multirow{2}{*}{$\begin{array}{c}\text { Raw } \\
\text { Result }\end{array}$} & \multirow{2}{*}{$\frac{\text { Rad }}{\text { Acc }}$} & \multirow{2}{*}{ Qual. } & \multirow[b]{2}{*}{$\mathbf{Q L}$} & \multirow{2}{*}{$\begin{array}{c}\text { January } \\
\text { Result }\end{array}$} & \multirow{2}{*}{$\begin{array}{c}\text { Exceedence } \\
\mathrm{Y} / \mathrm{N}\end{array}$} \\
\hline Constituent & Unit & & & & & & & \\
\hline Section I, INORGANICS & & 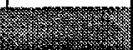 & 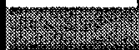 & & & & 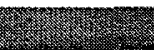 & 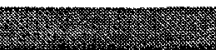 \\
\hline Antimony & $\mu g / L$ & 6 & 0.2 & & $\mathrm{U}$ & 0.2 & $<0.2$ & $\mathrm{~N}$ \\
\hline Arsenic & $\mu g h$ & 50 & 3 & & $\mathrm{U}$ & 3 & $<3$ & $\mathrm{~N}$ \\
\hline Barium & $\mu g / L$ & 2000 & 14.8 & & & 0.2 & 14.80 & $\mathbf{N}$ \\
\hline Cadmium & $\mu g / L$ & 5 & 0.254 & & Jl & 1 & $<1$ & $\mathbf{N}$ \\
\hline Chromium & $\mu g / L$ & 100 & 3 & & $\mathrm{U}$ & 3 & $<3$ & $\mathbf{N}$ \\
\hline Cobalt & $\mu g / L$ & 140 & 3.54 & & & 0.2 & 3.54 & $\mathbf{N}$ \\
\hline Copper & $\mu g / \mathrm{L}$ & 1300 & 1.94 & & & 0.2 & 1.94 & $N$ \\
\hline Cvanide & $\mu g / L$ & 200000 & 10 & & $\bar{U}$ & 10 & $<10$ & $\mathrm{~N}$ \\
\hline Lead & $\mu g / L$ & 50 & 2 & & $U$ & 2 & $<2$ & $\mathbf{N}$ \\
\hline Mercury & $\mu g / L$ & 2 & 0.2 & & $U$ & 0.2 & $<0.2$ & $\mathrm{~N}$ \\
\hline Nickel & $\mu g / L$ & 100 & 1.78 & & & 0.2 & 1.78 & $\mathrm{~N}$ \\
\hline Selenium & $\mu g / L$ & 50 & 5 & & $U$ & 5 & $<5$ & $\mathrm{~N}$ \\
\hline Silver & $\mu g / L$ & 50 & 1 & & $\bar{U}$ & 1 & $<1$ & $\mathbf{N}$ \\
\hline Thallium ${ }^{*}$ & $\mu g / L$ & 2 & 0.492 & & JIV & 2.5 & 2.5 & \\
\hline Vanadium & $\mu g /$ & 49 & 2 & & $\mathrm{U}$ & 2 & $<$ & $N$ \\
\hline Zinc & $\mu g / L$ & 5000 & 5 & & $U$ & 5 & $<5$ & $N$ \\
\hline \multicolumn{9}{|l|}{ Section II, ORGANICS } \\
\hline Benzene & $\mu g / L$ & 5 & 1 & & $U$ & 1 & $<1$ & $\mathrm{~N}$ \\
\hline B2EHP & $\mu g / L$ & 140 & 10.8 & & $\mathrm{U}$ & 10.8 & $<10.8$ & $\mathbf{N}$ \\
\hline Methylene Chloride & $\mu g / L$ & 5 & 5.41 & & UV & 5 & $<5$ & $N$ \\
\hline Phenol & $\mu g / L$ & 10 & 5 & & $\mathrm{U}$ & 5 & $<5$ & $\mathbf{N}$ \\
\hline Tetrachloroethylene & $\mu \mathrm{g} / \mathrm{L}$ & 5 & 1 & & $\mathrm{U}$ & 1 & $<1$ & $\mathbf{N}$ \\
\hline Trichloroethylene & $\mu g / L$ & 5 & 1 & & $U$ & 1 & $<1$ & $N$ \\
\hline Trichlorofluoromethane & $\mu \mathrm{g} / \mathrm{L}$ & 100 & 5 & & $U$ & 5 & $<5$ & $\mathbf{N}$ \\
\hline \multicolumn{9}{|c|}{ Section III, RADIONUCLIDES } \\
\hline Americium-241 & $\mathrm{pCi} i \mathrm{~L}$ & $<15$ & 0.821 & 0.377 & $\mathrm{JI}$ & 1.096 & $<1.096$ & $\mathrm{~N}$ \\
\hline Cesium-137 & $\mathrm{pCi} / \mathrm{L}$ & $<50$ & 0.231 & 1.93 & U & 7.41 & $<7.41$ & $\mathrm{~N}$ \\
\hline Curium-242 & $\mathrm{pCi} / \mathrm{L}$ & $<15$ & 0.137 & 0.162 & $U$ & 0.587 & $<0.587$ & $N$ \\
\hline Curium-243/244 & pCin & $<15$ & 0.61 & 0.301 & Jl & 0.81 & $<0.81$ & $\mathrm{~N}$ \\
\hline Curium-246 & $\mathrm{pCi} / \mathrm{L}$ & $<15$ & 0.0659 & 0.0936 & $U$ & 0.286 & $<0.286$ & $\mathbf{N}$ \\
\hline Carbon-14 & pCin & $<150$ & 26.3 & 4.68 & $J$ & 16.15 & $<50$ & $\mathrm{~N}$ \\
\hline Cobalt-60 & $\mathrm{pCi} / \mathrm{L}$ & $<50$ & -1.42 & 1.47 & $\mathrm{U}$ & 5.21 & $<5.21$ & $\mathrm{~N}$ \\
\hline lodine-129 & $\mathrm{pCi} / \mathrm{L}$ & $<60$ & 100 & 12.3 & & 25.67 & 100.00 & $\mathrm{Y}$ \\
\hline Plutonium-238 & $\mathrm{pCi} / \mathrm{L}$ & $<15$ & 0.0903 & 0.117 & $\bar{U}$ & 0.448 & $<0.448$ & $\mathbf{N}$ \\
\hline Plutonium-239/240 & $\mathrm{pCi} / \mathrm{L}$ & $<15$ & 0.00802 & 0.055 & $U$ & 0.254 & $<0.254$ & $\mathrm{~N}$ \\
\hline Radium-226 & $\mathrm{pCi} / \Omega$ & TR<25 & 0.712 & 0.435 & JI & 1.307 & $<1.307$ & $\mathbf{N}$ \\
\hline Radium-228 & pCin & $T R<25$ & -1.44 & 0.613 & UJ & 2.696 & $<.696$ & $\mathrm{~N}$ \\
\hline Strontium-90 & pCin & $<170$ & 5.38 & 0.799 & & 2 & 5.38 & $\mathbf{N}$ \\
\hline Technetium-99 & pCin & $<170$ & 38.6 & 11.8 & ال & 44.4 & $<44.4$ & $\mathbf{N}$ \\
\hline Thorium-228 & pCin & $<15$ & 0.819 & 0.665 & $U$ & 2.34 & $<.34$ & $\mathbf{N}$ \\
\hline Thorium-230 & pCi/L & $<15$ & 0.665 & 0.463 & Jl & 1.148 & $<1.148$ & $\mathbf{N}$ \\
\hline Thorium-232 & pCi/L & $<15$ & 0 & 0 & $U$ & 0.222 & $<0.222$ & $\bar{N}$ \\
\hline Uranium-233/234 & $p \mathrm{Ci} \Omega$ & $<25$ & 3.18 & 0.892 & & 2.116 & 3.18 & $\mathbf{N}$ \\
\hline Uranium-235 & $\mathrm{pCi} / \mathrm{L}$ & $<15$ & 0.237 & 0.243 & $\mathrm{U}$ & 0.819 & $<0.819$ & $\mathrm{~N}$ \\
\hline Uranium-238 & pCin & $<50$ & 6.21 & 1.32 & & 2.798 & 6.21 & $\mathrm{~N}$ \\
\hline
\end{tabular}

Notes: Samples collected 1/13/99

*Thallium DL is too high 
F-Area WTU UIC Sample Results: February 1999

\begin{tabular}{|c|c|c|c|c|c|c|c|c|}
\hline \multicolumn{2}{|c|}{ UIC Permitted Constituents } & Reg & Raw & Rad & & & February & Exceedence \\
\hline Constituent & Unit & Limit & Result & Acc & Qual. & $\mathbf{Q L}$ & Result & $\mathbf{Y} / \mathbf{N}$ \\
\hline \multicolumn{9}{|l|}{ Section I, INORGANICS } \\
\hline Antimony & $\mu g /$ & 6 & 0.2 & & $U$ & 0.2 & $<0.2$ & $\mathrm{~N}$ \\
\hline Arsenic & $\mu g / L$ & 50 & 3 & & U & 3 & $<3$ & $\mathbf{N}$ \\
\hline Barium & $\mu g / L$ & 2000 & 14.2 & & & 0.2 & 14.20 & $\mathbf{N}$ \\
\hline Cadmium & $\mu g / L$ & 5 & 1 & & $\mathrm{U}$ & 1 & $<1$ & $\mathbf{N}$ \\
\hline Chromium & $\mu g / L$ & 100 & 3 & & $U$ & 3 & $<3$ & $N$ \\
\hline Cobalt & $\mu g / L$ & 140 & 7.52 & & & 0.2 & 7.52 & $N$ \\
\hline Copper & $\mu g / L$ & 1300 & 6.96 & & & 0.2 & 6.96 & $\mathbf{N}$ \\
\hline Cyanide & $\mu g / L$ & 200000 & 10 & & $U$ & 10 & $<10$ & $\bar{N}$ \\
\hline Lead & $\mu g / L$ & 50 & 2 & & $\bar{U}$ & 2 & $<2$ & $\mathrm{~N}$ \\
\hline Mercury & $\mu g / L$ & 2 & 0.144 & & $\mathrm{~J}$ & 0.2 & $<0.2$ & $\mathrm{~N}$ \\
\hline Nickel & $\mu g / L$ & 100 & 2.33 & & & 0.2 & 2.33 & $\mathrm{~N}$ \\
\hline Selenium & $\mu g / L$ & 50 & 5 & & $U$ & 5 & $<5$ & $\mathrm{~N}$ \\
\hline Silver & $\mu g / L$ & 50 & 1 & & $U$ & 1 & $<1$ & $N$ \\
\hline Thallium ${ }^{\star}$ & $\mu \mathrm{g} / \mathrm{L}$ & 2 & 0.246 & & $\mathrm{JI}$ & 2.5 & $<2.5$ & \\
\hline Vanadium & $\mu g / L$ & 49 & 2 & & $\mathrm{U}$ & 2 & $<$ & $\mathbf{N}$ \\
\hline Zinc & $\mu g / L$ & 5000 & 5 & & $U$ & 5 & $<5$ & $N$ \\
\hline \multicolumn{9}{|l|}{ Section II, ORGANICS } \\
\hline Benzene & $\mu \mathrm{g} / \mathrm{L}$ & 5 & 1 & & UJ & 1 & $<1$ & $N$ \\
\hline B2EHP & $\mu g / L$ & 140 & 10 & & $\mathrm{U}$ & 10 & $<10$ & $\mathbf{N}$ \\
\hline Methylene Chloride & $\mu g / L$ & 5 & 2.29 & & UJ & 5 & $<5$ & $\mathbf{N}$ \\
\hline Phenol & $\mu g / L$ & 10 & 5 & & $\mathrm{U}$ & 5 & $<5$ & $\mathrm{~N}$ \\
\hline Tetrachloroethylene & $\mu g / L$ & 5 & 1 & & UJ & 1 & $<1$ & $\mathrm{~N}$ \\
\hline Trichloroethylene & $\mu g / L$ & 5 & 1 & & UJ & 1 & $<1$ & $\mathrm{~N}$ \\
\hline Trichlorofluoromethane & $\mu g / L$ & 100 & 5 & & UJ & 5 & $<5$ & $\mathbf{N}$ \\
\hline \multicolumn{9}{|c|}{ Section III, RADIONUCLIDES } \\
\hline Americium-241 & $\mathrm{pCi} / \mathrm{L}$ & $<15$ & 0.549 & 0.757 & $U$ & 2.934 & $<2.934$ & $N$ \\
\hline Cesium-137 & $\mathrm{pCin}$ & $<50$ & -0.7 & 1.96 & $U$ & 7.35 & $<7.35$ & $\mathrm{~N}$ \\
\hline Curium-242 & $\mathrm{pCi} / \mathrm{L}$ & $<15$ & -0.404 & 0.466 & $\bar{U}$ & 2.332 & $<2.332$ & $\mathrm{~N}$ \\
\hline Curium-243/244 & $\mathrm{pCi} / \mathrm{L}$ & $<15$ & 0.065 & 1.04 & $U$ & 4.3 & $<4.3$ & $\mathrm{~N}$ \\
\hline Curium-246 & $\mathrm{pCi} / \mathrm{L}$ & $<15$ & 0.0847 & 0.271 & $\mathrm{U}$ & 1.198 & $<1.198$ & $\mathrm{~N}$ \\
\hline Carbon-14 & $\mathrm{pCi} / \mathrm{L}$ & $<150$ & 16.9 & 6.18 & $\sqrt{\mathrm{J}}$ & 22.04 & $<2.04$ & $\mathbf{N}$ \\
\hline Cobalt-60 & $\mathrm{pCi} / \mathrm{L}$ & $<50$ & 1.55 & 1.96 & $U$ & 7.68 & $<7.68$ & $N$ \\
\hline lodine-129 & $\mathrm{pCi} / \mathrm{L}$ & $<60$ & 45.8 & 6.42 & & 14.72 & 45.80 & $N$ \\
\hline Plutonium-238 & $\mathrm{pCi} / \mathrm{L}$ & $<15$ & -0.118 & 0.123 & $\bar{U}$ & 0.678 & $<0.678$ & $N$ \\
\hline Plutonium-239/240 & $\mathrm{pCi} / \mathrm{L}$ & $<15$ & -0.051 & 0.101 & U & 0.549 & $<0.549$ & $N$ \\
\hline Radium-226 & $\mathrm{pCi} / \mathrm{L}$ & TR<25 & 1.03 & 0.673 & $\mathrm{JI}$ & 2.145 & $<2.145$ & $N$ \\
\hline Radium-228 & $\mathrm{pCi} / \mathrm{L}$ & $T R<25$ & -0.326 & 0.486 & $U$ & 1.897 & $<1.897$ & $N$ \\
\hline Strontium-90 & $\mathrm{pCi} / \mathrm{L}$ & $<170$ & 4.75 & 1.23 & & 4.47 & 4.75 & $\mathrm{~N}$ \\
\hline Technetium-99 & $\mathrm{pCi} / \mathrm{L}$ & $<170$ & 16 & 2.59 & & 9.58 & 16.00 & $N$ \\
\hline Thorium-228 & $\mathrm{pCi} / \mathrm{L}$ & $<15$ & 0.865 & 0.679 & $U$ & 2.418 & $<2.418$ & $\mathrm{~N}$ \\
\hline Thorium-230 & $\mathrm{pCi} / \mathrm{L}$ & $<15$ & 0.276 & 0.323 & $U$ & 1.133 & $<1.133$ & $N$ \\
\hline Thorium-232 & $\mathrm{pCi} / \mathrm{L}$ & $<15$ & 0.0815 & 0.235 & $U$ & 1.062 & $<1.062$ & $N$ \\
\hline Uranium-233/234 & $\mathrm{pCi} / \mathrm{L}$ & $<25$ & 4.57 & 1.23 & & 3.076 & 4.57 & $N$ \\
\hline Uranium-235 & $\mathrm{pCi} / \mathrm{L}$ & $<15$ & 1.07 & 0.574 & J & 1.739 & $<1.739$ & $\mathrm{~N}$ \\
\hline Uranium-238 & $\mathrm{pCi} / \mathrm{L}$ & $<50$ & 9.27 & 1.88 & & 3.956 & 9.27 & $\mathrm{~N}$ \\
\hline
\end{tabular}

Notes: Samples collected 2/17/99

*Thallium DL is too high 
F-Area WTU UIC Sample Results: March 1999

\begin{tabular}{|c|c|c|c|c|c|c|c|c|}
\hline \multicolumn{2}{|c|}{ UIC Permitted Constituents } & \multirow{2}{*}{\begin{tabular}{|l|} 
Reg \\
Limit
\end{tabular}} & \multirow{2}{*}{$\begin{array}{c}\text { Raw } \\
\text { Result }\end{array}$} & \multirow{2}{*}{$\begin{array}{l}\text { Rad } \\
\text { Acc }\end{array}$} & \multirow[b]{2}{*}{ Qual. } & \multirow[b]{2}{*}{ QL } & \multirow{2}{*}{$\begin{array}{l}\text { March } \\
\text { Result }\end{array}$} & \multirow{2}{*}{$\begin{array}{c}\text { Exceedence } \\
\text { Y/N }\end{array}$} \\
\hline Constituent & Unit & & & & & & & \\
\hline \multicolumn{9}{|l|}{ Section I, INORGANICS } \\
\hline Antimony & $\mu g / L$ & 6 & 0.168 & & J & 0.2 & $<0.2$ & $\mathrm{~N}$ \\
\hline Arsenic & $\mu g / L$ & 50 & 3 & & $\mathrm{U}$ & 3 & $<3$ & $N$ \\
\hline Barium & $\mu g / L$ & 2000 & 4.93 & & & 2 & 4.93 & $\mathbf{N}$ \\
\hline Cadmium & $\mu g /$ & 5 & 1 & & U & 1 & $<1$ & $\mathrm{~N}$ \\
\hline Chromium & $\mu g / L$ & 100 & 3 & & $U$ & 3 & $<3$ & $\mathbf{N}$ \\
\hline Cobalt & $\mu g / L$ & 140 & 2 & & & 0.2 & 2.00 & $\mathrm{~N}$ \\
\hline Copper & $\mu g / L$ & 1300 & 5.02 & & & 1 & 5.02 & $\mathbf{N}$ \\
\hline Cyanide & $\mu g / L$ & 200000 & 6.1 & & الد & 10 & $<10$ & $\mathrm{~N}$ \\
\hline Lead & $\mu g / L$ & 50 & 2 & & $\mathrm{U}$ & 2 & $<$ & $\mathbf{N}$ \\
\hline Mercury & $\mu g / L$ & 2 & 0.0648 & & J & 0.2 & $<0.2$ & $\mathrm{~N}$ \\
\hline Nickel & $\mu g / L$ & 100 & 2 & & $\mathrm{U}$ & 2 & $<$ & $\mathrm{N}$ \\
\hline Selenium & $\mu g / L$ & 50 & 5 & & $U$ & 5 & $<5$ & $\mathbf{N}$ \\
\hline Silver & $\mu g / L$ & 50 & 1 & & $\mathrm{U}$ & 1 & $<1$ & $\mathbf{N}$ \\
\hline Thallium* & $\mu g / L$ & 2 & 0.662 & & ل & 2.5 & 2.5 & \\
\hline Vanadium & $\mu g / L$ & 49 & 10 & & $\mathrm{U}$ & 10 & $<10$ & $\mathbf{N}$ \\
\hline Zinc & $\mu g / L$ & 5000 & 10 & & $\mathrm{U}$ & 10 & $<10$ & $\mathbf{N}$ \\
\hline \multicolumn{9}{|l|}{ Section II, ORGANICS } \\
\hline Benzene & $\mu g / L$ & 5 & 1 & & UJ & 1 & $<1$ & $\mathbf{N}$ \\
\hline B2EHP & $\mu g / L$ & 140 & 10 & & $\mathrm{U}$ & 10 & $<10$ & $\mathrm{~N}$ \\
\hline Methylene Chloride & $\mu g / L$ & 5 & 5 & & UJ & 5 & $<5$ & $\mathrm{~N}$ \\
\hline Phenol & $\mu g / L$ & 10 & 5 & & $\mathrm{U}$ & 5 & $<5$ & $\mathbf{N}$ \\
\hline Tetrachloroethylene & $\mu g / L$ & 5 & 1 & & UJ & 1 & $<1$ & $\mathbf{N}$ \\
\hline Trichloroethylene & $\mu g / L$ & 5 & 1 & & UJ & 1 & $<1$ & $\mathbf{N}$ \\
\hline Trichlorofluoromethane & $\mu g / L$ & 100 & 5 & & UJ & 5 & $<5$ & $\mathbf{N}$ \\
\hline \multicolumn{9}{|c|}{ Section III, RADIONUCLIDES } \\
\hline Americium-241 & $\mathrm{pCi} / \mathrm{L}$ & $<15$ & 0.601 & 0.687 & $U$ & 2.624 & $<2.624$ & $\mathbf{N}$ \\
\hline Cesium-137 & $\mathrm{pCi} / \mathrm{L}$ & $<50$ & 1.58 & 1.94 & $U$ & 7.7 & $<7.7$ & $\mathbf{N}$ \\
\hline Curium-242 & pCill & $<15$ & 0.23 & 0.519 & $\mathrm{U}$ & 2.098 & $<2.098$ & $\mathbf{N}$ \\
\hline Curium-243/244 & $\mathrm{pCil}$ & $<15$ & 0.513 & 0.368 & ل & 0.929 & $<0.929$ & $\mathbf{N}$ \\
\hline Curium-246 & pCill & $<15$ & 0.449 & 0.343 & Jl & 0.878 & $<0.878$ & $\mathbf{N}$ \\
\hline Carbon-14 & pCill & $<150$ & -0.695 & 10.5 & U & 39.2 & $<39.2$ & $\mathbf{N}$ \\
\hline Cobalt-60 & $\mathrm{pCi} / \mathrm{L}$ & $<50$ & 2.23 & 2.16 & $\mathrm{U}$ & 8.96 & $<8.96$ & $\mathbf{N}$ \\
\hline lodine-129 & pCill & $<60$ & 36 & 4.94 & & 11.24 & 36.00 & $\mathbf{N}$ \\
\hline Plutonium-238 & pCil & $<15$ & -0.239 & 0.304 & $\mathrm{U}$ & 1.512 & $<1.512$ & $\mathbf{N}$ \\
\hline Plutonium-239/240 & pCi/L & $<15$ & 0.176 & 0.251 & $\mathrm{U}$ & 0.953 & $<0.953$ & $\mathbf{N}$ \\
\hline Radium-226 & $\mathrm{pCi} / \mathrm{L}$ & TR $<25$ & 1.43 & 0.785 & ال & 2.403 & $<2.403$ & $\mathbf{N}$ \\
\hline Radium-228 & pCill & TR<25 & -0.556 & 0.452 & $\mathrm{U}$ & 1.994 & $<1.994$ & $N$ \\
\hline Strontium-90 & pCill & $<170$ & 1.76 & 0.711 & $\mathrm{Jl}$ & 2.682 & $<2.682$ & $N$ \\
\hline Technetium-99 & pCil & $<170$ & 3.49 & 2.83 & $U$ & 12.16 & $<12.16$ & $\mathbf{N}$ \\
\hline Thorium-228 & pCill & $<15$ & 0.775 & 0.713 & $\mathrm{U}$ & 2.586 & $<.586$ & $\mathbf{N}$ \\
\hline Thorium-230 & pCill & $<15$ & 0.641 & 0.583 & $\mathrm{U}$ & 2.057 & 2.057 & $N$ \\
\hline Thorium-232 & $\mathrm{pCi} / \mathrm{L}$ & $<15$ & -0.175 & 0.346 & $\mathrm{U}$ & 1.752 & $<1.752$ & $\mathbf{N}$ \\
\hline Uranium-233/234 & pCill & $<25$ & 3.78 & 1.38 & & 3.559 & 3.78 & $\mathbf{N}$ \\
\hline Uranium-235 & $\mathrm{pCil}$ & $<15$ & 0.223 & 0.316 & $\mathrm{U}$ & 0.966 & $<0.966$ & $\mathbf{N}$ \\
\hline Uranium-238 & pCill & $<50$ & 4 & 1.39 & & 3.113 & 4.00 & $\mathbf{N}$ \\
\hline
\end{tabular}

Notes: Samples collected 3/18/99

*Thallium DL is too high 
F-Area WTU UIC Sample Results: April 1999

\begin{tabular}{|c|c|c|c|c|c|c|c|c|}
\hline \multicolumn{2}{|c|}{ UIC Permitted Constituents } & \multirow{2}{*}{\begin{tabular}{|l|} 
Reg \\
Limit \\
\end{tabular}} & \multirow{2}{*}{$\begin{array}{l}\text { Raw } \\
\text { Result }\end{array}$} & \multirow{2}{*}{$\begin{array}{l}\text { Rad } \\
\text { Acc }\end{array}$} & \multirow{2}{*}{ Qual. } & \multirow[b]{2}{*}{$\mathbf{Q L}$} & \multirow{2}{*}{$\begin{array}{c}\text { April } \\
\text { Result }\end{array}$} & \multirow{2}{*}{$\begin{array}{c}\text { Exceedence } \\
\mathrm{Y} / \mathrm{N}\end{array}$} \\
\hline Constituent & Unit & & & & & & & \\
\hline Section I, INORGANICS & & & & & & $\sqrt{2}$ & jelog & \\
\hline Antimony & $\mu g / L$ & 6 & 0.26 & & U & 0.2 & $<0.2$ & $N$ \\
\hline Arsenic & $\mu g h$ & 50 & 3 & & $U$ & 3 & $<3$ & $\mathbf{N}$ \\
\hline Barium & $\mu g h$ & 2000 & 8.84 & & & 2 & 8.84 & $\mathrm{~N}$ \\
\hline Cadmium & $\mu g / L$ & 5 & 1 & & $U$ & 1 & $<1$ & $\mathbf{N}$ \\
\hline Chromium & $\mu g /$ & 100 & 0.851 & & $U$ & 3 & $<$ & $\mathbf{N}$ \\
\hline Cobalt & $\mu g / L$ & 140 & 3.57 & & & 0.2 & 3.57 & $\mathrm{~N}$ \\
\hline Copper & $\mu g /$ & 1300 & 2.52 & & & 1 & 2.52 & $N$ \\
\hline Cyanide & $\mu g / L$ & 200000 & 10 & & $U$ & 10 & $<10$ & $\mathbf{N}$ \\
\hline Lead & $\mu g / L$ & 50 & 0.374 & & $\mathrm{U}$ & 2 & $<$ & $\mathbf{N}$ \\
\hline Mercury & $\mu g /$ & 2 & 0.241 & & & 0.2 & 0.24 & $\mathbf{N}$ \\
\hline Nickel & $\mu g / L$ & 100 & 0.947 & & ال & 2 & $<2$ & $N$ \\
\hline Selenium & $\mu g / L$ & 50 & 5 & & $\bar{U}$ & 5 & $<5$ & $N$ \\
\hline Silver & $\mu g / L$ & 50 & 1 & & $\bar{U}$ & 1 & $<1$ & $\mathbf{N}$ \\
\hline Thallium ${ }^{*}$ & $\mu g /$ & 2 & 0.417 & & $U$ & 2.5 & $<2.5$ & \\
\hline Vanadium ${ }^{\star \star}$ & $\mu g / L$ & 49 & 100 & & $U$ & 100 & 100 & $?$ \\
\hline Zinc & $\mu g / L$ & 5000 & 26.9 & & & 10 & 26.90 & $\bar{N}$ \\
\hline \multicolumn{9}{|l|}{ Section II, ORGANICS } \\
\hline Benzene & $\mu g / \mathrm{L}$ & 5 & 1 & & $U$ & 1 & $<1$ & $\mathrm{~N}$ \\
\hline B2EHP & $\mu g / L$ & 140 & 9.9 & & UJ & 9.9 & $<9.9$ & $\mathrm{~N}$ \\
\hline Methylene Chloride & $\mu g / L$ & 5 & 5 & & $\mathrm{U}$ & 5 & $<5$ & $\mathrm{~N}$ \\
\hline Phenol & $\mu g / L$ & 10 & 5 & & $\mathrm{U}$ & 5 & $<5$ & $\mathrm{~N}$ \\
\hline Tetrachloroethylene & $\mu g / L$ & 5 & 1 & & $U$ & 1 & $<1$ & $\mathbf{N}$ \\
\hline Trichloroethylene & $\mu g / L$ & 5 & 1 & & UJ & 1 & $<1$ & $\mathrm{~N}$ \\
\hline Trichlorofluoromethane & $\mu g / L$ & 100 & 0.752 & & J & 1 & $<1$ & $\mathrm{~N}$ \\
\hline \multicolumn{9}{|c|}{ Section III, RADIONUCLIDES } \\
\hline Americium-241 & pCi/L & $<15$ & 1.73 & 0.74 & & 1.71 & 1.73 & $N$ \\
\hline Cesium-137 & $\mathrm{pCi} / \mathrm{L}$ & $<50$ & 0.0865 & 1.5 & $\mathrm{U}$ & 5.71 & $<5.71$ & $\mathrm{~N}$ \\
\hline Curium-242 & $\mathrm{pCi} / \mathrm{L}$ & $<15$ & 0.0791 & 0.158 & $U$ & 0.553 & $<0.553$ & $\mathbf{N}$ \\
\hline Curium-243/244 & $\mathrm{pCi} / \mathrm{L}$ & $<15$ & 0.982 & 0.624 & ال & 1.96 & $<1.96$ & $\mathrm{~N}$ \\
\hline Curium-246 & $\mathrm{pCi} / \mathrm{L}$ & $<15$ & 0.525 & 0.4 & لl & 1.03 & $<1.03$ & $\mathbf{N}$ \\
\hline Carbon-14 & $\mathrm{pCi} / \mathrm{L}$ & $<150$ & 61.9 & 6.03 & & 19.4 & 61.90 & $N$ \\
\hline Cobalt-60 & $\mathrm{pCi} / \mathrm{L}$ & $<50$ & -0.563 & 1.84 & $U$ & 6.47 & $<6.47$ & $\mathbf{N}$ \\
\hline lodine-129 & $\mathrm{pCi} / \mathrm{L}$ & $<60$ & 39.3 & 5.36 & & 12 & 39.30 & $\mathbf{N}$ \\
\hline Plutonium-238 & $\mathrm{pCi} / \mathrm{L}$ & $<15$ & 0.0283 & 0.314 & $U$ & 1.4 & $<1.4$ & $\mathrm{~N}$ \\
\hline Plutonium-239/240 & $\mathrm{pCi} / \mathrm{L}$ & $<15$ & 0.455 & 0.498 & $U$ & 1.76 & $<1.76$ & $\mathbf{N}$ \\
\hline Radium-226 & pCi/L & TR<25 & 1.17 & 0.611 & ال & 1.45 & $<1.45$ & $N$ \\
\hline Radium-228 & pCi/l & TR<25 & -0.289 & 0.439 & $U$ & 1.79 & $<1.79$ & $\mathbf{N}$ \\
\hline Strontium-90 & $\mathrm{pCi} / \mathrm{L}$ & $<170$ & 3.65 & 0.687 & & 2.39 & 3.65 & $\mathrm{~N}$ \\
\hline Technetium-99 & $\mathrm{pCi} / \mathrm{L}$ & $<170$ & 12.2 & 3.26 & & 11.7 & 12.20 & $\mathbf{N}$ \\
\hline Thorium-228 & $\mathrm{pCi} / \mathrm{L}$ & $<15$ & 1E-07 & 0.414 & $U$ & 1.92 & $<1.92$ & $N$ \\
\hline Thorium-230 & $\mathrm{pCi} / \mathrm{L}$ & $<15$ & 0.0389 & 0.158 & $U$ & 0.781 & $<0.781$ & $\mathbf{N}$ \\
\hline Thorium-232 & $\mathrm{pCi} / \mathrm{L}$ & $<15$ & -0.0179 & 0.0361 & $U$ & 0.467 & $<0.467$ & $N$ \\
\hline Uranium-233/234 & $\mathrm{pCi} / \mathrm{L}$ & $<25$ & 5.74 & 2.04 & ال & 6.1 & $<6.1$ & $\mathrm{~N}$ \\
\hline Uranium-235 & $\mathrm{pCi} / \mathrm{L}$ & $<15$ & 9.43 & 2.51 & & 6.43 & 9.43 & $\mathrm{~N}$ \\
\hline Uranium-238 & $\mathrm{pCi} / \mathrm{L}$ & $<50$ & 9.4 & 2.5 & & 6.41 & 6.41 & $N$ \\
\hline
\end{tabular}

Notes: Samples collected 4/8/99

*DL is too high

**Dilution factor of 100 
F-Area WTU UIC Sample Results: May 1999

\begin{tabular}{|c|c|c|c|c|c|c|c|c|}
\hline \multicolumn{2}{|c|}{ UIC Permitted Constituents } & $\operatorname{Reg}$ & Raw & $\operatorname{Rad}$ & & & May & Exceedence \\
\hline Constituent & Unit & Limit & Result & Acc & Qual. & $\mathbf{Q L}$ & Result & $Y / N$ \\
\hline Section 1, INORGANICS & & 20 & 25 & & & 30 & 20 & 1989 \\
\hline Antimony & $\mu g / L$ & 6 & 2 & & U & 2 & $<2$ & $\mathrm{~N}$ \\
\hline Arsenic & $\mu g / L$ & 50 & 2.75 & & J & 3 & $<3$ & $\mathbf{N}$ \\
\hline Barium & $\mu g / L$ & 2000 & 6.88 & & & 2 & 6.88 & $\mathbf{N}$ \\
\hline Cadmium & $\mu g / L$ & 5 & 1 & & $\mathrm{U}$ & 1 & $<1$ & $\bar{N}$ \\
\hline Chromium & $\mu g / L$ & 100 & 2.37 & & Jा & 3 & $\mathbf{3}$ & $\mathbf{N}$ \\
\hline Cobalt & $\mu g / L$ & 140 & 2.81 & & & 1 & 2.81 & $\mathbf{N}$ \\
\hline Copper & $\mu g / L$ & 1300 & 3.28 & & & 1 & 3.28 & $\mathbf{N}$ \\
\hline Cyanide & $\mu g / \mathrm{L}$ & 200000 & 10 & & $\mathrm{U}$ & 10 & $<10$ & $\mathbf{N}$ \\
\hline Lead & $\mu g / L$ & 50 & 2 & & $\bar{U}$ & 2 & 2 & $\bar{N}$ \\
\hline Mercury & $\mu g / L$ & 2 & 0.185 & & JI & 0.2 & $<0.2$ & $\mathbf{N}$ \\
\hline Nickel & $\mu g / L$ & 100 & 2 & & $\mathrm{U}$ & 2 & $<2$ & $\mathrm{~N}$ \\
\hline Selenium & $\mu g / L$ & 50 & 5 & & $\mathrm{U}$ & 5 & $<5$ & $\mathbf{N}$ \\
\hline Silver & $\mu g / L$ & 50 & 1 & & $U$ & 1 & $<1$ & $\mathbf{N}$ \\
\hline Thallium & $\mu g / L$ & 2 & 0.06 & & Jl & 0.5 & $<0.5$ & $N$ \\
\hline Vanadium & $\mu g / L$ & 49 & 10 & & $\bar{U}$ & 10 & $<10$ & $\bar{N}$ \\
\hline Zinc & $\mu g / L$ & 5000 & 10 & & $\mathrm{U}$ & 10 & $<10$ & $\mathbf{N}$ \\
\hline Section II, ORGANICS & & & & & & & and & \\
\hline Benzene & $\mu g / L$ & 5 & 1 & & UJ & 1 & $<1$ & $N$ \\
\hline B2EHP & $\mu g / L$ & 140 & 11.1 & & UJ & 11.1 & $<11.1$ & $\mathbf{N}$ \\
\hline Methylene Chloride & $\mu g / L$ & 5 & 5 & & UJ & 5 & $<5$ & $\bar{N}$ \\
\hline Phenol & $\mu g / L$ & 10 & 5 & & $\mathrm{U}$ & 5 & $<5$ & $\mathrm{~N}$ \\
\hline Tetrachloroethylene & $\mu g / L$ & 5 & 1 & & $\overline{U J}$ & 1 & $<1$ & $\bar{N}$ \\
\hline Trichloroethylene & $\mu g / L$ & 5 & 1 & & UJ & 1 & $<1$ & $\mathrm{~N}$ \\
\hline Trichlorofluoromethane & $\mu g / L$ & 100 & 5 & & UJ & 5 & $<5$ & $\mathrm{~N}$ \\
\hline - & & & & & & & & \\
\hline Section III, RADIONUC & DES & & & & & & & \\
\hline Americium-241 & pCin & $<15$ & 0.028 & 0.32 & $\mathrm{U}$ & 1.54 & $<1.54$ & N \\
\hline Cesium-137 & $\mathrm{pCi} / \mathrm{L}$ & $<50$ & 0.0102 & 1.94 & $\mathrm{U}$ & 7.42 & $<7.42$ & $\mathbf{N}$ \\
\hline Curium-242 & $\mathrm{pCi} / \mathrm{L}$ & $<15$ & 0.135 & 0.27 & $\mathrm{U}$ & 0.944 & $<0.944$ & $\mathbf{N}$ \\
\hline Curium-243/244 & $\mathrm{pCi} / \mathrm{L}$ & $<15$ & 0.533 & 0.601 & $\mathrm{U}$ & 2.1 & $<2.1$ & $\mathrm{~N}$ \\
\hline Curium-246 & $\mathrm{pCi} / \mathrm{L}$ & $<15$ & 0 & 0 & $U$ & 0.378 & $<0.378$ & $\overline{\mathbf{N}}$ \\
\hline Carbon-14 & $\mathrm{pCi} / \mathrm{L}$ & $<150$ & 10.9 & 6.48 & J & 23.6 & $<23.6$ & $\mathrm{~N}$ \\
\hline Cobalt-60 & $\mathrm{pCi} / \mathrm{L}$ & $<50$ & -0.386 & 2.1 & $\bar{U}$ & 8.1 & $<8.1$ & $N$ \\
\hline lodine-129 & $\mathrm{pCi} / \mathrm{L}$ & $<60$ & 6.99 & 1.45 & & 4.39 & 6.99 & $N$ \\
\hline Plutonium-238 & $\mathrm{pCi} / \mathrm{L}$ & $<15$ & -0.534 & 1.21 & $U$ & 6.33 & $<6.33$ & $\mathrm{~N}$ \\
\hline Plutonium-239/240 & $\mathrm{pCi} / \mathrm{L}$ & $<15$ & -0.0712 & 0.143 & $\mathrm{U}$ & 1.86 & $<1.86$ & $\mathbf{N}$ \\
\hline Radium-226 & $\mathrm{pCi} / \mathrm{L}$ & TR $<25$ & 0.94 & 0.689 & JI & 2.25 & $<2.25$ & $\mathbf{N}$ \\
\hline Radium-228 & $\mathrm{pCi} / \mathrm{L}$ & TR<25 & -0.279 & 0.431 & $U$ & 1.87 & $<1.87$ & $N$ \\
\hline Strontium-90 & $\mathrm{pCi} / \mathrm{L}$ & $<170$ & 5.49 & 1.08 & & 3.71 & 5.49 & $\bar{N}$ \\
\hline Technetium-99 & $\mathrm{pCi} / \mathrm{L}$ & $<170$ & 6.4 & 3.11 & JI & 11.3 & $<11.3$ & $N$ \\
\hline Thorium-228 & $\mathrm{pCi} / \mathrm{L}$ & $<15$ & 2.92 & 2.39 & $\bar{U}$ & 8.45 & $<8.45$ & $\bar{N}$ \\
\hline Thorium-230 & $\mathrm{pCi} / \mathrm{L}$ & $<15$ & 0.0122 & 0.679 & $U$ & 3.67 & $<3.67$ & $\mathbf{N}$ \\
\hline Thorium-232 & $\mathrm{pCi} / \mathrm{L}$ & $<15$ & 0 & 0 & $U$ & 0.918 & $<0.918$ & $\mathbf{N}$ \\
\hline Uranium-233/234 & $\mathrm{pCi} / \mathrm{L}$ & $<25$ & 3.18 & 1.95 & JI & 5.89 & $<5.89$ & $\mathbf{N}$ \\
\hline Uranium-235 & $\mathrm{pCi} / \mathrm{L}$ & $<15$ & 0.932 & 1.08 & $U$ & 3.81 & $<3.81$ & $\mathbf{N}$ \\
\hline Uranium-238 & $\mathrm{pCi} / \mathrm{L}$ & $<50$ & 3.96 & 2.09 & JI & 4.97 & $<4.97$ & $\mathbf{N}$ \\
\hline
\end{tabular}

Note: Samples collected 5/11/99 
F-Area WTU UIC Sample Results: June 1999

\begin{tabular}{|c|c|c|c|c|c|c|c|c|}
\hline \multicolumn{3}{|c|}{ UIC Permitted Constituer Reg } & \multirow{2}{*}{$\begin{array}{c}\text { Raw } \\
\text { Result }\end{array}$} & \multirow{2}{*}{$\begin{array}{l}\text { Rad } \\
\text { Acc }\end{array}$} & \multirow{2}{*}{ Qual. } & \multirow[b]{2}{*}{$\mathbf{Q L}$} & \multirow{2}{*}{$\begin{array}{l}\text { June } \\
\text { Result }\end{array}$} & \multirow{2}{*}{\begin{tabular}{|c|} 
Exceedence \\
$\mathrm{Y} / \mathrm{N}$ \\
\end{tabular}} \\
\hline Constituent & Unit & Limit & & & & & & \\
\hline \multicolumn{9}{|c|}{ Section I, INORG AWTCS } \\
\hline Antimony & $\mu \mathrm{g} / \mathrm{L}$ & 6 & 0.155 & & JI & 2 & $<2$ & $\mathrm{~N}$ \\
\hline Arsenic & $\mu \mathrm{g} / \mathrm{L}$ & 50 & 3 & & $\mathrm{U}$ & 3 & $<3$ & $\bar{N}$ \\
\hline Barium & $\mu g / L$ & 2000 & 6.74 & & & 2 & 6.74 & $\mathrm{~N}$ \\
\hline Cadmium & $\mu g / L$ & 5 & 1 & & $\mathrm{U}$ & 1 & $<1$ & $N$ \\
\hline Chromium & $\mu g / L$ & 100 & 3.42 & & & 3 & 3.42 & $\mathrm{~N}$ \\
\hline Cobalt & $\mu \mathrm{g} / \mathrm{L}$ & 140 & 2.5 & & & 1 & 2.50 & $N$ \\
\hline Copper & $\mu \mathrm{g} / \mathrm{L}$ & 1300 & 1.68 & & & 1 & 1.68 & $\mathrm{~N}$ \\
\hline Cyanide & $\mu g / L$ & 200000 & 10 & & $U$ & 10 & $<10$ & $N$ \\
\hline Lead & $\mu \mathrm{g} / \mathrm{L}$ & 50 & 2 & & $\mathrm{U}$ & 2 & $<2$ & $\mathrm{~N}$ \\
\hline Mercury & $\mu g / L$ & 2 & 0.28 & & & 0.2 & 0.28 & $\mathrm{~N}$ \\
\hline Nickel & $\mu g / L$ & 100 & 3.47 & & & 2 & 3.47 & $\mathbf{N}$ \\
\hline Selenium & $\mu g / L$ & 50 & 5 & & $U$ & 5 & $<5$ & $\mathbf{N}$ \\
\hline Silver & $\mu \mathrm{g} / \mathrm{L}$ & 50 & 0.355 & & JI & 1 & $<1$ & $N$ \\
\hline Thallium & $\mu g / L$ & 2 & 0.289 & & JI & 0.5 & $<0.5$ & $\mathbf{N}$ \\
\hline Vanadium & $\mu g / L$ & 49 & 10 & & $\mathrm{U}$ & 10 & $<10$ & $\mathrm{~N}$ \\
\hline Zinc & $\mu g / L$ & 5000 & 10 & & $\bar{U}$ & 10 & $<10$ & $N$ \\
\hline \multicolumn{9}{|c|}{ Section II, ORGANICS } \\
\hline Benzene & $\mu \mathrm{g} / \mathrm{L}$ & 5 & 1 & & UJ & 1 & $<1$ & $N$ \\
\hline B2EHP & $\mu g / L$ & 140 & 10 & & UJ & 10 & $<10$ & $\mathrm{~N}$ \\
\hline Methylene Chloride & $\mu \mathrm{g} / \mathrm{L}$ & 5 & 2.46 & & UJ & 5 & $<5$ & $\mathrm{~N}$ \\
\hline Phenol & $\mu g / L$ & 10 & 5 & & $\mathrm{U}$ & 5 & $<5$ & $\bar{N}$ \\
\hline Tetrachloroethylene & $\mu \mathrm{g} / \mathrm{L}$ & 5 & 1 & & UJ & 1 & $<1$ & $\mathrm{~N}$ \\
\hline Trichloroethylene & $\mu \mathrm{g} / \mathrm{L}$ & 5 & 1 & & UJ & 1 & $<1$ & $N$ \\
\hline Trichlorofluorometh & $\mu \mathrm{g} / \mathrm{L}$ & 100 & 5 & & UJ & 5 & $<5$ & $\mathrm{~N}$ \\
\hline \multicolumn{9}{|c|}{ Section III, RADIONUCLIDES } \\
\hline Americium-241 & $\mathrm{pCi} / \mathrm{L}$ & $<15$ & 0.812 & 0.598 & $U$ & 2.11 & $<2.11$ & $\mathrm{~N}$ \\
\hline Cesium-137 & $\mathrm{pCi} / \mathrm{L}$ & $<50$ & 0.653 & 2.09 & $\mathrm{U}$ & 8.03 & $<8.03$ & $\mathrm{~N}$ \\
\hline Curium-242 & $\mathrm{pCi} / \mathrm{L}$ & $<15$ & -0.0151 & 0.171 & $\mathrm{U}$ & 0.952 & $<0.952$ & $\bar{N}$ \\
\hline Curium-243/244 & $\mathrm{pCi} / \mathrm{L}$ & $<15$ & 0.614 & 0.645 & $\mathrm{U}$ & 2.51 & $<2.51$ & $\mathrm{~N}$ \\
\hline Curium-246 & $\mathrm{pCi} / \mathrm{L}$ & $<15$ & 0.236 & 0.331 & $\mathrm{U}$ & 1.3 & $<1.3$ & $\mathbf{N}$ \\
\hline Carbon-14 & $\mathrm{pCi} / \mathrm{L}$ & $<150$ & 18.8 & 4.96 & & 17.5 & 18.80 & $\mathbf{N}$ \\
\hline Cobalt-60 & $p C i / L$ & $<50$ & 0.716 & 1.92 & $\mathrm{U}$ & 7.71 & $<7.71$ & $\mathbf{N}$ \\
\hline lodine-129 & $\mathrm{pCi} / \mathrm{L}$ & $<60$ & 36.9 & 6.61 & & 16.1 & 36.90 & $\mathrm{~N}$ \\
\hline Plutonium-238 & $\mathrm{pCi} / \mathrm{L}$ & $<15$ & 0 & 0 & $\mathrm{U}$ & 0.321 & $<0.321$ & $\mathbf{N}$ \\
\hline Plutonium-239/240 & $\mathrm{pCi} / \mathrm{L}$ & $<15$ & 0 & 0 & $\mathrm{U}$ & 0.321 & $<0.321$ & $N$ \\
\hline Radium-226 & $\mathrm{pCi} / \mathrm{L}$ & TR $<25$ & 1.29 & 0.477 & & 1.08 & 1.29 & $\mathbf{N}$ \\
\hline Radium-228 & $\mathrm{pCi} / \mathrm{L}$ & $T R<25$ & -1.02 & 0.534 & $\mathrm{U}$ & 1.97 & $<1.97$ & $\mathbf{N}$ \\
\hline Strontium-90 & $\mathrm{pCi} / \mathrm{L}$ & $<170$ & 4.09 & 1.85 & Jl & 6.85 & $<6.85$ & $N$ \\
\hline Technetium-99 & $\mathrm{pCi} / \mathrm{L}$ & $<170$ & 27.1 & 6.16 & & 21.9 & 27.10 & $\mathbf{N}$ \\
\hline Thorium-228 & $p C i / L$ & $<15$ & -0.103 & 0.356 & $\mathrm{U}$ & 1.74 & $<1.74$ & $\mathbf{N}$ \\
\hline Thorium -230 & $p C i / L$ & $<15$ & -0.0825 & 0.0755 & $\mathrm{U}$ & 0.707 & $<0.707$ & $\mathrm{~N}$ \\
\hline Thorium-232 & $\mathrm{pCi} / \mathrm{L}$ & $<15$ & -0.0165 & 0.0322 & $U$ & 0.429 & $<0.429$ & $N$ \\
\hline Uranium-233/234 & pCi/L & $<25$ & 18 & 3.01 & & 6.46 & 18.00 & $\mathbf{N}$ \\
\hline Uranium-235 & $\mathrm{pCi} / \mathrm{L}$ & $<15$ & 2.17 & 0.762 & & 1.88 & 2.17 & $\mathbf{N}$ \\
\hline Uranium-238 & $\mathrm{pCi} / \mathrm{L}$ & $<50$ & 31.9 & 4.76 & & 9.88 & 31.90 & $\mathbf{N}$ \\
\hline
\end{tabular}

Note: Samples collected 6/7/99 
THIS PAGE WAS LEFT BLANK INTENTIONALLY. 


\section{APPENDIX G: OPERATIONAL DOWNTIME}

\section{Downtime During the First Half of 1999}

\begin{tabular}{|c|c|c|}
\hline Date & $\begin{array}{c}\text { Hours of } \\
\text { Operation }\end{array}$ & Downtime Explanation \\
\hline $1 / 1 / 99$ & 24.0 & WTU operated for 24 hours. \\
\hline $1 / 2 / 99$ & 20.0 & WTU down to clean the flocculation tank and clarifier. \\
\hline $1 / 3$ to $1 / 8 / 99$ & 144.0 & WTU operated for 24 hours. \\
\hline $1 / 9 / 99$ & 22.5 & $\begin{array}{l}\text { WTU system down due to high differential pressure across } \\
\text { the roughing filters. Backwashing was not effective. }\end{array}$ \\
\hline $1 / 10 / 99$ & 0.0 & $\begin{array}{l}\text { WTU down to change out the resin and modify the flow } \\
\text { transmitter. }\end{array}$ \\
\hline $1 / 11 / 99$ & 0.0 & WTU system down to relocate control valve. \\
\hline $1 / 12 / 99$ & 0.0 & $\begin{array}{l}\text { WTU system down to replace resin in ion-exchange columns } \\
\text { and roughing filters. }\end{array}$ \\
\hline $1 / 13 / 99$ & 0.0 & $\begin{array}{l}\text { WTU system down to replace resin in ion-exchange columns } \\
\text { and roughing filters and perform hydrostatic testing of } \\
\text { process lines and calibration of flow control valve. }\end{array}$ \\
\hline $1 / 14 / 99$ & 0.0 & $\begin{array}{l}\text { WTU system down to complete resin changeout, desludge the } \\
\text { system, and make final preparation for an extended run. }\end{array}$ \\
\hline $\begin{array}{l}1 / 15 \text { to } \\
1 / 18 / 99\end{array}$ & 0.0 & $\begin{array}{l}\text { WTU system down to desludge and make preparation for an } \\
\text { extended run. }\end{array}$ \\
\hline $\begin{array}{l}1 / 19 \text { to } \\
1 / 27 / 99\end{array}$ & 0.0 & $\begin{array}{l}\text { WTU system down to resolve nonconformance report (NCR) } \\
\text { dealing with chemical pump discharge lines and to modify } \\
\text { procedures to operate in a manual mode. }\end{array}$ \\
\hline $1 / 28 / 99$ & 15.0 & $\begin{array}{l}\text { WTU system restarted after resolution of NCR dealing with } \\
\text { chemical pump discharge line and working on procedure } \\
\text { modifications to operate in the manual mode. }\end{array}$ \\
\hline $1 / 29 / 99$ & 22.5 & $\begin{array}{l}\text { WTU system down due to failed bolts in the sludge pump } \\
\text { surge suppressor. }\end{array}$ \\
\hline $\begin{array}{l}1 / 30 \text { to } \\
1 / 31 / 99\end{array}$ & 0.0 & $\begin{array}{l}\text { WTU system down due to failed bolts in the sludge pump } \\
\text { surge suppressor. }\end{array}$ \\
\hline $\begin{array}{l}\text { January } \\
\text { Totals }\end{array}$ & 248.00 & \\
\hline $2 / 1 / 99$ & 0.0 & $\begin{array}{l}\text { WTU down due to failed bolts in the sludge pump surge } \\
\text { suppressor. }\end{array}$ \\
\hline $2 / 2 / 99$ & 7.0 & WTU down to replace the extruder on the small press. \\
\hline $2 / 3 / 99$ & 15.5 & WTU down to desludge and clean the small press. \\
\hline $2 / 4 / 99$ & 3.5 & WTU down to desludge and empty the small press. \\
\hline
\end{tabular}




\begin{tabular}{|c|c|c|}
\hline Date & $\begin{array}{r}\text { Hours of } \\
\text { Operation }\end{array}$ & Downtime Explanation \\
\hline $2 / 5 / 99$ & 4.5 & WTU down to desludge and empty the small press. \\
\hline $2 / 6 / 99$ & 20.5 & $\begin{array}{l}\text { WTU down due to failure of surge suppressor on sludge } \\
\text { pump. }\end{array}$ \\
\hline $2 / 7 / 99$ & 0.0 & $\begin{array}{l}\text { WTU down due to failure of surge suppressor on sludge } \\
\text { pump. }\end{array}$ \\
\hline $2 / 8 / 99$ & 0.0 & $\begin{array}{l}\text { WTU down due to failure of surge suppressor, sludge pump, } \\
\text { and changing out filter press media. }\end{array}$ \\
\hline $\begin{array}{l}2 / 9 \text { to } \\
2 / 10 / 99\end{array}$ & 0.0 & WTU down to desludge the clarifier and flocculation tank. \\
\hline $2 / 11 / 99$ & 1.0 & WTU down to desludge the clarifier and flocculation tank. \\
\hline $2 / 12 / 99$ & 2.5 & WTU down to desludge clarifier and flush process lines. \\
\hline $2 / 13 / 99$ & 14.5 & WTU down to desludge the process lines. \\
\hline $2 / 14 / 99$ & 21.0 & WTU down due to carryover from the flocculation tank. \\
\hline $2 / 15 / 99$ & 20.0 & WTU down due to carryover in the flocculation tank. \\
\hline $2 / 16 / 99$ & 24.0 & WTU operated for 24 hours. \\
\hline $2 / 17 / 99$ & 1.5 & $\begin{array}{l}\text { WTU down to clean clarifier/flocculation system } \\
\text { components. }\end{array}$ \\
\hline $2 / 18 / 99$ & 15.0 & WTU down to clean clarifier/flocculation system components \\
\hline $2 / 19 / 99$ & 0.0 & $\begin{array}{l}\text { WTU down to clean clarifier/flocculation system } \\
\text { components. }\end{array}$ \\
\hline $2 / 20 / 99$ & 0.0 & $\begin{array}{l}\text { WTU down to clean clarifier/flocculation system components } \\
\text { and prepare for planned outage. }\end{array}$ \\
\hline $2 / 21 / 99$ & 0.5 & $\begin{array}{l}\text { WTU down to clean clarifier/flocculation system components } \\
\text { and prepare for planned outage. Planned maintenance and } \\
\text { upgrade outage includes: replace resins, install lightning } \\
\text { protection, eliminate RO pump vibration, sludge pump } \\
\text { modifications, relocate air compressors, and chemical mixing } \\
\text { modifications. }\end{array}$ \\
\hline $\begin{array}{l}2 / 22 / 99 \text { to } \\
2 / 28 / 99\end{array}$ & 0.0 & WTU down for planned maintenance and upgrade outage. \\
\hline $\begin{array}{l}\text { February } \\
\text { Totals }\end{array}$ & 151.00 & \\
\hline $\begin{array}{l}3 / 1 / 99 \text { to } \\
3 / 14 / 99\end{array}$ & 0.0 & WTU down for planned maintenance and upgrade outage. \\
\hline $3 / 15 / 99$ & 0.0 & $\begin{array}{l}\text { WTU down for planned maintenance and upgrade outage. } \\
\text { Ops continued procedure walkdowns and valve tagging. }\end{array}$ \\
\hline
\end{tabular}




\begin{tabular}{|c|c|c|}
\hline Date & $\begin{array}{l}\text { Hours of } \\
\text { Operation }\end{array}$ & Downtime Explanation \\
\hline $3 / 16 / 99$ & 0.0 & $\begin{array}{l}\text { WTU down for planned maintenance and upgrade outage. } \\
\text { Engineering started loop verifications and software } \\
\text { installations of PLC. Ops started procedure validations. }\end{array}$ \\
\hline $3 / 17 / 99$ & 0.0 & $\begin{array}{l}\text { WTU down for planned maintenance and upgrade outage. } \\
\text { Engineering completed loop verifications and software } \\
\text { installation of the PLC. Ops completed procedure validation, } \\
\text { flushed preservative from the RO trains, and established } \\
\text { lockout for resin changeouts on } 3 / 18 / 99 \text {. }\end{array}$ \\
\hline $3 / 18 / 99$ & 0.0 & $\begin{array}{l}\text { WTU down for planned maintenance and upgrade outage. } \\
\text { Maintenance changed out resin in the roughing filter C\&D } \\
\text { and carbon column (INEX-3). }\end{array}$ \\
\hline $3 / 19 / 99$ & 0.0 & $\begin{array}{l}\text { WTU down for planned maintenance and upgrade outage. } \\
\text { Ops completed initial valve alignment per procedure and } \\
\text { cleaned out caustic and } \mathrm{FeCl}_{3} \text { mix tanks. Maintenance } \\
\text { changed out resin in roughing filters A\&B, zeolite column } \\
\text { (INEX-1), and dowex column (INEX-2). }\end{array}$ \\
\hline $3 / 20 / 99$ & 1.0 & $\begin{array}{l}\text { Shakedown testing conducted which included leak checks, } \\
\text { general operating tests, etc., of newly installed system } \\
\text { modifications. Following testing, operations backwashed } \\
\text { INEX-1, INEX-2, and INEX-3 and changed out prefilters and } \\
\text { bag filter. }\end{array}$ \\
\hline $3 / 21 / 99$ & 0.0 & $\begin{array}{l}\text { WTU system down to perform housekeeping on the pad and } \\
\text { in preparation for continued system testing on } 3 / 22 \text {. }\end{array}$ \\
\hline $3 / 22 / 99$ & 21.0 & $\begin{array}{l}\text { WTU system down in preparation for testing. Shakedown } \\
\text { testing began at } 0820 ; \text { injection began at } 0900 \text {. }\end{array}$ \\
\hline $3 / 23 / 99$ & 24.0 & WTU operated for 24 hours. \\
\hline $3 / 24 / 99$ & 16.0 & $\begin{array}{l}\text { WTU down following breakthrough of the resin columns. } \\
\text { Ops deinventoried system and made preparations for resin } \\
\text { changeouts. }\end{array}$ \\
\hline $\begin{array}{l}3 / 25 \text { to } \\
3 / 26 / 99\end{array}$ & 0.0 & $\begin{array}{l}\text { WTU down to replace resins for the ion exchange columns } \\
\text { and roughing filters. }\end{array}$ \\
\hline $3 / 27 / 99$ & 4.0 & $\begin{array}{l}\text { Shakedown testing conducted which included use of new } \\
\text { chemical addition tanks. WTU system down to troubleshoot } \\
\text { system components and delivery rate of chemicals. }\end{array}$ \\
\hline $3 / 28 / 99$ & 16.5 & $\begin{array}{l}\text { WTU system down to modify feed to top of } \mathrm{FeCl}_{3} \text { mixing } \\
\text { tank. }\end{array}$ \\
\hline $3 / 29 / 99$ & 16.0 & $\begin{array}{l}\text { WTU down due to } \mathrm{NaOH} \text { injection system repair caused by } \\
\text { computer data table error which caused loss of } \mathrm{pH} \text { in caustic } \\
\text { mix tank. }\end{array}$ \\
\hline
\end{tabular}




\begin{tabular}{|c|c|c|}
\hline Date & $\begin{array}{r}\text { Hours of } \\
\text { Operation }\end{array}$ & Downtime Explanation \\
\hline $3 / 30 / 99$ & 15.0 & $\begin{array}{l}\text { WTU down due to } \mathrm{NaOH} \text { injection system repair caused by } \\
\text { computer data table error which caused loss of } \mathrm{pH} \text { in caustic } \\
\text { mix tank. }\end{array}$ \\
\hline $3 / 31 / 99$ & 0.0 & $\begin{array}{l}\text { WTU system down due to electrical code violations that were } \\
\text { discovered in the MCC. }\end{array}$ \\
\hline $\begin{array}{l}\text { March } \\
\text { Totals }\end{array}$ & $\mathbf{1 1 3 . 5 0}$ & \\
\hline $4 / 1$ to $4 / 5 / 99$ & 0.0 & $\begin{array}{l}\text { WTU down due to electrical inspections to detect and correct } \\
\text { code violations resulting from WSRC subcontractor } \\
\text { equipment installation on the WTU pad. }\end{array}$ \\
\hline $4 / 6 / 99$ & 20.0 & $\begin{array}{l}\text { WTU down due to electrical inspections to detect and correct } \\
\text { code violations resulting from WSRC subcontractor } \\
\text { equipment installation on the WTU pad. }\end{array}$ \\
\hline $4 / 7 / 99$ & 11.5 & $\begin{array}{l}\text { WTU system down to repair/replace pumps and motors } \\
\text { (sludge pump, polyblend pump, and } \mathrm{FECl}_{3} \text { rupture disc). }\end{array}$ \\
\hline $4 / 8 / 99$ & 21.0 & $\begin{array}{l}\text { WTU system down to repair/replace pumps and motors } \\
\text { (sludge pump, polyblend pump, and } \mathrm{FECl}_{3} \text { rupture disc). }\end{array}$ \\
\hline $4 / 9 / 99$ & 19.5 & $\begin{array}{l}\text { WTU system down due to } \mathrm{FeCl}_{3} \text { injection system repair } \\
\text { (rupture disc). }\end{array}$ \\
\hline $4 / 10 / 99$ & 1.5 & $\begin{array}{l}\text { WTU system down to dissolve/remove plating material from } \\
\text { process piping/tanks. }\end{array}$ \\
\hline $4 / 11 / 99$ & 0.0 & $\begin{array}{l}\text { WTU system down to replace resin in the ion exchange } \\
\text { columns and roughing filters. }\end{array}$ \\
\hline $4 / 12 / 99$ & 14.75 & $\begin{array}{l}\text { WTU system down to replace resin in the ion exchange } \\
\text { columns and roughing filters. }\end{array}$ \\
\hline $\begin{array}{l}4 / 13 \text { to } \\
4 / 14 / 99\end{array}$ & 48.0 & WTU system operated for 24 hours. \\
\hline $4 / 15 / 99$ & 19.0 & $\begin{array}{l}\text { WTU system down to remove resin from process piping and } \\
\text { repair roughing filter lateral; system down for } 3 \text { hours due to } \\
\text { a tornado shelter alarm resulting in personnel evacuation to } \\
724-7 \mathrm{E} \text {. }\end{array}$ \\
\hline $4 / 16 / 99$ & 18.5 & $\begin{array}{l}\text { WTU system down to clean clarifier/flocculation system } \\
\text { components following an upset condition stemming from the } \\
\text { shutdown of the system for the tornado shelter alarm. }\end{array}$ \\
\hline $4 / 17 / 99$ & 22.0 & WTU system down to repair $\mathrm{NaOH}$ injection system. \\
\hline $4 / 18 / 99$ & 15.5 & WTU system down to repair $\mathrm{NaOH}$ injection system. \\
\hline $4 / 19 / 99$ & 24.0 & WTU system operated for 24 hours. \\
\hline $4 / 20 / 99$ & 21.5 & $\begin{array}{l}\text { WTU system down due to } \mathrm{FeCl}_{3} \text { injection system pump } \\
\text { rupture disc failure. }\end{array}$ \\
\hline
\end{tabular}




\begin{tabular}{|c|c|c|}
\hline Date & $\begin{array}{r}\text { Hours of } \\
\text { Operation }\end{array}$ & Downtime Explanation \\
\hline $4 / 21 / 99$ & 20.5 & $\begin{array}{l}\text { WTU system down due to repair/replacement of pumps and } \\
\text { motors }\left(\mathrm{FeCl}_{3} \text { injection system). }\right.\end{array}$ \\
\hline $\begin{array}{l}4 / 22 \text { to } \\
4 / 23 / 99\end{array}$ & 48.0 & WTU operated for 24 hours. \\
\hline $4 / 24 / 99$ & 22.0 & $\begin{array}{l}\text { WTU system down to evacuate the areas due to tornado } \\
\text { warning. }\end{array}$ \\
\hline $\begin{array}{l}4 / 25 \text { to } \\
4 / 27 / 99\end{array}$ & 72.0 & WTU operated for 24 hours. \\
\hline $4 / 28 / 99$ & 16.0 & WTU system down to clean clarifier/flocculation system. \\
\hline $4 / 29 / 99$ & 10.0 & WTU system down to clean clarifier/flocculation system. \\
\hline $4 / 30 / 99$ & 19.0 & $\begin{array}{l}\text { WTU system down for Polyblend injection sysem } \\
\text { maintenance. }\end{array}$ \\
\hline April Totals & 464.25 & \\
\hline $5 / 1$ to $5 / 2 / 99$ & 48.0 & WTU operated for 24 hours. \\
\hline $5 / 3 / 99$ & 21.5 & $\begin{array}{l}\text { WTU down to clean clarifier/flocculation system } \\
\text { components. }\end{array}$ \\
\hline $5 / 4 / 99$ & 12.25 & WTU down to repair polymer injection system. \\
\hline $5 / 5 / 99$ & 20.25 & WTU down to repair polymer injection system. \\
\hline $5 / 6 / 99$ & 16.5 & WTU down due to roughing filter A lateral screen failure. \\
\hline $\begin{array}{l}5 / 7 \text { to } \\
5 / 10 / 99\end{array}$ & 96.0 & WTU operated for 24 hours. \\
\hline $5 / 11 / 99$ & 11.5 & WTU down to repair polymer injection system. \\
\hline $5 / 12 / 99$ & 12.25 & WTU down to repair polymer injection system. \\
\hline 5/13/99 & 18.5 & WTU down to repair polymer injection system. \\
\hline $\begin{array}{l}5 / 14 \text { to } \\
5 / 16 / 99\end{array}$ & 72.0 & WTU system operated for 24 hours. \\
\hline $5 / 17 / 99$ & 9.5 & $\begin{array}{l}\text { WTU system down to clean clarifier/flocculation system } \\
\text { components and resin changeout. }\end{array}$ \\
\hline $5 / 18 / 99$ & 0.0 & $\begin{array}{l}\text { WTU system down to clean clarifier/flocculation system } \\
\text { components and resin changeout. }\end{array}$ \\
\hline $5 / 19 / 99$ & 7.0 & WTU system down to repair small filter press components. \\
\hline $5 / 20 / 99$ & 12.0 & $\begin{array}{l}\text { WTU system down to clean clarifier/flocculation system } \\
\text { components. }\end{array}$ \\
\hline $\begin{array}{l}5 / 21 \text { to } \\
5 / 24 / 99\end{array}$ & 96.0 & WTU operated for 24 hours. \\
\hline $5 / 25 / 99$ & 12.0 & $\begin{array}{l}\text { WTU system down to clean clarifier/flocculation system } \\
\text { components. }\end{array}$ \\
\hline
\end{tabular}




\begin{tabular}{|l|r|l|}
\hline Date & $\begin{array}{r}\text { Hours of } \\
\text { Operation }\end{array}$ & Downtime Explanation \\
\hline $5 / 26 / 99$ & 0.0 & $\begin{array}{l}\text { WTU system down to clean clarifier/flocculation system } \\
\text { components. }\end{array}$ \\
\hline $\begin{array}{l}5 / 27 \text { to } \\
5 / 28 / 99\end{array}$ & 0.0 & $\begin{array}{l}\text { WTU system down due to replacement of large filter press } \\
\text { screens. }\end{array}$ \\
\hline $\begin{array}{l}5 / 29 \text { to } \\
5 / 31 / 99\end{array}$ & 0.0 & $\begin{array}{l}\text { WTU system down to install inline polymer mixing vessel } \\
\text { and develop/approve test plan. }\end{array}$ \\
\hline May Totals & $\mathbf{4 6 5 . 2 5}$ & \\
\hline $6 / 1 / 99$ & 9.0 & $\begin{array}{l}\text { WTU system down due to poor flocculation quality and to } \\
\text { reassess the test plan path forward. Resin in roughing filters } \\
\text { C\&D was changed out. }\end{array}$ \\
\hline $6 / 2 / 99$ & 5.0 & $\begin{array}{l}\text { WTU system down due to poor flocculation quality and to } \\
\text { reassess the test plan path forward. }\end{array}$ \\
\hline $6 / 3 / 99$ & 13.33 & $\begin{array}{l}\text { WTU system down to install the split caustic/polymer } \\
\text { addition temporary modification. }\end{array}$ \\
\hline $6 / 4$ to $6 / 6 / 99$ & 72.0 & WTU operated for 24 hours. \\
\hline $6 / 7 / 99$ & 7.5 & $\begin{array}{l}\text { WTU system down for scheduled outage. Roughing filters } \\
\text { A\&B, C\&D were deinventoried. Preservative was added to } \\
\text { RO trains B and C, and the flocculation tank and clarifier } \\
\text { were desludged. }\end{array}$ \\
\hline $\begin{array}{l}\text { 6/8 to } \\
6 / 30 / 99\end{array}$ & $\mathbf{1 0 6 . 8 3}$ & $\begin{array}{l}\text { WTU down for scheduled outage to install RO pump } \\
\text { vibration modifications, Cuno filter addition, roughing filter } \\
\text { relocation, chemical addition modifications, PLC upgrades, } \\
\text { piping modifications, and RO pump rebuilding. }\end{array}$ \\
\hline $\begin{array}{l}\text { January to } \\
\text { June, 1999 }\end{array}$ & $\mathbf{1 5 4 8 . 8 3}$ & \\
\hline
\end{tabular}




\section{General Separations Area Maps For the F- And H-Area Hazardous Waste Management Facilities Semiannual Corrective Action Reports (U)}

Document Reference Numbers:

WSRC-TR-98-00147 F-Area HWMF

WSRC-TR-98-00148 H-Area HWMF

First and Second Quarter 1999

Volume II

Publication Date: October 1999

Westinghouse Savannah River Company, LLC

Savannah River Site

Aiken, SC 29808

Prepared for the U.S. Department of Energy under

Control Contract No. DE-AC09-96SR18500 


\section{DISCLAIMER}

This report was prepared as an account of work sponsored by an agency of the United States Government. Neither the United States Government nor any agency thereof, nor any of their employees, makes any warranty, express or implied, or assumes any legal liability or responsibility for the accuracy, completeness, or usefulness of any information, apparatus, product, or process disclosed, or represents that its use would not infringe privately owned rights. Reference herein to any specific commercial product, process, or service by trade name, trademark, manufacturer, or otherwise does not necessarily constitute or imply its endorsement, recommendation, or favoring by the United States Government or any agency thereof. The views and opinions of authors expressed herein do not necessarily state or reflect those of the United States Government or any agency thereof.

This report has been reproduced directly from the best available copy.

Available to DOE and DOE contractors from the Office of Scientific and Technical Information, P.O. Box 62, Oak Ridge, TN 37831; prices available from (615) 576-8401.

Available to the public from the National Technical Information Service, U.S. Department of Commerce; 5285 Port Royal Road, Springfield, VA 22161.

\begin{tabular}{|c|}
\hline $\begin{array}{c}\text { Printed in the United States of America } \\
\text { Prepared for } \\
\text { U. S. Department of Energy } \\
\text { and }\end{array}$ \\
Westinghouse Savannah River Company, LLC \\
\end{tabular}




\section{Contents}

\section{Volume II}

\section{Appendix H - General Separations Area Maps for the F- and H-Area Hazardous Waste Management Facilities Semiannual Corrective Action Reports}

1. Cadmium Concentrations at the General Separations Area, Second Quarter 1999 H-3

2. Gross Alpha Activities at the General Separations Area, Second Quarter 1999. H-4

3. Iodine-129 Activities at the General Separations Area, First Quarter 1999 $\mathrm{H}-5$

4. Lead Concentrations at the General Separations Area, Second Quarter 1999. H-6

5. Mercury Concentrations at the General Separations Area, Second Quarter 1999 $\mathrm{H}-7$

6. Nitrate Concentrations at the General Separations Area, Second Quarter 1999. $\mathrm{H}-8$

7. Nonvolatile Beta Activities at the General Separations Area, Second Quarter 1999

8. Strontium-90 Activities at the General Separations Area, First Quarter 1999 $\mathrm{H}-10$

9. Technetium-99 Activities at the General Separations Area, First Quarter 1999

10. Tritium Activities at the General Separations Area, Second Quarter 1999.

11. Uranium-233/234 Activities at the General Separations Area, First Quarter 1999.

12. Uranium-238 Activities at the General Separations Area, First Quarter 1999.

13. Potentiometric Surface Map of the UAZ of the UTRA at the General Separations Area, First Quarter 1999

14. Potentiometric Surface Map of the LAZ of the UTRA at the General Separations Area, First Quarter 1999

15. Potentiometric Surface Map of the GA at the General Separations Area, First Quarter 1999... H-17

16. Potentiometric Surface Map of the UAZ of the UTRA at the General Separations Area, Second Quarter 1999.

17. Potentiometric Surface Map of the LAZ of the UTRA at the General Separations Area, Second Quarter 1999.

18. Potentiometric Surface Map of the GA at the General Separations Area, Second Quarter 1999. 
WSRC-TR-99-00147

WSRC-TR-00148

Unclassified

THIS PAGE WAS LEFT BLANK INTENTIONALLY 
APPENDIX H: GENERAL SEPARATIONS AREA MAPS FOR THE F- AND H-AREA HAZARDOUS WASTE MANAGEMENT FACILITIES SEMIANNUAL CORRECTIVE ACTION REPORTS 
THIS PAGE WAS LEFT BLANK NTENTIONALLY. 


\section{Notice}

Page(s) size did not permit electronic reproduction. Information may be purchased by the general public from the National Technical Information Service, U.S. Department of Commerce, Springfield, VA 22161 (1800-553-6847). DOE and DOE contractors may purchase information by contacting DOE's Office of Scientific and Technical Information, P.O. Box 62, Oak Ridge, TN 37831-0062, Attn: Information Services (1865-576-8401). 